\title{
STREAMFLOW STATISTICS OF SELECTED STREAMS IN THE LOWER RED RIVER OF THE NORTH BASIN, NORTH DAKOTA, MINNESOTA, AND MANITOBA
}

By Tara Williams-Sether and Gregg J. Wiche

U.S. GEOLOGICAL SURVEY

Open-File Report 98-21

Prepared in cooperation with the

RED RIVER WATERSHED MANAGEMENT BOARD and the RED RIVER JOINT WATER RESOURCE BOARD 


\title{
U.S. DEPARTMENT OF THE INTERIOR BRUCE BABBITT, Secretary
}

\author{
U.S. GEOLOGICAL SURVEY \\ THOMAS J. CASADEVALL, Acting Director
}

Any use of trade, product, or firm names is for descriptive purposes only and does not imply endorsement by the U.S. Government.

For additional information write to:

\section{District Chief}

U.S. Geological Survey Water Resources Division 821 East Interstate Avenue Bismarck, ND 58501-1199
Copies of this report can be purchased from:

U.S. Geological Survey Branch of Information Services Box 25286

Denver, CO 80225-0286 


\section{CONTENTS}

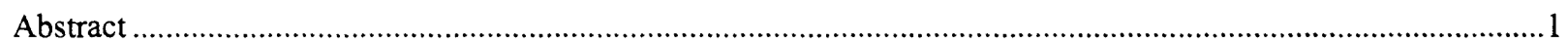

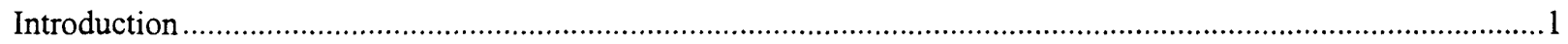

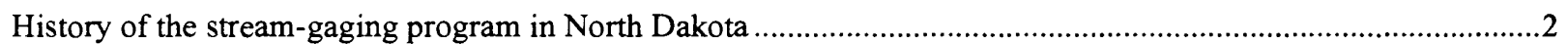

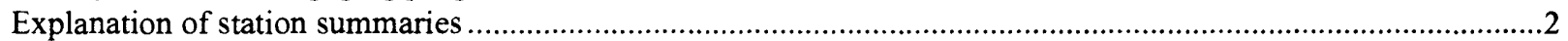

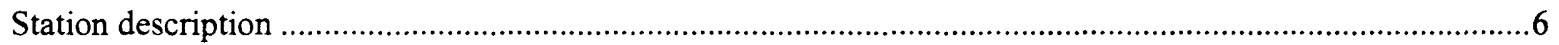

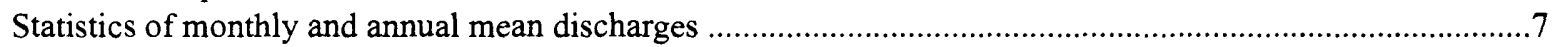

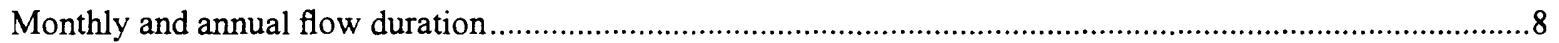

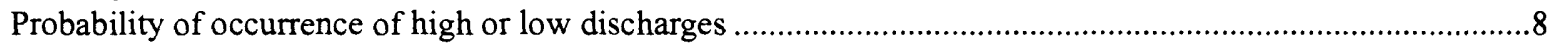

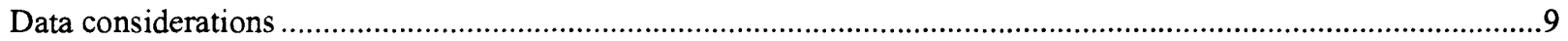

Period of record

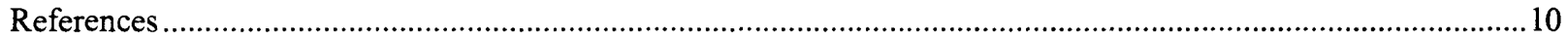

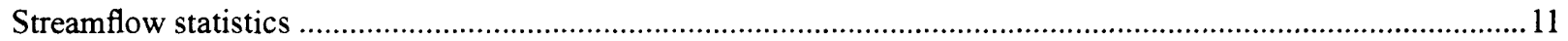

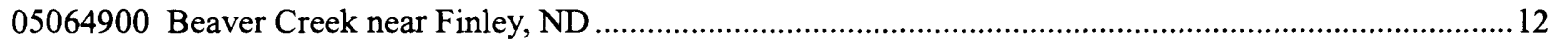

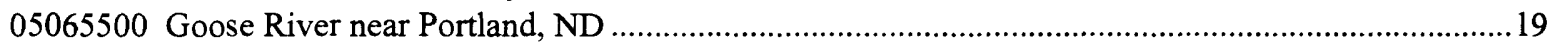

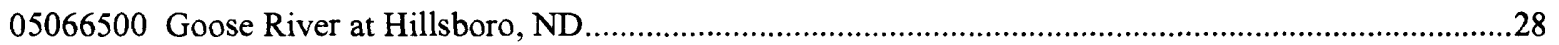

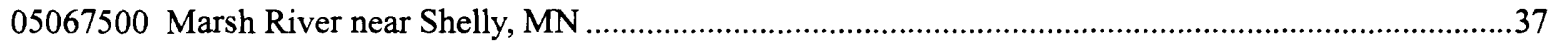

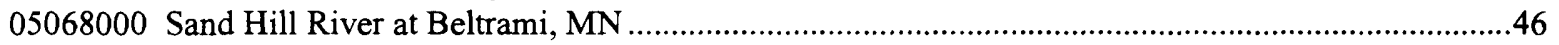

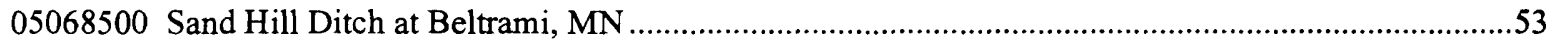

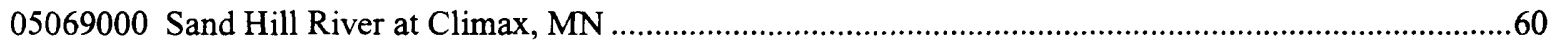

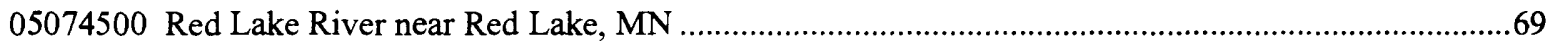

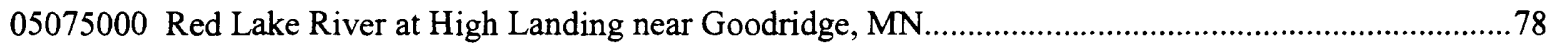

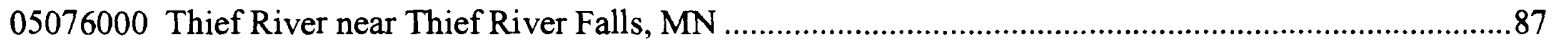

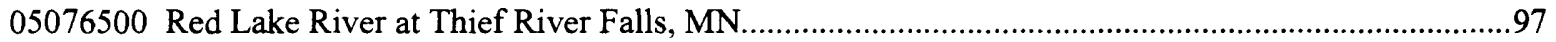

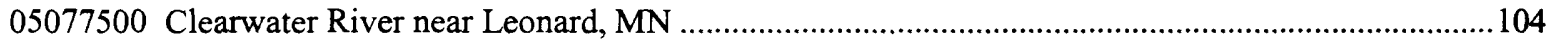

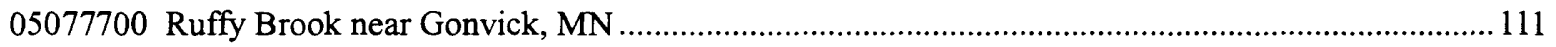

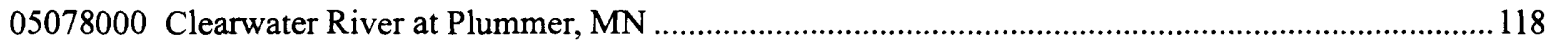

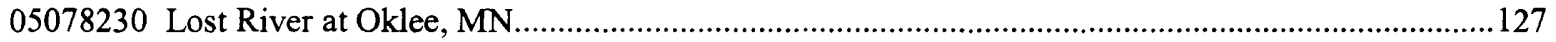

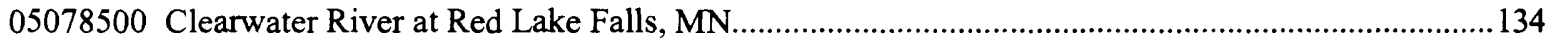

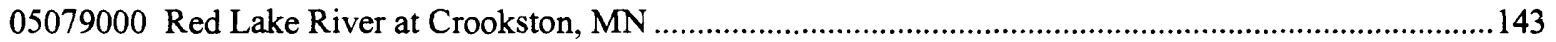

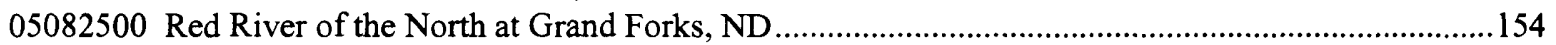

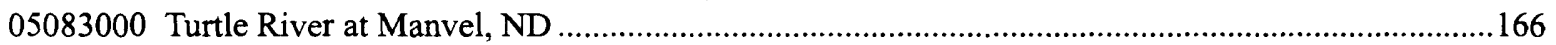

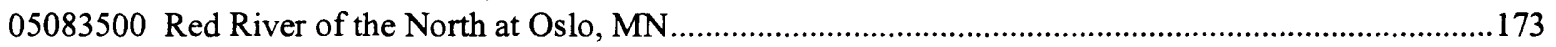

05083600 Middle Branch Forest River near Whitman, ND ...................................................................180

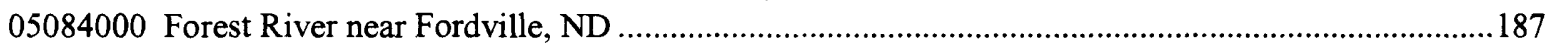

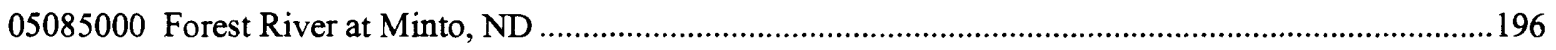

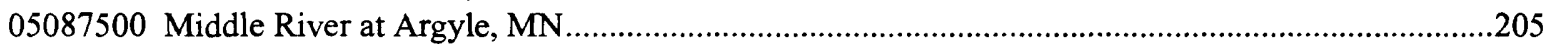

05088000 South Branch Park River near Park River, ND.........................................................................214

05089000 South Branch Park River below Homme Dam, ND ................................................................221

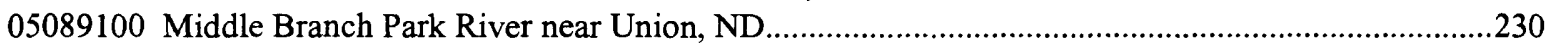

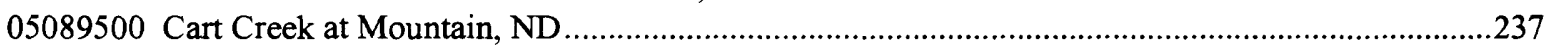

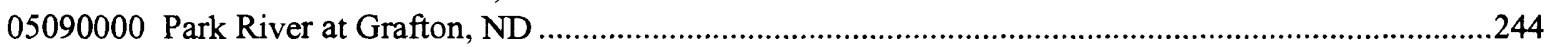

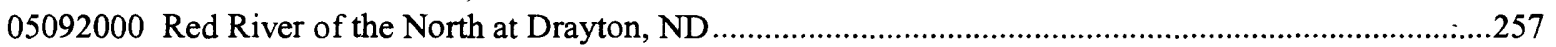

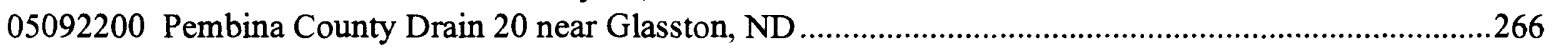

05093000 South Branch Two Rivers at Pelan, MN .................................................................................273

05094000 South Branch Two Rivers at Lake Bronson, MN .....................................................................28

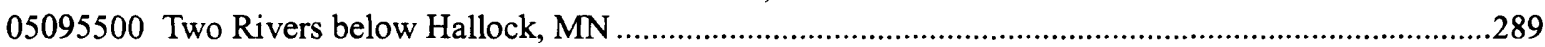

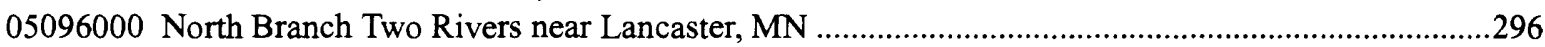

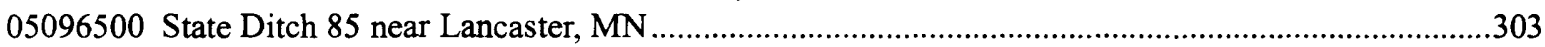

05098700 Hidden Island Coulee near Hansboro, ND ..............................................................................310

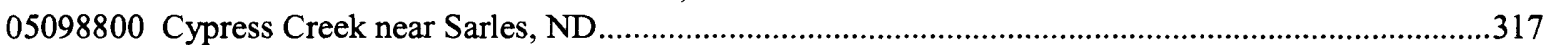

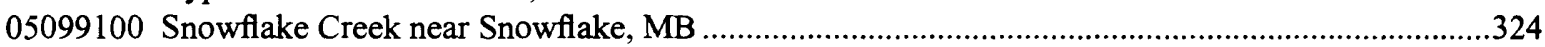

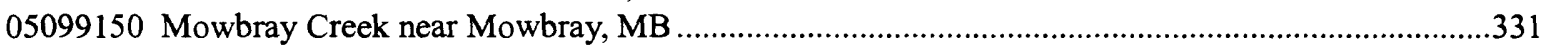




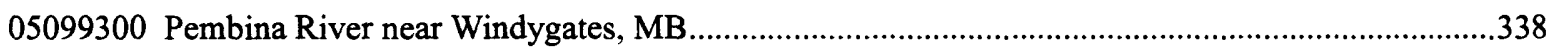

05099400 Little South Pembina River near Walhalla, ND ..........................................................................345

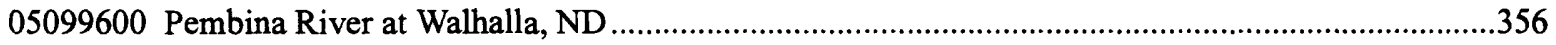

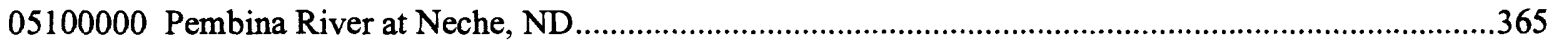

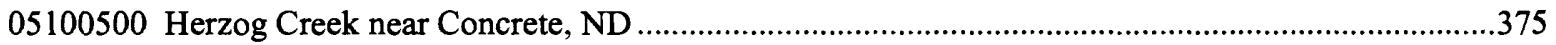

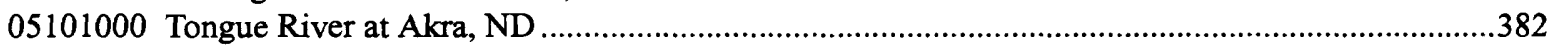

05101500 Tongue River at Cavalier, ND ..............................................................................................391

05102500 Red River Of The North at Emerson, MB ...............................................................................398

\section{FIGURES}

1. Map showing location of streamflow-gaging stations in the lower Red River of the North Basin for which streamflow statistics are published in this report .4

2. Graph showing number of streamflow-gaging stations in North Dakota, 1901-94.

\section{TABLES}

1. List of streamflow-gaging stations in the lower Red River of the North Basin for which streamflow statistics are published in this report. 


\section{DEFINITION OF TERMS}

Climatic year is the 12-month period April 1 through March 31 . The climatic year is designated by the calendar year in which it begins.

Cubic foot per second $\left(\mathrm{ft}^{3} / \mathrm{s}\right)$ is the rate of discharge representing a volume of 1 cubic foot passing a given point during 1 second and is equivalent to 7.48 gallons per second or 448.8 gallons per minute or 0.02832 cubic meters per second.

Discharge is the volume of water in the "natural" channel of a stream that passes a given point within a given period of time. Discharge often is used interchangeably with the term "streamflow".

Drainage area (of a stream at a streamflow-gaging station) is the area, measured in a horizontal plane, enclosed by a topographic divide from which direct surface runoff from precipitation normally drains by gravity into the stream upstream from the station.

Drainage basin is a part of the surface of the Earth that is occupied by a drainage system, which consists of a surface stream or a body of impounded surface water together with all tributary surface streams and bodies of impounded surface water.

Gage height is the water-surface elevation referred to some arbitrary gage datum. Gage height often is used interchangeably with the more general term "stage", although gage height is more appropriate when used with a reading on a gage.

Gaging station is a particular site on a stream, canal, lake, or reservoir where systematic observations of hydrologic data are obtained.

Ground water is the water in the ground that is in the zone of saturation, from which wells, springs, and ground-water runoff are supplied.

Hydrologic unit is a geographic area representing part or all of a surface drainage basin or distinct hydrologic feature as delineated by the Office of Water Data Coordination on the State Hydrologic Unit Maps; each hydrologic unit is identified by an eight-digit number.

Instantaneous discharge is the discharge at a particular instant of time.

Mean is the arithmetic average of a list of values.

Mean discharge is the arithmetic mean of individual discharges during a specific period.

Partial-record station is a particular site where limited streamflow data are collected systematically over a period of years.

Period of record is the time during which a stream-gaging station is in operation and for which the records are published.

Probability of occurrence is the likelihood that an event will occur. Probabilities are generally expressed as a decimal number between 0 and 1 . If the probability is 0 , the event will not occur; if the probability is 1 , the event will occur absolutely. Probability also can be expressed as a percent, where 0 percent corresponds to 0 probability and 100 percent corresponds to a probability of 1 . 
Recurrence interval is the average time interval between occurrences of a hydrologic event of a given or greater magnitude, usually expressed in years.

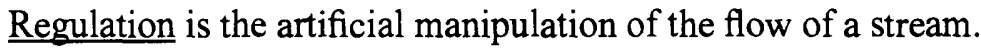

Stage see "gage height"

Standard deviation is a measure of the variability of the values in a list of values.

Stream-gaging station is a gaging station where a record of discharge of a stream is obtained.

Streamflow see "discharge"

Surface water is the water on the surface of the earth.

Water year is the 12-month period October 1 through September 30. The water year is designated by the calendar year in which it ends and which includes 9 of the 12 months. 


\title{
STREAMFLOW STATISTICS OF SELECTED STREAMS IN THE LOWER RED RIVER OF THE NORTH BASIN, NORTH DAKOTA, MINNESOTA, AND MANITOBA
}

\author{
By Tara Williams-Sether and Gregg J. Wiche
}

\section{ABSTRACT}

Statistical summaries of streamflow data through water year 1994 for selected active and discontinued U.S. Geological Survey gaging stations for the Red River of the North Basin downstream of Halstad, Minnesota, to and including Emerson, Manitoba, are presented in this report. The summaries for each streamflow-gaging station include (1) station description, (2) graph of the annual mean discharge for the period of record, (3) statistics of monthly and annual mean discharges, (4) graph of the annual flow duration, (5) monthly and annual flow duration, (6) probability of occurrence of annual high discharges, (7) probability of occurrence of annual low discharges, (8) probability of occurrence of seasonal low discharges, (9) annual peak discharge and corresponding gage height for the period of record, and (10) monthly and annual mean discharges for the period of record.

\section{INTRODUCTION}

A part of the mission of the United States Geological Survey is the collection of systematic data to determine the quantity, quality, and use of surface and ground water. A total of 7,292 streamflow-gaging stations (as of 1994) were operated by the U. S. Geological Survey in the United States, Puerto Rico, and the Trust Territories of the Pacific Islands (Wahl and others, 1995). Of the 7,292 streamflow-gaging stations, 60 were operated in the Red River of the North Basin upstream of Emerson, Manitoba, excluding the Devils Lake Basin.

At streamflow-gaging stations, the water level in the river is monitored continually. A relation between water level and discharge is developed by making periodic discharge measurements throughout the range in water level. This relation is referred to as a station rating. A continuous record of streamflow is computed for each gaging station by using the water level record and the station rating.

Knowledge of the magnitude and time distribution of streamflow is essential for all aspects of water management and environmental planning. Federal, State, and local agencies responsible for the development and management of North Dakota's surface-water resources use this knowledge for making safe, economical, and environmentally sound water-resource planning decisions.

Streamflow statistics published in annual state water reports by the U. S. Geological Survey include records of daily mean discharge, annual high and low discharge, and annual mean discharge. Other statistics can be retrieved from U.S. Geological Survey computer files. Water resource managers may go to various sources to obtain the necessary statistics. These sources may only include active gaging stations listed in the most recent annual report and, thus, overlook information available for many discontinued gaging stations. 
The purpose of this report is to provide a comprehensive publication summarizing streamflow statistics through water year 1994 for selected active and discontinued gaging stations for the Red River of the North Basin downstream of Halstad, Minnesota, to and including Emerson, Manitoba. Active and discontinued gaging stations listed in this report have a least 10 years of record. These stations are listed in table 1 and their locations are shown in figure 1.

\section{HISTORY OF THE STREAM-GAGING PROGRAM IN NORTH DAKOTA}

Much of the history of the stream-gaging program in North Dakota outlined in this report was written by Crosby (1970). However, the number of streamflow-gaging stations given in figure 2 may differ from the number given by Crosby (1970) because the type of gaging stations included may differ. The collection of systematic streamflow data began in 1882 when a gaging station was established on the Red River of the North at Grand Forks. This gaging station was a stage station; however, infrequent discharge measurements were made for navigational purposes. Stage data were obtained on the Missouri River at Bismarck in 1881-82 and in 1886-89 by the Missouri River Commission. As result of the National Reclamation Act of 1902 and the disastrous flood in 1897 in the Red River of the North Basin, the U.S. Geological Survey, in cooperation with the state of North Dakota, established and operated streamflowgaging stations from 1901-09 (fig. 2). Additional interest was created as problems with Canada concerning the division of waters along the international boundary resulted in the formation of the International Joint Commission in 1912. Eight streamflow-gaging stations were in operation in 1925 when State cooperation was discontinued (fig. 2). Only five federally operated gaging stations were continued. State cooperation resumed in 1931, but funds were limited from 1934-38. However, the Rivers and Harbors Act of 1927 and the Flood Control Acts of 1928 and 1936 resulted in the U.S. Army Corps of Engineers supporting a large expansion of the stream-gaging program. Forty-one gaging stations were in operation when the North Dakota-South Dakota U.S. Geological Survey Office was created on October 16, 1944. Plans for the coordinated development of the waters of the Missouri River Basin, with respect to flood control, navigation, power, and irrigation, were formulated in 1943-44 by the U.S. Army Corps of Engineers, the Bureau of Reclamation, and the States in the Basin. These plans resulted in a rapid increase in the stream-gaging program, and, by 1947, 64 gaging stations were in operation in North Dakota. The number of gaging stations grew steadily from the late 1940's until the late 1960's, and, by 1969, 109 gaging stations were in operation.

During 1969-76, the number of gaging stations in operation remained relatively stable. During the 1970 's, the U.S. Geological Survey established 25 additional gaging stations to monitor the quantity and quality of streamflow in drainage basins underlain by strippable lignite deposits (Haffield, 1981). By 1979, about 145 gaging stations were in operation in North Dakota. During 1981-83, the number of gaging stations in operation declined rapidly, and, during 1984-87, the number declined slowly to about 110. Since 1987, the number of gaging stations in operation has been relatively stable at about 105 to 110 .

\section{EXPLANATION OF STATION SUMMARIES}

Station summaries are presented so that each station description and tables of streamflow statistics and probabilities of occurrence are presented in the same order and format for each gaging station, including the same relative placement of the pages. Because the information and statistics in the tables were created by "data retrievals" or statistical program results, significant figures were not rounded to U.S. Geological Survey standards. The order of presentation is as follows:

1. station description,

2. graph of the annual mean discharge for the period of record,

3. table of statistics of monthly and annual mean discharges, 
Table 1. List of streamflow-gaging stations in the lower Red River of the North Basin for which streamflow statistics are published in this report

[ND, North Dakota; MN, Minnesota; MB, Manitoba]

\begin{tabular}{|c|c|}
\hline $\begin{array}{l}\text { Station } \\
\text { number }\end{array}$ & Station name \\
\hline 05064900 & Beaver Creek near Finley, ND \\
\hline 05065500 & Goose River near Portland, ND \\
\hline 05066500 & Goose River at Hillsboro, ND \\
\hline 05067500 & Marsh River near Shelly, MN \\
\hline 05068000 & Sand Hill River at Beltrami, MN \\
\hline 05068500 & Sand Hill Ditch at Beltrami, MN \\
\hline 05069000 & Sand Hill River at Climax, MN \\
\hline 05074500 & Red Lake River near Red Lake, MN \\
\hline 05075000 & Red Lake River at Highlanding near Goodridge, MN \\
\hline 05076000 & Thief River near Thief River Falls, MN \\
\hline 05076500 & Thief River at Thief River Falls, MN \\
\hline 05077500 & Clearwater River near Leonard, MN \\
\hline 05077700 & Ruffy Brook near Gonvick, MN \\
\hline 05078000 & Clearwater River at Plummer, MN \\
\hline 05078230 & Lost River at Oklee, MN \\
\hline 05078500 & Clearwater River at Red Lake Falls, MN \\
\hline 05079000 & Red Lake River at Crookston, MN \\
\hline 05082500 & Red River of the North at Grand Forks, ND \\
\hline 05083000 & Turtle River at Manvel, ND \\
\hline 05083500 & Red River of the North at Oslo, MN \\
\hline 05083600 & Middle Branch Forest River near Whitman, ND \\
\hline 05084000 & Forest River near Fordville, ND \\
\hline 05085000 & Forest River at Minto, ND \\
\hline 05087500 & Middle River at Argyle, MN \\
\hline 05088000 & South Branch Park River near Park River, ND \\
\hline 05089000 & South Branch Park River below Homme Dam, ND \\
\hline 05089100 & Middle Branch Park River near Union, ND \\
\hline 05089500 & Cart Creek at Mountain, ND \\
\hline 05090000 & Park River at Grafton, ND \\
\hline 05092000 & Red River of the North at Drayton, ND \\
\hline 05092200 & Pembina County Drain 20 near Glasston, ND \\
\hline 05093000 & South Branch Two Rivers at Pelon, MN \\
\hline 05094000 & South Branch Two Rivers at Lake Bronson, MN \\
\hline 05095500 & Two Rivers below Hallock, MN \\
\hline 05096000 & North Branch Two Rivers near Lancaster, MN \\
\hline 05096500 & State Ditch 85 near Lancaster, MN \\
\hline 05098700 & Hidden Island Coulee near Hansboro, ND \\
\hline 05098800 & Cypress Creek near Sarles, ND \\
\hline 05099100 & Snowflake Creek near Snowflake, MB \\
\hline 05099150 & Mowbray Creek near Mowbray, MB \\
\hline 05099300 & Pembina River near Windygates, MB \\
\hline 05099400 & Little South Pembina River near Walhalla, ND \\
\hline 05099600 & Pembina River at Walhalla, ND \\
\hline 05100000 & Pembina River at Neche, ND \\
\hline 05100500 & Herzog Creek near Concrete, ND \\
\hline 05101000 & Tongue River at Akra, ND \\
\hline 05101500 & Tongue River at Cavalier, ND \\
\hline 05102500 & Red River of the North at Emerson, MB \\
\hline
\end{tabular}




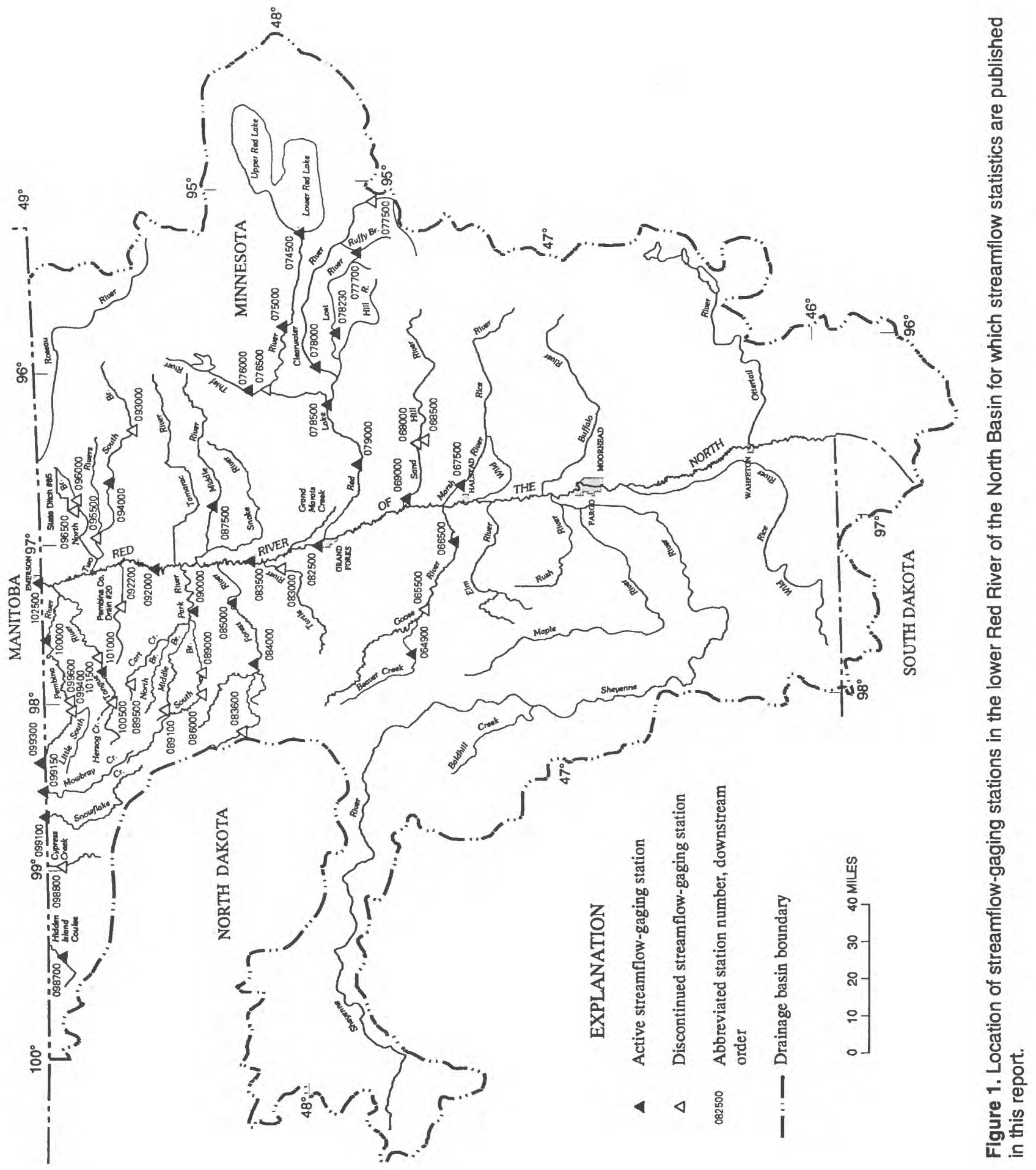




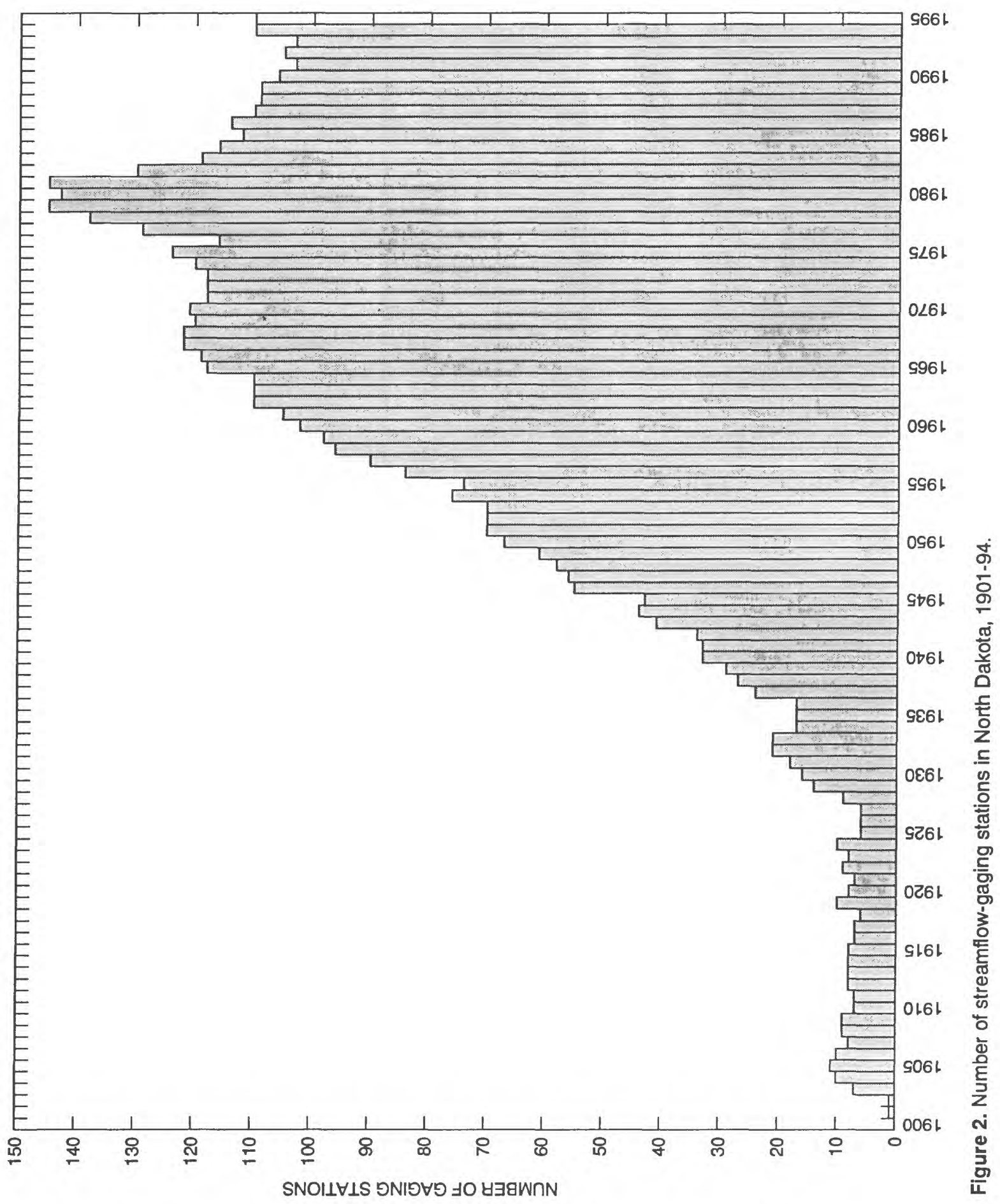


4. graph of the annual flow duration,

5. table of monthly and annual flow duration,

6. table of probability of occurrence of annual high discharges,

7. table of probability of occurrence of annual low discharges,

8. table of probability of occurrence of seasonal low discharges,

9. table of annual peak discharge and corresponding gage height for the period of record, and

10. table of monthly and annual mean discharges for the period of record.

Where both pre-regulation and post-regulation statistics are presented for a gaging station, the station description, graph of annual mean discharges, table of annual peak discharges and corresponding gage heights, and table of monthly and annual mean discharges are presented with the pre- and post-regulation data. The respective tables for the pre- and post-regulation data are presented in the same relative page format as non-regulated streams.

\section{Station Description}

The location, drainage area, period of record, and other information about each streamflow-gaging station are included in the station description. This information is compiled from records maintained by the U.S. Geological Survey and generally is presented in the same format as published in the annual state water report. The following comments clarify information presented under the various headings of the station description.

LOCATION.--Information on gaging station location is obtained from the most accurate maps available and is furnished with respect to cultural and physical features in the vicinity of the gaging station and the community or landmark included in the gaging station name. In the case of discontinued gaging stations, the location is furnished using features in the vicinity at the time the gaging station was in operation. In many instances, the identifying features have been altered since the gaging station was discontinued.

DRAINAGE AREA.--Drainage area is measured using U.S. Geological Survey 7.5-minute topographic quadrangle maps. However, 7.5-minute topographic maps for drainage area computations were not available when some gaging stations were installed; therefore, the accuracy of drainage areas also varies. Drainage areas of discontinued gaging stations are those determined while the gaging station was in operation.

PERIOD OF RECORD.--The period of record is the period for which there are published records for the gaging station or for an equivalent gaging station. An equivalent gaging station is a gaging station that was in operation in a different location prior to the subject gaging station, and whose location is such that records from it can reasonably be considered equivalent with records from the subject gaging station. This situation arises when a gaging station is relocated upstream or downstream and given a new gaging station number and name, but the changes in drainage area and other basin characteristics are not significantly different. Period of record to current year indicates that the station was in operation as of September 30 , 1994.

GAGE.--The type of gage or recorder that is or was used to collect data, the datum of the gage referred to sea level, and a condensed history of the types, locations, and datums of previous gages are given under this heading. 
EXTREMES FOR PERIOD OF RECORD.--Extremes may include maximum and minimum discharges and maximum and minimum gage heights. Unless otherwise qualified, the maximum discharge is the instantaneous maximum discharge corresponding to the highest gage height that occurred. If the maximum gage height did not occur on the same day as the maximum discharge, it is listed separately. Similarly, the minimum discharge is the instantaneous minimum discharge corresponding to the lowest gage height that occurred, unless qualified and listed otherwise.

EXTREMES OUTSIDE PERIOD OF RECORD.--Included is any information available concerning major floods or unusually low flows that occurred outside the stated period of record. The information may not have been obtained by the U.S. Geological Survey.

\section{Statistics of Monthly and Annual Mean Discharges}

Statistics of monthly and annual mean discharges presented for each gaging station include (1) the maximum, minimum, and mean monthly discharges and (2) the maximum, minimum, and mean annual discharges. The water years (October 1 through September 30) in which the maximum and minimum discharges occurred are listed with the respective values, and the standard deviation and coefficient of variation of the monthly and annual mean discharges are listed with the respective values. Also, the percentage of the annual discharge that is comprised by each monthly mean discharge is listed in the table.

Each of the statistics is explained in the following paragraphs. As an aid to the readers' understanding of how the monthly mean and annual mean discharges are determined, data for the gaging station Red Lake River at Crookston, MN (05079000, p. 143-153) are used as an example. The monthly mean value is the average of the daily values for the month. The annual mean value is the average of the daily values for the year. Months or years for which all daily values are not available are not included in the compilation of statistics.

The maximum monthly mean discharge is the maximum value of all the monthly mean values. The maximum mean value for October is 2,840 cubic feet per second $\left(\mathrm{ft}^{3} / \mathrm{s}\right)$, which occurred during water year 1972. Similarly, the minimum monthly mean discharge is the minimum value of all the monthly mean values. The minimum mean value for October is $8.02 \mathrm{ft}^{3} / \mathrm{s}$, which occurred during water year 1937. The maximum and minimum monthly mean values can be found in the statistics of monthly and annual mean discharges table or by searching the monthly and annual mean discharges table.

The mean monthly discharge is the mean of all the monthly mean discharges for a given month, and the standard deviation is a measure of the variability of the values. The mean monthly discharge for October is $805 \mathrm{ft}^{3} / \mathrm{s}$, and the standard deviation is $689 \mathrm{ft}^{3} / \mathrm{s}$. The monthly mean discharge for October (mean of the mean monthly values) is the same as the mean of all October daily values for the period of record used. However, the standard deviation is smaller than the standard deviation obtained using all daily values. The standard deviation is smaller because the monthly values have less variability than the daily values.

The coefficient of variation is the ratio of the standard deviation to the mean. The coefficient of variation is dimensionless. Because monthly mean discharges are generally much greater in spring than in winter, the standard deviations also are generally much greater in spring than in winter. However, dividing the standard deviation by the mean monthly discharge tends to equalize the measures for all months so a more meaningful comparison among months can be made. 
The percentage of the annual discharge is the percent of the annual discharge that occurred during each month. It is calculated by dividing the mean discharge for the month by the total of the 12 monthly mean discharges and multiplying by 100 . Because of rounding of the monthly percentage, the sum of the 12 percentages may not equal 100 percent.

The maximum, minimum, and mean annual discharges are selected or computed from the annual mean discharges for the period of record. The water years of occurrence of the maximum and minimum values are listed with the respective values, and the standard deviation of the mean of the annual mean values is listed with the mean value. The minimum annual mean discharge of $132 \mathrm{ft}^{3} / \mathrm{s}$ occurred in 1935 , and the maximum annual mean discharge of $3,130 \mathrm{ft}^{3} / \mathrm{s}$ occurred in 1950 . The mean annual discharge for the period of record is $1,110 \mathrm{ft}^{3} / \mathrm{s}$.

\section{Monthly and Annual Flow Duration}

The monthly and annual flow duration table is a magnitude and frequency analysis of daily discharge values. It is computed by tabulating the number of daily discharge values that fall within preselected class limits, computing the percentage of values within each class, and interpolating discharge values for the percentages shown in the table. Monthly values are calculated from daily values in all complete months in the record, and annual figures are calculated for all complete water years. For example, if the 90 -percent flow duration value for October is $101 \mathrm{ft}^{3} / \mathrm{s}$, then 90 percent of all October daily discharge values for the period of record were equal to or greater than $101 \mathrm{ft}^{3} / \mathrm{s}$.

\section{Probability of Occurrence of High or Low Discharges}

The probabilities of occurrence of annual high discharges, annual low discharges, and seasonal low discharges are presented in three tables for each gaging station. Probability of occurrence is an estimate of the likelihood that a particular discharge in a stream will be equaled or exceeded in 1 year or, in the case of low flows, the likelihood that the discharge will not be equaled or exceeded during the year. The probability of occurrence of a high flow is called the exceedance probability, and the probability of occurrence of low flow is called the nonexceedance probability. For example, if the maximum instantaneous discharge for the 0.20 exceedance probability is listed as $13,100 \mathrm{ft}^{3} / \mathrm{s}$, then a 20 percent chance exists that a discharge equal to or greater than $13,100 \mathrm{ft}^{3} / \mathrm{s}$ will occur once during the year.

Recurrence interval is another way of expressing annual probability and it is the reciprocal of probability of occurrence. The recurrence interval for an exceedance probability of 0.20 is 5 years ( 1 divided by 0.20 ). For a long discharge record the annual maximum discharge can be expected to equal or exceed $13,100 \mathrm{ft}^{3} / \mathrm{s}$ on average once every 5 years.

The table of probability of occurrence of annual high discharges for each gaging station lists the maximum instantaneous discharge and the maximum mean discharge for 3, 7, 15, and 30 consecutive-day periods for selected exceedance probabilities and recurrence intervals. Values for the maximum instantaneous discharge are computed from the streamflow record according to the guidelines established by the Hydrology Subcommittee of the Interagency Advisory Committee on Water Data (1982). According to the guidelines, adjustments are made for length of record and regional skew.

Values for the maximum mean discharges for $3,7,15$, and 30 consecutive-day periods are computed from the annual high mean values of the corresponding periods. The computations are based on the logPearson Type III distribution using values obtained for the water year. 
The table of probability of occurrence of annual low discharges for each gaging station lists the minimum mean discharge for $1,3,7,14,30,60,90,120$, and 183 consecutive-day periods for selected nonexceedance probabilities and recurrence intervals. Values for the minimum mean discharges are computed from the annual low discharge values of the corresponding periods using the log-Pearson Type III distribution. If the log-Pearson Type III distribution curve fails to fit the data at the lower end, a graphical interpretation is made. Probabilities of annual low discharges are computed using values obtained for the climatic year (April 1 through March 31).

The table of probability of occurrence of seasonal low discharges for each gaging station lists the minimum mean discharge for $1,7,14$, and 30 consecutive-day periods for selected probabilities and recurrence intervals. These values are computed from the seasonal low mean values of the corresponding periods using the log-Pearson Type III distribution.

The annual low discharge and the seasonal low discharges that occur in any given year are sensitive to natural-channel processes, such as evapotranspiration and human-induced hydrologic modifications, such as the operation of many small water-storage reservoirs; the effects of surface-water withdrawal for agricultural, municipal, and industrial use; and the effects of return flow to the river. Thus, the statistics in tables are given for recurrence intervals that generally are within twice the period of record.

\section{DATA CONSIDERATIONS}

\section{Period of Record}

The reliability of statistical data is related to the length of record for a stream. The Hydrology Subcommittee of the Interagency Advisory committee on Water Data (1982) recommends that at least 10 years of record be used for computing flood frequency estimates. Therefore, the length of record criterion for inclusion of a gaging station in this report is at least 10 years. Even with this criterion, the lengths and continuity of record for the gaging stations vary substantially. Subsequently, extreme high or low flows may be included in the streamflow record of one gaging station and not in another, resulting in inconsistencies in the streamflow statistics when comparing gaging station data. Also, longer record lengths for many of the gaging stations in this report may result in different streamflow statistics when comparing data in this report with data in previous publications.

Differences in statistical data for pre- and post-regulation periods may not be caused solely by regulation. Differences also can be attributed to the length of record and climatic variability as expressed by hydrologic variability. By comparing a statistic that easily can be affected by regulation, such as the 7-day low flow, and a statistic that generally is unaffected by regulation, such as the mean annual discharge, a determination can be made about the effect of regulation. As an example, the annual 7-day low flow with a 10-year recurrence interval for the Red River of the North at Fargo (Wiche and WilliamsSether, 1997) is $0 \mathrm{ft}^{3} / \mathrm{s}$ for the pre-regulation period (1901-41) and $17.9 \mathrm{ft}^{3} / \mathrm{s}$ for the post-regulation period (1942-94). The effect of regulation on the mean annual discharge of the Red River of the North can be assumed to be negligible; however, the mean annual discharge is $403 \mathrm{ft}^{3} / \mathrm{s}$ for the pre-regulation period and $741 \mathrm{ft}^{3} / \mathrm{s}$ for the post-regulation period. Although annual 7-day low flow for a 10 -year recurrence interval is much greater for the regulation period, the mean annual discharge for the regulated period also is much greater, indicating that regulation may happen to correspond to a relatively wet period in the Red River of the North Basin and may not be the sole factor for differences in statistical data for pre- and post-regulation periods. 


\section{REFERENCES}

Crosby, O.A, 1970, A proposed streamflow data program for North Dakota: U.S. Geological Survey Open-File Report, $68 \mathrm{p}$.

Haffield, N.D., 1981, Statistical summaries of streamflow and water-quality data for streams of western North Dakota, 1977-80: U.S. Geological Survey Open-File Report 81-1066, 78 p.

Hydrology Subcommittee of the Interagency Advisory Committee on Water Data, 1982, Guidelines for determining flood flow frequency: Hydrology Subcommittee Bulletin 17B, 28 p., 14 appendices.

Wahl, K.L., Thomas, W.O., Jr., and Hirsch, R.M., 1995, Stream-gaging program of the U.S. Geological Survey: U.S. Geological Survey Circular 1123, 22 p.

Wiche, G.J. and Williams-Sether T., 1997, Streamflow Characteristics of Streams in the Upper Red River of the North Basin, North Dakota, Minnesota, and South Dakota: U.S. Geological Survey Open-File Report 97-416, $374 \mathrm{p}$. 


\section{STREAMFLOW STATISTICS}




\section{BEAVER CREEK NEAR FINLEY, ND}

LOCATION.--Lat $47^{\circ} 35^{\prime} 40^{\prime \prime}$, long 97\%42'18’”, in NE $1 / 4$ sec.31, T.148 N., R.55 W., Steele County,

Hydrologic Unit 09020109 , on right bank $500 \mathrm{ft}$ upstream from bridge on county highway, and $7 \mathrm{mi}$ northeast of Finley.

DRAINAGE AREA.--160 $\mathrm{mi}^{2}$, approximately.

PERIOD OF RECORD.--October 1964 to current year.

GAGE.--Water-stage recorder and concrete broad-crested weir. Datum of gage is $1,170.08 \mathrm{ft}$ above sea level.

EXTREMES FOR PERIOD OF RECORD.--Maximum discharge, 1,900 $\mathrm{ft}^{3} / \mathrm{s}$, Apr. 19, 1979, gage height, $8.35 \mathrm{ft}$; maximum gage height, $9.70 \mathrm{ft}$, Mar. 14, 1966; no flow at times.

Annual mean discharge

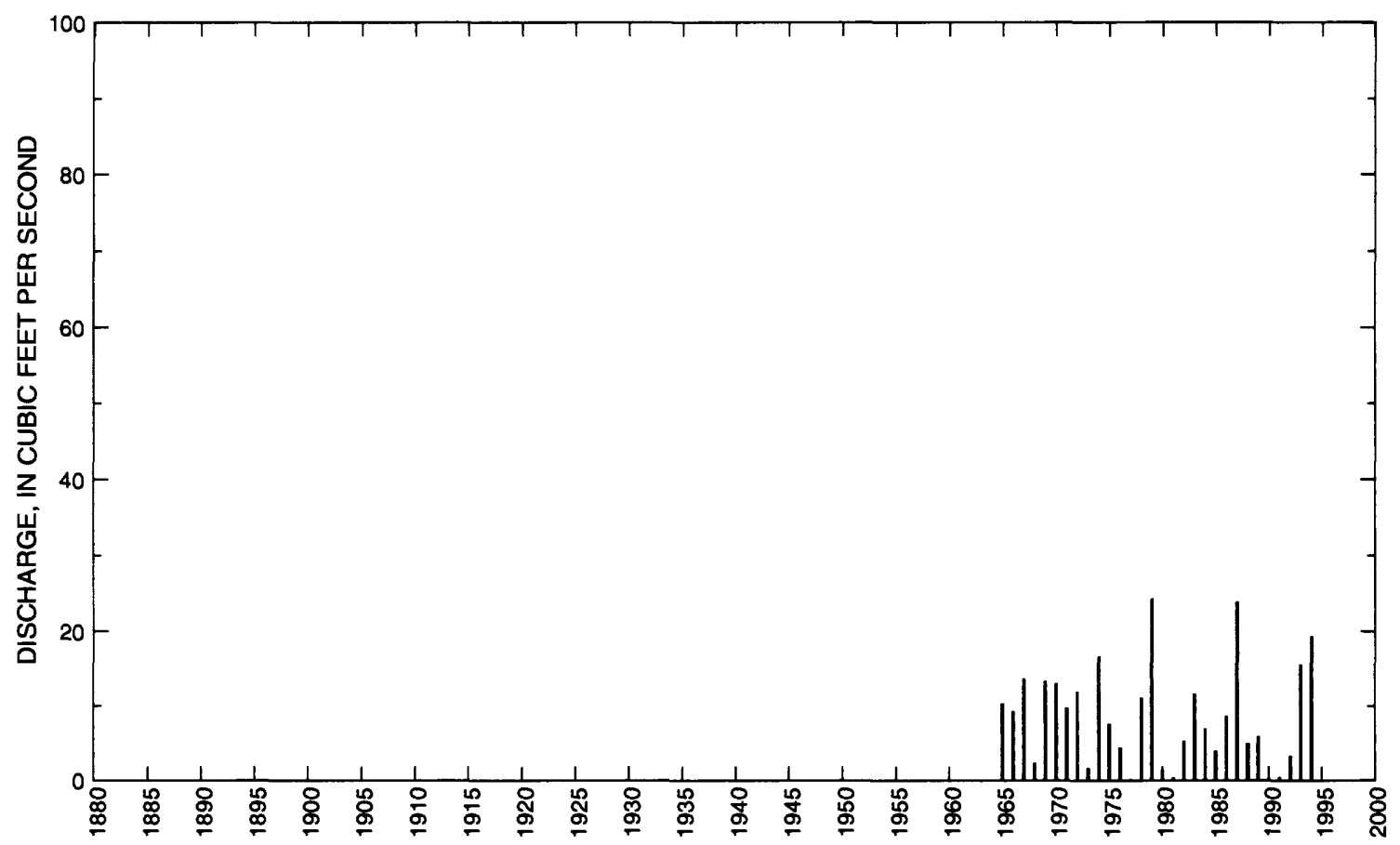


05064900 BEAVER CREEK NEAR FINLEY, ND--Continued

Statistics of monthly and annual mean discharges

[m, more than 1 year of occurrence]

\begin{tabular}{|c|c|c|c|c|c|c|c|c|}
\hline \multirow[b]{2}{*}{ Month } & \multicolumn{2}{|c|}{ Maximum } & \multicolumn{2}{|c|}{ Minimum } & \multicolumn{4}{|c|}{ Mean } \\
\hline & $\begin{array}{c}\text { Discharge } \\
\left(\mathrm{ft}^{3} / \mathrm{s}\right)\end{array}$ & $\begin{array}{c}\text { Water year } \\
\text { of } \\
\text { occurrence }\end{array}$ & $\begin{array}{c}\text { Discharge } \\
\left(\mathrm{ft}^{3} / \mathrm{s}\right)\end{array}$ & $\begin{array}{c}\text { Water year } \\
\text { of } \\
\text { occurrence }\end{array}$ & $\begin{array}{c}\text { Discharge } \\
\left(\mathrm{ft}^{3} / \mathrm{s}\right)\end{array}$ & $\begin{array}{c}\text { Standard } \\
\text { deviation } \\
\left(\mathrm{ft}^{3} / \mathrm{s}\right)\end{array}$ & $\begin{array}{l}\text { Coeffi- } \\
\text { cient of } \\
\text { variation }\end{array}$ & $\begin{array}{c}\text { Percentage } \\
\text { of annual } \\
\text { discharge }\end{array}$ \\
\hline October & 9.30 & 1983 & 0 & $\mathrm{~m}$ & 1.14 & 2.15 & 1.89 & 1.09 \\
\hline November & 5.04 & 1994 & 0 & $\mathrm{~m}$ & 0.520 & 1.02 & 1.95 & 0.50 \\
\hline December & 2.52 & 1994 & 0 & $\mathrm{~m}$ & 0.140 & 0.46 & 3.21 & 0.14 \\
\hline January & 0.796 & 1994 & 0 & $\mathrm{~m}$ & 0.030 & 0.15 & 5.20 & 0.03 \\
\hline February & 2.61 & 1984 & 0 & $\mathrm{~m}$ & 0.240 & 0.56 & 2.31 & 0.23 \\
\hline March & 64.6 & 1966 & 0 & $\mathrm{~m}$ & 23.2 & 21.4 & 0.92 & 22.16 \\
\hline April & 244 & 1979 & 0.192 & 1981 & 50.4 & 59.2 & 1.17 & 48.18 \\
\hline May & 47.0 & 1979 & 0.042 & 1977 & 8.95 & 12.2 & 1.36 & 8.55 \\
\hline June & 29.4 & 1994 & 0.001 & 1988 & 5.00 & 7.01 & 1.40 & 4.78 \\
\hline July & 104 & 1993 & 0 & $\mathrm{~m}$ & 8.67 & 21.6 & 2.49 & 8.28 \\
\hline August & 43.4 & 1994 & 0 & $\mathrm{~m}$ & 3.96 & 9.97 & 2.52 & 3.78 \\
\hline September & 21.2 & 1993 & 0 & $\mathrm{~m}$ & 2.41 & 5.49 & 2.28 & 2.30 \\
\hline Annual & 24.3 & 1979 & 0.121 & 1977 & 8.73 & 6.78 & 0.78 & 100 \\
\hline
\end{tabular}

Annual flow duration

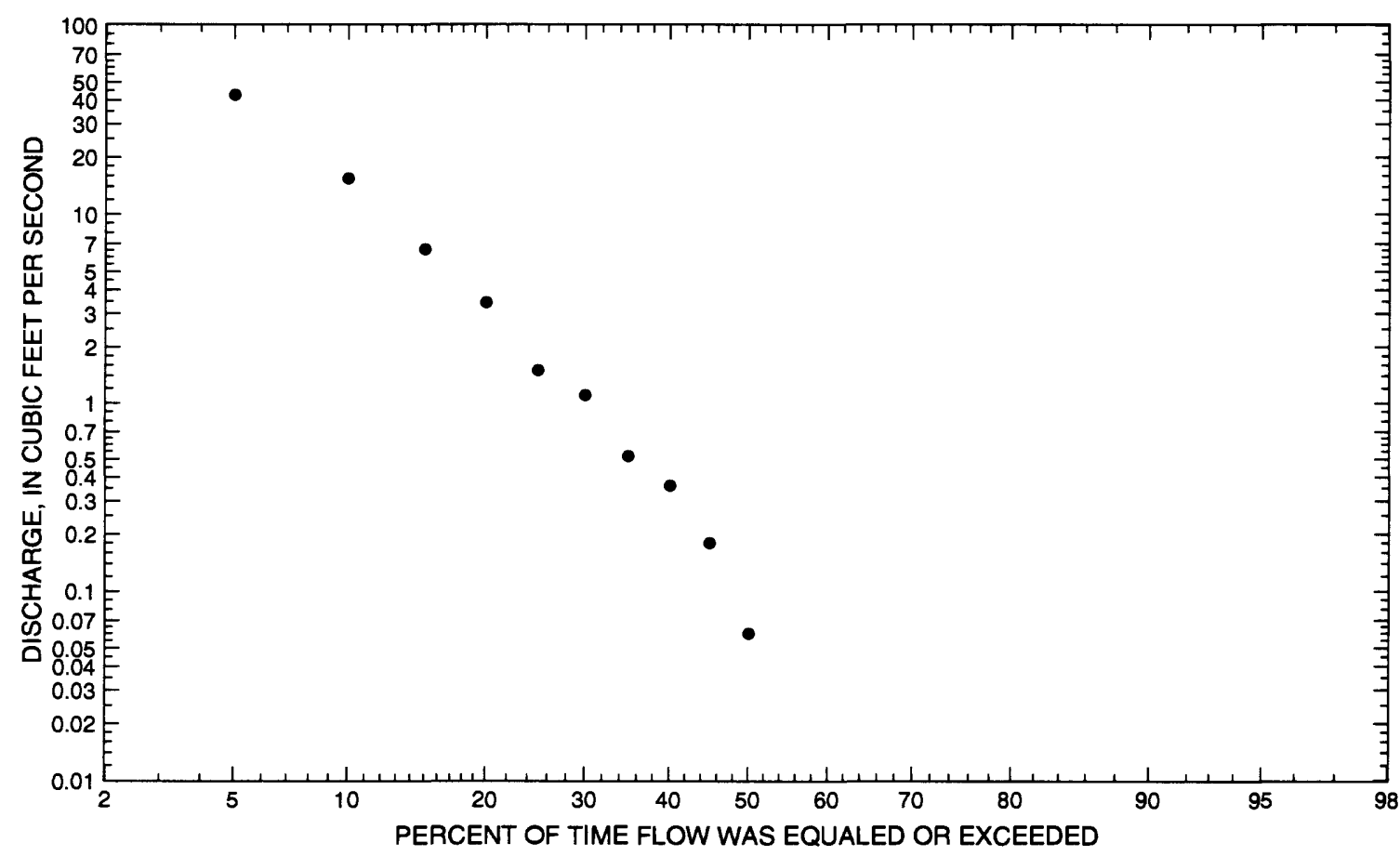




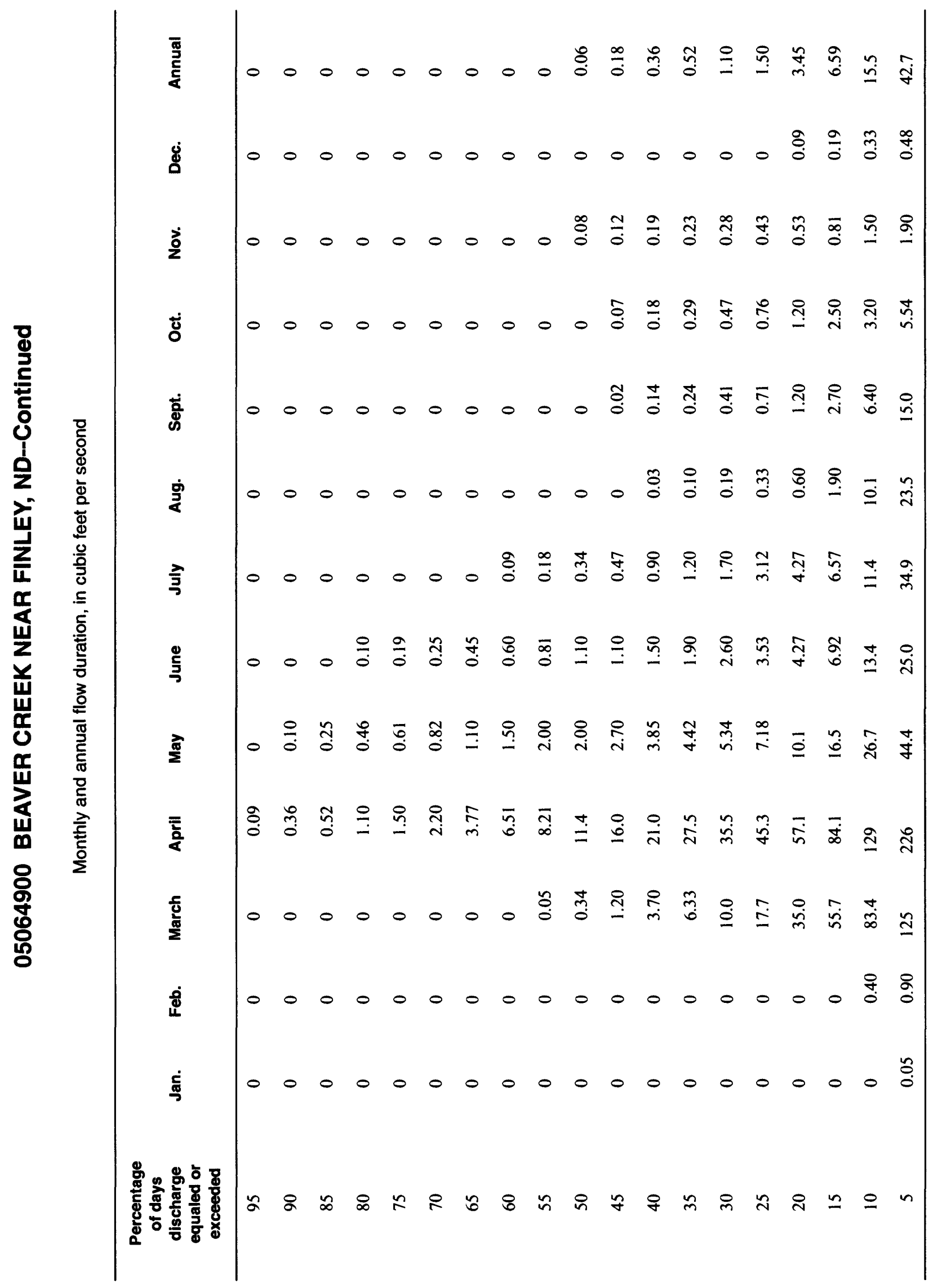




\section{BEAVER CREEK NEAR FINLEY, ND--Continued}

Probability of occurrence of annual high discharges

[ng, statistic not given]

\begin{tabular}{|c|c|c|c|c|c|c|}
\hline \multirow[b]{2}{*}{$\begin{array}{l}\text { Exceedance } \\
\text { probability }\end{array}$} & \multirow[b]{2}{*}{$\begin{array}{l}\text { Recurrence } \\
\text { interval } \\
\text { (years) }\end{array}$} & \multirow[b]{2}{*}{$\begin{array}{c}\text { Maximum } \\
\text { instantaneous } \\
\left(\mathrm{ft}^{3} / \mathrm{s}\right)\end{array}$} & \multicolumn{4}{|c|}{$\begin{array}{l}\text { Maximum mean discharge } \\
\left(\mathrm{ft}^{3} / \mathrm{s}\right)\end{array}$} \\
\hline & & & 3-day period & 7-day period & 15-day period & 30-day period \\
\hline 0.99 & 1.01 & 3.6 & 1.33 & 0.770 & 0.534 & 0.357 \\
\hline 0.95 & 1.05 & 16.0 & 7.72 & 5.11 & 3.62 & 2.42 \\
\hline 0.90 & 1.11 & 32.9 & 17.5 & 12.2 & 8.63 & 5.75 \\
\hline 0.80 & 1.25 & 73.4 & 42.2 & 30.6 & 21.7 & 14.3 \\
\hline 0.50 & 2 & 281 & 167 & 124 & 85.7 & 55.7 \\
\hline 0.20 & 5 & 843 & 457 & 328 & 216 & 137 \\
\hline 0.10 & 10 & 1,370 & 676 & 469 & 301 & 188 \\
\hline 0.04 & 25 & 2,150 & 941 & 625 & 389 & 240 \\
\hline 0.02 & 50 & 2,790 & 1,120 & 719 & 439 & 269 \\
\hline 0.01 & 100 & 3,440 & 1,270 & 795 & 478 & 291 \\
\hline 0.005 & 200 & 4,110 & 1,400 & 856 & 508 & 307 \\
\hline 0.002 & 500 & 5,000 & ng & ng & ng & ng \\
\hline
\end{tabular}

Probability of occurrence of annual low discharges

[ng, statistic not given]

\begin{tabular}{|c|c|c|c|c|c|c|c|c|c|c|}
\hline \multirow{3}{*}{$\begin{array}{l}\text { Non- } \\
\text { exceed- } \\
\text { ance } \\
\text { prob- } \\
\text { ability }\end{array}$} & \multirow{3}{*}{$\begin{array}{l}\text { Recur- } \\
\text { rence } \\
\text { inter- } \\
\text { val } \\
\text { (years) }\end{array}$} & \multicolumn{9}{|c|}{ Minimum mean discharge $\left(\mathrm{ft}^{3} / \mathrm{s}\right)$} \\
\hline & & \multicolumn{9}{|c|}{ Number of consecutlve days } \\
\hline & & 1 & 3 & 7 & 14 & 30 & 60 & 90 & 120 & 183 \\
\hline 0.05 & 20 & $\mathrm{ng}$ & ng & $\mathrm{ng}$ & ng & ng & $\mathrm{ng}$ & 0 & 0 & 0 \\
\hline 0.10 & 10 & $\mathrm{ng}$ & $\mathrm{ng}$ & $\mathrm{ng}$ & ng & ng & $\mathrm{ng}$ & 0 & 0 & 0 \\
\hline 0.20 & 5 & $\mathrm{ng}$ & $\mathrm{ng}$ & $\mathrm{ng}$ & $\mathrm{ng}$ & $\mathrm{ng}$ & ng & 0 & 0 & 0 \\
\hline 0.50 & 2 & $\mathrm{ng}$ & $\mathrm{ng}$ & $\mathrm{ng}$ & $\mathrm{ng}$ & $\mathrm{ng}$ & $\mathrm{ng}$ & 0 & 0.009 & 0.043 \\
\hline
\end{tabular}




\section{BEAVER CREEK NEAR FINLEY, ND--Continued}

Probability of occurrence of seasonal low discharges

[ng, statistic not given]

\begin{tabular}{|c|c|c|c|c|c|c|c|c|c|}
\hline \multirow[b]{3}{*}{$\begin{array}{l}\text { Non- } \\
\text { exceedance } \\
\text { probability }\end{array}$} & \multirow[b]{3}{*}{$\begin{array}{c}\text { Recurrence } \\
\text { interval } \\
\text { (years) }\end{array}$} & \multicolumn{8}{|c|}{ Minimum mean discharge $\left(\mathrm{ft}^{3} / \mathrm{s}\right)$} \\
\hline & & \multicolumn{8}{|c|}{ Number of consecutive days } \\
\hline & & 1 & 7 & 14 & 30 & 1 & 7 & 14 & 30 \\
\hline & & \multicolumn{4}{|c|}{ December-January-February } & \multicolumn{4}{|c|}{ March-April-May } \\
\hline 0.05 & 20 & $\mathrm{ng}$ & ng & $\mathrm{ng}$ & ng & 0 & 0 & 0 & 0 \\
\hline 0.10 & 10 & $\mathrm{ng}$ & $\mathrm{ng}$ & $\mathrm{ng}$ & $\mathrm{ng}$ & 0 & 0 & 0 & 0 \\
\hline 0.20 & 5 & $\mathrm{ng}$ & $\mathrm{ng}$ & $\mathrm{ng}$ & $\mathrm{ng}$ & 0 & 0 & 0 & 0.085 \\
\hline \multirow[t]{2}{*}{0.50} & 2 & $\mathrm{ng}$ & $\mathrm{ng}$ & $\mathrm{ng}$ & $\mathrm{ng}$ & 0 & 0 & 0 & 1.02 \\
\hline & & \multicolumn{4}{|c|}{ June-July-August } & \multicolumn{4}{|c|}{ September-October-November } \\
\hline 0.05 & 20 & 0 & 0 & 0 & 0 & 0 & 0 & 0 & 0 \\
\hline 0.10 & 10 & 0 & 0 & 0 & 0 & 0 & 0 & 0 & 0 \\
\hline 0.20 & 5 & 0 & 0 & 0 & 0 & 0 & 0 & 0 & 0 \\
\hline 0.50 & 2 & 0 & 0 & 0 & 0 & 0 & 0 & 0 & 0 \\
\hline
\end{tabular}


05064900 BEAVER CREEK NEAR FINLEY, ND--Continued

Annual peak discharge and corresponding gage height

$[--$, no data $]$

\begin{tabular}{|c|c|c|c|c|c|c|c|}
\hline $\begin{array}{l}\text { Water } \\
\text { year }\end{array}$ & Date & $\begin{array}{c}\text { Gage } \\
\text { height } \\
\text { (feet) }\end{array}$ & $\begin{array}{c}\text { Peak } \\
\text { discharge } \\
\left(\mathrm{ft}^{3} / \mathrm{s}\right)\end{array}$ & $\begin{array}{c}\text { Water } \\
\text { year }\end{array}$ & Date & $\begin{array}{c}\text { Gage } \\
\text { height } \\
\text { (feet) }\end{array}$ & $\begin{array}{c}\text { Peak } \\
\text { discharge } \\
\left(\mathrm{ft}^{3} / \mathrm{s}\right)\end{array}$ \\
\hline \multicolumn{8}{|c|}{ Annual peak discharge, by year, and corresponding gage height } \\
\hline 1965 & April 11 & 6.88 & 1,250 & 1980 & March 31 & 3.72 & 100 \\
\hline 1966 & March 20 & 7.33 & 400 & 1981 & July 17 & 2.89 & 10.0 \\
\hline 1967 & April 20 & 5.52 & 652 & 1982 & March 30 & 5.74 & 700 \\
\hline 1968 & June 7 & 3.55 & 47.0 & 1983 & June 21 & 4.53 & 259 \\
\hline 1969 & April 9 & 6.55 & 1,320 & 1984 & March 25 & -- & 150 \\
\hline 1970 & June 16 & 6.49 & 1,270 & 1985 & March 11 & 4.49 & 253 \\
\hline 1971 & April 5 & 4.77 & 392 & 1986 & May 12 & 4.60 & 282 \\
\hline 1972 & May 26 & 5.65 & 736 & 1987 & July 22 & 6.04 & 688 \\
\hline 1973 & March 14 & 4.35 & 170 & 1988 & March 24 & 4.07 & 130 \\
\hline 1974 & April 11 & -- & 550 & 1989 & April 5 & 4.55 & 230 \\
\hline 1975 & June 22 & 5.07 & 472 & 1990 & June 30 & 2.67 & 9.70 \\
\hline 1976 & March 24 & 5.26 & 290 & 1991 & May 23 & 2.65 & 7.80 \\
\hline 1977 & March 31 & 3.00 & 29.0 & 1992 & March 5 & -- & 95.0 \\
\hline 1978 & March 28 & 7.28 & 950 & 1993 & July 23 & 6.42 & 700 \\
\hline 1979 & April 19 & 8.35 & 1,900 & 1994 & April 24 & -- & 170 \\
\hline \multicolumn{8}{|c|}{ Annual peak discharge, from highest to lowest, and corresponding gage height } \\
\hline 1979 & April 19 & 8.35 & 1,900 & 1986 & May 12 & 4.60 & 282 \\
\hline 1969 & April 9 & 6.55 & 1,320 & 1983 & June 21 & 4.53 & 259 \\
\hline 1970 & June 16 & 6.49 & 1,270 & 1985 & March 11 & 4.49 & 253 \\
\hline 1965 & April 11 & 6.88 & 1,250 & 1989 & April 5 & 4.55 & 230 \\
\hline 1978 & March 28 & 7.28 & 950 & 1973 & March 14 & 4.35 & 170 \\
\hline 1972 & May 26 & 5.65 & 736 & 1994 & March 24 & -- & 170 \\
\hline 1982 & March 30 & 5.74 & 700 & 1984 & March 25 & -- & 150 \\
\hline 1993 & July 23 & 6.42 & 700 & 1988 & March 24 & 4.07 & 130 \\
\hline 1987 & July 22 & 6.04 & 688 & 1980 & March 31 & 3.72 & 100 \\
\hline 1967 & April 20 & 5.52 & 652 & 1992 & March 5 & -- & 95.0 \\
\hline 1974 & April 11 & -- & 550 & 1968 & June 7 & 3.55 & 47.0 \\
\hline 1975 & June 22 & 5.07 & 472 & 1977 & March 31 & 3.00 & 29.0 \\
\hline 1966 & March 20 & 7.33 & 400 & 1981 & July 17 & 2.89 & 10.0 \\
\hline 1971 & April 5 & 4.77 & 392 & 1990 & June 30 & 2.67 & 9.70 \\
\hline 1976 & March 24 & 5.26 & 290 & 1991 & May 23 & 2.65 & 7.80 \\
\hline
\end{tabular}




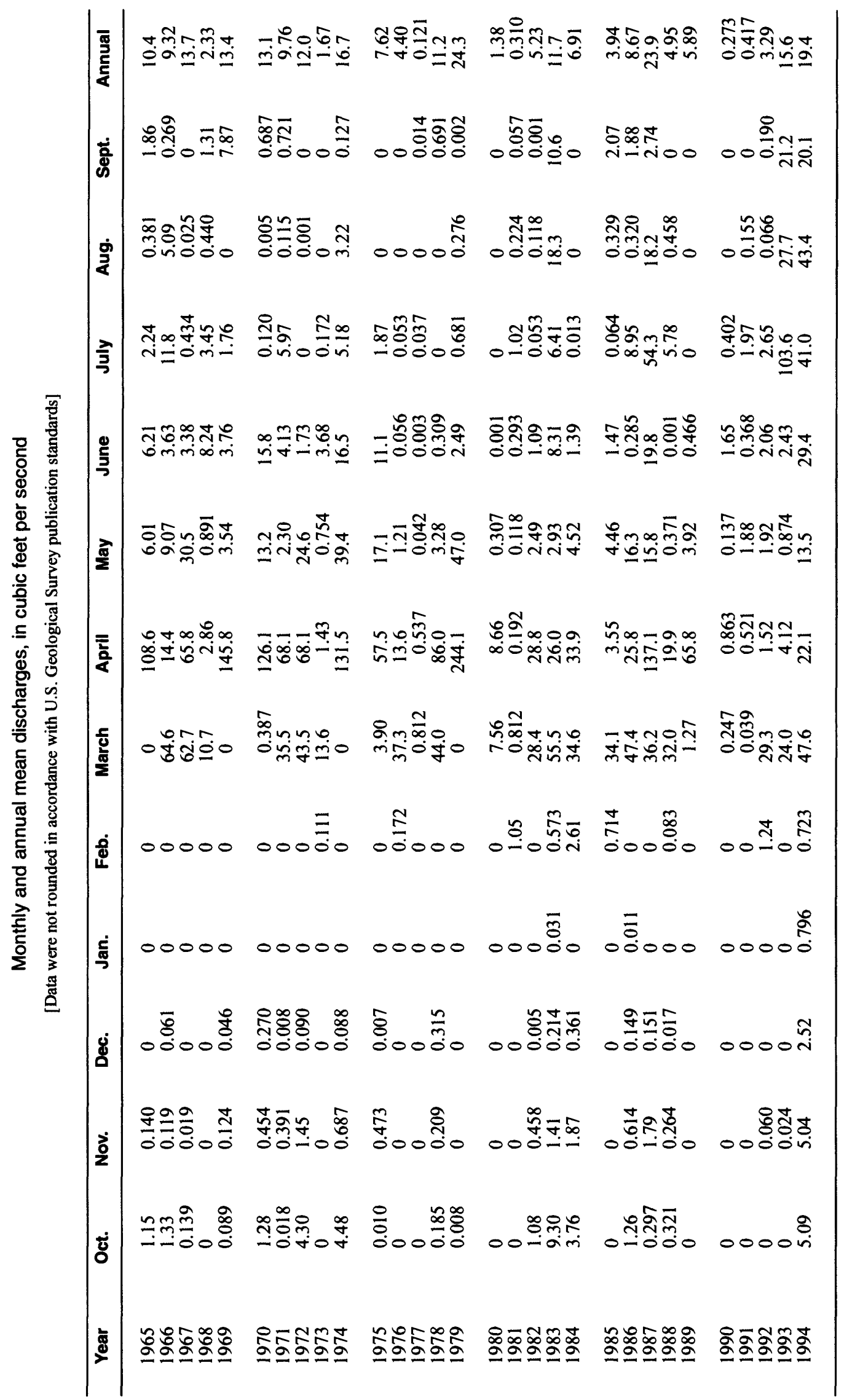




\section{GOOSE RIVER NEAR PORTLAND, ND}

LOCATION.--Lat $47^{\circ} 32^{\prime} 20^{\prime \prime}$, long $97^{\circ} 27^{\prime} 20^{\prime}$, in $\mathrm{SE}^{1} / 4 \mathrm{NE}^{1} / 4 \mathrm{sec} .19$, T.147 N., R.53 W., Traill County, Hydrologic Unit 09020101, on left bank $75 \mathrm{ft}$ upstream from bridge on State Highway 18, $1.2 \mathrm{mi}$ upstream from unnamed tributary, $4 \mathrm{mi}$ downstream from Beaver Creek, and 5 mi northwest of Portland.

DRAINAGE AREA.--517 $\mathrm{mi}^{2}$ of which about $110 \mathrm{mi}^{2}$ is probably noncontributing.

PERIOD OF RECORD.--October 1939 to September 1975, October 1980 to 1986 . Seasonal records only since 1983.

GAGE.--Water-stage recorder. Datum of gage is $967.48 \mathrm{ft}$ above National Geodetic Vertical Datum of 1929. Prior to Oct. 1, 1956, nonrecording gage at site $2 \mathrm{mi}$ upstream at datum $11.28 \mathrm{ft}$ higher.

EXTREMES FOR PERIOD OF RECORD.--Maximum discharge, 8,530 $\mathrm{ft}^{3} / \mathrm{s}$, May 9,1950 , gage height, $20.12 \mathrm{ft}$, on basis of contracted opening measurement, present site and datum; no flow at times most years.

EXTREMES OUTSIDE PERIOD OF RECORD.--Flood of Apr. 20-21, 1979, reached a stage of $20.96 \mathrm{ft}$, present datum, from floodmark; discharge not determined.

Annual mean discharge

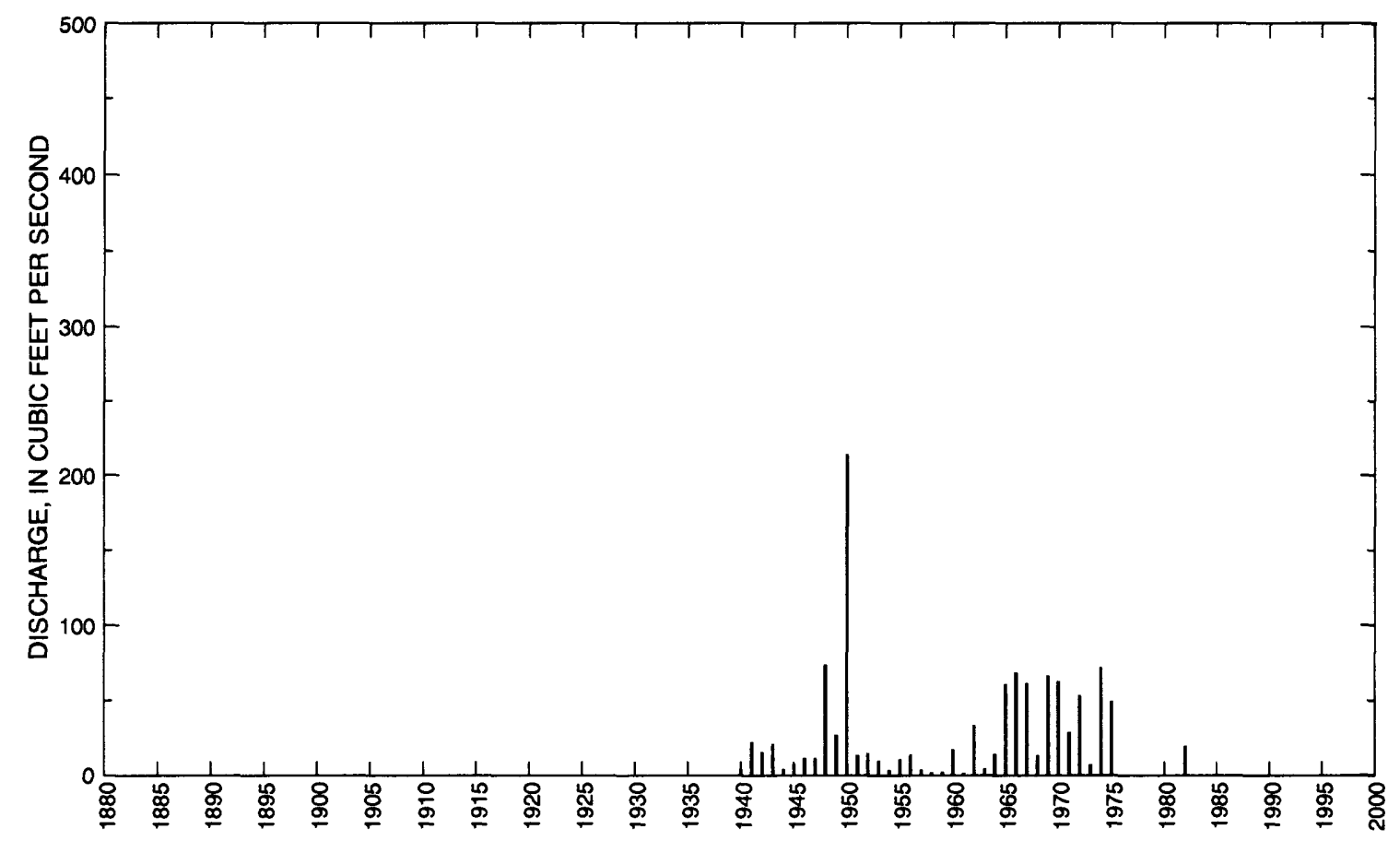




\section{GOOSE RIVER NEAR PORTLAND, ND--Continued}

Statistics of monthly and annual mean discharges

[m, more than 1 year of occurrence]

\begin{tabular}{|c|c|c|c|c|c|c|c|c|}
\hline \multirow[b]{2}{*}{ Month } & \multicolumn{2}{|c|}{ Maximum } & \multicolumn{2}{|c|}{ Minimum } & \multicolumn{4}{|c|}{ Mean } \\
\hline & $\begin{array}{c}\text { Discharge } \\
\left(\mathrm{ft}^{3} / \mathrm{s}\right)\end{array}$ & $\begin{array}{l}\text { Water year } \\
\text { of } \\
\text { occurrence }\end{array}$ & $\begin{array}{c}\text { Discharge } \\
\left(\mathrm{ft}^{3} / \mathrm{s}\right)\end{array}$ & $\begin{array}{l}\text { Water year } \\
\quad \text { of } \\
\text { occurrence }\end{array}$ & $\begin{array}{c}\text { Discharge } \\
\left(\mathrm{ft}^{3} / \mathrm{s}\right)\end{array}$ & $\begin{array}{c}\text { Standard } \\
\text { deviation } \\
\left(\mathrm{ft}^{3} / \mathrm{s}\right)\end{array}$ & $\begin{array}{l}\text { Coeffi- } \\
\text { cient of } \\
\text { variation }\end{array}$ & $\begin{array}{l}\text { Percentage } \\
\text { of annual } \\
\text { discharge }\end{array}$ \\
\hline October & 10.2 & 1965 & 0 & $\mathrm{~m}$ & 1.15 & 2.59 & 2.25 & 0.33 \\
\hline November & 6.92 & 1972 & 0 & $\mathrm{~m}$ & 0.830 & 1.61 & 1.95 & 0.23 \\
\hline December & 2.96 & 1970 & 0 & $\mathrm{~m}$ & 0.380 & 0.78 & 2.04 & 0.11 \\
\hline January & 1.77 & 1970 & 0 & $\mathrm{~m}$ & 0.190 & 0.41 & 2.22 & 0.05 \\
\hline February & 3.75 & 1954 & 0 & $\mathrm{~m}$ & 0.230 & 0.67 & 2.93 & 0.06 \\
\hline March & 408 & 1966 & 0 & $\mathrm{~m}$ & 47.2 & 79.64 & 1.69 & 13.3 \\
\hline April & 1,080 & 1950 & 0 & 1981 & 204 & 254 & 1.25 & 57.5 \\
\hline May & 1,390 & 1950 & 0 & 1981 & 64.0 & 215 & 3.36 & 18.1 \\
\hline June & 103 & 1964 & 0 & $\mathrm{~m}$ & 22.4 & 26.0 & 1.16 & 6.32 \\
\hline July & 52.5 & 1952 & 0 & $\mathrm{~m}$ & 8.98 & 12.6 & 1.40 & 2.54 \\
\hline August & 45.5 & 1966 & 0 & $\mathrm{~m}$ & 3.07 & 9.10 & 2.97 & 0.87 \\
\hline September & 29.9 & 1957 & 0 & $\mathrm{~m}$ & 2.09 & 5.77 & 2.75 & 0.59 \\
\hline Annual & 214 & 1950 & 0 & 1981 & 29.5 & 38.8 & 1.31 & 100 \\
\hline
\end{tabular}

Annual flow duration

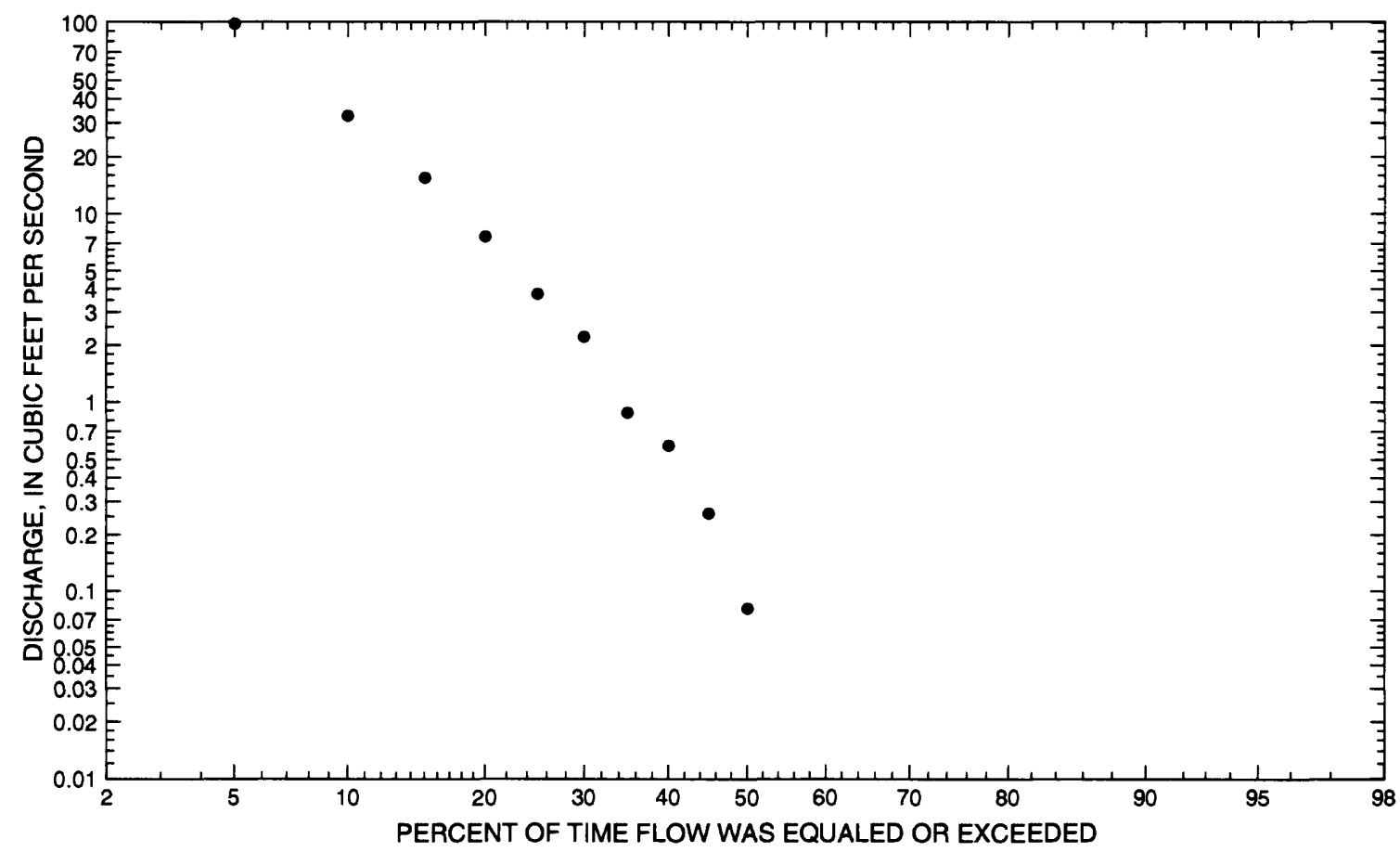




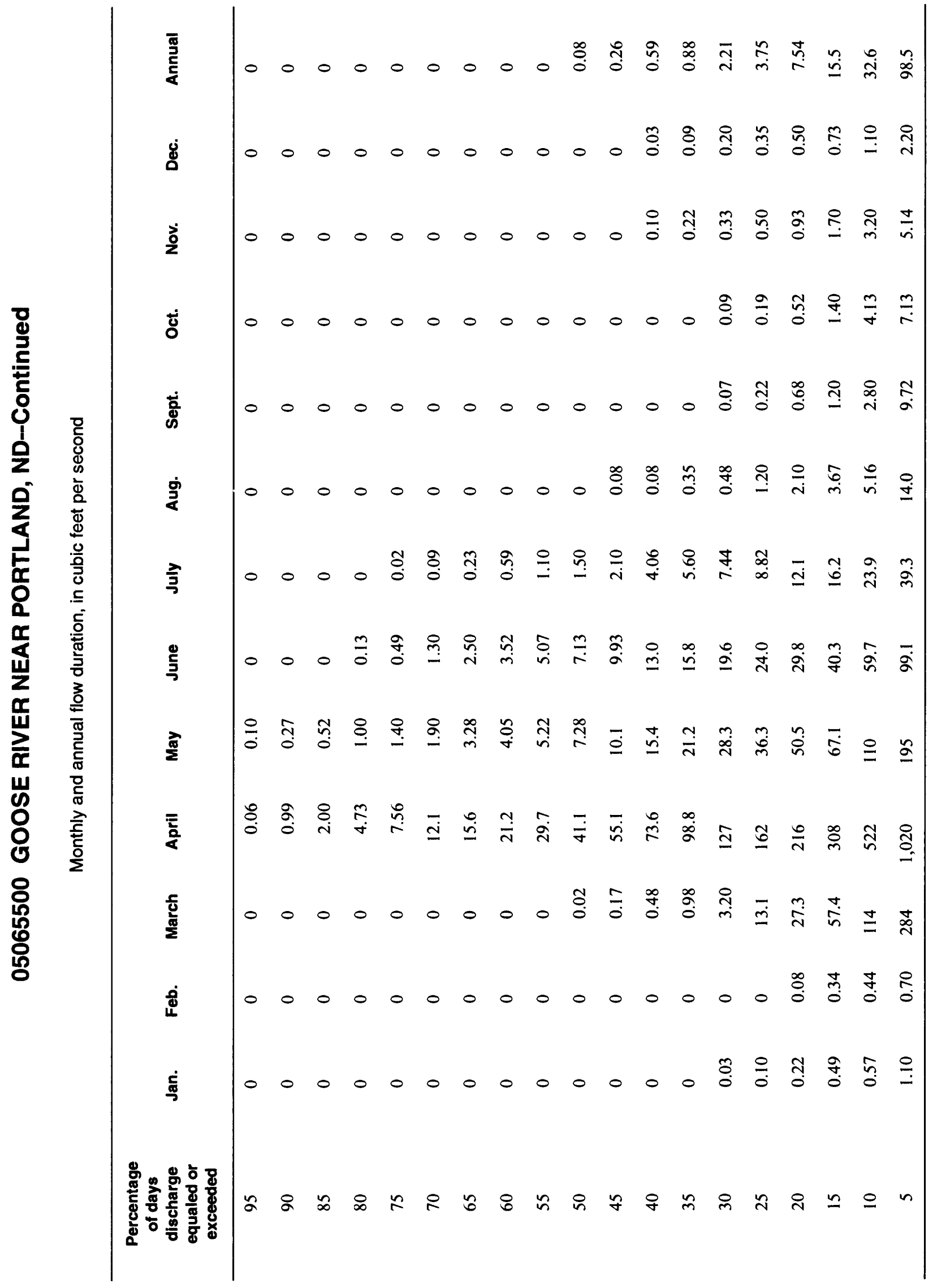




\section{GOOSE RIVER NEAR PORTLAND, ND--Continued}

Probability of occurrence of annual high discharges

[ng, statistic not given]

\begin{tabular}{|c|c|c|c|c|c|c|}
\hline \multirow[b]{2}{*}{$\begin{array}{l}\text { Exceedance } \\
\text { probability }\end{array}$} & \multirow[b]{2}{*}{$\begin{array}{l}\text { Recurrence } \\
\text { interval } \\
\text { (years) }\end{array}$} & \multirow[b]{2}{*}{$\begin{array}{c}\text { Maximum } \\
\text { instantaneous } \\
\left(\mathrm{ft}^{3} / \mathbf{s}\right)\end{array}$} & \multicolumn{4}{|c|}{$\begin{array}{l}\text { Maximum mean discharge } \\
\qquad\left(\mathrm{ft}^{3} / \mathrm{s}\right)\end{array}$} \\
\hline & & & 3-day period & 7-day period & 15-day perlod & 30-day period \\
\hline 0.99 & 1.01 & $\mathrm{ng}$ & 0 & 0 & 0 & 0 \\
\hline 0.95 & 1.05 & 86.1 & 26.4 & 19.2 & 13.9 & 9.94 \\
\hline 0.90 & 1.11 & 139 & 62.5 & 45.9 & 32.2 & 21.3 \\
\hline 0.80 & 1.25 & 242 & 142 & 105 & 71.2 & 44.8 \\
\hline 0.50 & 2 & 667 & 512 & 383 & 246 & 149 \\
\hline 0.20 & 5 & 1,710 & 1,500 & 1,120 & 689 & 424 \\
\hline 0.10 & 10 & 2,720 & 2,460 & 1,840 & 1,110 & 705 \\
\hline 0.04 & 25 & 4,360 & 4,010 & 2,990 & 1,770 & 1,170 \\
\hline 0.02 & 50 & 5,860 & 5,390 & 4,000 & 2,340 & 1,610 \\
\hline 0.01 & 100 & 7,580 & 6,940 & 5,140 & 2,970 & 2,130 \\
\hline 0.005 & 200 & 9,530 & 8630 & 6,380 & 3,660 & 2,710 \\
\hline 0.002 & 500 & 12,500 & ng & ng & ng & ng \\
\hline
\end{tabular}

Probability of occurrence of annual low discharges

Minimum mean discharge $\left(\mathrm{ft}^{3} / \mathrm{s}\right)$

Number of consecutive days

\begin{tabular}{ccccccccccc}
$\begin{array}{c}\text { Non- } \\
\text { exceed- } \\
\text { ance } \\
\text { prob- } \\
\text { ability }\end{array}$ & $\begin{array}{c}\text { Recur- } \\
\text { rence } \\
\text { inter- } \\
\text { val } \\
\text { (years) }\end{array}$ & $\mathbf{1}$ & $\mathbf{3}$ & $\mathbf{7}$ & $\mathbf{1 4}$ & $\mathbf{3 0}$ & $\mathbf{6 0}$ & $\mathbf{9 0}$ & $\mathbf{1 2 0}$ & $\mathbf{1 8 3}$ \\
\hline 0.05 & 20 & 0 & 0 & 0 & 0 & 0 & 0 & 0 & 0 & 0 \\
0.10 & 10 & 0 & 0 & 0 & 0 & 0 & 0 & 0 & 0 & 0 \\
0.20 & 5 & 0 & 0 & 0 & 0 & 0 & 0 & 0 & 0 & 0 \\
0.50 & 2 & 0 & 0 & 0 & 0 & 0 & 0 & 0 & 0 & 0.032 \\
\hline
\end{tabular}




\section{GOOSE RIVER NEAR PORTLAND, ND--Continued}

Probability of occurrence of seasonal low discharges

\begin{tabular}{|c|c|c|c|c|c|c|c|c|c|}
\hline \multirow[b]{3}{*}{$\begin{array}{c}\text { Non- } \\
\text { exceedance } \\
\text { probability }\end{array}$} & \multirow[b]{3}{*}{$\begin{array}{c}\text { Recurrence } \\
\text { interval } \\
\text { (years) }\end{array}$} & \multicolumn{8}{|c|}{ Minimum mean discharge $\left(\mathrm{ft}^{3} / \mathrm{s}\right)$} \\
\hline & & \multicolumn{8}{|c|}{ Number of consecutive days } \\
\hline & & 1 & 7 & 14 & 30 & 1 & 7 & 14 & 30 \\
\hline & & \multicolumn{4}{|c|}{ December-January-February } & \multicolumn{4}{|c|}{ March-April-May } \\
\hline 0.05 & 20 & 0 & 0 & 0 & 0 & 0 & 0 & 0 & 0 \\
\hline 0.10 & 10 & 0 & 0 & 0 & 0 & 0 & 0 & 0 & 0 \\
\hline 0.20 & 5 & 0 & 0 & 0 & 0 & 0 & 0 & 0 & 0.025 \\
\hline \multirow[t]{2}{*}{0.50} & 2 & 0 & 0 & 0 & 0 & 0 & 0 & 0 & 1.48 \\
\hline & & \multicolumn{4}{|c|}{ June-July-August } & \multicolumn{4}{|c|}{ September-October-November } \\
\hline 0.05 & 20 & 0 & 0 & 0 & 0 & 0 & 0 & 0 & 0 \\
\hline 0.10 & 10 & 0 & 0 & 0 & 0 & 0 & 0 & 0 & 0 \\
\hline 0.20 & 5 & 0 & 0 & 0 & 0 & 0 & 0 & 0 & 0 \\
\hline 0.50 & 2 & 0 & 0 & 0 & 0.090 & 0 & 0 & 0 & 0 \\
\hline
\end{tabular}


05065500 GOOSE RIVER NEAR PORTLAND, ND--Continued

Annual peak discharge and corresponding gage height

$[--$, no data $]$

\begin{tabular}{|c|c|c|c|c|c|c|c|}
\hline $\begin{array}{l}\text { Water } \\
\text { year }\end{array}$ & Date & $\begin{array}{c}\text { Gage } \\
\text { height } \\
\text { (feet) }\end{array}$ & $\begin{array}{c}\text { Peak } \\
\text { discharge } \\
\left(\mathrm{ft}^{3} / \mathrm{s}\right)\end{array}$ & $\begin{array}{c}\text { Water } \\
\text { year }\end{array}$ & Date & $\begin{array}{c}\text { Gage } \\
\text { height } \\
\text { (feet) }\end{array}$ & $\begin{array}{c}\text { Peak } \\
\text { discharge } \\
\left(\mathrm{ft}^{3} / \mathrm{s}\right)\end{array}$ \\
\hline \multicolumn{8}{|c|}{ Annual peak discharge, by year, and corresponding gage height } \\
\hline 1940 & April 16 & 7.88 & 487 & 1963 & April 8 & 5.83 & 150 \\
\hline 1941 & April 9 & 12.59 & 1,130 & 1964 & June 22 & 7.96 & 381 \\
\hline 1942 & April 5 & 11.51 & 850 & 1965 & April 13 & 18.22 & 3,740 \\
\hline 1943 & March 26 & 14.90 & 1,200 & 1966 & March 21 & 14.19 & 1,190 \\
\hline 1944 & April 10 & 5.10 & 169 & 1967 & April 23 & 16.05 & 2,080 \\
\hline 1945 & March 15 & 6.70 & 340 & 1968 & June 10 & 8.22 & 448 \\
\hline 1946 & March 20 & 9.14 & 530 & 1969 & April 13 & 18.17 & 3,660 \\
\hline 1947 & March 28 & 6.95 & 260 & 1970 & April 27 & 14.62 & 1,670 \\
\hline 1948 & April 21 & 21.30 & 4,700 & 1971 & April 9 & 12.63 & 1,150 \\
\hline 1949 & April 7 & 13.60 & 1,200 & 1972 & April 17 & 15.27 & 1,720 \\
\hline 1950 & May 9 & 20.12 & 8,530 & 1973 & March 17 & 7.71 & 247 \\
\hline 1951 & March 30 & 12.50 & 650 & 1974 & April 14 & 16.40 & 2,200 \\
\hline 1952 & April 3 & 11.45 & 600 & 1975 & April 19 & 14.92 & 1,340 \\
\hline 1953 & July 4 & 7.77 & 367 & 1976 & April 1 & 10.15 & 680 \\
\hline 1954 & June 15 & 4.28 & 58 & 1981 & -- & -- & 0 \\
\hline 1955 & March 31 & 10.80 & 600 & 1982 & April 1 & 12.32 & 810 \\
\hline 1956 & April 16 & 11.07 & 550 & 1983 & March 16 & 9.97 & 450 \\
\hline 1957 & September 5 & 5.95 & 134 & 1984 & March 27 & 13.09 & 1,170 \\
\hline 1958 & July 27 & 5.47 & 95.0 & 1985 & March 17 & 7.95 & 270 \\
\hline 1959 & April 3 & 4.94 & 72.0 & 1986 & March 25 & 10.71 & 818 \\
\hline 1960 & April 8 & 11.45 & 924 & 1987 & April 8 & 17.58 & 2,500 \\
\hline 1961 & March 10 & 4.13 & 71.0 & 1988 & March 29 & 11.20 & 500 \\
\hline 1962 & April 8 & 15.39 & 1,610 & & & & \\
\hline \multicolumn{8}{|c|}{ Annual peak discharge, from highest to lowest, and corresponding gage height } \\
\hline 1950 & May 9 & 20.12 & 8,530 & 1982 & April 1 & 12.32 & 810 \\
\hline 1948 & April 21 & 21.30 & 4,700 & 1976 & April 1 & 10.15 & 680 \\
\hline 1965 & April 13 & 18.22 & 3,740 & 1951 & March 30 & 12.50 & 650 \\
\hline 1969 & April 13 & 18.17 & 3,660 & 1952 & April 3 & 11.45 & 600 \\
\hline 1987 & April 8 & 17.58 & 2,500 & 1955 & March 31 & 10.80 & 600 \\
\hline 1974 & April 14 & 16.40 & 2,200 & 1956 & April 16 & 11.07 & 550 \\
\hline 1967 & April 23 & 16.05 & 2,080 & 1946 & March 20 & 9.14 & 530 \\
\hline 1972 & April 17 & 15.27 & 1,720 & 1988 & March 29 & 11.20 & 500 \\
\hline 1970 & April 27 & 14.62 & 1,670 & 1940 & April 16 & 7.88 & 487 \\
\hline 1962 & April 8 & 15.93 & 1,610 & 1983 & March 16 & 9.97 & 450 \\
\hline 1975 & April 19 & 14.92 & 1,340 & 1968 & June 10 & 8.22 & 448 \\
\hline 1943 & March 26 & 14.90 & 1,200 & 1964 & June 22 & 7.96 & 381 \\
\hline 1949 & April 7 & 13.60 & 1,200 & 1953 & July 4 & 7.77 & 367 \\
\hline 1966 & March 21 & 14.19 & 1,190 & 1945 & March 15 & 6.70 & 340 \\
\hline 1984 & March 27 & 13.09 & 1,170 & 1985 & March 17 & 7.95 & 270 \\
\hline 1971 & April 9 & 12.63 & 1,150 & 1947 & March 28 & 6.95 & 260 \\
\hline 1941 & April 9 & 12.59 & 1,130 & 1973 & March 17 & 7.71 & 247 \\
\hline 1960 & April 8 & 11.45 & 924 & 1944 & April 10 & 5.10 & 169 \\
\hline 1942 & April 5 & 11.51 & 850 & 1963 & April 8 & 5.83 & 150 \\
\hline 1986 & March 25 & 10.71 & 818 & 1957 & September 5 & 5.95 & 134 \\
\hline
\end{tabular}




\section{GOOSE RIVER NEAR PORTLAND, ND--Continued}

Annual peak discharge and corresponding gage height-Continued

$[--$, no data $]$

\begin{tabular}{|c|c|c|c|c|c|c|c|}
\hline $\begin{array}{l}\text { Water } \\
\text { year }\end{array}$ & Date & $\begin{array}{c}\text { Gage } \\
\text { height } \\
\text { (feet) }\end{array}$ & $\begin{array}{c}\text { Peak } \\
\text { discharge } \\
\left(\mathrm{ft}^{3} / \mathrm{s}\right)\end{array}$ & $\begin{array}{l}\text { Water } \\
\text { year }\end{array}$ & Date & $\begin{array}{c}\text { Gage } \\
\text { height } \\
\text { (feet) }\end{array}$ & $\begin{array}{c}\text { Peak } \\
\text { discharge } \\
\left(\mathrm{ft}^{3} / \mathrm{s}\right)\end{array}$ \\
\hline \multicolumn{8}{|c|}{ Annual peak discharge, from highest to lowest, and corresponding gage height-Continued } \\
\hline 1958 & July 27 & 5.47 & 95.0 & 1954 & June 15 & 4.28 & 58 \\
\hline 1959 & April 3 & 4.94 & 72.0 & 1981 & -- & -- & 0 \\
\hline 1961 & March 10 & 4.13 & 71.0 & & & & \\
\hline
\end{tabular}




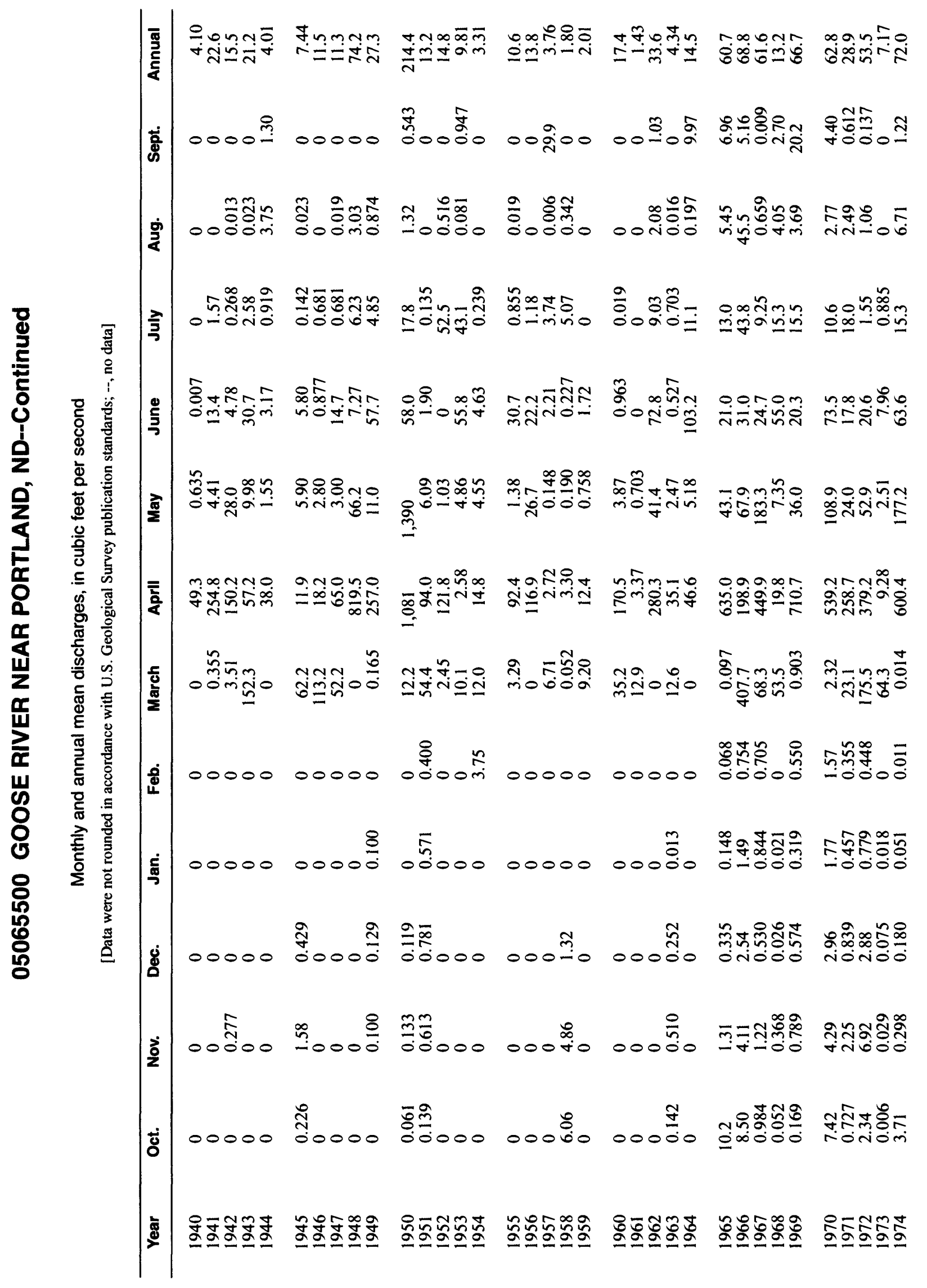




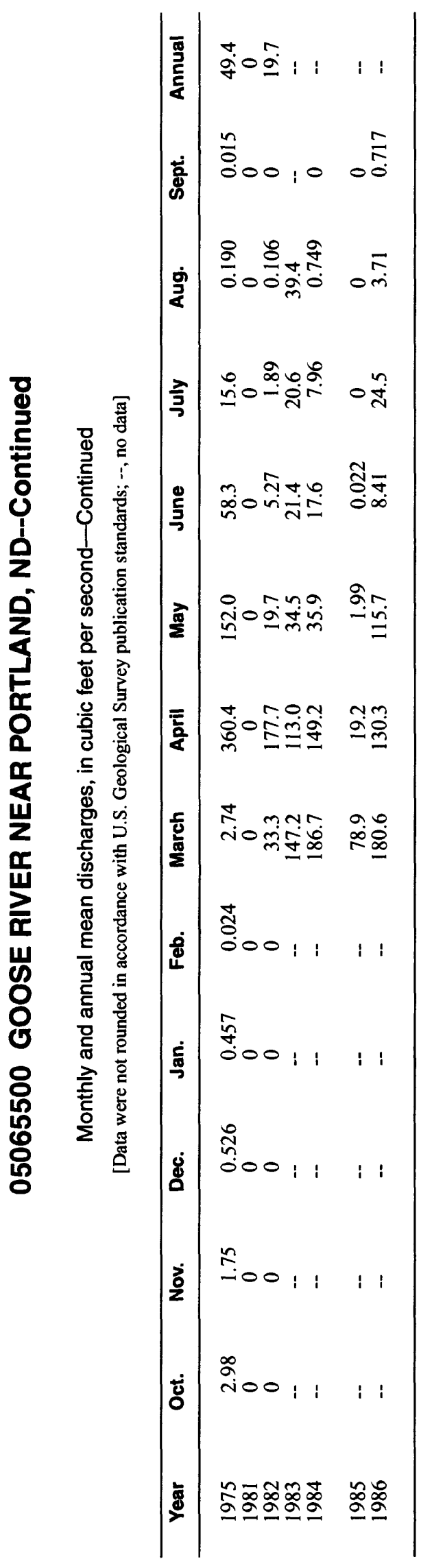




\section{GOOSE RIVER AT HILLSBORO, ND}

LOCATION.--Lat 47²4'34”, long 9703'39”, NW $1 / 4$ sec.5, T.145 N., R.50 W., Traill County, Hydrologic Unit 09020109, on right bank $600 \mathrm{ft}$ upstream from Foogman Dam in Hillsboro and $27.5 \mathrm{mi}$ upstream from mouth.

DRAINAGE AREA.--1,203 $\mathrm{mi}^{2}$ of which about $110 \mathrm{mi}^{2}$ is probably noncontributing.

PERIOD OF RECORD.--March 1931 to current year. No winter records during 1932-34. Monthly discharge only for some periods, published in WSP 1308.

GAGE.--Water-stage recorder and masonry dam. Datum of gage is $879.52 \mathrm{ft}$ above sea level. Sept. 26, 1941 , to Oct. 27, 1965, at site $600 \mathrm{ft}$ downstream at same datum. See WSP 1728 or 1913 for history of changes prior to Sept. 26, 1941.

EXTREMES FOR PERIOD OF RECORD.--Maximum discharge, 14,800 $\mathrm{ft}^{3} / \mathrm{s}$, Apr. 21, 1979, gage height, $16.76 \mathrm{ft}$; no flow at times.

Annual mean discharge

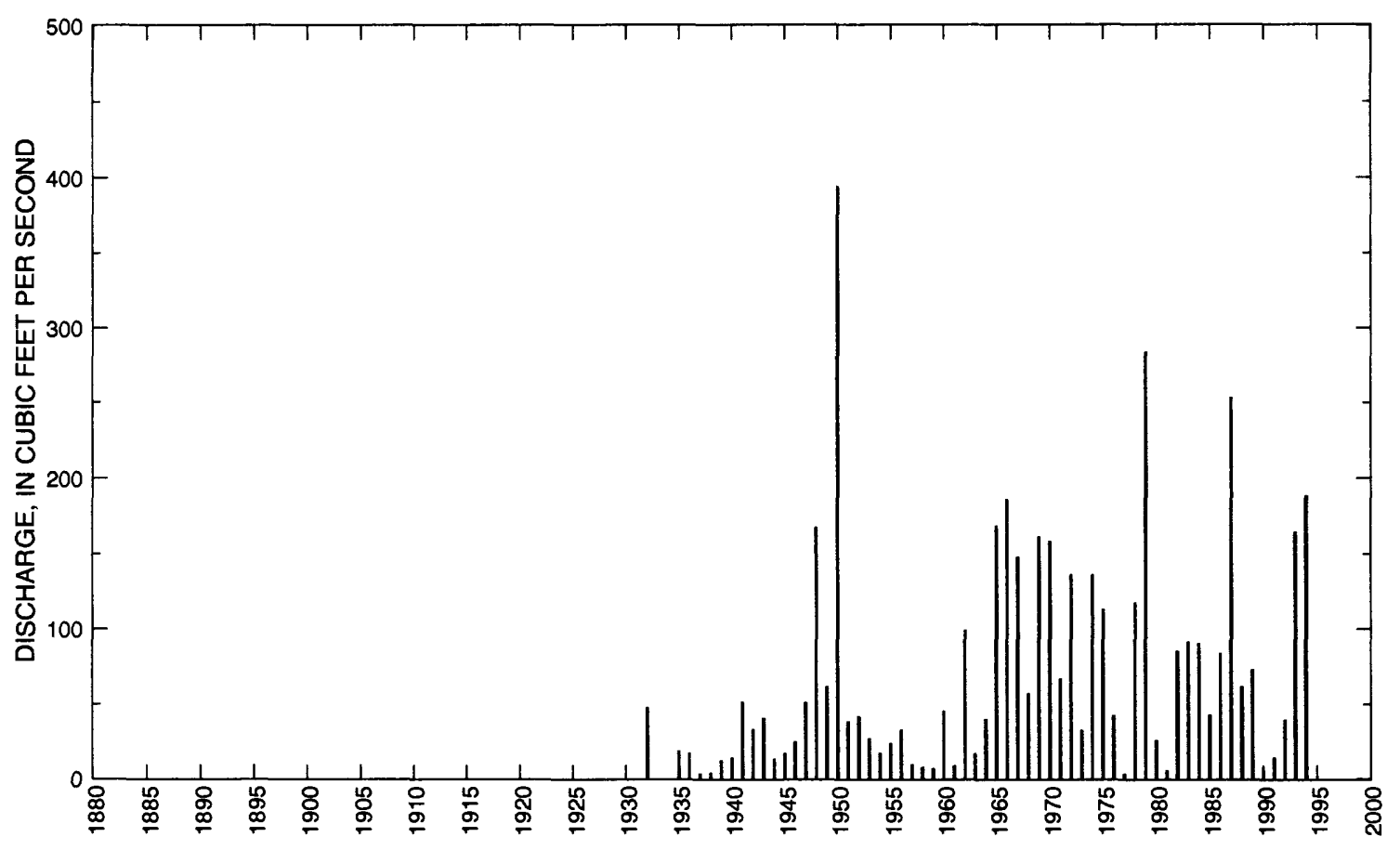




\section{GOOSE RIVER AT HILLSBORO, ND--Continued}

Statistics of monthly and annual mean discharges

[m, more than 1 year of occurrence]

\begin{tabular}{|c|c|c|c|c|c|c|c|c|}
\hline \multirow[b]{2}{*}{ Month } & \multicolumn{2}{|c|}{ Maximum } & \multicolumn{2}{|c|}{ Minimum } & \multicolumn{4}{|c|}{ Mean } \\
\hline & $\begin{array}{c}\text { Discharge } \\
\left(\mathrm{ft}^{3} / \mathrm{s}\right)\end{array}$ & $\begin{array}{c}\text { Water year } \\
\text { of } \\
\text { occurrence }\end{array}$ & $\begin{array}{c}\text { Discharge } \\
\left(\mathrm{ft}^{3} / \mathrm{s}\right)\end{array}$ & $\begin{array}{c}\text { Water year } \\
\text { of } \\
\text { occurrence }\end{array}$ & $\begin{array}{c}\text { Discharge } \\
\left(\mathrm{ft}^{3} / \mathrm{s}\right)\end{array}$ & $\begin{array}{c}\text { Standard } \\
\text { deviation } \\
\left(\mathrm{ft}^{3} / \mathrm{s}\right)\end{array}$ & $\begin{array}{l}\text { Coeffi- } \\
\text { cient of } \\
\text { variation }\end{array}$ & $\begin{array}{c}\text { Percentage } \\
\text { of annual } \\
\text { discharge }\end{array}$ \\
\hline October & 62.9 & 1983 & 0 & $\mathrm{~m}$ & 7.19 & 13.4 & 1.86 & 0.85 \\
\hline November & 46.5 & 1972 & 0 & $\mathrm{~m}$ & 7.12 & 9.49 & 1.33 & 0.84 \\
\hline December & 31.1 & 1994 & 0 & $\mathrm{~m}$ & 5.03 & 6.63 & 1.32 & 0.59 \\
\hline January & 23.2 & 1994 & 0 & $\mathrm{~m}$ & 3.11 & 4.22 & 1.36 & 0.37 \\
\hline February & 16.1 & 1954 & 0 & $\mathrm{~m}$ & 3.41 & 4.40 & 1.29 & 0.40 \\
\hline March & 1,110 & 1966 & 0 & 1940 & 139 & 215 & 1.55 & 16.4 \\
\hline April & 2,880 & 1979 & 6.51 & 1938 & 428 & 577 & 1.35 & 50.5 \\
\hline May & 2,280 & 1950 & 1.12 & 1939 & 107 & 292 & 2.73 & 12.6 \\
\hline June & 385 & 1968 & 1.35 & 1938 & 59.4 & 74.1 & 1.25 & 7.02 \\
\hline July & 729 & 1993 & 0 & 1940 & 49.7 & 123 & 2.47 & 5.87 \\
\hline August & 515 & 1993 & 0 & $\mathrm{~m}$ & 22.2 & 73.3 & 3.30 & 2.63 \\
\hline September & 326 & 1994 & 0 & $\mathrm{~m}$ & 16.3 & 50.1 & 3.08 & 1.92 \\
\hline Annual & 395 & 1950 & 3.47 & 1937 & 72.8 & 77.6 & 1.07 & 100 \\
\hline
\end{tabular}

Annual flow duration

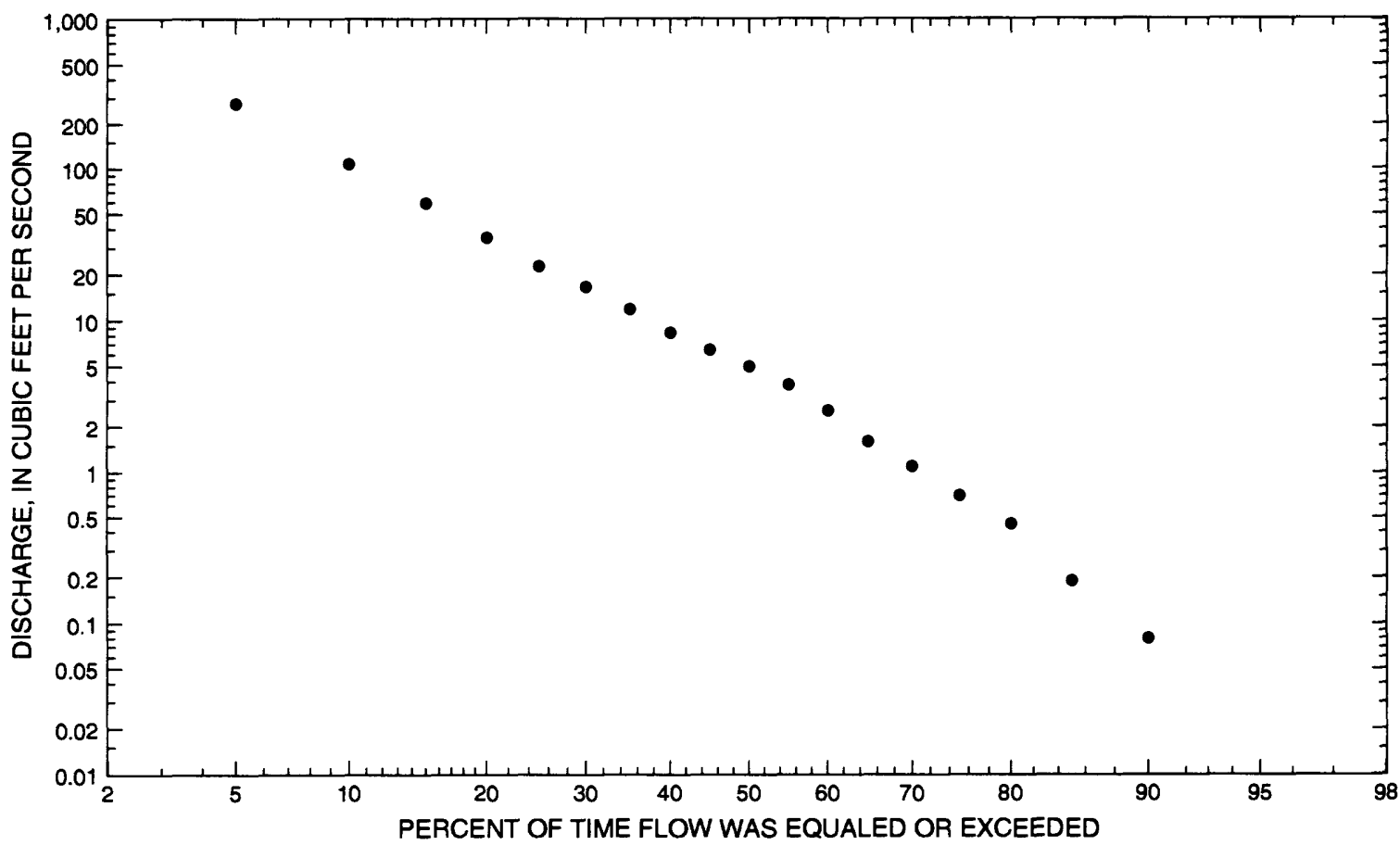




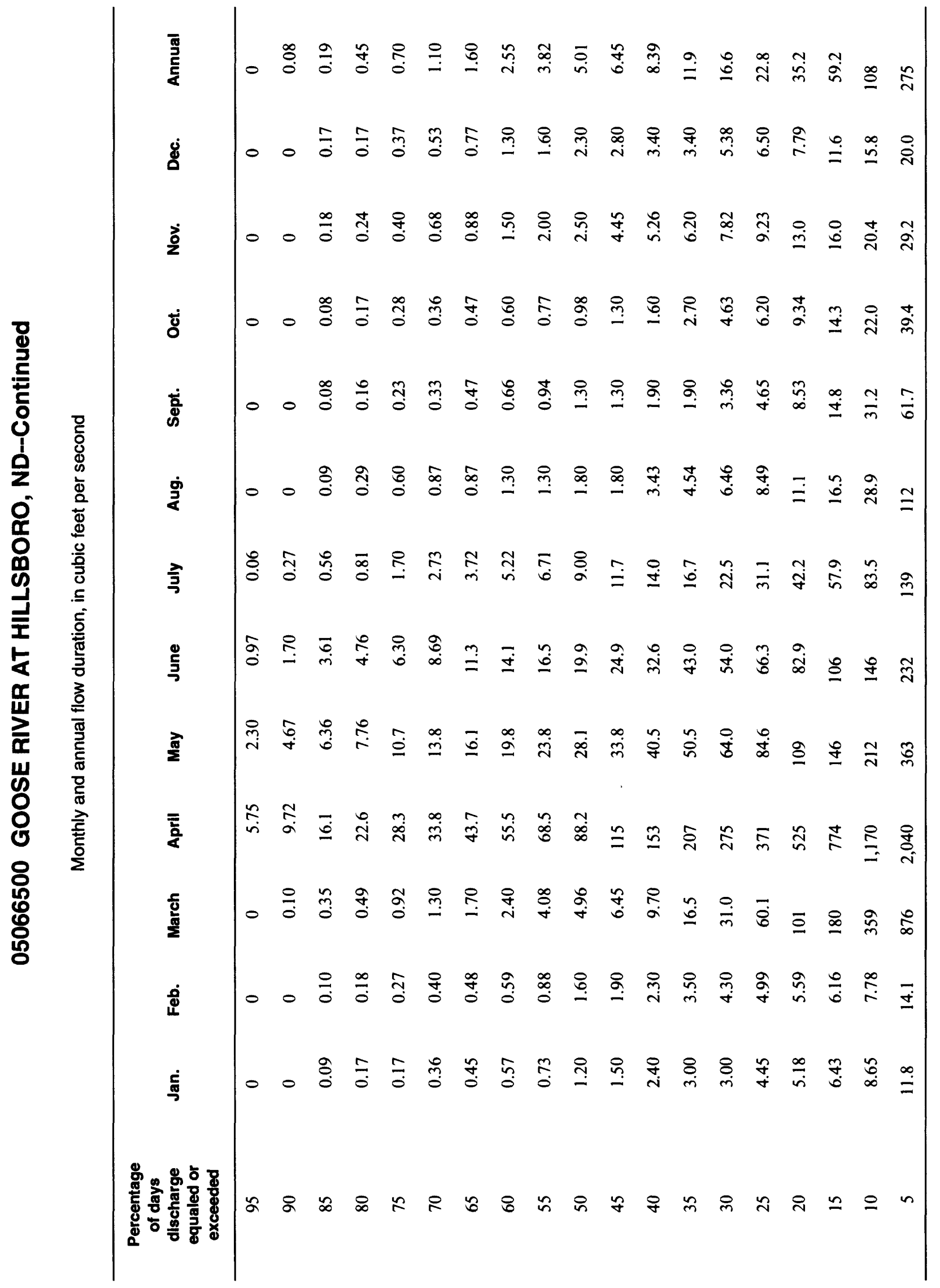




\section{GOOSE RIVER AT HILLSBORO, ND--Continued}

Probability of occurrence of annual high discharges

[ng, statistic not given]

\begin{tabular}{|c|c|c|c|c|c|c|}
\hline \multirow[b]{2}{*}{$\begin{array}{l}\text { Exceedance } \\
\text { probability }\end{array}$} & \multirow[b]{2}{*}{$\begin{array}{l}\text { Recurrence } \\
\text { interval } \\
\text { (years) }\end{array}$} & \multirow[b]{2}{*}{$\begin{array}{c}\text { Maximum } \\
\text { instantaneous } \\
\left(\mathrm{ft}^{3} / \mathrm{s}\right)\end{array}$} & \multicolumn{4}{|c|}{$\begin{array}{c}\text { Maximum mean discharge } \\
\left(\mathrm{ft}^{3} / \mathrm{s}\right)\end{array}$} \\
\hline & & & 3-day period & 7-day period & 15-day period & 30-day period \\
\hline 0.99 & 1.01 & 21.3 & 17.1 & 15.6 & 11.8 & 9.25 \\
\hline 0.95 & 1.05 & 78.2 & 69.7 & 59.6 & 43.3 & 30.5 \\
\hline 0.90 & 1.11 & 148 & 137 & 115 & 81.8 & 55.0 \\
\hline 0.80 & 1.25 & 307 & 292 & 238 & 167 & 108 \\
\hline 0.50 & 2 & 1,070 & 1,020 & 819 & 561 & 347 \\
\hline 0.20 & 5 & 3,110 & 2,840 & 2,280 & 1,540 & 960 \\
\hline 0.10 & 10 & 5,080 & 4,440 & 3,600 & 2,430 & 1,540 \\
\hline 0.04 & 25 & 8,160 & 6,720 & 5,540 & 3,750 & 2,440 \\
\hline 0.02 & 50 & 10,800 & 8,510 & 7,120 & 4,820 & 3,220 \\
\hline 0.01 & 100 & 13,700 & 10,300 & 8,750 & 5,940 & 4,070 \\
\hline 0.005 & 200 & 16,800 & 12,100 & 10,400 & 7,090 & 4,980 \\
\hline 0.002 & 500 & 21,100 & $n g$ & $n g$ & ng & ng \\
\hline
\end{tabular}

Probability of occurrence of annual low discharges

\begin{tabular}{|c|c|c|c|c|c|c|c|c|c|c|}
\hline \multirow[b]{3}{*}{$\begin{array}{l}\text { Non- } \\
\text { exceed- } \\
\text { ance } \\
\text { prob- } \\
\text { ability }\end{array}$} & \multirow[b]{3}{*}{$\begin{array}{c}\text { Recur- } \\
\text { rence } \\
\text { inter- } \\
\text { vai } \\
\text { (years) }\end{array}$} & \multicolumn{9}{|c|}{ Minimum mean discharge $\left(\mathrm{ft}^{3} / \mathrm{s}\right)$} \\
\hline & & \multicolumn{9}{|c|}{ Number of consecutive days } \\
\hline & & 1 & 3 & 7 & 14 & 30 & 60 & 90 & 120 & 183 \\
\hline 0.05 & 20 & 0 & 0 & 0 & 0 & 0 & 0 & 0 & 0 & 0.014 \\
\hline 0.10 & 10 & 0 & 0 & 0 & 0 & 0 & 0 & 0 & 0.016 & 0.176 \\
\hline 0.20 & 5 & 0 & 0 & 0 & 0 & 0 & 0.088 & 0.185 & 0.281 & 0.453 \\
\hline 0.50 & 2 & 0.071 & 0.083 & 0.142 & 0.207 & 0.310 & 0.620 & 0.961 & 1.43 & 1.97 \\
\hline
\end{tabular}




\section{GOOSE RIVER AT HILLSBORO, ND--Continued}

Probability of occurrence of seasonal low discharges

\begin{tabular}{|c|c|c|c|c|c|c|c|c|c|}
\hline \multirow[b]{3}{*}{$\begin{array}{c}\text { Non- } \\
\text { exceedance } \\
\text { probability }\end{array}$} & \multirow[b]{3}{*}{$\begin{array}{c}\text { Recurrence } \\
\text { interval } \\
\text { (years) }\end{array}$} & \multicolumn{8}{|c|}{ Minimum mean discharge $\left(\mathrm{ft}^{3} / \mathrm{s}\right)$} \\
\hline & & \multicolumn{8}{|c|}{ Number of consecutive days } \\
\hline & & 1 & 7 & 14 & 30 & 1 & 7 & 14 & 30 \\
\hline & & \multicolumn{4}{|c|}{ December-January-February } & \multicolumn{4}{|c|}{ March-April-May } \\
\hline 0.05 & 20 & 0 & 0 & 0 & 0 & 0 & 0 & 0 & 0.463 \\
\hline 0.10 & 10 & 0 & 0 & 0 & 0 & 0 & 0 & 0.016 & 1.04 \\
\hline 0.20 & 5 & 0 & 0 & 0.063 & 0.087 & 0.126 & 0.201 & 0.399 & 2.50 \\
\hline \multirow[t]{2}{*}{0.50} & 2 & 0.708 & 0.741 & 0.773 & 0.906 & 1.38 & 1.76 & 2.48 & 11.3 \\
\hline & & \multicolumn{4}{|c|}{ June-July-August } & \multicolumn{4}{|c|}{ September-October-November } \\
\hline 0.05 & 20 & 0 & 0 & 0 & 0.007 & 0 & 0 & 0 & 0 \\
\hline 0.10 & 10 & 0 & 0 & 0 & 0.098 & 0 & 0 & 0 & 0 \\
\hline 0.20 & 5 & 0.028 & 0.061 & 0.130 & 0.319 & 0 & 0 & 0.021 & 0.119 \\
\hline 0.50 & 2 & 0.614 & 0.837 & 1.16 & 2.02 & 0.194 & 0.350 & 0.440 & 0.746 \\
\hline
\end{tabular}




\section{GOOSE RIVER AT HILLSBORO, ND--Continued}

Annual peak discharge and corresponding gage height

$[--$, no data $]$

\begin{tabular}{|c|c|c|c|c|c|c|c|}
\hline $\begin{array}{l}\text { Water } \\
\text { year }\end{array}$ & Date & $\begin{array}{c}\text { Gage } \\
\text { height } \\
\text { (feet) }\end{array}$ & $\begin{array}{c}\text { Peak } \\
\text { discharge } \\
\left(\mathrm{ft}^{3} / \mathrm{s}\right)\end{array}$ & $\begin{array}{l}\text { Water } \\
\text { year }\end{array}$ & Date & $\begin{array}{c}\text { Gage } \\
\text { height } \\
\text { (feet) }\end{array}$ & $\begin{array}{c}\text { Peak } \\
\text { discharge } \\
\left(\mathrm{ft}^{3} / \mathrm{s}\right)\end{array}$ \\
\hline \multicolumn{8}{|c|}{ Annual peak discharge, by year, and corresponding gage height } \\
\hline${ }^{1} 1882$ & Apriil $^{2}$ & -- & 6,700 & 1961 & March 7 & 0.66 & 82.0 \\
\hline${ }^{1} 1897$ & April $^{2}$ & -- & 5,700 & 1962 & April 9 & 8.64 & 2,350 \\
\hline${ }^{1} 1904$ & April $^{2}$ & -- & 5,300 & 1963 & April 8 & 1.24 & 290 \\
\hline${ }^{1} 1916$ & Aprii $^{2}$ & -- & 4,700 & 1964 & June 14 & 3.61 & 1,110 \\
\hline 1931 & April 7 & 4.20 & 1.00 & 1965 & April 13 & 14.01 & 6,800 \\
\hline 1932 & March 3 & 15.14 & 959 & 1966 & March 23 & 12.17 & 3,290 \\
\hline 1933 & March $^{2}$ & 10.40 & 300 & 1967 & April 24 & 7.81 & 2,650 \\
\hline 1934 & April 2 & 4.95 & 107 & 1968 & June 12 & 4.48 & 1,420 \\
\hline 1935 & June 14 & 8.45 & 697 & 1969 & April 12 & 14.19 & 7,640 \\
\hline 1936 & April 16 & 13.06 & 1,660 & 1970 & April 9 & 9.18 & 3,040 \\
\hline 1937 & April 15 & 3.57 & 46.0 & 1971 & April 10 & 4.41 & 1,550 \\
\hline 1938 & March 15 & 4.44 & 104 & 1972 & April 15 & 6.62 & 2,380 \\
\hline 1939 & March 26 & 11.00 & 564 & 1973 & March 15 & 4.05 & 1,280 \\
\hline 1940 & April 17 & 1.66 & 710 & 1974 & April 15 & 9.43 & 3,450 \\
\hline 1941 & April 11 & 2.26 & 1,320 & 1975 & April 19 & 11.11 & 3,810 \\
\hline 1942 & April 6 & 2.27 & 1,140 & 1976 & March 29 & 3.98 & 1,260 \\
\hline 1943 & March 29 & 8.84 & 3,480 & 1977 & April 11 & 2.01 & 86.0 \\
\hline 1944 & April 10 & 1.11 & 304 & 1978 & April 1 & 10.56 & 3,800 \\
\hline 1945 & March 17 & 1.09 & 293 & 1979 & April 21 & 16.76 & 14,800 \\
\hline 1946 & March 22 & 3.22 & 1,300 & 1980 & March 31 & 3.55 & 790 \\
\hline 1947 & April 13 & 5.30 & 1,700 & 1981 & June 25 & 1.83 & 25.6 \\
\hline 1948 & April 16 & 10.63 & 4,180 & 1982 & April 3 & 8.89 & 2,900 \\
\hline 1949 & April 8 & 3.38 & 1,640 & 1983 & March 17 & -- & 1,140 \\
\hline 1950 & April 19 & 14.94 & 9,420 & 1984 & March 29 & 7.99 & 2,660 \\
\hline 1951 & March 31 & 3.48 & 1,130 & 1985 & March 16 & 4.17 & 1,240 \\
\hline 1952 & April 4 & 3.33 & 1,300 & 1986 & March 24 & 4.81 & 1,630 \\
\hline 1953 & July 6 & 1.36 & 408 & 1987 & March 26 & 10.22 & 3,570 \\
\hline 1954 & June 15 & 0.99 & 231 & 1988 & March 28 & 3.86 & 1,060 \\
\hline 1955 & April 3 & 2.44 & 1,220 & 1989 & April 8 & 9.80 & 3,000 \\
\hline 1956 & April 19 & 2.58 & 1,390 & 1990 & April 6 & 2.01 & 94.0 \\
\hline 1957 & September 7 & 1.17 & 200 & 1991 & June 1 & 2.75 & 351 \\
\hline 1958 & July 3 & 0.69 & 88.0 & 1992 & March 8 & 3.69 & 975 \\
\hline 1959 & April 5 & 0.84 & 143 & 1993 & July 30 & 12.90 & 4,360 \\
\hline 1960 & April 9 & 3.37 & 1,360 & 1994 & March 23 & 6.91 & 2,160 \\
\hline \multicolumn{8}{|c|}{ Annual peak discharge, from highest to lowest, and corresponding gage height } \\
\hline 1979 & April 21 & 16.76 & 14,800 & 1948 & April 16 & 10.63 & 4,180 \\
\hline 1950 & April 19 & 14.94 & 9,420 & 1975 & April 19 & 11.11 & 3,810 \\
\hline 1969 & April 12 & 14.19 & 7,640 & 1978 & April 1 & 10.56 & 3,800 \\
\hline 1965 & April 13 & 14.01 & 6,800 & 1987 & March 26 & 10.22 & 3,570 \\
\hline${ }^{1} 1882$ & April $^{2}$ & -- & 6,700 & 1943 & March 29 & 8.84 & 3,480 \\
\hline${ }^{1} 1897$ & April $^{2}$ & -- & 5,700 & 1974 & April 15 & 9.43 & 3,450 \\
\hline${ }^{1} 1904$ & April $^{2}$ & -- & 5,300 & 1966 & March 23 & 12.17 & 3,290 \\
\hline${ }^{\mathrm{l}} 1916$ & April $^{2}$ & -- & 4,700 & 1970 & April 9 & 9.18 & 3,040 \\
\hline 1993 & July 30 & 12.90 & 4,360 & 1989 & April 8 & 9.80 & 3,000 \\
\hline
\end{tabular}




\section{GOOSE RIVER AT HILLSBORO, ND--Continued}

Annual peak discharge and corresponding gage height-Continued

$$
[--, \text { no data }]
$$

\begin{tabular}{|c|c|c|c|c|c|c|c|}
\hline $\begin{array}{l}\text { Water } \\
\text { year }\end{array}$ & Date & $\begin{array}{c}\text { Gage } \\
\text { height } \\
\text { (feet) }\end{array}$ & $\begin{array}{c}\text { Peak } \\
\text { discharge } \\
\left(\mathrm{ft}^{3} / \mathrm{s}\right)\end{array}$ & $\begin{array}{l}\text { Water } \\
\text { year }\end{array}$ & Date & $\begin{array}{l}\text { Gage } \\
\text { helght } \\
\text { (feet) }\end{array}$ & $\begin{array}{c}\text { Peak } \\
\text { discharge } \\
\left(\mathrm{t}^{3} / \mathrm{s}\right)\end{array}$ \\
\hline \multicolumn{8}{|c|}{ Annual peak discharge, from highest to lowest, and corresponding gage height-Continued } \\
\hline 1982 & April 3 & 8.89 & 2,900 & 1988 & March 28 & 3.86 & 1,060 \\
\hline 1984 & March 29 & 7.99 & 2,660 & 1992 & March 8 & 3.69 & 975 \\
\hline 1967 & April 24 & 7.81 & 2,650 & 1932 & March 3 & 15.14 & 959 \\
\hline 1972 & April 15 & 6.62 & 2,380 & 1980 & March 31 & 3.55 & 790 \\
\hline 1962 & April 9 & 8.64 & 2,350 & 1940 & April 17 & 1.66 & 710 \\
\hline 1994 & March 23 & 6.91 & 2,160 & 1935 & June 14 & 8.45 & 697 \\
\hline 1947 & April 13 & 5.30 & 1,700 & 1939 & March 26 & 11.00 & 564 \\
\hline 1936 & April 16 & 13.06 & 1,660 & 1953 & July 6 & 1.36 & 408 \\
\hline 1949 & April 8 & 3.38 & 1,640 & 1991 & June 1 & 2.75 & 351 \\
\hline 1986 & March 24 & 4.81 & 1,630 & 1944 & April 10 & 1.11 & 304 \\
\hline 1971 & April 10 & 4.41 & 1,550 & 1933 & $\mathrm{March}^{2}$ & 10.40 & 300 \\
\hline 1968 & June 12 & 4.48 & 1,420 & 1945 & March 17 & 1.09 & 293 \\
\hline 1956 & April 19 & 2.58 & 1,390 & 1963 & April 8 & 1.24 & 290 \\
\hline 1960 & April 9 & 3.37 & 1,360 & 1954 & June 15 & 0.99 & 231 \\
\hline 1941 & April 11 & 2.26 & 1,320 & 1957 & September 7 & 1.17 & 200 \\
\hline 1946 & March 22 & 3.22 & 1,300 & 1959 & April 5 & 0.84 & 143 \\
\hline 1952 & April 4 & 3.33 & 1,300 & 1934 & April 2 & 4.95 & 107 \\
\hline 1973 & March 15 & 4.05 & 1,280 & 1938 & March 15 & 4.44 & 104 \\
\hline 1976 & March 29 & 3.98 & 1,260 & 1931 & April 7 & 4.20 & 100 \\
\hline 1985 & March 16 & 4.17 & 1,240 & 1990 & April 6 & 2.01 & 94.0 \\
\hline 1955 & April 3 & 2.44 & 1,220 & 1958 & July 3 & 0.69 & 88.0 \\
\hline 1942 & April 6 & 2.27 & 1,140 & 1977 & April 11 & 2.01 & 86.0 \\
\hline 1983 & March 17 & -- & 1,140 & 1961 & March 7 & 0.66 & 82.0 \\
\hline 1951 & March 31 & 3.48 & 1,130 & 1937 & April 15 & 3.57 & 46.0 \\
\hline 1964 & June 14 & 3.61 & 1,110 & 1981 & June 25 & 1.83 & 25.6 \\
\hline
\end{tabular}

${ }^{1}$ Determined by U.S. Army Corps of Engineers; not used in statistics.

${ }^{2}$ Day of month unknown. 


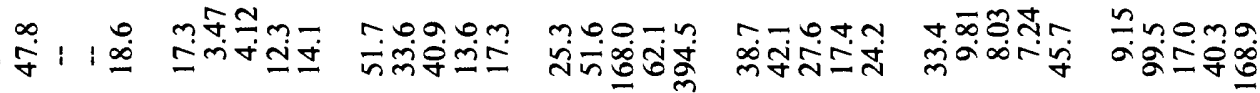

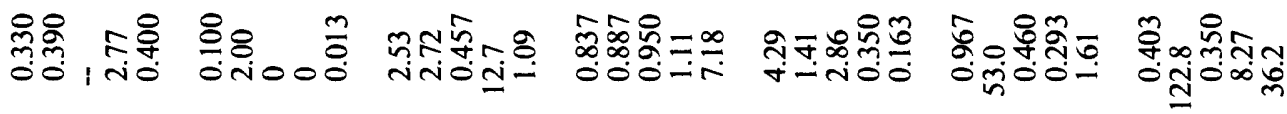

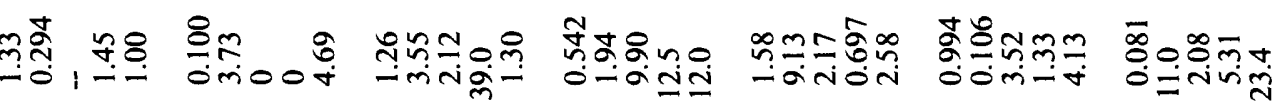

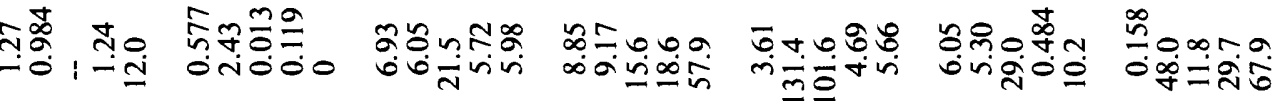

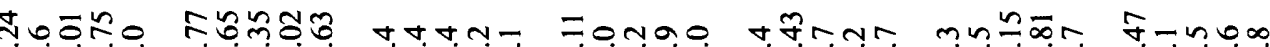

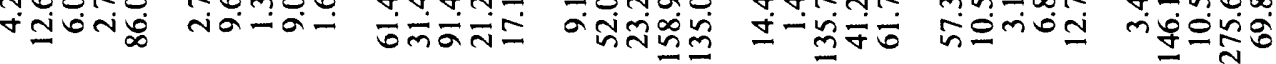

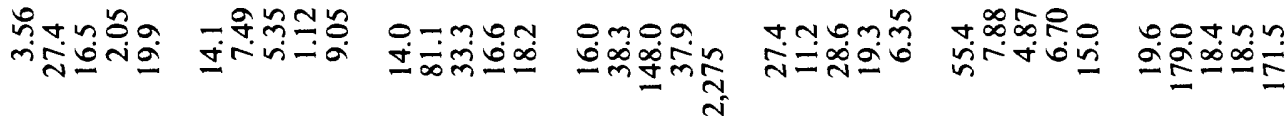

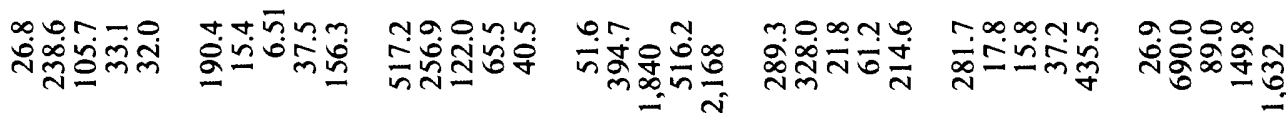

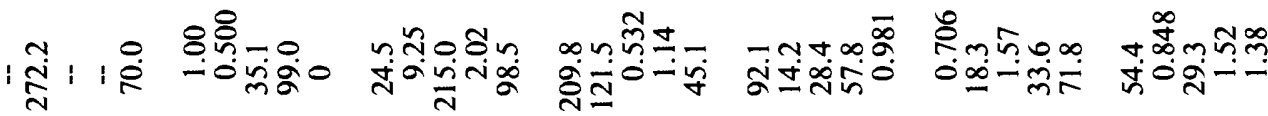
।

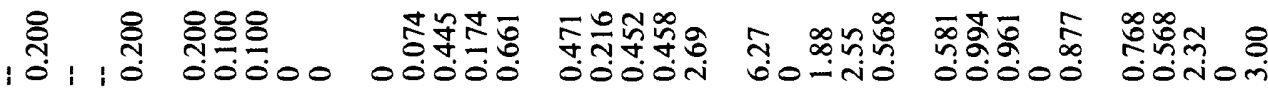

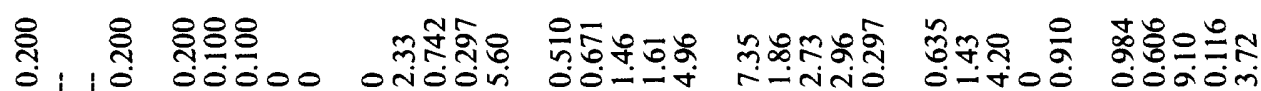

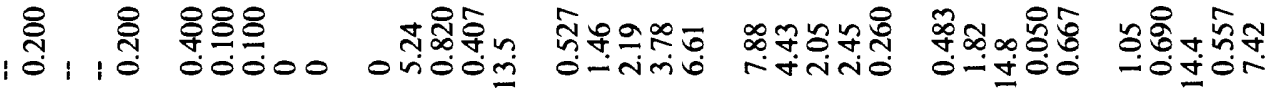

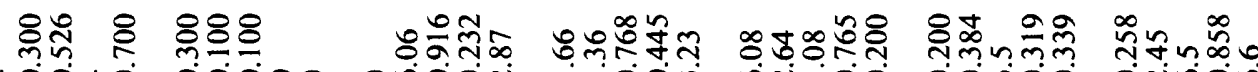
100,00000000004 -iom in-

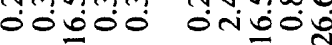

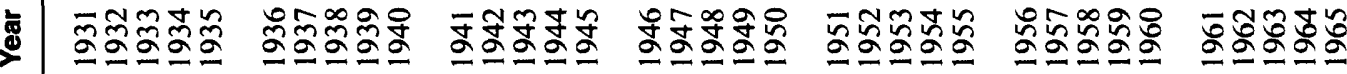




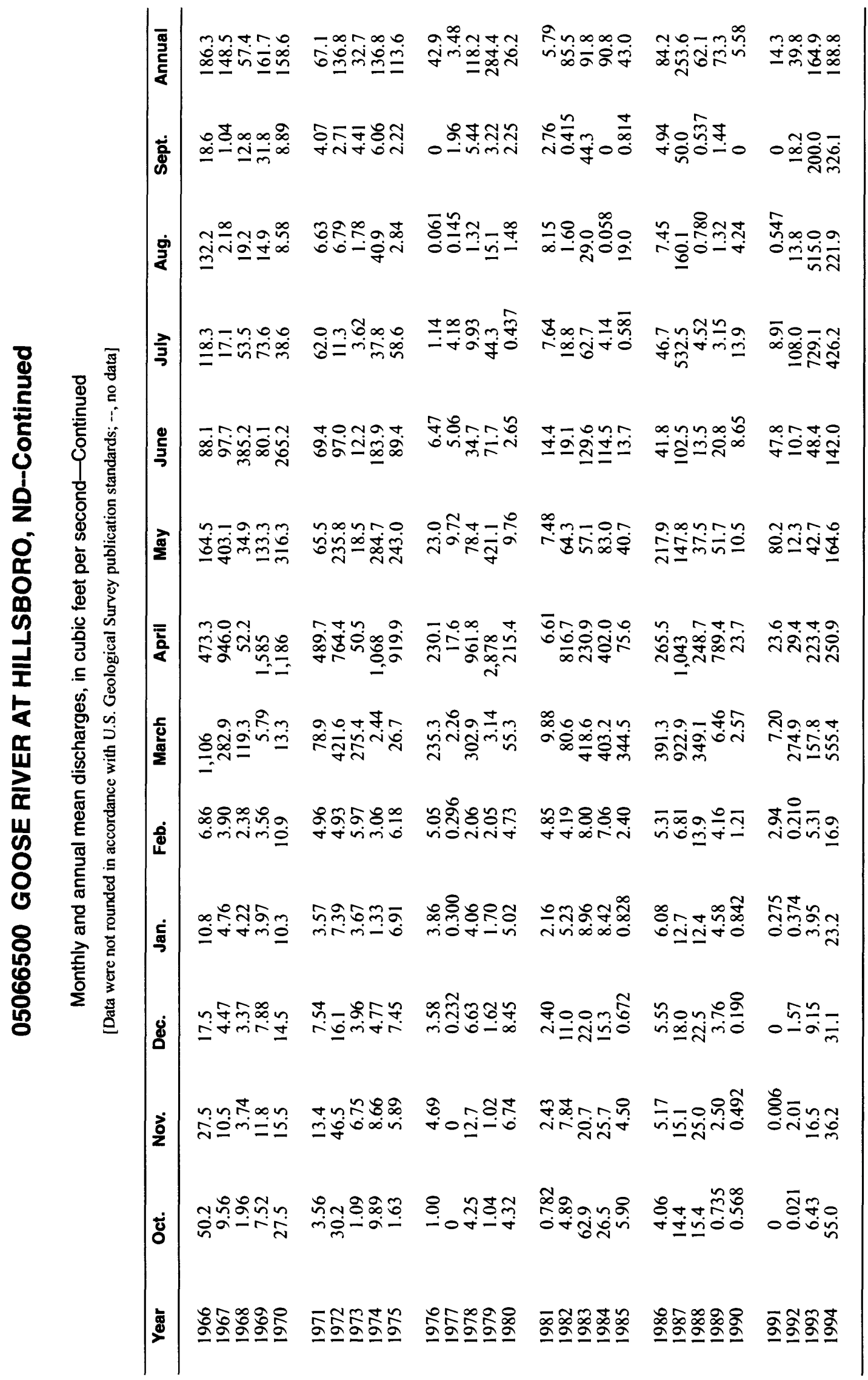




\section{MARSH RIVER NEAR SHELLY, MN}

LOCATION.--Lat $47^{\circ} 24^{\prime} 45^{\prime \prime}$, long $96^{\circ} 45^{\prime} 50^{\prime \prime}, \mathrm{NE}^{1} /{ }_{4} \mathrm{NW}^{1} / 4$ sec.3, T.145 N., R.48 W., Norman County, Hydrologic Unit 09020107, near center of span on downstream truss of bridge, $3.8 \mathrm{mi}$ southeast of Shelly and $10 \mathrm{mi}$ upstream from mouth.

DRAINAGE AREA.--151 $\mathrm{mi}^{2}$.

PERIOD OF RECORD.--March 1944 to September 1983, April 1985 to current year. No winter records since 1989. Monthly discharge only for March 1944, published in WSP 1308. Operated as a highflow partial-record station October 1983 to March 1985.

GAGE.--Water-stage recorder. Datum of gage is $841.14 \mathrm{ft}$ above mean sea level (levels by U.S. Army Corps of Engineers). Prior to Oct. 1, 1965, nonrecording gage at datum $3.0 \mathrm{ft}$ higher. Oct. 1, 1965, to May 17, 1989, nonrecording gage at present site and datum.

EXTREMES FOR PERIOD OF RECORD.--Maximum discharge, 4,880 $\mathrm{ft}^{3} / \mathrm{s}$, Apr. 19, 1979, gage height, $23.36 \mathrm{ft}$, from floodmark; no flow at times.

Annual mean discharge

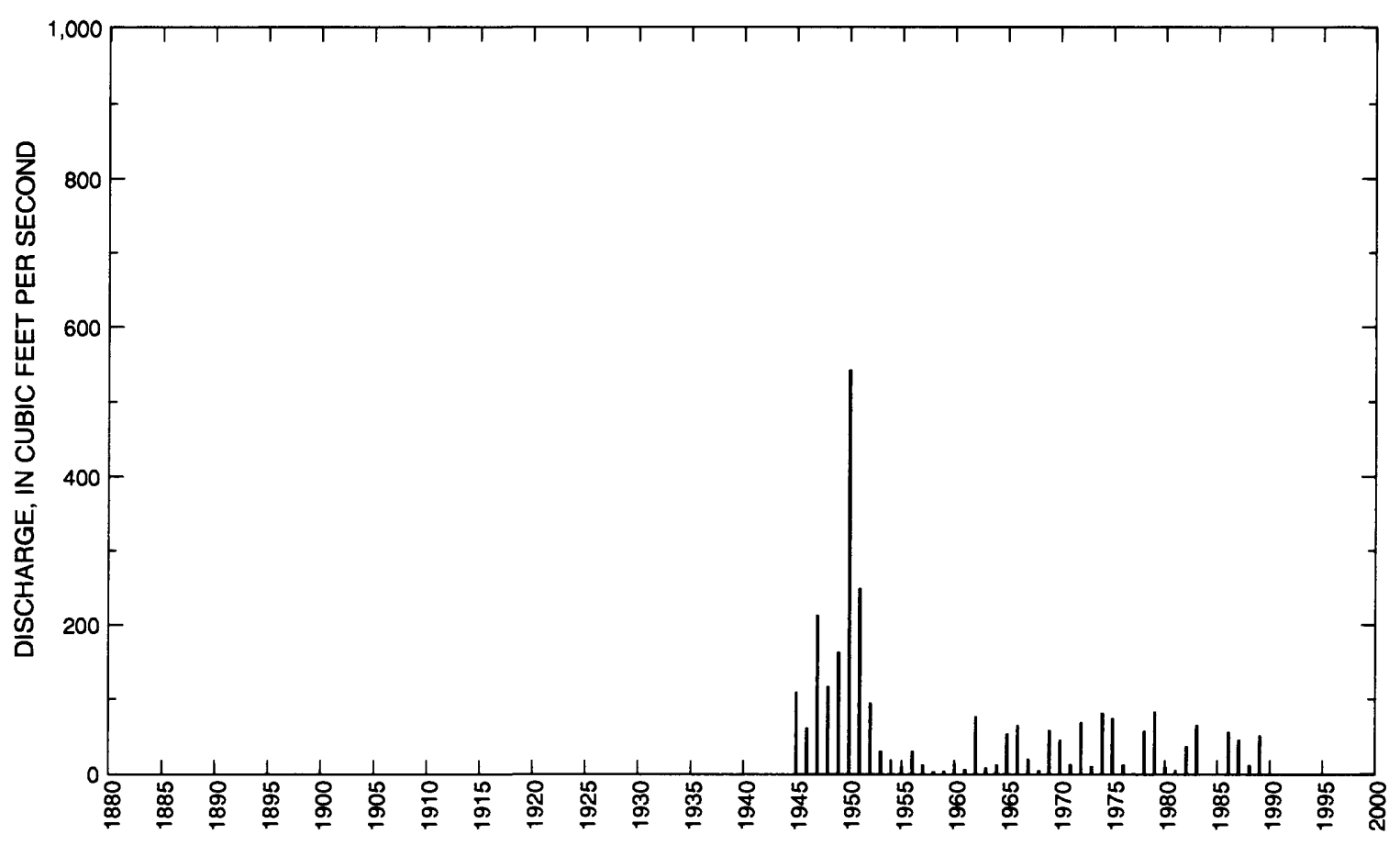




\section{MARSH RIVER NEAR SHELLY, MN--Continued}

Statistics of monthly and annual mean discharges

[m, more than 1 year of occurrence]

\begin{tabular}{|c|c|c|c|c|c|c|c|c|}
\hline \multirow[b]{2}{*}{ Month } & \multicolumn{2}{|c|}{ Maximum } & \multicolumn{2}{|c|}{ Minimum } & \multicolumn{4}{|c|}{ Mean } \\
\hline & $\begin{array}{c}\text { Discharge } \\
\left(\mathrm{ft}^{3} / \mathrm{s}\right)\end{array}$ & $\begin{array}{c}\text { Water year } \\
\text { of } \\
\text { occurrence }\end{array}$ & $\begin{array}{c}\text { Discharge } \\
\left(\mathrm{ft}^{3} / \mathrm{s}\right)\end{array}$ & $\begin{array}{c}\text { Water year } \\
\text { of } \\
\text { occurrence }\end{array}$ & $\begin{array}{c}\text { Discharge } \\
\left(\mathrm{ft}^{3} / \mathrm{s}\right)\end{array}$ & $\begin{array}{c}\text { Standard } \\
\text { deviation } \\
\left(\mathrm{ft}^{3} / \mathrm{s}\right)\end{array}$ & $\begin{array}{l}\text { Coeffi- } \\
\text { cient of } \\
\text { variation }\end{array}$ & $\begin{array}{l}\text { Percentage } \\
\text { of annual } \\
\text { discharge }\end{array}$ \\
\hline October & 130 & 1952 & 0 & $\mathrm{~m}$ & 12.4 & 29.0 & 2.34 & 1.75 \\
\hline November & 102 & 1952 & 0 & $\mathrm{~m}$ & 10.7 & 24.8 & 2.32 & 1.51 \\
\hline December & 77.1 & 1951 & 0 & $\mathrm{~m}$ & 5.60 & 16.1 & 2.87 & 0.79 \\
\hline January & 64.5 & 1951 & 0 & $\mathrm{~m}$ & 3.79 & 12.0 & 3.16 & 0.53 \\
\hline February & 62.1 & 1951 & 0 & $\mathrm{~m}$ & 3.29 & 10.8 & 3.29 & 0.46 \\
\hline March & 437 & 1945 & 0 & $\mathrm{~m}$ & 69.5 & 115 & 1.66 & 9.80 \\
\hline April & 1,270 & 1947 & 0.780 & 1991 & 289 & 360 & 1.25 & 40.72 \\
\hline May & 2,620 & 1950 & 1.35 & 1958 & 126 & 381 & 3.03 & 17.8 \\
\hline June & 1,030 & 1950 & 0 & 1980 & 81.6 & 182 & 2.23 & 11.5 \\
\hline July & 821 & 1950 & 0 & $\mathrm{~m}$ & 73.6 & 154 & 2.10 & 10.4 \\
\hline August & 363 & 1949 & 0 & $\mathrm{~m}$ & 20.8 & 58.9 & 2.84 & 2.93 \\
\hline September & 144 & 1944 & 0 & $\mathrm{~m}$ & 13.2 & 31.4 & 2.38 & 1.86 \\
\hline Annual & 543 & 1950 & 1.24 & 1977 & 63.3 & 92.7 & 1.46 & 100 \\
\hline
\end{tabular}

Annual flow duration

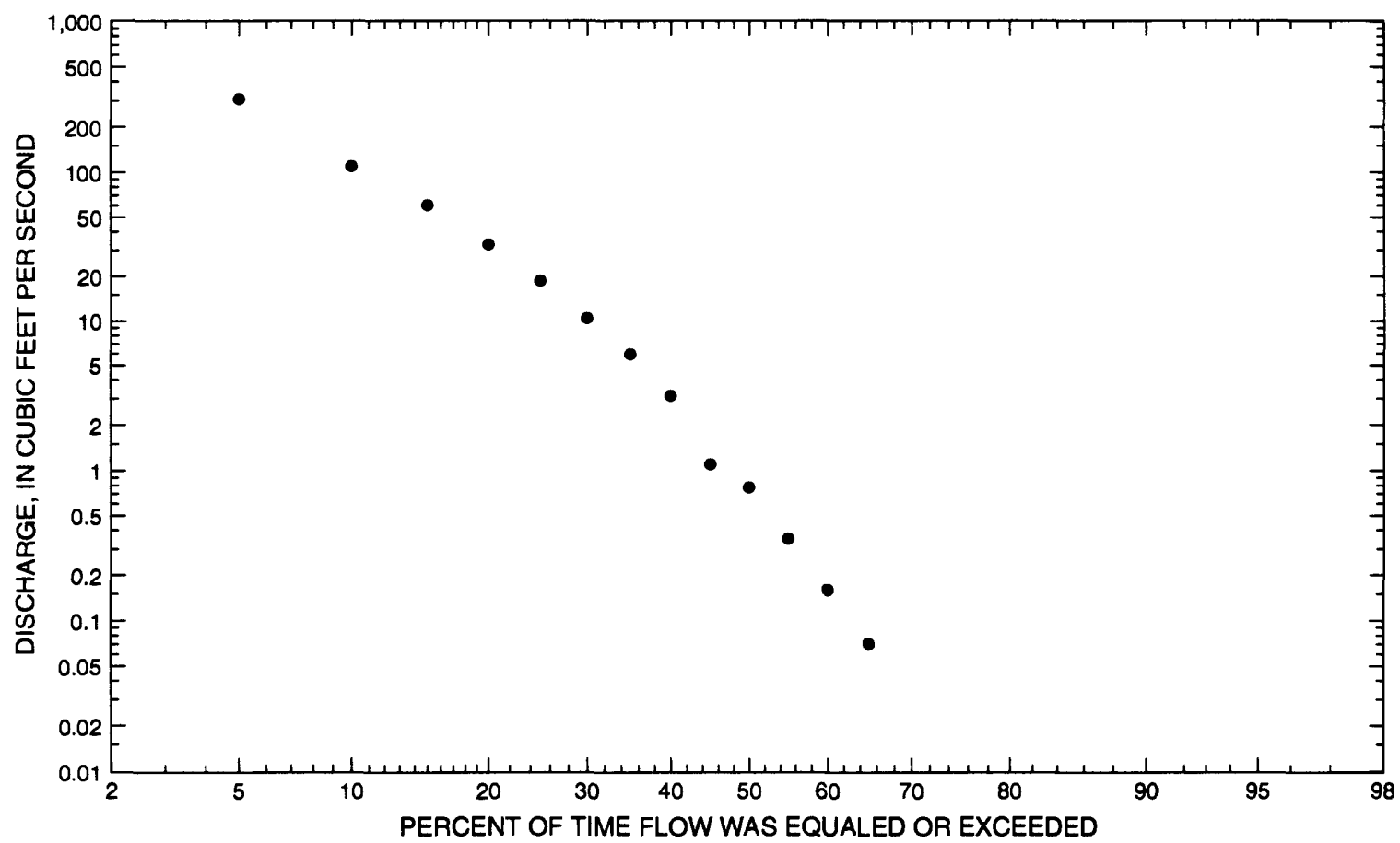




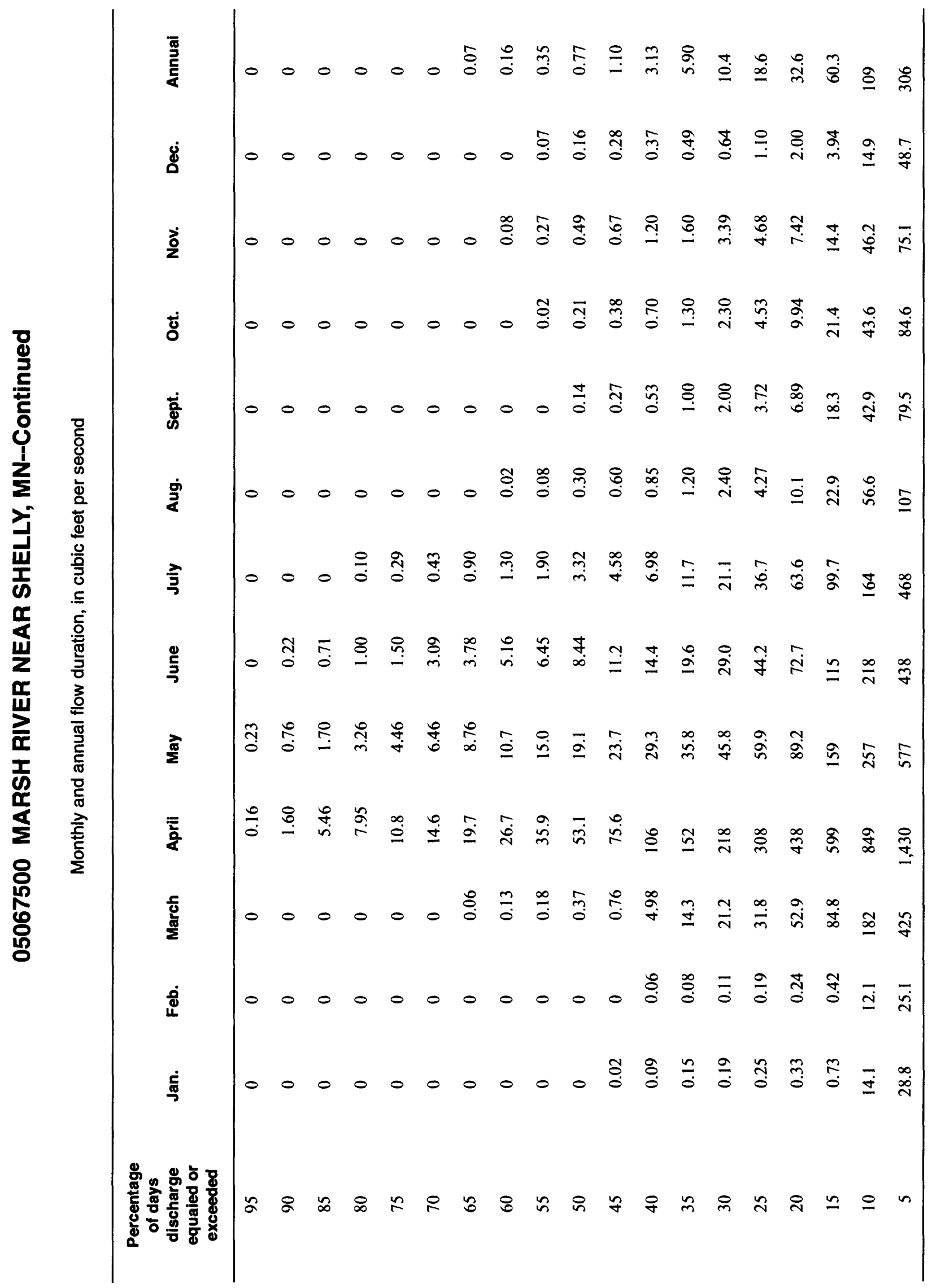




\section{MARSH RIVER NEAR SHELLY, MN--Continued}

Probability of occurrence of annual high discharges

[ng, statistic not given]

\begin{tabular}{|c|c|c|c|c|c|c|}
\hline \multirow[b]{2}{*}{$\begin{array}{l}\text { Exceedance } \\
\text { probability }\end{array}$} & \multirow[b]{2}{*}{$\begin{array}{l}\text { Recurrence } \\
\text { intervai } \\
\text { (years) }\end{array}$} & \multirow[b]{2}{*}{$\begin{array}{c}\text { Maximum } \\
\text { instantaneous } \\
\left(\mathrm{ft}^{3} / \mathrm{s}\right)\end{array}$} & \multicolumn{4}{|c|}{$\begin{array}{l}\text { Maximum mean discharge } \\
\left(\mathrm{ft}^{3} / \mathrm{s}\right)\end{array}$} \\
\hline & & & 3-day period & 7-day period & 15-day period & 30-day period \\
\hline 0.99 & 1.01 & 37.8 & 20.9 & 17.5 & 13.4 & 8.17 \\
\hline 0.95 & 1.05 & 112 & 72.5 & 56.6 & 40.8 & 25.8 \\
\hline 0.90 & 1.11 & 191 & 132 & 101 & 70.9 & 45.6 \\
\hline 0.80 & 1.25 & 346 & 258 & 193 & 133 & 87.2 \\
\hline 0.50 & 2 & 946 & 791 & 587 & 397 & 267 \\
\hline 0.20 & 5 & 2,180 & 1,980 & 1,500 & 1,030 & 706 \\
\hline 0.10 & 10 & 3,180 & 2,960 & 2,310 & 1,600 & 1,110 \\
\hline 0.04 & 25 & 4,530 & 4,320 & 3,480 & 2,460 & 1,720 \\
\hline 0.02 & 50 & 5,560 & 5,360 & 4,430 & 3,190 & 2,240 \\
\hline 0.01 & 100 & 6,600 & 6,390 & 5,420 & 3,970 & 2,790 \\
\hline 0.005 & 200 & 7,620 & 7,410 & 6,430 & 4,790 & 3,380 \\
\hline 0.002 & 500 & 8,940 & ng & ng & ng & ng \\
\hline
\end{tabular}

Probability of occurrence of annual low discharges

\begin{tabular}{|c|c|c|c|c|c|c|c|c|c|c|}
\hline \multirow{3}{*}{$\begin{array}{l}\text { Non- } \\
\text { exceed- } \\
\text { ance } \\
\text { prob- } \\
\text { abiilty }\end{array}$} & \multirow{3}{*}{$\begin{array}{l}\text { Recur- } \\
\text { rence } \\
\text { inter- } \\
\text { vai } \\
\text { (years) }\end{array}$} & \multicolumn{9}{|c|}{ Minimum mean discharge $\left(\mathrm{ft}^{3} / \mathrm{s}\right)$} \\
\hline & & \multicolumn{9}{|c|}{ Number of consecutive days } \\
\hline & & 1 & 3 & 7 & 14 & 30 & 60 & 90 & 120 & 183 \\
\hline 0.05 & 20 & 0 & 0 & 0 & 0 & 0 & 0 & 0 & 0 & 0 \\
\hline 0.10 & 10 & 0 & 0 & 0 & 0 & 0 & 0 & 0 & 0 & 0 \\
\hline 0.20 & 5 & 0 & 0 & 0 & 0 & 0 & 0 & 0 & 0 & 0 \\
\hline 0.50 & 2 & 0 & 0 & 0 & 0 & 0 & 0 & 0.019 & 0.119 & $0.41 \mathrm{C}$ \\
\hline
\end{tabular}




\section{MARSH RIVER NEAR SHELLY, MN--Continued}

Probability of occurrence of seasonal low discharges

\begin{tabular}{|c|c|c|c|c|c|c|c|c|c|}
\hline \multirow[b]{3}{*}{$\begin{array}{c}\text { Non- } \\
\text { exceedance } \\
\text { probability }\end{array}$} & \multirow[b]{3}{*}{$\begin{array}{c}\text { Recurrence } \\
\text { interval } \\
\text { (years) }\end{array}$} & \multicolumn{8}{|c|}{ Minimum mean discharge $\left(\mathrm{ft}^{3} / \mathrm{s}\right)$} \\
\hline & & \multicolumn{8}{|c|}{ Number of consecutive days } \\
\hline & & 1 & 7 & 14 & 30 & 1 & 7 & 14 & 30 \\
\hline & & \multicolumn{4}{|c|}{ December-January-February } & \multicolumn{4}{|c|}{ March-April-May } \\
\hline 0.05 & 20 & 0 & 0 & 0 & 0 & 0 & 0 & 0 & 0 \\
\hline 0.10 & 10 & 0 & 0 & 0 & 0 & 0 & 0 & 0 & 0 \\
\hline 0.20 & 5 & 0 & 0 & 0 & 0 & 0 & 0 & 0 & 0.182 \\
\hline \multirow[t]{2}{*}{0.50} & 2 & 0 & 0 & 0 & 0 & 0 & 0.013 & 0.140 & 3.98 \\
\hline & & \multicolumn{4}{|c|}{ June-July-August } & \multicolumn{4}{|c|}{ September-October-November } \\
\hline 0.05 & 20 & 0 & 0 & 0 & 0 & 0 & 0 & 0 & 0 \\
\hline 0.10 & 10 & 0 & 0 & 0 & 0 & 0 & 0 & 0 & 0 \\
\hline 0.20 & 5 & 0 & 0 & 0 & 0 & 0 & 0 & 0 & 0 \\
\hline 0.50 & 2 & 0 & 0 & 0.039 & 0.448 & 0 & 0 & 0 & 0.008 \\
\hline
\end{tabular}




\section{MARSH RIVER NEAR SHELLY, MN--Continued}

Annual peak discharge and corresponding gage height

$[--$, no data]

\begin{tabular}{|c|c|c|c|c|c|c|c|}
\hline $\begin{array}{l}\text { Water } \\
\text { year }\end{array}$ & Date & $\begin{array}{c}\text { Gage } \\
\text { height } \\
\text { (feet) }\end{array}$ & $\begin{array}{c}\text { Peak } \\
\text { discharge } \\
\left(\mathrm{ft}^{3} / \mathrm{s}\right)\end{array}$ & $\begin{array}{l}\text { Water } \\
\text { year }\end{array}$ & Date & $\begin{array}{c}\text { Gage } \\
\text { height } \\
\text { (feet) }\end{array}$ & $\begin{array}{c}\text { Peak } \\
\text { discharge } \\
\left(\mathrm{ft}^{3} / \mathrm{s}\right)\end{array}$ \\
\hline \multicolumn{8}{|c|}{ Annual peak discharge, by year, and corresponding gage height } \\
\hline 1944 & July 11 & 8.64 & 1,030 & 1970 & April 9 & 13.38 & 1,320 \\
\hline 1945 & March 18 & 8.48 & 1,000 & 1971 & April 1 & 10.11 & 619 \\
\hline 1946 & March 23 & - & 1,510 & 1972 & April 15 & 16.26 & 2,070 \\
\hline 1947 & April 14 & 17.80 & 4,150 & 1973 & March 16 & 9.30 & 366 \\
\hline 1948 & April 13 & -- & 1,040 & 1974 & April 15 & 18.87 & 2,460 \\
\hline 1949 & June 3 & 9.83 & 1,260 & 1975 & April 19 & 17.81 & 2,330 \\
\hline 1950 & May 11 & 18.96 & 4,660 & 1976 & March 31 & 11.32 & 785 \\
\hline 1951 & April 8 & 12.56 & 2,100 & 1977 & April 10 & 4.13 & 42 \\
\hline 1952 & July 21 & 7.80 & 979 & 1978 & April 9 & 19.22 & 2,240 \\
\hline 1953 & June 17 & 4.11 & 389 & 1979 & April 19 & 23.36 & 4,880 \\
\hline 1954 & April 13 & 4.63 & 376 & 1980 & April 3 & 9.99 & 615 \\
\hline 1955 & April 5 & 4.10 & 289 & 1981 & May 23 & 11.03 & 896 \\
\hline 1956 & April 13 & 12.50 & 1,960 & 1982 & April 2 & 13.06 & 1,070 \\
\hline 1957 & June 23 & - & 304 & 1983 & March 8 & 13.84 & 1,240 \\
\hline 1958 & July 10 & 1.48 & 47 & 1984 & June 11 & 16.17 & 2,260 \\
\hline 1959 & March 31 & 2.42 & 96 & 1985 & May 13 & 13.23 & 1,380 \\
\hline 1960 & April 7 & 6.04 & 492 & 1986 & March 30 & 14.59 & 1,720 \\
\hline 1961 & March 9 & 3.51 & 100 & 1987 & July 24 & 15.80 & 1,730 \\
\hline 1962 & June 11 & 9.87 & 1,240 & 1988 & March 26 & 7.86 & 250 \\
\hline 1963 & April 8 & 4.60 & 274 & 1989 & April 7 & 21.18 & 3,490 \\
\hline 1964 & April 22 & 5.41 & 450 & 1990 & June 2 & 6.65 & 254 \\
\hline 1965 & April 13 & 16.87 & 3,120 & 1991 & May 23 & 11.88 & 1,120 \\
\hline 1966 & April 2 & 13.85 & 1,460 & 1992 & March 8 & 10.36 & 430 \\
\hline 1967 & March 30 & 11.54 & 866 & 1993 & April 2 & 13.78 & 660 \\
\hline 1968 & March 27 & 7.35 & 221 & 1994 & September 19 & 11.50 & 995 \\
\hline 1969 & April 12 & 22.28 & 3,910 & & & & \\
\hline \multicolumn{8}{|c|}{ Annual peak discharge, from highest to lowest, and corresponding gage height } \\
\hline 1979 & April 19 & 23.36 & 4,880 & 1970 & April 9 & 13.38 & 1,320 \\
\hline 1950 & May 11 & 18.96 & 4,660 & 1962 & June 11 & 9.87 & 1,240 \\
\hline 1969 & April 12 & 22.28 & 3,910 & 1983 & March 8 & 13.84 & 1,240 \\
\hline 1989 & April 7 & 21.18 & 3,490 & 1991 & May 23 & 11.88 & 1,120 \\
\hline 1965 & April 13 & 16.87 & 3,120 & 1982 & April 2 & 13.06 & 1,070 \\
\hline 1974 & April 15 & 18.87 & 2,460 & 1948 & April 13 & -. & 1,040 \\
\hline 1975 & April 19 & 17.81 & 2,330 & 1944 & July 11 & 8.64 & 1,030 \\
\hline 1984 & June 11 & 16.17 & 2,260 & 1945 & March 18 & 8.48 & 1,000 \\
\hline 1978 & April 9 & 19.22 & 2,240 & 1994 & September 19 & 11.50 & 995 \\
\hline 1951 & April 8 & 12.56 & 2,100 & 1952 & July 21 & 7.80 & 979 \\
\hline 1972 & April 15 & 16.26 & 2,070 & 1981 & May 23 & 11.03 & 896 \\
\hline 1956 & April 13 & 12.50 & 1,960 & 1967 & March 30 & 11.54 & 866 \\
\hline 1987 & July 24 & 15.80 & 1,730 & 1976 & March 31 & 11.32 & 785 \\
\hline 1986 & March 30 & 14.59 & 1,720 & 1993 & April 2 & 13.78 & 660 \\
\hline 1946 & March 23 & - & 1,510 & 1971 & April 1 & 10.11 & 619 \\
\hline 1966 & April 2 & 13.85 & 1,460 & 1980 & April 3 & 9.99 & 615 \\
\hline 1985 & May 13 & 13.23 & 1,380 & 1960 & April 7 & 6.04 & 492 \\
\hline
\end{tabular}




\section{MARSH RIVER NEAR SHELLY, MN--Continued}

Annual peak discharge and corresponding gage height-Continued

$$
[-, \text { no data }]
$$

\begin{tabular}{|c|c|c|c|c|c|c|c|}
\hline $\begin{array}{c}\text { Water } \\
\text { year }\end{array}$ & Date & $\begin{array}{c}\text { Gage } \\
\text { height } \\
\text { (feet) }\end{array}$ & $\begin{array}{c}\text { Peak } \\
\text { discharge } \\
\left(\mathrm{ft}^{3} / \mathrm{s}\right)\end{array}$ & $\begin{array}{c}\text { Water } \\
\text { year }\end{array}$ & Date & $\begin{array}{c}\text { Gage } \\
\text { height } \\
\text { (feet) }\end{array}$ & $\begin{array}{c}\text { Peak } \\
\text { discharge } \\
\left(\mathrm{ft}^{3} / \mathrm{s}\right)\end{array}$ \\
\hline \multicolumn{8}{|c|}{ Annual peak discharge, from highest to lowest, and corresponding gage height--Continued } \\
\hline 1964 & April 22 & 5.41 & 450 & 1990 & June 2 & 6.65 & 254 \\
\hline 1992 & March 8 & 10.36 & 430 & 1988 & March 26 & 7.86 & 250 \\
\hline 1953 & June 17 & 4.11 & 389 & 1968 & March 27 & 7.35 & 221 \\
\hline 1954 & April 13 & 4.63 & 376 & 1961 & March 9 & 3.51 & 100 \\
\hline 1973 & March 16 & 9.30 & 366 & 1959 & March 31 & 2.42 & 96 \\
\hline 1957 & June 23 & -- & 304 & 1958 & July 10 & 1.48 & 47 \\
\hline 1955 & April 5 & 4.10 & 289 & 1977 & April 10 & 4.13 & 42 \\
\hline 1963 & April 8 & 4.60 & 274 & & & & \\
\hline
\end{tabular}




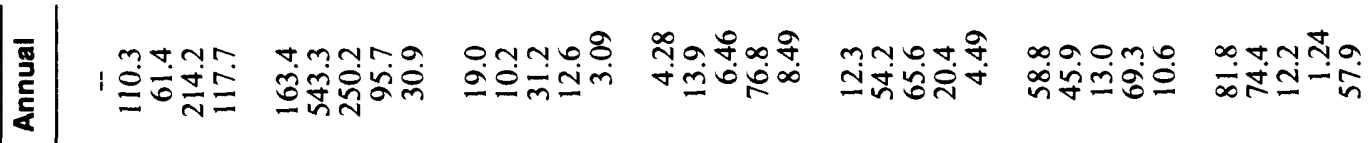

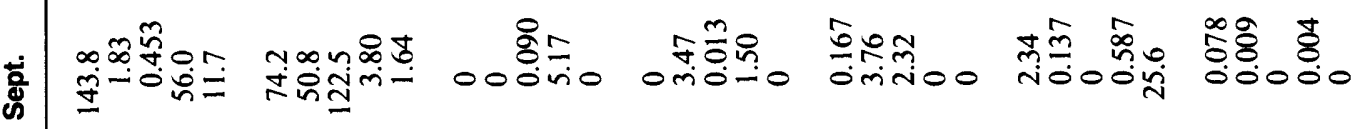

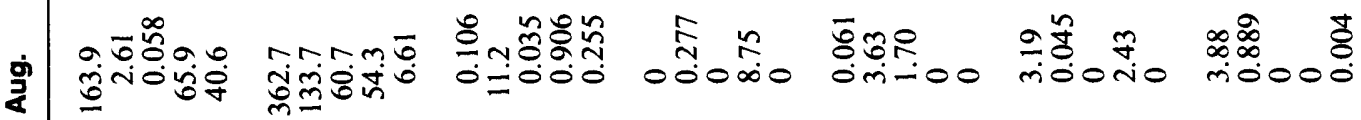

豙

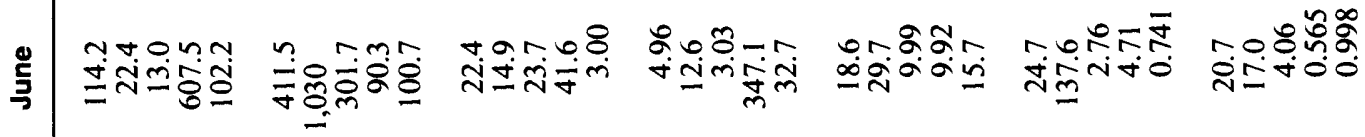

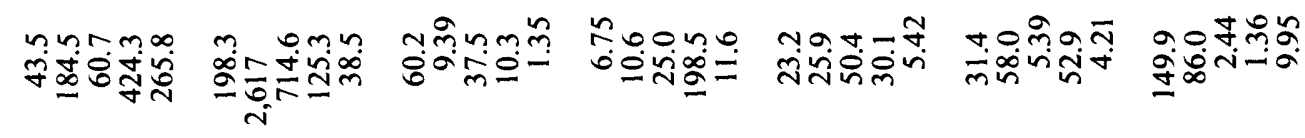

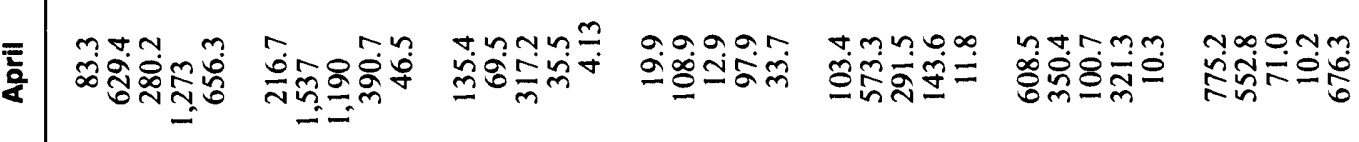

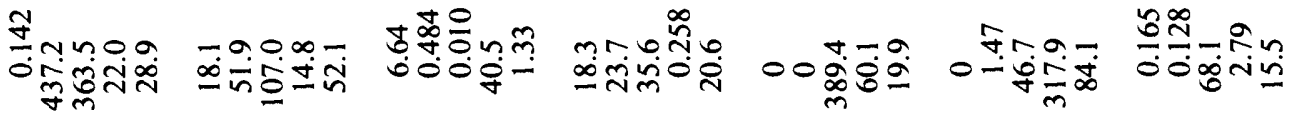
în i injơn

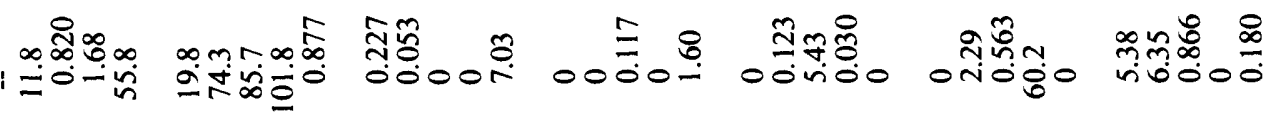
.

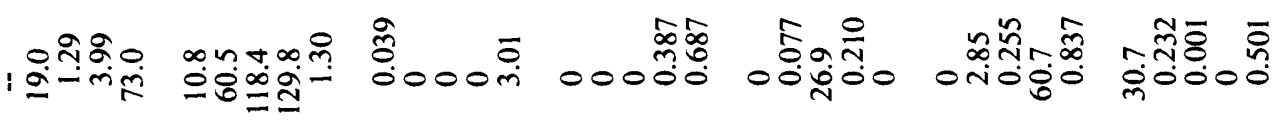

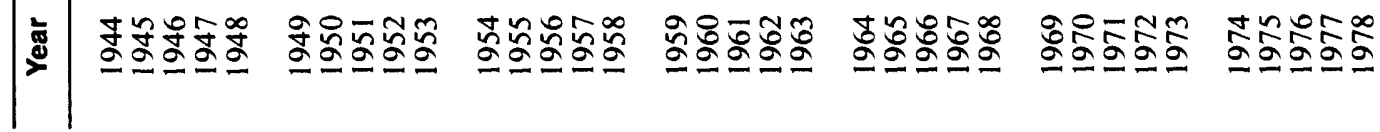




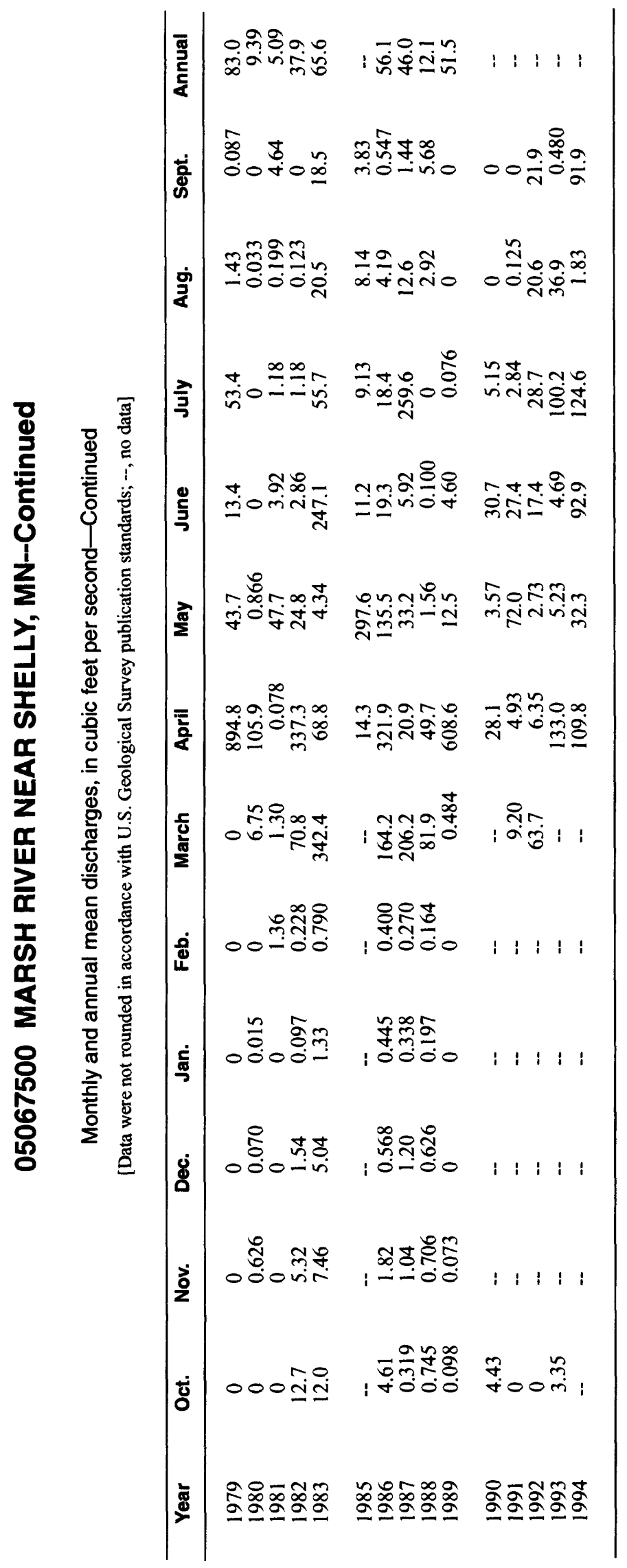




\section{SAND HILL RIVER AT BELTRAMI, MN}

LOCATION.--Lat $47^{\circ} 32^{\prime} 50^{\prime \prime}$, long $96^{\circ} 32^{\prime} 00^{\prime \prime}$, in $\mathrm{NE}^{1 / 1} \mathrm{SW}^{1 / 1} 4 \mathrm{sec} .16, \mathrm{~T} .147 \mathrm{~N}$., R.46 W., on upstream side of bridge abutment on U.S. Highway 75 in Beltrami, $150 \mathrm{ft}$ upstream from Great Northern Railway bridge and $0.25 \mathrm{mi}$ north of post office.

DRAINAGE AREA.--324 $\mathrm{mi}^{2}$ includes that of Sand Hill ditch.

PERIOD OF RECORD.--April to November 1943, March 1944 to September 1958. Monthly discharge only for some periods, published in WSP 1308.

GAGE.--Staff gage. Datum of gage is $896.80 \mathrm{ft}$ above mean sea level, adjustment of 1912 (levels by U.S. Army Corps of Engineers). Prior to Aug. 28, 1944, reference point at same site and datum.

EXTREMES FOR PERIOD OF RECORD.--Maximum discharge, $291 \mathrm{ft}^{3} / \mathrm{s}$, Apr. 19, 1950, gage height, $5.97 \mathrm{ft}$, from floodmark; no flow for many days most years.

Annual mean discharge

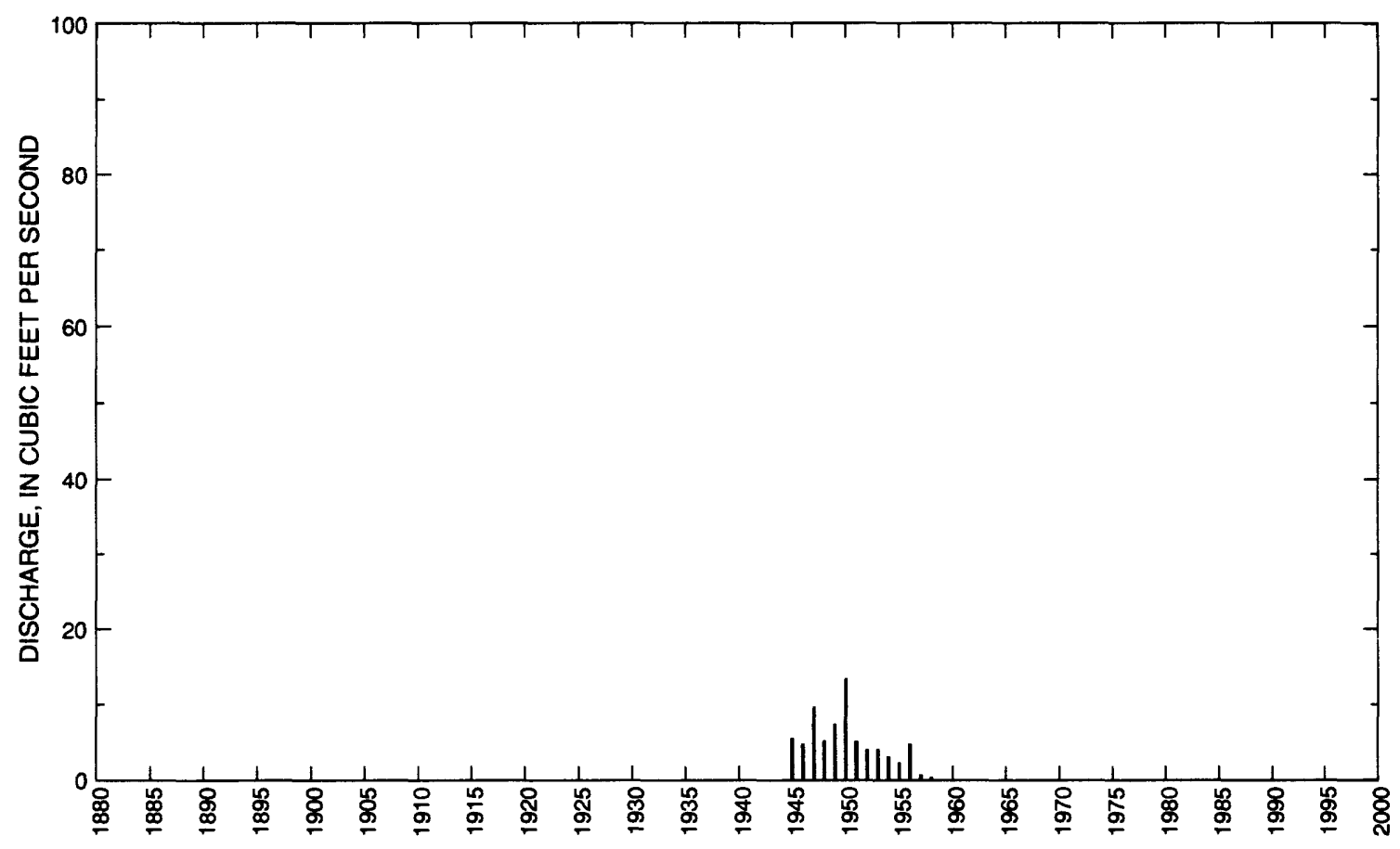




\section{SAND HILL RIVER AT BELTRAMI, MN--Continued}

Statistics of monthly and annual mean discharges

[m, more than 1 year of occurrence]

\begin{tabular}{|c|c|c|c|c|c|c|c|c|}
\hline \multirow[b]{2}{*}{ Month } & \multicolumn{2}{|c|}{ Maximum } & \multicolumn{2}{|c|}{ Minimum } & \multicolumn{4}{|c|}{ Mean } \\
\hline & $\begin{array}{c}\text { Discharge } \\
\left(\mathrm{ft}^{3} / \mathrm{s}\right)\end{array}$ & $\begin{array}{c}\text { Water year } \\
\text { of } \\
\text { occurrence }\end{array}$ & $\begin{array}{c}\text { Discharge } \\
\left(\mathrm{ft}^{3} / \mathrm{s}\right)\end{array}$ & $\begin{array}{c}\text { Water year } \\
\text { of } \\
\text { occurrence }\end{array}$ & $\begin{array}{c}\text { Discharge } \\
\left(\mathrm{ft}^{3} / \mathrm{s}\right)\end{array}$ & $\begin{array}{l}\text { Standard } \\
\text { deviation } \\
\left(\mathrm{ft}^{3} / \mathbf{s}\right)\end{array}$ & $\begin{array}{l}\text { Coeffi- } \\
\text { cient of } \\
\text { variation }\end{array}$ & $\begin{array}{c}\text { Percentage } \\
\text { of annual } \\
\text { discharge }\end{array}$ \\
\hline October & 5.32 & 1947 & 0.071 & 1957 & 2.09 & 1.67 & 0.80 & 3.52 \\
\hline November & 5.43 & 1950 & 0.500 & 1956 & 1.88 & 1.26 & 0.67 & 3.17 \\
\hline December & 1.43 & 1945 & 0 & $\mathrm{~m}$ & 0.650 & 0.47 & 0.73 & 1.09 \\
\hline January & 0.877 & 1947 & 0 & $\mathrm{~m}$ & 0.310 & 0.34 & 1.12 & 0.52 \\
\hline February & 0.804 & 1947 & 0 & $\mathrm{~m}$ & 0.220 & 0.31 & 1.44 & 0.36 \\
\hline March & 12.9 & 1945 & 0 & $\mathrm{~m}$ & 2.05 & 3.64 & 1.78 & 3.45 \\
\hline April & 43.1 & 1947 & 0.103 & 1958 & 19.5 & 12.9 & 0.66 & 32.8 \\
\hline May & 84.2 & 1950 & 0.113 & 1958 & 15.0 & 20.1 & 1.34 & 25.3 \\
\hline June & 30.4 & 1947 & 0.820 & 1952 & 9.29 & 7.66 & 0.82 & 15.6 \\
\hline July & 22.7 & 1949 & 0.229 & 1957 & 4.98 & 5.63 & 1.13 & 8.38 \\
\hline August & 7.96 & 1944 & 0 & $\mathrm{~m}$ & 2.27 & 2.00 & 0.88 & 3.82 \\
\hline September & 3.38 & 1945 & 0 & $\mathrm{~m}$ & 1.20 & 0.98 & 0.81 & 2.03 \\
\hline Annual & 13.4 & 1950 & 0.368 & 1958 & 5.04 & 3.41 & 0.68 & 100 \\
\hline
\end{tabular}

Annual flow duration

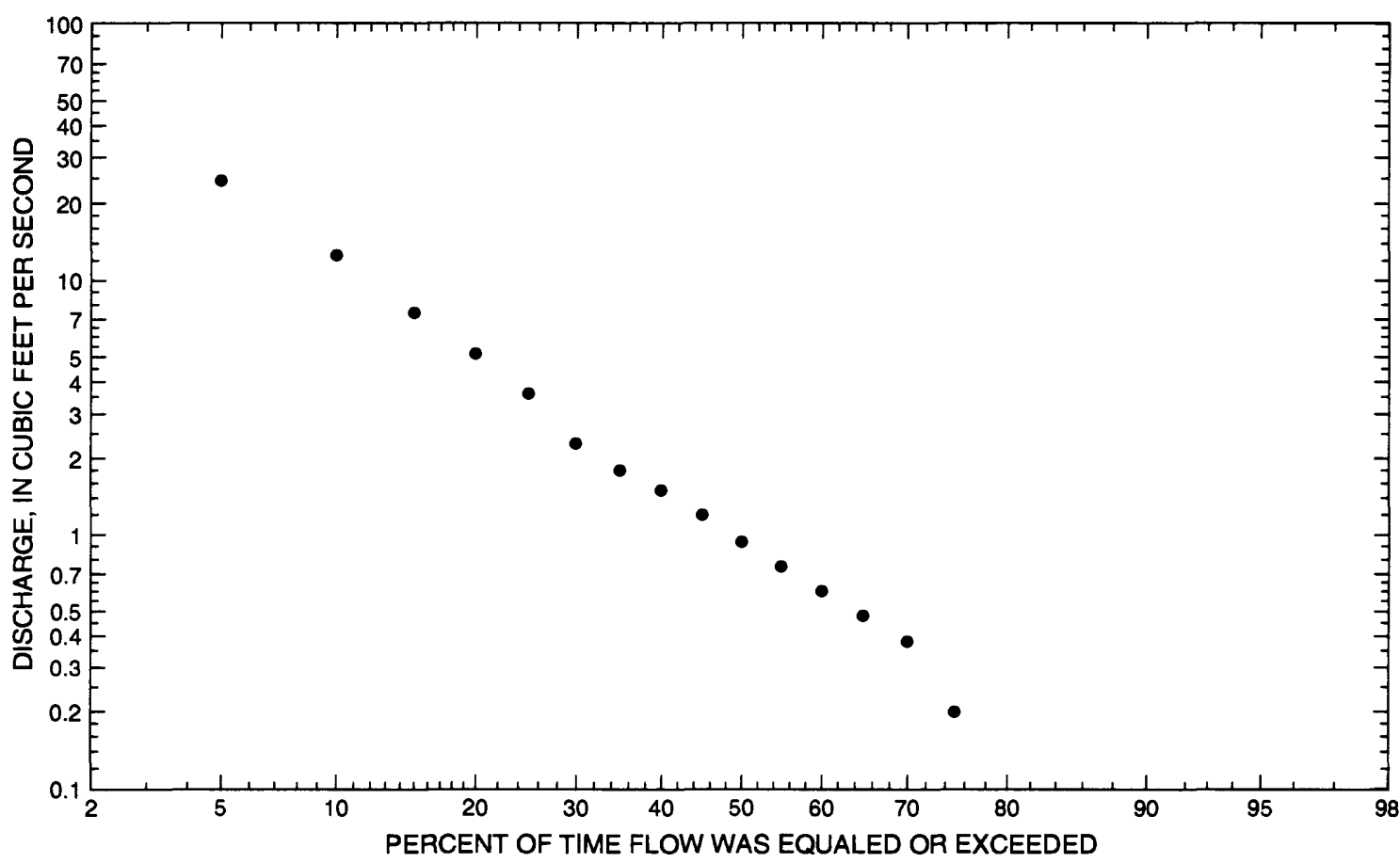




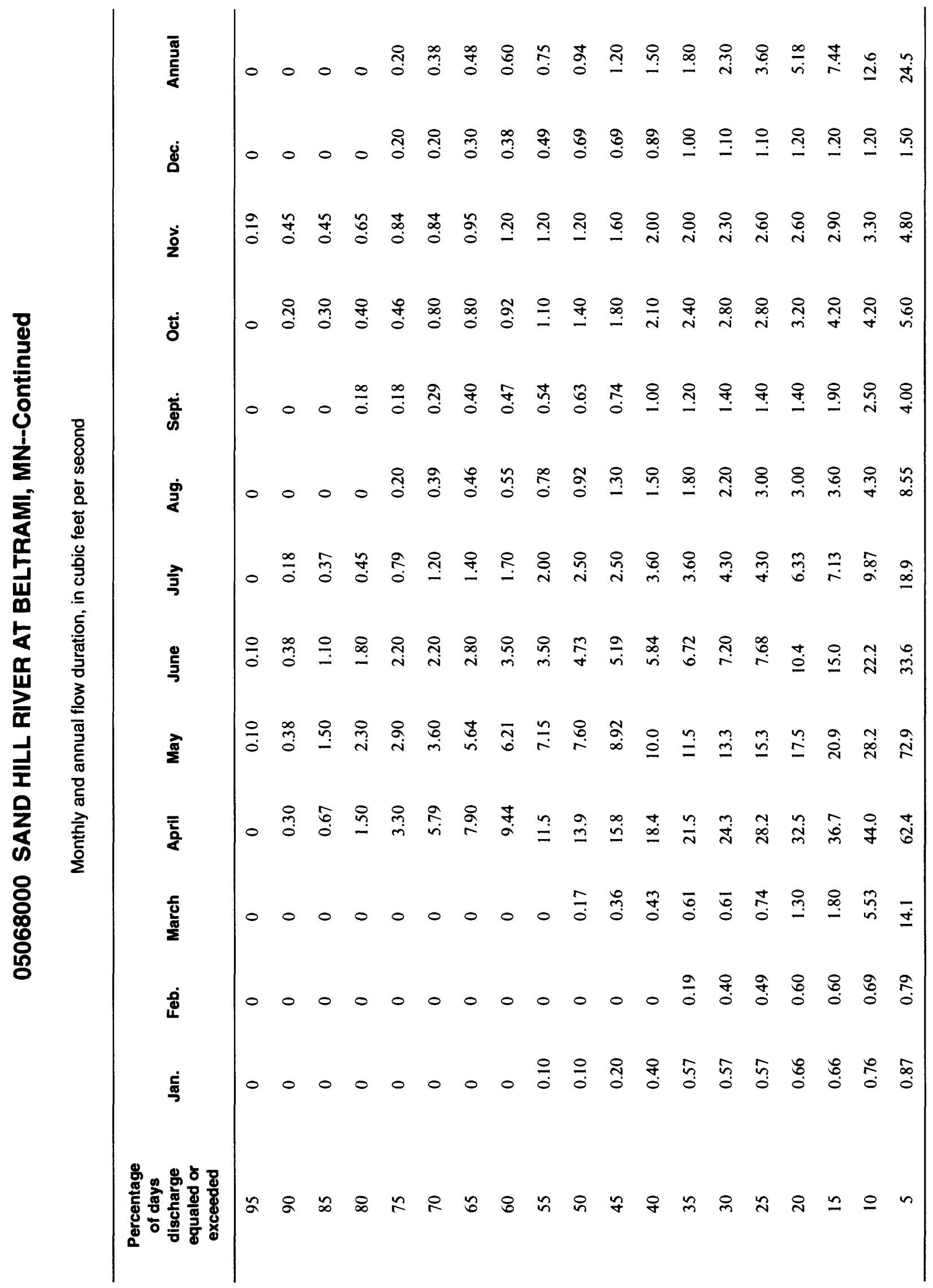




\section{SAND HILL RIVER AT BELTRAMI, MN-Continued}

Probability of occurrence of annual high discharges

[ng, statistic not given]

\begin{tabular}{lcccccc}
\hline & & & \multicolumn{5}{c}{$\begin{array}{c}\text { Maximum mean discharge } \\
\left(\mathbf{f t}^{3} / \mathbf{s}\right)\end{array}$} \\
\cline { 5 - 7 } $\begin{array}{c}\text { Exceedance } \\
\text { probability }\end{array}$ & $\begin{array}{c}\text { Recurrence } \\
\text { intervai } \\
\text { (years) }\end{array}$ & $\begin{array}{c}\text { Maximum } \\
\text { instantaneous } \\
\left(\mathbf{f t}^{3} / \mathbf{s}\right)\end{array}$ & 3-day period & 7-day period & 15-day period & 30-day period \\
\hline 0.99 & 1.01 & 13.9 & 7.29 & 4.26 & 2.17 & 1.08 \\
0.95 & 1.05 & 22.4 & 13.6 & 9.10 & 5.56 & 3.26 \\
0.90 & 1.11 & 28.9 & 18.7 & 13.2 & 8.71 & 5.48 \\
0.80 & 1.25 & 39.4 & 27.1 & 20.3 & 14.3 & 9.66 \\
0.50 & 2 & 71.1 & 52.9 & 42.1 & 31.8 & 23.8 \\
0.20 & 5 & 129 & 97.6 & 79.1 & 59.5 & 47.1 \\
0.10 & 10 & 176 & 132 & 106 & 77.6 & 62.2 \\
0.04 & 25 & 245 & 179 & 140 & 98.6 & 79.3 \\
0.02 & 50 & 304 & 216 & 165 & 112 & 90.3 \\
0.01 & 100 & 369 & 254 & 190 & 125 & 99.8 \\
0.005 & 200 & 441 & 294 & 215 & 136 & 108 \\
0.002 & 500 & 548 & $\mathrm{ng}$ & $\mathrm{ng}$ & $\mathrm{ng}$ & $\mathrm{ng}$ \\
\hline
\end{tabular}

Probability of occurrence of annual low discharges

[ng, statistic not given]

\begin{tabular}{|c|c|c|c|c|c|c|c|c|c|c|}
\hline \multirow{3}{*}{$\begin{array}{c}\text { Non- } \\
\text { exceed- } \\
\text { ance } \\
\text { prob- } \\
\text { ability }\end{array}$} & \multirow{3}{*}{$\begin{array}{l}\text { Recur- } \\
\text { rence } \\
\text { inter- } \\
\text { vai } \\
\text { (years) }\end{array}$} & \multicolumn{9}{|c|}{ Minimum mean discharge $\left(\mathrm{ft}^{3} / \mathrm{s}\right)$} \\
\hline & & \multicolumn{9}{|c|}{ Number of consecutive days } \\
\hline & & 1 & 3 & 7 & 14 & 30 & 60 & 90 & 120 & 183 \\
\hline 0.01 & 100 & $\mathrm{ng}$ & $\mathrm{ng}$ & 0 & 0 & 0 & 0 & 0 & 0.003 & 0.057 \\
\hline 0.02 & 50 & $\mathrm{ng}$ & $\mathrm{ng}$ & 0 & 0 & 0 & 0 & 0 & 0.006 & 0.082 \\
\hline 0.05 & 20 & $\mathrm{ng}$ & $\mathrm{ng}$ & 0 & 0 & 0 & 0 & 0 & 0.014 & 0.138 \\
\hline 0.10 & 10 & $\mathrm{ng}$ & $\mathrm{ng}$ & 0 & 0 & 0 & 0 & 0 & 0.030 & 0.213 \\
\hline 0.20 & 5 & $\mathrm{ng}$ & $\mathrm{ng}$ & 0 & 0 & 0 & 0 & 0 & 0.068 & 0.347 \\
\hline 0.50 & 2 & $\mathrm{ng}$ & $\mathrm{ng}$ & 0 & 0 & 0 & 0 & 0.120 & 0.262 & 0.788 \\
\hline
\end{tabular}




\section{SAND HILL RIVER AT BELTRAMI, MN--Continued}

Probability of occurrence of seasonal low discharges

\begin{tabular}{|c|c|c|c|c|c|c|c|c|c|}
\hline \multirow[b]{3}{*}{$\begin{array}{c}\text { Non- } \\
\text { exceedance } \\
\text { probability }\end{array}$} & \multirow[b]{3}{*}{$\begin{array}{c}\text { Recurrence } \\
\text { interval } \\
\text { (years) }\end{array}$} & \multicolumn{8}{|c|}{ Minimum mean discharge $\left(\mathrm{ft}^{3} / \mathrm{s}\right)$} \\
\hline & & \multicolumn{8}{|c|}{ Number of consecutive days } \\
\hline & & 1 & 7 & 14 & 30 & 1 & 7 & 14 & 30 \\
\hline & & \multicolumn{4}{|c|}{ December-January-February } & \multicolumn{4}{|c|}{ March-April-May } \\
\hline 0.05 & 20 & 0 & 0 & 0 & 0 & 0 & 0 & 0 & 0 \\
\hline 0.10 & 10 & 0 & 0 & 0 & 0 & 0 & 0 & 0 & 0 \\
\hline 0.20 & 5 & 0 & 0 & 0 & 0 & 0 & 0 & 0 & 0 \\
\hline \multirow[t]{2}{*}{0.50} & 2 & 0 & 0 & 0 & 0 & 0 & 0 & 0 & 0.346 \\
\hline & & \multicolumn{4}{|c|}{ June-July-August } & \multicolumn{4}{|c|}{ September-October-November } \\
\hline 0.05 & 20 & 0 & 0 & 0 & 0 & 0 & 0 & 0 & 0 \\
\hline 0.10 & 10 & 0 & 0 & 0 & 0 & 0 & 0 & 0 & 0.046 \\
\hline 0.20 & 5 & 0 & 0 & 0.031 & 0.224 & 0 & 0 & 0.083 & 0.159 \\
\hline 0.50 & 2 & 0.152 & 0.185 & 0.480 & 0.987 & 0.213 & 0.274 & 0.453 & 0.644 \\
\hline
\end{tabular}


05068000 SAND HILL RIVER AT BELTRAMI, MN-Continued

Annual peak discharge and corresponding gage height

$[--$, no data $]$

\begin{tabular}{|c|c|c|c|c|c|c|c|}
\hline $\begin{array}{l}\text { Water } \\
\text { year }\end{array}$ & Date & $\begin{array}{c}\text { Gage } \\
\text { height } \\
\text { (feet) }\end{array}$ & $\begin{array}{c}\text { Peak } \\
\text { discharge } \\
\left(\mathrm{ft}^{3} / \mathrm{s}\right)\end{array}$ & $\begin{array}{l}\text { Water } \\
\text { year }\end{array}$ & Date & $\begin{array}{c}\text { Gage } \\
\text { height } \\
\text { (feet) }\end{array}$ & $\begin{array}{c}\text { Peak } \\
\text { discharge } \\
\left(\mathrm{ft}^{3} / \mathrm{s}\right)\end{array}$ \\
\hline \multicolumn{8}{|c|}{ Annual peak discharge, by year, and corresponding gage height } \\
\hline 1943 & April 5 & -- & 179 & 1951 & April 8 & 4.10 & 71 \\
\hline 1944 & August 12 & -- & 30 & 1952 & April 9 & 3.20 & 46 \\
\hline 1945 & April 12 & 3.88 & 53 & 1953 & June 17 & 3.60 & 62 \\
\hline 1946 & March 28 & -- & 42 & 1954 & June 16 & -- & 49 \\
\hline 1947 & June 12 & 5.20 & 167 & 1955 & April 4 & 4.51 & 54 \\
\hline 1948 & April 13 & -- & 75 & 1956 & May 13 & 4.62 & 105 \\
\hline 1949 & June 2 & 5.00 & 163 & 1957 & June 23 & -- & 50 \\
\hline 1950 & April 19 & 5.97 & 291 & 1958 & July 5 & 3.21 & 22 \\
\hline \multicolumn{8}{|c|}{ Annual peak discharge, from highest to lowest, and corresponding gage height } \\
\hline 1950 & April 19 & 5.97 & 291 & 1955 & April 4 & 4.51 & 54 \\
\hline 1943 & April 13 & -- & 179 & 1945 & April 12 & 3.88 & 53 \\
\hline 1947 & June 12 & 5.20 & 167 & 1957 & June 23 & -- & 50 \\
\hline 1949 & June 2 & 5.00 & 163 & 1954 & June 16 & -- & 49 \\
\hline 1956 & May 13 & 4.62 & 105 & 1952 & April 9 & 3.20 & 46 \\
\hline 1948 & April 13 & -- & 75 & 1946 & March 28 & -- & 42 \\
\hline 1951 & April 8 & 4.10 & 71 & 1944 & August 12 & -- & 30 \\
\hline 1953 & June 17 & 3.60 & 62 & 1958 & July 5 & 3.21 & 22 \\
\hline
\end{tabular}




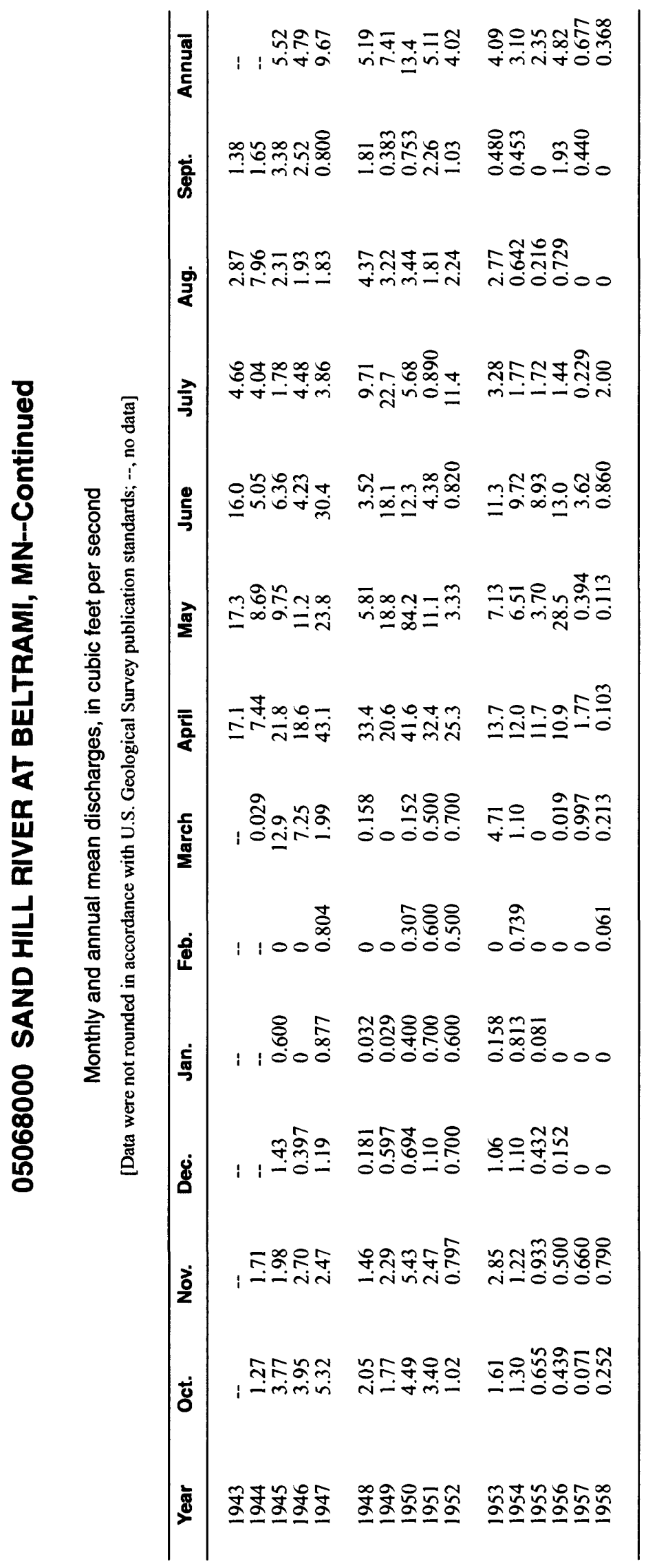




\section{SAND HILL DITCH AT BELTRAMI, MN}

LOCATION.--Lat $47^{\circ} 32^{\prime} 10^{\prime \prime}$, long $96^{\circ} 32^{\prime} 00^{\prime \prime}$, in SE $1 /{ }_{4} \mathrm{NW}^{1} / 4$ sec. 21 , T.147 N., R.46 W., near center of span on downstream side of bridge on U.S. Highway 75, $150 \mathrm{ft}$ upstream from Great Northern Railway bridge and $0.25 \mathrm{mi}$ south of post office in Beltrami.

DRAINAGE AREA.--Not available.

PERIOD OF RECORD.--March 1943 to September 1958. Winter records incomplete some years. Monthly discharge only for some periods, published in WSP 1308.

GAGE.--Chain gage. Datum of gage is $883.50 \mathrm{ft}$ above mean sea level, adjustment of 1912 (levels by U.S. Army Corps of Engineers). Prior to Aug. 26, 1944, reference point and Aug. 26, 1944, to Nov. 21, 1948 , chain gage, at same site at datum $12.62 \mathrm{ft}$ higher. Nov. 22, 1948, to Sept. 30, 1956, chain gage at same site at datum $10.00 \mathrm{ft}$ higher.

EXTREMES FOR PERIOD OF RECORD.--Maximum discharge, 2,460 $\mathrm{ft}^{3} / \mathrm{s}$, Apr. 20, 1950, gage height, $20.99 \mathrm{ft}$, present datum; maximum gage height, $21.59 \mathrm{ft}$, present datum, Apr. 19,1950, from floodmark, backwater from ice; no flow at times.

Annual mean discharge

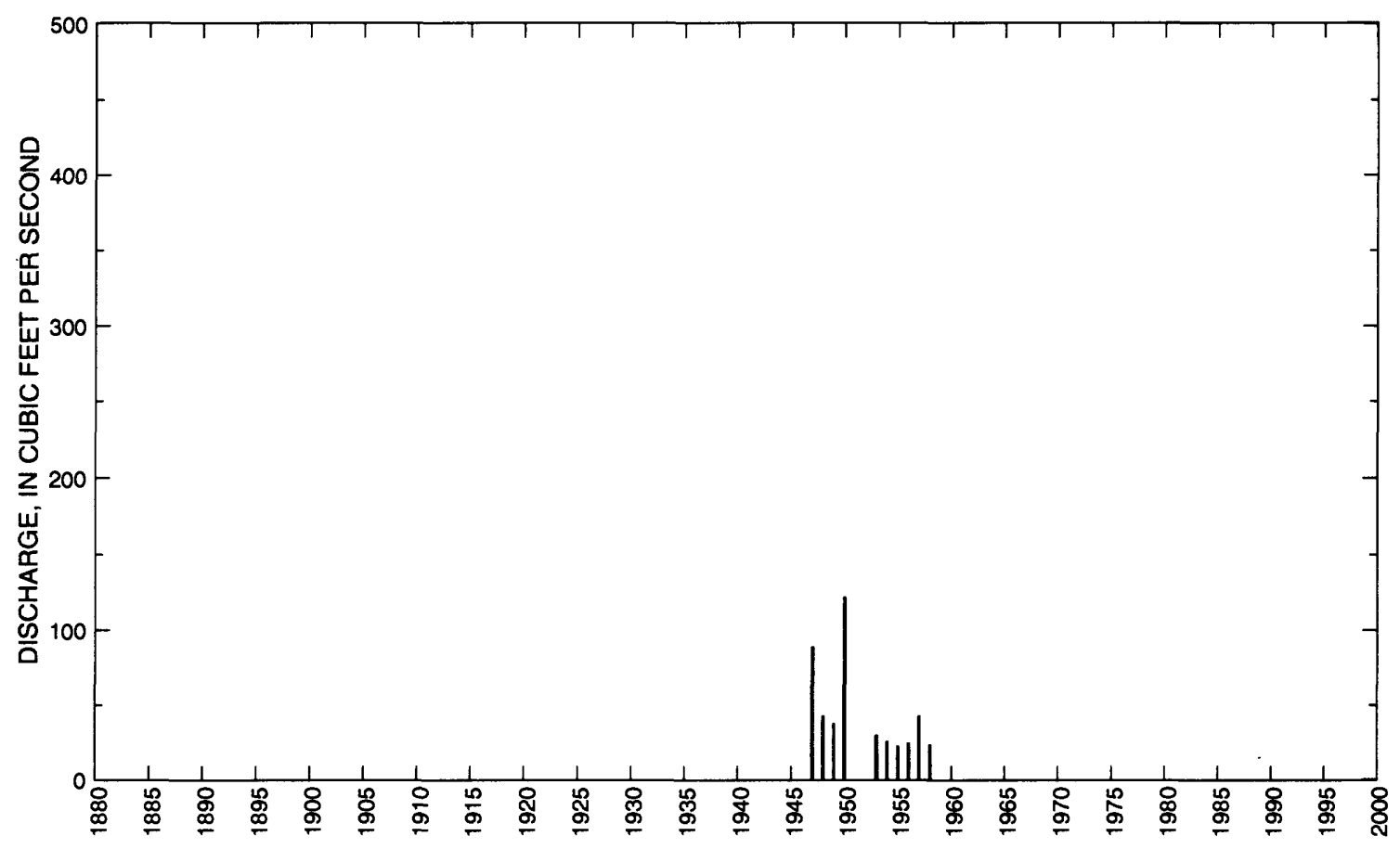


05068500 SAND HILL DITCH AT BELTRAMI, MN--Continued

Statistics of monthly and annual mean discharges

\begin{tabular}{|c|c|c|c|c|c|c|c|c|}
\hline \multirow[b]{2}{*}{ Month } & \multicolumn{2}{|c|}{ Maximum } & \multicolumn{2}{|c|}{ Minimum } & \multicolumn{4}{|c|}{ Mean } \\
\hline & $\begin{array}{c}\text { Discharge } \\
\left(\mathrm{ft}^{3} / \mathrm{s}\right)\end{array}$ & $\begin{array}{c}\text { Water year } \\
\text { of } \\
\text { occurrence }\end{array}$ & $\begin{array}{c}\text { Discharge } \\
\left(\mathrm{ft}^{3} / \mathrm{s}\right)\end{array}$ & $\begin{array}{l}\text { Water year } \\
\text { of } \\
\text { occurrence }\end{array}$ & $\begin{array}{c}\text { Discharge } \\
\left(\mathrm{ft}^{3} / \mathrm{s}\right)\end{array}$ & $\begin{array}{l}\text { Standard } \\
\text { deviation } \\
\left(\mathrm{ft}^{3} / \mathrm{s}\right)\end{array}$ & $\begin{array}{l}\text { Coeffi- } \\
\text { cient of } \\
\text { variation }\end{array}$ & $\begin{array}{l}\text { Percentage } \\
\text { of annual } \\
\text { discharge }\end{array}$ \\
\hline October & 53.4 & 1946 & 9.57 & 1955 & 21.9 & 12.3 & 0.56 & 3.79 \\
\hline November & 41.7 & 1958 & 8.43 & 1956 & 21.0 & 9.32 & 0.44 & 3.63 \\
\hline December & 25.0 & 1945 & 5.09 & 1956 & 13.1 & 5.16 & 0.39 & 2.27 \\
\hline January & 14.0 & 1947 & 4.90 & 1948 & 8.58 & 2.94 & 0.34 & 1.48 \\
\hline February & 14.4 & 1958 & 0.790 & 1948 & 8.32 & 4.01 & 0.48 & 1.44 \\
\hline March & 150 & 1946 & 0 & 1944 & 37.6 & 48.8 & 1.30 & 6.49 \\
\hline April & 493 & 1947 & 27.5 & 1958 & 196 & 144 & 0.74 & 33.8 \\
\hline May & 588 & 1950 & 23.4 & 1958 & 98.9 & 136 & 1.37 & 17.1 \\
\hline June & 222 & 1947 & 20.6 & 1952 & 76.0 & 62.0 & 0.82 & 13.1 \\
\hline July & 178 & 1950 & 14.1 & 1951 & 51.6 & 42.8 & 0.83 & 8.91 \\
\hline August & 113 & 1944 & 8.97 & 1955 & 25.8 & 25.2 & 0.97 & 4.46 \\
\hline September & 52.3 & 1945 & 8.49 & 1954 & 20.4 & 16.0 & 0.79 & 3.52 \\
\hline Annual & 122 & 1950 & 22.6 & 1955 & 46.2 & 33.2 & 0.72 & 100 \\
\hline
\end{tabular}

Annual flow duration

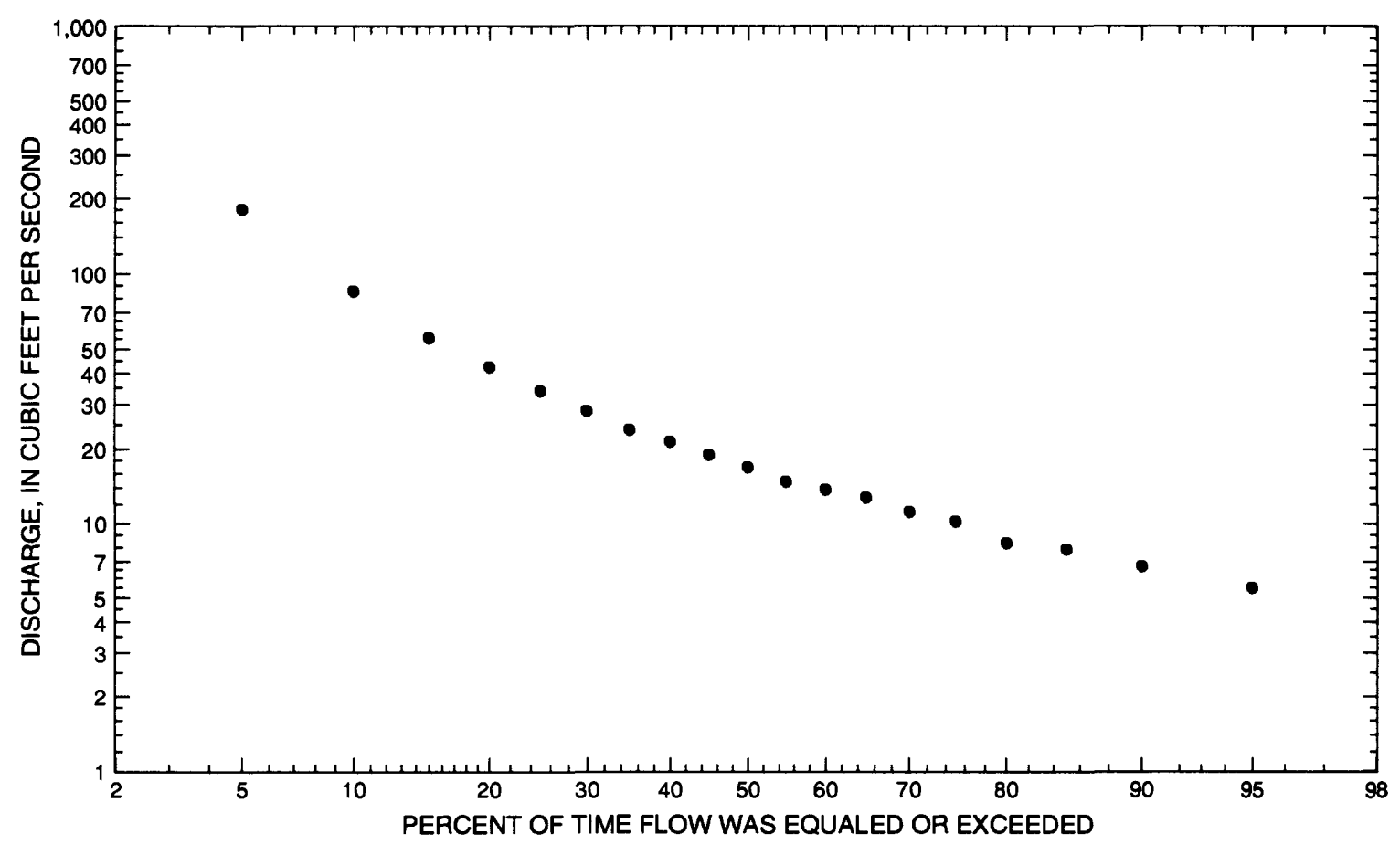




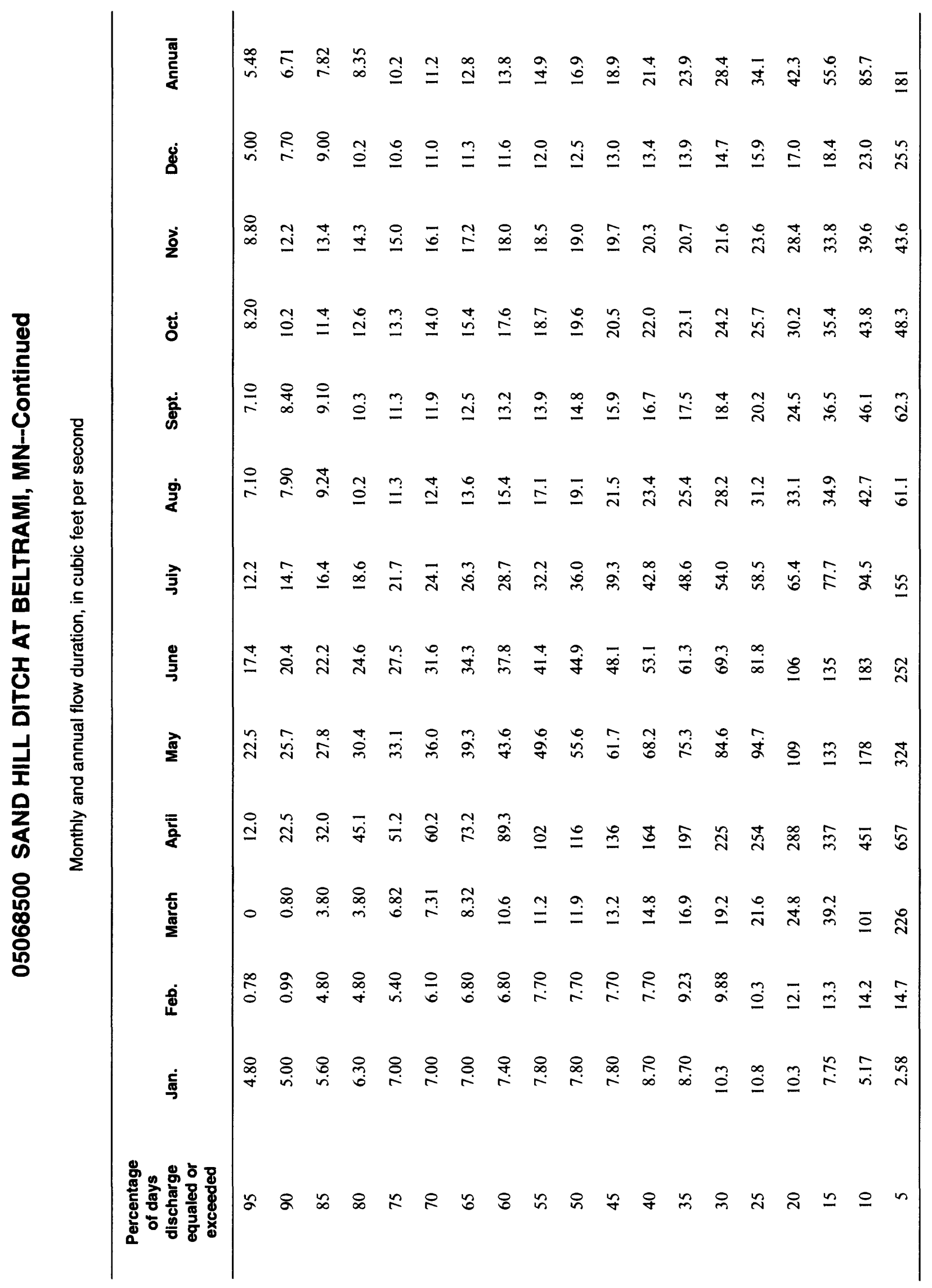




\section{SAND HILL DITCH AT BELTRAMI, MN--Continued}

Probability of occurrence of annual high discharges

[ng, statistic not given]

\begin{tabular}{ccccccc}
\hline & & & \multicolumn{5}{c}{$\begin{array}{c}\text { Maximum mean discharge } \\
\left(\mathbf{f t}^{3} / \mathbf{s}\right)\end{array}$} \\
\cline { 5 - 7 } $\begin{array}{c}\text { Exceedance } \\
\text { probability }\end{array}$ & $\begin{array}{c}\text { Recurrence } \\
\text { interval } \\
\text { (years) }\end{array}$ & $\begin{array}{c}\text { Maximum } \\
\text { instantaneous } \\
\left(\mathbf{f t}^{3} / \mathbf{s}\right)\end{array}$ & 3-day period & 7-day period & 15-day period & 30-day period \\
\hline 0.99 & 1.01 & 68.8 & 50.1 & 51.8 & 46.2 & 34.6 \\
0.95 & 1.05 & 124 & 92.9 & 87.0 & 71.0 & 49.5 \\
0.90 & 1.11 & 168 & 129 & 116 & 90.7 & 61.7 \\
0.80 & 1.25 & 243 & 192 & 164 & 124 & 82.9 \\
0.50 & 2 & 478 & 408 & 328 & 235 & 160 \\
0.20 & 5 & 918 & 866 & 673 & 476 & 351 \\
0.10 & 10 & 1,280 & 1,280 & 992 & 706 & 559 \\
0.04 & 25 & 1,800 & 1,950 & 1,510 & 1,100 & 958 \\
0.02 & 50 & 2,250 & 2,550 & 2,000 & 1,480 & 1,390 \\
0.01 & 100 & 2,730 & 3,240 & 2,570 & 1,940 & 1,970 \\
0.005 & 200 & 3,250 & 4,040 & 3,250 & 2,510 & 2,760 \\
0.002 & 500 & 4,010 & ng & ng & ng & ng \\
\hline
\end{tabular}

Probability of occurrence of annual low discharges

Minimum mean discharge $\left(\mathrm{ft}^{3} / \mathrm{s}\right)$

\begin{tabular}{|c|c|c|c|c|c|c|c|c|c|c|}
\hline \multirow[b]{2}{*}{$\begin{array}{l}\text { Non- } \\
\text { exceed- } \\
\text { ance } \\
\text { prob- } \\
\text { ability }\end{array}$} & \multirow[b]{2}{*}{$\begin{array}{c}\text { Recur- } \\
\text { rence } \\
\text { inter- } \\
\text { vai } \\
\text { (years) }\end{array}$} & \multicolumn{9}{|c|}{ Number of consecutive days } \\
\hline & & 1 & 3 & 7 & 14 & 30 & 60 & 90 & 120 & 183 \\
\hline 0.05 & 20 & 1.09 & 1.09 & 1.11 & 1.18 & 1.23 & 1.71 & 3.10 & 5.34 & 7.62 \\
\hline 0.10 & 10 & 1.86 & 1.87 & 1.92 & 2.07 & 2.18 & 2.76 & 4.07 & 6.05 & 8.18 \\
\hline 0.20 & 5 & 3.16 & 3.19 & 3.31 & 3.58 & 3.80 & 4.42 & 5.46 & 7.05 & 9.02 \\
\hline 0.50 & 2 & 6.25 & 6.34 & 6.59 & 7.12 & 7.59 & 8.17 & 8.65 & 9.41 & 11.3 \\
\hline
\end{tabular}




\section{SAND HILL DITCH AT BELTRAMI, MN--Continued}

Probability of occurrence of seasonal low discharges

\begin{tabular}{|c|c|c|c|c|c|c|c|c|c|}
\hline \multirow[b]{3}{*}{$\begin{array}{c}\text { Non- } \\
\text { exceedance } \\
\text { probability }\end{array}$} & \multirow[b]{3}{*}{$\begin{array}{c}\text { Recurrence } \\
\text { interval } \\
\text { (years) }\end{array}$} & \multicolumn{8}{|c|}{ Minimum mean discharge $\left(\mathrm{ft}^{3} / \mathrm{s}\right)$} \\
\hline & & \multicolumn{8}{|c|}{ Number of consecutive days } \\
\hline & & 1 & 7 & 14 & 30 & 1 & 7 & 14 & 30 \\
\hline & & \multicolumn{4}{|c|}{ December-January-February } & \multicolumn{4}{|c|}{ March-April-May } \\
\hline 0.05 & 20 & 1.14 & 1.14 & 1.20 & 1.32 & 0 & 0 & 0 & 0 \\
\hline 0.10 & 10 & 2.10 & 2.10 & 2.17 & 2.32 & 0.820 & 0.825 & 0.919 & 1.95 \\
\hline 0.20 & 5 & 3.80 & 3.80 & 3.88 & 4.06 & 3.08 & 3.10 & 3.41 & 4.91 \\
\hline \multirow[t]{2}{*}{0.50} & 2 & 8.08 & 8.08 & 8.12 & 8.24 & 9.30 & 9.37 & 10.0 & 15.6 \\
\hline & & \multicolumn{4}{|c|}{ June-July-August } & \multicolumn{4}{|c|}{ September-October-November } \\
\hline 0.05 & 20 & 5.30 & 5.70 & 6.16 & 7.28 & 5.00 & 5.34 & 5.99 & 7.15 \\
\hline 0.10 & 10 & 6.50 & 7.00 & 7.51 & 8.94 & 5.89 & 6.28 & 6.95 & 8.11 \\
\hline 0.20 & 5 & 8.30 & 8.97 & 9.56 & 11.5 & 7.23 & 7.71 & 8.42 & 9.60 \\
\hline 0.50 & 2 & 13.1 & 14.4 & 15.3 & 18.5 & 10.9 & 11.8 & 12.6 & 14.0 \\
\hline
\end{tabular}


05068500 SAND HILL DITCH AT BELTRAMI, MN--Continued

Annual peak discharge and corresponding gage height

$[--$, no data $]$

\begin{tabular}{|c|c|c|c|c|c|c|c|}
\hline $\begin{array}{c}\text { Water } \\
\text { year }\end{array}$ & Date & $\begin{array}{c}\text { Gage } \\
\text { height } \\
\text { (feet) }\end{array}$ & $\begin{array}{c}\text { Peak } \\
\text { discharge } \\
\left(\mathrm{ft}^{3} / \mathrm{s}\right)\end{array}$ & $\begin{array}{c}\text { Water } \\
\text { year }\end{array}$ & Date & $\begin{array}{c}\text { Gage } \\
\text { height } \\
\text { (feet) }\end{array}$ & $\begin{array}{c}\text { Peak } \\
\text { discharge } \\
\left(\mathrm{ft}^{3} / \mathrm{s}\right)\end{array}$ \\
\hline \multicolumn{8}{|c|}{ Annual peak discharge, by year, and corresponding gage height } \\
\hline 1943 & April 5 & -- & 741 & 1951 & April 9 & 8.46 & 823 \\
\hline 1944 & August 15 & -- & 226 & 1952 & April 12 & -- & 315 \\
\hline 1945 & April 1 & - & 344 & 1953 & March 29 & -- & 190 \\
\hline 1946 & March 25 & -- & 564 & 1954 & April 14 & - & 278 \\
\hline 1947 & April 16 & 7.57 & 1,220 & 1955 & April 7 & 10.07 & 551 \\
\hline 1948 & April 11 & 7.20 & 1,050 & 1956 & April 19 & -- & 480 \\
\hline 1949 & June 2 & -- & 425 & 1957 & June 28 & -- & 370 \\
\hline 1950 & April 20 & -- & 2,460 & 1958 & July 5 & - & 95 \\
\hline \multicolumn{8}{|c|}{ Annual peak discharge, from highest to lowest, and corresponding gage height } \\
\hline 1950 & April 20 & -- & 2,460 & 1949 & June 2 & -- & 425 \\
\hline 1947 & April 16 & 7.57 & 1,220 & 1957 & June 28 & -- & 370 \\
\hline 1948 & April 11 & 7.20 & 1,050 & 1945 & April 1 & -- & 344 \\
\hline 1951 & April 9 & 8.46 & 823 & 1952 & April 12 & - & 315 \\
\hline 1943 & April 5 & -- & 741 & 1954 & April 14 & -- & 278 \\
\hline 1946 & March 25 & -- & 564 & 1944 & August 15 & -- & 226 \\
\hline 1955 & April 7 & 10.07 & 551 & 1953 & March 29 & -- & 190 \\
\hline 1956 & April 19 & -- & 480 & 1958 & July 5 & -- & 95 \\
\hline
\end{tabular}




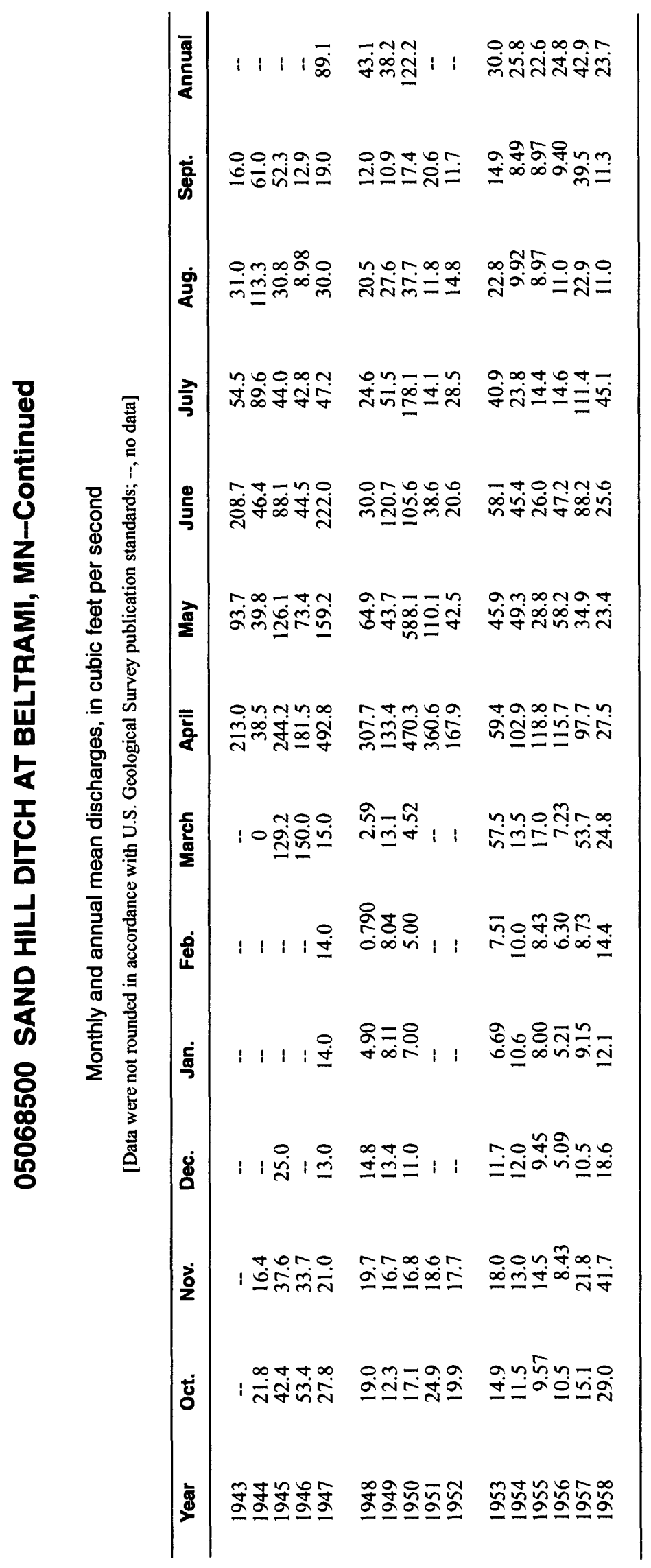




\section{SAND HILL RIVER AT CLIMAX, MN}

LOCATION.--Lat $47^{\circ} 36^{\prime} 43^{\prime}$, long $96^{\circ} 48^{\prime} 52^{\prime}$, in $\mathrm{NE}^{1 /}{ }_{4} \mathrm{NE}^{1 / 4}$ sec.30, T.148 N., R.48 W., Polk County, Hydrologic Unit 09020301 , on left bank $25 \mathrm{ft}$ upstream from bridge on U.S. Highway 75 in Climax and 3.7 mi upstream from mouth.

DRAINAGE AREA.--426 $\mathrm{mi}^{2}$.

PERIOD OF RECORD.--March 1943 to September 1984, June 1985 to current year. Winter records incomplete prior to 1947. Monthly discharge only for some periods, published in WSP 1308 and 1728. October 1984 to May 1985, operated as a high-flow partial-record station.

GAGE.--Water-stage recorder. Datum of gage is $820.10 \mathrm{ft}$ above mean sea level (levels by U.S. Army Corps of Engineers). Prior to Oct. 1, 1966, nonrecording gage at site $3.2 \mathrm{mi}$ upstream at datum $12.78 \mathrm{ft}$ higher. Oct. 1, 1966, to Sept. 5, 1989, nonrecording gage at present site and datum.

EXTREMES FOR PERIOD OF RECORD.--Maximum discharge, 4,560 $\mathrm{ft}^{3} / \mathrm{s}$, Apr. 14, 1965, gage height, $17.81 \mathrm{ft}$; maximum gage height, $32.79 \mathrm{ft}$, Apr. 23, 1979, from floodmark, backwater from Red River of the North; minimum daily discharge, $1.0 \mathrm{ft}^{3} / \mathrm{s}$, Jan. 17-18, 1962.

Annual mean discharge

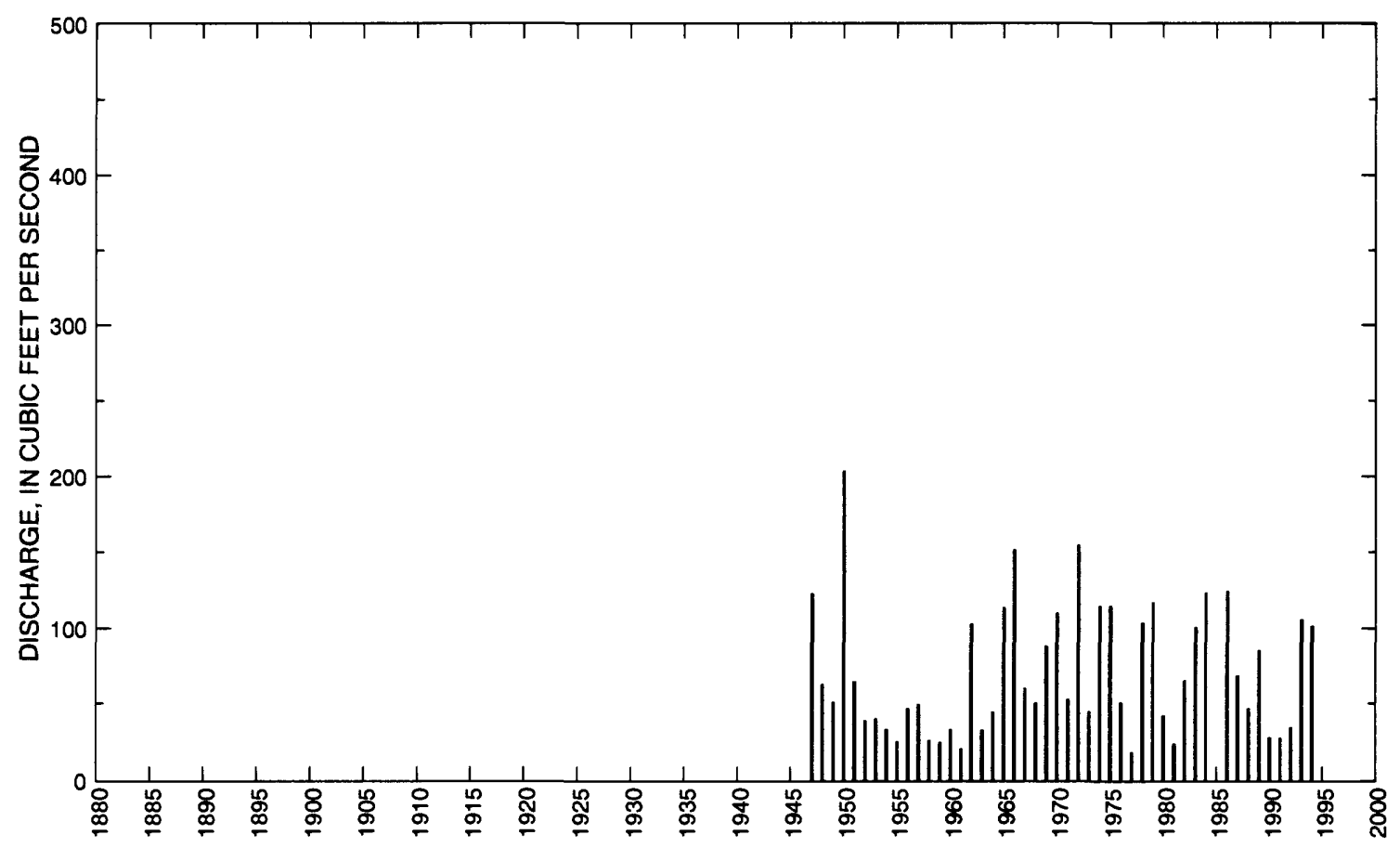




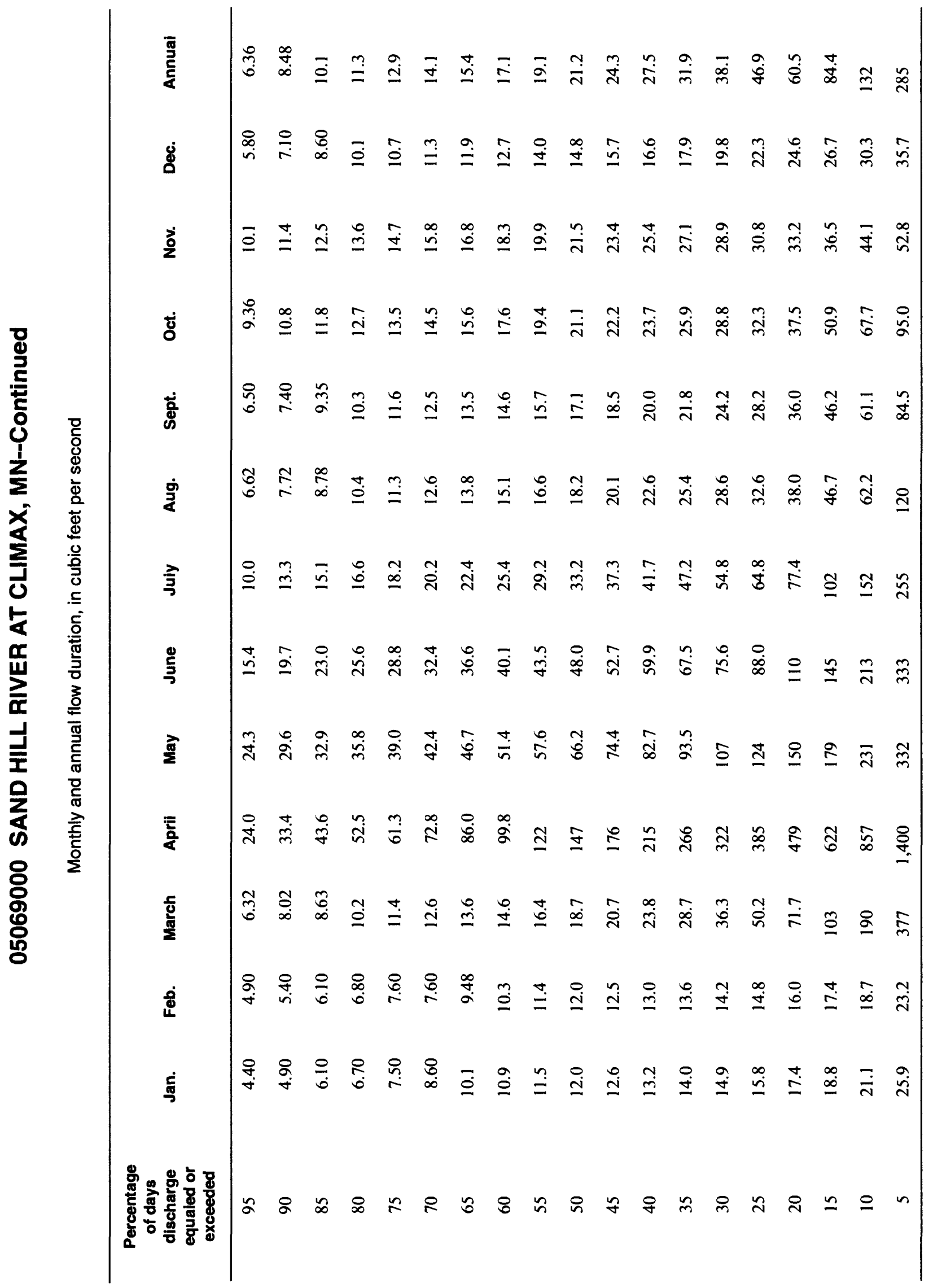




\section{SAND HILL RIVER AT CLIMAX, MN--Continued}

Statistics of monthly and annual mean discharges

[m, more than 1 year of occurrence]

\begin{tabular}{|c|c|c|c|c|c|c|c|c|}
\hline \multirow[b]{2}{*}{ Month } & \multicolumn{2}{|c|}{ Maximum } & \multicolumn{2}{|c|}{ Minimum } & \multicolumn{4}{|c|}{ Mean } \\
\hline & $\begin{array}{c}\text { Discharge } \\
\left(\mathrm{ft}^{3} / \mathrm{s}\right)\end{array}$ & $\begin{array}{c}\text { Water year } \\
\text { of } \\
\text { occurrence }\end{array}$ & $\begin{array}{c}\text { Discharge } \\
\left(\mathrm{ft}^{3} / \mathrm{s}\right)\end{array}$ & $\begin{array}{c}\text { Water year } \\
\text { of } \\
\text { occurrence }\end{array}$ & $\begin{array}{c}\text { Discharge } \\
\left(\mathrm{ft}^{3} / \mathrm{s}\right)\end{array}$ & $\begin{array}{c}\text { Standard } \\
\text { deviation } \\
\left(\mathrm{ft}^{3} / \mathrm{s}\right)\end{array}$ & $\begin{array}{l}\text { Coeffi- } \\
\text { cient of } \\
\text { variation }\end{array}$ & $\begin{array}{l}\text { Percentage } \\
\text { of annual } \\
\text { discharge }\end{array}$ \\
\hline October & 224 & 1972 & 9.43 & 1977 & 31.7 & 34.9 & 1.10 & 3.67 \\
\hline November & 209 & 1972 & 8.64 & 1956 & 27.1 & 28.4 & 1.05 & 3.14 \\
\hline December & 48.7 & 1972 & 5.11 & 1964 & 16.9 & 9.18 & 0.54 & 1.96 \\
\hline January & 30.1 & 1986 & 2.02 & 1962 & 12.4 & 5.96 & 0.48 & 1.44 \\
\hline February & 46.8 & 1984 & 3.55 & 1962 & 12.2 & 6.80 & 0.56 & 1.42 \\
\hline March & 385 & 1966 & 5.81 & 1948 & 74.2 & 91.8 & 1.24 & 8.60 \\
\hline April & 946 & 1978 & 25.3 & 1981 & 343 & 285 & 0.83 & 39.7 \\
\hline May & 1,160 & 1950 & 23.7 & 1958 & 117 & 163 & 1.39 & 13.6 \\
\hline June & 596 & 1984 & 11.5 & 1980 & 95.6 & 105 & 1.10 & 11.1 \\
\hline July & 376 & 1994 & 8.95 & 1980 & 67.5 & 75.8 & 1.12 & 7.82 \\
\hline August & 426 & 1993 & 6.30 & 1961 & 38.2 & 67.3 & 1.76 & 4.42 \\
\hline September & 124 & 1994 & 6.49 & 1955 & 27.1 & 25.2 & 0.93 & 3.14 \\
\hline Annual & 204 & 1950 & 18.4 & 1977 & 71.3 & 43.1 & 0.60 & 100 \\
\hline
\end{tabular}

Annual flow duration

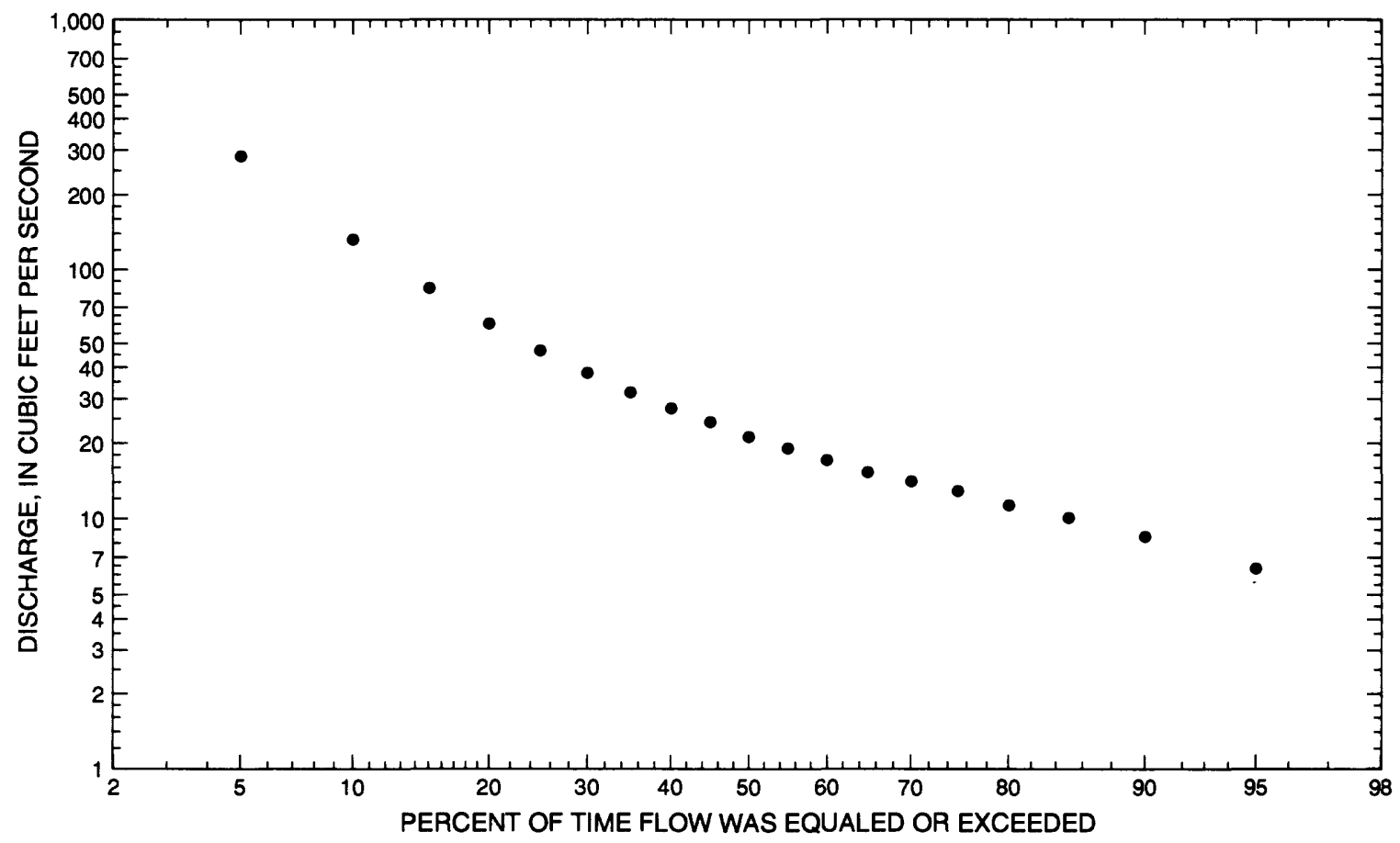




\section{SAND HILL RIVER AT CLIMAX, MN--Continued}

Probability of occurrence of annual high discharges

[ng, statistic not given]

\begin{tabular}{|c|c|c|c|c|c|c|}
\hline \multirow[b]{2}{*}{$\begin{array}{l}\text { Exceedance } \\
\text { probability }\end{array}$} & \multirow{2}{*}{$\begin{array}{l}\text { Recurrence } \\
\text { interval } \\
\text { (years) }\end{array}$} & \multirow{2}{*}{$\begin{array}{c}\text { Maximum } \\
\text { instantaneous } \\
\left(\mathrm{ft}^{3} / \mathrm{s}\right)\end{array}$} & \multicolumn{4}{|c|}{$\begin{array}{l}\text { Maximum mean discharge } \\
\qquad\left(\mathrm{ft}^{3} / \mathrm{s}\right)\end{array}$} \\
\hline & & & 3-day perlod & 7-day period & 15-day perlod & 30-day perlod \\
\hline 0.99 & 1.01 & 106 & 73.3 & 60.1 & 45.7 & 34.4 \\
\hline 0.95 & 1.05 & 218 & 158 & 128 & 95.7 & 69.6 \\
\hline 0.90 & 1.11 & 314 & 233 & 188 & 140 & 99.9 \\
\hline 0.80 & 1.25 & 481 & 369 & 296 & 217 & 153 \\
\hline 0.50 & 2 & 1,040 & 847 & 673 & 482 & 328 \\
\hline 0.20 & 5 & 2,120 & 1,830 & 1,440 & 1,010 & 670 \\
\hline 0.10 & 10 & 3,010 & 2,660 & 2,110 & 1,450 & 951 \\
\hline 0.04 & 25 & 4,290 & 3,920 & 3,100 & 2,100 & 1,360 \\
\hline 0.02 & 50 & 5,350 & 4,980 & 3,930 & 2,640 & 1,700 \\
\hline 0.01 & 100 & 6,490 & 6,140 & 4,850 & 3,220 & 2,070 \\
\hline 0.005 & 200 & 7,700 & 7,400 & 5,850 & 3,850 & 2,460 \\
\hline 0.002 & 500 & 9,420 & ng & ng & ng & ng \\
\hline
\end{tabular}

Probability of occurrence of annual low discharges

\begin{tabular}{|c|c|c|c|c|c|c|c|c|c|c|}
\hline \multirow[b]{3}{*}{$\begin{array}{l}\text { Non- } \\
\text { exceed- } \\
\text { ance } \\
\text { prob- } \\
\text { ability }\end{array}$} & \multirow[b]{3}{*}{$\begin{array}{c}\text { Recur- } \\
\text { rence } \\
\text { inter- } \\
\text { val } \\
\text { (years) }\end{array}$} & \multicolumn{9}{|c|}{ Minimum mean discharge $\left(\mathrm{ft}^{3} / \mathrm{s}\right)$} \\
\hline & & \multicolumn{9}{|c|}{ Number of consecutive days } \\
\hline & & 1 & 3 & 7 & 14 & 30 & 60 & 90 & 120 & 183 \\
\hline 0.05 & 20 & 2.55 & 2.68 & 2.88 & 3.22 & 3.71 & 4.56 & 5.37 & 6.52 & 8.13 \\
\hline 0.10 & 10 & 3.30 & 3.48 & 3.70 & 4.05 & 4.60 & 5.45 & 6.30 & 7.46 & 9.13 \\
\hline 0.20 & 5 & 4.40 & 4.65 & 4.90 & 5.24 & 5.86 & 6.71 & 7.64 & 8.84 & 10.7 \\
\hline 0.50 & 2 & 7.12 & 7.46 & 7.79 & 8.09 & 8.84 & 9.75 & 11.0 & 12.4 & 15.0 \\
\hline
\end{tabular}




\section{SAND HILL RIVER AT CLIMAX, MN--Continued}

Probability of occurrence of seasonal low discharges

\begin{tabular}{|c|c|c|c|c|c|c|c|c|c|}
\hline \multirow[b]{3}{*}{$\begin{array}{c}\text { Non- } \\
\text { exceedance } \\
\text { probability }\end{array}$} & \multirow[b]{3}{*}{$\begin{array}{c}\text { Recurrence } \\
\text { intervai } \\
\text { (years) }\end{array}$} & \multicolumn{8}{|c|}{ Minimum mean discharge $\left(\mathrm{ft}^{3} / \mathrm{s}\right)$} \\
\hline & & \multicolumn{8}{|c|}{ Number of consecutive days } \\
\hline & & 1 & 7 & 14 & 30 & 1 & 7 & 14 & 30 \\
\hline & & \multicolumn{4}{|c|}{ December-January-February } & \multicolumn{4}{|c|}{ March-April-May } \\
\hline 0.05 & 20 & 2.80 & 3.01 & 3.34 & 3.77 & 5.01 & 5.69 & 6.08 & 7.22 \\
\hline 0.10 & 10 & 3.81 & 4.06 & 4.37 & 4.79 & 6.19 & 6.77 & 7.26 & 9.35 \\
\hline 0.20 & 5 & 5.34 & 5.64 & 5.89 & 6.28 & 7.88 & 8.36 & 9.04 & 13.0 \\
\hline \multirow[t]{2}{*}{0.50} & 2 & 9.14 & 9.48 & 9.61 & 9.91 & 12.0 & 12.6 & 14.0 & 25.6 \\
\hline & & \multicolumn{4}{|c|}{ June-July-August } & \multicolumn{4}{|c|}{ September-October-November } \\
\hline 0.05 & 20 & 4.67 & 5.57 & 6.10 & 7.57 & 4.83 & 5.64 & 6.42 & 7.34 \\
\hline 0.10 & 10 & 5.57 & 6.50 & 7.14 & 8.75 & 5.67 & 6.61 & 7.42 & 8.52 \\
\hline 0.20 & 5 & 7.00 & 7.99 & 8.80 & 10.7 & 6.97 & 8.10 & 8.96 & 10.3 \\
\hline 0.50 & 2 & 11.3 & 12.6 & 14.0 & 17.1 & 10.7 & 12.3 & 13.4 & 15.6 \\
\hline
\end{tabular}




\section{SAND HILL RIVER AT CLIMAX, MN--Continued}

Annual peak discharge and corresponding gage height

$$
[--, \text { no data }]
$$

\begin{tabular}{|c|c|c|c|c|c|c|c|}
\hline $\begin{array}{l}\text { Water } \\
\text { year }\end{array}$ & Date & $\begin{array}{l}\text { Gage } \\
\text { height } \\
\text { (feet) }\end{array}$ & $\begin{array}{c}\text { Peak } \\
\text { discharge } \\
\left(\mathrm{tt}^{3} / \mathrm{s}\right)\end{array}$ & $\begin{array}{l}\text { Water } \\
\text { year }\end{array}$ & Date & $\begin{array}{c}\text { Gage } \\
\text { height } \\
\text { (feet) }\end{array}$ & $\begin{array}{c}\text { Peak } \\
\text { discharge } \\
\left(\mathrm{ft}^{3} / \mathrm{s}\right)\end{array}$ \\
\hline \multicolumn{8}{|c|}{ Annual peak discharge, by year, and corresponding gage height } \\
\hline 1943 & April 7 & 10.48 & 941 & 1969 & April 14 & 24.57 & 4,180 \\
\hline 1944 & August 18 & -- & 226 & 1970 & April 16 & 13.64 & 1,980 \\
\hline 1945 & April 11 & 9.18 & 767 & 1971 & April 8 & 13.60 & 1,460 \\
\hline 1946 & March 27 & - & 675 & 1972 & April 13 & 14.03 & 2,160 \\
\hline 1947 & April 19 & 13.28 & 1,840 & 1973 & March 17 & 11.25 & 897 \\
\hline 1948 & April 14 & -- & 1,640 & 1974 & April 16 & 15.87 & 1,890 \\
\hline 1949 & June 1 & 10.40 & 990 & 1975 & April 20 & 19.24 & 2,550 \\
\hline 1950 & April 22 & 16.31 & 3,040 & 1976 & April 2 & 13.60 & 1,390 \\
\hline 1951 & April 11 & 11.90 & 1,250 & 1977 & May 31 & -- & 298 \\
\hline 1952 & April 12 & 9.52 & 544 & 1978 & April 10 & 27.21 & 3,060 \\
\hline 1953 & March 29 & -- & 219 & 1979 & April 20 & -- & 3,400 \\
\hline 1954 & June 15 & -- & 489 & 1980 & April 5 & 10.10 & 879 \\
\hline 1955 & April 8 & 10.35 & 842 & 1981 & May 24 & 7.20 & 362 \\
\hline 1956 & April 20 & 10.72 & 1,370 & 1982 & April 15 & 10.00 & 820 \\
\hline 1957 & June 29 & 7.06 & 481 & 1983 & June 14 & 11.00 & 1,230 \\
\hline 1958 & July 5 & -- & 168 & 1984 & June 9 & 15.60 & 2,850 \\
\hline 1959 & April 2 & 7.64 & 310 & 1985 & August 17 & 10.50 & 974 \\
\hline 1960 & April 6 & 8.80 & 460 & 1986 & March 29 & 16.21 & 2,000 \\
\hline 1961 & March 25 & 4.86 & 140 & 1987 & July 29 & 7.95 & 492 \\
\hline 1962 & July 8 & 11.70 & 1,570 & 1988 & March 5 & 8.94 & 610 \\
\hline 1963 & April 7 & 6.34 & 300 & 1989 & March 10 & 25.00 & 2,430 \\
\hline 1964 & April 17 & 9.40 & 730 & 1990 & June 2 & 7.52 & 405 \\
\hline 1965 & April 14 & 17.81 & 4,560 & 1991 & May 23 & 8.71 & 613 \\
\hline 1966 & April 2 & 17.33 & 4,220 & 1992 & August 25 & -- & 312 \\
\hline 1967 & March 30 & 14.46 & 2,060 & 1993 & August 3 & 16.13 & 1,320 \\
\hline 1968 & June 7 & 12.71 & 1,400 & 1994 & July 8 & 12.67 & 1,660 \\
\hline \multicolumn{8}{|c|}{ Annual peak discharge, from highest to lowest, and corresponding gage height } \\
\hline 1965 & April 14 & 17.81 & 4,560 & 1962 & July 8 & 11.70 & 1,570 \\
\hline 1966 & April 2 & 17.33 & 4,220 & 1971 & April 8 & 13.60 & 1,460 \\
\hline 1969 & April 14 & 24.57 & 4,180 & 1968 & June 7 & 12.71 & 1,400 \\
\hline 1979 & April 20 & -- & 3,400 & 1976 & April 2 & 13.60 & 1,390 \\
\hline 1978 & April 10 & 27.21 & 3,060 & 1956 & April 20 & 10.72 & 1,370 \\
\hline 1950 & April 22 & 16.31 & 3,040 & 1993 & August 3 & 16.13 & 1,320 \\
\hline 1984 & June 9 & 15.60 & 2,850 & 1951 & April 11 & 11.90 & 1,250 \\
\hline 1975 & April 20 & 19.24 & 2,550 & 1983 & June 14 & 11.00 & 1,230 \\
\hline 1989 & March 10 & 25.00 & 2,430 & 1949 & June 1 & 10.40 & 990 \\
\hline 1972 & April 13 & 14.03 & 2,160 & 1985 & August 17 & 10.50 & 974 \\
\hline 1967 & March 30 & 14.46 & 2,060 & 1943 & April 7 & 10.48 & 941 \\
\hline 1986 & March 29 & 16.27 & 2,000 & 1973 & March 17 & 11.25 & 897 \\
\hline 1970 & April 16 & 13.64 & 1,980 & 1980 & April 5 & 10.10 & 879 \\
\hline 1974 & April 16 & 15.87 & 1,890 & 1955 & April 8 & 10.35 & 842 \\
\hline 1947 & April 19 & 13.28 & 1,840 & 1982 & April 15 & 10.00 & 820 \\
\hline 1994 & July 8 & 12.67 & 1,660 & 1945 & April 11 & 9.18 & 767 \\
\hline 1948 & April 14 & -- & 1,640 & 1964 & April 17 & 9.40 & 730 \\
\hline
\end{tabular}




\section{SAND HILL RIVER AT CLIMAX, MN--Continued}

Annual peak discharge and corresponding gage height-Continued

$$
[-, \text { no data }]
$$

\begin{tabular}{|c|c|c|c|c|c|c|c|}
\hline $\begin{array}{l}\text { Water } \\
\text { year }\end{array}$ & Date & $\begin{array}{c}\text { Gage } \\
\text { helght } \\
\text { (feet) }\end{array}$ & $\begin{array}{c}\text { Peak } \\
\text { discharge } \\
\left(\mathrm{ft}^{3} / \mathrm{s}\right)\end{array}$ & $\begin{array}{l}\text { Water } \\
\text { year }\end{array}$ & Date & $\begin{array}{c}\text { Gage } \\
\text { height } \\
\text { (feet) }\end{array}$ & $\begin{array}{c}\text { Peak } \\
\text { discharge } \\
\left(\mathrm{ft}^{3} / \mathrm{s}\right)\end{array}$ \\
\hline \multicolumn{8}{|c|}{ Annual peak discharge, from highest to lowest, and corresponding gage height-Continued } \\
\hline 1946 & March 27 & -- & 675 & 1981 & May 24 & 7.20 & 362 \\
\hline 1991 & May 23 & 8.71 & 613 & 1992 & August 25 & -- & 312 \\
\hline 1988 & March 5 & 8.94 & 610 & 1959 & April 2 & 7.64 & 310 \\
\hline 1952 & April 12 & 9.52 & 544 & 1963 & April 7 & 6.34 & 300 \\
\hline 1987 & July 29 & 7.95 & 492 & 1977 & May 31 & -- & 298 \\
\hline 1954 & June 15 & -- & 489 & 1944 & August 18 & -- & 226 \\
\hline 1957 & June 29 & 7.06 & 481 & 1953 & March 29 & -- & 219 \\
\hline 1960 & April 6 & 8.80 & 460 & 1958 & July 5 & -- & 168 \\
\hline 1990 & June 2 & 7.52 & 405 & 1961 & March 25 & 4.86 & 140 \\
\hline
\end{tabular}




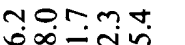

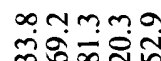

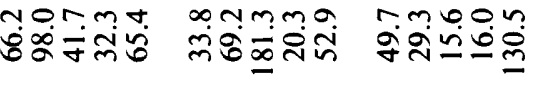

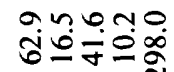

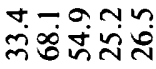

nonmo

T.m.

Foron

$m o+-\infty$

onomo

nmorico

mกmun nnamo

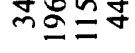

กิ우웅

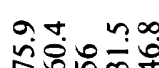

$\infty \infty \bar{m} 8=$

งกิ์ที่

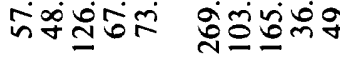

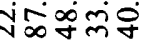

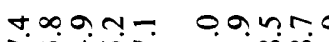

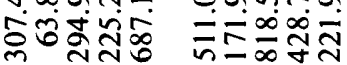

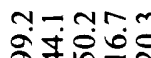

ทำำ=

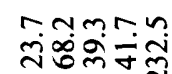

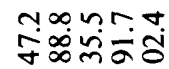

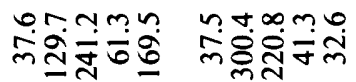

: :

singo

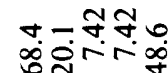

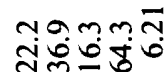

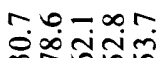

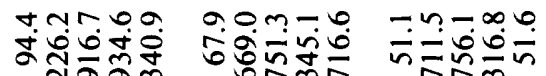

$\stackrel{0}{\underline{1}}$

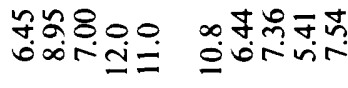

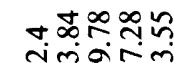

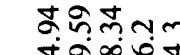

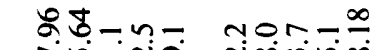

$1+119$

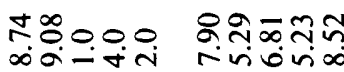

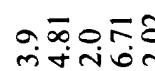

$\approx 8 \infty-n$

$+\infty .00$

onn-mn tonos

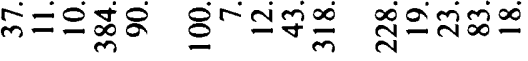

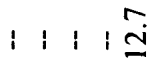
$\infty \sigma= \pm \pm$

$\min$

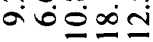

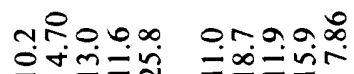

:

宅争导

o. $\sin \infty$

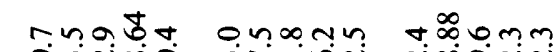

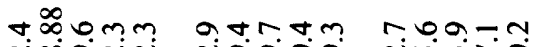
电六

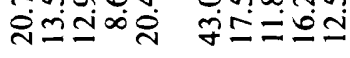
moñ

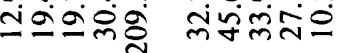

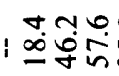

minon

$\infty \operatorname{nos}+0$

$\tan \underset{\infty}{\infty} \stackrel{0}{=}$

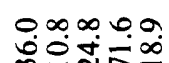

tonon nong? - $\sin$

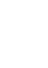




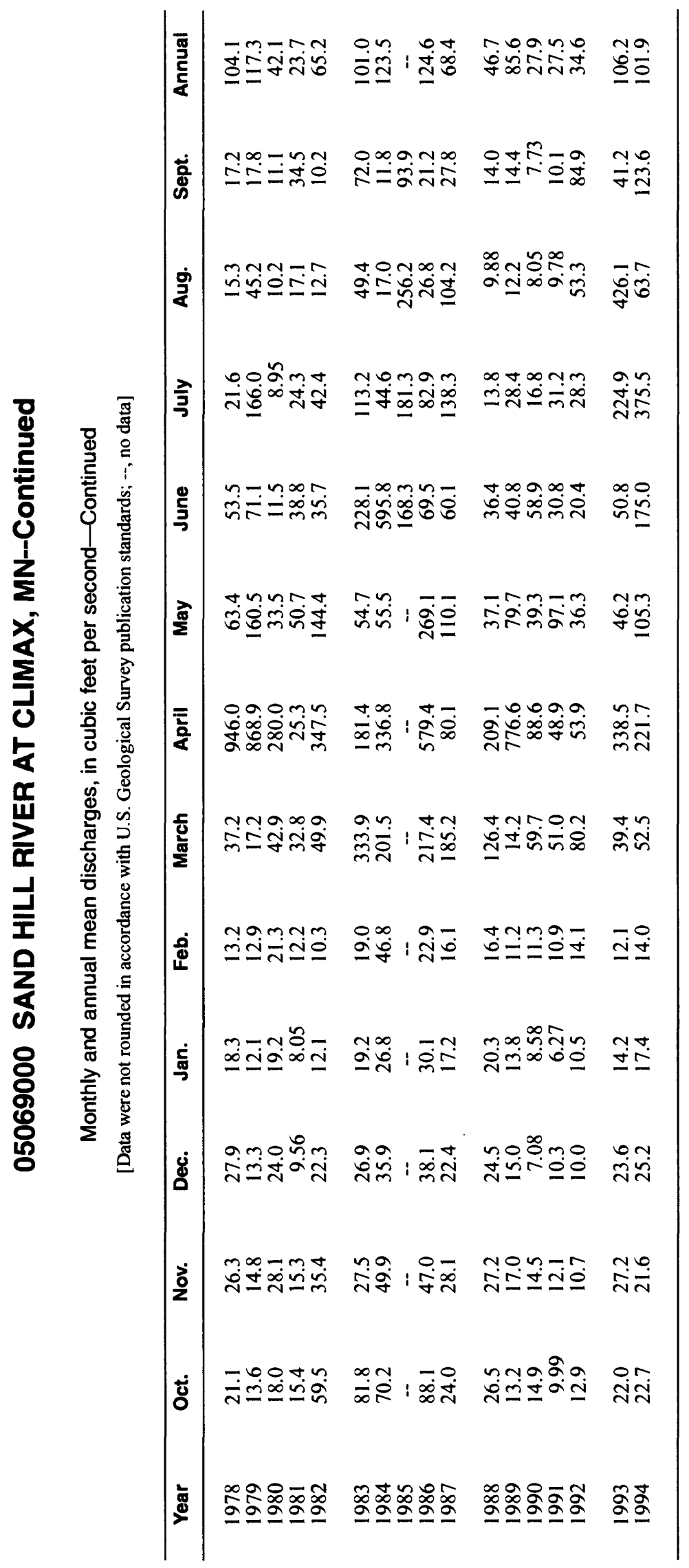




\section{RED LAKE RIVER NEAR RED LAKE, MN}

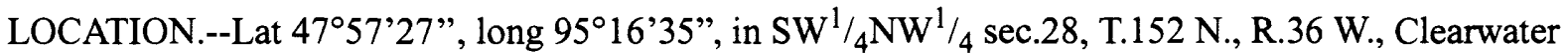
County, Hydrologic Unit 09020302, on Red Lake Indian Reservation, on left bank $50 \mathrm{ft}$ downstream from dam at outlet of Lower Red Lake and 13 mi northwest of city of Red Lake.

DRAINAGE AREA.--1,950 $\mathrm{mi}^{2}$, approximately.

PERIOD OF RECORD.--May 1933 to September 1994. Monthly discharge only for May 1933, published WSP 1308.

GAGE.--Water-stage recorder. Datum of gage is $1,100.00 \mathrm{ft}$, adjustment of 1912 (levels by U.S. Army Corps of Engineers). Prior to Sept. 7, 1934, nonrecording gage at site $50 \mathrm{ft}$ upstream at datum $69.00 \mathrm{ft}$ higher. Sept. 7, 1934, to Nov. 26, 1951, water-stage recorder at present site at datum $69.00 \mathrm{ft}$ higher. Nov. 27, 1951, to Sept. 30, 1986, water-stage recorder at present site at datum $67.00 \mathrm{ft}$ higher.

EXTREMES FOR PERIOD OF RECORD.--Maximum discharge, 3,600 $\mathrm{ft}^{3} / \mathrm{s}$, June 25 , 1950, gage height, $78.19 \mathrm{ft}$; no flow at times.

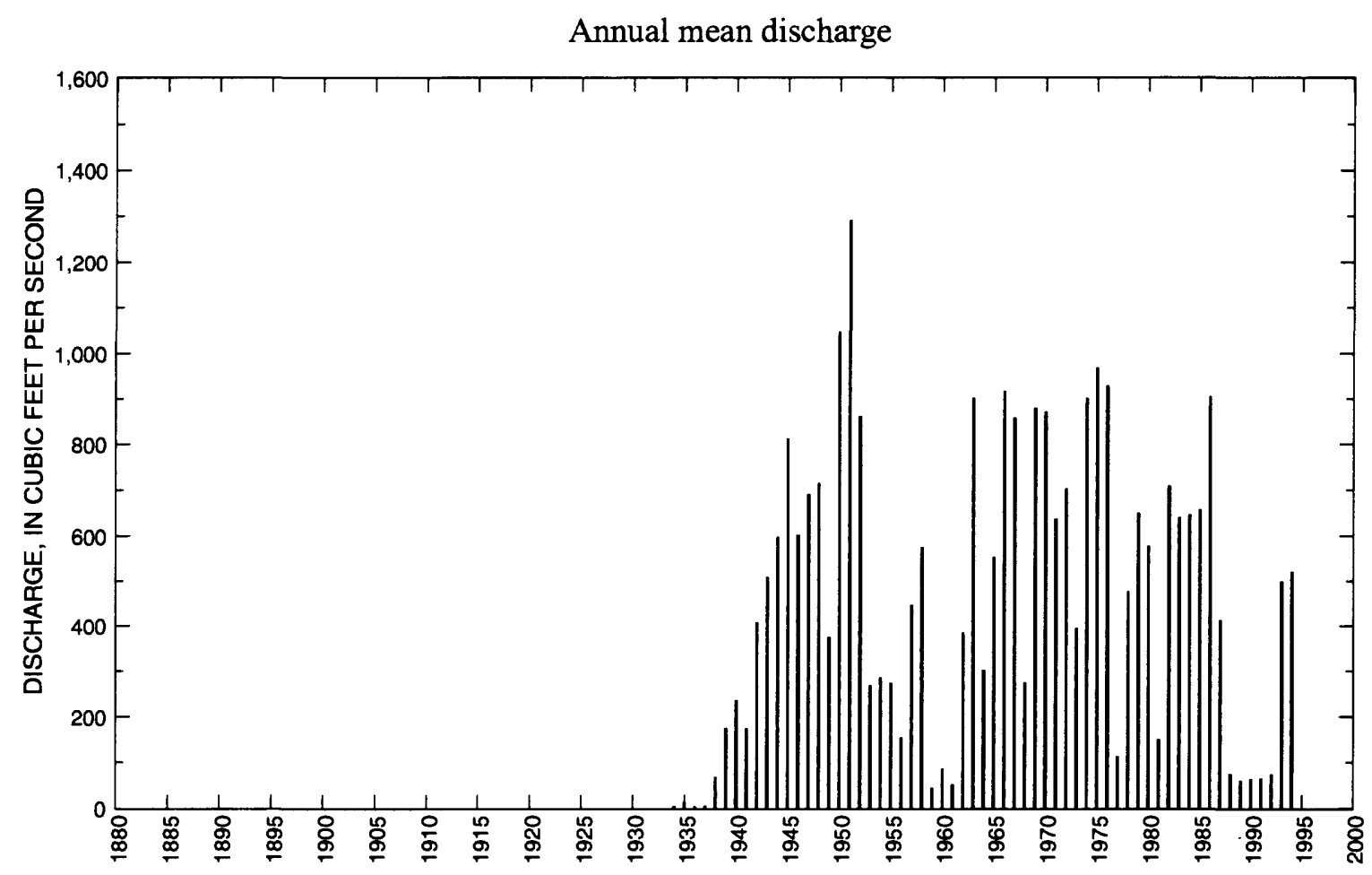




\section{RED LAKE RIVER NEAR RED LAKE, MN--Continued}

Statistics of monthly and annual mean discharges

\begin{tabular}{|c|c|c|c|c|c|c|c|c|}
\hline \multirow[b]{2}{*}{ Month } & \multicolumn{2}{|c|}{ Maximum } & \multicolumn{2}{|c|}{ Minimum } & \multicolumn{4}{|c|}{ Mean } \\
\hline & $\begin{array}{c}\text { Discharge } \\
\left(\mathrm{ft}^{3} / \mathbf{s}\right)\end{array}$ & $\begin{array}{l}\text { Water year } \\
\text { of } \\
\text { occurrence }\end{array}$ & $\begin{array}{c}\text { Discharge } \\
\left(\mathrm{ft}^{3} / \mathrm{s}\right)\end{array}$ & $\begin{array}{c}\text { Water year } \\
\text { of } \\
\text { occurrence }\end{array}$ & $\begin{array}{c}\text { Discharge } \\
\left(\mathrm{ft}^{3} / \mathrm{s}\right)\end{array}$ & $\begin{array}{c}\text { Standard } \\
\text { deviation } \\
\left(\mathrm{ft}^{3} / \mathbf{s}\right)\end{array}$ & $\begin{array}{l}\text { Coeffi- } \\
\text { clent of } \\
\text { variation }\end{array}$ & $\begin{array}{l}\text { Percentage } \\
\text { of annual } \\
\text { discharge }\end{array}$ \\
\hline October & 2,070 & 1951 & 5.10 & 1934 & 471 & 439 & 0.93 & 8.43 \\
\hline November & 1,650 & 1951 & 3.57 & 1934 & 456 & 368 & 0.81 & 8.16 \\
\hline December & 1,500 & 1951 & 0.952 & 1934 & 453 & 339 & 0.75 & 8.11 \\
\hline January & 1,420 & 1951 & 0.355 & 1934 & 466 & 356 & 0.76 & 8.35 \\
\hline February & 1,340 & 1951 & 0.400 & 1934 & 461 & 351 & 0.76 & 8.26 \\
\hline March & 1,400 & 1951 & 0.597 & 1936 & 423 & 326 & 0.77 & 7.58 \\
\hline April & 1,200 & 1951 & 4.00 & 1936 & 332 & 299 & 0.90 & 5.95 \\
\hline May & 1,620 & 1950 & 0.603 & 1933 & 483 & 442 & 0.92 & 8.65 \\
\hline June & 2,020 & 1950 & 2.15 & 1933 & 571 & 481 & 0.84 & 10.2 \\
\hline July & 1,840 & 1950 & 4.63 & 1934 & 534 & 432 & 0.81 & 9.57 \\
\hline August & 1,460 & 1975 & 2.73 & 1936 & 469 & 388 & 0.83 & 8.40 \\
\hline September & 1,710 & 1950 & 1.61 & 1934 & 464 & 413 & 0.89 & 8.31 \\
\hline Annual & 1,290 & 1951 & 5.55 & 1936 & 469 & 330 & 0.70 & 100 \\
\hline
\end{tabular}

Annual flow duration

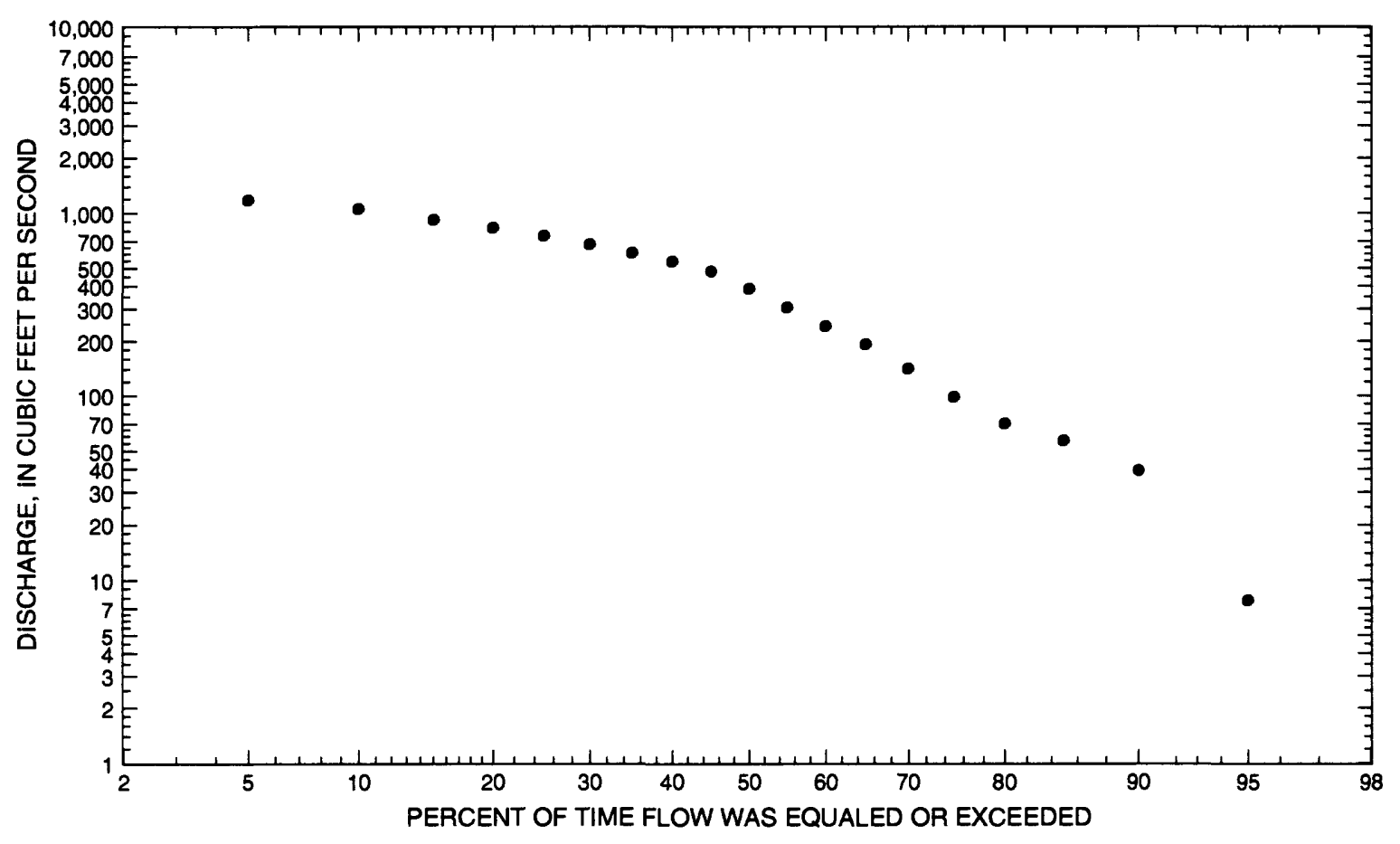




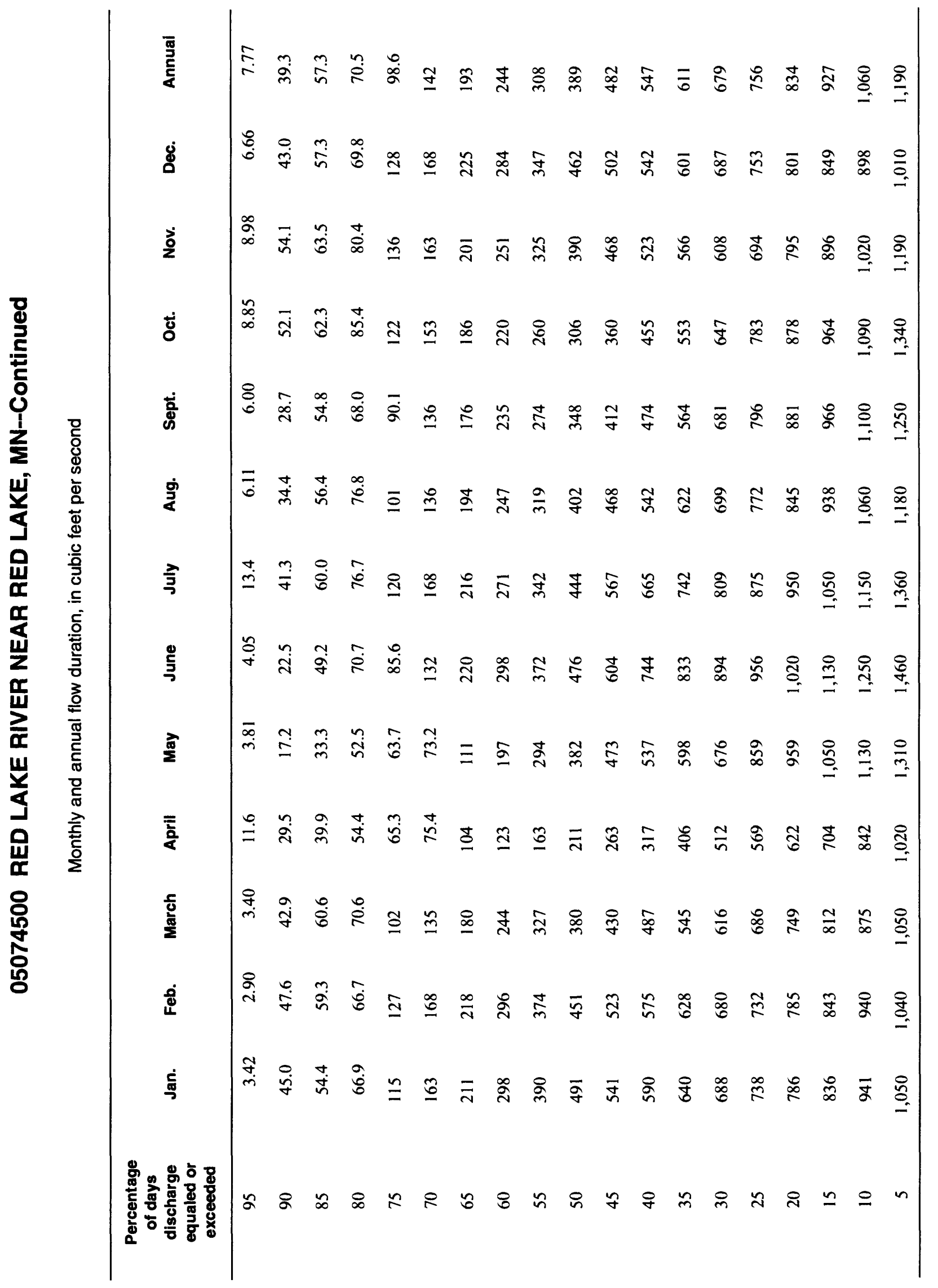


05074500 RED LAKE RIVER NEAR RED LAKE, MN--Continued

Probability of occurrence of annual high discharges

[ng, statistic not given]

\begin{tabular}{|c|c|c|c|c|c|c|}
\hline \multirow[b]{2}{*}{$\begin{array}{l}\text { Exceedance } \\
\text { probability }\end{array}$} & \multirow[b]{2}{*}{$\begin{array}{l}\text { Recurrence } \\
\text { interval } \\
\text { (years) }\end{array}$} & \multirow[b]{2}{*}{$\begin{array}{c}\text { Maximum } \\
\text { instantaneous } \\
\left(\mathrm{ft}^{3} / \mathbf{s}\right)\end{array}$} & \multicolumn{4}{|c|}{$\begin{array}{l}\text { Maximum mean discharge } \\
\qquad\left(\mathrm{ft}^{3} / \mathrm{s}\right)\end{array}$} \\
\hline & & & 3-day period & 7-day period & 15-day period & 30-day period \\
\hline 0.99 & 1.01 & 24.3 & 16.7 & 15.0 & 13.3 & 10.5 \\
\hline 0.95 & 1.05 & 76.7 & 65.1 & 60.0 & 55.5 & 47.6 \\
\hline 0.90 & 1.11 & 133 & 122 & 114 & 107 & 95.4 \\
\hline 0.80 & 1.25 & 247 & 240 & 228 & 217 & 200 \\
\hline 0.50 & 2 & 685 & 683 & 662 & 646 & 620 \\
\hline 0.20 & 5 & 1,560 & 1,440 & 1,420 & 1,400 & 1,360 \\
\hline 0.10 & 10 & 2,240 & 1,920 & 1,910 & 1,880 & 1,830 \\
\hline 0.04 & 25 & 3,120 & 2,430 & 2,440 & 2,400 & 2,320 \\
\hline 0.02 & 50 & 3,770 & 2,740 & 2,770 & 2,710 & 2,600 \\
\hline 0.01 & 100 & 4,400 & 3,000 & 3,030 & 2,970 & 2,830 \\
\hline 0.005 & 200 & 5,000 & 3,210 & 3,250 & 3,180 & 3,010 \\
\hline 0.002 & 500 & 5,740 & $\mathrm{ng}$ & ng & ng & $\mathrm{ng}$ \\
\hline
\end{tabular}

Probability of occurrence of annual low discharges

\begin{tabular}{|c|c|c|c|c|c|c|c|c|c|c|}
\hline \multirow[b]{3}{*}{$\begin{array}{l}\text { Non- } \\
\text { exceed- } \\
\text { ance } \\
\text { prob- } \\
\text { ability }\end{array}$} & \multirow[b]{3}{*}{$\begin{array}{l}\text { Recur- } \\
\text { rence } \\
\text { inter- } \\
\text { val } \\
\text { (years) }\end{array}$} & \multicolumn{9}{|c|}{ Minimum mean discharge $\left(\mathrm{ft}^{3} / \mathrm{s}\right)$} \\
\hline & & \multicolumn{9}{|c|}{ Number of consecutive days } \\
\hline & & 1 & 3 & 7 & 14 & 30 & 60 & 90 & 120 & 183 \\
\hline 0.05 & 20 & 0.700 & 0.773 & ${ }^{1} 1.35$ & 1.95 & 3.66 & 4.97 & 8.27 & 10.3 & 14.2 \\
\hline 0.10 & 10 & 3.46 & 3.96 & ${ }^{1} 4.90$ & 5.93 & 9.89 & 12.9 & 20.1 & 24.5 & 31.2 \\
\hline 0.20 & 5 & 11.5 & 13.3 & 14.9 & 18.8 & 28.0 & 35.8 & 51.4 & 61.4 & 72.3 \\
\hline 0.50 & 2 & 59.6 & 67.9 & 91.8 & 98.0 & 129 & 165 & 210 & 241 & 265 \\
\hline
\end{tabular}

${ }^{1}$ Graphical interpretation. 


\section{RED LAKE RIVER NEAR RED LAKE, MN--Continued}

Probability of occurrence of seasonal low discharges

\begin{tabular}{|c|c|c|c|c|c|c|c|c|c|}
\hline \multirow[b]{3}{*}{$\begin{array}{l}\text { Non- } \\
\text { exceedance } \\
\text { probability }\end{array}$} & \multirow[b]{3}{*}{$\begin{array}{c}\text { Recurrence } \\
\text { intervai } \\
\text { (years) }\end{array}$} & \multicolumn{8}{|c|}{ Minimum mean discharge $\left(f t^{3} / s\right)$} \\
\hline & & \multicolumn{8}{|c|}{ Number of consecutive days } \\
\hline & & 1 & 7 & 14 & 30 & 1 & 7 & 14 & 30 \\
\hline & & \multicolumn{4}{|c|}{ December-January-February } & \multicolumn{4}{|c|}{ March-April-May } \\
\hline 0.05 & 20 & 5.64 & 6.15 & 6.42 & 6.75 & 1.83 & ${ }^{1} 1.90$ & 2.77 & 4.17 \\
\hline 0.10 & 10 & 20.0 & 21.2 & 21.8 & 22.7 & 5.87 & 5.91 & 8.48 & 12.0 \\
\hline 0.20 & 5 & 69.6 & 72.2 & 73.7 & 76.2 & 18.3 & 22.0 & 27.2 & 36.2 \\
\hline \multirow[t]{2}{*}{0.50} & 2 & 342 & 354 & 361 & 374 & 94.9 & 134 & 147 & 182 \\
\hline & & \multicolumn{4}{|c|}{ June-July-August } & \multicolumn{4}{|c|}{ September-October-November } \\
\hline 0.05 & 20 & 1.81 & 2.62 & 4.18 & 9.11 & 1.12 & 3.86 & 5.96 & 10.1 \\
\hline 0.10 & 10 & 5.92 & 8.70 & 12.8 & 23.4 & 7.01 & 12.7 & 16.1 & 23.5 \\
\hline 0.20 & 5 & 20.7 & 30.3 & 41.2 & 63.0 & 27.0 & 39.9 & 45.9 & 58.2 \\
\hline 0.50 & 2 & 137 & 185 & 222 & 274 & 153 & 194 & 217 & 238 \\
\hline
\end{tabular}

${ }^{1}$ Graphical interpretation. 
05074500 RED LAKE RIVER NEAR RED LAKE, MN--Continued

Annual peak discharge and corresponding gage height

$[--$, no data]

\begin{tabular}{|c|c|c|c|c|c|c|c|}
\hline $\begin{array}{l}\text { Water } \\
\text { year }\end{array}$ & Date & $\begin{array}{c}\text { Gage } \\
\text { height } \\
\text { (feet) }\end{array}$ & $\begin{array}{c}\text { Peak } \\
\text { discharge } \\
\left(\mathrm{ft}^{3} / \mathrm{s}\right) \\
\end{array}$ & $\begin{array}{l}\text { Water } \\
\text { year }\end{array}$ & Date & $\begin{array}{c}\text { Gage } \\
\text { height } \\
\text { (feet) }\end{array}$ & $\begin{array}{c}\text { Peak } \\
\text { discharge } \\
\left(\mathrm{ft}^{3} / \mathbf{s}\right) \\
\end{array}$ \\
\hline \multicolumn{8}{|c|}{ Annual peak discharge, by year, and corresponding gage height } \\
\hline 1933 & July 4 & 72.02 & 46.0 & 1964 & October 23 & -- & 1,030 \\
\hline 1934 & May 1 & 71.52 & 57.0 & 1965 & July 22 & -- & 848 \\
\hline 1935 & June 13 & 72.02 & 140 & 1966 & May 19 & -- & 1,480 \\
\hline 1936 & September 14 & -- & 52.0 & 1967 & July 2 & -- & 1,240 \\
\hline 1937 & September 30 & 72.14 & 61.0 & 1968 & July 16 & -- & 705 \\
\hline 1938 & July 5 & 74.80 & 317 & 1969 & June 5 & -- & 1,200 \\
\hline 1939 & June 16 & 74.76 & 432 & 1970 & October 20 & -- & 1,190 \\
\hline 1940 & June 2 & 75.18 & 534 & 1971 & June 19 & -- & 889 \\
\hline 1941 & June 7 & -- & 449 & 1972 & May 17 & -- & 1,220 \\
\hline 1942 & May 13 & 75.58 & 926 & 1973 & October 21 & -- & 808 \\
\hline 1943 & June 3 & -- & 1,510 & 1974 & July 10 & -- & 1,440 \\
\hline 1944 & June 17 & -- & 1,420 & 1975 & July 31 & 75.02 & 1,680 \\
\hline 1945 & June 1 & 76.27 & 1,650 & 1976 & October 14 & 73.06 & 1,560 \\
\hline 1946 & May 31 & 75.81 & 1,400 & 1977 & October 1 & 70.30 & 252 \\
\hline 1947 & June 10 & 76.41 & 1,960 & 1978 & July 5 & 72.88 & 996 \\
\hline 1948 & May 23 & -- & 1,210 & 1979 & July 3 & 72.21 & 1,260 \\
\hline 1949 & April 24 & -- & 561 & 1980 & November 29 & 71.97 & 742 \\
\hline 1950 & June 25 & 78.19 & 3,600 & 1981 & August 31 & 71.46 & 461 \\
\hline 1951 & October 6 & 75.57 & 2,320 & 1982 & July 4 & 72.94 & 1,170 \\
\hline 1952 & May 18 & 72.97 & 1,610 & 1983 & October 14 & 72.23 & 961 \\
\hline 1953 & June 15 & 70.97 & 492 & 1984 & July 13 & 73.28 & 1,100 \\
\hline 1954 & June 29 & - & 725 & 1985 & May 31 & 72.41 & 1,200 \\
\hline 1955 & April 5 & -- & 384 & 1986 & May 28 & 72.59 & 1,470 \\
\hline 1956 & October 12 & 69.53 & 252 & 1987 & October 3 & 72.45 & 940 \\
\hline 1957 & July 20 & -- & 1,870 & 1988 & August 23 & 69.54 & 108 \\
\hline 1958 & October 1 & 73.10 & 1,360 & 1989 & July 20 & 70.44 & 72.0 \\
\hline 1959 & November 18 & - & 68.0 & 1990 & July 3 & -- & 72.0 \\
\hline 1960 & November 19 & -- & 162 & 1991 & June 26 & 70.19 & 76.0 \\
\hline 1961 & June 24 & -- & 240 & 1992 & September 19 & -- & 225 \\
\hline 1962 & August 23 & -- & 1,260 & 1993 & August 30 & 73.81 & 955 \\
\hline 1963 & October 5 & -- & 1,250 & 1994 & June 28 & -- & 1,040 \\
\hline \multicolumn{8}{|c|}{ Annual peak discharge, from highest to lowest, and corresponding gage height } \\
\hline 1950 & June 25 & 78.19 & 3,600 & 1944 & June 17 & -- & 1,420 \\
\hline 1951 & October 6 & 75.57 & 2,320 & 1946 & May 31 & 75.81 & 1,400 \\
\hline 1947 & June 10 & 76.41 & 1,960 & 1958 & October 1 & 73.10 & 1,360 \\
\hline 1957 & July 20 & - & 1,870 & 1962 & August 23 & -- & 1,260 \\
\hline 1975 & July 31 & 75.02 & 1,680 & 1979 & July 3 & 72.21 & 1,260 \\
\hline 1945 & June 1 & 76.27 & 1,650 & 1963 & October 5 & -- & 1,250 \\
\hline 1952 & May 18 & 72.97 & 1,610 & 1967 & July 2 & -- & 1,240 \\
\hline 1976 & October 14 & 73.06 & 1,560 & 1972 & May 17 & -- & 1,220 \\
\hline 1943 & June 3 & -- & 1,510 & 1948 & May 23 & -- & 1,210 \\
\hline 1966 & May 19 & -- & 1,480 & 1969 & June 5 & -- & 1,200 \\
\hline 1986 & May 28 & 72.59 & 1,470 & 1985 & May 31 & 72.41 & 1,200 \\
\hline 1974 & July 10 & -- & 1,440 & 1970 & October 20 & - & 1,190 \\
\hline
\end{tabular}




\section{RED LAKE RIVER NEAR RED LAKE, MN--Continued}

Annual peak discharge and corresponding gage height--Continued

$$
[--, \text { no data }]
$$

\begin{tabular}{|c|c|c|c|c|c|c|c|}
\hline $\begin{array}{l}\text { Water } \\
\text { year }\end{array}$ & Date & $\begin{array}{c}\text { Gage } \\
\text { height } \\
\text { (feet) }\end{array}$ & $\begin{array}{c}\text { Peak } \\
\text { discharge } \\
\left(\mathrm{ft}^{3} / \mathrm{s}\right)\end{array}$ & $\begin{array}{c}\text { Water } \\
\text { year }\end{array}$ & Date & $\begin{array}{c}\text { Gage } \\
\text { height } \\
\text { (feet) }\end{array}$ & $\begin{array}{c}\text { Peak } \\
\text { discharge } \\
\left(\mathrm{ft}^{3} / \mathrm{s}\right)\end{array}$ \\
\hline \multicolumn{8}{|c|}{ Annual peak discharge, from highest to lowest, and corresponding gage height-Continued } \\
\hline 1982 & July 4 & 72.94 & 1,170 & 1941 & June 7 & - & 449 \\
\hline 1984 & July 13 & 73.28 & 1,100 & 1939 & June 16 & 74.76 & 432 \\
\hline 1994 & June 28 & -- & 1,040 & 1955 & April 5 & -- & 384 \\
\hline 1964 & October 23 & - & 1,030 & 1938 & July 5 & 74.80 & 317 \\
\hline 1978 & July 5 & 72.88 & 996 & 1956 & October 12 & 69.53 & 252 \\
\hline 1983 & October 14 & 72.23 & 961 & 1977 & October 1 & 70.30 & 252 \\
\hline 1993 & August 30 & 73.81 & 955 & 1961 & June 24 & -- & 240 \\
\hline 1987 & October 3 & 72.45 & 940 & 1992 & September 19 & -- & 225 \\
\hline 1942 & May 13 & 75.58 & 926 & 1960 & November 19 & -- & 162 \\
\hline 1971 & June 19 & - & 889 & 1935 & June 13 & 72.02 & 140 \\
\hline 1965 & July 22 & -- & 848 & 1988 & August 23 & 69.54 & 108 \\
\hline 1973 & October 21 & - & 808 & 1991 & June 26 & 70.19 & 76.0 \\
\hline 1980 & November 29 & 71.97 & 742 & 1989 & July 20 & 70.44 & 72.0 \\
\hline 1954 & June 29 & - & 725 & 1990 & July 3 & -- & 72.0 \\
\hline 1968 & July 16 & -- & 705 & 1959 & November 18 & -- & 68.0 \\
\hline 1949 & April 24 & -- & 561 & 1937 & September 30 & 72.14 & 61.0 \\
\hline 1940 & June 2 & 75.18 & 534 & 1934 & May 1 & 71.52 & 57.0 \\
\hline 1953 & June 15 & 70.97 & 492 & 1936 & September 14 & -- & 52.0 \\
\hline 1981 & August 31 & 71.46 & 461 & 1933 & July 4 & 72.02 & 46.0 \\
\hline
\end{tabular}




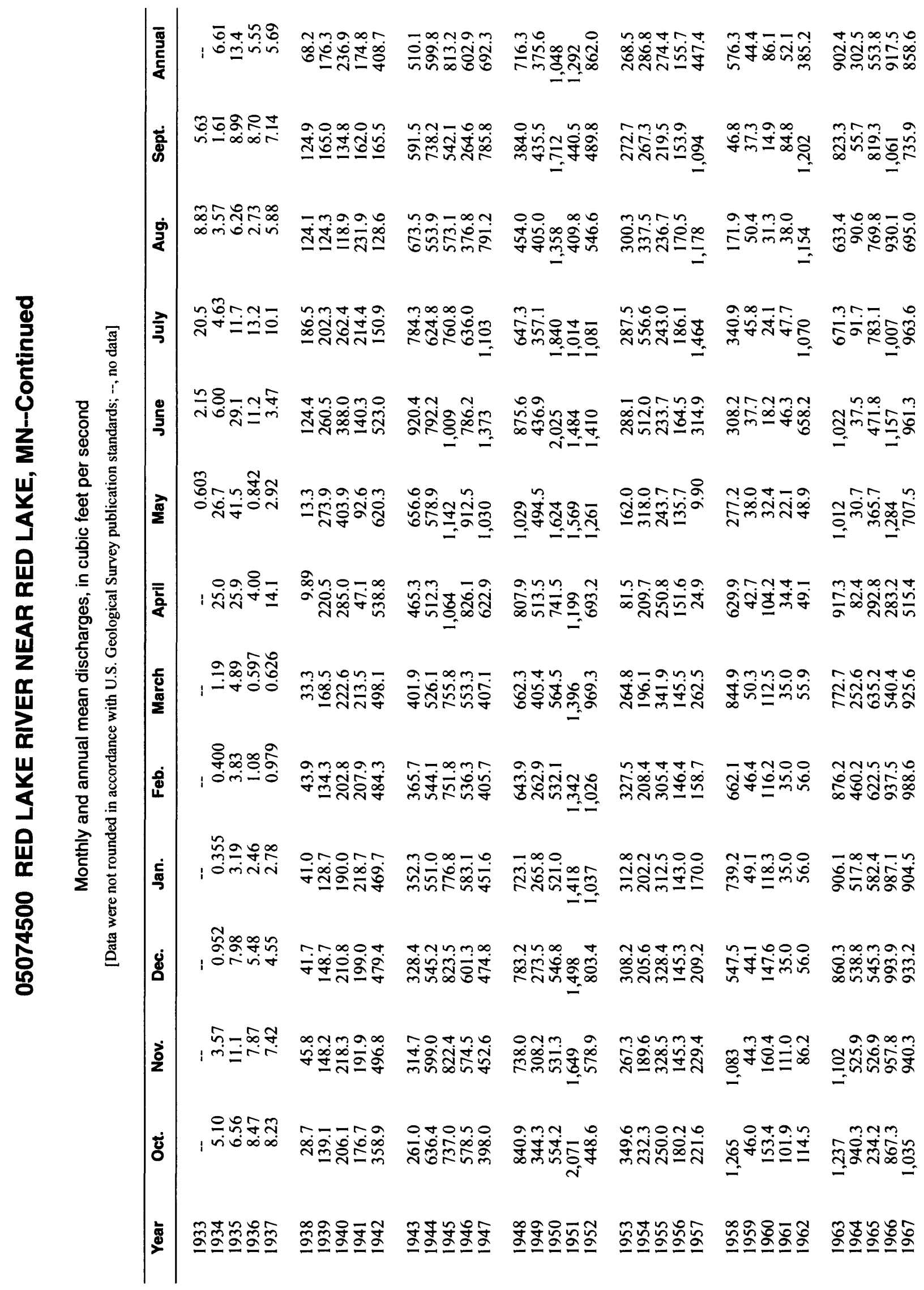




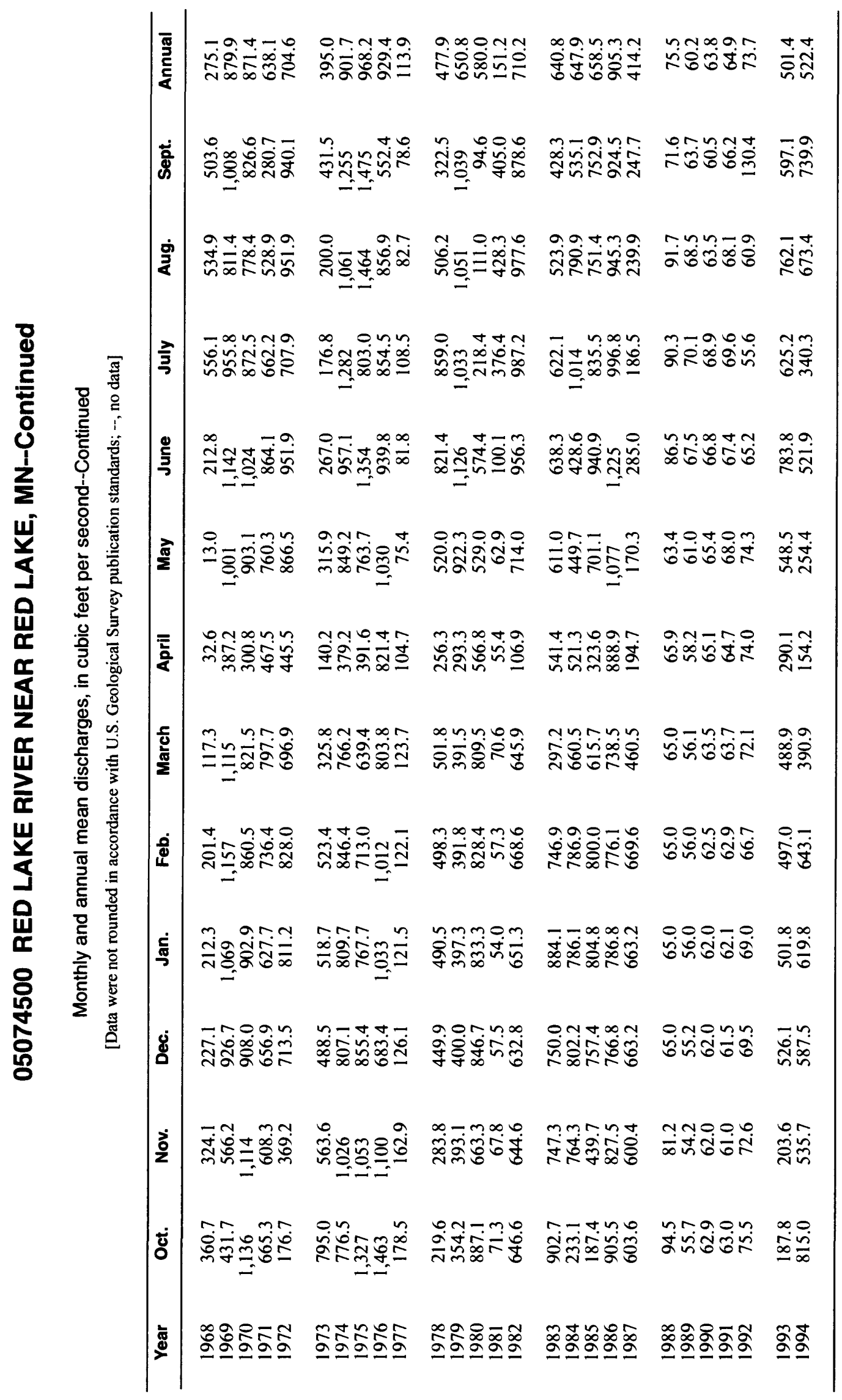




\section{RED LAKE RIVER AT HIGH LANDING NEAR GOODRIDGE, MN}

LOCATION.--Lat $48^{\circ} 02^{\prime} 34^{\prime \prime}$, long $95^{\circ} 48^{\prime} 28^{\prime \prime}$, in NW $1 / 4 \mathrm{NW}^{1} / 4$ sec.28, T.153 N., R.40 W., Pennington

County, Hydrologic Unit 09020303 , on left bank $50 \mathrm{ft}$ upstream from highway bridge at High Landing, $7 \mathrm{mi}$ south of Goodridge and $33 \mathrm{mi}$ upstream from Thief River.

DRAINAGE AREA.--2,300 $\mathrm{mi}^{2}$, approximately.

PERIOD OF RECORD.--September 1929 to current year. Prior to October 1930, published as "at Kratka”.

GAGE.--Water-stage recorder. Datum of gage is $1,141.57 \mathrm{ft}$ above sea level, adjustment of 1912 (levels by U.S. Army Corps of Engineers). See WSP 1308 or 1738 for history of changes prior to Oct. 1, 1949.

EXTREMES FOR PERIOD OF RECORD.--Maximum discharge, 4,060 $\mathrm{ft}^{3} / \mathrm{s}$, July 7, 1975, gage height, $13.39 \mathrm{ft}$; maximum gage height, $13.44 \mathrm{ft}$, July 3, 1975; no flow at times.

Annual mean discharge

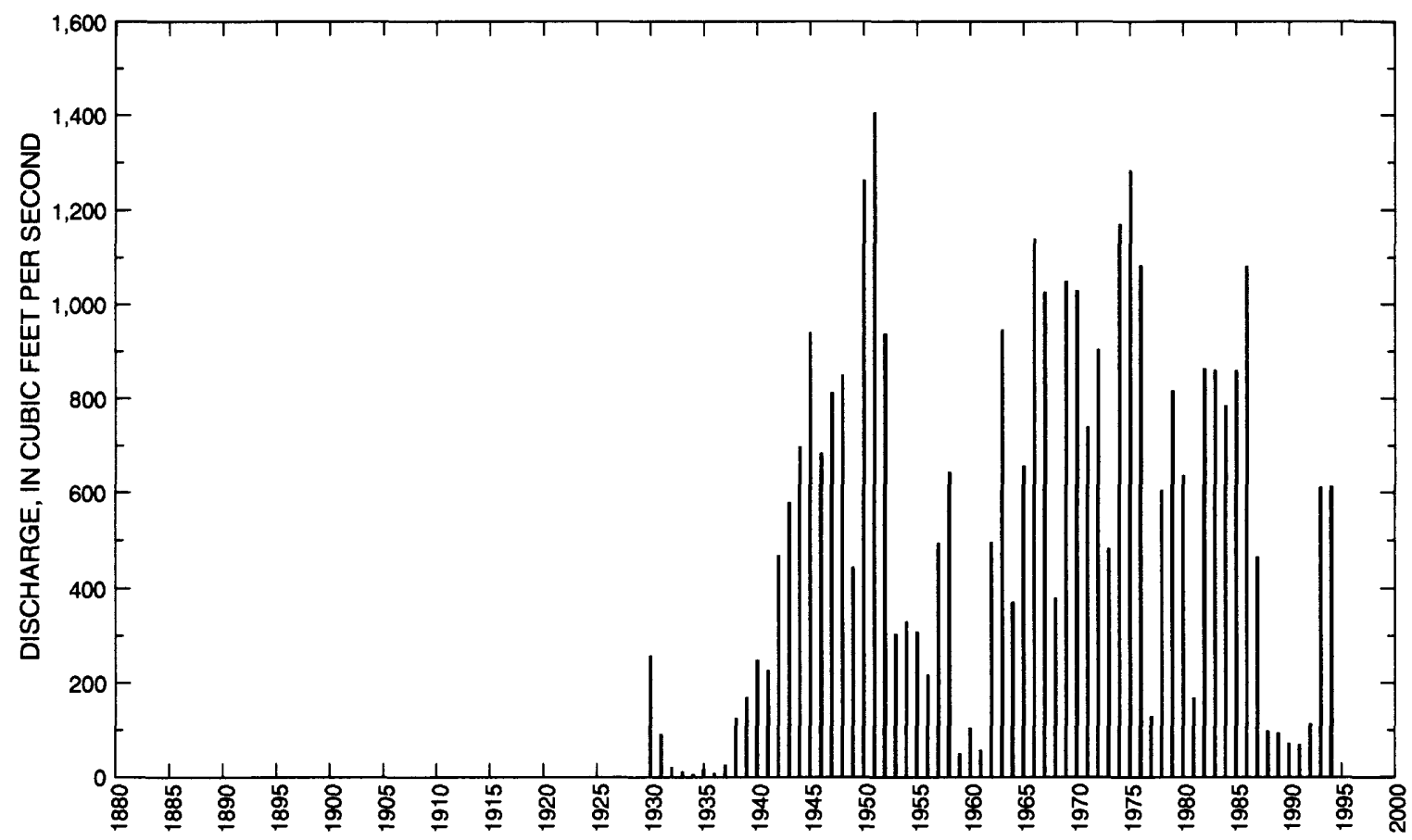


05075000 RED LAKE RIVER AT HIGH LANDING NEAR

GOODRIDGE, MN--Continued

Statistics of monthly and annual mean discharges

[m, more than 1 year of occurrence]

\begin{tabular}{|c|c|c|c|c|c|c|c|c|}
\hline \multirow[b]{2}{*}{ Month } & \multicolumn{2}{|c|}{ Maximum } & \multicolumn{2}{|c|}{ Minimum } & \multicolumn{4}{|c|}{ Mean } \\
\hline & $\begin{array}{c}\text { Discharge } \\
\left(\mathrm{ft}^{3} / \mathrm{s}\right)\end{array}$ & $\begin{array}{l}\text { Water year } \\
\text { of } \\
\text { occurrence }\end{array}$ & $\begin{array}{c}\text { Discharge } \\
\left(\mathrm{ft}^{3} / \mathrm{s}\right)\end{array}$ & $\begin{array}{l}\text { Water year } \\
\text { of } \\
\text { occurrence }\end{array}$ & $\begin{array}{c}\text { Discharge } \\
\left(\left(\mathrm{tt}^{3} / \mathbf{s}\right)\right)\end{array}$ & $\begin{array}{c}\text { Standard } \\
\text { deviation } \\
\left(\mathrm{ft}^{3} / \mathrm{s}\right)\end{array}$ & $\begin{array}{l}\text { Coeffi- } \\
\text { cient of } \\
\text { variation }\end{array}$ & $\begin{array}{l}\text { Percentage } \\
\text { of annuai } \\
\text { discharge }\end{array}$ \\
\hline October & 1,960 & 1951 & 2.11 & 1934 & 523 & 490 & 0.94 & 8.20 \\
\hline November & 1,730 & 1951 & 1.61 & 1934 & 492 & 422 & 0.86 & 7.72 \\
\hline December & 1,540 & 1951 & 0 & 1934 & 447 & 363 & 0.81 & 7.02 \\
\hline January & 1,420 & 1951 & 0 & 1934 & 446 & 366 & 0.82 & 7.00 \\
\hline February & 1,370 & 1951 & 0 & $\mathrm{~m}$ & 443 & 365 & 0.82 & 6.95 \\
\hline March & 1,450 & 1951 & 0 & $\mathrm{~m}$ & 476 & 381 & 0.80 & 7.47 \\
\hline April & 1,980 & 1951 & 24.7 & 1933 & 652 & 531 & 0.81 & 10.2 \\
\hline May & 3,180 & 1950 & 5.58 & 1933 & 658 & 617 & 0.94 & 10.3 \\
\hline June & 2,160 & 1950 & 1.04 & 1936 & 658 & 542 & 0.82 & 10.3 \\
\hline July & 2,470 & 1975 & 5.92 & 1934 & 567 & 511 & 0.90 & 8.90 \\
\hline August & 1,480 & 1975 & 0.026 & 1934 & 495 & 421 & 0.85 & 7.76 \\
\hline September & 1,730 & 1950 & 0 & 1934 & 516 & 445 & 0.86 & 8.10 \\
\hline Annual & 1,410 & 1951 & 6.21 & 1934 & 531 & 396 & 0.75 & 100 \\
\hline
\end{tabular}

Annual flow duration

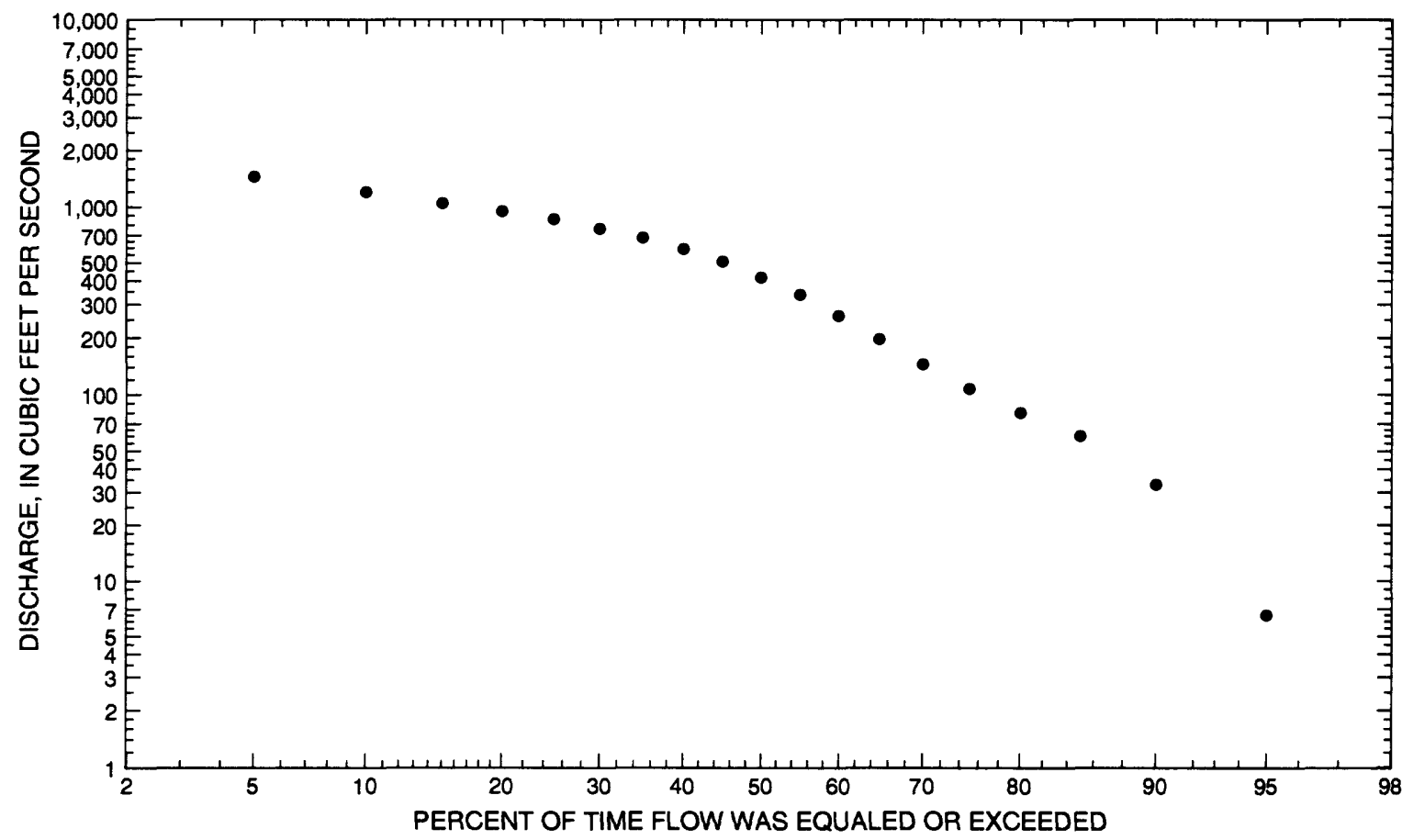


要

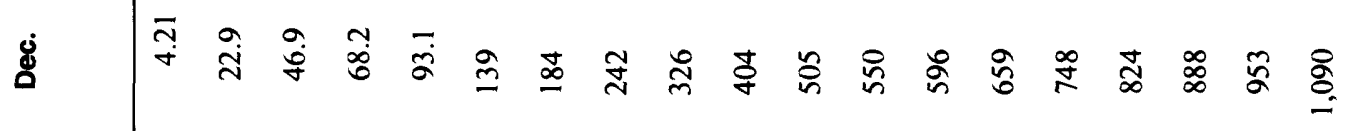

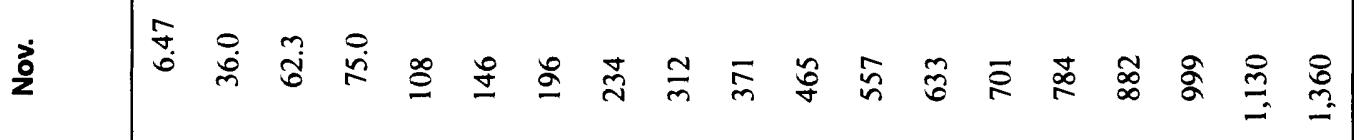

。

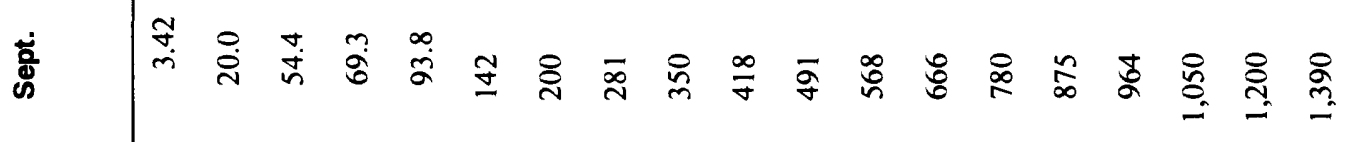

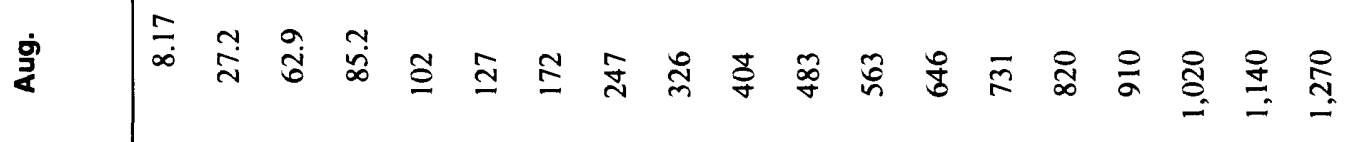

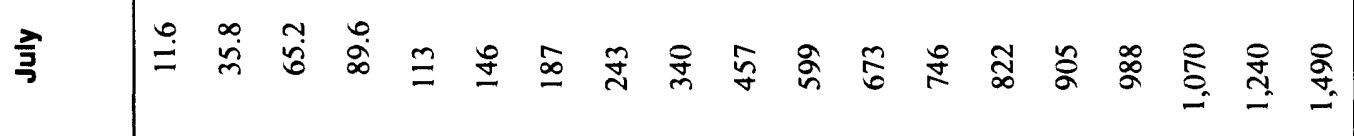

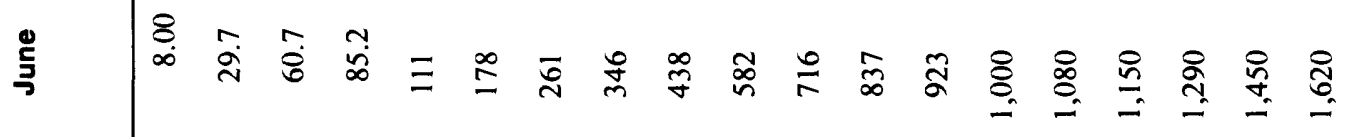

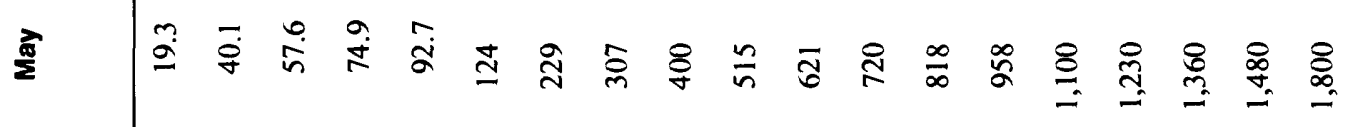

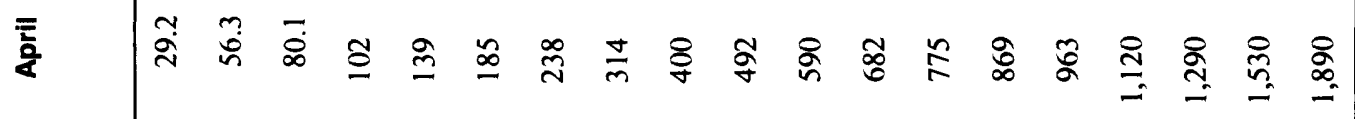

帝

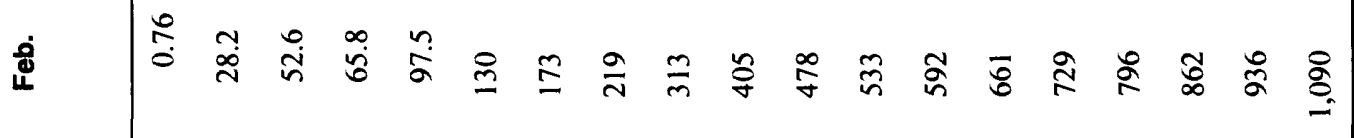

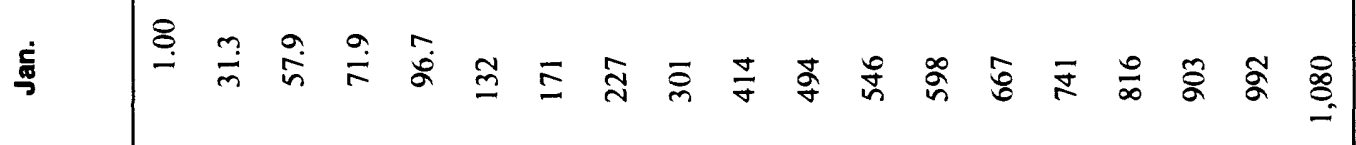

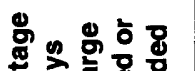

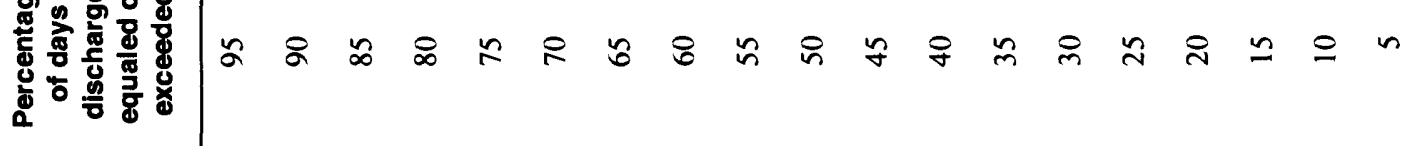




\section{RED LAKE RIVER AT HIGH LANDING NEAR \\ GOODRIDGE, MN--Continued}

Probability of occurrence of annual high discharges

[ng, statistic not given]

\begin{tabular}{|c|c|c|c|c|c|c|}
\hline \multirow[b]{2}{*}{$\begin{array}{l}\text { Exceedance } \\
\text { probability }\end{array}$} & \multirow[b]{2}{*}{$\begin{array}{l}\text { Recurrence } \\
\text { intervai } \\
\text { (years) }\end{array}$} & \multirow[b]{2}{*}{$\begin{array}{c}\text { Maximum } \\
\text { instantaneous } \\
\left(\mathrm{ft}^{3} / \mathrm{s}\right)\end{array}$} & \multicolumn{4}{|c|}{$\begin{array}{l}\text { Maximum mean discharge } \\
\qquad\left(\mathrm{ft}^{3} / \mathrm{s}\right)\end{array}$} \\
\hline & & & 3-day period & 7-day period & 15-day period & 30-day period \\
\hline 0.99 & 1.01 & $\mathrm{ng}$ & 40.5 & 33.7 & 25.0 & 20.4 \\
\hline 0.95 & 1.05 & 194 & 139 & 119 & 94.6 & 76.9 \\
\hline 0.90 & 1.11 & 314 & 247 & 214 & 175 & 142 \\
\hline 0.80 & 1.25 & 535 & 459 & 402 & 337 & 277 \\
\hline 0.50 & 2 & 1,280 & 1,200 & 1,070 & 928 & 782 \\
\hline 0.20 & 5 & 2,580 & 2,400 & 2,170 & 1,910 & 1,660 \\
\hline 0.10 & 10 & 3,470 & 3,140 & 2,850 & 2,510 & 2,230 \\
\hline 0.04 & 25 & 4,560 & 3,930 & 3,590 & 3,140 & 2,860 \\
\hline 0.02 & 50 & 5,310 & 4,410 & 4,040 & 3,520 & 3,250 \\
\hline 0.01 & 100 & 6,000 & 4,810 & 4,400 & 3,820 & 3,580 \\
\hline 0.005 & 200 & 6,630 & 5,140 & 4,710 & 4,070 & 3,850 \\
\hline 0.002 & 500 & 7,390 & ng & $\mathrm{ng}$ & $\mathrm{ng}$ & ng \\
\hline
\end{tabular}

Probability of occurrence of annual low discharges

\begin{tabular}{|c|c|c|c|c|c|c|c|c|c|c|}
\hline \multirow[b]{3}{*}{$\begin{array}{l}\text { Non- } \\
\text { exceed- } \\
\text { ance } \\
\text { prob- } \\
\text { abiiity }\end{array}$} & \multirow[b]{3}{*}{$\begin{array}{c}\text { Recur- } \\
\text { rence } \\
\text { inter- } \\
\text { vai } \\
\text { (years) }\end{array}$} & \multicolumn{9}{|c|}{ Minimum mean discharge $\left(\mathrm{ft}^{3} / \mathrm{s}\right)$} \\
\hline & & \multicolumn{9}{|c|}{ Number of consecutive days } \\
\hline & & 1 & 3 & 7 & 14 & 30 & 60 & 90 & 120 & 183 \\
\hline 0.05 & 20 & 0 & 0 & 0 & 0 & 0 & 0 & 1.12 & 3.80 & 7.63 \\
\hline 0.10 & 10 & 0 & 0 & 1.76 & 2.47 & 6.34 & ${ }^{1} 8.50$ & ${ }^{1} 11.0$ & 14.8 & 21.2 \\
\hline 0.20 & 5 & ${ }^{1} 10.0$ & ${ }^{1} 13.0$ & 17.8 & 23.5 & 32.8 & 141.0 & ${ }^{1} 51.0$ & 52.9 & 61.3 \\
\hline 0.50 & 2 & 108 & ${ }^{1} 125$ & 161 & ${ }^{1} 180$ & 191 & $1_{205}$ & $1_{240}$ & 274 & 288 \\
\hline
\end{tabular}

${ }^{1}$ Graphical interpretation. 


\section{RED LAKE RIVER AT HIGH LANDING NEAR GOODRIDGE, MN--Continued}

Probability of occurrence of seasonal low discharges

\begin{tabular}{|c|c|c|c|c|c|c|c|c|c|}
\hline \multirow[b]{3}{*}{$\begin{array}{c}\text { Non- } \\
\text { exceedance } \\
\text { probability }\end{array}$} & \multirow[b]{3}{*}{$\begin{array}{l}\text { Recurrence } \\
\text { intervai } \\
\text { (years) }\end{array}$} & \multicolumn{8}{|c|}{ Minimum mean discharge $\left(\mathrm{ft}^{3} / \mathrm{s}\right)$} \\
\hline & & \multicolumn{8}{|c|}{ Number of consecutive days } \\
\hline & & 1 & 7 & 14 & 30 & 1 & 7 & 14 & 30 \\
\hline & & \multicolumn{4}{|c|}{ December-January-February } & \multicolumn{4}{|c|}{ March-April-May } \\
\hline 0.05 & 20 & 0.184 & 0.306 & 0.438 & 0.569 & 0.072 & 0.204 & 0.500 & 2.23 \\
\hline 0.10 & 10 & 11.4 & 13.6 & 15.6 & 17.3 & 5.92 & 9.04 & 12.8 & 14.6 \\
\hline 0.20 & 5 & 53.5 & 58.2 & 62.3 & 65.8 & 31.5 & 39.9 & 47.7 & 59.9 \\
\hline \multirow[t]{2}{*}{0.50} & 2 & 305 & 310 & 315 & 323 & 213 & 233 & 246 & 319 \\
\hline & & \multicolumn{4}{|c|}{ June-July-August } & \multicolumn{4}{|c|}{ September-October-November } \\
\hline 0.05 & 20 & 0 & 0.199 & 0.539 & 3.01 & 0 & 0.020 & 0.395 & 3.43 \\
\hline 0.10 & 10 & 3.33 & 3.40 & 4.65 & 13.0 & 4.19 & 5.20 & ${ }^{1} 7.00$ & 13.4 \\
\hline 0.20 & 5 & 27.8 & ${ }^{1} 30.0$ & 32.2 & 55.0 & 29.9 & 33.4 & ${ }^{1} 40.0$ & 49.0 \\
\hline 0.50 & 2 & 235 & 273 & 309 & 348 & 181 & 253 & ${ }^{1} 263$ & 280 \\
\hline
\end{tabular}

${ }^{\mathrm{I}}$ Graphical interpretation. 


\section{RED LAKE RIVER AT HIGH LANDING NEAR GOODRIDGE, MN--Continued}

Annual peak discharge and corresponding gage height

$[--$, no data $]$

\begin{tabular}{|c|c|c|c|c|c|c|c|}
\hline $\begin{array}{l}\text { Water } \\
\text { year }\end{array}$ & Date & $\begin{array}{c}\text { Gage } \\
\text { helght } \\
\text { (feet) }\end{array}$ & $\begin{array}{c}\text { Peak } \\
\text { discharge } \\
\left(\mathrm{ft}^{3} / \mathrm{s}\right)\end{array}$ & $\begin{array}{l}\text { Water } \\
\text { year }\end{array}$ & Date & $\begin{array}{c}\text { Gage } \\
\text { height } \\
\text { (feet) }\end{array}$ & $\begin{array}{c}\text { Peak } \\
\text { discharge } \\
\left(\mathrm{ft}^{3} / \mathrm{s}\right)\end{array}$ \\
\hline \multicolumn{8}{|c|}{ Annual peak discharge, by year, and corresponding gage height } \\
\hline 1930 & April 5 & 4.08 & 912 & 1963 & April 8 & 8.31 & 1,600 \\
\hline 1931 & April 3 & 2.38 & 254 & 1964 & October 24 & 7.32 & 1,170 \\
\hline 1932 & April 9 & 3.45 & 241 & 1965 & April 13 & 11.42 & 2,740 \\
\hline 1933 & April 18 & 3.84 & 85.0 & 1966 & April 3 & 12.68 & 3,340 \\
\hline 1934 & May 3 & 1.60 & 58.0 & 1967 & March 30 & 10.84 & 2,480 \\
\hline 1935 & April 13 & 2.68 & 111 & 1968 & July 18 & 11.98 & 2,770 \\
\hline 1936 & April 17 & 3.80 & 248 & 1969 & April 10 & 10.49 & 2,320 \\
\hline 1937 & July 15 & 5.08 & 285 & 1970 & June 16 & 9.90 & 2,120 \\
\hline 1938 & May 13 & 7.18 & 1,460 & 1971 & April 8 & 10.54 & 1,610 \\
\hline 1939 & April 21 & 7.44 & 1,300 & 1972 & May 29 & 8.75 & 1,580 \\
\hline 1940 & April 19 & 6.22 & 1,300 & 1973 & September 25 & 7.93 & 1,290 \\
\hline 1941 & June 15 & -- & 912 & 1974 & April 28 & 11.07 & 2,670 \\
\hline 1942 & April 2 & 7.81 & 1,800 & 1975 & July 7 & 13.39 & 4,060 \\
\hline 1943 & April 8 & 7.22 & 2,010 & 1976 & October 25 & 8.56 & 1,890 \\
\hline 1944 & April 11 & 9.20 & 1,320 & 1977 & May 19 & 5.31 & 667 \\
\hline 1945 & March 27 & 7.91 & 2,540 & 1978 & April 8 & 11.93 & 2,440 \\
\hline 1946 & March 30 & 7.67 & 2,310 & 1979 & April 25 & 12.30 & 3,660 \\
\hline 1947 & June 15 & 8.30 & 2,660 & 1980 & April 5 & 9.05 & 1,500 \\
\hline 1948 & April 20 & 9.20 & 3,390 & 1981 & June 29 & 5.71 & 577 \\
\hline 1949 & April 13 & 5.70 & 1,360 & 1982 & April 15 & 10.37 & 1,800 \\
\hline 1950 & May 11 & 13.42 & 3,720 & 1983 & October 12 & 8.54 & 1,510 \\
\hline 1951 & April 29 & 10.24 & 2,170 & 1984 & June 9 & 10.03 & 2,210 \\
\hline 1952 & May 19 & 8.76 & 1,730 & 1985 & August 18 & 11.61 & 2,490 \\
\hline 1953 & June 9 & 5.12 & 670 & 1986 & April 29 & 10.82 & 2,470 \\
\hline 1954 & June 23 & 5.31 & 742 & 1987 & May 26 & 6.48 & 961 \\
\hline 1955 & April 8 & 8.04 & 1,240 & 1988 & April 4 & 7.75 & 600 \\
\hline 1956 & April 20 & 10.29 & 1,330 & 1989 & April 14 & 7.55 & 600 \\
\hline 1957 & June 28 & 9.91 & 1,960 & 1990 & March 13 & 5.65 & 150 \\
\hline 1958 & October 17 & 7.95 & 1,520 & 1991 & July 13 & 5.32 & 154 \\
\hline 1959 & April 3 & 5.42 & 326 & 1992 & April 21 & -- & 390 \\
\hline 1960 & April 6 & 7.45 & 530 & 1993 & July 26 & 10.74 & 1,860 \\
\hline 1961 & April 20 & 2.92 & 282 & 1994 & July 8 & 10.66 & 2,020 \\
\hline 1962 & June 11 & 12.10 & 3,060 & & & & \\
\hline \multicolumn{8}{|c|}{ Annual peak discharge, from highest to lowest, and corresponding gage height } \\
\hline 1975 & July 7 & 13.39 & 4,060 & 1947 & June 15 & 8.30 & 2,660 \\
\hline 1950 & May 11 & 13.42 & 3,720 & 1945 & March 27 & 7.91 & 2,540 \\
\hline 1979 & April 25 & 12.30 & 3,660 & 1985 & August 18 & 11.61 & 2,490 \\
\hline 1948 & April 20 & 9.20 & 3,390 & 1967 & March 30 & 10.84 & 2,480 \\
\hline 1966 & April 3 & 12.68 & 3,340 & 1986 & April 29 & 10.82 & 2,470 \\
\hline 1962 & June 11 & 12.10 & 3,060 & 1978 & April 8 & 11.93 & 2,440 \\
\hline 1968 & July 18 & 11.98 & 2,770 & 1969 & April 10 & 10.49 & 2,320 \\
\hline 1965 & April 13 & 11.42 & 2,740 & 1946 & March 30 & 7.67 & 2,310 \\
\hline 1974 & April 28 & 11.07 & 2,670 & 1984 & June 9 & 10.03 & 2,210 \\
\hline
\end{tabular}




\section{RED LAKE RIVER AT HIGH LANDING NEAR GOODRIDGE, MN--Continued}

Annual peak discharge and corresponding gage height--Continued

$$
[--, \text { no data] }
$$

\begin{tabular}{|c|c|c|c|c|c|c|c|}
\hline $\begin{array}{l}\text { Water } \\
\text { year }\end{array}$ & Date & $\begin{array}{c}\text { Gage } \\
\text { height } \\
\text { (feet) }\end{array}$ & $\begin{array}{c}\text { Peak } \\
\text { discharge } \\
\left(\mathrm{ft}^{3} / \mathrm{s}\right)\end{array}$ & $\begin{array}{l}\text { Water } \\
\text { year }\end{array}$ & Date & $\begin{array}{c}\text { Gage } \\
\text { height } \\
\text { (feet) }\end{array}$ & $\begin{array}{c}\text { Peak } \\
\text { discharge } \\
\left(\mathrm{ft}^{3} / \mathbf{s}\right)\end{array}$ \\
\hline \multicolumn{8}{|c|}{ Annual peak discharge, from highest to lowest, and corresponding gage height-Continued } \\
\hline 1951 & April 29 & 10.24 & 2,170 & 1964 & October 24 & 7.32 & 1,170 \\
\hline 1970 & June 16 & 9.90 & 2,120 & 1987 & May 26 & 6.48 & 961 \\
\hline 1994 & July 8 & 10.66 & 2,020 & 1930 & April 5 & 4.08 & 912 \\
\hline 1943 & April 8 & 7.22 & 2,010 & 1941 & June 15 & - & 912 \\
\hline 1957 & June 28 & 9.91 & 1,960 & 1954 & June 23 & 5.31 & 742 \\
\hline 1976 & October 25 & 8.56 & 1,890 & 1977 & May 19 & 5.31 & 667 \\
\hline 1993 & July 26 & 10.74 & 1,860 & 1953 & June 9 & 5.12 & 670 \\
\hline 1942 & April 2 & 7.81 & 1,800 & 1988 & April 4 & 7.75 & 600 \\
\hline 1982 & April 15 & 10.37 & 1,800 & 1989 & April 14 & 7.55 & 600 \\
\hline 1952 & May 19 & 8.76 & 1,730 & 1981 & June 29 & 5.71 & 577 \\
\hline 1971 & April 8 & 10.54 & 1,610 & 1960 & April 6 & 7.45 & 530 \\
\hline 1963 & April 8 & 8.31 & 1,600 & 1992 & April 21 & -- & 390 \\
\hline 1972 & May 29 & 8.75 & 1,580 & 1959 & April 3 & 5.42 & 326 \\
\hline 1958 & October 17 & 7.95 & 1,520 & 1937 & July 15 & 5.08 & 285 \\
\hline 1983 & October 12 & 8.54 & 1,510 & 1961 & April 20 & 2.92 & 282 \\
\hline 1980 & April 5 & 9.05 & 1,500 & 1931 & April 3 & 2.38 & 254 \\
\hline 1938 & May 13 & 7.18 & 1,460 & 1936 & April 17 & 3.80 & 248 \\
\hline 1949 & April 13 & 5.70 & 1,360 & 1932 & April 9 & 3.45 & 241 \\
\hline 1956 & April 20 & 10.29 & 1,330 & 1991 & July 13 & 5.32 & 154 \\
\hline 1944 & April 11 & 9.20 & 1,320 & 1990 & March 13 & 5.65 & 150 \\
\hline 1939 & April 21 & 7.44 & 1,300 & 1935 & April 13 & 2.68 & 111 \\
\hline 1940 & April 19 & 6.22 & 1,300 & 1933 & April 18 & 3.84 & 85.0 \\
\hline 1973 & September 25 & 7.93 & 1,290 & 1934 & May 3 & 1.60 & 58.0 \\
\hline 1955 & April 8 & 8.04 & 1,240 & & & & \\
\hline
\end{tabular}


产

it

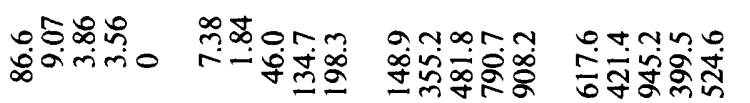

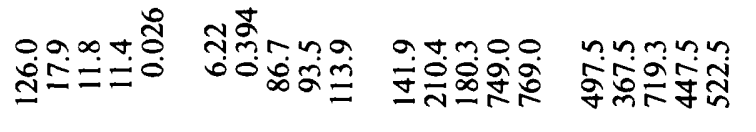
$-\pi \infty+$

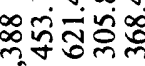

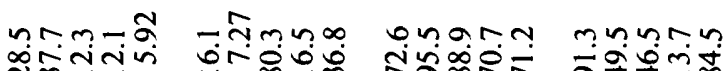

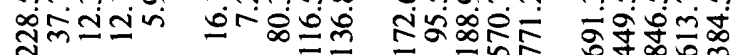

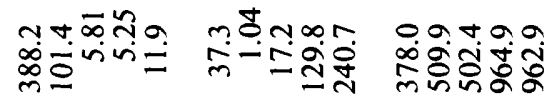

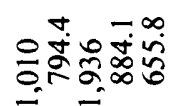

西

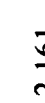

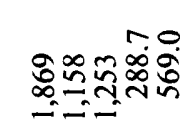

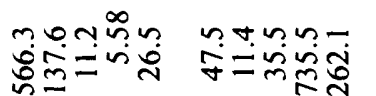

rane

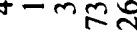

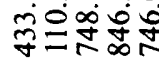

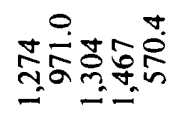

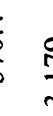

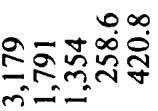

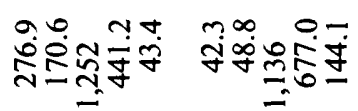

marrom

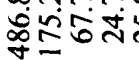

ar.e-o

rmm

亗

$0,000 \%$

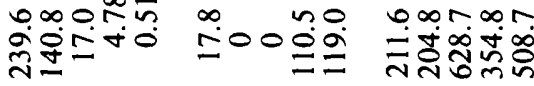

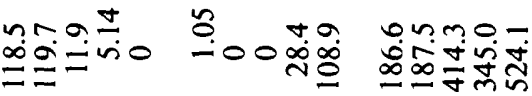

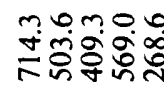

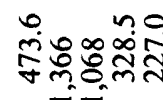

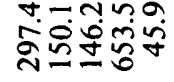

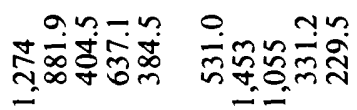

habet tatint

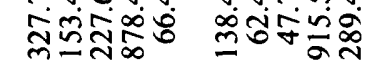

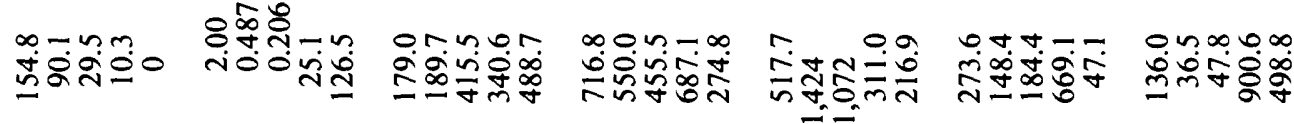

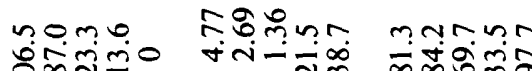

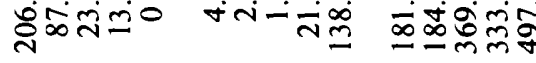

anj-nas

o $\infty a-$

m-rom omreno

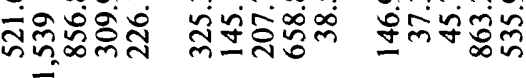

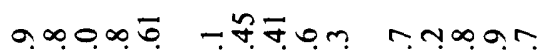

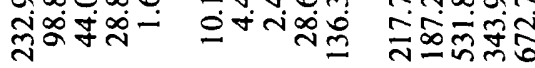

\section{$\sin -\pi$}

orom

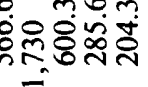

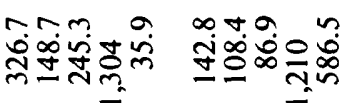

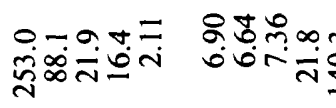

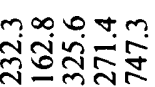

opo

วินีำ

nor. tor

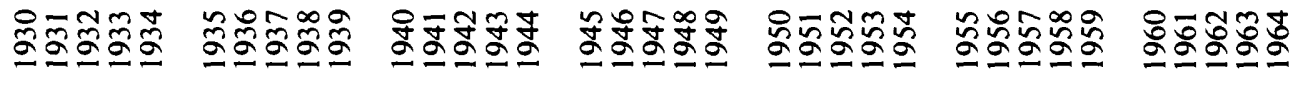




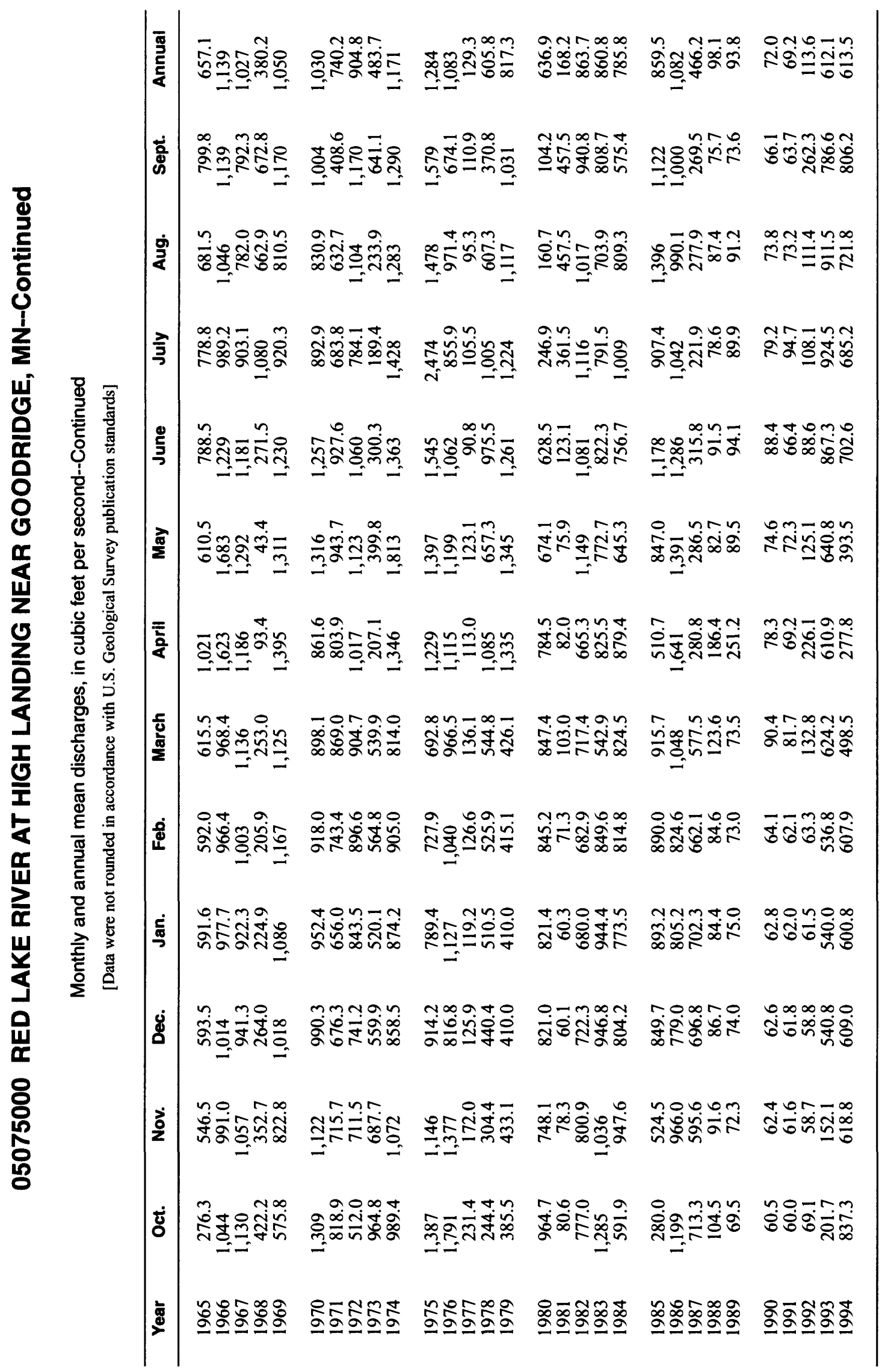




\section{THIEF RIVER NEAR THIEF RIVER FALLS, MN}

LOCATION.--Lat $48^{\circ} 11^{\prime} 08^{\prime \prime}$, long 96 $10^{\prime} 11^{\prime \prime}$, in NW $1 / 4 \mathrm{SW}^{1} / 4$ sec.3, T.154 N., R.43 W., Marshall County, Hydrologic Unit 09020304, on right bank, 0.2 mi upstream from highway bridge, 5 mi north of Thief River Falls, $7 \mathrm{mi}$ upstream from mouth, and $9 \mathrm{mi}$ downstream from Mud Lake National Wildlife Refuge.

DRAINAGE AREA.--959 $\mathrm{mi}^{2}$.

PERIOD OF RECORD.--July 1909 to September 1917, April 1920 to September 1921, October 1922 to September 1924, October 1928 to September 1981, March 1982 to current year. Monthly discharge only for some periods, annual maximums for water years 1919,1922, 1925, 1926, published in WSP 1308. October 1981 to February 1982, operated as a high-flow partial-record station.

GAGE.--Water-stage recorder and control of grouted boulders. Datum of gage is $1,112.33 \mathrm{ft}$ above mean sea level (levels by Minnesota Department of Transportation). Prior to May 4, 1939, nonrecording gages as same site and datum.

EXTREMES FOR PERIOD OF RECORD.--Maximum discharge, 5,610 $\mathrm{ft}^{3} / \mathrm{s}$, May 13, 1950, gage height, $17.38 \mathrm{ft}$; no flow at times.

Annual mean discharge

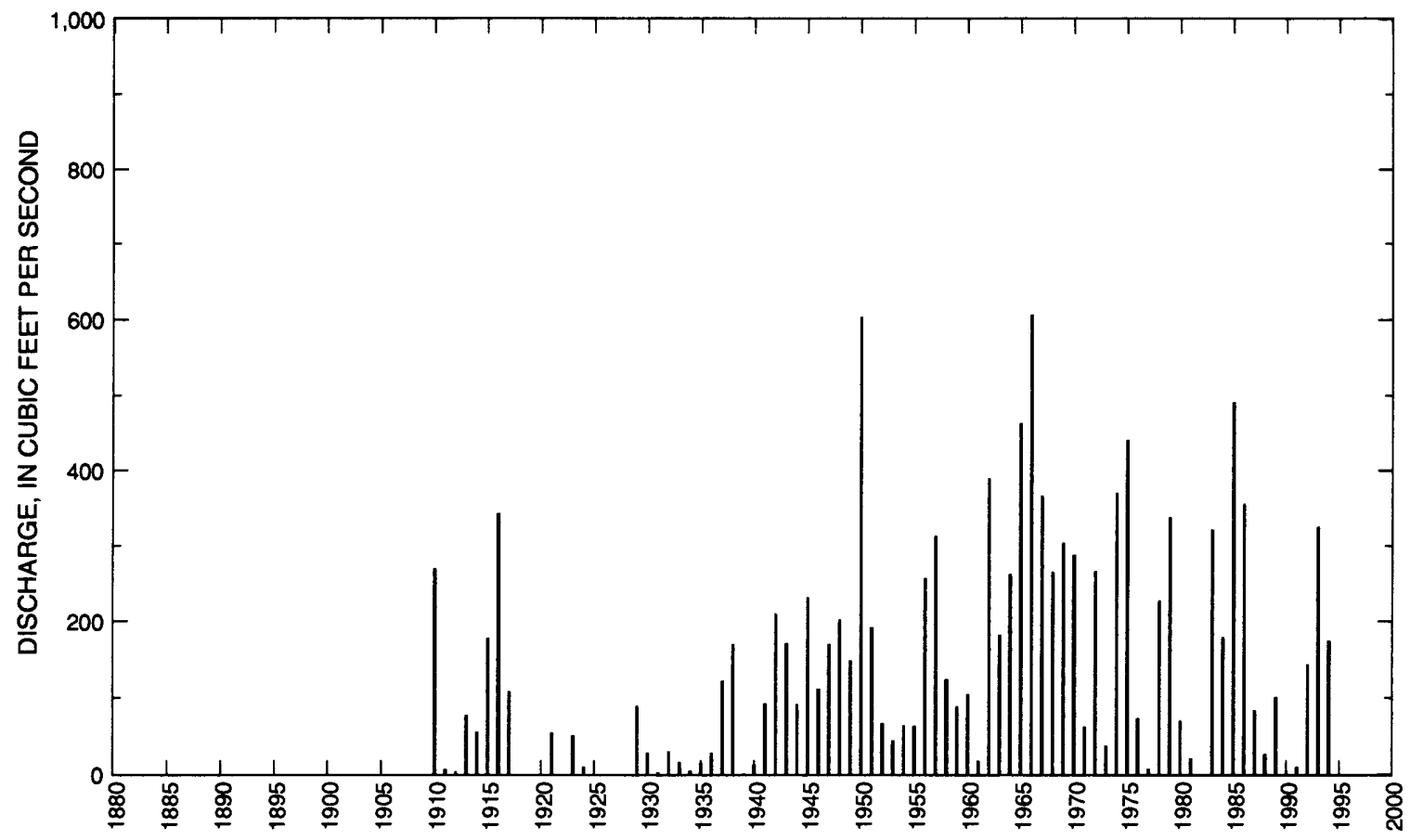


05076000 THIEF RIVER NEAR THIEF RIVER FALLS, MN--Continued

Statistics of monthly and annual mean discharges

[m, more than 1 year of occurrence]

\begin{tabular}{|c|c|c|c|c|c|c|c|c|}
\hline \multirow[b]{2}{*}{ Month } & \multicolumn{2}{|c|}{ Maximum } & \multicolumn{2}{|c|}{ Minimum } & \multicolumn{4}{|c|}{ Mean } \\
\hline & $\begin{array}{c}\text { Discharge } \\
\left(\mathrm{ft}^{3} / \mathrm{s}\right)\end{array}$ & $\begin{array}{c}\text { Water year } \\
\text { of } \\
\text { occurrence }\end{array}$ & $\begin{array}{c}\text { Discharge } \\
\left(\mathrm{ft}^{3} / \mathrm{s}\right)\end{array}$ & $\begin{array}{c}\text { Water year } \\
\text { of } \\
\text { occurrence }\end{array}$ & $\begin{array}{c}\text { Discharge } \\
\left(\mathrm{ft}^{3} / \mathrm{s}\right)\end{array}$ & $\begin{array}{l}\text { Standard } \\
\text { deviation } \\
\left(\mathrm{ft}^{3} / \mathrm{s}\right)\end{array}$ & $\begin{array}{l}\text { Coeffi- } \\
\text { cient of } \\
\text { variation }\end{array}$ & $\begin{array}{l}\text { Percentage } \\
\text { of annual } \\
\text { discharge }\end{array}$ \\
\hline October & 637 & 1986 & 0 & $\mathrm{~m}$ & 86.6 & 151 & 1.74 & 4.43 \\
\hline November & 844 & 1972 & 0 & $\mathrm{~m}$ & 62.8 & 126 & 2.00 & 3.21 \\
\hline December & 206 & 1945 & 0 & $\mathrm{~m}$ & 19.1 & 42.1 & 2.20 & 0.98 \\
\hline January & 100 & 1910 & 0 & $\mathrm{~m}$ & 5.55 & 14.9 & 2.69 & 0.28 \\
\hline February & 45.0 & 1910 & 0 & $\mathrm{~m}$ & 3.50 & 8.76 & 2.50 & 0.18 \\
\hline March & 608 & 1983 & 0 & $\mathrm{~m}$ & 67.4 & 123 & 1.82 & 3.45 \\
\hline April & 2,830 & 1966 & 7.75 & 1981 & 573 & 564 & 0.98 & 29.3 \\
\hline May & 4,270 & 1950 & 1.83 & 1990 & 454 & 653 & 1.44 & 23.2 \\
\hline June & 1,770 & 1962 & 0.032 & 1980 & 282 & 376 & 1.33 & 14.4 \\
\hline July & 2,100 & 1975 & 0 & $\mathrm{~m}$ & 208 & 343 & 1.64 & 10.7 \\
\hline August & 1,010 & 1993 & 0 & $\mathrm{~m}$ & 94.5 & 200 & 2.12 & 4.83 \\
\hline September & 1,010 & 1993 & 0 & $\mathrm{~m}$ & 99.3 & 200 & 2.02 & 5.08 \\
\hline Annual & 607 & 1966 & 1.28 & 1939 & 162 & 149 & 0.92 & 100 \\
\hline
\end{tabular}

Annual flow duration

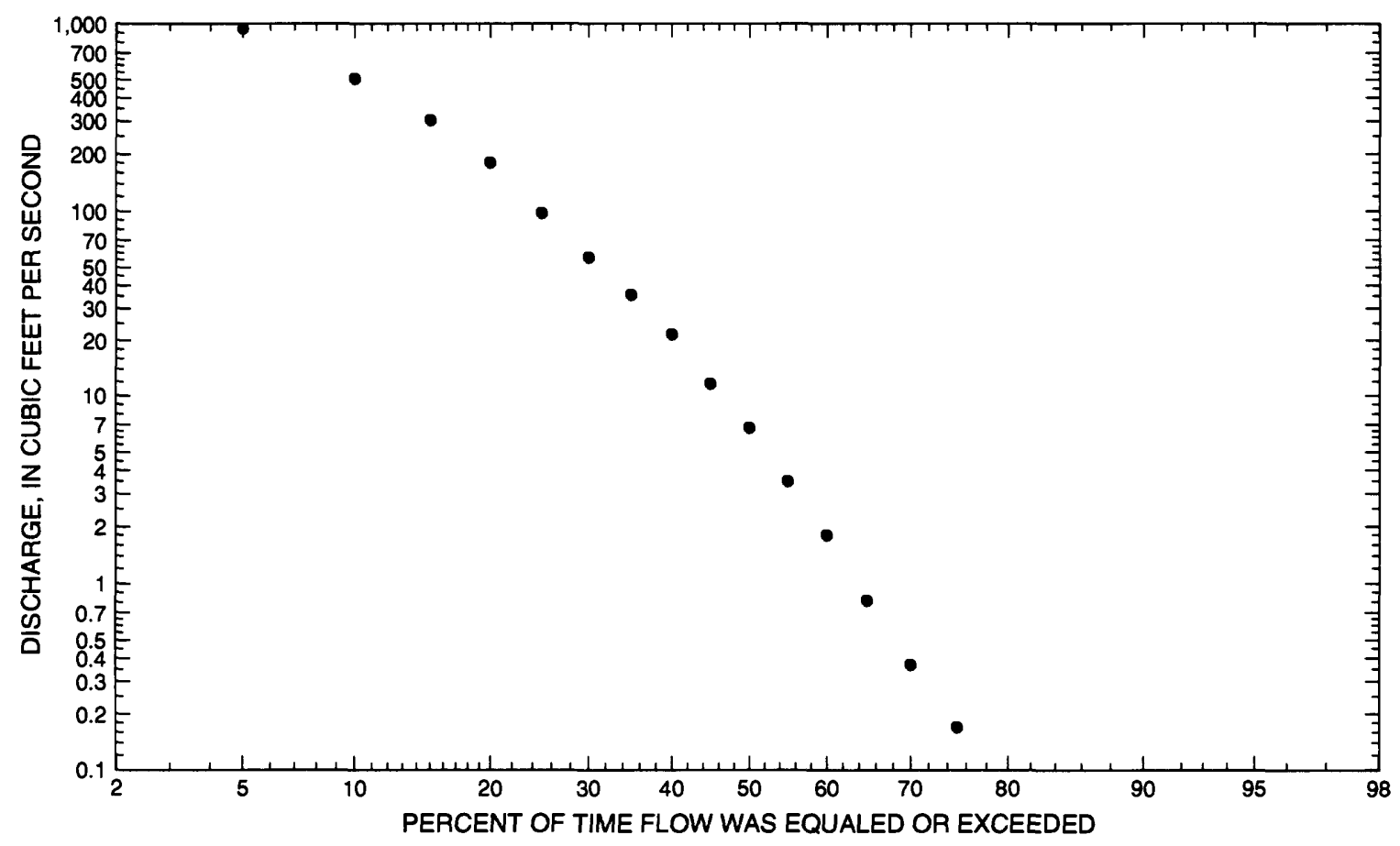




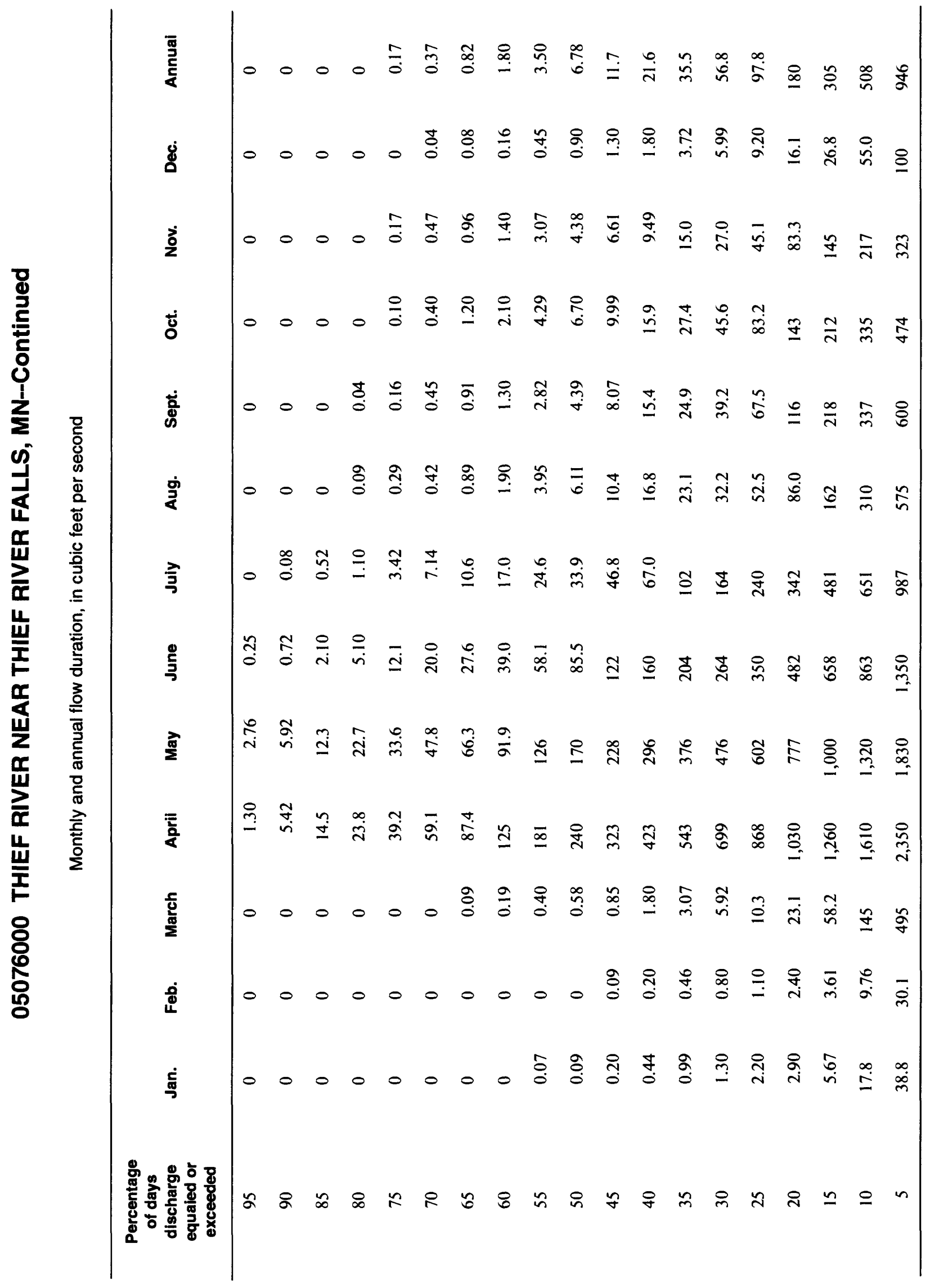


05076000 THIEF RIVER NEAR THIEF RIVER FALLS, MN--Continued

Probability of occurrence of annual high discharges

[ng, statistic not given]

\begin{tabular}{|c|c|c|c|c|c|c|}
\hline \multirow[b]{2}{*}{$\begin{array}{l}\text { Exceedance } \\
\text { probability }\end{array}$} & \multirow[b]{2}{*}{$\begin{array}{l}\text { Recurrence } \\
\text { interval } \\
\text { (years) }\end{array}$} & \multirow[b]{2}{*}{$\begin{array}{c}\text { Maximum } \\
\text { instantaneous } \\
\left(\mathrm{ft}^{3} / \mathrm{s}\right)\end{array}$} & \multicolumn{4}{|c|}{$\begin{array}{l}\text { Maximum mean discharge } \\
\left(\mathrm{ft}^{3} / \mathrm{s}\right)\end{array}$} \\
\hline & & & 3-day period & 7-day period & 15-day period & 30-day period \\
\hline 0.99 & 1.01 & ng & 28.2 & 23.7 & 15.8 & 9.76 \\
\hline 0.95 & 1.05 & 259 & 120 & 99.3 & 67.8 & 44.2 \\
\hline 0.90 & 1.11 & 400 & 231 & 191 & 133 & 89.6 \\
\hline 0.80 & 1.25 & 647 & 459 & 381 & 276 & 192 \\
\hline 0.50 & 2 & 1,420 & 1,250 & 1,080 & 853 & 638 \\
\hline 0.20 & 5 & 2,620 & 2,410 & 2,190 & 1,920 & 1,550 \\
\hline 0.10 & 10 & 3,400 & 3,010 & 2,830 & 2,630 & 2,200 \\
\hline 0.04 & 25 & 4,310 & 3,560 & 3,470 & 3,410 & 2,960 \\
\hline 0.02 & 50 & 4,910 & 3,840 & 3,820 & 3,900 & 3,470 \\
\hline 0.01 & 100 & 5,450 & 4,040 & 4,090 & 4,310 & 3,910 \\
\hline 0.005 & 200 & 5,930 & 4,180 & 4,290 & 4,650 & 4,280 \\
\hline 0.002 & 500 & 6,490 & ng & ng & ng & ng \\
\hline
\end{tabular}

Probability of occurrence of annual low discharges

\begin{tabular}{|c|c|c|c|c|c|c|c|c|c|c|}
\hline \multirow[b]{3}{*}{$\begin{array}{l}\text { Non- } \\
\text { exceed- } \\
\text { ance } \\
\text { prob- } \\
\text { abiiity }\end{array}$} & \multirow[b]{3}{*}{$\begin{array}{l}\text { Recur- } \\
\text { rence } \\
\text { inter- } \\
\text { val } \\
\text { (years) }\end{array}$} & \multicolumn{9}{|c|}{ Minimum mean discharge $\left(\mathrm{ft}^{3} / \mathrm{s}\right)$} \\
\hline & & \multicolumn{9}{|c|}{ Number of consecutive days } \\
\hline & & 1 & 3 & 7 & 14 & 30 & 60 & 90 & 120 & 183 \\
\hline 0.05 & 20 & 0 & 0 & 0 & 0 & 0 & 0 & 0 & 0 & 0 \\
\hline 0.10 & 10 & 0 & 0 & 0 & 0 & 0 & 0 & 0 & 0 & 0 \\
\hline 0.20 & 5 & 0 & 0 & 0 & 0 & 0 & 0 & 0 & 0 & 0.082 \\
\hline 0.50 & 2 & 0 & 0 & 0 & 0 & 0 & 0.037 & 0.163 & 0.761 & 4.64 \\
\hline
\end{tabular}


05076000 THIEF RIVER NEAR THIEF RIVER FALLS, MN--Continued

Probability of occurrence of seasonal low discharges

\begin{tabular}{|c|c|c|c|c|c|c|c|c|c|}
\hline \multirow[b]{3}{*}{$\begin{array}{c}\text { Non- } \\
\text { exceedance } \\
\text { probability }\end{array}$} & \multirow[b]{3}{*}{$\begin{array}{c}\text { Recurrence } \\
\text { intervai } \\
\text { (years) }\end{array}$} & \multicolumn{8}{|c|}{ Minimum mean discharge $\left(\mathrm{ft}^{3} / \mathrm{s}\right)$} \\
\hline & & \multicolumn{8}{|c|}{ Number of consecutive days } \\
\hline & & 1 & 7 & 14 & 30 & 1 & 7 & 14 & 30 \\
\hline & & \multicolumn{4}{|c|}{ December-January-February } & \multicolumn{4}{|c|}{ March-April-May } \\
\hline 0.05 & 20 & 0 & 0 & 0 & 0 & 0 & 0 & 0 & 0 \\
\hline 0.10 & 10 & 0 & 0 & 0 & 0 & 0 & 0 & 0 & 0 \\
\hline 0.20 & 5 & 0 & 0 & 0 & 0 & 0 & 0 & 0 & 0.175 \\
\hline \multirow[t]{2}{*}{0.50} & 2 & 0 & 0 & 0 & 0.011 & 0.017 & 0.108 & 0.215 & 4.80 \\
\hline & & \multicolumn{4}{|c|}{ June-July-August } & \multicolumn{4}{|c|}{ September-October-November } \\
\hline 0.05 & 20 & 0 & 0 & 0 & 0 & 0 & 0 & 0 & 0 \\
\hline 0.10 & 10 & 0 & 0 & 0 & 0 & 0 & 0 & 0 & 0 \\
\hline 0.20 & 5 & 0 & 0 & 0 & 0.182 & 0 & 0 & 0 & 0 \\
\hline 0.50 & 2 & 0.873 & 1.55 & 2.52 & 5.91 & 0.360 & 0.566 & 0.999 & 2.61 \\
\hline
\end{tabular}




\section{THIEF RIVER NEAR THIEF RIVER FALLS, MN--Continued}

Annual peak discharge and corresponding gage height

$[--$, no data $]$

\begin{tabular}{|c|c|c|c|c|c|c|c|}
\hline $\begin{array}{l}\text { Water } \\
\text { year }\end{array}$ & Date & $\begin{array}{c}\text { Gage } \\
\text { height } \\
\text { (feet) }\end{array}$ & $\begin{array}{c}\text { Peak } \\
\text { discharge } \\
\left(\mathrm{ft}^{3} / \mathrm{s}\right)\end{array}$ & $\begin{array}{l}\text { Water } \\
\text { year }\end{array}$ & Date & $\begin{array}{c}\text { Gage } \\
\text { height } \\
\text { (feet) }\end{array}$ & $\begin{array}{c}\text { Peak } \\
\text { discharge } \\
\left(\mathrm{ft}^{3} / \mathrm{s}\right)\end{array}$ \\
\hline \multicolumn{8}{|c|}{ Annual peak discharge, by year, and corresponding gage height } \\
\hline 1909 & July 19 & 10.60 & 1,970 & 1954 & April 12 & 7.61 & 624 \\
\hline 1910 & April 3 & 9.05 & 1,440 & 1955 & April 8 & 10.72 & 958 \\
\hline 1911 & April 26 & 5.40 & 127 & 1956 & April 21 & 10.57 & 1,840 \\
\hline 1912 & September 30 & 5.60 & 164 & 1957 & September 3 & 9.97 & 1,630 \\
\hline 1913 & April 7 & 14.00 & 1,530 & 1958 & October 17 & 7.67 & 828 \\
\hline 1914 & June 11 & 7.42 & 795 & 1959 & April 5 & 8.48 & 950 \\
\hline 1915 & June 30 & 10.66 & 1,920 & 1960 & April 6 & 12.11 & 1,100 \\
\hline 1916 & April 23 & 14.50 & 4,080 & 1961 & March 24 & 7.49 & 441 \\
\hline 1917 & April 11 & 12.72 & 2,600 & 1962 & June 10 & 12.68 & 2,800 \\
\hline 1919 & July 10 & 16.30 & 5,040 & 1963 & April 8 & 11.43 & 2,180 \\
\hline 1920 & April 8 & -- & 1,780 & 1964 & August 2 & 12.05 & 2,480 \\
\hline 1921 & April 6 & -- & 1,700 & 1965 & April 12 & 14.99 & 4,110 \\
\hline 1922 & May 12 & 11.90 & 2,680 & 1966 & April 3 & 15.66 & 3,320 \\
\hline 1923 & April 21 & 8.40 & 1,160 & 1967 & April 21 & 13.26 & 3,100 \\
\hline 1924 & April 21 & 5.30 & 145 & 1968 & July 18 & 10.84 & 1,950 \\
\hline 1925 & June 11 & 9.10 & 1,420 & 1969 & April 13 & 13.55 & 3,190 \\
\hline 1926 & June 25 & -- & 1,660 & 1970 & June 17 & 12.72 & 2,820 \\
\hline 1929 & March 18 & 13.71 & 1,870 & 1971 & April 9 & 11.40 & 1,780 \\
\hline 1930 & May 12 & 7.26 & 776 & 1972 & April 18 & 11.73 & 2,340 \\
\hline 1931 & April 15 & - & 39.0 & 1973 & September 29 & 7.86 & 871 \\
\hline 1932 & April 8 & 9.25 & 1,340 & 1974 & April 21 & 13.37 & 3,160 \\
\hline 1933 & April 18 & 6.35 & 470 & 1975 & July 2 & 14.17 & 3,260 \\
\hline 1934 & April 7 & 5.67 & 150 & 1976 & April 1 & 12.75 & 1,350 \\
\hline 1935 & April 13 & - & 318 & 1977 & May 19 & 6.35 & 403 \\
\hline 1936 & April 19 & -. & 890 & 1978 & April 18 & 12.57 & 2,740 \\
\hline 1937 & August 3 & 9.75 & 1,160 & 1979 & April 24 & 14.11 & 3,590 \\
\hline 1938 & May 19 & 9.00 & 1,130 & 1980 & April 7 & 10.45 & 1,710 \\
\hline 1939 & April 27 & 4.82 & 35.0 & 1981 & June 28 & 7.08 & 620 \\
\hline 1940 & April 15 & 7.85 & 728 & 1982 & May 4 & 11.27 & 2,130 \\
\hline 1941 & June 12 & 7.82 & 822 & 1983 & March 7 & 14.19 & 1,500 \\
\hline 1942 & May 2 & 9.69 & 1,480 & 1984 & June 9 & 9.80 & 1,520 \\
\hline 1943 & April 8 & 9.43 & 1,060 & 1985 & June 28 & 11.27 & 2,130 \\
\hline 1944 & June 6 & 7.15 & 666 & 1986 & May 1 & 11.82 & 2,420 \\
\hline 1945 & April 11 & 10.24 & 1,650 & 1987 & March 26 & 13.23 & 1,570 \\
\hline 1946 & April 6 & 8.77 & 1,170 & 1988 & April 4 & 10.37 & 1,170 \\
\hline 1947 & June 11 & 9.84 & 1,560 & 1989 & April 16 & 14.31 & 2,300 \\
\hline 1948 & April 19 & 10.67 & 1,850 & 1990 & April 1 & 6.11 & 150 \\
\hline 1949 & June 1 & 10.00 & 1,620 & 1991 & May 23 & 6.07 & 314 \\
\hline 1950 & May 13 & 17.38 & 5,610 & 1992 & April 22 & -- & 1,220 \\
\hline 1951 & May 2 & 9.81 & 1,630 & 1993 & August 31 & - & 2,180 \\
\hline 1952 & April 8 & 10.72 & 1,500 & 1994 & July 9 & 11.38 & 1,960 \\
\hline 1953 & June 2 & 6.37 & 429 & & & & \\
\hline
\end{tabular}


05076000 THIEF RIVER NEAR THIEF RIVER FALLS, MN--Continued

Annual peak discharge and corresponding gage height--Continued

$$
[--, \text { no data }]
$$

\begin{tabular}{|c|c|c|c|c|c|c|c|}
\hline $\begin{array}{l}\text { Water } \\
\text { year }\end{array}$ & Date & $\begin{array}{c}\text { Gage } \\
\text { height } \\
\text { (feet) }\end{array}$ & $\begin{array}{c}\text { Peak } \\
\text { discharge } \\
\left(\mathrm{ft}^{3} / \mathrm{s}\right)\end{array}$ & $\begin{array}{l}\text { Water } \\
\text { year }\end{array}$ & Date & $\begin{array}{c}\text { Gage } \\
\text { helght } \\
\text { (feet) }\end{array}$ & $\begin{array}{c}\text { Peak } \\
\text { discharge } \\
\left(\mathrm{ft}^{3} / \mathbf{s}\right)\end{array}$ \\
\hline \multicolumn{8}{|c|}{ Annual peak discharge, from highest to lowest, and corresponding gage height } \\
\hline 1950 & May 13 & 17.38 & 5,610 & 1984 & June 9 & 9.80 & 1,520 \\
\hline 1919 & July 10 & 16.30 & 5,040 & 1952 & April 8 & 10.72 & 1,500 \\
\hline 1965 & April 12 & 14.99 & 4,110 & 1983 & March 7 & 14.19 & 1,500 \\
\hline 1916 & April 23 & 14.50 & 4,080 & 1942 & May 2 & 9.69 & 1,480 \\
\hline 1979 & April 24 & 14.11 & 3,590 & 1910 & April 3 & 9.05 & 1,440 \\
\hline 1966 & April 3 & 15.66 & 3,320 & 1925 & June 11 & 9.10 & 1,420 \\
\hline 1975 & July 2 & 14.17 & 3,260 & 1976 & April 1 & 12.75 & 1,350 \\
\hline 1969 & April 13 & 13.55 & 3,190 & 1932 & April 8 & 9.25 & 1,340 \\
\hline 1974 & April 21 & 13.37 & 3,160 & 1992 & April 22 & -- & 1,220 \\
\hline 1967 & April 21 & 13.26 & 3,100 & 1946 & April 6 & 8.77 & 1,170 \\
\hline 1970 & June 17 & 12.72 & 2,820 & 1988 & April 4 & 10.37 & 1,170 \\
\hline 1962 & June 10 & 12.68 & 2,800 & 1923 & April 21 & 8.40 & 1,160 \\
\hline 1978 & April 18 & 12.57 & 2,740 & 1937 & August 3 & 9.75 & 1,160 \\
\hline 1922 & May 12 & 11.90 & 2,680 & 1938 & May 19 & 9.00 & 1,130 \\
\hline 1917 & April 11 & 12.72 & 2,600 & 1960 & April 6 & 12.11 & 1,100 \\
\hline 1964 & August 2 & 12.05 & 2,480 & 1943 & April 8 & 9.43 & 1,060 \\
\hline 1986 & May 1 & 11.82 & 2,420 & 1955 & April 8 & 10.72 & 958 \\
\hline 1972 & April 18 & 11.73 & 2,340 & 1959 & April 5 & 8.48 & 950 \\
\hline 1989 & April 16 & 14.31 & 2,300 & 1936 & April 19 & -- & 890 \\
\hline 1963 & April 8 & 11.43 & 2,180 & 1973 & September 29 & 7.86 & 871 \\
\hline 1993 & August 31 & -- & 2,180 & 1958 & October 17 & 7.67 & 828 \\
\hline 1982 & May 4 & 11.27 & 2,130 & 1941 & June 12 & 7.82 & 822 \\
\hline 1985 & June 28 & 11.27 & 2,130 & 1914 & June 11 & 7.42 & 795 \\
\hline 1909 & July 19 & 10.60 & 1,970 & 1930 & May 12 & 7.26 & 776 \\
\hline 1994 & July 9 & 11.38 & 1,960 & 1940 & April 15 & 7.85 & 728 \\
\hline 1968 & July 18 & 10.84 & 1,950 & 1944 & June 6 & 7.15 & 666 \\
\hline 1915 & June 30 & 10.66 & 1,920 & 1954 & April 12 & 7.61 & 624 \\
\hline 1929 & March 18 & 13.71 & 1,870 & 1981 & June 28 & 7.08 & 620 \\
\hline 1948 & April 19 & 10.67 & 1,850 & 1933 & April 18 & 6.35 & 470 \\
\hline 1956 & April 21 & 10.57 & 1,840 & 1961 & March 24 & 7.49 & 441 \\
\hline 1920 & April 8 & -- & 1,780 & 1953 & June 2 & 6.37 & 429 \\
\hline 1971 & April 9 & 11.40 & 1,780 & 1977 & May 19 & 6.35 & 403 \\
\hline 1980 & April 7 & 10.45 & 1,710 & 1935 & April 13 & -- & 318 \\
\hline 1921 & April 6 & -- & 1,700 & 1991 & May 23 & 6.07 & 314 \\
\hline 1926 & June 25 & -- & 1,660 & 1912 & September 30 & 5.60 & 164 \\
\hline 1945 & April 11 & 10.24 & 1,650 & 1934 & April 7 & 5.67 & 150 \\
\hline 1951 & May 2 & 9.81 & 1,630 & 1990 & April 1 & 6.11 & 150 \\
\hline 1957 & September 3 & 9.97 & 1,630 & 1924 & April 21 & 5.30 & 145 \\
\hline 1949 & June 1 & 10.00 & 1,620 & 1911 & April 26 & 5.40 & 127 \\
\hline 1987 & March 26 & 13.23 & 1,570 & 1931 & April 15 & -- & 39.0 \\
\hline 1947 & June 11 & 9.84 & 1,560 & 1939 & April 27 & 4.82 & 35.0 \\
\hline 1913 & April 7 & 14.00 & 1,530 & & & & \\
\hline
\end{tabular}


|

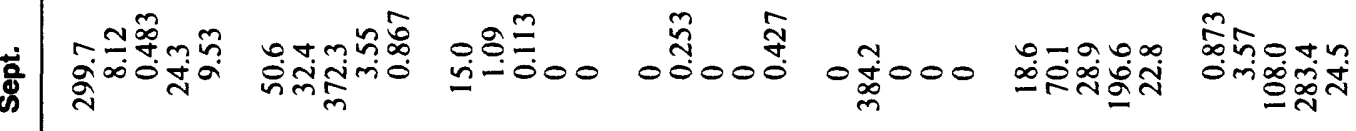

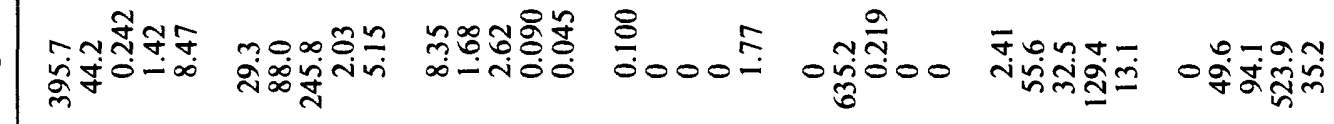
|

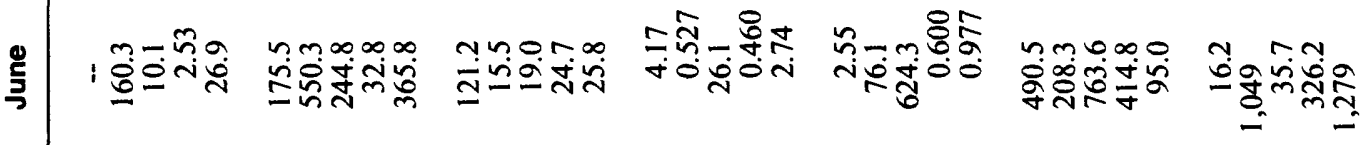

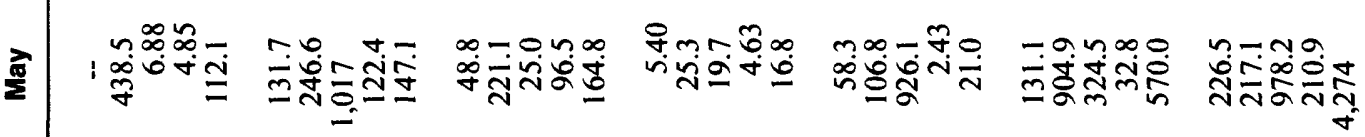
产

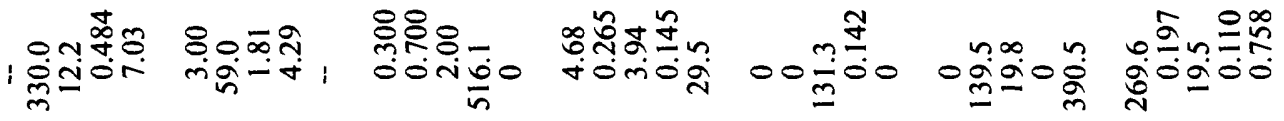

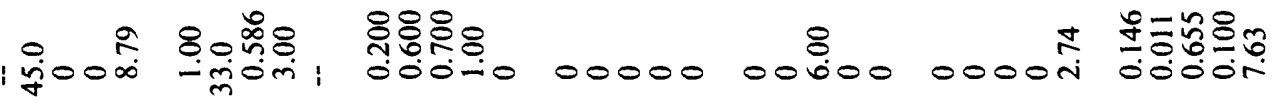

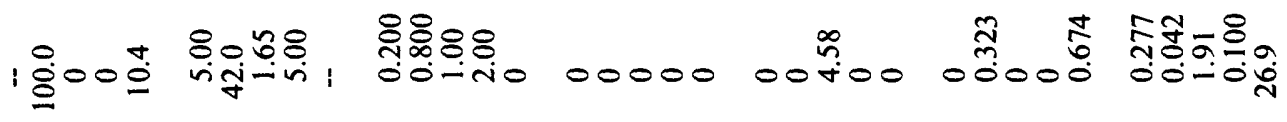

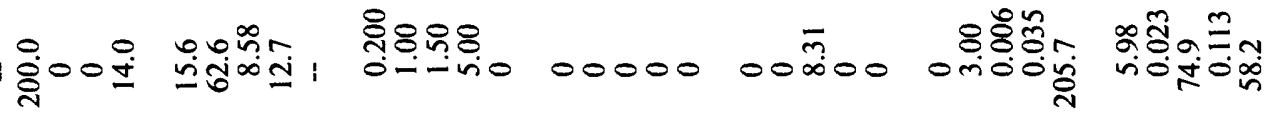

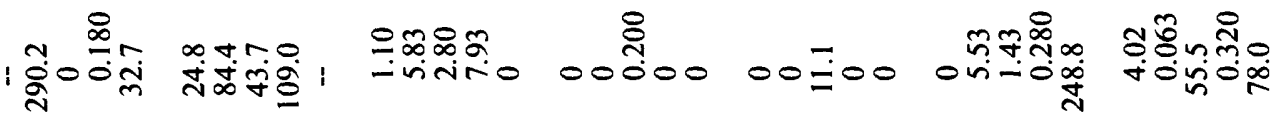

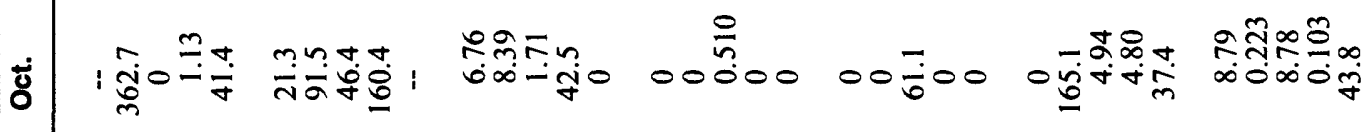

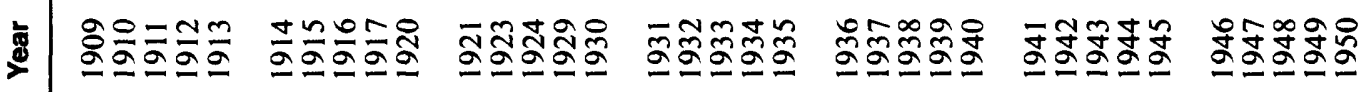




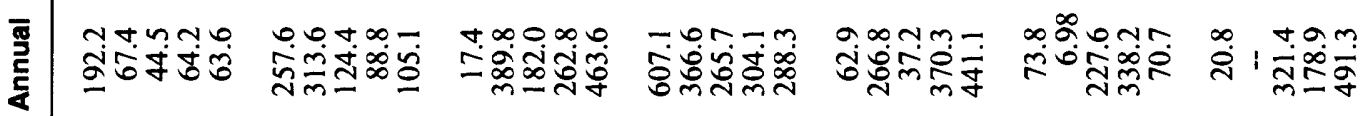

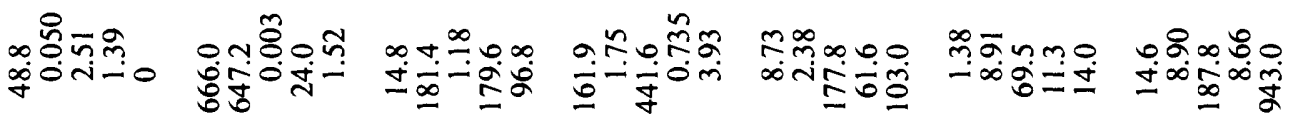

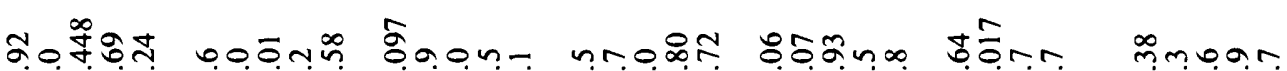

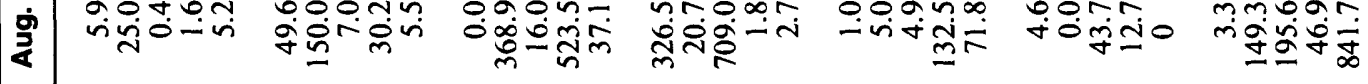

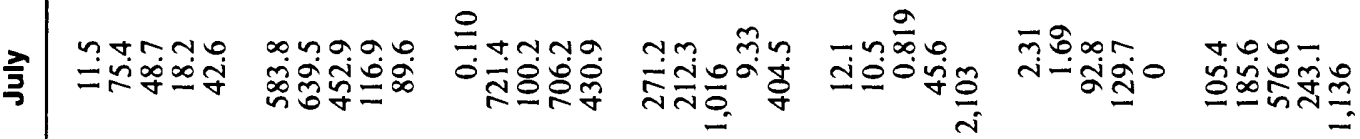

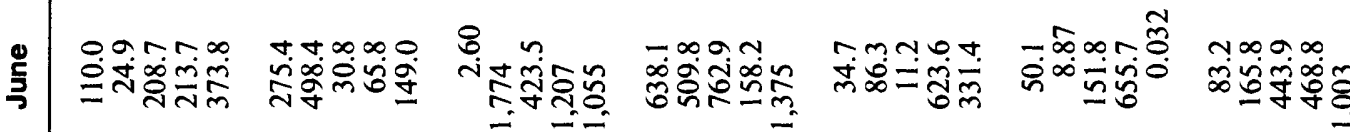

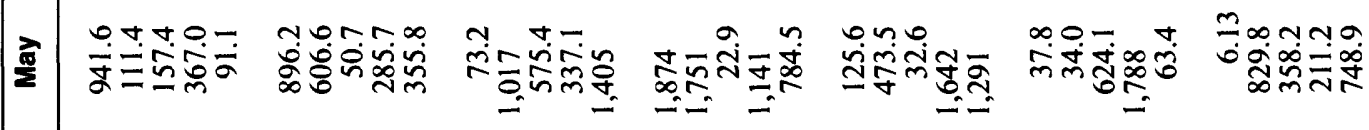

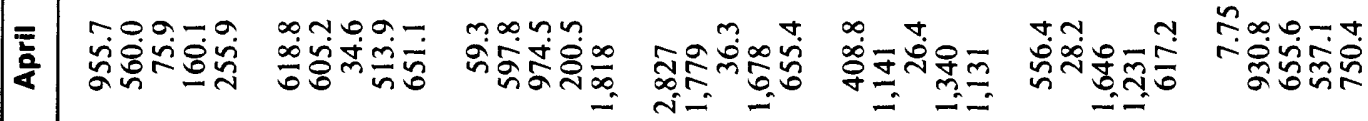

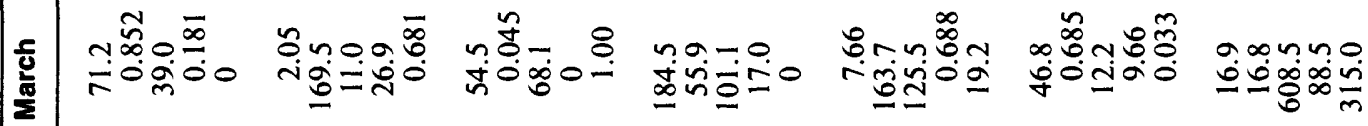

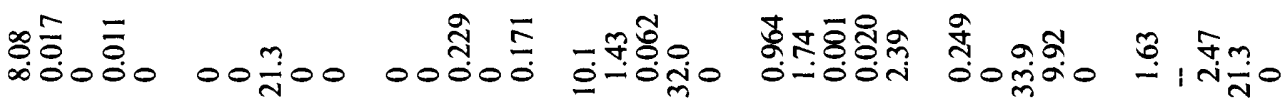

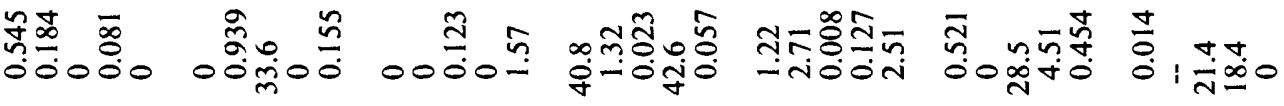

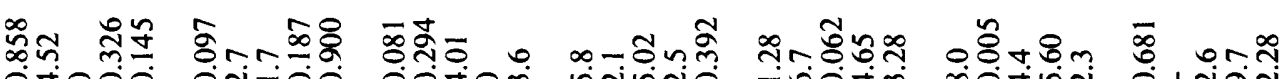

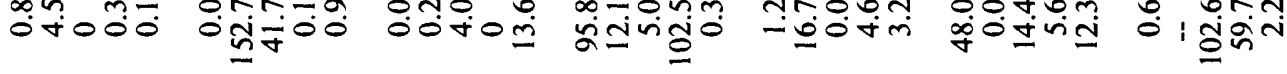

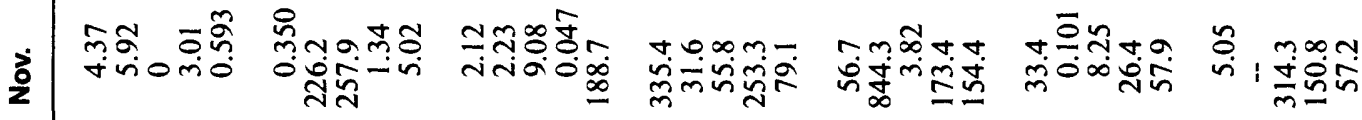

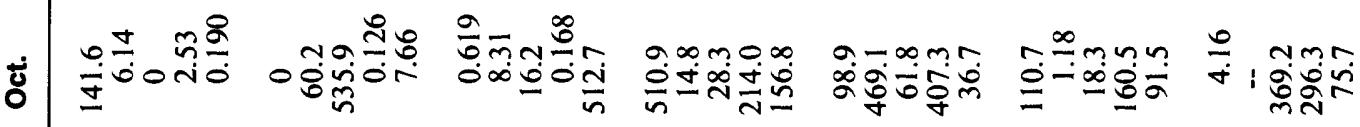

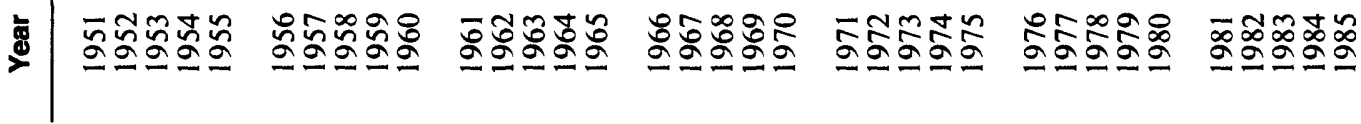




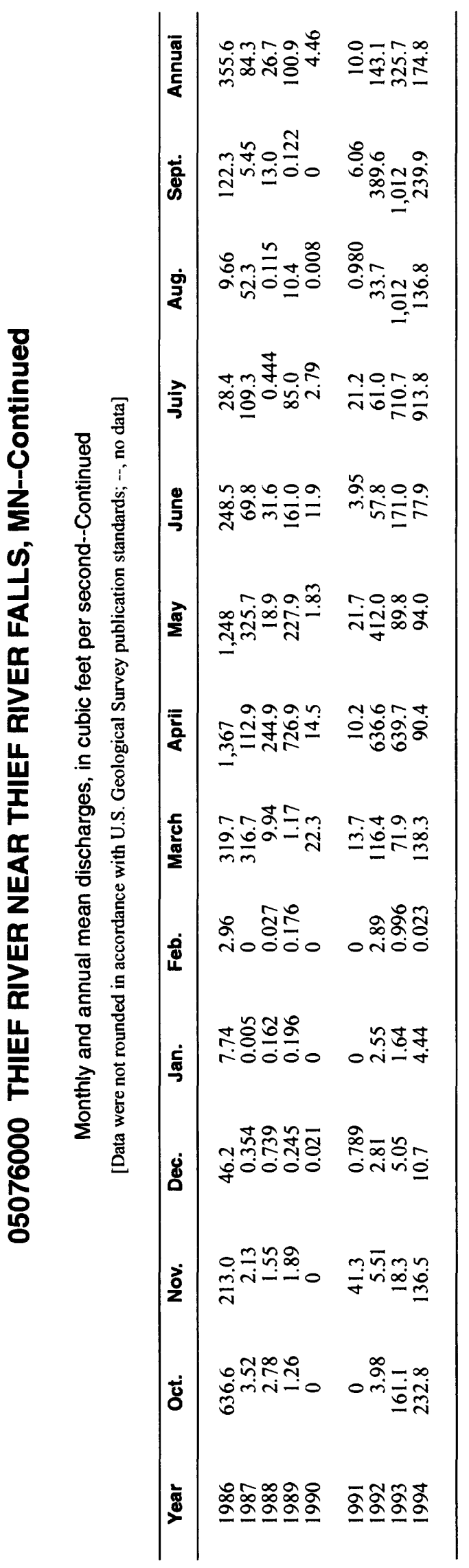




\section{RED LAKE RIVER AT THIEF RIVER FALLS, MN}

LOCATION.--Lat 48 06'40”, long 96¹0'50”, in sec.33, T.154 N., R.43 W., at Thief River Falls, Pennington County, Hydrologic Unit 09020303, $0.3 \mathrm{mi}$ downstream from Thief River Falls dam and $1 \mathrm{mi}$ downstream from Thief River.

DRAINAGE AREA.--3,450 $\mathrm{mi}^{2}$, approximately.

PERIOD OF RECORD.--July 1909 to September 1918, April 1920 to September 1930 (operated as continuous-record station). October 1918 to September 1919 (peak flow and gage height only).

GAGE.--Staff gage. Datum of gage is $1,093.6 \mathrm{ft}$ above mean sea level, datum of 1929. Prior to Aug. 19, 1920 , staff gage at site $100 \mathrm{ft}$ upstream at same datum.

EXTREMES FOR PERIOD OF RECORD.--Maximum discharge, 8,000 $\mathrm{ft}^{3} / \mathrm{s}$, Apr. 16, 1916, gage height, $15.0 \mathrm{ft}$, backwater from ice; no flow at times due to regulation.

Annual mean discharge

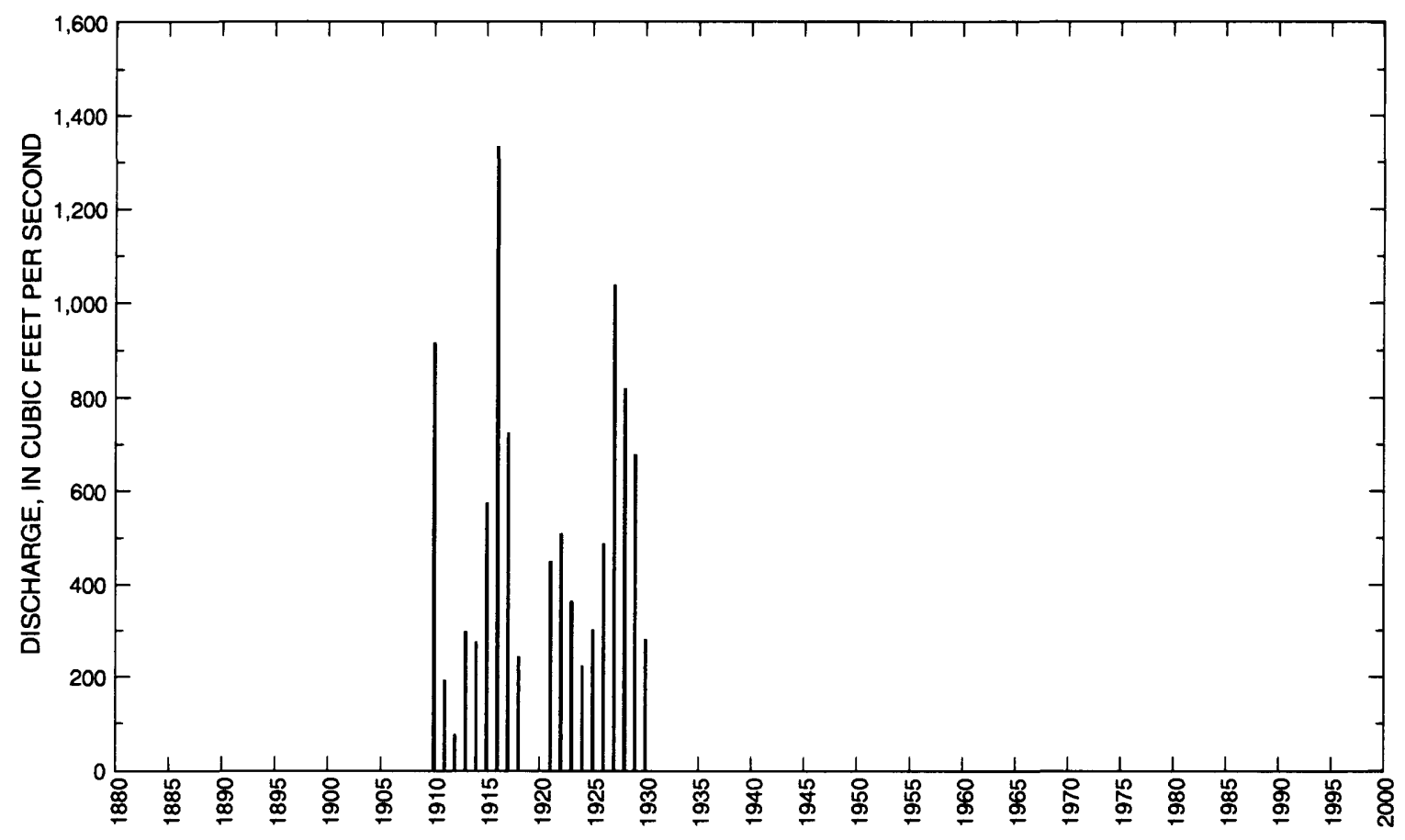




\section{RED LAKE RIVER AT THIEF RIVER FALLS, MN--Continued}

Statistics of monthly and annual mean discharges

\begin{tabular}{|c|c|c|c|c|c|c|c|c|}
\hline \multirow[b]{2}{*}{ Month } & \multicolumn{2}{|c|}{ Maximum } & \multicolumn{2}{|c|}{ Minimum } & \multicolumn{4}{|c|}{ Mean } \\
\hline & $\begin{array}{c}\text { Discharge } \\
\left(\mathrm{ft}^{3} / \mathrm{s}\right)\end{array}$ & $\begin{array}{c}\text { Water year } \\
\text { of } \\
\text { occurrence }\end{array}$ & $\begin{array}{c}\text { Discharge } \\
\left(\mathrm{ft}^{3} / \mathrm{s}\right)\end{array}$ & $\begin{array}{c}\text { Water year } \\
\text { of } \\
\text { occurrence }\end{array}$ & $\begin{array}{c}\text { Discharge } \\
\left(\mathrm{ft}^{3} / \mathrm{s}\right)\end{array}$ & $\begin{array}{c}\text { Standard } \\
\text { deviation } \\
\left(\mathrm{ft}^{3} / \mathrm{s}\right)\end{array}$ & $\begin{array}{l}\text { Coeffi- } \\
\text { cient of } \\
\text { variation }\end{array}$ & $\begin{array}{l}\text { Percentage } \\
\text { of annual } \\
\text { discharge }\end{array}$ \\
\hline October & 1,040 & 1917 & 32.8 & 1912 & 414 & 273 & 0.66 & 6.46 \\
\hline November & 828 & 1910 & 13.8 & 1912 & 341 & 222 & 0.65 & 5.32 \\
\hline December & 700 & 1910 & 9.00 & 1912 & 283 & 188 & 0.66 & 4.43 \\
\hline January & 530 & 1910 & 4.00 & 1912 & 220 & 148 & 0.67 & 3.43 \\
\hline February & 530 & 1910 & 4.00 & 1912 & 225 & 159 & 0.71 & 3.51 \\
\hline March & 2,200 & 1910 & 7.00 & 1912 & 507 & 551 & 1.09 & 7.92 \\
\hline April & 3,580 & 1916 & 85.5 & 1912 & 1,260 & 969 & 0.77 & 19.6 \\
\hline May & 3,750 & 1927 & 93.1 & 1912 & 990 & 935 & 0.94 & 15.5 \\
\hline June & 2,320 & 1916 & 98.3 & 1912 & 859 & 571 & 0.66 & 13.4 \\
\hline July & 1,890 & 1916 & 64.4 & 1911 & 562 & 467 & 0.83 & 8.78 \\
\hline August & 1,100 & 1916 & 30.8 & 1911 & 363 & 304 & 0.84 & 5.67 \\
\hline September & 1,300 & 1916 & 26.0 & 1911 & 379 & 336 & 0.89 & 5.92 \\
\hline Annual & 1,330 & 1916 & 77.4 & 1912 & 516 & 330 & 0.64 & 100 \\
\hline
\end{tabular}

Annual flow duration

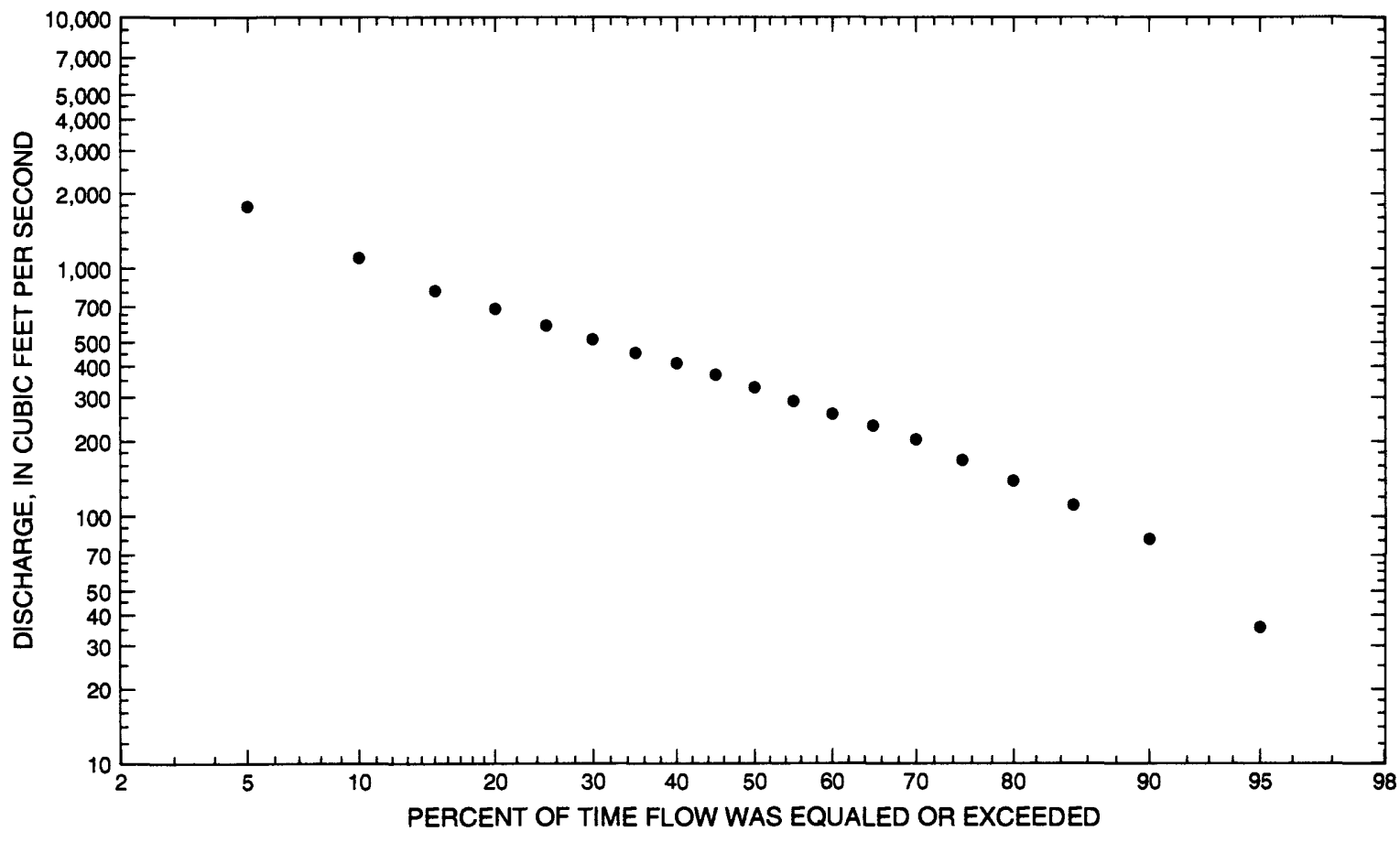




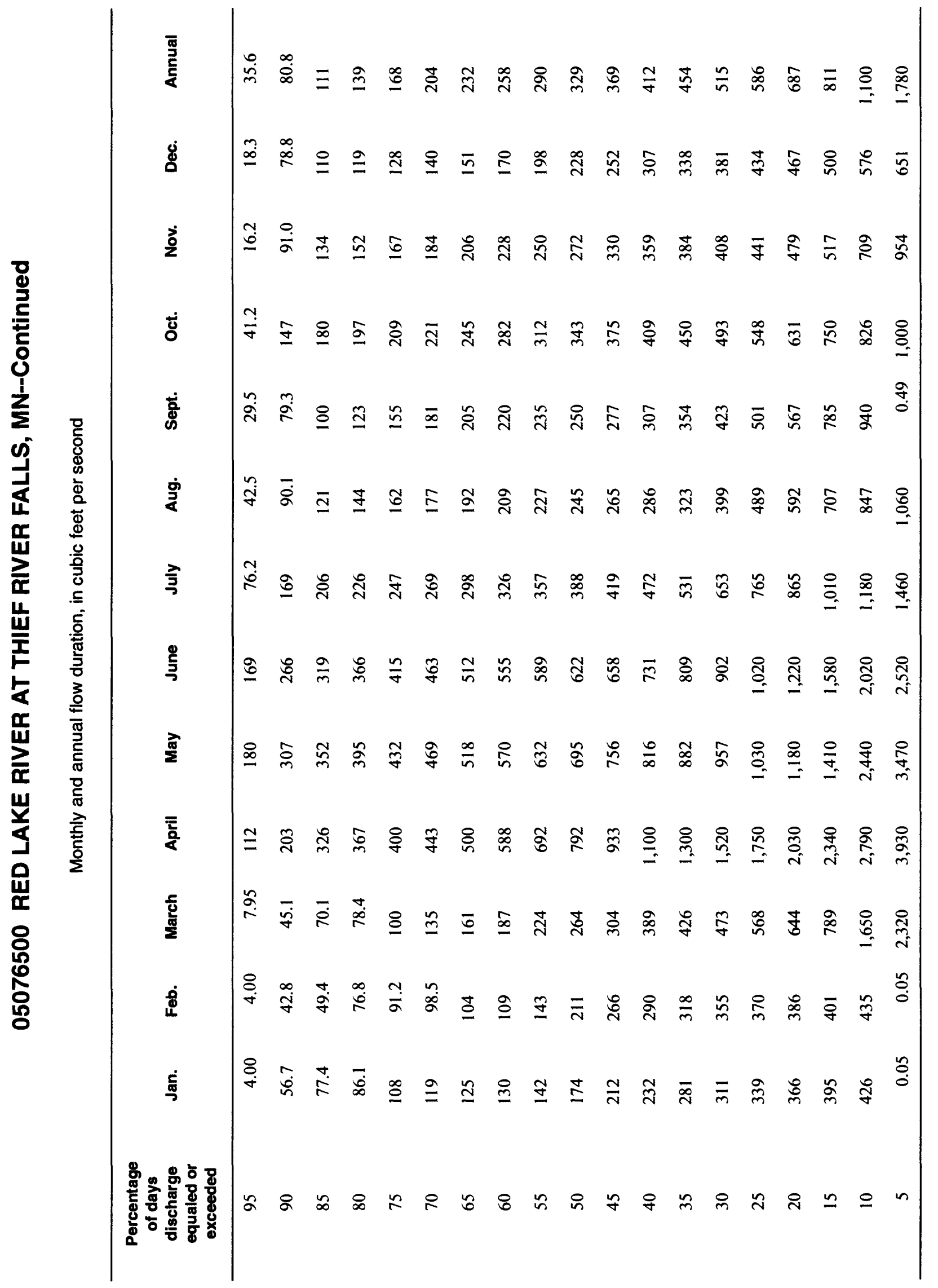




\section{RED LAKE RIVER AT THIEF RIVER FALLS, MN--Continued}

Probability of occurrence of annual high discharges

[ng, statistic not given]

\begin{tabular}{|c|c|c|c|c|c|c|}
\hline \multirow[b]{2}{*}{$\begin{array}{l}\text { Exceedance } \\
\text { probability }\end{array}$} & \multirow[b]{2}{*}{$\begin{array}{l}\text { Recurrence } \\
\text { interval } \\
\text { (years) }\end{array}$} & \multirow[b]{2}{*}{$\begin{array}{c}\text { Maximum } \\
\text { instantaneous } \\
\left(\mathrm{ft}^{3} / \mathbf{s}\right)\end{array}$} & \multicolumn{4}{|c|}{$\begin{array}{l}\text { Maximum mean discharge } \\
\left(\mathrm{ft}^{3} / \mathrm{s}\right)\end{array}$} \\
\hline & & & 3-day period & 7-day period & 15-day period & 30-day period \\
\hline 0.99 & 1.01 & 561 & 331 & 312 & 248 & 205 \\
\hline 0.95 & 1.05 & 998 & 667 & 573 & 448 & 360 \\
\hline 0.90 & 1.11 & 1,330 & 934 & 777 & 605 & 482 \\
\hline 0.80 & 1.25 & 1,840 & 1,360 & 1,100 & 861 & 679 \\
\hline 0.50 & 2 & 3,240 & 2,520 & 2,040 & 1,630 & 1,280 \\
\hline 0.20 & 5 & 5,280 & 4,150 & 3,520 & 2,930 & 2,330 \\
\hline 0.10 & 10 & 6,630 & 5,150 & 4,560 & 3,920 & 3,150 \\
\hline 0.04 & 25 & 8,280 & 6,280 & 5,890 & 5,270 & 4,300 \\
\hline 0.02 & 50 & 9,450 & 7,030 & 6,880 & 6,330 & 5,230 \\
\hline 0.01 & 100 & 10,600 & 7,700 & 7,850 & 7,430 & 6,220 \\
\hline 0.005 & 200 & 11,600 & 8,310 & 8,820 & 8,570 & 7,270 \\
\hline 0.002 & 500 & 13,000 & ng & ng & ng & $\mathrm{ng}$ \\
\hline
\end{tabular}

Probability of occurrence of annual low discharges

\begin{tabular}{|c|c|c|c|c|c|c|c|c|c|c|}
\hline \multirow[b]{3}{*}{$\begin{array}{l}\text { Non- } \\
\text { exceed- } \\
\text { ance } \\
\text { prob- } \\
\text { ability }\end{array}$} & \multirow[b]{3}{*}{$\begin{array}{l}\text { Recur- } \\
\text { rence } \\
\text { inter- } \\
\text { vai } \\
\text { (years) }\end{array}$} & \multicolumn{9}{|c|}{ Minimum mean discharge $\left(\mathrm{ft}^{3} / \mathrm{s}\right)$} \\
\hline & & \multicolumn{9}{|c|}{ Number of consecutive days } \\
\hline & & 1 & 3 & 7 & 14 & 30 & 60 & 90 & 120 & 183 \\
\hline 0.05 & 20 & 0 & 3.21 & 7.46 & 10.6 & 12.4 & 14.5 & 17.3 & 20.5 & 34.5 \\
\hline 0.10 & 10 & 3.93 & 7.52 & 15.1 & 20.0 & 24.6 & 30.5 & 35.6 & 42.3 & 62.6 \\
\hline 0.20 & 5 & 10.2 & 18.5 & 31.8 & 39.5 & 50.3 & 64.6 & 73.9 & 87.3 & 115 \\
\hline 0.50 & 2 & 39.1 & 70.9 & 98.7 & 114 & 142 & 178 & 198 & 225 & 265 \\
\hline
\end{tabular}


05076500 RED LAKE RIVER AT THIEF RIVER FALLS, MN--Continued

Probability of occurrence of seasonal low discharges

\begin{tabular}{|c|c|c|c|c|c|c|c|c|c|}
\hline \multirow[b]{3}{*}{$\begin{array}{c}\text { Non- } \\
\text { exceedance } \\
\text { probability }\end{array}$} & \multirow[b]{3}{*}{$\begin{array}{c}\text { Recurrence } \\
\text { intervai } \\
\text { (years) }\end{array}$} & \multicolumn{8}{|c|}{ Minimum mean discharge $\left(\mathrm{ft}^{3} / \mathrm{s}\right)$} \\
\hline & & \multicolumn{8}{|c|}{ Number of consecutive days } \\
\hline & & 1 & 7 & 14 & 30 & 1 & 7 & 14 & 30 \\
\hline & & \multicolumn{4}{|c|}{ December-January-February } & \multicolumn{4}{|c|}{ March-April-May } \\
\hline 0.05 & 20 & 8.20 & 12.1 & 12.8 & 13.9 & 7.50 & 15.5 & 18.1 & 24.4 \\
\hline 0.10 & 10 & 16.0 & 23.8 & 26.0 & 28.7 & 14.6 & 28.0 & 33.5 & 51.3 \\
\hline 0.20 & 5 & 33.1 & 48.7 & 54.4 & 60.5 & 31.2 & 54.2 & 65.8 & 111 \\
\hline \multirow[t]{2}{*}{0.50} & 2 & 104 & 143 & 160 & 176 & 115 & 164 & 194 & 340 \\
\hline & & \multicolumn{4}{|c|}{ June-July-August } & \multicolumn{4}{|c|}{ September-October-November } \\
\hline 0.05 & 20 & 0 & 13.8 & 33.4 & 50.1 & 2.86 & 15.2 & 21.0 & 41.7 \\
\hline 0.10 & 10 & 12.1 & 31.6 & 56.1 & 75.9 & 7.03 & 37.6 & 46.9 & 73.7 \\
\hline 0.20 & 5 & 24.9 & 73.3 & 98.5 & 120 & 18.2 & 88.2 & 102 & 133 \\
\hline 0.50 & 2 & 83.1 & 230 & 237 & 260 & 74.2 & 232 & 265 & 306 \\
\hline
\end{tabular}


05076500 RED LAKE RIVER AT THIEF RIVER FALLS, MN--Continued

Annual peak discharge and corresponding gage height

$[--$, no data $]$

\begin{tabular}{|c|c|c|c|c|c|c|c|}
\hline $\begin{array}{l}\text { Water } \\
\text { years }\end{array}$ & Date & $\begin{array}{c}\text { Gage } \\
\text { height } \\
\text { (feet) }\end{array}$ & $\begin{array}{c}\text { Peak } \\
\text { discharge } \\
\left(\mathrm{ft}^{3} / \mathrm{s}\right)\end{array}$ & $\begin{array}{c}\text { Water } \\
\text { year }\end{array}$ & Date & $\begin{array}{c}\text { Gage } \\
\text { height } \\
\text { (feet) }\end{array}$ & $\begin{array}{c}\text { Peak } \\
\text { discharge } \\
\left(\mathrm{ft}^{3} / \mathrm{s}\right)\end{array}$ \\
\hline \multicolumn{8}{|c|}{ Annual peak discharge, by year, and corresponding gage height } \\
\hline 1910 & March 24 & 8.70 & 3,220 & 1921 & April 8 & 8.80 & 3,300 \\
\hline 1911 & June 9 & 10.00 & 4,550 & 1922 & April 13 & 9.70 & 4,200 \\
\hline 1912 & September 24 & 6.30 & 1,080 & 1923 & April 21 & 9.80 & 4,300 \\
\hline 1913 & April 7 & -- & 3,820 & 1924 & April 20 & 5.70 & 895 \\
\hline 1914 & June 11 & 6.30 & 1,230 & 1925 & June 9 & 9.00 & 3,500 \\
\hline 1915 & June 29 & 8.40 & 2,880 & 1926 & June 22 & 8.10 & 2,640 \\
\hline 1916 & April 16 & 15.00 & 8,000 & 1927 & April 12 & 11.40 & 6,080 \\
\hline 1917 & April 10 & 10.70 & 5,270 & 1928 & April 6 & 8.10 & 2,640 \\
\hline 1918 & March 26 & 5.90 & 995 & 1929 & March 18 & 11.80 & 4,200 \\
\hline 1919 & July 4 & 12.70 & 7,600 & 1930 & May 13 & 7.45 & 2,020 . \\
\hline 1920 & April 16 & 9.20 & 3,700 & & & & \\
\hline \multicolumn{8}{|c|}{ Annual peak discharge, from highest to lowest, and corresponding gage height } \\
\hline 1916 & April 16 & 15.00 & 8,000 & 1921 & April 8 & 8.80 & 3,300 \\
\hline 1919 & July 4 & 12.70 & 7,600 & 1910 & March 24 & 8.70 & 3,220 \\
\hline 1927 & April 12 & 11.40 & 6,080 & 1915 & June 29 & 8.40 & 2,880 \\
\hline 1917 & April 10 & 10.70 & 5,270 & 1926 & June 22 & 8.10 & 2,640 \\
\hline 1911 & June 9 & 10.00 & 4,550 & 1928 & April 6 & 8.10 & 2,640 \\
\hline 1923 & April 21 & 9.80 & 4,300 & 1930 & May 13 & 7.45 & 2,020 \\
\hline 1922 & April 13 & 9.70 & 4,200 & 1914 & June 11 & 6.30 & 1,230 \\
\hline 1929 & March 18 & 11.80 & 4,200 & 1912 & September 24 & 6.30 & 1,080 \\
\hline 1913 & April 7 & 9.40 & 3,820 & 1918 & March 26 & 5.90 & 995 \\
\hline 1920 & April 16 & 9.20 & 3,700 & 1924 & April 20 & 5.70 & 895 \\
\hline 1925 & June 9 & 9.00 & 3,500 & & & & \\
\hline
\end{tabular}




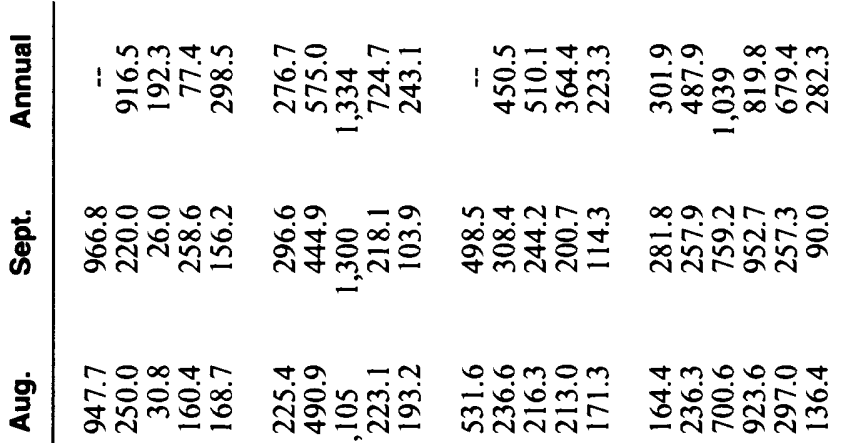
วิ)

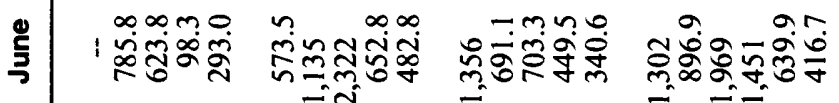

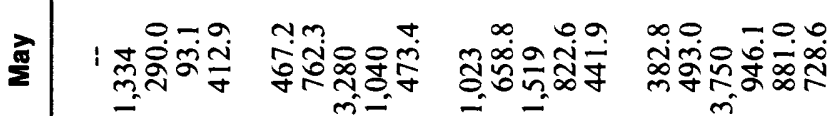

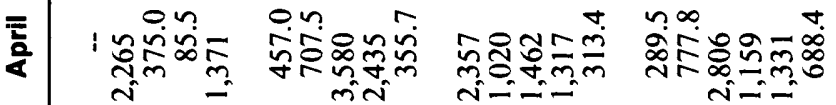

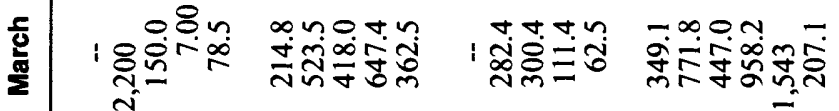

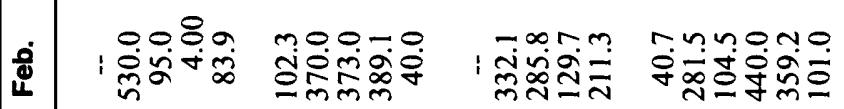
⿷匚 - Oo: bonno o-no bonoro

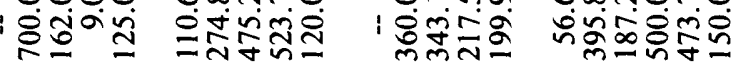

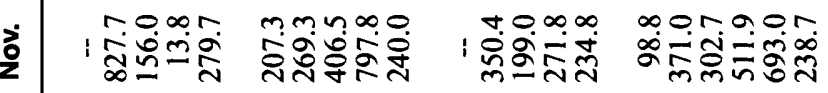

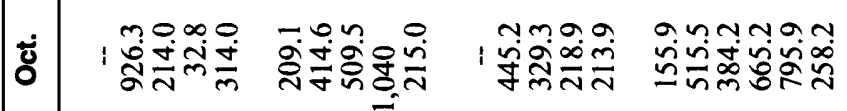

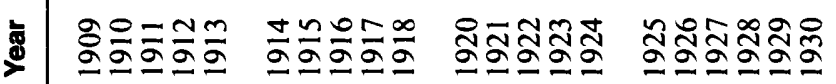


LOCATION.--Lat $47^{\circ} 44^{\prime} 00^{\prime \prime}$, long $95^{\circ} 13^{\prime} 00^{\prime \prime}$, in E ${ }^{1} / 2 \mathrm{SW}^{1} / 4$ sec.12, T.149 N., R.36 W., Clearwater County, Hydrologic Unit $09020305,300 \mathrm{ft}$ downstream from dam at outlet of Clearwater Lake and $8 \mathrm{mi}$ northeast of Leonard.

DRAINAGE AREA.--153 $\mathrm{mi}^{2}$.

PERIOD OF RECORD.--July 1934 to September 1947.

GAGE.--Staff gage. Datum of gage is $1,261.80 \mathrm{ft}$ above mean sea level, adjustment of 1912 (levels by U.S. Army Corps of Engineers). Prior to Sept. 10, 1934, at site $400 \mathrm{ft}$ downstream at different datum.

EXTREMES FOR PERIOD OF RECORD.--Maximum discharge, $655 \mathrm{ft}^{3} / \mathrm{s}$, Apr. 21, 1947, gage height, $3.19 \mathrm{ft}$; minimum daily discharge, $2 \mathrm{ft}^{3} / \mathrm{s}$, Mar. 25 to Apr. 3, 1940, estimated leakage through stop logs.

Annual mean discharge

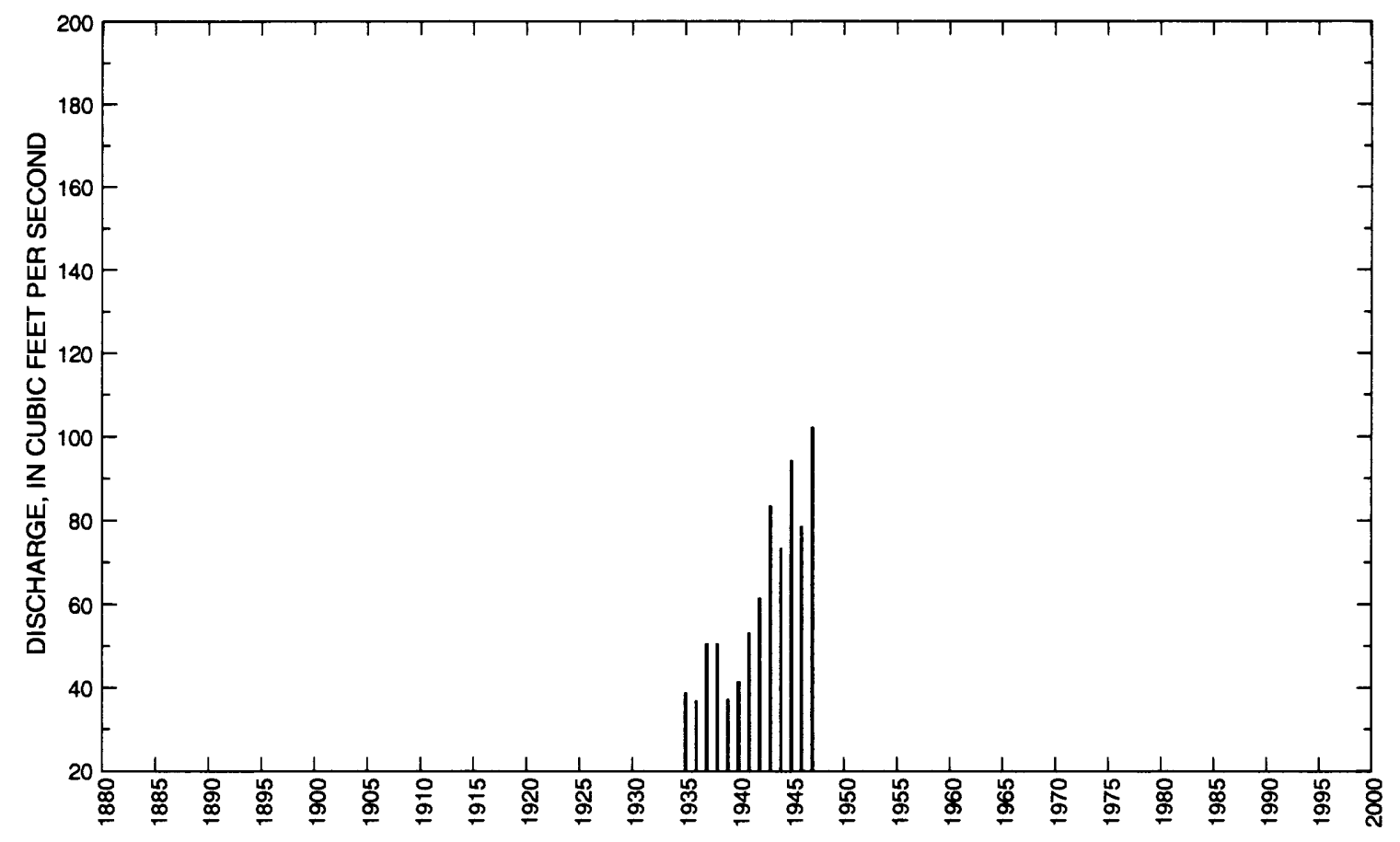


05077500 CLEARWATER RIVER NEAR LEONARD, MN-Continued

Statistics of monthly and annual mean discharges

\begin{tabular}{|c|c|c|c|c|c|c|c|c|}
\hline \multirow[b]{2}{*}{ Month } & \multicolumn{2}{|c|}{ Maximum } & \multicolumn{2}{|c|}{ Minimum } & \multicolumn{4}{|c|}{ Mean } \\
\hline & $\begin{array}{c}\text { Discharge } \\
\left(\mathbf{f t}^{3} / \mathbf{s}\right)\end{array}$ & $\begin{array}{l}\text { Water year } \\
\text { of } \\
\text { occurrence }\end{array}$ & $\begin{array}{c}\text { Discharge } \\
\left(\mathrm{ft}^{3} / \mathrm{s}\right)\end{array}$ & $\begin{array}{l}\text { Water year } \\
\text { of } \\
\text { occurrence }\end{array}$ & $\begin{array}{c}\text { Discharge } \\
\left(\mathrm{ft}^{3} / \mathrm{s}\right)\end{array}$ & $\begin{array}{l}\text { Standard } \\
\text { deviation } \\
\left(\mathrm{ft}^{3} / \mathrm{s}\right)\end{array}$ & $\begin{array}{l}\text { Coeffi- } \\
\text { clent of } \\
\text { variation }\end{array}$ & $\begin{array}{c}\text { Percentage } \\
\text { of annual } \\
\text { discharge }\end{array}$ \\
\hline October & 87.4 & 1947 & 20.3 & 1941 & 48.2 & 27.3 & 0.57 & 6.57 \\
\hline November & 82.7 & 1945 & 25.5 & 1935 & 44.8 & 18.1 & 0.40 & 6.11 \\
\hline December & 63.6 & 1945 & 24.4 & 1935 & 37.6 & 11.4 & 0.30 & 5.13 \\
\hline January & 47.9 & 1946 & 19.9 & 1940 & 33.1 & 7.66 & 0.23 & 4.51 \\
\hline February & 48.9 & 1945 & 16.9 & 1937 & 30.7 & 8.71 & 0.28 & 4.19 \\
\hline March & 178 & 1945 & 12.4 & 1937 & 55.1 & 50.7 & 0.92 & 7.52 \\
\hline April & 363 & 1947 & 57.1 & 1938 & 142 & 81.7 & 0.58 & 19.4 \\
\hline May & 236 & 1947 & 53.5 & 1935 & 119 & 54.4 & 0.46 & 16.2 \\
\hline June & 219 & 1943 & 25.7 & 1936 & 85.9 & 54.5 & 0.63 & 11.7 \\
\hline July & 81.5 & 1944 & 15.1 & 1936 & 43.4 & 19.2 & 0.44 & 5.91 \\
\hline August & 114 & 1944 & 6.09 & 1936 & 41.5 & 28.9 & 0.70 & 5.66 \\
\hline September & 118 & 1944 & 9.53 & 1934 & 52.3 & 34.5 & 0.66 & 7.13 \\
\hline Annual & 102 & 1947 & 36.9 & 1936 & 61.8 & 22.5 & 0.36 & 100 \\
\hline
\end{tabular}

Annual flow duration

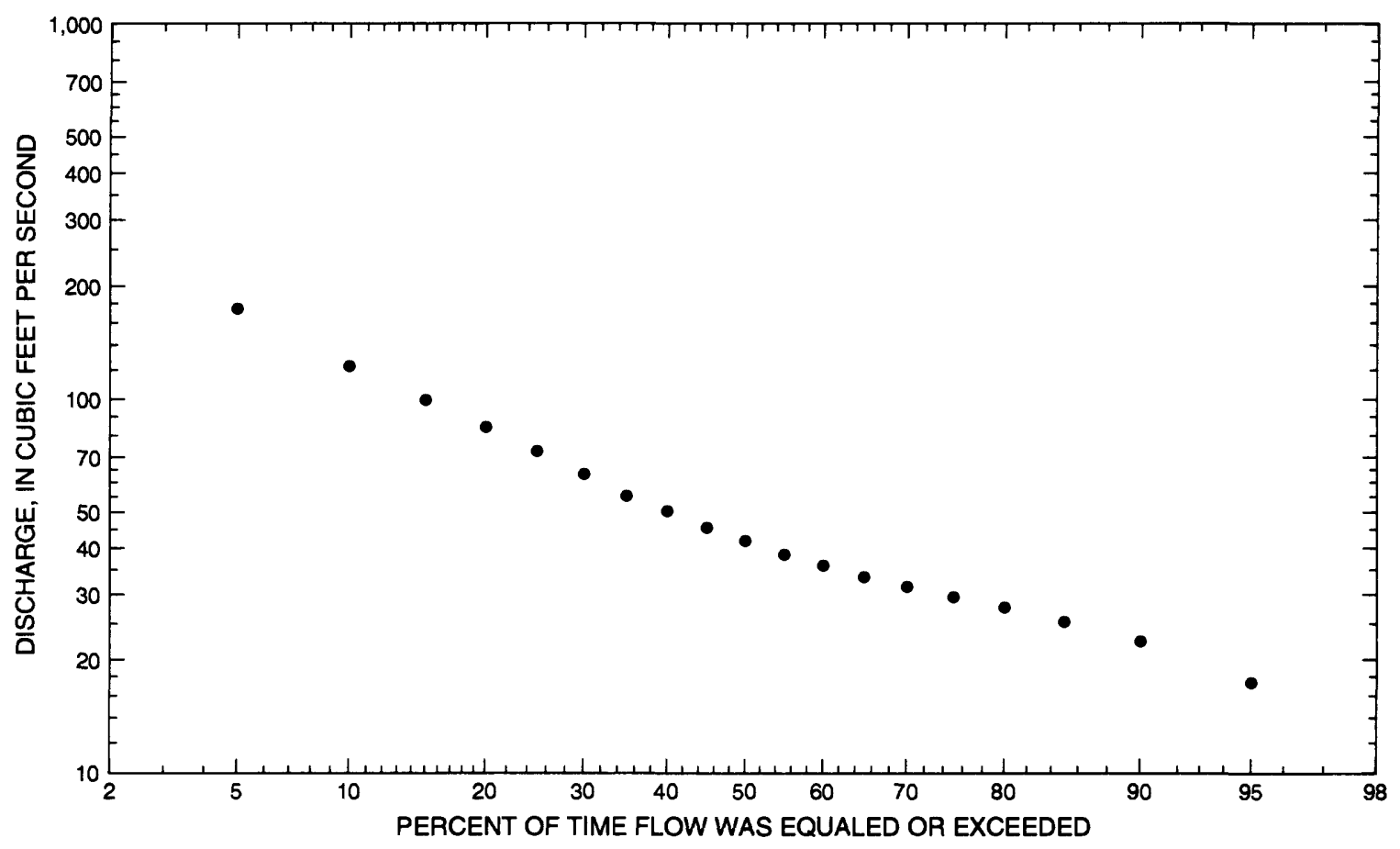




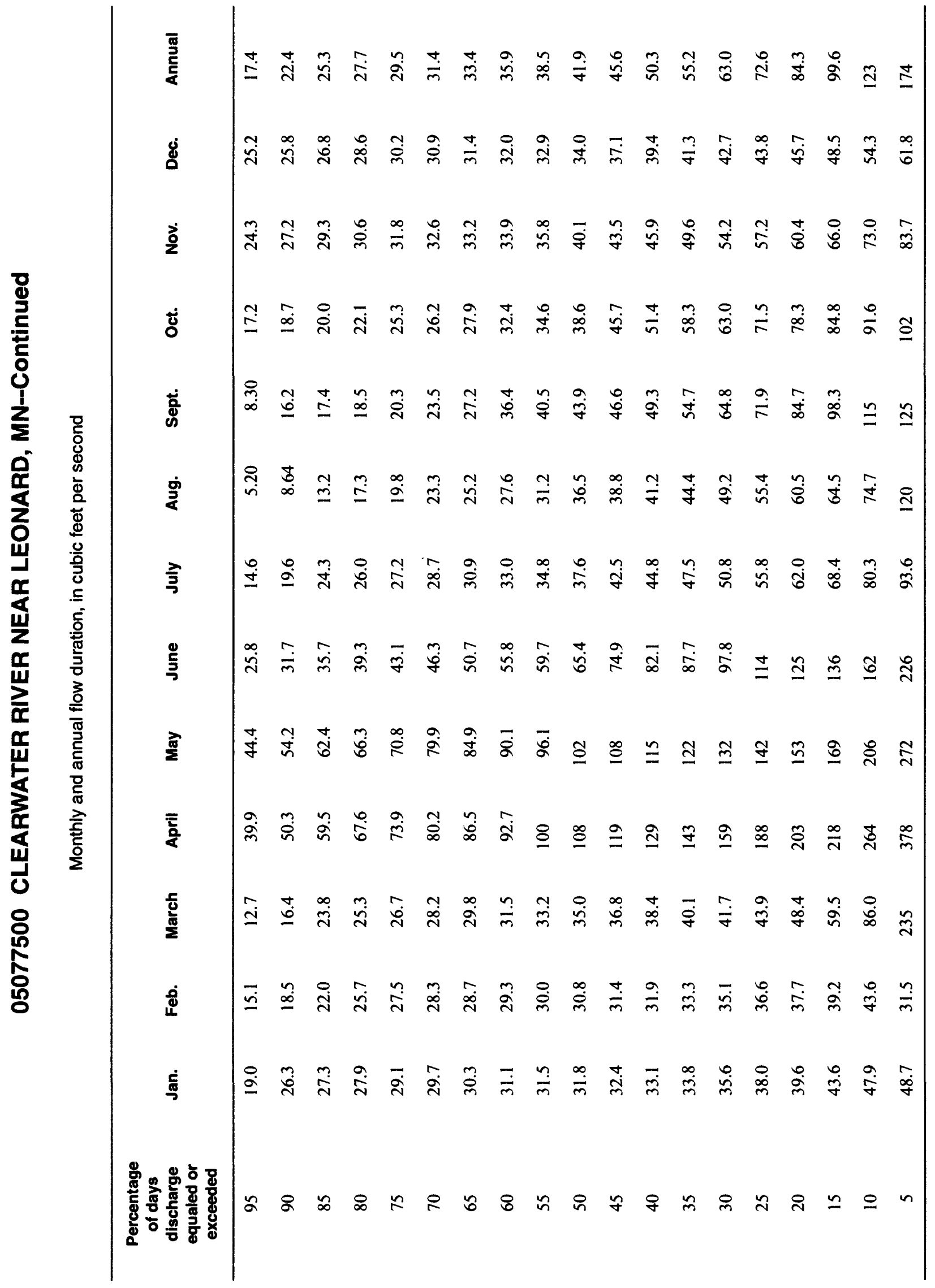


05077500 CLEARWATER RIVER NEAR LEONARD, MN--Continued

Probability of occurrence of annual high discharges

[ng, statistic not given]

\begin{tabular}{lcccccc}
\hline & & & \multicolumn{5}{c}{$\begin{array}{c}\text { Maximum mean discharge } \\
\left(\mathbf{f t}^{3} / \mathbf{s}\right)\end{array}$} \\
\cline { 5 - 7 } $\begin{array}{c}\text { Exceedance } \\
\text { probabiiity }\end{array}$ & $\begin{array}{c}\text { Recurrence } \\
\text { interval } \\
\text { (year) }\end{array}$ & $\begin{array}{c}\text { Maximum } \\
\text { instantaneous } \\
\left(\mathbf{f t}^{3} / \mathbf{s}\right)\end{array}$ & 3-day period & 7-day period & 15-day period & 30-day period \\
\hline 0.99 & 1.01 & 67.7 & 65.1 & 63.8 & 58.3 & 63.3 \\
0.95 & 1.05 & 104 & 99.9 & 95.8 & 85.1 & 81.5 \\
0.90 & 1.11 & 130 & 124 & 118 & 104 & 94.2 \\
0.80 & 1.25 & 168 & 161 & 152 & 132 & 113 \\
0.50 & 2 & 266 & 256 & 240 & 205 & 165 \\
0.20 & 5 & 408 & 395 & 371 & 317 & 251 \\
0.10 & 10 & 503 & 490 & 462 & 396 & 318 \\
0.04 & 25 & 622 & 610 & 581 & 500 & 413 \\
0.02 & 50 & 709 & 699 & 670 & 580 & 493 \\
0.01 & 100 & 795 & 788 & 761 & 662 & 580 \\
0.005 & 200 & 880 & 877 & 852 & 747 & 676 \\
0.002 & 500 & 992 & ng & ng & ng & ng \\
\hline
\end{tabular}

Probability of occurrence of annual low discharges

Minimum mean discharge $\left(\mathrm{ft}^{3} / \mathrm{s}\right)$

Number of consecutive days

\begin{tabular}{|c|c|c|c|c|c|c|c|c|c|c|}
\hline $\begin{array}{c}\text { Non- } \\
\text { exceed- } \\
\text { ance } \\
\text { prob- } \\
\text { ability }\end{array}$ & $\begin{array}{c}\text { Recur- } \\
\text { rence } \\
\text { inter- } \\
\text { val } \\
\text { (years) }\end{array}$ & 1 & 3 & 7 & 14 & 30 & 60 & 90 & 120 & 183 \\
\hline 0.05 & 20 & 1.66 & 1.86 & 4.12 & 5.98 & 7.59 & 11.3 & 14.8 & 16.6 & 19.0 \\
\hline 0.10 & 10 & 3.03 & 3.32 & 6.37 & 8.83 & 10.9 & 14.4 & 17.4 & 19.0 & 21.7 \\
\hline 0.20 & 5 & 5.80 & 6.20 & 10.2 & 13.3 & 15.8 & 18.8 & 21.0 & 22.5 & 25.7 \\
\hline 0.50 & 2 & 16.2 & 16.7 & 20.8 & 24.1 & 26.9 & 28.5 & 29.2 & 30.8 & 35.7 \\
\hline
\end{tabular}




\section{CLEARWATER RIVER NEAR LEONARD, MN-Continued}

Probability of occurrence of seasonal low discharges

\begin{tabular}{|c|c|c|c|c|c|c|c|c|c|}
\hline \multirow[b]{3}{*}{$\begin{array}{c}\text { Non- } \\
\text { exceedance } \\
\text { probability }\end{array}$} & \multirow[b]{3}{*}{$\begin{array}{c}\text { Recurrence } \\
\text { interval } \\
\text { (years) }\end{array}$} & \multicolumn{8}{|c|}{ Minimum mean discharge $\left(\mathrm{ft}^{3} / \mathrm{s}\right)$} \\
\hline & & \multicolumn{8}{|c|}{ Number of consecutive days } \\
\hline & & 1 & 7 & 14 & 30 & 1 & 7 & 14 & 30 \\
\hline & & \multicolumn{4}{|c|}{ December-January-February } & \multicolumn{4}{|c|}{ March-April-May } \\
\hline 0.05 & 20 & 10.0 & 12.1 & 13.8 & 17.4 & 3.76 & 4.25 & 9.97 & 14.3 \\
\hline 0.10 & 10 & 13.0 & 14.8 & 16.4 & 19.8 & 6.85 & 7.90 & 13.4 & 17.4 \\
\hline 0.20 & 5 & 17.1 & 18.6 & 19.8 & 22.8 & 12.5 & 14.4 & 18.4 & 22.3 \\
\hline \multirow[t]{2}{*}{0.50} & 2 & 26.2 & 26.7 & 27.4 & 29.5 & 27.6 & 30.4 & $1_{33.5}$ & 37.2 \\
\hline & & \multicolumn{4}{|c|}{ June-July-August } & \multicolumn{4}{|c|}{ September-October-November } \\
\hline 0.05 & 20 & 4.96 & 5.70 & 6.29 & 8.07 & 6.90 & 8.65 & 9.04 & 10.5 \\
\hline 0.10 & 10 & 7.67 & 8.56 & 9.47 & 11.8 & 9.40 & 11.5 & 12.0 & 13.6 \\
\hline 0.20 & 5 & 12.2 & 13.2 & 14.6 & 17.6 & 13.4 & 15.9 & 16.6 & 18.4 \\
\hline 0.50 & 2 & 24.7 & 25.9 & 28.2 & 32.6 & 25.3 & 28.1 & 29.4 & 31.8 \\
\hline
\end{tabular}

${ }^{1}$ Graphical interpretation. 
05077500 CLEARWATER RIVER NEAR LEONARD, MN--Continued

Annual peak discharge and corresponding gage height

\begin{tabular}{|c|c|c|c|c|c|c|c|}
\hline $\begin{array}{l}\text { Water } \\
\text { year }\end{array}$ & Date & $\begin{array}{c}\text { Gage } \\
\text { height } \\
\text { (feet) }\end{array}$ & $\begin{array}{c}\text { Peak } \\
\text { discharge } \\
\left(\mathrm{ft}^{3} / \mathrm{s}\right) \\
\end{array}$ & $\begin{array}{l}\text { Water } \\
\text { year }\end{array}$ & Date & $\begin{array}{c}\text { Gage } \\
\text { height } \\
\text { (feet) }\end{array}$ & $\begin{array}{c}\text { Peak } \\
\text { discharge } \\
\left(\mathrm{ft}^{3} / \mathrm{s}\right) \\
\end{array}$ \\
\hline \multicolumn{8}{|c|}{ Annual peak discharge, by year, and corresponding gage height } \\
\hline 1935 & May 15 & 1.49 & 100 & 1942 & April 1 & 2.04 & 198 \\
\hline 1936 & April 19 & 2.06 & 275 & 1943 & April 8 & 2.57 & 382 \\
\hline 1937 & September 3 & 1.96 & 236 & 1944 & June 9 & 1.84 & 174 \\
\hline 1938 & May 11 & 2.43 & 347 & 1945 & March 27 & 2.70 & 435 \\
\hline 1939 & April 5 & 1.56 & 115 & 1946 & March 25 & 2.77 & 399 \\
\hline 1940 & April 16 & 2.22 & 266 & 1947 & April 21 & 3.19 & 655 \\
\hline 1941 & April 15 & 2.06 & 237 & & & & \\
\hline \multicolumn{8}{|c|}{ Annual peak discharge, from highest to lowest, and corresponding gage height } \\
\hline 1947 & April 21 & 3.19 & 655 & 1941 & April 15 & 2.06 & 237 \\
\hline 1945 & March 27 & 2.70 & 435 & 1937 & September 3 & 1.96 & 236 \\
\hline 1946 & March 25 & 2.77 & 399 & 1942 & April 1 & 2.04 & 198 \\
\hline 1943 & April 8 & 2.57 & 382 & 1944 & June 9 & 1.84 & 174 \\
\hline 1938 & May 11 & 2.43 & 347 & 1939 & April 5 & 1.56 & 115 \\
\hline 1936 & April 19 & 2.06 & 275 & 1935 & May 15 & 1.49 & 100 \\
\hline 1940 & April 16 & 2.22 & 266 & & & & \\
\hline
\end{tabular}




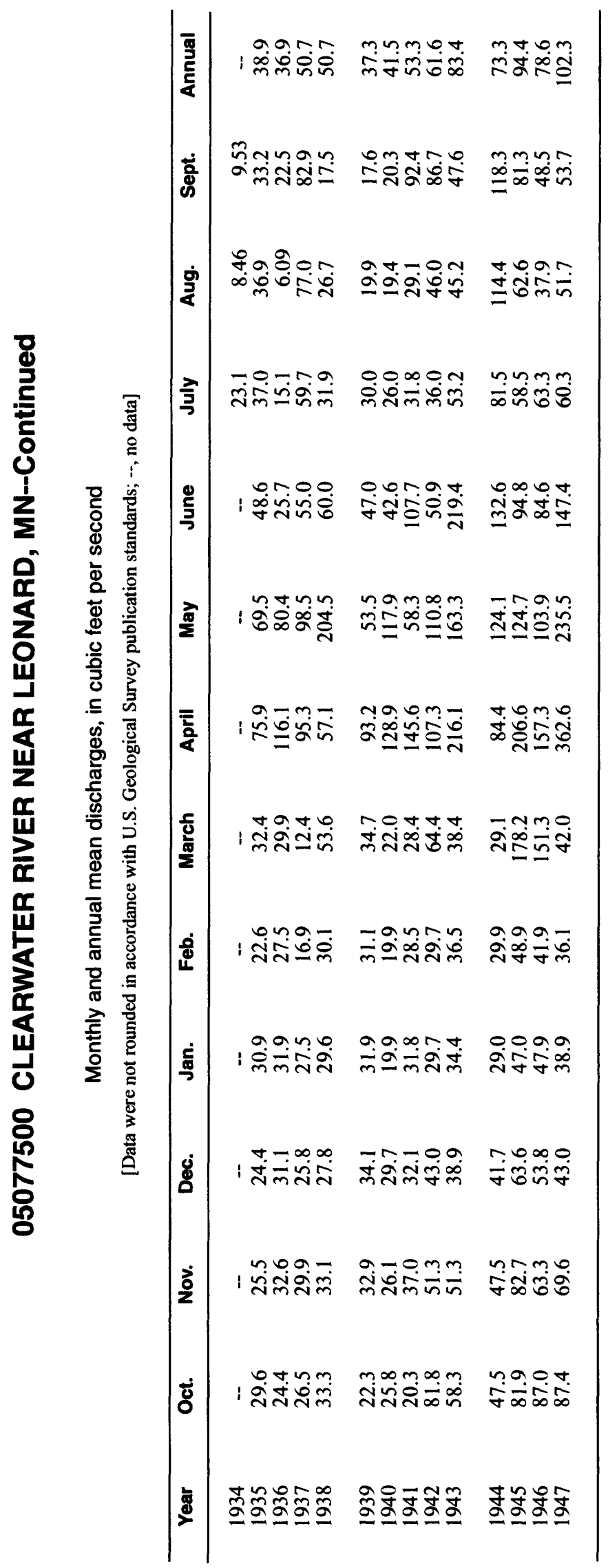




\section{RUFFY BROOK NEAR GONVICK, MN}

LOCATION.--Lat $47^{\circ} 44^{\prime} 50^{\prime \prime}$, long $95^{\circ} 24^{\prime} 45^{\prime \prime}$, in $\mathrm{SE}^{1} / 4 \mathrm{SE}^{1} / 4$ sec.5, T.149 N., R.37 W., Clearwater County, Hydrologic Unit 09020305, at culvert on County Highway 67, 4.0 mi upstream from mouth, and $4.8 \mathrm{mi}$ east of Gonvick.

DRAINAGE AREA.--45.2 $\mathrm{mi}^{2}$.

PERIOD OF RECORD.--April 1960 to current. April 1960 to September 1978, operated as a continuousrecord station. October 1978 to January 1986, operated as a high-flow partial-record station. February 1986 to October 1986, operated as a continuous-record station. November 1986 to current, operated as a high-flow partial record station. Monthly and daily figures for Apr. 1, 1960, to June 30, 1960, published in WSP 1913.

GAGE.--Nonrecording gage and crest-stage gage. Datum of gage is $1,227.93 \mathrm{ft}$, adjustment of 1912 (levels by U.S. Army Corps of Engineers). Prior to Sept. 9, 1960, reference point at same site and datum.

EXTREMES FOR PERIOD OF RECORD.--Maximum discharge, $453 \mathrm{ft}^{3} / \mathrm{s}$, Mar. 30, 1967, gage height, $6.35 \mathrm{ft}$; maximum gage height, $6.62 \mathrm{ft}$, Apr. 9, 1969, backwater from ice; no flow Feb. 20 to Mar. 6, 1968.

Annual mean discharge

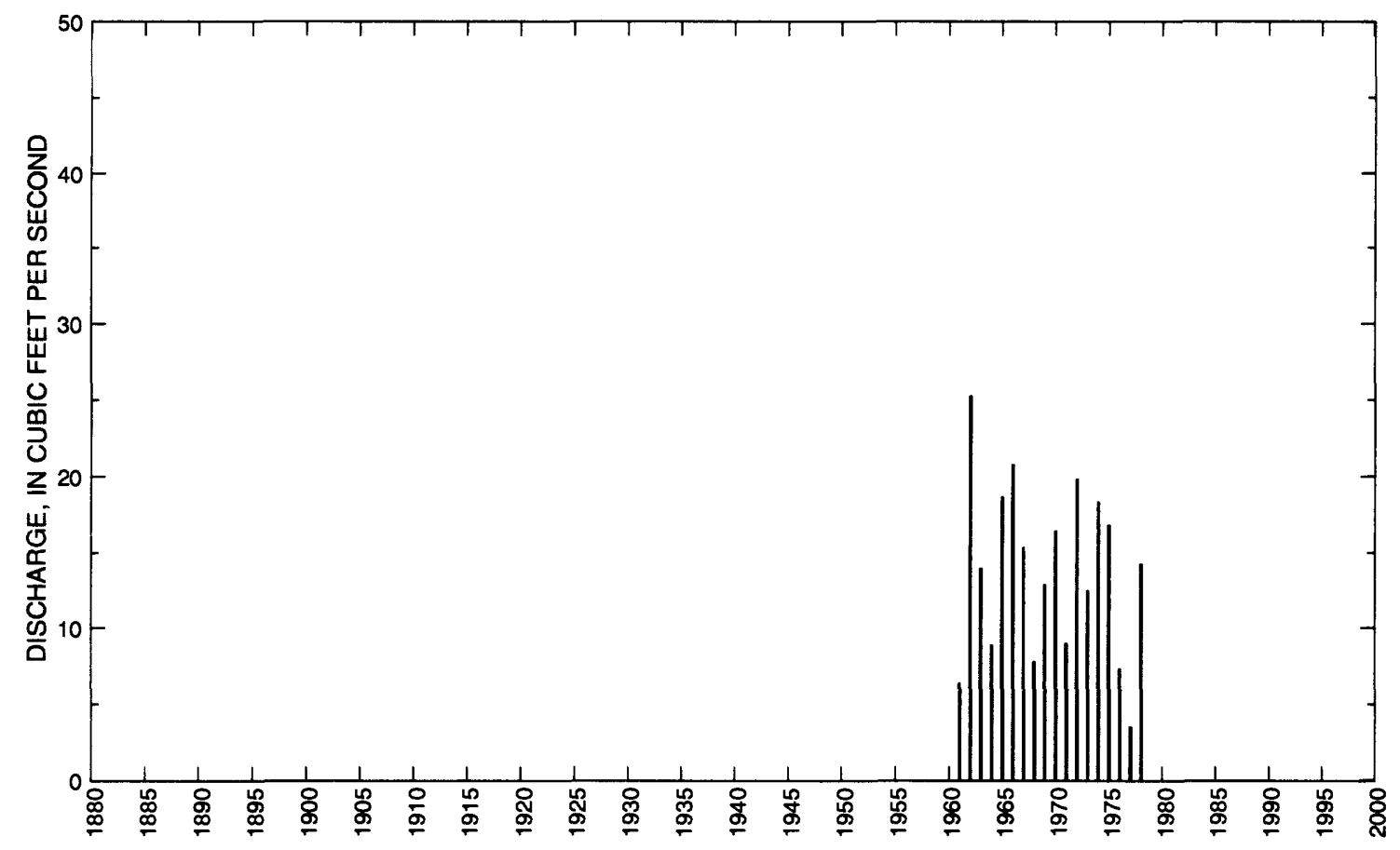


05077700 RUFFY BROOK NEAR GONVICK, MN--Continued

Statistics of monthly and annual mean discharges

\begin{tabular}{|c|c|c|c|c|c|c|c|c|}
\hline \multirow[b]{2}{*}{ Month } & \multicolumn{2}{|c|}{ Maximum } & \multicolumn{2}{|c|}{ Minimum } & \multicolumn{4}{|c|}{ Mean } \\
\hline & $\begin{array}{c}\text { Discharge } \\
\left(\mathrm{ft}^{3} / \mathrm{s}\right)\end{array}$ & $\begin{array}{c}\text { Water year } \\
\text { of } \\
\text { occurrence }\end{array}$ & $\begin{array}{c}\text { Discharge } \\
\left(\mathrm{ft}^{3} / \mathrm{s}\right)\end{array}$ & $\begin{array}{c}\text { Water year } \\
\text { of } \\
\text { occurrence }\end{array}$ & $\begin{array}{c}\text { Discharge } \\
\left(\mathrm{ft}^{3} / \mathrm{s}\right)\end{array}$ & $\begin{array}{l}\text { Standard } \\
\text { deviation } \\
\left(\mathrm{ft}^{3} / \mathrm{s}\right)\end{array}$ & $\begin{array}{l}\text { Coeffi- } \\
\text { cient of } \\
\text { variation }\end{array}$ & $\begin{array}{c}\text { Percentage } \\
\text { of annual } \\
\text { discharge }\end{array}$ \\
\hline October & 34.7 & 1972 & 1.15 & 1968 & 7.93 & 9.12 & 1.15 & 4.87 \\
\hline November & 31.4 & 1972 & 1.45 & 1977 & 6.87 & 6.88 & 1.00 & 4.22 \\
\hline December & 13.6 & 1978 & 0.314 & 1977 & 4.53 & 3.44 & 0.76 & 2.78 \\
\hline January & 8.99 & 1974 & 0.396 & 1977 & 3.17 & 2.20 & 0.69 & 1.95 \\
\hline February & 7.38 & 1974 & 0.393 & 1968 & 2.77 & 1.84 & 0.66 & 1.70 \\
\hline March & 37.5 & 1966 & 2.07 & 1971 & 12.4 & 11.9 & 0.97 & 7.60 \\
\hline April & 118 & 1966 & 6.91 & 1977 & 58.2 & 30.9 & 0.53 & 35.7 \\
\hline May & 105 & 1962 & 4.61 & 1977 & 29.1 & 22.4 & 0.77 & 17.9 \\
\hline June & 54.4 & 1962 & 2.29 & 1961 & 18.2 & 16.8 & 0.92 & 11.2 \\
\hline July & 57.3 & 1962 & 2.10 & 1961 & 9.31 & 13.7 & 1.47 & 5.72 \\
\hline August & 18.7 & 1966 & 1.09 & 1976 & 3.79 & 3.97 & 1.05 & 2.33 \\
\hline September & 59.3 & 1973 & 0.623 & 1967 & 6.57 & 12.8 & 1.94 & 4.04 \\
\hline Annual & 25.3 & 1962 & 3.56 & 1977 & 13.8 & 5.79 & 0.42 & 100 \\
\hline
\end{tabular}

Annual flow duration

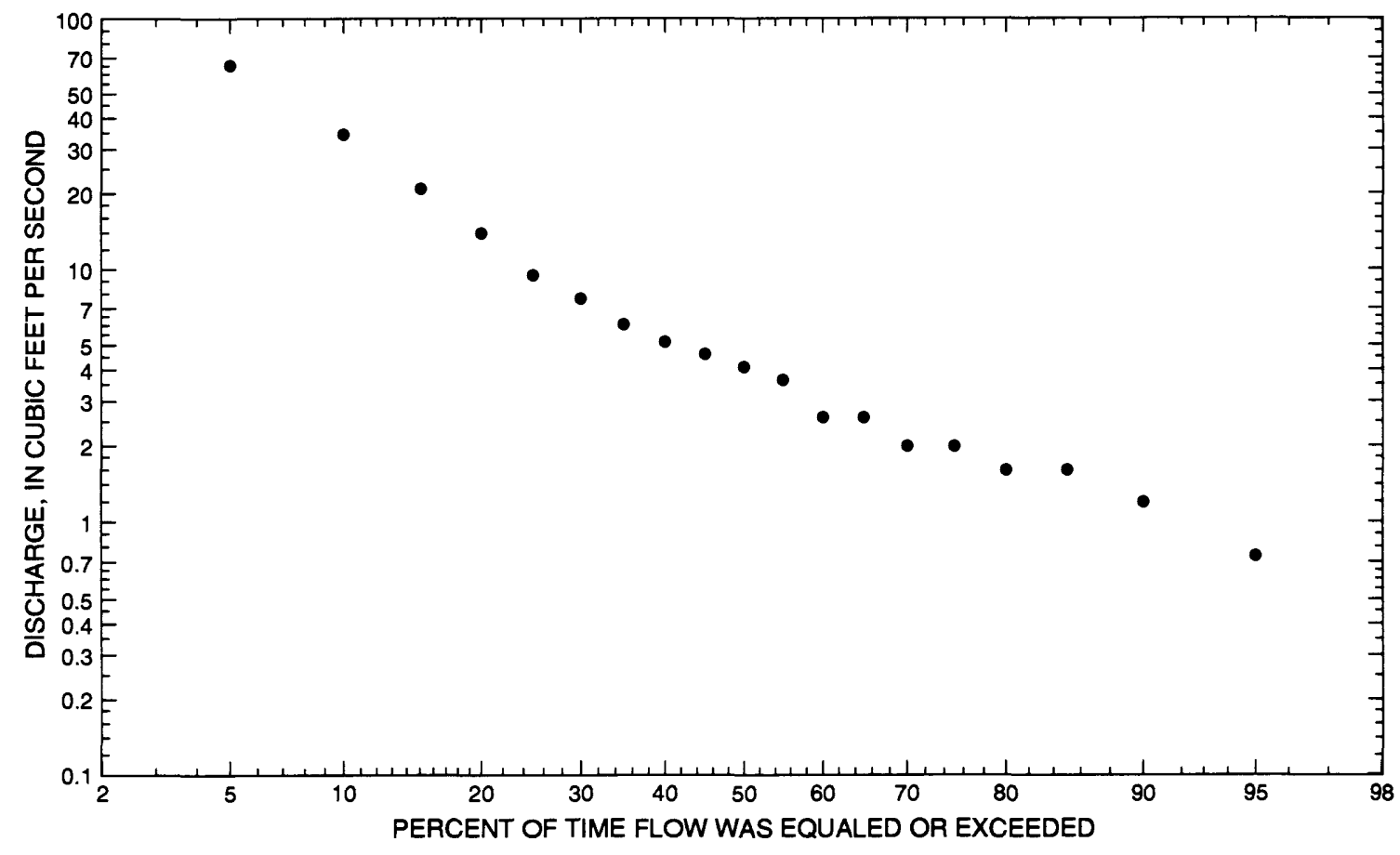




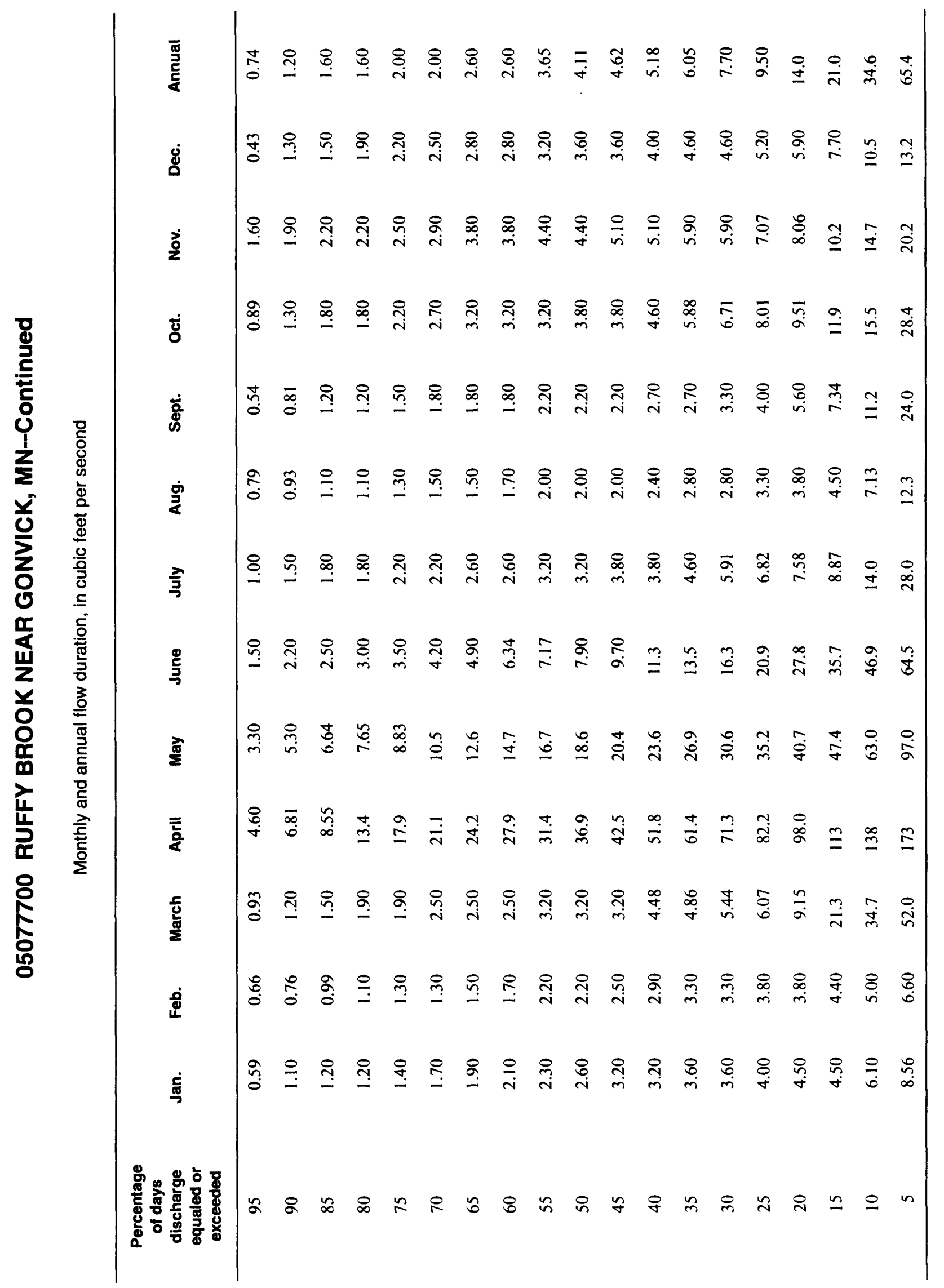




\section{RUFFY BROOK NEAR GONVICK, MN--Continued}

Probability of occurrence of annual high discharges

[ng, statistic not given]

\begin{tabular}{|c|c|c|c|c|c|c|}
\hline \multirow[b]{2}{*}{$\begin{array}{l}\text { Exceedance } \\
\text { probability }\end{array}$} & \multirow[b]{2}{*}{$\begin{array}{l}\text { Recurrence } \\
\text { interval } \\
\text { (years) }\end{array}$} & \multirow[b]{2}{*}{$\begin{array}{c}\text { Maximum } \\
\text { instantaneous } \\
\left(\mathrm{ft}^{3} / \mathrm{s}\right)\end{array}$} & \multicolumn{4}{|c|}{$\begin{array}{c}\text { Maximum mean discharge } \\
\qquad\left(\mathrm{ft}^{3} / \mathrm{s}\right)\end{array}$} \\
\hline & & & 3-day period & 7-day period & 15-day period & 30-day period \\
\hline 0.99 & 1.01 & ng & 22.5 & 21.3 & 16.6 & 10.2 \\
\hline 0.95 & 1.05 & 62.6 & 47.2 & 43.2 & 33.0 & 22.1 \\
\hline 0.90 & 1.11 & 81.5 & 66.8 & 59.8 & 45.3 & 31.4 \\
\hline 0.80 & 1.25 & 110 & 97.5 & 84.7 & 63.4 & 45.1 \\
\hline 0.50 & 2 & 187 & 177 & 144 & 106 & 76.7 \\
\hline 0.20 & 5 & 296 & 276 & 210 & 152 & 108 \\
\hline 0.10 & 10 & 367 & 329 & 242 & 174 & 121 \\
\hline 0.04 & 25 & 454 & 383 & 271 & 193 & 132 \\
\hline 0.02 & 50 & 515 & 416 & 287 & 204 & 136 \\
\hline 0.01 & 100 & 574 & 442 & 299 & 212 & 140 \\
\hline 0.005 & 200 & 630 & 464 & 308 & 218 & 142 \\
\hline 0.002 & 500 & 702 & $\mathrm{ng}$ & $\mathrm{ng}$ & ng & ng \\
\hline
\end{tabular}

Probability of occurrence of annual low discharges

\begin{tabular}{|c|c|c|c|c|c|c|c|c|c|c|}
\hline \multirow[b]{3}{*}{$\begin{array}{l}\text { Non- } \\
\text { exceed- } \\
\text { ance } \\
\text { prob- } \\
\text { abiiity }\end{array}$} & \multirow[b]{3}{*}{$\begin{array}{c}\text { Recur- } \\
\text { rence } \\
\text { inter- } \\
\text { vai } \\
\text { (years) }\end{array}$} & \multicolumn{9}{|c|}{ Minimum mean discharge $\left(\mathrm{ft}^{3} / \mathrm{s}\right)$} \\
\hline & & \multicolumn{9}{|c|}{ Number of consecutive days } \\
\hline & & 1 & 3 & 7 & 14 & 30 & 60 & 90 & 120 & 183 \\
\hline 0.05 & 20 & 0 & 0 & 0 & 0 & 0.279 & 0.553 & 0.742 & 0.983 & 1.13 \\
\hline 0.10 & 10 & 0.310 & 0.348 & 0.389 & 0.394 & 0.447 & 0.773 & 0.987 & 1.26 & 1.46 \\
\hline 0.20 & 5 & 0.476 & 0.528 & 0.623 & 0.662 & 0.729 & 1.10 & 1.35 & 1.66 & 1.98 \\
\hline 0.50 & 2 & 0.830 & 0.900 & 1.09 & 1.24 & 1.46 & 1.92 & 2.24 & 2.66 & 3.48 \\
\hline
\end{tabular}




\section{RUFFY BROOK NEAR GONVICK, MN--Continued}

Probability of occurrence of seasonal low discharges

\begin{tabular}{|c|c|c|c|c|c|c|c|c|c|}
\hline \multirow[b]{3}{*}{$\begin{array}{c}\text { Non- } \\
\text { exceedance } \\
\text { probability }\end{array}$} & \multirow[b]{3}{*}{$\begin{array}{l}\text { Recurrence } \\
\text { interval } \\
\text { (years) }\end{array}$} & \multicolumn{8}{|c|}{ Minimum mean discharge $\left(t^{3} / s\right)$} \\
\hline & & \multicolumn{8}{|c|}{ Number of consecutive days } \\
\hline & & 1 & 7 & 14 & 30 & 1 & 7 & 14 & 30 \\
\hline & & \multicolumn{4}{|c|}{ December-January-February } & \multicolumn{4}{|c|}{ March-April-May } \\
\hline 0.05 & 20 & 0 & 0 & 0 & 0.391 & 0 & 0 & 0.775 & 1.81 \\
\hline 0.10 & 10 & 0.109 & 0.408 & 0.414 & 0.585 & 0.720 & 0.720 & 1.06 & 2.26 \\
\hline 0.20 & 5 & 0.779 & 0.783 & 0.802 & 0.921 & 1.13 & 1.13 & 1.49 & 3.04 \\
\hline \multirow[t]{2}{*}{0.50} & 2 & 1.83 & 1.85 & 1.91 & 1.99 & 2.13 & 2.15 & 2.62 & 5.74 \\
\hline & & \multicolumn{4}{|c|}{ June-July-August } & \multicolumn{4}{|c|}{ September-October-November } \\
\hline 0.05 & 20 & 0.453 & 0.730 & 0.848 & 1.03 & 0.297 & 0.438 & 0.697 & 0.764 \\
\hline 0.10 & 10 & 0.540 & 0.823 & 0.967 & 1.19 & 0.430 & 0.600 & 0.907 & 1.03 \\
\hline 0.20 & 5 & 0.671 & 0.960 & 1.14 & 1.42 & 0.647 & 0.856 & 1.23 & 1.45 \\
\hline 0.50 & 2 & 1.03 & 1.32 & 1.59 & 2.06 & 1.26 & 1.56 & 2.07 & 2.72 \\
\hline
\end{tabular}




\section{RUFFY BROOK NEAR GONVICK, MN--Continued}

Annual peak discharge and corresponding gage height

$$
[--, \text { no data }]
$$

\begin{tabular}{|c|c|c|c|c|c|c|c|}
\hline $\begin{array}{l}\text { Water } \\
\text { year }\end{array}$ & Date & $\begin{array}{c}\text { Gage } \\
\text { height } \\
\text { (feet) }\end{array}$ & $\begin{array}{c}\text { Peak } \\
\text { discharge } \\
\left(\mathrm{ft}^{3} / \mathrm{s}\right)\end{array}$ & $\begin{array}{c}\text { Water } \\
\text { year }\end{array}$ & Date & $\begin{array}{c}\text { Gage } \\
\text { height } \\
\text { (feet) }\end{array}$ & $\begin{array}{c}\text { Peak } \\
\text { discharge } \\
\left(\mathrm{ft}^{3} / \mathbf{s}\right)\end{array}$ \\
\hline \multicolumn{8}{|c|}{ Annual peak discharge, by year, and corresponding gage height } \\
\hline 1960 & April 17 & 4.45 & 74.0 & 1978 & April 7 & 5.67 & 357 \\
\hline 1961 & April 20 & 3.74 & 66.0 & 1979 & April 20 & 4.94 & 284 \\
\hline 1962 & July 7 & 6.70 & 364 & 1980 & April 5 & 3.36 & 137 \\
\hline 1963 & May 28 & 5.48 & 225 & 1981 & June 28 & -- & 150 \\
\hline 1964 & April 16 & 4.45 & 134 & 1982 & April 15 & 4.59 & 249 \\
\hline 1965 & April 13 & 6.38 & 412 & 1983 & March 7 & 4.81 & 175 \\
\hline 1966 & April 2 & 5.53 & 265 & 1984 & March 26 & 4.03 & 195 \\
\hline 1967 & March 30 & 6.35 & 453 & 1985 & May 13 & 4.91 & 281 \\
\hline 1968 & June 10 & 2.70 & 76.0 & 1986 & March 29 & 4.61 & 190 \\
\hline 1969 & April 9 & 6.62 & 232 & 1987 & July 22 & 6.05 & 395 \\
\hline 1970 & April 29 & 5.28 & 279 & 1988 & April 4 & 5.20 & 165 \\
\hline 1971 & April 8 & 5.40 & 176 & 1989 & April 5 & 4.44 & 178 \\
\hline 1972 & April 17 & 4.87 & 237 & 1990 & June 20 & -- & 30.0 \\
\hline 1973 & September 2 & 3.94 & 156 & 1991 & July 3 & 2.29 & 66.0 \\
\hline 1974 & April 12 & 5.80 & 268 & 1992 & March 7 & 3.25 & 98.0 \\
\hline 1975 & April 17 & 5.74 & 364 & 1993 & March 29 & 3.74 & 169 \\
\hline 1976 & March 29 & 5.25 & 147 & 1994 & July 19 & 2.85 & 112 \\
\hline 1977 & September 24 & 2.05 & 47.0 & 1995 & March 13 & 6.10 & 200 \\
\hline \multicolumn{8}{|c|}{ Annual peak discharge, from highest to lowest, and corresponding gage height } \\
\hline 1967 & March 30 & 6.35 & 453 & 1989 & April 5 & 4.44 & 178 \\
\hline 1965 & April 13 & 6.38 & 412 & 1971 & April 8 & 5.40 & 176 \\
\hline 1987 & July 22 & 6.05 & 395 & 1983 & March 7 & 4.81 & 175 \\
\hline 1962 & July 7 & 6.70 & 364 & 1993 & March 29 & 3.74 & 169 \\
\hline 1975 & April 17 & 5.74 & 364 & 1988 & April 4 & 5.20 & 165 \\
\hline 1978 & April 7 & 5.67 & 357 & 1973 & September 2 & 3.94 & 156 \\
\hline 1979 & April 20 & 4.94 & 284 & 1981 & June 28 & -- & 150 \\
\hline 1985 & May 13 & 4.91 & 281 & 1976 & March 29 & 5.25 & 147 \\
\hline 1970 & April 29 & 5.28 & 279 & 1980 & April 5 & 3.36 & 137 \\
\hline 1974 & April 12 & 5.80 & 268 & 1964 & April 16 & 4.45 & 134 \\
\hline 1966 & April 2 & 5.53 & 265 & 1994 & July 19 & 2.85 & 112 \\
\hline 1982 & April 15 & 4.59 & 249 & 1992 & March 7 & 3.25 & 98.0 \\
\hline 1972 & April 17 & 4.87 & 237 & 1968 & June 10 & 2.70 & 76.0 \\
\hline 1969 & April 9 & 6.62 & 232 & 1960 & April 17 & 4.45 & 74.0 \\
\hline 1963 & May 28 & 5.48 & 225 & 1961 & April 20 & 3.74 & 66.0 \\
\hline 1995 & March 13 & 6.10 & 200 & 1991 & July 3 & 2.29 & 66.0 \\
\hline 1984 & March 26 & 4.03 & 195 & 1977 & September 24 & 2.05 & 47.0 \\
\hline 1986 & March 29 & 4.61 & 190 & 1990 & June 20 & -- & 30.0 \\
\hline
\end{tabular}




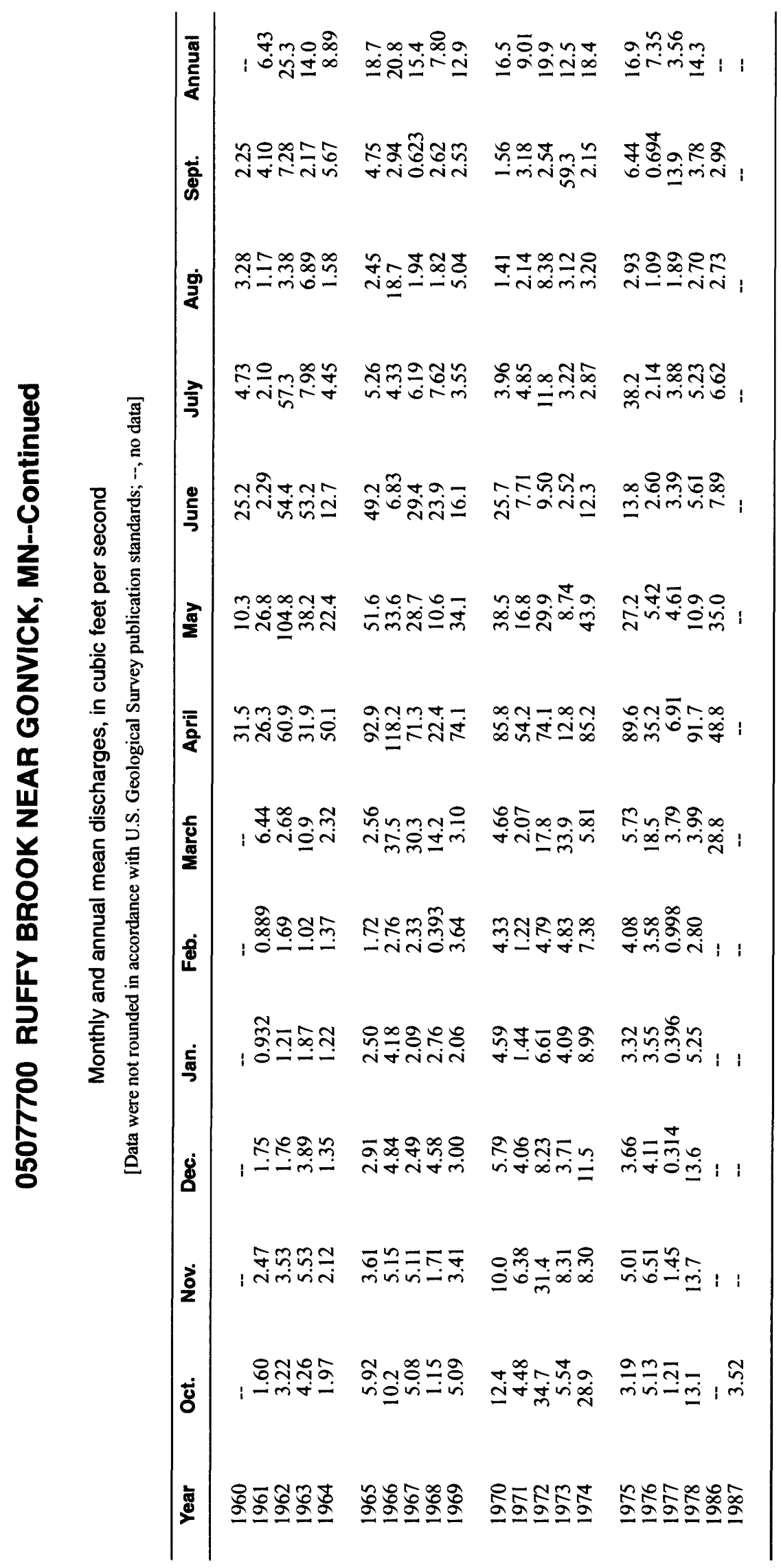




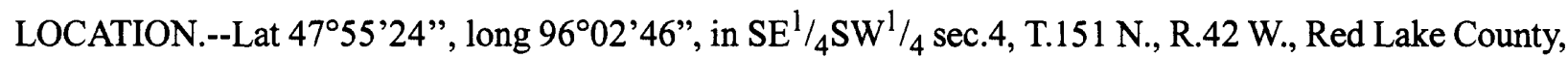
Hydrologic Unit 09020305, on right bank $200 \mathrm{ft}$ downstream from Soo Line Railroad bridge, $300 \mathrm{ft}$ downstream from bridge on U.S. Highway 59, $0.9 \mathrm{mi}$ northwest of railroad depot in Plummer, and 8 nii upstream from Hill River.

DRAINAGE AREA.--512 $\mathrm{mi}^{2}$.

PERIOD OF RECORD.--April 1939 to September 1979, March 1982 to current year. Annual maximums only, October 1979 to February 1982.

GAGE.--Water-stage recorder. Datum of gage is $1,098.57 \mathrm{ft}$ above sea level (levels by U.S. Army Corps of Engineers). Prior to Nov. 10, 1939, nonrecording gage at site $100 \mathrm{ft}$ upstream at same datum.

EXTREMES FOR PERIOD OF RECORD.--Maximum discharge, 3,940 ft3/s, Apr. 25, 1979, gage height, $12.31 \mathrm{ft}$; maximun gage height, $12.37 \mathrm{ft}$, Apr. 18, 1979, backwater from ice; minimum daily discharge, $2.6 \mathrm{ft}^{3} / \mathrm{s}$, May 16, 1977, gage height, 1.71 .

Annual mean discharge

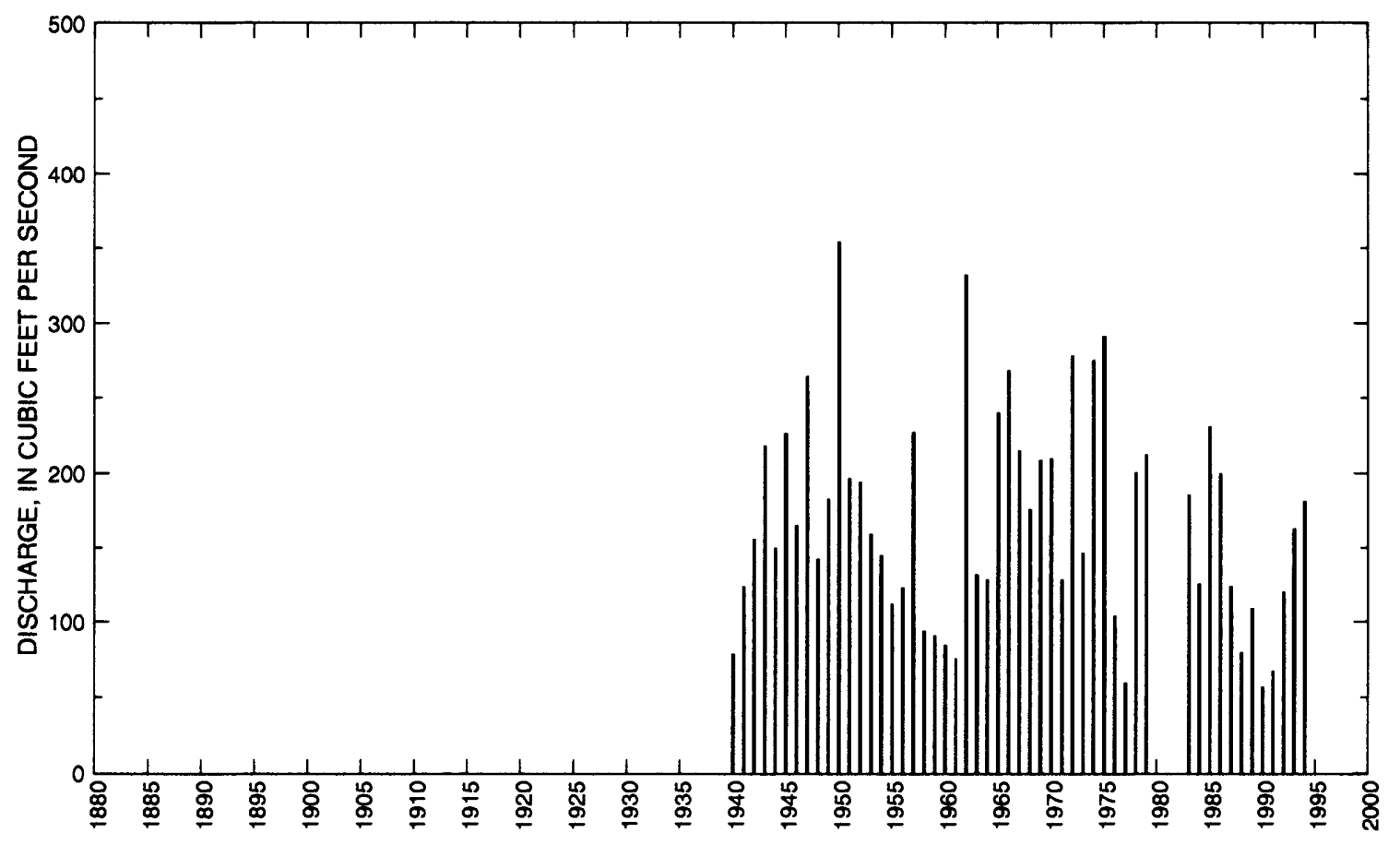


05078000 CLEARWATER RIVER AT PLUMMER, MN--Continued

Statistics of monthly and annual mean discharges

\begin{tabular}{|c|c|c|c|c|c|c|c|c|}
\hline \multirow[b]{2}{*}{ Month } & \multicolumn{2}{|c|}{ Maximum } & \multicolumn{2}{|c|}{ Minimum } & \multicolumn{4}{|c|}{ Mean } \\
\hline & $\begin{array}{c}\text { Discharge } \\
\left(\mathrm{ft}^{3} / \mathrm{s}\right)\end{array}$ & $\begin{array}{c}\text { Water year } \\
\text { of } \\
\text { occurrence }\end{array}$ & $\begin{array}{c}\text { Discharge } \\
\left(\mathrm{ft}^{3} / \mathrm{s}\right)\end{array}$ & $\begin{array}{c}\text { Water year } \\
\text { of } \\
\text { occurrence }\end{array}$ & $\begin{array}{c}\text { Discharge } \\
\left(\mathrm{ft}^{3} / \mathrm{s}\right)\end{array}$ & $\begin{array}{c}\text { Standard } \\
\text { deviation } \\
\left(\mathrm{ft}^{3} / \mathrm{s}\right)\end{array}$ & $\begin{array}{l}\text { Coeffi- } \\
\text { cient of } \\
\text { variation }\end{array}$ & $\begin{array}{l}\text { Percentage } \\
\text { of annuai } \\
\text { discharge }\end{array}$ \\
\hline October & 483 & 1972 & 21.5 & 1941 & 113 & 95.8 & 0.85 & 5.59 \\
\hline November & 503 & 1972 & 23.8 & 1991 & 91.0 & 71.2 & 0.78 & 4.49 \\
\hline December & 140 & 1978 & 24.4 & 1990 & 63.3 & 25.6 & 0.40 & 3.12 \\
\hline January & 90.1 & 1952 & 18.4 & 1940 & 50.7 & 17.2 & 0.34 & 2.50 \\
\hline February & 98.4 & 1974 & 19.0 & 1940 & 47.0 & 16.9 & 0.36 & 2.32 \\
\hline March & 351 & 1945 & 22.8 & 1940 & 109 & 89.6 & 0.82 & 5.39 \\
\hline April & 1,390 & 1966 & 26.8 & 1977 & 516 & 347 & 0.67 & 25.5 \\
\hline May & 1,970 & 1950 & 7.52 & 1977 & 347 & 321 & 0.93 & 17.1 \\
\hline June & 1,140 & 1962 & 30.1 & 1991 & 254 & 228 & 0.90 & 12.5 \\
\hline July & 844 & 1975 & 16.0 & 1940 & 206 & 182 & 0.88 & 10.2 \\
\hline August & 507 & 1985 & 13.3 & 1940 & 123 & 107 & 0.87 & 6.05 \\
\hline September & 666 & 1973 & 14.1 & 1940 & 106 & 101 & 0.95 & 5.25 \\
\hline Annual & 354 & 1950 & 57.0 & 1990 & 170 & 70.7 & 0.42 & 100 \\
\hline
\end{tabular}

Annual flow duration

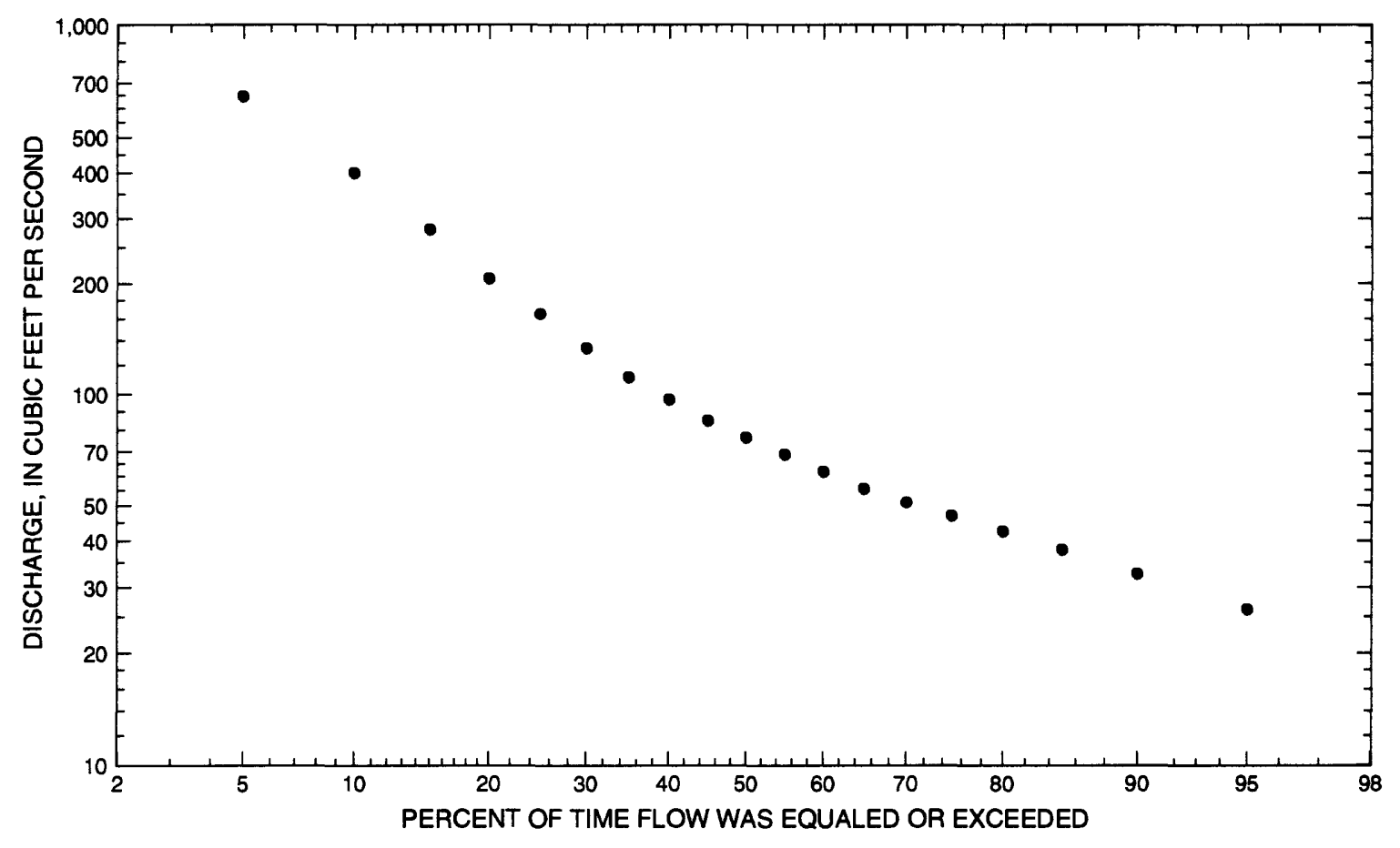




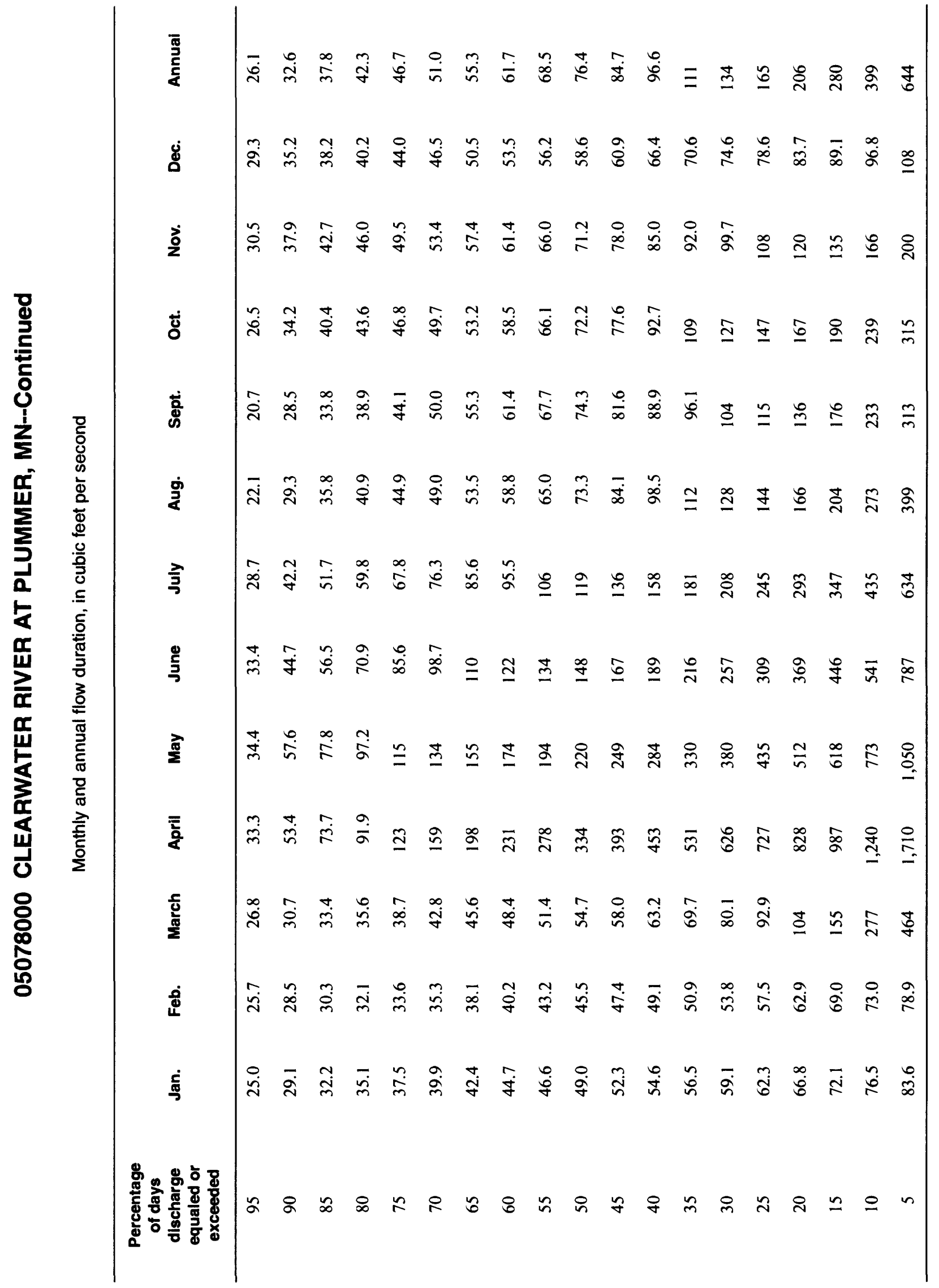




\section{CLEARWATER RIVER AT PLUMMER, MN--Continued}

Probability of occurrence of annual high discharges

[ng, statistic not given]

\begin{tabular}{|c|c|c|c|c|c|c|}
\hline \multirow[b]{2}{*}{$\begin{array}{l}\text { Exceedance } \\
\text { probability }\end{array}$} & \multirow[b]{2}{*}{$\begin{array}{l}\text { Recurrence } \\
\text { intervai } \\
\text { (years) }\end{array}$} & \multirow[b]{2}{*}{$\begin{array}{c}\text { Maximum } \\
\text { instantaneous } \\
\left(\mathrm{ft}^{3} / \mathrm{s}\right)\end{array}$} & \multicolumn{4}{|c|}{$\begin{array}{c}\text { Maximum mean discharge } \\
\left(\mathrm{ft}^{3} / \mathrm{s}\right)\end{array}$} \\
\hline & & & 3-day period & 7-day period & 15-day period & 30-day period \\
\hline 0.99 & 1.01 & 311 & 313 & 286 & 227 & 169 \\
\hline 0.95 & 1.05 & 484 & 461 & 410 & 328 & 251 \\
\hline 0.90 & 1.11 & 611 & 567 & 498 & 400 & 309 \\
\hline 0.80 & 1.25 & 806 & 731 & 634 & 510 & 397 \\
\hline 0.50 & 2 & 1,360 & 1,200 & 1,020 & 817 & 637 \\
\hline 0.20 & 5 & 2,250 & 1,980 & 1,660 & 1,320 & 1,010 \\
\hline 0.10 & 10 & 2,920 & 2,580 & 2,160 & 1,700 & 1,290 \\
\hline 0.04 & 25 & 3,840 & 3,440 & 2,880 & 2,240 & 1,660 \\
\hline 0.02 & 50 & 4,570 & 4,140 & 3,470 & 2,670 & 1,950 \\
\hline 0.01 & 100 & 5,340 & 4,900 & 4,120 & 3,140 & 2,250 \\
\hline 0.005 & 200 & 6,140 & 5,720 & 4,820 & 3,640 & 2,570 \\
\hline 0.002 & 500 & 7,280 & $\mathrm{ng}$ & $\mathrm{ng}$ & ng & ng \\
\hline
\end{tabular}

Probability of occurrence of annual low discharges

Minimum mean discharge $\left(\mathrm{ft}^{3} / \mathrm{s}\right)$

Number of consecutive days

\begin{tabular}{|c|c|c|c|c|c|c|c|c|c|c|}
\hline $\begin{array}{l}\text { Non- } \\
\text { exceed- } \\
\text { ance } \\
\text { prob- } \\
\text { ability }\end{array}$ & $\begin{array}{l}\text { Recur- } \\
\text { rence } \\
\text { inter- } \\
\text { val } \\
\text { (years) }\end{array}$ & 1 & 3 & 7 & 14 & 30 & 60 & 90 & 120 & 183 \\
\hline 0.05 & 20 & 7.08 & 8.03 & 9.19 & 11.0 & 13.7 & 19.5 & 23.1 & 26.0 & 29.8 \\
\hline 0.10 & 10 & 10.0 & 11.4 & 13.2 & 15.3 & 18.3 & 23.4 & 27.3 & 30.6 & 35.4 \\
\hline 0.20 & 5 & 14.6 & 16.6 & 19.2 & 21.4 & 24.8 & 28.7 & 32.9 & 36.8 & 43.5 \\
\hline 0.50 & 2 & 26.3 & 29.0 & 32.7 & 34.9 & 38.6 & 40.7 & 45.3 & 50.7 & 63.7 \\
\hline
\end{tabular}




\section{CLEARWATER RIVER AT PLUMMER, MN-Continued}

Probability of occurrence of seasonal low discharges

\begin{tabular}{|c|c|c|c|c|c|c|c|c|c|}
\hline \multirow[b]{3}{*}{$\begin{array}{c}\text { Non- } \\
\text { exceedance } \\
\text { probability }\end{array}$} & \multirow[b]{3}{*}{$\begin{array}{c}\text { Recurrence } \\
\text { intervai } \\
\text { (years) }\end{array}$} & \multicolumn{8}{|c|}{ Minimum mean discharge $\left(\mathrm{ft}^{3} / \mathrm{s}\right)$} \\
\hline & & \multicolumn{8}{|c|}{ Number of consecutive days } \\
\hline & & 1 & 7 & 14 & 30 & 1 & 7 & 14 & 30 \\
\hline & & \multicolumn{4}{|c|}{ December-January-February } & \multicolumn{4}{|c|}{ March-April-May } \\
\hline 0.05 & 20 & 16.1 & 19.8 & 21.3 & 23.1 & 8.46 & 11.1 & 13.5 & 17.9 \\
\hline 0.10 & 10 & 19.8 & 23.1 & 24.6 & 26.6 & 12.7 & 16.6 & 19.2 & 24.4 \\
\hline 0.20 & 5 & 24.9 & 27.7 & 29.2 & 31.5 & 19.6 & 25.1 & 27.7 & 35.1 \\
\hline \multirow[t]{2}{*}{0.50} & 2 & 37.3 & 38.7 & 40.1 & 42.7 & 37.1 & 44.9 & 47.8 & 67.7 \\
\hline & & \multicolumn{4}{|c|}{ June-July-August } & \multicolumn{4}{|c|}{ September-October-November } \\
\hline 0.05 & 20 & 10.9 & 14.5 & 17.6 & 22.0 & 12.3 & 15.8 & 17.6 & 20.9 \\
\hline 0.10 & 10 & 14.9 & 18.8 & 22.1 & 27.6 & 15.8 & 19.8 & 22.2 & 26.4 \\
\hline 0.20 & 5 & 21.3 & 25.5 & 29.0 & 36.8 & 21.2 & 26.0 & 29.2 & 34.8 \\
\hline 0.50 & 2 & 39.3 & 44.6 & 49.5 & 64.7 & 36.8 & 43.0 & 48.0 & 57.3 \\
\hline
\end{tabular}


05078000 CLEARWATER RIVER AT PLUMMER, MN--Continued

Annual peak discharge and corresponding gage height

$[--$, no data $]$

\begin{tabular}{|c|c|c|c|c|c|c|c|}
\hline $\begin{array}{l}\text { Water } \\
\text { year }\end{array}$ & Date & $\begin{array}{c}\text { Gage } \\
\text { height } \\
\text { (feet) }\end{array}$ & $\begin{array}{c}\text { Peak } \\
\text { discharge } \\
\left(\mathrm{ft}^{3} / \mathrm{s}\right) \\
\end{array}$ & $\begin{array}{l}\text { Water } \\
\text { year }\end{array}$ & Date & $\begin{array}{c}\text { Gage } \\
\text { height } \\
\text { (feet) }\end{array}$ & $\begin{array}{c}\text { Peak } \\
\text { discharge } \\
\left(\mathrm{ft}^{3} / \mathbf{s}\right)\end{array}$ \\
\hline \multicolumn{8}{|c|}{ Annual peak discharge, by year, and corresponding gage height } \\
\hline 1939 & April 27 & -- & 380 & 1967 & April 3 & 10.19 & 2,470 \\
\hline 1940 & April 16 & 6.98 & 840 & 1968 & June 18 & 11.00 & 3,000 \\
\hline 1941 & June 8 & 6.57 & 756 & 1969 & April 11 & 11.89 & 3,630 \\
\hline 1942 & April 3 & 6.12 & 722 & 1970 & April 26 & 9.48 & 2,080 \\
\hline 1943 & April 21 & 6.43 & 800 & 1971 & April 10 & 8.32 & 1,520 \\
\hline 1944 & August 10 & 8.12 & 1,160 & 1972 & April 16 & 10.33 & 2,550 \\
\hline 1945 & March 28 & 6.76 & 952 & 1973 & September 6 & 7.98 & 1,270 \\
\hline 1946 & March 23 & 8.09 & 1,030 & 1974 & April 22 & 10.31 & 2,540 \\
\hline 1947 & June 11 & 8.34 & 1,420 & 1975 & July 4 & 11.19 & 2,960 \\
\hline 1948 & April 25 & 6.80 & 929 & 1976 & April 1 & 8.86 & 1,250 \\
\hline 1949 & June 1 & 9.08 & 1,870 & 1977 & September 27 & 4.85 & 429 \\
\hline 1950 & May 6 & 11.33 & 3,630 & 1978 & April 13 & 11.40 & 3,270 \\
\hline 1951 & May 3 & 7.22 & 1,110 & 1979 & April 25 & 12.31 & 3,940 \\
\hline 1952 & April 15 & 8.10 & 1,440 & 1980 & April 8 & 6.57 & 898 \\
\hline 1953 & July 5 & 6.23 & 834 & 1981 & June 29 & 7.37 & 1,150 \\
\hline 1954 & April 13 & 8.52 & 1,640 & 1982 & April 16 & 8.71 & 1,700 \\
\hline 1955 & April 6 & 9.64 & 1,800 & 1983 & March 7 & 10.17 & 1,200 \\
\hline 1956 & April 21 & 9.58 & 2,240 & 1984 & June 9 & 9.07 & 1,880 \\
\hline 1957 & June 27 & 11.84 & 3,570 & 1985 & August 19 & 8.59 & 1,650 \\
\hline 1958 & July 6 & 6.08 & 822 & 1986 & May 1 & 8.03 & 1,430 \\
\hline 1959 & April 6 & 6.37 & 702 & 1987 & July 27 & 7.62 & 1,290 \\
\hline 1960 & April 7 & 8.18 & 710 & 1988 & April 7 & 8.22 & 900 \\
\hline 1961 & April 25 & 4.88 & 461 & 1989 & April 15 & 9.34 & 1,200 \\
\hline 1962 & June 9 & 11.90 & 3,640 & 1990 & June 22 & 4.67 & 393 \\
\hline 1963 & April 4 & 7.54 & 966 & 1991 & July 7 & 5.39 & 565 \\
\hline 1964 & April 17 & 8.57 & 1,640 & 1992 & August 26 & 6.60 & 776 \\
\hline 1965 & April 12 & 11.88 & 3,620 & 1993 & July 28 & 7.20 & 954 \\
\hline 1966 & April 3 & 10.73 & 2,000 & 1994 & July 9 & 9.22 & 1,440 \\
\hline \multicolumn{8}{|c|}{ Annual peak discharge, from highest to lowest, and corresponding gage height } \\
\hline 1979 & April 25 & 12.31 & 3,940 & 1966 & April 3 & 10.73 & 2,000 \\
\hline 1962 & June 9 & 11.90 & 3,640 & 1984 & June 9 & 9.07 & 1,880 \\
\hline 1950 & May 6 & 11.33 & 3,630 & 1949 & June 1 & 9.08 & 1,870 \\
\hline 1969 & April 11 & 11.89 & 3,630 & 1955 & April 6 & 9.64 & 1,800 \\
\hline 1965 & April 12 & 11.88 & 3,620 & 1982 & April 16 & 8.71 & 1,700 \\
\hline 1957 & June 27 & 11.84 & 3,570 & 1985 & August 19 & 8.59 & 1,650 \\
\hline 1978 & April 13 & 11.40 & 3,270 & 1954 & April 13 & 8.52 & 1,640 \\
\hline 1968 & June 18 & 11.00 & 3,000 & 1964 & April 17 & 8.57 & 1,640 \\
\hline 1975 & July 4 & 11.19 & 2,960 & 1971 & April 10 & 8.32 & 1,520 \\
\hline 1972 & April 16 & 10.33 & 2,550 & 1952 & April 15 & 8.10 & 1,440 \\
\hline 1974 & April 22 & 10.31 & 2,540 & 1994 & July 9 & 9.22 & 1,440 \\
\hline 1967 & April 3 & 10.19 & 2,470 & 1986 & May 1 & 8.03 & 1,430 \\
\hline 1956 & April 21 & 9.58 & 2,240 & 1947 & June 11 & 8.34 & 1,420 \\
\hline 1970 & April 26 & 9.48 & 2,080 & 1987 & July 27 & 7.62 & 1,290 \\
\hline
\end{tabular}




\section{CLEARWATER RIVER AT PLUMMER, MN--Continued}

Annual peak discharge and corresponding gage height--Continued

$$
[--, \text { no data }]
$$

\begin{tabular}{|c|c|c|c|c|c|c|c|}
\hline $\begin{array}{c}\text { Water } \\
\text { year }\end{array}$ & Date & $\begin{array}{c}\text { Gage } \\
\text { height } \\
\text { (feet) }\end{array}$ & $\begin{array}{c}\text { Peak } \\
\text { discharge } \\
\left(\mathrm{ft}^{3} / \mathrm{s}\right)\end{array}$ & $\begin{array}{c}\text { Water } \\
\text { year }\end{array}$ & Date & $\begin{array}{c}\text { Gage } \\
\text { height } \\
\text { (feet) }\end{array}$ & $\begin{array}{c}\text { Peak } \\
\text { discharge } \\
\left(\mathrm{ft}^{3} / \mathrm{s}\right)\end{array}$ \\
\hline \multicolumn{8}{|c|}{ Annual peak discharge, from highest to lowest, and corresponding gage height-Continued } \\
\hline 1973 & September 6 & 7.98 & 1,270 & 1940 & April 16 & 6.98 & 840 \\
\hline 1976 & April 1 & 8.86 & 1,250 & 1953 & July 5 & 6.23 & 834 \\
\hline 1983 & March 7 & 10.17 & 1,200 & 1958 & July 6 & 6.08 & 822 \\
\hline 1989 & April 15 & 9.34 & 1,200 & 1943 & April 21 & 6.43 & 800 \\
\hline 1944 & August 10 & 8.12 & 1,160 & 1992 & August 26 & 6.60 & 776 \\
\hline 1981 & June 29 & 7.37 & 1,150 & 1941 & June 8 & 6.57 & 756 \\
\hline 1951 & May 3 & 7.22 & 1,110 & 1942 & April 3 & 6.12 & 722 \\
\hline 1946 & March 23 & 8.09 & 1,030 & 1960 & April 7 & 8.18 & 710 \\
\hline 1963 & April 4 & 7.54 & 966 & 1959 & April 6 & 6.37 & 702 \\
\hline 1993 & July 28 & 7.20 & 954 & 1991 & July 7 & 5.39 & 565 \\
\hline 1945 & March 28 & 6.76 & 952 & 1961 & April 25 & 4.88 & 461 \\
\hline 1948 & April 25 & 6.80 & 929 & 1977 & September 27 & 4.85 & 429 \\
\hline 1988 & April 7 & 8.22 & 900 & 1990 & June 22 & 4.67 & 393 \\
\hline 1980 & April 8 & 6.57 & 898 & 1939 & April 27 & -- & 380 \\
\hline
\end{tabular}




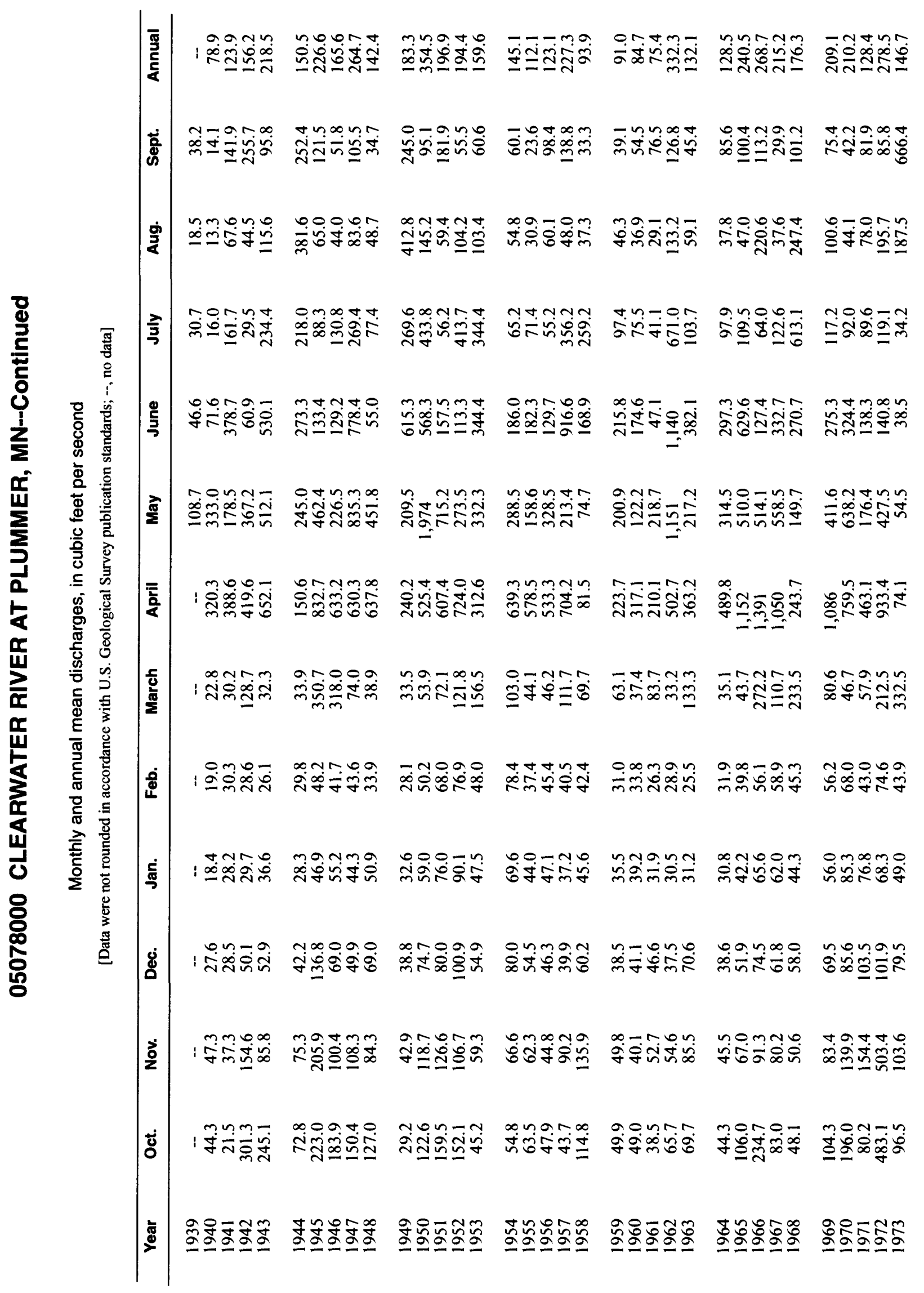




\begin{tabular}{|c|c|c|c|c|c|}
\hline & 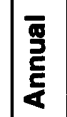 & 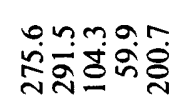 & $\stackrel{\infty}{\stackrel{\sim}{\sim}}: \mid \begin{array}{l}\infty \\
\stackrel{\infty}{\infty} \\
\infty\end{array}$ & 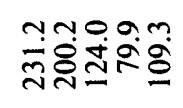 & 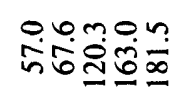 \\
\hline & 㝘 & 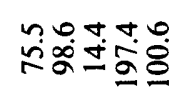 & 我 : & 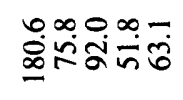 & 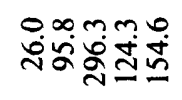 \\
\hline & 安 & 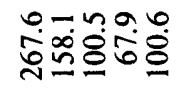 & 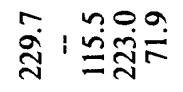 & 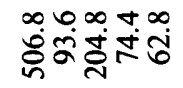 & 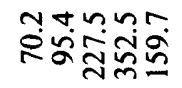 \\
\hline 哥 & $\frac{2}{3}$ & 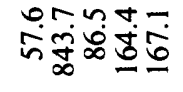 & 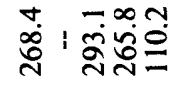 & 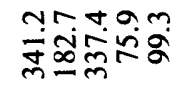 & 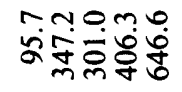 \\
\hline 信 & $\stackrel{0}{5}$ & 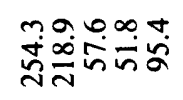 & 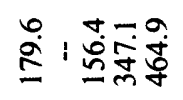 & 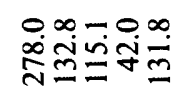 & 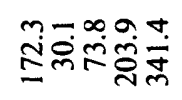 \\
\hline 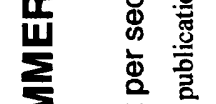 & 产 & 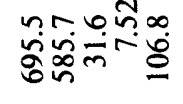 & 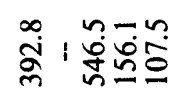 & 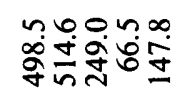 & 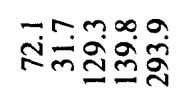 \\
\hline 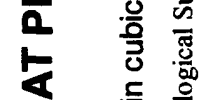 & $\overline{\bar{z}}$ & 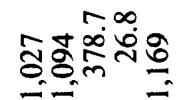 & 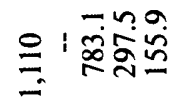 & 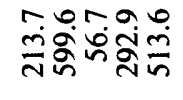 & 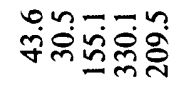 \\
\hline 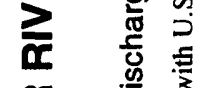 & 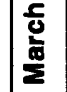 & 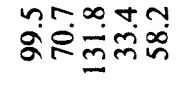 & $\begin{array}{l}0 \\
\dot{\sigma}\end{array}$ & 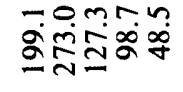 & 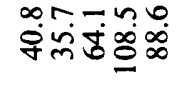 \\
\hline 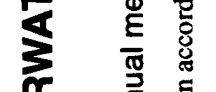 & 要 & 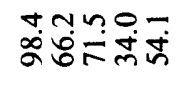 & & 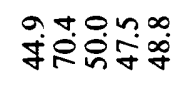 & 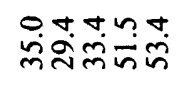 \\
\hline 恖 & 站 & 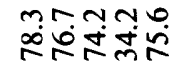 & $\begin{array}{l}\infty \\
\infty \\
\infty\end{array}$ & 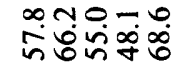 & 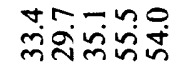 \\
\hline & 8 & 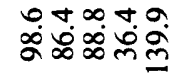 & 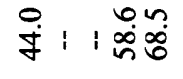 & $\begin{array}{l}n \infty \\
\infty \infty \\
\infty \\
\infty\end{array}$ & 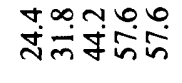 \\
\hline & iे & 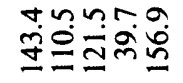 & $\begin{array}{l}\infty \\
\dot{\sigma}+1 \\
\dot{q}\end{array}$ & 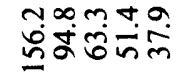 & 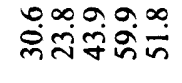 \\
\hline & ठั̊ & 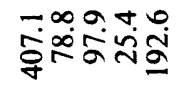 & 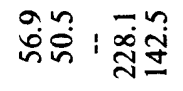 & 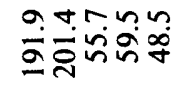 & 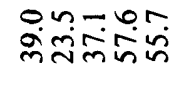 \\
\hline & 离 & 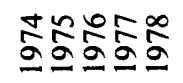 & 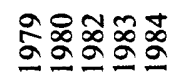 & 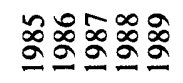 & 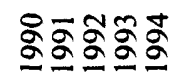 \\
\hline
\end{tabular}




\section{LOST RIVER AT OKLEE, MN}

LOCATION.--Lat 47 50'35”, long 9551'30”, in $\mathrm{SE}^{1} / 4 \mathrm{NE}^{1} / 4 \mathrm{sec} .2, \mathrm{~T} .150$ N., R.41 W., Red Lake County, Hydrologic Unit 09020305, on downstream side of bridge on State Highway 222 at northwest edge of Oklee, 12 mi upstream from mouth.

DRAINAGE AREA.--266 $\mathrm{mi}^{2}$.

PERIOD OF RECORD.--April 1960 to September 1981, February 1982 to current year. Monthly and daily figures for April 1960, to June 1960, published in WSP 2113.

GAGE.--Water-stage recorder. Datum of gage is $1,126.94 \mathrm{ft}$ above sea level, adjustment of 1912 (levels by U.S. Army Corps of Engineers). Prior to Sept. 9, 1960, reference points at same site at datum $8.00 \mathrm{ft}$ higher. Sept. 9, 1960, to Sept. 30, 1964, nonrecording gage at same site at datum $8.00 \mathrm{ft}$ higher.

Oct. 1, 1964, to Sept. 30, 1981, and Feb. 24, 1982, to Sept. 6, 1989, nonrecording gage at same site and datum.

EXTREMES FOR PERIOD OF RECORD.--Maximum discharge, 3,210 ft $3 / \mathrm{s}$, Apr. 11, 1969, gage height, $14.91 \mathrm{ft}$; maximum gage height, $16.72 \mathrm{ft}$, May 24, 1962; no flow at times.

EXTREMES OUTSIDE PERIOD OF RECORD.--Maximum stage known since at least 1897, $18.39 \mathrm{ft}$, present datum, Apr. 21, 1950, from floodmarks.

Annual mean discharge

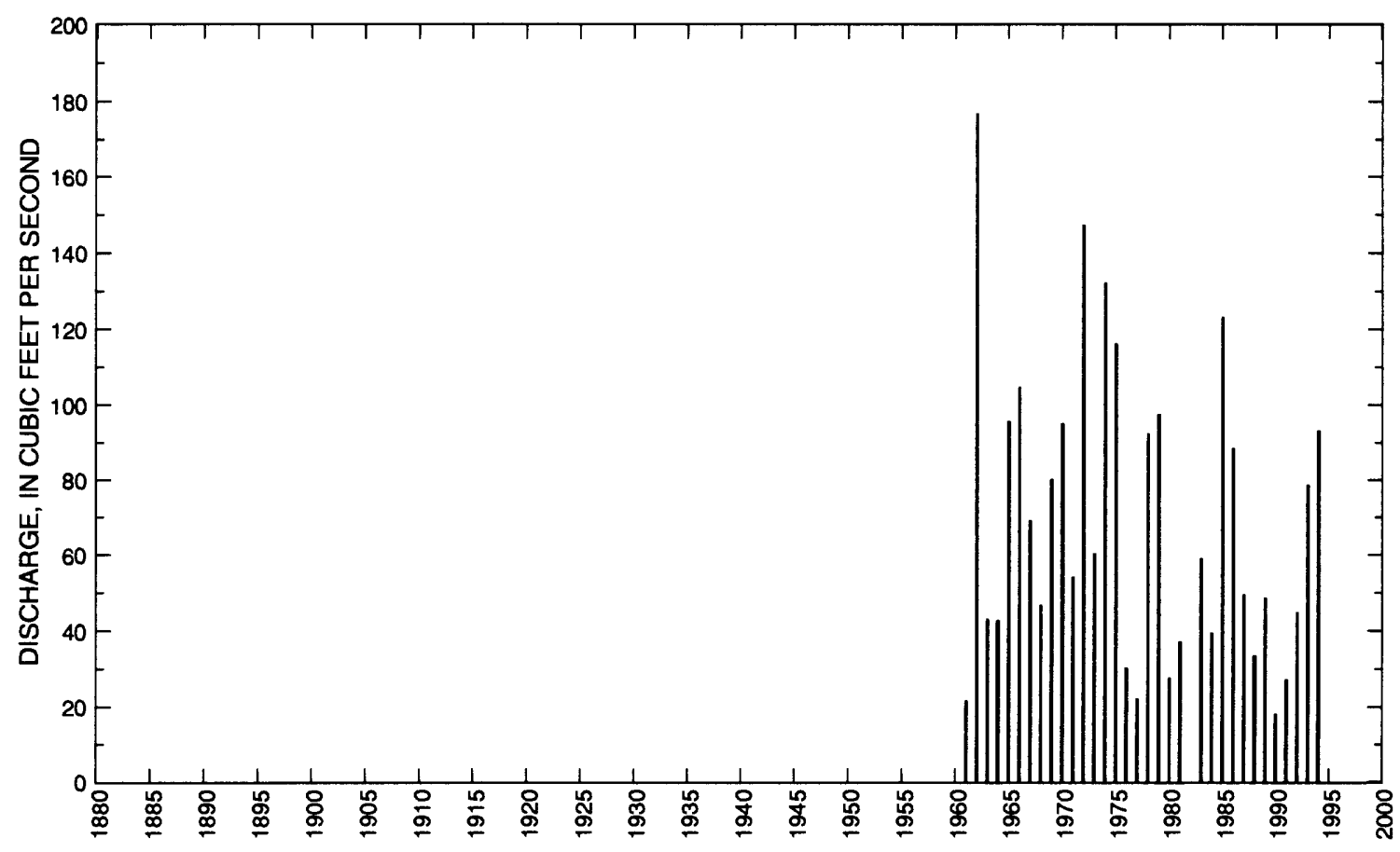




\section{LOST RIVER AT OKLEE, MN--Continued}

Statistics of monthly and annual mean discharges

\begin{tabular}{|c|c|c|c|c|c|c|c|c|}
\hline \multirow[b]{2}{*}{ Month } & \multicolumn{2}{|c|}{ Maximum } & \multicolumn{2}{|c|}{ Minimum } & \multicolumn{4}{|c|}{ Mean } \\
\hline & $\begin{array}{c}\text { Discharge } \\
\left(\mathrm{ft}^{3} / \mathrm{s}\right)\end{array}$ & $\begin{array}{c}\text { Water year } \\
\text { of } \\
\text { occurrence }\end{array}$ & $\begin{array}{c}\text { Discharge } \\
\left(\mathrm{ft}^{3} / \mathrm{s}\right)\end{array}$ & $\begin{array}{c}\text { Water year } \\
\text { of } \\
\text { occurrence }\end{array}$ & $\begin{array}{c}\text { Discharge } \\
\left(\mathrm{ft}^{3} / \mathrm{s}\right)\end{array}$ & $\begin{array}{c}\text { Standard } \\
\text { deviation } \\
\left(\mathrm{ft}^{3} / \mathbf{s}\right)\end{array}$ & $\begin{array}{l}\text { Coeffi- } \\
\text { cient of } \\
\text { variation }\end{array}$ & $\begin{array}{l}\text { Percentage } \\
\text { of annuai } \\
\text { discharge }\end{array}$ \\
\hline October & 470 & 1972 & 1.02 & 1991 & 45.1 & 83.8 & 1.86 & 5.37 \\
\hline November & 232 & 1972 & 1.11 & 1977 & 29.9 & 40.2 & 1.35 & 3.56 \\
\hline December & 56.6 & 1978 & 0.050 & 1977 & 13.4 & 11.3 & 0.85 & 1.59 \\
\hline January & 19.8 & 1986 & 0.002 & 1977 & 7.94 & 5.72 & 0.72 & 0.95 \\
\hline February & 25.8 & 1984 & 0 & 1977 & 7.65 & 5.92 & 0.77 & 0.91 \\
\hline March & 242 & 1986 & 0.190 & 1964 & 71.0 & 66.5 & 0.94 & 8.46 \\
\hline April & 745 & 1966 & 29.5 & 1991 & 293 & 234 & 0.80 & 34.9 \\
\hline May & 622 & 1962 & 10.5 & 1980 & 127 & 120 & 0.94 & 15.1 \\
\hline June & 657 & 1962 & 8.20 & 1980 & 87.8 & 115 & 1.31 & 10.5 \\
\hline July & 442 & 1962 & 1.99 & 1961 & 78.4 & 104 & 1.33 & 9.34 \\
\hline August & 351 & 1985 & 1.17 & 1961 & 40.9 & 75.6 & 1.85 & 4.87 \\
\hline September & 330 & 1973 & 0 & 1990 & 37.4 & 62.1 & 1.66 & 4.45 \\
\hline Annual & 177 & 1962 & 18.2 & 1990 & 69.7 & 39.9 & 0.57 & 100 \\
\hline
\end{tabular}

Annual flow duration

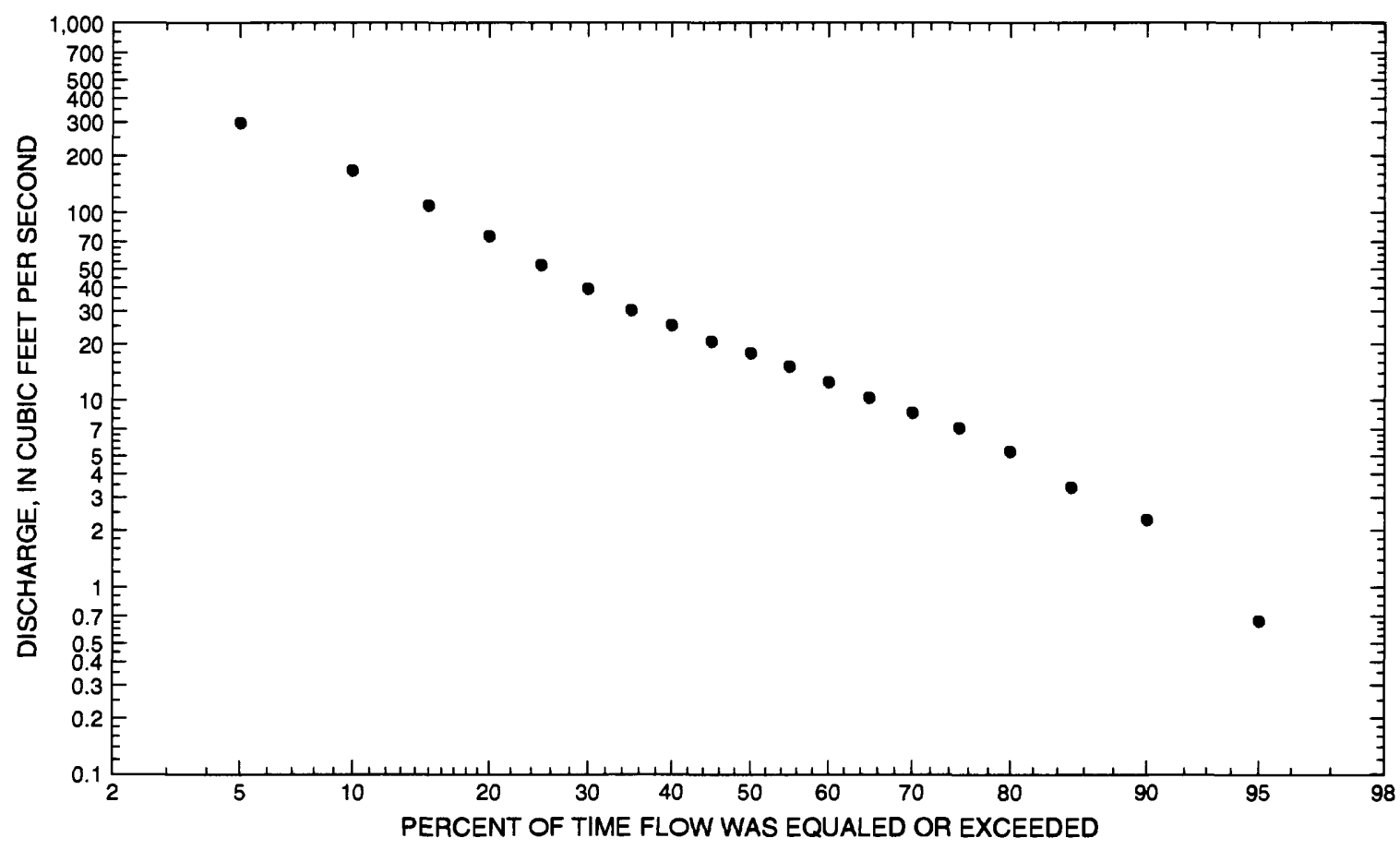




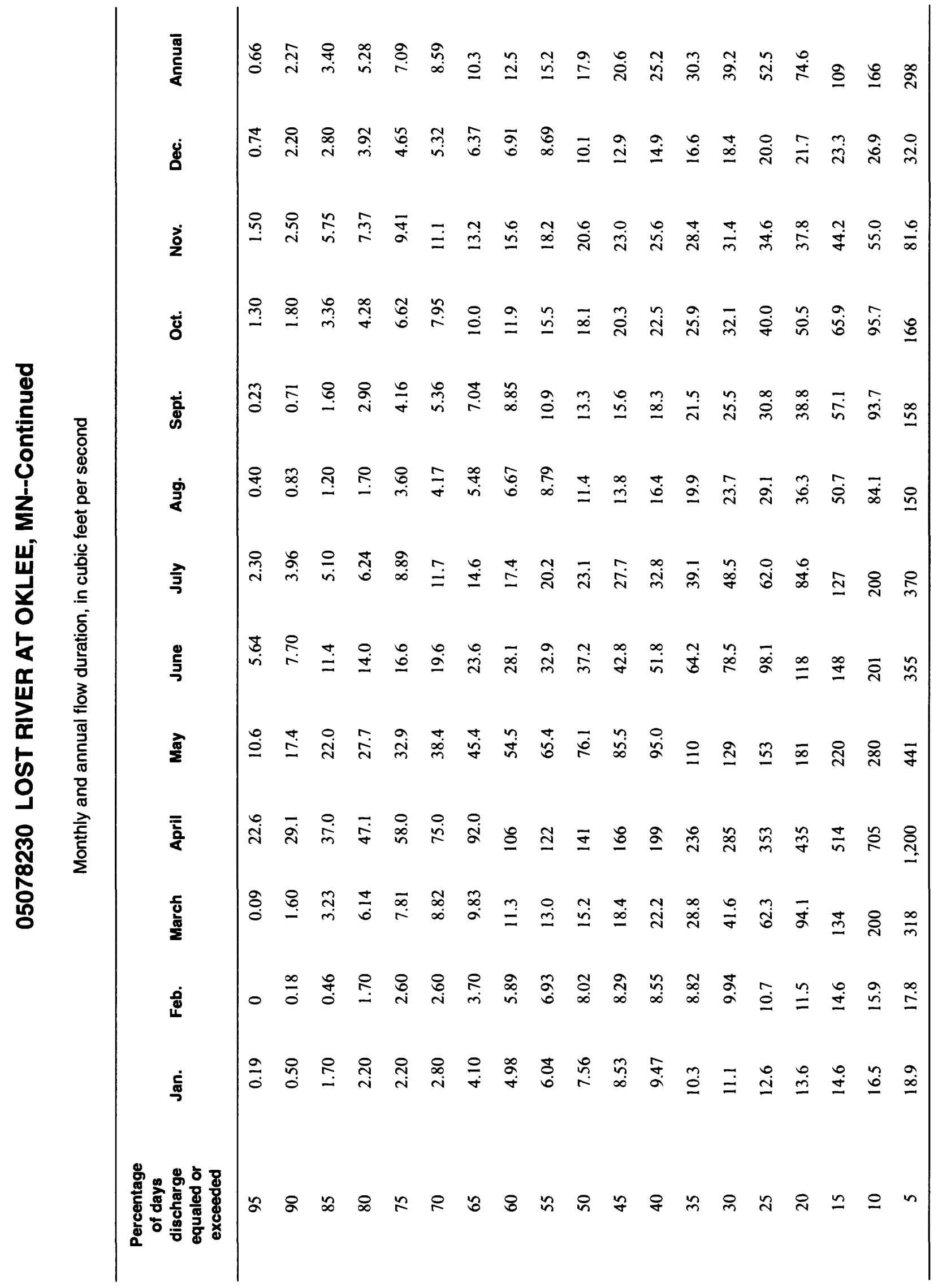




\section{LOST RIVER AT OKLEE, MN-Continued}

Probability of occurrence of annual high discharges

[ng, statistic not given]

\begin{tabular}{|c|c|c|c|c|c|c|}
\hline \multirow[b]{2}{*}{$\begin{array}{l}\text { Exceedance } \\
\text { probablility }\end{array}$} & \multirow[b]{2}{*}{$\begin{array}{l}\text { Recurrence } \\
\text { interval } \\
\text { (years) }\end{array}$} & \multirow[b]{2}{*}{$\begin{array}{c}\text { Maximum } \\
\text { instantaneous } \\
\left(\mathrm{ft}^{3} / \mathrm{s}\right)\end{array}$} & \multicolumn{4}{|c|}{$\begin{array}{l}\text { Maximum mean discharge } \\
\qquad\left(\mathrm{ft}^{3} / \mathrm{s}\right)\end{array}$} \\
\hline & & & 3-day period & 7-day period & 15-day period & 30-day period \\
\hline 0.99 & 1.01 & 124 & 103 & 90.4 & 63.8 & 48.8 \\
\hline 0.95 & 1.05 & 258 & 207 & 170 & 120 & 88.4 \\
\hline 0.90 & 1.11 & 371 & 293 & 234 & 165 & 120 \\
\hline 0.80 & 1.25 & 562 & 436 & 339 & 238 & 170 \\
\hline 0.50 & 2 & 1,150 & 878 & 656 & 460 & 321 \\
\hline 0.20 & 5 & 2,120 & 1,630 & 1,200 & 835 & 576 \\
\hline 0.10 & 10 & 2,810 & 2,180 & 1,600 & 1,110 & 765 \\
\hline 0.04 & 25 & 3,710 & 2,920 & 2,150 & 1,490 & 1,020 \\
\hline 0.02 & 50 & 4,370 & 3,470 & 2,580 & 1,780 & 1,220 \\
\hline 0.01 & 100 & 5,020 & 4,030 & 3,020 & 2,070 & 1,430 \\
\hline 0.005 & 200 & 5,650 & 4,590 & 3,470 & 2,370 & 1,640 \\
\hline 0.002 & 500 & 6,470 & ng & ng & ng & ng \\
\hline
\end{tabular}

Probability of occurrence of annual low discharges

\begin{tabular}{|c|c|c|c|c|c|c|c|c|c|c|}
\hline \multirow[b]{3}{*}{$\begin{array}{l}\text { Non- } \\
\text { exceed- } \\
\text { ance } \\
\text { prob- } \\
\text { ability }\end{array}$} & \multirow[b]{3}{*}{$\begin{array}{c}\text { Recur- } \\
\text { rence } \\
\text { inter- } \\
\text { val } \\
\text { (years) }\end{array}$} & \multicolumn{9}{|c|}{ Minimum mean discharge $\left(\mathrm{ft}^{3} / \mathrm{s}\right)$} \\
\hline & & \multicolumn{9}{|c|}{ Number of consecutive days } \\
\hline & & 1 & 3 & 7 & 14 & 30 & 60 & 90 & 120 & 183 \\
\hline 0.05 & 20 & 0 & 0 & 0 & 0 & 0 & 0.102 & 0.278 & 0.592 & 1.27 \\
\hline 0.10 & 10 & 0 & 0 & 0 & 0 & 0.032 & 0.385 & 0.855 & 1.35 & 2.32 \\
\hline 0.20 & 5 & 0.171 & 0.206 & 0.282 & 0.408 & 0.558 & 1.12 & 2.10 & 3.11 & 4.51 \\
\hline 0.50 & 2 & 1.31 & 1.51 & 1.85 & 2.44 & 3.48 & 4.59 & 6.68 & 9.70 & 13.1 \\
\hline
\end{tabular}




\section{LOST RIVER AT OKLEE, MN--Continued}

Probability of occurrence of seasonal low discharges

\begin{tabular}{|c|c|c|c|c|c|c|c|c|c|}
\hline \multirow[b]{3}{*}{$\begin{array}{l}\text { Non- } \\
\text { exceedance } \\
\text { probability }\end{array}$} & \multirow[b]{3}{*}{$\begin{array}{l}\text { Recurrence } \\
\text { interval } \\
\text { (years) }\end{array}$} & \multicolumn{8}{|c|}{ Minimum mean discharge $\left(\mathrm{ft}^{3} / \mathrm{s}\right)$} \\
\hline & & \multicolumn{8}{|c|}{ Number of consecutive days } \\
\hline & & 1 & 7 & 14 & 30 & 1 & 7 & 14 & 30 \\
\hline & & \multicolumn{4}{|c|}{ December-January-February } & \multicolumn{4}{|c|}{ March-April-May } \\
\hline 0.05 & 20 & 0 & 0 & 0 & 0.063 & 0 & 0 & 0.047 & 2.01 \\
\hline 0.10 & 10 & 0.080 & 0.078 & 0.082 & 0.303 & 0.155 & 0.435 & ${ }^{1} 1.00$ & 4.44 \\
\hline 0.20 & 5 & 1.00 & 1.04 & 1.07 & ${ }^{1} 1.10$ & 1.25 & 1.63 & $1_{3.00}$ & 10.0 \\
\hline \multirow[t]{2}{*}{0.50} & 2 & 4.91 & 5.18 & 5.27 & ${ }^{1} 5.40$ & 5.53 & 7.32 & ${ }^{1} 15.0$ & 31.4 \\
\hline & & \multicolumn{4}{|c|}{ June-July-August } & \multicolumn{4}{|c|}{ September-October-November } \\
\hline 0.05 & 20 & 0.052 & 0.238 & 0.483 & 1.51 & 0.054 & 0.082 & 0.226 & 0.517 \\
\hline 0.10 & 10 & 0.164 & 0.442 & 0.800 & 2.14 & 0.200 & 0.307 & 0.678 & 1.28 \\
\hline 0.20 & 5 & 0.466 & 0.910 & 1.46 & 3.33 & 0.615 & 0.940 & 1.72 & 2.83 \\
\hline 0.50 & 2 & 2.33 & 3.31 & 4.47 & 8.28 & 3.22 & 4.64 & 6.56 & 9.29 \\
\hline
\end{tabular}

${ }^{1}$ Graphical interpretation. 
05078230 LOST RIVER AT OKLEE, MN--Continued

Annual peak discharge and corresponding gage height

$[--$, no data $]$

\begin{tabular}{|c|c|c|c|c|c|c|c|}
\hline $\begin{array}{l}\text { Water } \\
\text { year }\end{array}$ & Date & $\begin{array}{c}\text { Gage } \\
\text { height } \\
\text { (feet) }\end{array}$ & $\begin{array}{c}\text { Peak } \\
\text { discharge } \\
\left(\mathrm{ft}^{3} / \mathrm{s}\right)\end{array}$ & $\begin{array}{l}\text { Water } \\
\text { year }\end{array}$ & Date & $\begin{array}{c}\text { Gage } \\
\text { height } \\
\text { (feet) }\end{array}$ & $\begin{array}{c}\text { Peak } \\
\text { discharge } \\
\left(\mathrm{ft}^{3} / \mathbf{s}\right)\end{array}$ \\
\hline \multicolumn{8}{|c|}{ Annual peak discharge, by year, and corresponding gage height } \\
\hline 1950 & April 21 & 18.39 & 2,790 & 1978 & April 9 & 16.64 & 3,140 \\
\hline 1961 & April 23 & ${ }^{1} 4.34$ & 279 & 1979 & April 20 & 14.63 & 2,140 \\
\hline 1962 & May 24 & ${ }^{1} 8.72$ & 1,490 & 1980 & April 3 & 9.95 & 670 \\
\hline 1963 & June 4 & ${ }^{1} 5.13$ & 355 & 1981 & September 8 & 12.98 & 1,560 \\
\hline 1964 & April 16 & ${ }^{1} 6.07$ & 472 & 1982 & April 16 & 12.04 & 1,320 \\
\hline 1965 & April 12 & 11.12 & 1,780 & 1983 & March 7 & 13.29 & 520 \\
\hline 1966 & April 2 & 12.60 & 2,240 & 1984 & June 9 & 9.13 & 626 \\
\hline 1967 & March 31 & 14.17 & 2,880 & 1985 & August 18 & 15.03 & 2,320 \\
\hline 1968 & July 17 & 7.35 & 551 & 1986 & March 30 & 13.50 & 1,720 \\
\hline 1969 & April 11 & 14.91 & 3,210 & 1987 & July 24 & 9.00 & 661 \\
\hline 1970 & April 25 & 12.76 & 2,300 & 1988 & April 4 & 9.68 & 620 \\
\hline 1971 & April 8 & 12.34 & 1,430 & 1989 & April 6 & 13.57 & 940 \\
\hline 1972 & April 15 & 13.98 & 2,070 & 1990 & June 21 & 5.89 & 134 \\
\hline 1973 & September 4 & 11.27 & 1,030 & 1991 & September 10 & 6.60 & 247 \\
\hline 1974 & August 15 & 12.91 & 2,270 & 1992 & March 9 & 12.33 & 500 \\
\hline 1975 & April 19 & 14.66 & 2,120 & 1993 & March 30 & 12.47 & 1,000 \\
\hline 1976 & March 31 & 11.70 & 920 & 1994 & July 8 & -- & 1,270 \\
\hline 1977 & July 3 & 9.40 & 855 & & & & \\
\hline \multicolumn{8}{|c|}{ Annual peak discharge, from highest to lowest, and corresponding gage height } \\
\hline 1969 & April 11 & 14.91 & 3,210 & 1973 & September 4 & 11.27 & 1,030 \\
\hline 1978 & April 9 & 16.64 & 3,140 & 1993 & March 30 & 12.47 & 1,000 \\
\hline 1967 & March 31 & 14.17 & 2,880 & 1989 & April 6 & 13.57 & 940 \\
\hline 1950 & April 21 & 18.39 & 2,790 & 1976 & March 31 & 11.70 & 920 \\
\hline 1985 & August 18 & 15.03 & 2,320 & 1977 & July 3 & 9.40 & 855 \\
\hline 1970 & April 25 & 12.76 & 2,300 & 1980 & April 3 & 9.95 & 670 \\
\hline 1974 & August 15 & 12.91 & 2,270 & 1987 & July 24 & 9.00 & 661 \\
\hline 1966 & April 2 & 12.60 & 2,240 & 1984 & June 9 & 9.13 & 626 \\
\hline 1979 & April 20 & 14.63 & 2,140 & 1988 & April 4 & 9.68 & 620 \\
\hline 1975 & April 19 & 14.66 & 2,120 & 1968 & July 17 & 7.35 & 551 \\
\hline 1972 & April 15 & 13.98 & 2,070 & 1983 & March 7 & 13.29 & 520 \\
\hline 1965 & April 12 & 11.12 & 1,780 & 1992 & March 9 & 12.33 & 500 \\
\hline 1986 & March 30 & 13.50 & 1,720 & 1964 & April 16 & ${ }^{1} 6.07$ & 472 \\
\hline 1981 & September 8 & 12.98 & 1,560 & 1963 & June 4 & ${ }^{1} 5.13$ & 355 \\
\hline 1962 & May 24 & ${ }^{1} 8.72$ & 1,490 & 1961 & April 23 & ${ }^{1} 4.34$ & 279 \\
\hline 1971 & April 8 & 12.34 & 1,430 & 1991 & September 10 & 6.60 & 247 \\
\hline 1982 & April 16 & 12.04 & 1,320 & 1990 & June 21 & 5.89 & 134 \\
\hline 1994 & July 8 & -- & 1,270 & & & & \\
\hline
\end{tabular}

${ }^{1}$ Gage height recorded using datum 8 feet higher. 


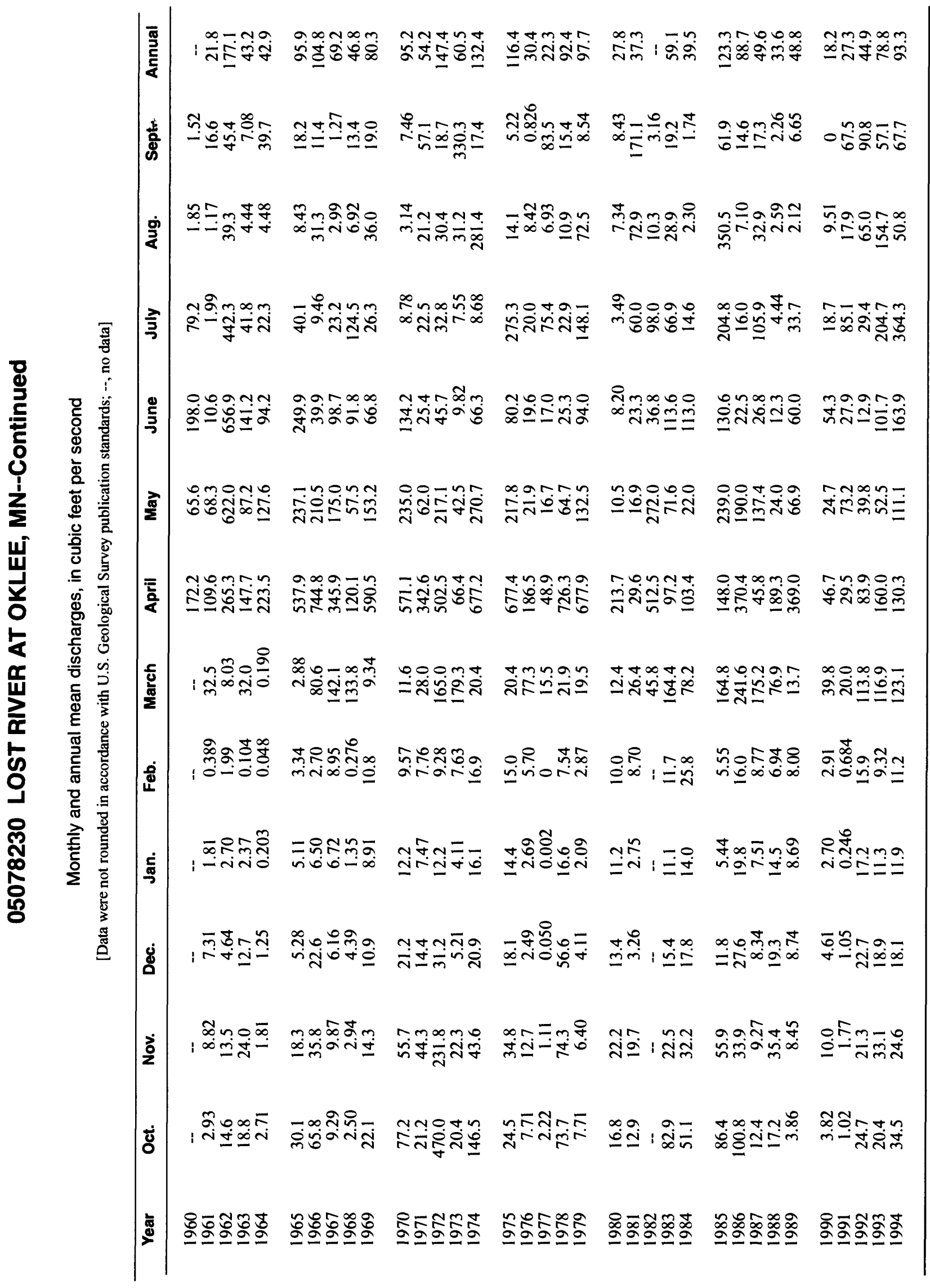




\section{CLEARWATER RIVER AT RED LAKE FALLS, MN}

LOCATION.--Lat 47 $53^{\prime} 15^{\prime \prime}$, long $96^{\circ} 16^{\prime} 25^{\prime \prime}$, in $\mathrm{NW}^{1} /{ }_{4} \mathrm{NE}^{1 / 4}$ sec.22, T.151 N., R.44 W., Red Lake County, Hydrologic Unit 09020305, on left bank $40 \mathrm{ft}$ downstream from Great Northern Railroad bridge in Red Lake Falls, 1.4 mi upstream from mouth, and 3 mi downstream from Badger Creek.

DRAINAGE AREA.--1,370 $\mathrm{mi}^{2}$, approximately.

PERIOD OF RECORD.--June 1909 to September 1917, October 1934 to September 1981, March 1982 to current year. Annual maximum only for October 1918 to September 1919. Monthly discharge only for October, November, 1934, published in WSP 1308. October 1981 to February 1982, operated as a high-flow partial-record station.

GAGE.--Water-stage recorder. Datum of gage is $948.94 \mathrm{ft}$ above sea level (levels by U.S. Army Corps of Engineers). Prior to Sept. 12, 1911, nonrecording gage at site $0.5 \mathrm{mi}$ upstream, and Sept. 12, 1911, to Sept. 30, 1917, nonrecording gage at site $40 \mathrm{ft}$ upstream at different datum.

EXTREMES FOR PERIOD OF RECORD.--Maximum discharge, 10,300 $\mathrm{ft}^{3} / \mathrm{s}$, Apr. 25, 1979, gage height, $12.38 \mathrm{ft}$; maximum gage height, $15.85 \mathrm{ft}$, Mar. 6, 1983, highwater mark, backwater from ice; no flow at times.

Annual mean discharge

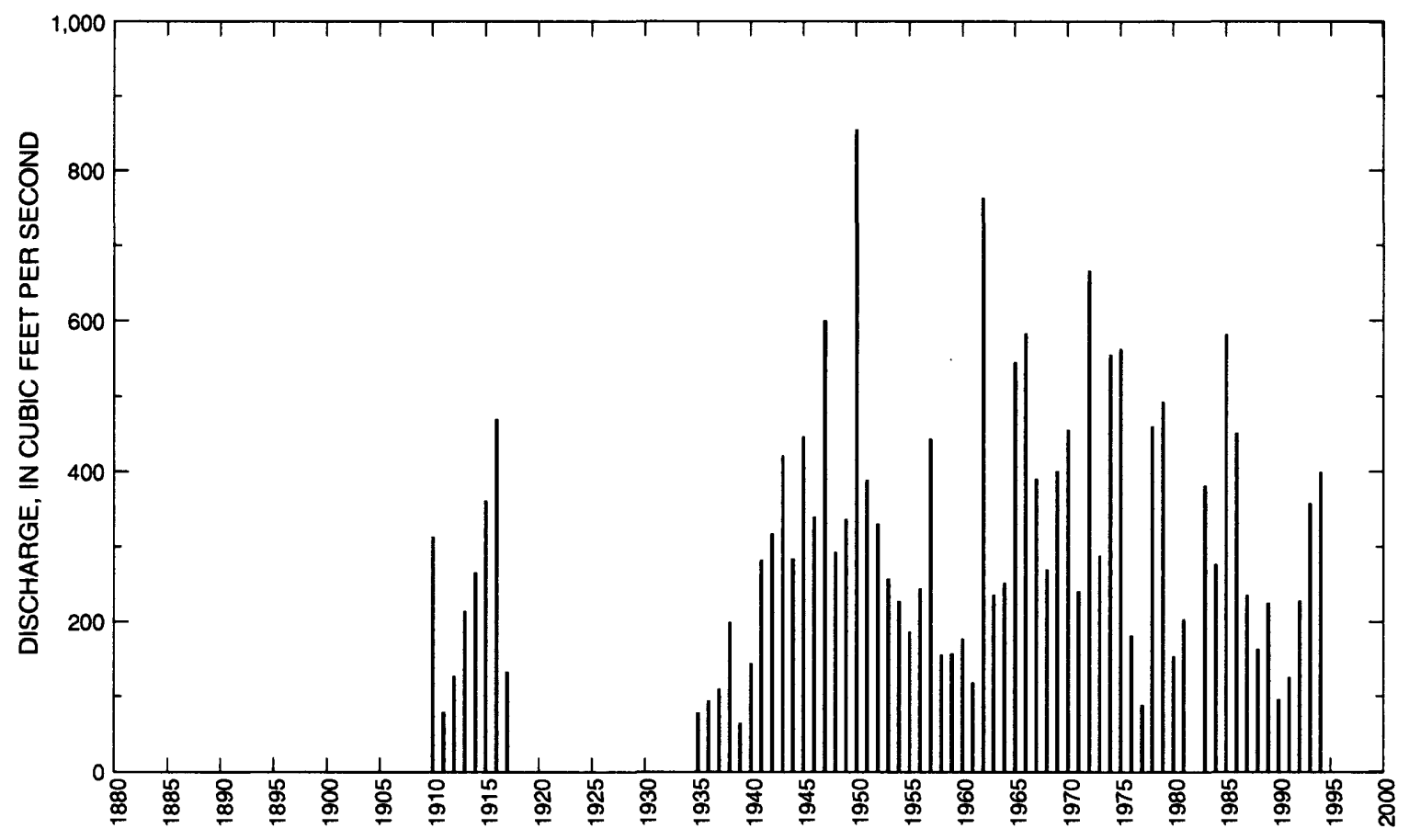


05078500 CLEARWATER RIVER AT RED LAKE FALLS, MN--Continued

Statistics of monthly and annual mean discharges

\begin{tabular}{|c|c|c|c|c|c|c|c|c|}
\hline \multirow[b]{2}{*}{ Month } & \multicolumn{2}{|c|}{ Maximum } & \multicolumn{2}{|c|}{ Minimum } & \multicolumn{4}{|c|}{ Mean } \\
\hline & $\begin{array}{c}\text { Discharge } \\
\left(\mathrm{ft}^{3} / \mathrm{s}\right)\end{array}$ & $\begin{array}{c}\text { Water year } \\
\text { of } \\
\text { occurrence }\end{array}$ & $\begin{array}{c}\text { Discharge } \\
\left(\mathrm{ft}^{3} / \mathrm{s}\right)\end{array}$ & $\begin{array}{c}\text { Water year } \\
\text { of } \\
\text { occurrence }\end{array}$ & $\begin{array}{c}\text { Discharge } \\
\left(\mathrm{ft}^{3} / \mathbf{s}\right)\end{array}$ & $\begin{array}{c}\text { Standard } \\
\text { devlation } \\
\left(\mathrm{ft}^{3} / \mathrm{s}\right)\end{array}$ & $\begin{array}{l}\text { Coeffi- } \\
\text { cient of } \\
\text { variation }\end{array}$ & $\begin{array}{l}\text { Percentage } \\
\text { of annuai } \\
\text { discharge }\end{array}$ \\
\hline October & 1,350 & 1972 & 10.0 & 1935 & 178 & 210 & 1.18 & 4.72 \\
\hline November & 1,230 & 1972 & 19.0 & 1935 & 134 & 155 & 1.15 & 3.56 \\
\hline December & 260 & 1910 & 21.4 & 1937 & 85.6 & 49.1 & 0.57 & 2.27 \\
\hline January & 220 & 1910 & 21.4 & 1940 & 68.0 & 34.7 & 0.51 & 1.80 \\
\hline February & 150 & 1984 & 19.1 & 1937 & 62.8 & 29.3 & 0.47 & 1.67 \\
\hline March & 993 & 1946 & 13.6 & 1937 & 225 & 252 & 1.12 & 5.97 \\
\hline April & 3,460 & 1966 & 61.0 & 1981 & 1,120 & 870 & 0.78 & 29.8 \\
\hline May & 5,060 & 1950 & 32.2 & 1977 & 663 & 723 & 1.09 & 17.6 \\
\hline June & 3,040 & 1962 & 26.5 & 1980 & 481 & 508 & 1.06 & 12.8 \\
\hline July & 1,670 & 1994 & 8.34 & 1936 & 367 & 386 & 1.05 & 9.74 \\
\hline August & 1,690 & 1985 & 1.49 & 1936 & 204 & 279 & 1.36 & 5.42 \\
\hline September & 1,270 & 1973 & 2.92 & 1936 & 179 & 195 & 1.09 & 4.75 \\
\hline Annual & 855 & 1950 & 64.4 & 1939 & 311 & 174 & 0.56 & 100 \\
\hline
\end{tabular}

Annual flow duration

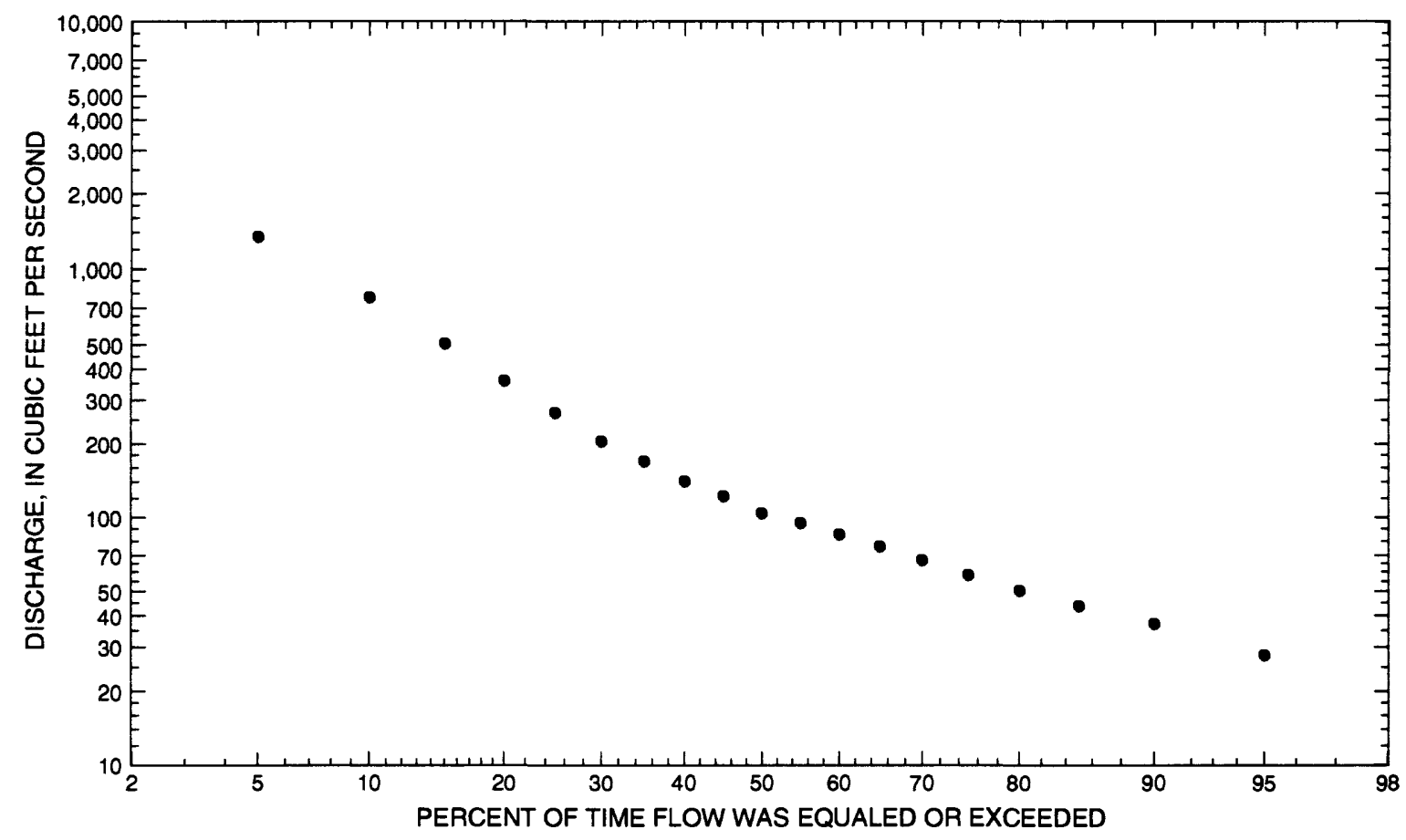




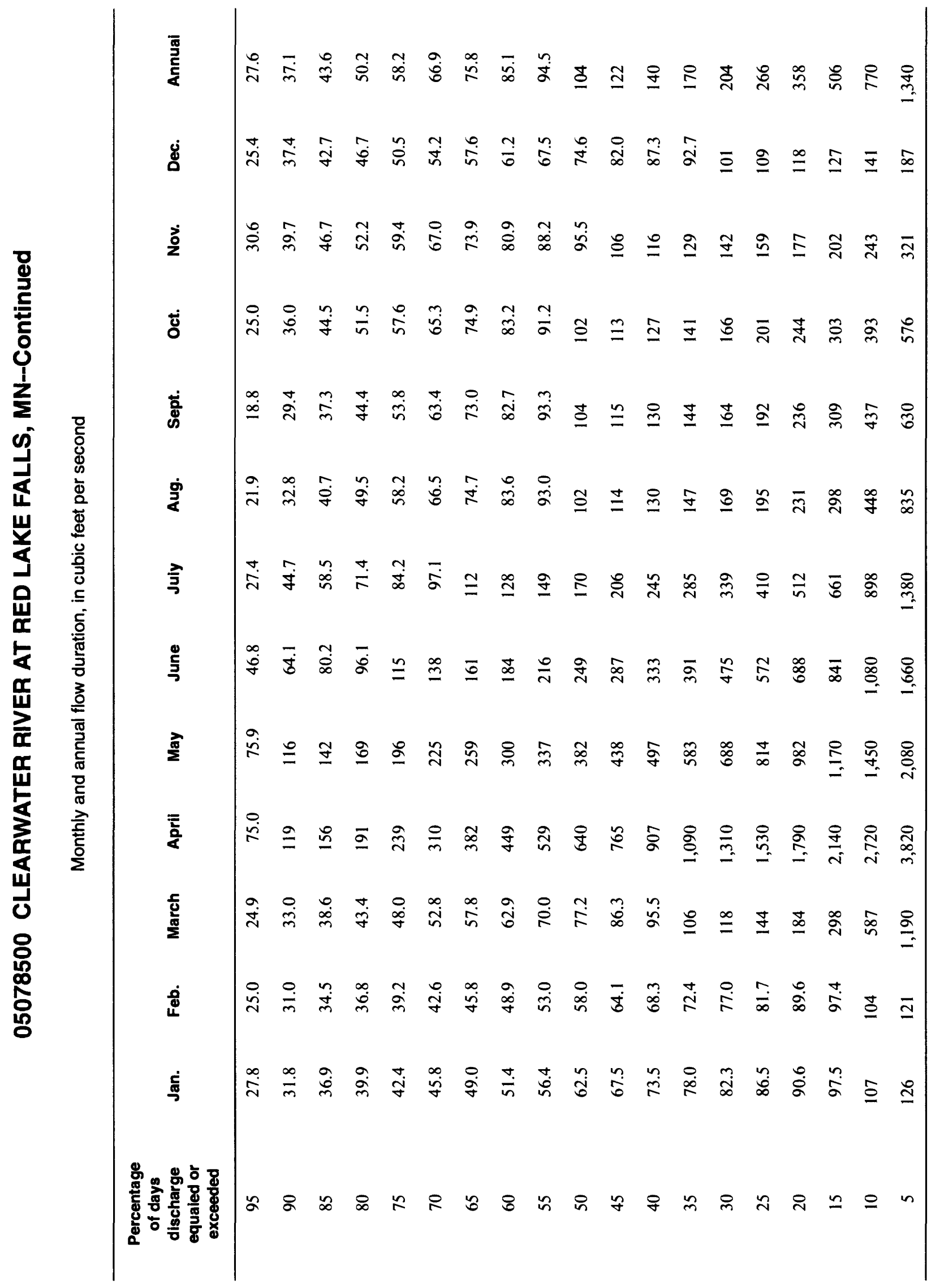


05078500 CLEARWATER RIVER AT RED LAKE FALLS, MN--Continued

Probability of occurrence of annual high discharges

[ng, statistic not given]

\begin{tabular}{|c|c|c|c|c|c|c|}
\hline \multirow[b]{2}{*}{$\begin{array}{l}\text { Exceedance } \\
\text { probability }\end{array}$} & \multirow[b]{2}{*}{$\begin{array}{l}\text { Recurrence } \\
\text { intervai } \\
\text { (years) }\end{array}$} & \multirow[b]{2}{*}{$\begin{array}{c}\text { Maximum } \\
\text { instantaneous } \\
\left(\mathrm{ft}^{3} / \mathbf{s}\right)\end{array}$} & \multicolumn{4}{|c|}{$\begin{array}{l}\text { Maximum mean discharge } \\
\left(\mathrm{ft}^{3} / \mathrm{s}\right)\end{array}$} \\
\hline & & & 3-day period & 7-day period & 15-day period & 30-day period \\
\hline 0.99 & 1.01 & 480 & 416 & 363 & 270 & 197 \\
\hline 0.95 & 1.05 & 860 & 714 & 615 & 472 & 347 \\
\hline 0.90 & 1.11 & 1,160 & 947 & 810 & 629 & 466 \\
\hline 0.80 & 1.25 & 1,640 & 1,330 & 1,130 & 882 & 657 \\
\hline 0.50 & 2 & 3,070 & 2,480 & 2,080 & 1,640 & 1,230 \\
\hline 0.20 & 5 & 5,510 & 4,540 & 3,770 & 2,960 & 2,230 \\
\hline 0.10 & 10 & 7,340 & 6,170 & 5,100 & 3,970 & 3,000 \\
\hline 0.04 & 25 & 9,830 & 8,510 & 7,010 & 5,380 & 4,060 \\
\hline 0.02 & 50 & 11,800 & 10,400 & 8,570 & 6,510 & 4,910 \\
\hline 0.01 & 100 & 13,800 & 12,500 & 10,300 & 7,700 & 5,810 \\
\hline 0.005 & 200 & 15,900 & 14,700 & 12,100 & 8,960 & 6,740 \\
\hline 0.002 & 500 & 18,800 & ng & ng & ng & ng \\
\hline
\end{tabular}

Probability of occurrence of annual low discharges

\begin{tabular}{|c|c|c|c|c|c|c|c|c|c|c|}
\hline \multirow[b]{3}{*}{$\begin{array}{l}\text { Non- } \\
\text { exceed- } \\
\text { ance } \\
\text { prob- } \\
\text { ability }\end{array}$} & \multirow[b]{3}{*}{$\begin{array}{c}\text { Recur- } \\
\text { rence } \\
\text { inter- } \\
\text { vai } \\
\text { (years) }\end{array}$} & \multicolumn{9}{|c|}{ Minimum mean discharge $\left(\mathrm{ft}^{3} / \mathrm{s}\right)$} \\
\hline & & \multicolumn{9}{|c|}{ Number of consecutive days } \\
\hline & & 1 & 3 & 7 & 14 & 30 & 60 & 90 & 120 & 183 \\
\hline 0.05 & 20 & 1.80 & 4.49 & 5.87 & 6.77 & 8.54 & 12.9 & 17.5 & 22.4 & 28.4 \\
\hline 0.10 & 10 & 4.90 & 9.47 & 12.0 & 13.5 & 16.3 & 21.4 & 25.4 & 29.8 & 36.7 \\
\hline 0.20 & 5 & 12.7 & 19.1 & 23.0 & 25.3 & 29.5 & 34.5 & 37.2 & 40.9 & 49.5 \\
\hline 0.50 & 2 & 38.4 & 42.3 & 46.1 & 49.2 & 54.1 & 59.7 & 63.1 & 67.4 & 84.6 \\
\hline
\end{tabular}




\section{CLEARWATER RIVER AT RED LAKE FALLS, MN-Continued}

Probability of occurrence of seasonal low discharges

\begin{tabular}{|c|c|c|c|c|c|c|c|c|c|}
\hline \multirow[b]{3}{*}{$\begin{array}{c}\text { Non- } \\
\text { exceedance } \\
\text { probability }\end{array}$} & \multirow[b]{3}{*}{$\begin{array}{c}\text { Recurrence } \\
\text { interval } \\
\text { (years) }\end{array}$} & \multicolumn{8}{|c|}{ Minimum mean discharge $\left(\mathrm{ft}^{3} / \mathrm{s}\right)$} \\
\hline & & \multicolumn{8}{|c|}{ Number of consecutive days } \\
\hline & & 1 & 7 & 14 & 30 & 1 & 7 & 14 & 30 \\
\hline & & \multicolumn{4}{|c|}{ December-January-February } & \multicolumn{4}{|c|}{ March-April-May } \\
\hline 0.05 & 20 & 8.16 & 17.7 & 19.9 & 22.9 & 10.3 & 18.8 & 20.6 & 25.5 \\
\hline 0.10 & 10 & 13.2 & 22.7 & 24.8 & 27.7 & 16.5 & 24.3 & 26.4 & 34.0 \\
\hline 0.20 & 5 & 21.9 & 30.1 & 31.9 & 34.7 & 26.8 & 32.5 & 35.4 & 48.6 \\
\hline \multirow[t]{2}{*}{0.50} & 2 & 46.7 & 48.9 & 50.1 & 52.8 & 53.9 & 54.9 & 59.9 & 100 \\
\hline & & \multicolumn{4}{|c|}{ June-July-August } & \multicolumn{4}{|c|}{ September-October-November } \\
\hline 0.05 & 20 & 2.84 & 8.16 & 9.81 & 13.4 & 3.59 & 7.24 & 8.62 & 16.2 \\
\hline 0.10 & 10 & 7.43 & 15.0 & 17.4 & 22.9 & 8.93 & 15.5 & 18.1 & 24.8 \\
\hline 0.20 & 5 & 19.0 & 28.1 & 31.8 & 41.0 & 21.4 & 32.0 & 36.7 & ${ }^{1} 41.0$ \\
\hline 0.50 & 2 & 62.0 & 69.0 & 76.9 & 101 & 60.8 & 74.2 & 83.0 & ${ }^{1} 88.0$ \\
\hline
\end{tabular}

${ }^{1}$ Graphical interpretation. 
05078500 CLEARWATER RIVER AT RED LAKE FALLS, MN--Continued

Annual peak discharge and corresponding gage height

$$
[--, \text { no data }]
$$

\begin{tabular}{|c|c|c|c|c|c|c|c|}
\hline $\begin{array}{l}\text { Water } \\
\text { year }\end{array}$ & Date & $\begin{array}{c}\text { Gage } \\
\text { height } \\
\text { (feet) }\end{array}$ & $\begin{array}{c}\text { Peak } \\
\text { discharge } \\
\left(\mathrm{ft}^{3} / \mathrm{s}\right)\end{array}$ & $\begin{array}{c}\text { Water } \\
\text { year }\end{array}$ & Date & $\begin{array}{c}\text { Gage } \\
\text { height } \\
\text { (feet) }\end{array}$ & $\begin{array}{c}\text { Peak } \\
\text { discharge } \\
\left(\mathrm{ft}^{3} / \mathrm{s}\right)\end{array}$ \\
\hline \multicolumn{8}{|c|}{ Annual peak discharge, by year, and corresponding gage height } \\
\hline 1910 & March 22 & 9.50 & 1,960 & 1961 & April 26 & 3.97 & 884 \\
\hline 1911 & June 9 & 7.92 & 793 & 1962 & June 10 & 10.96 & 8,600 \\
\hline 1912 & September 29 & - & 1,110 & 1963 & July 13 & 6.15 & 2,300 \\
\hline 1913 & April 8 & -- & 3,320 & 1964 & April 17 & 6.58 & 3,050 \\
\hline 1914 & June 12 & 4.70 & 1,340 & 1965 & April 13 & 10.86 & 8,680 \\
\hline 1915 & June 29 & 6.91 & 3,320 & 1966 & April 3 & 11.02 & 8,920 \\
\hline 1916 & April 15 & -- & 3,990 & 1967 & March 31 & 9.32 & 5,820 \\
\hline 1917 & April 11 & - & 1,250 & 1968 & July 18 & 7.30 & 3,550 \\
\hline 1919 & -- & - & 6,700 & 1969 & April 12 & 11.82 & 9,740 \\
\hline 1935 & March 27 & 5.02 & 696 & 1970 & April 26 & 9.32 & 5,630 \\
\hline 1936 & April 17 & -- & 1,260 & 1971 & April 10 & 7.58 & 3,500 \\
\hline 1937 & May 1 & - & 1,010 & 1972 & April 16 & 9.95 & 6,720 \\
\hline 1938 & May 14 & 5.95 & 2,220 & 1973 & March 15 & 7.42 & 2,900 \\
\hline 1939 & April 20 & 3.80 & 830 & 1974 & April 22 & 10.08 & 6,920 \\
\hline 1940 & April 15 & 11.43 & 3,100 & 1975 & April 19 & 10.34 & 7,310 \\
\hline 1941 & June 8 & - & 3,290 & 1976 & April 3 & 6.84 & 3,120 \\
\hline 1942 & March 27 & 10.04 & 2,200 & 1977 & July 5 & 4.13 & 866 \\
\hline 1943 & April 7 & - & 2,780 & 1978 & April 9 & 11.56 & 9,890 \\
\hline 1944 & August 10 & 7.42 & 3,210 & 1979 & April 25 & 12.38 & 10,300 \\
\hline 1945 & March 27 & -- & 2,680 & 1980 & April 8 & 5.59 & 1,910 \\
\hline 1946 & March 22 & -- & 3,380 & 1981 & September 8 & 6.97 & 3,160 \\
\hline 1947 & April 15 & -- & 5,430 & 1982 & April 15 & 7.76 & 4,160 \\
\hline 1948 & April 7 & - & 3,000 & 1983 & June 13 & 7.05 & 3,190 \\
\hline 1949 & June 2 & 7.30 & 3,360 & 1984 & June 9 & 8.85 & 5,450 \\
\hline 1950 & May 6 & 11.28 & 9,310 & 1985 & August 19 & 9.86 & 7,120 \\
\hline 1951 & April 10 & 6.52 & 2,880 & 1986 & April 30 & 7.39 & 3,720 \\
\hline 1952 & April 16 & 6.17 & 2,550 & 1987 & May 27 & 5.93 & 2,170 \\
\hline 1953 & July 5 & 4.44 & 1,120 & 1988 & April 7 & 6.05 & 2,270 \\
\hline 1954 & April 14 & 6.16 & 2,540 & 1989 & April 7 & 7.62 & 2,550 \\
\hline 1955 & April 8 & 7.25 & 3,660 & 1990 & June 22 & 3.77 & 652 \\
\hline 1956 & April 21 & 8.67 & 5,560 & 1991 & September 16 & 4.45 & 1,040 \\
\hline 1957 & June 28 & 9.98 & 6,840 & 1992 & August 25 & -- & 1,640 \\
\hline 1958 & July 7 & 4.65 & 1,320 & 1993 & March 30 & 9.88 & 3,200 \\
\hline 1959 & April 4 & 6.30 & 1,960 & 1994 & July 8 & 8.99 & 5,350 \\
\hline 1960 & April 6 & 8.47 & 4,010 & & & & \\
\hline \multicolumn{8}{|c|}{ Annual peak discharge, from highest to lowest, and corresponding gage height } \\
\hline 1979 & April 25 & 12.38 & 10,300 & 1975 & April 19 & 10.34 & 7,310 \\
\hline 1978 & April 9 & 11.56 & 9,890 & 1985 & August 19 & 9.86 & 7,120 \\
\hline 1969 & April 12 & 11.82 & 9,740 & 1974 & April 22 & 10.08 & 6,920 \\
\hline 1950 & May 6 & 11.28 & 9,310 & 1957 & June 28 & 9.98 & 6,840 \\
\hline 1966 & April 3 & 11.02 & 8,920 & 1919 & & -- & 6,700 \\
\hline 1965 & April 13 & 10.86 & 8,680 & 1967 & March 31 & 9.32 & 5,820 \\
\hline 1962 & June 10 & 10.96 & 8,600 & 1970 & April 26 & 9.32 & 5,630 \\
\hline
\end{tabular}


05078500 CLEARWATER RIVER AT RED LAKE FALLS, MN--Continued

Annual peak discharge and corresponding gage height-Continued

$$
[--, \text { no data }]
$$

\begin{tabular}{|c|c|c|c|c|c|c|c|}
\hline $\begin{array}{c}\text { Water } \\
\text { year }\end{array}$ & Date & $\begin{array}{c}\text { Gage } \\
\text { height } \\
\text { (feet) }\end{array}$ & $\begin{array}{c}\text { Peak } \\
\text { discharge } \\
\left(\mathrm{ft}^{3} / \mathrm{s}\right)\end{array}$ & $\begin{array}{c}\text { Water } \\
\text { year }\end{array}$ & Date & $\begin{array}{c}\text { Gage } \\
\text { helght } \\
\text { (feet) }\end{array}$ & $\begin{array}{c}\text { Peak } \\
\text { discharge } \\
\left(\mathrm{ft}^{3} / \mathrm{s}\right)\end{array}$ \\
\hline \multicolumn{8}{|c|}{ Annual peak discharge, from highest to lowest, and corresponding gage height--Continued } \\
\hline 1956 & April 21 & 8.67 & 5,560 & 1945 & March 27 & -- & 2,680 \\
\hline 1984 & June 9 & 8.85 & 5,450 & 1952 & April 16 & 6.17 & 2,550 \\
\hline 1947 & April 15 & -- & 5,430 & 1989 & April 7 & 7.62 & 2,550 \\
\hline 1994 & July 8 & 8.99 & 5,350 & 1954 & April 14 & 6.16 & 2,540 \\
\hline 1982 & April 15 & 7.76 & 4,160 & 1963 & July 13 & 6.15 & 2,300 \\
\hline 1960 & April 6 & 8.47 & 4,010 & 1988 & April 7 & 6.05 & 2,270 \\
\hline 1916 & April 15 & - & 3,990 & 1938 & May 14 & 5.95 & 2,220 \\
\hline 1986 & April 30 & 7.39 & 3,720 & 1942 & March 27 & 10.04 & 2,200 \\
\hline 1955 & April 8 & 7.25 & 3,660 & 1987 & May 27 & 5.93 & 2,170 \\
\hline 1968 & July 18 & 7.30 & 3,550 & 1910 & March 22 & 9.50 & 1,960 \\
\hline 1971 & April 10 & 7.58 & 3,500 & 1959 & April 4 & 6.30 & 1,960 \\
\hline 1946 & March 22 & -- & 3,380 & 1992 & August 25 & -- & 1,640 \\
\hline 1949 & June 2 & 7.30 & 3,360 & 1914 & June 12 & 4.70 & 1,340 \\
\hline 1913 & April 8 & - & 3,320 & 1958 & July 7 & 4.65 & 1,320 \\
\hline 1915 & June 29 & 6.91 & 3,320 & 1936 & April 17 & -- & 1,260 \\
\hline 1941 & June 8 & -- & 3,290 & 1917 & April 11 & -- & 1,250 \\
\hline 1944 & August 10 & 7.42 & 3,210 & 1953 & July 5 & 4.44 & 1,120 \\
\hline 1993 & March 30 & 9.88 & 3,200 & 1912 & September 29 & -- & 1,110 \\
\hline 1983 & June 13 & 7.05 & 3,190 & 1991 & September 16 & 4.45 & 1,040 \\
\hline 1981 & September 8 & 6.97 & 3,160 & 1937 & May 1 & -- & 1,010 \\
\hline 1976 & April 3 & 6.84 & 3,120 & 1961 & April 26 & 3.97 & 884 \\
\hline 1940 & April 15 & 11.43 & 3,100 & 1977 & July 5 & 4.13 & 866 \\
\hline 1964 & April 17 & 6.58 & 3,050 & 1939 & April 20 & 3.80 & 830 \\
\hline 1948 & April 7 & -- & 3,000 & 1911 & June 9 & 7.92 & 793 \\
\hline 1973 & March 15 & 7.42 & 2,900 & 1935 & March 27 & 5.02 & 696 \\
\hline 1951 & April 10 & 6.52 & 2,880 & 1990 & June 22 & 3.77 & 652 \\
\hline 1943 & April 7 & -- & 2,780 & & & & \\
\hline
\end{tabular}




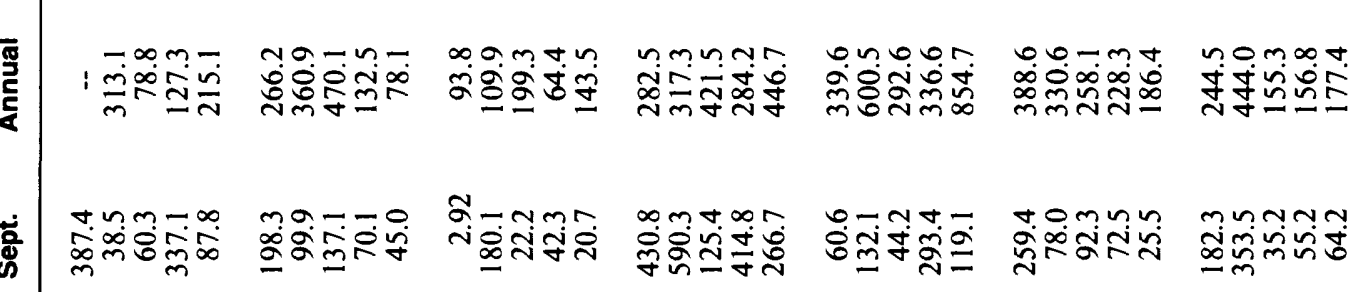

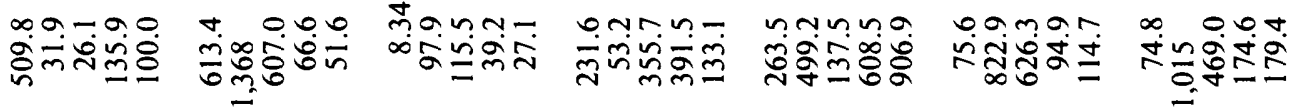

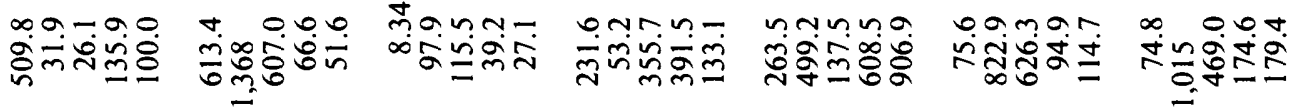

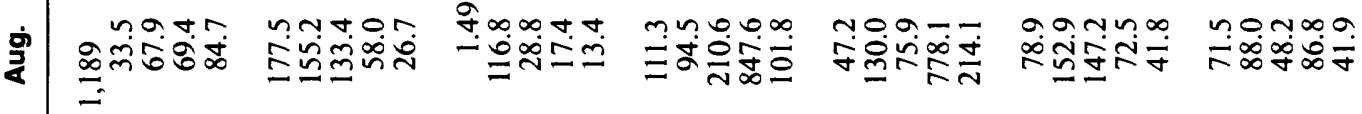

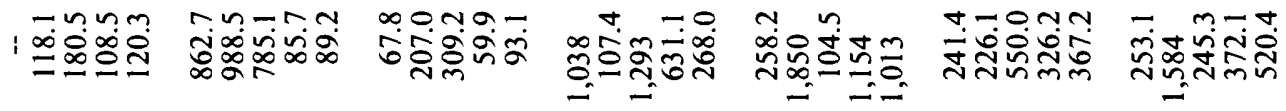

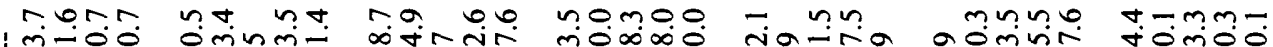

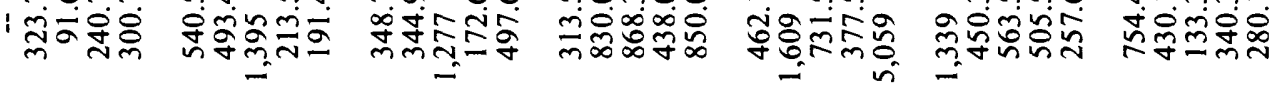

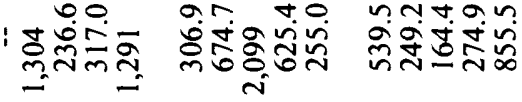

ذِํำ

$\cos$

(2)

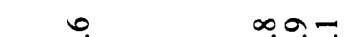

o으 nont momo

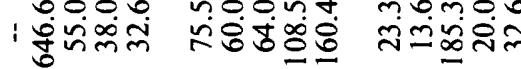

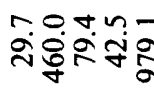

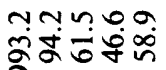

ㄱำษ

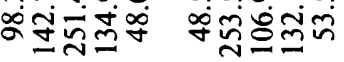

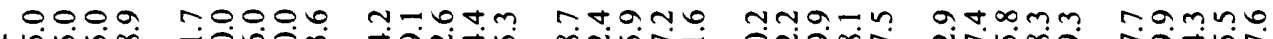

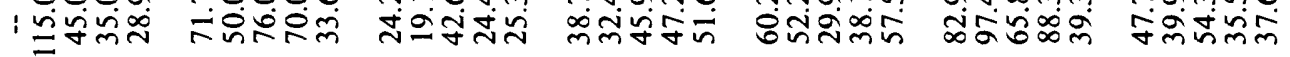
i 워ํํำ

눙ํำ

$\min \infty \mathrm{n}$

$\infty, 0 \infty m$

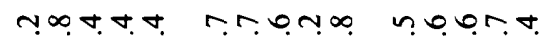
nูmone

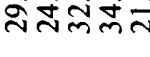

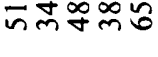

œ

कํำำ

वेंद्य

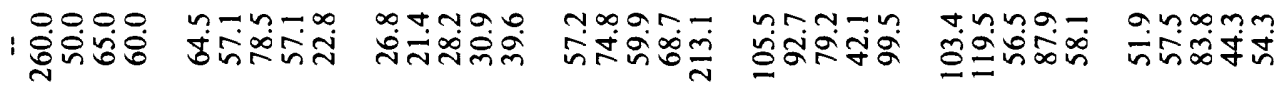

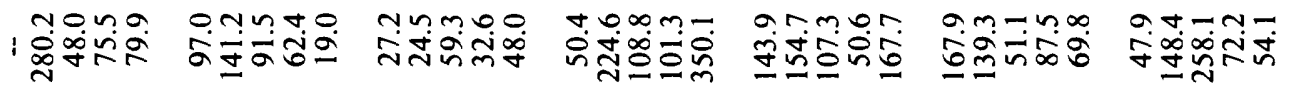

:

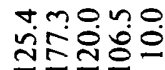

$\infty 0090$

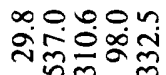

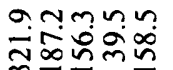

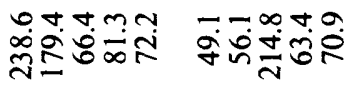

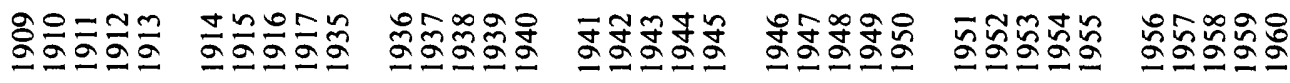




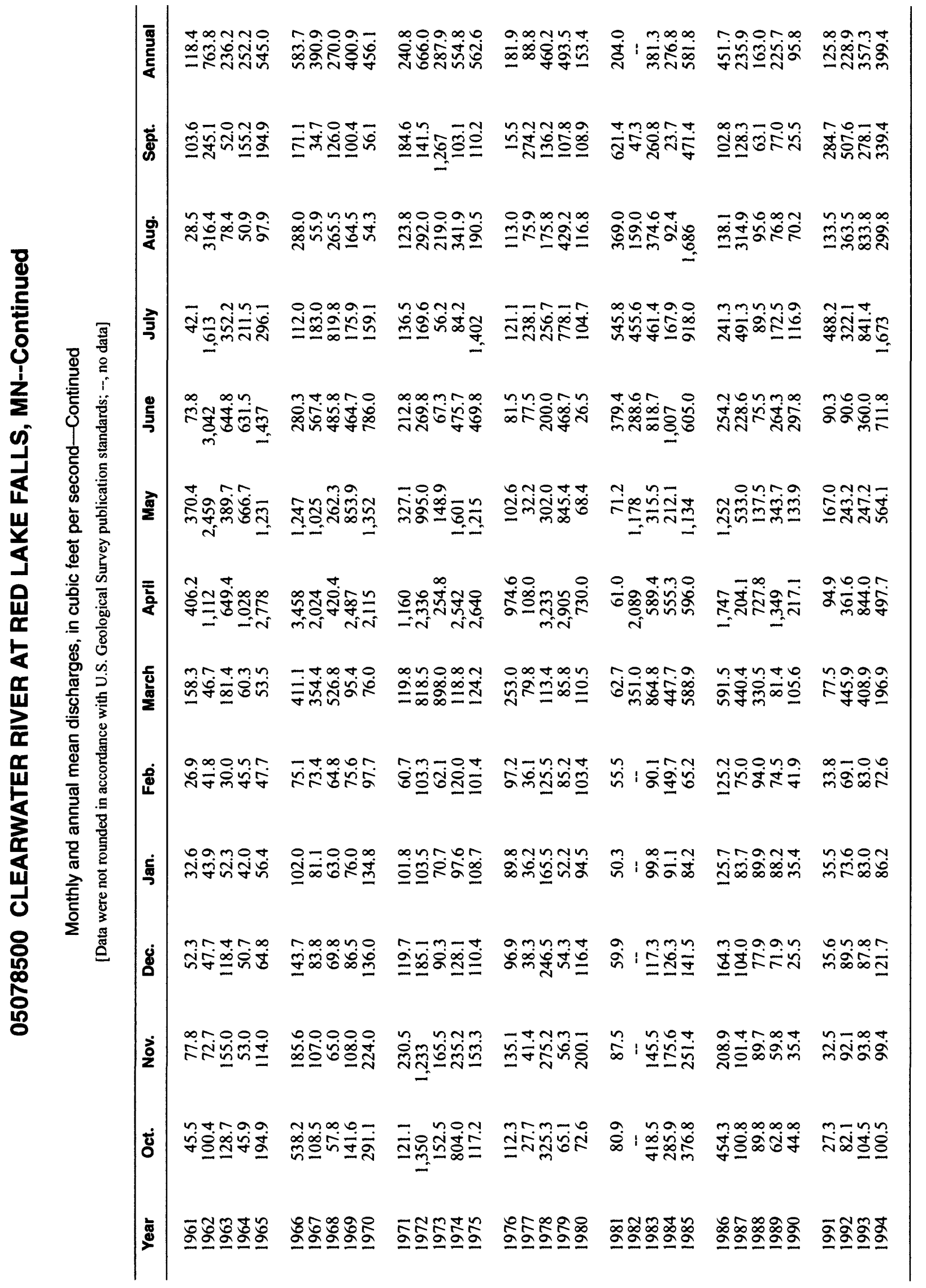




\section{RED LAKE RIVER AT CROOKSTON, MN}

LOCATION.--Lat 4746'32”, long 96³6'33”, in SW $1 / 4 \mathrm{SW}^{1} / 4$ sec.30, T.150 N., R.46 W., Polk County, Hydrologic Unit 09020303, on right bank $100 \mathrm{ft}$ upstream from Sargent Street bridge in Crookston, $0.3 \mathrm{mi}$ downstream from Interstate Power Co.'s dam, $0.6 \mathrm{mi}$ downstream from bridge on U.S.

Highway 75, and $53 \mathrm{mi}$ upstream from mouth.

DRAINAGE AREA.--5,280 $\mathrm{mi}^{2}$, approximately.

PERIOD OF RECORD.--May 1901 to current year. Monthly discharge only for some periods, published in WSP 1308. Figures of daily discharge for Apr. 3-30, 1904, published in WSP 130, have been found unreliable and should not be used.

GAGE.--Water-stage recorder. Datum of gage is $832.72 \mathrm{ft}$ above sea level. May 18,1901 , to June 30 , 1909, nonrecording gage at bridge $300 \mathrm{ft}$ upstream at same datum. July 1, 1909, to Sept. 25, 1911, nonrecording gage, Sept. 26, 1911, to Sept. 30, 1919, water-stage recorder, Oct. 1, 1919, to

Sept. 30, 1930, nonrecording gage, at present site and datum.

EXTREMES FOR PERIOD OF RECORD.--Maximum discharge, 28,400 $\mathrm{ft}^{3} / \mathrm{s}$, Apr. 12, 1969, gage height, $27.33 \mathrm{ft}$; minimum daily discharge, $0.0 \mathrm{ft}^{3} / \mathrm{s}$, July 13, 1960, caused by regulation of powerplant upstream.

EXTREMES OUTSIDE PERIOD OF RECORD.--Flood of April 1897 reached a stage of 25.20 on April 11, discharge 18,900 $\mathrm{ft}^{3} / \mathrm{s}$.

Annual mean discharge

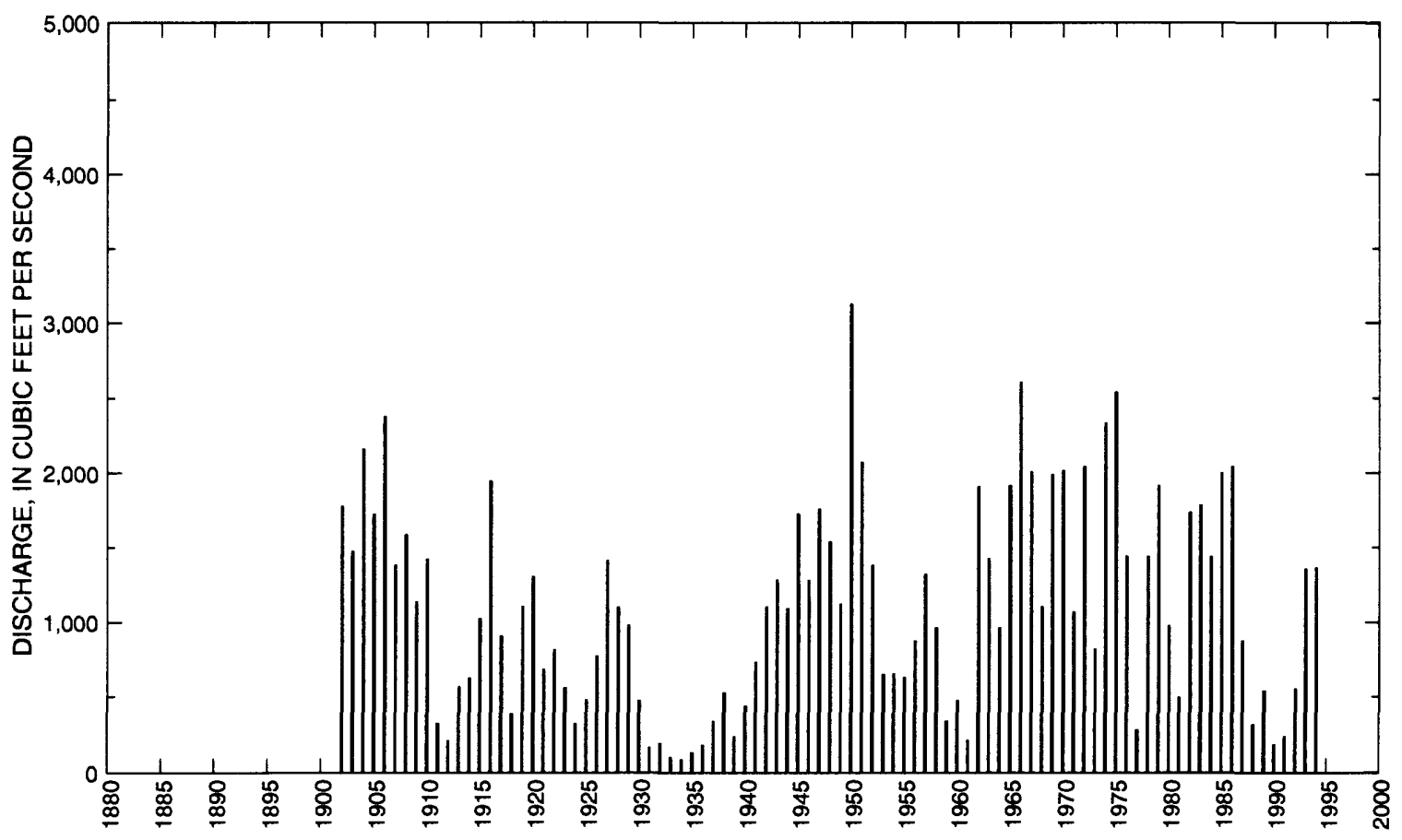


05079000 RED LAKE RIVER AT CROOKSTON, MN--Continued

Statistics of monthly and annual mean discharges

\begin{tabular}{|c|c|c|c|c|c|c|c|c|}
\hline \multirow[b]{2}{*}{ Month } & \multicolumn{2}{|c|}{ Maximum } & \multicolumn{2}{|c|}{ Minimum } & \multicolumn{4}{|c|}{ Mean } \\
\hline & $\begin{array}{c}\text { Discharge } \\
\left(\mathrm{ft}^{3} / \mathrm{s}\right)\end{array}$ & $\begin{array}{c}\text { Water year } \\
\text { of } \\
\text { occurrence }\end{array}$ & $\begin{array}{c}\text { Discharge } \\
\left(\mathrm{ft}^{3} / \mathbf{s}\right)\end{array}$ & $\begin{array}{l}\text { Water year } \\
\quad \text { of } \\
\text { occurrence }\end{array}$ & $\begin{array}{c}\text { Discharge } \\
\left(\mathrm{ft}^{3} / \mathrm{s}\right)\end{array}$ & $\begin{array}{l}\text { Standard } \\
\text { deviation } \\
\left(\mathrm{ft}^{3} / \mathrm{s}\right)\end{array}$ & $\begin{array}{l}\text { Coeffi- } \\
\text { cient of } \\
\text { variation }\end{array}$ & $\begin{array}{l}\text { Percentage } \\
\text { of annual } \\
\text { discharge }\end{array}$ \\
\hline October & 2,840 & 1972 & 8.02 & 1937 & 805 & 689 & 0.86 & 6.00 \\
\hline November & 3,170 & 1972 & 10.1 & 1937 & 660 & 548 & 0.83 & 4.92 \\
\hline December & 1,900 & 1904 & 5.34 & 1937 & 547 & 425 & 0.78 & 4.08 \\
\hline January & 1,500 & 1906 & 15.6 & 1934 & 494 & 385 & 0.78 & 3.68 \\
\hline February & 1,460 & 1951 & 17.8 & 1937 & 470 & 357 & 0.76 & 3.50 \\
\hline March & 3,630 & 1910 & 24.9 & 1936 & 938 & 854 & 0.91 & 6.99 \\
\hline April & 10,300 & 1966 & 232 & 1981 & 2,920 & 2,270 & 0.78 & 21.8 \\
\hline May & 15,300 & 1950 & 154 & 1934 & 2,030 & 2,050 & 1.01 & 15.1 \\
\hline June & 7,200 & 1962 & 80.4 & 1934 & 1,640 & 1,300 & 0.79 & 12.2 \\
\hline July & 6,850 & 1975 & 26.2 & 1936 & 1,290 & 1,240 & 0.96 & 9.58 \\
\hline August & 3,870 & 1985 & 12.3 & 1934 & 815 & 743 & 0.91 & 6.07 \\
\hline September & 3,010 & 1905 & 8.87 & 1934 & 810 & 649 & 0.80 & 6.03 \\
\hline Annual & 3,130 & 1950 & 132 & 1935 & 1,110 & 695 & 0.62 & 100 \\
\hline
\end{tabular}

Annual flow duration

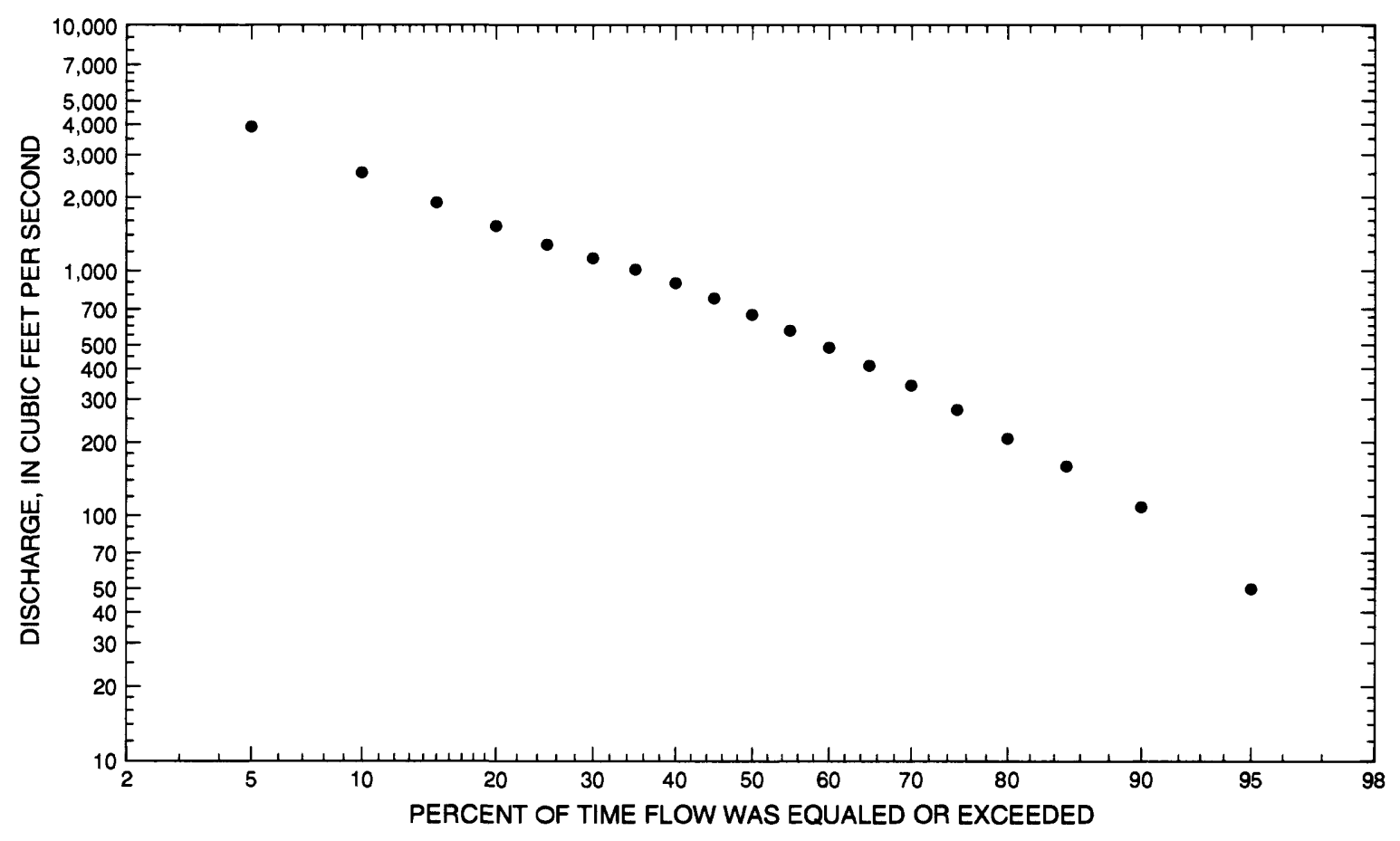




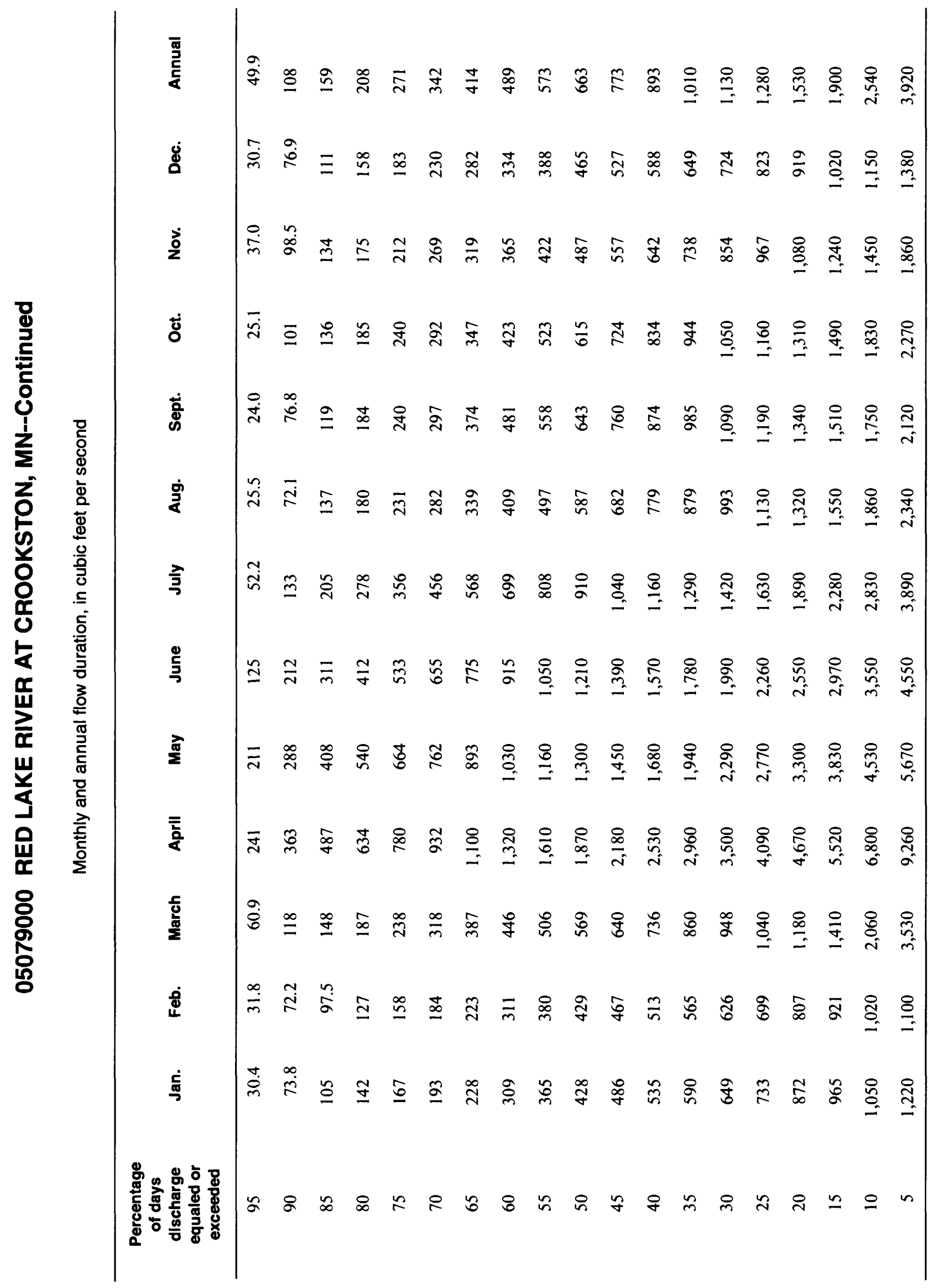


05079000 RED LAKE RIVER AT CROOKSTON, MN--Continued

Probability of occurrence of annual high discharges

[ng, statistic not given]

\begin{tabular}{|c|c|c|c|c|c|c|}
\hline \multirow[b]{2}{*}{$\begin{array}{l}\text { Exceedance } \\
\text { probability }\end{array}$} & \multirow[b]{2}{*}{$\begin{array}{l}\text { Recurrence } \\
\text { interval } \\
\text { (years) }\end{array}$} & \multirow[b]{2}{*}{$\begin{array}{c}\text { Maximum } \\
\text { instantaneous } \\
\left(\mathrm{ft}^{3} / \mathrm{s}\right)^{1}\end{array}$} & \multicolumn{4}{|c|}{$\begin{array}{l}\text { Maximum mean discharge } \\
\qquad\left(\mathrm{ft}^{3} / \mathrm{s}\right)\end{array}$} \\
\hline & & & 3-day period & 7-day perlod & 15-day period & 30-day period \\
\hline 0.99 & 1.01 & 870 & 647 & 528 & 395 & 319 \\
\hline 0.95 & 1.05 & 1,770 & 1,380 & 1,150 & 878 & 690 \\
\hline 0.90 & 1.11 & 2,510 & 2,000 & 1,670 & 1,290 & 1,010 \\
\hline 0.80 & 1.25 & 3,730 & 3,030 & 2,550 & 2,000 & 1,540 \\
\hline 0.50 & 2 & 7,360 & 6,140 & 5,210 & 4,140 & 3,190 \\
\hline 0.20 & 5 & 13,100 & 11,100 & 9,430 & 7,560 & 5,890 \\
\hline 0.10 & 10 & 17,100 & 14,500 & 12,300 & 9,870 & 7,780 \\
\hline 0.04 & 25 & 22,100 & 18,700 & 15,800 & 12,700 & 10,200 \\
\hline 0.02 & 50 & 25,700 & 21,700 & 18,300 & 14,700 & 11,900 \\
\hline 0.01 & 100 & 29,100 & 24,600 & 20,700 & 16,600 & 13,500 \\
\hline 0.005 & 200 & 32,400 & 27,300 & 22,900 & 18,300 & 15,100 \\
\hline 0.002 & 500 & 36,700 & $\mathrm{ng}$ & ng & ng & ng \\
\hline
\end{tabular}

${ }^{1}$ Historic adjustment applied.

Probability of occurrence of annual low discharges

\begin{tabular}{|c|c|c|c|c|c|c|c|c|c|c|}
\hline \multirow[b]{3}{*}{$\begin{array}{l}\text { Non- } \\
\text { exceed- } \\
\text { ance } \\
\text { prob- } \\
\text { ability }\end{array}$} & \multirow[b]{3}{*}{$\begin{array}{c}\text { Recur- } \\
\text { rence } \\
\text { inter- } \\
\text { val } \\
\text { (years) }\end{array}$} & \multicolumn{9}{|c|}{ Minimum mean discharge $\left(\mathrm{ft}^{3} / \mathrm{s}\right)$} \\
\hline & & \multicolumn{9}{|c|}{ Number of consecutive days } \\
\hline & & 1 & 3 & 7 & 14 & 30 & 60 & 90 & 120 & 183 \\
\hline 0.05 & 20 & 12.4 & 15.1 & 17.2 & 19.5 & 23.8 & 28.2 & 31.8 & 36.1 & 43.8 \\
\hline 0.10 & 10 & 24.7 & 29.8 & 33.9 & 38.2 & 45.6 & 53.8 & 60.8 & 68.5 & 82.2 \\
\hline 0.20 & 5 & 52.1 & 62.5 & 70.7 & 78.9 & 92.2 & 108 & 122 & 136 & 161 \\
\hline 0.50 & 2 & 171 & 198 & 224 & 246 & 277 & 317 & 354 & 387 & 456 \\
\hline
\end{tabular}




\section{RED LAKE RIVER AT CROOKSTON, MN--Continued}

Probability of occurrence of seasonal low discharges

\begin{tabular}{|c|c|c|c|c|c|c|c|c|c|}
\hline \multirow[b]{3}{*}{$\begin{array}{c}\text { Non- } \\
\text { exceedance } \\
\text { probability }\end{array}$} & \multirow[b]{3}{*}{$\begin{array}{l}\text { Recurrence } \\
\text { interval } \\
\text { (years) }\end{array}$} & \multicolumn{8}{|c|}{ Minimum mean discharge $\left(\mathrm{ft}^{3} / \mathrm{s}\right)$} \\
\hline & & \multicolumn{8}{|c|}{ Number of consecutive days } \\
\hline & & 1 & 7 & 14 & 30 & 1 & 7 & 14 & 30 \\
\hline & & \multicolumn{4}{|c|}{ December-January-February } & \multicolumn{4}{|c|}{ March-April-May } \\
\hline 0.05 & 20 & 23.6 & 25.7 & 27.6 & 30.7 & 34.2 & 46.7 & 52.4 & 84.6 \\
\hline 0.10 & 10 & 46.0 & 50.8 & 53.7 & 58.8 & 61.1 & 79.9 & 89.2 & 139 \\
\hline 0.20 & 5 & 94.7 & 105 & 110 & 118 & 116 & 144 & 160 & 239 \\
\hline \multirow[t]{2}{*}{0.50} & 2 & 291 & 323 & 332 & 350 & 330 & 374 & 409 & 579 \\
\hline & & \multicolumn{4}{|c|}{ June-July-August } & \multicolumn{4}{|c|}{ September-October-November } \\
\hline 0.05 & 20 & 18.3 & 26.1 & 31.5 & 41.4 & 16.7 & 23.2 & 26.0 & 30.9 \\
\hline 0.10 & 10 & 38.0 & 52.4 & 62.9 & 80.0 & 32.9 & 47.1 & 53.3 & 63.6 \\
\hline 0.20 & 5 & 84.2 & 112 & 133 & 163 & 68.6 & 100 & 114 & 137 \\
\hline 0.50 & 2 & 300 & 369 & 424 & 501 & 219 & 317 & 364 & 440 \\
\hline
\end{tabular}


05079000 RED LAKE RIVER AT CROOKSTON, MN--Continued

Annual peak discharge and corresponding gage height

$[--$, no data $]$

\begin{tabular}{|c|c|c|c|c|c|c|c|}
\hline $\begin{array}{l}\text { Water } \\
\text { year }\end{array}$ & Date & $\begin{array}{c}\text { Gage } \\
\text { height } \\
\text { (feet) }\end{array}$ & $\begin{array}{c}\text { Peak } \\
\text { discharge } \\
\left(\mathrm{ft}^{3} / \mathrm{s}\right)\end{array}$ & $\begin{array}{l}\text { Water } \\
\text { year }\end{array}$ & Date & $\begin{array}{c}\text { Gage } \\
\text { height } \\
\text { (feet) }\end{array}$ & $\begin{array}{c}\text { Peak } \\
\text { discharge } \\
\left(\mathrm{ft}^{3} / \mathrm{s}\right)\end{array}$ \\
\hline \multicolumn{8}{|c|}{ Annual peak discharge, by year, and corresponding gage height } \\
\hline${ }^{1} 1897$ & April 11 & 25.20 & 18,900 & 1947 & June 12 & 18.08 & 12,400 \\
\hline 1902 & May 21 & 10.00 & 5,170 & 1948 & April 19 & -- & 9,520 \\
\hline 1904 & April 24 & 20.42 & 13,700 & 1949 & June 2 & 17.43 & 10,700 \\
\hline 1905 & May 13 & 14.50 & 8,730 & 1950 & May 7 & 25.70 & 27,400 \\
\hline 1906 & April 15 & 21.00 & 14,600 & 1951 & April 7 & 19.00 & 12,600 \\
\hline 1907 & April 4 & 12.04 & 6,330 & 1952 & April 11 & 12.65 & 6,320 \\
\hline 1908 & April 10 & 17.00 & 10,700 & 1953 & March 24 & 8.01 & 2,560 \\
\hline 1909 & July 21 & 8.77 & 3,680 & 1954 & April 12 & 11.37 & 5,330 \\
\hline 1910 & March 20 & 14.20 & 7,920 & 1955 & April 8 & 18.30 & 12,400 \\
\hline 1911 & June 10 & 8.45 & 3,620 & 1956 & April 20 & 19.78 & 14,000 \\
\hline 1912 & September 29 & -- & 2,120 & 1957 & June 29 & 18.10 & 11,800 \\
\hline 1913 & April 8 & - & 7,170 & 1958 & July 7 & 8.62 & 3,370 \\
\hline 1914 & June 12 & 7.40 & 2,630 & 1959 & April 5 & 11.72 & 5,630 \\
\hline 1915 & June 29 & 14.25 & 7,860 & 1960 & April 6 & 12.56 & 5,520 \\
\hline 1916 & April 17 & 21.80 & 15,900 & 1961 & March 27 & 5.67 & 1,450 \\
\hline 1917 & April 11 & -- & 5,480 & 1962 & June 11 & 21.90 & 16,700 \\
\hline 1918 & April 2 & 6.50 & 1,950 & 1963 & April 9 & 13.25 & 6,820 \\
\hline 1919 & July 5 & 21.10 & 14,900 & 1964 & June 20 & 11.74 & 5,550 \\
\hline 1920 & March 25 & 23.30 & 9,520 & 1965 & April 14 & 23.51 & 19,400 \\
\hline 1922 & May 13 & 13.00 & 6,910 & 1966 & April 3 & 24.41 & 21,500 \\
\hline 1923 & April 20 & -- & 5,820 & 1967 & April 1 & 23.49 & 19,300 \\
\hline 1924 & April 23 & 5.20 & 1,140 & 1968 & July 19 & 17.17 & 11,100 \\
\hline 1925 & June 9 & 13.50 & 7,300 & 1969 & April 12 & 27.33 & 28,400 \\
\hline 1926 & March 24 & 12.30 & 6,500 & 1970 & April 26 & 19.05 & 13,300 \\
\hline 1927 & April 13 & 14.00 & 7,700 & 1971 & April 10 & 20.74 & 15,300 \\
\hline 1928 & April 8 & -- & 3,910 & 1972 & April 16 & 20.28 & 14,700 \\
\hline 1929 & March 19 & 14.90 & 7,620 & 1973 & September 26 & 10.86 & 4,960 \\
\hline 1930 & May 13 & 10.30 & 4,770 & 1974 & April 23 & 22.89 & 16,400 \\
\hline 1931 & March 26 & 4.93 & 1,030 & 1975 & April 18 & 21.97 & 15,600 \\
\hline 1932 & April 9 & 9.78 & 4,390 & 1976 & April 3 & 19.45 & 12,500 \\
\hline 1933 & April 2 & 5.92 & 1,440 & 1977 & May 20 & 8.66 & 3,440 \\
\hline 1934 & April 8 & 6.89 & 1,490 & 1978 & April 7 & 23.11 & 18,100 \\
\hline 1935 & March 27 & 8.38 & 2,490 & 1979 & April 26 & 24.99 & 21,900 \\
\hline 1936 & April 18 & 11.33 & 4,540 & 1980 & April 9 & 12.31 & 6,600 \\
\hline 1937 & August 4 & 10.25 & 3,750 & 1981 & June 29 & 13.56 & 7,120 \\
\hline 1938 & May 10 & 12.62 & 5,910 & 1982 & April 17 & 16.12 & 9,320 \\
\hline 1939 & April 24 & 8.92 & 3,050 & 1983 & June 23 & 13.98 & 7,330 \\
\hline 1940 & April 16 & 14.73 & 6,000 & 1984 & June 10 & 20.71 & 14,400 \\
\hline 1941 & June 9 & -- & 6,190 & 1985 & August 19 & 16.38 & 9,580 \\
\hline 1942 & March 28 & -- & 7,090 & 1986 & April 1 & 19.47 & 11,500 \\
\hline 1943 & April 8 & 16.88 & 9,420 & 1987 & May 28 & 11.22 & 5,360 \\
\hline 1944 & August 11 & 12.20 & 5,770 & 1988 & April 8 & 10.90 & 5,090 \\
\hline 1945 & March 28 & 15.96 & 9,130 & 1989 & April 17 & 16.11 & 8,800 \\
\hline 1946 & March 24 & -- & 9,020 & 1990 & June 23 & 4.60 & 916 \\
\hline
\end{tabular}


05079000 RED LAKE RIVER AT CROOKSTON, MN--Continued

Annual peak discharge and corresponding gage height--Continued

$$
[--, \text { no data }]
$$

\begin{tabular}{|c|c|c|c|c|c|c|c|}
\hline $\begin{array}{l}\text { Water } \\
\text { year }\end{array}$ & Date & $\begin{array}{c}\text { Gage } \\
\text { height } \\
\text { (feet) }\end{array}$ & $\begin{array}{c}\text { Peak } \\
\text { discharge } \\
\left(\mathrm{ft}^{3} / \mathrm{s}\right)\end{array}$ & $\begin{array}{l}\text { Water } \\
\text { year }\end{array}$ & Date & $\begin{array}{c}\text { Gage } \\
\text { height } \\
\text { (feet) }\end{array}$ & $\begin{array}{c}\text { Peak } \\
\text { discharge } \\
\left(\mathrm{ft}^{3} / \mathrm{s}\right)\end{array}$ \\
\hline \multicolumn{8}{|c|}{ Annual peak discharge, by year, and corresponding gage height--Continued } \\
\hline 1991 & June 13 & 6.99 & 2,200 & 1993 & April 1 & 18.24 & 7,400 \\
\hline 1992 & April 28 & -- & 2,460 & 1994 & July 10 & 20.41 & 13,600 \\
\hline \multicolumn{8}{|c|}{ Annual peak discharge, from highest to lowest, and corresponding gage height } \\
\hline 1969 & April 12 & 27.33 & 28,400 & 1927 & April 13 & 14.00 & 7,700 \\
\hline 1950 & May 7 & 25.70 & 27,400 & 1929 & March 19 & 14.90 & 7,620 \\
\hline 1979 & April 26 & 24.99 & 21,900 & 1993 & April 1 & 18.24 & 7,400 \\
\hline 1966 & April 3 & 24.41 & 21,500 & 1983 & June 23 & 13.98 & 7,330 \\
\hline 1965 & April 14 & 23.51 & 19,400 & 1925 & June 9 & 13.50 & 7,300 \\
\hline 1967 & April 1 & 23.49 & 19,300 & 1913 & April 8 & -- & 7,170 \\
\hline${ }^{1} 1897$ & April 11 & 25.20 & 18,900 & 1981 & June 29 & 13.56 & 7,120 \\
\hline 1978 & April 7 & 23.11 & 18,100 & 1942 & March 28 & -- & 7,090 \\
\hline 1962 & June 11 & 21.90 & 16,700 & 1922 & May 13 & 13.00 & 6,910 \\
\hline 1974 & April 23 & 22.89 & 16,400 & 1963 & April 9 & 13.25 & 6,820 \\
\hline 1916 & April 17 & 21.80 & 15,900 & 1980 & April 9 & 12.31 & 6,600 \\
\hline 1975 & April 18 & 21.97 & 15,600 & 1926 & March 24 & 12.30 & 6,500 \\
\hline 1971 & April 10 & 20.74 & 15,300 & 1907 & April 4 & 12.04 & 6,330 \\
\hline 1919 & July 5 & 21.10 & 14,900 & 1952 & April 11 & 12.65 & 6,320 \\
\hline 1972 & April 16 & 20.28 & 14,700 & 1941 & June 9 & -- & 6,190 \\
\hline 1906 & April 15 & 21.00 & 14,600 & 1940 & April 16 & 14.73 & 6,000 \\
\hline 1984 & June 10 & 20.71 & 14,400 & 1938 & May 10 & 12.62 & 5,910 \\
\hline 1956 & April 20 & 19.78 & 14,000 & 1923 & April 20 & -- & 5,820 \\
\hline 1904 & April 24 & 20.42 & 13,700 & 1944 & August 11 & 12.20 & 5,770 \\
\hline 1994 & July 10 & 20.41 & 13,600 & 1959 & April 5 & 11.72 & 5,630 \\
\hline 1970 & April 26 & 19.05 & 13,300 & 1964 & June 20 & 11.74 & 5,550 \\
\hline 1951 & April 7 & 19.00 & 12,600 & 1960 & April 6 & 12.56 & 5,520 \\
\hline 1976 & April 3 & 19.45 & 12,500 & 1917 & April 11 & -- & 5,480 \\
\hline 1947 & June 12 & 18.08 & 12,400 & 1987 & May 28 & 11.22 & 5,360 \\
\hline 1955 & April 8 & 18.30 & 12,400 & 1954 & April 12 & 11.37 & 5,330 \\
\hline 1957 & June 29 & 18.10 & 11,800 & 1902 & May 21 & 10.00 & 5,170 \\
\hline 1986 & April 1 & 19.47 & 11,500 & 1988 & April 8 & 10.90 & 5,090 \\
\hline 1968 & July 19 & 17.17 & 11,100 & 1973 & September 26 & 10.86 & 4,960 \\
\hline 1908 & April 10 & 17.00 & 10,700 & 1930 & May 13 & 10.30 & 4,770 \\
\hline 1949 & June 2 & 17.43 & 10,700 & 1936 & April 18 & 11.33 & 4,540 \\
\hline 1985 & August 19 & 16.38 & 9,580 & 1932 & April 9 & 9.78 & 4,390 \\
\hline 1920 & March 25 & 23.30 & 9,520 & 1928 & April 8 & -- & 3,910 \\
\hline 1948 & April 19 & -- & 9,520 & 1937 & August 4 & 10.25 & 3,750 \\
\hline 1943 & April 8 & 16.88 & 9,420 & 1909 & July 21 & 8.77 & 3,680 \\
\hline 1982 & April 17 & 16.12 & 9,320 & 1911 & June 10 & 8.45 & 3,620 \\
\hline 1945 & March 28 & 15.96 & 9,130 & 1977 & May 20 & 8.66 & 3,440 \\
\hline 1946 & March 24 & -- & 9,020 & 1958 & July 7 & 8.62 & 3,370 \\
\hline 1989 & April 17 & 16.11 & 8,800 & 1939 & April 24 & 8.92 & 3,050 \\
\hline 1905 & May 13 & 14.50 & 8,730 & 1914 & June 12 & 7.40 & 2,630 \\
\hline 1910 & March 20 & 14.20 & 7,920 & 1953 & March 24 & 8.01 & 2,560 \\
\hline 1915 & June 29 & 14.25 & 7,860 & 1935 & March 27 & 8.38 & 2,490 \\
\hline
\end{tabular}




\section{RED LAKE RIVER AT CROOKSTON, MN--Continued}

Annual peak discharge and corresponding gage height--Continued

$$
[--, \text { no data }]
$$

\begin{tabular}{|c|c|c|c|c|c|c|c|}
\hline $\begin{array}{l}\text { Water } \\
\text { year }\end{array}$ & Date & $\begin{array}{c}\text { Gage } \\
\text { height } \\
\text { (feet) }\end{array}$ & $\begin{array}{c}\text { Peak } \\
\text { discharge } \\
\left(\mathrm{ft}^{3} / \mathrm{s}\right)\end{array}$ & $\begin{array}{l}\text { Water } \\
\text { year }\end{array}$ & Date & $\begin{array}{l}\text { Gage } \\
\text { height } \\
\text { (feet) }\end{array}$ & $\begin{array}{c}\text { Peak } \\
\text { discharge } \\
\left(\mathrm{ft}^{3} / \mathrm{s}\right)\end{array}$ \\
\hline \multicolumn{8}{|c|}{ Annual peak discharge, from highest to lowest, and corresponding gage height-Continued } \\
\hline 1992 & April 28 & -- & 2,460 & 1961 & March 27 & 5.67 & 1,450 \\
\hline 1991 & June 13 & 6.99 & 2,200 & 1933 & April 2 & 5.92 & 1,440 \\
\hline 1912 & September 29 & -- & 2,120 & 1924 & April 23 & 5.20 & 1,140 \\
\hline 1918 & April 2 & 6.50 & 1,950 & 1931 & March 26 & 4.93 & 1,030 \\
\hline 1934 & April 8 & 6.89 & 1,490 & 1990 & June 23 & 4.60 & 916 \\
\hline
\end{tabular}

${ }^{1}$ Reported by Professor E.F. Chandler. 


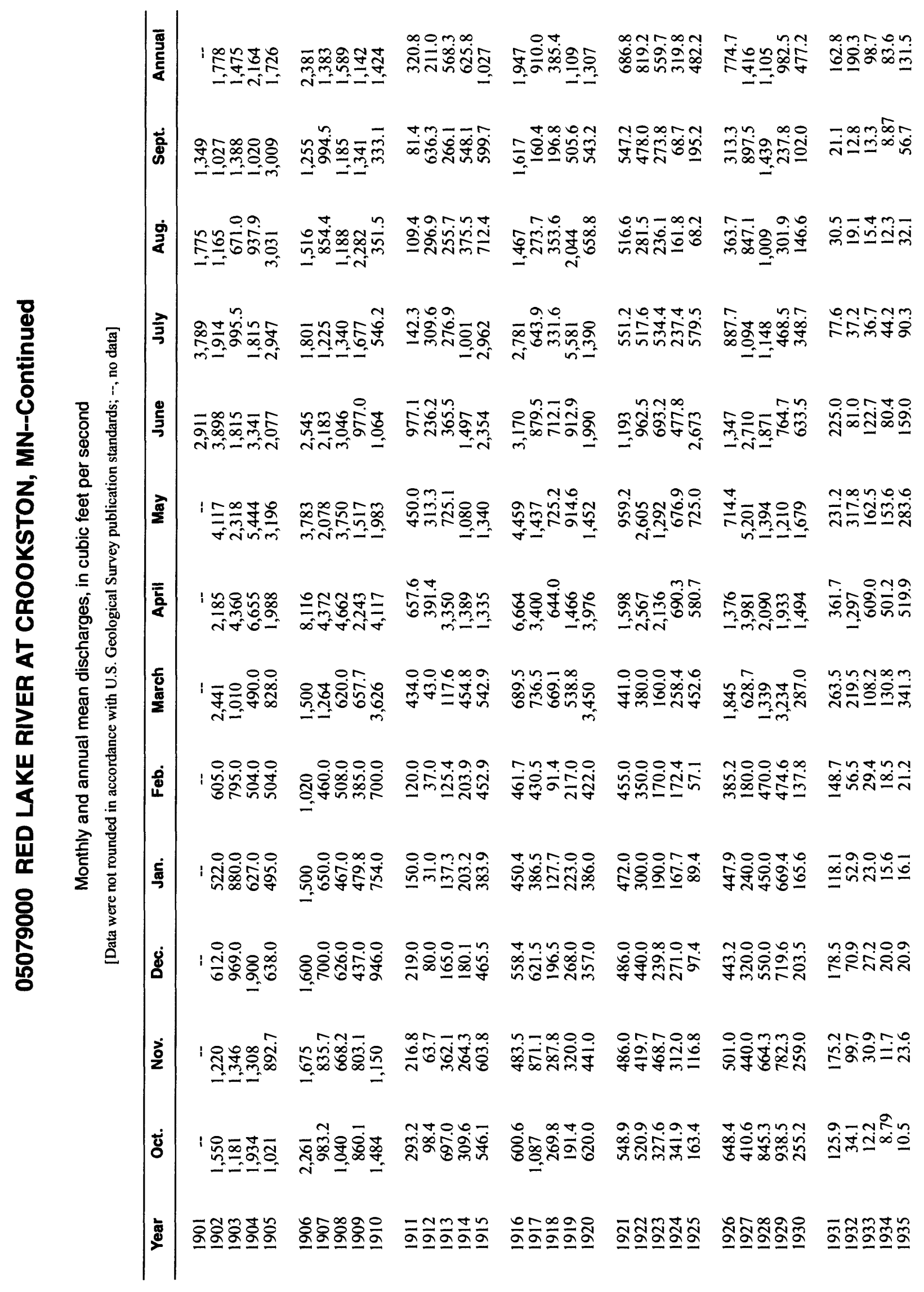




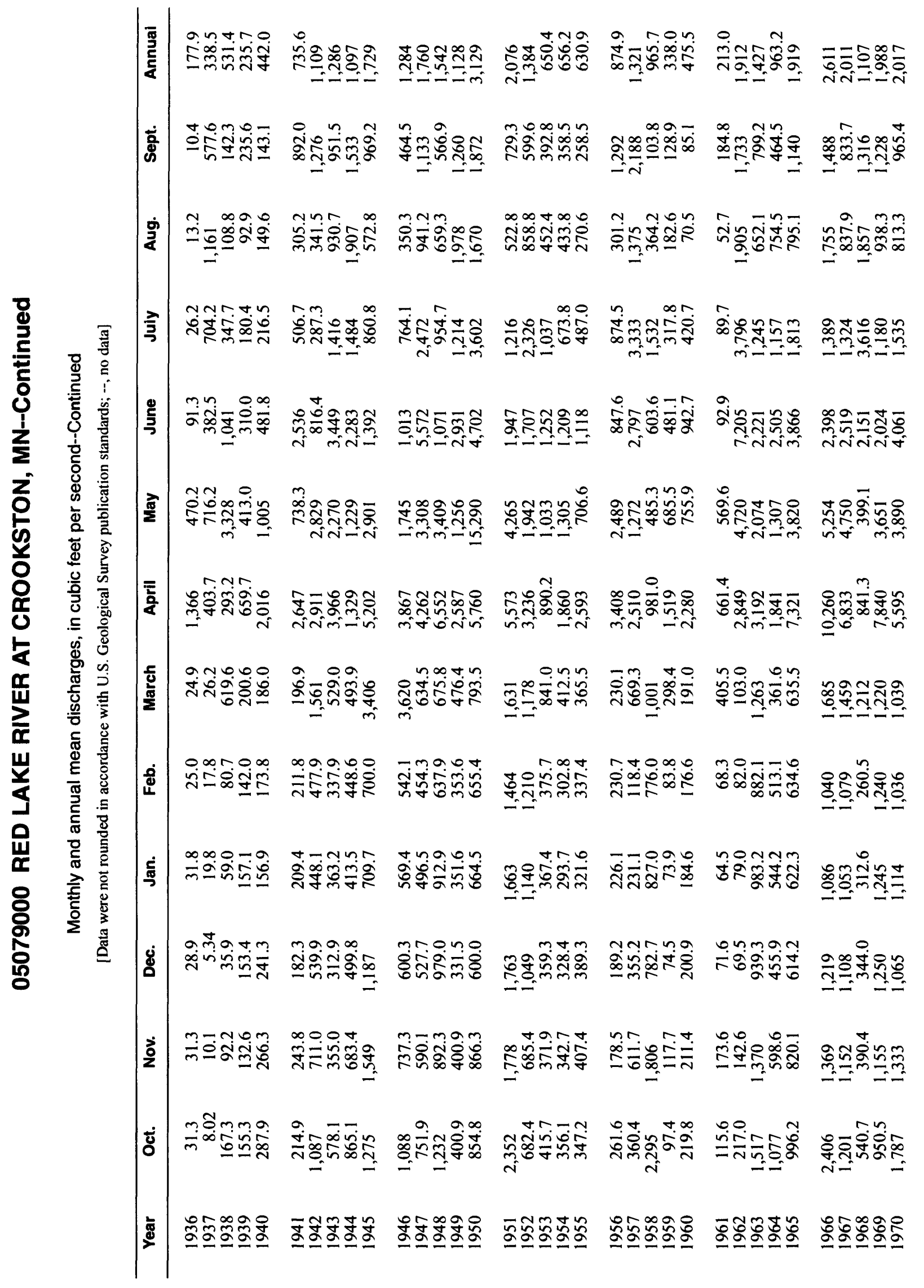




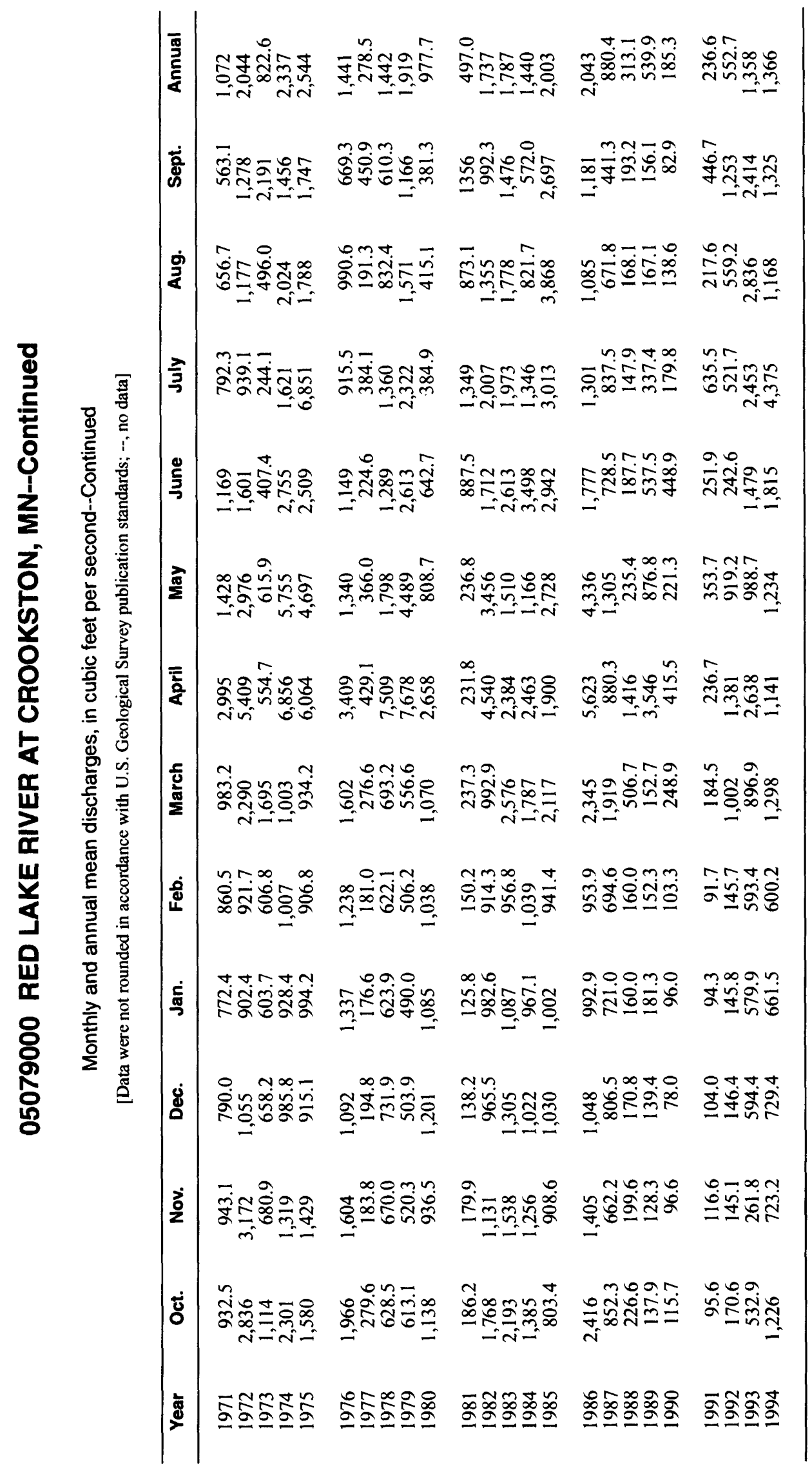


LOCATION.--Lat 4755'38', long 9701'34", in sec.2, T.151 N., R.50 W., Grand Forks County, Hydrologic Unit 09020301, on right bank $200 \mathrm{ft}$ upstream from the DeMers Avenue bridge, $0.4 \mathrm{mi}$ downstream from Red Lake River, and at mile 297.6.

DRAINAGE AREA.--30,100 $\mathrm{mi}^{2}$, approximately, including $3,800 \mathrm{mi}^{2}$ in closed basins.

PERIOD OF RECORD.--April 1882 to current year. Prior to January 1904 monthly discharge only, published in WSP 1308.

GAGE.--Water-stage recorder. Datum of gage is $779.00 \mathrm{ft}$ above sea level. Oct.1, 1983, to Sept. 30, 1986, datum of gage was $780.00 \mathrm{ft}$ at same site. Apr. 14, 1965, to Sept. 30, 1983, water-stage recorder $1.9 \mathrm{mi}$ downstream at a datum of $778.35 \mathrm{ft}$. Nov. 3, 1933, to Apr. 13, 1965, water-stage recorder $0.3 \mathrm{mi}$ upstream at $778.35 \mathrm{ft}$ datum. See WSP 1728 or 1913 for history of changes prior to Nov. 3, 1933.

EXTREMES FOR PERIOD OF RECORD.--Maximum discharge, $85,000 \mathrm{ft}^{3} / \mathrm{s}$, Apr. 10, 1897, gage height, $50.20 \mathrm{ft}$; minimum daily discharge, $1.8 \mathrm{ft}^{3} / \mathrm{s}$, Sept. 2,1977 , caused by unusual regulation during repair of dam at Grand Forks.

Annual mean discharge

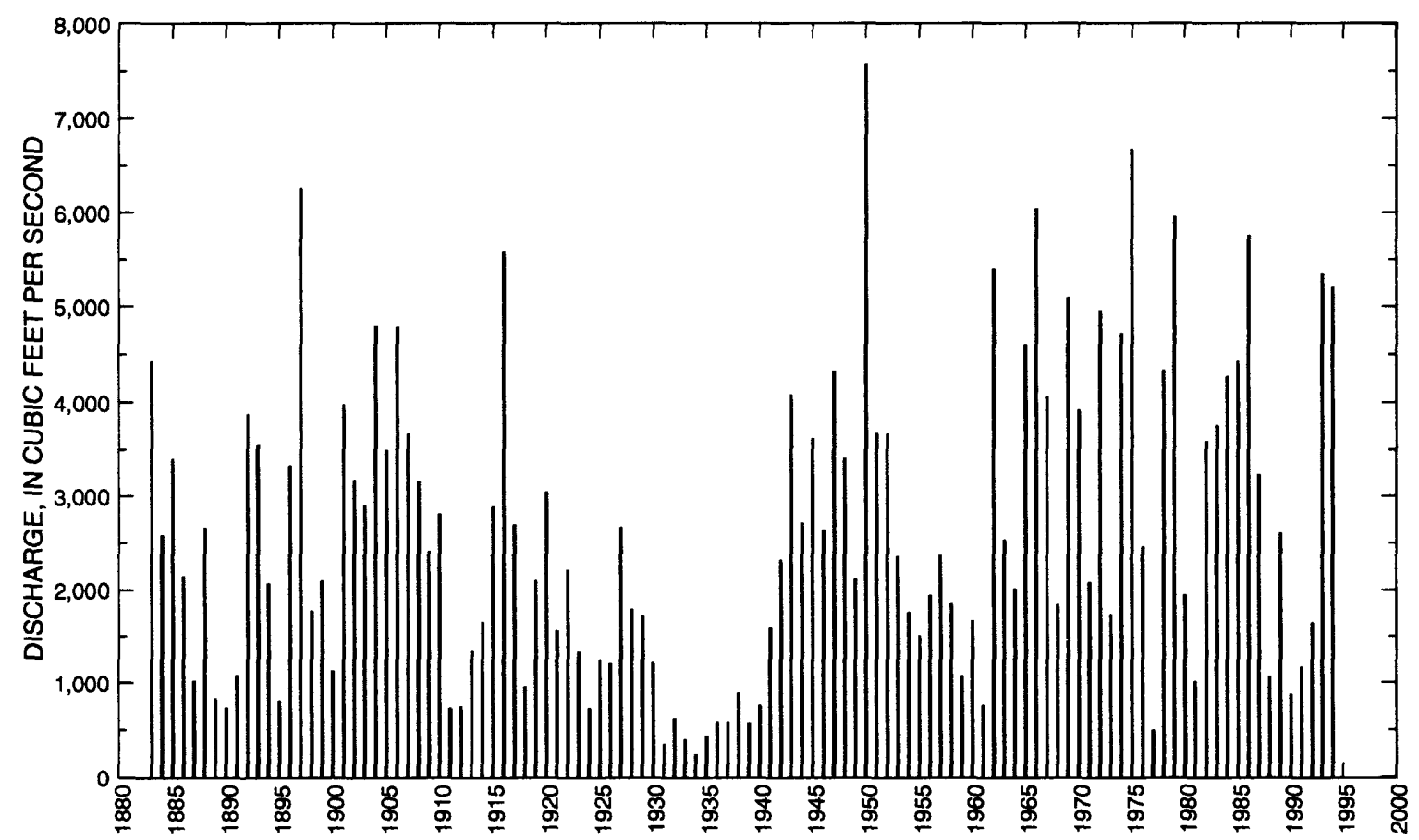


05082500 RED RIVER OF THE NORTH AT GRAND FORKS, ND--Continued

Statistics of monthly and annual mean discharges

\begin{tabular}{|c|c|c|c|c|c|c|c|c|}
\hline \multirow[b]{2}{*}{ Month } & \multicolumn{2}{|c|}{ Maximum } & \multicolumn{2}{|c|}{ Minimum } & \multicolumn{4}{|c|}{ Mean } \\
\hline & $\begin{array}{c}\text { Discharge } \\
\left(\mathrm{ft}^{3} / \mathbf{s}\right)\end{array}$ & $\begin{array}{c}\text { Water year } \\
\text { of } \\
\text { occurrence }\end{array}$ & $\begin{array}{c}\text { Discharge } \\
\left(\mathrm{ft}^{3} / \mathrm{s}\right)\end{array}$ & $\begin{array}{c}\text { Water year } \\
\text { of } \\
\text { occurrence }\end{array}$ & $\begin{array}{c}\text { Discharge } \\
\left(\mathrm{ft}^{3} / \mathrm{s}\right)\end{array}$ & $\begin{array}{c}\text { Standard } \\
\text { deviation } \\
\left(\mathrm{ft}^{3} / \mathbf{s}\right)\end{array}$ & $\begin{array}{l}\text { Coeffi- } \\
\text { cient of } \\
\text { variation }\end{array}$ & $\begin{array}{c}\text { Percentage } \\
\text { of annuai } \\
\text { discharge }\end{array}$ \\
\hline October & 5,690 & 1901 & 12.1 & 1937 & 1,380 & 1,080 & 0.78 & 4.33 \\
\hline November & 5,220 & 1972 & 30.5 & 1937 & 1,210 & 877 & 0.73 & 3.79 \\
\hline December & 3,070 & 1972 & 17.8 & 1937 & 967 & 665 & 0.69 & 3.04 \\
\hline January & 1,930 & 1951 & 18.8 & 1937 & 795 & 542 & 0.68 & 2.50 \\
\hline February & 1,870 & 1952 & 2.87 & 1937 & 758 & 514 & 0.68 & 2.38 \\
\hline March & 10,200 & 1966 & 42.1 & 1937 & 2,120 & 2,220 & 1.05 & 6.66 \\
\hline April & 39,800 & 1897 & 954 & 1938 & 9,270 & 7,980 & 0.86 & 29.1 \\
\hline May & 36,500 & 1950 & 373 & 1934 & 5,080 & 4,910 & 0.96 & 16.0 \\
\hline June & 19,300 & 1962 & 151 & 1934 & 3,890 & 3,070 & 0.79 & 12.2 \\
\hline July & 25,300 & 1975 & 88.8 & 1936 & 3,190 & 3,450 & 1.08 & 10.0 \\
\hline August & 17,000 & 1993 & 30.6 & 1934 & 1,760 & 2,070 & 1.18 & 5.53 \\
\hline September & 6,250 & 1993 & 20.3 & 1936 & 1,410 & 1,130 & 0.81 & 4.42 \\
\hline Annual & 7,580 & 1950 & 244 & 1934 & 2,630 & 1,650 & 0.63 & 100 \\
\hline
\end{tabular}

Annual flow duration

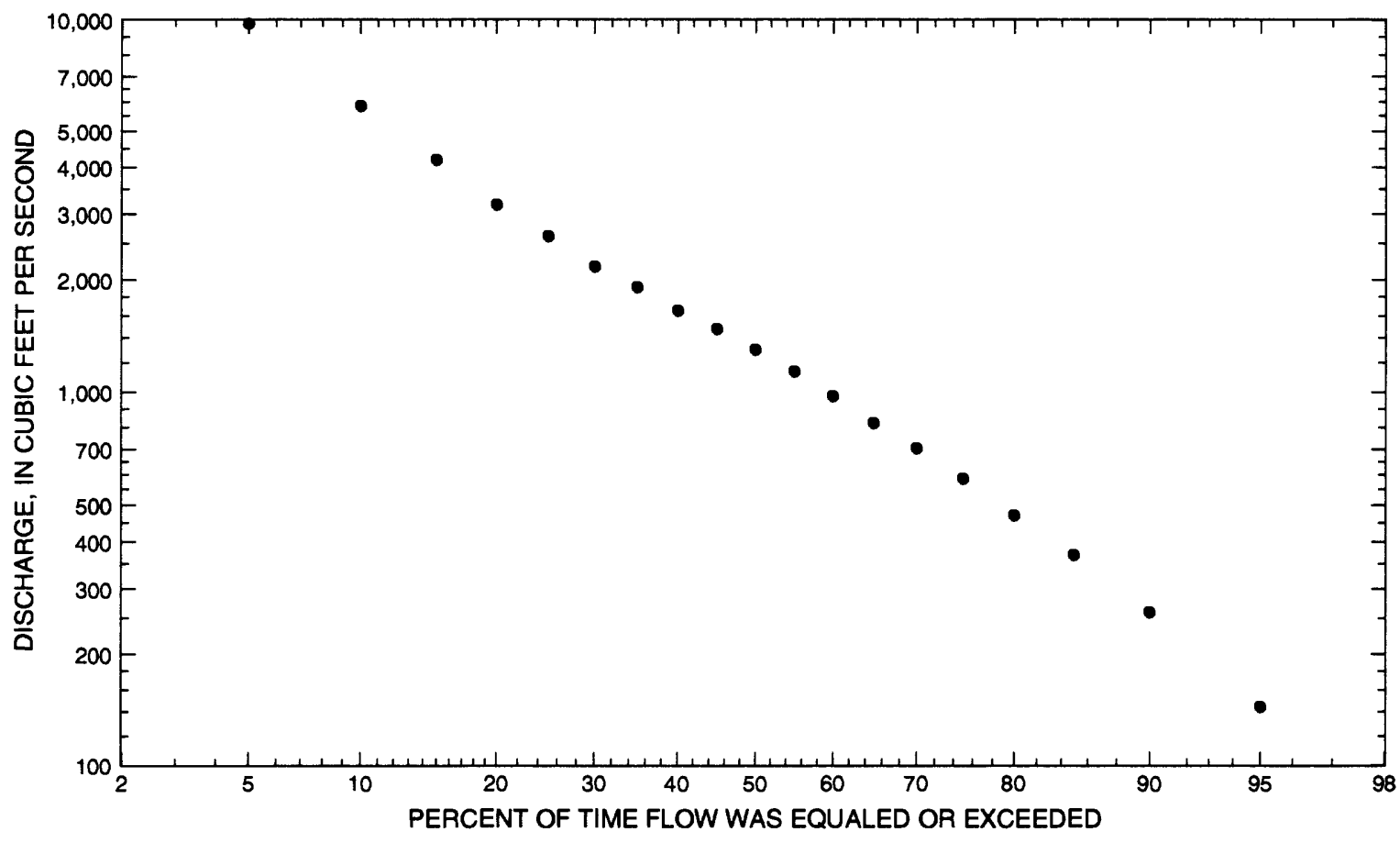




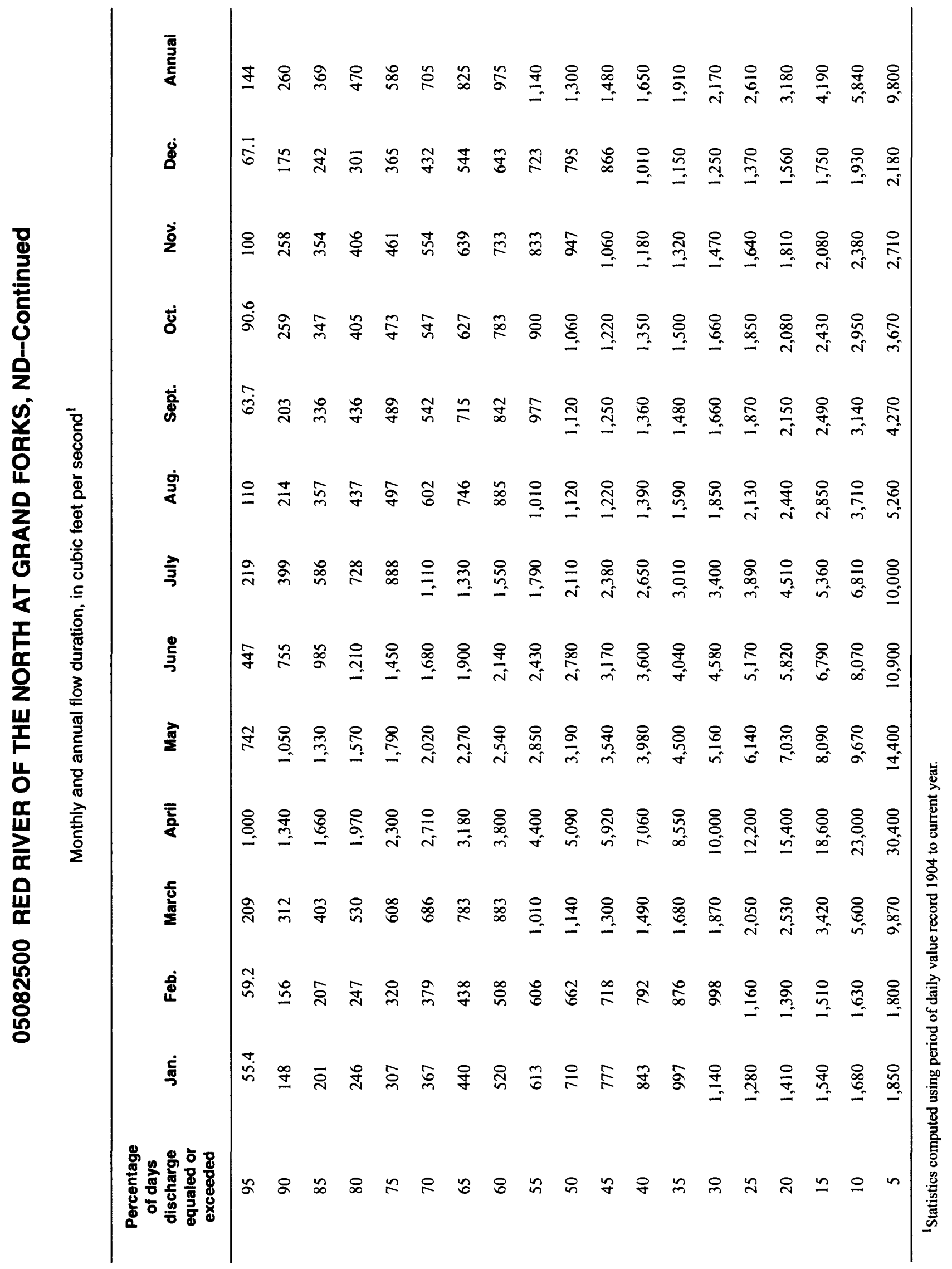


05082500 RED RIVER OF THE NORTH AT GRAND FORKS, ND--Continued

Probability of occurrence of annual high discharges

[ng, statistic not given]

\begin{tabular}{|c|c|c|c|c|c|c|}
\hline \multirow[b]{2}{*}{$\begin{array}{l}\text { Exceedance } \\
\text { probability }\end{array}$} & \multirow[b]{2}{*}{$\begin{array}{l}\text { Recurrence } \\
\text { Interval } \\
\text { (years) }\end{array}$} & \multirow[b]{2}{*}{$\begin{array}{c}\text { Maximum } \\
\text { Instantaneous } \\
\left(\mathrm{ft}^{3} / \mathbf{s}\right)\end{array}$} & \multicolumn{4}{|c|}{$\begin{array}{l}\text { Maximum mean discharge } \\
\qquad\left(\mathrm{ft}^{3} / \mathbf{s}\right)^{1}\end{array}$} \\
\hline & & & 3-day period & 7-day period & 15-day perlod & 30-day period \\
\hline 0.99 & 1.01 & 1,640 & 1,600 & 1,450 & 1,220 & 1,010 \\
\hline 0.95 & 1.05 & 3,260 & 3,240 & 2,940 & 2,430 & 1,950 \\
\hline 0.90 & 1.11 & 4,640 & 4,620 & 4,190 & 3,450 & 2,730 \\
\hline 0.80 & 1.25 & 6,990 & 6,960 & 6,340 & 5,200 & 4,060 \\
\hline 0.50 & 2 & 14,700 & 14,300 & 13,200 & 11,000 & 8,400 \\
\hline 0.20 & 5 & 29,200 & 27,400 & 25,800 & 21,900 & 16,700 \\
\hline 0.10 & 10 & 41,000 & 37,400 & 35,600 & 30,800 & 23,400 \\
\hline 0.04 & 25 & 58,000 & 51,000 & 49,300 & 43,700 & 33,200 \\
\hline 0.02 & 50 & 71,900 & 61,500 & 60,300 & 54,400 & 41,400 \\
\hline 0.01 & 100 & 86,700 & 72,400 & 71,600 & 65,700 & 50,200 \\
\hline 0.005 & 200 & 102,000 & 83,500 & 83,500 & 77,900 & 59,700 \\
\hline 0.002 & 500 & 125,000 & $\mathrm{ng}$ & ng & ng & ng \\
\hline
\end{tabular}

${ }^{1}$ Statistics computed using period of daily value record 1904 to current year.

Probability of occurrence of annual low discharges

\begin{tabular}{|c|c|c|c|c|c|c|c|c|c|c|}
\hline \multirow[b]{3}{*}{$\begin{array}{l}\text { Non- } \\
\text { exceed- } \\
\text { ance } \\
\text { prob- } \\
\text { abiilty }\end{array}$} & \multirow[b]{3}{*}{$\begin{array}{c}\text { Recur- } \\
\text { rence } \\
\text { inter- } \\
\text { val } \\
\text { (years) }\end{array}$} & \multicolumn{9}{|c|}{ Minimum mean discharge $\left(\mathrm{ft}^{3} / \mathrm{s}\right)^{1}$} \\
\hline & & \multicolumn{9}{|c|}{ Number of consecutive days } \\
\hline & & 1 & 3 & 7 & 14 & 30 & 60 & 90 & 120 & 183 \\
\hline 0.05 & 20 & 24.5 & 34.2 & 36.1 & 38.8 & 44.4 & 62.0 & 72.7 & 88.9 & 98.9 \\
\hline 0.10 & 10 & 58.5 & 72.5 & 76.3 & 81.3 & 91.4 & 115 & 132 & 156 & 179 \\
\hline 0.20 & 5 & 142 & 158 & 166 & 175 & 193 & 223 & 250 & 284 & 335 \\
\hline 0.50 & 2 & 479 & 482 & 507 & 531 & 568 & 601 & 654 & 707 & 854 \\
\hline
\end{tabular}

${ }^{1}$ Statistics computed using period of daily value record 1904 to current year. 


\section{RED RIVER OF THE NORTH AT GRAND FORKS, ND--Continued}

Probability of occurrence of seasonal low discharges

\begin{tabular}{|c|c|c|c|c|c|c|c|c|c|}
\hline \multirow[b]{3}{*}{$\begin{array}{c}\text { Non- } \\
\text { exceedance } \\
\text { probability }\end{array}$} & \multirow[b]{3}{*}{$\begin{array}{l}\text { Recurrence } \\
\text { intervai } \\
\text { (years) }\end{array}$} & \multicolumn{8}{|c|}{ Minimum mean discharge $\left(\mathrm{ft}^{3} / \mathrm{s}\right)^{1}$} \\
\hline & & \multicolumn{8}{|c|}{ Number of consecutive days } \\
\hline & & 1 & 7 & 14 & 30 & 1 & 7 & 14 & 30 \\
\hline & & \multicolumn{4}{|c|}{ December-January-February } & \multicolumn{4}{|c|}{ March-April-May } \\
\hline 0.05 & 20 & 39.8 & 43.0 & 46.1 & 51.5 & 81.1 & 106 & 143 & 229 \\
\hline 0.10 & 10 & 85.1 & 91.3 & 96.8 & 105 & 158 & 192 & 233 & 361 \\
\hline 0.20 & 5 & 187 & 199 & 208 & 221 & 312 & 354 & 393 & 598 \\
\hline \multirow[t]{2}{*}{0.50} & 2 & 572 & 599 & 616 & 639 & 791 & 841 & 875 & 1,380 \\
\hline & & \multicolumn{4}{|c|}{ June-July-August } & \multicolumn{4}{|c|}{ September-October-November } \\
\hline 0.05 & 20 & 79.0 & 89.0 & 96.6 & 114 & 34.2 & 56.4 & 62.4 & 74.9 \\
\hline 0.10 & 10 & 146 & 162 & 176 & 206 & 79.9 & 114 & 126 & 150 \\
\hline 0.20 & 5 & 283 & 312 & 336 & 392 & 189 & 237 & 261 & 310 \\
\hline 0.50 & 2 & 804 & 871 & 936 & 1,090 & 620 & 685 & 755 & 874 \\
\hline
\end{tabular}

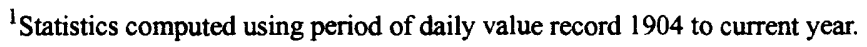


05082500 RED RIVER OF THE NORTH AT GRAND FORKS, ND--Continued

Annual peak discharge and corresponding gage height

$[--$, no data $]$

\begin{tabular}{|c|c|c|c|c|c|c|c|}
\hline $\begin{array}{l}\text { Water } \\
\text { year }\end{array}$ & Date & $\begin{array}{c}\text { Gage } \\
\text { height } \\
\text { (feet) }\end{array}$ & $\begin{array}{c}\text { Peak } \\
\text { discharge } \\
\left(\mathrm{ft}^{3} / \mathbf{s}\right)\end{array}$ & $\begin{array}{l}\text { Water } \\
\text { year }\end{array}$ & Date & $\begin{array}{c}\text { Gage } \\
\text { height } \\
\text { (feet) }\end{array}$ & $\begin{array}{c}\text { Peak } \\
\text { discharge } \\
\left(\mathrm{ft}^{3} / \mathbf{s}\right)\end{array}$ \\
\hline \multicolumn{8}{|c|}{ Annual peak discharge, by year, and corresponding gage height } \\
\hline 1882 & April 18 & 48.00 & 75,000 & 1927 & April 13 & 20.00 & 10,600 \\
\hline 1883 & April 26 & 42.20 & 38,600 & 1928 & April 2 & 21.80 & 12,200 \\
\hline 1884 & April 16 & 31.10 & 20,600 & 1929 & March 24 & -- & 17,100 \\
\hline 1885 & April 17 & 23.10 & 13,000 & 1930 & April 7 & 18.90 & 9,610 \\
\hline 1886 & May 3 & 20.60 & 10,800 & 1931 & April 10 & 6.48 & 1,630 \\
\hline 1887 & April 15 & 16.30 & 7,300 & 1932 & April 10 & 22.07 & 10,400 \\
\hline 1888 & April 19 & 29.50 & 19,000 & 1933 & April 3 & 15.18 & 4,380 \\
\hline 1889 & April 1 & 12.00 & 3,000 & 1934 & April 12 & 10.02 & 3,210 \\
\hline 1890 & April 15 & 10.60 & 3,470 & 1935 & March 29 & 13.07 & 2,920 \\
\hline 1891 & April 13 & 17.70 & 6,000 & 1936 & April 18 & 25.00 & 14,500 \\
\hline 1892 & April 17 & 33.40 & 23,000 & 1937 & May 4 & 11.57 & 4,180 \\
\hline 1893 & April 24 & 45.50 & 53,300 & 1938 & May 12 & 15.49 & 6,600 \\
\hline 1894 & April 24 & 26.90 & 16,400 & 1939 & April 6 & 20.13 & 6,720 \\
\hline 1895 & April 6 & 9.90 & 2,000 & 1940 & April 18 & 21.88 & 10,000 \\
\hline 1896 & May 30 & 32.00 & 21,600 & 1941 & April 12 & 27.86 & 13,400 \\
\hline 1897 & April 10 & 50.20 & 85,000 & 1942 & April 5 & 24.10 & 11,000 \\
\hline 1898 & April 14 & 15.00 & 4,500 & 1943 & April 12 & 38.16 & 28,200 \\
\hline 1899 & April 17 & 20.90 & 9,000 & 1944 & August 13 & 19.79 & 10,400 \\
\hline 1900 & April 10 & 13.20 & 4,000 & 1945 & March 29 & -- & 21,300 \\
\hline 1901 & April 7 & 26.30 & 14,000 & 1946 & March 27 & 33.10 & 22,000 \\
\hline 1902 & March 30 & 26.00 & 15,000 & 1947 & April 21 & 40.60 & 35,000 \\
\hline 1903 & April 11 & 28.00 & 18,800 & 1948 & April 16 & 41.68 & 34,200 \\
\hline 1904 & April 27 & 40.65 & 33,000 & 1949 & April 10 & 29.11 & 15,200 \\
\hline 1905 & May 16 & 26.11 & 16,800 & 1950 & May 12 & 45.61 & 54,000 \\
\hline 1906 & April 18 & 36.00 & 27,600 & 1951 & April 12 & 33.52 & 23,600 \\
\hline 1907 & April 7 & 39.95 & 30,400 & 1952 & April 20 & -- & 23,900 \\
\hline 1908 & April 11 & 32.80 & 20,500 & 1953 & June 25 & 24.63 & 14,600 \\
\hline 1909 & July 30 & 18.80 & 9,260 & 1954 & April 15 & 18.63 & 9,620 \\
\hline 1910 & March 22 & 30.70 & 18,500 & 1955 & April 10 & 26.17 & 15,400 \\
\hline 1911 & June 12 & 10.70 & 3,520 & 1956 & April 23 & 32.43 & 21,400 \\
\hline 1912 & April 8 & 12.73 & 4,730 & 1957 & July 2 & 24.67 & 14,700 \\
\hline 1913 & April 8 & 26.70 & 17,200 & 1958 & July 9 & 16.03 & 7,500 \\
\hline 1914 & June 16 & 17.50 & 8,240 & 1959 & April 6 & -- & 6,300 \\
\hline 1915 & July 3 & 30.80 & 21,500 & 1960 & April 12 & 28.88 & 17,200 \\
\hline 1916 & April 23 & 37.70 & 29,000 & 1961 & March 28 & 9.75 & 3,400 \\
\hline 1917 & April 8 & 33.90 & 21,600 & 1962 & June 16 & 34.45 & 26,600 \\
\hline 1918 & March 28 & 11.30 & 4,480 & 1963 & April II & 21.23 & 10,800 \\
\hline 1919 & July 8 & 23.20 & 13,600 & 1964 & April 19 & 22.71 & 13,200 \\
\hline 1920 & March 31 & -- & 30,300 & 1965 & April 17 & 44.92 & 52,000 \\
\hline 1921 & April 10 & 20.90 & 11,500 & 1966 & April 4 & 45.55 & 55,000 \\
\hline 1922 & April 11 & 28.72 & 19,000 & 1967 & April 4 & 37.50 & 28,200 \\
\hline 1923 & April 22 & 26.15 & 16,200 & 1968 & June 11 & 20.03 & 9,420 \\
\hline 1924 & May 2 & 8.20 & 2,530 & 1969 & April 16 & 45.69 & 53,500 \\
\hline 1925 & June 12 & 19.00 & 9,690 & 1970 & April 28 & 34.30 & 23,700 \\
\hline 1926 & March 28 & 18.10 & 7,720 & 1971 & April 11 & 27.86 & 15,800 \\
\hline
\end{tabular}




\section{RED RIVER OF THE NORTH AT GRAND FORKS, ND--Continued}

Annual peak discharge and corresponding gage height-Continued

$$
[--, \text { no data }]
$$

\begin{tabular}{|c|c|c|c|c|c|c|c|}
\hline $\begin{array}{l}\text { Water } \\
\text { year }\end{array}$ & Date & $\begin{array}{c}\text { Gage } \\
\text { height } \\
\text { (feet) }\end{array}$ & $\begin{array}{c}\text { Peak } \\
\text { discharge } \\
\left(\mathrm{ft}^{3} / \mathrm{s}\right)\end{array}$ & $\begin{array}{l}\text { Water } \\
\text { year }\end{array}$ & Date & $\begin{array}{c}\text { Gage } \\
\text { height } \\
\text { (feet) }\end{array}$ & $\begin{array}{c}\text { Peak } \\
\text { discharge } \\
\left(\mathrm{ft}^{3} / \mathrm{s}\right)\end{array}$ \\
\hline \multicolumn{8}{|c|}{ Annual peak discharge, by year, and corresponding gage height-Continued } \\
\hline 1972 & April 17 & 38.50 & 31,400 & 1984 & April 2 & 37.06 & 32,300 \\
\hline 1973 & March 20 & 27.32 & 11,300 & 1985 & May 19 & 25.90 & 17,800 \\
\hline 1974 & April 19 & 40.25 & 34,300 & 1986 & April 2 & 37.00 & 31,900 \\
\hline 1975 & July 14 & 43.08 & 42,800 & 1987 & March 29 & 33.19 & 17,500 \\
\hline 1976 & April 3 & 34.58 & 23,600 & 1988 & April 5 & 21.16 & 8,500 \\
\hline 1977 & April 10 & 8.52 & 2,190 & 1989 & April 13 & 43.21 & 39,600 \\
\hline 1978 & April 11 & 45.73 & 54,200 & 1990 & April 5 & 17.56 & 5,040 \\
\hline 1979 & April 23 & 48.63 & 82,000 & 1991 & July 8 & 17.63 & 4,870 \\
\hline 1980 & April 6 & 31.01 & 22,000 & 1992 & March 12 & 23.30 & 8,000 \\
\hline 1981 & July 1 & 14.68 & 6,710 & 1993 & August 3 & 36.39 & 26,200 \\
\hline 1982 & April 12 & 37.18 & 23,900 & 1994 & July 12 & 34.30 & 26,800 \\
\hline 1983 & April 6 & 29.17 & 14,300 & & & & \\
\hline \multicolumn{8}{|c|}{ Annual peak discharge, from highest to lowest, and corresponding gage height } \\
\hline 1897 & April 10 & 50.20 & 85,000 & 1951 & April 12 & 33.52 & 23,600 \\
\hline 1979 & April 23 & 48.63 & 82,000 & 1976 & April 3 & 34.58 & 23,600 \\
\hline 1882 & April 18 & 48.00 & 75,000 & 1892 & April 17 & 33.40 & 23,000 \\
\hline 1966 & April 4 & 45.55 & 55,000 & 1946 & March 27 & 33.10 & 22,000 \\
\hline 1978 & April 11 & 45.73 & 54,200 & 1980 & April 6 & 31.01 & 22,000 \\
\hline 1950 & May 12 & 45.61 & 54,000 & 1896 & May 30 & 32.00 & 21,600 \\
\hline 1969 & April 16 & 45.69 & 53,500 & 1917 & April 8 & 33.90 & 21,600 \\
\hline 1893 & April 24 & 45.50 & 53,300 & 1915 & July 3 & 30.80 & 21,500 \\
\hline 1965 & April 17 & 44.92 & 52,000 & 1956 & April 23 & 32.43 & 21,400 \\
\hline 1975 & July 14 & 43.08 & 42,800 & 1945 & March 29 & -- & 21,300 \\
\hline 1989 & April 13 & 43.21 & 39,600 & 1884 & April 16 & 31.10 & 20,600 \\
\hline 1883 & April 26 & 42.20 & 38,600 & 1908 & April 11 & 32.80 & 20,500 \\
\hline 1947 & April 21 & 40.60 & 35,000 & 1888 & April 19 & 29.50 & 19,000 \\
\hline 1974 & April 19 & 40.25 & 34,300 & 1922 & April 11 & 28.72 & 19,000 \\
\hline 1948 & April 16 & 41.68 & 34,200 & 1903 & April 11 & 28.00 & 18,800 \\
\hline 1904 & April 27 & 40.65 & 33,000 & 1910 & March 22 & 30.70 & 18,500 \\
\hline 1984 & April 2 & 37.06 & 32,300 & 1985 & May 19 & 25.90 & 17,800 \\
\hline 1986 & April 2 & 37.00 & 31,900 & 1987 & March 29 & 33.19 & 17,500 \\
\hline 1972 & April 17 & 38.50 & 31,400 & 1913 & April 8 & 26.70 & 17,200 \\
\hline 1907 & April 7 & 39.95 & 30,400 & 1960 & April 12 & 28.88 & 17,200 \\
\hline 1920 & March 31 & -- & 30,300 & 1929 & March 24 & -- & 17,100 \\
\hline 1916 & April 23 & 37.70 & 29,000 & 1905 & May 16 & 26.11 & 16,800 \\
\hline 1943 & April 12 & 38.16 & 28,200 & 1894 & April 24 & 26.90 & 16,400 \\
\hline 1967 & April 4 & 37.50 & 28,200 & 1923 & April 22 & 26.15 & 16,200 \\
\hline 1906 & April 18 & 36.00 & 27,600 & 1971 & April 11 & 27.86 & 15,800 \\
\hline 1994 & July 12 & 34.30 & 26,800 & 1955 & April 10 & 26.17 & 15,400 \\
\hline 1962 & June 16 & 34.45 & 26,600 & 1949 & April 10 & 29.11 & 15,200 \\
\hline 1993 & August 3 & 36.39 & 26,200 & 1902 & March 30 & 26.00 & 15,000 \\
\hline 1952 & April 20 & -- & 23,900 & 1957 & July 2 & 24.67 & 14,700 \\
\hline 1982 & April 12 & 37.18 & 23,900 & 1953 & June 25 & 24.63 & 14,600 \\
\hline 1970 & April 28 & 34.30 & 23,700 & 1936 & April 18 & 25.00 & 14,500 \\
\hline
\end{tabular}


05082500 RED RIVER OF THE NORTH AT GRAND FORKS, ND--Continued

Annual peak discharge and corresponding gage height-Continued

$[--$ no data $]$

\begin{tabular}{|c|c|c|c|c|c|c|c|}
\hline $\begin{array}{l}\text { Water } \\
\text { year }\end{array}$ & Date & $\begin{array}{c}\text { Gage } \\
\text { height } \\
\text { (feet) }\end{array}$ & $\begin{array}{c}\text { Peak } \\
\text { discharge } \\
\left(\mathrm{ft}^{3} / \mathrm{s}\right)\end{array}$ & $\begin{array}{l}\text { Water } \\
\text { year }\end{array}$ & Date & $\begin{array}{c}\text { Gage } \\
\text { height } \\
\text { (feet) }\end{array}$ & $\begin{array}{c}\text { Peak } \\
\text { discharge } \\
\left(\mathrm{ft}^{3} / \mathrm{s}\right)\end{array}$ \\
\hline \multicolumn{8}{|c|}{ Annual peak discharge, from highest to lowest, and corresponding gage height-Continued } \\
\hline 1983 & April 6 & 29.17 & 14,300 & 1958 & July 9 & 16.03 & 7,500 \\
\hline 1901 & April 7 & 26.30 & 14,000 & 1887 & April 15 & 16.30 & 7,300 \\
\hline 1919 & July 8 & 23.20 & 13,600 & 1939 & April 6 & 20.13 & 6,720 \\
\hline 1941 & April 12 & 27.86 & 13,400 & 1981 & July 1 & 14.68 & 6,710 \\
\hline 1964 & April 19 & 22.71 & 13,200 & 1938 & May 12 & 15.49 & 6,600 \\
\hline 1885 & April 17 & 23.10 & 13,000 & 1959 & April 6 & - & 6,300 \\
\hline 1928 & April 2 & 21.80 & 12,200 & 1891 & April 13 & 17.70 & 6,000 \\
\hline 1921 & April 10 & 20.90 & 11,500 & 1990 & April 5 & 17.56 & 5,040 \\
\hline 1973 & March 20 & 27.32 & 11,300 & 1991 & July 8 & 17.63 & 4,870 \\
\hline 1942 & April 5 & 24.10 & 11,000 & 1912 & April 8 & 12.73 & 4,730 \\
\hline 1886 & May 3 & 20.60 & 10,800 & 1898 & April 14 & 15.00 & 4,500 \\
\hline 1963 & April 11 & 21.23 & 10,800 & 1918 & March 28 & 11.30 & 4,480 \\
\hline 1927 & April 13 & 20.00 & 10,600 & 1933 & April 3 & 15.18 & 4,380 \\
\hline 1932 & April 10 & 22.07 & 10,400 & 1937 & May 4 & 11.57 & 4,180 \\
\hline 1944 & August 13 & 19.79 & 10,400 & 1900 & April 10 & 13.20 & 4,000 \\
\hline 1940 & April 18 & 21.88 & 10,000 & 1911 & June 12 & 10.70 & 3,520 \\
\hline 1925 & June 12 & 19.00 & 9,690 & 1890 & April 15 & 10.60 & 3,470 \\
\hline 1954 & April 15 & 18.63 & 9,620 & 1961 & March 28 & 9.75 & 3,400 \\
\hline 1930 & April 7 & 18.90 & 9,610 & 1934 & April 12 & 10.02 & 3,210 \\
\hline 1968 & June 11 & 20.03 & 9,420 & 1889 & April 1 & 12.00 & 3,000 \\
\hline 1909 & July 30 & 18.80 & 9,260 & 1935 & March 29 & 13.07 & 2,920 \\
\hline 1899 & April 17 & 20.90 & 9,000 & 1924 & May 2 & 8.20 & 2,530 \\
\hline 1988 & April 5 & 21.16 & 8,500 & 1977 & April 10 & 8.52 & 2,190 \\
\hline 1914 & June 16 & 17.50 & 8,240 & 1895 & April 6 & 9.90 & 2,000 \\
\hline 1992 & March 12 & 23.30 & 8,000 & 1931 & April 10 & 6.48 & 1,630 \\
\hline 1926 & March 28 & 18.10 & 7,720 & & & & \\
\hline
\end{tabular}




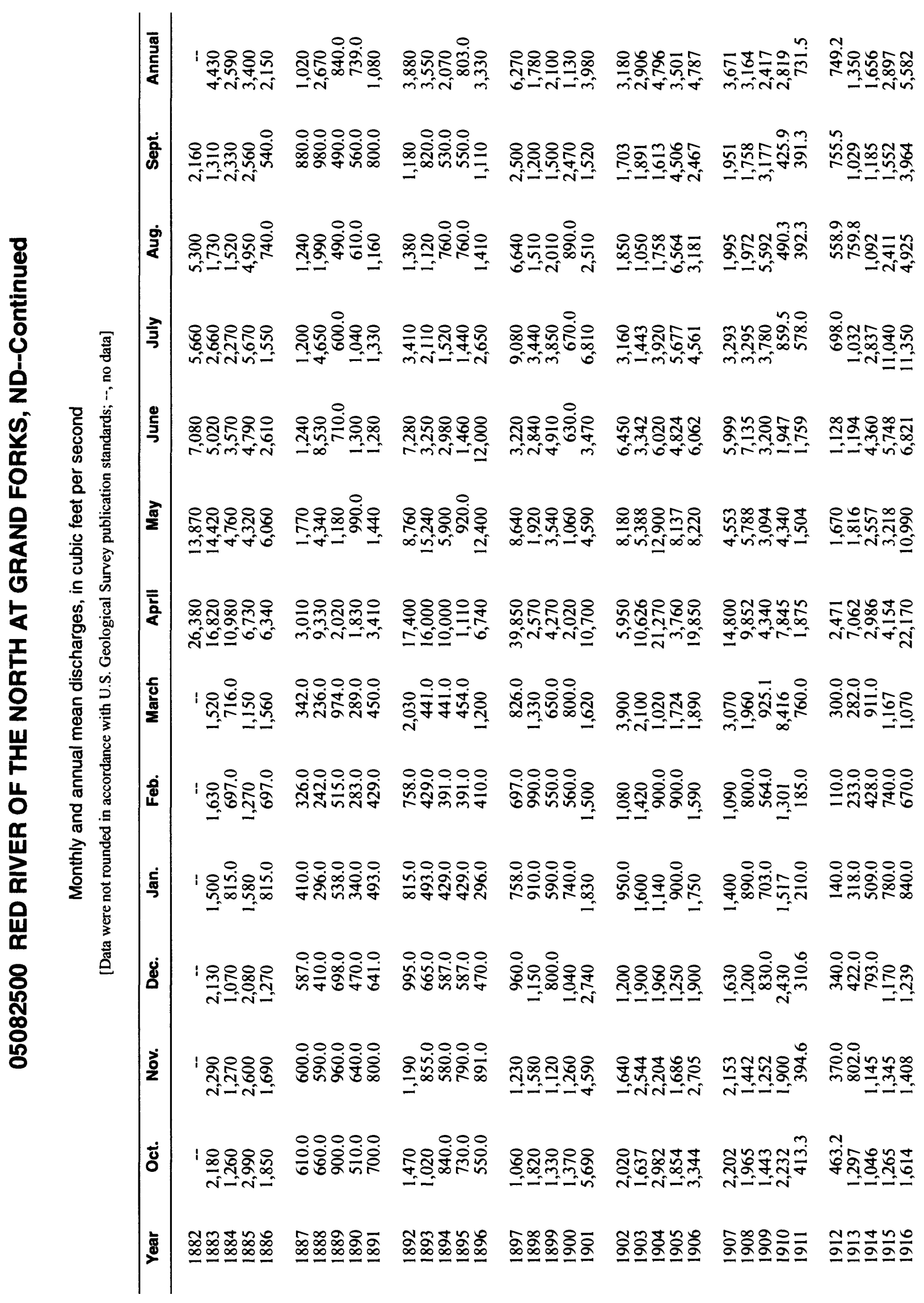




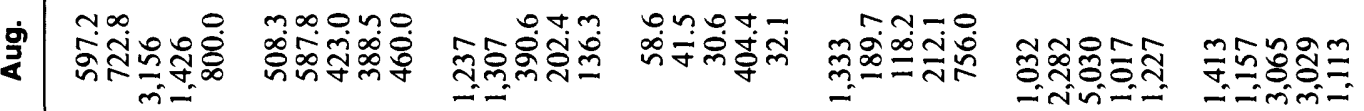

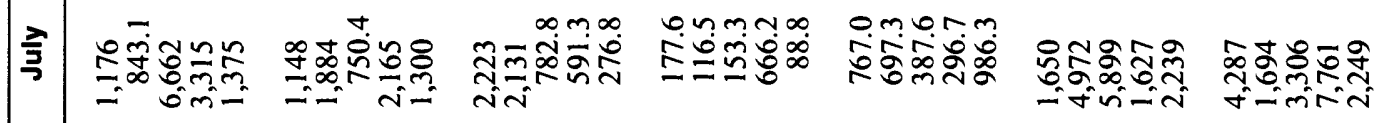

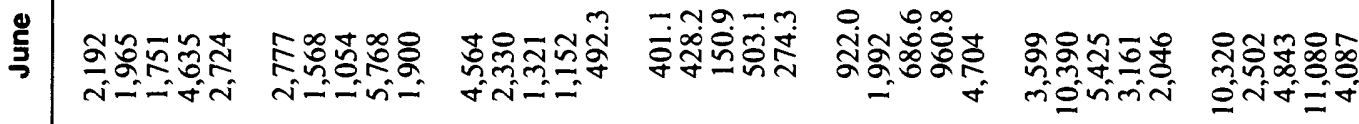

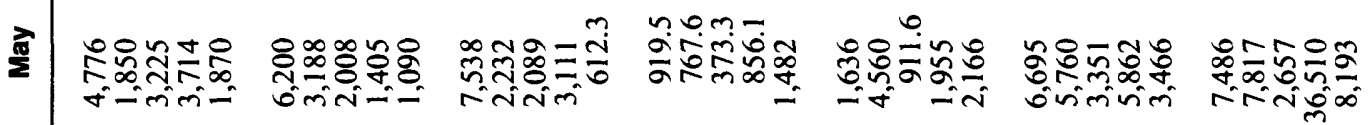

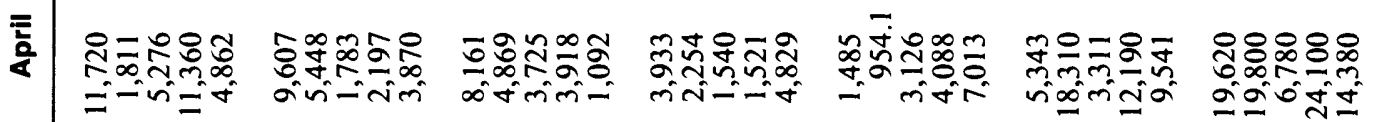

遂

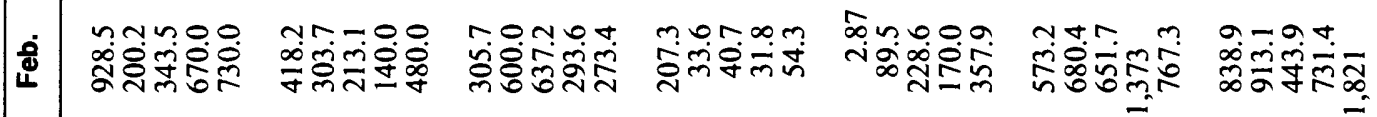

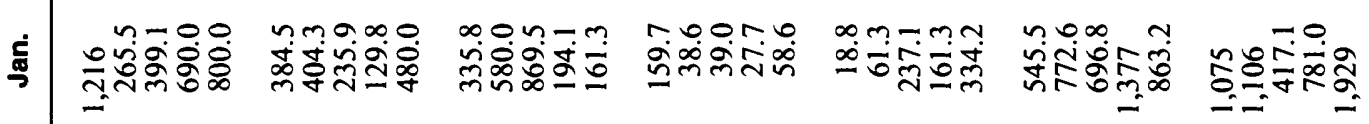

\&

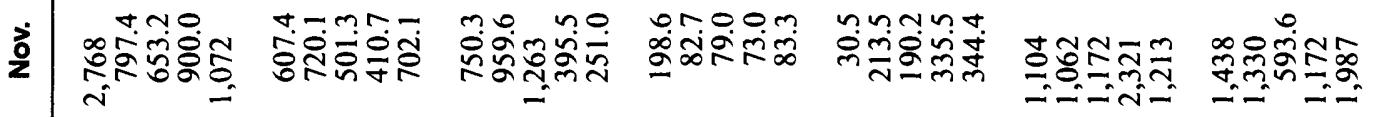

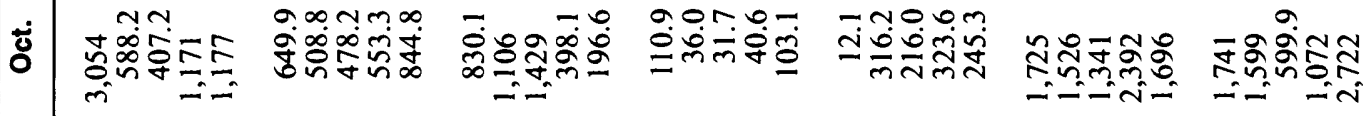

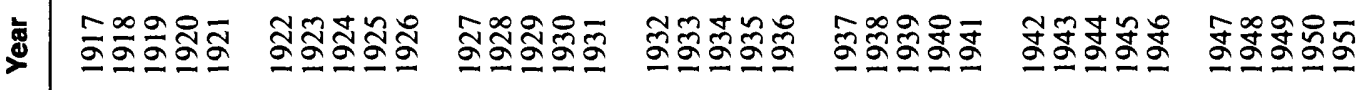


$\infty$ nivi=i⿻जivito

अ-inimi

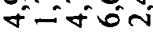

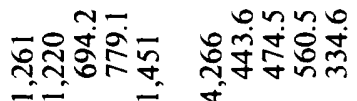

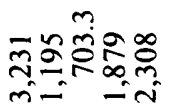

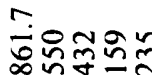

นrmo oี ma항

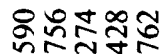

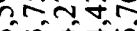

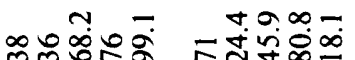

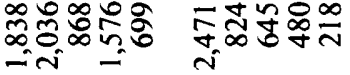

$r$

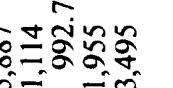

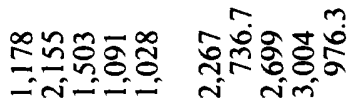

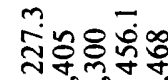

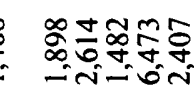

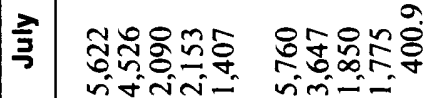

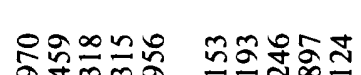
miniti

ํํํ융으

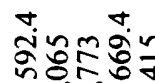

윻ํㅇㅇ응

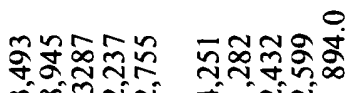
웜ํㅇㅇ mंर्यंत्रत in mini

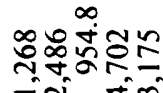
moñरत $\forall-N$ i

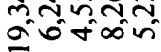

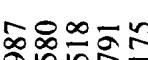

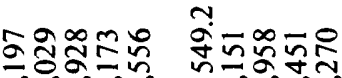
min-

గํㅜ윰 min $=-20$ 주응용 45iñ

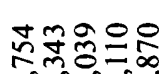

유옹 ஸ̃

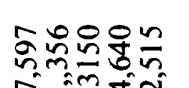

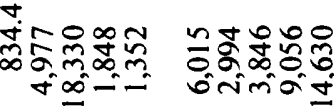

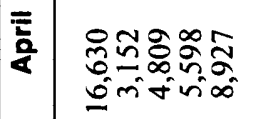

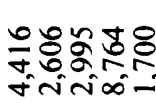

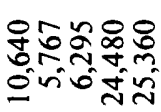

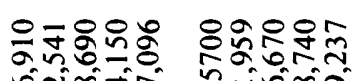
ñ.

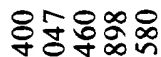
ジロデす

\%웅유.

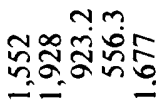

n nN 수워

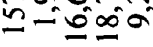

inm (1)

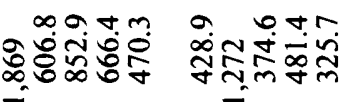

$=0 \infty$

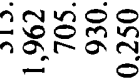

ㅇㅗㅀㅠำ

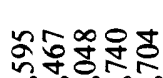

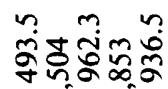

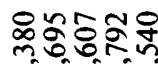
irivino

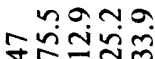

- $\infty \infty-$ 드융요

ชิํํำำกี้

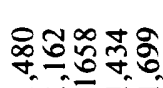

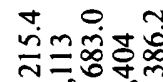

ริำ $\frac{m}{\infty} \infty$

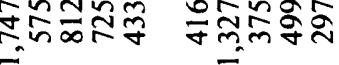

$0-0$

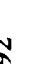

9

$2=8$

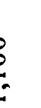

똥뭉

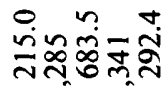

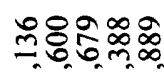

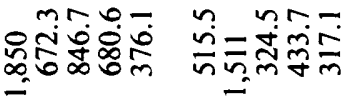

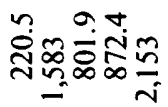

$\stackrel{\infty}{\infty} \stackrel{\infty}{\sim} 0$

ํํㅇำำำำ

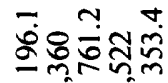

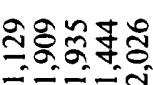

ํำำำ

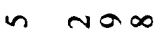

0

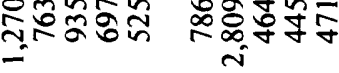

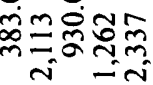

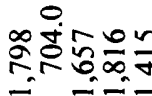

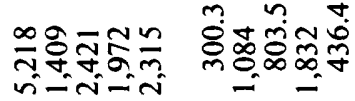

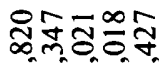

$9 \infty n \infty$ ก ก ก

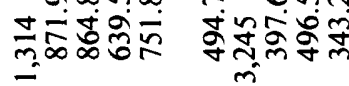

ㄴㅗㅀㅜㅇㅛ

ñ

กั่

요ำำ

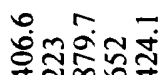
-ninin

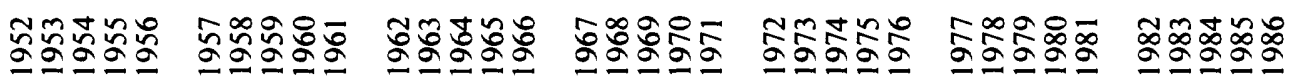




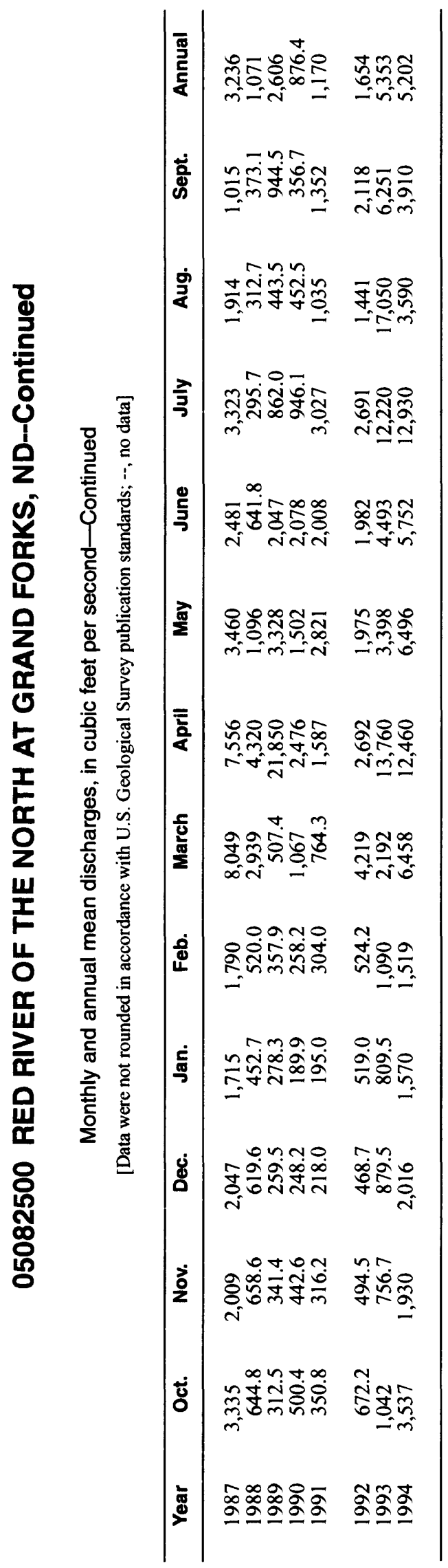




\section{TURTLE RIVER AT MANVEL, ND}

LOCATION.--Lat $48^{\circ} 04^{\prime} 43^{\prime \prime}$, long $97^{\circ} 11^{\prime} 03^{\prime \prime}$, in SE $1 / 4 \mathrm{sec} .10$, T.153 N., R.51 W., Grand Forks County, Hydrologic Unit 09020307, on left bank $10 \mathrm{ft}$ downstream from bridge on State Highway No. 33, $0.3 \mathrm{mi}$ west of Manvel, and $10 \mathrm{mi}$ upstream from mouth.

DRAINAGE AREA.--613 $\mathrm{mi}^{2}$, of which $57 \mathrm{mi}^{2}$ is probably noncontributing.

PERIOD OF RECORD.--October 1945 to September 1970 (continuous record); water year 1972-73 and December 1979 to September 1982 (gage heights and annual maximum discharge only).

GAGE.--Water-stage recorder. Datum of gage is $799.28 \mathrm{ft}$ above sea level. Prior to June 29, 1959, nonrecording gage at same site and datum.

EXTREMES FOR PERIOD OF RECORD.--Maximum discharge, $28,000 \mathrm{ft}^{3} / \mathrm{s}$, Apr. 19, 1950, from rating curve extended above $4,300 \mathrm{ft}^{3} / \mathrm{s}$ on basis of contracted-opening measurement of peak flow, gage height, $21.5 \mathrm{ft}$ from floodmark; no flow at times some years.

Annual mean discharge

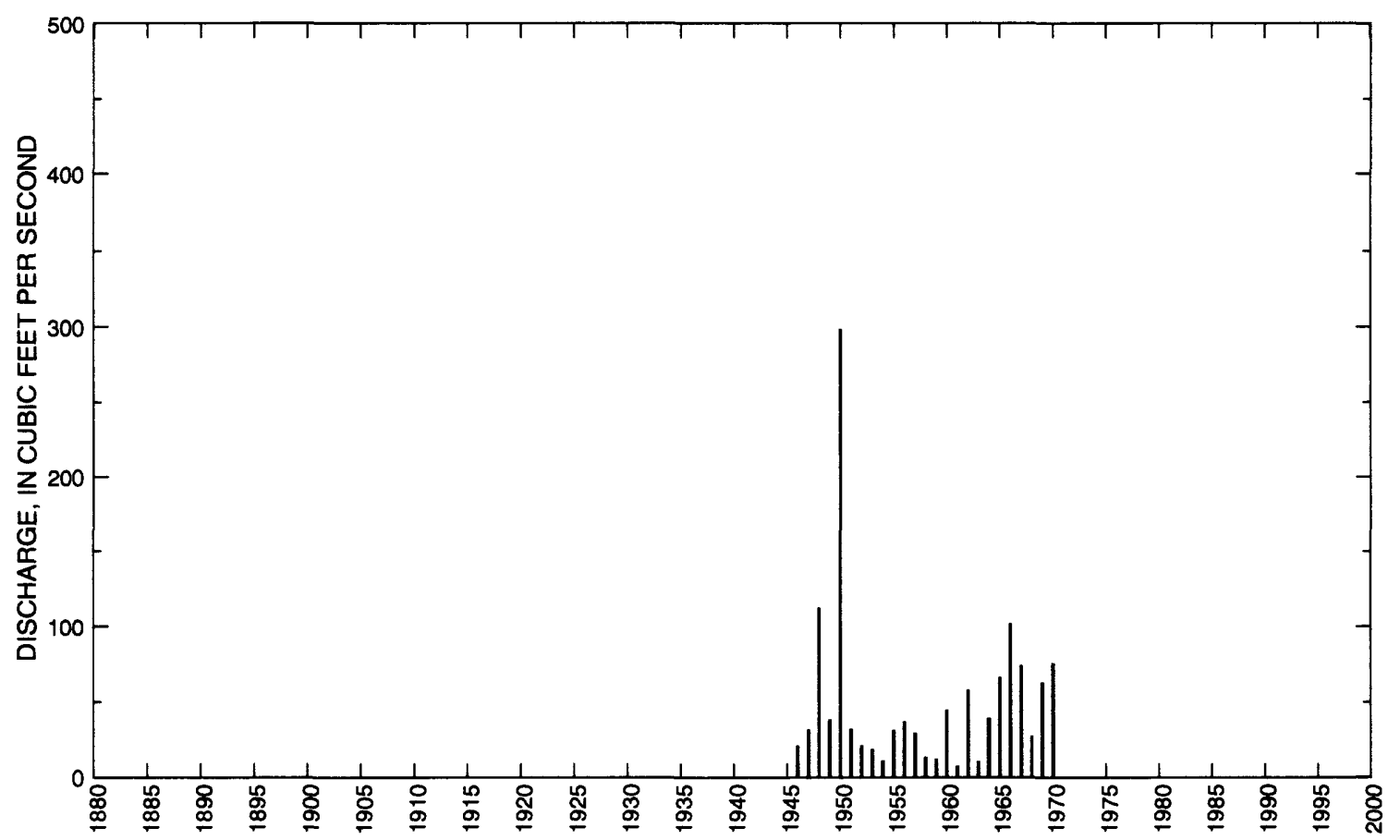




\section{TURTLE RIVER AT MANVEL, ND--Continued}

Statistics of monthly and annual mean discharges

[m, more than 1 year of occurrence]

\begin{tabular}{|c|c|c|c|c|c|c|c|c|}
\hline \multirow[b]{2}{*}{ Month } & \multicolumn{2}{|c|}{ Maximum } & \multicolumn{2}{|c|}{ Minimum } & \multicolumn{4}{|c|}{ Mean } \\
\hline & $\begin{array}{c}\text { Discharge } \\
\left(\mathrm{ft}^{3} / \mathrm{s}\right)\end{array}$ & $\begin{array}{c}\text { Water year } \\
\text { of } \\
\text { occurrence }\end{array}$ & $\begin{array}{c}\text { Discharge } \\
\left(\mathrm{ft}^{3} / \mathrm{s}\right)\end{array}$ & $\begin{array}{l}\text { Water year } \\
\text { of } \\
\text { occurrence }\end{array}$ & $\begin{array}{c}\text { Discharge } \\
\left(\mathrm{ft}^{3} / \mathrm{s}\right)\end{array}$ & $\begin{array}{c}\text { Standard } \\
\text { deviation } \\
\left(\mathrm{ft}^{3} / \mathrm{s}\right)\end{array}$ & $\begin{array}{l}\text { Coeffi- } \\
\text { cient of } \\
\text { variation }\end{array}$ & $\begin{array}{l}\text { Percentage } \\
\text { of annuail } \\
\text { discharge }\end{array}$ \\
\hline October & 49.1 & 1966 & 0.626 & 1961 & 8.86 & 11.4 & 1.29 & 1.44 \\
\hline November & 24.9 & 1958 & 0.643 & 1964 & 7.75 & 5.21 & 0.67 & 1.26 \\
\hline December & 10.2 & 1966 & 0.097 & 1964 & 3.66 & 2.76 & 0.75 & 0.59 \\
\hline January & 5.10 & 1951 & 0 & $\mathrm{~m}$ & 1.45 & 1.62 & 1.12 & 0.24 \\
\hline February & 4.84 & 1967 & 0 & $\mathrm{~m}$ & 0.940 & 1.27 & 1.34 & 0.15 \\
\hline March & 479 & 1966 & 0 & $\mathrm{~m}$ & 46.9 & 97.6 & 2.08 & 7.61 \\
\hline April & 1990 & 1950 & 23.2 & 1958 & 348 & 437 & 1.26 & 56.4 \\
\hline May & 1460 & 1950 & 7.87 & 1946 & 105 & 286 & 2.72 & 17.1 \\
\hline June & 269 & 1964 & 3.43 & 1961 & 52.8 & 59.6 & 1.13 & 8.56 \\
\hline July & 57.9 & 1968 & 1.15 & 1961 & 19.7 & 16.5 & 0.83 & 3.20 \\
\hline August & 22.1 & 1966 & 0.287 & 1946 & 5.91 & 5.10 & 0.86 & 0.96 \\
\hline September & 215 & 1957 & 0.180 & 1959 & 15.8 & 42.9 & 2.72 & 2.55 \\
\hline Annual & 299 & 1950 & 7.51 & 1961 & 51.2 & 58.8 & 1.15 & 100 \\
\hline
\end{tabular}

Annual flow duration

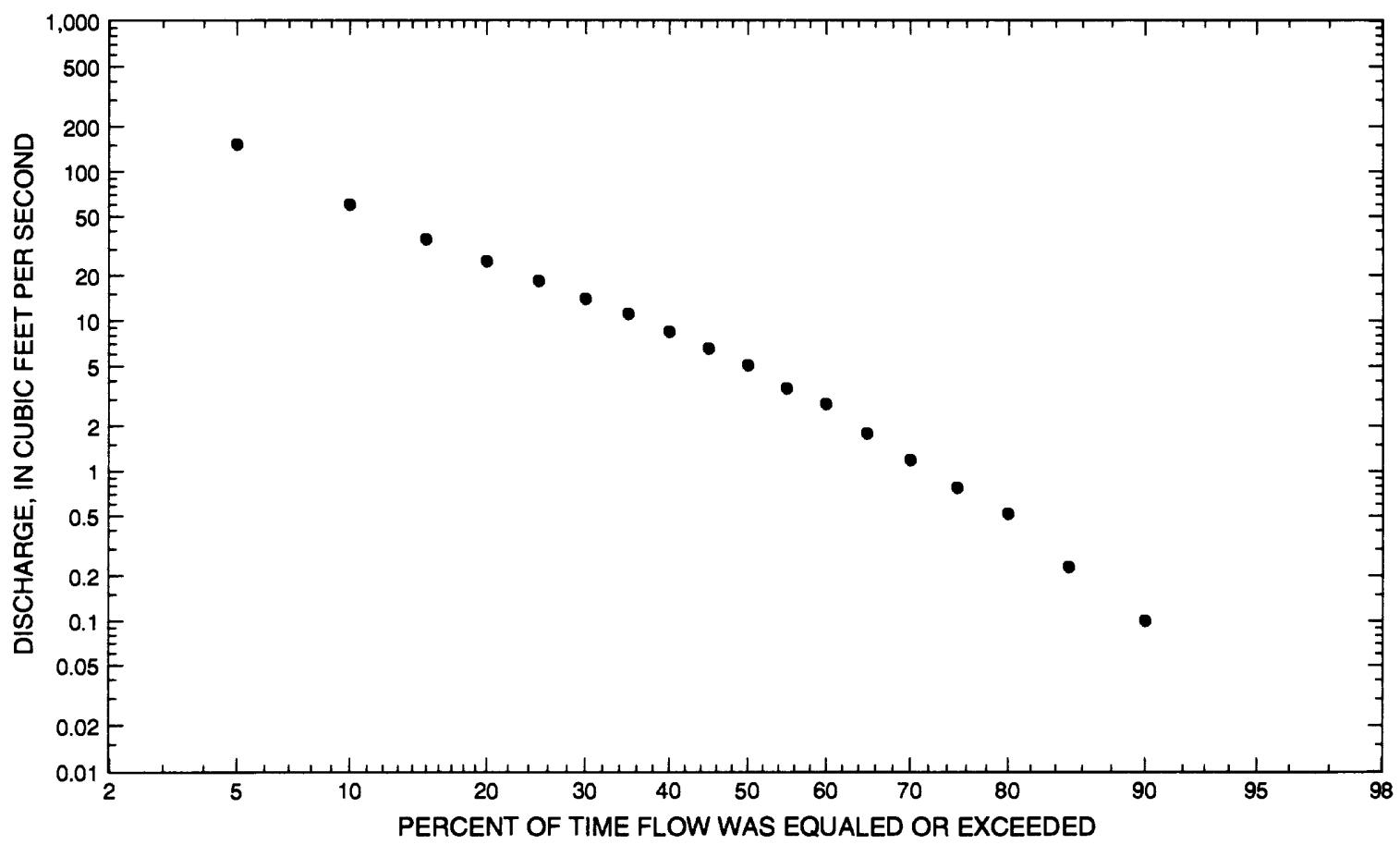




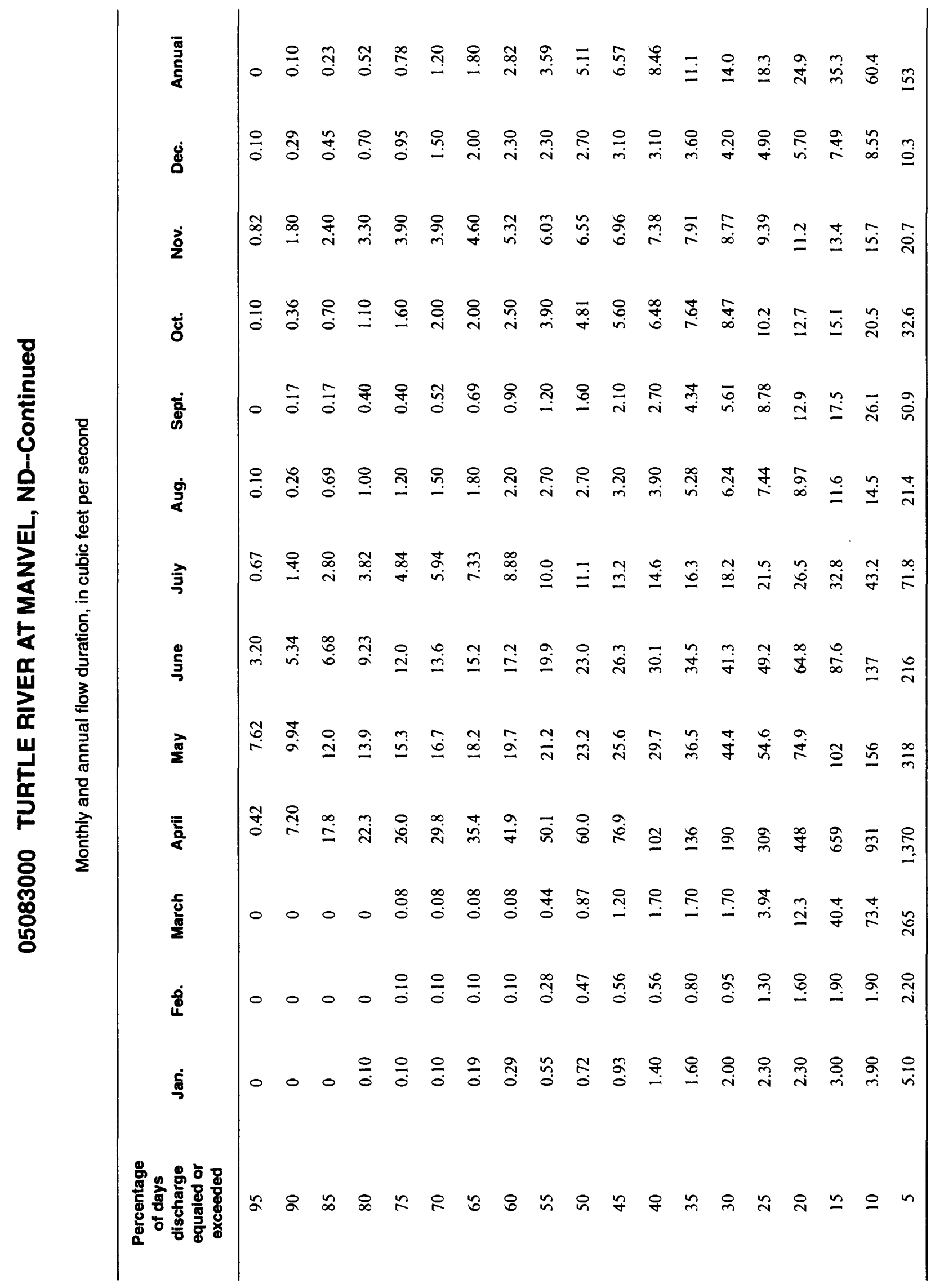




\section{TURTLE RIVER AT MANVEL, ND--Continued}

Probability of occurrence of annual high discharges

[ng, statistic not given]

\begin{tabular}{|c|c|c|c|c|c|c|}
\hline \multirow[b]{2}{*}{$\begin{array}{l}\text { Exceedance } \\
\text { probability }\end{array}$} & \multirow[b]{2}{*}{$\begin{array}{c}\text { Recurrence } \\
\text { intervai } \\
\text { (years) }\end{array}$} & \multirow{2}{*}{$\begin{array}{c}\text { Maximum } \\
\text { instantaneous } \\
\left(\mathrm{ft}^{3} / \mathrm{s}\right)\end{array}$} & \multicolumn{4}{|c|}{$\begin{array}{l}\text { Maximum mean discharge } \\
\left(\mathrm{ft}^{3} / \mathrm{s}\right)\end{array}$} \\
\hline & & & 3-day period & 7-day period & 15-day period & 30-day period \\
\hline 0.99 & 1.01 & 36.9 & 45.2 & 34.5 & 26.9 & 24.5 \\
\hline 0.95 & 1.05 & 87.8 & 100 & 82.8 & 62.6 & 47.2 \\
\hline 0.90 & 1.11 & 140 & 154 & 131 & 96.9 & 67.7 \\
\hline 0.80 & 1.25 & 249 & 262 & 225 & 163 & 106 \\
\hline 0.50 & 2 & 757 & 739 & 620 & 423 & 255 \\
\hline 0.20 & 5 & 2,360 & 2,150 & 1,650 & 1,050 & 638 \\
\hline 0.10 & 10 & 4,320 & 3,820 & 2,710 & 1,660 & 1,050 \\
\hline 0.04 & 25 & 8,270 & 7,090 & 4,550 & 2,680 & 1,800 \\
\hline 0.02 & 50 & 12,600 & 10,600 & 6,330 & 3,610 & 2,570 \\
\hline 0.01 & 100 & 18,600 & 15,400 & 8,480 & 4,710 & 3,560 \\
\hline 0.005 & 200 & 26,500 & 21,600 & 11,100 & 5,980 & 4,810 \\
\hline 0.002 & 500 & 40,700 & ng & $\mathrm{ng}$ & ng & ng \\
\hline
\end{tabular}

Probability of occurrence of annual low discharges

Minimum mean discharge $\left(\mathrm{ft}^{3} / \mathrm{s}\right)$

\begin{tabular}{|c|c|c|c|c|c|c|c|c|c|c|}
\hline \multirow[b]{3}{*}{$\begin{array}{c}\text { Non- } \\
\text { exceed- } \\
\text { ance } \\
\text { prob- } \\
\text { ability }\end{array}$} & \multirow[b]{3}{*}{$\begin{array}{l}\text { Recur- } \\
\text { rence } \\
\text { inter- } \\
\text { val } \\
\text { (years) }\end{array}$} & \multicolumn{9}{|c|}{ Minimum mean discharge $\left(\mathrm{ft}^{3} / \mathrm{s}\right)$} \\
\hline & & \multicolumn{9}{|c|}{ Number of consecutive days } \\
\hline & & 1 & 3 & 7 & 14 & 30 & 60 & 90 & 120 & 183 \\
\hline 0.05 & 20 & 0 & 0 & 0 & 0 & 0 & 0 & 0 & 0.144 & 0.701 \\
\hline 0.10 & 10 & 0 & 0 & 0 & 0 & 0 & 0 & 0.013 & 0.288 & 1.00 \\
\hline 0.20 & 5 & 0 & 0 & 0 & 0 & 0 & 0.024 & 0.112 & 0.598 & 1.52 \\
\hline 0.50 & 2 & 0 & 0 & 0.035 & 0.086 & 0.152 & 0.425 & 0.730 & 1.77 & 3.23 \\
\hline
\end{tabular}




\section{TURTLE RIVER AT MANVEL, ND--Continued}

Probability of occurrence of seasonal low discharges

\begin{tabular}{|c|c|c|c|c|c|c|c|c|c|}
\hline \multirow[b]{3}{*}{$\begin{array}{c}\text { Non- } \\
\text { exceedance } \\
\text { probability }\end{array}$} & \multirow[b]{3}{*}{$\begin{array}{c}\text { Recurrence } \\
\text { intervai } \\
\text { (years) }\end{array}$} & \multicolumn{8}{|c|}{ Minimum mean discharge $\left(\mathrm{ft}^{3} / \mathrm{s}\right)$} \\
\hline & & \multicolumn{8}{|c|}{ Number of consecutive days } \\
\hline & & 1 & 7 & 14 & 30 & 1 & 7 & 14 & 30 \\
\hline & & \multicolumn{4}{|c|}{ December-January-February } & \multicolumn{4}{|c|}{ March-April-May } \\
\hline 0.05 & 20 & 0 & 0 & 0 & 0 & 0 & 0 & 0 & 0 \\
\hline 0.10 & 10 & 0 & 0 & 0 & 0 & 0 & 0 & 0 & 0.009 \\
\hline 0.20 & 5 & 0 & 0 & 0 & 0 & 0 & 0 & 0 & 0.186 \\
\hline \multirow[t]{2}{*}{0.50} & 2 & 0.203 & 0.223 & 0.267 & 0.339 & 0.321 & ${ }^{1} 0.375$ & 0.423 & 3.53 \\
\hline & & \multicolumn{4}{|c|}{ June-July-August } & \multicolumn{4}{|c|}{ September-October-November } \\
\hline 0.05 & 20 & 0 & 0.037 & 0.137 & 0.464 & 0 & 0 & 0.006 & 0.039 \\
\hline 0.10 & 10 & 0.047 & 0.138 & 0.255 & 0.759 & 0 & 0 & 0.039 & 0.145 \\
\hline 0.20 & 5 & 0.184 & 0.332 & 0.515 & 1.33 & 0 & 0.082 & 0.129 & 0.361 \\
\hline 0.50 & 2 & 0.788 & 1.20 & 1.68 & 3.45 & 0.334 & 0.590 & 0.838 & 1.61 \\
\hline
\end{tabular}

${ }^{1}$ Graphical interpretation. 


\section{TURTLE RIVER AT MANVEL, ND--Continued}

Annual peak discharge and corresponding gage height

\begin{tabular}{|c|c|c|c|c|c|c|c|}
\hline $\begin{array}{l}\text { Water } \\
\text { year }\end{array}$ & Date & $\begin{array}{c}\text { Gage } \\
\text { height } \\
\text { (feet) }\end{array}$ & $\begin{array}{c}\text { Peak } \\
\text { discharge } \\
\left(\mathrm{ft}^{3} / \mathrm{s}\right)\end{array}$ & $\begin{array}{l}\text { Water } \\
\text { year }\end{array}$ & Date & $\begin{array}{c}\text { Gage } \\
\text { height } \\
\text { (feet) }\end{array}$ & $\begin{array}{c}\text { Peak } \\
\text { discharge } \\
\left(\mathrm{ft}^{3} / \mathrm{s}\right)\end{array}$ \\
\hline \multicolumn{8}{|c|}{ Annual peak discharge, by year, and corresponding gage height } \\
\hline 1946 & March 23 & 14.38 & 700 & 1961 & March 24 & 9.53 & 110 \\
\hline 1947 & April 13 & 8.79 & 431 & 1962 & April 14 & 16.22 & 970 \\
\hline 1948 & April 19 & 17.88 & 7,130 & 1963 & April 6 & 7.97 & 150 \\
\hline 1949 & April 10 & 15.83 & 1,600 & 1964 & June 23 & 11.40 & 661 \\
\hline 1950 & April 19 & 21.50 & 28,000 & 1965 & April 13 & 16.92 & 3,150 \\
\hline 1951 & April 6 & 13.90 & 940 & 1966 & March 22 & 17.95 & 1,680 \\
\hline 1952 & April 7 & 13.90 & 600 & 1967 & March 31 & 16.64 & 2,000 \\
\hline 1953 & June 22 & 7.48 & 219 & 1968 & March 29 & 12.45 & 450 \\
\hline 1954 & April 11 & 8.45 & 100 & 1969 & April 13 & 17.38 & 3,470 \\
\hline 1955 & April 5 & 16.27 & 1,460 & 1970 & April 11 & 17.37 & 2,430 \\
\hline 1956 & April 20 & 16.20 & 1,500 & 1972 & March 19 & 16.75 & 2,000 \\
\hline 1957 & September 5 & 13.15 & 900 & 1973 & March 12 & 8.69 & 150 \\
\hline 1958 & October 9 & 6.04 & 135 & 1980 & April 3 & 13.08 & 500 \\
\hline 1959 & March 30 & 13.31 & 370 & 1981 & June 17 & 6.39 & 84.0 \\
\hline 1960 & April 7 & 13.90 & 1,080 & 1982 & April 2 & 15.74 & 578 \\
\hline \multicolumn{8}{|c|}{ Annual peak discharge, from highest to lowest, and corresponding gage height } \\
\hline 1950 & April 19 & 21.50 & 28,000 & 1946 & March 23 & 14.38 & 700 \\
\hline 1948 & April 19 & 17.88 & 7,130 & 1964 & June 23 & 11.40 & 661 \\
\hline 1969 & April 13 & 17.38 & 3,470 & 1952 & April 7 & 13.90 & 600 \\
\hline 1965 & April 13 & 16.92 & 3,150 & 1982 & April 2 & 15.74 & 578 \\
\hline 1970 & April 11 & 17.37 & 2,430 & 1980 & April 3 & 13.08 & 500 \\
\hline 1967 & March 31 & 16.64 & 2,000 & 1968 & March 29 & 12.45 & 450 \\
\hline 1972 & March 19 & 16.75 & 2,000 & 1947 & April 13 & 8.79 & 431 \\
\hline 1966 & March 22 & 17.95 & 1,680 & 1959 & March 30 & 13.31 & 370 \\
\hline 1949 & April 10 & 15.83 & 1,600 & 1953 & June 22 & 7.48 & 219 \\
\hline 1956 & April 20 & 16.20 & 1,500 & 1963 & April 6 & 7.97 & 150 \\
\hline 1955 & April 5 & 16.27 & 1,460 & 1973 & March 12 & 8.69 & 150 \\
\hline 1960 & April 7 & 13.90 & 1,080 & 1958 & October 9 & 6.04 & 135 \\
\hline 1962 & April 14 & 16.22 & 970 & 1961 & March 24 & 9.53 & 110 \\
\hline 1957 & September 5 & 13.15 & 900 & 1954 & April 11 & 8.45 & 100 \\
\hline 1951 & April 6 & 13.90 & 940 & 1981 & June 17 & 6.39 & 84.0 \\
\hline
\end{tabular}


焉

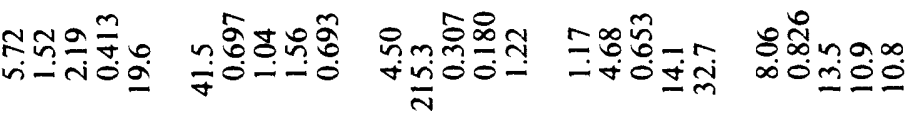

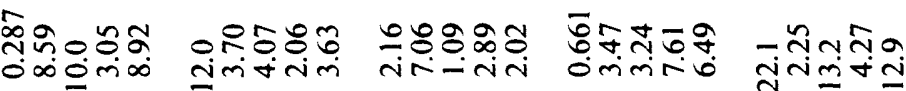

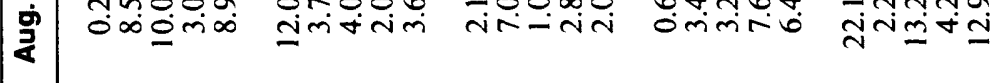

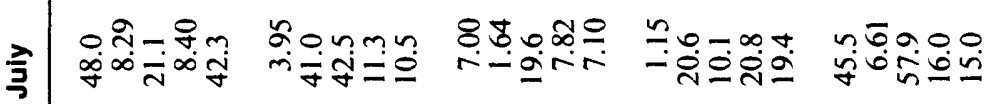

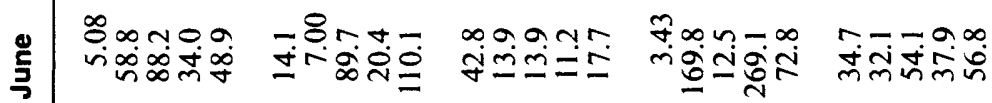

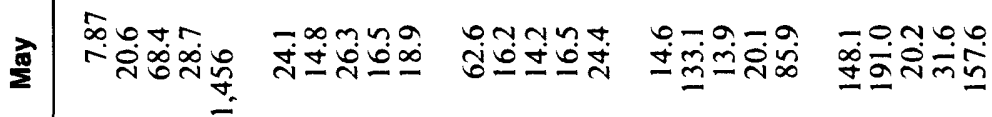

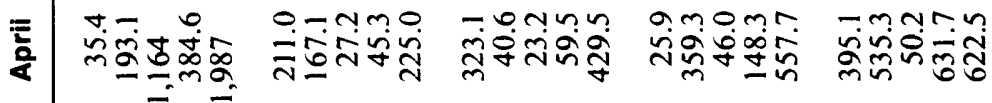

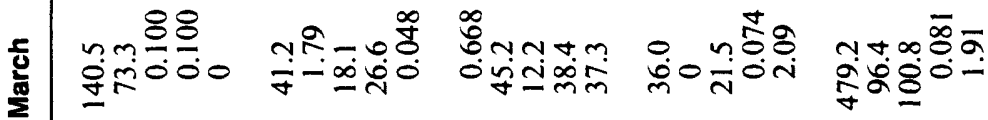

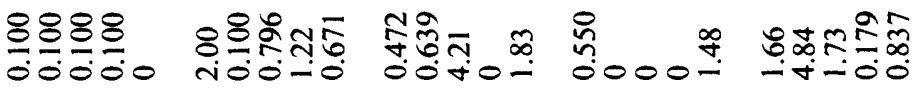

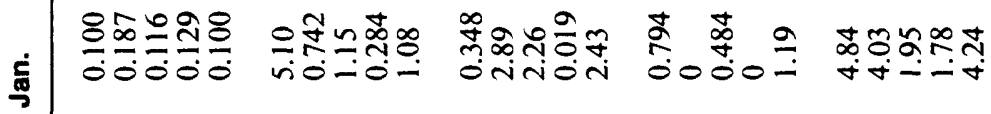
\&

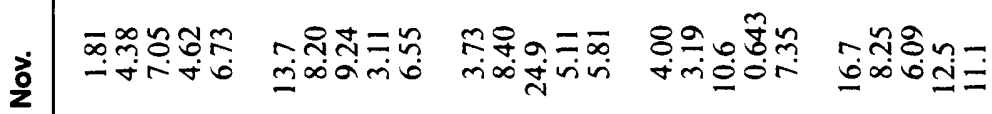

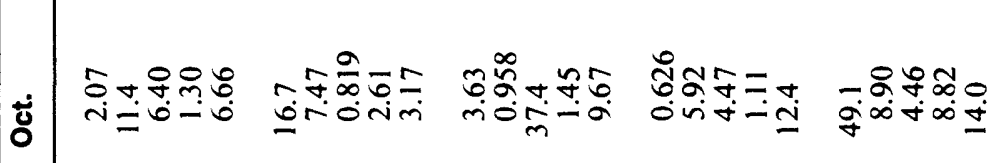

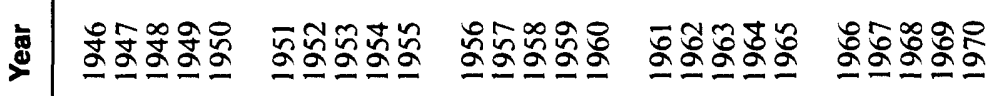




\section{RED RIVER OF THE NORTH AT OSLO, MN}

LOCATION.--Lat $48^{\circ} 11^{\prime} 40^{\prime \prime}$, long 9708'30', in SW $1 / 4 \mathrm{SW}^{1 / 4}$ sec.36, T.155 N., R.51 W., Walsh County, Hydrologic Unit 09020306, on bridge crossing the Red River 0.5 mi west of Oslo, Minn.

DRAINAGE AREA.--31,200 $\mathrm{mi}^{2}$, approximately, including $3,800 \mathrm{mi}^{2}$ in closed basins.

PERIOD OF RECORD.-- 1936 to 1937,1941 to 1943,1945 to 1960,1974 to 1976 , operated as a continuous-record gaging station; 1985 to current, operated as a crest-stage station.

GAGE.--Wire-weight gage. Datum of gage is $772.65 \mathrm{ft}$ above mean sea level, datum of 1929. Prior to Apr. 2, 1948, staff gage on railroad bridge $200 \mathrm{ft}$ upstream and Apr. 3, 1948, to Sept. 8, 1959, wireweight gage at bridge $620 \mathrm{ft}$ downstream, both at datum $5.00 \mathrm{ft}$ higher.

EXTREMES FOR PERIOD OF RECORD.--Maximum discharge, 63,000 $\mathrm{ft}^{3} / \mathrm{s}$, May 10, 1950, gage height, $31.83 \mathrm{ft}$, site and datum then in use; maximum gage height, $36.72 \mathrm{ft}$, Apr. 14, 1989; no flow at times.

EXTREMES OUTSIDE PERIOD OF RECORD.--Maximum stage known, about $37.5 \mathrm{ft}$ in 1897, present datum.

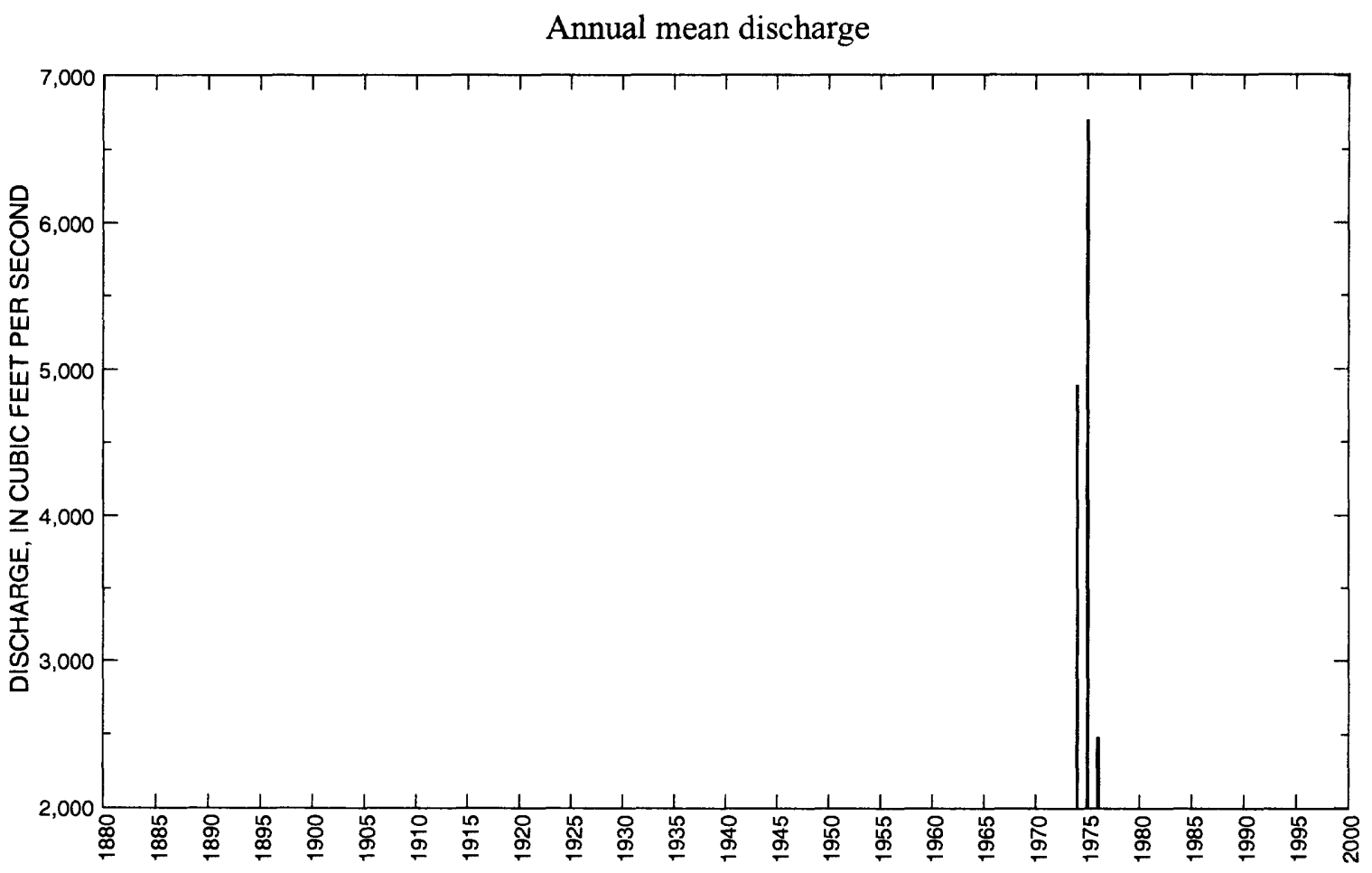


05083500 RED RIVER OF THE NORTH AT OSLO, MN--Continued

Statistics of monthly and annual mean discharges

\begin{tabular}{|c|c|c|c|c|c|c|c|c|}
\hline \multirow[b]{2}{*}{ Month } & \multicolumn{2}{|c|}{ Maximum } & \multicolumn{2}{|c|}{ Minimum } & \multicolumn{4}{|c|}{ Mean } \\
\hline & $\begin{array}{c}\text { Discharge } \\
\left(\mathrm{ft}^{3} / \mathrm{s}\right)\end{array}$ & $\begin{array}{c}\text { Water year } \\
\text { of } \\
\text { occurrence }\end{array}$ & $\begin{array}{c}\text { Discharge } \\
\left(\mathrm{ft}^{3} / \mathrm{s}\right)\end{array}$ & $\begin{array}{c}\text { Water year } \\
\text { of } \\
\text { occurrence }\end{array}$ & $\begin{array}{c}\text { Discharge } \\
\left(\mathrm{ft}^{3} / \mathrm{s}\right)\end{array}$ & $\begin{array}{c}\text { Standard } \\
\text { deviation } \\
\left(\mathrm{ft}^{3} / \mathbf{s}\right)\end{array}$ & $\begin{array}{l}\text { Coeffi- } \\
\text { cient of } \\
\text { varlation }\end{array}$ & $\begin{array}{l}\text { Percentage } \\
\text { of annual } \\
\text { discharge }\end{array}$ \\
\hline October & 3,770 & 1974 & 13.8 & 1937 & 2,020 & 1,550 & 0.77 & 5.14 \\
\hline November & 2,440 & 1974 & 2,020 & 1975 & 2,270 & 222 & 0.10 & 5.79 \\
\hline December & 1,940 & 1974 & 1,240 & 1975 & 1,650 & 368 & 0.22 & 4.22 \\
\hline January & 1,740 & 1974 & 1,300 & 1975 & 1,580 & 244 & 0.15 & 4.03 \\
\hline February & 1,680 & 1976 & 1,430 & 1975 & 1,590 & 139 & 0.09 & 4.06 \\
\hline March & 3,310 & 1976 & 1,730 & 1975 & 2,350 & 837 & 0.36 & 6.00 \\
\hline April & 27,000 & 1950 & 1,520 & 1937 & 9,710 & 6,700 & 0.69 & 24.8 \\
\hline May & 40,900 & 1950 & 1,430 & 1958 & 6,780 & 8,540 & 1.26 & 17.3 \\
\hline June & 11,800 & 1950 & 286 & 1936 & 4,150 & 3,090 & 0.74 & 10.6 \\
\hline July & 25,600 & 1975 & 92.9 & 1936 & 4,560 & 5,790 & 1.27 & 11.6 \\
\hline August & 3,170 & 1949 & 31.2 & 1936 & 1,630 & 1,040 & 0.64 & 4.16 \\
\hline September & 2,240 & 1975 & 18.9 & 1936 & 904 & 808 & 0.89 & 2.31 \\
\hline Annual & 6,700 & 1975 & 2,480 & 1976 & 4,690 & 2,120 & 0.45 & 100 \\
\hline
\end{tabular}

Annual flow duration

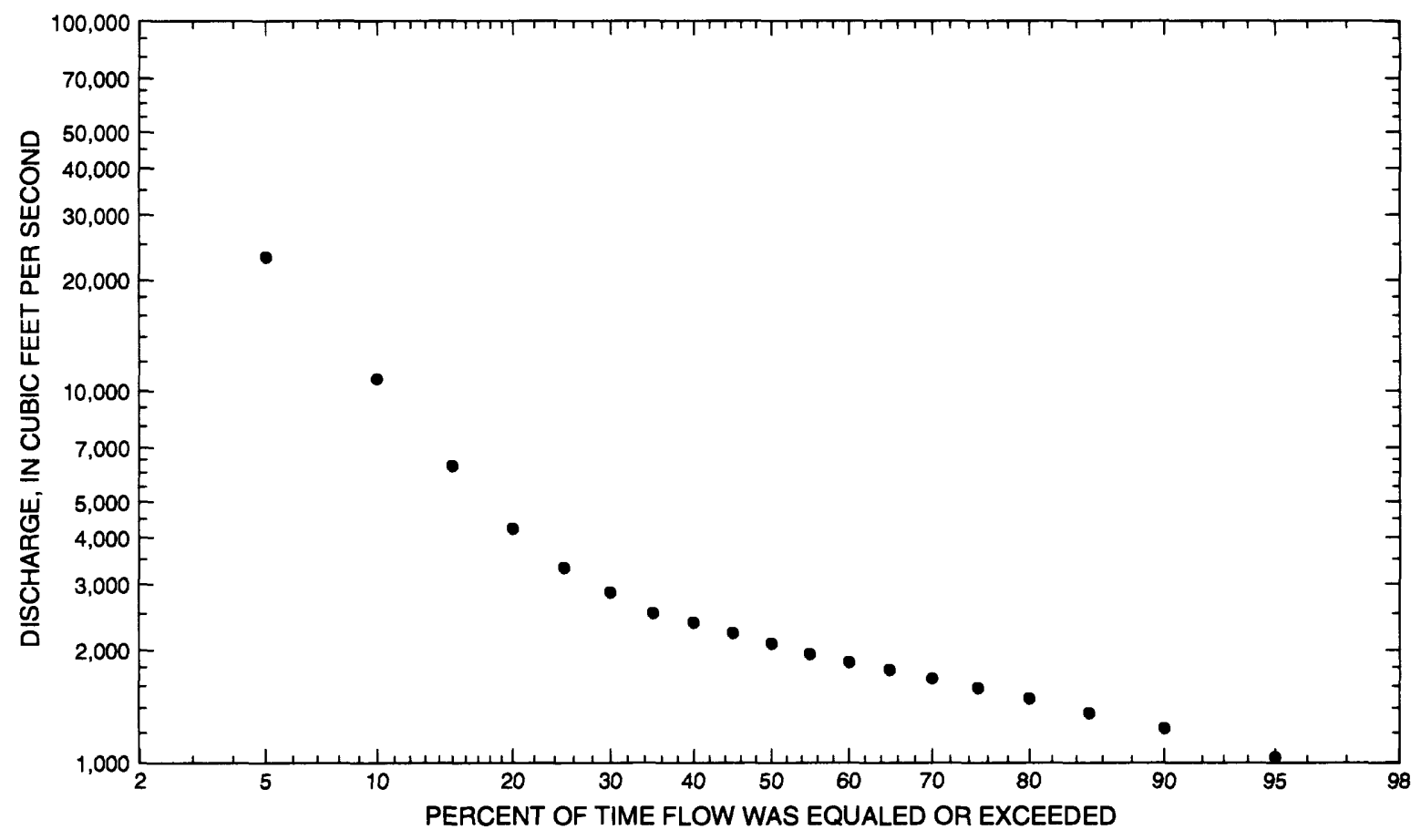




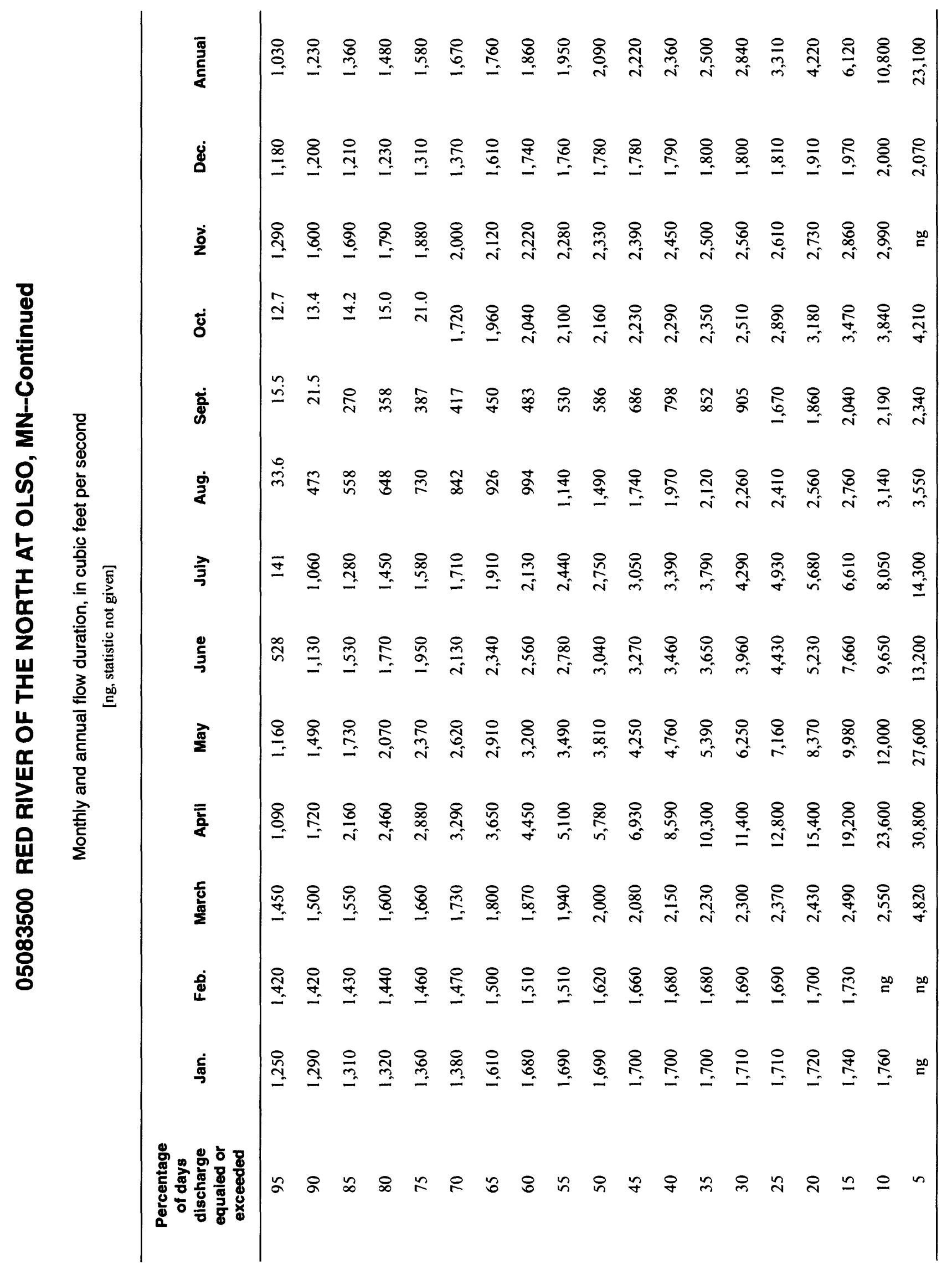




\section{RED RIVER OF THE NORTH AT OSLO, MN--Continued}

Probability of occurrence of annual high discharges

[ng, statistic not given]

\begin{tabular}{lccccccc}
\hline & & & \multicolumn{5}{c}{$\begin{array}{c}\text { Maximum mean discharge } \\
\text { (ft } \mathbf{3}^{3 / \mathbf{s})}\end{array}$} \\
\cline { 6 - 8 } $\begin{array}{c}\text { Exceedance } \\
\text { probability }\end{array}$ & $\begin{array}{c}\text { Recurrence } \\
\text { Interval } \\
\text { (years) }\end{array}$ & $\begin{array}{c}\text { Maximum } \\
\text { instantaneous } \\
\left(\mathbf{f t}^{3} / \mathbf{s}\right)\end{array}$ & 3-day period & 7-day period & 15-day period & 30-day period \\
\hline 0.99 & 1.01 & 2,990 & $\mathrm{ng}$ & $\mathrm{ng}$ & $\mathrm{ng}$ & $\mathrm{ng}$ \\
0.95 & 1.05 & 5,440 & $\mathrm{ng}$ & $\mathrm{ng}$ & $\mathrm{ng}$ & $\mathrm{ng}$ \\
0.90 & 1.11 & 7,320 & $\mathrm{ng}$ & $\mathrm{ng}$ & $\mathrm{ng}$ & $\mathrm{ng}$ \\
0.80 & 1.25 & 10,300 & $\mathrm{ng}$ & $\mathrm{ng}$ & $\mathrm{ng}$ & $\mathrm{ng}$ \\
0.50 & 2 & 18,600 & $\mathrm{ng}$ & $\mathrm{ng}$ & $\mathrm{ng}$ & $\mathrm{ng}$ \\
0.20 & 5 & 31,400 & $\mathrm{ng}$ & $\mathrm{ng}$ & $\mathrm{ng}$ & $\mathrm{ng}$ \\
0.10 & 10 & 40,100 & $\mathrm{ng}$ & $\mathrm{ng}$ & $\mathrm{ng}$ & $\mathrm{ng}$ \\
0.04 & 25 & 51,000 & $\mathrm{ng}$ & $\mathrm{ng}$ & $\mathrm{ng}$ & $\mathrm{ng}$ \\
0.02 & 50 & 59,000 & $\mathrm{ng}$ & $\mathrm{ng}$ & $\mathrm{ng}$ & $\mathrm{ng}$ \\
0.01 & 100 & 66,700 & $\mathrm{ng}$ & $\mathrm{ng}$ & $\mathrm{ng}$ & $\mathrm{ng}$ \\
0.005 & 200 & 74,300 & $\mathrm{ng}$ & $\mathrm{ng}$ & $\mathrm{ng}$ & $\mathrm{ng}$ \\
0.002 & 500 & 84,000 & $\mathrm{ng}$ & $\mathrm{ng}$ & $\mathrm{ng}$ & $\mathrm{ng}$ \\
\hline
\end{tabular}

Probability of occurrence of annual low discharges

[ng, statistic not given]

\begin{tabular}{|c|c|c|c|c|c|c|c|c|c|c|}
\hline \multirow{3}{*}{$\begin{array}{l}\text { Non- } \\
\text { exceed- } \\
\text { ance } \\
\text { prob- } \\
\text { ability }\end{array}$} & \multirow{3}{*}{$\begin{array}{c}\text { Recur- } \\
\text { rence } \\
\text { inter- } \\
\text { val } \\
\text { (years) }\end{array}$} & \multicolumn{9}{|c|}{ Minimum mean discharge $\left(\mathrm{ft}^{3} / \mathrm{s}\right)$} \\
\hline & & \multicolumn{9}{|c|}{ Number of consecutive days } \\
\hline & & 1 & 3 & 7 & 14 & 30 & 60 & 90 & 120 & 183 \\
\hline 0.05 & 20 & $\mathrm{ng}$ & $\mathrm{ng}$ & $\mathrm{ng}$ & $\mathrm{ng}$ & $\mathrm{ng}$ & $\mathrm{ng}$ & $\mathrm{ng}$ & $\mathrm{ng}$ & $\mathrm{ng}$ \\
\hline 0.10 & 10 & $\mathrm{ng}$ & $\mathrm{ng}$ & ng & $\mathrm{ng}$ & $\mathrm{ng}$ & $\mathrm{ng}$ & $\mathrm{ng}$ & $\mathrm{ng}$ & ng \\
\hline 0.20 & 5 & $\mathrm{ng}$ & $\mathrm{ng}$ & $\mathrm{ng}$ & $\mathrm{ng}$ & $\mathrm{ng}$ & $\mathrm{ng}$ & $\mathrm{ng}$ & $\mathrm{ng}$ & $\mathrm{ng}$ \\
\hline 0.50 & 2 & $\mathrm{ng}$ & $\mathrm{ng}$ & $\mathrm{ng}$ & $\mathrm{ng}$ & $\mathrm{ng}$ & $\mathrm{ng}$ & $\mathrm{ng}$ & $\mathrm{ng}$ & $\mathrm{ng}$ \\
\hline
\end{tabular}


05083500 RED RIVER OF THE NORTH AT OSLO, MN--Continued

Probability of occurrence of seasonal low discharges

[ng, statistic not given]

\begin{tabular}{|c|c|c|c|c|c|c|c|c|c|}
\hline \multirow[b]{3}{*}{$\begin{array}{l}\text { Non- } \\
\text { exceedance } \\
\text { probability }\end{array}$} & \multirow[b]{3}{*}{$\begin{array}{c}\text { Recurrence } \\
\text { interval } \\
\text { (years) }\end{array}$} & \multicolumn{8}{|c|}{ Minimum mean discharge $\left(\mathrm{ft}^{3} / \mathrm{s}\right)$} \\
\hline & & \multicolumn{8}{|c|}{ Number of consecutive days } \\
\hline & & 1 & 7 & 14 & 30 & 1 & 7 & 14 & 30 \\
\hline & & \multicolumn{4}{|c|}{ December-January-February } & \multicolumn{4}{|c|}{ March-April-May } \\
\hline 0.05 & 20 & ng & ng & ng & ng & ng & ng & ng & ng \\
\hline 0.10 & 10 & ng & ng & ng & ng & ng & ng & ng & ng \\
\hline 0.20 & 5 & ng & ng & $\mathrm{ng}$ & ng & ng & ng & ng & ng \\
\hline \multirow[t]{2}{*}{0.50} & 2 & ng & ng & ng & ng & ng & $\mathrm{ng}$ & ng & ng \\
\hline & & \multicolumn{4}{|c|}{ June-July-August } & \multicolumn{4}{|c|}{ September-October-November } \\
\hline 0.05 & 20 & 73.5 & 78.5 & 87.3 & 93.8 & ng & ng & ng & ng \\
\hline 0.10 & 10 & 171 & 185 & 205 & 229 & ng & ng & ng & ng \\
\hline 0.20 & 5 & 392 & 426 & 473 & 546 & ng & $\mathrm{ng}$ & ng & $\mathrm{ng}$ \\
\hline 0.50 & 2 & 1,130 & 1,220 & 1,340 & 1,630 & ng & ng & ng & ng \\
\hline
\end{tabular}




\section{RED RIVER OF THE NORTH AT OSLO, MN-Continued}

Annual peak discharge and corresponding gage height

$$
[-, \text { no data }]
$$

\begin{tabular}{|c|c|c|c|c|c|c|c|}
\hline $\begin{array}{l}\text { Water } \\
\text { year }\end{array}$ & Date & $\begin{array}{c}\text { Gage } \\
\text { height } \\
\text { (feet) }\end{array}$ & $\begin{array}{c}\text { Peak } \\
\text { discharge } \\
\left(\mathrm{ft}^{3} / \mathrm{s}\right)\end{array}$ & $\begin{array}{l}\text { Water } \\
\text { year }\end{array}$ & Date & $\begin{array}{c}\text { Gage } \\
\text { height } \\
\text { (feet) }\end{array}$ & $\begin{array}{c}\text { Peak } \\
\text { discharge } \\
\left(\mathrm{ft}^{3} / \mathrm{s}\right)\end{array}$ \\
\hline \multicolumn{8}{|c|}{ Annual peak discharge, by year, and corresponding gage height } \\
\hline 1936 & April 18 & -- & 15,000 & 1958 & July 10 & 10.29 & 7,890 \\
\hline 1937 & May 4 & 6.47 & 4,070 & 1959 & April 7 & 10.78 & 7,200 \\
\hline 1942 & April 4 & -- & 12,500 & 1960 & April 12 & -. & 17,100 \\
\hline 1943 & April 13 & 29.16 & 31,500 & 1974 & April 19 & -- & $1_{33,000}^{-}$ \\
\hline 1945 & March 26 & - & 24,000 & 1975 & April 23 & -- & 142,500 \\
\hline 1946 & March 30 & 25.11 & - & 1976 & April 5 & -. & $1_{23,200}$ \\
\hline 1947 & April 22 & - & 33,800 & 1985 & May 20 & 24.43 & 17,800 \\
\hline 1948 & April 17 & - & 41,400 & 1986 & April 3 & 34.20 & 30,000 \\
\hline 1949 & April 10 & 24.08 & 18,700 & 1987 & March 30 & 31.76 & 18,500 \\
\hline 1950 & May 10 & 31.83 & 63,000 & 1988 & April 6 & 20.10 & 11,500 \\
\hline 1951 & April 12 & 25.46 & 24,800 & 1989 & April 14 & 36.72 & 33,500 \\
\hline 1952 & April 21 & - & 24,800 & 1990 & April 5 & 15.64 & 4,900 \\
\hline 1953 & June 25 & 17.55 & 14,900 & 1991 & July 10 & 12.04 & 5,200 \\
\hline 1954 & April 15 & 12.39 & 9,790 & 1992 & March 15 & 22.47 & 8,200 \\
\hline 1955 & April 10 & 19.46 & 16,400 & 1993 & April 7 & -- & 28,100 \\
\hline 1956 & April 24 & 25.50 & 22,500 & 1994 & July 13 & 30.86 & 26,600 \\
\hline 1957 & July 2 & 17.42 & 14,900 & & & & \\
\hline \multicolumn{8}{|c|}{ Annual peak discharge, from highest to lowest, and corresponding gage height } \\
\hline 1950 & May 10 & 31.83 & 63,000 & 1985 & May 20 & 24.43 & 17,800 \\
\hline 1975 & April 23 & - & $1_{42,500}$ & 1960 & April 12 & - & 17,100 \\
\hline 1948 & April 17 & - & 41,400 & 1955 & April 10 & 19.46 & 16,400 \\
\hline 1947 & April 22 & - & 33,800 & 1936 & April 18 & - & 15,000 \\
\hline 1989 & April 14 & 36.72 & 33,500 & 1953 & June 25 & 17.55 & 14,900 \\
\hline 1974 & April 19 & - & $1_{33,000}$ & 1957 & July 2 & 17.42 & 14,900 \\
\hline 1943 & April 13 & 29.16 & 31,500 & 1942 & April 4 & -- & 11,900 \\
\hline 1986 & April 3 & 34.20 & 30,000 & 1988 & April 6 & 20.10 & 11,500 \\
\hline 1993 & April 7 & -- & 28,100 & 1954 & April 15 & 12.39 & 9,790 \\
\hline 1994 & July 13 & 30.86 & 26,600 & 1992 & March 15 & 22.47 & 8,200 \\
\hline 1951 & April 12 & 25.46 & 24,800 & 1958 & July 10 & 10.29 & 7,890 \\
\hline 1952 & April 21 & - & 24,800 & 1959 & April 7 & 10.78 & 7,200 \\
\hline 1945 & March 26 & -- & 24,000 & 1991 & July 10 & 12.04 & 5,200 \\
\hline 1976 & April 5 & - & $\mathrm{l}_{23,200}$ & 1990 & April 5 & 15.64 & 4,900 \\
\hline 1956 & April 24 & 25.50 & 22,500 & 1937 & May 4 & 6.47 & 4,070 \\
\hline 1949 & April 10 & 24.08 & 18,700 & 1946 & March 30 & 25.11 & - \\
\hline 1987 & March 30 & 31.76 & 18,500 & & & & \\
\hline
\end{tabular}

${ }^{1}$ Daily mean. 


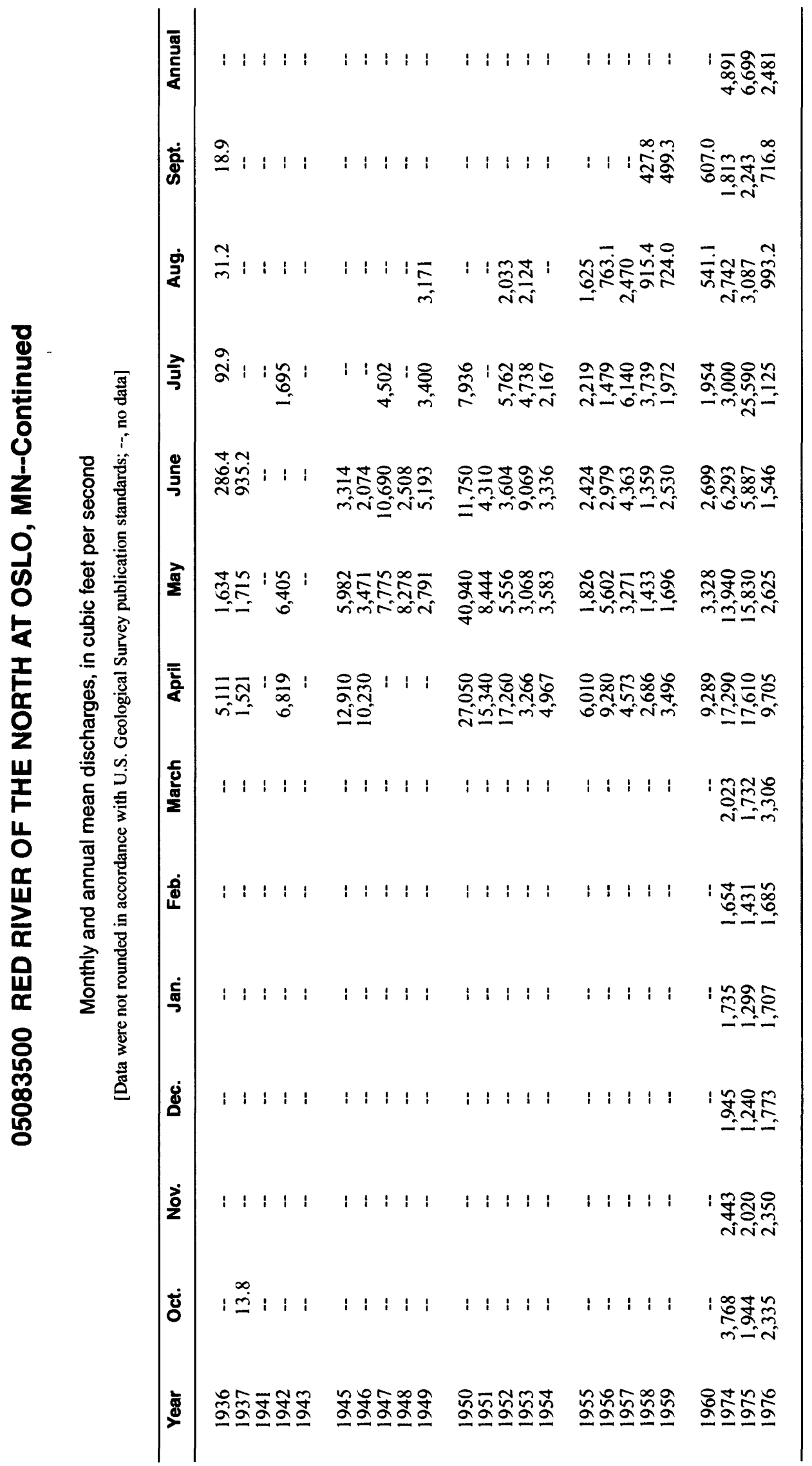




\section{MIDDLE BRANCH FOREST RIVER NEAR WHITMAN, ND}

LOCATION.--Lat $48^{\circ} 14^{\prime} 50^{\prime \prime}$, long $98^{\circ} 07^{\prime} 00^{\prime \prime}$, in $\mathrm{SE}^{1} / 4^{\mathrm{NW}}{ }^{1} / 4$ sec.16, T.155 N., R.58 W., Walsh County, Hydrologic Unit 09020308, $150 \mathrm{ft}$ downstream from bridge on State Highway 35, and 6 mi north of Whitman.

DRAINAGE AREA.--47.7 $\mathrm{mi}^{2}$, of which about $9 \mathrm{mi}^{2}$ is probably noncontributing.

PERIOD OF RECORD.--October 1960 to September 1990.

GAGE.--Water-stage recorder. Datum of gage is $1,510 \mathrm{ft}$ above sea level, from topographic map.

EXTREMES FOR PERIOD OF RECORD.--Maximum discharge, $984 \mathrm{ft}^{3} / \mathrm{s}$, May 19, 1974, gage height, $7.11 \mathrm{ft}$; maximum gage height, $7.96 \mathrm{ft}$, Apr. 4, 1987; no flow for many months each year.

Annual mean discharge

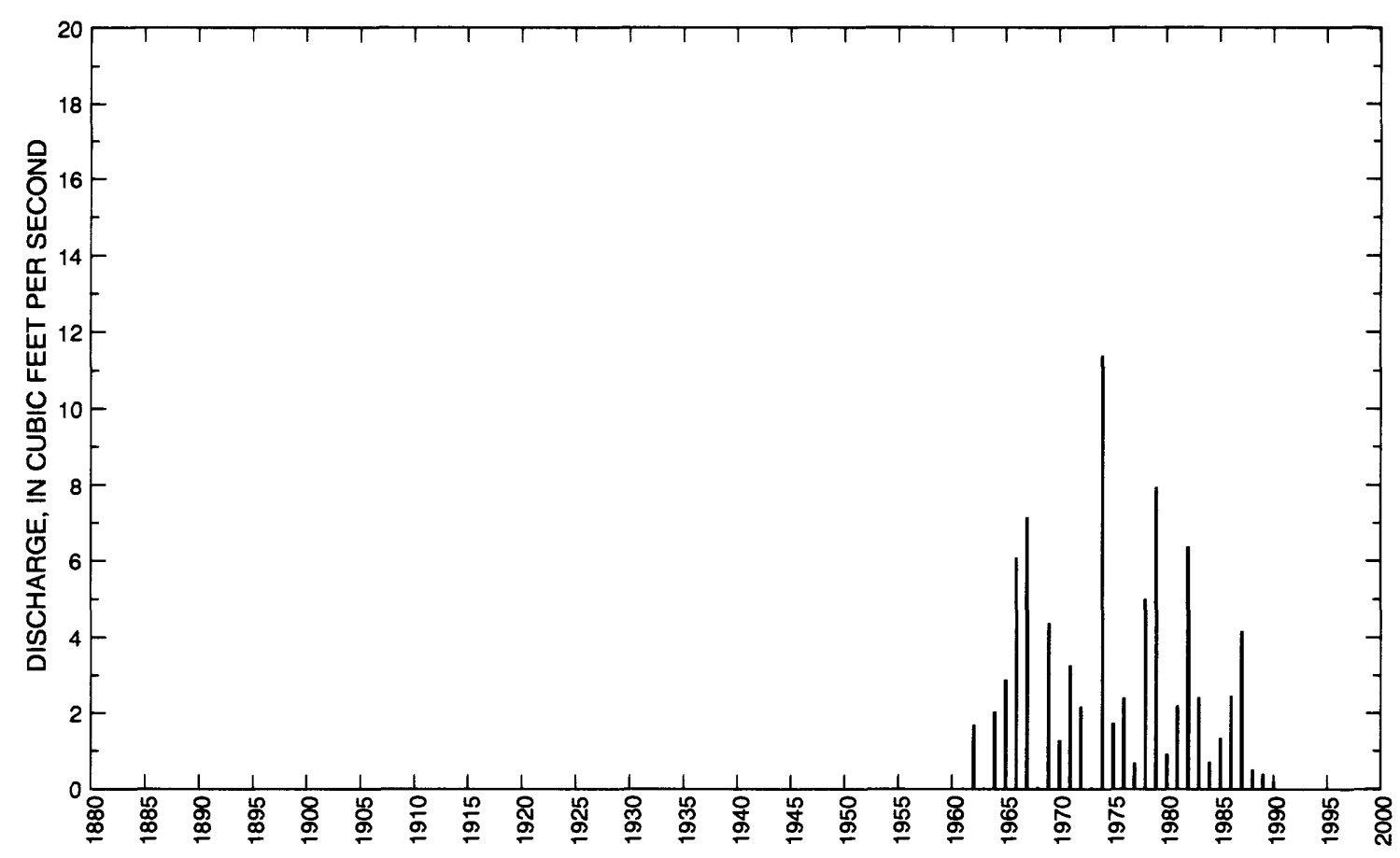


05083600 MIDDLE BRANCH FOREST RIVER NEAR WHITMAN, ND--Continued

Statistics of monthly and annual mean discharges

[ $\mathrm{m}$, more than 1 year of occurrence; ng, statistic not given]

\begin{tabular}{|c|c|c|c|c|c|c|c|c|}
\hline \multirow[b]{2}{*}{ Month } & \multicolumn{2}{|c|}{ Maximum } & \multicolumn{2}{|c|}{ Minimum } & \multicolumn{4}{|c|}{ Mean } \\
\hline & $\begin{array}{c}\text { Discharge } \\
\left(\mathrm{ft}^{3 / s}\right)\end{array}$ & $\begin{array}{l}\text { Water year } \\
\text { of } \\
\text { occurrence }\end{array}$ & $\begin{array}{c}\text { Discharge } \\
\left(\mathrm{ft}^{3} / \mathrm{s}\right)\end{array}$ & $\begin{array}{c}\text { Water year } \\
\text { of } \\
\text { occurrence }\end{array}$ & $\begin{array}{c}\text { Discharge } \\
\left(\mathrm{ft}^{3} / \mathrm{s}\right)\end{array}$ & $\begin{array}{c}\text { Standard } \\
\text { deviation } \\
\left(\mathrm{ft}^{3} / \mathbf{s}\right)\end{array}$ & $\begin{array}{l}\text { Coeffi- } \\
\text { cient of } \\
\text { variation }\end{array}$ & $\begin{array}{l}\text { Percentage } \\
\text { of annual } \\
\text { discharge }\end{array}$ \\
\hline October & 3.45 & 1983 & 0 & $\mathrm{~m}$ & 0.210 & 0.67 & 3.26 & 0.62 \\
\hline November & 0.439 & 1983 & 0 & $\mathrm{~m}$ & 0.040 & 0.10 & 2.63 & 0.11 \\
\hline December & 0.029 & 1982 & 0 & $\mathrm{~m}$ & 0 & 0.01 & 3.80 & 0 \\
\hline January & 0 & $\mathrm{~m}$ & 0 & $\mathrm{~m}$ & 0 & 0 & ng & 0 \\
\hline February & 7.10 & 1981 & 0 & $\mathrm{~m}$ & 0.240 & 1.30 & 5.48 & 0.72 \\
\hline March & 15.1 & 1966 & 0 & $\mathrm{~m}$ & 3.09 & 4.68 & 1.51 & 9.40 \\
\hline April & 87.9 & 1979 & 0 & $\mathrm{~m}$ & 19.5 & 22.5 & 1.15 & 59.4 \\
\hline May & 68.8 & 1974 & 0 & $\mathrm{~m}$ & 5.22 & 14.2 & 2.73 & 15.9 \\
\hline June & 19.5 & 1964 & 0 & $\mathrm{~m}$ & 1.75 & 4.36 & 2.48 & 5.33 \\
\hline July & 33.3 & 1982 & 0 & $\mathrm{~m}$ & 2.13 & 6.23 & 2.93 & 6.48 \\
\hline August & 7.02 & 1966 & 0 & $\mathrm{~m}$ & 0.560 & 1.63 & 2.94 & 1.69 \\
\hline September & 1.23 & 1981 & 0 & $\mathrm{~m}$ & 0.110 & 0.30 & 2.66 & 0.34 \\
\hline Annual & 11.4 & 1974 & 0.009 & 1963 & 2.73 & 2.77 & 1.01 & 100 \\
\hline
\end{tabular}

Annual flow duration

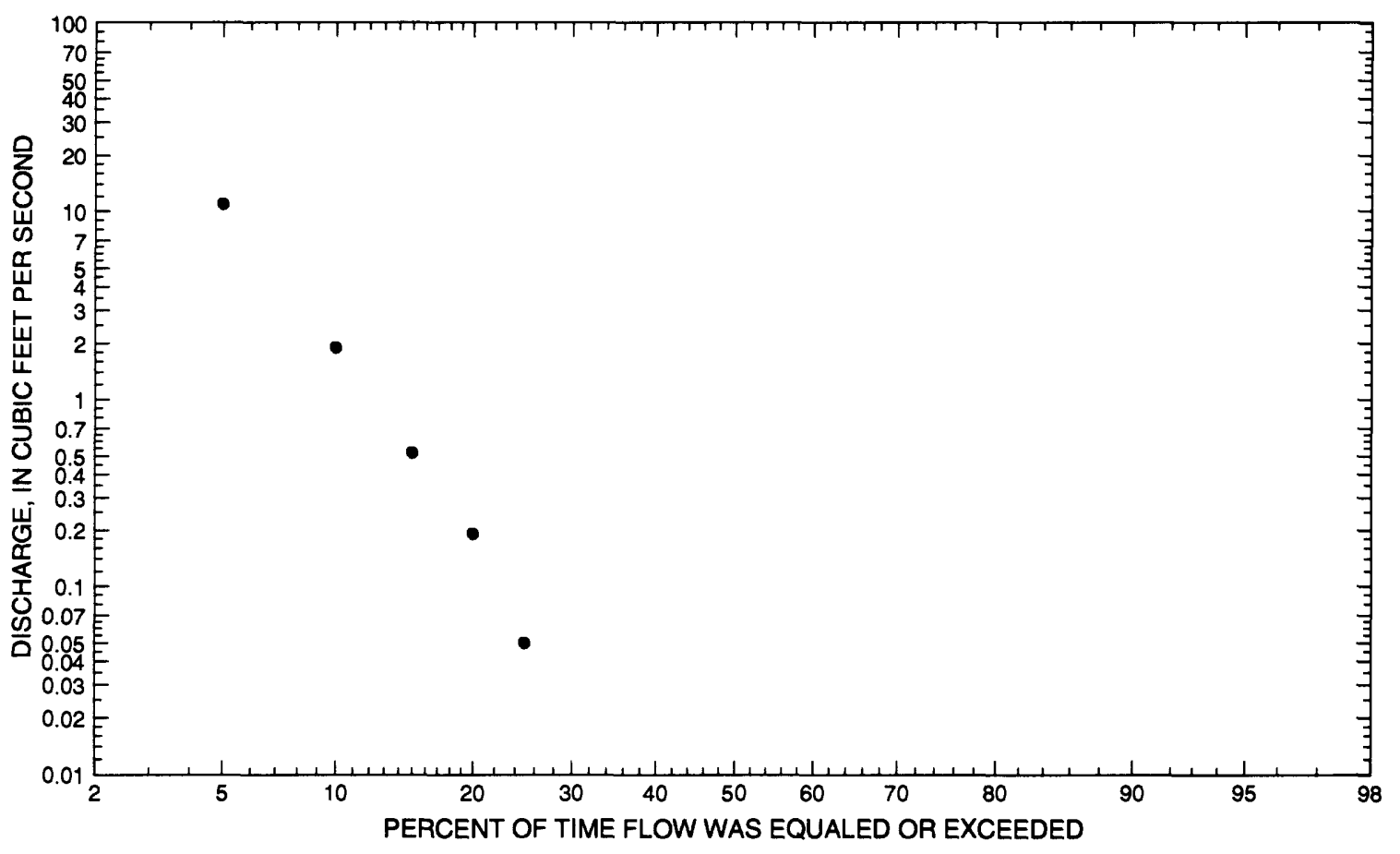




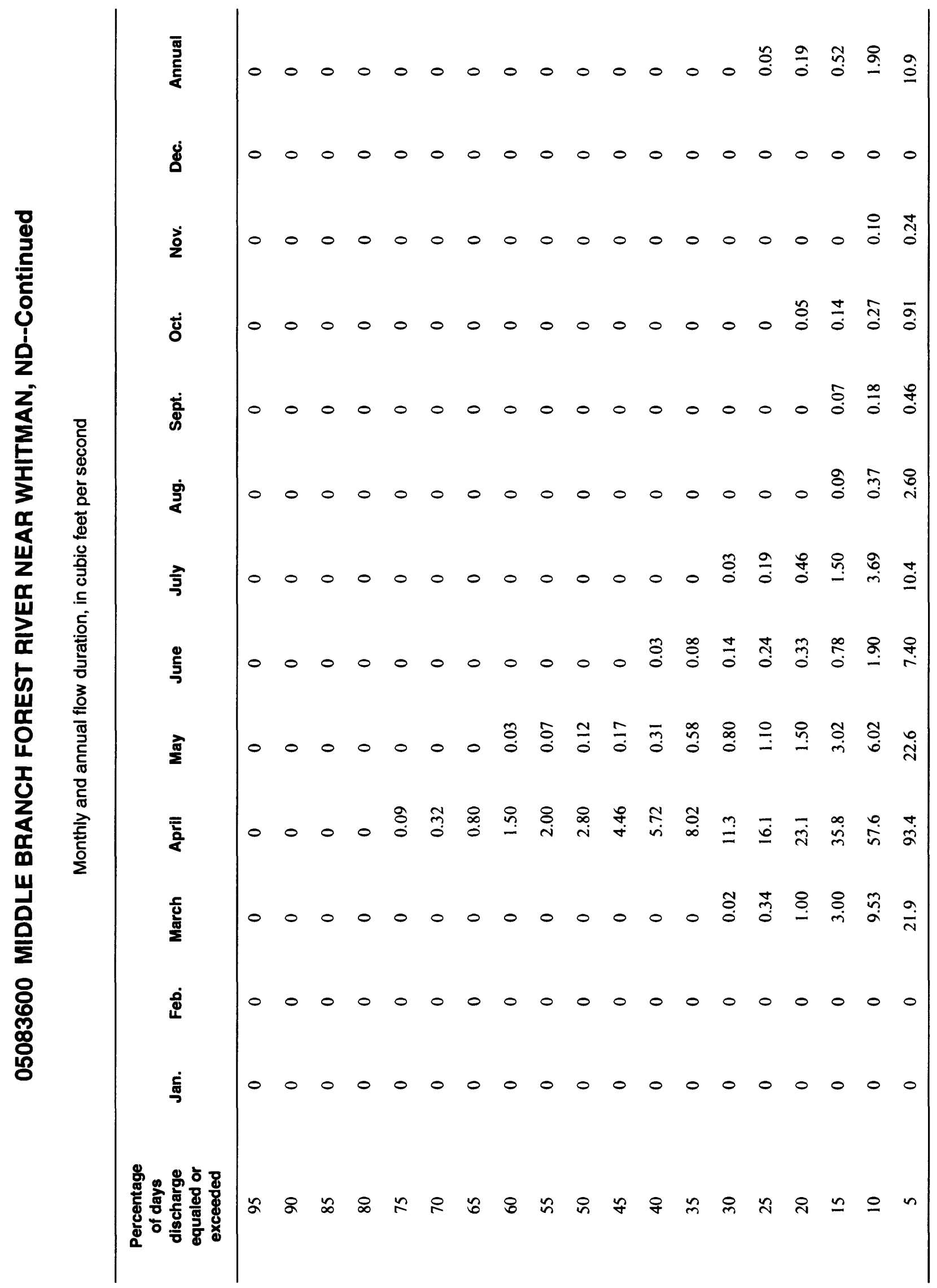


05083600 MIDDLE BRANCH FOREST RIVER NEAR WHITMAN, ND--Continued

Probability of occurrence of annual high discharges

[ng, statistic not given]

\begin{tabular}{|c|c|c|c|c|c|c|}
\hline \multirow[b]{2}{*}{$\begin{array}{l}\text { Exceedance } \\
\text { probability }\end{array}$} & \multirow[b]{2}{*}{$\begin{array}{l}\text { Recurrence } \\
\text { intervai } \\
\text { (years) }\end{array}$} & \multirow[b]{2}{*}{$\begin{array}{c}\text { Maximum } \\
\text { instantaneous } \\
\left(\mathrm{ft}^{3} / \mathrm{s}\right)\end{array}$} & \multicolumn{4}{|c|}{$\begin{array}{l}\text { Maximum mean discharge } \\
\left(\mathrm{ft}^{3} / \mathrm{s}\right)\end{array}$} \\
\hline & & & 3-day period & 7-day period & 15-day period & 30-day period \\
\hline 0.99 & 1.01 & 0.900 & 0.173 & 0.078 & 0.043 & 0.023 \\
\hline 0.95 & 1.05 & 4.10 & 1.32 & 0.765 & 0.489 & 0.282 \\
\hline 0.90 & 1.11 & 8.60 & 3.41 & 2.19 & 1.47 & 0.874 \\
\hline 0.80 & 1.25 & 20.2 & 9.58 & 6.73 & 4.69 & 2.85 \\
\hline 0.50 & 2 & 88.2 & 49.1 & 37.6 & 26.0 & 15.9 \\
\hline 0.20 & 5 & 315 & 167 & 126 & 80.0 & 47.7 \\
\hline 0.10 & 10 & 569 & 273 & 200 & 118 & 68.9 \\
\hline 0.04 & 25 & 1,010 & 417 & 290 & 159 & 90.2 \\
\hline 0.02 & 50 & 1,420 & 523 & 349 & 182 & 102 \\
\hline 0.01 & 100 & 1,900 & 622 & 399 & 200 & 110 \\
\hline 0.005 & 200 & 2,440 & 712 & 442 & 213 & 116 \\
\hline 0.002 & 500 & 3,240 & ng & ng & ng & ng \\
\hline
\end{tabular}

Probability of occurrence of annual low discharges

[ng, statistic not given]

\begin{tabular}{|c|c|c|c|c|c|c|c|c|c|c|}
\hline \multirow{3}{*}{$\begin{array}{l}\text { Non- } \\
\text { exceed- } \\
\text { ance } \\
\text { prob- } \\
\text { abiiity }\end{array}$} & \multirow{3}{*}{$\begin{array}{l}\text { Recur- } \\
\text { rence } \\
\text { inter- } \\
\text { val } \\
\text { (years) }\end{array}$} & \multicolumn{9}{|c|}{ Minimum mean discharge $\left(\mathrm{tt}^{3} / \mathrm{s}\right)$} \\
\hline & & \multicolumn{9}{|c|}{ Number of consecutive days } \\
\hline & & 1 & 3 & 7 & 14 & 30 & 60 & 90 & 120 & 183 \\
\hline 0.05 & 20 & $\mathrm{ng}$ & ng & ng & $\mathrm{ng}$ & $\mathrm{ng}$ & ng & $\mathrm{ng}$ & $\mathrm{ng}$ & 0 \\
\hline 0.10 & 10 & $\mathrm{ng}$ & ng & ng & $\mathrm{ng}$ & $\mathrm{ng}$ & ng & $\mathrm{ng}$ & $\mathrm{ng}$ & 0 \\
\hline 0.20 & 5 & $\mathrm{ng}$ & ng & ng & $\mathrm{ng}$ & $\mathrm{ng}$ & ng & $\mathrm{ng}$ & $\mathrm{ng}$ & 0 \\
\hline 0.50 & 2 & ng & $\mathrm{ng}$ & ng & $\mathrm{ng}$ & $\mathrm{ng}$ & ng & $\mathrm{ng}$ & $\mathrm{ng}$ & 0 \\
\hline
\end{tabular}




\section{MIDDLE BRANCH FOREST RIVER NEAR WHITMAN, ND--Continued}

Probability of occurrence of seasonal low discharges

[ng, statistic not given]

\begin{tabular}{|c|c|c|c|c|c|c|c|c|c|}
\hline \multirow[b]{3}{*}{$\begin{array}{c}\text { Non- } \\
\text { exceedance } \\
\text { probabiliity }\end{array}$} & \multirow[b]{3}{*}{$\begin{array}{c}\text { Recurrence } \\
\text { interval } \\
\text { (years) }\end{array}$} & \multicolumn{8}{|c|}{ Minimum mean discharge $\left(t^{3} / \mathrm{s}\right)$} \\
\hline & & \multicolumn{8}{|c|}{ Number of consecutive days } \\
\hline & & 1 & 7 & 14 & 30 & 1 & 7 & 14 & 30 \\
\hline & & \multicolumn{4}{|c|}{ December-January-February } & \multicolumn{4}{|c|}{ March-April-May } \\
\hline 0.05 & 20 & ng & ng & $\mathrm{ng}$ & ng & ng & $\mathrm{ng}$ & 0 & 0 \\
\hline 0.10 & 10 & ng & ng & $\mathrm{ng}$ & ng & $\mathrm{ng}$ & $\mathrm{ng}$ & 0 & 0 \\
\hline 0.20 & 5 & ng & ng & $\mathrm{ng}$ & ng & ng & $\mathrm{ng}$ & 0 & 0 \\
\hline \multirow[t]{2}{*}{0.50} & 2 & ng & ng & ng & ng & ng & ng & 0 & 0 \\
\hline & & \multicolumn{4}{|c|}{ June-July-August } & \multicolumn{4}{|c|}{ September-October-November } \\
\hline 0.05 & 20 & ng & ng & $\mathrm{ng}$ & 0 & ng & $\mathrm{ng}$ & $\mathrm{ng}$ & 0 \\
\hline 0.10 & 10 & ng & ng & ng & 0 & $\mathrm{ng}$ & $\mathrm{ng}$ & $\mathrm{ng}$ & 0 \\
\hline 0.20 & 5 & ng & ng & $\mathrm{ng}$ & 0 & ng & $\mathrm{ng}$ & $\mathrm{ng}$ & 0 \\
\hline 0.50 & 2 & ng & ng & $\mathrm{ng}$ & 0 & $\mathrm{ng}$ & $\mathrm{ng}$ & ng & 0 \\
\hline
\end{tabular}


05083600 MIDDLE BRANCH FOREST RIVER NEAR WHITMAN, ND--Continued

Annual peak discharge and corresponding gage height

$[-$, no data $]$

\begin{tabular}{|c|c|c|c|c|c|c|c|}
\hline $\begin{array}{l}\text { Water } \\
\text { year }\end{array}$ & Date & $\begin{array}{c}\text { Gage } \\
\text { height } \\
\text { (feet) }\end{array}$ & $\begin{array}{c}\text { Peak } \\
\text { discharge } \\
\left(\mathrm{ft}^{3} / \mathrm{s}\right)\end{array}$ & $\begin{array}{l}\text { Water } \\
\text { year }\end{array}$ & Date & $\begin{array}{c}\text { Gage } \\
\text { height } \\
\text { (feet) }\end{array}$ & $\begin{array}{c}\text { Peak } \\
\text { discharge } \\
\left(\mathrm{ft}^{3} / \mathrm{s}\right)\end{array}$ \\
\hline \multicolumn{8}{|c|}{ Annual peak discharge, by year, and corresponding gage height } \\
\hline 1961 & March 23 & 3.11 & 1.60 & 1976 & June 12 & 5.30 & 140 \\
\hline 1962 & April 21 & 4.62 & 100 & 1977 & April 10 & 4.10 & 37.0 \\
\hline 1963 & June 25 & 3.44 & 7.00 & 1978 & April 9 & 5.82 & 282 \\
\hline 1964 & June 19 & 5.23 & 192 & 1979 & April 22 & 7.17 & 737 \\
\hline 1965 & April 11 & 5.76 & 240 & 1980 & May 27 & 6.10 & 420 \\
\hline 1966 & April 5 & 4.91 & 134 & 1981 & April 2 & 4.47 & 65.0 \\
\hline 1967 & May 10 & 5.33 & 192 & 1982 & July 24 & 6.38 & 506 \\
\hline 1968 & March 8 & 4.27 & 5.00 & 1983 & April 8 & 5.03 & 67.0 \\
\hline 1969 & April 11 & 6.82 & 425 & 1984 & March 30 & 4.41 & 25.0 \\
\hline 1970 & April 7 & 4.84 & 74.0 & 1985 & March 16 & 5.19 & 62.0 \\
\hline 1971 & April 8 & 6.54 & 235 & 1986 & March 25 & 5.10 & 86.0 \\
\hline 1972 & April 16 & 4.12 & 53.0 & 1987 & April 7 & 6.85 & 654 \\
\hline 1973 & March 12 & -- & 4.00 & 1988 & April 4 & 4.62 & 22.0 \\
\hline 1974 & May 19 & 7.11 & 984 & 1989 & April 22 & 4.62 & 34.0 \\
\hline 1975 & July 1 & 4.55 & 71.0 & 1990 & April 3 & -- & 7.00 \\
\hline \multicolumn{8}{|c|}{ Annual peak discharge, from highest to lowest, and corresponding gage height } \\
\hline 1974 & May 19 & 7.11 & 984 & 1970 & April 7 & 4.84 & 74.0 \\
\hline 1979 & April 22 & 7.17 & 737 & 1975 & July 1 & 4.55 & 71.0 \\
\hline 1987 & April 7 & 6.85 & 654 & 1983 & April 8 & 5.03 & 67.0 \\
\hline 1982 & July 24 & 6.38 & 506 & 1981 & April 2 & 4.47 & 65.0 \\
\hline 1969 & April 11 & 6.82 & 425 & 1985 & March 16 & 5.19 & 62.0 \\
\hline 1980 & May 27 & 6.10 & 420 & 1972 & April 16 & 4.12 & 53.0 \\
\hline 1978 & April 9 & 5.82 & 282 & 1977 & April 10 & 4.10 & 37.0 \\
\hline 1965 & April 11 & 5.76 & 240 & 1989 & April 22 & 4.62 & 34.0 \\
\hline 1971 & April 8 & 6.54 & 235 & 1984 & March 30 & 4.41 & 25.0 \\
\hline 1964 & June 19 & 5.23 & 192 & 1988 & April 4 & 4.62 & 22.0 \\
\hline 1967 & May 10 & 5.33 & 192 & 1963 & June 25 & 3.44 & 7.00 \\
\hline 1976 & June 12 & 5.30 & 140 & 1990 & April 3 & -- & 7.00 \\
\hline 1966 & April 5 & 4.91 & 134 & 1968 & March 8 & 4.27 & 5.00 \\
\hline 1962 & April 21 & 4.62 & 100 & 1973 & March 12 & -- & 4.00 \\
\hline 1986 & March 25 & 5.10 & 86.0 & 1961 & March 23 & 3.11 & 1.60 \\
\hline
\end{tabular}




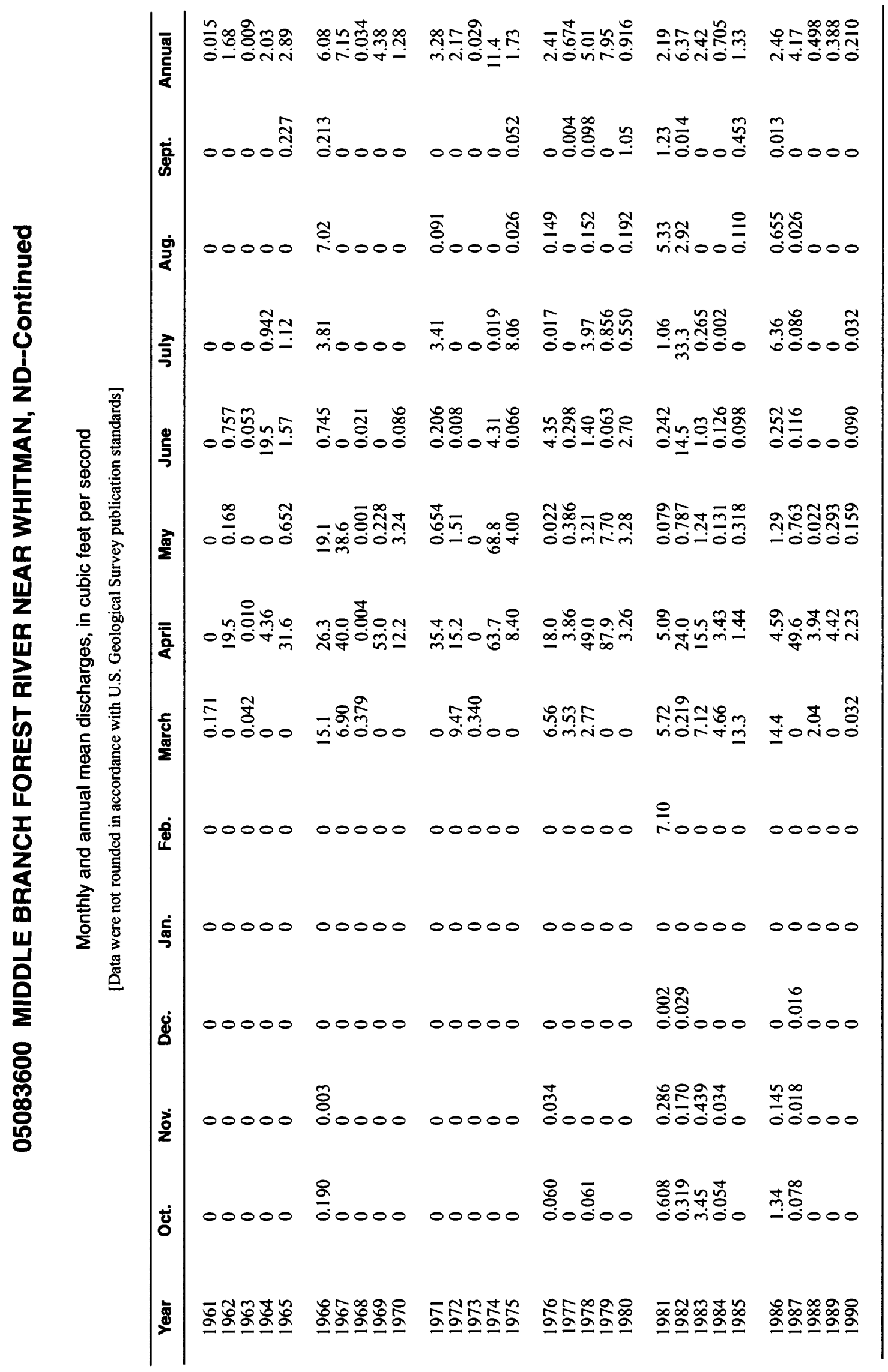


LOCATION.--Lat $48^{\circ} 11^{\prime} 50^{\prime \prime}$, long 97'43'49', on line between secs.32 and 33, T.155 N., R.55 W., Walsh County, Hydrologic Unit 09020308 , on right bank $50 \mathrm{ft}$ upstream from highway bridge, $0.5 \mathrm{mi}$ downstream from South Branch, and $3 \mathrm{mi}$ southeast of Fordville.

DRAINAGE AREA.--456 $\mathrm{mi}^{2}$, of which about $120 \mathrm{mi}^{2}$ is probably noncontributing.

PERIOD OF RECORD.--April 1940 to current year.

GAGE.--Water-stage recorder. Datum of gage is $1,035 \mathrm{ft}$ above sea level, from topographic map. Prior to July 21,1951 , nonrecording gage at site $50 \mathrm{ft}$ downstream at same datum.

EXTREMES FOR PERIOD OF RECORD.--Maximum discharge, 16,400 $\mathrm{ft}^{3} / \mathrm{s}$, Apr. 18, 1950, gage height, $14.48 \mathrm{ft}$; no flow at times.

Annual mean discharge

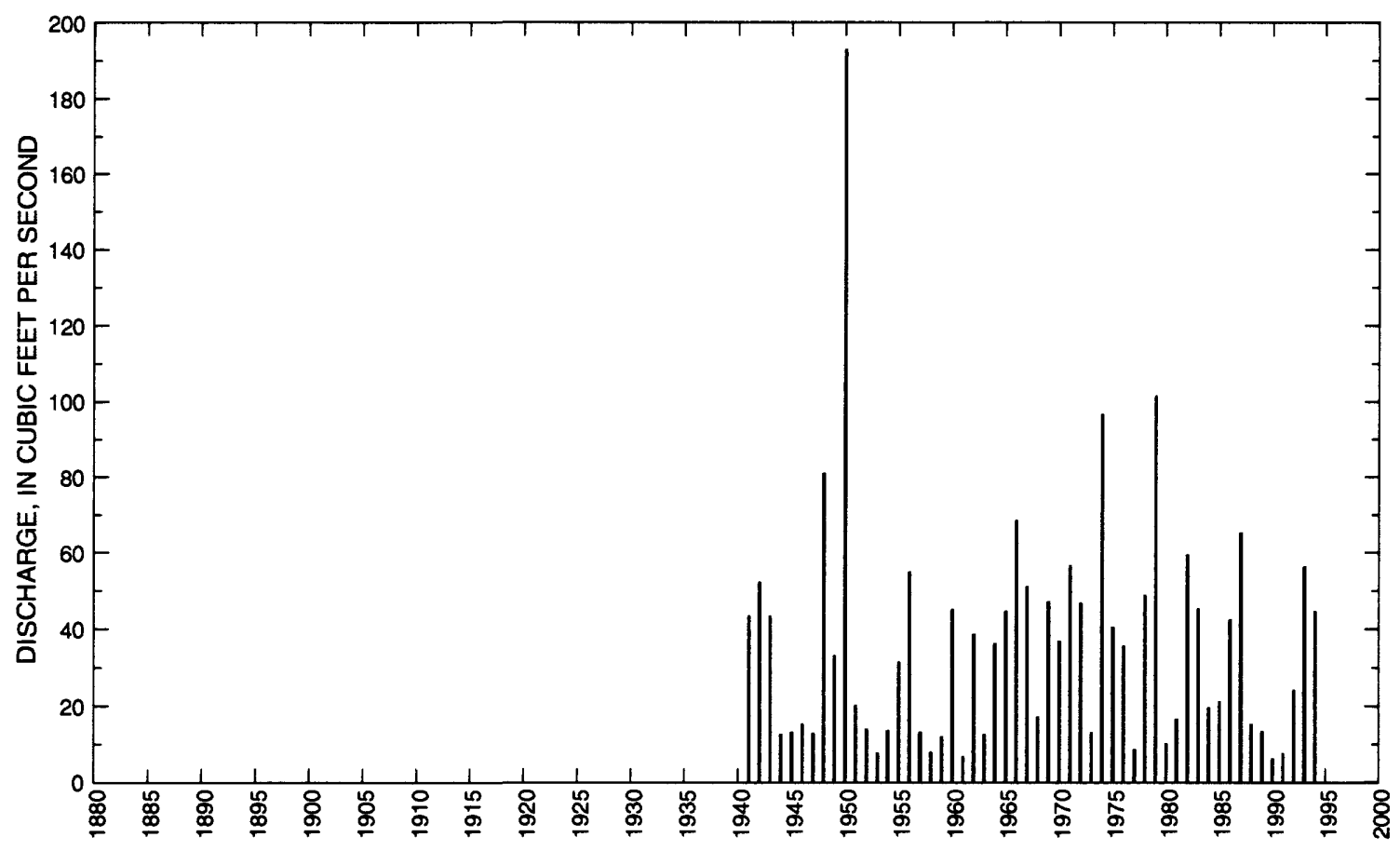


05084000 FOREST RIVER NEAR FORDVILLE, ND--Continued

Statistics of monthly and annual mean discharges

[m, more than 1 year of occurrence]

\begin{tabular}{|c|c|c|c|c|c|c|c|c|}
\hline \multirow[b]{2}{*}{ Month } & \multicolumn{2}{|c|}{ Maximum } & \multicolumn{2}{|c|}{ Minimum } & \multicolumn{4}{|c|}{ Mean } \\
\hline & $\begin{array}{c}\text { Discharge } \\
\left(\mathrm{ft}^{3} / \mathrm{s}\right)\end{array}$ & $\begin{array}{c}\text { Water year } \\
\text { of } \\
\text { occurrence }\end{array}$ & $\begin{array}{c}\text { Discharge } \\
\left(\mathrm{ft}^{3} / \mathrm{s}\right)\end{array}$ & $\begin{array}{l}\text { Water year } \\
\text { of } \\
\text { occurrence }\end{array}$ & $\begin{array}{c}\text { Discharge } \\
\left(\mathrm{ft}^{3} / \mathrm{s}\right)\end{array}$ & $\begin{array}{l}\text { Standard } \\
\text { deviation } \\
\left(\mathrm{ft}^{3} / \mathrm{s}\right)\end{array}$ & $\begin{array}{l}\text { Coeffi- } \\
\text { cient of } \\
\text { variation }\end{array}$ & $\begin{array}{l}\text { Percentage } \\
\text { of annual } \\
\text { discharge }\end{array}$ \\
\hline October & 57.9 & 1983 & 1.52 & 1941 & 10.0 & 8.43 & 0.84 & 2.30 \\
\hline November & 23.7 & 1983 & 2.03 & 1941 & 8.73 & 4.24 & 0.49 & 2.00 \\
\hline December & 19.2 & 1983 & 2.06 & 1941 & 7.24 & 3.53 & 0.49 & 1.66 \\
\hline January & 16.3 & 1986 & 2.70 & 1941 & 6.20 & 2.53 & 0.41 & 1.42 \\
\hline February & 29.9 & 1981 & 1.21 & 1963 & 7.23 & 4.54 & 0.63 & 1.66 \\
\hline March & 278 & 1966 & 4.07 & 1941 & 59.7 & 65.5 & 1.10 & 13.7 \\
\hline April & 1,180 & 1950 & 9.46 & 1991 & 196 & 241 & 1.23 & 45.0 \\
\hline May & 1,040 & 1950 & 7.07 & 1961 & 61.2 & 154 & 2.52 & 14.1 \\
\hline June & 255 & 1964 & 2.74 & 1940 & 33.2 & 45.4 & 1.37 & 7.62 \\
\hline July & 232 & 1982 & 3.34 & $\mathrm{~m}$ & 24.9 & 42.8 & 1.72 & 5.72 \\
\hline August & 280 & 1993 & 1.64 & 1945 & 12.5 & 37.4 & 2.98 & 2.88 \\
\hline September & 53.3 & 1993 & 0.913 & 1940 & 8.81 & 7.62 & 0.87 & 2.02 \\
\hline Annual & 193 & 1950 & 6.37 & 1990 & 36.7 & 31.6 & 0.86 & 100 \\
\hline
\end{tabular}

Annual flow duration

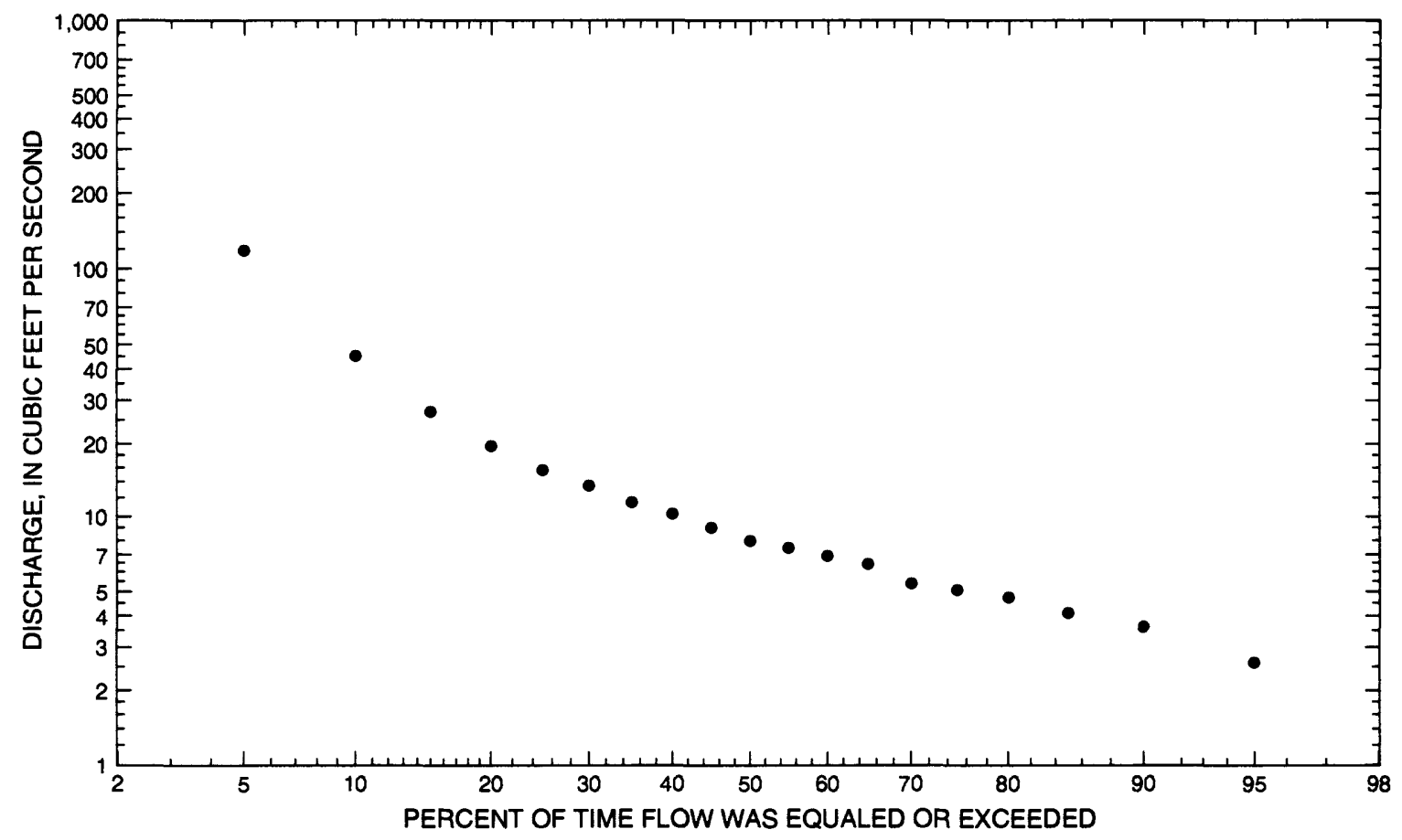




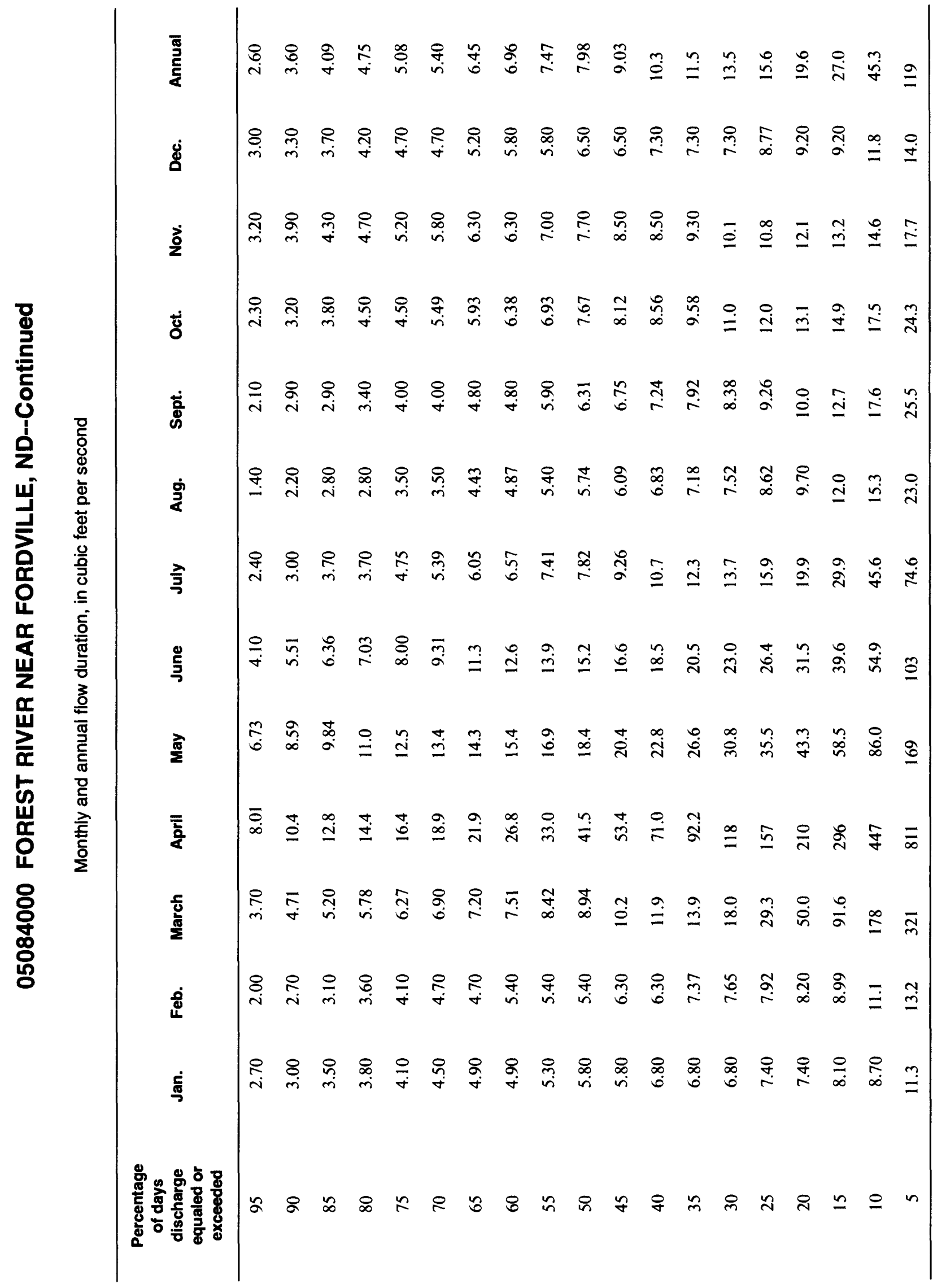




\section{FOREST RIVER NEAR FORDVILLE, ND--Continued}

Probability of occurrence of annual high discharges

[ng, statistic not given]

\begin{tabular}{|c|c|c|c|c|c|c|}
\hline \multirow[b]{2}{*}{$\begin{array}{l}\text { Exceedance } \\
\text { probability }\end{array}$} & \multirow[b]{2}{*}{$\begin{array}{l}\text { Recurrence } \\
\text { interval } \\
\text { (years) }\end{array}$} & \multirow[b]{2}{*}{$\begin{array}{c}\text { Maximum } \\
\text { instantaneous } \\
\left(\mathrm{ft}^{3} / \mathrm{s}\right)\end{array}$} & \multicolumn{4}{|c|}{$\begin{array}{l}\text { Maximum mean discharge } \\
\qquad\left(\mathrm{ft}^{3} / \mathrm{s}\right)\end{array}$} \\
\hline & & & 3-day period & 7-day period & 15-day period & 30-day period \\
\hline 0.99 & 1.01 & 15.3 & 11.2 & 9.91 & 7.79 & 7.30 \\
\hline 0.95 & 1.05 & 58.6 & 41.4 & 33.6 & 25.5 & 20.0 \\
\hline 0.90 & 1.11 & 114 & 78.6 & 61.1 & 45.4 & 33.3 \\
\hline 0.80 & 1.25 & 244 & 162 & 121 & 87.3 & 59.7 \\
\hline 0.50 & 2 & 908 & 559 & 387 & 265 & 168 \\
\hline 0.20 & 5 & 2,850 & 1,590 & 1,040 & 675 & 424 \\
\hline 0.10 & 10 & 4,850 & 2,570 & 1,650 & 1,030 & 660 \\
\hline 0.04 & 25 & 8,160 & 4,060 & 2,550 & 1,540 & 1,030 \\
\hline 0.02 & 50 & 11,100 & 5,320 & 3,310 & 1,960 & 1,340 \\
\hline 0.01 & 100 & 14,500 & 6,660 & 4,110 & 2,380 & 1,690 \\
\hline 0.005 & 200 & 18,200 & 8,080 & 4,950 & 2,820 & 2,070 \\
\hline 0.002 & 500 & 23,600 & ng & ng & ng & ng \\
\hline
\end{tabular}

Probability of occurrence of annual low discharges

\begin{tabular}{|c|c|c|c|c|c|c|c|c|c|c|}
\hline \multirow[b]{3}{*}{$\begin{array}{c}\text { Non- } \\
\text { exceed- } \\
\text { ance } \\
\text { prob- } \\
\text { abiiity }\end{array}$} & \multirow[b]{3}{*}{$\begin{array}{l}\text { Recur- } \\
\text { rence } \\
\text { inter- } \\
\text { val } \\
\text { (years) }\end{array}$} & \multicolumn{9}{|c|}{ Minimum mean dlscharge $\left(\mathrm{ft}^{3} / \mathrm{s}\right)$} \\
\hline & & \multicolumn{9}{|c|}{ Number of consecutive days } \\
\hline & & 1 & 3 & 7 & 14 & 30 & 60 & 90 & 120 & 183 \\
\hline 0.05 & 20 & 0.524 & 0.657 & 0.930 & 1.08 & 1.46 & 1.99 & 2.43 & 2.78 & 3.18 \\
\hline 0.10 & 10 & 0.848 & 0.994 & 1.28 & 1.45 & 1.88 & 2.44 & 2.92 & 3.33 & 3.76 \\
\hline 0.20 & 5 & 1.36 & 1.50 & 1.78 & 2.01 & 2.48 & 3.08 & 3.61 & 4.09 & 4.61 \\
\hline 0.50 & 2 & 2.73 & 2.85 & 3.06 & 3.43 & 3.96 & 4.63 & 5.28 & 5.94 & 6.74 \\
\hline
\end{tabular}


05084000 FOREST RIVER NEAR FORDVILLE, ND-Continued

Probability of occurrence of seasonal low discharges

\begin{tabular}{|c|c|c|c|c|c|c|c|c|c|}
\hline \multirow[b]{3}{*}{$\begin{array}{c}\text { Non- } \\
\text { exceedance } \\
\text { probability }\end{array}$} & \multirow[b]{3}{*}{$\begin{array}{c}\text { Recurrence } \\
\text { interval } \\
\text { (years) }\end{array}$} & \multicolumn{8}{|c|}{ Minimum mean discharge $\left(t^{3} / \mathrm{s}\right)$} \\
\hline & & \multicolumn{8}{|c|}{ Number of consecutive days } \\
\hline & & 1 & 7 & 14 & 30 & 1 & 7 & 14 & 30 \\
\hline & & \multicolumn{4}{|c|}{ December-January-February } & \multicolumn{4}{|c|}{ March-April-May } \\
\hline 0.05 & 20 & 1.58 & 1.65 & 1.77 & 2.12 & 2.75 & 3.28 & 3.57 & 5.12 \\
\hline 0.10 & 10 & 1.99 & 2.09 & 2.22 & 2.61 & 3.26 & 3.79 & 4.12 & 5.85 \\
\hline 0.20 & 5 & 2.58 & 2.72 & 2.87 & 3.31 & 4.01 & 4.54 & 4.96 & 7.15 \\
\hline \multirow[t]{2}{*}{0.50} & 2 & 4.01 & 4.22 & 4.42 & 4.93 & 5.92 & 6.54 & 7.38 & 12.0 \\
\hline & & \multicolumn{4}{|c|}{ June-July-August } & \multicolumn{4}{|c|}{ September-October-November } \\
\hline 0.05 & 20 & 0.687 & 1.12 & 1.49 & 1.96 & 1.30 & 1.42 & 1.74 & 2.12 \\
\hline 0.10 & 10 & 1.04 & 1.50 & 1.87 & 2.33 & 1.70 & 1.91 & 2.30 & 2.80 \\
\hline 0.20 & 5 & 1.63 & 2.11 & 2.46 & 2.95 & 2.25 & 2.64 & 3.14 & 3.78 \\
\hline 0.50 & 2 & 3.38 & 3.86 & 4.24 & 4.96 & 3.67 & 4.49 & 5.14 & 6.13 \\
\hline
\end{tabular}




\section{FOREST RIVER NEAR FORDVILLE, ND--Continued}

Annual peak discharge and corresponding gage height

$$
[-, \text { no data }]
$$

\begin{tabular}{|c|c|c|c|c|c|c|c|}
\hline $\begin{array}{l}\text { Water } \\
\text { year }\end{array}$ & Date & $\begin{array}{c}\text { Gage } \\
\text { height } \\
\text { (feet) }\end{array}$ & $\begin{array}{c}\text { Peak } \\
\text { discharge } \\
\left(\mathrm{ft}^{3} / \mathrm{s}\right)\end{array}$ & $\begin{array}{l}\text { Water } \\
\text { year }\end{array}$ & Date & $\begin{array}{c}\text { Gage } \\
\text { height } \\
\text { (feet) }\end{array}$ & $\begin{array}{c}\text { Peak } \\
\text { discharge } \\
\left(\mathrm{ft}^{3} / \mathrm{s}\right)\end{array}$ \\
\hline \multicolumn{8}{|c|}{ Annual peak discharge, by year, and corresponding gage height } \\
\hline 1940 & April 17 & 3.60 & 130 & 1968 & March 8 & 4.48 & 500 \\
\hline 1941 & April 8 & 8.03 & 2,250 & 1969 & April 11 & 8.80 & 3,290 \\
\hline 1942 & April 4 & 9.73 & 3,650 & 1970 & April 8 & 7.68 & 2,380 \\
\hline 1943 & June 9 & 6.72 & 1,620 & 1971 & April 8 & 8.39 & 2,800 \\
\hline 1944 & April 5 & 5.89 & 400 & 1972 & April 14 & 6.43 & 1,500 \\
\hline 1945 & March 27 & 3.15 & 243 & 1973 & June 17 & 3.56 & 384 \\
\hline 1946 & March 20 & 6.14 & 950 & 1974 & May 20 & 9.86 & 5,050 \\
\hline 1947 & $\operatorname{March} 23$ & 7.40 & 700 & 1975 & April 12 & 5.68 & 1,270 \\
\hline 1948 & April 18 & 14.15 & 14,600 & 1976 & March 29 & 5.46 & 1,100 \\
\hline 1949 & April 7 & 5.64 & 1,470 & 1977 & July 14 & 2.43 & 100 \\
\hline 1950 & April 18 & 14.48 & 16,400 & 1978 & April 6 & 5.50 & 1,200 \\
\hline 1951 & March 29 & -- & 500 & 1979 & April 20 & 9.98 & 5,200 \\
\hline 1952 & July 2 & 3.94 & 825 & 1980 & April 2 & 2.55 & 105 \\
\hline 1953 & May 30 & 2.04 & 130 & 1981 & April 2 & 2.73 & 180 \\
\hline 1954 & June 15 & 4.29 & 1,020 & 1982 & July 25 & 6.49 & 1,760 \\
\hline 1955 & March 31 & 8.46 & 3,000 & 1983 & March 7 & 5.23 & 995 \\
\hline 1956 & June 6 & 8.14 & 3,370 & 1984 & March 24 & 3.92 & 386 \\
\hline 1957 & March 22 & 3.53 & 356 & 1985 & March 13 & 5.45 & 625 \\
\hline 1958 & July 4 & 1.87 & 17.0 & 1986 & March 17 & 5.79 & 1,380 \\
\hline 1959 & April 4 & 2.91 & 321 & 1987 & April 5 & 6.00 & 1,410 \\
\hline 1960 & April 7 & 7.55 & 2,810 & 1988 & March 24 & 3.23 & 229 \\
\hline 1961 & March 19 & 2.69 & 65.0 & 1989 & April 16 & 2.79 & 158 \\
\hline 1962 & April 6 & 7.69 & 2,600 & 1990 & June 3 & 1.67 & 17.0 \\
\hline 1963 & July 8 & 3.67 & 590 & 1991 & September 18 & -- & 77.0 \\
\hline 1964 & June 19 & 9.03 & 3,960 & 1992 & March 7 & -- & 530 \\
\hline 1965 & April 11 & 10.22 & 4,730 & 1993 & July 26 & 5.72 & 1,090 \\
\hline 1966 & March 21 & 7.14 & 1,580 & 1994 & March 21 & 4.66 & 721 \\
\hline 1967 & March 30 & 7.40 & 2,030 & & & & \\
\hline \multicolumn{8}{|c|}{ Annual peak discharge, from highest to lowest, and corresponding gage height } \\
\hline 1950 & April 18 & 14.48 & 16,400 & 1967 & March 30 & 7.40 & 2,030 \\
\hline 1948 & April 18 & 14.15 & 14,600 & 1982 & July 25 & 6.49 & 1,760 \\
\hline 1979 & April 20 & 9.98 & 5,200 & 1943 & June 9 & 6.72 & 1,620 \\
\hline 1974 & May 20 & 9.86 & 5,050 & 1966 & March 21 & 7.14 & 1,580 \\
\hline 1965 & April 11 & 10.22 & 4,730 & 1972 & April 14 & 6.43 & 1,500 \\
\hline 1964 & June 19 & 9.03 & 3,960 & 1949 & April 7 & 5.64 & 1,470 \\
\hline 1942 & April 4 & 9.73 & 3,650 & 1987 & April 5 & 6.00 & 1,410 \\
\hline 1956 & June 6 & 8.14 & 3,370 & 1986 & March 17 & 5.79 & 1,380 \\
\hline 1969 & April 11 & 8.80 & 3,290 & 1975 & April 12 & 5.68 & 1,270 \\
\hline 1955 & March 31 & 8.46 & 3,000 & 1978 & April 6 & 5.50 & 1,200 \\
\hline 1960 & April 7 & 7.55 & 2,810 & 1976 & March 29 & 5.46 & 1,100 \\
\hline 1971 & April 8 & 8.39 & 2,800 & 1993 & July 26 & 5.72 & 1,090 \\
\hline 1962 & April 6 & 7.69 & 2,600 & 1973 & June 17 & 3.56 & 384 \\
\hline 1970 & April 8 & 7.68 & 2,380 & 1983 & March 7 & 5.23 & 995 \\
\hline 1941 & April 8 & 8.03 & 2,250 & 1946 & March 20 & 6.14 & 950 \\
\hline
\end{tabular}




\section{FOREST RIVER NEAR FORDVILLE, ND--Continued}

Annual peak discharge and corresponding gage height--Continued

$$
[--, \text { no data] }
$$

\begin{tabular}{llllllll}
\hline $\begin{array}{c}\text { Water } \\
\text { year }\end{array}$ & \multicolumn{1}{c}{ Date } & $\begin{array}{c}\text { Gage } \\
\text { height } \\
\text { (feet) }\end{array}$ & $\begin{array}{c}\text { Peak } \\
\text { discharge } \\
\left(\mathbf{f t}^{3} / \mathbf{s}\right)\end{array}$ & $\begin{array}{c}\text { Water } \\
\text { year }\end{array}$ & Date & $\begin{array}{c}\text { Gage } \\
\text { height } \\
\text { (feet) }\end{array}$ & $\begin{array}{c}\text { Peak } \\
\text { discharge } \\
\left(\mathbf{f t}^{3} / \mathbf{s}\right)\end{array}$ \\
\hline \multicolumn{7}{c}{ Annual peak discharge, from highest to lowest, and corresponding gage height-Continued } \\
1952 & July 2 & 3.94 & 825 & 1945 & March 27 & 3.15 & 243 \\
1994 & March 21 & 4.66 & 721 & 1988 & March 24 & 3.23 & 229 \\
1947 & March 23 & 7.40 & 700 & 1981 & April 2 & 2.73 & 180 \\
1985 & March 13 & 5.45 & 625 & 1989 & April 16 & 2.79 & 158 \\
1963 & July 8 & 3.67 & 590 & 1940 & April 17 & 3.60 & 130 \\
1992 & March 7 & - & 530 & 1953 & May 30 & 2.04 & 130 \\
1951 & March 29 & - & 500 & 1980 & April 2 & 2.55 & 105 \\
1968 & March 8 & 4.48 & 500 & 1977 & July 14 & 2.43 & 100 \\
1944 & April 5 & 5.89 & 400 & 1991 & September 18 & - & 77.0 \\
1984 & March 24 & 3.92 & 386 & 1961 & March 19 & 2.69 & 65.0 \\
1973 & June 17 & 3.56 & 384 & 1958 & July 4 & 1.87 & 17.0 \\
1957 & March 22 & 3.53 & 356 & 1990 & June 3 & 1.67 & 17.0 \\
1959 & April 4 & 2.91 & 321 & & & & \\
\hline
\end{tabular}




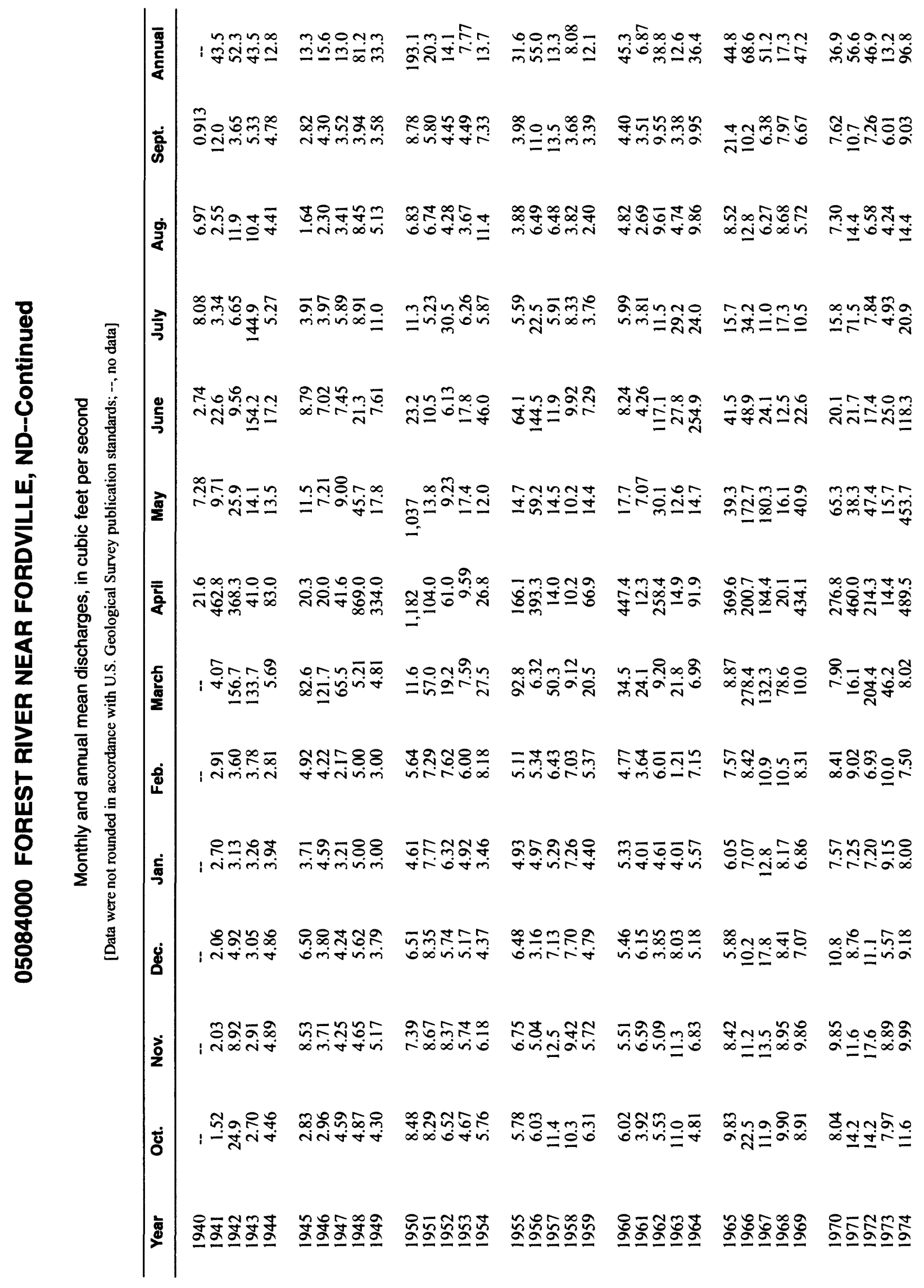




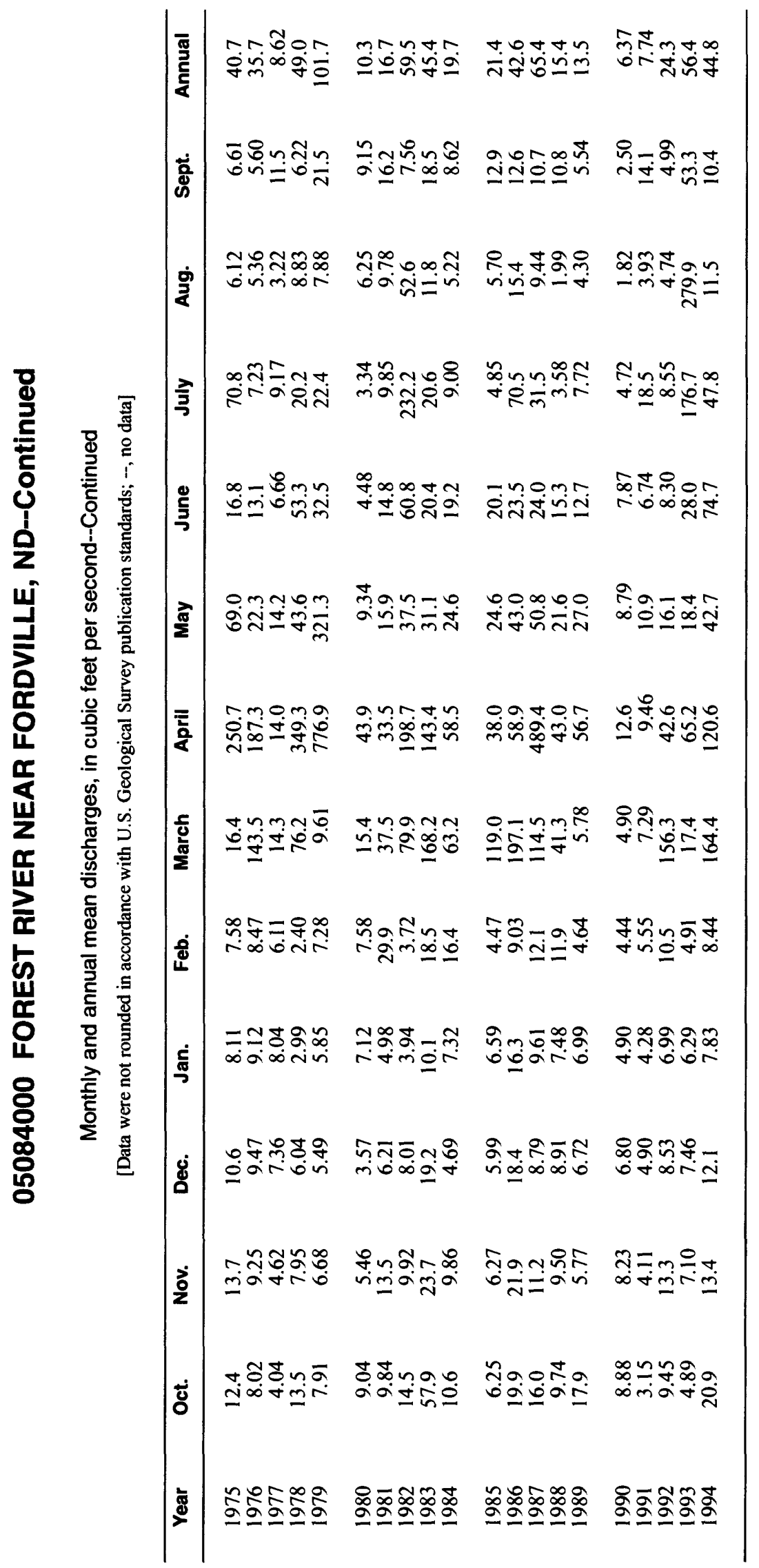




\section{FOREST RIVER AT MINTO, ND}

LOCATION.--Lat $48^{\circ} 16^{\prime} 10^{\prime \prime}$, long $97^{\circ} 22^{\prime} 10^{\prime \prime}$, in $\mathrm{SE}^{1} / 4$ sec.51, T.156 N., R.52 W., Walsh County, Hydrologic Unit 09020308, on right bank $30 \mathrm{ft}$ upstream from dam in Minto, $150 \mathrm{ft}$ upstream from Burlington Northern Railway bridge, and $900 \mathrm{ft}$ east of U.S. Highway 81 .

DRAINAGE AREA.--740 $\mathrm{mi}^{2}$, of which about $120 \mathrm{mi}^{2}$ is probably noncontributing.

PERIOD OF RECORD.--April 1944 to current year.

GAGE.--Water-stage recorder. Datum of gage is $806.95 \mathrm{ft}$ above sea level. Prior to July 15, 1954, nonrecording gage at site $400 \mathrm{ft}$ upstream at same datum.

EXTREMES FOR PERIOD OF RECORD.--Maximum discharge, 16,600 $\mathrm{ft}^{3} / \mathrm{s}$, Apr. 18, 1950; maximum gage height, $11.80 \mathrm{ft}$, Apr. 19, 1948, and Apr. 18, 1950; no flow at times.

Annual mean discharge

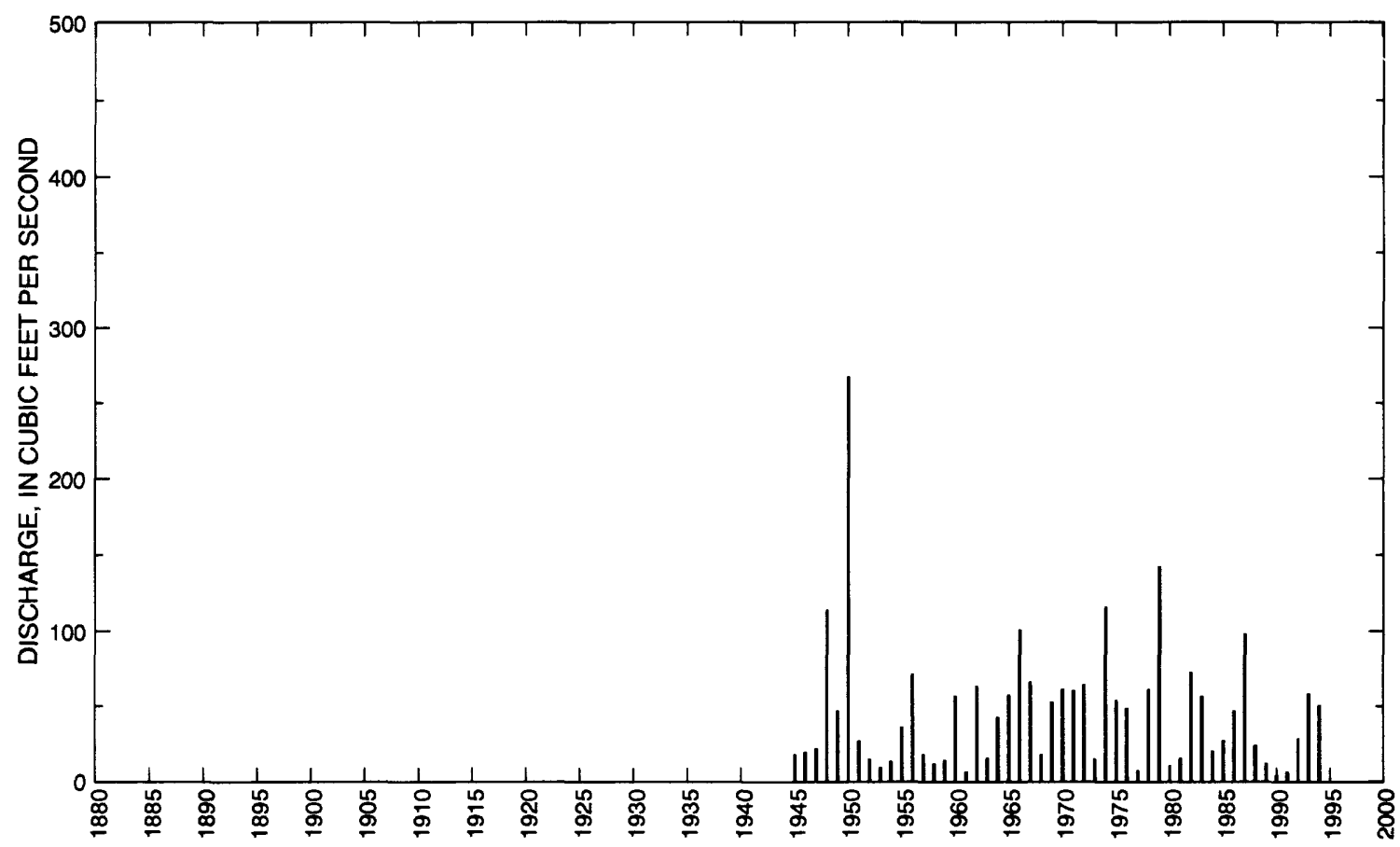




\section{FOREST RIVER AT MINTO, ND--Continued}

Statistics of monthly and annual mean discharges

[m, more than 1 year of occurrence]

\begin{tabular}{|c|c|c|c|c|c|c|c|c|}
\hline \multirow[b]{2}{*}{ Month } & \multicolumn{2}{|c|}{ Maximum } & \multicolumn{2}{|c|}{ Minimum } & \multicolumn{4}{|c|}{ Mean } \\
\hline & $\begin{array}{c}\text { Discharge } \\
\left(\mathrm{ft}^{3} / \mathrm{s}\right)\end{array}$ & $\begin{array}{c}\text { Water year } \\
\text { of } \\
\text { occurrence }\end{array}$ & $\begin{array}{c}\text { Discharge } \\
\left(\mathrm{ft}^{3} / \mathrm{s}\right)\end{array}$ & $\begin{array}{l}\text { Water year } \\
\text { of } \\
\text { occurrence }\end{array}$ & $\begin{array}{c}\text { Discharge } \\
\left(\mathrm{ft}^{3} / \mathrm{s}\right)\end{array}$ & $\begin{array}{l}\text { Standard } \\
\text { deviation } \\
\left(\mathrm{ft}^{3} / \mathrm{s}\right)\end{array}$ & $\begin{array}{l}\text { Coeffi- } \\
\text { cient of } \\
\text { variation }\end{array}$ & $\begin{array}{l}\text { Percentage } \\
\text { of annual } \\
\text { discharge }\end{array}$ \\
\hline October & 59.1 & 1983 & 0 & 1991 & 9.20 & 9.20 & 1.00 & 1.64 \\
\hline November & 23.6 & 1983 & 0.971 & 1991 & 8.85 & 5.16 & 0.58 & 1.58 \\
\hline December & 13.4 & 1983 & 0.291 & 1990 & 4.81 & 3.40 & 0.71 & 0.86 \\
\hline January & 9.66 & 1983 & 0 & $\mathrm{~m}$ & 2.41 & 2.49 & 1.03 & 0.43 \\
\hline February & 17.3 & 1981 & 0 & $\mathrm{~m}$ & 2.01 & 3.23 & 1.61 & 0.36 \\
\hline March & 438 & 1966 & 0 & 1962 & 59.4 & 88.0 & 1.48 & 10.6 \\
\hline April & 1,570 & 1950 & 21.9 & 1953 & 285 & 335 & 1.17 & 50.9 \\
\hline May & 1,520 & 1950 & 10.6 & 1946 & 92.3 & 222 & 2.40 & 16.5 \\
\hline June & 267 & 1964 & 4.21 & 1991 & 45.6 & 59.0 & 1.29 & 8.13 \\
\hline July & 236 & 1982 & 1.87 & 1980 & 27.3 & 39.6 & 1.45 & 4.87 \\
\hline August & 328 & 1993 & 0 & 1946 & 15.2 & 46.7 & 3.08 & 2.70 \\
\hline September & 69.0 & 1993 & 0 & $\mathrm{~m}$ & 8.65 & 12.2 & 1.41 & 1.54 \\
\hline Annual & 268 & 1950 & 4.36 & 1990 & 47.2 & 45.4 & 0.96 & 100 \\
\hline
\end{tabular}

Annual flow duration

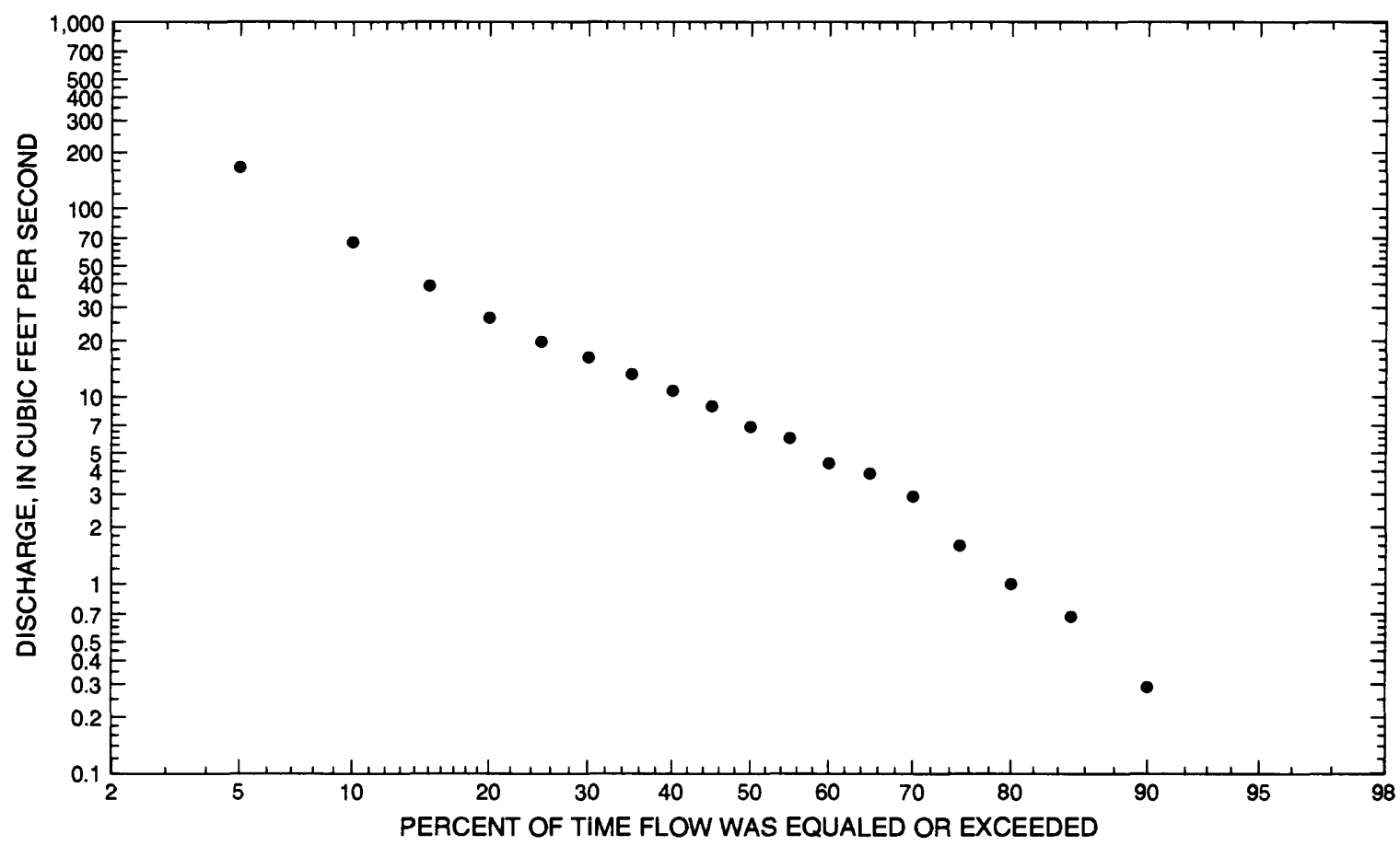




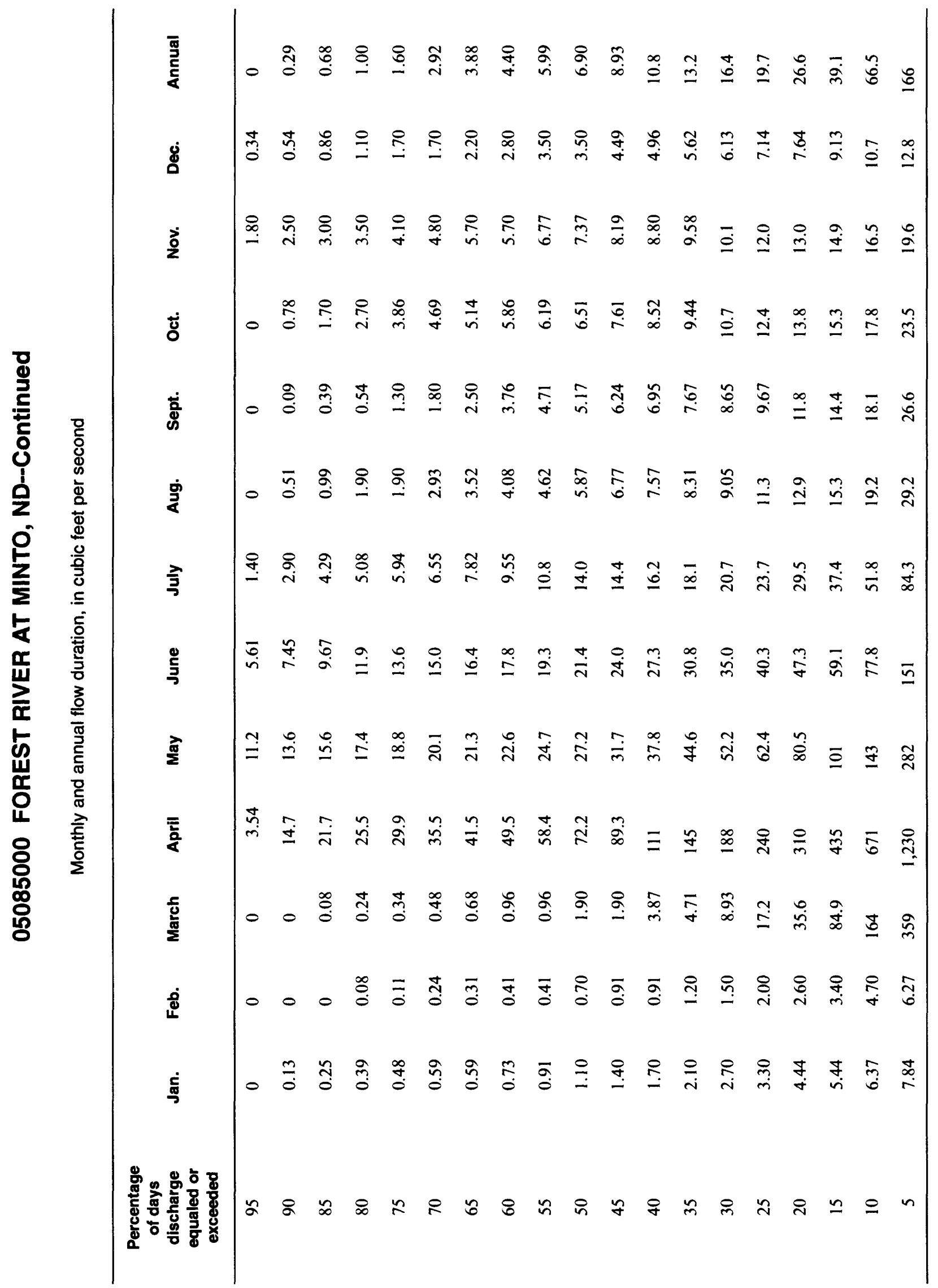




\section{FOREST RIVER AT MINTO, ND--Continued}

Probability of occurrence of annual high discharges

[ng, statistic not given]

\begin{tabular}{|c|c|c|c|c|c|c|}
\hline \multirow[b]{2}{*}{$\begin{array}{l}\text { Exceedance } \\
\text { probability }\end{array}$} & \multirow[b]{2}{*}{$\begin{array}{l}\text { Recurrence } \\
\text { interval } \\
\text { (years) }\end{array}$} & \multirow[b]{2}{*}{$\begin{array}{c}\text { Maximum } \\
\text { instantaneous } \\
\left(\mathrm{ft}^{3} / \mathrm{s}\right)\end{array}$} & \multicolumn{4}{|c|}{$\begin{array}{l}\text { Maximum mean discharge } \\
\left(\mathrm{ft}^{3} / \mathrm{s}\right)\end{array}$} \\
\hline & & & 3-day period & 7-day period & 15-day period & 30-day period \\
\hline 0.99 & 1.01 & 39.5 & 26.9 & 23.5 & 17.7 & 14.2 \\
\hline 0.95 & 1.05 & 104 & 71.6 & 58.9 & 44.1 & 32.6 \\
\hline 0.90 & 1.11 & 172 & 119 & 94.9 & 70.5 & 50.3 \\
\hline 0.80 & 1.25 & 313 & 217 & 167 & 123 & 84.3 \\
\hline 0.50 & 2 & 939 & 657 & 479 & 337 & 222 \\
\hline 0.20 & 5 & 2,680 & 1,880 & 1,310 & 870 & 564 \\
\hline 0.10 & 10 & 4,530 & 3,200 & 2,190 & 1,390 & 909 \\
\hline 0.04 & 25 & 7,830 & 5,550 & 3,730 & 2,270 & 1,500 \\
\hline 0.02 & 50 & 11,000 & 7,840 & 5,220 & 3,070 & 2,060 \\
\hline 0.01 & 100 & 15,000 & 10,700 & 7,030 & 4,010 & 2,730 \\
\hline 0.005 & 200 & 19,700 & 14,000 & 9,210 & 5,090 & 3,520 \\
\hline 0.002 & 500 & 27,300 & ng & ng & ng & $\mathrm{ng}$ \\
\hline
\end{tabular}

Probability of occurrence of annual low discharges

\begin{tabular}{|c|c|c|c|c|c|c|c|c|c|c|}
\hline \multirow[b]{3}{*}{$\begin{array}{l}\text { Non- } \\
\text { exceed- } \\
\text { ance } \\
\text { prob- } \\
\text { ability }\end{array}$} & \multirow[b]{3}{*}{$\begin{array}{c}\text { Recur- } \\
\text { rence } \\
\text { inter- } \\
\text { vai } \\
\text { (years) }\end{array}$} & \multicolumn{9}{|c|}{ Minimum mean discharge $\left(\mathrm{ft}^{3} / \mathrm{s}\right)$} \\
\hline & & \multicolumn{9}{|c|}{ Number of consecutive days } \\
\hline & & 1 & 3 & 7 & 14 & 30 & 60 & 90 & 120 & 183 \\
\hline 0.05 & 20 & 0 & 0 & 0 & 0 & 0 & 0 & 0.058 & 0.314 & 0.823 \\
\hline 0.10 & 10 & 0 & 0 & 0 & 0 & 0 & 0.039 & 0.179 & 0.521 & 1.23 \\
\hline 0.20 & 5 & 0 & 0 & 0 & 0 & 0 & 0.157 & 0.389 & 0.915 & 1.94 \\
\hline 0.50 & 2 & 0.242 & 0.264 & 0.286 & 0.337 & 0.465 & 0.728 & 1.27 & 2.32 & 4.14 \\
\hline
\end{tabular}


05085000 FOREST RIVER AT MINTO, ND--Continued

Probability of seasonal low discharges

\begin{tabular}{|c|c|c|c|c|c|c|c|c|c|}
\hline \multirow[b]{3}{*}{$\begin{array}{c}\text { Non- } \\
\text { exceedance } \\
\text { probability }\end{array}$} & \multirow[b]{3}{*}{$\begin{array}{l}\text { Recurrence } \\
\text { interval } \\
\text { (years) }\end{array}$} & \multicolumn{8}{|c|}{ Minimum mean discharge $\left(\mathrm{ft}^{3} / \mathrm{s}\right)$} \\
\hline & & \multicolumn{8}{|c|}{ Number of consecutive days } \\
\hline & & 1 & 7 & 14 & 30 & 1 & 7 & 14 & 30 \\
\hline & & \multicolumn{4}{|c|}{ December-January-February } & \multicolumn{4}{|c|}{ March-April-May } \\
\hline 0.05 & 20 & 0 & 0 & 0 & 0 & 0 & 0 & 0 & 0.080 \\
\hline 0.10 & 10 & 0 & 0 & 0 & 0 & 0 & 0 & 0 & 0.434 \\
\hline 0.20 & 5 & 0 & 0 & 0 & 0.116 & 0 & 0.059 & 0.153 & 1.37 \\
\hline \multirow[t]{2}{*}{0.50} & 2 & 0.451 & 0.471 & 0.546 & 0.709 & 0.470 & 0.597 & 1.05 & 7.64 \\
\hline & & \multicolumn{4}{|c|}{ June-July-August } & \multicolumn{4}{|c|}{ September-October-November } \\
\hline 0.05 & 20 & 0 & 0 & 0 & 0.140 & 0 & 0 & 0 & 0 \\
\hline 0.10 & 10 & 0.026 & 0.097 & 0.315 & 0.529 & 0 & 0 & 0 & 0.234 \\
\hline 0.20 & 5 & 0.544 & 0.940 & 1.12 & 1.68 & 0 & 0 & 0.237 & 0.886 \\
\hline 0.50 & 2 & 2.44 & 3.18 & 3.97 & 6.59 & 1.81 & 2.98 & $1_{3.50}$ & 4.03 \\
\hline
\end{tabular}

${ }^{1}$ Graphical interpretation. 


\section{FOREST RIVER AT MINTO, ND--Continued}

Annual peak discharge and corresponding gage height

$$
[--, \text { no data }]
$$

\begin{tabular}{|c|c|c|c|c|c|c|c|}
\hline $\begin{array}{l}\text { Water } \\
\text { year }\end{array}$ & Date & $\begin{array}{c}\text { Gage } \\
\text { height } \\
\text { (feet) }\end{array}$ & $\begin{array}{c}\text { Peak } \\
\text { discharge } \\
\left(\mathrm{ft}^{3} / \mathrm{s}\right)\end{array}$ & $\begin{array}{l}\text { Water } \\
\text { year }\end{array}$ & Date & $\begin{array}{c}\text { Gage } \\
\text { height } \\
\text { (feet) }\end{array}$ & $\begin{array}{c}\text { Peak } \\
\text { discharge } \\
\left(\mathrm{ft}^{3} / \mathrm{s}\right)\end{array}$ \\
\hline \multicolumn{8}{|c|}{ Annual peak discharge, by year, and corresponding gage height } \\
\hline${ }^{1} 1882$ & April & -- & 2,200 & 1968 & March 27 & 2.31 & 315 \\
\hline${ }^{1} 1897$ & April & -- & 1,850 & 1969 & April 12 & 7.67 & 3,960 \\
\hline${ }^{1} 1907$ & April & - & 1,750 & 1970 & April 9 & 7.04 & 2,220 \\
\hline${ }^{1} 1916$ & April & -- & 1,600 & 1971 & April 10 & 5.84 & 2,460 \\
\hline 1944 & April 10 & 5.00 & 650 & 1972 & March 17 & 5.96 & 2,120 \\
\hline 1945 & March 27 & 2.11 & 250 & 1973 & June 19 & 1.93 & 208 \\
\hline 1946 & March 22 & - & 1,000 & 1974 & April 16 & 7.79 & 4,580 \\
\hline 1947 & March 25 & 4.12 & 1,100 & 1975 & April 15 & 6.73 & 1,600 \\
\hline 1948 & April 19 & 11.80 & 11,500 & 1976 & March 31 & 7.03 & 1,500 \\
\hline 1949 & April 7 & 8.19 & 2,020 & 1977 & April 10 & 1.58 & 77.0 \\
\hline 1950 & April 18 & 11.80 & 16,600 & 1978 & March 31 & 6.52 & 1,600 \\
\hline 1951 & April 5 & 3.60 & 900 & 1979 & April 20 & 8.93 & 6,730 \\
\hline 1952 & April 2 & 2.78 & 370 & 1980 & April 1 & 2.01 & 167 \\
\hline 1953 & June 4 & 3.53 & 910 & 1981 & June 28 & 2.04 & 176 \\
\hline 1954 & June 16 & 2.61 & 391 & 1982 & July 26 & 4.07 & 1,140 \\
\hline 1955 & April 2 & 8.56 & 4,200 & 1983 & March 15 & 3.52 & 820 \\
\hline 1956 & April 2l & 6.63 & 2,930 & 1984 & March 26 & 2.77 & 394 \\
\hline 1957 & March 23 & 2.72 & 461 & 1985 & March 14 & 3.39 & 640 \\
\hline 1958 & June 10 & 2.72 & 463 & 1986 & March 19 & 3.40 & 690 \\
\hline 1959 & April 2 & 2.45 & 338 & 1987 & April 4 & 6.91 & 2,360 \\
\hline 1960 & April 6 & 5.60 & 2,050 & 1988 & May 29 & 3.71 & 945 \\
\hline 1961 & March 22 & 1.90 & 147 & 1989 & April 18 & 2.49 & 371 \\
\hline 1962 & April 19 & 6.68 & 2,400 & 1990 & April 15 & 1.56 & 58.0 \\
\hline 1963 & March 24 & 2.54 & 250 & 1991 & April 4 & 1.54 & 60.0 \\
\hline 1964 & June 21 & 4.31 & 1,460 & 1992 & March 8 & 3.34 & 640 \\
\hline 1965 & April 12 & 7.48 & 3,710 & 1993 & July 28 & 3.70 & 705 \\
\hline 1966 & March 21 & 7.82 & 3,100 & 1994 & March 26 & -- & 533 \\
\hline 1967 & March 31 & 7.22 & 3,070 & & & & \\
\hline \multicolumn{8}{|c|}{ Annual peak discharge, from highest to lowest, and corresponding gage height } \\
\hline 1950 & April 18 & 11.80 & 16,600 & 1972 & March 17 & 5.96 & 2,120 \\
\hline 1948 & April 19 & 11.80 & 11,500 & 1960 & April 6 & 5.60 & 2,050 \\
\hline 1979 & April 20 & 8.93 & 6,730 & 1949 & April 7 & 8.19 & 2,020 \\
\hline 1974 & April 16 & 7.79 & 4,580 & ${ }^{1} 1897$ & April & -- & 1,850 \\
\hline 1955 & April 2 & 8.56 & 4,200 & ${ }^{1} 1907$ & April & -- & 1,750 \\
\hline 1969 & April 12 & 7.67 & 3,960 & ${ }^{1} 1916$ & April & -- & 1,600 \\
\hline 1965 & April 12 & 7.48 & 3,710 & 1975 & April 15 & 6.73 & 1,600 \\
\hline 1966 & March 21 & 7.82 & 3,100 & 1978 & March 31 & 6.52 & 1,600 \\
\hline 1967 & March 31 & 7.22 & 3,070 & 1976 & March 31 & 7.03 & 1,500 \\
\hline 1956 & April 2l & 6.63 & 2,930 & 1964 & June 21 & 4.31 & 1,460 \\
\hline 1971 & April 10 & 5.84 & 2,460 & 1982 & July 26 & 4.07 & 1,140 \\
\hline 1962 & April 19 & 6.68 & 2,400 & 1947 & March 25 & 4.12 & 1,100 \\
\hline 1987 & April 4 & 6.91 & 2,360 & 1946 & March 22 & -- & 1,000 \\
\hline 1970 & April 9 & 7.04 & 2,220 & 1988 & May 29 & 3.71 & 945 \\
\hline${ }^{1} 1882$ & April & -- & 2,200 & 1953 & June 4 & 3.53 & 910 \\
\hline
\end{tabular}




\section{FOREST RIVER AT MINTO, ND--Continued}

Annual peak discharge and corresponding gage height--Continued

$$
[-, \text { no data }]
$$

\begin{tabular}{llllllll}
\hline $\begin{array}{c}\text { Water } \\
\text { year }\end{array}$ & \multicolumn{1}{c}{ Date } & $\begin{array}{c}\text { Gage } \\
\text { height } \\
\text { (feet) }\end{array}$ & $\begin{array}{c}\text { Peak } \\
\text { discharge } \\
\left(\mathbf{f t}^{\mathbf{3}} / \mathbf{s}\right)\end{array}$ & $\begin{array}{c}\text { Water } \\
\text { year }\end{array}$ & Date & $\begin{array}{c}\text { Gage } \\
\text { height } \\
\text { (feet) }\end{array}$ & $\begin{array}{c}\text { Peak } \\
\text { discharge } \\
\left.\mathbf{( t t}^{3} / \mathbf{s}\right)\end{array}$ \\
\hline \multicolumn{7}{c}{ Annual peak discharge, from highest to lowest, and corresponding gage height-Continued } \\
1951 & April 5 & 3.60 & 900 & 1952 & April 2 & 2.78 & 370 \\
1983 & March 15 & 3.52 & 820 & 1959 & April 2 & 2.45 & 338 \\
1993 & July 28 & 3.70 & 705 & 1968 & March 27 & 2.31 & 315 \\
1986 & March 19 & 3.40 & 690 & 1945 & March 27 & 2.11 & 250 \\
1944 & April 10 & 5.00 & 650 & 1963 & March 24 & 2.54 & 250 \\
1985 & March 14 & 3.39 & 640 & 1973 & June 19 & 1.93 & 208 \\
1992 & March 8 & 3.34 & 640 & 1981 & June 28 & 2.04 & 176 \\
1994 & March 26 & - & 533 & 1980 & April 1 & 2.01 & 167 \\
1958 & June 10 & 2.72 & 463 & 1961 & March 22 & 1.90 & 147 \\
1957 & March 23 & 2.72 & 461 & 1977 & April 10 & 1.58 & 77.0 \\
1984 & March 26 & 2.77 & 394 & 1991 & April 4 & 1.54 & 60.0 \\
1954 & June 16 & 2.61 & 391 & 1990 & April 15 & 1.56 & 58.0 \\
1989 & April 18 & 2.49 & 371 & & & & \\
\hline
\end{tabular}

${ }^{1}$ Determined by U.S. Army Corps of Engineers; not used in statistics. 


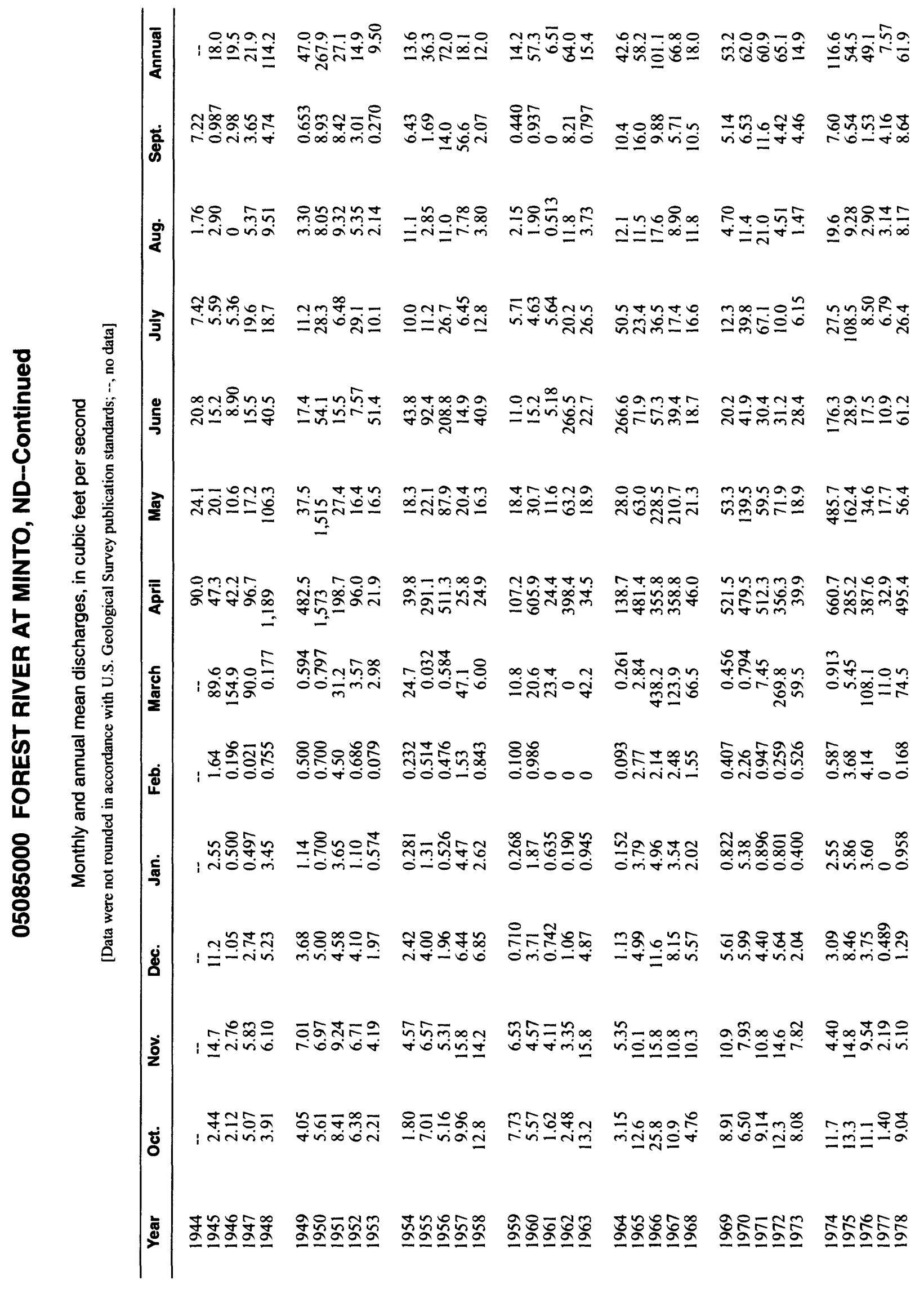




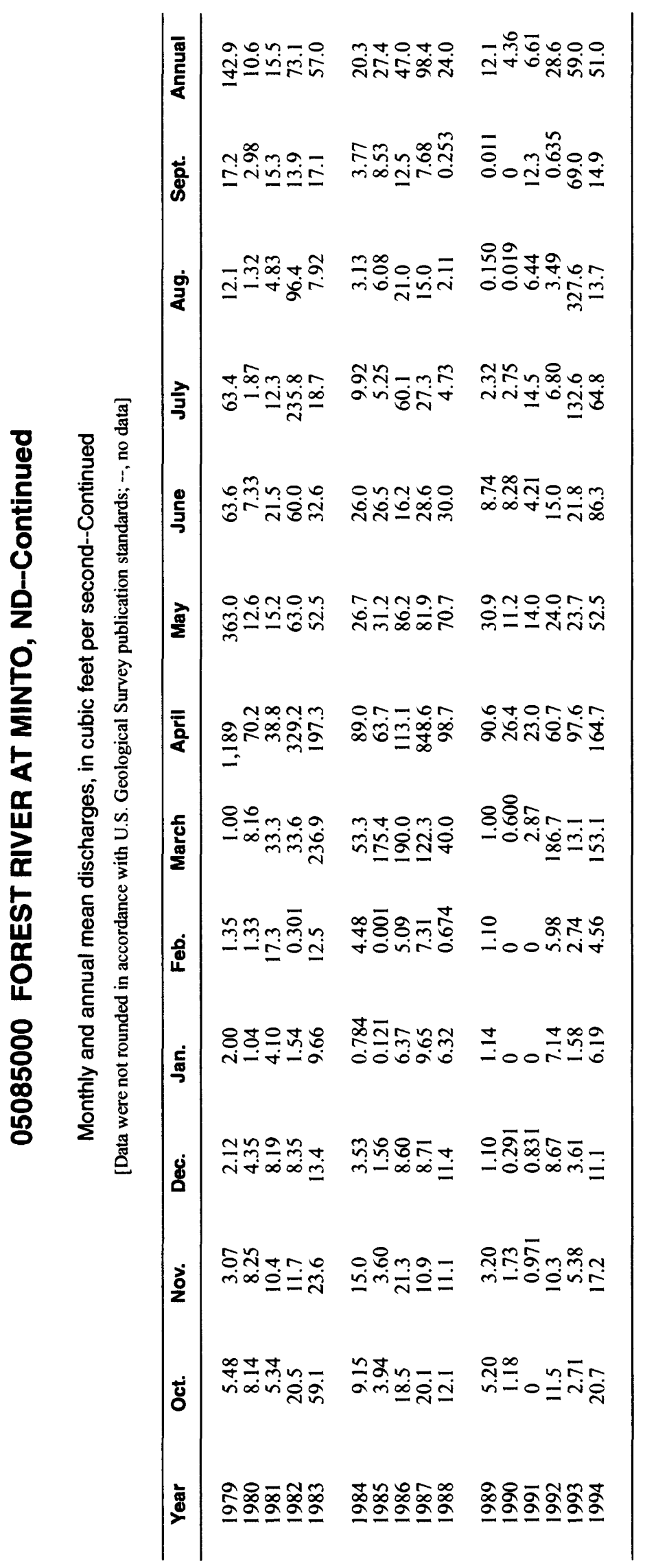




\section{MIDDLE RIVER AT ARGYLE, MN}

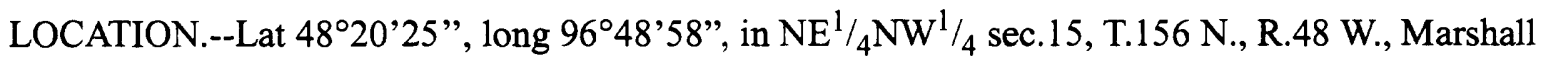
County, Hydrologic Unit 09020309, on left bank $30 \mathrm{ft}$ upstream of bridge on County Highway 4 in Argyle and $14 \mathrm{mi}$ upstream from mouth.

DRAINAGE AREA.--265 $\mathrm{mi}^{2}$.

PERIOD OF RECORD.--March to September 1945, October 1950 to September 1981, February 1982 to current year. Monthly discharge only for some periods, published in WSP 1728. October 1981 to January 1982, operated as a high-flow partial-record station.

GAGE.--Water-stage recorder. Datum of gage is $828.53 \mathrm{ft}$ above mean sea level. Prior to Nov. 8, 1951, nonrecording gage and Nov. 8,1951 , to Sept. 18, 1952, water-stage recorder at site $800 \mathrm{ft}$ downstream at datum $1.0 \mathrm{ft}$ higher. Sept. 19,1952 , to June 28,1982 , recording gage at site $800 \mathrm{ft}$ downstream at present datum. June 29, 1982, to Sept. 20,1983, nonrecording gage at present site and datum.

EXTREMES FOR PERIOD OF RECORD.--Maximum discharge, 4,260 $\mathrm{ft}^{3} / \mathrm{s}$, July 3, 1975, gage height, $16.59 \mathrm{ft}$, present datum; no flow at times.

EXTREMES OUTSIDE PERIOD OF RECORD.--Flood of April 1950 reached a stage of $15.25 \mathrm{ft}$ present datum, site then in use, from floodmarks.

Annual mean discharge

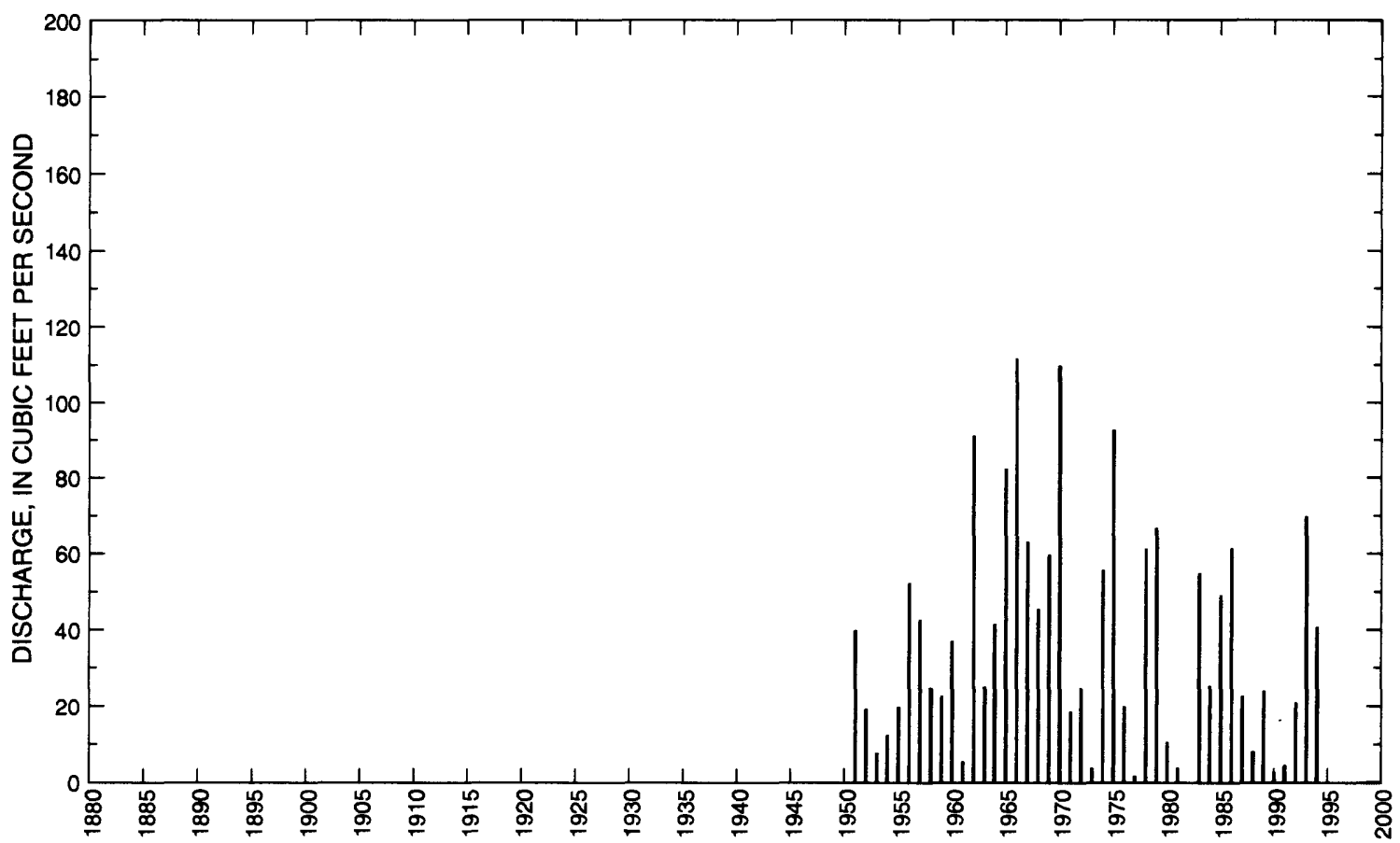




\section{MIDDLE RIVER AT ARGYLE, MN--Continued}

Statistics of monthly and annual mean discharges

$[\mathrm{m}$, more than 1 year of occurrence]

\begin{tabular}{|c|c|c|c|c|c|c|c|c|}
\hline \multirow[b]{2}{*}{ Month } & \multicolumn{2}{|c|}{ Maximum } & \multicolumn{2}{|c|}{ Minimum } & \multicolumn{4}{|c|}{ Mean } \\
\hline & $\begin{array}{c}\text { Discharge } \\
\left(\mathrm{ft}^{3} / \mathrm{s}\right)\end{array}$ & $\begin{array}{c}\text { Water year } \\
\text { of } \\
\text { occurrence }\end{array}$ & $\begin{array}{c}\text { Discharge } \\
\left(\mathrm{ft}^{3} / \mathrm{s}\right)\end{array}$ & $\begin{array}{c}\text { Water year } \\
\text { of } \\
\text { occurrence }\end{array}$ & $\begin{array}{c}\text { Discharge } \\
\left(\mathrm{ft}^{3} / \mathrm{s}\right)\end{array}$ & $\begin{array}{c}\text { Standard } \\
\text { deviation } \\
\left(\mathrm{ft}^{3} / \mathrm{s}\right)\end{array}$ & $\begin{array}{l}\text { Coeffi- } \\
\text { cient of } \\
\text { variation }\end{array}$ & $\begin{array}{l}\text { Percentage } \\
\text { of annual } \\
\text { discharge }\end{array}$ \\
\hline October & 94.1 & 1983 & 0 & $\mathrm{~m}$ & 8.96 & 19.5 & 2.18 & 1.93 \\
\hline November & 33.4 & 1957 & 0 & $\mathrm{~m}$ & 5.18 & 7.72 & 1.49 & 1.12 \\
\hline December & 15.8 & 1983 & 0 & $\mathrm{~m}$ & 2.33 & 3.09 & 1.33 & 0.50 \\
\hline January & 4.65 & 1983 & 0 & $\mathrm{~m}$ & 1.02 & 1.22 & 1.21 & 0.22 \\
\hline February & 3.32 & 1983 & 0 & $\mathrm{~m}$ & 0.750 & 0.91 & 1.21 & 0.16 \\
\hline March & 217 & 1983 & 0 & $\mathrm{~m}$ & 25.1 & 43.5 & 1.73 & 5.42 \\
\hline April & 747 & 1966 & 0.197 & 1991 & 200 & 184 & 0.92 & 43.0 \\
\hline May & 330 & 1970 & 2.12 & 1981 & 70.0 & 82.0 & 1.17 & 15.1 \\
\hline June & 660 & 1970 & 0.366 & 1973 & 70.0 & 129 & 1.84 & 15.1 \\
\hline July & 688 & 1975 & 0 & 1961 & 54.1 & 116 & 2.15 & 11.7 \\
\hline August & 264 & 1993 & 0 & 1961 & 11.0 & 39.6 & 3.60 & 2.36 \\
\hline September & 272 & 1993 & 0 & $\mathrm{~m}$ & 16.3 & 50.4 & 3.09 & 3.51 \\
\hline Annual & 112 & 1966 & 1.60 & 1977 & 38.5 & 29.6 & 0.77 & 100 \\
\hline
\end{tabular}

Annual flow duration

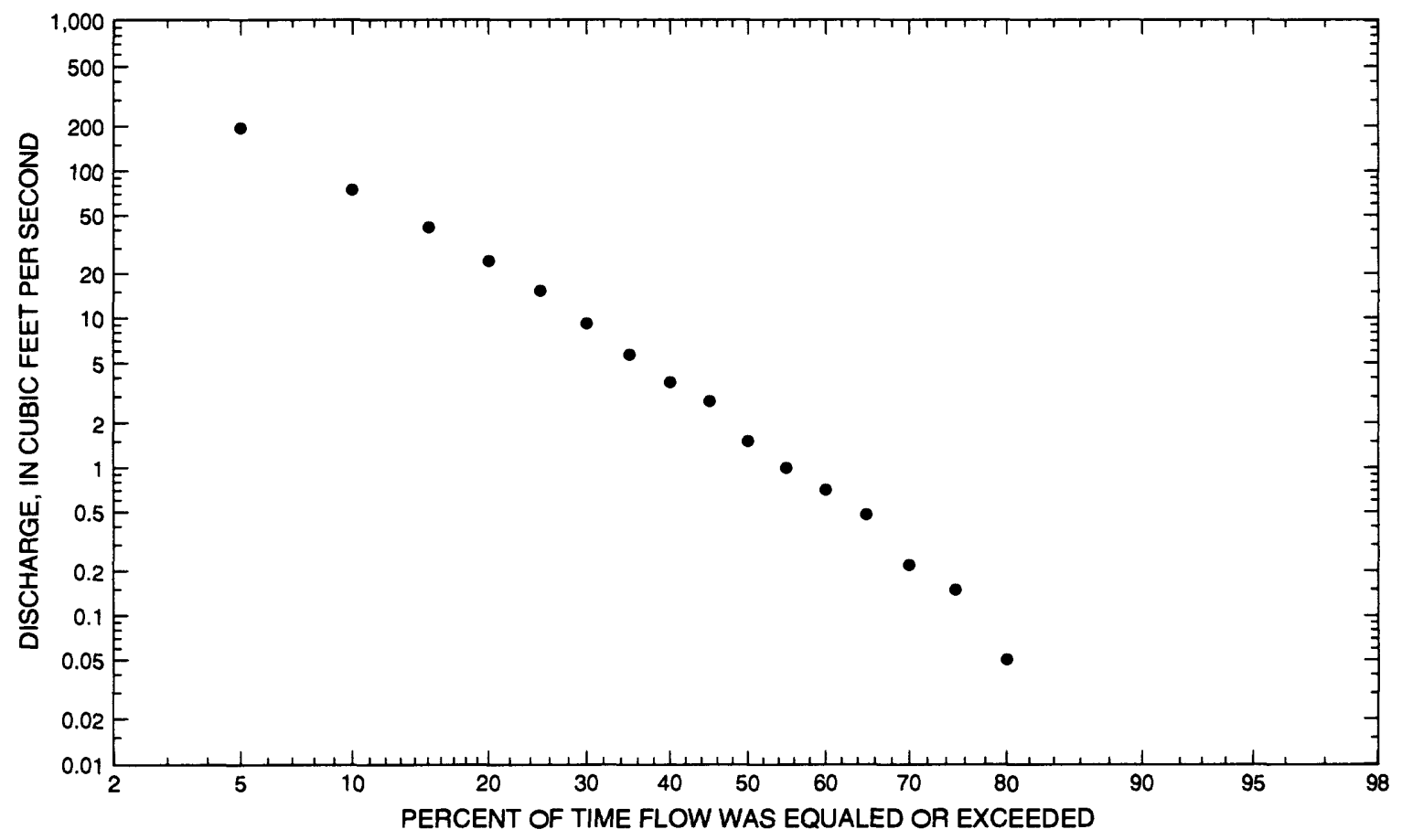




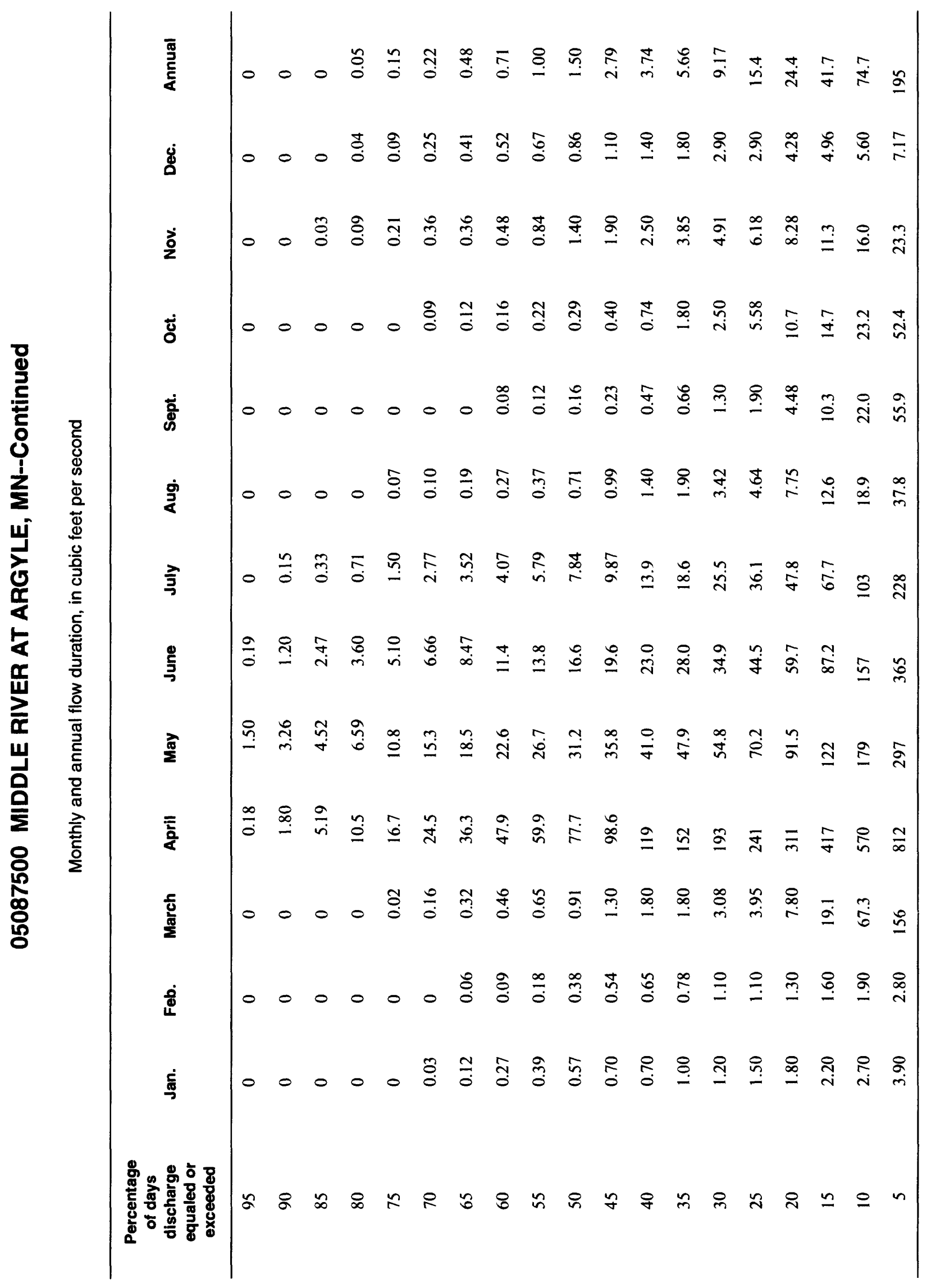




\section{MIDDLE RIVER AT ARGYLE, MN--Continued}

Probability of occurrence of annual high discharges

[ng, statistic not given]

\begin{tabular}{|c|c|c|c|c|c|c|}
\hline \multirow[b]{2}{*}{$\begin{array}{l}\text { Exceedance } \\
\text { probability }\end{array}$} & \multirow[b]{2}{*}{$\begin{array}{c}\text { Recurrence } \\
\text { interval } \\
\text { (years) }\end{array}$} & \multirow[b]{2}{*}{$\begin{array}{c}\text { Maximum } \\
\text { instantaneous } \\
\left(\mathrm{ft}^{3} / \mathrm{s}\right)\end{array}$} & \multicolumn{4}{|c|}{$\begin{array}{c}\text { Maximum mean discharge } \\
\left(\mathrm{ft}^{3} / \mathrm{s}\right)\end{array}$} \\
\hline & & & 3-day period & 7-day period & 15-day period & 30-day period \\
\hline 0.99 & 1.01 & ng & 16.4 & 15.0 & 11.9 & 8.78 \\
\hline 0.95 & 1.05 & 104 & 62.9 & 54.6 & 39.8 & 27.3 \\
\hline 0.90 & 1.11 & 171 & 117 & 99.5 & 70.2 & 46.9 \\
\hline 0.80 & 1.25 & 300 & 229 & 190 & 130 & 84.7 \\
\hline 0.50 & 2 & 771 & 652 & 520 & 349 & 221 \\
\hline 0.20 & 5 & 1,680 & 1,390 & 1,080 & 732 & 465 \\
\hline 0.10 & 10 & 2,380 & 1,860 & 1,440 & 990 & 635 \\
\hline 0.04 & 25 & 3,310 & 2,390 & 1,830 & 1,290 & 839 \\
\hline 0.02 & 50 & 4,000 & 2,710 & 2,080 & 1,480 & 978 \\
\hline 0.01 & 100 & 4,680 & 2,980 & 2,280 & 1,650 & 1,100 \\
\hline 0.005 & 200 & 5,340 & 3,200 & 2,450 & 1,800 & 1,220 \\
\hline 0.002 & 500 & 6,170 & ng & $\mathrm{ng}$ & $\mathrm{ng}$ & $\mathrm{ng}$ \\
\hline
\end{tabular}

Probability of occurrence of annual low discharges

Minimum mean discharge $\left(\mathrm{ft}^{3} / \mathrm{s}\right)$

Number of consecutive days

\begin{tabular}{|c|c|c|c|c|c|c|c|c|c|c|}
\hline $\begin{array}{l}\text { Non- } \\
\text { exceed- } \\
\text { ance } \\
\text { prob- } \\
\text { abillty }\end{array}$ & $\begin{array}{c}\text { Recur- } \\
\text { rence } \\
\text { inter- } \\
\text { val } \\
\text { (years) }\end{array}$ & 1 & 3 & 7 & 14 & 30 & 60 & 90 & 120 & 183 \\
\hline 0.05 & 20 & 0 & 0 & 0 & 0 & 0 & 0 & 0 & 0 & 0 \\
\hline 0.10 & 10 & 0 & 0 & 0 & 0 & 0 & 0 & 0 & 0 & 0 \\
\hline 0.20 & 5 & 0 & 0 & 0 & 0 & 0 & 0 & 0 & 0.043 & 0.093 \\
\hline 0.50 & 2 & 0 & 0 & 0 & 0 & 0.051 & 0.135 & 0.313 & 0.471 & 0.816 \\
\hline
\end{tabular}




\section{MIDDLE RIVER AT ARGYLE, MN--Continued}

Probability of occurrence of seasonal low discharges

\begin{tabular}{|c|c|c|c|c|c|c|c|c|c|}
\hline \multirow[b]{3}{*}{$\begin{array}{c}\text { Non- } \\
\text { exceedance } \\
\text { probability }\end{array}$} & \multirow[b]{3}{*}{$\begin{array}{l}\text { Recurrence } \\
\text { interval } \\
\text { (years) }\end{array}$} & \multicolumn{8}{|c|}{ Minimum mean discharge $\left(\mathrm{ft}^{3} / \mathrm{s}\right)$} \\
\hline & & \multicolumn{8}{|c|}{ Number of consecutive days } \\
\hline & & 1 & 7 & 14 & 30 & 1 & 7 & 14 & 30 \\
\hline & & \multicolumn{4}{|c|}{ December-January-February } & \multicolumn{4}{|c|}{ March-April-May } \\
\hline 0.05 & 20 & 0 & 0 & 0 & 0 & 0 & 0 & 0 & 0 \\
\hline 0.10 & 10 & 0 & 0 & 0 & 0 & 0 & 0 & 0 & 0 \\
\hline 0.20 & 5 & 0 & 0 & 0 & 0 & 0 & 0 & 0 & 0.057 \\
\hline \multirow[t]{2}{*}{0.50} & 2 & 0.246 & 0.249 & ${ }^{1} 0.252$ & 0.255 & 0.317 & 0.353 & 0.472 & 3.67 \\
\hline & & \multicolumn{4}{|c|}{ June-July-August } & \multicolumn{4}{|c|}{ September-October-November } \\
\hline 0.05 & 20 & 0 & 0 & 0 & 0 & 0 & 0 & 0 & 0 \\
\hline 0.10 & 10 & 0 & 0 & 0 & 0.015 & 0 & 0 & 0 & 0 \\
\hline 0.20 & 5 & 0 & 0 & 0 & 0.086 & 0 & 0 & 0 & 0 \\
\hline 0.50 & 2 & 0.154 & 0.284 & 0.411 & 0.879 & 0 & 0.026 & 0.070 & 0.209 \\
\hline
\end{tabular}

${ }^{1}$ Graphical interpretation. 
05087500 MIDDLE RIVER AT ARGYLE, MN--Continued

Annual peak discharge and corresponding gage height

$[--$, no data $]$

\begin{tabular}{|c|c|c|c|c|c|c|c|}
\hline $\begin{array}{c}\text { Water } \\
\text { year }\end{array}$ & Date & $\begin{array}{c}\text { Gage } \\
\text { height } \\
\text { (feet) }\end{array}$ & $\begin{array}{c}\text { Peak } \\
\text { discharge } \\
\left(\mathrm{ft}^{3} / \mathrm{s}\right)\end{array}$ & $\begin{array}{c}\text { Water } \\
\text { year }\end{array}$ & Date & $\begin{array}{c}\text { Gage } \\
\text { height } \\
\text { (feet) }\end{array}$ & $\begin{array}{c}\text { Peak } \\
\text { discharge } \\
\left(\mathrm{ft}^{3} / \mathrm{s}\right)\end{array}$ \\
\hline \multicolumn{8}{|c|}{ Annual peak discharge, by year, and corresponding gage height } \\
\hline 1945 & March 30 & 10.80 & 939 & 1972 & April 17 & 10.12 & 729 \\
\hline 1950 & April & 15.25 & 2,790 & 1973 & March 15 & 5.10 & 93.0 \\
\hline 1951 & April 9 & 11.75 & 1,220 & 1974 & April 23 & 14.65 & 2,070 \\
\hline 1952 & April 11 & 9.89 & 612 & 1975 & July 3 & 16.59 & 4,260 \\
\hline 1953 & March 31 & 6.65 & 112 & 1976 & April 3 & 10.21 & 631 \\
\hline 1954 & June 18 & -- & 128 & 1977 & May 28 & 2.50 & 24.0 \\
\hline 1955 & June 9 & 9.87 & 527 & 1978 & April 10 & 14.45 & 1,320 \\
\hline 1956 & July 11 & 13.18 & 1,390 & 1979 & April 20 & 15.29 & 2,140 \\
\hline 1957 & September 7 & 10.73 & 734 & 1980 & April 9 & 6.91 & 357 \\
\hline 1958 & July 9 & 11.38 & 846 & 1981 & July 3 & 3.97 & 107 \\
\hline 1959 & April 5 & 11.23 & 570 & 1982 & April 18 & 9.96 & 711 \\
\hline 1960 & April 10 & 12.60 & 903 & 1983 & March 9 & 14.17 & 1,020 \\
\hline 1961 & March 27 & 6.77 & 135 & 1984 & June 12 & 9.10 & 513 \\
\hline 1962 & June 12 & 14.12 & 1,620 & 1985 & June 29 & 12.58 & 939 \\
\hline 1963 & April 11 & 11.28 & 825 & 1986 & March 31 & 13.43 & 1,040 \\
\hline 1964 & June 22 & 12.40 & 900 & 1987 & March 28 & 11.78 & 550 \\
\hline 1965 & April 12 & 15.29 & 2,590 & 1988 & June 1 & 7.21 & 357 \\
\hline 1966 & April 3 & 16.00 & 1,820 & 1989 & April 18 & 15.25 & 1,550 \\
\hline 1967 & April 23 & 13.41 & 1,320 & 1990 & April 4 & 4.86 & 60.0 \\
\hline 1968 & July 20 & 12.87 & 1,120 & 1991 & July 8 & -- & 87.0 \\
\hline 1969 & April 11 & 15.92 & 2,530 & 1992 & March 9 & 9.87 & 350 \\
\hline 1970 & May 31 & 14.82 & 2,200 & 1993 & September 3 & 14.18 & 1,180 \\
\hline 1971 & April 9 & 11.74 & 773 & 1994 & September 19 & 11.30 & 707 \\
\hline \multicolumn{8}{|c|}{ Annual peak discharge, from highest to lowest, and corresponding gage height } \\
\hline 1975 & July 3 & 16.59 & 4,260 & 1985 & June 29 & 12.58 & 939 \\
\hline 1950 & April & 15.25 & 2,790 & 1960 & April 10 & 12.60 & 903 \\
\hline 1965 & April 12 & 15.29 & 2,590 & 1964 & June 22 & 12.40 & 900 \\
\hline 1969 & April 11 & 15.92 & 2,530 & 1958 & July 9 & 11.38 & 846 \\
\hline 1970 & May 31 & 14.82 & 2,200 & 1963 & April 11 & 11.28 & 825 \\
\hline 1979 & April 20 & 15.29 & 2,140 & 1971 & April 9 & 11.74 & 773 \\
\hline 1974 & April 23 & 14.65 & 2,070 & 1957 & September 7 & 10.73 & 734 \\
\hline 1966 & April 3 & 16.00 & 1,820 & 1972 & April 17 & 10.12 & 729 \\
\hline 1962 & June 12 & 14.12 & 1,620 & 1982 & April 18 & 9.96 & 711 \\
\hline 1989 & April 18 & 15.25 & 1,550 & 1994 & September 19 & 11.30 & 707 \\
\hline 1956 & July 11 & 13.18 & 1,390 & 1976 & April 3 & 10.21 & 631 \\
\hline 1967 & April 23 & 13.41 & 1,320 & 1952 & April 11 & 9.89 & 612 \\
\hline 1978 & April 10 & 14.45 & 1,320 & 1959 & April 5 & 11.23 & 570 \\
\hline 1951 & April 9 & 11.75 & 1,220 & 1987 & March 28 & 11.78 & 550 \\
\hline 1993 & September 3 & 14.18 & 1,180 & 1955 & June 9 & 9.87 & 527 \\
\hline 1968 & July 20 & 12.87 & 1,120 & 1984 & June 12 & 9.10 & 513 \\
\hline 1986 & March 31 & 13.43 & 1,040 & 1980 & April 9 & 6.91 & 357 \\
\hline 1983 & March 9 & 14.17 & 1,020 & 1988 & June 1 & 7.21 & 357 \\
\hline 1945 & March 30 & 10.80 & 939 & 1992 & March 9 & 9.87 & 350 \\
\hline
\end{tabular}




\section{MIDDLE RIVER AT ARGYLE, MN--Continued}

Annual peak discharge and corresponding gage height--Continued

$$
[--, \text { no data }]
$$

\begin{tabular}{|c|c|c|c|c|c|c|c|}
\hline $\begin{array}{l}\text { Water } \\
\text { year }\end{array}$ & Date & $\begin{array}{c}\text { Gage } \\
\text { height } \\
\text { (feet) }\end{array}$ & $\begin{array}{c}\text { Peak } \\
\text { discharge } \\
\left(\mathrm{ft}^{3} / \mathrm{s}\right)\end{array}$ & $\begin{array}{c}\text { Water } \\
\text { year }\end{array}$ & Date & $\begin{array}{l}\text { Gage } \\
\text { height } \\
\text { (feet) }\end{array}$ & $\begin{array}{c}\text { Peak } \\
\text { discharge } \\
\left(\mathrm{ft}^{3} / \mathrm{s}\right)\end{array}$ \\
\hline \multicolumn{8}{|c|}{ Annual peak discharge, from highest to lowest, and corresponding gage height-Continued } \\
\hline 1961 & March 27 & 6.77 & 135 & 1973 & March 15 & 5.10 & 93.0 \\
\hline 1954 & June 18 & -- & 128 & 1991 & July 8 & -- & 87.0 \\
\hline 1953 & March 31 & 6.65 & 112 & 1990 & April 4 & 4.86 & 60.0 \\
\hline 1981 & July 3 & 3.97 & 107 & 1977 & May 28 & 2.50 & 24.0 \\
\hline
\end{tabular}




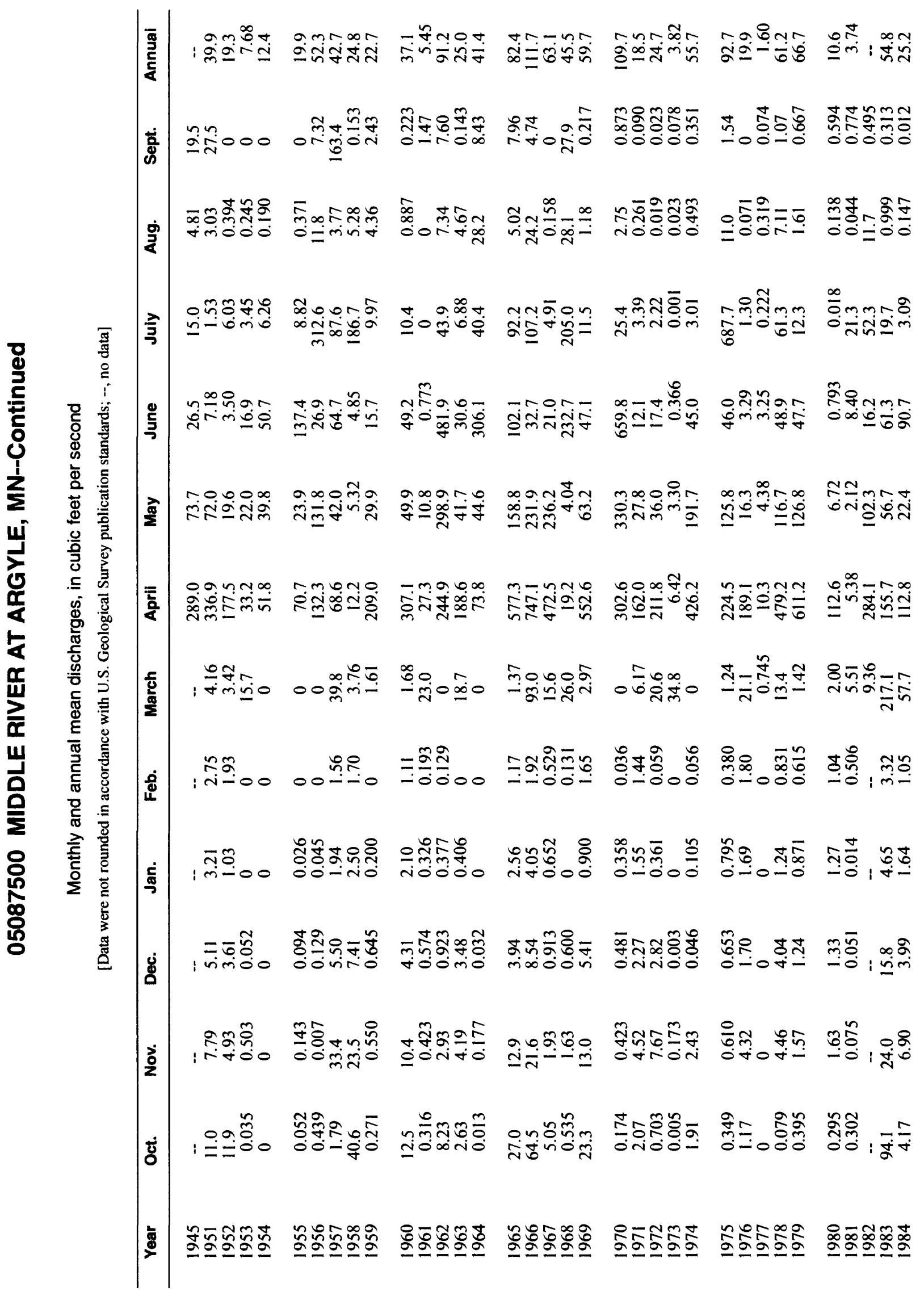




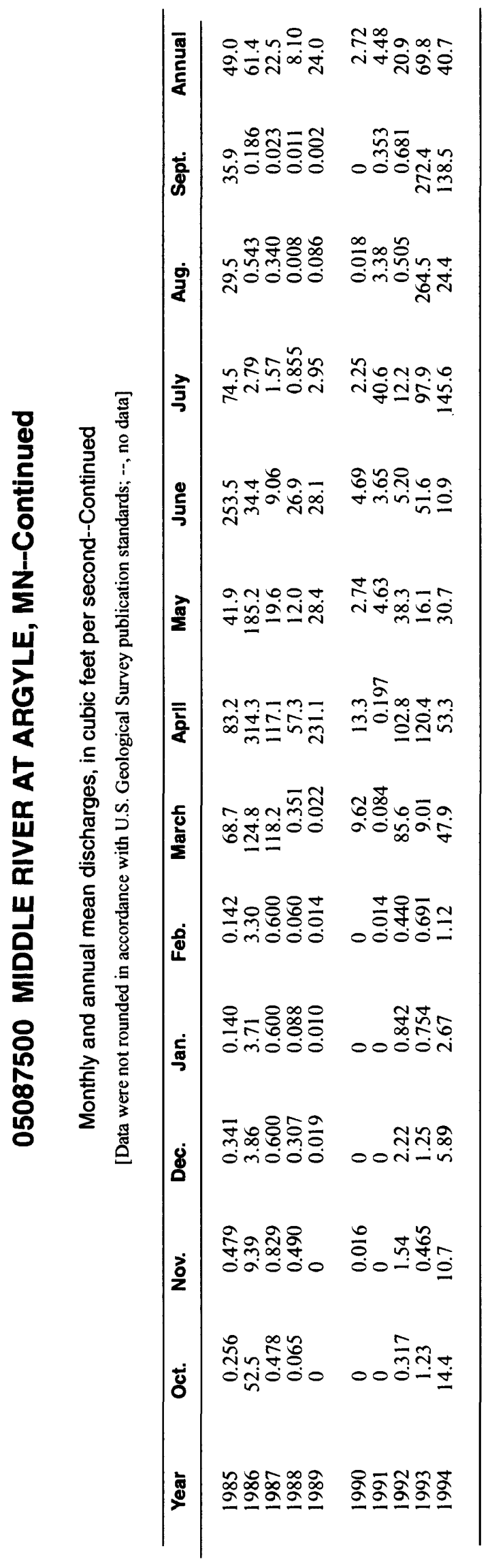




\section{SOUTH BRANCH PARK RIVER NEAR PARK RIVER, ND}

LOCATION.--Lat 48 $24^{\prime} 50^{\prime \prime}$, long 97'51'40" (revised), on line between sec.15 and 16, T.157 N., R.56 W., Walsh County, Hydrologic Unit 09020310, at bridge on State Highway 32, half a mile upstream from small tributary and 4.5 miles northwest of town of Park River.

DRAINAGE AREA.--214 $\mathrm{mi}^{2}$.

PERIOD OF RECORD.--October 1939 to September 1950. October 1939 to March 1940 estimated on basis of weather records and records for Park River at Grafton, North Dakota.

GAGE.--Chain gage.

EXTREMES FOR PERIOD OF RECORD.--Maximum discharge, 11,000 $\mathrm{ft}^{3} / \mathrm{s}$, Apr. 18, 1948, gage height, $11.80 \mathrm{ft}$, from rating curve extended above $6,600 \mathrm{ft}^{3} / \mathrm{s}$; no flow at times most years.

Annual mean discharge

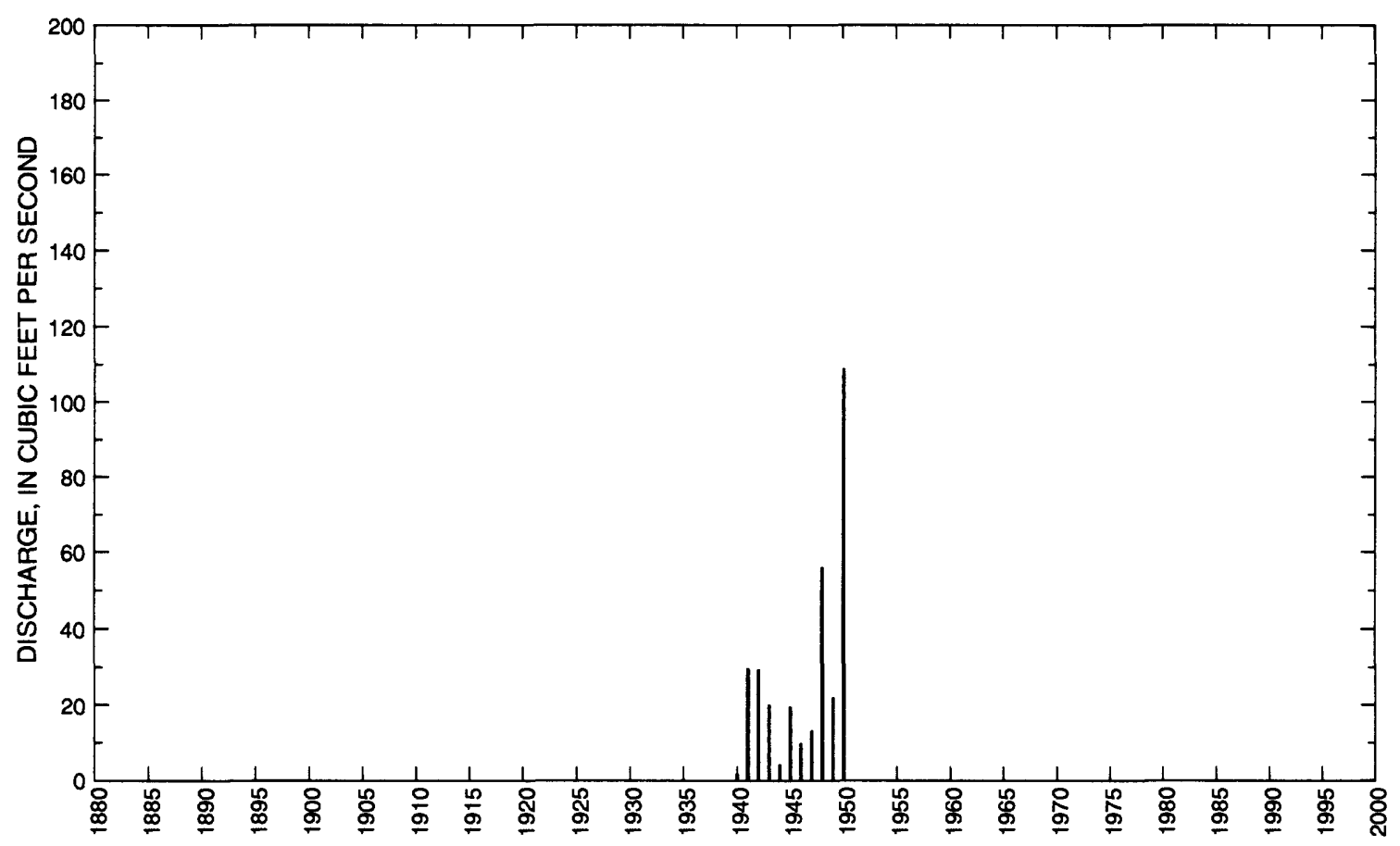




\section{SOUTH BRANCH PARK RIVER NEAR PARK RIVER, ND--Continued}

Statistics of monthly and annual mean dischargesvariation

[m, more than 1 year of occurrence]

\begin{tabular}{|c|c|c|c|c|c|c|c|c|}
\hline \multirow[b]{2}{*}{ Month } & \multicolumn{2}{|c|}{ Maximum } & \multicolumn{2}{|c|}{ Minimum } & \multicolumn{4}{|c|}{ Mean } \\
\hline & $\begin{array}{c}\text { Discharge } \\
\left(\mathrm{ft}^{3} / \mathrm{s}\right)\end{array}$ & $\begin{array}{c}\text { Water year } \\
\text { of } \\
\text { occurrence }\end{array}$ & $\begin{array}{c}\text { Discharge } \\
\left(\mathrm{ft}^{3} / \mathbf{s}\right)\end{array}$ & $\begin{array}{l}\text { Water year } \\
\text { of } \\
\text { occurrence }\end{array}$ & $\begin{array}{c}\text { Discharge } \\
\left(\mathrm{ft}^{3} / \mathbf{s}\right)\end{array}$ & $\begin{array}{l}\text { Standard } \\
\text { deviation } \\
\left(\mathrm{ft}^{3} / \mathrm{s}\right)\end{array}$ & $\begin{array}{l}\text { Coeffi- } \\
\text { cient of } \\
\text { variation }\end{array}$ & $\begin{array}{l}\text { Percentage } \\
\text { of annual } \\
\text { discharge }\end{array}$ \\
\hline October & 19.6 & 1942 & 0 & $\mathrm{~m}$ & 2.20 & 5.79 & 2.63 & 0.64 \\
\hline November & 3.74 & 1942 & 0 & $\mathrm{~m}$ & 0.870 & 1.43 & 1.65 & 0.25 \\
\hline December & 2.78 & 1945 & 0 & $\mathrm{~m}$ & 0.490 & 0.86 & 1.76 & 0.14 \\
\hline January & 0.274 & 1945 & 0 & $\mathrm{~m}$ & 0.090 & 0.09 & 1.06 & 0.03 \\
\hline February & 0.200 & $\mathrm{~m}$ & 0 & $\mathrm{~m}$ & 0.070 & 0.08 & 1.08 & 0.02 \\
\hline March & 181 & 1945 & 0 & $\mathrm{~m}$ & 41.7 & 59.7 & 1.43 & 12.1 \\
\hline April & 642 & 1950 & 17.7 & $\mathrm{~m}$ & 208 & 228 & 1.10 & 60.3 \\
\hline May & 639 & 1950 & 2.28 & 1940 & 68.3 & 190 & 2.78 & 19.8 \\
\hline June & 56.2 & 1943 & 0.180 & 1940 & 11.2 & 15.9 & 1.42 & 3.24 \\
\hline July & 23.9 & 1947 & 0.019 & 1940 & 6.07 & 8.99 & 1.48 & 1.76 \\
\hline August & 21.7 & 1948 & 0.042 & $\mathrm{~m}$ & 3.13 & 6.38 & 2.04 & 0.91 \\
\hline September & 17.4 & 1941 & 0.023 & 1940 & 2.76 & 5.23 & 1.89 & 0.80 \\
\hline Annual & 109 & 1950 & 1.67 & 1940 & 28.7 & 30.5 & 1.07 & 100 \\
\hline
\end{tabular}

Annual flow duration

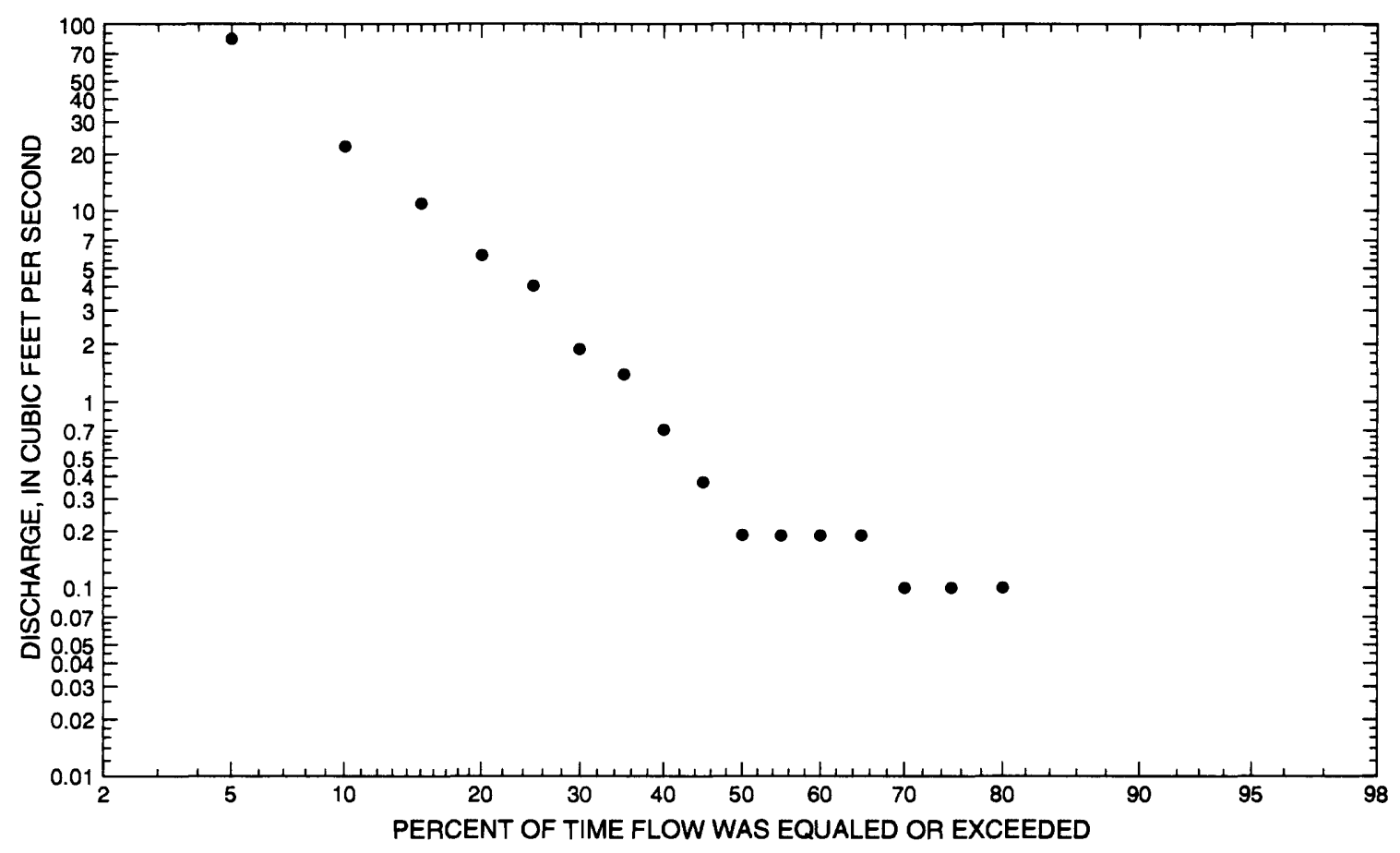




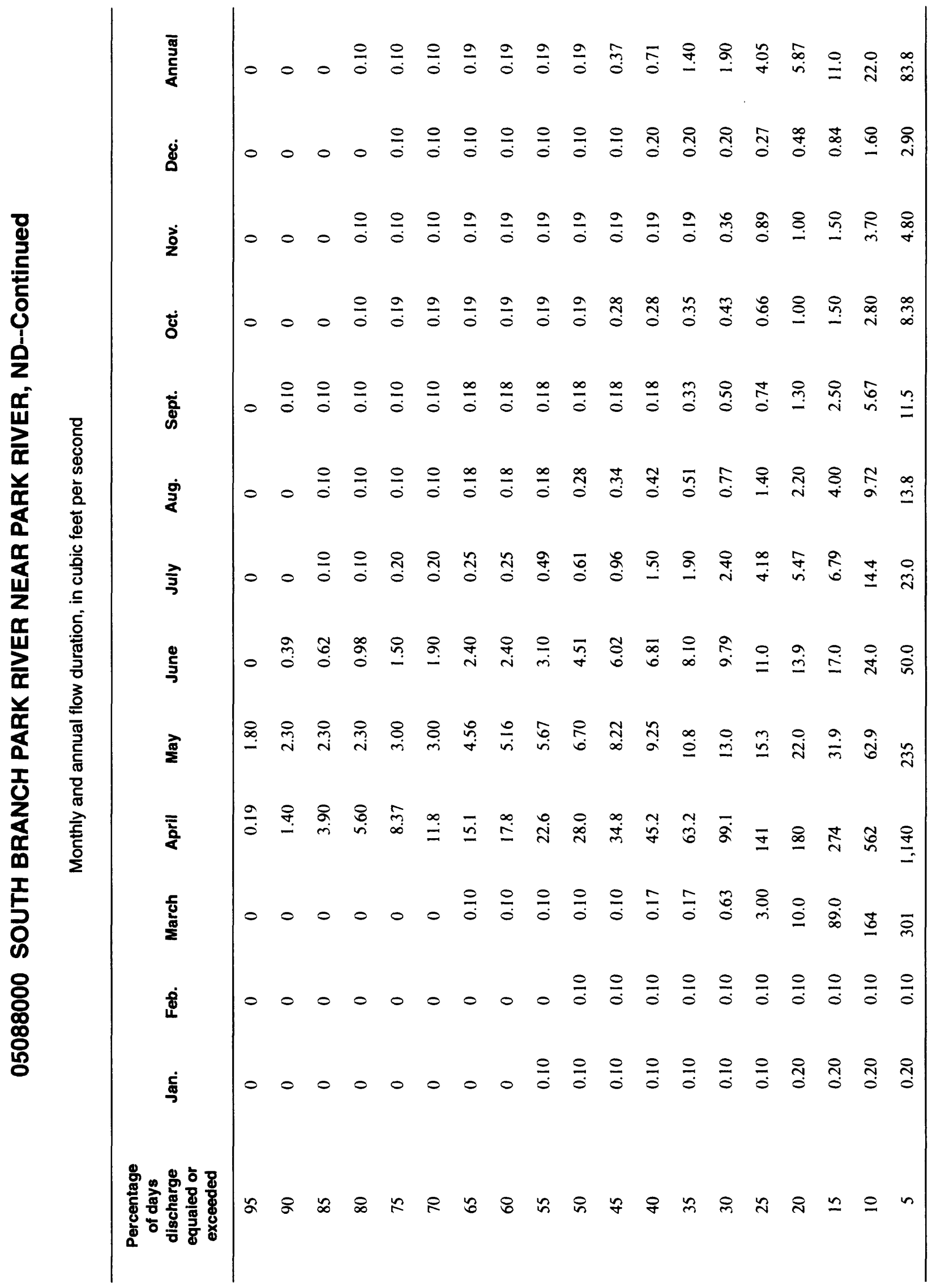


05088000 SOUTH BRANCH PARK RIVER NEAR PARK RIVER, ND--Continued

Probability of occurrence of annual high discharges

[ng, statistic not given]

\begin{tabular}{lcccccc}
\hline & & & \multicolumn{5}{c}{$\begin{array}{c}\text { Maximum mean discharge } \\
\left(\mathbf{f t}^{\mathbf{3}} \mathbf{s}\right)\end{array}$} \\
\cline { 5 - 7 } $\begin{array}{c}\text { Exceedance } \\
\text { probability }\end{array}$ & $\begin{array}{c}\text { Recurrence } \\
\text { intervai } \\
\text { (years) }\end{array}$ & $\begin{array}{c}\text { Maximum } \\
\text { instantaneous } \\
\left(\mathbf{f t}^{3} / \mathbf{s}\right)\end{array}$ & 3-day period & 7-day period & 15-day period & 30-day period \\
\hline 0.99 & 1.01 & 40.6 & 48.5 & 29.1 & 14.7 & 7.85 \\
0.95 & 1.05 & 110 & 98.1 & 65.5 & 40.0 & 21.7 \\
0.90 & 1.11 & 186 & 144 & 101 & 65.7 & 36.3 \\
0.80 & 1.25 & 346 & 233 & 168 & 116 & 65.6 \\
0.50 & 2 & 1,100 & 600 & 446 & 311 & 187 \\
0.20 & 5 & 3,380 & 1,620 & 1,160 & 737 & 482 \\
0.10 & 10 & 5,980 & 2,760 & 1,910 & 1,100 & 758 \\
0.04 & 25 & 10,800 & 4,950 & 3,240 & 1,640 & 1,190 \\
0.02 & 50 & 15,900 & 7,280 & 4,530 & 2,080 & 1,570 \\
0.01 & 100 & 22,200 & 10,300 & 6,130 & 2,550 & 2,000 \\
0.005 & 200 & 30,100 & 14,300 & 8,070 & 3,030 & 2,460 \\
0.002 & 500 & 43,400 & ng & ng & ng & ng \\
\hline
\end{tabular}

Probability of occurrence of annual low discharges

[ng, statistic not given]

\begin{tabular}{|c|c|c|c|c|c|c|c|c|c|c|}
\hline \multirow{3}{*}{$\begin{array}{l}\text { Non- } \\
\text { exceed- } \\
\text { ance } \\
\text { prob- } \\
\text { abiiity }\end{array}$} & \multirow{3}{*}{$\begin{array}{c}\text { Recur- } \\
\text { rence } \\
\text { Inter- } \\
\text { vai } \\
\text { (years) }\end{array}$} & \multicolumn{9}{|c|}{ Minimum mean discharge $\left(\mathrm{ft}^{3} / \mathrm{s}\right)$} \\
\hline & & \multicolumn{9}{|c|}{ Number of consecutive days } \\
\hline & & 1 & 3 & 7 & 14 & 30 & 60 & 90 & 120 & 183 \\
\hline 0.05 & 20 & ng & ng & 0 & 0 & 0 & 0 & 0 & 0 & 0 \\
\hline 0.10 & 10 & ng & ng & 0 & 0 & 0 & 0 & 0 & 0 & 0 \\
\hline 0.20 & 5 & ng & ng & 0 & 0 & 0 & 0 & 0 & 0.032 & 0.093 \\
\hline 0.50 & 2 & $\mathrm{ng}$ & ng & 0 & 0.034 & 0.054 & 0.087 & 0.109 & 0.125 & 0.234 \\
\hline
\end{tabular}


05088000 SOUTH BRANCH PARK RIVER NEAR PARK RIVER, ND--Continued

Probability of occurrence of seasonal low discharges

[ng, statistic not given]

\begin{tabular}{|c|c|c|c|c|c|c|c|c|c|}
\hline \multirow[b]{3}{*}{$\begin{array}{c}\text { Non- } \\
\text { exceedance } \\
\text { probability }\end{array}$} & \multirow[b]{3}{*}{$\begin{array}{c}\text { Recurrence } \\
\text { interval } \\
\text { (years) }\end{array}$} & \multicolumn{8}{|c|}{ Minimum mean discharge $\left(\mathrm{ft}^{3} / \mathrm{s}\right)$} \\
\hline & & \multicolumn{8}{|c|}{ Number of consecutive days } \\
\hline & & 1 & 7 & 14 & 30 & 1 & 7 & 14 & 30 \\
\hline & & \multicolumn{4}{|c|}{ December-January-February } & \multicolumn{4}{|c|}{ March-April-May } \\
\hline 0.05 & 20 & ng & 0 & 0 & 0 & 0 & 0 & 0 & 0 \\
\hline 0.10 & 10 & ng & 0 & 0 & 0 & 0 & 0 & 0 & 0 \\
\hline 0.20 & 5 & ng & 0 & 0 & 0 & 0 & 0 & 0 & 0 \\
\hline \multirow[t]{2}{*}{0.50} & 2 & ng & 0.084 & ${ }^{1} 0.087$ & ${ }^{1} 0.090$ & 0.088 & ${ }^{1} 0.425$ & ${ }^{1} 0.760$ & 1.10 \\
\hline & & \multicolumn{4}{|c|}{ June-July-August } & \multicolumn{4}{|c|}{ September-October-November } \\
\hline 0.05 & 20 & 0 & 0 & 0 & 0 & 0 & 0 & 0 & 0 \\
\hline 0.10 & 10 & 0 & 0 & 0.004 & 0.025 & 0 & 0 & 0 & 0 \\
\hline 0.20 & 5 & 0 & 0 & 0.023 & 0.071 & 0 & 0.050 & 0.087 & 0.114 \\
\hline 0.50 & 2 & 0.103 & 0.115 & 0.147 & 0.292 & 0.102 & 0.117 & 0.121 & 0.207 \\
\hline
\end{tabular}

${ }^{1}$ Graphical interpretation. 


\section{SOUTH BRANCH PARK RIVER NEAR PARK RIVER, ND--Continued}

Annual peak discharge and corresponding gage height

$$
[--, \text { no data }]
$$

\begin{tabular}{|c|c|c|c|c|c|c|c|}
\hline $\begin{array}{l}\text { Water } \\
\text { year }\end{array}$ & Date & $\begin{array}{l}\text { Gage } \\
\text { height } \\
\text { (feet) }\end{array}$ & $\begin{array}{c}\text { Peak } \\
\text { discharge } \\
\left(\mathrm{ft}^{3} / \mathrm{s}\right)\end{array}$ & $\begin{array}{c}\text { Water } \\
\text { year }\end{array}$ & Date & $\begin{array}{l}\text { Gage } \\
\text { height } \\
\text { (feet) }\end{array}$ & $\begin{array}{c}\text { Peak } \\
\text { discharge } \\
\left(\mathrm{ft}^{3} / \mathrm{s}\right)\end{array}$ \\
\hline \multicolumn{8}{|c|}{ Annual peak discharge, by year, and corresponding gage height } \\
\hline 1940 & April 19 & 3.16 & 171 & 1946 & March 22 & 5.66 & 400 \\
\hline 1941 & April 10 & 8.85 & 3,340 & 1947 & July 25 & 4.30 & 518 \\
\hline 1942 & April 4 & - & 1,880 & 1948 & April 18 & 11.80 & 11,000 \\
\hline 1943 & March 24 & 7.60 & 900 & 1949 & April 9 & 5.93 & 1,200 \\
\hline 1944 & April 11 & -- & 170 & 1950 & April 19 & 10.10 & 5,970 \\
\hline 1945 & March 14 & 6.60 & 800 & & & & \\
\hline \multicolumn{8}{|c|}{ Annual peak discharge, from highest to lowest, and corresponding gage height } \\
\hline 1948 & April 18 & 11.80 & 11,000 & 1945 & March 14 & 6.60 & 800 \\
\hline 1950 & April 19 & 10.10 & 5,970 & 1947 & July 25 & 4.30 & 518 \\
\hline 1941 & April 10 & 8.85 & 3,340 & 1946 & March 22 & 5.66 & 400 \\
\hline 1942 & April 4 & -- & 1,880 & 1940 & April 19 & 3.16 & 171 \\
\hline 1949 & April 9 & 5.93 & 1,200 & 1944 & April 11 & -- & 170 \\
\hline 1943 & March 24 & 7.60 & 900 & & & & \\
\hline
\end{tabular}




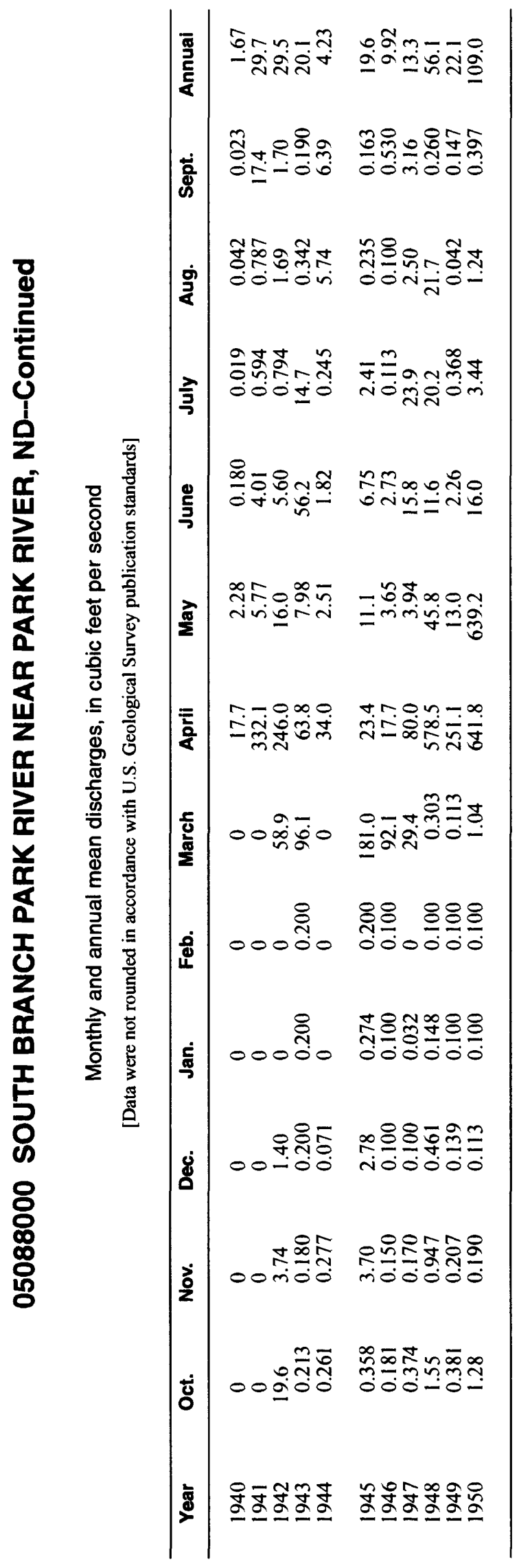




\section{SOUTH BRANCH PARK RIVER BELOW HOMME DAM, ND}

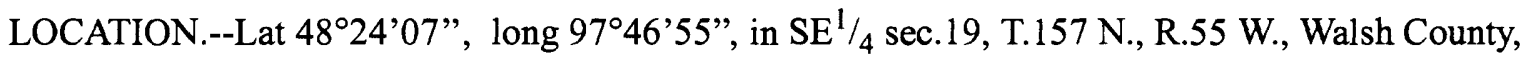
Hydrologic Unit 09020310, on right bank 0.5 mi downstream from Homme Dam, and 2 mi west of town of Park River.

DRAINAGE AREA.--226 $\mathrm{mi}^{2}$.

PERIOD OF RECORD.--October 1949 to September 1994. Monthly discharge only for October and November 1949, published in WSP 1308.

GAGE.--Water-stage recorder. Datum of gage is $1,000.00 \mathrm{ft}$ above sea level.

EXTREMES FOR PERIOD OF RECORD.--Maximum discharge, about 13,000 $\mathrm{ft}^{3} / \mathrm{s}$, Apr. 24, 1950, gage height, $37.52 \mathrm{ft}$, dam failure; no flow at times.

Annual mean discharge

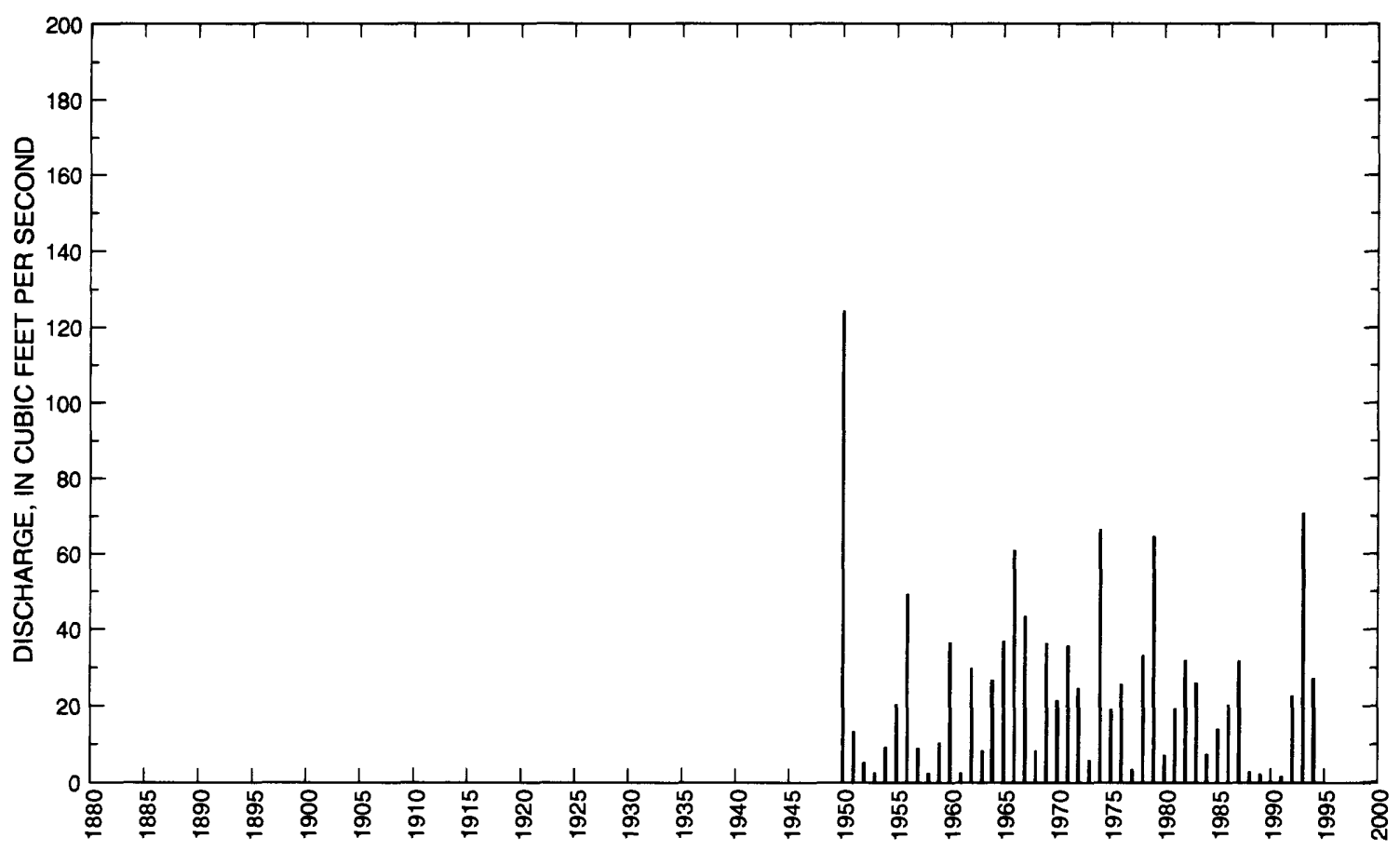


05089000 SOUTH BRANCH PARK RIVER BELOW HOMME DAM, ND--Continued

Statistics of monthly and annual mean discharges

\begin{tabular}{|c|c|c|c|c|c|c|c|c|}
\hline \multirow[b]{2}{*}{ Month } & \multicolumn{2}{|c|}{ Maximum } & \multicolumn{2}{|c|}{ Minimum } & \multicolumn{4}{|c|}{ Mean } \\
\hline & $\begin{array}{c}\text { Discharge } \\
\left(\mathrm{ft}^{3 / \mathbf{s}}\right)\end{array}$ & $\begin{array}{c}\text { Water year } \\
\text { of } \\
\text { occurrence }\end{array}$ & $\begin{array}{c}\text { Discharge } \\
\left(\mathrm{ft}^{3 / \mathrm{s})}\right.\end{array}$ & $\begin{array}{l}\text { Water year } \\
\text { of } \\
\text { occurrence }\end{array}$ & $\begin{array}{c}\text { Discharge } \\
\left(\mathrm{ft}^{3} / \mathrm{s}\right)\end{array}$ & $\begin{array}{c}\text { Standard } \\
\text { deviation } \\
\left(\mathrm{ft}^{3} / \mathrm{s}\right)\end{array}$ & $\begin{array}{l}\text { Coeffl- } \\
\text { cient of } \\
\text { variation }\end{array}$ & $\begin{array}{c}\text { Percentage } \\
\text { of annual } \\
\text { discharge }\end{array}$ \\
\hline October & 25.7 & 1983 & 0 & 1950 & 3.97 & 5.98 & 1.50 & 1.32 \\
\hline November & 16.0 & 1985 & 0.015 & 1989 & 3.59 & 3.34 & 0.93 & 1.19 \\
\hline December & 12.2 & 1983 & 0.010 & 1990 & 4.21 & 3.18 & 0.75 & 1.40 \\
\hline January & 31.2 & 1974 & 0 & 1990 & 5.39 & 5.06 & 0.94 & 1.79 \\
\hline February & 28.6 & 1981 & 0 & 1990 & 5.75 & 5.49 & 0.95 & 1.91 \\
\hline March & 234 & 1966 & 0.059 & 1991 & 31.8 & 48.4 & 1.52 & 10.6 \\
\hline April & 749 & 1950 & 0.608 & 1977 & 144 & 174 & 1.20 & 47.9 \\
\hline May & 706 & 1950 & 0.727 & 1977 & 48.1 & 114 & 2.37 & 16.0 \\
\hline June & 182 & 1964 & 0.776 & 1989 & 19.5 & 30.2 & 1.54 & 6.49 \\
\hline July & 500 & 1993 & 0.028 & 1990 & 21.6 & 75.1 & 3.48 & 7.18 \\
\hline August & 194 & 1993 & 0 & 1990 & 7.34 & 28.6 & 3.90 & 2.44 \\
\hline September & 36.2 & 1951 & 0 & 1990 & 5.28 & 8.17 & 1.55 & 1.76 \\
\hline Annual & 124.5 & 1950 & 0.516 & 1990 & 25.0 & 24.0 & 0.96 & 100 \\
\hline
\end{tabular}

Annual flow duration

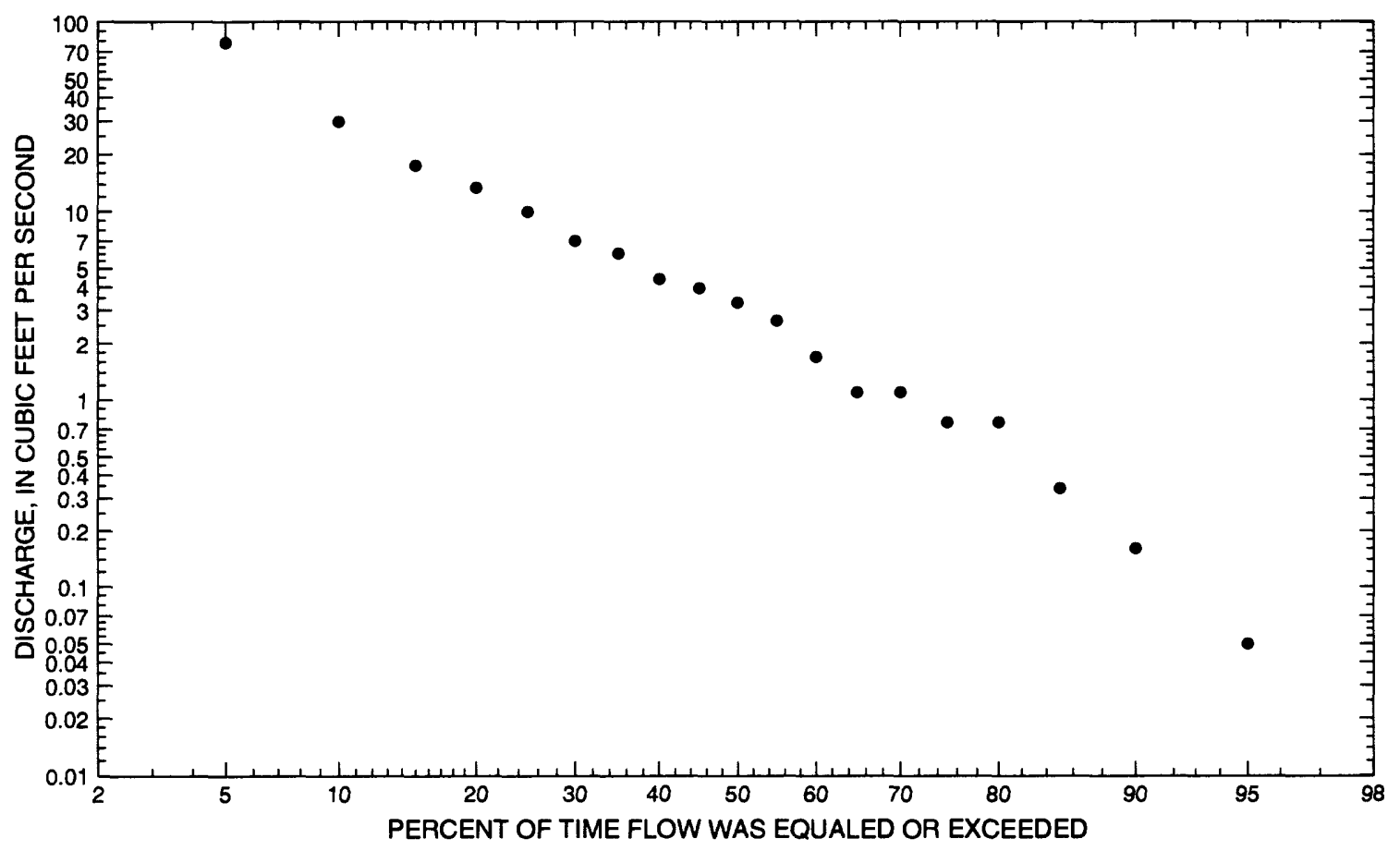




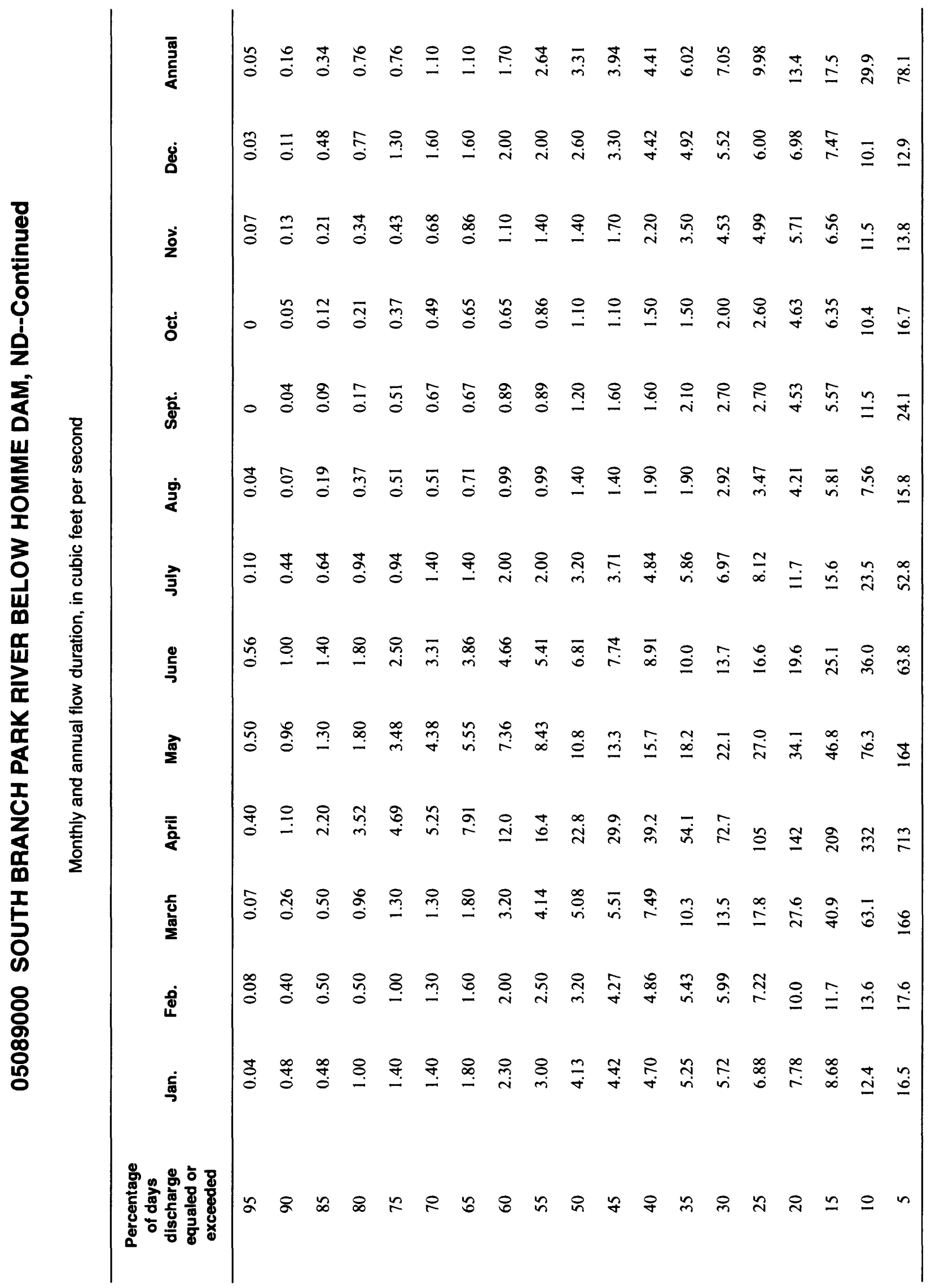


05089000 SOUTH BRANCH PARK RIVER BELOW HOMME DAM, ND--Continued

Probability of occurrence of annual high discharges

[ng, statistic not given]

\begin{tabular}{|c|c|c|c|c|c|c|}
\hline \multirow[b]{2}{*}{$\begin{array}{l}\text { Exceedance } \\
\text { probability }\end{array}$} & \multirow[b]{2}{*}{$\begin{array}{l}\text { Recurrence } \\
\text { interval } \\
\text { (years) }\end{array}$} & \multirow[b]{2}{*}{$\begin{array}{c}\text { Maximum } \\
\text { instantaneous } \\
\left(\mathrm{ft}^{3} / \mathrm{s}\right)\end{array}$} & \multicolumn{4}{|c|}{$\begin{array}{l}\text { Maximum mean discharge } \\
\qquad\left(\mathrm{ft}^{3} / \mathrm{s}\right)\end{array}$} \\
\hline & & & 3-day period & 7-day perlod & 15-day period & 30-day period \\
\hline 0.99 & 1.01 & 5.60 & 2.35 & 2.08 & 1.84 & 1.56 \\
\hline 0.95 & 1.05 & 27.3 & 13.6 & 11.0 & 8.58 & 6.43 \\
\hline 0.90 & 1.11 & 59.1 & 31.8 & 24.5 & 18.1 & 12.9 \\
\hline 0.80 & 1.25 & 141 & 81.5 & 60.0 & 41.9 & 28.2 \\
\hline 0.50 & 2 & 614 & 388 & 267 & 171 & 108 \\
\hline 0.20 & 5 & 2,110 & 1,370 & 903 & 544 & 337 \\
\hline 0.10 & 10 & 3,670 & 2,380 & 1,540 & 910 & 568 \\
\hline 0.04 & 25 & 6,220 & 3,970 & 2,540 & 1,480 & 936 \\
\hline 0.02 & 50 & 8,470 & 5,300 & 3,380 & 1,950 & 1,260 \\
\hline 0.01 & 100 & 10,900 & 6,700 & 4,270 & 2,460 & 1,610 \\
\hline 0.005 & 200 & 13,600 & 8,150 & 5,180 & 2,980 & 1,990 \\
\hline 0.002 & 500 & 17,300 & $\mathrm{ng}$ & ng & $\mathrm{ng}$ & ng \\
\hline
\end{tabular}

Probability of occurrence of annual low discharges

\begin{tabular}{|c|c|c|c|c|c|c|c|c|c|c|}
\hline \multirow[b]{3}{*}{$\begin{array}{l}\text { Non- } \\
\text { exceed- } \\
\text { ance } \\
\text { prob- } \\
\text { ability }\end{array}$} & \multirow[b]{3}{*}{$\begin{array}{c}\text { Recur- } \\
\text { rence } \\
\text { inter- } \\
\text { val } \\
\text { (years) }\end{array}$} & \multicolumn{9}{|c|}{ Minimum mean discharge $\left(t^{3} / s\right)$} \\
\hline & & \multicolumn{9}{|c|}{ Number of consecutive days } \\
\hline & & 1 & 3 & 7 & 14 & 30 & 60 & 90 & 120 & 183 \\
\hline 0.05 & 20 & 0 & 0 & 0 & 0.003 & 0.005 & ${ }^{1} 0.008$ & 0.011 & 0.041 & 0.167 \\
\hline 0.10 & 10 & 0 & 0 & 0.006 & 0.026 & 0.044 & 0.076 & 0.150 & 0.178 & 0.425 \\
\hline 0.20 & 5 & 0.033 & 0.037 & 0.054 & 0.075 & 0.129 & 0.253 & 0.448 & 0.598 & 1.06 \\
\hline 0.50 & 2 & 0.148 & 0.174 & 0.250 & 0.312 & 0.525 & 1.04 & 1.59 & 2.27 & 3.29 \\
\hline
\end{tabular}

${ }^{1}$ Graphical interpretation. 


\section{SOUTH BRANCH PARK RIVER BELOW HOMME DAM, ND--Continued}

Probability of occurrence of seasonal low discharges

\begin{tabular}{|c|c|c|c|c|c|c|c|c|c|}
\hline \multirow[b]{3}{*}{$\begin{array}{c}\text { Non- } \\
\text { exceedance } \\
\text { probability }\end{array}$} & \multirow[b]{3}{*}{$\begin{array}{l}\text { Recurrence } \\
\text { intervai } \\
\text { (years) }\end{array}$} & \multicolumn{8}{|c|}{ Minimum mean discharge $\left(\mathrm{ft}^{3} / \mathrm{s}\right)$} \\
\hline & & \multicolumn{8}{|c|}{ Number of consecutive days } \\
\hline & & 1 & 7 & 14 & 30 & 1 & 7 & 14 & 30 \\
\hline & & \multicolumn{4}{|c|}{ December-January-February } & \multicolumn{4}{|c|}{ March-April-May } \\
\hline 0.05 & 20 & 0.005 & 0.029 & 0.031 & 0.052 & 0.035 & 0.044 & 0.072 & 0.160 \\
\hline 0.10 & 10 & 0.055 & 0.085 & 0.095 & 0.170 & 0.084 & 0.124 & 0.188 & 0.396 \\
\hline 0.20 & 5 & 0.177 & 0.235 & 0.270 & 0.493 & 0.208 & 0.337 & 0.481 & 1.07 \\
\hline \multirow[t]{2}{*}{0.50} & 2 & 0.874 & 1.06 & 1.23 & 1.99 & 0.910 & 1.62 & 2.14 & 5.26 \\
\hline & & \multicolumn{4}{|c|}{ June-July-August } & \multicolumn{4}{|c|}{ September-October-November } \\
\hline 0.05 & 20 & 0 & 0.012 & 0.024 & 0.078 & 0 & 0 & 0.004 & 0.006 \\
\hline 0.10 & 10 & 0.030 & 0.071 & 0.113 & 0.277 & 0 & 0.006 & 0.034 & 0.064 \\
\hline 0.20 & 5 & 0.094 & 0.182 & 0.267 & 0.565 & 0.049 & 0.067 & 0.102 & 0.191 \\
\hline 0.50 & 2 & 0.391 & 0.700 & 0.928 & 1.64 & 0.256 & 0.374 & 0.462 & 0.802 \\
\hline
\end{tabular}


05089000 SOUTH BRANCH PARK RIVER BELOW HOMME DAM, ND--Continued

Annual peak discharge and corresponding gage height

\begin{tabular}{|c|c|c|c|c|c|c|c|}
\hline $\begin{array}{l}\text { Water } \\
\text { year }\end{array}$ & Date & $\begin{array}{c}\text { Gage } \\
\text { height } \\
\text { (feet) }\end{array}$ & $\begin{array}{c}\text { Peak } \\
\text { discharge } \\
\left(\mathrm{ft}^{3} / \mathrm{s}\right)\end{array}$ & $\begin{array}{l}\text { Water } \\
\text { year }\end{array}$ & Date & $\begin{array}{c}\text { Gage } \\
\text { height } \\
\text { (feet) }\end{array}$ & $\begin{array}{c}\text { Peak } \\
\text { discharge } \\
\left(\mathrm{ft}^{3} / \mathrm{s}\right)\end{array}$ \\
\hline \multicolumn{8}{|c|}{ Annual peak discharge, by year, and corresponding gage height } \\
\hline 1950 & April 24 & 37.52 & 13,000 & 1973 & March 24 & 23.68 & 101 \\
\hline 1951 & April 4 & 28.42 & 900 & 1974 & May 20 & 29.12 & 2,610 \\
\hline 1952 & September 10 & 24.75 & 106 & 1975 & July 1 & 27.19 & 1,300 \\
\hline 1953 & August 28 & 23.64 & 17.0 & 1976 & April 2 & 27.08 & 1,240 \\
\hline 1954 & June 14 & 25.73 & 386 & 1977 & September 17 & 23.53 & 12.0 \\
\hline 1955 & April 1 & 28.50 & 1,600 & 1978 & April 7 & 28.56 & 2,170 \\
\hline 1956 & April 25 & 29.16 & 2,000 & 1979 & April 20 & 31.49 & 5,380 \\
\hline 1957 & March 23 & 25.54 & 324 & 1980 & May 28 & 24.67 & 211 \\
\hline 1958 & March 21 & 23.98 & 58.0 & 1981 & April 2 & 25.49 & 429 \\
\hline 1959 & April 3 & 25.48 & 368 & 1982 & July 24 & 27.40 & 1,290 \\
\hline 1960 & April 12 & 29.43 & 2,580 & 1983 & March 6 & 27.10 & 1,200 \\
\hline 1961 & April 26 & 23.91 & 86.0 & 1984 & March 25 & 24.83 & 244 \\
\hline 1962 & April 18 & 27.99 & 1,780 & 1985 & March 19 & 25.71 & 473 \\
\hline 1963 & June 25 & 24.90 & 316 & 1986 & March 23 & 25.90 & 620 \\
\hline 1964 & June 19 & 28.71 & 2,300 & 1987 & April 6 & 28.36 & 2,000 \\
\hline 1965 & April 11 & 30.68 & 3,100 & 1988 & April 3 & 23.39 & 29.0 \\
\hline 1966 & April 1 & 27.81 & 1,670 & 1989 & April 24 & 23.45 & 61.0 \\
\hline 1967 & May 7 & 28.37 & 1,950 & 1990 & April 5 & 23.46 & 24.0 \\
\hline 1968 & March 26 & 24.69 & 233 & 1991 & July 6 & 23.56 & 32.0 \\
\hline 1969 & April 11 & 29.45 & 2,900 & 1992 & March 29 & 25.62 & 933 \\
\hline 1970 & April 8 & 26.63 & 980 & 1993 & July 28 & 29.53 & 4,210 \\
\hline 1971 & April 9 & 28.99 & 2,850 & 1994 & March 22 & 25.62 & 500 \\
\hline 1972 & March 17 & 27.20 & 1,210 & & & & \\
\hline \multicolumn{8}{|c|}{ Annual peak discharge, from highest to lowest, and corresponding gage height } \\
\hline 1950 & April 24 & 37.52 & 13,000 & 1970 & April 8 & 26.63 & 980 \\
\hline 1979 & April 20 & 31.49 & 5,380 & 1992 & March 29 & 25.62 & 933 \\
\hline 1993 & July 28 & 29.53 & 4,210 & 1951 & April 4 & 28.42 & 900 \\
\hline 1965 & April 11 & 30.68 & 3,100 & 1986 & March 23 & 25.90 & 620 \\
\hline 1969 & April 11 & 29.45 & 2,900 & 1994 & March 22 & 25.62 & 500 \\
\hline 1971 & April 9 & 28.99 & 2,850 & 1985 & March 19 & 25.71 & 473 \\
\hline 1974 & May 20 & 29.12 & 2,610 & 1981 & April 2 & 25.49 & 429 \\
\hline 1960 & April 12 & 29.43 & 2,580 & 1954 & June 14 & 25.73 & 386 \\
\hline 1964 & June 19 & 28.71 & 2,300 & 1959 & April 3 & 25.48 & 368 \\
\hline 1978 & April 7 & 28.56 & 2,170 & 1957 & March 23 & 25.54 & 324 \\
\hline 1956 & April 25 & 29.16 & 2,000 & 1963 & June 25 & 24.90 & 316 \\
\hline 1987 & April 6 & 28.36 & 2,000 & 1984 & March 25 & 24.83 & 244 \\
\hline 1967 & May 7 & 28.37 & 1,950 & 1968 & March 26 & 24.69 & 233 \\
\hline 1962 & April 18 & 27.99 & 1,780 & 1980 & May 28 & 24.67 & 211 \\
\hline 1966 & April 1 & 27.81 & 1,670 & 1952 & September 10 & 24.75 & 106 \\
\hline 1955 & April 1 & 28.50 & 1,600 & 1973 & March 24 & 23.68 & 101 \\
\hline 1975 & July 1 & 27.19 & 1,300 & 1961 & April 26 & 23.91 & 86.0 \\
\hline 1982 & July 24 & 27.40 & 1,290 & 1989 & April 24 & 23.45 & 61.0 \\
\hline 1976 & April 2 & 27.08 & 1,240 & 1958 & March 21 & 23.98 & 58.0 \\
\hline 1972 & March 17 & 27.20 & 1,210 & 1991 & July 6 & 23.56 & 32.0 \\
\hline 1983 & March 6 & 27.10 & 1,200 & 1988 & April 3 & 23.39 & 29.0 \\
\hline
\end{tabular}




\section{SOUTH BRANCH PARK RIVER BELOW HOMME DAM, ND--Continued}

Annual peak discharge and corresponding gage height--Continued

\begin{tabular}{|c|c|c|c|c|c|c|c|}
\hline $\begin{array}{l}\text { Water } \\
\text { year }\end{array}$ & Date & $\begin{array}{c}\text { Gage } \\
\text { height } \\
\text { (feet) }\end{array}$ & $\begin{array}{c}\text { Peak } \\
\text { discharge } \\
\left(\mathrm{ft}^{3} / \mathrm{s}\right)\end{array}$ & $\begin{array}{c}\text { Water } \\
\text { year }\end{array}$ & Date & $\begin{array}{c}\text { Gage } \\
\text { height } \\
\text { (feet) }\end{array}$ & $\begin{array}{c}\text { Peak } \\
\text { discharge } \\
\left(\mathrm{ft}^{3} / \mathrm{s}\right)\end{array}$ \\
\hline \multicolumn{8}{|c|}{ Annual peak discharge, from highest to lowest, and corresponding gage height-Continued } \\
\hline 1990 & April 5 & 23.46 & 24.0 & 1977 & September 17 & 23.53 & 12.0 \\
\hline 1953 & August 28 & 23.64 & 17.0 & & & & \\
\hline
\end{tabular}




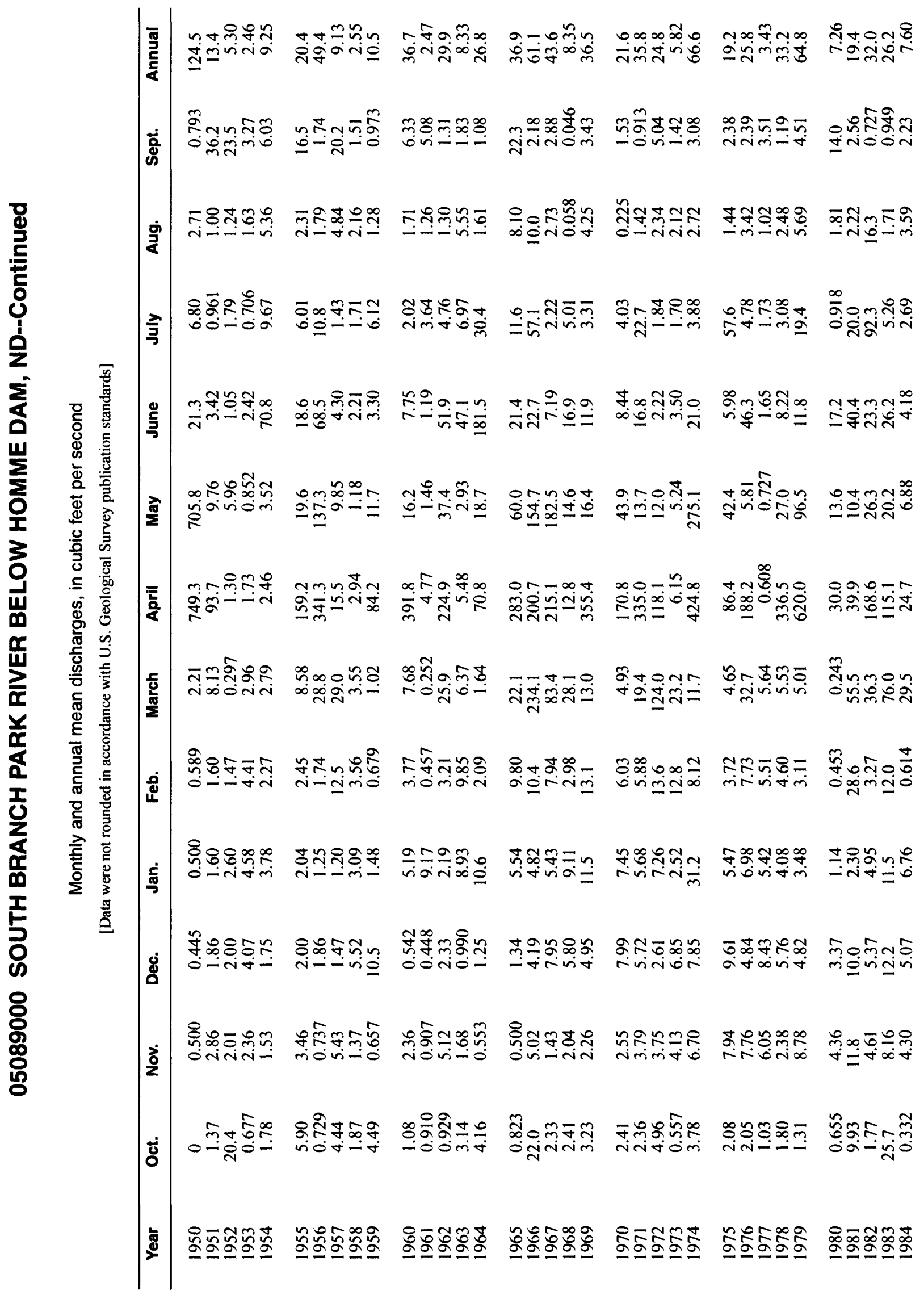




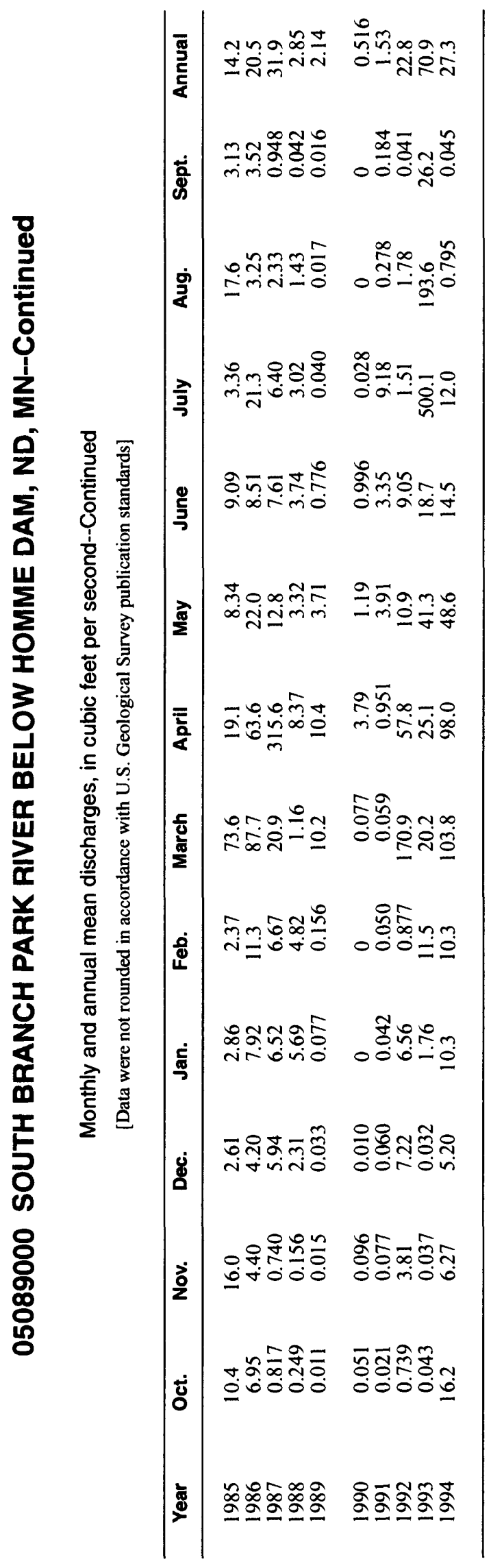




\section{MIDDLE BRANCH PARK RIVER NEAR UNION, ND}

LOCATION.--Lat $48^{\circ} 32^{\prime} 32^{\prime \prime}$, long $98^{\circ} 01^{\prime} 10^{\prime}$, on north line of sec. 5 , T.158 N., R.57 W., Walsh County, Hydrologic Unit 09020310 , on left bank $150 \mathrm{ft}$ downstream from bridge on county highway between Walsh and Cavalier counties, and $3.5 \mathrm{mi}$ southwest of Union.

DRAINAGE AREA.--15.3 $\mathrm{mi}^{2}$.

PERIOD OF RECORD.--October 1965 to September 1986. Seasonal records only since 1983.

GAGE.--Water-stage recorder. Datum of gage is $1,495 \mathrm{ft}$ above sea level, from topographic map.

EXTREMES FOR PERIOD OF RECORD.--Maximum discharge, $960 \mathrm{ft}^{3} / \mathrm{s}$, Apr. 20, 1979, gage height, $6.16 \mathrm{ft}$, backwater from ice; maximum gage height, $7.51 \mathrm{ft}$, May 4, 1966, from floodmark, backwater from snowdrift; no flow for several months each year.

Annual mean discharge

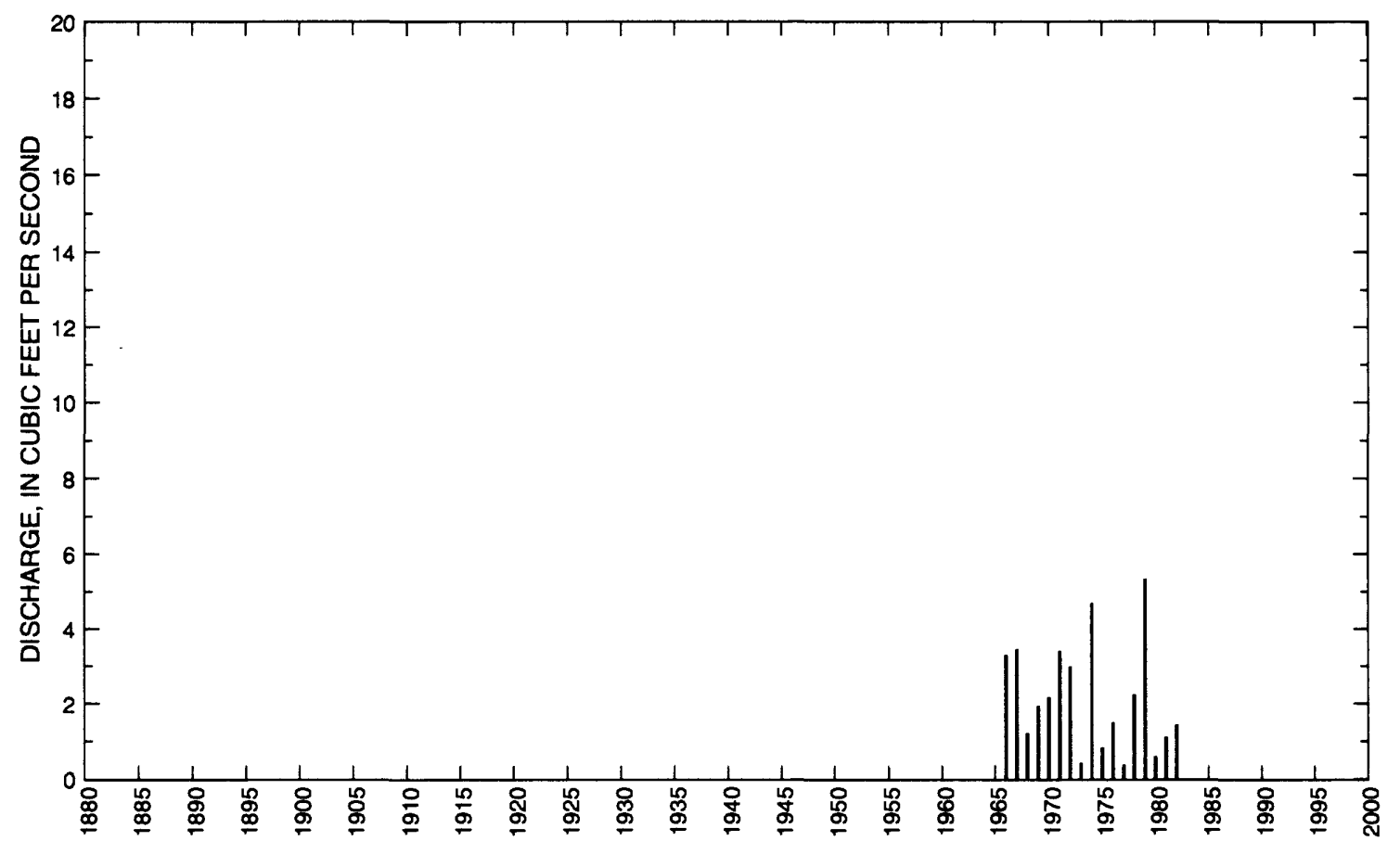


05089100 MIDDLE BRANCH PARK RIVER NEAR UNION, ND--Continued

Statistics of monthly and annual mean discharges

[m, more than 1 year of occurrence]

\begin{tabular}{|c|c|c|c|c|c|c|c|c|}
\hline \multirow[b]{2}{*}{ Month } & \multicolumn{2}{|c|}{ Maximum } & \multicolumn{2}{|c|}{ Minimum } & \multicolumn{4}{|c|}{ Mean } \\
\hline & $\begin{array}{c}\text { Discharge } \\
\left(\mathrm{ft}^{3} / \mathrm{s}\right)\end{array}$ & $\begin{array}{c}\text { Water year } \\
\text { of } \\
\text { occurrence }\end{array}$ & $\begin{array}{c}\text { Discharge } \\
\left(\mathrm{ft}^{3} / \mathrm{s}\right)\end{array}$ & $\begin{array}{c}\text { Water year } \\
\text { of } \\
\text { occurrence }\end{array}$ & $\begin{array}{c}\text { Discharge } \\
\left(\mathrm{ft}^{3} / \mathrm{s}\right)\end{array}$ & $\begin{array}{c}\text { Standard } \\
\text { deviation } \\
\left(\mathrm{ft}^{3} / \mathrm{s}\right)\end{array}$ & $\begin{array}{l}\text { Coeffi- } \\
\text { cient of } \\
\text { variation }\end{array}$ & $\begin{array}{l}\text { Percentage } \\
\text { of annuai } \\
\text { discharge }\end{array}$ \\
\hline October & 1.45 & 1966 & 0.032 & 1968 & 0.260 & 0.36 & 1.39 & 1.09 \\
\hline November & 0.539 & 1966 & 0.021 & 1977 & 0.120 & 0.16 & 1.29 & 0.51 \\
\hline December & 0.164 & 1978 & 0 & $\mathrm{~m}$ & 0.030 & 0.05 & 1.47 & 0.14 \\
\hline January & 0.055 & 1978 & 0 & $\mathrm{~m}$ & 0 & 0.01 & 3.15 & 0.02 \\
\hline February & 2.91 & 1981 & 0 & $\mathrm{~m}$ & 0.170 & 0.71 & 4.12 & 0.71 \\
\hline March & 23.0 & 1972 & 0 & $\mathrm{~m}$ & 3.95 & 5.82 & 1.47 & 16.5 \\
\hline April & 60.0 & 1979 & 0.242 & 1973 & 14.6 & 15.9 & 1.09 & 60.9 \\
\hline May & 17.8 & 1967 & 0.039 & 1973 & 2.46 & 4.26 & 1.73 & 10.3 \\
\hline June & 6.12 & 1986 & 0.018 & 1973 & 1.13 & 1.71 & 1.51 & 4.73 \\
\hline July & 5.46 & 1966 & 0.010 & 1973 & 0.950 & 1.65 & 1.74 & 3.95 \\
\hline August & 0.351 & 1980 & 0.351 & 1980 & 0.120 & 0.11 & 0.93 & 0.50 \\
\hline September & 1.31 & 1980 & 0 & 1984 & 0.160 & 0.28 & 1.78 & 0.66 \\
\hline Annual & 5.35 & 1979 & 0.381 & 1977 & 2.18 & 1.48 & 0.68 & 100 \\
\hline
\end{tabular}

Annual flow duration

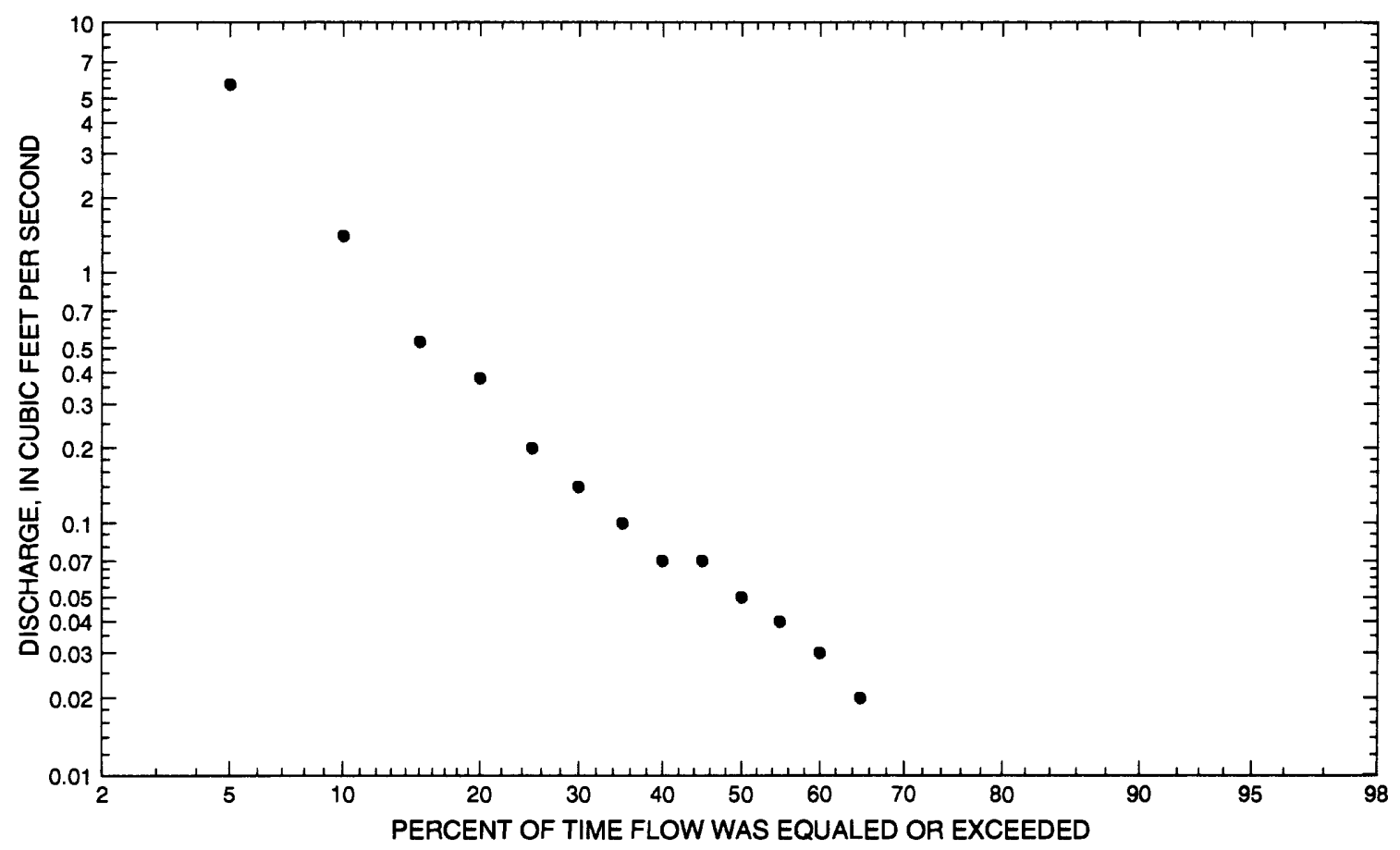




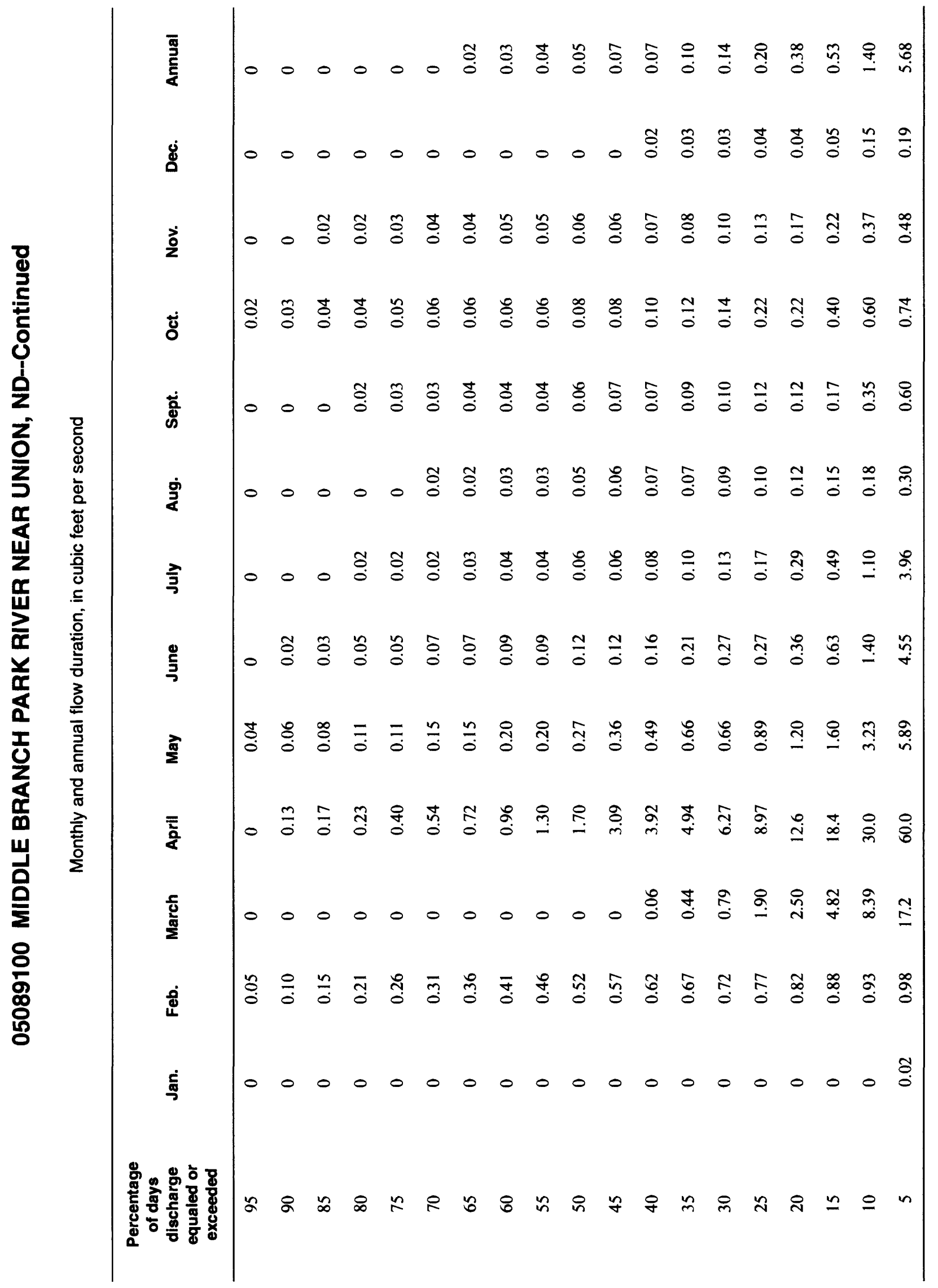


05089100 MIDDLE BRANCH PARK RIVER NEAR UNION, ND--Continued

Probability of occurrence of annual high discharges

[ng, statistic not given]

\begin{tabular}{ccccccc}
\hline & & & \multicolumn{5}{c}{$\begin{array}{c}\text { Maximum mean discharge } \\
\left(\mathbf{f t}^{3} / \mathbf{s}\right)\end{array}$} \\
\cline { 5 - 7 } $\begin{array}{c}\text { Exceedance } \\
\text { probability }\end{array}$ & $\begin{array}{c}\text { Recurrence } \\
\text { interval } \\
\text { (years) }\end{array}$ & $\begin{array}{c}\text { Maximum } \\
\text { instantaneous } \\
\left(\mathbf{f t}^{3} / \mathbf{s}\right)\end{array}$ & 3-day period & 7-day period & 15-day period & 30-day period \\
\hline 0.99 & 1.01 & 22.5 & 4.83 & 2.71 & 1.60 & 1.08 \\
0.95 & 1.05 & 42.9 & 10.1 & 6.39 & 3.92 & 2.60 \\
0.90 & 1.11 & 60.3 & 15.0 & 9.92 & 6.14 & 4.03 \\
0.80 & 1.25 & 90.8 & 24.3 & 16.6 & 10.3 & 6.65 \\
0.50 & 2 & 197 & 62.1 & 42.5 & 25.5 & 15.8 \\
0.20 & 5 & 422 & 162 & 102 & 57.3 & 33.5 \\
0.10 & 10 & 626 & 268 & 158 & 84.1 & 47.6 \\
0.04 & 25 & 949 & 463 & 246 & 123 & 67.0 \\
0.02 & 50 & 1,240 & 661 & 324 & 155 & 82.3 \\
0.01 & 100 & 1,570 & 913 & 413 & 189 & 97.8 \\
0.005 & 200 & 1,960 & 1,230 & 513 & 225 & 114 \\
0.002 & 500 & 2,540 & $\mathrm{ng}$ & $\mathrm{ng}$ & $\mathrm{ng}$ & $\mathrm{ng}$ \\
\hline
\end{tabular}

Probability of occurrence of annual low discharges

[ng, statistic not given]

\begin{tabular}{|c|c|c|c|c|c|c|c|c|c|c|}
\hline \multirow{3}{*}{$\begin{array}{l}\text { Non- } \\
\text { exceed- } \\
\text { ance } \\
\text { prob- } \\
\text { ability }\end{array}$} & \multirow{3}{*}{$\begin{array}{l}\text { Recur- } \\
\text { rence } \\
\text { inter- } \\
\text { val } \\
\text { (years) }\end{array}$} & \multicolumn{9}{|c|}{ Minimum mean discharge $\left(\mathrm{ft}^{3} / \mathrm{s}\right)$} \\
\hline & & \multicolumn{9}{|c|}{ Number of consecutive days } \\
\hline & & 1 & 3 & 7 & 14 & 30 & 60 & 90 & 120 & 183 \\
\hline 0.05 & 20 & ng & ng & $\mathrm{ng}$ & $\mathrm{ng}$ & $\mathrm{ng}$ & ng & $\mathrm{ng}$ & 0 & 0.015 \\
\hline 0.10 & 10 & ng & $\mathrm{ng}$ & $\mathrm{ng}$ & $\mathrm{ng}$ & $\mathrm{ng}$ & ng & $\mathrm{ng}$ & 0 & 0.017 \\
\hline 0.20 & 5 & ng & $\mathrm{ng}$ & $\mathrm{ng}$ & $\mathrm{ng}$ & $\mathrm{ng}$ & ng & $\mathrm{ng}$ & 0 & 0.021 \\
\hline 0.50 & 2 & ng & $\mathrm{ng}$ & ng & ng & ng & ng & ng & 0 & 0.036 \\
\hline
\end{tabular}




\section{MIDDLE BRANCH PARK RIVER NEAR UNION, ND--Continued}

Probability of occurrence of seasonal low discharges

[ng, statistic not given]

\begin{tabular}{|c|c|c|c|c|c|c|c|c|c|}
\hline \multirow[b]{3}{*}{$\begin{array}{c}\text { Non- } \\
\text { exceedance } \\
\text { probability }\end{array}$} & \multirow[b]{3}{*}{$\begin{array}{l}\text { Recurrence } \\
\text { interval } \\
\text { (years) }\end{array}$} & \multicolumn{8}{|c|}{ Minimum mean discharge $\left(\mathrm{ft}^{3} / \mathrm{s}\right)$} \\
\hline & & \multicolumn{8}{|c|}{ Number of consecutive days } \\
\hline & & 1 & 7 & 14 & 30 & 1 & 7 & 14 & 30 \\
\hline & & \multicolumn{4}{|c|}{ December-January-February } & \multicolumn{4}{|c|}{ March-April-May } \\
\hline 0.05 & 20 & ng & ng & ng & ng & ng & 0 & 0 & 0 \\
\hline 0.10 & 10 & ng & ng & ng & ng & $\mathrm{ng}$ & 0 & 0 & 0 \\
\hline 0.20 & 5 & ng & $\mathrm{ng}$ & ng & ng & $\mathrm{ng}$ & 0 & 0 & 0.029 \\
\hline \multirow[t]{2}{*}{0.50} & 2 & ng & $\mathrm{ng}$ & ng & ng & $\mathrm{ng}$ & 0 & 0 & 0.129 \\
\hline & & \multicolumn{4}{|c|}{ June-July-August } & \multicolumn{4}{|c|}{ September-October-November } \\
\hline 0.05 & 20 & 0 & 0 & 0 & 0 & 0 & 0 & 0 & 0.011 \\
\hline 0.10 & 10 & 0 & 0 & 0 & 0 & 0 & 0 & 0 & 0.015 \\
\hline 0.20 & 5 & 0 & 0 & 0 & 0.009 & 0 & 0 & 0 & 0.020 \\
\hline 0.50 & 2 & 0.008 & ${ }^{1} 0.013$ & 0.018 & 0.030 & 0.008 & 0.015 & 0.025 & 0.043 \\
\hline
\end{tabular}

${ }^{1}$ Graphical interpretation. 
05089100 MIDDLE BRANCH PARK RIVER NEAR UNION, ND--Continued

Annual peak discharge and corresponding gage height

\begin{tabular}{|c|c|c|c|c|c|c|c|}
\hline $\begin{array}{l}\text { Water } \\
\text { year }\end{array}$ & Date & $\begin{array}{c}\text { Gage } \\
\text { height } \\
\text { (feet) }\end{array}$ & $\begin{array}{c}\text { Peak } \\
\text { discharge } \\
\left(\mathrm{ft}^{3} / \mathrm{s}\right)\end{array}$ & $\begin{array}{l}\text { Water } \\
\text { year }\end{array}$ & Date & $\begin{array}{c}\text { Gage } \\
\text { height } \\
\text { (feet) }\end{array}$ & $\begin{array}{c}\text { Peak } \\
\text { discharge } \\
\left(\mathrm{ft}^{3} / \mathrm{s}\right)\end{array}$ \\
\hline \multicolumn{8}{|c|}{ Annual peak discharge, by year, and corresponding gage height } \\
\hline 1966 & May 4 & 7.51 & 200 & 1977 & May 19 & 3.33 & 108 \\
\hline 1967 & May 6 & 7.22 & 687 & 1978 & April 6 & 5.91 & 300 \\
\hline 1968 & May 14 & 5.44 & 75.0 & 1979 & April 20 & 6.16 & 960 \\
\hline 1969 & April 11 & 7.10 & 506 & 1980 & May 26 & 3.67 & 112 \\
\hline 1970 & April 24 & 6.07 & 243 & 1981 & June 27 & 3.42 & 90.0 \\
\hline 1971 & April 9 & 7.00 & 650 & 1982 & July 24 & 3.71 & 94.0 \\
\hline 1972 & March 16 & 6.07 & 385 & 1983 & April 5 & 3.83 & 91.0 \\
\hline 1973 & March 13 & 4.55 & 51.0 & 1984 & July 14 & 3.38 & 71.0 \\
\hline 1974 & April 15 & 6.75 & 450 & 1985 & March 17 & 4.07 & 85.0 \\
\hline 1975 & April 15 & 5.00 & 74.0 & 1986 & June 20 & 5.38 & 507 \\
\hline 1976 & June 12 & 3.37 & 285 & & & & \\
\hline \multicolumn{8}{|c|}{ Annual peak discharge, from highest to lowest, and corresponding gage height } \\
\hline 1979 & April 20 & 6.16 & 960 & 1980 & May 26 & 3.67 & 112 \\
\hline 1967 & May 6 & 7.22 & 687 & 1977 & May 19 & 3.33 & 108 \\
\hline 1971 & April 9 & 7.00 & 650 & 1982 & July 24 & 3.71 & 94.0 \\
\hline 1986 & June 20 & 5.38 & 507 & 1983 & April 5 & 3.83 & 91.0 \\
\hline 1969 & April 11 & 7.10 & 506 & 1981 & June 27 & 3.42 & 90.0 \\
\hline 1974 & April 15 & 6.75 & 450 & 1985 & March 17 & 4.07 & 85.0 \\
\hline 1972 & March 16 & 6.07 & 385 & 1968 & May 14 & 5.44 & 75.0 \\
\hline 1978 & April 6 & 5.91 & 300 & 1975 & April 15 & 5.00 & 74.0 \\
\hline 1976 & June 12 & 3.37 & 285 & 1984 & July 14 & 3.38 & 71.0 \\
\hline 1970 & April 24 & 6.07 & 243 & 1973 & March 13 & 4.55 & 51.0 \\
\hline 1966 & May 4 & 7.51 & 200 & & & & \\
\hline
\end{tabular}




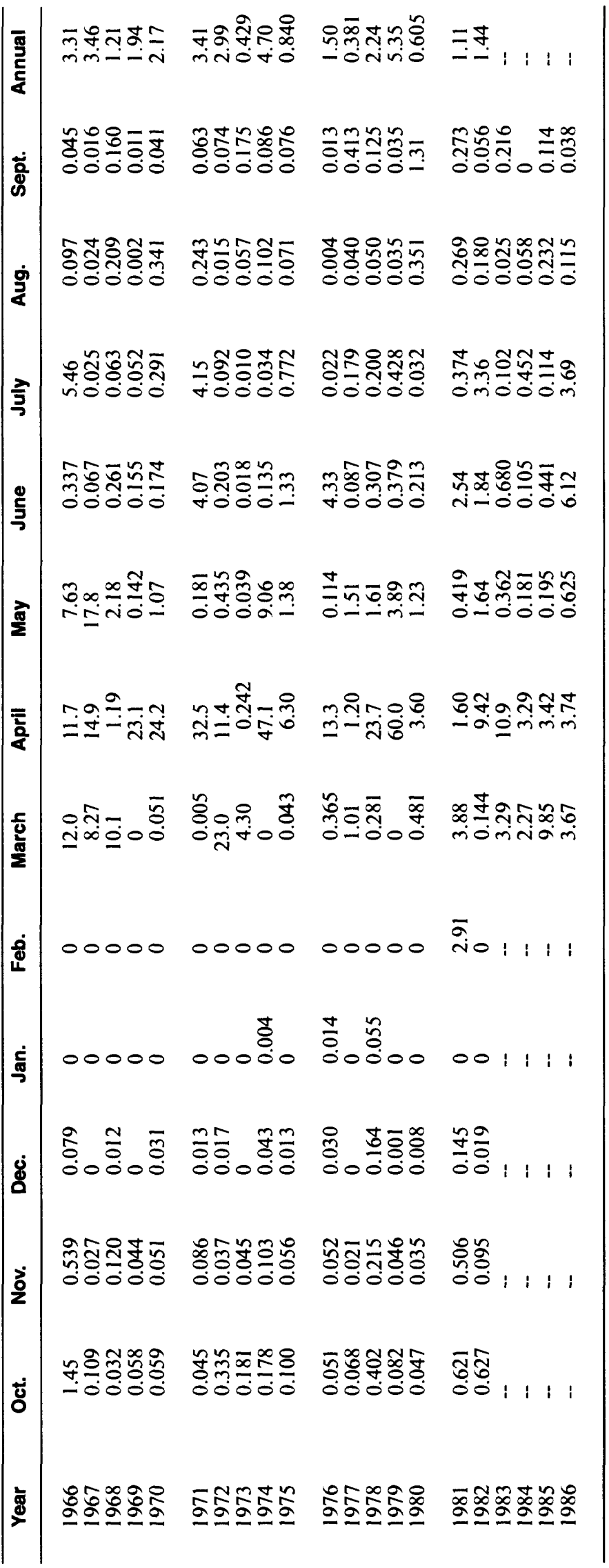




\section{CART CREEK AT MOUNTAIN, ND}

LOCATION.--Lat 48 40'37', long 9751'41', in SWl/4 sec.15, T.160 N., R.56 W., Pembina County, Hydrologic Unit 09020310, on right bank $50 \mathrm{ft}$ downstream from bridge on State Highway 32, and $0.7 \mathrm{mi}$ south of Mountain.

DRAINAGE AREA.--16.9 $\mathrm{mi}^{2}$.

PERIOD OF RECORD.--June 1954 to September 1984.

GAGE.--Water-stage recorder and wooden control. Datum of gage is $1,027.40 \mathrm{ft}$ above sea level.

EXTREMES FOR PERIOD OF RECORD.--Maxinuum discharge, 1,300 $\mathrm{ft}^{3} / \mathrm{s}$, June 18, 1964, gage height, $9.18 \mathrm{ft}$; maximum gage height, $9.51 \mathrm{ft}$, Apr. 19, 1979; no flow at times in some years.

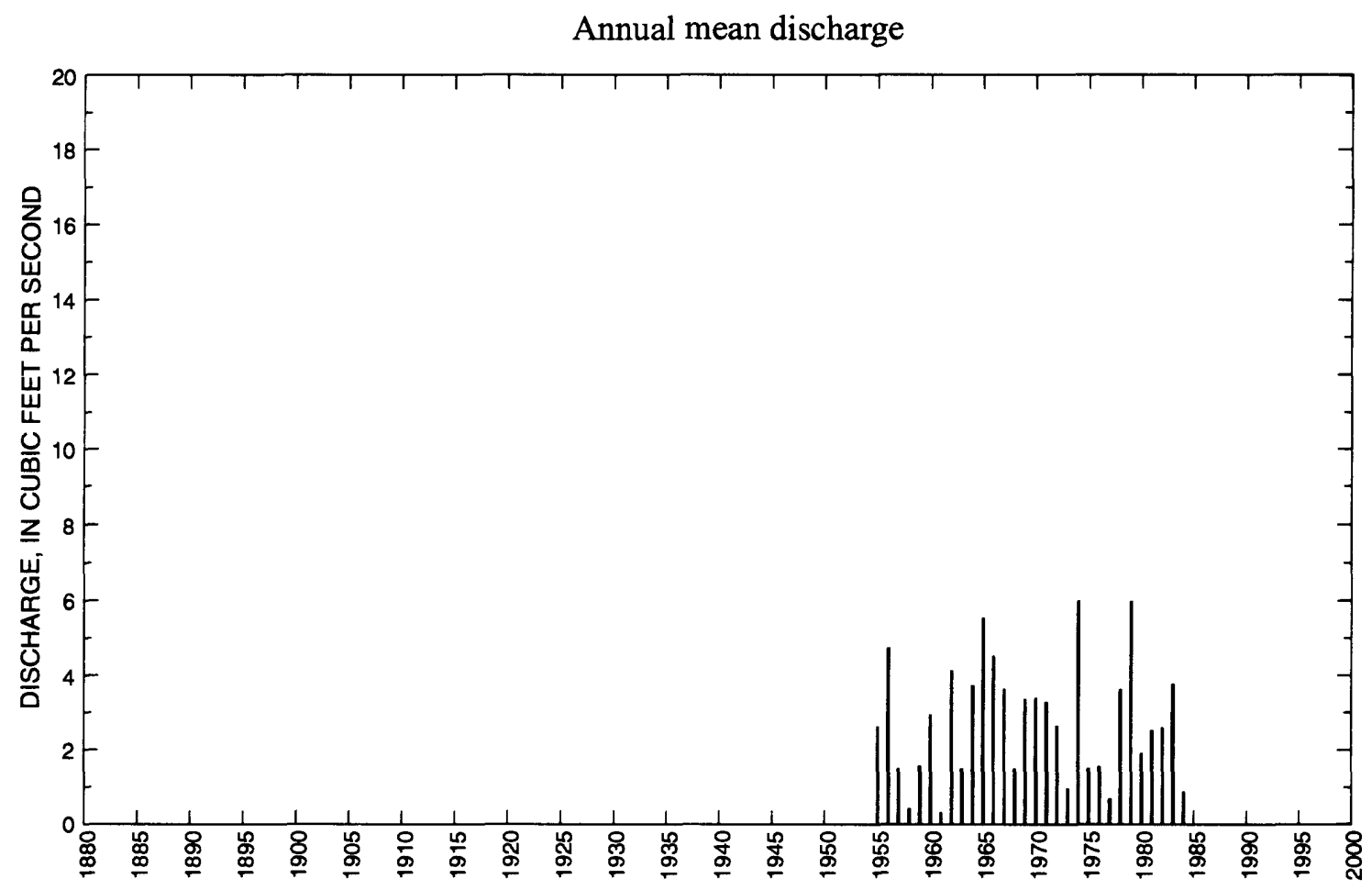




\section{CART CREEK AT MOUNTAIN, ND--Continued}

Statistics of monthly and annual mean discharges

[m, more than 1 year of occurrence]

\begin{tabular}{|c|c|c|c|c|c|c|c|c|}
\hline \multirow[b]{2}{*}{ Month } & \multicolumn{2}{|c|}{ Maximum } & \multicolumn{2}{|c|}{ Minimum } & \multicolumn{4}{|c|}{ Mean } \\
\hline & $\begin{array}{c}\text { Discharge } \\
\left(\mathrm{ft}^{3} / \mathrm{s}\right)\end{array}$ & $\begin{array}{c}\text { Water year } \\
\text { of } \\
\text { occurrence }\end{array}$ & $\begin{array}{c}\text { Discharge } \\
\left(\mathrm{ft}^{3} / \mathrm{s}\right)\end{array}$ & $\begin{array}{c}\text { Water year } \\
\text { of } \\
\text { occurrence }\end{array}$ & $\begin{array}{c}\text { Discharge } \\
\left(\mathrm{ft}^{3} / \mathrm{s}\right)\end{array}$ & $\begin{array}{c}\text { Standard } \\
\text { deviation } \\
\left(\mathrm{ft}^{3} / \mathrm{s}\right)\end{array}$ & $\begin{array}{l}\text { Coeffi- } \\
\text { cient of } \\
\text { variation }\end{array}$ & $\begin{array}{l}\text { Percentage } \\
\text { of annual } \\
\text { discharge }\end{array}$ \\
\hline October & 4.20 & 1981 & 0 & $\mathrm{~m}$ & 0.680 & 0.95 & 1.41 & 2.02 \\
\hline November & 2.10 & 1981 & 0 & $\mathrm{~m}$ & 0.460 & 0.44 & 0.97 & 1.36 \\
\hline December & 2.54 & 1983 & 0 & $\mathrm{~m}$ & 0.260 & 0.49 & 1.92 & 0.76 \\
\hline January & 0.684 & 1965 & 0 & $\mathrm{~m}$ & 0.110 & 0.20 & 1.84 & 0.32 \\
\hline February & 1.69 & 1983 & 0 & $\mathrm{~m}$ & 0.190 & 0.43 & 2.32 & 0.56 \\
\hline March & 19.8 & 1983 & 0 & $\mathrm{~m}$ & 3.32 & 4.55 & 1.37 & 9.92 \\
\hline April & 55.6 & 1979 & 0.940 & 1961 & 17.8 & 15.0 & 0.84 & 53.1 \\
\hline May & 21.0 & 1974 & 0.294 & 1961 & 5.70 & 5.84 & 1.02 & 17.0 \\
\hline June & 25.5 & 1964 & 0.013 & 1961 & 3.12 & 4.84 & 1.55 & 9.31 \\
\hline July & 3.20 & 1966 & 0 & 1961 & 0.800 & 0.86 & 1.08 & 2.39 \\
\hline August & 3.37 & 1980 & 0 & $\mathrm{~m}$ & 0.460 & 0.81 & 1.77 & 1.37 \\
\hline September & 5.39 & 1980 & 0 & $\mathrm{~m}$ & 0.610 & 1.13 & 1.83 & 1.83 \\
\hline Annual & 6.00 & 1974 & 0.311 & 1961 & 2.78 & 1.61 & 0.58 & 100 \\
\hline
\end{tabular}

Annual flow duration

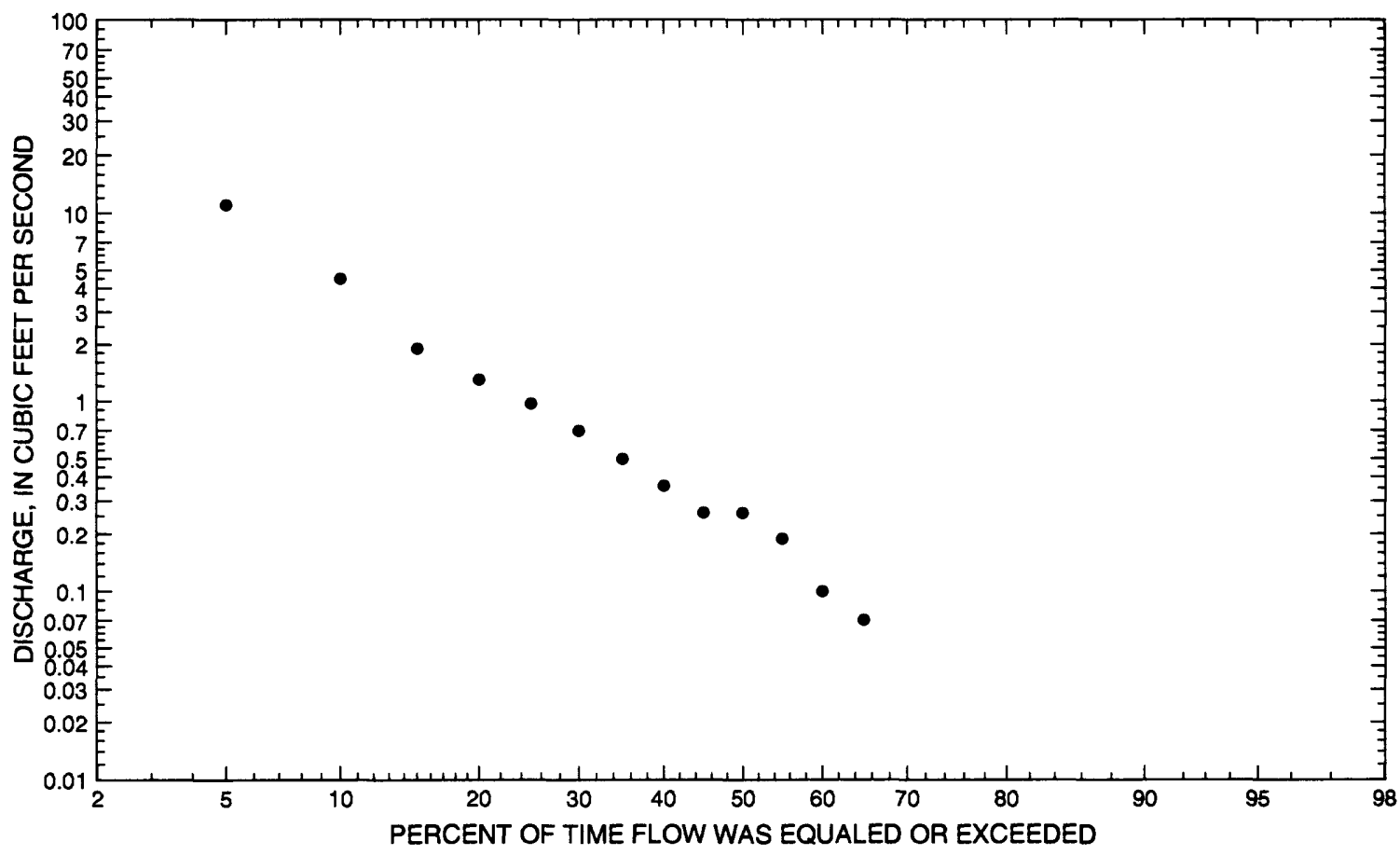




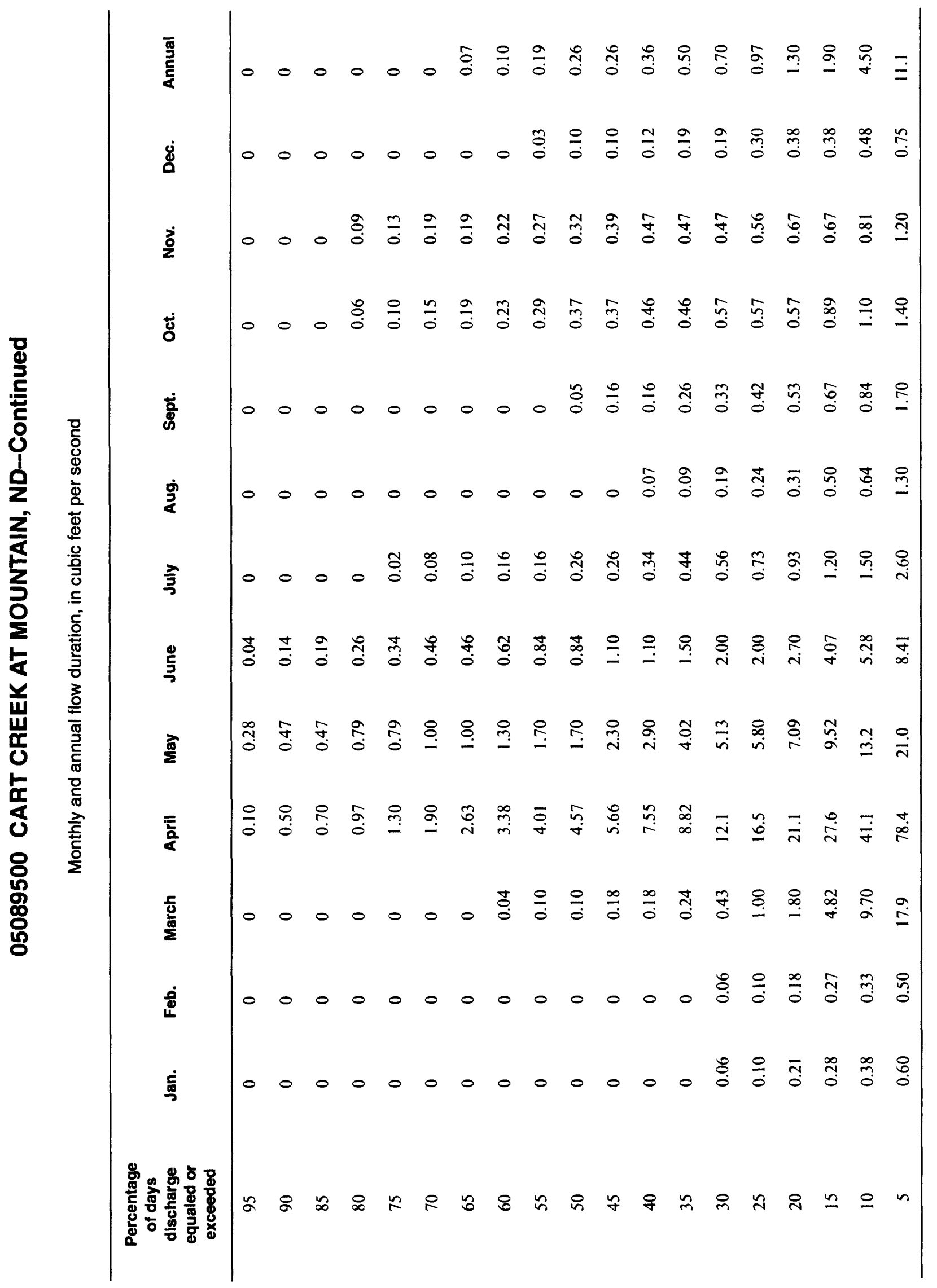




\section{CART CREEK AT MOUNTAIN, ND--Continued}

Probability of occurrence of annual high discharges

[ng, statistic not given]

\begin{tabular}{|c|c|c|c|c|c|c|}
\hline \multirow[b]{2}{*}{$\begin{array}{l}\text { Exceedance } \\
\text { probability }\end{array}$} & \multirow[b]{2}{*}{$\begin{array}{l}\text { Recurrence } \\
\text { intervai } \\
\text { (years) }\end{array}$} & \multirow[b]{2}{*}{$\begin{array}{c}\text { Maximum } \\
\text { instantaneous } \\
\left(\mathrm{ft}^{3} / \mathrm{s}\right)\end{array}$} & \multicolumn{4}{|c|}{$\begin{array}{l}\text { Maximum mean discharge } \\
\left(\mathrm{ft}^{3} / \mathrm{s}\right)\end{array}$} \\
\hline & & & 3-day period & 7-day period & 15-day period & 30-day period \\
\hline 0.99 & 1.01 & 11.4 & 4.84 & 2.95 & 1.58 & 1.12 \\
\hline 0.95 & 1.05 & 28.9 & 11.2 & 7.32 & 4.40 & 3.02 \\
\hline 0.90 & 1.11 & 46.2 & 17.2 & 11.4 & 7.15 & 4.81 \\
\hline 0.80 & 1.25 & 79.1 & 28.1 & 18.9 & 12.2 & 8.04 \\
\hline 0.50 & 2 & 204 & 67.6 & 44.7 & 29.0 & 18.3 \\
\hline 0.20 & 5 & 475 & 150 & 92.8 & 56.9 & 34.6 \\
\hline 0.10 & 10 & 710 & 221 & 129 & 75.4 & 45.0 \\
\hline 0.04 & 25 & 1,060 & 325 & 178 & 97.2 & 56.8 \\
\hline 0.02 & 50 & 1,350 & 413 & 215 & 112 & 64.5 \\
\hline 0.01 & 100 & 1,660 & 507 & 252 & 125 & 71.3 \\
\hline 0.005 & 200 & 1,990 & 609 & 288 & 136 & 77.2 \\
\hline 0.002 & 500 & 2,460 & $\mathrm{ng}$ & ng & $\mathrm{ng}$ & $\mathrm{ng}$ \\
\hline
\end{tabular}

Probability of occurrence of annual low discharges

[ng, statistic not given]

\begin{tabular}{|c|c|c|c|c|c|c|c|c|c|c|}
\hline \multirow{3}{*}{$\begin{array}{c}\text { Non- } \\
\text { exceed- } \\
\text { ance } \\
\text { prob- } \\
\text { ability }\end{array}$} & \multirow{3}{*}{$\begin{array}{c}\text { Recur- } \\
\text { rence } \\
\text { inter- } \\
\text { val } \\
\text { (years) }\end{array}$} & \multicolumn{9}{|c|}{ Minimum mean discharge $\left(\mathrm{ft}^{3} / \mathrm{s}\right)$} \\
\hline & & \multicolumn{9}{|c|}{ Number of consecutive days } \\
\hline & & 1 & 3 & 7 & 14 & 30 & 60 & 90 & 120 & 183 \\
\hline 0.05 & 20 & $\mathrm{ng}$ & $\mathrm{ng}$ & ng & 0 & 0 & 0 & 0 & 0 & 0 \\
\hline 0.10 & 10 & $\mathrm{ng}$ & $\mathrm{ng}$ & ng & 0 & 0 & 0 & 0 & 0 & 0 \\
\hline 0.20 & 5 & $\mathrm{ng}$ & $\mathrm{ng}$ & ng & 0 & 0 & 0 & 0 & 0 & 0.039 \\
\hline 0.50 & 2 & $\mathrm{ng}$ & ng & ng & 0 & 0 & 0 & 0 & 0.072 & 0.175 \\
\hline
\end{tabular}




\section{CART CREEK AT MOUNTAIN, ND--Continued}

Probability of occurrence of seasonal low discharges

\begin{tabular}{|c|c|c|c|c|c|c|c|c|c|}
\hline \multirow[b]{3}{*}{$\begin{array}{c}\text { Non- } \\
\text { exceedance } \\
\text { probability }\end{array}$} & \multirow[b]{3}{*}{$\begin{array}{l}\text { Recurrence } \\
\text { intervai } \\
\text { (years) }\end{array}$} & \multicolumn{8}{|c|}{ Minimum mean discharge $\left(\mathrm{ft}^{3} / \mathrm{s}\right)$} \\
\hline & & \multicolumn{8}{|c|}{ Number of consecutive days } \\
\hline & & 1 & 7 & 14 & 30 & 1 & 7 & 14 & 30 \\
\hline & & \multicolumn{4}{|c|}{ December-January-February } & \multicolumn{4}{|c|}{ March-April-May } \\
\hline 0.05 & 20 & 0 & 0 & 0 & 0 & 0 & 0 & 0 & 0 \\
\hline 0.10 & 10 & 0 & 0 & 0 & 0 & 0 & 0 & 0 & 0 \\
\hline 0.20 & 5 & 0 & 0 & 0 & 0 & 0 & 0 & 0 & 0.100 \\
\hline \multirow[t]{2}{*}{0.50} & 2 & 0 & 0 & 0 & 0 & 0 & 0 & 0.041 & 0.598 \\
\hline & & \multicolumn{4}{|c|}{ June-July-August } & \multicolumn{4}{|c|}{ September-October-November } \\
\hline 0.05 & 20 & 0 & 0 & 0 & 0 & 0 & 0 & 0 & 0 \\
\hline 0.10 & 10 & 0 & 0 & 0 & 0 & 0 & 0 & 0 & 0 \\
\hline 0.20 & 5 & 0 & 0 & 0 & 0 & 0 & 0 & 0 & 0 \\
\hline 0.50 & 2 & 0 & 0 & 0 & 0.042 & 0 & 0 & 0 & 0.153 \\
\hline
\end{tabular}




\section{CART CREEK AT MOUNTAIN, ND--Continued}

Annual peak discharge and corresponding gage height

$[-$, no data $]$

\begin{tabular}{|c|c|c|c|c|c|c|c|}
\hline $\begin{array}{l}\text { Water } \\
\text { year }\end{array}$ & Date & $\begin{array}{c}\text { Gage } \\
\text { height } \\
\text { (feet) }\end{array}$ & $\begin{array}{c}\text { Peak } \\
\text { discharge } \\
\left(\mathrm{ft}^{3} / \mathrm{s}\right)\end{array}$ & $\begin{array}{l}\text { Water } \\
\text { year }\end{array}$ & Date & $\begin{array}{c}\text { Gage } \\
\text { height } \\
\text { (feet) }\end{array}$ & $\begin{array}{c}\text { Peak } \\
\text { discharge } \\
\left(\mathrm{ft}^{3} / \mathrm{s}\right)\end{array}$ \\
\hline \multicolumn{8}{|c|}{ Annual peak discharge, by year, and corresponding gage height } \\
\hline 1954 & June 21 & 3.63 & 64.0 & 1970 & April 25 & 7.45 & 603 \\
\hline 1955 & April 1 & 6.88 & 392 & 1971 & April 9 & 5.63 & 337 \\
\hline 1956 & April 26 & 5.96 & 340 & 1972 & March 16 & 4.85 & 230 \\
\hline 1957 & September 2 & 3.83 & 146 & 1973 & March 24 & 2.69 & 36.0 \\
\hline 1958 & July 4 & 2.43 & 27.0 & 1974 & April 13 & 8.10 & 220 \\
\hline 1959 & April 2 & 5.63 & 160 & 1975 & April 28 & 2.85 & 49.0 \\
\hline 1960 & April 11 & 7.42 & 570 & 1976 & June 12 & 3.88 & 188 \\
\hline 1961 & March 21 & 3.28 & 30.0 & 1977 & May 29 & 4.93 & 267 \\
\hline 1962 & August 10 & 6.22 & 422 & 1978 & April 6 & 4.97 & 380 \\
\hline 1963 & June 3 & 4.94 & 218 & 1979 & April 19 & 9.51 & 734 \\
\hline 1964 & June 18 & 9.18 & 1,300 & 1980 & April 3 & 3.38 & 74.0 \\
\hline 1965 & April 10 & 8.22 & 927 & 1981 & March 27 & 3.28 & 62.0 \\
\hline 1966 & May 4 & 6.26 & 329 & 1982 & May 18 & 3.80 & 165 \\
\hline 1967 & May 6 & 3.96 & 205 & 1983 & March 30 & -- & 215 \\
\hline 1968 & May 15 & 3.01 & 126 & 1984 & March 26 & 3.04 & 22.0 \\
\hline 1969 & April 9 & 7.80 & 528 & & & & \\
\hline \multicolumn{8}{|c|}{ Annual peak discharge, from highest to lowest, and corresponding gage height } \\
\hline 1964 & June 18 & -- & 1,300 & 1983 & March 30 & -- & 215 \\
\hline 1965 & April 10 & 8.22 & 927 & 1967 & May 6 & 3.96 & 205 \\
\hline 1979 & April 19 & 9.51 & 734 & 1976 & June 12 & 3.88 & 188 \\
\hline 1970 & April 25 & 7.45 & 603 & 1982 & May 18 & 3.80 & 165 \\
\hline 1960 & April 11 & 7.42 & 570 & 1959 & April 2 & 5.63 & 160 \\
\hline 1969 & April 9 & 7.80 & 528 & 1957 & September 2 & 3.83 & 146 \\
\hline 1962 & August 10 & 6.22 & 422 & 1968 & May 15 & 3.01 & 126 \\
\hline 1955 & April 1 & 6.88 & 392 & 1980 & April 3 & 3.38 & 74.0 \\
\hline 1978 & April 6 & 4.97 & 380 & 1954 & June 21 & 3.63 & 64.0 \\
\hline 1956 & April 26 & 5.96 & 340 & 1981 & March 27 & 3.28 & 62.0 \\
\hline 1971 & April 9 & 5.63 & 337 & 1975 & April 28 & 2.85 & 49.0 \\
\hline 1966 & May 4 & 6.26 & 329 & 1973 & March 24 & 2.69 & 36.0 \\
\hline 1977 & May 29 & 4.93 & 267 & 1961 & March 21 & 3.28 & 30.0 \\
\hline 1972 & March 16 & 4.85 & 230 & 1958 & July 4 & 2.43 & 27.0 \\
\hline 1974 & April 13 & 8.10 & 220 & 1984 & March 26 & 3.04 & 22.0 \\
\hline 1963 & June 3 & 4.94 & 218 & & & & \\
\hline
\end{tabular}




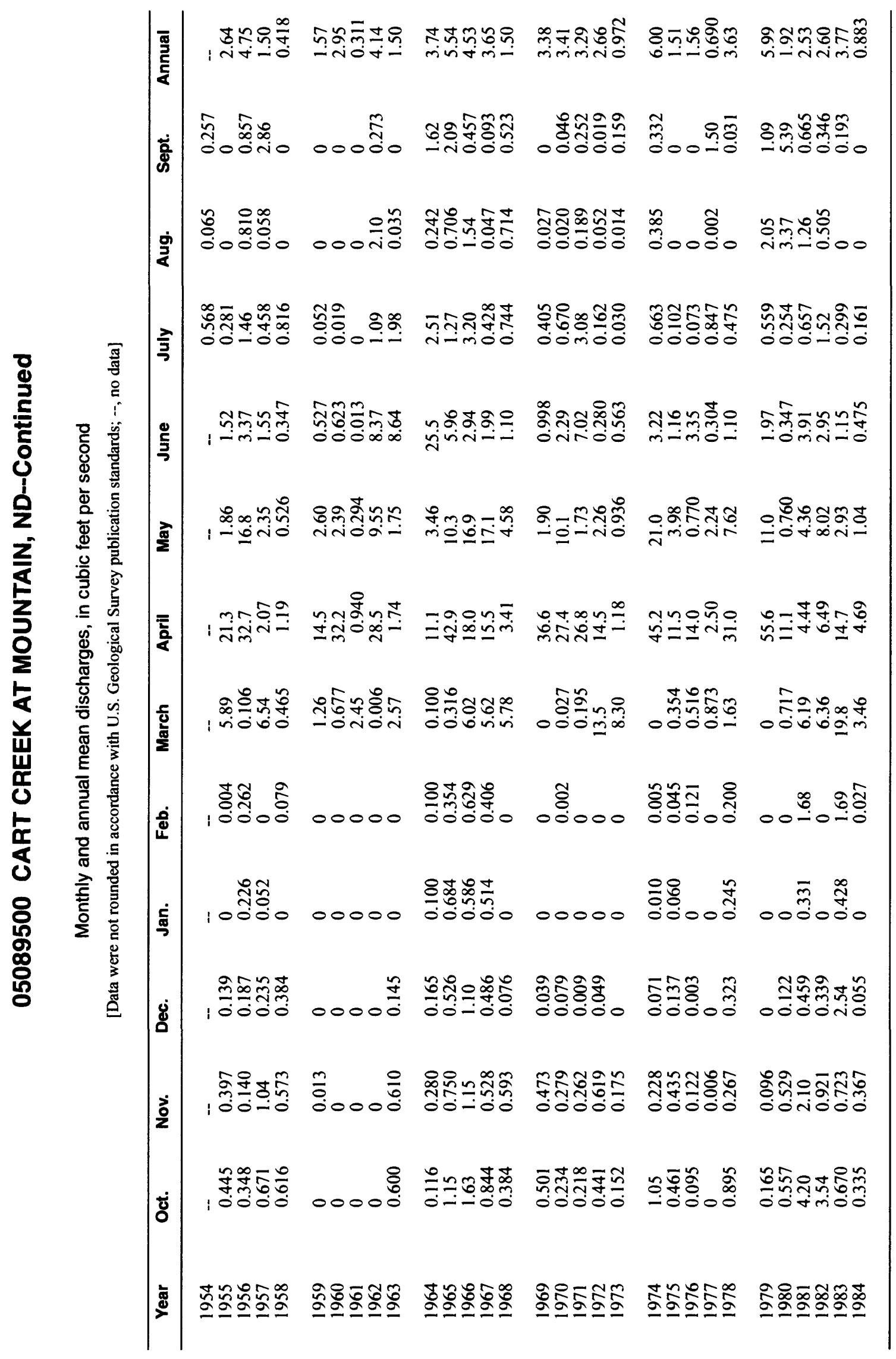




\section{PARK RIVER AT GRAFTON, ND}

LOCATION.--Lat $48^{\circ} 25^{\prime} 29^{\prime \prime}$, long $97^{\circ} 24^{\prime} 42^{\prime \prime}$, in NE $1 / 4$ sec.13, T.157 N., R.53 W., Walsh County, Hydrologic Unit 09020310, on right bank just upstream of U.S. Highway 81 bridge in Grafton, and $3.5 \mathrm{mi}$ downstream from South Branch.

DRAINAGE AREA.--695 $\mathrm{mi}^{2}$, approximately.

PERIOD OF RECORD.--April 1931 to current year. Monthly discharge only for some periods, published in WSP 1308.

GAGE.--Water-stage recorder. Datum of gage is $811.00 \mathrm{ft}$ above sea level. Prior to Oct.1, 1984, gage located on right bank $30 \mathrm{ft}$ upstream of Wakeman Avenue bridge. Datum of gage was $807.39 \mathrm{ft}$. Prior to Sept. 30, 1940, nonrecording gage at site $30 \mathrm{ft}$ downstream at same datum. Oct. 1, 1940, to Sept. 17, 1946, nonrecording gage at site $2 \mathrm{mi}$ downstream above masonry dam at same datum. Sept. 18,1946 , to July 25,1952 , nonrecording gage at site $30 \mathrm{ft}$ downstream at same datum.

EXTREMES FOR PERIOD OF RECORD.--Maximum discharge, 12,600 $\mathrm{ft}^{3} / \mathrm{s}$, Apr. 19, 1950, gage height, $20.13 \mathrm{ft}$; no flow at times.

Annual mean discharge

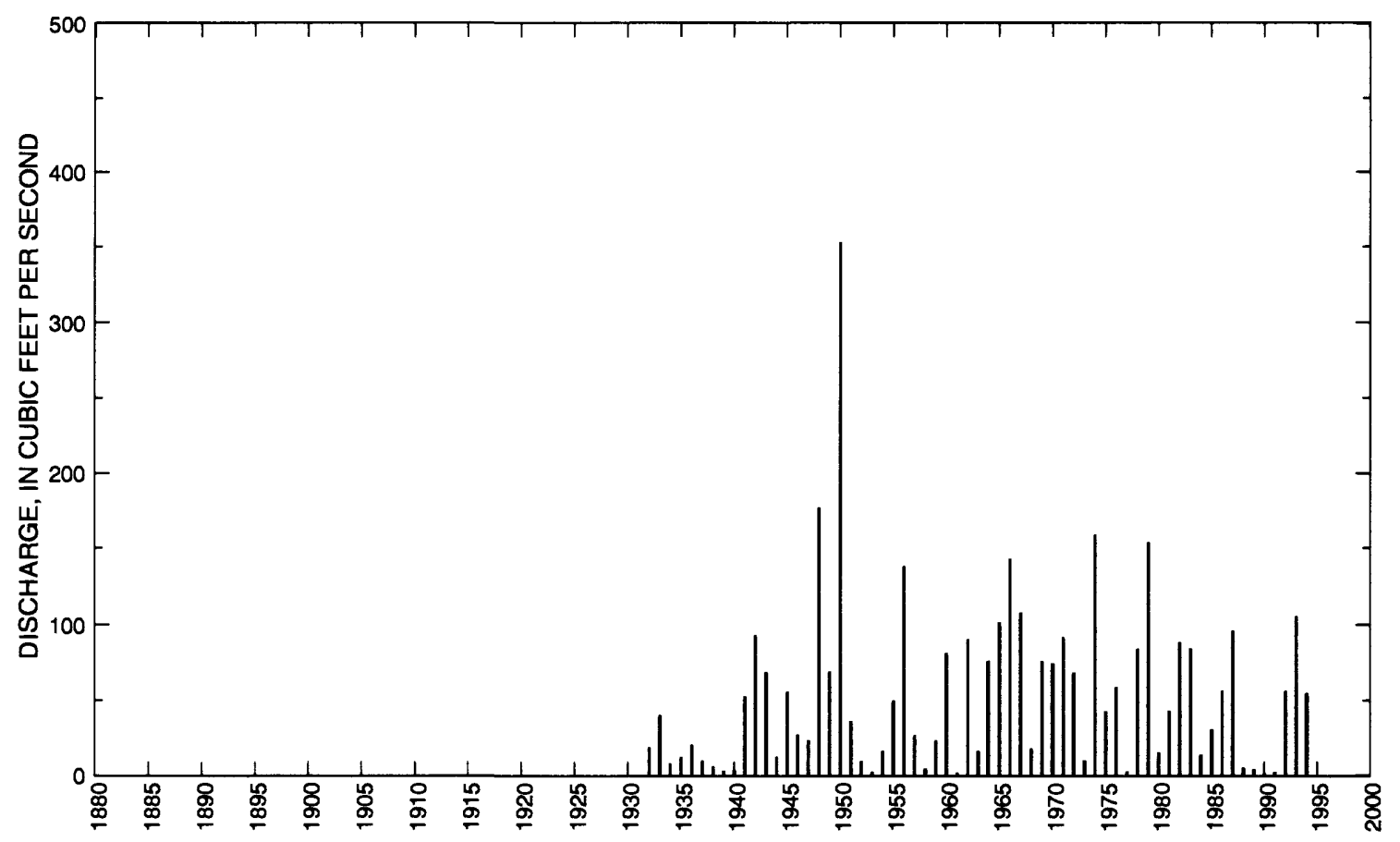




\section{PARK RIVER AT GRAFTON, ND--Continued}

Pre-regulation period, $1932-49$

Statistics of monthly and annual mean discharges, pre-regulation period

[m, more than 1 year of occurrence]

\begin{tabular}{|c|c|c|c|c|c|c|c|c|}
\hline \multirow[b]{2}{*}{ Month } & \multicolumn{2}{|c|}{ Maximum } & \multicolumn{2}{|c|}{ Minimum } & \multicolumn{4}{|c|}{ Mean } \\
\hline & $\begin{array}{c}\text { Discharge } \\
\left(\mathrm{ft}^{3} / \mathrm{s}\right)\end{array}$ & $\begin{array}{l}\text { Water year } \\
\text { of } \\
\text { occurrence }\end{array}$ & $\begin{array}{c}\text { Discharge } \\
\left(\mathrm{ft}^{3} / \mathrm{s}\right)\end{array}$ & $\begin{array}{l}\text { Water year } \\
\text { of } \\
\text { occurrence }\end{array}$ & $\begin{array}{c}\text { Discharge } \\
\left(\mathrm{ft}^{3} / \mathrm{s}\right)\end{array}$ & $\begin{array}{l}\text { Standard } \\
\text { deviation } \\
\left(\mathrm{ft}^{3} / \mathbf{s}\right)\end{array}$ & $\begin{array}{l}\text { Coeffi- } \\
\text { clent of } \\
\text { variation }\end{array}$ & $\begin{array}{l}\text { Percentage } \\
\text { of annual } \\
\text { discharge }\end{array}$ \\
\hline October & 32.7 & 1942 & 0 & $\mathrm{~m}$ & 2.07 & 7.65 & 3.70 & 0.44 \\
\hline November & 14.5 & 1945 & 0 & $\mathrm{~m}$ & 1.64 & 3.77 & 2.30 & 0.35 \\
\hline December & 6.99 & 1945 & 0 & $\mathrm{~m}$ & 0.770 & 1.79 & 2.31 & 0.17 \\
\hline January & 0.484 & 1948 & 0 & $\mathrm{~m}$ & 0.070 & 0.13 & 2.02 & 0.01 \\
\hline February & 2.00 & 1932 & 0 & $\mathrm{~m}$ & 0.160 & 0.47 & 2.97 & 0.03 \\
\hline March & 410 & 1945 & 0 & $\mathrm{~m}$ & 68.4 & 116 & 1.70 & 14.6 \\
\hline April & 1,810 & 1948 & 5.40 & 1938 & 323 & 451 & 1.40 & 68.9 \\
\hline May & 218 & 1948 & 2.05 & 1939 & 30.0 & 48.8 & 1.63 & 6.40 \\
\hline June & 202 & 1943 & 0.347 & 1938 & 20.6 & 45.2 & 2.20 & 4.40 \\
\hline July & 151 & 1943 & 0.013 & 1934 & 15.2 & 36.7 & 2.41 & 3.26 \\
\hline August & 30.5 & 1948 & 0 & $\mathrm{~m}$ & 3.97 & 7.81 & 1.97 & 0.85 \\
\hline September & 31.8 & 1941 & 0 & $\mathrm{~m}$ & 2.66 & 7.34 & 2.76 & 0.57 \\
\hline Annual & 177 & 1948 & 3.11 & 1939 & 39.0 & 43.5 & 1.12 & 100 \\
\hline
\end{tabular}

Annual flow duration

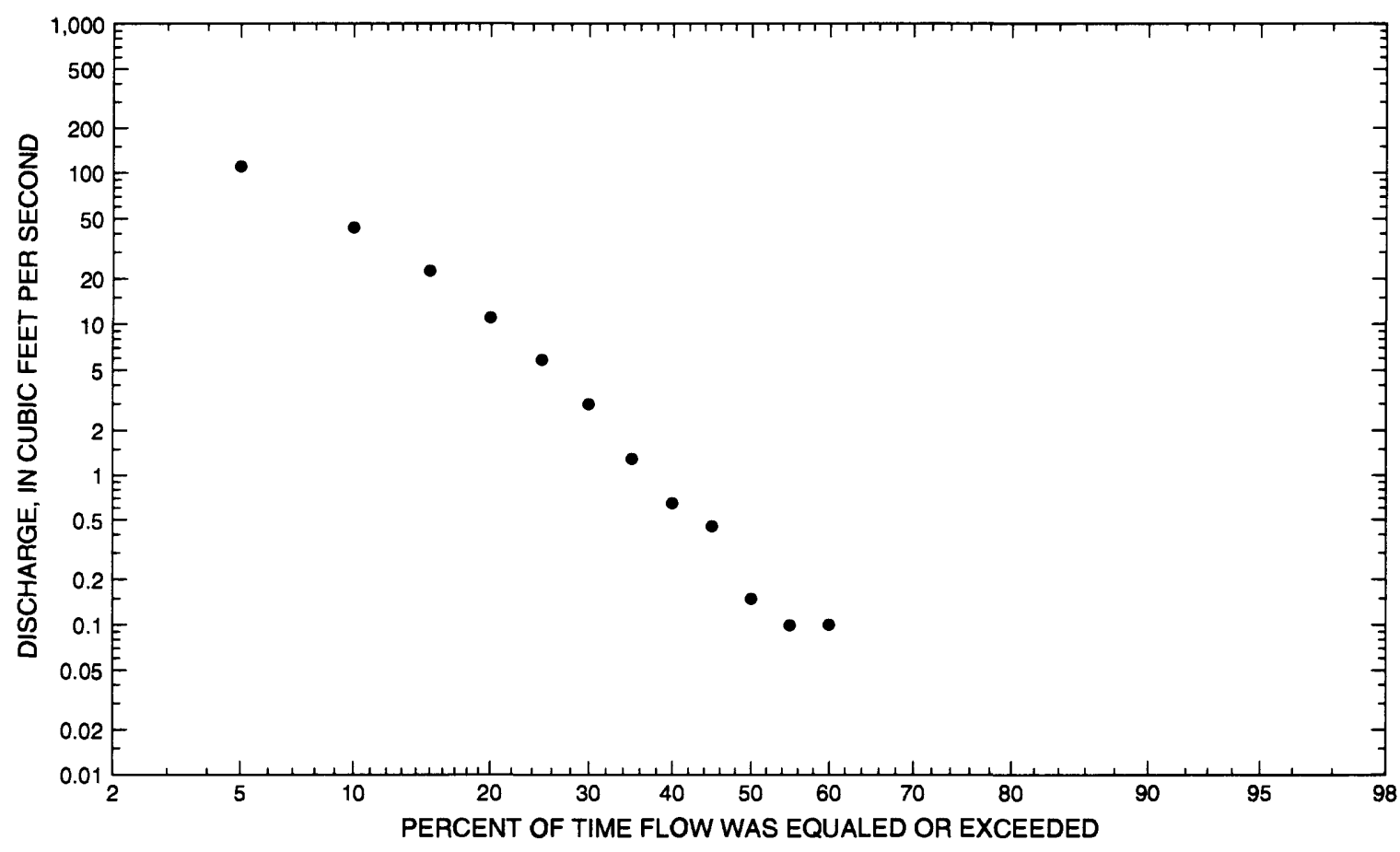




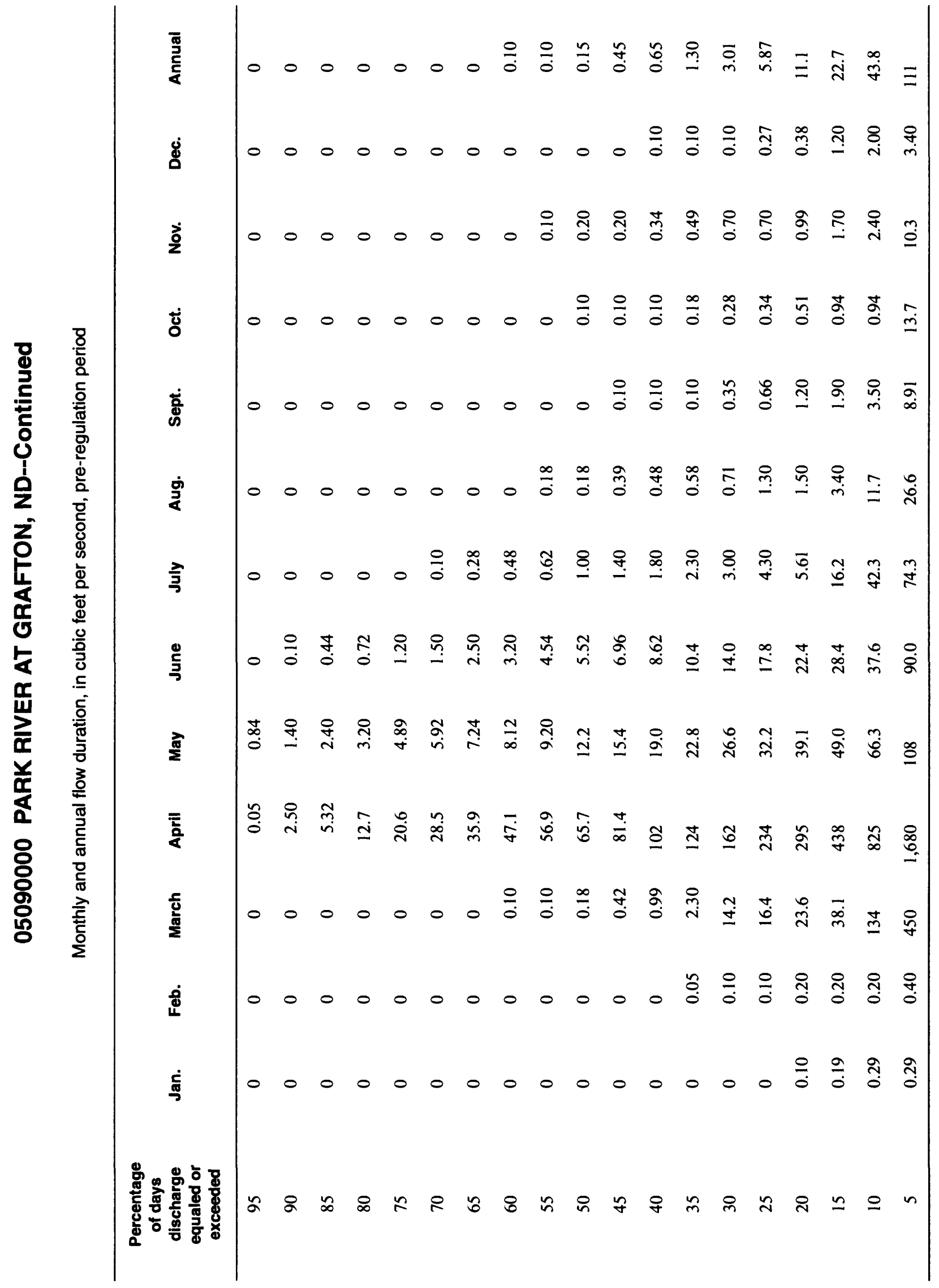


05090000 PARK RIVER AT GRAFTON, ND--Continued

Probability of occurrence of annual high discharges, pre-regulation period

[ng, statistic not given]

\begin{tabular}{|c|c|c|c|c|c|c|}
\hline \multirow[b]{2}{*}{$\begin{array}{l}\text { Exceedance } \\
\text { probability }\end{array}$} & \multirow[b]{2}{*}{$\begin{array}{l}\text { Recurrence } \\
\text { intervai } \\
\text { (years) }\end{array}$} & \multirow[b]{2}{*}{$\begin{array}{c}\text { Maximum } \\
\text { instantaneous } \\
\left(\mathrm{ft}^{3} / \mathrm{s}\right)\end{array}$} & \multicolumn{4}{|c|}{$\begin{array}{l}\text { Maximum mean discharge } \\
\left(\mathrm{ft}^{3} / \mathrm{s}\right)\end{array}$} \\
\hline & & & 3-day period & 7-day period & 15-day period & 30-day period \\
\hline 0.99 & 1.01 & 70.9 & 62.7 & 47.6 & 26.4 & 16.3 \\
\hline 0.95 & 1.05 & 148 & 123 & 94.8 & 55.9 & 34.3 \\
\hline 0.90 & 1.11 & 221 & 178 & 139 & 83.8 & 51.3 \\
\hline 0.80 & 1.25 & 358 & 284 & 222 & 138 & 83.9 \\
\hline 0.50 & 2 & 909 & 722 & 565 & 362 & 217 \\
\hline 0.20 & 5 & 2,340 & 1,940 & 1,510 & 974 & 572 \\
\hline 0.10 & 10 & 3,840 & 3,320 & 2,560 & 1,650 & 953 \\
\hline 0.04 & 25 & 6,550 & 6,010 & 4,580 & 2,900 & 1,650 \\
\hline 0.02 & 50 & 9,260 & 8,900 & 6,720 & 4,200 & 2,370 \\
\hline 0.01 & 100 & 12,700 & 12,700 & 9,540 & 5,880 & 3,270 \\
\hline 0.005 & 200 & 16,900 & 17,800 & 13,200 & 8,010 & 4,400 \\
\hline 0.002 & 500 & 23,900 & ng & ng & ng & ng \\
\hline
\end{tabular}

Probability of occurrence of annual low discharges, pre-regulation period

[ng, statistic not given]

\begin{tabular}{|c|c|c|c|c|c|c|c|c|c|c|}
\hline \multirow{3}{*}{$\begin{array}{c}\text { Non- } \\
\text { exceed- } \\
\text { ance } \\
\text { prob- } \\
\text { ability }\end{array}$} & \multirow{3}{*}{$\begin{array}{c}\text { Recur- } \\
\text { rence } \\
\text { inter- } \\
\text { val } \\
\text { (years) }\end{array}$} & \multicolumn{9}{|c|}{ Minimum mean discharge $\left(\mathrm{ft}^{3} / \mathrm{s}\right)$} \\
\hline & & \multicolumn{9}{|c|}{ Number of consecutive days } \\
\hline & & 1 & 3 & 7 & 14 & 30 & 60 & 90 & 120 & 183 \\
\hline 0.05 & 20 & ng & $\mathrm{ng}$ & $\mathrm{ng}$ & 0 & 0 & 0 & 0 & 0 & 0 \\
\hline 0.10 & 10 & ng & ng & ng & 0 & 0 & 0 & 0 & 0 & 0 \\
\hline 0.20 & 5 & ng & ng & $\mathrm{ng}$ & 0 & 0 & 0 & 0 & 0 & 0 \\
\hline 0.50 & 2 & $\mathrm{ng}$ & $\mathrm{ng}$ & $\mathrm{ng}$ & 0 & 0 & 0 & 0 & 0.024 & 0.051 \\
\hline
\end{tabular}




\section{PARK RIVER AT GRAFTON, ND--Continued}

Probability of occurrence of seasonal low discharges, pre-regulation period

\begin{tabular}{|c|c|c|c|c|c|c|c|c|c|}
\hline \multirow[b]{3}{*}{$\begin{array}{c}\text { Non- } \\
\text { exceedance } \\
\text { probability }\end{array}$} & \multirow[b]{3}{*}{$\begin{array}{c}\text { Recurrence } \\
\text { interval } \\
\text { (years) }\end{array}$} & \multicolumn{8}{|c|}{ Minimum mean discharge $\left(\mathrm{ft}^{3} / \mathrm{s}\right)$} \\
\hline & & \multicolumn{8}{|c|}{ Number of consecutive days } \\
\hline & & 1 & 7 & 14 & 30 & 1 & 7 & 14 & 30 \\
\hline & & \multicolumn{4}{|c|}{ December-January-February } & \multicolumn{4}{|c|}{ March-April-May } \\
\hline 0.05 & 20 & 0 & 0 & 0 & 0 & 0 & 0 & 0 & 0 \\
\hline 0.10 & 10 & 0 & 0 & 0 & 0 & 0 & 0 & 0 & 0 \\
\hline 0.20 & 5 & 0 & 0 & 0 & 0 & 0 & 0 & 0 & 0.137 \\
\hline \multirow[t]{2}{*}{0.50} & 2 & 0 & 0 & 0 & 0 & 0 & 0 & 0.024 & 2.60 \\
\hline & & \multicolumn{4}{|c|}{ June-July-August } & \multicolumn{4}{|c|}{ September-October-November } \\
\hline 0.05 & 20 & 0 & 0 & 0 & 0 & 0 & 0 & 0 & 0 \\
\hline 0.10 & 10 & 0 & 0 & 0 & 0 & 0 & 0 & 0 & 0 \\
\hline 0.20 & 5 & 0 & 0 & 0 & 0 & 0 & 0 & 0 & 0 \\
\hline 0.50 & 2 & 0 & 0 & 0.092 & 0.332 & 0 & 0 & 0 & 0 \\
\hline
\end{tabular}


05090000 PARK RIVER AT GRAFTON, ND--Continued

Post-regulation period, $1950-94$

Statistics of monthly and annual mean discharges, post-regulation period

$[\mathrm{m}$, more than 1 year of occurrence]

\begin{tabular}{|c|c|c|c|c|c|c|c|c|}
\hline \multirow[b]{2}{*}{ Month } & \multicolumn{2}{|c|}{ Maximum } & \multicolumn{2}{|c|}{ Minimum } & \multicolumn{4}{|c|}{ Mean } \\
\hline & $\begin{array}{c}\text { Discharge } \\
\left(\mathrm{ft}^{3} / \mathrm{s}\right)\end{array}$ & $\begin{array}{c}\text { Water year } \\
\text { of } \\
\text { occurrence }\end{array}$ & $\begin{array}{c}\text { Discharge } \\
\left(\mathrm{ft}^{3} / \mathrm{s}\right)\end{array}$ & $\begin{array}{c}\text { Water year } \\
\text { of } \\
\text { occurrence }\end{array}$ & $\begin{array}{c}\text { Discharge } \\
\left(\mathrm{ft}^{3} / \mathrm{s}\right)\end{array}$ & $\begin{array}{c}\text { Standard } \\
\text { deviation } \\
\left(\mathrm{ft}^{3} / \mathrm{s}\right)\end{array}$ & $\begin{array}{c}\text { Coeffi- } \\
\text { cient of } \\
\text { variation }\end{array}$ & $\begin{array}{c}\text { Percentage } \\
\text { of annuai } \\
\text { discharge }\end{array}$ \\
\hline October & 69.9 & 1983 & 0 & $\mathrm{~m}$ & 6.41 & 14.4 & 2.25 & 0.86 \\
\hline November & 31.3 & 1981 & 0 & $\mathrm{~m}$ & 4.12 & 6.25 & 1.52 & 0.56 \\
\hline December & 17.4 & 1983 & 0 & $\mathrm{~m}$ & 2.89 & 4.09 & 1.41 & 0.39 \\
\hline January & 13.9 & 1983 & 0 & $\mathrm{~m}$ & 1.79 & 3.04 & 1.70 & 0.24 \\
\hline February & 45.7 & 1981 & 0 & $\mathrm{~m}$ & 3.04 & 7.09 & 2.33 & 0.41 \\
\hline March & 400 & 1983 & 0 & 1991 & 62.8 & 106 & 1.69 & 8.47 \\
\hline April & 2,050 & 1950 & 0 & 1991 & 407 & 456 & 1.12 & 54.9 \\
\hline May & 2,070 & 1950 & 2.33 & 1961 & 140 & 330 & 2.36 & 19.9 \\
\hline June & 576 & 1964 & 0 & 1961 & 54.2 & 93.5 & 1.72 & 7.30 \\
\hline July & 398 & 1993 & 0 & 1990 & 32.0 & 71.6 & 2.23 & 4.32 \\
\hline August & 569 & 1993 & 0 & $\mathrm{~m}$ & 17.8 & 84.4 & 4.76 & 2.39 \\
\hline September & 151 & 1957 & 0 & $\mathrm{~m}$ & 9.59 & 26.0 & 2.71 & 1.29 \\
\hline Annual & 353 & 1950 & 1.38 & 1990 & 61.7 & 63.1 & 1.02 & 100 \\
\hline
\end{tabular}

Annual flow duration

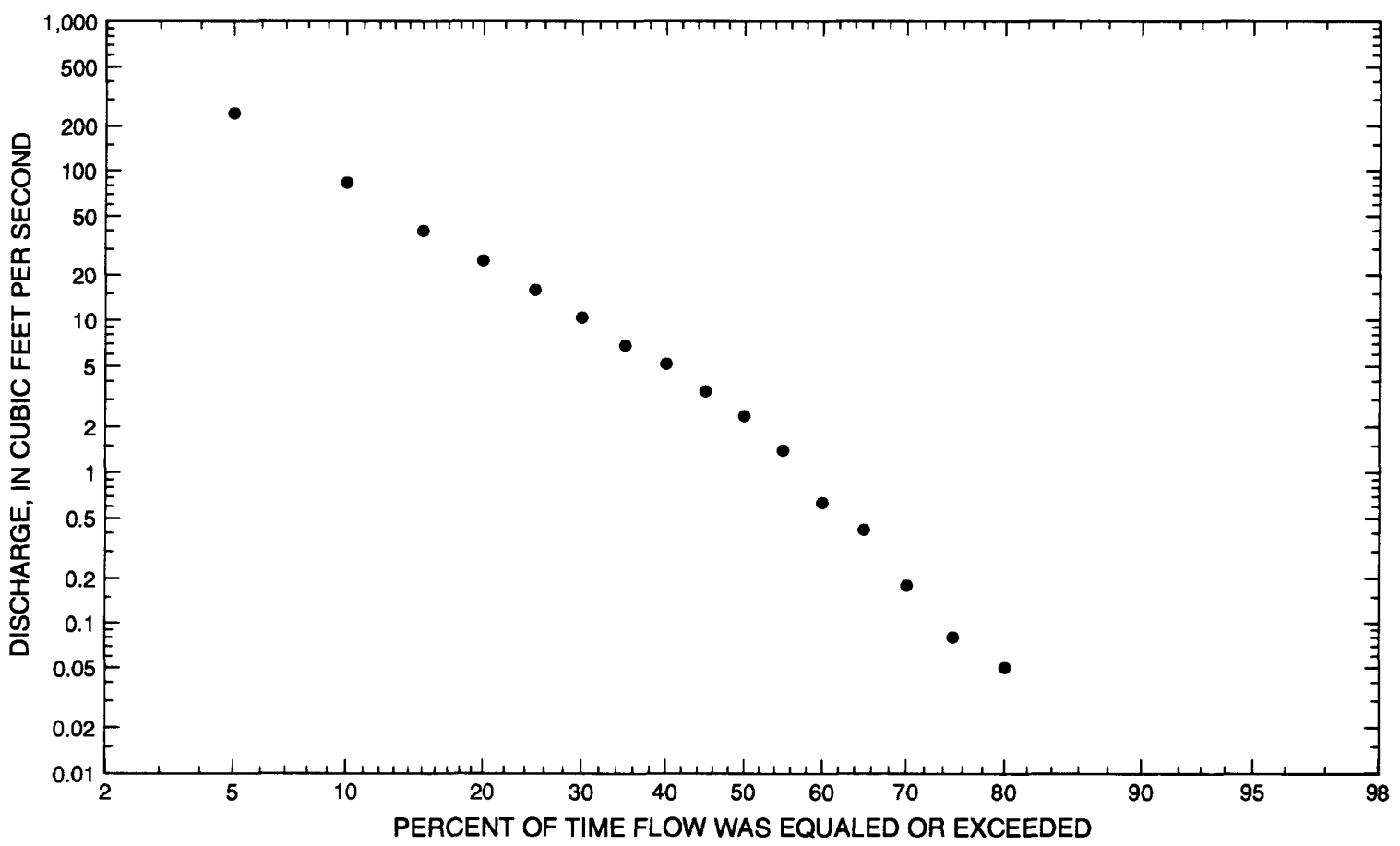




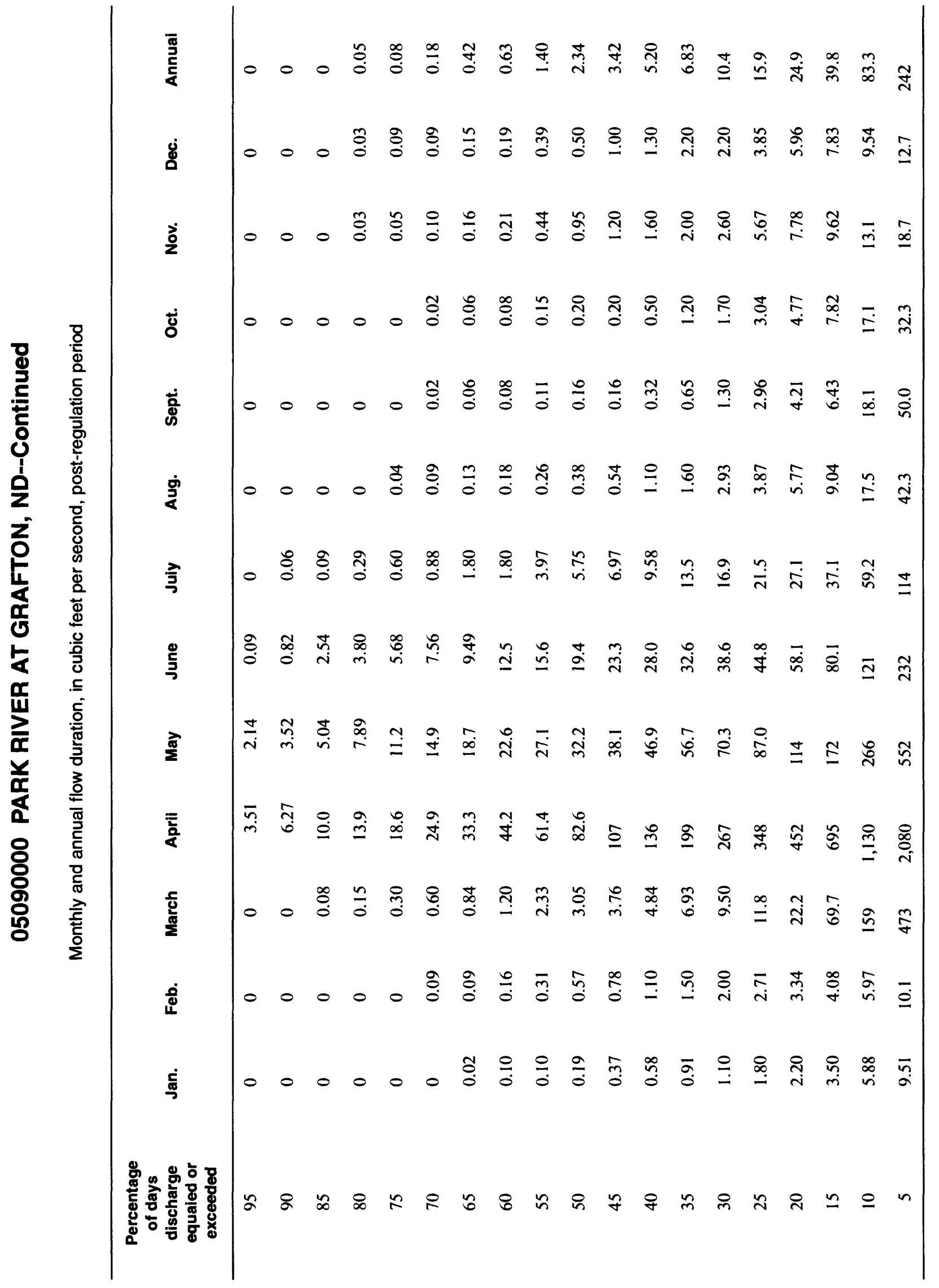


05090000 PARK RIVER AT GRAFTON, ND--Continued

Probability of occurrence of annual high discharges, post-regulation period

[ng, statistic not given]

\begin{tabular}{|c|c|c|c|c|c|c|}
\hline \multirow[b]{2}{*}{$\begin{array}{l}\text { Exceedance } \\
\text { probability }\end{array}$} & \multirow[b]{2}{*}{$\begin{array}{l}\text { Recurrence } \\
\text { intervai } \\
\text { (years) }\end{array}$} & \multirow[b]{2}{*}{$\begin{array}{c}\text { Maximum } \\
\text { instantaneous } \\
\left(\mathrm{ft}^{3} / \mathrm{s}\right)\end{array}$} & \multicolumn{4}{|c|}{$\begin{array}{c}\text { Maximum mean discharge } \\
\qquad\left(\mathrm{ft}^{3} / \mathrm{s}\right)\end{array}$} \\
\hline & & & 3-day period & 7-day period & 15-day period & 30-day period \\
\hline 0.99 & 1.01 & 11.6 & 4.90 & 4.30 & 3.76 & 2.99 \\
\hline 0.95 & 1.05 & 51.5 & 29.5 & 24.8 & 19.8 & 14.4 \\
\hline 0.90 & 1.11 & 107 & 69.5 & 57.3 & 43.8 & 30.7 \\
\hline 0.80 & 1.25 & 246 & 179 & 145 & 106 & 71.6 \\
\hline 0.50 & 2 & 1,010 & 838 & 658 & 451 & 293 \\
\hline 0.20 & 5 & 3,380 & 2,840 & 2,190 & 1,440 & 922 \\
\hline 0.10 & 10 & 5,860 & 4,770 & 3,660 & 2,360 & 1,520 \\
\hline 0.04 & 25 & 9,950 & 7,630 & 5,830 & 3,730 & 2,430 \\
\hline 0.02 & 50 & 13,600 & 9,910 & 7,570 & 4,820 & 3,180 \\
\hline 0.01 & 100 & 17,600 & 12,200 & 9,340 & 5,920 & 3,950 \\
\hline 0.005 & 200 & 22,000 & 14,500 & 11,100 & 7,020 & 4,730 \\
\hline 0.002 & 500 & 28,300 & ng & ng & $\mathrm{ng}$ & ng \\
\hline
\end{tabular}

Probability of occurrence of annual low discharges, post-regulation period

\begin{tabular}{|c|c|c|c|c|c|c|c|c|c|c|}
\hline \multirow[b]{3}{*}{$\begin{array}{c}\text { Non- } \\
\text { exceed- } \\
\text { ance } \\
\text { prob- } \\
\text { ability }\end{array}$} & \multirow[b]{3}{*}{$\begin{array}{c}\text { Recur- } \\
\text { rence } \\
\text { inter- } \\
\text { vai } \\
\text { (years) }\end{array}$} & \multicolumn{9}{|c|}{ Minimum mean discharge $\left(\mathrm{ft}^{3} / \mathrm{s}\right)$} \\
\hline & & \multicolumn{9}{|c|}{ Number of consecutive days } \\
\hline & & 1 & 3 & 7 & 14 & 30 & 60 & 90 & 120 & 183 \\
\hline 0.05 & 20 & 0 & 0 & 0 & 0 & 0 & 0 & 0 & 0 & 0.023 \\
\hline 0.10 & 10 & 0 & 0 & 0 & 0 & 0 & 0 & 0 & 0.010 & 0.063 \\
\hline 0.20 & 5 & 0 & 0 & 0 & 0 & 0 & 0 & 0.017 & 0.083 & 0.174 \\
\hline 0.50 & 2 & 0 & 0 & 0 & 0 & 0.042 & 0.097 & 0.243 & 0.537 & 0.947 \\
\hline
\end{tabular}




\section{PARK RIVER AT GRAFTON, ND--Continued}

Probability of occurrence of seasonal low discharges, post-regulation period

\begin{tabular}{|c|c|c|c|c|c|c|c|c|c|}
\hline \multirow[b]{3}{*}{$\begin{array}{c}\text { Non- } \\
\text { exceedance } \\
\text { probability }\end{array}$} & \multirow[b]{3}{*}{$\begin{array}{c}\text { Recurrence } \\
\text { interval } \\
\text { (years) }\end{array}$} & \multicolumn{8}{|c|}{ Minimum mean discharge $\left(\mathrm{ft}^{3} / \mathrm{s}\right)$} \\
\hline & & \multicolumn{8}{|c|}{ Number of consecutive days } \\
\hline & & 1 & 7 & 14 & 30 & 1 & 7 & 14 & 30 \\
\hline & & \multicolumn{4}{|c|}{ December-January-February } & \multicolumn{4}{|c|}{ March-April-May } \\
\hline 0.05 & 20 & 0 & 0 & 0 & 0 & 0 & 0 & 0 & 0.059 \\
\hline 0.10 & 10 & 0 & 0 & 0 & 0 & 0 & 0 & 0 & 0.250 \\
\hline 0.20 & 5 & 0 & 0 & 0 & 0 & 0.029 & 0.036 & 0.085 & 0.980 \\
\hline \multirow[t]{2}{*}{0.50} & 2 & 0.009 & 0.073 & 0.074 & 0.140 & 0.691 & 0.945 & 1.23 & 7.33 \\
\hline & & \multicolumn{4}{|c|}{ June-July-August } & \multicolumn{4}{|c|}{ September-October-November } \\
\hline 0.05 & 20 & 0 & 0 & 0 & 0 & 0 & 0 & 0 & 0 \\
\hline 0.10 & 10 & 0 & 0 & 0 & 0.007 & 0 & 0 & 0 & 0 \\
\hline 0.20 & 5 & 0 & 0 & 0 & 0.081 & 0 & 0 & 0 & 0 \\
\hline 0.50 & 2 & 0.045 & 0.120 & 0.217 & 0.812 & 0.026 & ${ }^{1} 0.032$ & 0.038 & 0.088 \\
\hline
\end{tabular}

${ }^{1}$ Graphical interpretation. 


\section{PARK RIVER AT GRAFTON, ND--Continued}

Annual peak discharge and corresponding gage height

$[--$, no data $]$

\begin{tabular}{|c|c|c|c|c|c|c|c|}
\hline $\begin{array}{l}\text { Water } \\
\text { year }\end{array}$ & Date & $\begin{array}{c}\text { Gage } \\
\text { height } \\
\text { (feet) }\end{array}$ & $\begin{array}{c}\text { Peak } \\
\text { discharge } \\
\left(\mathrm{ft}^{3} / \mathrm{s}\right)\end{array}$ & $\begin{array}{c}\text { Water } \\
\text { year }\end{array}$ & Date & $\begin{array}{c}\text { Gage } \\
\text { height } \\
\text { (feet) }\end{array}$ & $\begin{array}{c}\text { Peak } \\
\text { discharge } \\
\left(\mathrm{ft}^{3} / \mathrm{s}\right)\end{array}$ \\
\hline \multicolumn{8}{|c|}{ Annual peak discharge, by year, and corresponding gage height } \\
\hline${ }^{1} 1882$ & -- & 16.00 & -- & 1962 & April 20 & 18.27 & 5,900 \\
\hline${ }^{1} 1897$ & April 15 & 18.14 & 3,480 & 1963 & June 6 & 8.78 & 344 \\
\hline${ }^{1} 1916$ & April & 17.64 & 3,140 & 1964 & June 20 & 14.01 & 2,140 \\
\hline 1932 & April 10 & -- & 750 & 1965 & April 13 & 18.21 & 5,710 \\
\hline 1933 & April 2 & 15.20 & 2,200 & 1966 & April 4 & 16.08 & 2,790 \\
\hline 1934 & April 9 & 6.61 & 393 & 1967 & May 9 & 14.46 & 2,290 \\
\hline 1935 & March 28 & 8.34 & 443 & 1968 & March 28 & 9.85 & 630 \\
\hline 1936 & April 14 & 13.68 & 1,200 & 1969 & April 13 & 18.13 & 4,990 \\
\hline 1937 & April 10 & 8.21 & 380 & 1970 & April 29 & 12.09 & 1,520 \\
\hline 1938 & March 21 & 6.12 & 291 & 1971 & April 10 & 16.42 & 3,600 \\
\hline 1939 & March 30 & 7.68 & 150 & 1972 & April 14 & 14.30 & 2,150 \\
\hline 1940 & April 20 & 5.83 & 210 & 1973 & March 27 & 8.82 & 251 \\
\hline 1941 & April 13 & 13.04 & 1,830 & 1974 & April 16 & 17.03 & 3,660 \\
\hline 1942 & April 6 & 15.46 & 4,310 & 1975 & April 17 & 11.10 & 900 \\
\hline 1943 & March 28 & 13.15 & 1,430 & 1976 & April 4 & 14.06 & 2,000 \\
\hline 1944 & April 12 & 5.55 & 563 & 1977 & May 5 & 7.57 & 40.0 \\
\hline 1945 & March 16 & 10.88 & 1,180 & 1978 & April 8 & 17.10 & 3,700 \\
\hline 1946 & March 23 & 11.40 & 1,490 & 1979 & April 22 & 19.56 & 8,740 \\
\hline 1947 & April 4 & 9.70 & 520 & 1980 & April 7 & 9.09 & 399 \\
\hline 1948 & April 19 & 20.06 & 11,700 & 1981 & April 3 & 9.51 & 471 \\
\hline 1949 & April 11 & 16.94 & 2,530 & 1982 & April 1 & 12.26 & 1,420 \\
\hline 1950 & April 19 & 20.13 & 12,600 & 1983 & March 7 & 12.34 & 1,360 \\
\hline 1951 & April 6 & 13.34 & 1,640 & 1984 & March 27 & 8.72 & 269 \\
\hline 1952 & April 5 & 6.66 & 180 & 1985 & March 19 & 9.74 & 1,000 \\
\hline 1953 & June 5 & 5.45 & 125 & 1986 & March 24 & 9.48 & 859 \\
\hline 1954 & June 16 & 7.24 & 478 & 1987 & April 7 & 13.44 & 3,220 \\
\hline 1955 & April 3 & 16.84 & 2,100 & 1988 & April 6 & 7.79 & 143 \\
\hline 1956 & April 22 & 16.25 & 4,200 & 1989 & April 24 & 7.79 & 143 \\
\hline 1957 & September 3 & 10.96 & 1,300 & 1990 & April 21 & 7.28 & 24.0 \\
\hline 1958 & April 7 & 5.70 & 41.0 & 1991 & May 23 & 7.47 & 48.0 \\
\hline 1959 & April 6 & 12.29 & 1,200 & 1992 & March 8 & 9.90 & 1,030 \\
\hline 1960 & April 15 & 15.40 & 2,770 & 1993 & July 30 & 12.22 & 2,420 \\
\hline 1961 & April 27 & 7.51 & 40.0 & 1994 & March 22 & 10.49 & 800 \\
\hline \multicolumn{8}{|c|}{ Annual peak discharge, from highest to lowest, and corresponding gage height } \\
\hline 1950 & April 19 & 20.13 & 12,600 & 1971 & April 10 & 16.42 & 3,600 \\
\hline 1948 & April 19 & 20.06 & 11,700 & ${ }^{1} 1897$ & April 15 & 18.14 & 3,480 \\
\hline 1979 & April 22 & 19.56 & 8,740 & 1987 & April 7 & 13.44 & 3,220 \\
\hline 1962 & April 20 & 18.27 & 5,900 & ${ }^{1} 1916$ & April & 17.64 & 3,140 \\
\hline 1965 & April 13 & 18.21 & 5,710 & 1966 & April 4 & 16.08 & 2,790 \\
\hline 1969 & April 13 & 18.13 & 4,990 & 1960 & April 15 & 15.40 & 2,770 \\
\hline 1942 & April 6 & 15.46 & 4,310 & 1949 & April 11 & 16.94 & 2,530 \\
\hline 1956 & April 22 & 16.25 & 4,200 & 1993 & July 30 & 12.22 & 2,420 \\
\hline 1978 & April 8 & 17.10 & 3,700 & 1967 & May 9 & 14.46 & 2,290 \\
\hline 1974 & April 16 & 17.03 & 3,660 & 1933 & April 2 & 15.20 & 2,200 \\
\hline
\end{tabular}




\section{PARK RIVER AT GRAFTON, ND--Continued}

Annual peak discharge and corresponding gage height--Continued

$$
\text { [-, no data] }
$$

\begin{tabular}{|c|c|c|c|c|c|c|c|}
\hline $\begin{array}{l}\text { Water } \\
\text { year }\end{array}$ & Date & $\begin{array}{c}\text { Gage } \\
\text { height } \\
\text { (feet) }\end{array}$ & $\begin{array}{c}\text { Peak } \\
\text { discharge } \\
\left(\mathrm{ft}^{3} / \mathrm{s}\right)\end{array}$ & $\begin{array}{l}\text { Water } \\
\text { year }\end{array}$ & Date & $\begin{array}{c}\text { Gage } \\
\text { height } \\
\text { (feet) }\end{array}$ & $\begin{array}{c}\text { Peak } \\
\text { discharge } \\
\left(\mathrm{ft}^{3} / \mathrm{s}\right)\end{array}$ \\
\hline \multicolumn{8}{|c|}{ Annual peak discharge, from highest to lowest, and corresponding gage height-Continued } \\
\hline 1972 & April 14 & 14.30 & 2,150 & 1947 & April 4 & 9.70 & 520 \\
\hline 1964 & June 20 & 14.01 & 2,140 & 1954 & June 16 & 7.24 & 478 \\
\hline 1955 & April 3 & 16.84 & 2,100 & 1981 & April 3 & 9.51 & 471 \\
\hline 1976 & April 4 & 14.06 & 2,000 & 1935 & March 28 & 8.34 & 443 \\
\hline 1941 & April 13 & 13.04 & 1,830 & 1980 & April 7 & 9.09 & 399 \\
\hline 1951 & April 6 & 13.34 & 1,640 & 1934 & April 9 & 6.61 & 393 \\
\hline 1970 & April 29 & 12.09 & 1,520 & 1937 & April 10 & 8.21 & 380 \\
\hline 1946 & March 23 & 11.40 & 1,490 & 1963 & June 6 & 8.78 & 344 \\
\hline 1943 & March 28 & 13.15 & 1,430 & 1938 & March 21 & 6.12 & 291 \\
\hline 1982 & April 1 & 12.26 & 1,420 & 1984 & March 27 & 8.72 & 269 \\
\hline 1983 & March 7 & 12.34 & 1,360 & 1973 & March 27 & 8.82 & 251 \\
\hline 1957 & September 3 & 10.96 & 1,300 & 1940 & April 20 & 5.83 & 210 \\
\hline 1936 & April 14 & 13.68 & 1,200 & 1952 & April 5 & 6.66 & 180 \\
\hline 1959 & April 6 & 12.29 & 1,200 & 1939 & March 30 & 7.68 & 150 \\
\hline 1945 & March 16 & 10.88 & 1,180 & 1988 & April 6 & 7.79 & 143 \\
\hline 1992 & March 8 & 9.90 & 1,030 & 1989 & April 24 & 7.79 & 143 \\
\hline 1985 & March 19 & 9.74 & 1,000 & 1953 & June 5 & 5.45 & 125 \\
\hline 1975 & April 17 & 11.10 & 900 & 1991 & May 23 & 7.47 & 48.0 \\
\hline 1986 & March 24 & 9.48 & 859 & 1958 & April 7 & 5.70 & 41.0 \\
\hline 1994 & March 22 & 10.49 & 800 & 1961 & April 27 & 7.51 & 40.0 \\
\hline 1932 & April 10 & -- & 750 & 1977 & May 5 & 7.57 & 40.0 \\
\hline 1968 & March 28 & 9.85 & 630 & 1990 & April 21 & 7.28 & 24.0 \\
\hline 1944 & April 12 & 5.55 & 563 & ${ }^{1} 1882$ & - & 16.00 & -- \\
\hline
\end{tabular}

${ }^{1}$ Determined by U.S. Army Corps of Engineers; not used in statistics. 


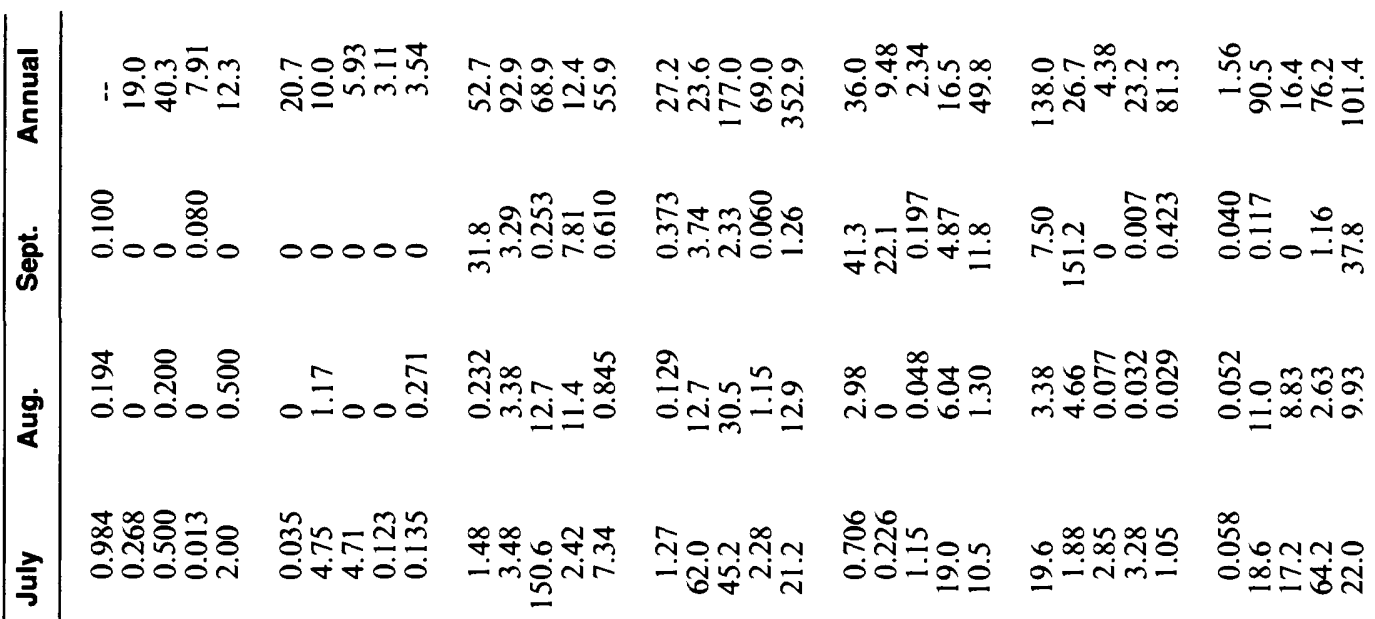

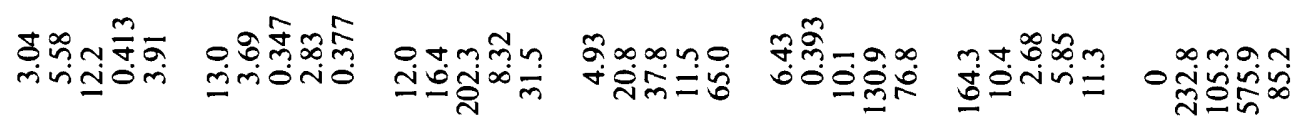

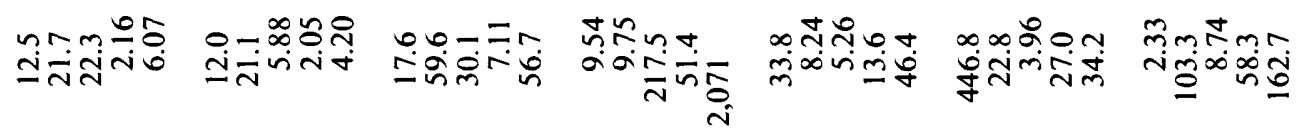

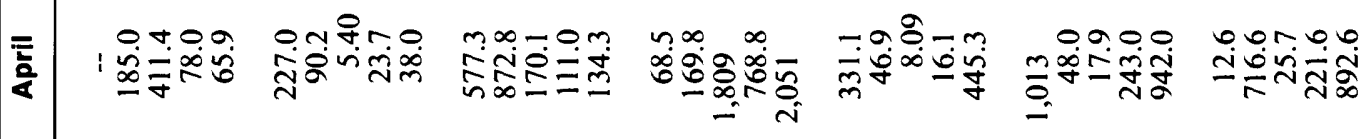

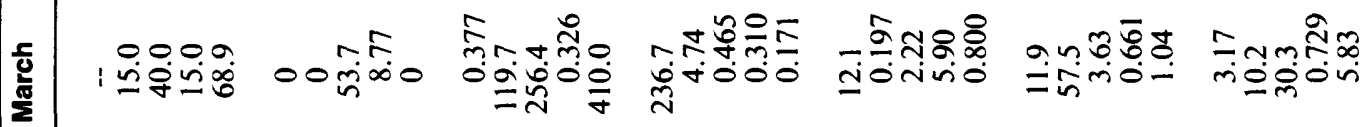
i 10000 00000 0000் i

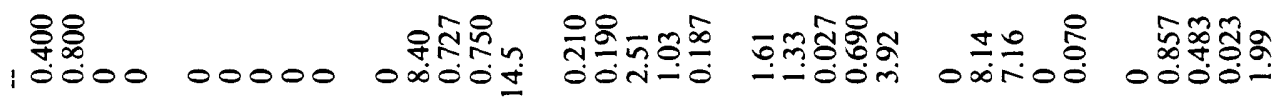

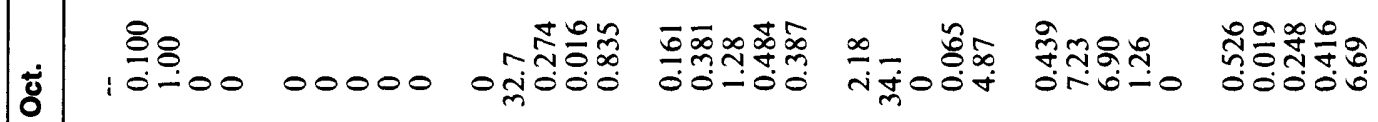

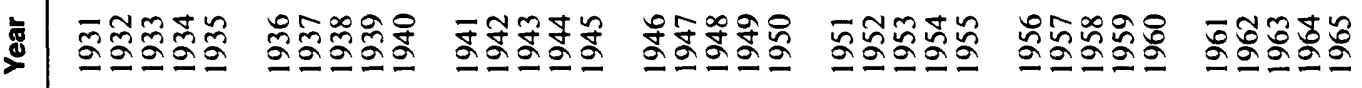




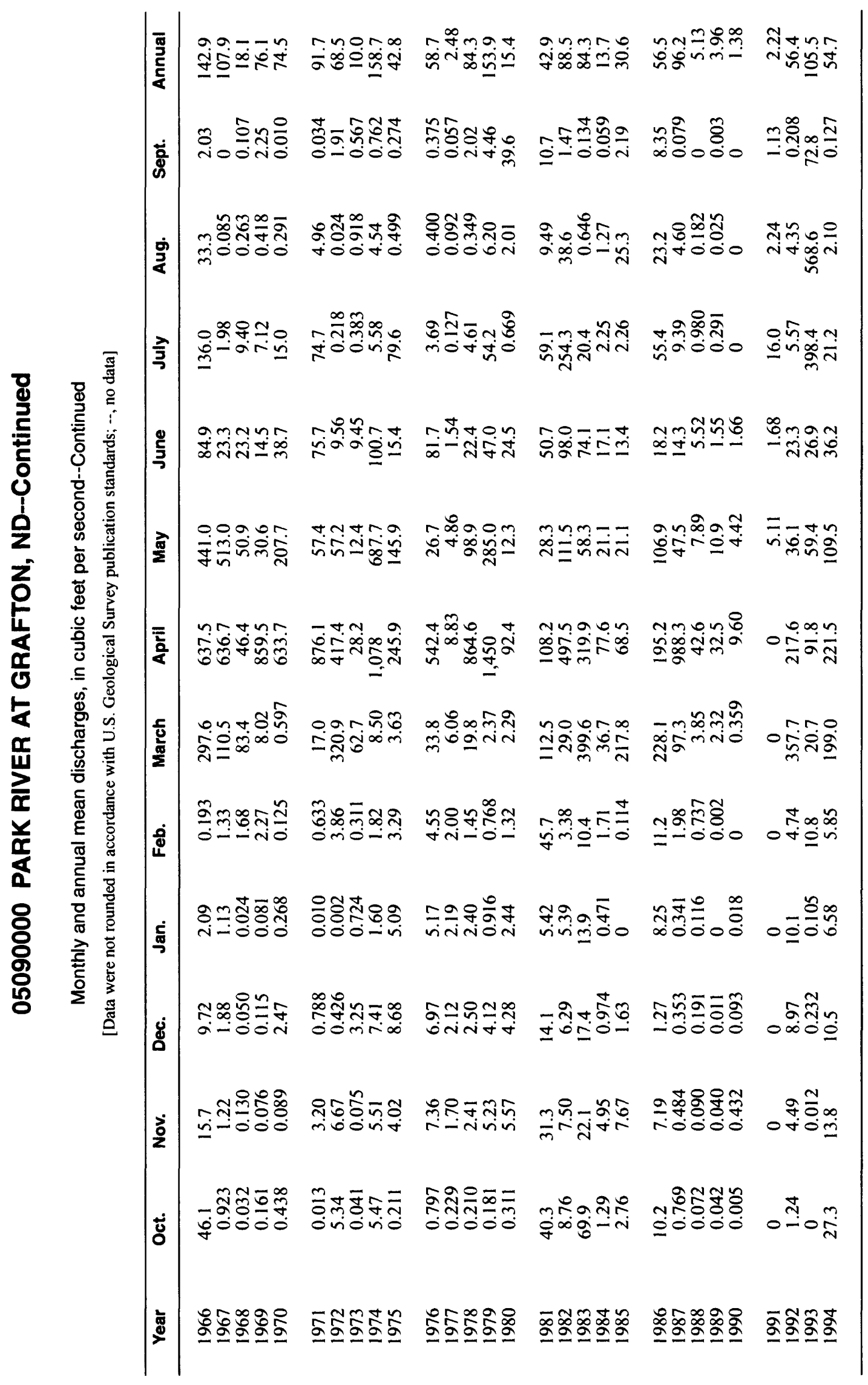


LOCATION.--Lat 48³4'20”, long 9708'50”, in $\mathrm{SE}^{1} /{ }_{4} \mathrm{SE}^{1 / 4} \mathrm{SE}^{1 / 4} \mathrm{sec} .24, \mathrm{~T} .159$ N., R.51 W., Pembina County, Hydrologic Unit 09020311 , on downstream side of bridge on North Dakota State Highway 11, at the North Dakota-Minnesota border, 1.5 mi northeast of Drayton, and at mile 206.7.

DRAINAGE AREA.--34,800 $\mathrm{mi}^{2}$, approximately, includes $3,800 \mathrm{mi}^{2}$ in closed basins.

PERIOD OF RECORD.--April 1936 to June 1937, April 1941 to current year (fragmentary prior to April 1949).

GAGE.--Water-stage recorder and concrete control. Datum of gage is $755.00 \mathrm{ft}$ above sea level (Minnesota highway benchmark). Prior to Nov. 30, 1954, nonrecording gage at site 1.5 mi upstream at datum $1.59 \mathrm{ft}$ higher.

EXTREMES FOR PERIOD OF RECORD.--Maximum discharge, 92,900 $\mathrm{ft}^{3} / \mathrm{s}$, Apr. 28, 1979, gage height, $43.66 \mathrm{ft}$; minimum daily discharge, $7.7 \mathrm{ft}^{3} / \mathrm{s}$, Oct. 16, 1936, gage height, $1.75 \mathrm{ft}$.

EXTREMES OUTSIDE PERIOD OF RECORD.--Flood of April 1897 reached a stage of about $41 \mathrm{ft}$ at site and datum in use prior to Nov. 30, 1954.

Annual mean discharge

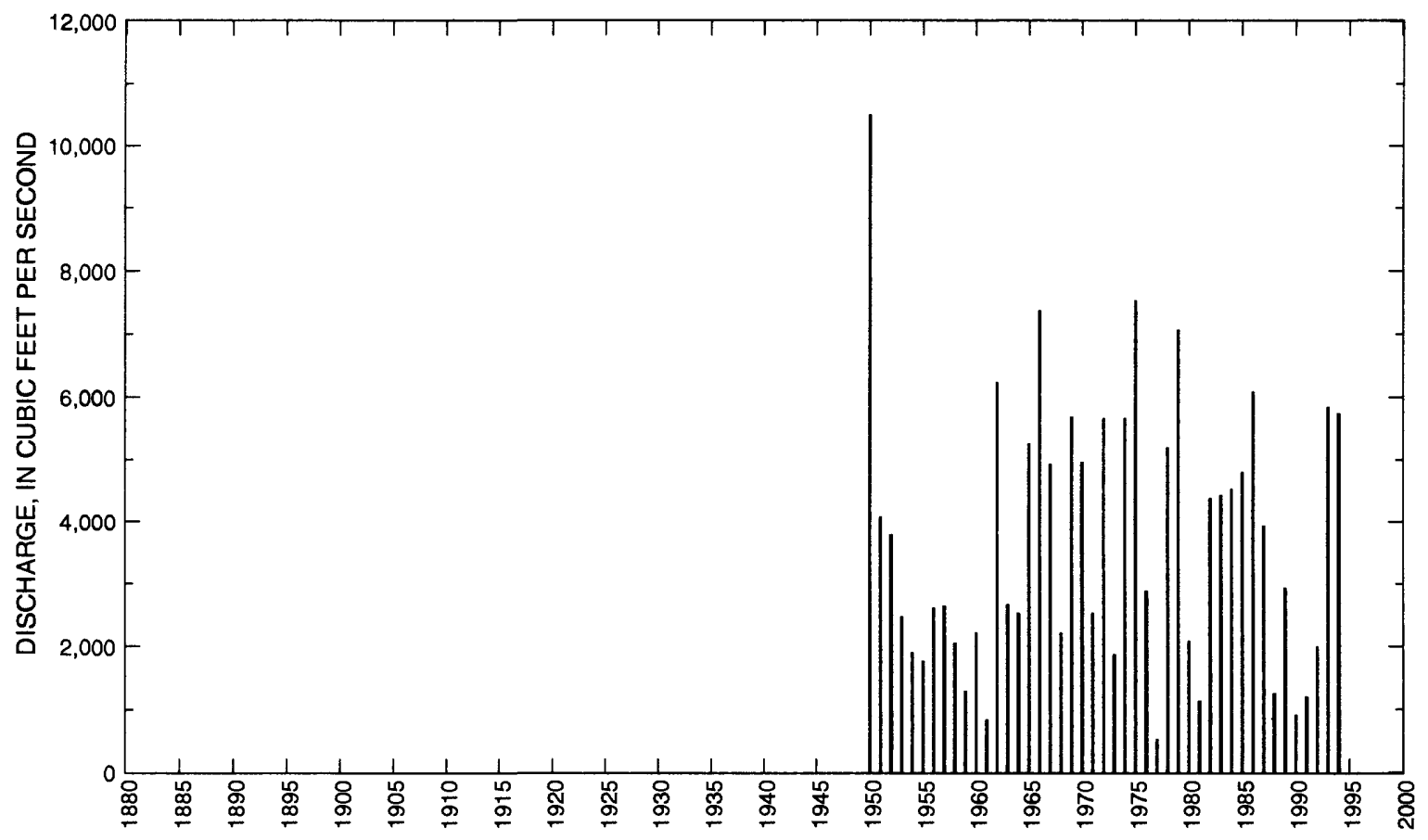




\section{RED RIVER OF THE NORTH AT DRAYTON, ND--Continued}

Statistics of monthly and annual mean discharges

\begin{tabular}{|c|c|c|c|c|c|c|c|c|}
\hline \multirow[b]{2}{*}{ Month } & \multicolumn{2}{|c|}{ Maximum } & \multicolumn{2}{|c|}{ Minimum } & \multicolumn{4}{|c|}{ Mean } \\
\hline & $\begin{array}{c}\text { Discharge } \\
\left(\mathrm{ft}^{3} / \mathrm{s}\right)\end{array}$ & $\begin{array}{c}\text { Water year } \\
\text { of } \\
\text { occurrence }\end{array}$ & $\begin{array}{c}\text { Discharge } \\
\left(\mathrm{ft}^{3} / \mathrm{s}\right)\end{array}$ & $\begin{array}{c}\text { Water year } \\
\text { of } \\
\text { occurrence }\end{array}$ & $\begin{array}{c}\text { Discharge } \\
\left(\mathrm{ft}^{3} / \mathrm{s}\right)\end{array}$ & $\begin{array}{c}\text { Standard } \\
\text { deviation } \\
\left(\mathrm{ft}^{3} / \mathrm{s}\right)\end{array}$ & $\begin{array}{l}\text { Coeffi- } \\
\text { cient of } \\
\text { variation }\end{array}$ & $\begin{array}{c}\text { Percentage } \\
\text { of annuai } \\
\text { discharge }\end{array}$ \\
\hline October & 4,460 & 1972 & 13.8 & 1937 & 1,700 & 1,270 & 0.75 & 3.89 \\
\hline November & 5,650 & 1972 & 277 & 1977 & 1,460 & 1,010 & 0.69 & 3.36 \\
\hline December & 3,070 & 1972 & 149 & 1977 & 1,180 & 713 & 0.61 & 2.69 \\
\hline January & 2,060 & 1966 & 174 & 1990 & 1,040 & 584 & 0.56 & 2.37 \\
\hline February & 1,880 & 1952 & 201 & 1977 & 1,010 & 534 & 0.53 & 2.32 \\
\hline March & 9,330 & 1983 & 280 & 1962 & 2,620 & 2,380 & 0.91 & 6.01 \\
\hline April & 38,400 & 1966 & 1,280 & 1981 & 13,200 & 9,460 & 0.72 & 30.2 \\
\hline May & 58,900 & 1950 & 938 & 1977 & 7,900 & 9,630 & 1.22 & 18.1 \\
\hline June & 23,400 & 1962 & 399 & 1936 & 5,010 & 4,170 & 0.83 & 11.5 \\
\hline July & 28,200 & 1975 & 118 & 1936 & 4,410 & 4,650 & 1.05 & 10.1 \\
\hline August & 21,600 & 1993 & 50.1 & 1936 & 2,350 & 3,210 & 1.37 & 5.39 \\
\hline September & 7,910 & 1993 & 27.4 & 1936 & 1,790 & 1,480 & 0.82 & 4.11 \\
\hline Annual & 10,500 & 1950 & 536 & 1977 & 3,740 & 2,190 & 0.59 & 100 \\
\hline
\end{tabular}

Annual flow duration

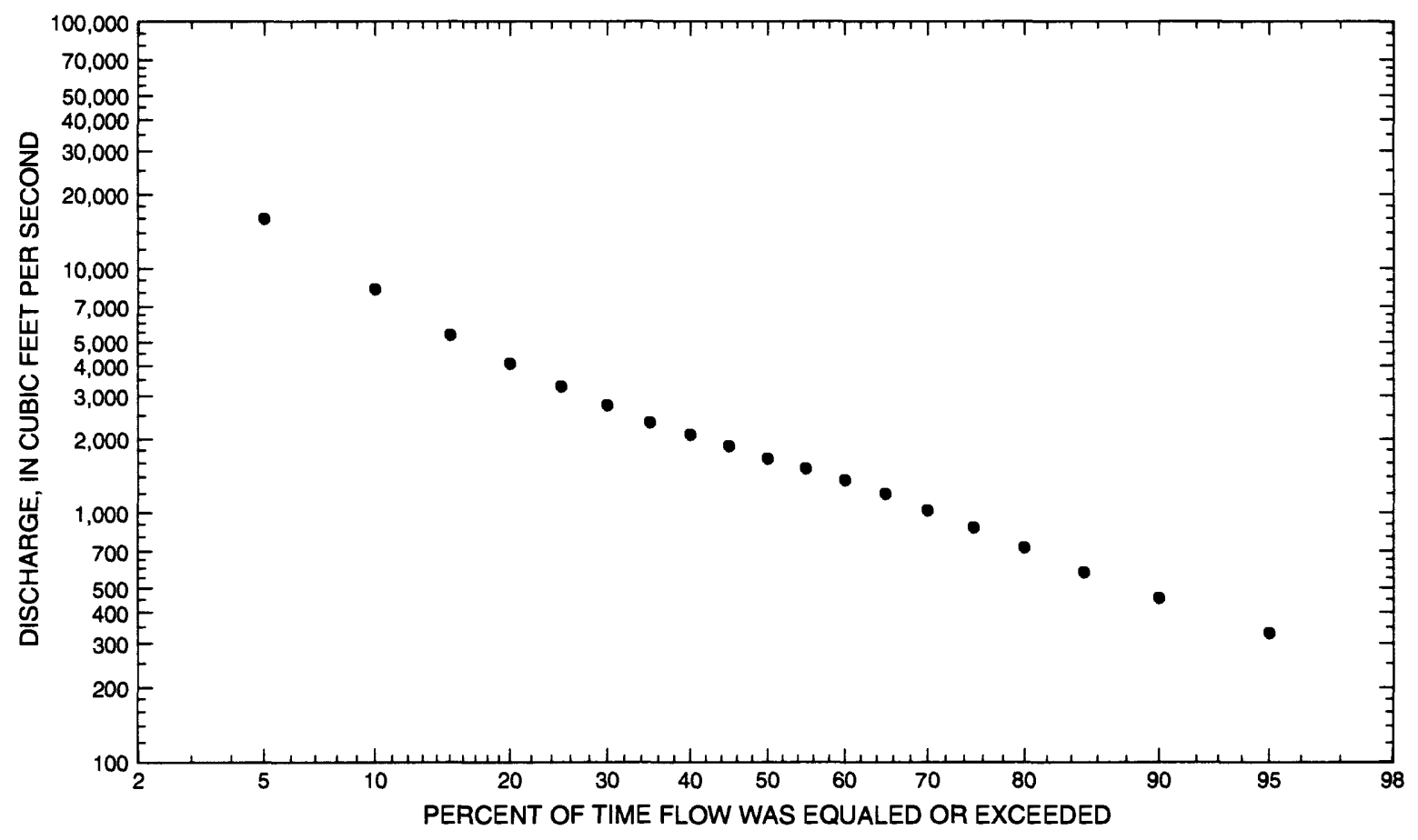




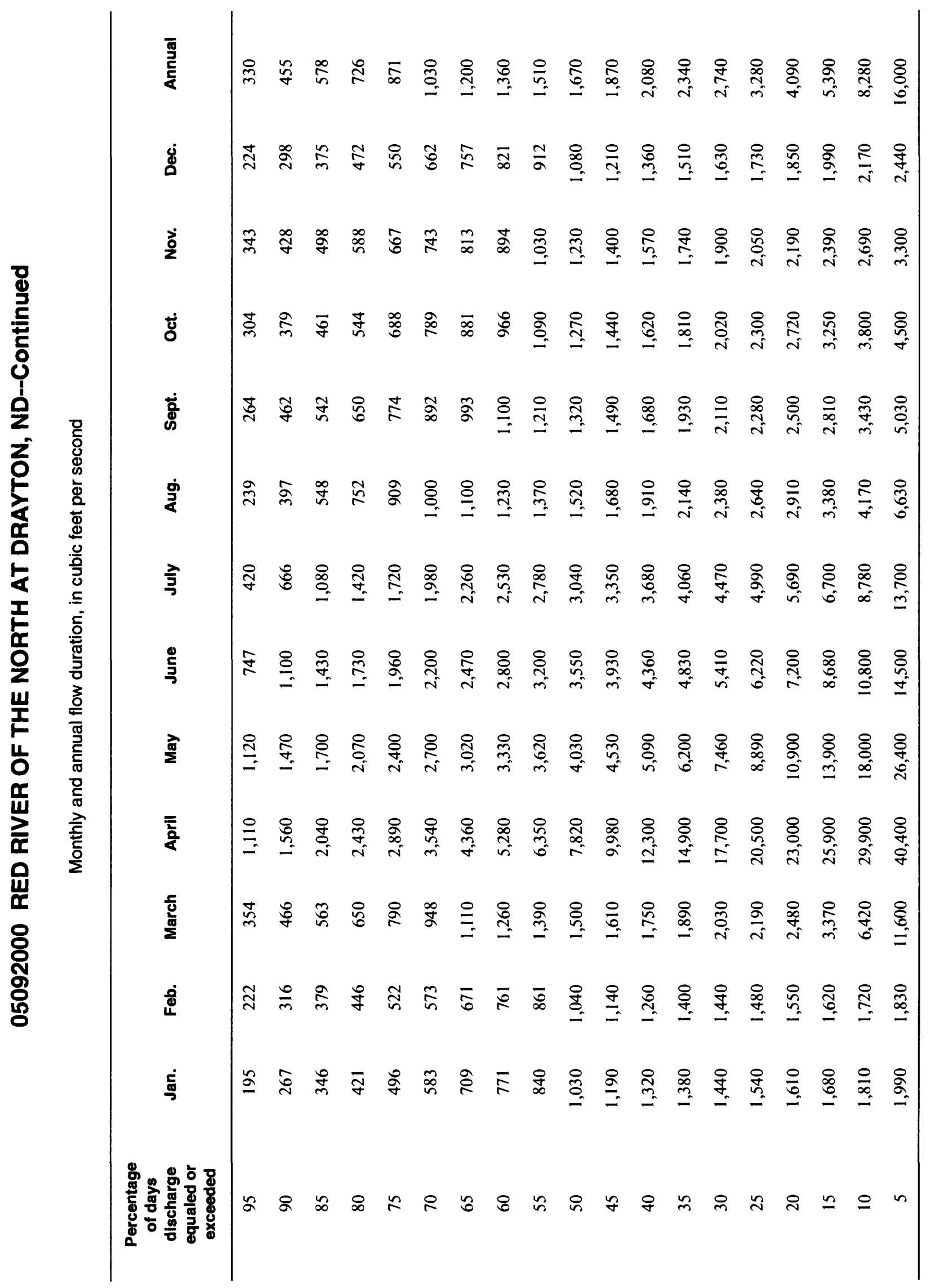




\section{RED RIVER OF THE NORTH AT DRAYTON, ND--Continued}

Probability of occurrence of annual high discharges

[ng, statistic not given]

\begin{tabular}{|c|c|c|c|c|c|c|}
\hline \multirow[b]{2}{*}{$\begin{array}{l}\text { Exceedance } \\
\text { probability }\end{array}$} & \multirow[b]{2}{*}{$\begin{array}{l}\text { Recurrence } \\
\text { interval } \\
\text { (years) }\end{array}$} & \multirow[b]{2}{*}{$\begin{array}{c}\text { Maximum } \\
\text { instantaneous } \\
\left(\mathrm{ft}^{3} / \mathrm{s}\right)\end{array}$} & \multicolumn{4}{|c|}{$\begin{array}{l}\text { Maximum mean discharge } \\
\qquad\left(\mathrm{ft}^{3} / \mathrm{s}\right)\end{array}$} \\
\hline & & & 3-day period & 7-day period & 15-day period & 30-day period \\
\hline 0.99 & 1.01 & 2,830 & 2,450 & 2,230 & 1,790 & 1,360 \\
\hline 0.95 & 1.05 & 5,580 & 5,050 & 4,640 & 3,770 & 2,850 \\
\hline 0.90 & 1.11 & 7,810 & 7,220 & 6,680 & 5,460 & 4,130 \\
\hline 0.80 & 1.25 & 11,500 & 10,900 & 10,100 & 8,370 & 6,340 \\
\hline 0.50 & 2 & 22,400 & 22,000 & 20,700 & 17,600 & 13,600 \\
\hline 0.20 & 5 & 40,100 & 40,500 & 38,700 & 34,000 & 26,900 \\
\hline 0.10 & 10 & 52,600 & 53,800 & 51,700 & 46,400 & 37,300 \\
\hline 0.04 & 25 & 68,700 & 71,000 & 68,700 & 63,000 & 51,700 \\
\hline 0.02 & 50 & 80,600 & 83,800 & 81,400 & 75,800 & 63,200 \\
\hline 0.01 & 100 & 92,300 & 96,400 & 93,900 & 88,700 & 75,000 \\
\hline 0.005 & 200 & 104,000 & 109,000 & 106,000 & 102,000 & 87,200 \\
\hline 0.002 & 500 & 119,000 & ng & ng & $\mathrm{ng}$ & ng \\
\hline
\end{tabular}

Probability of occurrence of annual low discharges

\begin{tabular}{|c|c|c|c|c|c|c|c|c|c|c|}
\hline \multirow[b]{3}{*}{$\begin{array}{l}\text { Non- } \\
\text { exceed- } \\
\text { ance } \\
\text { prob- } \\
\text { ability }\end{array}$} & \multirow[b]{3}{*}{$\begin{array}{c}\text { Recur- } \\
\text { rence } \\
\text { inter- } \\
\text { val } \\
\text { (years) }\end{array}$} & \multicolumn{9}{|c|}{ Minimum mean discharge $\left(\mathrm{ft}^{3} / \mathrm{s}\right)$} \\
\hline & & \multicolumn{9}{|c|}{ Number of consecutive days } \\
\hline & & 1 & 3 & 7 & 14 & 30 & 60 & 90 & 120 & 183 \\
\hline 0.05 & 20 & 136 & 138 & 147 & 155 & 178 & 212 & 235 & 260 & 312 \\
\hline 0.10 & 10 & 194 & 198 & 209 & 222 & 252 & 294 & 322 & 354 & 419 \\
\hline 0.20 & 5 & 290 & 296 & 314 & 333 & 372 & 425 & 461 & 501 & 589 \\
\hline 0.50 & 2 & 577 & 593 & 626 & 666 & 724 & 800 & 855 & 913 & 1,070 \\
\hline
\end{tabular}




\section{RED RIVER OF THE NORTH AT DRAYTON, ND--Continued}

Probability of occurrence of seasonal low discharges

\begin{tabular}{|c|c|c|c|c|c|c|c|c|c|}
\hline \multirow[b]{3}{*}{$\begin{array}{l}\text { Non- } \\
\text { exceedance } \\
\text { probabiiity }\end{array}$} & \multirow[b]{3}{*}{$\begin{array}{l}\text { Recurrence } \\
\text { interval } \\
\text { (years) }\end{array}$} & \multicolumn{8}{|c|}{ Minimum mean discharge $\left(\mathrm{ft}^{3} / \mathrm{s}\right)$} \\
\hline & & \multicolumn{8}{|c|}{ Number of consecutive days } \\
\hline & & 1 & 7 & 14 & 30 & 1 & 7 & 14 & 30 \\
\hline & & \multicolumn{4}{|c|}{ December-January-February } & \multicolumn{4}{|c|}{ March-April-May } \\
\hline 0.05 & 20 & 169 & 174 & 180 & 196 & 268 & 281 & 305 & 436 \\
\hline 0.10 & 10 & 248 & 257 & 265 & 285 & 363 & 383 & 417 & 576 \\
\hline 0.20 & 5 & 379 & 394 & 408 & 431 & 512 & 541 & 594 & 807 \\
\hline \multirow[t]{2}{*}{0.50} & 2 & 759 & 793 & 820 & 849 & 916 & 971 & 1,080 & 1,530 \\
\hline & & \multicolumn{4}{|c|}{ June-July-August } & \multicolumn{4}{|c|}{ September-October-November } \\
\hline 0.05 & 20 & 161 & 183 & 200 & 233 & 183 & 211 & 230 & 308 \\
\hline 0.10 & 10 & 274 & 303 & 328 & 390 & 252 & 288 & 314 & 401 \\
\hline 0.20 & 5 & 482 & 522 & 561 & 676 & 363 & 412 & 449 & 548 \\
\hline 0.50 & 2 & 1,140 & 1,200 & 1,300 & 1,590 & 685 & 767 & 851 & 978 \\
\hline
\end{tabular}




\section{RED RIVER OF THE NORTH AT DRAYTON, ND--Continued}

Annual peak discharge and corresponding gage height

$$
[-, \text { no data }]
$$

\begin{tabular}{|c|c|c|c|c|c|c|c|}
\hline $\begin{array}{l}\text { Water } \\
\text { year }\end{array}$ & Date & $\begin{array}{c}\text { Gage } \\
\text { helght } \\
\text { (feet) }\end{array}$ & $\begin{array}{c}\text { Peak } \\
\text { discharge } \\
\left(\mathrm{ft}^{3} / \mathrm{s}\right)\end{array}$ & $\begin{array}{l}\text { Water } \\
\text { year }\end{array}$ & Date & $\begin{array}{c}\text { Gage } \\
\text { height } \\
\text { (feet) }\end{array}$ & $\begin{array}{c}\text { Peak } \\
\text { discharge } \\
\left(\mathrm{ft}^{3} / \mathrm{s}\right)\end{array}$ \\
\hline \multicolumn{8}{|c|}{ Annual peak discharge, by year, and corresponding gage height } \\
\hline 1897 & April & 41.00 & -- & 1967 & April 8 & 36.70 & 32,200 \\
\hline 1936 & April 19 & -- & 16,600 & 1968 & July 23 & 20.41 & 12,500 \\
\hline 1937 & May 5 & -- & 4,530 & 1969 & April 19 & 41.08 & 59,000 \\
\hline 1941 & April 15 & 32.00 & 22,800 & 1970 & April 29 & 38.20 & 31,700 \\
\hline 1942 & April 7 & -- & 21,900 & 1971 & April 11 & 29.50 & 23,400 \\
\hline 1943 & April 17 & 33.66 & 28,700 & 1972 & April 20 & 34.75 & 31,100 \\
\hline 1944 & April 18 & 21.05 & 12,300 & 1973 & March 25 & 24.49 & 13,400 \\
\hline 1945 & April 2 & 31.70 & 24,600 & 1974 & April 25 & -- & 43,900 \\
\hline 1946 & March 30 & -- & 23,000 & 1975 & May 4 & 39.80 & 44,000 \\
\hline 1947 & April 28 & 33.12 & 29,300 & 1976 & April 7 & 35.00 & 27,600 \\
\hline 1948 & April 21 & 39.81 & 57,000 & 1977 & April 9 & 12.12 & 3,400 \\
\hline 1949 & April 12 & -- & 27,900 & 1978 & April 16 & 41.19 & 56,200 \\
\hline 1950 & May 12 & 41.58 & 86,500 & 1979 & April 28 & 43.66 & 92,900 \\
\hline 1951 & April 15 & 30.10 & 24,600 & 1980 & April 10 & 29.00 & 22,400 \\
\hline 1952 & April 25 & -- & 23,900 & 1981 & July 3 & 13.96 & 7,520 \\
\hline 1953 & June 26 & 20.00 & 14,700 & 1982 & April 17 & 36.78 & 35,500 \\
\hline 1954 & April 15 & 16.38 & 11,100 & 1983 & April 9 & 30.88 & 21,300 \\
\hline 1955 & April 11 & 27.28 & 18,000 & 1984 & April 6 & -- & 32,400 \\
\hline 1956 & April 27 & 35.16 & 28,000 & 1985 & May 21 & 28.12 & 17,700 \\
\hline 1957 & July 4 & 22.33 & 14,100 & 1986 & April 7 & 36.59 & 29,700 \\
\hline 1958 & July 12 & 14.53 & 7,850 & 1987 & April 7 & 36.61 & 27,600 \\
\hline 1959 & April 8 & 23.78 & 11,200 & 1988 & April 7 & 22.12 & 13,900 \\
\hline 1960 & April 14 & 33.71 & 24,700 & 1989 & April 19 & 39.35 & 41,800 \\
\hline 1961 & March 31 & 12.98 & 3,600 & 1990 & April 7 & 15.54 & 5,080 \\
\hline 1962 & April 24 & 36.26 & 32,300 & 1991 & July 11 & 13.26 & 4,940 \\
\hline 1963 & April 12 & 20.42 & 12,900 & 1992 & March 16 & 23.28 & 8,800 \\
\hline 1964 & April 20 & 23.60 & 15,600 & 1993 & August 14 & 36.48 & 27,600 \\
\hline 1965 & April 22 & 40.43 & 47,200 & 1994 & April 6 & 33.57 & 27,900 \\
\hline 1966 & April 8 & 42.15 & 67,500 & & & & \\
\hline \multicolumn{8}{|c|}{ Annual peak discharge, from highest to lowest, and corresponding gage height } \\
\hline 1979 & April 28 & 43.66 & 92,900 & 1970 & April 29 & 38.20 & 31,700 \\
\hline 1950 & May 12 & 41.58 & 86,500 & 1972 & April 20 & 34.75 & 31,100 \\
\hline 1966 & April 8 & 42.15 & 67,500 & 1986 & April 7 & 36.59 & 29,700 \\
\hline 1969 & April 19 & 41.08 & 59,000 & 1947 & April 28 & 33.12 & 29,300 \\
\hline 1948 & April 21 & 39.81 & 57,000 & 1943 & April 17 & 33.66 & 28,700 \\
\hline 1978 & April 16 & 41.19 & 56,200 & 1956 & April 27 & 35.16 & 28,000 \\
\hline 1965 & April 22 & 40.43 & 47,200 & 1949 & April 12 & -- & 27,900 \\
\hline 1975 & May 4 & 39.80 & 44,000 & 1994 & April 6 & 33.57 & 27,900 \\
\hline 1974 & April 25 & -- & 43,900 & 1976 & April 7 & 35.00 & 27,600 \\
\hline 1989 & April 19 & 39.35 & 41,800 & 1987 & April 7 & 36.61 & 27,600 \\
\hline 1982 & April 17 & 36.78 & 35,500 & 1993 & August 14 & 36.48 & 27,600 \\
\hline 1984 & April 6 & -- & 32,400 & 1960 & April 14 & 33.71 & 24,700 \\
\hline 1962 & April 24 & 36.26 & 32,300 & 1945 & April 2 & 31.70 & 24,600 \\
\hline 1967 & April 8 & 36.70 & 32,200 & 1951 & April 15 & 30.10 & 24,600 \\
\hline
\end{tabular}


05092000 RED RIVER OF THE NORTH AT DRAYTON, ND--Continued

Annual peak discharge and corresponding gage height-Continued

$$
[--, \text { no data }]
$$

\begin{tabular}{|c|c|c|c|c|c|c|c|}
\hline $\begin{array}{l}\text { Water } \\
\text { year }\end{array}$ & Date & $\begin{array}{c}\text { Gage } \\
\text { height } \\
\text { (feet) }\end{array}$ & $\begin{array}{c}\text { Peak } \\
\text { discharge } \\
\left(\mathrm{ft}^{3} / \mathrm{s}\right)\end{array}$ & $\begin{array}{l}\text { Water } \\
\text { year }\end{array}$ & Date & $\begin{array}{c}\text { Gage } \\
\text { height } \\
\text { (feet) }\end{array}$ & $\begin{array}{c}\text { Peak } \\
\text { discharge } \\
\left(\mathrm{ft}^{3} / \mathrm{s}\right)\end{array}$ \\
\hline \multicolumn{8}{|c|}{ Annual peak discharge, from highest to lowest, and corresponding gage height--Continued } \\
\hline 1952 & April 25 & -- & 23,900 & 1963 & April 12 & 20.42 & 12,900 \\
\hline 1971 & April 11 & 29.50 & 23,400 & 1968 & July 23 & 20.41 & 12,500 \\
\hline 1946 & March 30 & -- & 23,000 & 1944 & April 18 & 21.05 & 12,300 \\
\hline 1941 & April 15 & 32.00 & 22,800 & 1959 & April 8 & 23.78 & 11,200 \\
\hline 1980 & April 10 & 29.00 & 22,400 & 1954 & April 15 & 16.38 & 11,100 \\
\hline 1942 & April 7 & -- & 21,900 & 1992 & March 16 & 23.28 & 8,800 \\
\hline 1983 & April 9 & 30.88 & 21,300 & 1958 & July 12 & 14.53 & 7,850 \\
\hline 1955 & April 11 & 27.28 & 18,000 & 1981 & July 3 & 13.96 & 7,520 \\
\hline 1985 & May 21 & 28.12 & 17,700 & 1990 & April 7 & 15.54 & 5,080 \\
\hline 1936 & April 19 & -- & 16,600 & 1991 & July 11 & 13.26 & 4,940 \\
\hline 1964 & April 20 & 23.60 & 15,600 & 1937 & May 5 & -- & 4,530 \\
\hline 1953 & June 26 & 20.00 & 14,700 & 1961 & March 31 & 12.98 & 3,600 \\
\hline 1957 & July 4 & 22.33 & 14,100 & 1977 & April 9 & 12.12 & 3,400 \\
\hline 1988 & April 7 & 22.12 & 13,900 & 1897 & April & 41.00 & -- \\
\hline 1973 & March 25 & 24.49 & 13,400 & & & & \\
\hline
\end{tabular}




\begin{tabular}{|c|c|c|c|c|c|c|c|}
\hline $\begin{array}{l}\bar{\Phi} \\
\stackrel{5}{5} \\
\frac{5}{4}\end{array}$ & $\begin{array}{lllll}1 & 1 & 1 & 1 & 1\end{array}$ & $\begin{array}{lllll}1 & 1 & 1 & 1 & 1\end{array}$ & 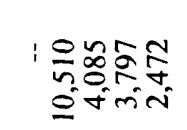 & 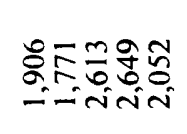 & 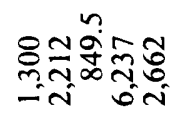 & 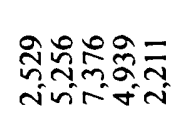 & 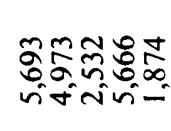 \\
\hline চே. & $\underset{\sim}{\stackrel{\nabla}{N}:}: 1:$ & 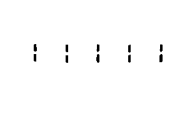 & 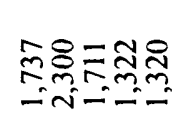 & 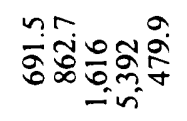 & 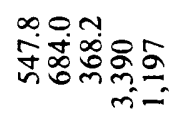 & 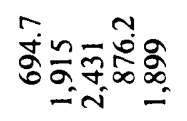 & 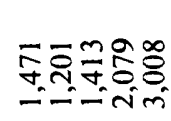 \\
\hline$\frac{9}{8}$ & $\overline{\dot{n}} 1: 11$ & 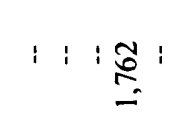 & 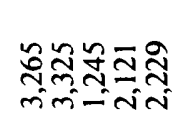 & 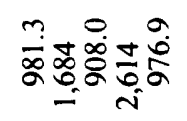 & 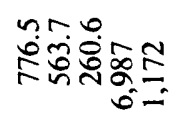 & 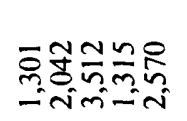 & 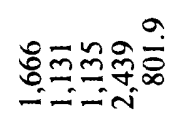 \\
\hline$\frac{2}{3}$ & 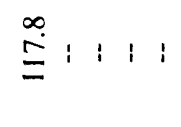 & 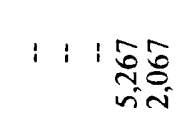 & 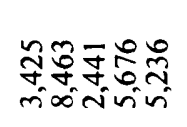 & 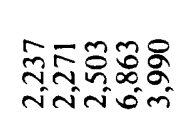 & 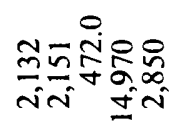 & 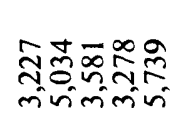 & 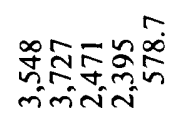 \\
\hline 苞 & 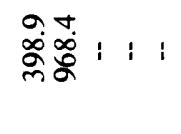 & 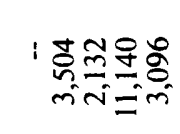 & 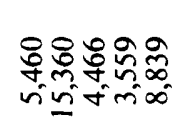 & 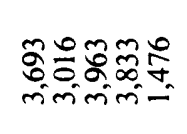 & 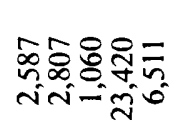 & 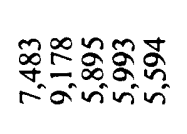 & 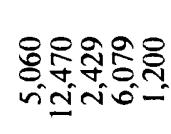 \\
\hline$\frac{10}{2}$ & $\begin{array}{l}\mathscr{N} \tilde{\kappa}: ! \\
\infty \\
-=-\end{array}$ & 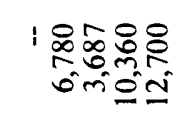 & 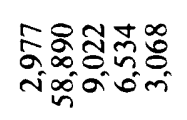 & 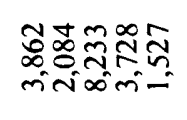 & 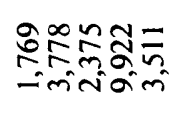 & 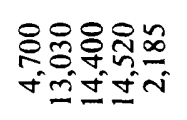 & 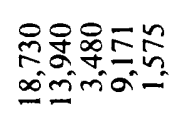 \\
\hline 衣 & 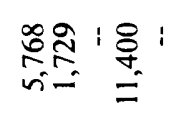 & 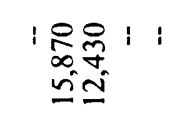 & 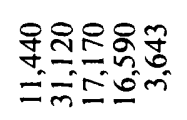 & 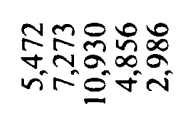 & 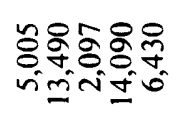 & 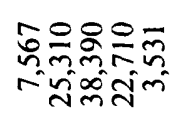 & 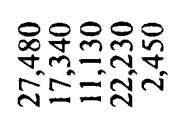 \\
\hline 䍃 & $\begin{array}{lllll}1 & 1 & 1 & 1 & 1\end{array}$ & $\begin{array}{lllll}1 & 1 & 1 & 1 & 1\end{array}$ & 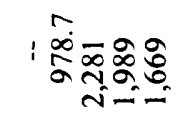 & 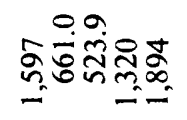 & 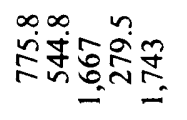 & 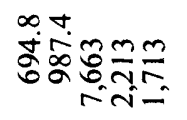 & 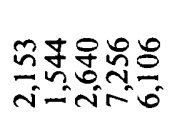 \\
\hline 80ं & $\begin{array}{lllll}1 & 1 & 1 & 1 & 1\end{array}$ & $\begin{array}{lllll}1 & 1 & 1 & 1 & 1\end{array}$ & 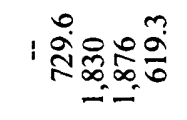 & 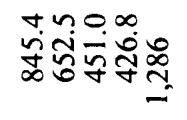 & 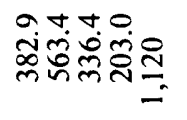 & 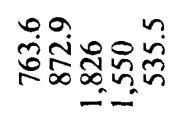 & 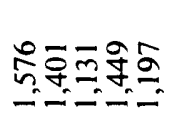 \\
\hline 寽 & 11111 & $\begin{array}{lllll}1 & 1 & 1 & 1 & 1\end{array}$ & : & 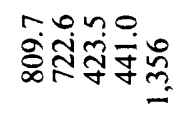 & 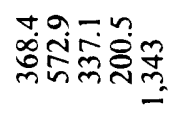 & 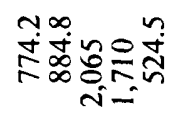 & 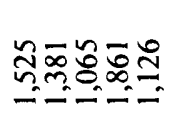 \\
\hline 巡 & $\begin{array}{lllll}1 & 1 & 1 & 1 & 1\end{array}$ & $\begin{array}{lllll}1 & 1 & 1 & 1 & 1\end{array}$ & 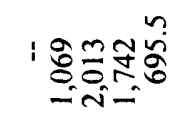 & 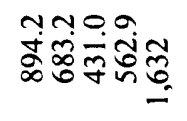 & 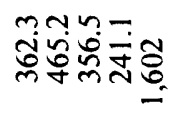 & 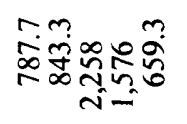 & B유유요 \\
\hline के & $\begin{array}{lllll} & 1 & 1 & 1 & 1\end{array}$ & $\begin{array}{lllll}1 & 1 & 1 & 1 & 1\end{array}$ & 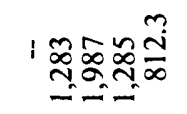 & 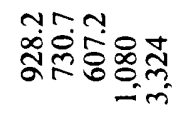 & 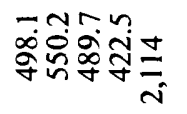 & 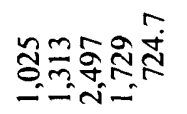 & 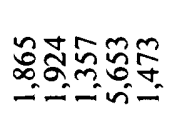 \\
\hline ठัँ & $\stackrel{\infty}{\cong}: \mid 1$ & $\begin{array}{lllll}1 & 1 & 1 & 1 & 1\end{array}$ & 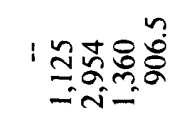 & 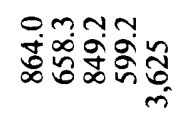 & 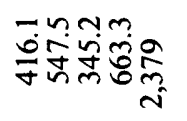 & 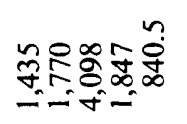 & 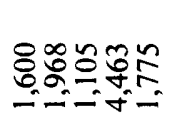 \\
\hline 。 & 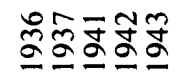 & 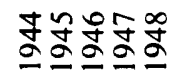 & 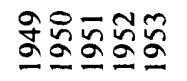 & 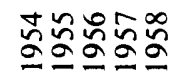 & 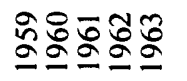 & 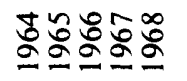 & ㅇํㅇ으으옹 \\
\hline
\end{tabular}




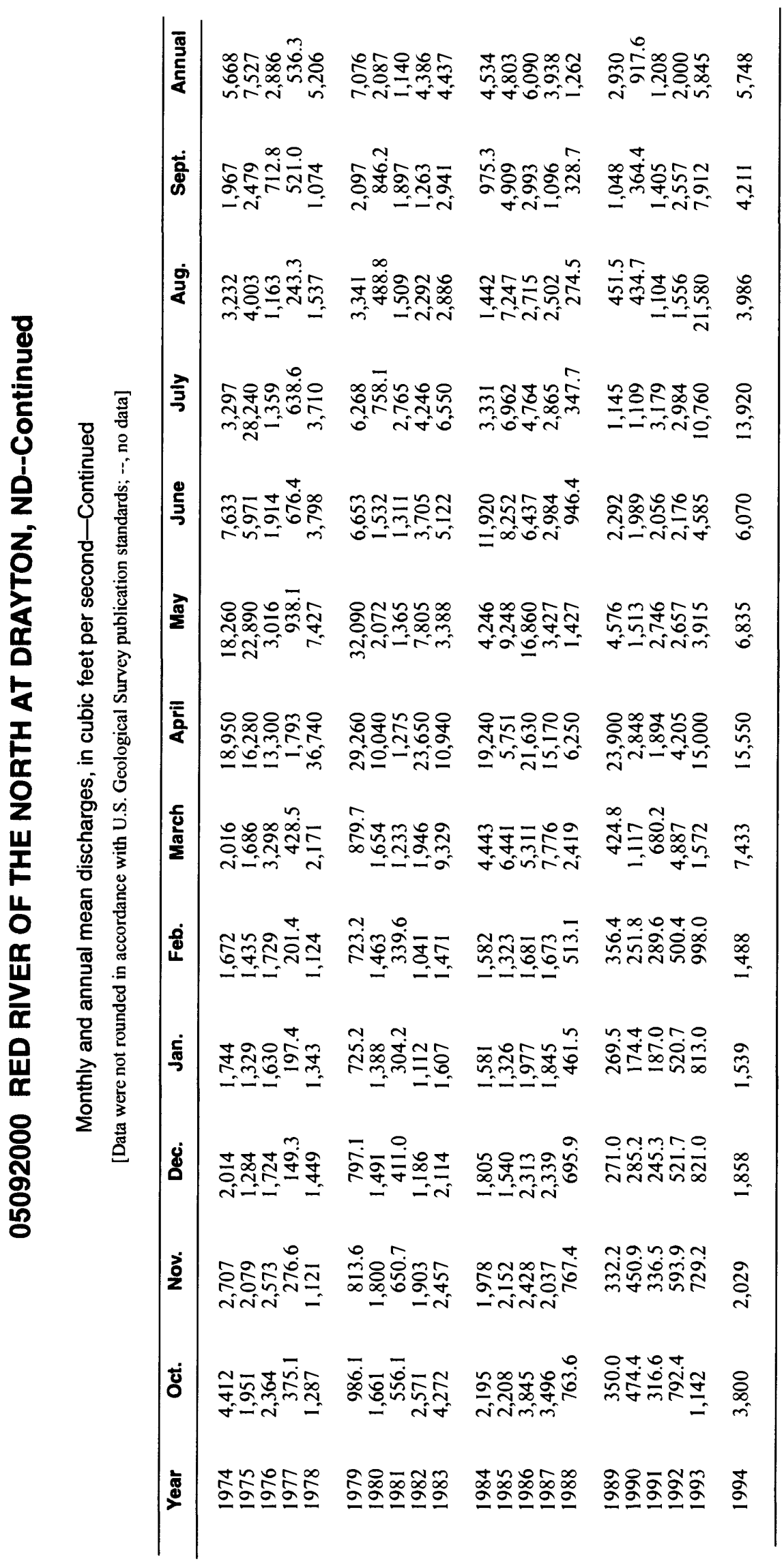


LOCATION.--Lat $48^{\circ} 41^{\prime} 49^{\prime \prime}$, long $97^{\circ} 23^{\prime} 03^{\prime}$, in NW $1 / 4$ sec.8, T.160 N., R.52 W., Pembina County, Hydrologic Unit 09020311 , on left bank $50 \mathrm{ft}$ downstream from bridge on county highway, and $3 \mathrm{mi}$ southeast of Glasston.

DRAINAGE AREA.--80 $\mathrm{mi}^{2}$.

PERIOD OF RECORD.--October 1971 to September 1986. Seasonal records only since 1983.

GAGE.--Water-stage recorder. Datum of gage is $808 \mathrm{ft}$ above sea level, from topographic map.

EXTREMES FOR PERIOD OF RECORD.--Maximum discharge, $940 \mathrm{ft}^{3} / \mathrm{s}$, Apr. 20, 1979, gage height, $9.3 \mathrm{ft}$; maximum gage height, $14.64 \mathrm{ft}$, Apr. 19, 1979, backwater from ice; no flow most of time.

Annual mean discharge

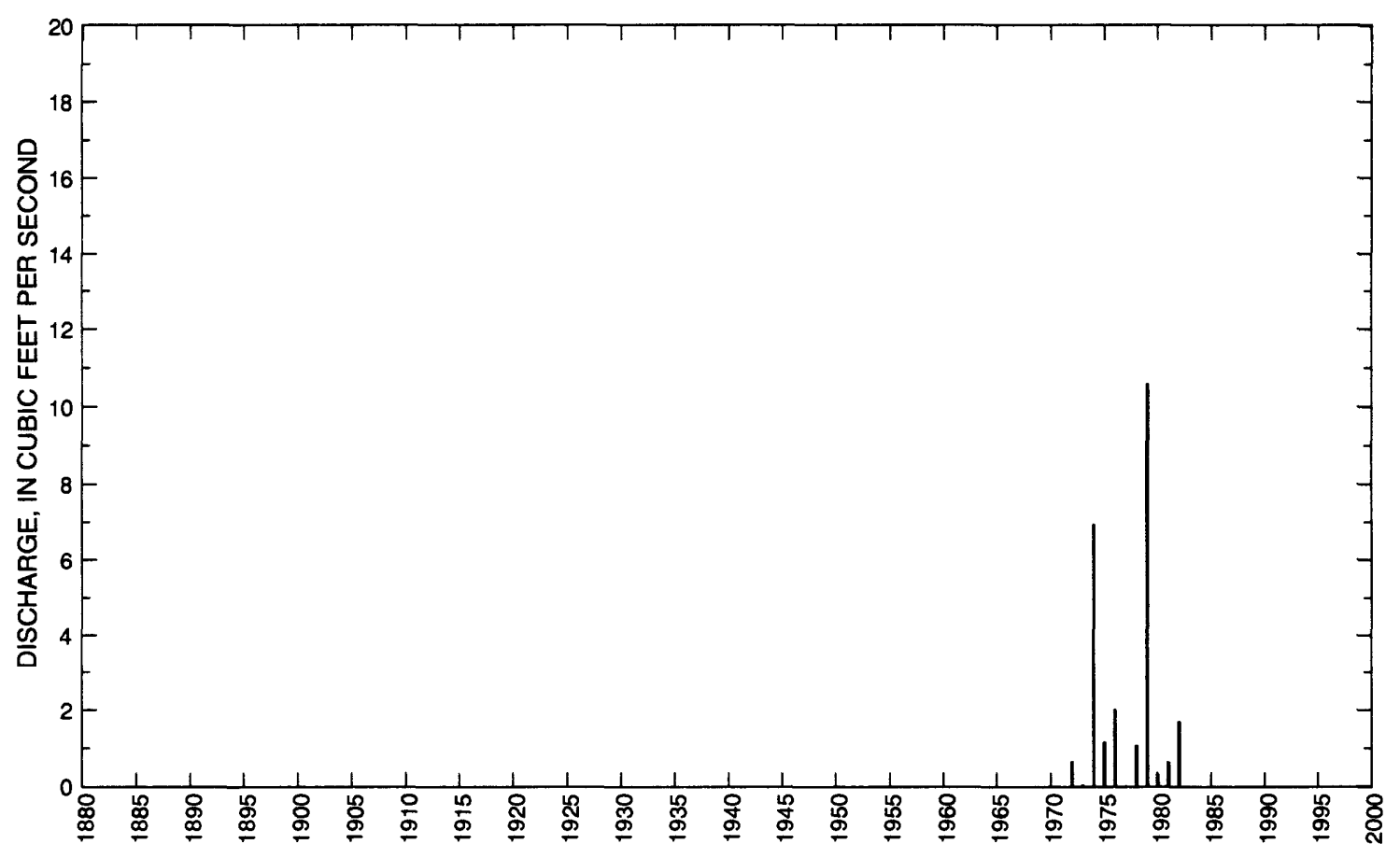


05092200 PEMBINA COUNTY DRAIN 20 NEAR GLASSTON, ND--Continued

Statistics of monthly and annual mean discharges

[m, more than 1 year of occurrence; ng, statistic not given]

\begin{tabular}{|c|c|c|c|c|c|c|c|c|}
\hline \multirow[b]{2}{*}{ Month } & \multicolumn{2}{|c|}{ Maximum } & \multicolumn{2}{|c|}{ Minimum } & \multicolumn{4}{|c|}{ Mean } \\
\hline & $\begin{array}{c}\text { Discharge } \\
\left(\mathrm{ft}^{3} / \mathrm{s}\right)\end{array}$ & $\begin{array}{c}\text { Water year } \\
\text { of } \\
\text { occurrence }\end{array}$ & $\begin{array}{c}\text { Discharge } \\
\left(\mathrm{ft}^{3} / \mathrm{s}\right)\end{array}$ & $\begin{array}{c}\text { Water year } \\
\text { of } \\
\text { occurrence }\end{array}$ & $\begin{array}{c}\text { Discharge } \\
\left(\mathrm{ft}^{3} / \mathrm{s}\right)\end{array}$ & $\begin{array}{c}\text { Standard } \\
\text { deviation } \\
\left(\mathrm{ft}^{3} / \mathrm{s}\right)\end{array}$ & $\begin{array}{l}\text { Coeffi- } \\
\text { cient of } \\
\text { variation }\end{array}$ & $\begin{array}{l}\text { Percentage } \\
\text { of annuai } \\
\text { discharge }\end{array}$ \\
\hline October & 0.224 & 1981 & 0 & $\mathrm{~m}$ & 0.030 & 0.07 & 2.40 & 0.11 \\
\hline November & 0.125 & 1981 & 0 & $\mathrm{~m}$ & 0.010 & 0.04 & 3.32 & 0.04 \\
\hline December & 0 & $\mathrm{~m}$ & 0 & $\mathrm{~m}$ & 0 & 0 & ng & 0 \\
\hline January & 0 & $\mathrm{~m}$ & 0 & $\mathrm{~m}$ & 0 & 0 & ng & 0 \\
\hline February & 0 & $\mathrm{~m}$ & 0 & $\mathrm{~m}$ & 0 & 0 & ng & 0 \\
\hline March & 7.92 & 1985 & 0 & $\mathrm{~m}$ & 2.03 & 2.54 & 1.26 & 7.83 \\
\hline April & 124 & 1979 & 0 & $\mathrm{~m}$ & 19.6 & 32.5 & 1.66 & 75.7 \\
\hline May & 38.1 & 1974 & 0 & $\mathrm{~m}$ & 3.21 & 9.74 & 3.03 & 12.4 \\
\hline June & 2.36 & 1983 & 0 & $\mathrm{~m}$ & 0.370 & 0.64 & 1.73 & 1.43 \\
\hline July & 6.17 & 1986 & 0 & $\mathrm{~m}$ & 0.450 & 1.58 & 3.53 & 1.74 \\
\hline August & 1.27 & 1985 & 0 & $\mathrm{~m}$ & 0.100 & 0.32 & 3.15 & 0.40 \\
\hline September & 0.670 & 1985 & 0 & $\mathrm{~m}$ & 0.080 & 0.18 & 2.29 & 0.31 \\
\hline Annual & 10.6 & 1979 & 0.005 & 1977 & 2.29 & 3.37 & 1.47 & 100 \\
\hline
\end{tabular}

Annual flow duration

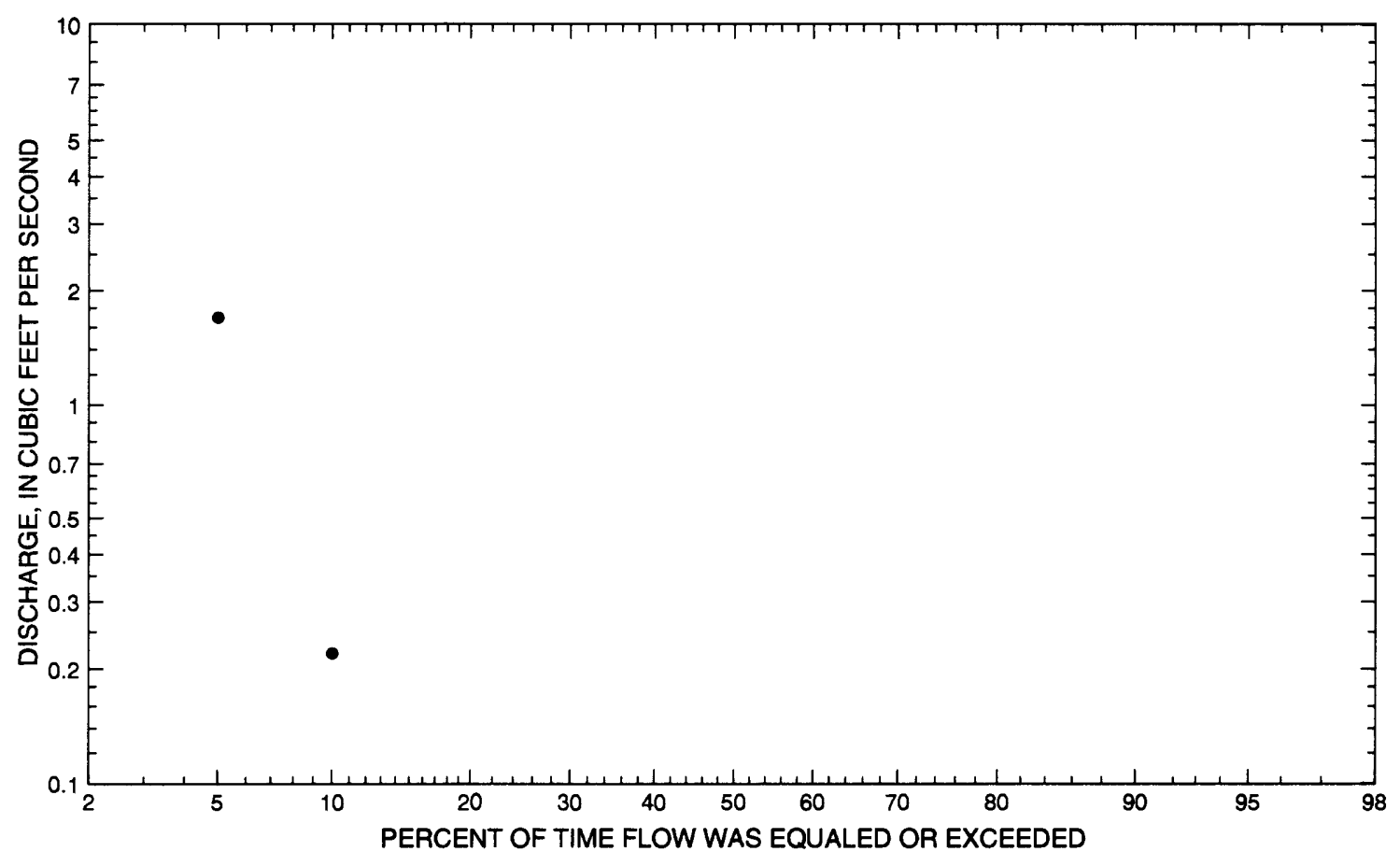




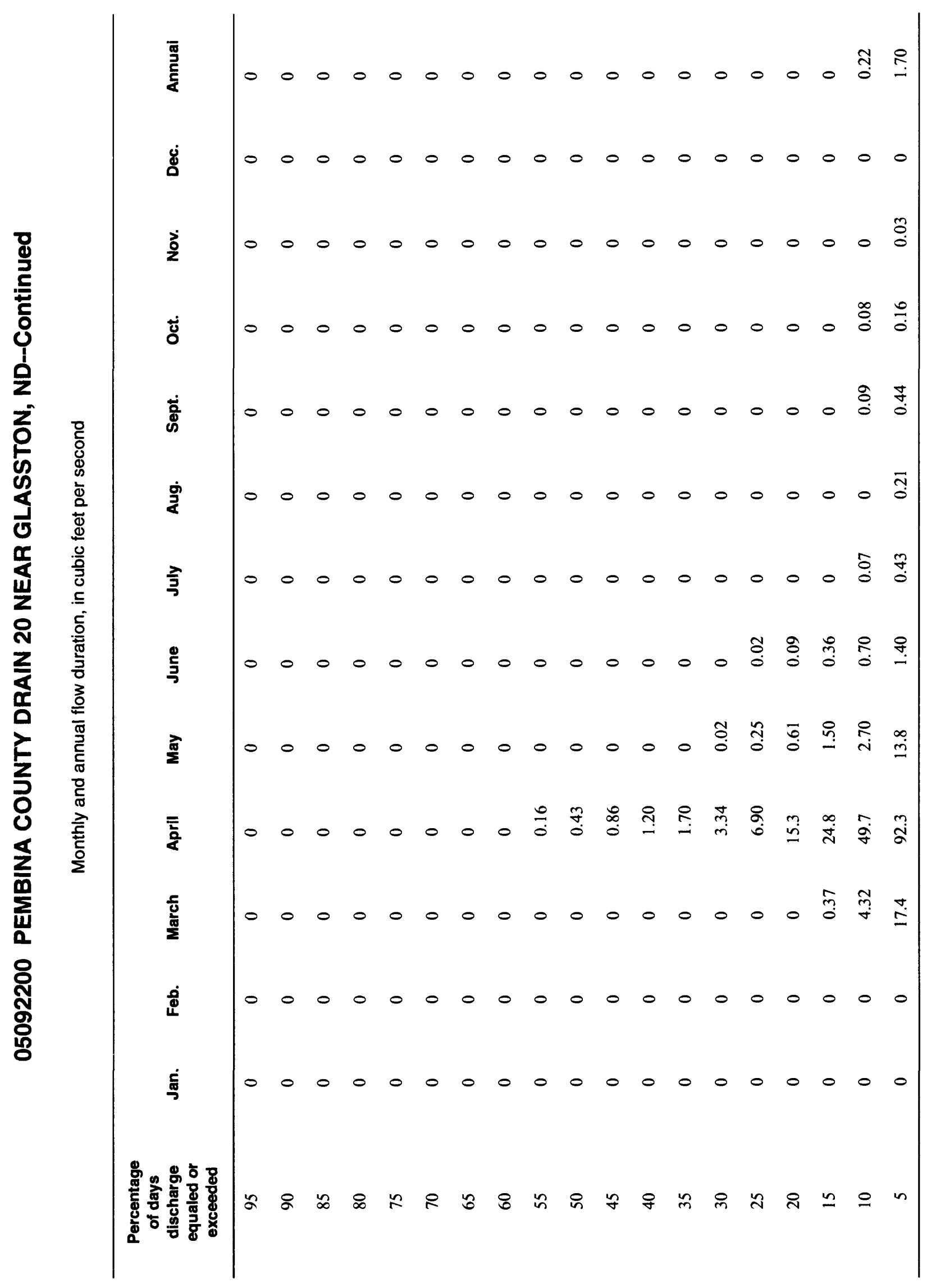


05092200 PEMBINA COUNTY DRAIN 20 NEAR GLASSTON, ND--Continued

Probability of occurrence of annual high discharges

[ng, statistic not given]

\begin{tabular}{lcccccc}
\hline & & & \multicolumn{5}{c}{$\begin{array}{c}\text { Maximum mean discharge } \\
\left(\mathbf{f t}^{\mathbf{3}} / \mathbf{s}\right)\end{array}$} \\
\cline { 5 - 7 } $\begin{array}{c}\text { Exceedance } \\
\text { probabiity }\end{array}$ & $\begin{array}{c}\text { Recurrence } \\
\text { intervai } \\
\text { (years) }\end{array}$ & $\begin{array}{c}\text { Maximum } \\
\text { instantaneous } \\
\left(\mathbf{f t}^{3} / \mathbf{s}\right)\end{array}$ & 3-day period & 7-day period & 15-day period & 30-day period \\
\hline 0.99 & 1.01 & $\mathrm{ng}$ & 0.029 & 0.014 & 0.005 & 0.005 \\
0.95 & 1.05 & $\mathrm{ng}$ & 0.449 & 0.250 & 0.112 & 0.085 \\
0.90 & 1.11 & 15.7 & 1.60 & 0.946 & 0.467 & 0.310 \\
0.80 & 1.25 & 28.8 & 6.21 & 3.90 & 2.11 & 1.24 \\
0.50 & 2 & 85.6 & 50.0 & 33.7 & 19.8 & 10.4 \\
0.20 & 5 & 232 & 219 & 151 & 88.2 & 46.5 \\
0.10 & 10 & 376 & 384 & 264 & 149 & 81.7 \\
0.04 & 25 & 614 & 609 & 415 & 224 & 129 \\
0.02 & 50 & 830 & 768 & 517 & 271 & 163 \\
0.01 & 100 & 1,080 & 909 & 607 & 308 & 192 \\
0.005 & 200 & 1,360 & 1,030 & 682 & 338 & 218 \\
0.002 & 500 & 1,780 & $\mathrm{ng}$ & $\mathrm{ng}$ & $\mathrm{ng}$ & $\mathrm{ng}$ \\
\hline
\end{tabular}

Probability of occurrence of annual low discharges

[ng, statistic not given]

\begin{tabular}{|c|c|c|c|c|c|c|c|c|c|c|}
\hline \multirow[b]{3}{*}{$\begin{array}{l}\text { Non- } \\
\text { exceed- } \\
\text { ance } \\
\text { prob- } \\
\text { abiiity }\end{array}$} & \multirow[b]{3}{*}{$\begin{array}{c}\text { Recur- } \\
\text { rence } \\
\text { inter- } \\
\text { vai } \\
\text { (years) }\end{array}$} & \multicolumn{9}{|c|}{ Minimum mean discharge $\left(\mathrm{ft}^{3} / \mathrm{s}\right)$} \\
\hline & & \multicolumn{9}{|c|}{ Number of consecutive days } \\
\hline & & 1 & 3 & 7 & 14 & 30 & 60 & 90 & 120 & 183 \\
\hline 0.05 & 20 & ng & ng & $\mathrm{ng}$ & ng & $\mathrm{ng}$ & $\mathrm{ng}$ & $\mathrm{ng}$ & $\mathrm{ng}$ & ng \\
\hline 0.10 & 10 & $\mathrm{ng}$ & $\mathrm{ng}$ & $\mathrm{ng}$ & ng & $\mathrm{ng}$ & $\mathrm{ng}$ & $\mathrm{ng}$ & ng & ng \\
\hline 0.20 & 5 & $\mathrm{ng}$ & $\mathrm{ng}$ & $\mathrm{ng}$ & $\mathrm{ng}$ & $\mathrm{ng}$ & $\mathrm{ng}$ & $\mathrm{ng}$ & $\mathrm{ng}$ & $\mathrm{ng}$ \\
\hline 0.50 & 2 & $\mathrm{ng}$ & $\mathrm{ng}$ & ng & ng & $\mathrm{ng}$ & $\mathrm{ng}$ & $\mathrm{ng}$ & ng & ng \\
\hline
\end{tabular}




\section{PEMBINA COUNTY DRAIN 20 NEAR GLASSTON, ND--Continued}

Probability of occurrence of seasonal low discharges

[ng, statistic not given]

\begin{tabular}{|c|c|c|c|c|c|c|c|c|c|}
\hline \multirow[b]{3}{*}{$\begin{array}{c}\text { Non- } \\
\text { exceedance } \\
\text { probability }\end{array}$} & \multirow[b]{3}{*}{$\begin{array}{c}\text { Recurrence } \\
\text { intervai } \\
\text { (years) }\end{array}$} & \multicolumn{8}{|c|}{ Minimum mean discharge $\left(\mathrm{ft}^{3} / \mathrm{s}\right)$} \\
\hline & & \multicolumn{8}{|c|}{ Number of consecutive days } \\
\hline & & 1 & 7 & 14 & 30 & 1 & 7 & 14 & 30 \\
\hline & & \multicolumn{4}{|c|}{ December-January-February } & \multicolumn{4}{|c|}{ March-April-May } \\
\hline 0.05 & 20 & ng & $\mathrm{ng}$ & ng & ng & ng & $\mathrm{ng}$ & ng & 0 \\
\hline 0.10 & 10 & ng & $\mathrm{ng}$ & ng & ng & ng & $\mathrm{ng}$ & ng & 0 \\
\hline 0.20 & 5 & ng & ng & ng & ng & ng & $\mathrm{ng}$ & $\mathrm{ng}$ & 0 \\
\hline \multirow[t]{2}{*}{0.50} & 2 & ng & $\mathrm{ng}$ & ng & ng & ng & ng & ng & 0 \\
\hline & & \multicolumn{4}{|c|}{ June-July-August } & \multicolumn{4}{|c|}{ September-October-November } \\
\hline 0.05 & 20 & ng & ng & ng & ng & ng & $\mathrm{ng}$ & ng & ng \\
\hline 0.10 & 10 & ng & ng & ng & ng & ng & ng & ng & ng \\
\hline 0.20 & 5 & $\mathrm{ng}$ & $\mathrm{ng}$ & $\mathrm{ng}$ & $\mathrm{ng}$ & $\mathrm{ng}$ & $\mathrm{ng}$ & ng & ng \\
\hline 0.50 & 2 & ng & $\mathrm{ng}$ & ng & ng & ng & $\mathrm{ng}$ & $\mathrm{ng}$ & ng \\
\hline
\end{tabular}




\section{PEMBINA COUNTY DRAIN 20 NEAR GLASSTON, ND--Continued}

Annual peak discharge and corresponding gage height

$$
[-- \text { no data }]
$$

\begin{tabular}{|c|c|c|c|c|c|c|c|}
\hline $\begin{array}{l}\text { Water } \\
\text { year }\end{array}$ & Date & $\begin{array}{c}\text { Gage } \\
\text { height } \\
\text { (feet) }\end{array}$ & $\begin{array}{c}\text { Peak } \\
\text { discharge } \\
\left(\mathrm{ft}^{3} / \mathrm{s}\right)\end{array}$ & $\begin{array}{l}\text { Water } \\
\text { year }\end{array}$ & Date & $\begin{array}{c}\text { Gage } \\
\text { height } \\
\text { (feet) }\end{array}$ & $\begin{array}{c}\text { Peak } \\
\text { discharge } \\
\left(\mathrm{ft}^{3} / \mathrm{s}\right)\end{array}$ \\
\hline \multicolumn{8}{|c|}{ Annual peak discharge, by year, and corresponding gage height } \\
\hline 1972 & April 13 & 6.02 & 85.0 & 1980 & March 30 & 8.92 & 40.0 \\
\hline 1973 & June 19 & 3.94 & 4.60 & 1981 & April 1 & 6.53 & 80.0 \\
\hline 1974 & April 23 & 7.19 & 212 & 1982 & April 13 & -- & 90.0 \\
\hline 1975 & April 30 & 5.70 & 73.0 & 1983 & April 4 & 7.95 & 166 \\
\hline 1976 & April 3 & 7.10 & 268 & 1984 & March 29 & 8.66 & 88.0 \\
\hline 1977 & July 2 & 4.72 & 2.20 & 1985 & March 14 & 11.25 & 59.0 \\
\hline 1978 & March 27 & 11.60 & 73.0 & 1986 & July 12 & 7.50 & 90.0 \\
\hline 1979 & April 20 & 9.30 & 940 & & & & \\
\hline \multicolumn{8}{|c|}{ Annual peak discharge, from highest to lowest, and corresponding gage height } \\
\hline 1979 & April 20 & 9.30 & 940 & 1981 & April 1 & 6.53 & 80.0 \\
\hline 1976 & April 3 & 7.10 & 268 & 1975 & April 30 & 5.70 & 73.0 \\
\hline 1974 & April 23 & 7.19 & 212 & 1978 & March 27 & 11.60 & 73.0 \\
\hline 1983 & April 4 & 7.95 & 166 & 1985 & March 14 & 11.25 & 59.0 \\
\hline 1982 & April 13 & -- & 90.0 & 1980 & March 30 & 8.92 & 40.0 \\
\hline 1986 & July 12 & 7.50 & 90.0 & 1973 & June 19 & 3.94 & 4.60 \\
\hline 1984 & March 29 & 8.66 & 88.0 & 1977 & July 2 & 4.72 & 2.20 \\
\hline 1972 & April 13 & 6.02 & 85.0 & & & & \\
\hline
\end{tabular}




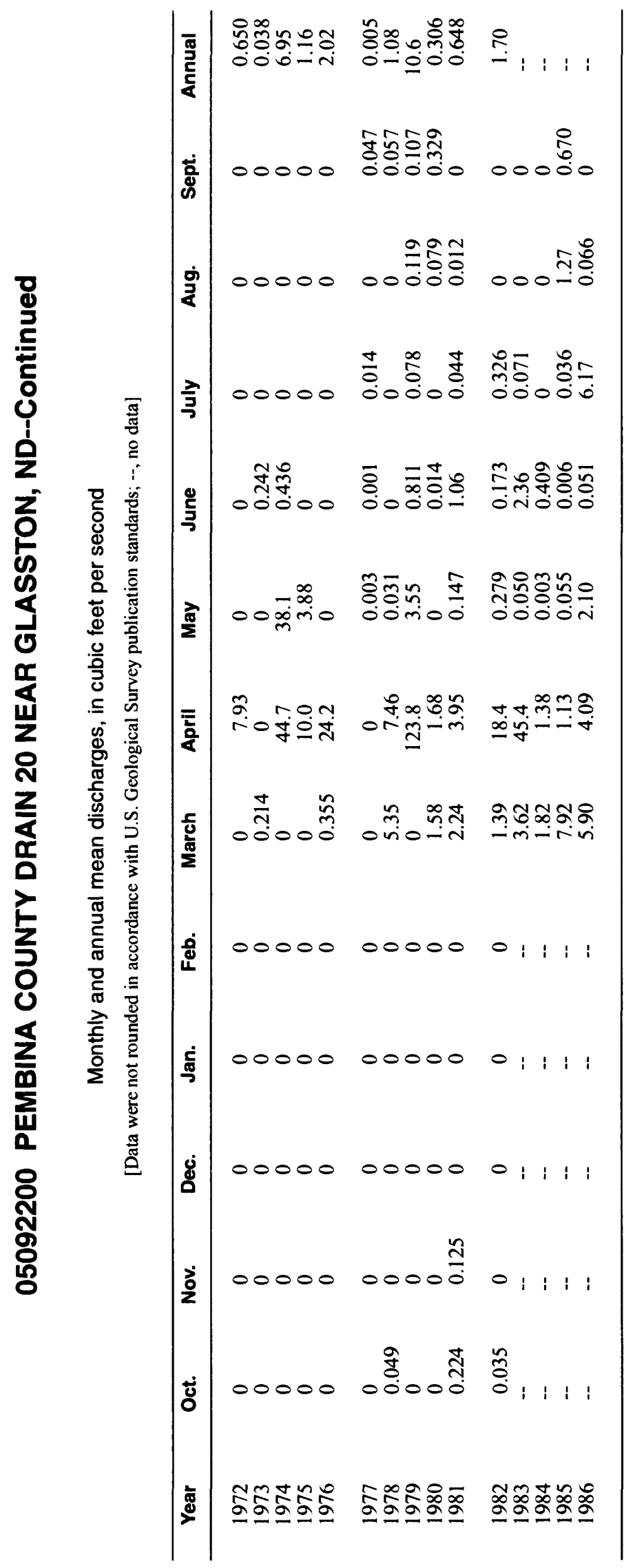




\section{SOUTH BRANCH TWO RIVERS AT PELAN, MN}

LOCATION.--Lat $48^{\circ} 38^{\prime} 45^{\prime \prime}$, long 96 $23^{\prime} 15^{\prime \prime}$, in NW $1 / 4$ sec.31, T.160 N., R.44 W., Kittson County, Hydrologic Unit 09020312, on left bank $40 \mathrm{ft}$ downstream from bridge on State Highway 11 and 0.25 mi west of Pelan.

DRAINAGE AREA.--281 $\mathrm{mi}^{2}$.

PERIOD OF RECORD.--August 1928 to September 1938, October 1953 to November 1956. Monthly discharge only for some periods, published in WSP 1308. Published as South Fork Two Rivers at Pelan 1928-38.

GAGE.--Water-stage recorder. Datum of gage is 1,028.23 ft above mean sea level. Prior to Mar. 24, 1936, chain gage at site $500 \mathrm{ft}$ upstream at same datum. Mar. 24, 1936, to Sept. 30, 1938, chain gage at site $40 \mathrm{ft}$ upstream at same datum.

EXTREMES FOR PERIOD OF RECORD.--Maximum discharge, 2,040 $\mathrm{ft}^{3} / \mathrm{s}$, July 10, 1956, gage height, $10.90 \mathrm{ft}$; no flow at times each year.

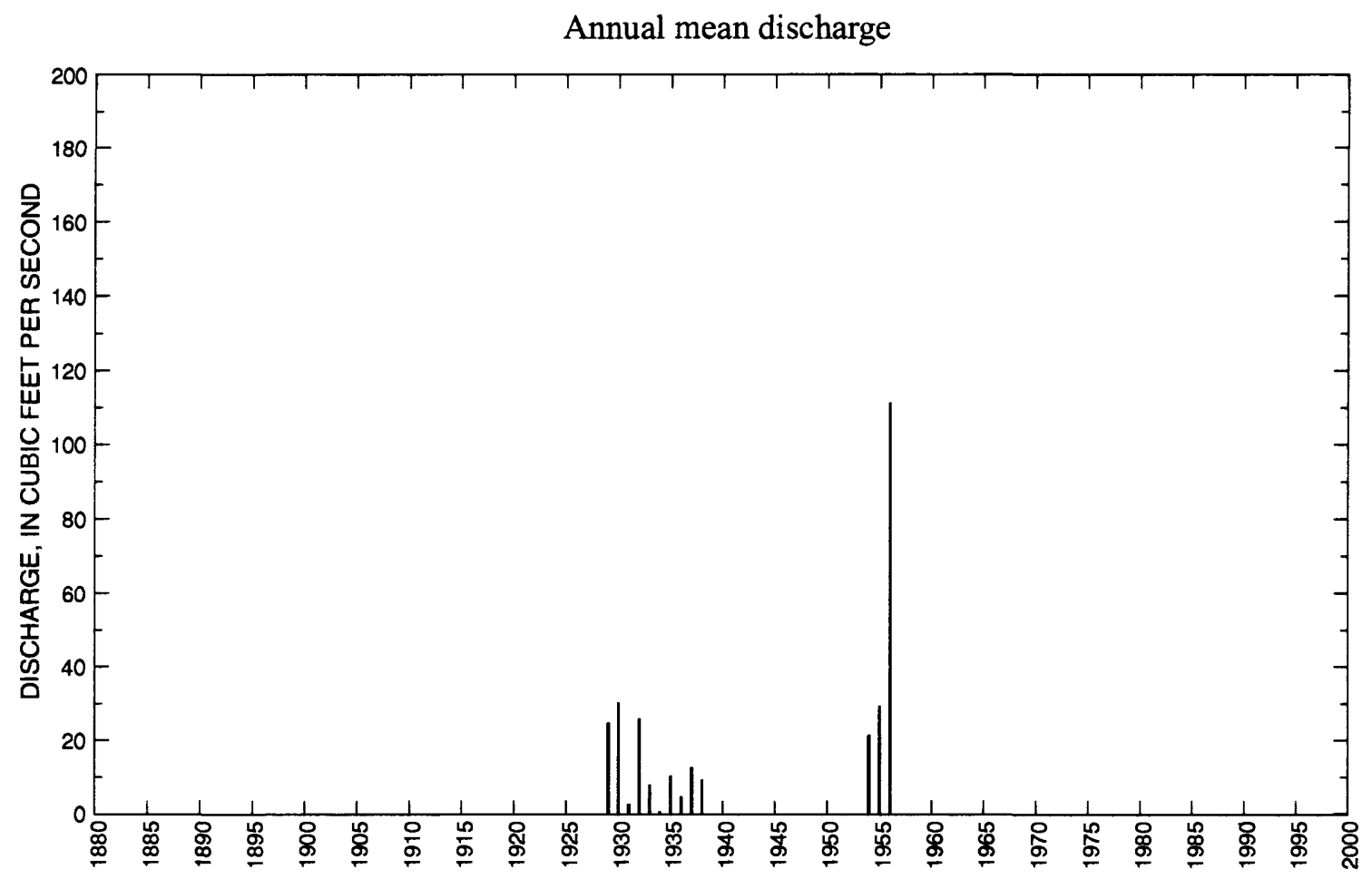




\section{SOUTH BRANCH TWO RIVERS AT PELAN, MN--Continued}

Statistics of monthly and annual mean discharges

[m, more than 1 year of occurrence; ng, statistic not given]

\begin{tabular}{|c|c|c|c|c|c|c|c|c|}
\hline \multirow[b]{2}{*}{ Month } & \multicolumn{2}{|c|}{ Maximum } & \multicolumn{2}{|c|}{ Minimum } & \multicolumn{4}{|c|}{ Mean } \\
\hline & $\begin{array}{c}\text { Discharge } \\
\left(\mathrm{ft}^{3} / \mathbf{s}\right)\end{array}$ & $\begin{array}{c}\text { Water year } \\
\text { of } \\
\text { occurrence }\end{array}$ & $\begin{array}{c}\text { Discharge } \\
\left(\mathrm{ft}^{3} / \mathbf{s}\right)\end{array}$ & $\begin{array}{c}\text { Water year } \\
\text { of } \\
\text { occurrence }\end{array}$ & $\begin{array}{c}\text { Discharge } \\
\left(\mathrm{ft}^{3} / \mathrm{s}\right)\end{array}$ & $\begin{array}{c}\text { Standard } \\
\text { deviation } \\
\left(\mathrm{ft}^{3} / \mathrm{s}\right)\end{array}$ & $\begin{array}{l}\text { Coeffl- } \\
\text { cient of } \\
\text { variation }\end{array}$ & $\begin{array}{l}\text { Percentage } \\
\text { of annuai } \\
\text { discharge }\end{array}$ \\
\hline October & 5.22 & 1929 & 0 & $\mathrm{~m}$ & 0.670 & 1.57 & 2.35 & 0.24 \\
\hline November & 54.9 & 1957 & 0 & $\mathrm{~m}$ & 4.12 & 14.6 & 3.55 & 1.50 \\
\hline December & 1.00 & 1929 & 0 & $\mathrm{~m}$ & 0.090 & 0.28 & 3.14 & 0.03 \\
\hline January & 0 & $\mathrm{~m}$ & 0 & $\mathrm{~m}$ & 0 & 0 & ng & 0 \\
\hline February & 0 & $\mathrm{~m}$ & 0 & $\mathrm{~m}$ & 0 & 0 & ng & 0 \\
\hline March & 193 & 1929 & 0 & $\mathrm{~m}$ & 17.5 & 53.0 & 3.03 & 6.36 \\
\hline April & 345 & 1956 & 7.40 & 1938 & 89.8 & 97.1 & 1.08 & 32.6 \\
\hline May & 278 & 1930 & 0.477 & 1934 & 68.2 & 80.7 & 1.18 & 24.7 \\
\hline June & 243 & 1955 & 0.040 & 1934 & 32.8 & 68.1 & 2.08 & 11.9 \\
\hline July & 699 & 1956 & 0 & 1934 & 55.8 & 193 & 3.46 & 20.2 \\
\hline August & 18.2 & 1956 & 0 & $\mathrm{~m}$ & 2.75 & 6.18 & 2.25 & 1.00 \\
\hline September & 43.5 & 1956 & 0 & $\mathrm{~m}$ & 3.97 & 11.8 & 2.97 & 1.44 \\
\hline Annual & 111 & 1956 & 0.766 & 1934 & 22.5 & 28.6 & 1.27 & 100 \\
\hline
\end{tabular}

Annual flow duration

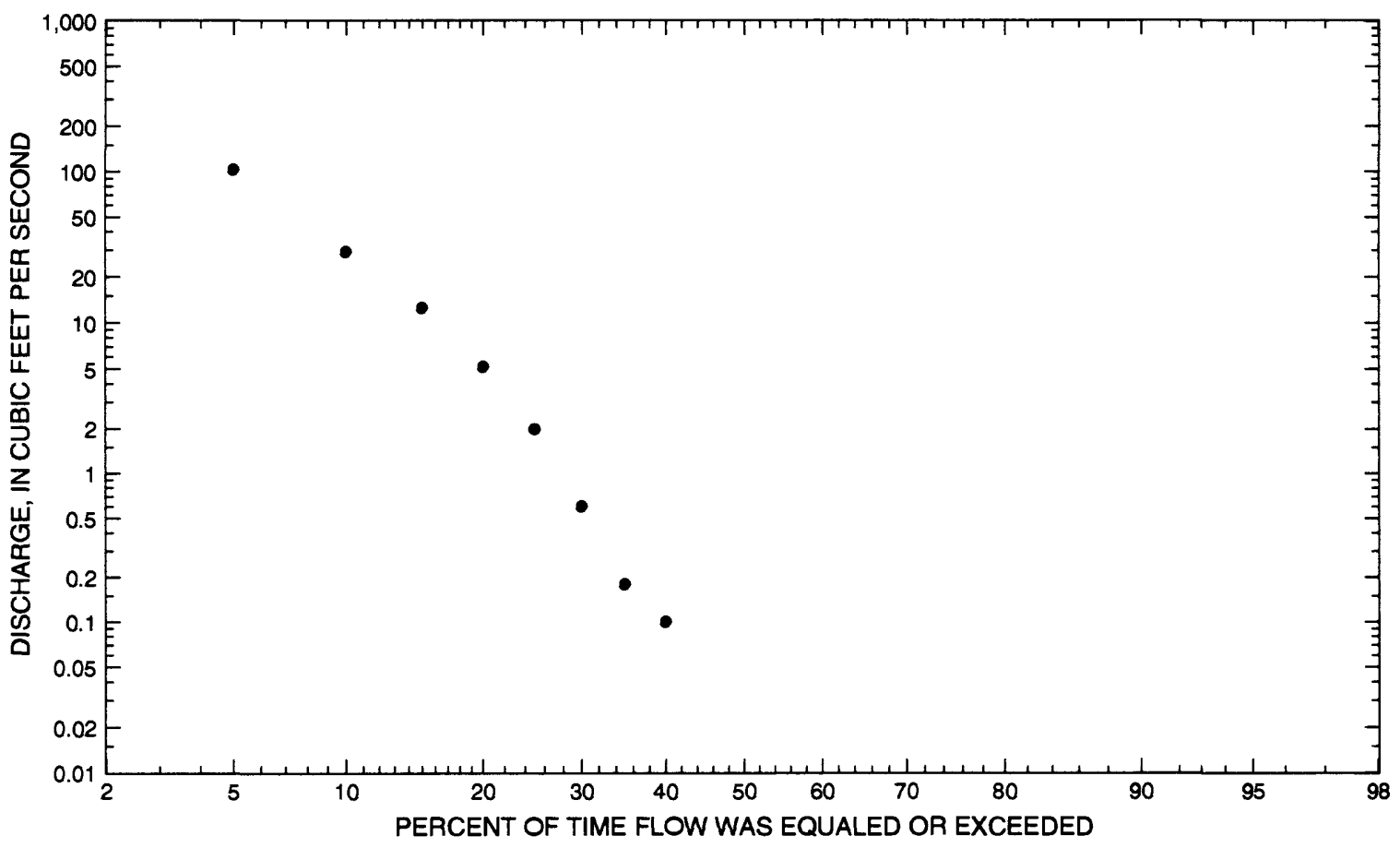




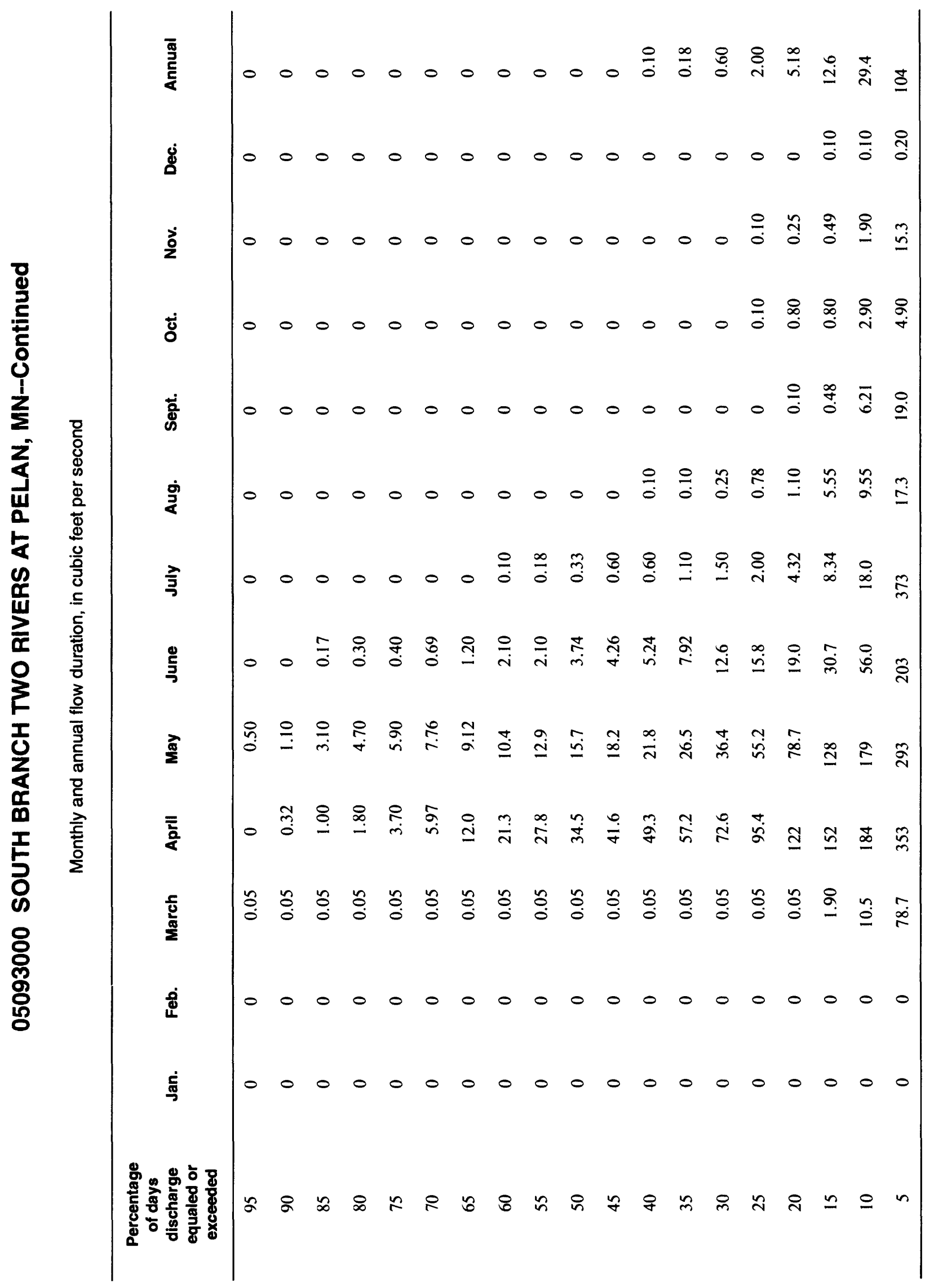




\section{SOUTH BRANCH TWO RIVERS AT PELAN, MN--Continued}

Probability of occurrence of annual high discharges

[ng, statistic not given]

\begin{tabular}{lcccccc}
\hline & & & \multicolumn{5}{c}{$\begin{array}{c}\text { Maximum mean discharge } \\
\left.\text { (ft } \mathbf{3}^{3} / \mathbf{s}\right)\end{array}$} \\
\cline { 5 - 7 } $\begin{array}{c}\text { Exceedance } \\
\text { probability }\end{array}$ & $\begin{array}{c}\text { Recurrence } \\
\text { interval } \\
\text { (years) }\end{array}$ & $\begin{array}{c}\text { Maximum } \\
\text { instantaneous } \\
\left(\mathbf{f t}^{3} / \mathbf{s}\right)\end{array}$ & 3-day period & 7-day period & 15-day period & 30-day period \\
\hline 0.99 & 1.01 & 20.4 & 18.0 & 13.9 & 7.56 & 3.95 \\
0.95 & 1.05 & 52.5 & 45.7 & 37.4 & 22.8 & 13.4 \\
0.90 & 1.11 & 84.9 & 73.6 & 61.4 & 39.2 & 24.0 \\
0.80 & 1.25 & 149 & 129 & 109 & 72.4 & 45.9 \\
0.50 & 2 & 410 & 358 & 301 & 205 & 132 \\
0.20 & 5 & 1,040 & 931 & 748 & 496 & 306 \\
0.10 & 10 & 1,650 & 1,490 & 1,160 & 742 & 439 \\
0.04 & 25 & 2,620 & 2,430 & 1,790 & 1,090 & 609 \\
0.02 & 50 & 3,500 & 3,290 & 2,330 & 1,370 & 731 \\
0.01 & 100 & 4,500 & 4,290 & 2,930 & 1,650 & 846 \\
0.005 & 200 & 5,620 & 5,450 & 3,590 & 1,940 & 954 \\
0.002 & 500 & 7,310 & $n g$ & $n g$ & $n g$ & $\mathrm{ng}$ \\
\hline
\end{tabular}

Probability of occurrence of annual low discharges

[ng, statistic not given]

\begin{tabular}{|c|c|c|c|c|c|c|c|c|c|c|}
\hline \multirow{3}{*}{$\begin{array}{c}\text { Non- } \\
\text { exceed- } \\
\text { ance } \\
\text { prob- } \\
\text { ability }\end{array}$} & \multirow{3}{*}{$\begin{array}{c}\text { Recur- } \\
\text { rence } \\
\text { inter- } \\
\text { val } \\
\text { (years) }\end{array}$} & \multicolumn{9}{|c|}{ Minimum mean discharge $\left(\mathrm{tt}^{3} / \mathrm{s}\right)$} \\
\hline & & \multicolumn{9}{|c|}{ Number of consecutive days } \\
\hline & & 1 & 3 & 7 & 14 & 30 & 60 & 90 & 120 & 183 \\
\hline 0.05 & 20 & ng & $\mathrm{ng}$ & ng & $\mathrm{ng}$ & $\mathrm{ng}$ & $\mathrm{ng}$ & $\mathrm{ng}$ & $\mathrm{ng}$ & 0 \\
\hline 0.10 & 10 & $\mathrm{ng}$ & $\mathrm{ng}$ & $\mathrm{ng}$ & $\mathrm{ng}$ & $\mathrm{ng}$ & $\mathrm{ng}$ & $\mathrm{ng}$ & $\mathrm{ng}$ & 0 \\
\hline 0.20 & 5 & $\mathrm{ng}$ & $\mathrm{ng}$ & $\mathrm{ng}$ & $\mathrm{ng}$ & $\mathrm{ng}$ & $\mathrm{ng}$ & $\mathrm{ng}$ & $\mathrm{ng}$ & 0 \\
\hline 0.50 & 2 & $\mathrm{ng}$ & $\mathrm{ng}$ & $\mathrm{ng}$ & $\mathrm{ng}$ & $\mathrm{ng}$ & $\mathrm{ng}$ & $\mathrm{ng}$ & $\mathrm{ng}$ & 0 \\
\hline
\end{tabular}


05093000 SOUTH BRANCH TWO RIVERS AT PELAN, MN--Continued

Probability of occurrence of seasonal low discharges

[ng, statistic not given]

\begin{tabular}{|c|c|c|c|c|c|c|c|c|c|}
\hline \multirow[b]{3}{*}{$\begin{array}{c}\text { Non- } \\
\text { exceedance } \\
\text { probability }\end{array}$} & \multirow[b]{3}{*}{$\begin{array}{c}\text { Recurrence } \\
\text { intervai } \\
\text { (years) }\end{array}$} & \multicolumn{8}{|c|}{ Minimum mean discharge $\left(\mathrm{ft}^{3} / \mathrm{s}\right)$} \\
\hline & & \multicolumn{8}{|c|}{ Number of consecutive days } \\
\hline & & 1 & 7 & 14 & 30 & 1 & 7 & 14 & 30 \\
\hline & & \multicolumn{4}{|c|}{ December-January-February } & \multicolumn{4}{|c|}{ March-April-May } \\
\hline 0.05 & 20 & ng & ng & $\mathrm{ng}$ & ng & ng & $\mathrm{ng}$ & $\mathrm{ng}$ & 0 \\
\hline 0.10 & 10 & ng & ng & $\mathrm{ng}$ & ng & ng & ng & $\mathrm{ng}$ & 0 \\
\hline 0.20 & 5 & $\mathrm{ng}$ & ng & $\mathrm{ng}$ & ng & ng & $\mathrm{ng}$ & $\mathrm{ng}$ & 0 \\
\hline \multirow[t]{2}{*}{0.50} & 2 & $\mathrm{ng}$ & ng & $\mathrm{ng}$ & ng & ng & ng & $\mathrm{ng}$ & 0 \\
\hline & & \multicolumn{4}{|c|}{ June-July-August } & \multicolumn{4}{|c|}{ September-October-November } \\
\hline 0.05 & 20 & $\mathrm{ng}$ & $\mathrm{ng}$ & 0 & 0 & $\mathrm{ng}$ & ng & $\mathrm{ng}$ & ng \\
\hline 0.10 & 10 & $\mathrm{ng}$ & $\mathrm{ng}$ & 0 & 0 & ng & ng & ng & $\mathrm{ng}$ \\
\hline 0.20 & 5 & $\mathrm{ng}$ & $\mathrm{ng}$ & 0 & 0 & $\mathrm{ng}$ & $\mathrm{ng}$ & $\mathrm{ng}$ & $\mathrm{ng}$ \\
\hline 0.50 & 2 & $\mathrm{ng}$ & ng & 0 & 0.017 & $\mathrm{ng}$ & $\mathrm{ng}$ & ng & $\mathrm{ng}$ \\
\hline
\end{tabular}




\section{SOUTH BRANCH TWO RIVERS AT PELAN, MN--Continued}

Annual peak discharge and corresponding gage height

$$
[--, \text { no data] }
$$

\begin{tabular}{|c|c|c|c|c|c|c|c|}
\hline $\begin{array}{l}\text { Water } \\
\text { year }\end{array}$ & Date & $\begin{array}{c}\text { Gage } \\
\text { height } \\
\text { (feet) }\end{array}$ & $\begin{array}{c}\text { Peak } \\
\text { discharge } \\
\left(\mathrm{ft}^{3} / \mathrm{s}\right)\end{array}$ & $\begin{array}{l}\text { Water } \\
\text { year }\end{array}$ & Date & $\begin{array}{c}\text { Gage } \\
\text { height } \\
\text { (feet) }\end{array}$ & $\begin{array}{c}\text { Peak } \\
\text { discharge } \\
\left(\mathrm{ft}^{3} / \mathrm{s}\right)\end{array}$ \\
\hline \multicolumn{8}{|c|}{ Annual peak discharge, by year, and corresponding gage height } \\
\hline 1929 & March 21 & 6.40 & 718 & 1936 & April 20 & 3.25 & 178 \\
\hline 1930 & May 13 & 10.18 & 1,810 & 1937 & May 3 & 5.00 & 506 \\
\hline 1931 & April 7 & - & 77.0 & 1938 & May 13 & 4.04 & 285 \\
\hline 1932 & April 9 & 8.02 & 1,140 & 1954 & June 18 & 3.70 & 266 \\
\hline 1933 & April 3 & 4.90 & 242 & 1955 & June 7 & 8.07 & 1,040 \\
\hline 1934 & April 8 & 2.44 & 46.0 & 1956 & July 10 & 10.90 & 2,040 \\
\hline 1935 & March 29 & 5.24 & 242 & & & & \\
\hline \multicolumn{8}{|c|}{ Annual peak discharge, from highest to lowest, and corresponding gage height } \\
\hline 1956 & July 10 & 10.90 & 2,040 & 1954 & June 18 & 3.70 & 266 \\
\hline 1930 & May 13 & 10.18 & 1,810 & 1933 & April 3 & 4.90 & 242 \\
\hline 1932 & April 9 & 8.02 & 1,140 & 1935 & March 29 & 5.24 & 242 \\
\hline 1955 & June 7 & 8.07 & 1,040 & 1936 & April 20 & 3.25 & 178 \\
\hline 1929 & March 21 & 6.40 & 718 & 1931 & April 7 & -- & 77.0 \\
\hline 1937 & May 3 & 5.00 & 506 & 1934 & April 8 & 2.44 & 46.0 \\
\hline 1938 & May 13 & 4.04 & 285 & & & & \\
\hline
\end{tabular}




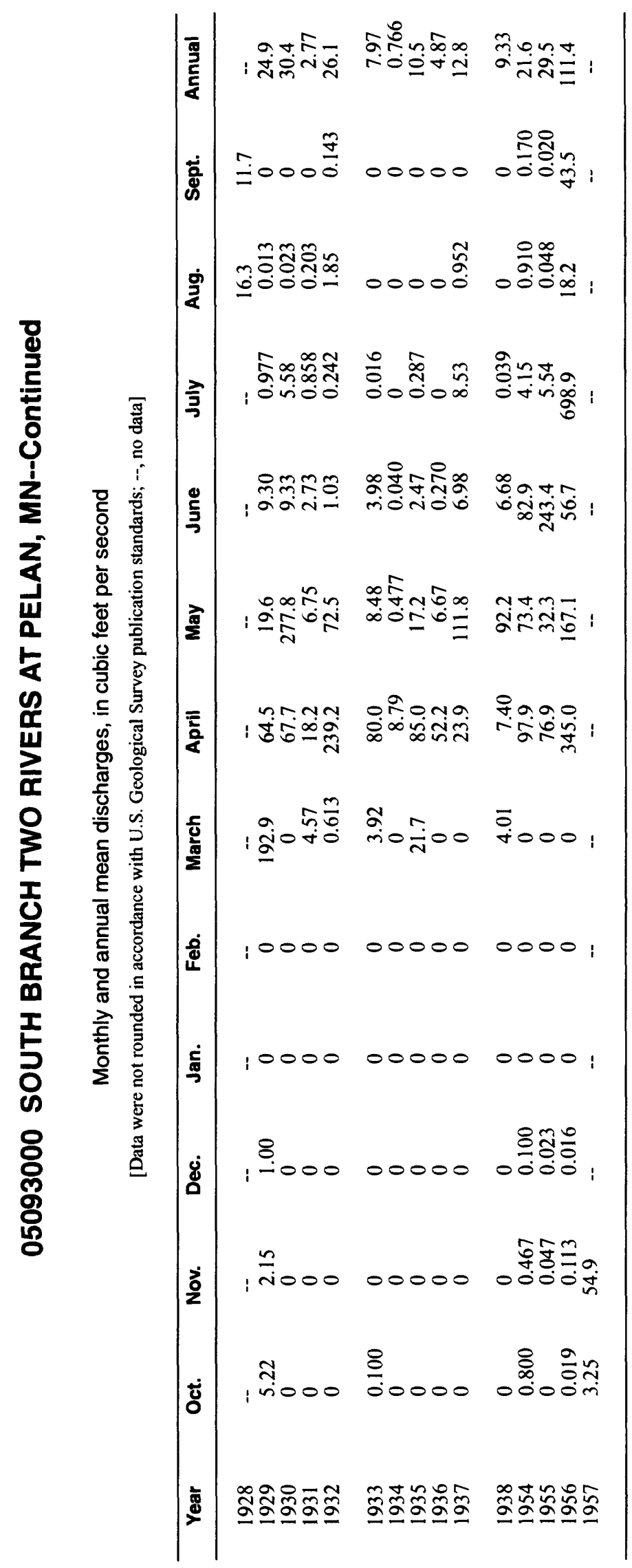




\section{SOUTH BRANCH TWO RIVERS AT LAKE BRONSON, MN}

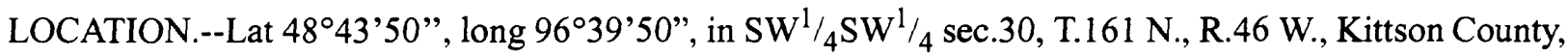
Hydrologic Unit 09020312, on left bank $70 \mathrm{ft}$ upstream from culvert on U.S. Highway 59 at Lake Bronson and 3.4 mi downstream from dam at outlet of Bronson Lake.

DRAINAGE AREA.--444 $\mathrm{mi}^{2}$.

PERIOD OF RECORD.--September 1928 to November 1936, April to September 1937, April 1941 to October 1943, April to December 1944, April 1945 to September 1947, October 1953 to September 1981, April 1985 to current year. Monthly discharge only for some periods, published in WSP 1308. October 1981 to March 1985, annual maximums only. Published as South Fork Two Rivers at Bronson prior to 1941.

GAGE.--Water-stage recorder. Datum of gage is $928.53 \mathrm{ft}$ above mean sea level (Minnesota Department of Transportation benchmark). Prior to Nov. 23, 1953, nonrecording gage at bridge $100 \mathrm{ft}$ downstream at datum $2.00 \mathrm{ft}$ higher. Nov. 23, 1953, to Oct. 5, 1963, water-stage recorder at same site at datum 2.00 $\mathrm{ft}$ higher.

EXTREMES FOR PERIOD OF RECORD.--Maximum discharge, 5,410 $\mathrm{ft}^{3} / \mathrm{s}$, Apr. 5, 1966, gage height, $18.23 \mathrm{ft}$; no flow at times.

Annual mean discharge

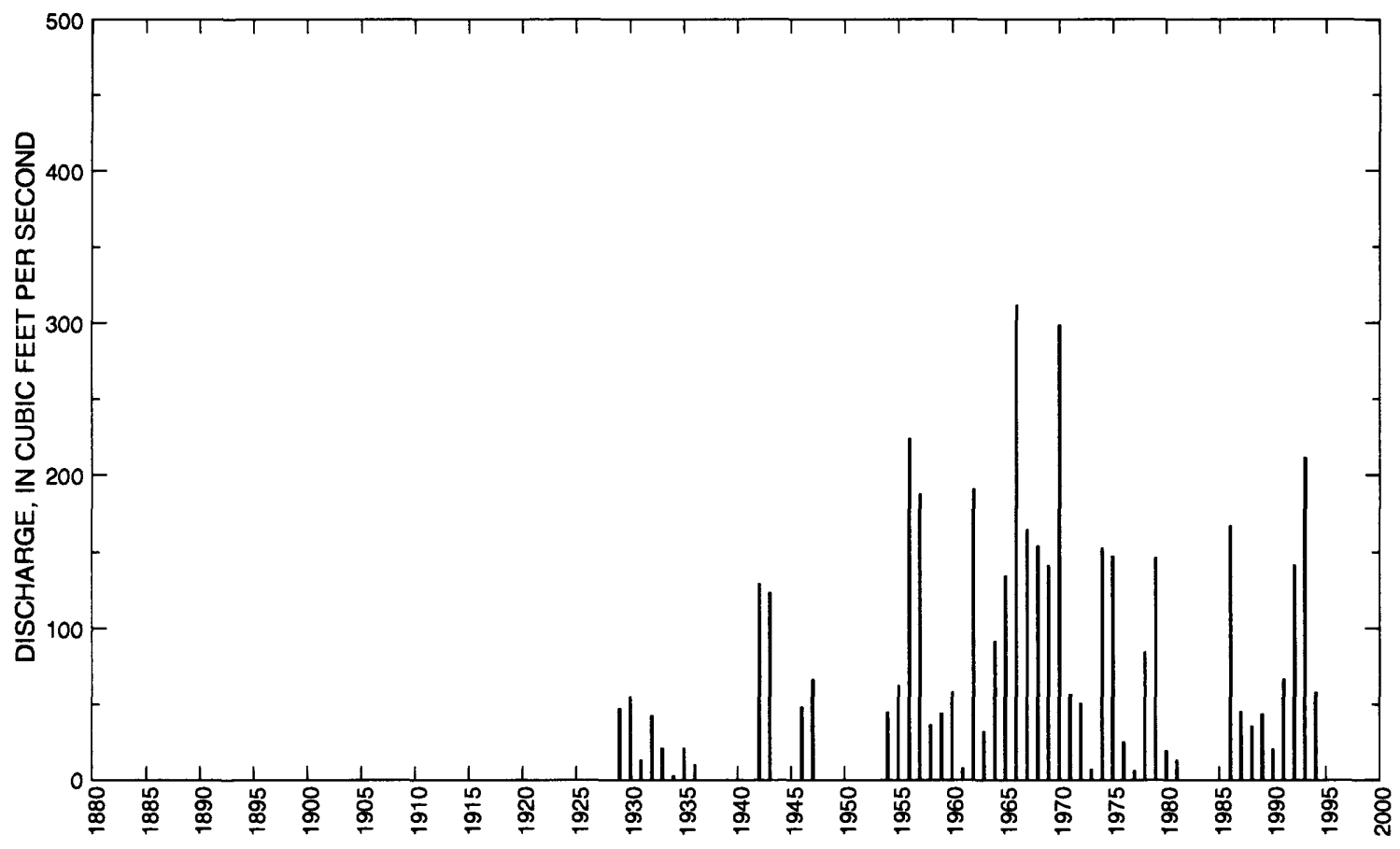


05094000 SOUTH BRANCH TWO RIVERS AT LAKE BRONSON, MN--Continued

Statistics of monthly and annual mean discharges

\begin{tabular}{|c|c|c|c|c|c|c|c|c|}
\hline \multirow[b]{2}{*}{ Month } & \multicolumn{2}{|c|}{ Maximum } & \multicolumn{2}{|c|}{ Minimum } & \multicolumn{4}{|c|}{ Mean } \\
\hline & $\begin{array}{c}\text { Discharge } \\
\left(\mathrm{ft}^{3} / \mathrm{s}\right)\end{array}$ & $\begin{array}{l}\text { Water year } \\
\text { of } \\
\text { occurrence }\end{array}$ & $\begin{array}{c}\text { Discharge } \\
\left(\mathrm{ft}^{3} / \mathrm{s}\right)\end{array}$ & $\begin{array}{c}\text { Water year } \\
\text { of } \\
\text { occurrence }\end{array}$ & $\begin{array}{c}\text { Discharge } \\
\left.\left(\mathrm{ft}^{3} / \mathrm{s}\right)\right)\end{array}$ & $\begin{array}{l}\text { Standard } \\
\text { deviation } \\
\left(\mathrm{ft}^{3} / \mathrm{s}\right)\end{array}$ & $\begin{array}{l}\text { Coeffi- } \\
\text { cient of } \\
\text { variation }\end{array}$ & $\begin{array}{l}\text { Percentage } \\
\text { of annual } \\
\text { discharge }\end{array}$ \\
\hline October & 153 & 1958 & 0.400 & 1991 & 19.0 & 34.5 & 1.81 & 1.82 \\
\hline November & 87.5 & 1957 & 0.381 & 1990 & 11.2 & 19.3 & 1.72 & 1.07 \\
\hline December & 34.5 & 1992 & 0.131 & 1987 & 4.59 & 5.74 & 1.25 & 0.44 \\
\hline January & 12.1 & 1992 & 0.116 & 1987 & 2.81 & 2.56 & 0.91 & 0.27 \\
\hline February & 23.6 & 1981 & 0.120 & 1987 & 3.20 & 4.07 & 1.27 & 0.31 \\
\hline March & 362 & 1986 & 0.658 & 1934 & 59.8 & 89.6 & 1.50 & 5.70 \\
\hline April & 1,980 & 1966 & 0.539 & 1991 & 398 & 387 & 0.97 & 37.9 \\
\hline May & 1,340 & 1970 & 0.984 & 1991 & 189 & 274 & 1.45 & 18.1 \\
\hline June & 1,340 & 1970 & 1.43 & 1980 & 161 & 268 & 1.66 & 15.4 \\
\hline July & 1,140 & 1956 & 0.437 & 1988 & 106 & 223 & 2.11 & 10.1 \\
\hline August & 1,350 & 1993 & 0.089 & 1988 & 48.2 & 189 & 3.93 & 4.60 \\
\hline September & 525 & 1957 & 0 & 1937 & 45.7 & 107 & 2.34 & 4.36 \\
\hline Annual & 312 & 1966 & 2.89 & 1934 & 87.5 & 76.6 & 0.88 & 100 \\
\hline
\end{tabular}

Annual flow duration

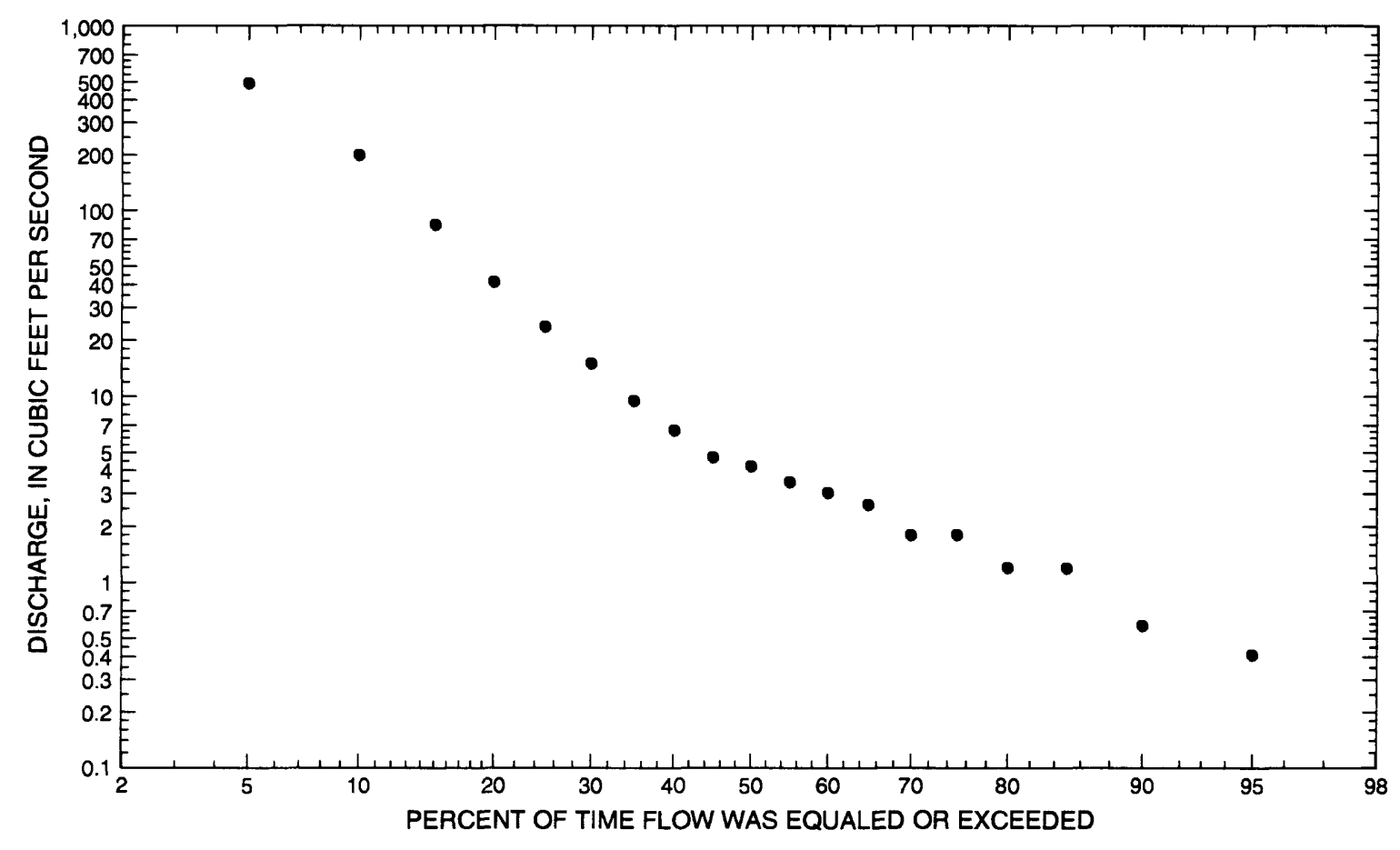




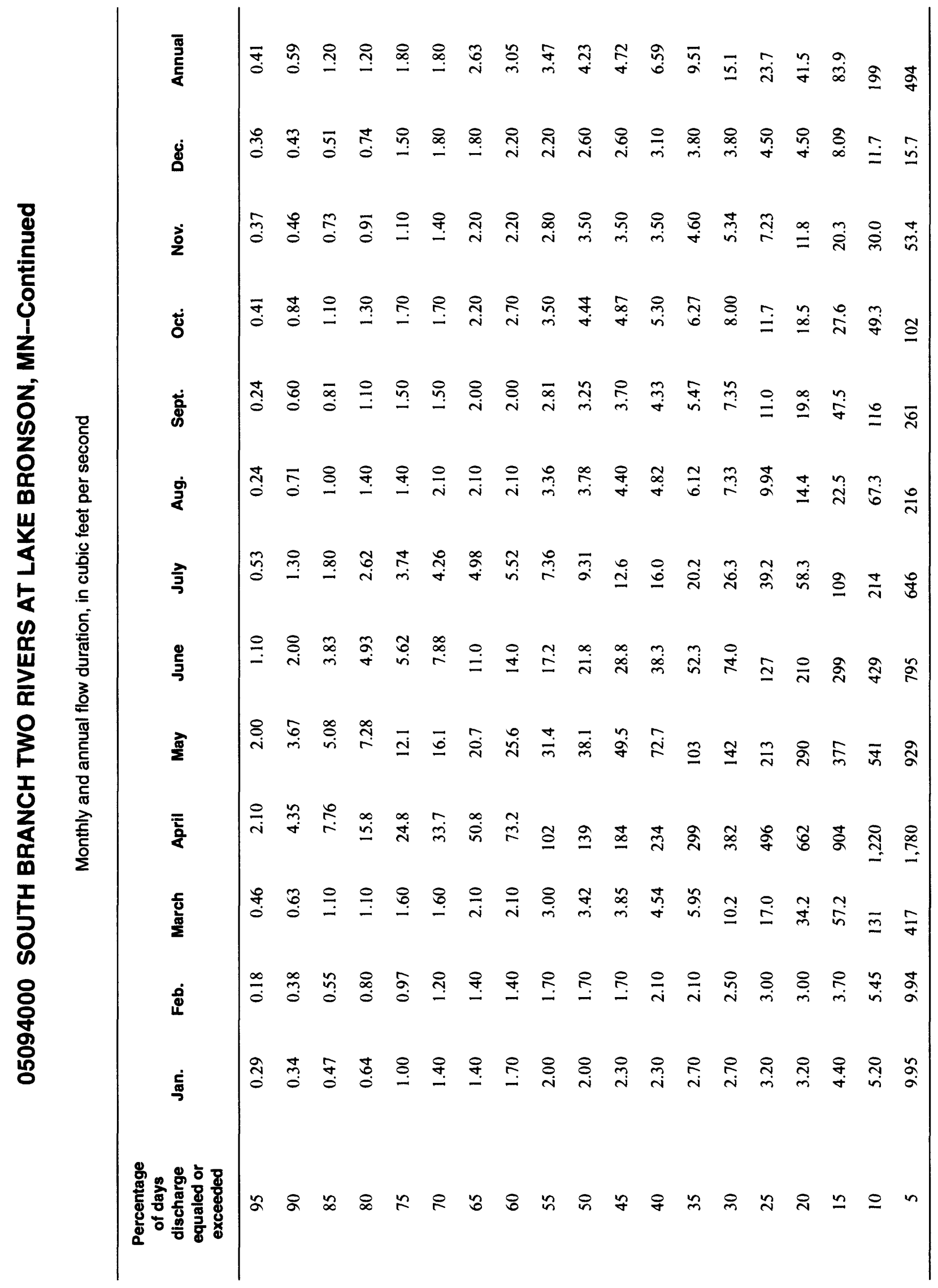


05094000 SOUTH BRANCH TWO RIVERS AT LAKE BRONSON, MN--Continued

Probability of occurrence of annual high discharges

[ng, statistic not given]

\begin{tabular}{|c|c|c|c|c|c|c|}
\hline \multirow[b]{2}{*}{$\begin{array}{l}\text { Exceedance } \\
\text { probabillty }\end{array}$} & \multirow[b]{2}{*}{$\begin{array}{l}\text { Recurrence } \\
\text { interval } \\
\text { (years) }\end{array}$} & \multirow[b]{2}{*}{$\begin{array}{c}\text { Maximum } \\
\text { instantaneous } \\
\left(\mathrm{ft}^{3} / \mathbf{s}\right)\end{array}$} & \multicolumn{4}{|c|}{$\begin{array}{l}\text { Maximum mean discharge } \\
\left(\mathrm{ft}^{3} / \mathrm{s}\right)\end{array}$} \\
\hline & & & 3-day period & 7-day period & 15-day period & 30-day period \\
\hline 0.99 & 1.01 & $\mathrm{ng}$ & 57.3 & 41.0 & 25.0 & 15.8 \\
\hline 0.95 & 1.05 & 337 & 166 & 128 & 83.9 & 53.3 \\
\hline 0.90 & 1.11 & 462 & 275 & 219 & 148 & 94.5 \\
\hline 0.80 & 1.25 & 667 & 478 & 394 & 274 & 176 \\
\hline 0.50 & 2 & 1,280 & 1,170 & 1,000 & 730 & 477 \\
\hline 0.20 & 5 & 2,330 & 2,320 & 2,040 & 1,520 & 1,020 \\
\hline 0.10 & 10 & 3,100 & 3,090 & 2,730 & 2,040 & 1,380 \\
\hline 0.04 & 25 & 4,140 & 3,990 & 3,520 & 2,630 & 1,810 \\
\hline 0.02 & 50 & 4,940 & 4,590 & 4,040 & 3,010 & 2,090 \\
\hline 0.01 & 100 & 5,770 & 5,120 & 4,490 & 3,350 & 2,340 \\
\hline 0.005 & 200 & 6,610 & 5,590 & 4,890 & 3,630 & 2,560 \\
\hline 0.002 & 500 & 7,740 & ng & $\mathrm{ng}$ & $\mathrm{ng}$ & ng \\
\hline
\end{tabular}

Probability of occurrence of annual low discharges

\begin{tabular}{|c|c|c|c|c|c|c|c|c|c|c|}
\hline \multirow[b]{3}{*}{$\begin{array}{l}\text { Non- } \\
\text { exceed- } \\
\text { ance } \\
\text { prob- } \\
\text { ability }\end{array}$} & \multirow[b]{3}{*}{$\begin{array}{c}\text { Recur- } \\
\text { rence } \\
\text { inter- } \\
\text { val } \\
\text { (years) }\end{array}$} & \multicolumn{9}{|c|}{ Minimum mean discharge $\left(t^{3} / s\right)$} \\
\hline & & \multicolumn{9}{|c|}{ Number of consecutive days } \\
\hline & & 1 & 3 & 7 & 14 & 30 & 60 & 90 & 120 & 183 \\
\hline 0.05 & 20 & 0.015 & 0.015 & 0.050 & 0.092 & 0.187 & 0.302 & 0.387 & 0.458 & 0.947 \\
\hline 0.10 & 10 & 0.098 & 0.103 & 0.123 & 0.183 & 0.295 & 0.451 & 0.569 & 0.647 & 1.21 \\
\hline 0.20 & 5 & 0.247 & 0.264 & 0.287 & 0.354 & 0.492 & 0.710 & 0.884 & 0.982 & 1.71 \\
\hline 0.50 & 2 & 0.836 & 0.890 & 0.939 & 0.978 & 1.18 & 1.54 & 1.90 & 2.17 & 3.81 \\
\hline
\end{tabular}




\section{SOUTH BRANCH TWO RIVERS AT LAKE BRONSON, MN-Continued}

Probability of occurrence of seasonal low discharges

\begin{tabular}{|c|c|c|c|c|c|c|c|c|c|}
\hline \multirow[b]{3}{*}{$\begin{array}{c}\text { Non- } \\
\text { exceedance } \\
\text { probabiiity }\end{array}$} & \multirow[b]{3}{*}{$\begin{array}{c}\text { Recurrence } \\
\text { intervai } \\
\text { (years) }\end{array}$} & \multicolumn{8}{|c|}{ Minimum mean discharge $\left(\mathrm{ft}^{3} / \mathrm{s}\right)$} \\
\hline & & \multicolumn{8}{|c|}{ Number of consecutive days } \\
\hline & & 1 & 7 & 14 & 30 & 1 & 7 & 14 & 30 \\
\hline & & \multicolumn{4}{|c|}{ December-January-February } & \multicolumn{4}{|c|}{ March-April-May } \\
\hline 0.05 & 20 & 0.252 & 0.258 & 0.264 & 0.298 & 0.177 & 0.202 & 0.343 & 0.679 \\
\hline 0.10 & 10 & 0.375 & 0.385 & 0.392 & 0.441 & 0.338 & 0.393 & 0.514 & 1.10 \\
\hline 0.20 & 5 & 0.592 & 0.608 & 0.619 & 0.694 & 0.631 & 0.753 & 0.814 & 2.01 \\
\hline \multirow[t]{2}{*}{0.50} & 2 & 1.30 & 1.35 & 1.39 & 1.55 & 1.60 & 2.02 & $1_{3.00}$ & 6.85 \\
\hline & & \multicolumn{4}{|c|}{ June-July-August } & \multicolumn{4}{|c|}{ September-October-November } \\
\hline 0.05 & 20 & 0.042 & 0.103 & 0.235 & 0.544 & 0.153 & 0.214 & 0.294 & 0.353 \\
\hline 0.10 & 10 & 0.193 & 0.282 & 0.431 & 0.803 & 0.246 & 0.338 & 0.429 & 0.525 \\
\hline 0.20 & 5 & 0.515 & 0.726 & 0.845 & 1.35 & 0.434 & 0.587 & 0.695 & 0.876 \\
\hline 0.50 & 2 & 1.84 & 2.70 & 2.89 & 4.20 & 1.25 & 1.66 & 1.91 & 2.58 \\
\hline
\end{tabular}

${ }^{1}$ Graphical interpretation. 
Annual peak discharge and corresponding gage height

$$
[--, \text { no data }]
$$

\begin{tabular}{|c|c|c|c|c|c|c|c|}
\hline $\begin{array}{l}\text { Water } \\
\text { year }\end{array}$ & Date & $\begin{array}{c}\text { Gage } \\
\text { height } \\
\text { (feet) }\end{array}$ & $\begin{array}{c}\text { Peak } \\
\text { discharge } \\
\left(\mathrm{ft}^{3} / \mathrm{s}\right)\end{array}$ & $\begin{array}{l}\text { Water } \\
\text { year }\end{array}$ & Date & $\begin{array}{c}\text { Gage } \\
\text { height } \\
\text { (feet) }\end{array}$ & $\begin{array}{c}\text { Peak } \\
\text { discharge } \\
\left(\mathrm{ft}^{3} / \mathrm{s}\right)\end{array}$ \\
\hline \multicolumn{8}{|c|}{ Annual peak discharge, by year, and corresponding gage height } \\
\hline 1929 & March 20 & 9.00 & 940 & 1967 & April 21 & 11.43 & 2,430 \\
\hline 1930 & May 15 & 10.90 & 1,820 & 1968 & July 19 & 11.08 & 2,290 \\
\hline 1931 & April 9 & 6.00 & 300 & 1969 & April 14 & 14.01 & 3,520 \\
\hline 1932 & April 11 & 9.70 & 1,310 & 1970 & April 14 & 14.51 & 4,140 \\
\hline 1933 & April 4 & 7.58 & 415 & 1971 & April 8 & 9.00 & 1,500 \\
\hline 1934 & April 10 & 5.00 & 64.0 & 1972 & April 15 & 8.50 & 1,480 \\
\hline 1935 & April 13 & 7.21 & 565 & 1973 & March 14 & 10.18 & 800 \\
\hline 1936 & April 19 & 6.64 & 358 & 1974 & April 24 & 11.35 & 2,460 \\
\hline 1937 & May 4 & 7.02 & 594 & 1975 & July 6 & 10.37 & 1,960 \\
\hline 1941 & April 11 & 10.09 & 1,580 & 1976 & April 1 & 7.24 & 980 \\
\hline 1942 & April 1 & 12.05 & 2,210 & 1977 & April 8 & 5.06 & 219 \\
\hline 1943 & April 11 & 8.31 & 1,050 & 1978 & April 12 & 12.12 & 2,770 \\
\hline 1944 & June 12 & 7.46 & 820 & 1979 & April 22 & 13.17 & 3,340 \\
\hline 1945 & April 16 & 6.96 & 670 & 1980 & April 8 & 6.90 & 820 \\
\hline 1946 & March 26 & 7.03 & 668 & 1981 & June 30 & 5.39 & 340 \\
\hline 1947 & June 17 & 9.07 & 1,290 & 1982 & April 2 & 7.94 & 1,040 \\
\hline 1954 & June 16 & 7.03 & 567 & 1983 & March 8 & 9.33 & 1,530 \\
\hline 1955 & June 9 & 8.33 & 1,020 & 1984 & April 3 & 6.45 & 623 \\
\hline 1956 & April 24 & 12.79 & 2,650 & 1985 & June 26 & 12.16 & 2,790 \\
\hline 1957 & July 8 & 10.52 & 1,810 & 1986 & March 29 & 11.47 & 2,510 \\
\hline 1958 & September 23 & 6.29 & 355 & 1987 & March 27 & 7.62 & 996 \\
\hline 1959 & April 8 & 8.60 & 1,110 & 1988 & March 24 & 8.46 & 1,170 \\
\hline 1960 & April 11 & 8.72 & 1,270 & 1989 & April 19 & 10.78 & 2,100 \\
\hline 1961 & March 26 & 6.86 & 451 & 1990 & April 1 & 7.10 & 784 \\
\hline 1962 & June 13 & 12.82 & 2,960 & 1991 & July 13 & 11.35 & 2,160 \\
\hline 1963 & April 8 & 7.56 & 1,570 & 1992 & April 2 & 10.82 & 1,870 \\
\hline 1964 & June 14 & 10.88 & 2,210 & 1993 & August 10 & 12.87 & 3,050 \\
\hline 1965 & April 15 & 12.30 & 2,780 & 1994 & March 26 & -- & 850 \\
\hline 1966 & April 5 & 18.23 & 5,410 & & & & \\
\hline \multicolumn{8}{|c|}{ Annual peak discharge, from highest to lowest, and corresponding gage height } \\
\hline 1966 & April 5 & 18.23 & 5,410 & 1942 & April 1 & 12.05 & 2,210 \\
\hline 1970 & April 14 & 14.51 & 4,140 & 1964 & June 14 & 10.88 & 2,210 \\
\hline 1969 & April 14 & 14.01 & 3,520 & 1991 & July 13 & 11.35 & 2,160 \\
\hline 1979 & April 22 & 13.17 & 3,340 & 1989 & April 19 & 10.78 & 2,100 \\
\hline 1993 & August 10 & 12.87 & 3,050 & 1975 & July 6 & 10.37 & 1,960 \\
\hline 1962 & June 13 & 12.82 & 2,960 & 1992 & April 2 & 10.82 & 1,870 \\
\hline 1985 & June 26 & 12.16 & 2,790 & 1930 & May 15 & 10.90 & 1,820 \\
\hline 1965 & April 15 & 12.30 & 2,780 & 1957 & July 8 & 10.52 & 1,810 \\
\hline 1978 & April 12 & 12.12 & 2,770 & 1941 & April 11 & 10.09 & 1,580 \\
\hline 1956 & April 24 & 12.79 & 2,650 & 1963 & April 8 & 7.56 & 1,570 \\
\hline 1986 & March 29 & 11.47 & 2,510 & 1983 & March 8 & 9.33 & 1,530 \\
\hline 1974 & April 24 & 11.35 & 2,460 & 1971 & April 8 & 9.00 & 1,500 \\
\hline 1967 & April 21 & 11.43 & 2,430 & 1972 & April 15 & 8.50 & 1,480 \\
\hline 1968 & July 19 & 11.08 & 2,290 & 1932 & April 11 & 9.70 & 1,310 \\
\hline
\end{tabular}


05094000 SOUTH BRANCH TWO RIVERS AT LAKE BRONSON, MN--Continued

Annual peak discharge and corresponding gage height--Continued

$$
[--, \text { no data }]
$$

\begin{tabular}{|c|c|c|c|c|c|c|c|}
\hline $\begin{array}{l}\text { Water } \\
\text { year }\end{array}$ & Date & $\begin{array}{c}\text { Gage } \\
\text { height } \\
\text { (feet) }\end{array}$ & $\begin{array}{c}\text { Peak } \\
\text { discharge } \\
\left(\mathrm{ft}^{3} / \mathrm{s}\right)\end{array}$ & $\begin{array}{c}\text { Water } \\
\text { year }\end{array}$ & Date & $\begin{array}{c}\text { Gage } \\
\text { height } \\
\text { (feet) }\end{array}$ & $\begin{array}{c}\text { Peak } \\
\text { discharge } \\
\left(\mathrm{ft}^{3} / \mathrm{s}\right)\end{array}$ \\
\hline \multicolumn{8}{|c|}{ Annual peak discharge, from highest to lowest, and corresponding gage height-Continued } \\
\hline 1947 & June 17 & 9.07 & 1,290 & 1945 & April 16 & 6.96 & 670 \\
\hline 1960 & April 11 & 8.72 & 1,270 & 1946 & March 26 & 7.03 & 668 \\
\hline 1988 & March 24 & 8.46 & 1,170 & 1984 & April 3 & 6.45 & 623 \\
\hline 1959 & April 8 & 8.60 & 1,110 & 1937 & May 4 & 7.02 & 594 \\
\hline 1943 & April 11 & 8.31 & 1,050 & 1954 & June 16 & 7.03 & 567 \\
\hline 1982 & April 2 & 7.94 & 1,040 & 1935 & April 13 & 7.21 & 565 \\
\hline 1955 & June 9 & 8.33 & 1,020 & 1961 & March 26 & 6.86 & 451 \\
\hline 1987 & March 27 & 7.62 & 996 & 1933 & April 4 & 7.58 & 415 \\
\hline 1976 & April 1 & 7.24 & 980 & 1936 & April 19 & 6.64 & 358 \\
\hline 1929 & March 20 & 9.00 & 940 & 1958 & September 23 & 6.29 & 355 \\
\hline 1994 & March 26 & -- & 850 & 1981 & June 30 & 5.39 & 340 \\
\hline 1944 & June 12 & 7.46 & 820 & 1931 & April 9 & 6.00 & 300 \\
\hline 1980 & April 8 & 6.90 & 820 & 1977 & April 8 & 5.06 & 219 \\
\hline 1973 & March 14 & 10.18 & 800 & 1934 & April 10 & 5.00 & 64.0 \\
\hline 1990 & April 1 & 7.10 & 784 & & & & \\
\hline
\end{tabular}




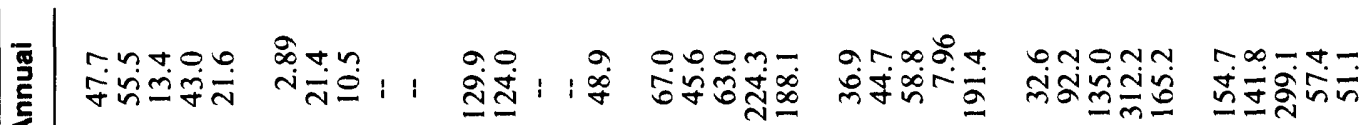

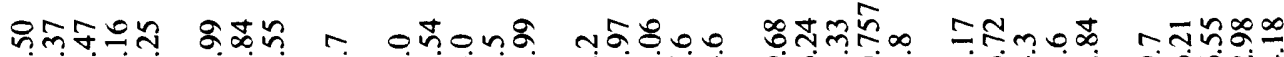

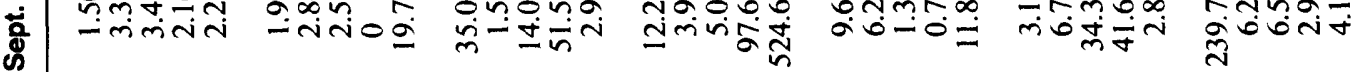

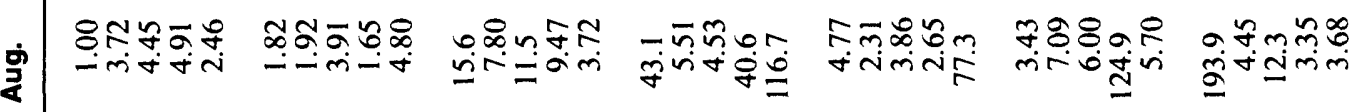

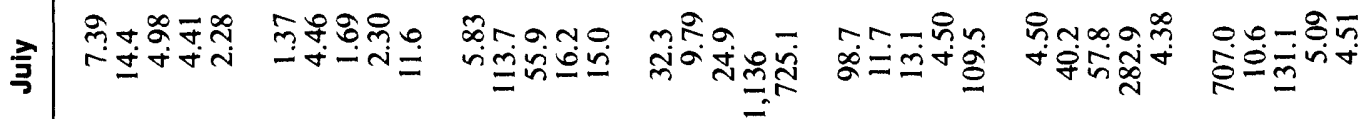

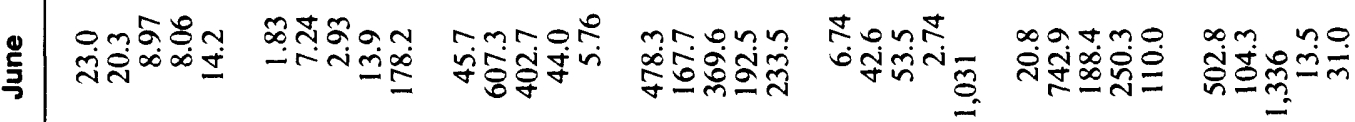

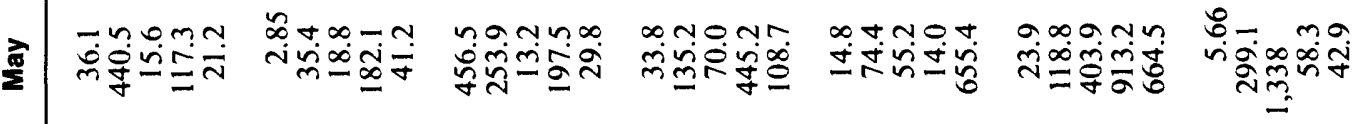

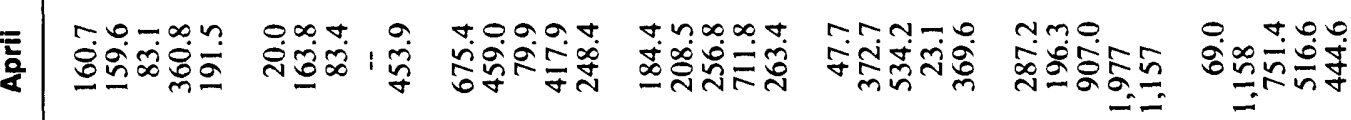

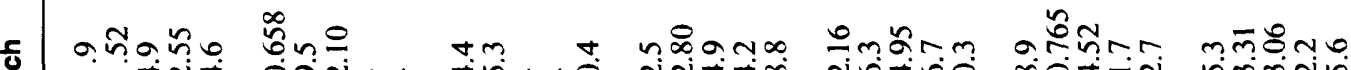

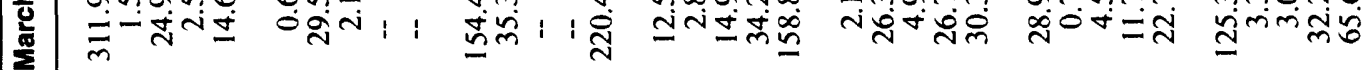

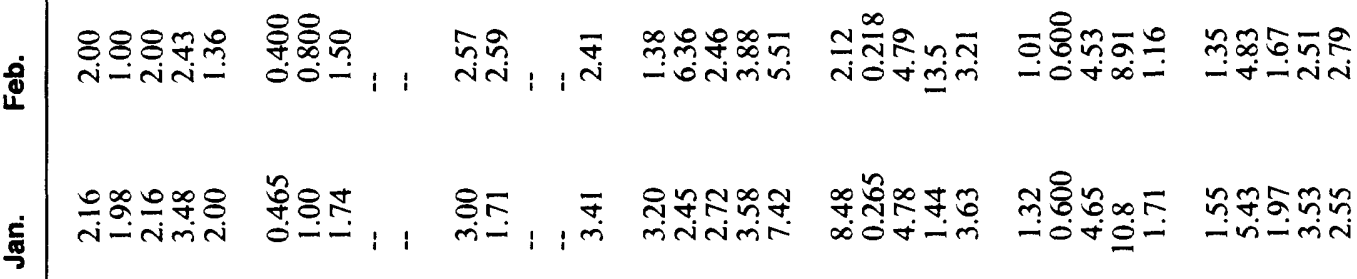

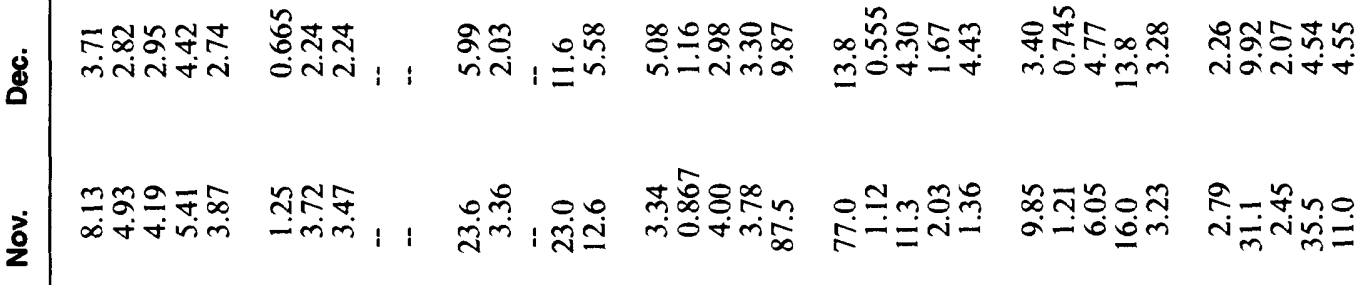

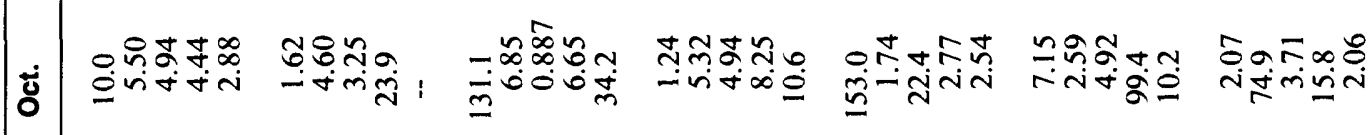

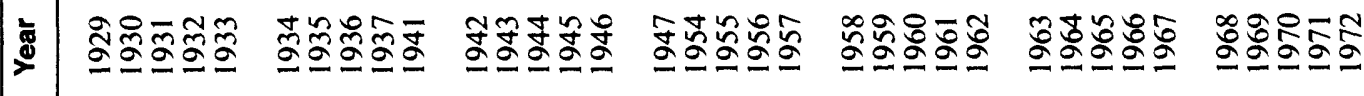




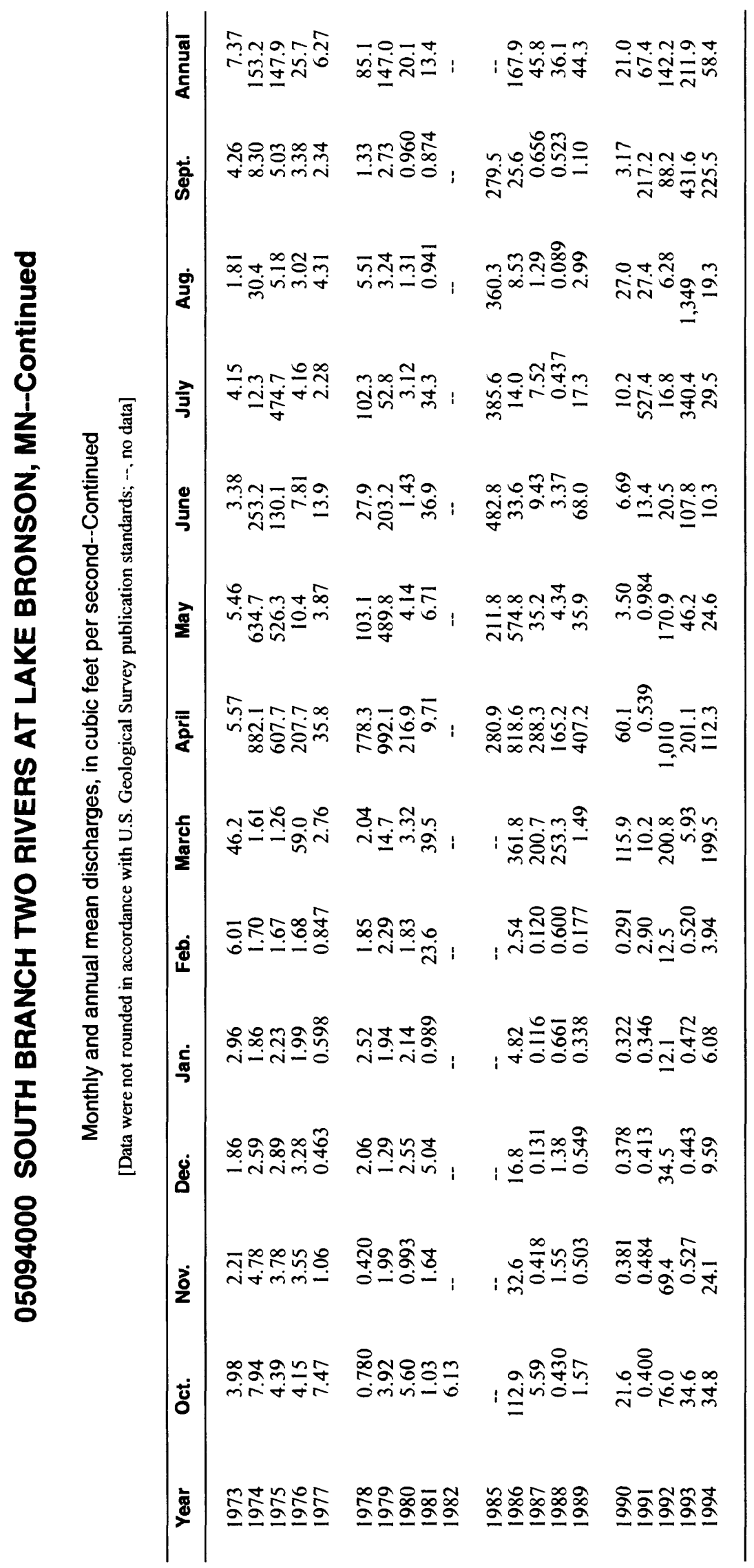




\section{TWO RIVERS BELOW HALLOCK, MN}

LOCATION.--Lat 4846'50', long 9702'25', in NE1/4 $\mathrm{SE}^{1 / 4}$ sec.7, T.161 N., R.49 W., Kittson County, Hydrologic Unit 09020312 , on downstream side of highway bridge, 4 mi west of Hallock and $5 \mathrm{mi}$ upstream from North Branch Two Rivers.

DRAINAGE AREA.--644 $\mathrm{mi}^{2}$.

PERIOD OF RECORD.--March 1945 to September 1955.

GAGE.--Chain gage. Datum of gage is $780 \mathrm{ft}$, from topographic map.

EXTREMES FOR PERIOD OF RECORD.--Maximum discharge, 3,690 $\mathrm{ft}^{3} / \mathrm{s}$, May 13, 1950, gage height, $25.78 \mathrm{ft}$, from graph based on gage readings, backwater from Red River of the North; no flow at times in many years.

Annual mean discharge

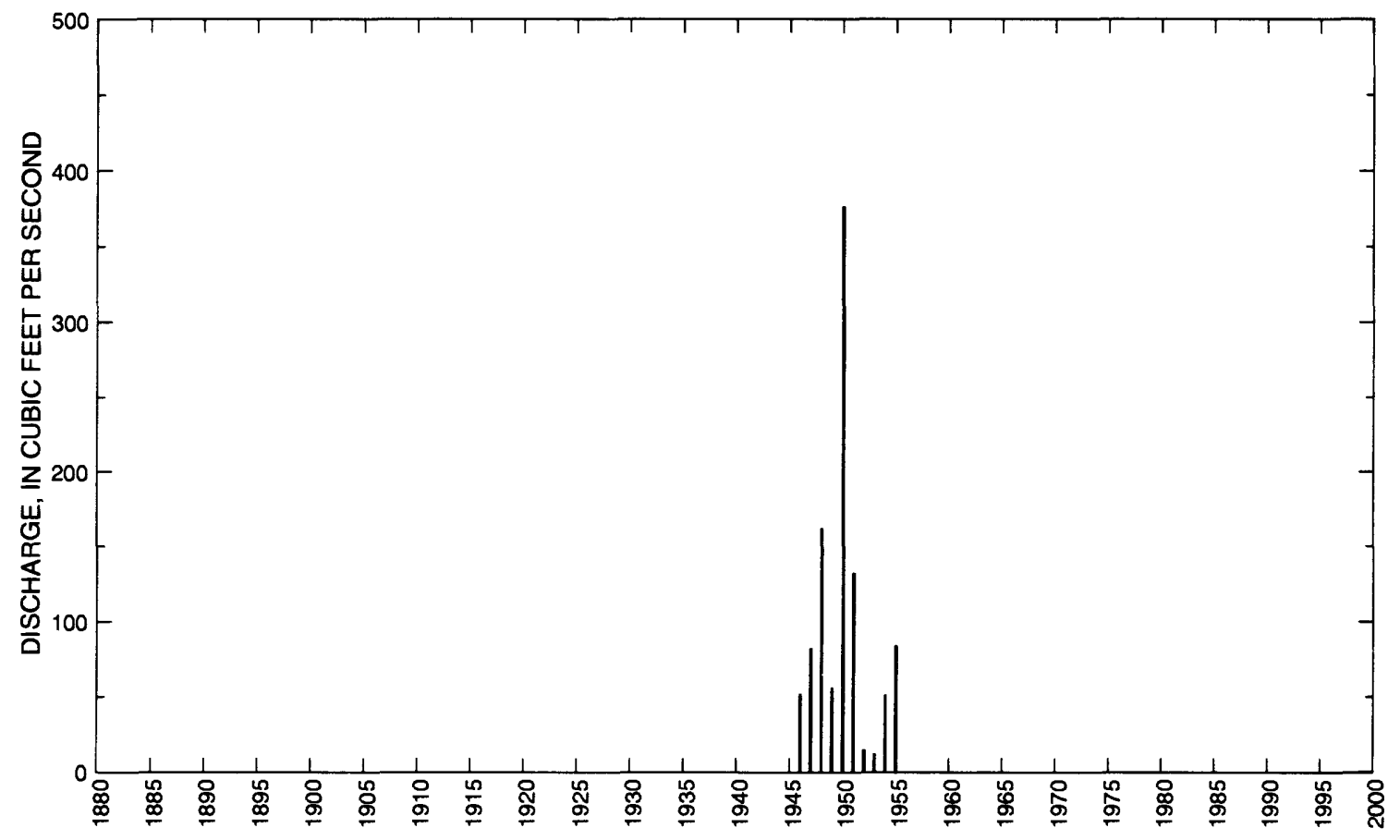


05095500 TWO RIVERS BELOW HALLOCK, MN--Continued

Statistics of monthly and annual mean discharges

\begin{tabular}{|c|c|c|c|c|c|c|c|c|}
\hline \multirow[b]{2}{*}{ Month } & \multicolumn{2}{|c|}{ Maximum } & \multicolumn{2}{|c|}{ Minimum } & \multicolumn{4}{|c|}{ Mean } \\
\hline & $\begin{array}{c}\text { Discharge } \\
\left(\mathrm{ft}^{3} / \mathrm{s}\right)\end{array}$ & $\begin{array}{c}\text { Water year } \\
\text { of } \\
\text { occurrence }\end{array}$ & $\begin{array}{c}\text { Discharge } \\
\left(\mathrm{ft}^{3} / \mathrm{s}\right)\end{array}$ & $\begin{array}{c}\text { Water year } \\
\text { of } \\
\text { occurrence }\end{array}$ & $\begin{array}{c}\text { Discharge } \\
\left(\mathrm{ft}^{3} / \mathrm{s}\right)\end{array}$ & $\begin{array}{l}\text { Standard } \\
\text { deviation } \\
\left(\mathrm{ft}^{3} / \mathrm{s}\right)\end{array}$ & $\begin{array}{l}\text { Coeffi- } \\
\text { cient of } \\
\text { variation }\end{array}$ & $\begin{array}{l}\text { Percentage } \\
\text { of annual } \\
\text { discharge }\end{array}$ \\
\hline October & 168 & 1951 & 0.171 & 1949 & 28.3 & 51.1 & 1.80 & 2.30 \\
\hline November & 25.9 & 1948 & 0.570 & 1954 & 11.3 & 9.88 & 0.87 & 0.92 \\
\hline December & 12.0 & 1948 & 0.261 & 1954 & 5.04 & 4.45 & 0.88 & 0.41 \\
\hline January & 8.40 & 1951 & 0.048 & 1953 & 2.24 & 2.98 & 1.33 & 0.18 \\
\hline February & 6.61 & 1953 & 0 & 1949 & 2.13 & 2.41 & 1.13 & 0.17 \\
\hline March & 299 & 1945 & 0.448 & 1955 & 49.7 & 104 & 2.08 & 4.04 \\
\hline April & 900 & 1951 & 30.0 & 1953 & 436 & 304 & 0.70 & 35.4 \\
\hline May & 2,520 & 1950 & 6.79 & 1952 & 378 & 732 & 1.93 & 30.7 \\
\hline June & 612 & 1950 & 4.42 & 1952 & 198 & 230 & 1.16 & 16.1 \\
\hline July & 354 & 1950 & 0.913 & 1952 & 80.2 & 127 & 1.58 & 6.52 \\
\hline August & 108 & 1948 & 0.910 & 1952 & 21.4 & 32.5 & 1.52 & 1.74 \\
\hline September & 85.1 & 1950 & 0 & 1952 & 17.7 & 29.8 & 1.68 & 1.44 \\
\hline Annual & 377 & 1950 & 12.2 & 1953 & 103 & 107 & 1.05 & 100 \\
\hline
\end{tabular}

Annual flow duration

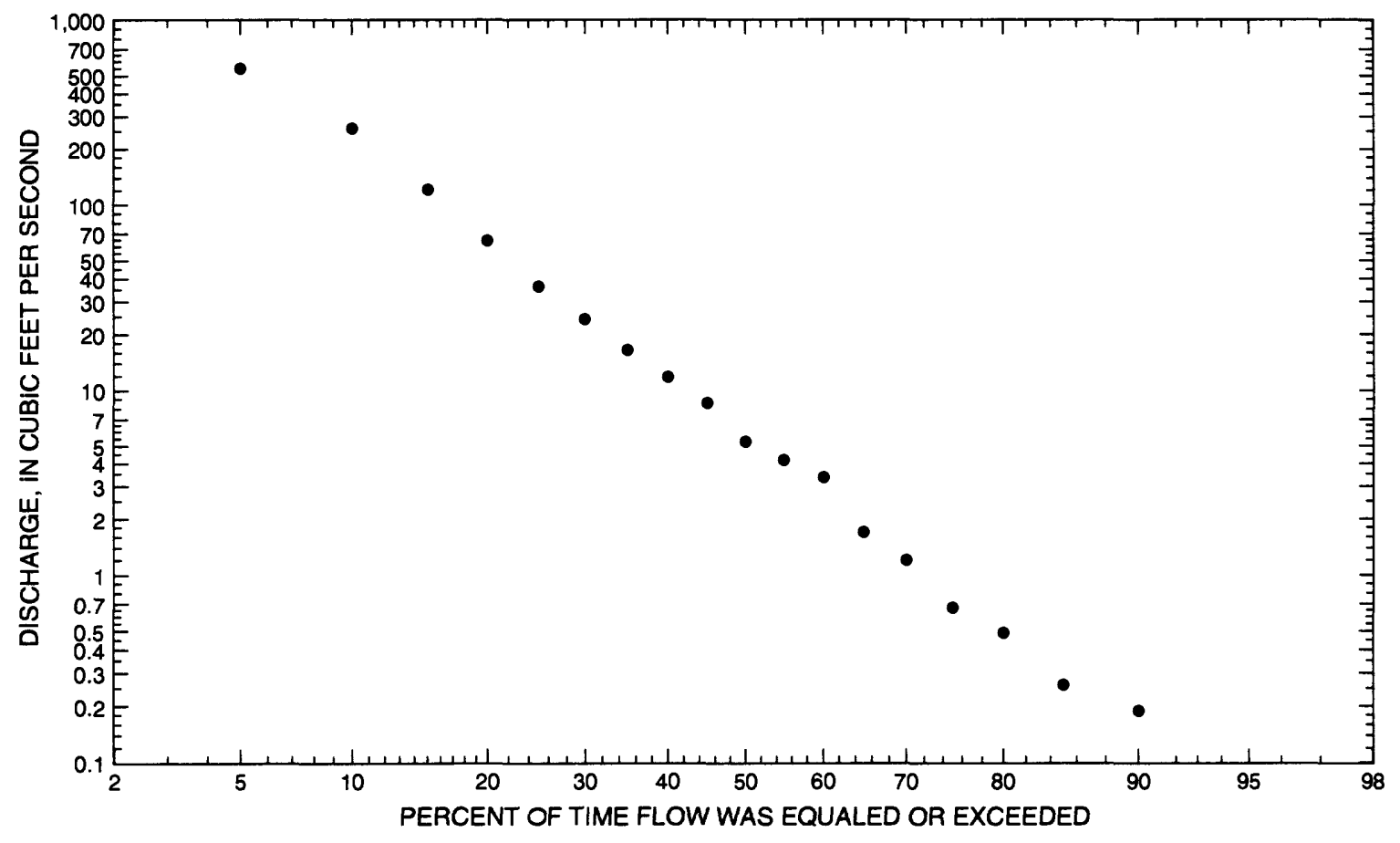




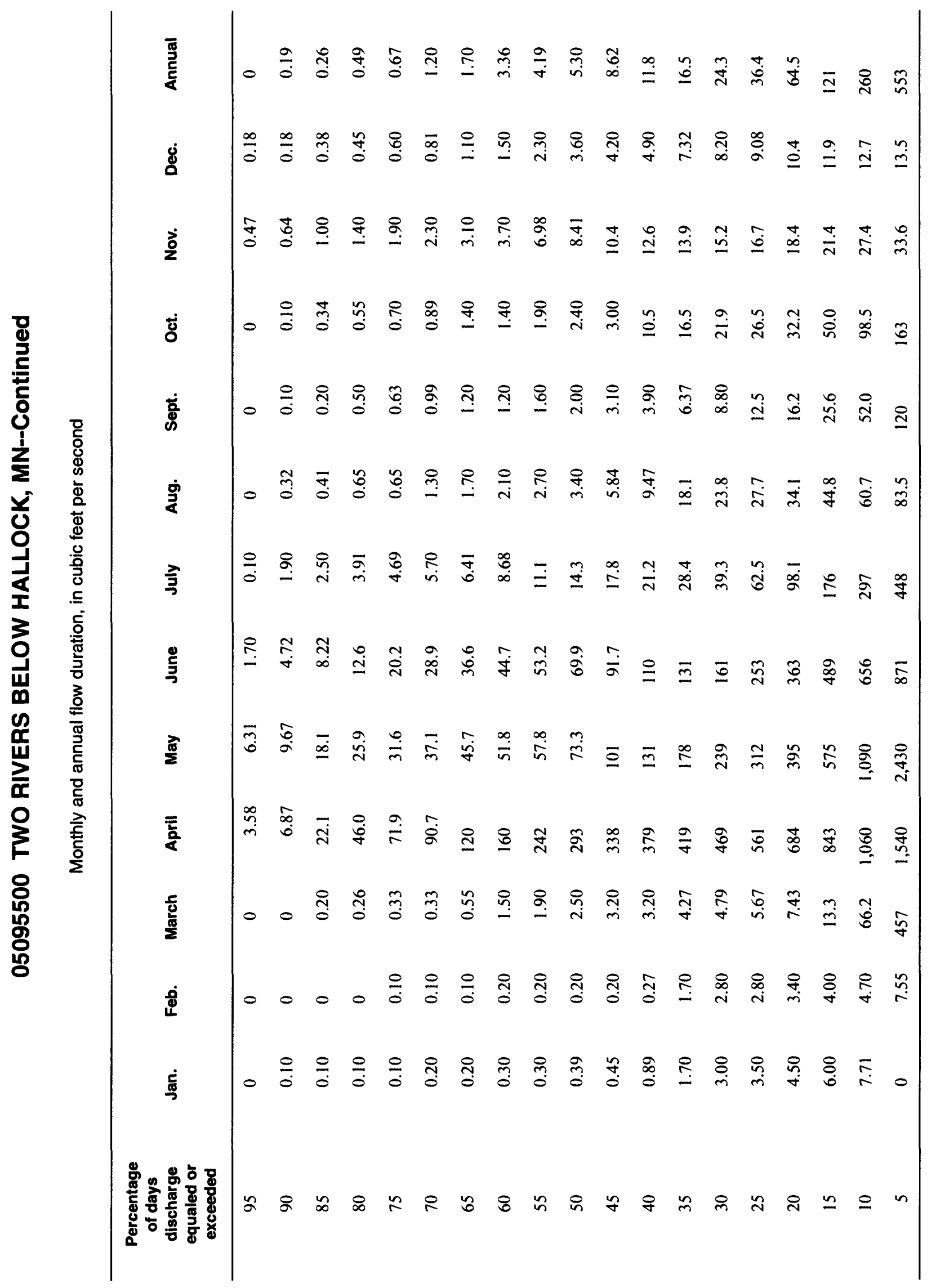




\section{TWO RIVERS BELOW HALLOCK, MN--Continued}

Probability of occurrence of annual high discharges

[ng, statistic not given]

\begin{tabular}{|c|c|c|c|c|c|c|}
\hline \multirow[b]{2}{*}{$\begin{array}{l}\text { Exceedance } \\
\text { probability }\end{array}$} & \multirow[b]{2}{*}{$\begin{array}{l}\text { Recurrence } \\
\text { intervai } \\
\text { (years) }\end{array}$} & \multirow[b]{2}{*}{$\begin{array}{c}\text { Maximum } \\
\text { instantaneous } \\
\left(\mathrm{ft}^{3} / \mathrm{s}\right)\end{array}$} & \multicolumn{4}{|c|}{$\begin{array}{l}\text { Maximum mean discharge } \\
\left(\mathrm{ft}^{3} / \mathrm{s}\right)\end{array}$} \\
\hline & & & 3-day period & 7-day period & 15-day period & 30-day period \\
\hline 0.99 & 1.01 & 92.1 & 72.5 & 63.1 & 44.2 & 33.1 \\
\hline 0.95 & 1.05 & 194 & 167 & 147 & 107 & 77.2 \\
\hline 0.90 & 1.11 & 282 & 253 & 225 & 166 & 119 \\
\hline 0.80 & 1.25 & 434 & 406 & 364 & 278 & 198 \\
\hline 0.50 & 2 & 932 & 919 & 846 & 686 & 500 \\
\hline 0.20 & 5 & 1,840 & 1,870 & 1,770 & 1,540 & 1,180 \\
\hline 0.10 & 10 & 2,560 & 2,610 & 2,510 & 2,280 & 1,820 \\
\hline 0.04 & 25 & 3,540 & 3,600 & 3,540 & 3,350 & 2,810 \\
\hline 0.02 & 50 & 4,310 & 4,370 & 4,350 & 4,240 & 3,700 \\
\hline 0.01 & 100 & 5,110 & 5,150 & 5,180 & 5,200 & 4,700 \\
\hline 0.005 & 200 & 5,940 & 5,940 & 6,030 & 6,210 & 5,820 \\
\hline 0.002 & 500 & 7,060 & ng & ng & ng & ng \\
\hline
\end{tabular}

Probability of occurrence of annual low discharges

\begin{tabular}{|c|c|c|c|c|c|c|c|c|c|c|}
\hline \multirow[b]{3}{*}{$\begin{array}{l}\text { Non- } \\
\text { exceed- } \\
\text { ance } \\
\text { prob- } \\
\text { abiiity }\end{array}$} & \multirow[b]{3}{*}{$\begin{array}{l}\text { Recur- } \\
\text { rence } \\
\text { inter- } \\
\text { vai } \\
\text { (years) }\end{array}$} & \multicolumn{9}{|c|}{ Minimum mean discharge $\left(\mathrm{ft}^{3} / \mathrm{s}\right)$} \\
\hline & & \multicolumn{9}{|c|}{ Number of consecutive days } \\
\hline & & 1 & 3 & 7 & 14 & 30 & 60 & 90 & 120 & 183 \\
\hline 0.05 & 20 & 0 & 0 & 0 & 0 & 0 & 0 & 0.130 & 0.223 & 0.482 \\
\hline 0.10 & 10 & 0 & 0 & 0 & 0 & 0 & 0 & 0.183 & 0.354 & 0.766 \\
\hline 0.20 & 5 & 0 & 0 & 0 & 0 & 0 & 0.055 & 0.286 & 0.608 & 1.36 \\
\hline 0.50 & 2 & 0.080 & 0.083 & 0.107 & 0.116 & 0.153 & 0.262 & 0.739 & 1.62 & 4.17 \\
\hline
\end{tabular}


05095500 TWO RIVERS BELOW HALLOCK, MN--Continued

Probability of occurrence of seasonal low discharges

\begin{tabular}{|c|c|c|c|c|c|c|c|c|c|}
\hline \multirow[b]{3}{*}{$\begin{array}{c}\text { Non- } \\
\text { exceedance } \\
\text { probability }\end{array}$} & \multirow[b]{3}{*}{$\begin{array}{l}\text { Recurrence } \\
\text { interval } \\
\text { (years) }\end{array}$} & \multicolumn{8}{|c|}{ Minimum mean discharge $\left(\mathrm{ft}^{3} / \mathrm{s}\right)$} \\
\hline & & \multicolumn{8}{|c|}{ Number of consecutive days } \\
\hline & & 1 & 7 & 14 & 30 & 1 & 7 & 14 & 30 \\
\hline & & \multicolumn{4}{|c|}{ December-January-February } & \multicolumn{4}{|c|}{ March-April-May } \\
\hline 0.05 & 20 & 0 & 0 & 0 & 0 & 0 & 0 & 0 & 0.273 \\
\hline 0.10 & 10 & 0 & 0 & 0 & 0 & 0 & 0 & 0 & 0.426 \\
\hline 0.20 & 5 & 0 & 0 & 0 & 0 & 0.066 & 0.072 & 0.075 & 0.789 \\
\hline \multirow[t]{2}{*}{0.50} & 2 & 0.160 & 0.180 & 0.188 & 0.229 & 0.989 & 1.10 & 1.27 & 3.31 \\
\hline & & \multicolumn{4}{|c|}{ June-July-August } & \multicolumn{4}{|c|}{ September-October-November } \\
\hline 0.05 & 20 & 0 & 0 & 0 & 0 & 0 & 0 & 0 & 0 \\
\hline 0.10 & 10 & 0.094 & 0.094 & 0.118 & 0.469 & 0 & 0 & 0 & 0 \\
\hline 0.20 & 5 & 0.314 & 0.351 & 0.477 & 1.22 & 0 & 0 & 0.046 & 0.265 \\
\hline 0.50 & 2 & 1.32 & 1.74 & 2.44 & 4.48 & 0.461 & 0.650 & 0.863 & 1.77 \\
\hline
\end{tabular}


05095500 TWO RIVERS BELOW HALLOCK, MN--Continued

Annual peak discharge and corresponding gage height

$$
[--, \text { no data }]
$$

\begin{tabular}{|c|c|c|c|c|c|c|c|}
\hline $\begin{array}{l}\text { Water } \\
\text { year }\end{array}$ & Date & $\begin{array}{c}\text { Gage } \\
\text { height } \\
\text { (feet) }\end{array}$ & $\begin{array}{c}\text { Peak } \\
\text { discharge } \\
\left(\mathrm{ft}^{3} / \mathrm{s}\right)\end{array}$ & $\begin{array}{c}\text { Water } \\
\text { year }\end{array}$ & Date & $\begin{array}{l}\text { Gage } \\
\text { height } \\
\text { (feet) }\end{array}$ & $\begin{array}{c}\text { Peak } \\
\text { discharge } \\
\left(\mathrm{ft}^{3} / \mathrm{s}\right)\end{array}$ \\
\hline \multicolumn{8}{|c|}{ Annual peak discharge, by year, and corresponding gage height } \\
\hline 1945 & March 28 & -- & 856 & 1951 & April 17 & 15.64 & 1,350 \\
\hline 1946 & March 26 & 11.07 & 670 & 1952 & April 14 & 10.24 & 392 \\
\hline 1947 & June 19 & 14.74 & 1,280 & 1953 & June 8 & 5.42 & 146 \\
\hline 1948 & April 26 & 22.84 & 2,270 & 1954 & June 19 & 10.80 & 553 \\
\hline 1949 & April 15 & 14.47 & 1,040 & 1955 & June 13 & 12.97 & 902 \\
\hline 1950 & May 13 & 25.78 & 3,690 & & & & \\
\hline \multicolumn{8}{|c|}{ Annual peak discharge, from highest to lowest, and corresponding gage height } \\
\hline 1950 & May 13 & 25.78 & 3,690 & 1945 & March 28 & -- & 856 \\
\hline 1948 & April 26 & 22.84 & 2,270 & 1946 & March 26 & 11.07 & 670 \\
\hline 1951 & April 17 & 15.64 & 1,350 & 1954 & June 19 & 10.80 & 553 \\
\hline 1947 & June 19 & 14.74 & 1,280 & 1952 & April 14 & 10.24 & 392 \\
\hline 1949 & April 15 & 14.47 & 1,040 & 1953 & June 8 & 5.42 & 146 \\
\hline 1955 & June 13 & 12.97 & 902 & & & & \\
\hline
\end{tabular}




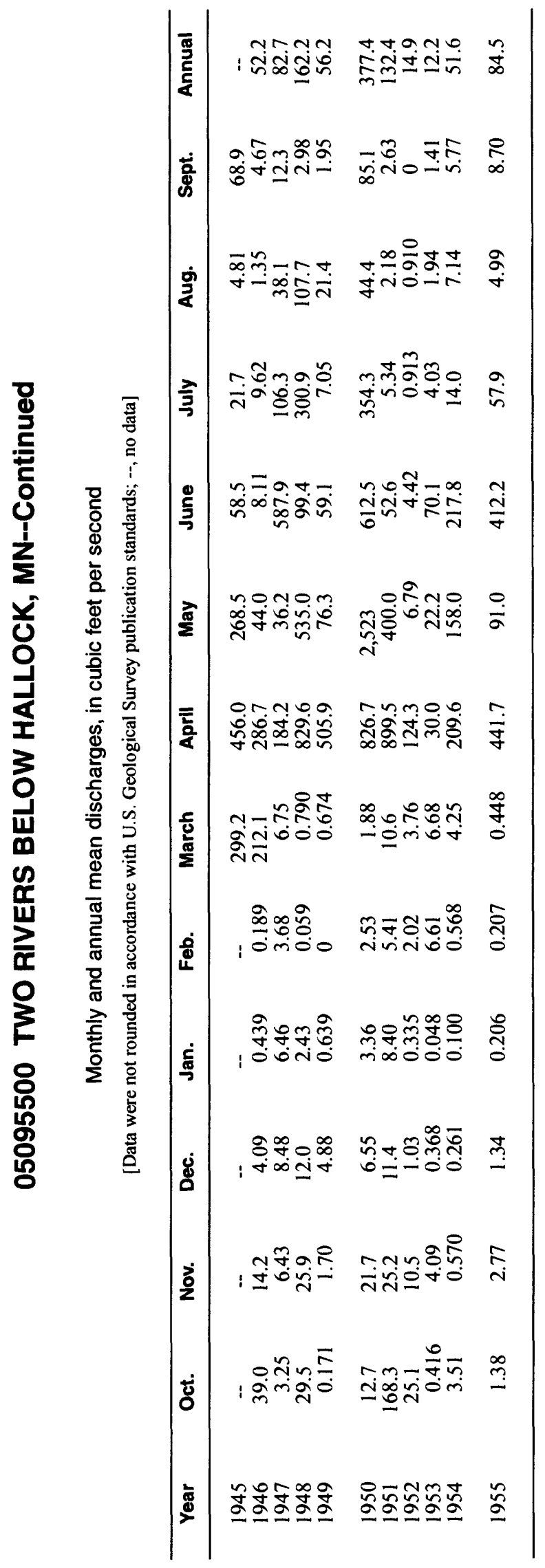


LOCATION.--Lat 48 53'21", long 96 $40^{\prime} 01^{\prime \prime}$, in NE $1 / 4$ sec.1, T.162 N., R.47 W., Kittson County, Hydrologic Unit 09020312 , on downstream side of highway bridge, $0.5 \mathrm{mi}$ upstream from State ditch 85 and 7 mi northeast of Lancaster.

DRAINAGE AREA.--32 $\mathrm{mi}^{2}$, approximately.

PERIOD OF RECORD.--April 1929 to September 1938, April 1941 to September 1955. Prior to 1941, published as North Fork Two Rivers near Lancaster.

GAGE.--Staff gage. Datum of gage is $963.69 \mathrm{ft}$ above mean sea level, adjustment of 1912 (levels by U.S. Army Corps of Engineers).

EXTREMES FOR PERIOD OF RECORD.--Maximum discharge, $912 \mathrm{ft}^{3} / \mathrm{s}$, May 20, 1950, gage height, $6.25 \mathrm{ft}$; no flow during several months each year.

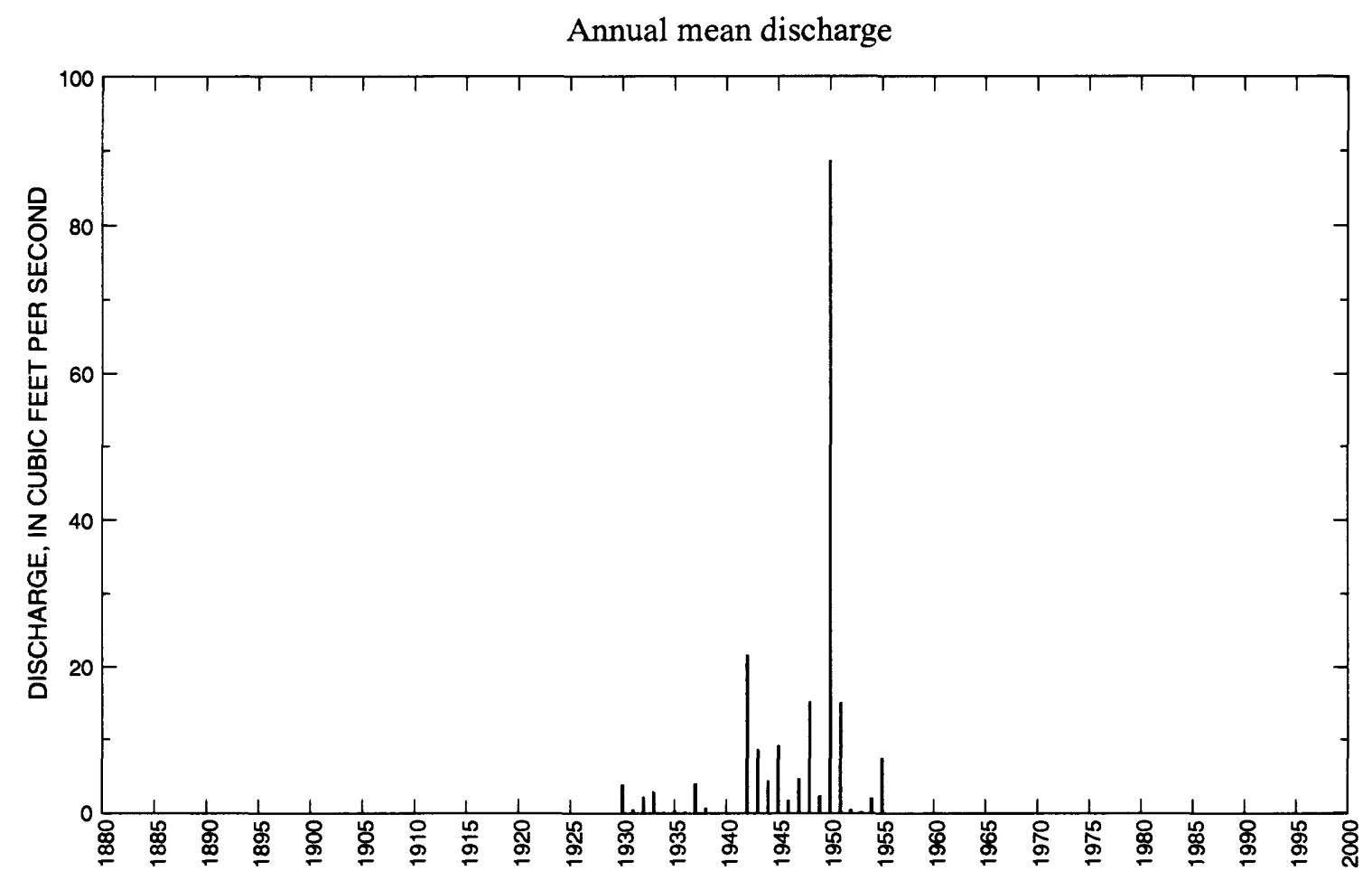


05096000 NORTH BRANCH TWO RIVERS NEAR LANCASTER, MN--Continued

Statistics of monthly and annual mean discharges

[m, more than 1 year of occurrence]

\begin{tabular}{|c|c|c|c|c|c|c|c|c|}
\hline \multirow[b]{2}{*}{ Month } & \multicolumn{2}{|c|}{ Maximum } & \multicolumn{2}{|c|}{ Minimum } & \multicolumn{4}{|c|}{ Mean } \\
\hline & $\begin{array}{c}\text { Discharge } \\
\left(\mathrm{ft}^{3} / \mathrm{s}\right)\end{array}$ & $\begin{array}{l}\text { Water year } \\
\text { of } \\
\text { occurrence }\end{array}$ & $\begin{array}{c}\text { Discharge } \\
\left(\mathrm{ft}^{3} / \mathrm{s}\right)\end{array}$ & $\begin{array}{c}\text { Water year } \\
\text { of } \\
\text { occurrence }\end{array}$ & $\begin{array}{c}\text { Discharge } \\
\left(\mathrm{ft}^{3} / \mathrm{s}\right)\end{array}$ & $\begin{array}{l}\text { Standard } \\
\text { deviation } \\
\left(\mathrm{ft}^{3} / \mathrm{s}\right)\end{array}$ & $\begin{array}{l}\text { Coeffi- } \\
\text { cient of } \\
\text { variation }\end{array}$ & $\begin{array}{l}\text { Percentage } \\
\text { of annual } \\
\text { discharge }\end{array}$ \\
\hline October & 57.7 & 1942 & 0 & $\mathrm{~m}$ & 3.46 & 12.2 & 3.51 & 3.52 \\
\hline November & 8.60 & 1945 & 0 & $\mathrm{~m}$ & 0.900 & 2.11 & 2.36 & 0.91 \\
\hline December & 0.397 & 1945 & 0 & $\mathrm{~m}$ & 0.060 & 0.12 & 1.93 & 0.07 \\
\hline January & 0.213 & 1948 & 0 & $\mathrm{~m}$ & 0.010 & 0.05 & 3.93 & 0.01 \\
\hline February & 0.048 & 1948 & 0 & $\mathrm{~m}$ & 0 & 0.01 & 4.80 & 0 \\
\hline March & 38.6 & 1942 & 0 & $\mathrm{~m}$ & 3.48 & 9.81 & 2.82 & 3.54 \\
\hline April & 108 & 1942 & 0.100 & 1953 & 26.7 & 33.2 & 1.24 & 27.2 \\
\hline May & 635 & 1950 & 0.006 & 1934 & 38.8 & 126 & 3.24 & 39.5 \\
\hline June & 235 & 1950 & 0 & $\mathrm{~m}$ & 16.4 & 47.0 & 2.86 & 16.7 \\
\hline July & 86.6 & 1950 & 0 & $\mathrm{~m}$ & 5.24 & 17.5 & 3.34 & 5.33 \\
\hline August & 5.76 & 1950 & 0 & $\mathrm{~m}$ & 0.630 & 1.48 & 2.37 & 0.64 \\
\hline September & 47.1 & 1941 & 0 & $\mathrm{~m}$ & 2.57 & 9.39 & 3.65 & 2.61 \\
\hline Annual & 88.7 & 1950 & 0.096 & 1934 & 8.52 & 18.4 & 2.16 & 100 \\
\hline
\end{tabular}

Annual flow duration

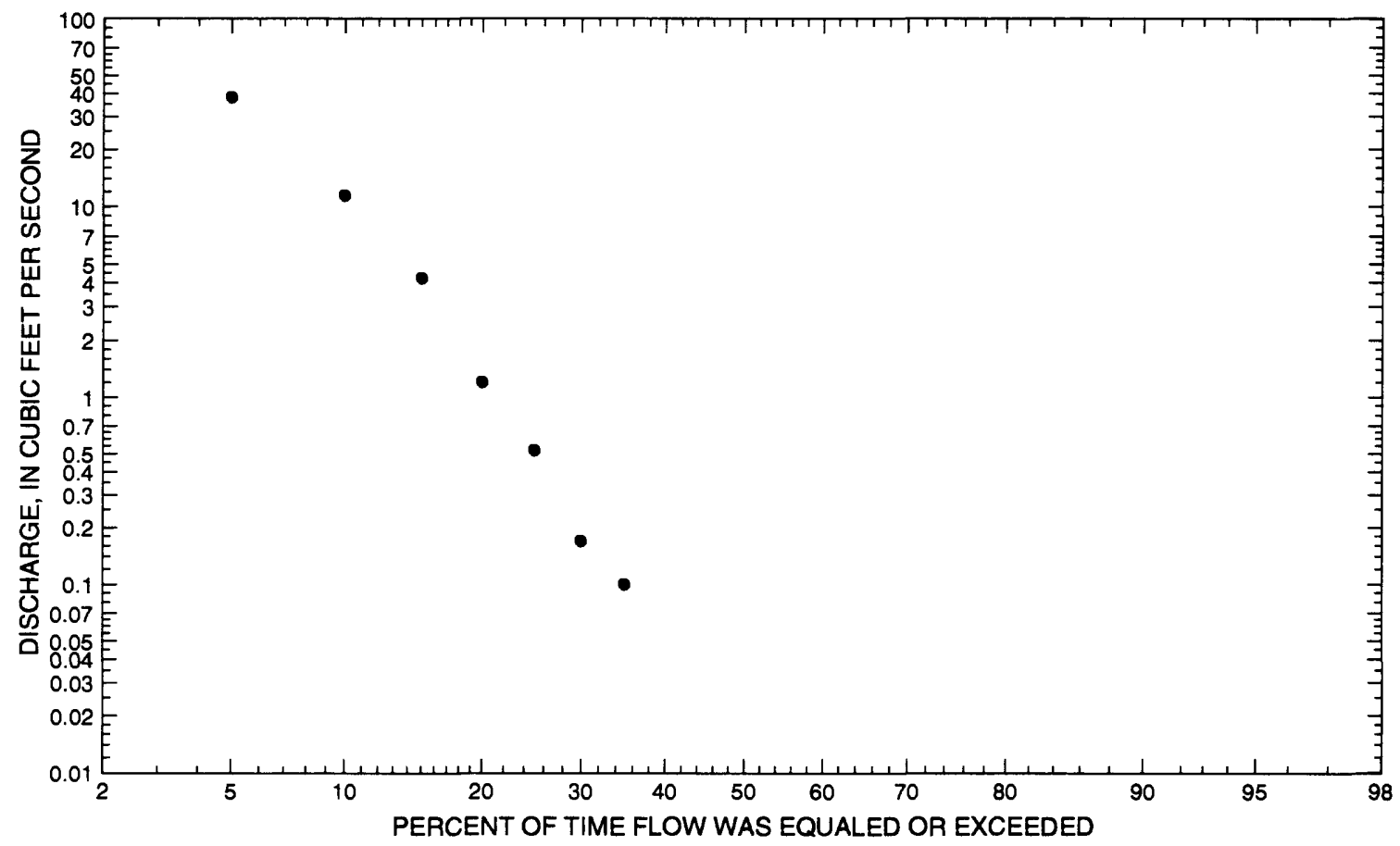




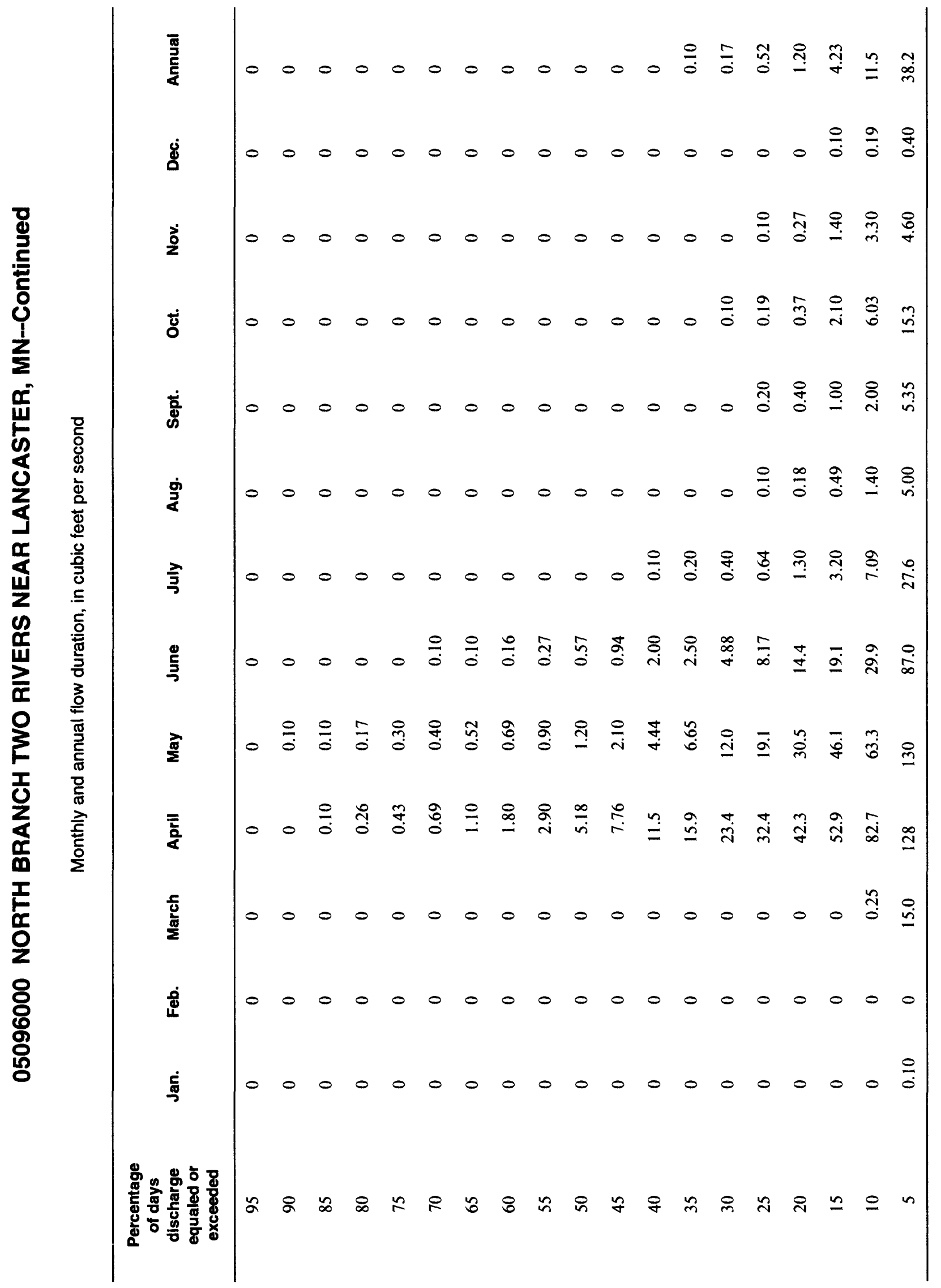


05096000 NORTH BRANCH TWO RIVERS NEAR LANCASTER, MN--Continued

Probability of occurrence of annual high discharges

[ng, statistic not given]

\begin{tabular}{|c|c|c|c|c|c|c|}
\hline \multirow[b]{2}{*}{$\begin{array}{l}\text { Exceedance } \\
\text { probability }\end{array}$} & \multirow[b]{2}{*}{$\begin{array}{l}\text { Recurrence } \\
\text { intervai } \\
\text { (years) }\end{array}$} & \multirow[b]{2}{*}{$\begin{array}{c}\text { Maximum } \\
\text { instantaneous } \\
\left(\mathrm{ft}^{3} / \mathrm{s}\right)\end{array}$} & \multicolumn{4}{|c|}{$\begin{array}{l}\text { Maximum mean discharge } \\
\left(\mathrm{ft}^{3} / \mathrm{s}\right)\end{array}$} \\
\hline & & & 3-day period & 7-day period & 15-day period & 30-day period \\
\hline 0.99 & 1.01 & 1.80 & 1.22 & 0.916 & 0.598 & 0.328 \\
\hline 0.95 & 1.05 & 6.30 & 4.38 & 3.20 & 2.07 & 1.17 \\
\hline 0.90 & 1.11 & 11.7 & 8.30 & 6.03 & 3.91 & 2.27 \\
\hline 0.80 & 1.25 & 23.9 & 17.3 & 12.6 & 8.25 & 4.94 \\
\hline 0.50 & 2 & 83.4 & 62.9 & 46.8 & 32.2 & 20.7 \\
\hline 0.20 & 5 & 252 & 197 & 155 & 115 & 80.5 \\
\hline 0.10 & 10 & 426 & 338 & 276 & 216 & 159 \\
\hline 0.04 & 25 & 716 & 576 & 496 & 411 & 322 \\
\hline 0.02 & 50 & 980 & 796 & 711 & 616 & 501 \\
\hline 0.01 & 100 & 1,280 & 1,050 & 970 & 878 & 742 \\
\hline 0.005 & 200 & 1,620 & 1,340 & 1,280 & 1,200 & 1,050 \\
\hline 0.002 & 500 & 2,120 & $\mathrm{ng}$ & ng & ng & $\mathrm{ng}$ \\
\hline
\end{tabular}

Probability of occurrence of annual low discharges

[ng, statistic not given]

\begin{tabular}{|c|c|c|c|c|c|c|c|c|c|c|}
\hline \multirow{3}{*}{$\begin{array}{l}\text { Non- } \\
\text { exceed- } \\
\text { ance } \\
\text { prob- } \\
\text { ability }\end{array}$} & \multirow{3}{*}{$\begin{array}{c}\text { Recur- } \\
\text { rence } \\
\text { inter- } \\
\text { vai } \\
\text { (years) }\end{array}$} & \multicolumn{9}{|c|}{ Minimum mean discharge $\left(\mathrm{ft}^{3} / \mathrm{s}\right)$} \\
\hline & & \multicolumn{9}{|c|}{ Number of consecutive days } \\
\hline & & 1 & 3 & 7 & 14 & 30 & 60 & 90 & 120 & 183 \\
\hline 0.05 & 20 & $\mathrm{ng}$ & $\mathrm{ng}$ & ng & ng & $\mathrm{ng}$ & $\mathrm{ng}$ & $\mathrm{ng}$ & 0 & 0 \\
\hline 0.10 & 10 & $\mathrm{ng}$ & ng & ng & $\mathrm{ng}$ & $\mathrm{ng}$ & ng & $\mathrm{ng}$ & 0 & 0 \\
\hline 0.20 & 5 & ng & ng & ng & $\mathrm{ng}$ & $\mathrm{ng}$ & ng & ng & 0 & 0 \\
\hline 0.50 & 2 & $\mathrm{ng}$ & $\mathrm{ng}$ & $\mathrm{ng}$ & $\mathrm{ng}$ & $\mathrm{ng}$ & $\mathrm{ng}$ & $\mathrm{ng}$ & 0 & 0 \\
\hline
\end{tabular}


05096000 NORTH BRANCH TWO RIVERS NEAR LANCASTER, MN--Continued

Probability of occurrence of seasonal low discharges

[ng, statistic not given]

\begin{tabular}{|c|c|c|c|c|c|c|c|c|c|}
\hline \multirow[b]{3}{*}{$\begin{array}{c}\text { Non- } \\
\text { exceedance } \\
\text { probability }\end{array}$} & \multirow[b]{3}{*}{$\begin{array}{c}\text { Recurrence } \\
\text { intervai } \\
\text { (years) }\end{array}$} & \multicolumn{8}{|c|}{ Minimum mean discharge $\left(\mathrm{ft}^{3} / \mathrm{s}\right)$} \\
\hline & & \multicolumn{8}{|c|}{ Number of consecutive days } \\
\hline & & 1 & 7 & 14 & 30 & 1 & 7 & 14 & 30 \\
\hline & & \multicolumn{4}{|c|}{ December-January-February } & \multicolumn{4}{|c|}{ March-April-May } \\
\hline 0.05 & 20 & ng & ng & ng & ng & ng & ng & ng & 0 \\
\hline 0.10 & 10 & ng & ng & ng & ng & ng & ng & ng & 0 \\
\hline 0.20 & 5 & ng & ng & ng & ng & ng & ng & ng & 0 \\
\hline \multirow[t]{2}{*}{0.50} & 2 & ng & ng & ng & ng & ng & ng & ng & 0 \\
\hline & & \multicolumn{4}{|c|}{ June-July-August } & \multicolumn{4}{|c|}{ September-October-November } \\
\hline 0.05 & 20 & ng & ng & 0 & 0 & 0 & 0 & 0 & 0 \\
\hline 0.10 & 10 & ng & ng & 0 & 0 & 0 & 0 & 0 & 0 \\
\hline 0.20 & 5 & ng & ng & 0 & 0 & 0 & 0 & 0 & 0 \\
\hline 0.50 & 2 & ng & ng & 0 & 0 & 0 & 0 & 0 & 0 \\
\hline
\end{tabular}


05096000 NORTH BRANCH TWO RIVERS NEAR LANCASTER, MN--Continued

Annual peak discharge and corresponding gage height

$$
[-, \text { no data }]
$$

\begin{tabular}{|c|c|c|c|c|c|c|c|}
\hline $\begin{array}{l}\text { Water } \\
\text { year }\end{array}$ & Date & $\begin{array}{c}\text { Gage } \\
\text { height } \\
\text { (feet) }\end{array}$ & $\begin{array}{c}\text { Peak } \\
\text { discharge } \\
\left(\mathrm{ft}^{3} / \mathrm{s}\right)\end{array}$ & $\begin{array}{l}\text { Water } \\
\text { year }\end{array}$ & Date & $\begin{array}{c}\text { Gage } \\
\text { height } \\
\text { (feet) }\end{array}$ & $\begin{array}{c}\text { Peak } \\
\text { discharge } \\
\left(\mathrm{ft}^{3} / \mathrm{s}\right)\end{array}$ \\
\hline \multicolumn{8}{|c|}{ Annual peak discharge, by year, and corresponding gage height } \\
\hline 1930 & May 12 & 3.10 & 226 & 1944 & June 6 & 3.52 & 203 \\
\hline 1931 & April 8 & 1.90 & 38.0 & 1945 & March 27 & 2.88 & 124 \\
\hline 1932 & April 15 & 1.90 & 59.0 & 1946 & March 20 & 2.38 & 56.0 \\
\hline 1933 & May 26 & 2.68 & 126 & 1947 & June 11 & 3.40 & 217 \\
\hline 1934 & April 9 & 1.06 & 15.0 & 1948 & April 18 & 3.68 & 281 \\
\hline 1935 & April 15 & -- & 12.0 & 1949 & April 13 & - & 50.0 \\
\hline 1936 & April 14 & 1.20 & 8.00 & 1950 & May 20 & 6.25 & 912 \\
\hline 1937 & April 30 & 3.80 & 277 & 1951 & April 30 & 2.91 & 173 \\
\hline 1938 & June 2 & 1.20 & 8.00 & 1952 & April 8 & 1.22 & 21.0 \\
\hline 1941 & September 25 & 4.06 & 290 & 1953 & June 29 & 0.75 & 4.00 \\
\hline 1942 & April 4 & -- & 253 & 1954 & June 15 & 1.97 & 50.0 \\
\hline 1943 & April 5 & 3.50 & 175 & 1955 & April 22 & 2.92 & 151 \\
\hline \multicolumn{8}{|c|}{ Annual peak discharge, from highest to lowest, and corresponding gage height } \\
\hline 1950 & May 20 & 6.25 & 912 & 1945 & March 27 & 2.88 & 124 \\
\hline 1941 & September 25 & 4.06 & 290 & 1932 & April 15 & 1.90 & 59.0 \\
\hline 1948 & April 18 & 3.68 & 281 & 1946 & March 20 & 2.38 & 56.0 \\
\hline 1937 & April 30 & 3.80 & 277 & 1949 & April 13 & - & 50.0 \\
\hline 1942 & April 4 & - & 253 & 1954 & June 15 & 1.97 & 50.0 \\
\hline 1930 & May 12 & 3.10 & 226 & 1931 & April 8 & 1.90 & 38.0 \\
\hline 1947 & June 11 & 3.40 & 217 & 1952 & April 8 & 1.22 & 21.0 \\
\hline 1944 & June 6 & 3.52 & 203 & 1934 & April 9 & 1.06 & 15.0 \\
\hline 1943 & April 5 & 3.50 & 175 & 1935 & April 15 & -- & 12.0 \\
\hline 1951 & April 30 & 2.91 & 173 & 1936 & April 14 & 1.20 & 8.00 \\
\hline 1955 & April 22 & 2.92 & 151 & 1938 & June 2 & 1.20 & 8.00 \\
\hline 1933 & May 26 & 2.68 & 126 & 1953 & June 29 & 0.75 & 4.00 \\
\hline
\end{tabular}




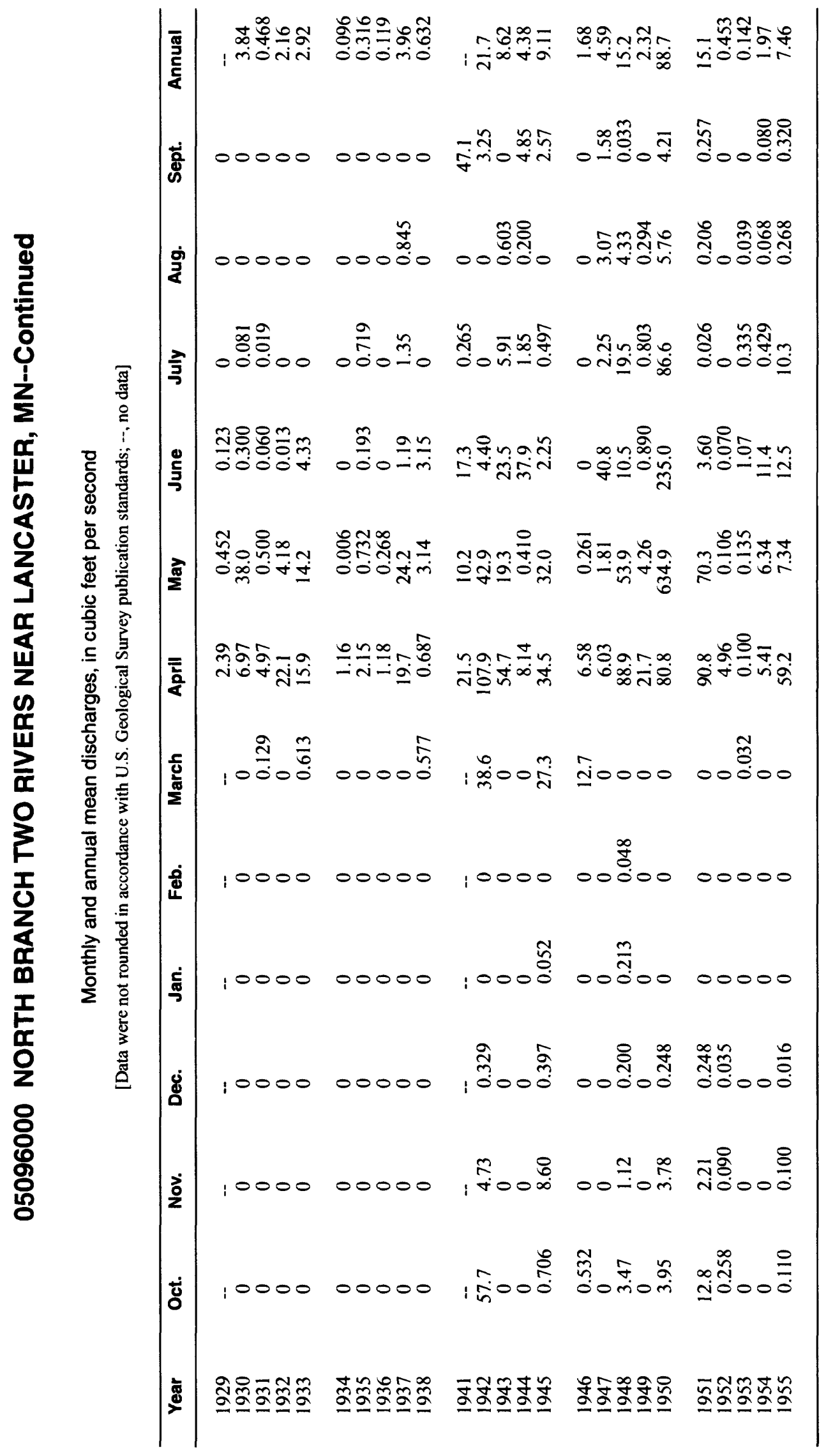




\section{STATE DITCH 85 NEAR LANCASTER, MN}

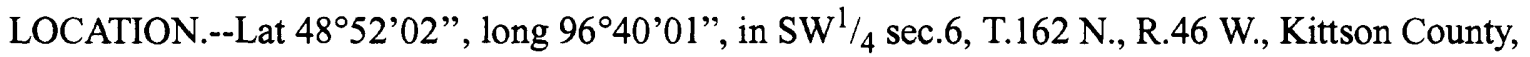
Hydrologic Unit 09020312, on left bank at upstream side of highway bridge, 1 mi upstream from North Branch Two Rivers, and $7 \mathrm{mi}$ northeast of Lancaster.

DRAINAGE AREA.--95 $\mathrm{mi}^{2}$, approximately.

PERIOD OF RECORD.--April 1929 to September 1938, March 1942 to September 1955.

GAGE.--Staff gage. Datum of gage is $969.28 \mathrm{ft}$ above mean sea level, adjustment of 1912 (levels by U.S. Army Corps of Engineers).

EXTREMES FOR PERIOD OF RECORD.--Maximum discharge, 1,480 $\mathrm{ft}^{3} / \mathrm{s}$, May 20, 1950; gage height, $5.90 \mathrm{ft}$; maximum gage height, $6.30 \mathrm{ft}$, Mar. 29, 1942, backwater from ice; no flow for several months each year.

Annual mean discharge

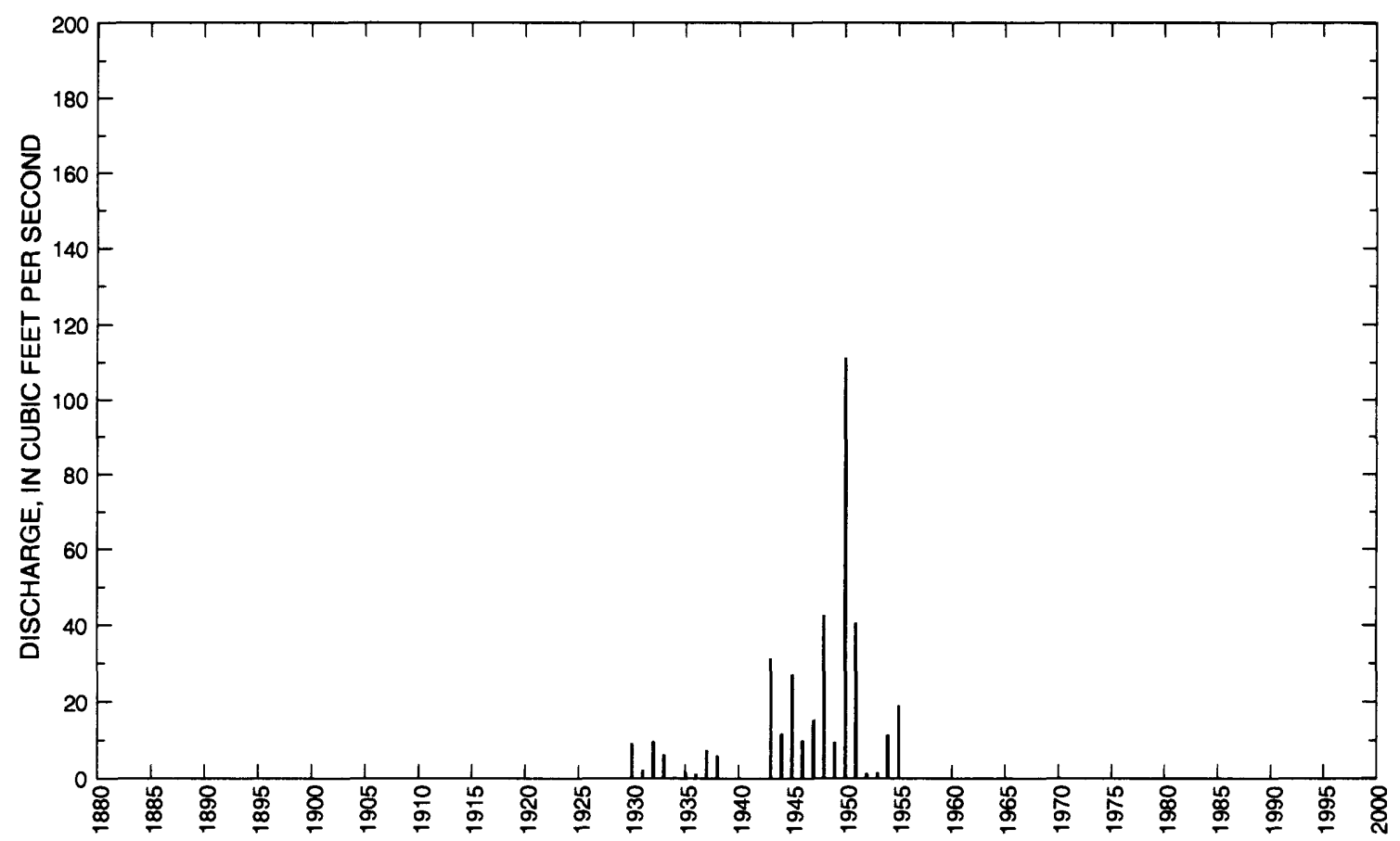




\section{STATE DITCH 85 NEAR LANCASTER, MN--Continued}

Statistics of monthly and annual mean discharges

[ $\mathrm{m}$, more than 1 year of occurrence; $\mathrm{ng}$, statistic not given]

\begin{tabular}{|c|c|c|c|c|c|c|c|c|}
\hline \multirow[b]{2}{*}{ Month } & \multicolumn{2}{|c|}{ Maximum } & \multicolumn{2}{|c|}{ Minimum } & \multicolumn{4}{|c|}{ Mean } \\
\hline & $\begin{array}{c}\text { Discharge } \\
\left(\mathrm{ft}^{3} / \mathbf{s}\right)\end{array}$ & $\begin{array}{c}\text { Water year } \\
\text { of } \\
\text { occurrence }\end{array}$ & $\begin{array}{c}\text { Discharge } \\
\left(\mathrm{ft}^{3} / \mathrm{s}\right)\end{array}$ & $\begin{array}{c}\text { Water year } \\
\text { of } \\
\text { occurrence }\end{array}$ & $\begin{array}{c}\text { Discharge } \\
\left(\mathrm{ft}^{3} / \mathbf{s}\right)\end{array}$ & $\begin{array}{c}\text { Standard } \\
\text { deviation } \\
\left(\mathrm{ft}^{3} / \mathrm{s}\right)\end{array}$ & $\begin{array}{l}\text { Coeffi- } \\
\text { cient of } \\
\text { variation }\end{array}$ & $\begin{array}{l}\text { Percentage } \\
\text { of annuai } \\
\text { discharge }\end{array}$ \\
\hline October & 43.5 & 1951 & 0 & $\mathrm{~m}$ & 3.70 & 9.74 & 2.63 & 1.74 \\
\hline November & 7.88 & 1945 & 0 & $\mathrm{~m}$ & 1.37 & 2.57 & 1.88 & 0.64 \\
\hline December & 0.945 & 1948 & 0 & $\mathrm{~m}$ & 0.070 & 0.21 & 2.87 & 0.04 \\
\hline January & 0.084 & 1948 & 0 & $\mathrm{~m}$ & 0 & 0.02 & 4.69 & 0 \\
\hline February & 0 & $\mathrm{~m}$ & 0 & $\mathrm{~m}$ & 0 & 0 & ng & 0 \\
\hline March & 55.2 & 1945 & 0 & $\mathrm{~m}$ & 5.35 & 14.0 & 2.62 & 2.52 \\
\hline April & 245 & 1942 & 0.267 & 1953 & 62.2 & 64.2 & 1.03 & 29.3 \\
\hline May & 714 & 1950 & 0.081 & 1934 & 78.2 & 151 & 1.94 & 36.8 \\
\hline June & 297 & 1950 & 0.107 & 1952 & 39.6 & 65.0 & 1.64 & 18.6 \\
\hline July & 175 & 1950 & 0 & $\mathrm{~m}$ & 14.3 & 36.6 & 2.56 & 6.71 \\
\hline August & 34.0 & 1950 & 0 & $\mathrm{~m}$ & 3.60 & 7.89 & 2.19 & 1.70 \\
\hline September & 39.8 & 1942 & 0 & $\mathrm{~m}$ & 4.24 & 9.84 & 2.32 & 2.00 \\
\hline Annual & 111 & 1950 & 0.276 & 1934 & 17.1 & 24.4 & 1.43 & 100 \\
\hline
\end{tabular}

Annual flow duration

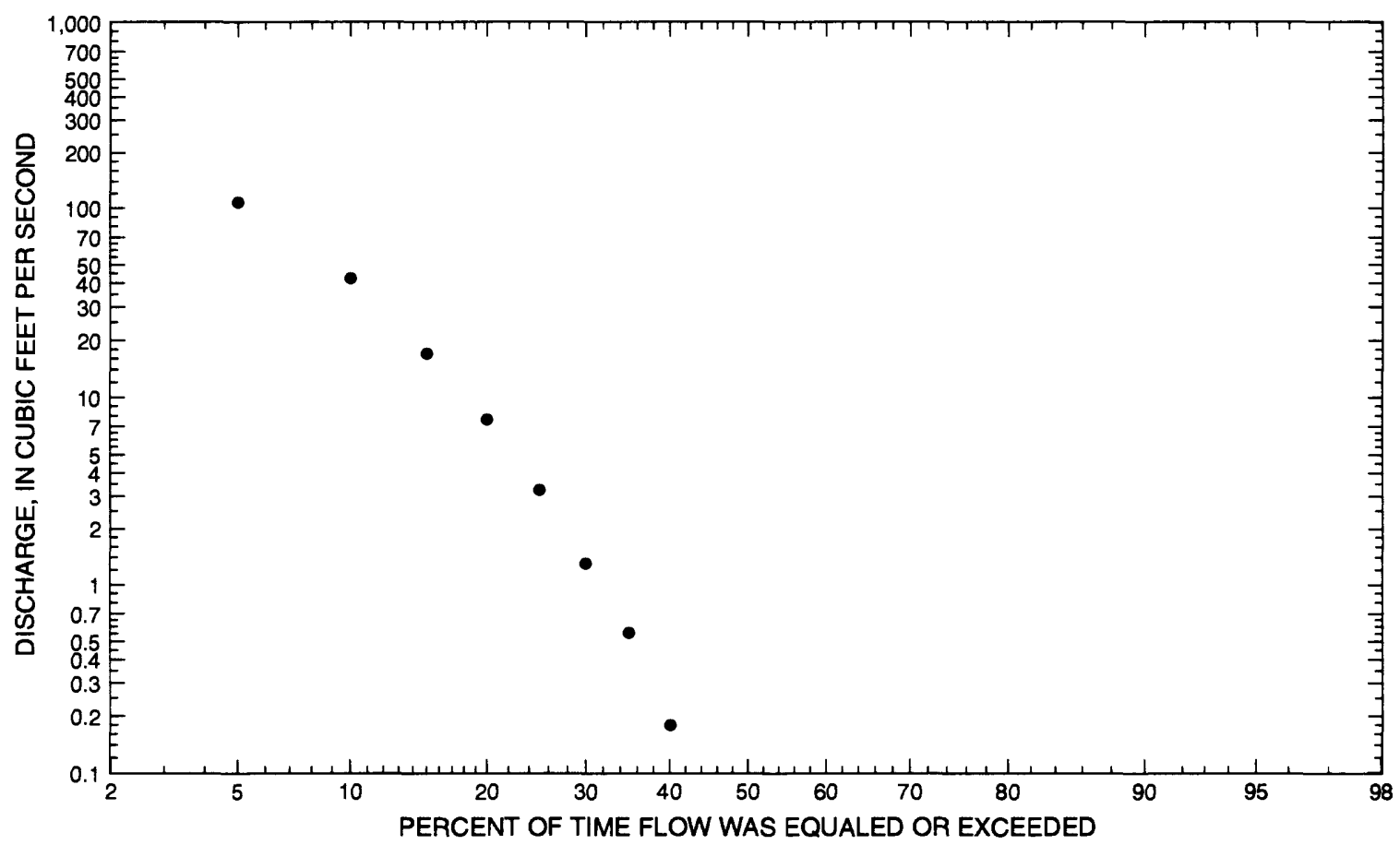




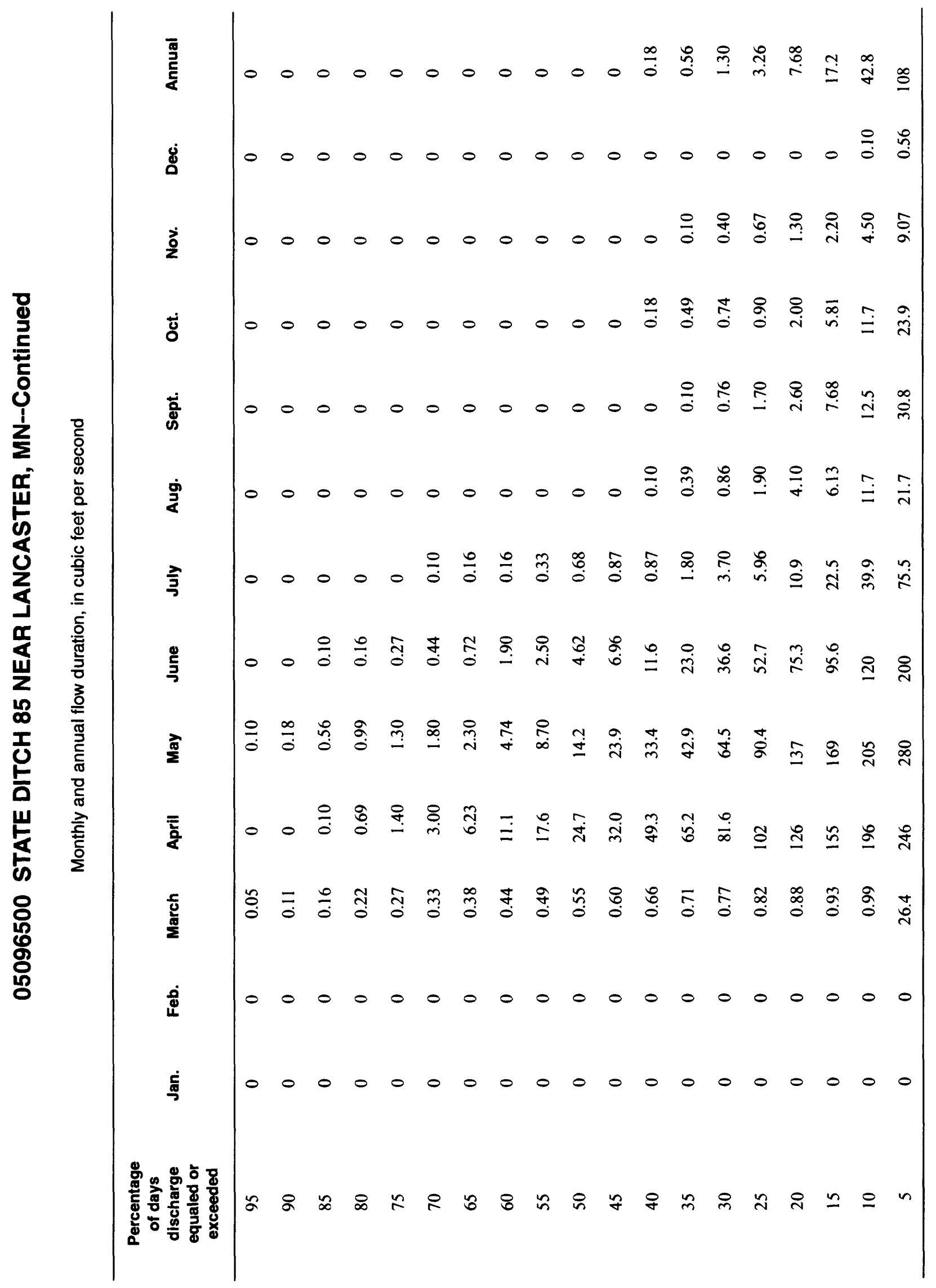




\section{STATE DITCH 85 NEAR LANCASTER, MN--Continued}

Probability of occurrence of annual high discharges

[ng, statistic not given]

\begin{tabular}{|c|c|c|c|c|c|c|}
\hline \multirow[b]{2}{*}{$\begin{array}{l}\text { Exceedance } \\
\text { probability }\end{array}$} & \multirow[b]{2}{*}{$\begin{array}{l}\text { Recurrence } \\
\text { interval } \\
\text { (years) }\end{array}$} & \multirow[b]{2}{*}{$\begin{array}{c}\text { Maximum } \\
\text { instantaneous } \\
\left(\mathrm{ft}^{3} / \mathbf{s}\right)\end{array}$} & \multicolumn{4}{|c|}{$\begin{array}{l}\text { Maximum mean discharge } \\
\qquad\left(\mathrm{ft}^{3} / \mathrm{s}\right)\end{array}$} \\
\hline & & & 3-day period & 7-day period & 15-day period & 30-day period \\
\hline 0.99 & 1.01 & ng & 7.38 & 6.67 & 4.34 & 2.24 \\
\hline 0.95 & 1.05 & 33.5 & 18.3 & 15.9 & 11.4 & 6.86 \\
\hline 0.90 & 1.11 & 45.2 & 28.8 & 24.7 & 18.5 & 11.9 \\
\hline 0.80 & 1.25 & 65.2 & 48.4 & 41.3 & 32.4 & 22.4 \\
\hline 0.50 & 2 & 132 & 120 & 104 & 87.0 & 67.0 \\
\hline 0.20 & 5 & 272 & 266 & 241 & 210 & 173 \\
\hline 0.10 & 10 & 399 & 387 & 364 & 319 & 269 \\
\hline 0.04 & 25 & 602 & 560 & 552 & 485 & 415 \\
\hline 0.02 & 50 & 786 & 700 & 713 & 626 & 536 \\
\hline 0.01 & 100 & 1,000 & 846 & 892 & 778 & 667 \\
\hline 0.005 & 200 & 1,250 & 998 & 1,090 & 943 & 806 \\
\hline 0.002 & 500 & 1,640 & $n g$ & ng & ng & ng \\
\hline
\end{tabular}

Probability of occurrence of annual low discharges

[ng, statistic not given]

\begin{tabular}{|c|c|c|c|c|c|c|c|c|c|c|}
\hline \multirow{3}{*}{$\begin{array}{l}\text { Non- } \\
\text { exceed- } \\
\text { ance } \\
\text { prob- } \\
\text { ability }\end{array}$} & \multirow{3}{*}{$\begin{array}{c}\text { Recur- } \\
\text { rence } \\
\text { inter- } \\
\text { val } \\
\text { (years) }\end{array}$} & \multicolumn{9}{|c|}{ Minimum mean discharge $\left(\mathrm{ft}^{3} / \mathrm{s}\right)$} \\
\hline & & \multicolumn{9}{|c|}{ Number of consecutive days } \\
\hline & & 1 & 3 & 7 & 14 & 30 & 60 & 90 & 120 & 183 \\
\hline 0.05 & 20 & ng & ng & $\mathrm{ng}$ & $\mathrm{ng}$ & $\mathrm{ng}$ & $\mathrm{ng}$ & $\mathrm{ng}$ & 0 & 0 \\
\hline 0.10 & 10 & ng & ng & ng & ng & $\mathrm{ng}$ & ng & $\mathrm{ng}$ & 0 & 0 \\
\hline 0.20 & 5 & ng & $\mathrm{ng}$ & ng & ng & ng & ng & ng & 0 & 0 \\
\hline 0.50 & 2 & $\mathrm{ng}$ & $\mathrm{ng}$ & $\mathrm{ng}$ & $\mathrm{ng}$ & $\mathrm{ng}$ & $\mathrm{ng}$ & $\mathrm{ng}$ & 0 & 0 \\
\hline
\end{tabular}




\section{STATE DITCH 85 NEAR LANCASTER, MN--Continued}

Probability of occurrence of seasonal low discharges

[ng, statistic not given]

\begin{tabular}{|c|c|c|c|c|c|c|c|c|c|}
\hline \multirow[b]{3}{*}{$\begin{array}{l}\text { Non- } \\
\text { exceedance } \\
\text { probability }\end{array}$} & \multirow[b]{3}{*}{$\begin{array}{l}\text { Recurrence } \\
\text { interval } \\
\text { (years) }\end{array}$} & \multicolumn{8}{|c|}{ Minimum mean discharge $\left(\mathrm{ft}^{3} / \mathrm{s}\right)$} \\
\hline & & \multicolumn{8}{|c|}{ Number of consecutive days } \\
\hline & & 1 & 7 & 14 & 30 & 1 & 7 & 14 & 30 \\
\hline & & \multicolumn{4}{|c|}{ December-January-February } & \multicolumn{4}{|c|}{ March-April-May } \\
\hline 0.05 & 20 & ng & ng & ng & ng & ng & ng & ng & 0 \\
\hline 0.10 & 10 & ng & ng & ng & ng & ng & ng & ng & 0 \\
\hline 0.20 & 5 & ng & ng & ng & ng & ng & ng & ng & 0 \\
\hline \multirow[t]{2}{*}{0.50} & 2 & ng & ng & ng & ng & ng & ng & ng & 0 \\
\hline & & \multicolumn{4}{|c|}{ June-July-August } & \multicolumn{4}{|c|}{ September-October-November } \\
\hline 0.05 & 20 & 0 & 0 & 0 & 0 & 0 & 0 & 0 & 0 \\
\hline 0.10 & 10 & 0 & 0 & 0 & 0 & 0 & 0 & 0 & 0 \\
\hline 0.20 & 5 & 0 & 0 & 0 & 0 & 0 & 0 & 0 & 0 \\
\hline 0.50 & 2 & 0 & 0 & 0 & 0 & 0 & 0 & 0 & 0 \\
\hline
\end{tabular}


05096500 STATE DITCH 85 NEAR LANCASTER, MN--Continued

Annual peak discharge and corresponding gage height

$$
[--, \text { no data }]
$$

\begin{tabular}{|c|c|c|c|c|c|c|c|}
\hline $\begin{array}{l}\text { Water } \\
\text { Year }\end{array}$ & Date & $\begin{array}{c}\text { Gage } \\
\text { height } \\
\text { (feet) }\end{array}$ & $\begin{array}{c}\text { Peak } \\
\text { discharge } \\
\left(\mathrm{ft}^{3} / \mathrm{s}\right)\end{array}$ & $\begin{array}{l}\text { Water } \\
\text { year }\end{array}$ & Date & $\begin{array}{c}\text { Gage } \\
\text { height } \\
\text { (feet) }\end{array}$ & $\begin{array}{c}\text { Peak } \\
\text { discharge } \\
\left(\mathrm{tt}^{3} / \mathrm{s}\right)\end{array}$ \\
\hline \multicolumn{8}{|c|}{ Annual peak discharge, by year, and corresponding gage height } \\
\hline 1929 & April 8 & 3.28 & 102 & 1944 & June 6 & 4.48 & 190 \\
\hline 1930 & May 19 & 4.23 & 160 & 1945 & May 3 & 4.50 & 180 \\
\hline 1931 & April 9 & 3.12 & 99.0 & 1946 & March 22 & 4.00 & 113 \\
\hline 1932 & April 18 & 4.90 & 202 & 1947 & June 13 & 4.74 & 218 \\
\hline 1933 & April 18 & 3.52 & 112 & 1948 & May 2 & 5.26 & 288 \\
\hline 1934 & April 12 & 1.14 & 11.0 & 1949 & April 13 & 4.12 & 136 \\
\hline 1935 & Apirl 22 & -- & 47.0 & 1950 & May 20 & 5.90 & 1,480 \\
\hline 1936 & April 25 & -- & 22.0 & 1951 & April 30 & 4.90 & 250 \\
\hline 1937 & May 1 & -- & 180 & 1952 & April 8 & 2.20 & 57.0 \\
\hline 1938 & June 3 & 3.42 & 60.0 & 1953 & June 7 & 2.28 & 56.0 \\
\hline 1942 & April 5 & -- & 298 & 1954 & June 17 & 3.70 & 124 \\
\hline 1943 & June 6 & 4.47 & 190 & 1955 & April 24 & 4.76 & 211 \\
\hline \multicolumn{8}{|c|}{ Annual peak discharge, from highest to lowest, and corresponding gage height } \\
\hline 1950 & May 20 & 5.90 & 1,480 & 1949 & April 13 & 4.12 & 136 \\
\hline 1942 & April 5 & -- & 298 & 1954 & June 17 & 3.70 & 124 \\
\hline 1948 & May 2 & 5.26 & 288 & 1946 & March 22 & 4.00 & 113 \\
\hline 1951 & April 30 & 4.90 & 250 & 1933 & April 18 & 3.52 & 112 \\
\hline 1947 & June 13 & 4.74 & 218 & 1929 & April 8 & 3.28 & 102 \\
\hline 1955 & April 24 & 4.76 & 211 & 1931 & April 9 & 3.12 & 99.0 \\
\hline 1932 & April 18 & 4.90 & 202 & 1938 & June 3 & 3.42 & 60.0 \\
\hline 1943 & June 6 & 4.47 & 190 & 1952 & April 8 & 2.20 & 57.0 \\
\hline 1944 & June 6 & 4.28 & 190 & 1953 & June 7 & 2.28 & 56.0 \\
\hline 1937 & May 1 & -- & 180 & 1935 & Apirl 22 & -- & 47.0 \\
\hline 1945 & May 3 & 4.50 & 180 & 1936 & April 25 & -- & 22.0 \\
\hline 1930 & May 19 & 4.23 & 160 & 1934 & April 12 & 1.14 & 11.0 \\
\hline
\end{tabular}




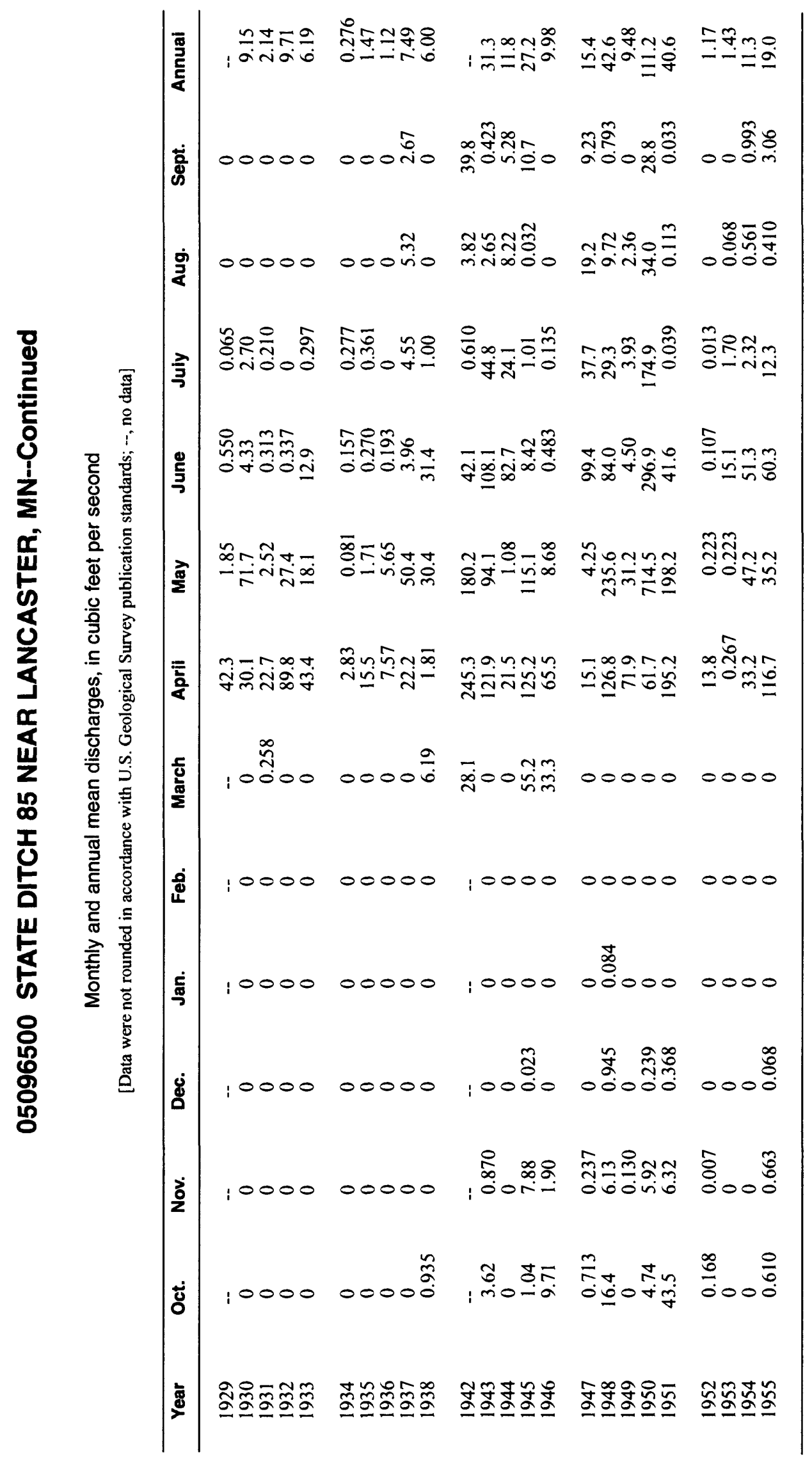


LOCATION.--Lat 4857'10”, long 99²5'35”, in SE $1 / 4 \mathrm{SW}^{1 / 4} \mathrm{sec} .11$, T.163 N., R.68 W., Towner County, Hydrologic Unit 09020313, on right bank $400 \mathrm{ft}$ downstream from bridge on county highway, and $2.5 \mathrm{mi}$ west of Hansboro.

DRAINAGE AREA.--38 $\mathrm{mi}^{2}$, approximately.

PERIOD OF RECORD.--October 1961 to current year.

GAGE.--Water-stage recorder. Datum of gage is $1,615 \mathrm{ft}$ above sea level, from topographic map. Prior to May 20, 1962, nonrecording gage $400 \mathrm{ft}$ upstream at same datum.

EXTREMES FOR PERIOD OF RECORD.--Maximum discharge, 1,200 $\mathrm{ft}^{3} / \mathrm{s}$, Apr. 23, 1979, gage height, $10.50 \mathrm{ft}$, from floodmark, backwater from ice; no flow at times.

Annual mean discharge

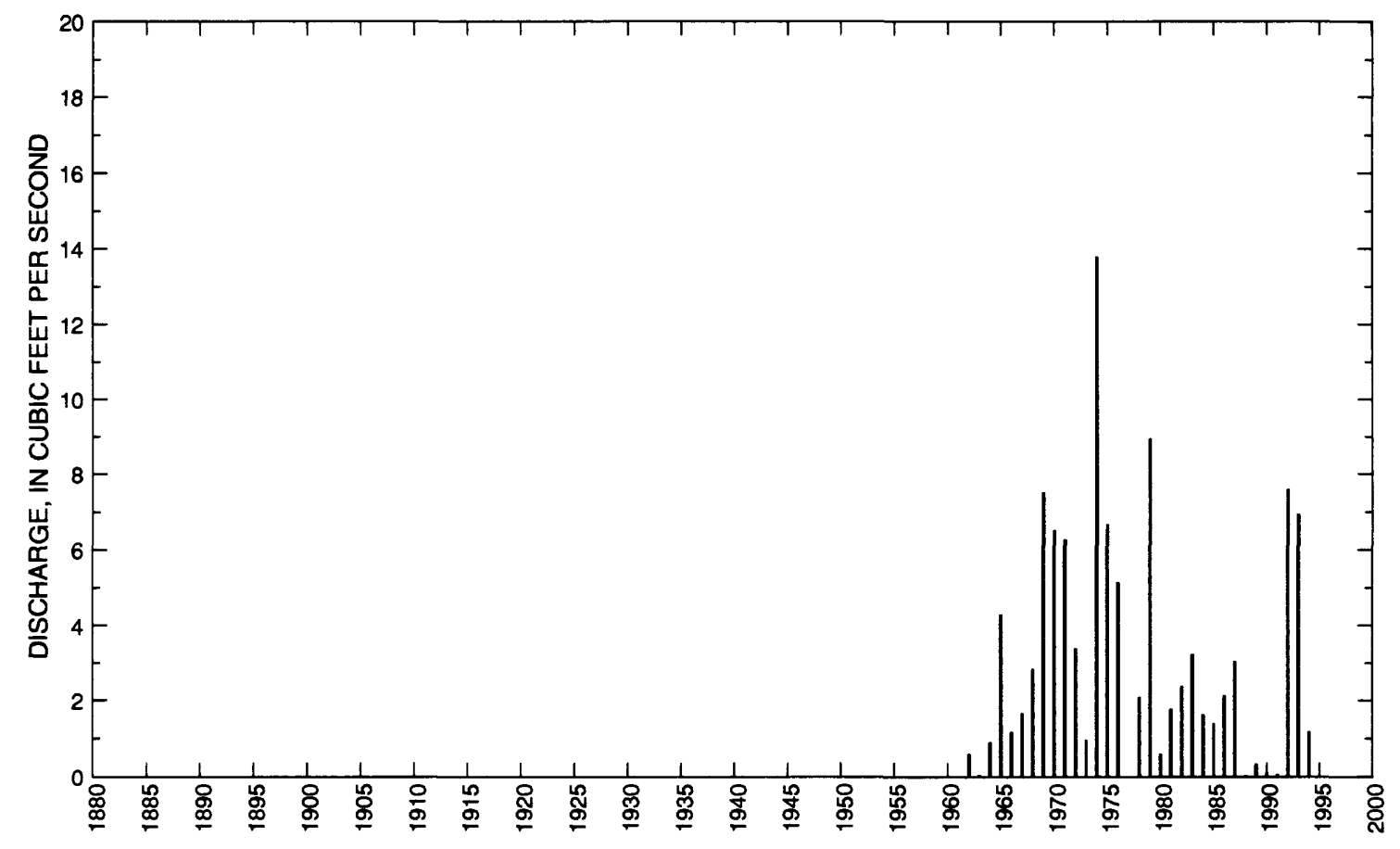




\section{HIDDEN ISLAND COULEE NEAR HANSBORO, ND--Continued}

Statistics of monthly and annual mean discharges

[ $\mathrm{m}$, more than 1 year of occurrence]

\begin{tabular}{|c|c|c|c|c|c|c|c|c|}
\hline \multirow[b]{2}{*}{ Month } & \multicolumn{2}{|c|}{ Maximum } & \multicolumn{2}{|c|}{ Minimum } & \multicolumn{4}{|c|}{ Mean } \\
\hline & $\begin{array}{c}\text { Discharge } \\
\left(\mathrm{ft}^{3} / \mathrm{s}\right)\end{array}$ & $\begin{array}{c}\text { Water year } \\
\text { of } \\
\text { occurrence }\end{array}$ & $\begin{array}{c}\text { Discharge } \\
\left(\mathrm{ft}^{3} / \mathrm{s}\right)\end{array}$ & $\begin{array}{c}\text { Water year } \\
\text { of } \\
\text { occurrence }\end{array}$ & $\begin{array}{c}\text { Discharge } \\
\left(\mathrm{ft}^{3} / \mathrm{s}\right)\end{array}$ & $\begin{array}{c}\text { Standard } \\
\text { deviation } \\
\left(\mathrm{ft}^{3} / \mathrm{s}\right)\end{array}$ & $\begin{array}{l}\text { Coeffi- } \\
\text { cient of } \\
\text { variation }\end{array}$ & $\begin{array}{c}\text { Percentage } \\
\text { of annual } \\
\text { discharge }\end{array}$ \\
\hline October & 1.05 & 1981 & 0 & $\mathrm{~m}$ & 0.070 & 0.21 & 2.92 & 0.18 \\
\hline November & 0.538 & 1981 & 0 & $\mathrm{~m}$ & 0.030 & 0.10 & 3.34 & 0.08 \\
\hline December & 1.02 & 1983 & 0 & $\mathrm{~m}$ & 0.030 & 0.18 & 5.47 & 0.08 \\
\hline January & 0.012 & 1994 & 0 & $\mathrm{~m}$ & 0 & 0 & 5.74 & 0 \\
\hline February & 11.4 & 1981 & 0 & $\mathrm{~m}$ & 0.350 & 1.98 & 5.74 & 0.90 \\
\hline March & 77.5 & 1992 & 0 & $\mathrm{~m}$ & 5.56 & 14.0 & 2.51 & 14.4 \\
\hline April & 117 & 1974 & 0.003 & 1963 & 22.0 & 30.7 & 1.39 & 57.3 \\
\hline May & 43.9 & 1974 & 0 & $\mathrm{~m}$ & 4.44 & 9.31 & 2.09 & 11.6 \\
\hline June & 9.34 & 1986 & 0 & $\mathrm{~m}$ & 1.14 & 2.35 & 2.06 & 2.97 \\
\hline July & 57.4 & 1993 & 0 & $\mathrm{~m}$ & 3.09 & 10.5 & 3.41 & 8.04 \\
\hline August & 17.1 & 1968 & 0 & $\mathrm{~m}$ & 1.07 & 3.49 & 3.26 & 2.78 \\
\hline September & 16.7 & 1968 & 0 & $\mathrm{~m}$ & 0.640 & 2.92 & 4.55 & 1.67 \\
\hline Annual & 13.8 & 1974 & 0.003 & 1977 & 3.19 & 3.25 & 1.02 & 100 \\
\hline
\end{tabular}

Annual flow duration

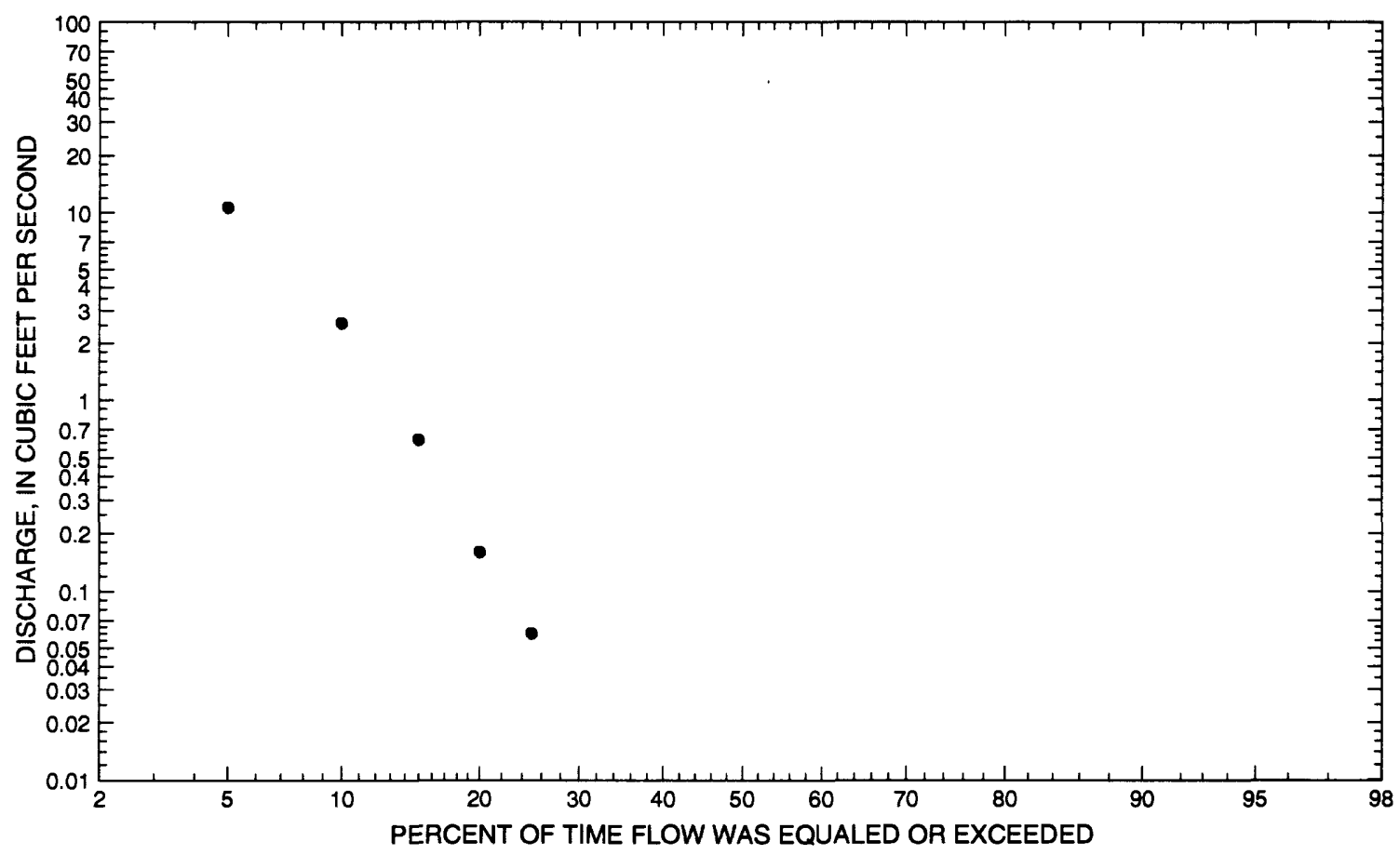




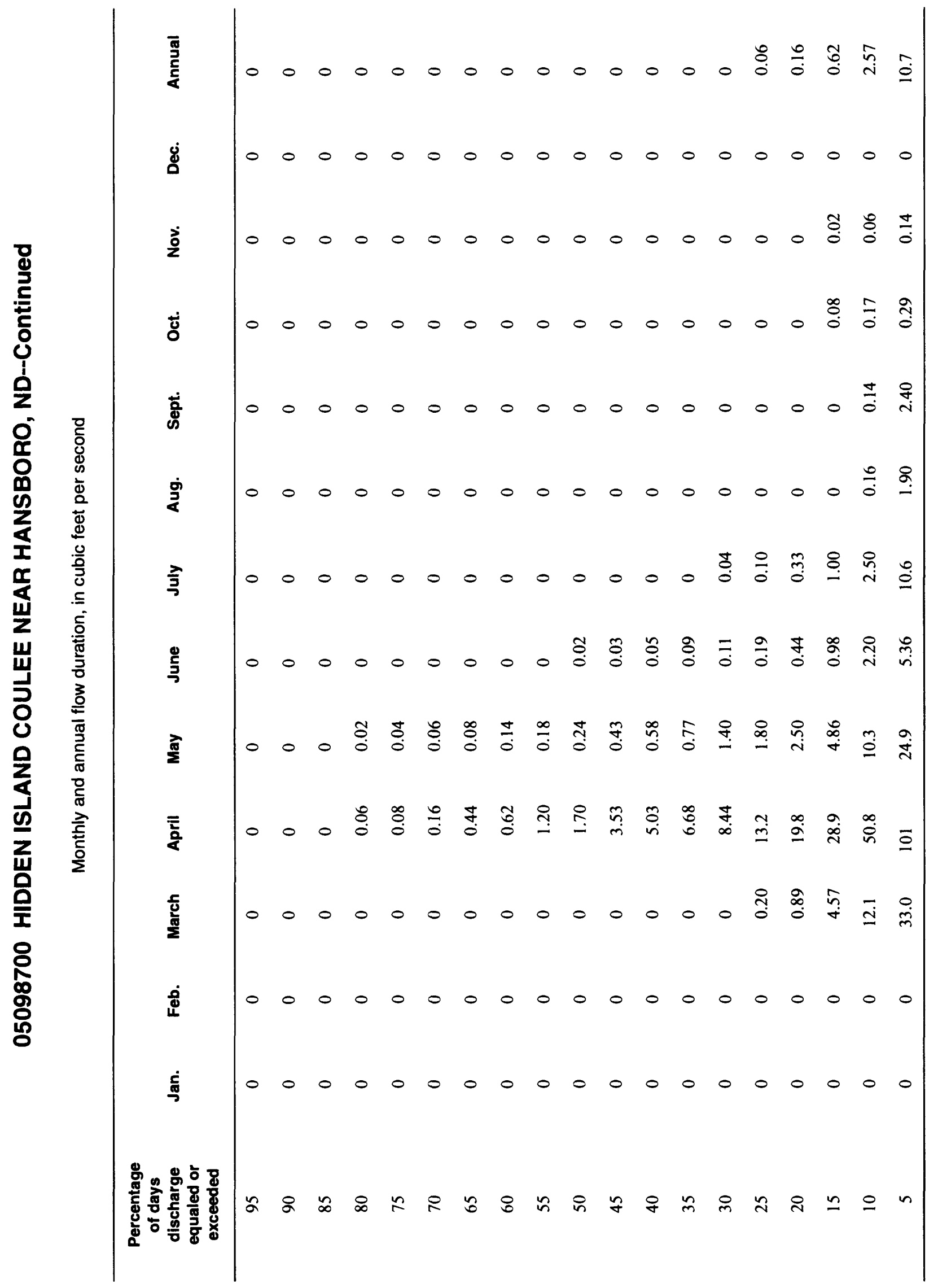


05098700 HIDDEN ISLAND COULEE NEAR HANSBORO, ND--Continued

Probability of occurrence of annual high discharges

[ng, statistic not given]

\begin{tabular}{|c|c|c|c|c|c|c|}
\hline \multirow[b]{2}{*}{$\begin{array}{l}\text { Exceedance } \\
\text { probability }\end{array}$} & \multirow[b]{2}{*}{$\begin{array}{l}\text { Recurrence } \\
\text { interval } \\
\text { (years) }\end{array}$} & \multirow[b]{2}{*}{$\begin{array}{c}\text { Maximum } \\
\text { instantaneous } \\
\left(\mathrm{ft}^{3} / \mathbf{s}\right)\end{array}$} & \multicolumn{4}{|c|}{$\begin{array}{l}\text { Maximum mean discharge } \\
\qquad\left(\mathrm{ft}^{3} / \mathrm{s}\right)\end{array}$} \\
\hline & & & 3-day period & 7-day period & 15-day period & 30-day period \\
\hline 0.99 & 1.01 & ng & 0.069 & 0.064 & 0.041 & 0.021 \\
\hline 0.95 & 1.05 & 7.40 & 1.03 & 0.834 & 0.540 & 0.295 \\
\hline 0.90 & 1.11 & 15.4 & 3.44 & 2.66 & 1.72 & 0.963 \\
\hline 0.80 & 1.25 & 35.2 & 12.1 & 8.92 & 5.74 & 3.31 \\
\hline 0.50 & 2 & 140 & 75.2 & 52.6 & 33.4 & 20.0 \\
\hline 0.20 & 5 & 433 & 238 & 165 & 103 & 62.6 \\
\hline 0.10 & 10 & 715 & 349 & 243 & 150 & 91.8 \\
\hline 0.04 & 25 & 1,140 & 461 & 323 & 197 & 121 \\
\hline 0.02 & 50 & 1,500 & 520 & 368 & 223 & 137 \\
\hline 0.01 & 100 & 1,870 & 563 & 400 & 242 & 149 \\
\hline 0.005 & 200 & 2,250 & 593 & 424 & 255 & 157 \\
\hline 0.002 & 500 & 2,760 & ng & ng & ng & ng \\
\hline
\end{tabular}

Probability of occurrence of annual low discharges

[ng, statistic not given]

\begin{tabular}{|c|c|c|c|c|c|c|c|c|c|c|}
\hline \multirow{3}{*}{$\begin{array}{c}\text { Non- } \\
\text { exceed- } \\
\text { ance } \\
\text { prob- } \\
\text { ability }\end{array}$} & \multirow{3}{*}{$\begin{array}{c}\text { Recur- } \\
\text { rence } \\
\text { inter- } \\
\text { vai } \\
\text { (years) }\end{array}$} & \multicolumn{9}{|c|}{ Minimum mean discharge $\left(\mathrm{ft}^{3} / \mathrm{s}\right)$} \\
\hline & & \multicolumn{9}{|c|}{ Number of consecutive days } \\
\hline & & 1 & 3 & 7 & 14 & 30 & 60 & 90 & 120 & 183 \\
\hline 0.05 & 20 & ng & ng & $\mathrm{ng}$ & $\mathrm{ng}$ & ng & ng & ng & ng & 0 \\
\hline 0.10 & 10 & ng & ng & ng & ng & ng & ng & ng & ng & 0 \\
\hline 0.20 & 5 & ng & ng & ng & ng & ng & ng & ng & ng & 0 \\
\hline 0.50 & 2 & $\mathrm{ng}$ & $\mathrm{ng}$ & $\mathrm{ng}$ & $\mathrm{ng}$ & $\mathrm{ng}$ & $\mathrm{ng}$ & $\mathrm{ng}$ & ng. & 0 \\
\hline
\end{tabular}




\section{HIDDEN ISLAND COULEE NEAR HANSBORO, ND--Continued}

Probability of occurrence of seasonal low discharges

[ng, statistic not given]

\begin{tabular}{|c|c|c|c|c|c|c|c|c|c|}
\hline \multirow[b]{3}{*}{$\begin{array}{c}\text { Non- } \\
\text { exceedance } \\
\text { probability }\end{array}$} & \multirow[b]{3}{*}{$\begin{array}{c}\text { Recurrence } \\
\text { interval } \\
\text { (years) }\end{array}$} & \multicolumn{8}{|c|}{ Minimum mean discharge $\left(\mathrm{ft}^{3} / \mathrm{s}\right)$} \\
\hline & & \multicolumn{8}{|c|}{ Number of consecutive days } \\
\hline & & 1 & 7 & 14 & 30 & 1 & 7 & 14 & 30 \\
\hline & & \multicolumn{4}{|c|}{ December-January-February } & \multicolumn{4}{|c|}{ March-April-May } \\
\hline 0.05 & 20 & ng & ng & $\mathrm{ng}$ & ng & ng & 0 & 0 & 0 \\
\hline 0.10 & 10 & ng & $\mathrm{ng}$ & ng & $\mathrm{ng}$ & ng & 0 & 0 & 0 \\
\hline 0.20 & 5 & $\mathrm{ng}$ & ng & $\mathrm{ng}$ & $\mathrm{ng}$ & ng & 0 & 0 & 0 \\
\hline \multirow[t]{2}{*}{0.50} & 2 & $\mathrm{ng}$ & ng & ng & ng & ng & 0 & 0 & 0.033 \\
\hline & & \multicolumn{4}{|c|}{ June-July-August } & \multicolumn{4}{|c|}{ September-October-November } \\
\hline 0.05 & 20 & ng & ng & $\mathrm{ng}$ & 0 & ng & ng & 0 & 0 \\
\hline 0.10 & 10 & $\mathrm{ng}$ & ng & $\mathrm{ng}$ & 0 & ng & $\mathrm{ng}$ & 0 & 0 \\
\hline 0.20 & 5 & $\mathrm{ng}$ & $\mathrm{ng}$ & $\mathrm{ng}$ & 0 & $\mathrm{ng}$ & $\mathrm{ng}$ & 0 & 0 \\
\hline 0.50 & 2 & ng & $\mathrm{ng}$ & $\mathrm{ng}$ & 0 & $\mathrm{ng}$ & $\mathrm{ng}$ & 0 & 0 \\
\hline
\end{tabular}


05098700 HIDDEN ISLAND COULEE NEAR HANSBORO, ND-Continued

Annual peak discharge and corresponding gage height

$[--$, no data]

\begin{tabular}{|c|c|c|c|c|c|c|c|}
\hline $\begin{array}{l}\text { Water } \\
\text { year }\end{array}$ & Date & $\begin{array}{c}\text { Gage } \\
\text { height } \\
\text { (feet) }\end{array}$ & $\begin{array}{c}\text { Peak } \\
\text { discharge } \\
\left(\mathrm{ft}^{3} / \mathrm{s}\right)\end{array}$ & $\begin{array}{l}\text { Water } \\
\text { year }\end{array}$ & Date & $\begin{array}{c}\text { Gage } \\
\text { height } \\
\text { (feet) }\end{array}$ & $\begin{array}{c}\text { Peak } \\
\text { discharge } \\
\left(\mathrm{ft}^{3} / \mathrm{s}\right)\end{array}$ \\
\hline \multicolumn{8}{|c|}{ Annual peak discharge, by year, and corresponding gage height } \\
\hline 1962 & April 6 & -- & 40.0 & 1979 & April 23 & 10.50 & 1,200 \\
\hline 1963 & July 25 & 5.23 & 8.90 & 1980 & April 3 & 7.51 & 90.0 \\
\hline 1964 & April 9 & -. & 32.0 & 1981 & February 21 & 7.65 & 95.0 \\
\hline 1965 & April 11 & 7.09 & 124 & 1982 & March 30 & 8.01 & 214 \\
\hline 1966 & March 14 & 6.92 & 66.0 & 1983 & April 2 & 7.99 & 155 \\
\hline 1967 & April 21 & 6.73 & 128 & 1984 & March 24 & 7.70 & 264 \\
\hline 1968 & August 24 & 7.17 & 218 & 1985 & March 17 & 7.69 & 60.0 \\
\hline 1969 & April 12 & 8.80 & 700 & 1986 & June 21 & 7.45 & 276 \\
\hline 1970 & April 29 & 7.20 & 317 & 1987 & April 5 & 8.14 & 281 \\
\hline 1971 & April 10 & 8.11 & 637 & 1988 & April 4 & 6.12 & 4.60 \\
\hline 1972 & March 16 & 7.62 & 354 & 1989 & April 8 & 6.23 & 66.0 \\
\hline 1973 & June 20 & 6.51 & 52.0 & 1990 & April 3 & 5.79 & 5.10 \\
\hline 1974 & April 21 & 8.61 & 1,060 & 1991 & August 6 & -- & 6.00 \\
\hline 1975 & April 28 & 7.93 & 405 & 1992 & March 29 & -- & 264 \\
\hline 1976 & April 1 & 8.12 & 330 & 1993 & July 19 & 8.30 & 355 \\
\hline 1977 & April 11 & 5.42 & 0.10 & 1994 & March 24 & -- & 224 \\
\hline 1978 & April 6 & 7.73 & 200 & & & & \\
\hline \multicolumn{8}{|c|}{ Annual peak discharge, from highest to lowest, and corresponding gage height } \\
\hline 1979 & April 23 & 10.50 & 1,200 & 1983 & April 2 & 7.99 & 155 \\
\hline 1974 & April 21 & 8.61 & 1,060 & 1967 & April 21 & 6.73 & 128 \\
\hline 1969 & April 12 & 8.80 & 700 & 1965 & April 11 & 7.09 & 124 \\
\hline 1971 & April 10 & 8.11 & 637 & 1981 & February 21 & 7.65 & 95.0 \\
\hline 1975 & April 28 & 7.93 & 405 & 1980 & April 3 & 7.51 & 90.0 \\
\hline 1993 & July 19 & 8.30 & 355 & 1966 & March 14 & 6.92 & 66.0 \\
\hline 1972 & March 16 & 7.62 & 354 & 1989 & April 8 & 6.23 & 66.0 \\
\hline 1976 & April 1 & 8.12 & 330 & 1985 & March 17 & 7.69 & 60.0 \\
\hline 1970 & April 29 & 7.20 & 317 & 1973 & June 20 & 6.51 & 52.0 \\
\hline 1987 & April 5 & 8.14 & 281 & 1962 & April 6 & - & 40.0 \\
\hline 1986 & June 21 & 7.45 & 276 & 1964 & April 9 & -- & 32.0 \\
\hline 1984 & March 24 & 7.70 & 264 & 1963 & July 25 & 5.23 & 8.90 \\
\hline 1992 & March 29 & -- & 264 & 1991 & August 6 & - & 6.00 \\
\hline 1994 & March 24 & -- & 224 & 1990 & April 3 & 5.79 & 5.10 \\
\hline 1968 & August 24 & 7.17 & 218 & 1988 & April 4 & 6.12 & 4.60 \\
\hline 1982 & March 30 & 8.01 & 214 & 1977 & April 11 & 5.42 & 0.100 \\
\hline 1978 & April 6 & 7.73 & 200 & & & & \\
\hline
\end{tabular}




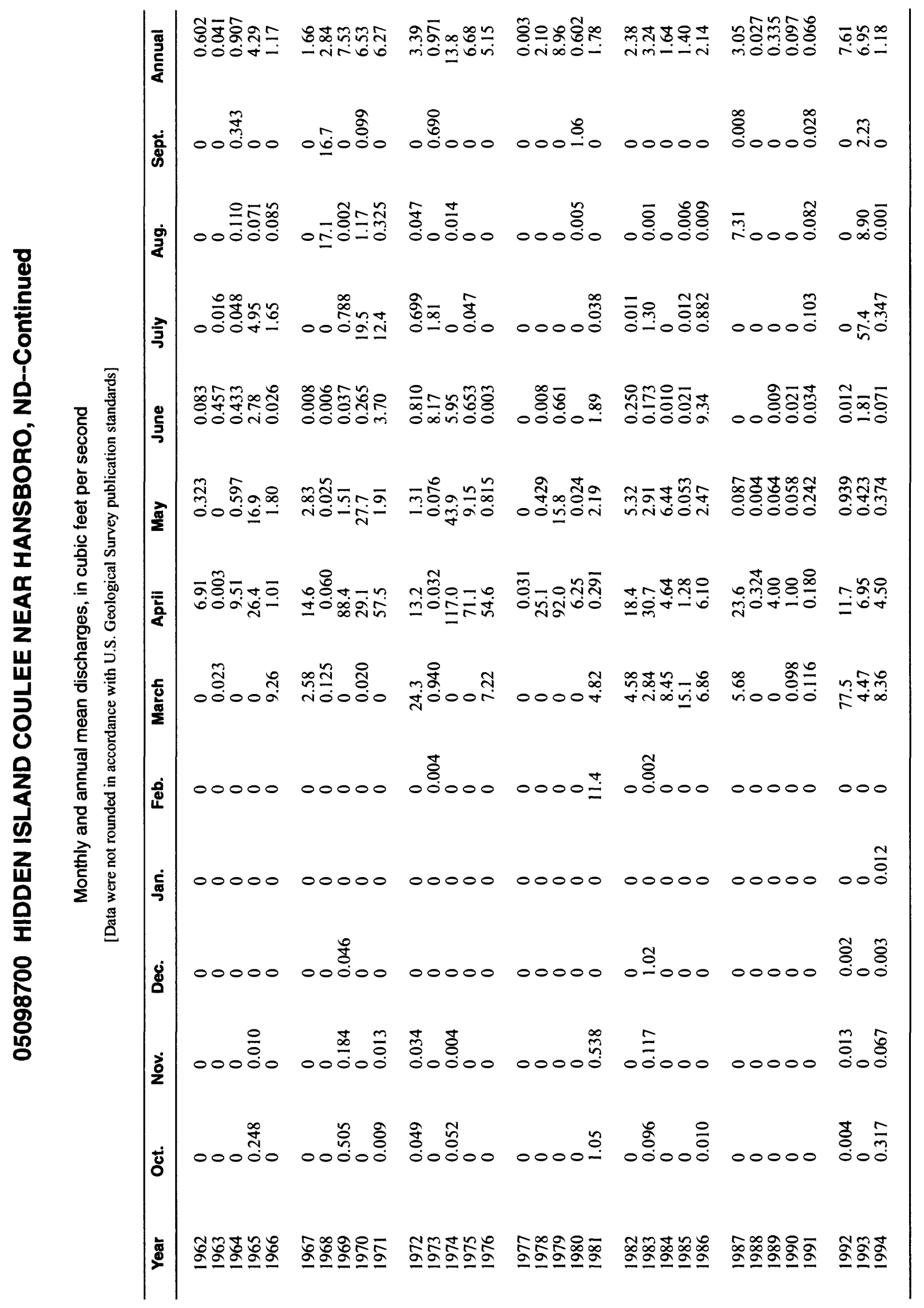




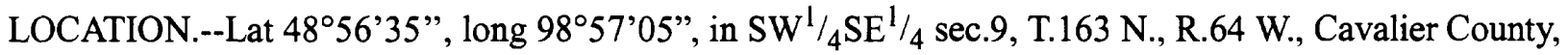
Hydrologic Unit 09020313, on right bank $150 \mathrm{ft}$ downstream from twin multiplate culverts on county highway, and $2.5 \mathrm{mi}$ east of Sarles.

DRAINAGE AREA.--71 $\mathrm{mi}^{2}$, approximately.

PERIOD OF RECORD.--May 1961 to September 1988. Prior to October 1973, published as Long River near Sarles.

GAGE.--Water-stage recorder. Datum of gage is $1,545 \mathrm{ft}$ above sea level, from topographic map.

EXTREMES FOR PERIOD OF RECORD.--Maximum discharge, 2,000 $\mathrm{ft}^{3} / \mathrm{s}$, Apr. 21, 1979; maximum gage height, $10.35 \mathrm{ft}$, Apr. 21, 1979, backwater from ice and snow; no flow for several months each year.

Annual mean discharge

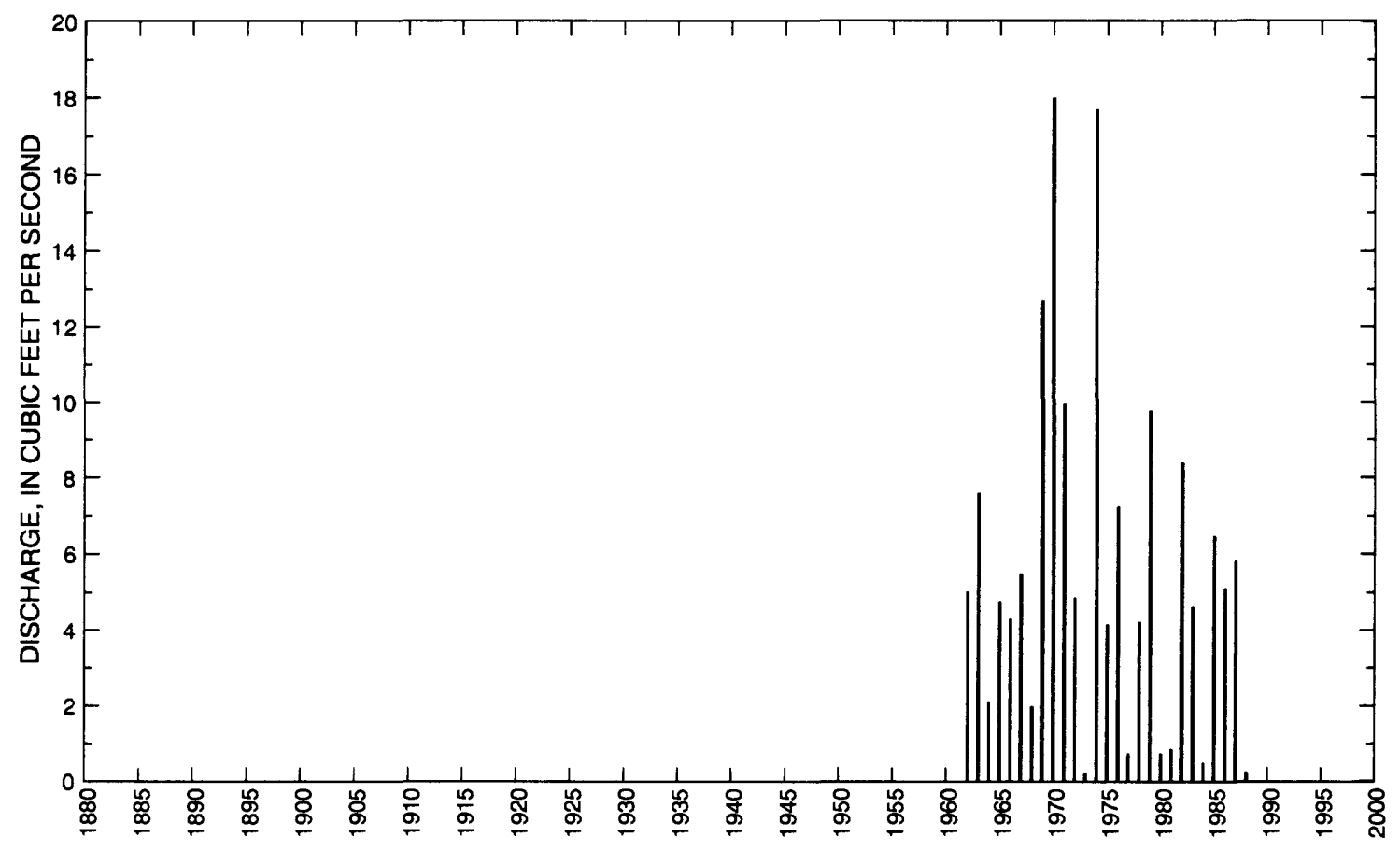


05098800 CYPRESS CREEK NEAR SARLES, ND--Continued

Statistics of monthly and annual mean discharges

[m, more than 1 year of occurrence; ng, statistic not given]

\begin{tabular}{|c|c|c|c|c|c|c|c|c|}
\hline \multirow[b]{2}{*}{ Month } & \multicolumn{2}{|c|}{ Maximum } & \multicolumn{2}{|c|}{ Minimum } & \multicolumn{4}{|c|}{ Mean } \\
\hline & $\begin{array}{c}\text { Discharge } \\
\left(\mathrm{ft}^{3} / \mathbf{s}\right)\end{array}$ & $\begin{array}{c}\text { Water year } \\
\text { of } \\
\text { occurrence }\end{array}$ & $\begin{array}{c}\text { Discharge } \\
\left(\mathrm{ft}^{3} / \mathrm{s}\right)\end{array}$ & $\begin{array}{l}\text { Water year } \\
\text { of } \\
\text { occurrence }\end{array}$ & $\begin{array}{c}\text { Discharge } \\
\left(\mathrm{ft}^{3} / \mathrm{s}\right)\end{array}$ & $\begin{array}{c}\text { Standard } \\
\text { deviation } \\
\left(\mathrm{ft}^{3} / \mathrm{s}\right)\end{array}$ & $\begin{array}{l}\text { Coeffi- } \\
\text { cient of } \\
\text { variation }\end{array}$ & $\begin{array}{c}\text { Percentage } \\
\text { of annuai } \\
\text { discharge }\end{array}$ \\
\hline October & 2.25 & 1983 & 0 & $\mathrm{~m}$ & 0.110 & 0.43 & 4.06 & 0.15 \\
\hline November & 0.162 & 1983 & 0 & $\mathrm{~m}$ & 0.010 & 0.03 & 4.20 & 0.01 \\
\hline December & 0.337 & 1983 & 0 & $\mathrm{~m}$ & 0.010 & 0.06 & 5.29 & 0.02 \\
\hline January & 0 & $\mathrm{~m}$ & 0 & $\mathrm{~m}$ & 0 & 0 & ng & 0 \\
\hline February & 4.71 & 1981 & 0 & $\mathrm{~m}$ & 0.170 & 0.91 & 5.20 & 0.26 \\
\hline March & 49.2 & 1985 & 0 & $\mathrm{~m}$ & 7.28 & 13.6 & 1.87 & 10.7 \\
\hline April & 171 & 1974 & 0.037 & 1973 & 42.2 & 48.8 & 1.16 & 61.9 \\
\hline May & 42.6 & 1974 & 0 & $\mathrm{~m}$ & 4.10 & 8.56 & 2.09 & 6.02 \\
\hline June & 122 & 1970 & 0 & $\mathrm{~m}$ & 9.79 & 25.9 & 2.64 & 14.4 \\
\hline July & 30.4 & 1965 & 0 & $\mathrm{~m}$ & 3.04 & 7.74 & 2.55 & 4.46 \\
\hline August & 16.5 & 1963 & 0 & $\mathrm{~m}$ & 1.04 & 3.44 & 3.30 & 1.53 \\
\hline September & 11.9 & 1968 & 0 & $\mathrm{~m}$ & 0.450 & 2.25 & 4.97 & 0.66 \\
\hline Annual & 18.0 & 1970 & 0.219 & 1973 & 5.69 & 4.76 & 0.84 & 100 \\
\hline
\end{tabular}

Annual flow duration

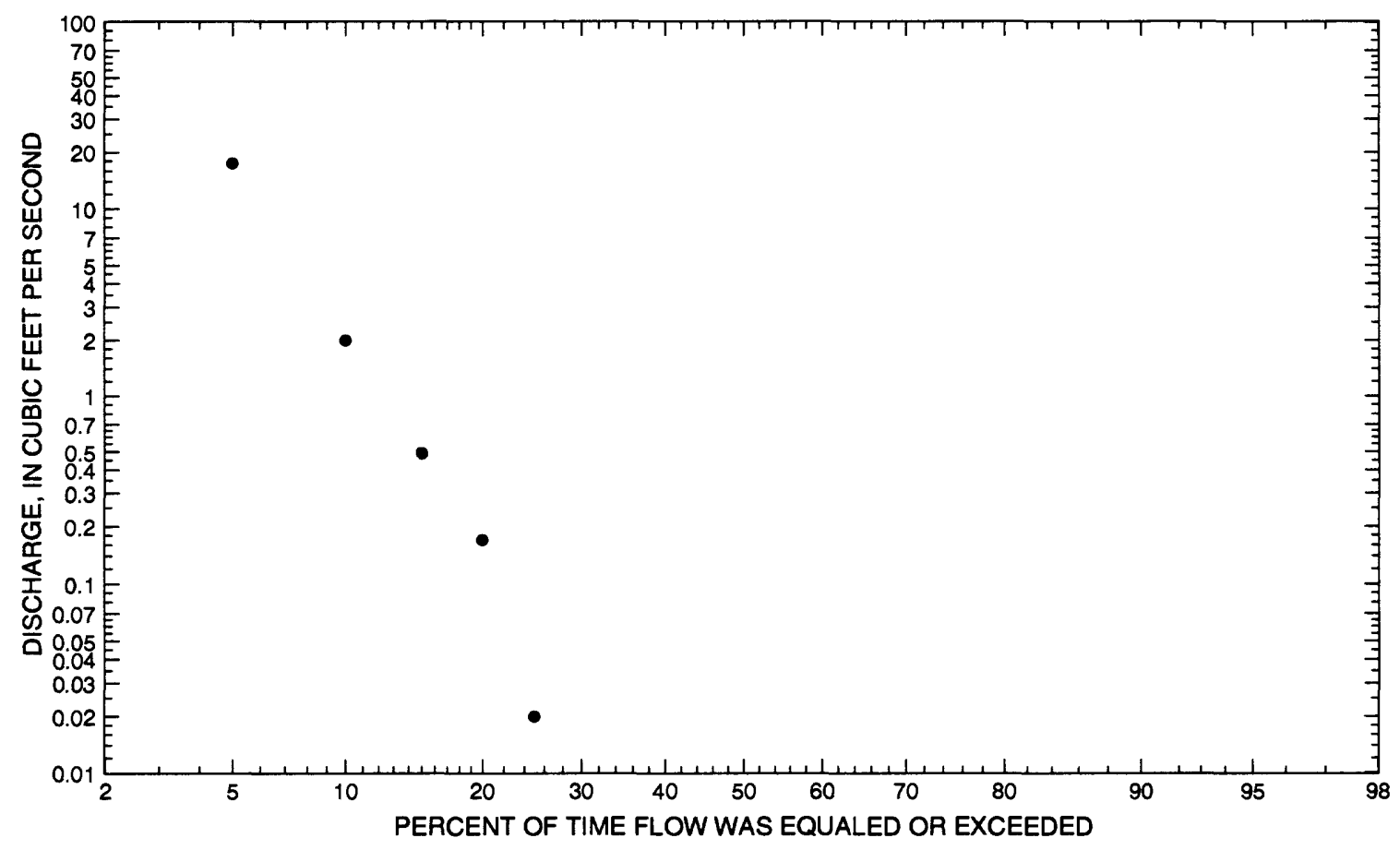




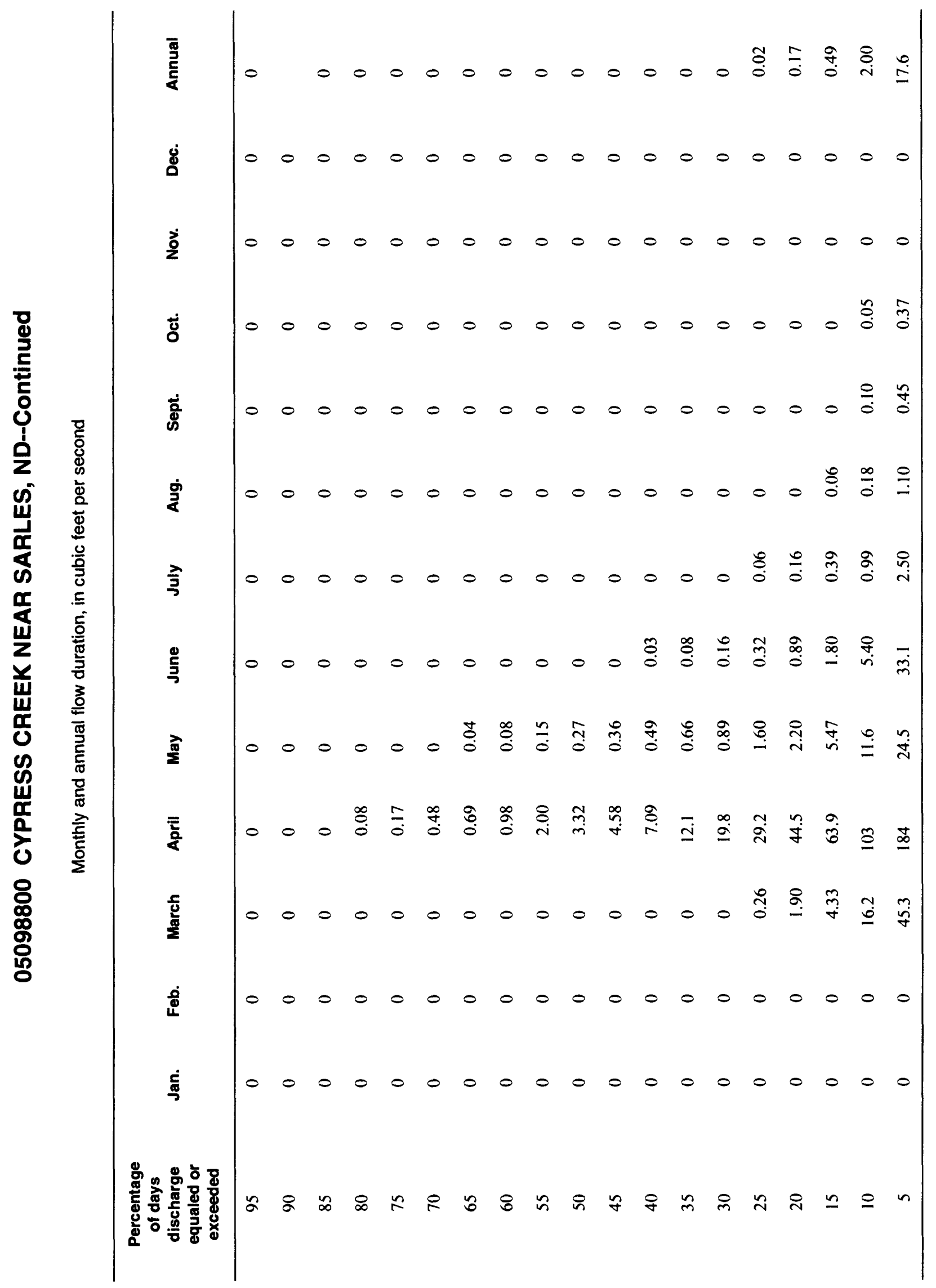




\section{CYPRESS CREEK NEAR SARLES, ND--Continued}

Probability of occurrence of annual high discharges

[ng, statistic not given]

\begin{tabular}{|c|c|c|c|c|c|c|}
\hline \multirow[b]{2}{*}{$\begin{array}{l}\text { Exceedance } \\
\text { probability }\end{array}$} & \multirow[b]{2}{*}{$\begin{array}{l}\text { Recurrence } \\
\text { intervai } \\
\text { (years) }\end{array}$} & \multirow[b]{2}{*}{$\begin{array}{c}\text { Maximum } \\
\text { instantaneous } \\
\left(\mathrm{ft}^{3} / \mathbf{s}\right)\end{array}$} & \multicolumn{4}{|c|}{$\begin{array}{c}\text { Maximum mean discharge } \\
\left(\mathrm{ft}^{3} / \mathrm{s}\right)\end{array}$} \\
\hline & & & 3-day period & 7-day period & 15-day period & 30-day period \\
\hline 0.99 & 1.01 & ng & 3.55 & 2.99 & 2.02 & 1.15 \\
\hline 0.95 & 1.05 & 30.8 & 13.2 & 10.4 & 6.93 & 3.97 \\
\hline 0.90 & 1.11 & 53.9 & 25.0 & 19.1 & 12.5 & 7.18 \\
\hline 0.80 & 1.25 & 103 & 51.3 & 37.7 & 24.1 & 13.8 \\
\hline 0.50 & 2 & 323 & 173 & 118 & 70.8 & 39.7 \\
\hline 0.20 & 5 & 903 & 478 & 300 & 167 & 91.2 \\
\hline 0.10 & 10 & 1,480 & 755 & 455 & 242 & 130 \\
\hline 0.04 & 25 & 2,410 & 1,160 & 672 & 340 & 178 \\
\hline 0.02 & 50 & 3,250 & 1,500 & 842 & 412 & 212 \\
\hline 0.01 & 100 & 4,200 & 1,850 & 1,010 & 480 & 244 \\
\hline 0.005 & 200 & 5,260 & 2,210 & 1,180 & 545 & 273 \\
\hline 0.002 & 500 & 6,840 & ng & ng & ng & ng \\
\hline
\end{tabular}

Probability of occurrence of annual low discharges

[ng, statistic not given]

\begin{tabular}{|c|c|c|c|c|c|c|c|c|c|c|}
\hline \multirow{3}{*}{$\begin{array}{l}\text { Non- } \\
\text { exceed- } \\
\text { ance } \\
\text { prob- } \\
\text { ability }\end{array}$} & \multirow{3}{*}{$\begin{array}{c}\text { Recur- } \\
\text { rence } \\
\text { inter- } \\
\text { val } \\
\text { (years) }\end{array}$} & \multicolumn{9}{|c|}{ Minimum mean discharge $\left(f^{3} / \mathrm{s}\right)$} \\
\hline & & \multicolumn{9}{|c|}{ Number of consecutive days } \\
\hline & & 1 & 3 & 7 & 14 & 30 & 60 & 90 & 120 & 183 \\
\hline 0.05 & 20 & ng & ng & ng & $\mathrm{ng}$ & ng & ng & ng & $\mathrm{ng}$ & 0 \\
\hline 0.10 & 10 & $\mathrm{ng}$ & ng & $\mathrm{ng}$ & ng & ng & ng & ng & $\mathrm{ng}$ & 0 \\
\hline 0.20 & 5 & $\mathrm{ng}$ & ng & ng & $\mathrm{ng}$ & $\mathrm{ng}$ & $\mathrm{ng}$ & ng & $\mathrm{ng}$ & 0 \\
\hline 0.50 & 2 & $\mathrm{ng}$ & $\mathrm{ng}$ & $\mathrm{ng}$ & $\mathrm{ng}$ & ng & ng & $\mathrm{ng}$ & $\mathrm{ng}$ & 0 \\
\hline
\end{tabular}




\section{CYPRESS CREEK NEAR SARLES, ND--Continued}

Probability of occurrence of seasonal low discharges

[ng, statistic not given]

\begin{tabular}{|c|c|c|c|c|c|c|c|c|c|}
\hline \multirow[b]{3}{*}{$\begin{array}{c}\text { Non- } \\
\text { exceedance } \\
\text { probability }\end{array}$} & \multirow[b]{3}{*}{$\begin{array}{c}\text { Recurrence } \\
\text { interval } \\
\text { (years) }\end{array}$} & \multicolumn{8}{|c|}{ Minimum mean discharge $\left(\mathrm{ft}^{3} / \mathrm{s}\right)$} \\
\hline & & \multicolumn{8}{|c|}{ Number of consecutive days } \\
\hline & & 1 & 7 & 14 & 30 & 1 & 7 & 14 & 30 \\
\hline & & \multicolumn{4}{|c|}{ December-January-February } & \multicolumn{4}{|c|}{ March-April-May } \\
\hline 0.05 & 20 & $\mathrm{ng}$ & ng & ng & $\mathrm{ng}$ & ng & ng & ng & 0 \\
\hline 0.10 & 10 & ng & ng & ng & ng & ng & ng & ng & 0 \\
\hline 0.20 & 5 & ng & ng & ng & ng & $\mathrm{ng}$ & ng & $\mathrm{ng}$ & 0 \\
\hline \multirow[t]{2}{*}{0.50} & 2 & ng & ng & ng & ng & ng & ng & ng & 0 \\
\hline & & \multicolumn{4}{|c|}{ June-July-August } & \multicolumn{4}{|c|}{ September-October-November } \\
\hline 0.05 & 20 & ng & ng & ng & 0 & ng & ng & ng & ng \\
\hline 0.10 & 10 & ng & ng & ng & 0 & $\mathrm{ng}$ & ng & $\mathrm{ng}$ & ng \\
\hline 0.20 & 5 & $\mathrm{ng}$ & $\mathrm{ng}$ & $\mathrm{ng}$ & 0 & $\mathrm{ng}$ & $\mathrm{ng}$ & $\mathrm{ng}$ & ng \\
\hline 0.50 & 2 & $\mathrm{ng}$ & ng & ng & 0 & ng & $\mathrm{ng}$ & $\mathrm{ng}$ & ng \\
\hline
\end{tabular}


05098800 CYPRESS CREEK NEAR SARLES, ND--Continued

Annual peak discharge and corresponding gage height

\begin{tabular}{|c|c|c|c|c|c|c|c|}
\hline $\begin{array}{l}\text { Water } \\
\text { year }\end{array}$ & Date & $\begin{array}{c}\text { Gage } \\
\text { height } \\
\text { (feet) }\end{array}$ & $\begin{array}{c}\text { Peak } \\
\text { discharge } \\
\left(\mathrm{ft}^{3} / \mathrm{s}\right)\end{array}$ & $\begin{array}{l}\text { Water } \\
\text { year }\end{array}$ & Date & $\begin{array}{c}\text { Gage } \\
\text { height } \\
\text { (feet) }\end{array}$ & $\begin{array}{c}\text { Peak } \\
\text { discharge } \\
\left(\mathrm{ft}^{3} / \mathrm{s}\right)\end{array}$ \\
\hline \multicolumn{8}{|c|}{ Annual peak discharge, by year, and corresponding gage height } \\
\hline 1962 & July 8 & 4.37 & 393 & 1976 & April 4 & 7.30 & 650 \\
\hline 1963 & June 2 & 4.70 & 572 & 1977 & May 20 & 3.42 & 50.0 \\
\hline 1964 & April 10 & 3.03 & 132 & 1978 & April 7 & 5.84 & 392 \\
\hline 1965 & July 22 & 5.00 & 360 & 1979 & April 21 & 10.35 & 2,000 \\
\hline 1966 & March 17 & 6.18 & 670 & 1980 & April 5 & 4.05 & 85.0 \\
\hline 1967 & April 21 & 4.57 & 310 & 1981 & February 24 & 5.00 & 31.0 \\
\hline 1968 & August 25 & 3.08 & 98.0 & 1982 & June 5 & 8.41 & 1,440 \\
\hline 1969 & April 12 & 7.90 & 1,130 & 1983 & April 2 & 4.88 & 148 \\
\hline 1970 & June 11 & 8.55 & 1,740 & 1984 & March 28 & 3.99 & 38.0 \\
\hline 1971 & April 10 & 8.56 & 1,920 & 1985 & March 13 & 6.22 & 470 \\
\hline 1972 & March 19 & 4.98 & 245 & 1986 & March 17 & 5.23 & 198 \\
\hline 1973 & March 12 & 3.40 & 40.0 & 1987 & April 5 & 7.20 & 605 \\
\hline 1974 & April 17 & 7.62 & 1,260 & 1988 & March 29 & 3.00 & 6.30 \\
\hline 1975 & April 13 & 5.75 & 389 & & & & \\
\hline \multicolumn{8}{|c|}{ Annual peak discharge, from highest to lowest, and corresponding gage height } \\
\hline 1979 & April 21 & 10.35 & 2,000 & 1965 & July 22 & 5.00 & 360 \\
\hline 1971 & April 10 & 8.56 & 1,920 & 1967 & April 21 & 4.57 & 310 \\
\hline 1970 & June 11 & 8.55 & 1,740 & 1972 & March 19 & 4.98 & 245 \\
\hline 1982 & June 5 & 8.41 & 1,440 & 1986 & March 17 & 5.23 & 198 \\
\hline 1974 & April 17 & 7.62 & 1,260 & 1983 & April 2 & 4.88 & 148 \\
\hline 1969 & April 12 & 7.90 & 1,130 & 1964 & April 10 & 3.03 & 132 \\
\hline 1966 & March 17 & 6.18 & 670 & 1968 & August 25 & 3.08 & 98.0 \\
\hline 1976 & April 4 & 7.30 & 650 & 1980 & April 5 & 4.05 & 85.0 \\
\hline 1987 & April 5 & 7.20 & 605 & 1977 & May 20 & 3.42 & 50.0 \\
\hline 1963 & June 2 & 4.70 & 572 & 1973 & March 12 & 3.40 & 40.0 \\
\hline 1985 & March 13 & 6.22 & 470 & 1984 & $\operatorname{March} 28$ & 3.99 & 38.0 \\
\hline 1962 & July 8 & 4.37 & 393 & 1981 & February 24 & 5.00 & 31.0 \\
\hline 1978 & April 7 & 5.84 & 392 & 1988 & March 29 & 3.00 & 6.30 \\
\hline 1975 & April 13 & 5.75 & 389 & & & & \\
\hline
\end{tabular}




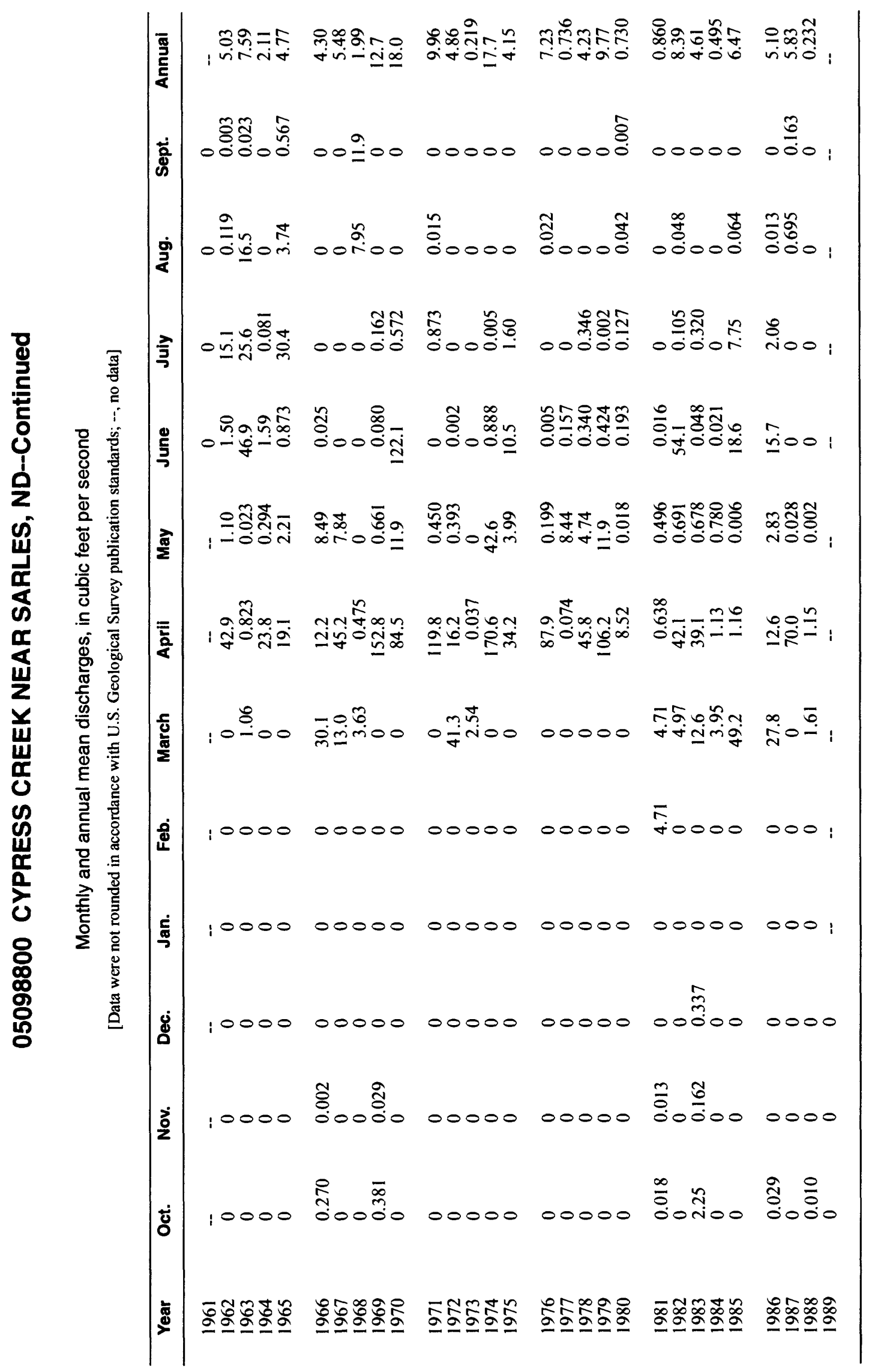




\section{SNOWFLAKE CREEK NEAR SNOWFLAKE, MB}

LOCATION.--Lat 49 $01^{\prime} 17^{\prime \prime}$, long 98 36'13", in SW $1 / 4$ sec.10, T.1., R.9 W., first meridian, Hydrologic Unit 09020313, at traffic bridge, $2.5 \mathrm{mi}$ east, and $1.5 \mathrm{mi}$ south of Snowflake, Manitoba.

DRAINAGE AREA.--348 $\mathrm{mi}^{2}$.

PERIOD OF RECORD.--March 1961 to current year.

GAGE.--Water-stage recorder since March 1968 and nonrecording gage prior thereto. Datum of gage is Geodetic Survey of Canada Datum of 1929. Prior to Jan. 1, 1987, recording gage at same site at datum of 1,221.66 ft above Geodetic Survey of Canada Datum of 1929. Prior to Apr. 2, 1964, nonrecording gage at present site and datum. Apr. 2, 1964, to May 10, 1965, nonrecording gage at site $0.5 \mathrm{mi}$ downstream at present datum.

EXTREMES FOR PERIOD OF RECORD.--Maximum discharge, 1,130 $\mathrm{ft}^{3} / \mathrm{s}$, Apr. 21, 1979, gage height, $1,229.94 \mathrm{ft}$; no flow at times.

Annual mean discharge

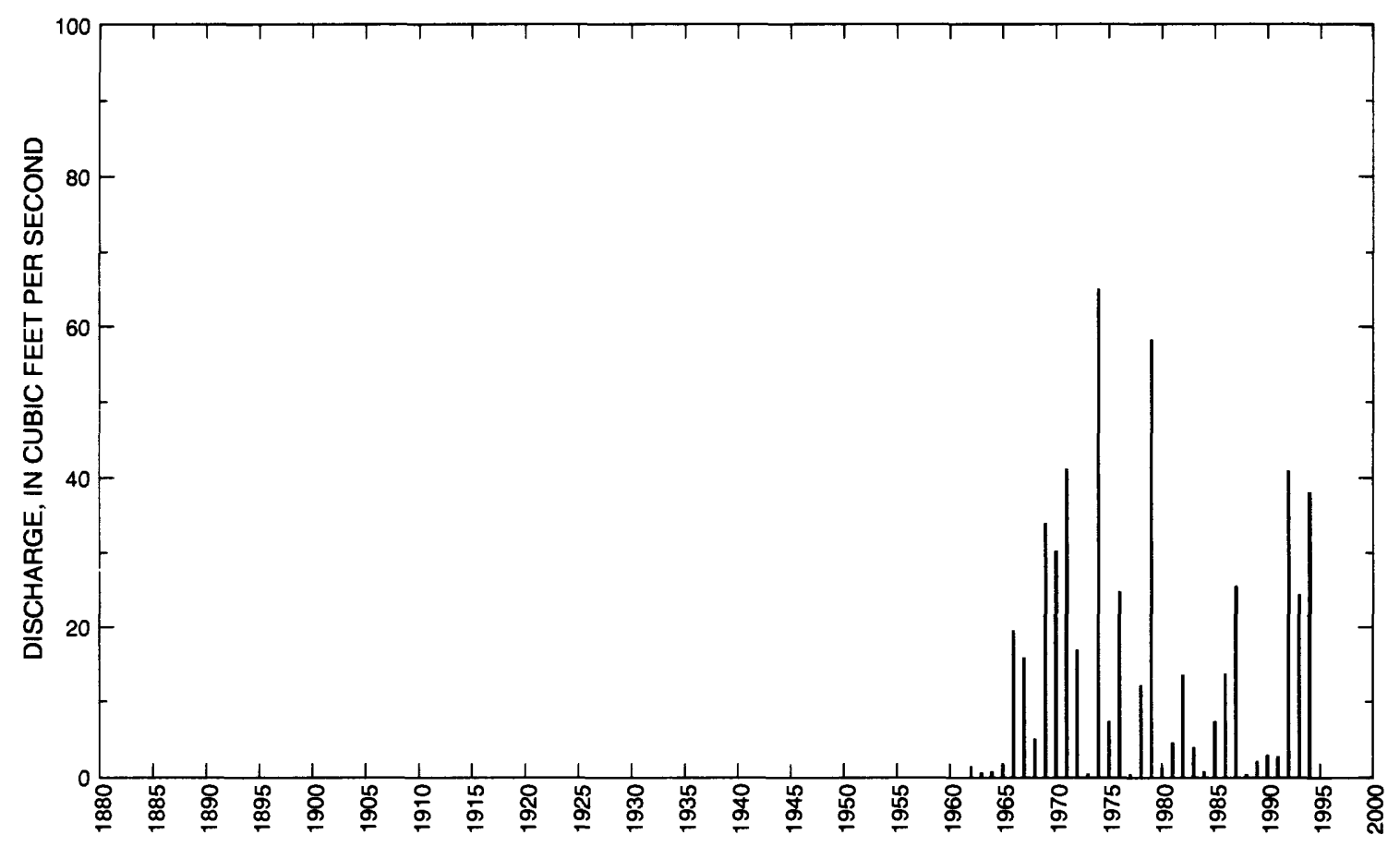


05099100 SNOWFLAKE CREEK NEAR SNOWFLAKE, MB--Continued

Statistics of monthly and annual mean discharges

[m, more than 1 year of occurrence]

\begin{tabular}{|c|c|c|c|c|c|c|c|c|}
\hline \multirow[b]{2}{*}{ Month } & \multicolumn{2}{|c|}{ Maximum } & \multicolumn{2}{|c|}{ Minimum } & \multicolumn{4}{|c|}{ Mean } \\
\hline & $\begin{array}{c}\text { Discharge } \\
\left(\mathrm{ft}^{3} / \mathrm{s}\right)\end{array}$ & $\begin{array}{c}\text { Water year } \\
\text { of } \\
\text { occurrence }\end{array}$ & $\begin{array}{c}\text { Discharge } \\
\left(\mathrm{ft}^{3} / \mathrm{s}\right)\end{array}$ & $\begin{array}{c}\text { Water year } \\
\text { of } \\
\text { occurrence }\end{array}$ & $\begin{array}{c}\text { Discharge } \\
\left(\mathrm{ft}^{3} / \mathbf{s}\right)\end{array}$ & $\begin{array}{c}\text { Standard } \\
\text { deviation } \\
\left(\mathrm{ft}^{3} / \mathrm{s}\right)\end{array}$ & $\begin{array}{l}\text { Coeffi- } \\
\text { cient of } \\
\text { variation }\end{array}$ & $\begin{array}{l}\text { Percentage } \\
\text { of annual } \\
\text { discharge }\end{array}$ \\
\hline October & 68.6 & 1994 & 0 & $\mathrm{~m}$ & 3.04 & 12.0 & 3.94 & 1.66 \\
\hline November & 25.7 & 1994 & 0 & $\mathrm{~m}$ & 1.28 & 5.54 & 3.55 & 0.70 \\
\hline December & 1.51 & 1994 & 0 & $\mathrm{~m}$ & 0.090 & 0.30 & 3.14 & 0.05 \\
\hline January & 0.078 & 1994 & 0 & $\mathrm{~m}$ & 0 & 0.02 & 3.94 & 0 \\
\hline February & 4.90 & 1981 & 0 & $\mathrm{~m}$ & 0.150 & 0.85 & 5.61 & 0.08 \\
\hline March & 42.5 & 1992 & 0 & $\mathrm{~m}$ & 6.01 & 9.43 & 1.57 & 3.27 \\
\hline April & 353 & 1992 & 0.225 & 1973 & 85.7 & 103 & 1.21 & 46.7 \\
\hline May & 390 & 1979 & 0.061 & 1988 & 54.7 & 94.1 & 1.72 & 29.8 \\
\hline June & 123 & 1974 & 0 & $\mathrm{~m}$ & 17.2 & 27.3 & 1.59 & 9.40 \\
\hline July & 35.7 & 1993 & 0 & $\mathrm{~m}$ & 6.83 & 9.90 & 1.45 & 3.72 \\
\hline August & 70.4 & 1993 & 0 & $\mathrm{~m}$ & 4.08 & 12.2 & 2.98 & 2.22 \\
\hline September & 99.7 & 1993 & 0 & $\mathrm{~m}$ & 4.29 & 17.1 & 3.99 & 2.34 \\
\hline Annual & 65.1 & 1974 & 0.378 & 1988 & 15.7 & 17.6 & 1.12 & 100 \\
\hline
\end{tabular}

Annual flow duration

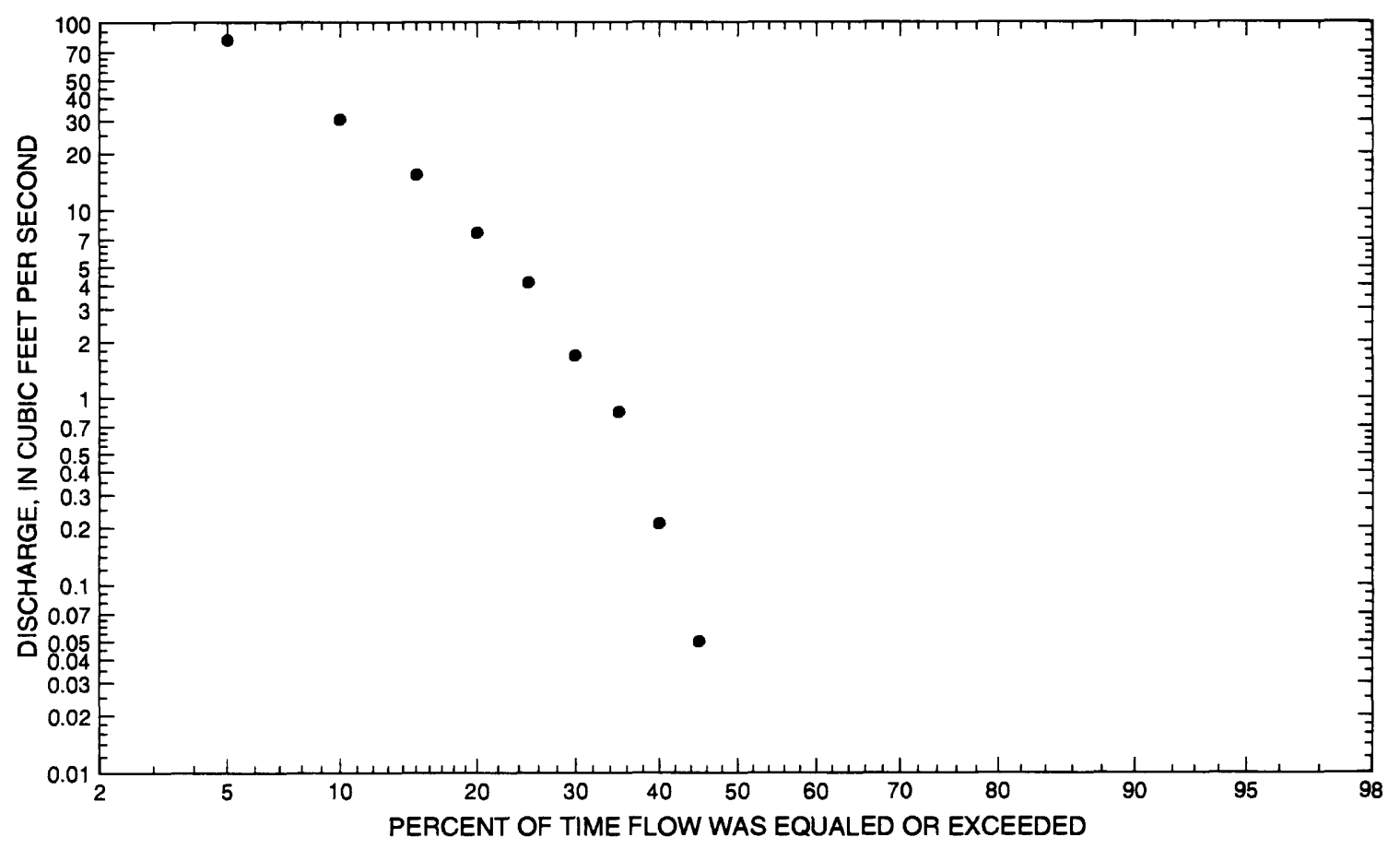




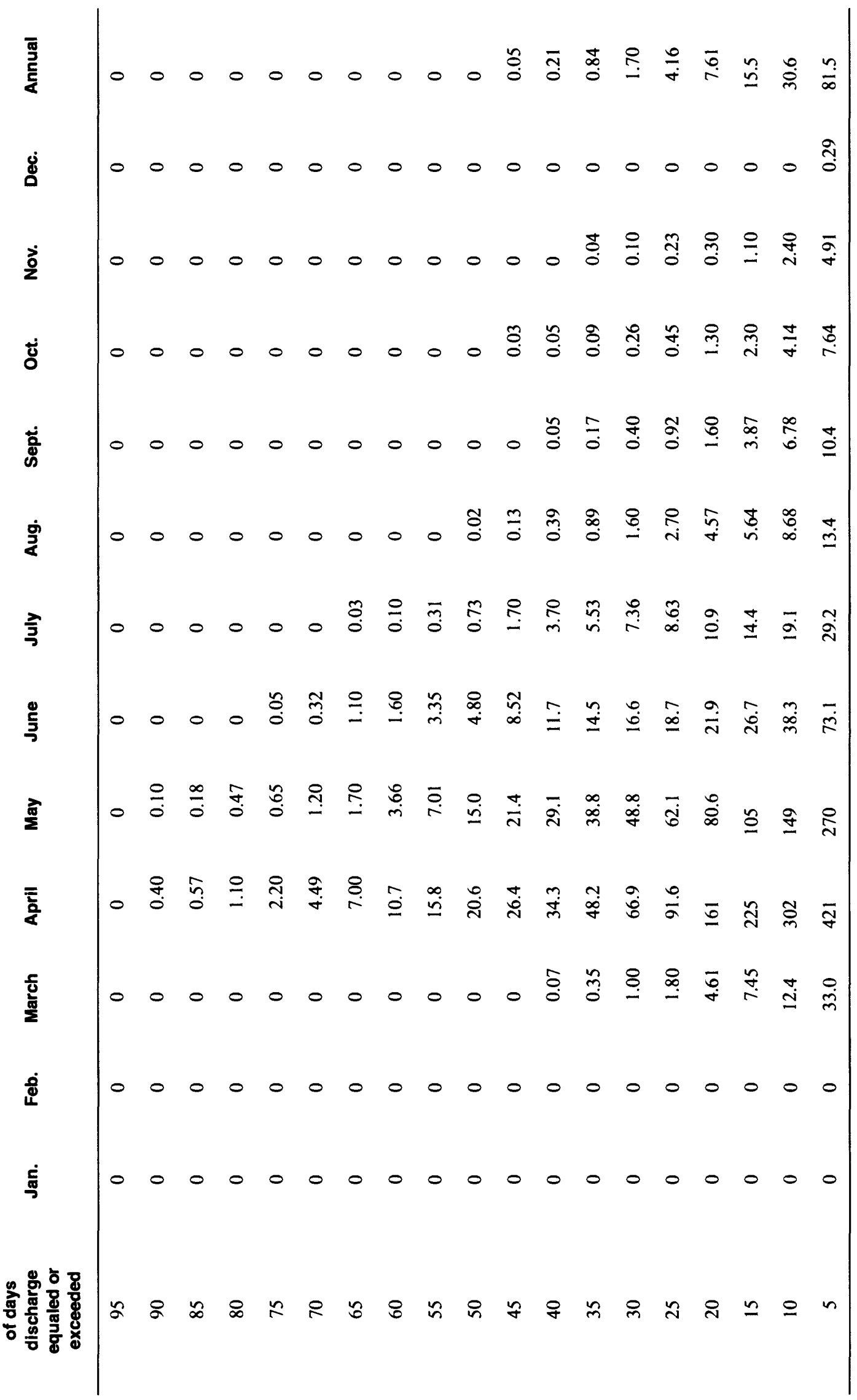




\section{SNOWFLAKE CREEK NEAR SNOWFLAKE, MB--Continued}

Probability of occurrence of annual high discharges

[ng, statistic not given]

\begin{tabular}{lcccccc}
\hline & & & \multicolumn{5}{c}{$\begin{array}{c}\text { Maximum mean discharge } \\
\left(\mathbf{f t}^{3} / \mathbf{s}\right)\end{array}$} \\
\cline { 5 - 7 } $\begin{array}{c}\text { Exceedance } \\
\text { probability }\end{array}$ & $\begin{array}{c}\text { Recurrence } \\
\text { interval } \\
\text { (years) }\end{array}$ & $\begin{array}{c}\text { Maximum } \\
\text { instantaneous } \\
\left(\mathbf{f t}^{3} / \mathbf{s}\right)\end{array}$ & 3-day period & 7-day period & 15-day period & 30-day period \\
\hline 0.99 & 1.01 & 4.40 & 3.15 & 2.18 & 1.51 & 0.962 \\
0.95 & 1.05 & 14.1 & 9.57 & 6.81 & 4.83 & 3.20 \\
0.90 & 1.11 & 25.1 & 16.8 & 12.2 & 8.80 & 5.97 \\
0.80 & 1.25 & 48.8 & 32.5 & 24.2 & 17.9 & 12.5 \\
0.50 & 2 & 156 & 106 & 83.5 & 65.6 & 48.5 \\
0.20 & 5 & 432 & 314 & 265 & 224 & 176 \\
0.10 & 10 & 698 & 533 & 467 & 415 & 338 \\
0.04 & 25 & 1,120 & 911 & 836 & 784 & 662 \\
0.02 & 50 & 1,490 & 1,270 & 1,200 & 1,170 & 1,010 \\
0.01 & 100 & 1,910 & 1,690 & 1,650 & 1,660 & 1,470 \\
0.005 & 200 & 2,360 & 2,180 & 2,190 & 2,280 & 2,060 \\
0.002 & 500 & 3,010 & ng & ng & ng & ng \\
\hline
\end{tabular}

Probability of occurrence of annual low discharges

[ng, statistic not given]

\begin{tabular}{|c|c|c|c|c|c|c|c|c|c|c|}
\hline \multirow{3}{*}{$\begin{array}{l}\text { Non- } \\
\text { exceed- } \\
\text { ance } \\
\text { prob- } \\
\text { ability }\end{array}$} & \multirow{3}{*}{$\begin{array}{l}\text { Recur- } \\
\text { rence } \\
\text { inter- } \\
\text { val } \\
\text { (years) }\end{array}$} & \multicolumn{9}{|c|}{ Minimum mean discharge $\left(\mathrm{tt}^{3} / \mathrm{s}\right)$} \\
\hline & & \multicolumn{9}{|c|}{ Number of consecutive days } \\
\hline & & 1 & 3 & 7 & 14 & 30 & 60 & 90 & 120 & 183 \\
\hline 0.05 & 20 & $\mathrm{ng}$ & $\mathrm{ng}$ & $\mathrm{ng}$ & $\mathrm{ng}$ & $\mathrm{ng}$ & $\mathrm{ng}$ & 0 & 0 & 0 \\
\hline 0.10 & 10 & $\mathrm{ng}$ & ng & $\mathrm{ng}$ & $\mathrm{ng}$ & $\mathrm{ng}$ & $\mathrm{ng}$ & 0 & 0 & 0 \\
\hline 0.20 & 5 & $\mathrm{ng}$ & ng & $\mathrm{ng}$ & $\mathrm{ng}$ & $\mathrm{ng}$ & $\mathrm{ng}$ & 0 & 0 & 0 \\
\hline 0.50 & 2 & $\mathrm{ng}$ & $\mathrm{ng}$ & $\mathrm{ng}$ & $\mathrm{ng}$ & $\mathrm{ng}$ & $\mathrm{ng}$ & 0 & 0 & 0.024 \\
\hline
\end{tabular}


05099100 SNOWFLAKE CREEK NEAR SNOWFLAKE, MB--Continued

Probability of occurrence of seasonal low discharges

[ng, statistic not given]

\begin{tabular}{|c|c|c|c|c|c|c|c|c|c|}
\hline \multirow[b]{3}{*}{$\begin{array}{c}\text { Non- } \\
\text { exceedance } \\
\text { probability }\end{array}$} & \multirow[b]{3}{*}{$\begin{array}{c}\text { Recurrence } \\
\text { intervai } \\
\text { (years) }\end{array}$} & \multicolumn{8}{|c|}{ Minimum mean discharge $\left(\mathrm{ft}^{3} / \mathrm{s}\right)$} \\
\hline & & \multicolumn{8}{|c|}{ Number of consecutive days } \\
\hline & & 1 & 7 & 14 & 30 & 1 & 7 & 14 & 30 \\
\hline & & \multicolumn{4}{|c|}{ December-January-February } & \multicolumn{4}{|c|}{ March-April-May } \\
\hline 0.05 & 20 & ng & ng & ng & ng & ng & 0 & 0 & 0 \\
\hline 0.10 & 10 & ng & ng & ng & ng & ng & 0 & 0 & 0 \\
\hline 0.20 & 5 & ng & ng & ng & ng & ng & 0 & 0 & 0 \\
\hline \multirow[t]{2}{*}{0.50} & 2 & ng & ng & ng & $\mathrm{ng}$ & ng & 0 & 0 & 0.643 \\
\hline & & \multicolumn{4}{|c|}{ June-July-August } & \multicolumn{4}{|c|}{ September-October-November } \\
\hline 0.05 & 20 & 0 & 0 & 0 & 0 & 0 & 0 & 0 & 0 \\
\hline 0.10 & 10 & 0 & 0 & 0 & 0 & 0 & 0 & 0 & 0 \\
\hline 0.20 & 5 & 0 & 0 & 0 & 0 & 0 & 0 & 0 & 0 \\
\hline 0.50 & 2 & 0 & 0 & 0 & 0.116 & 0 & 0 & 0 & 0 \\
\hline
\end{tabular}


05099100 SNOWFLAKE CREEK NEAR SNOWFLAKE, MB--Continued

Annual peak discharge and corresponding gage height

$[--$, no data $]$

\begin{tabular}{|c|c|c|c|c|c|c|c|}
\hline $\begin{array}{l}\text { Water } \\
\text { years }\end{array}$ & Date & $\begin{array}{c}\text { Gage } \\
\text { height } \\
\text { (feet) }\end{array}$ & $\begin{array}{c}\text { Peak } \\
\text { discharge } \\
\left(\mathrm{ft}^{3} / \mathrm{s}\right)\end{array}$ & $\begin{array}{c}\text { Water } \\
\text { year }\end{array}$ & Date & $\begin{array}{l}\text { Gage } \\
\text { height } \\
\text { (feet) }\end{array}$ & $\begin{array}{c}\text { Peak } \\
\text { discharge } \\
\left(\mathrm{ft}^{3} / \mathrm{s}\right)\end{array}$ \\
\hline \multicolumn{8}{|c|}{ Annual peak discharge, by year, and corresponding gage height } \\
\hline 1961 & March 27 & $1,224.21$ & 13.6 & 1978 & April 27 & $1,226.31$ & 247 \\
\hline 1962 & April 18 & $1,226.41$ & 101 & 1979 & April 21 & $1,229.94$ & 1,130 \\
\hline 1963 & March 21 & $1,226.39$ & 9.90 & 1980 & April 5 & $1,225.71$ & 67.0 \\
\hline 1964 & April 9 & $1,224.18$ & 21.7 & 1981 & May 22 & $1,225.67$ & 82.0 \\
\hline 1965 & April 11 & $1,225.12$ & 44.0 & 1982 & June 6 & $1,228.38$ & 505 \\
\hline 1966 & April 14 & $1,226.36$ & 227 & 1983 & April 6 & $1,225.87$ & 56.0 \\
\hline 1967 & April 20 & $1,226.54$ & 310 & 1984 & March 26 & $1,224.75$ & 14.9 \\
\hline 1968 & August 25 & $1,224.01$ & 79.4 & 1985 & April 8 & $1,226.68$ & 190 \\
\hline 1969 & April 11 & $1,227.39$ & 653 & 1986 & June 3 & $1,228.07$ & 427 \\
\hline 1970 & April 25 & $1,226.27$ & 279 & 1987 & April 5 & $1,228.77$ & 438 \\
\hline 1971 & April 12 & - & 658 & 1988 & April 3 & $1,225.34$ & 29.0 \\
\hline 1972 & April 16 & - & 402 & 1989 & April 14 & $1,226.64$ & 185 \\
\hline 1973 & March 18 & - & 21.2 & 1990 & April 3 & $1,226.57$ & 155 \\
\hline 1974 & May 21 & $1,227.64$ & 823 & 1991 & July 12 & $1,226.63$ & 188 \\
\hline 1975 & April 28 & $1,224.64$ & 158 & 1992 & April 8 & $1,228.86$ & 600 \\
\hline 1976 & April 8 & - & 344 & 1993 & July 28 & $1,226.69$ & 155 \\
\hline 1977 & May 19 & $1,225.43$ & 29.0 & 1994 & April 15 & $1,227.84$ & 378 \\
\hline \multicolumn{8}{|c|}{ Annual peak discharge, from highest to lowest, and corresponding gage height } \\
\hline 1979 & April 21 & $1,229.94$ & 1,130 & 1989 & April 14 & $1,226.64$ & 185 \\
\hline 1974 & May 21 & $1,227.64$ & 823 & 1975 & April 28 & $1,224.64$ & 158 \\
\hline 1971 & April 12 & -- & 658 & 1990 & April 3 & $1,226.57$ & 155 \\
\hline 1969 & April 11 & $1,227.39$ & 653 & 1993 & July 28 & $1,226.69$ & 155 \\
\hline 1992 & April 8 & $1,228.86$ & 600 & 1962 & April 18 & $1,226.41$ & 101 \\
\hline 1982 & June 6 & $1,228.38$ & 505 & 1981 & May 22 & $1,225.67$ & 82.0 \\
\hline 1987 & April 5 & $1,228.77$ & 438 & 1968 & August 25 & $1,224.01$ & 79.4 \\
\hline 1986 & June 3 & $1,228.07$ & 427 & 1980 & April 5 & $1,225.71$ & 67.0 \\
\hline 1972 & April 16 & -- & 402 & 1983 & April 6 & $1,225.87$ & 56.0 \\
\hline 1994 & April 15 & $1,227.84$ & 378 & 1965 & April 11 & $1,225.12$ & 44.0 \\
\hline 1976 & April 8 & -- & 344 & 1977 & May 19 & $1,225.43$ & 29.0 \\
\hline 1967 & April 20 & $1,226.54$ & 310 & 1988 & April 3 & $1,225.34$ & 29.0 \\
\hline 1970 & April 25 & $1,226.27$ & 279 & 1964 & April 9 & $1,224.18$ & 21.7 \\
\hline 1978 & April 27 & $1,226.31$ & 247 & 1973 & March 18 & - & 21.2 \\
\hline 1966 & April 14 & $1,226.36$ & 227 & 1984 & March 26 & $1,224.75$ & 14.9 \\
\hline 1985 & April 8 & $1,226.68$ & 190 & 1961 & March 27 & $1,224.21$ & 13.6 \\
\hline 1991 & July 12 & $1,226.63$ & 188 & 1963 & March 21 & $1,226.39$ & 9.90 \\
\hline
\end{tabular}




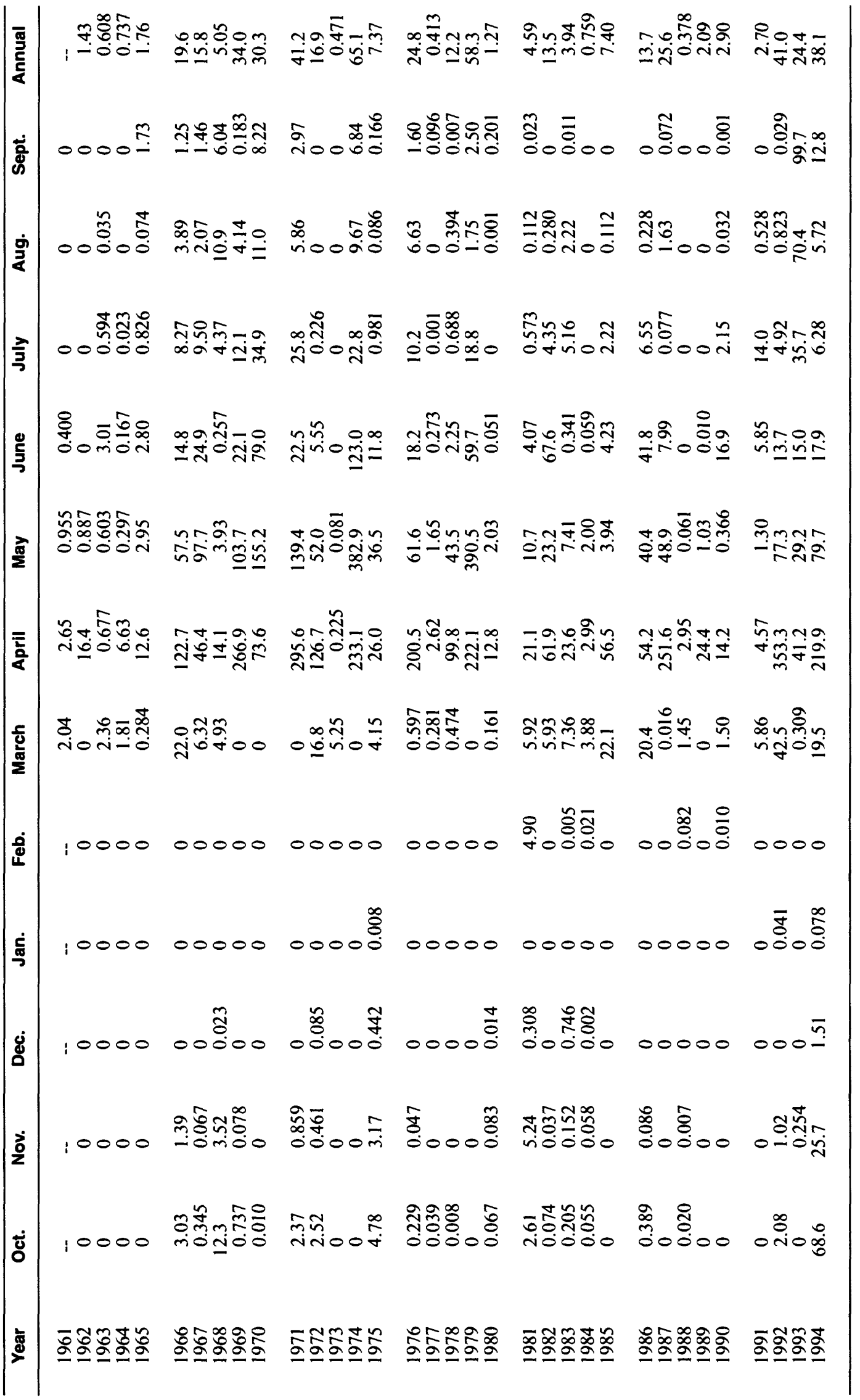




\section{MOWBRAY CREEK NEAR MOWBRAY, MB}

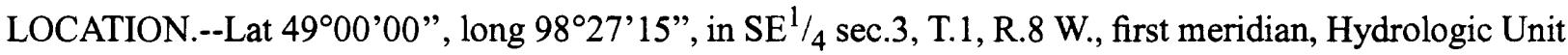
09020313, on downstream side of bridge on Municipal Road on international boundary, and $1.5 \mathrm{mi}$ east of Mowbray, Manitoba.

DRAINAGE AREA.--93.9 mi².

PERIOD OF RECORD.--March 1962 to current year. Seasonal records only most years.

GAGE.--Water-stage recorder. Datum of gage is Geodetic Survey of Canada Datum of 1929.

Nonrecording gage prior to 1971 .

EXTREMES FOR PERIOD OF RECORD.--Maximum discharge, $943 \mathrm{ft}^{3} / \mathrm{s}$, Apr. 6, 1987, gage height, $1,534.57 \mathrm{ft}$; no flow at times.

Annual mean discharge

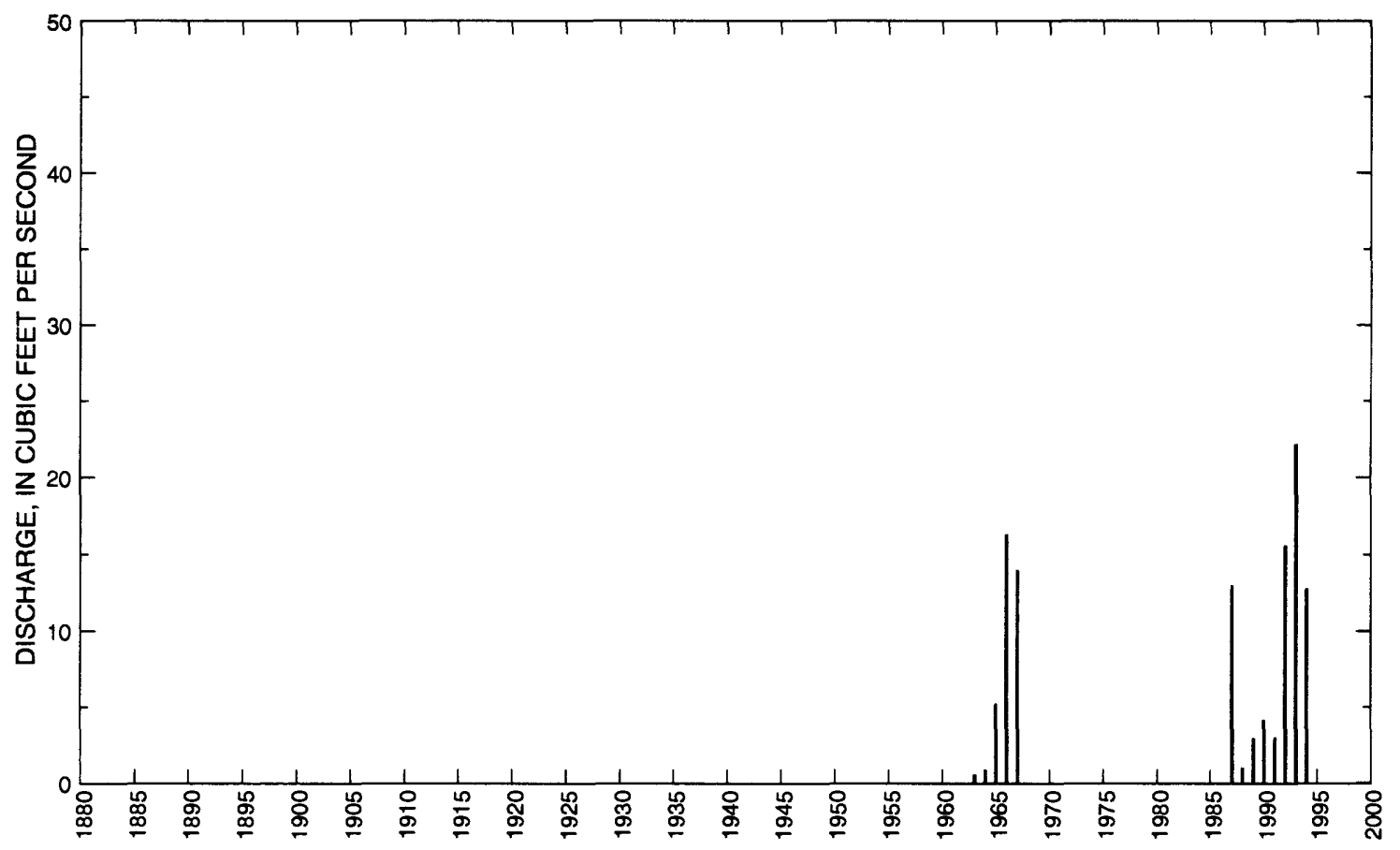


05099150 MOWBRAY CREEK NEAR MOWBRAY, MB--Continued

Statistics of monthly and annual mean discharges

[m, more than 1 year of occurrence; ng, statistic not given]

\begin{tabular}{|c|c|c|c|c|c|c|c|c|}
\hline \multirow[b]{2}{*}{ Month } & \multicolumn{2}{|c|}{ Maximum } & \multicolumn{2}{|c|}{ Minimum } & \multicolumn{4}{|c|}{ Mean } \\
\hline & $\begin{array}{c}\text { Discharge } \\
\left(\mathrm{ft}^{3} / \mathrm{s}\right)\end{array}$ & $\begin{array}{c}\text { Water year } \\
\text { of } \\
\text { occurrence }\end{array}$ & $\begin{array}{c}\text { Discharge } \\
\left(\mathrm{ft}^{3} / \mathrm{s}\right)\end{array}$ & $\begin{array}{c}\text { Water year } \\
\text { of } \\
\text { occurrence }\end{array}$ & $\begin{array}{c}\text { Discharge } \\
\left(\mathrm{ft}^{3} / \mathrm{s}\right)\end{array}$ & $\begin{array}{c}\text { Standard } \\
\text { deviation } \\
\left(\mathrm{ft}^{3} / \mathbf{s}\right)\end{array}$ & $\begin{array}{l}\text { Coeffi- } \\
\text { cient of } \\
\text { variation }\end{array}$ & $\begin{array}{l}\text { Percentage } \\
\text { of annual } \\
\text { discharge }\end{array}$ \\
\hline October & 5.15 & 1981 & 0 & $\mathrm{~m}$ & 0.600 & 1.36 & 2.27 & 0.56 \\
\hline November & 0.634 & 1988 & 0 & $\mathrm{~m}$ & 0.090 & 0.21 & 2.37 & 0.08 \\
\hline December & 0.036 & 1988 & 0 & $\mathrm{~m}$ & 0.010 & 0.01 & 2.44 & 0.01 \\
\hline January & 0 & $\mathrm{~m}$ & 0 & $\mathrm{~m}$ & 0 & 0 & ng & 0 \\
\hline February & 5.68 & 1981 & 0 & $\mathrm{~m}$ & 0.390 & 1.46 & 3.80 & 0.36 \\
\hline March & 62.0 & 1985 & 0 & $\mathrm{~m}$ & 9.91 & 17.3 & 1.75 & 9.33 \\
\hline April & 208 & 1974 & 1.05 & 1973 & 64.4 & 63.4 & 0.98 & 60.6 \\
\hline May & 159 & 1974 & 0.009 & 1973 & 15.0 & 31.7 & 2.12 & 14.1 \\
\hline June & 32.0 & 1986 & 0 & $\mathrm{~m}$ & 5.45 & 7.86 & 1.44 & 5.13 \\
\hline July & 55.6 & 1993 & 0 & $\mathrm{~m}$ & 4.02 & 10.4 & 2.58 & 3.79 \\
\hline August & 140 & 1993 & 0 & $\mathrm{~m}$ & 5.09 & 24.4 & 4.79 & 4.80 \\
\hline September & 16.9 & 1994 & 0 & $\mathrm{~m}$ & 1.29 & 3.43 & 2.67 & 1.21 \\
\hline Annual & 22.2 & 1993 & 0.589 & 1963 & 8.60 & 7.27 & 0.85 & 100 \\
\hline
\end{tabular}

Annual flow duration

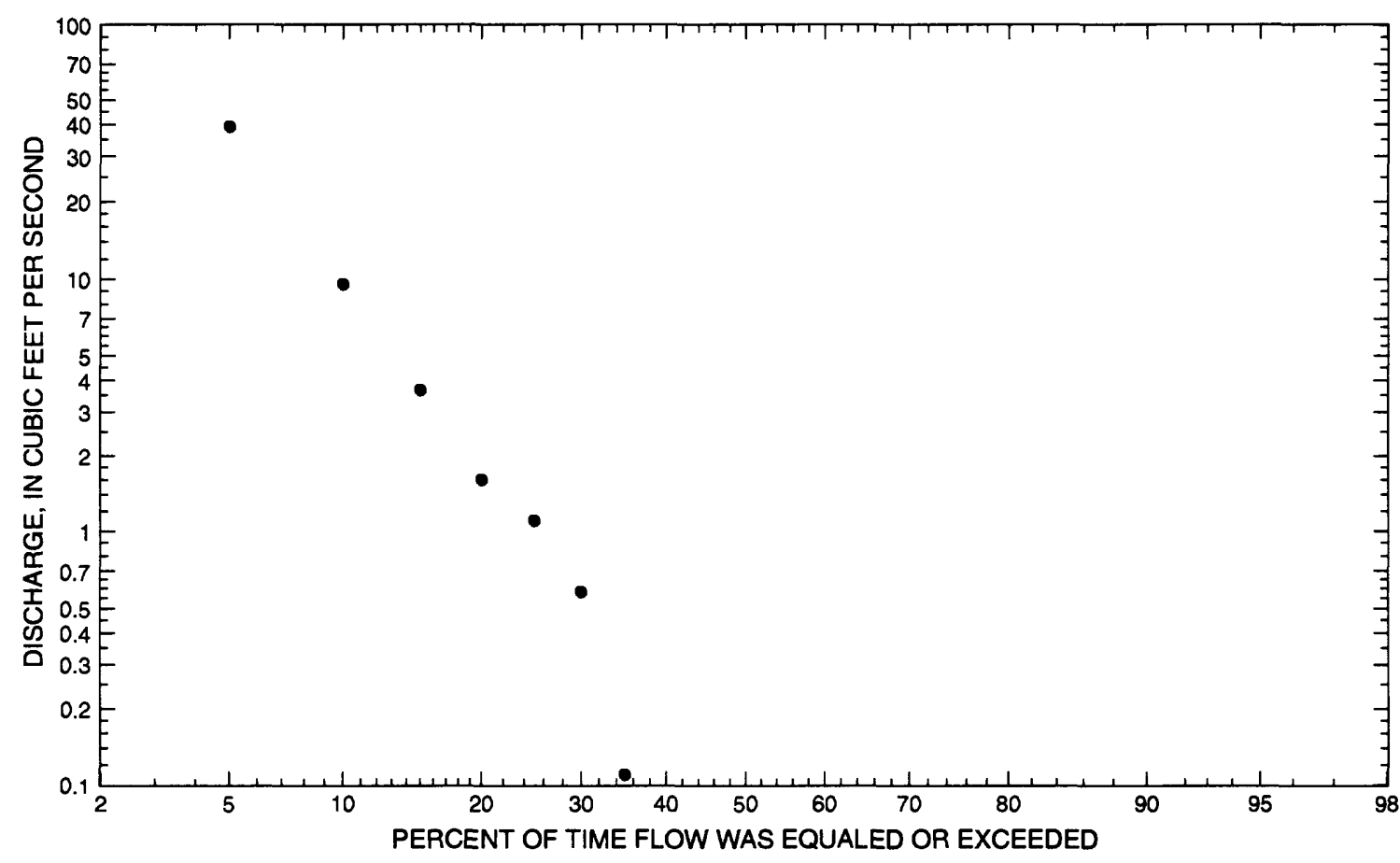




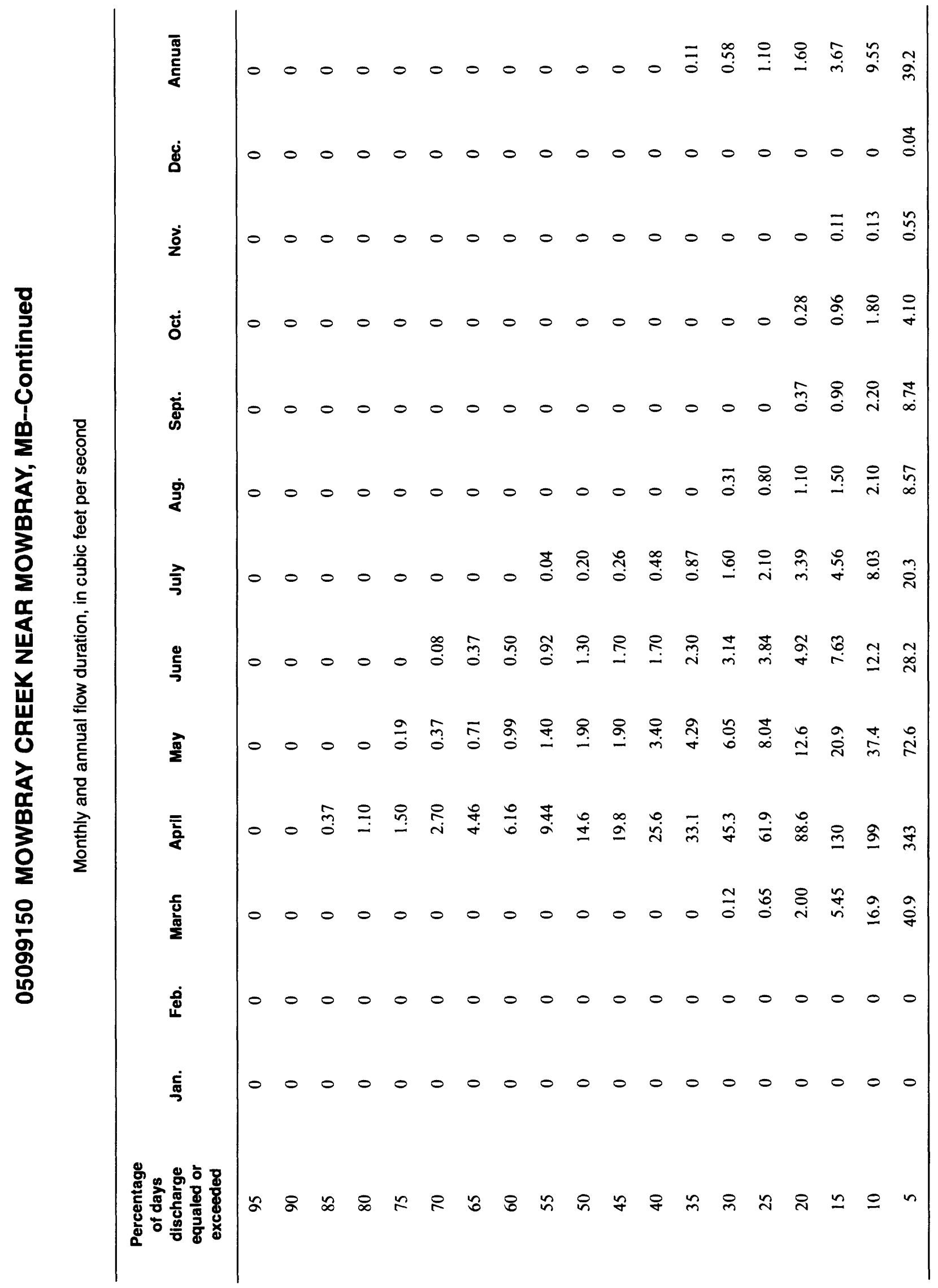


05099150 MOWBRAY CREEK NEAR MOWBRAY, MB--Continued

Probability of occurrence of annual high discharges

[ng, statistic not given]

\begin{tabular}{lcccccc}
\hline & & & \multicolumn{5}{c}{$\begin{array}{c}\text { Maximum mean discharge } \\
\left(\mathbf{f t}^{3} / \mathbf{s}\right)\end{array}$} \\
\cline { 6 - 7 } $\begin{array}{c}\text { Exceedance } \\
\text { probabiity }\end{array}$ & $\begin{array}{c}\text { Recurrence } \\
\text { intervai } \\
\text { (years) }\end{array}$ & $\begin{array}{c}\text { Maximum } \\
\text { instantaneous } \\
\left(\mathbf{f t}^{\mathbf{3} / \mathbf{s})}\right.\end{array}$ & 3-day period & 7-day period & 15-day period & 30-day period \\
\hline 0.99 & 1.01 & 6.70 & 2.86 & 2.67 & 1.94 & 1.37 \\
0.95 & 1.05 & 22.5 & 11.7 & 9.88 & 6.77 & 4.60 \\
0.90 & 1.11 & 40.3 & 23.0 & 18.6 & 12.5 & 8.36 \\
0.80 & 1.25 & 77.4 & 48.4 & 38.1 & 25.1 & 16.5 \\
0.50 & 2 & 230 & 163 & 126 & 82.3 & 52.5 \\
0.20 & 5 & 564 & 428 & 340 & 227 & 141 \\
0.10 & 10 & 837 & 645 & 528 & 360 & 222 \\
0.04 & 25 & 1,210 & 936 & 800 & 563 & 343 \\
0.02 & 50 & 1,500 & 1,150 & 1,020 & 732 & 444 \\
0.01 & 100 & 1,790 & 1,360 & 1,240 & 912 & 551 \\
0.005 & 200 & 2,070 & 1,560 & 1,470 & 1,100 & 662 \\
0.002 & 500 & 2,430 & $\mathrm{ng}$ & $\mathrm{ng}$ & $\mathrm{ng}$ & $\mathrm{ng}$ \\
\hline
\end{tabular}

Probability of occurrence of annual low discharges

[ng, statistic not given]

\begin{tabular}{|c|c|c|c|c|c|c|c|c|c|c|}
\hline \multirow{3}{*}{$\begin{array}{l}\text { Non- } \\
\text { exceed- } \\
\text { ance } \\
\text { prob- } \\
\text { ability }\end{array}$} & \multirow{3}{*}{$\begin{array}{c}\text { Recur- } \\
\text { rence } \\
\text { inter- } \\
\text { vai } \\
\text { (years) }\end{array}$} & \multicolumn{9}{|c|}{ Minimum mean discharge $\left(\mathrm{ft}^{3} / \mathrm{s}\right)$} \\
\hline & & \multicolumn{9}{|c|}{ Number of consecutive days } \\
\hline & & 1 & 3 & 7 & 14 & 30 & 60 & 90 & 120 & 183 \\
\hline 0.05 & 20 & ng & ng & ng & $\mathrm{ng}$ & $\mathrm{ng}$ & ng & $\mathrm{ng}$ & $\mathrm{ng}$ & ng \\
\hline 0.10 & 10 & ng & ng & ng & $\mathrm{ng}$ & $\mathrm{ng}$ & $\mathrm{ng}$ & ng & $\mathrm{ng}$ & $\mathrm{ng}$ \\
\hline 0.20 & 5 & ng & ng & ng & ng & $\mathrm{ng}$ & ng & ng & ng & ng \\
\hline 0.50 & 2 & $\mathrm{ng}$ & $\mathrm{ng}$ & $\mathrm{ng}$ & $\mathrm{ng}$ & ng & ng & $\mathrm{ng}$ & $\mathrm{ng}$ & $\mathrm{ng}$ \\
\hline
\end{tabular}




\section{MOWBRAY CREEK NEAR MOWBRAY, MB--Continued}

Probability of occurrence of seasonal low discharges

[ng, statistic not given]

\begin{tabular}{|c|c|c|c|c|c|c|c|c|c|}
\hline \multirow[b]{3}{*}{$\begin{array}{c}\text { Non- } \\
\text { exceedance } \\
\text { probability }\end{array}$} & \multirow[b]{3}{*}{$\begin{array}{c}\text { Recurrence } \\
\text { intervai } \\
\text { (years) }\end{array}$} & \multicolumn{8}{|c|}{ Minimum mean discharge $\left(\mathrm{ft}^{3} / \mathrm{s}\right)$} \\
\hline & & \multicolumn{8}{|c|}{ Number of consecutive days } \\
\hline & & 1 & 7 & 14 & 30 & 1 & 7 & 14 & 30 \\
\hline & & \multicolumn{4}{|c|}{ December-January-February } & \multicolumn{4}{|c|}{ March-April-May } \\
\hline 0.05 & 20 & $\mathrm{ng}$ & ng & ng & ng & $\mathrm{ng}$ & ng & 0 & 0 \\
\hline 0.10 & 10 & ng & ng & ng & ng & $\mathrm{ng}$ & ng & 0 & 0 \\
\hline 0.20 & 5 & ng & ng & ng & ng & ng & ng & 0 & 0 \\
\hline \multirow[t]{2}{*}{0.50} & 2 & ng & ng & ng & ng & ng & ng & 0 & 0.096 \\
\hline & & \multicolumn{4}{|c|}{ June-July-August } & \multicolumn{4}{|c|}{ September-October-November } \\
\hline 0.05 & 20 & 0 & 0 & 0 & 0 & ng & ng & ng & ng \\
\hline 0.10 & 10 & 0 & 0 & 0 & 0 & ng & ng & ng & ng \\
\hline 0.20 & 5 & 0 & 0 & 0 & 0 & $\mathrm{ng}$ & $\mathrm{ng}$ & ng & $\mathrm{ng}$ \\
\hline 0.50 & 2 & 0 & 0 & 0 & 0 & $\mathrm{ng}$ & ng & $\mathrm{ng}$ & ng \\
\hline
\end{tabular}




\section{MOWBRAY CREEK NEAR MOWBRAY, MB--Continued}

Annual peak discharge and corresponding gage height

$$
\text { [--, no data] }
$$

\begin{tabular}{|c|c|c|c|c|c|c|c|}
\hline $\begin{array}{l}\text { Water } \\
\text { year }\end{array}$ & Date & $\begin{array}{l}\text { Gage } \\
\text { height } \\
\text { (feet) }\end{array}$ & $\begin{array}{c}\text { Peak } \\
\text { discharge } \\
\left(\mathrm{ft}^{3} / \mathrm{s}\right)\end{array}$ & $\begin{array}{l}\text { Water } \\
\text { year }\end{array}$ & Date & $\begin{array}{l}\text { Gage } \\
\text { height } \\
\text { (feet) }\end{array}$ & $\begin{array}{c}\text { Peak } \\
\text { discharge } \\
\left(\mathrm{tt}^{3} / \mathrm{s}\right)\end{array}$ \\
\hline \multicolumn{8}{|c|}{ Annual peak discharge, by year, and corresponding gage height } \\
\hline 1962 & April 20 & $1,531.73$ & 305 & 1979 & April 24 & $1,533.00$ & 777 \\
\hline 1963 & June 25 & $1,528.95$ & 9.20 & 1980 & May 29 & $1,532.27$ & 310 \\
\hline 1964 & April 16 & $1,529.57$ & 22.8 & 1981 & May 22 & $1,530.96$ & 126 \\
\hline 1965 & April 13 & $1,531.36$ & 202 & 1982 & April 14 & $1,532.80$ & 350 \\
\hline 1966 & March 29 & $1,533.86$ & 305 & 1983 & April 7 & $1,531.49$ & 155 \\
\hline 1967 & April 24 & $1,532.42$ & 392 & 1984 & June 10 & $1,530.45$ & 68.0 \\
\hline 1968 & April 9 & $1,529.33$ & 17.6 & 1985 & March 22 & $1,532.92$ & 484 \\
\hline 1969 & April 15 & $1,532.23$ & 377 & 1986 & March 28 & $1,533.79$ & 643 \\
\hline 1970 & April 27 & $1,532.23$ & 372 & 1987 & April 6 & $1,534.57$ & 943 \\
\hline 1971 & April 12 & $1,532.93$ & 630 & 1988 & April 3 & $1,530.80$ & 55.0 \\
\hline 1972 & March 21 & $1,531.35$ & 138 & 1989 & April 19 & $1,532.59$ & 392 \\
\hline 1973 & March 18 & -- & 67.0 & 1990 & April 7 & $1,531.64$ & 137 \\
\hline 1974 & April 21 & $1,533.00$ & 670 & 1991 & May 31 & $1,531.75$ & 133 \\
\hline 1975 & April 15 & $1,530.47$ & 59.0 & 1992 & April 1 & $1,534.10$ & 685 \\
\hline 1976 & April 7 & $1,532.47$ & 455 & 1993 & August 3 & $1,533.25$ & 317 \\
\hline 1977 & September 8 & $1,529.91$ & 29.0 & 1994 & April 2 & $1,533.99$ & 784 \\
\hline 1978 & April 11 & $1,532.44$ & 378 & & & & \\
\hline \multicolumn{8}{|c|}{ Annual peak discharge, from highest to lowest, and corresponding gage height } \\
\hline 1987 & April 6 & $1,534.57$ & 943 & 1962 & April 20 & $1,531.73$ & 305 \\
\hline 1994 & April 2 & $1,533.99$ & 784 & 1966 & March 29 & $1,533.86$ & 305 \\
\hline 1979 & April 24 & $1,533.00$ & 777 & 1965 & April 13 & $1,531.36$ & 202 \\
\hline 1992 & April 1 & $1,534.10$ & 685 & 1983 & April 7 & $1,531.49$ & 155 \\
\hline 1974 & April 21 & $1,533.00$ & 670 & 1972 & March 21 & $1,531.35$ & 138 \\
\hline 1986 & March 28 & $1,533.79$ & 643 & 1990 & April 7 & $1,531.64$ & 137 \\
\hline 1971 & April 12 & $1,532.93$ & 630 & 1991 & May 31 & $1,531.75$ & 133 \\
\hline 1985 & March 22 & $1,532.92$ & 484 & 1981 & May 22 & $1,530.96$ & 126 \\
\hline 1976 & April 7 & $1,532.47$ & 455 & 1984 & June 10 & $1,530.45$ & 68.0 \\
\hline 1967 & April 24 & $1,532.42$ & 392 & 1973 & March 18 & -- & 67.0 \\
\hline 1989 & April 19 & $1,532.59$ & 392 & 1975 & April 15 & $1,530.47$ & 59.0 \\
\hline 1978 & April 11 & $1,532.44$ & 378 & 1988 & April 3 & $1,530.80$ & 55.0 \\
\hline 1969 & April 15 & $1,532.23$ & 377 & 1977 & September 8 & $1,529.91$ & 29.0 \\
\hline 1970 & April 27 & $1,532.23$ & 372 & 1964 & April 16 & $1,529.57$ & 22.8 \\
\hline 1982 & April 14 & $1,532.80$ & 350 & 1968 & April 9 & $1,529.33$ & 17.6 \\
\hline 1993 & August 3 & $1,533.25$ & 317 & 1963 & June 25 & $1,528.95$ & 9.20 \\
\hline 1980 & May 29 & $1,532.27$ & 310 & & & & \\
\hline
\end{tabular}




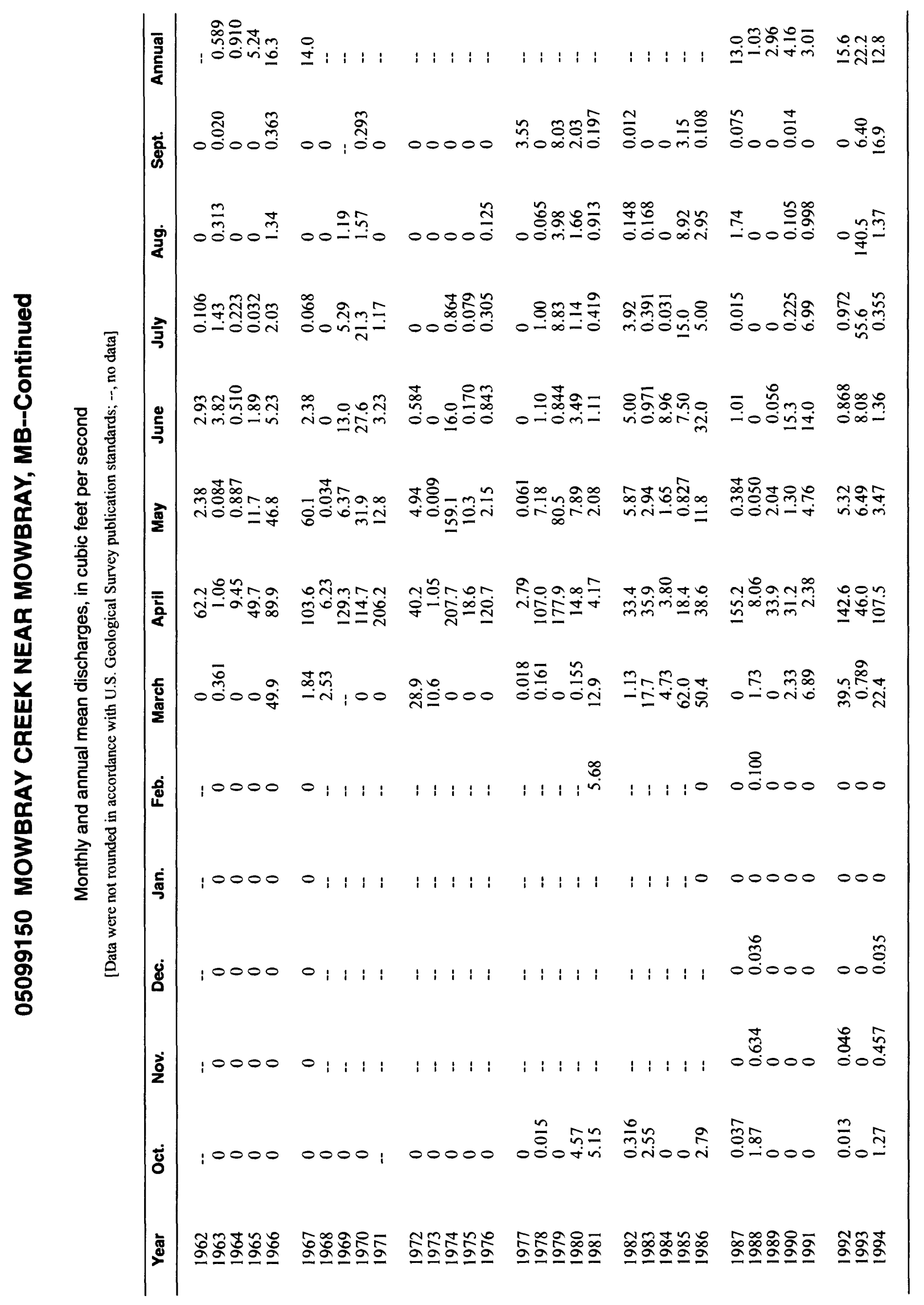




\section{PEMBINA RIVER NEAR WINDYGATES, MB}

LOCATION.--Lat 4901'53', long 98 $16^{\prime} 40^{\prime \prime}$, in SE $1 / 4$ sec.13, T.1, R.7 W., first meridian, Hydrologic Unit 09020313 , on left bank $0.2 \mathrm{mi}$ downstream from bridge, and $3 \mathrm{mi}$ northeast of Windygates, Manitoba.

DRAINAGE AREA.--3,020 $\mathrm{mi}^{2}$.

PERIOD OF RECORD.--April 1962 to current year.

GAGE.--Water-stage recorder. Datum of gage is Geodetic Survey of Canada Datum of 1929. Prior to Jan. 1, 1985, datum of gage at 1,102.02 ft above Geodetic Survey of Canada Datum of 1929.

EXTREMES FOR PERIOD OF RECORD.--Maximum discharge, 11,500 $\mathrm{ft}^{3} / \mathrm{s}$, Apr. 26, 1974, gage height, $1,121.52 \mathrm{ft}$; no flow at times.

Annual mean discharge

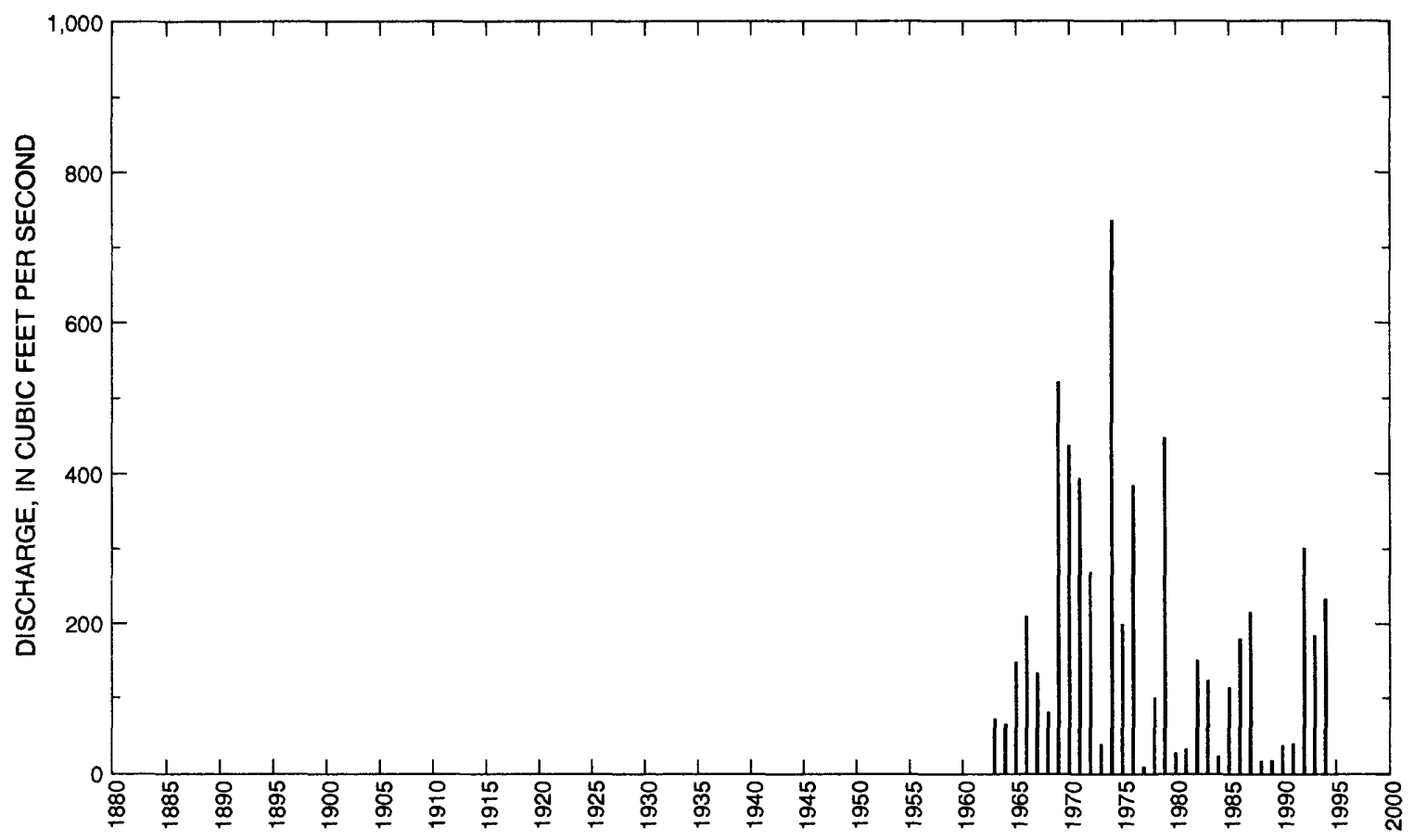


05099300 PEMBINA RIVER NEAR WINDYGATES, MB--Continued

Statistics of monthly and annual mean discharges

[m, more than 1 year of occurrence]

\begin{tabular}{|c|c|c|c|c|c|c|c|c|}
\hline \multirow[b]{2}{*}{ Month } & \multicolumn{2}{|c|}{ Maximum } & \multicolumn{2}{|c|}{ Minimum } & \multicolumn{4}{|c|}{ Mean } \\
\hline & $\begin{array}{c}\text { Discharge } \\
\left(\mathrm{ft}^{3} / \mathrm{s}\right)\end{array}$ & $\begin{array}{c}\text { Water year } \\
\text { of } \\
\text { occurrence }\end{array}$ & $\begin{array}{c}\text { Discharge } \\
\left(\mathrm{ft}^{3} / \mathrm{s}\right)\end{array}$ & $\begin{array}{c}\text { Water year } \\
\text { of } \\
\text { occurrence }\end{array}$ & $\begin{array}{c}\text { Discharge } \\
\left(\mathrm{ft}^{3} / \mathrm{s}\right)\end{array}$ & $\begin{array}{l}\text { Standard } \\
\text { deviation } \\
\left(\mathrm{ft}^{3} / \mathrm{s}\right)\end{array}$ & $\begin{array}{l}\text { Coeffi- } \\
\text { cient of } \\
\text { variation }\end{array}$ & $\begin{array}{c}\text { Percentage } \\
\text { of annual } \\
\text { discharge }\end{array}$ \\
\hline October & 343 & 1969 & 0 & $\mathrm{~m}$ & 45.1 & 71.9 & 1.59 & 2.05 \\
\hline November & 170 & 1969 & 0 & $\mathrm{~m}$ & 24.5 & 36.0 & 1.47 & 1.11 \\
\hline December & 56.9 & 1994 & 0 & $\mathrm{~m}$ & 10.6 & 14.2 & 1.34 & 0.480 \\
\hline January & 27.0 & 1994 & 0 & $\mathrm{~m}$ & 5.30 & 6.90 & 1.30 & 0.240 \\
\hline February & 44.5 & 1976 & 0 & $\mathrm{~m}$ & 4.86 & 8.81 & 1.81 & 0.220 \\
\hline March & 320 & 1985 & 0 & $\mathrm{~m}$ & 70.5 & 107 & 1.51 & 3.20 \\
\hline April & 3,110 & 1969 & 21.3 & 1977 & 821 & 890 & 1.08 & 37.3 \\
\hline May & 3,620 & 1974 & 27.0 & 1988 & 669 & 830 & 1.24 & 30.4 \\
\hline June & 1,510 & 1974 & 4.03 & 1988 & 274 & 322 & 1.18 & 12.5 \\
\hline July & 633 & 1970 & 0.070 & 1988 & 124 & 135 & 1.09 & 5.62 \\
\hline August & 719 & 1993 & 0 & 1988 & 86.1 & 134 & 1.55 & 3.91 \\
\hline September & 544 & 1993 & 0 & $\mathrm{~m}$ & 66.8 & 107 & 1.60 & 3.03 \\
\hline Annual & 736 & 1974 & 9.61 & 1977 & 186 & 174 & 0.940 & 100 \\
\hline
\end{tabular}

Annual flow duration

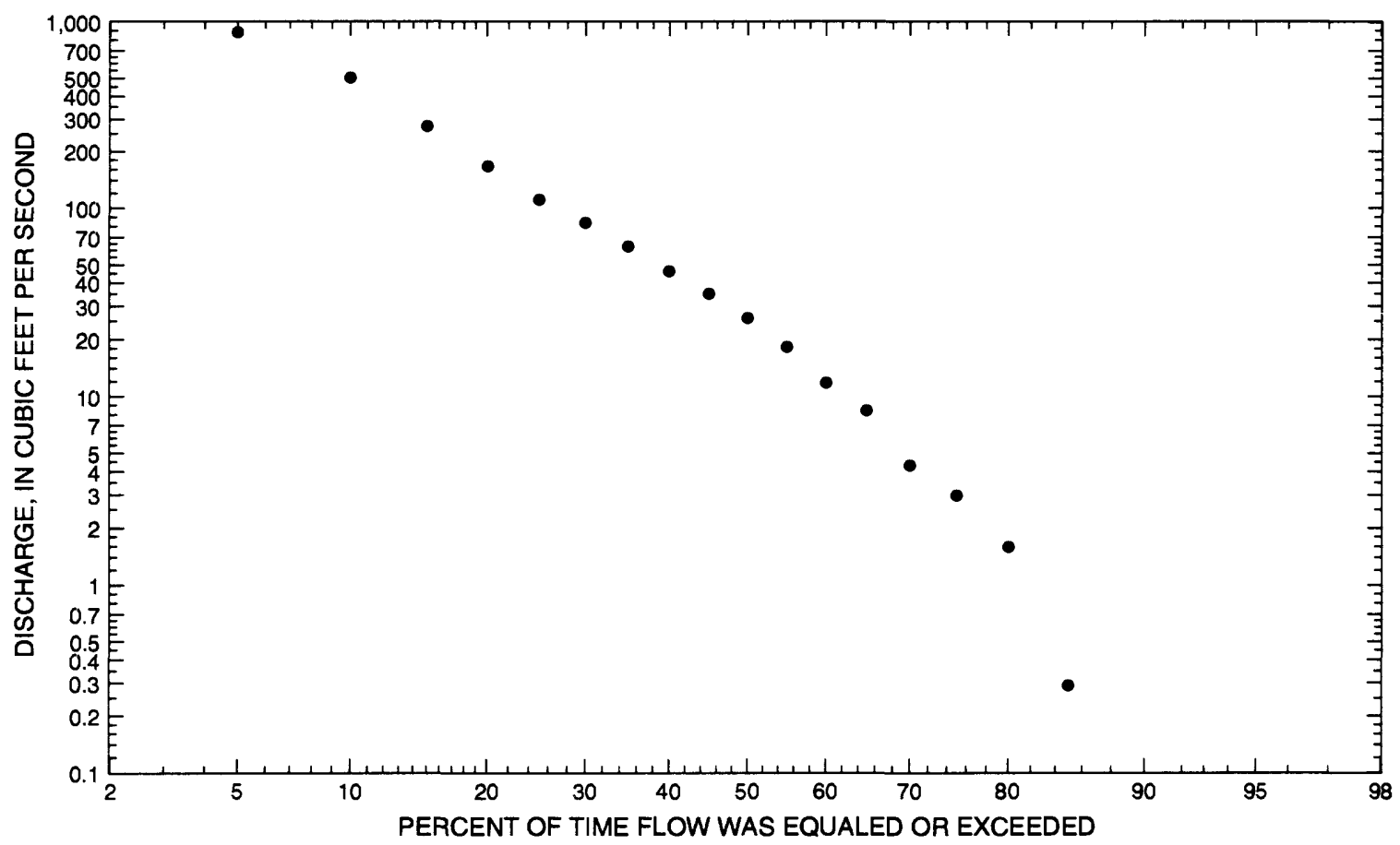




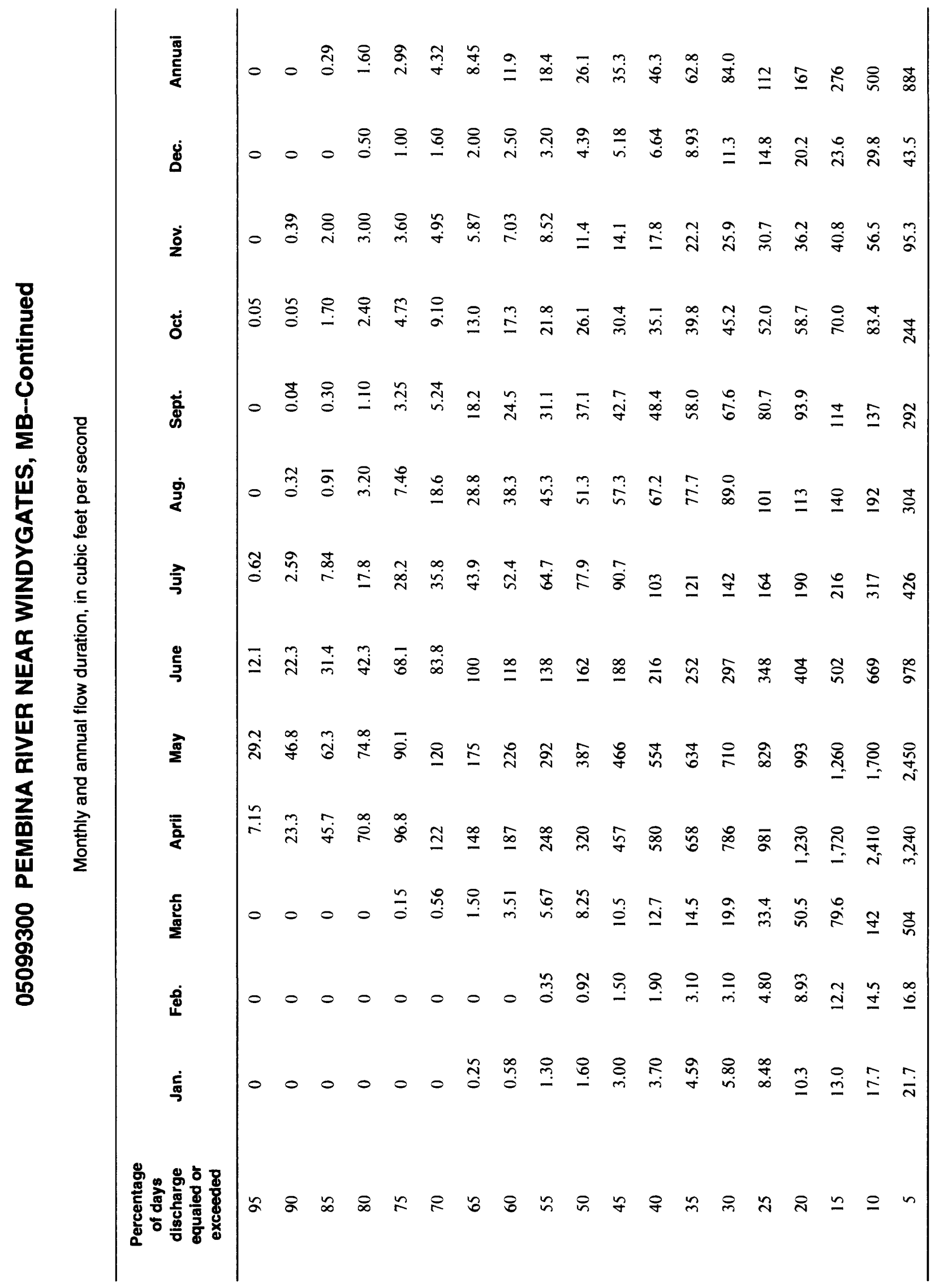




\section{PEMBINA RIVER NEAR WINDYGATES, MB--Continued}

Probability of occurrence of annual high discharges

[ng, statistic not given]

\begin{tabular}{|c|c|c|c|c|c|c|}
\hline \multirow[b]{2}{*}{$\begin{array}{l}\text { Exceedance } \\
\text { probability }\end{array}$} & \multirow[b]{2}{*}{$\begin{array}{l}\text { Recurrence } \\
\text { interval } \\
\text { (years) }\end{array}$} & \multirow[b]{2}{*}{$\begin{array}{l}\text { Maximum } \\
\text { instantaneous } \\
\left(\mathrm{ft}^{3} / \mathrm{s}\right)\end{array}$} & \multicolumn{4}{|c|}{$\begin{array}{l}\text { Maximum mean discharge } \\
\qquad\left(\mathrm{ft}^{3} / \mathrm{s}\right)\end{array}$} \\
\hline & & & 3-day period & 7-day period & 15-day period & 30-day period \\
\hline 0.99 & 1.01 & 74.7 & 52.3 & 43.8 & 32.5 & 22.2 \\
\hline 0.95 & 1.05 & 174 & 122 & 105 & 84.0 & 64.1 \\
\hline 0.90 & 1.11 & 270 & 191 & 168 & 137 & 110 \\
\hline 0.80 & 1.25 & 454 & 330 & 293 & 247 & 206 \\
\hline 0.50 & 2 & 1,190 & 937 & 841 & 728 & 634 \\
\hline 0.20 & 5 & 3,020 & 2,660 & 2,380 & 2,050 & 1,780 \\
\hline 0.10 & 10 & 4,830 & 4,600 & 4,080 & 3,460 & 2,950 \\
\hline 0.04 & 25 & 7,880 & 8,240 & 7,200 & 5,950 & 4,930 \\
\hline 0.02 & 50 & 10,700 & 12,000 & 10,400 & 8,400 & 6,760 \\
\hline 0.01 & 100 & 14,100 & 16,800 & 14,400 & 11,400 & 8,890 \\
\hline 0.005 & 200 & 18,100 & 23,000 & 19,400 & 15,000 & 11,300 \\
\hline 0.002 & 500 & 24,300 & ng & ng & ng & ng \\
\hline
\end{tabular}

Probability of occurrence of annual low discharges

\begin{tabular}{|c|c|c|c|c|c|c|c|c|c|c|}
\hline \multirow[b]{3}{*}{$\begin{array}{l}\text { Non- } \\
\text { exceed- } \\
\text { ance } \\
\text { prob- } \\
\text { ability }\end{array}$} & \multirow[b]{3}{*}{$\begin{array}{c}\text { Recur- } \\
\text { rence } \\
\text { inter- } \\
\text { val } \\
\text { (years) }\end{array}$} & \multicolumn{9}{|c|}{ Minimum mean discharge $\left(\mathrm{ft}^{3} / \mathrm{s}\right)$} \\
\hline & & \multicolumn{9}{|c|}{ Number of consecutlve days } \\
\hline & & 1 & 3 & 7 & 14 & 30 & 60 & 90 & 120 & 183 \\
\hline 0.05 & 20 & 0 & 0 & 0 & 0 & 0 & 0 & 0 & 0 & 0 \\
\hline 0.10 & 10 & 0 & 0 & 0 & 0 & 0 & 0 & 0 & 0.030 & 0.192 \\
\hline 0.20 & 5 & 0 & 0 & 0 & 0 & 0 & 0 & 0.048 & 0.472 & 1.32 \\
\hline 0.50 & 2 & 0.139 & 0.146 & 0.168 & 0.441 & 0.646 & 0.983 & 1.93 & 3.18 & 10.5 \\
\hline
\end{tabular}




\section{PEMBINA RIVER NEAR WINDYGATES, MB--Continued}

Probability of occurrence of seasonal low discharges

\begin{tabular}{|c|c|c|c|c|c|c|c|c|c|}
\hline \multirow[b]{3}{*}{$\begin{array}{c}\text { Non- } \\
\text { exceedance } \\
\text { probability }\end{array}$} & \multirow[b]{3}{*}{$\begin{array}{c}\text { Recurrence } \\
\text { intervai } \\
\text { (years) }\end{array}$} & \multicolumn{8}{|c|}{ Minimum mean discharge $\left(\mathrm{ft}^{3} / \mathrm{s}\right)$} \\
\hline & & \multicolumn{8}{|c|}{ Number of consecutive days } \\
\hline & & 1 & 7 & 14 & 30 & 1 & 7 & 14 & 30 \\
\hline & & \multicolumn{4}{|c|}{ December-January-February } & \multicolumn{4}{|c|}{ March-April-May } \\
\hline 0.05 & 20 & 0 & 0 & 0 & 0 & 0 & 0 & 0 & 0 \\
\hline 0.10 & 10 & 0 & 0 & 0 & 0 & 0 & 0 & 0 & 0.246 \\
\hline 0.20 & 5 & 0 & 0 & 0 & 0 & 0 & 0 & 0 & 2.37 \\
\hline \multirow[t]{2}{*}{0.50} & 2 & 0.284 & 0.356 & 0.459 & 0.715 & 1.35 & 2.15 & 2.82 & 15.2 \\
\hline & & \multicolumn{4}{|c|}{ June-July-August } & \multicolumn{4}{|c|}{ September-October-November } \\
\hline 0.05 & 20 & 0 & 0 & 0 & 0 & 0 & 0 & 0 & 0 \\
\hline 0.10 & 10 & 0.006 & 0.035 & 0.076 & 0.755 & 0 & 0 & 0 & 0.012 \\
\hline 0.20 & 5 & 0.998 & 1.79 & 2.48 & 4.35 & 0.298 & 0.576 & 0.913 & 0.968 \\
\hline 0.50 & 2 & 21.6 & 23.1 & 25.9 & 33.2 & 5.33 & 6.70 & 7.78 & 11.5 \\
\hline
\end{tabular}




\section{PEMBINA RIVER NEAR WINDYGATES, MB--Continued}

Annual peak discharge and corresponding gage height

$$
[--, \text { no data }]
$$

\begin{tabular}{|c|c|c|c|c|c|c|c|}
\hline $\begin{array}{c}\text { Water } \\
\text { year }\end{array}$ & Date & $\begin{array}{c}\text { Gage } \\
\text { height } \\
\text { (feet) }\end{array}$ & $\begin{array}{c}\text { Peak } \\
\text { discharge } \\
\left(\mathrm{ft}^{3} / \mathrm{s}\right)\end{array}$ & $\begin{array}{c}\text { Water } \\
\text { year }\end{array}$ & Date & $\begin{array}{l}\text { Gage } \\
\text { height } \\
\text { (feet) }\end{array}$ & $\begin{array}{c}\text { Peak } \\
\text { discharge } \\
\left(\mathrm{ft}^{3} / \mathrm{s}\right)\end{array}$ \\
\hline \multicolumn{8}{|c|}{ Annual peak discharge, by year, and corresponding gage height } \\
\hline 1962 & April 21 & $1,108.51$ & 1,610 & 1979 & May 2 & $1,116.63$ & 5,440 \\
\hline 1963 & June 6 & $1,105.17$ & 255 & 1980 & April 6 & -- & 327 \\
\hline 1964 & May 10 & $1,105.62$ & 323 & 1981 & May 22 & $1,107.31$ & 526 \\
\hline 1965 & April 14 & $1,109.75$ & 1,460 & 1982 & June 6 & $1,110.89$ & 1,920 \\
\hline 1966 & April 3 & $1,110.94$ & 1,700 & 1983 & April 8 & $1,109.29$ & 1,160 \\
\hline 1967 & April 21 & $1,111.06$ & 1,890 & 1984 & June 16 & $1,103.63$ & 491 \\
\hline 1968 & March 24 & $1,108.51$ & 744 & 1985 & April 5 & $1,108.93$ & 1,220 \\
\hline 1969 & April 19 & $1,119.31$ & 8,170 & 1986 & March 28 & $1,109.49$ & 1,230 \\
\hline 1970 & April 28 & $1,115.01$ & 3,800 & 1987 & April 8 & $1,111.90$ & 2,540 \\
\hline 1971 & April 10 & $1,115.04$ & 5,910 & 1988 & April 4 & $1,106.75$ & 178 \\
\hline 1972 & April 12 & -- & 1,620 & 1989 & April 16 & $1,106.79$ & 441 \\
\hline 1973 & August 8 & -- & 145 & 1990 & April 3 & $1,108.50$ & 794 \\
\hline 1974 & April 26 & $1,121.52$ & 11,500 & 1991 & July 12 & $1,107.33$ & 487 \\
\hline 1975 & May 14 & $1,109.76$ & 1,510 & 1992 & April 8 & $1,113.73$ & 3,080 \\
\hline 1976 & April 17 & $1,115.13$ & 4,210 & 1993 & July 28 & $1,109.73$ & 1,140 \\
\hline 1977 & May 21 & $1,105.47$ & 153 & 1994 & April 13 & $1,109.94$ & 1,380 \\
\hline 1978 & April 9 & $1,110.88$ & 1,090 & & & & \\
\hline \multicolumn{8}{|c|}{ Annual peak discharge, from highest to lowest, and corresponding gage height } \\
\hline 1974 & April 26 & $1,121.52$ & 11,500 & 1985 & April 5 & $1,108.93$ & 1,220 \\
\hline 1969 & April 19 & $1,119.31$ & 8,170 & 1983 & April 8 & $1,109.29$ & 1,160 \\
\hline 1971 & April 10 & $1,115.04$ & 5,910 & 1993 & July 28 & $1,109.73$ & 1,140 \\
\hline 1979 & May 2 & $1,116.63$ & 5,440 & 1978 & April 9 & $1,110.88$ & 1,090 \\
\hline 1976 & April 17 & $1,115.13$ & 4,210 & 1990 & April 3 & $1,108.50$ & 794 \\
\hline 1970 & April 28 & $1,115.01$ & 3,800 & 1968 & March 24 & $1,108.51$ & 744 \\
\hline 1992 & April 8 & $1,113.73$ & 3,080 & 1981 & May 22 & $1,107.31$ & 526 \\
\hline 1987 & April 8 & $1,111.90$ & 2,540 & 1984 & June 16 & $1,103.63$ & 491 \\
\hline 1982 & June 6 & $1,110.89$ & 1,920 & 1991 & July 12 & $1,107.33$ & 487 \\
\hline 1967 & April 21 & $1,111.06$ & 1,890 & 1989 & April 16 & $1,106.79$ & 441 \\
\hline 1966 & April 3 & $1,110.94$ & 1,700 & 1980 & April 6 & -- & 327 \\
\hline 1972 & April 12 & - & 1,620 & 1964 & May 10 & $1,105.62$ & 323 \\
\hline 1962 & April 21 & $1,108.51$ & 1,610 & 1963 & June 6 & $1,105.17$ & 255 \\
\hline 1975 & May 14 & $1,109.76$ & 1,510 & 1988 & April 4 & $1,106.75$ & 178 \\
\hline 1965 & April 14 & $1,109.75$ & 1,460 & 1977 & May 21 & $1,105.47$ & 153 \\
\hline 1994 & April 13 & $1,109.94$ & 1,380 & 1973 & August 8 & -- & 145 \\
\hline 1986 & March 28 & $1,109.49$ & 1,230 & & & & \\
\hline
\end{tabular}




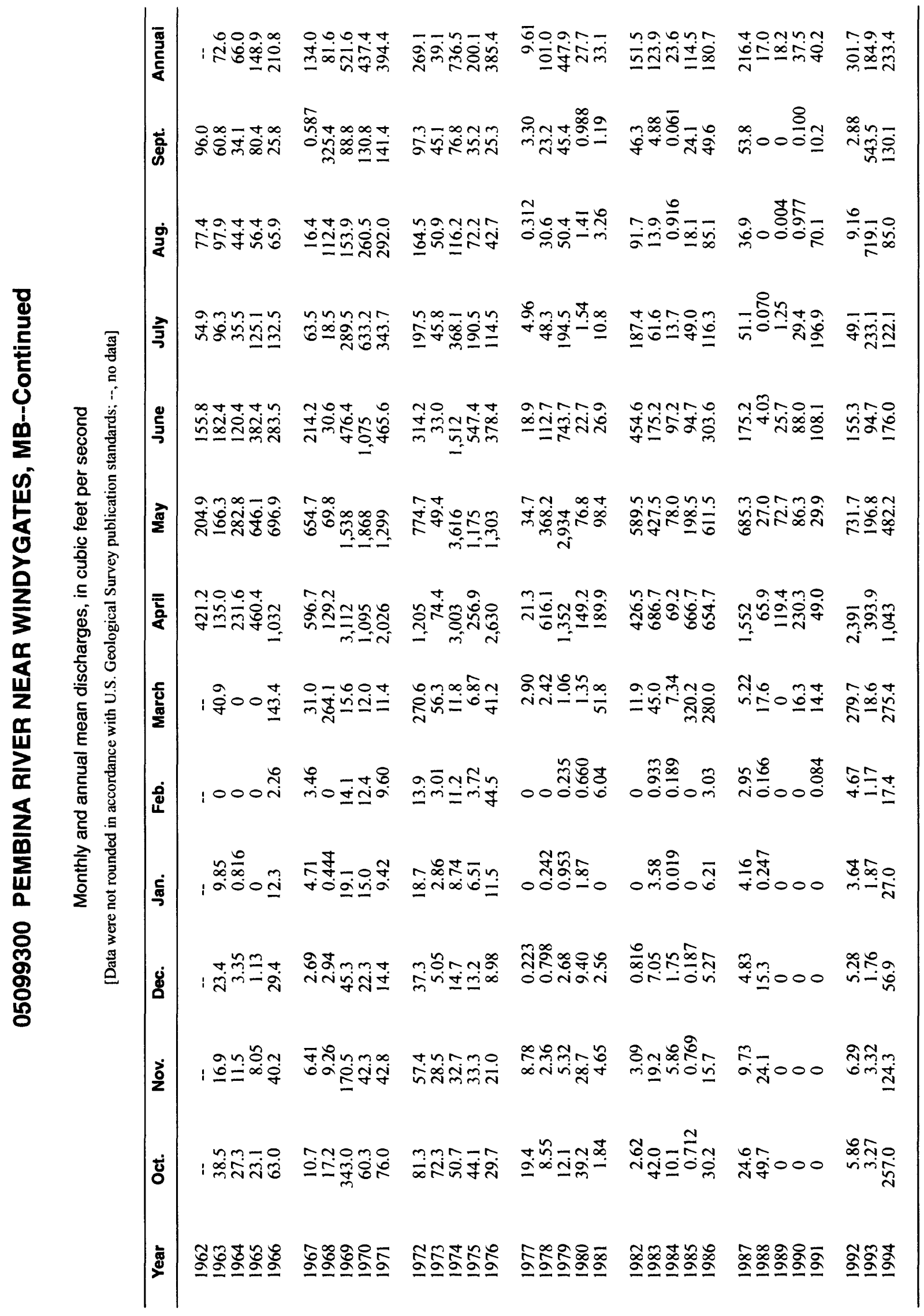




\section{LITTLE SOUTH PEMBINA RIVER NEAR WALHALLA, ND}

LOCATION.--Lat 48 51'55”, long 9800'20”, in SW1/4 sec.10, T.162 N., R.57 W., Cavalier County, Hydrologic Unit 09020313, on right bank $25 \mathrm{ft}$ upstream from county bridge, $3.5 \mathrm{mi}$ above mouth, and 6 mi southwest of Walhalla.

DRAINAGE AREA.--182 $\mathrm{mi}^{2}$, of which $10 \mathrm{mi}^{2}$ is noncontributing.

PERIOD OF RECORD.--April 1956 to September 1982. Prior to October 1973, published as Little Pembina River near Walhalla.

GAGE.--Water-stage recorder. Datum of gage is $1,099.48 \mathrm{ft}$ above sea level. Prior to Sept. 10, 1956, nonrecording gage at bridge $25 \mathrm{ft}$ downstream at same datum.

EXTREMES FOR PERIOD OF RECORD.--Maximum discharge, 6,600 $\mathrm{ft}^{3} / \mathrm{s}$, Apr. 25,1970 , gage height, $13.95 \mathrm{ft}$; maximum gage height, $14.38 \mathrm{ft}$, Apr. 17, 1962, backwater from ice; no flow at times in some years.

Annual mean discharge

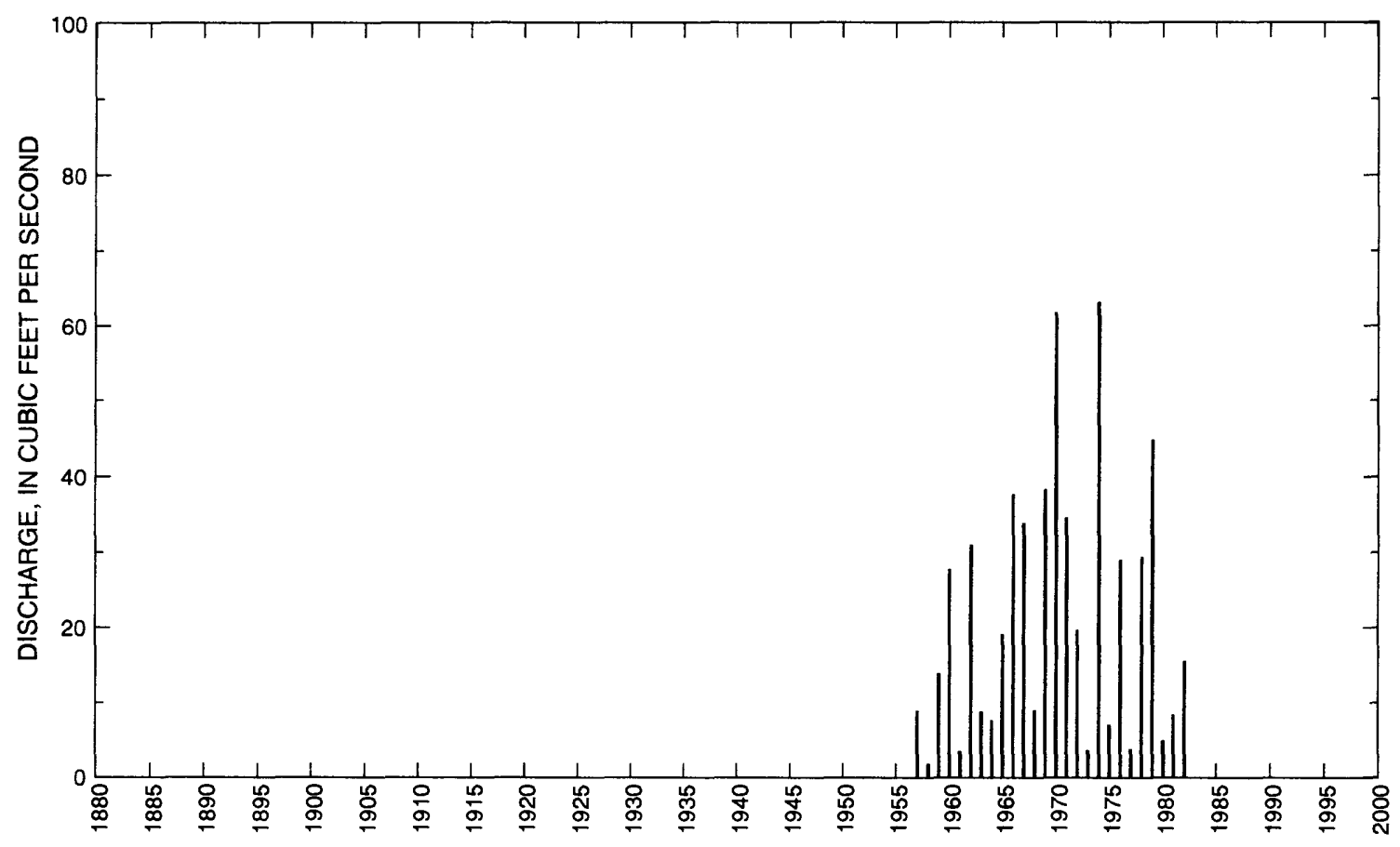


05099400 LITTLE SOUTH PEMBINA RIVER NEAR WALHALLA, ND--Continued

Pre-regulation period, $1956-70$

Statistics of monthly and annual mean discharges, pre-regulation period

[m, more than 1 year of occurrence]

\begin{tabular}{|c|c|c|c|c|c|c|c|c|}
\hline \multirow[b]{2}{*}{ Month } & \multicolumn{2}{|c|}{ Maximum } & \multicolumn{2}{|c|}{ Minimum } & \multicolumn{4}{|c|}{ Mean } \\
\hline & $\begin{array}{c}\text { Discharge } \\
\left(\mathrm{ft}^{3} / \mathrm{s}\right)\end{array}$ & $\begin{array}{c}\text { Water year } \\
\text { of } \\
\text { occurrence }\end{array}$ & $\begin{array}{c}\text { Discharge } \\
\left(\mathrm{ft}^{3} / \mathrm{s}\right)\end{array}$ & $\begin{array}{c}\text { Water year } \\
\text { of } \\
\text { occurrence }\end{array}$ & $\begin{array}{c}\text { Discharge } \\
\left(\mathrm{ft}^{3} / \mathrm{s}\right)\end{array}$ & $\begin{array}{c}\text { Standard } \\
\text { deviation } \\
\left(\mathrm{ft}^{3} / \mathrm{s}\right)\end{array}$ & $\begin{array}{l}\text { Coeffi- } \\
\text { cient of } \\
\text { variation }\end{array}$ & $\begin{array}{l}\text { Percentage } \\
\text { of annual } \\
\text { discharge }\end{array}$ \\
\hline October & 3.26 & 1969 & 0.177 & 1962 & 1.27 & 0.98 & 0.77 & 0.45 \\
\hline November & 2.86 & 1957 & 0.177 & 1962 & 1.04 & 0.75 & 0.73 & 0.37 \\
\hline December & 1.05 & 1966 & 0.229 & 1962 & 0.620 & 0.28 & 0.45 & 0.22 \\
\hline January & 0.834 & 1967 & 0.016 & 1958 & 0.420 & 0.27 & 0.64 & 0.15 \\
\hline February & 0.946 & 1969 & 0 & $\mathrm{~m}$ & 0.340 & 0.28 & 0.83 & 0.12 \\
\hline March & 139 & 1966 & 0 & 1962 & 25.7 & 38.6 & 1.50 & 9.07 \\
\hline April & 461 & 1970 & 6.05 & 1958 & 172 & 162 & 0.94 & 60.6 \\
\hline May & 150 & 1967 & 2.34 & 1958 & 43.5 & 50.0 & 1.15 & 15.3 \\
\hline June & 102 & 1970 & 0.440 & 1958 & 19.6 & 24.8 & 1.27 & 6.90 \\
\hline July & 62.6 & 1970 & 0.181 & 1961 & 11.8 & 18.9 & 1.60 & 4.16 \\
\hline August & 21.6 & 1957 & 0.010 & 1961 & 4.26 & 5.76 & 1.35 & 1.50 \\
\hline September & 17.0 & 1970 & 0.090 & 1961 & 3.15 & 5.11 & 1.62 & 1.11 \\
\hline Annual & 61.8 & 1970 & 1.78 & 1958 & 21.6 & 17.3 & 0.80 & 100 \\
\hline
\end{tabular}

Annual flow duration

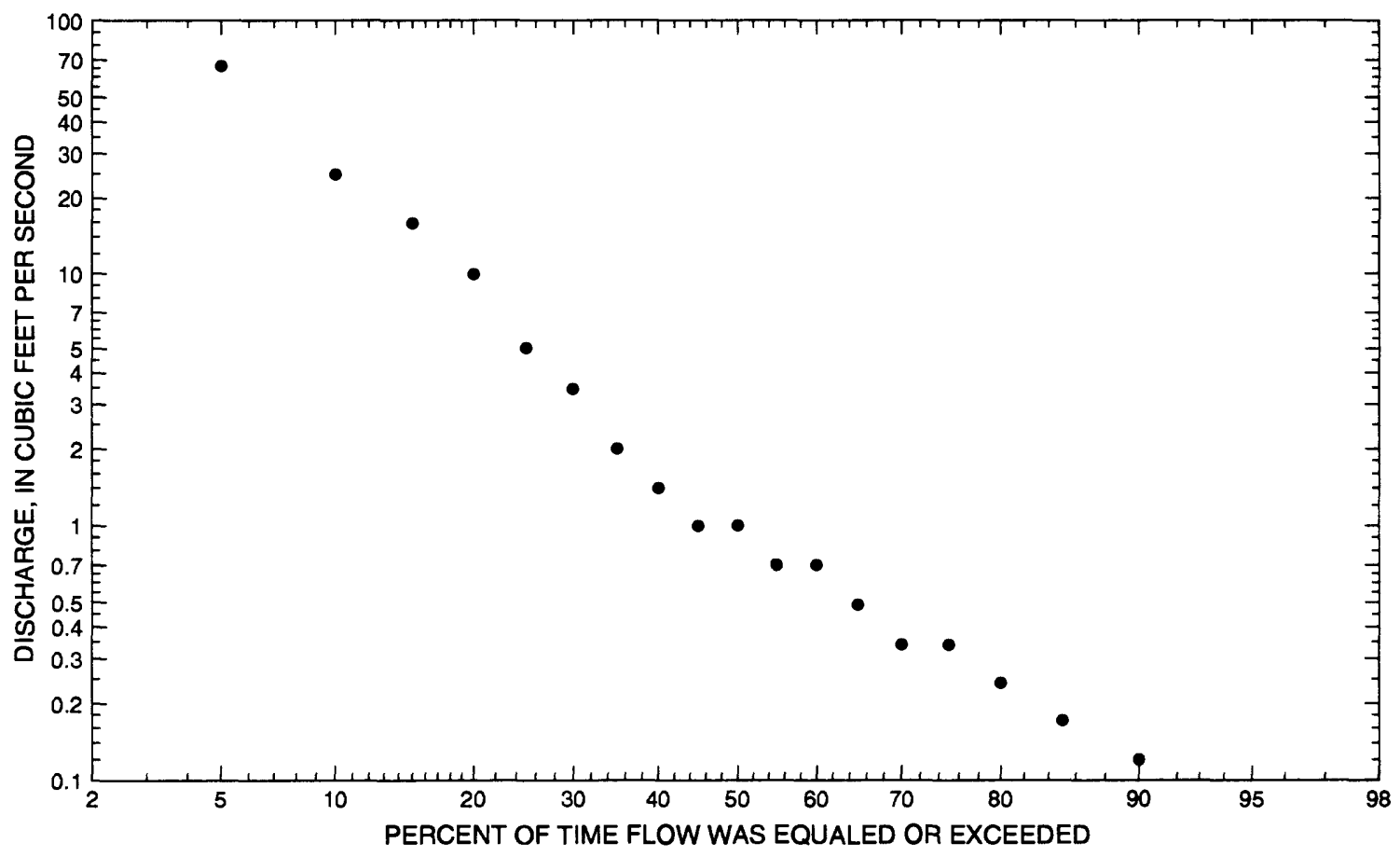




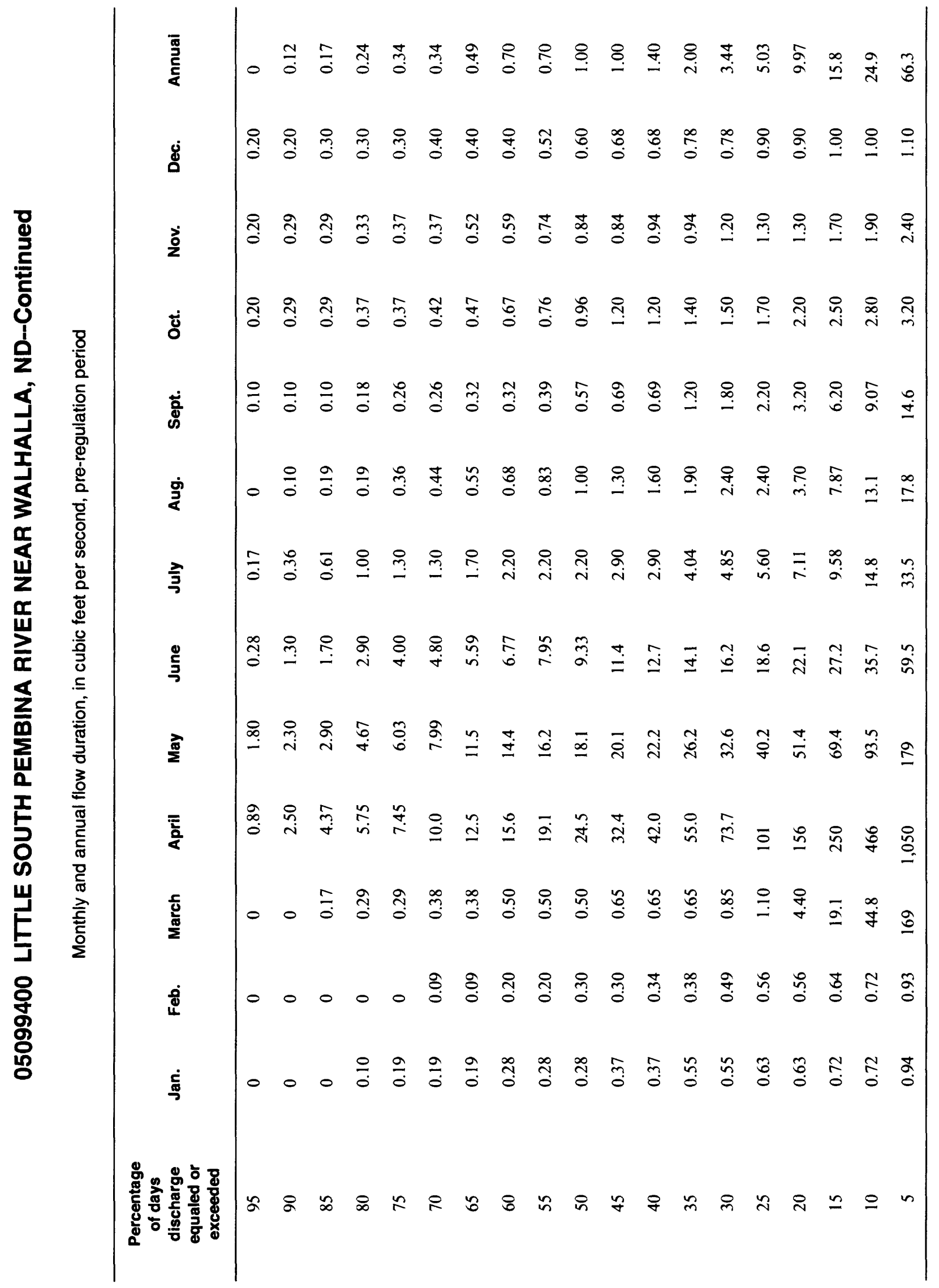


05099400 LITTLE SOUTH PEMBINA RIVER NEAR WALHALLA, ND--Continued

Probability of occurrence of annual high discharges, pre-regulation period

[ng, statistic not given]

\begin{tabular}{|c|c|c|c|c|c|c|}
\hline \multirow[b]{2}{*}{$\begin{array}{l}\text { Exceedance } \\
\text { probability }\end{array}$} & \multirow[b]{2}{*}{$\begin{array}{l}\text { Recurrence } \\
\text { intervai } \\
\text { (years) }\end{array}$} & \multirow[b]{2}{*}{$\begin{array}{c}\text { Maximum } \\
\text { instantaneous } \\
\left(\mathrm{ft}^{3} / \mathrm{s}\right)\end{array}$} & \multicolumn{4}{|c|}{$\begin{array}{l}\text { Maximum mean discharge } \\
\qquad\left(\mathrm{ft}^{3} / \mathrm{s}\right)\end{array}$} \\
\hline & & & 3-day period & 7-day period & 15-day period & 30-day period \\
\hline 0.99 & 1.01 & ng & 6.81 & 5.29 & 5.19 & 3.69 \\
\hline 0.95 & 1.05 & $\mathrm{ng}$ & 36.2 & 26.1 & 20.0 & 13.0 \\
\hline 0.90 & 1.11 & 283 & 78.3 & 54.7 & 37.8 & 23.6 \\
\hline 0.80 & 1.25 & 514 & 179 & 120 & 75.8 & 45.9 \\
\hline 0.50 & 2 & 1,470 & 640 & 407 & 230 & 136 \\
\hline 0.20 & 5 & 3,720 & 1,590 & 965 & 536 & 323 \\
\hline 0.10 & 10 & 5,780 & 2,250 & 1,340 & 758 & 468 \\
\hline 0.04 & 25 & 8,940 & 2,990 & 1,760 & 1,030 & 655 \\
\hline 0.02 & 50 & 11,600 & 3,450 & 2,020 & 1,210 & 790 \\
\hline 0.01 & 100 & 14,600 & 3,840 & 2,230 & 1,380 & 918 \\
\hline 0.005 & 200 & 17,800 & 4,160 & 2,400 & 1,520 & 1,040 \\
\hline 0.002 & 500 & 22,300 & ng & ng & ng & ng \\
\hline
\end{tabular}

Probability of occurrence of annual low discharges, pre-regulation period

\begin{tabular}{|c|c|c|c|c|c|c|c|c|c|c|}
\hline \multirow[b]{3}{*}{$\begin{array}{l}\text { Non- } \\
\text { exceed- } \\
\text { ance } \\
\text { prob- } \\
\text { abiilty }\end{array}$} & \multirow[b]{3}{*}{$\begin{array}{c}\text { Recur- } \\
\text { rence } \\
\text { inter- } \\
\text { vai } \\
\text { (years) }\end{array}$} & \multicolumn{9}{|c|}{ Minimum mean discharge $\left(\mathrm{ft}^{3} / \mathrm{s}\right)$} \\
\hline & & \multicolumn{9}{|c|}{ Number of consecutive days } \\
\hline & & 1 & 3 & 7 & 14 & 30 & 60 & 90 & 120 & 183 \\
\hline 0.05 & 20 & 0 & 0 & 0 & 0 & 0 & 0 & 0.044 & 0.109 & 0.142 \\
\hline 0.10 & 10 & 0 & 0 & 0 & 0 & 0 & 0 & 0.077 & 0.157 & 0.208 \\
\hline 0.20 & 5 & 0 & 0 & 0 & 0 & 0 & 0.049 & 0.139 & 0.235 & 0.316 \\
\hline 0.50 & 2 & 0.061 & 0.070 & 0.098 & 0.135 & 0.179 & 0.239 & 0.337 & 0.442 & 0.623 \\
\hline
\end{tabular}


05099400 LITTLE SOUTH PEMBINA RIVER NEAR WALHALLA, ND--Continued

Probability of occurrence of seasonal low discharges, pre-regulation period

\begin{tabular}{|c|c|c|c|c|c|c|c|c|c|}
\hline \multirow[b]{3}{*}{$\begin{array}{l}\text { Non- } \\
\text { exceedance } \\
\text { probability }\end{array}$} & \multirow[b]{3}{*}{$\begin{array}{c}\text { Recurrence } \\
\text { interval } \\
\text { (years) }\end{array}$} & \multicolumn{8}{|c|}{ Minimum mean discharge $\left(\mathrm{ft}^{3} / \mathrm{s}\right)$} \\
\hline & & \multicolumn{8}{|c|}{ Number of consecutive days } \\
\hline & & 1 & 7 & 14 & 30 & 1 & 7 & 14 & 30 \\
\hline & & \multicolumn{4}{|c|}{ December-January-February } & \multicolumn{4}{|c|}{ March-April-May } \\
\hline 0.05 & 20 & 0 & 0 & 0 & 0 & 0 & 0 & 0 & 0 \\
\hline 0.10 & 10 & 0 & 0 & 0 & 0 & 0 & 0 & 0 & 0.144 \\
\hline 0.20 & 5 & 0 & 0 & 0 & 0 & 0 & 0 & 0 & 0.433 \\
\hline \multirow[t]{2}{*}{0.50} & 2 & 0.072 & 0.119 & 0.171 & 0.227 & 0.358 & 0.396 & 0.400 & 2.33 \\
\hline & & \multicolumn{4}{|c|}{ June-July-August } & \multicolumn{4}{|c|}{ September-October-November } \\
\hline 0.05 & 20 & 0 & 0 & 0 & 0 & 0 & 0 & 0.046 & 0.087 \\
\hline 0.10 & 10 & 0.071 & 0.083 & 0.100 & 0.115 & 0.058 & 0.064 & 0.081 & 0.130 \\
\hline 0.20 & 5 & 0.149 & 0.196 & 0.245 & 0.339 & 0.130 & 0.148 & 0.152 & 0.208 \\
\hline 0.50 & 2 & 0.372 & 0.534 & 0.698 & 1.30 & 0.349 & 0.401 & 0.431 & 0.495 \\
\hline
\end{tabular}


05099400 LITTLE SOUTH PEMBINA RIVER NEAR WALHALLA, ND--Continued

Post-regulation period, 1971-82

Statistics of monthly and annual mean discharges, post-regulation period

[m, more than 1 year of occurrence]

\begin{tabular}{|c|c|c|c|c|c|c|c|c|}
\hline \multirow[b]{2}{*}{ Month } & \multicolumn{2}{|c|}{ Maximum } & \multicolumn{2}{|c|}{ Minimum } & \multicolumn{4}{|c|}{ Mean } \\
\hline & $\begin{array}{c}\text { Discharge } \\
\left(\mathrm{ft}^{3} / \mathrm{s}\right)\end{array}$ & $\begin{array}{c}\text { Water year } \\
\text { of } \\
\text { occurrence }\end{array}$ & $\begin{array}{c}\text { Discharge } \\
\left(\mathrm{ft}^{3} / \mathrm{s}\right)\end{array}$ & $\begin{array}{c}\text { Water year } \\
\text { of } \\
\text { occurrence }\end{array}$ & $\begin{array}{c}\text { Discharge } \\
\left(\mathrm{ft}^{3} / \mathrm{s}\right)\end{array}$ & $\begin{array}{c}\text { Standard } \\
\text { deviation } \\
\left(\mathrm{ft}^{3} / \mathbf{s}\right)\end{array}$ & $\begin{array}{l}\text { Coeffi- } \\
\text { cient of } \\
\text { variation }\end{array}$ & $\begin{array}{l}\text { Percentage } \\
\text { of annual } \\
\text { discharge }\end{array}$ \\
\hline October & 5.45 & 1981 & 0.356 & 1976 & 1.99 & 1.68 & 0.850 & 0.750 \\
\hline November & 3.94 & 1971 & 0.283 & 1977 & 1.42 & 1.12 & 0.790 & 0.530 \\
\hline December & 1.29 & 1971 & 0.053 & 1977 & 0.550 & 0.400 & 0.730 & 0.210 \\
\hline January & 0.629 & 1971 & 0 & 1973 & 0.260 & 0.220 & 0.840 & 0.100 \\
\hline February & 30.1 & 1981 & 0 & $\mathrm{~m}$ & 2.73 & 8.62 & 3.16 & 1.03 \\
\hline March & 93.4 & 1972 & 0.319 & 1975 & 20.2 & 28.4 & 1.41 & 7.60 \\
\hline April & 457 & 1974 & 4.92 & 1973 & 176 & 169 & 0.960 & 66.2 \\
\hline May & 255 & 1974 & 2.85 & 1973 & 41.6 & 72.2 & 1.74 & 15.7 \\
\hline June & 37.6 & 1974 & 1.09 & 1980 & 10.9 & 11.4 & 1.05 & 4.10 \\
\hline July & 35.8 & 1976 & 0.451 & 1973 & 6.50 & 10.1 & 1.55 & 2.45 \\
\hline August & 6.25 & 1976 & 0.379 & 1981 & 1.69 & 1.63 & 0.960 & 0.640 \\
\hline September & 6.84 & 1977 & 0.238 & 1972 & 1.98 & 2.22 & 1.12 & 0.750 \\
\hline Annual & 63.2 & 1974 & 3.57 & 1973 & 22.0 & 18.8 & 0.860 & 100 \\
\hline
\end{tabular}

Annual flow duration

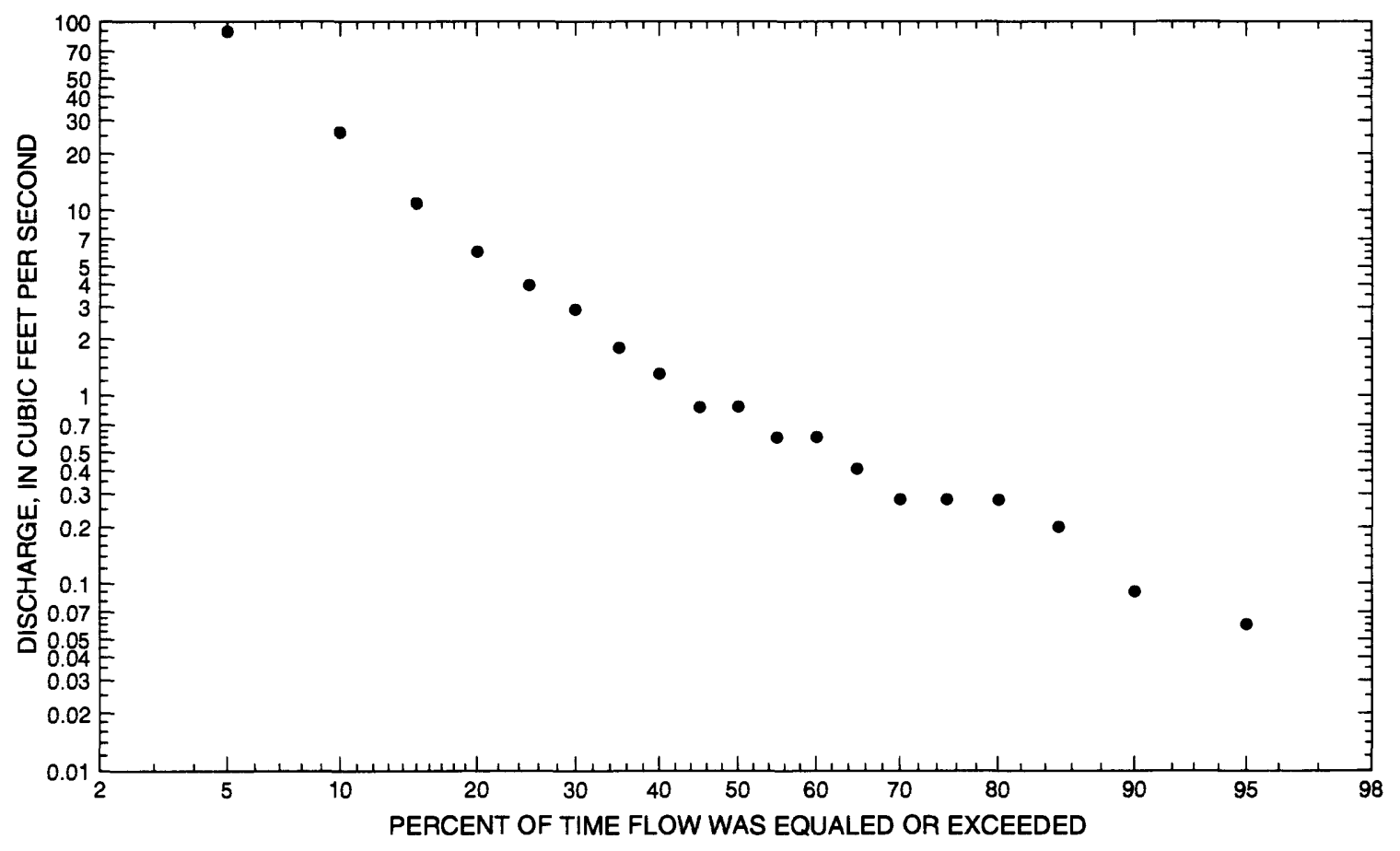




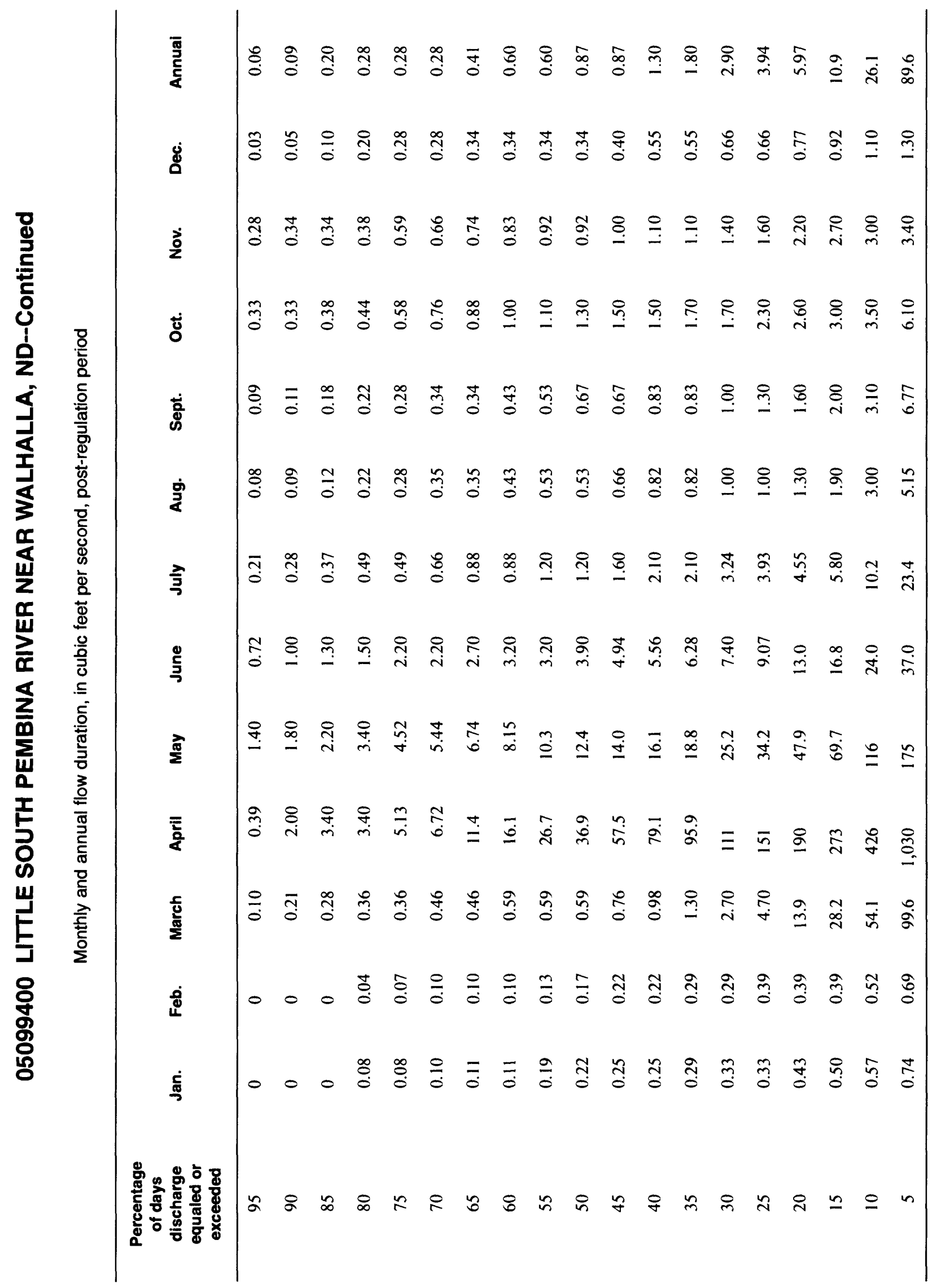


05099400 LITTLE SOUTH PEMBINA RIVER NEAR WALHALLA, ND--Continued

Probability of occurrence of annual high discharges, post-regulation period

[ng, statistic not given]

\begin{tabular}{|c|c|c|c|c|c|c|}
\hline \multirow[b]{2}{*}{$\begin{array}{l}\text { Exceedance } \\
\text { probability }\end{array}$} & \multirow[b]{2}{*}{$\begin{array}{c}\text { Recurrence } \\
\text { interval } \\
\text { (years) }\end{array}$} & \multirow[b]{2}{*}{$\begin{array}{c}\text { Maximum } \\
\text { instantaneous } \\
\left(\mathrm{ft}^{3} / \mathrm{s}\right)\end{array}$} & \multicolumn{4}{|c|}{$\begin{array}{l}\text { Maximum mean discharge } \\
\qquad\left(\mathrm{ft}^{3} / \mathrm{s}\right)\end{array}$} \\
\hline & & & 3-day period & 7-day period & 15-day period & 30-day period \\
\hline 0.99 & 1.01 & 37.5 & 23.5 & 16.9 & 11.0 & 6.78 \\
\hline 0.95 & 1.05 & 94.6 & 52.4 & 39.0 & 25.6 & 17.4 \\
\hline 0.90 & 1.11 & 153 & 80.9 & 61.0 & 40.0 & 28.0 \\
\hline 0.80 & 1.25 & 272 & 138 & 105 & 68.5 & 48.8 \\
\hline 0.50 & 2 & 786 & 389 & 298 & 189 & 132 \\
\hline 0.20 & 5 & 2,180 & 1,130 & 848 & 518 & 329 \\
\hline 0.10 & 10 & 3,660 & 1,990 & 1,470 & 872 & 512 \\
\hline 0.04 & 25 & 6,280 & 3,680 & 2,650 & 1,510 & 802 \\
\hline 0.02 & 50 & 8,850 & 5,490 & 3,870 & 2,160 & 1,060 \\
\hline 0.01 & 100 & 12,000 & 7,900 & 5,450 & 2,960 & 1,340 \\
\hline 0.005 & 200 & 15,800 & 11,000 & 7,470 & 3,960 & 1,660 \\
\hline 0.002 & 500 & 21,800 & ng & $\mathrm{ng}$ & $\mathrm{ng}$ & $\mathrm{ng}$ \\
\hline
\end{tabular}

Probability of occurrence of annual low discharges, post-regulation period

\begin{tabular}{|c|c|c|c|c|c|c|c|c|c|c|}
\hline \multirow[b]{3}{*}{$\begin{array}{l}\text { Non- } \\
\text { exceed- } \\
\text { ance } \\
\text { prob- } \\
\text { ability }\end{array}$} & \multirow[b]{3}{*}{$\begin{array}{c}\text { Recur- } \\
\text { rence } \\
\text { inter- } \\
\text { val } \\
\text { (years) }\end{array}$} & \multicolumn{9}{|c|}{ Minimum mean discharge $\left(\mathrm{ft}^{3} / \mathrm{s}\right)$} \\
\hline & & \multicolumn{9}{|c|}{ Number of consecutive days } \\
\hline & & 1 & 3 & 7 & 14 & 30 & 60 & 90 & 120 & 183 \\
\hline 0.05 & 20 & 0 & 0 & 0 & 0 & 0 & 0 & 0.015 & 0.087 & 0.199 \\
\hline 0.10 & 10 & 0 & 0 & 0 & 0 & 0 & 0 & 0.033 & 0.126 & 0.256 \\
\hline 0.20 & 5 & 0.006 & 0.012 & 0.013 & 0.015 & 0.035 & 0.067 & 0.074 & 0.190 & 0.350 \\
\hline 0.50 & 2 & 0.050 & 0.057 & 0.070 & 0.092 & 0.131 & 0.175 & 0.241 & 0.387 & 0.646 \\
\hline
\end{tabular}


05099400 LITTLE SOUTH PEMBINA RIVER NEAR WALHALLA, ND--Continued

Probability of occurrence of seasonal low discharges, post-regulation period

\begin{tabular}{|c|c|c|c|c|c|c|c|c|c|}
\hline \multirow[b]{3}{*}{$\begin{array}{c}\text { Non- } \\
\text { exceedance } \\
\text { probability }\end{array}$} & \multirow[b]{3}{*}{$\begin{array}{c}\text { Recurrence } \\
\text { interval } \\
\text { (years) }\end{array}$} & \multicolumn{8}{|c|}{ Minimum mean discharge $\left(\mathrm{ft}^{3} / \mathrm{s}\right)$} \\
\hline & & \multicolumn{8}{|c|}{ Number of consecutive days } \\
\hline & & 1 & 7 & 14 & 30 & 1 & 7 & 14 & 30 \\
\hline & & \multicolumn{4}{|c|}{ December-January-February } & \multicolumn{4}{|c|}{ March-April-May } \\
\hline 0.05 & 20 & 0 & 0 & 0 & 0 & 0 & 0 & 0.128 & 0.299 \\
\hline 0.10 & 10 & 0 & 0 & 0 & 0 & 0.089 & 0.084 & 0.163 & 0.461 \\
\hline 0.20 & 5 & 0.013 & 0.023 & 0.023 & 0.044 & 0.137 & 0.156 & 0.227 & 0.781 \\
\hline \multirow[t]{2}{*}{0.50} & 2 & 0.142 & 0.139 & 0.144 & 0.155 & 0.258 & 0.358 & 0.466 & 2.17 \\
\hline & & \multicolumn{4}{|c|}{ June-July-August } & \multicolumn{4}{|c|}{ September-October-November } \\
\hline 0.05 & 20 & 0.021 & 0.044 & 0.063 & 0.172 & 0.028 & 0.038 & 0.130 & 0.218 \\
\hline 0.10 & 10 & 0.035 & 0.066 & 0.099 & 0.224 & 0.044 & 0.062 & 0.161 & 0.265 \\
\hline 0.20 & 5 & 0.065 & 0.108 & 0.163 & 0.312 & 0.075 & 0.107 & 0.213 & 0.342 \\
\hline 0.50 & 2 & 0.190 & 0.267 & 0.385 & 0.610 & 0.196 & 0.272 & 0.377 & 0.597 \\
\hline
\end{tabular}


05099400 LITTLE SOUTH PEMBINA RIVER NEAR WALHALLA, ND--Continued

Annual peak discharge and corresponding gage height

\begin{tabular}{|c|c|c|c|c|c|c|c|}
\hline $\begin{array}{l}\text { Water } \\
\text { year }\end{array}$ & Date & $\begin{array}{c}\text { Gage } \\
\text { height } \\
\text { (feet) }\end{array}$ & $\begin{array}{c}\text { Peak } \\
\text { discharge } \\
\left(\mathrm{ft}^{3} / \mathrm{s}\right)\end{array}$ & $\begin{array}{l}\text { Water } \\
\text { year }\end{array}$ & Date & $\begin{array}{c}\text { Gage } \\
\text { height } \\
\text { (feet) }\end{array}$ & $\begin{array}{c}\text { Peak } \\
\text { discharge } \\
\left(\mathrm{ft}^{3} / \mathrm{s}\right)\end{array}$ \\
\hline \multicolumn{8}{|c|}{ Annual peak discharge, by year, and corresponding gage height } \\
\hline 1956 & April 21 & 10.15 & 2,800 & 1970 & April 25 & 13.95 & 6,600 \\
\hline 1957 & August 1 & 7.73 & 447 & 1971 & April 9 & 13.26 & 4,480 \\
\hline 1958 & July 5 & 6.75 & 20.0 & 1972 & March 17 & 9.56 & 626 \\
\hline 1959 & April 3 & 9.38 & 996 & 1973 & August 9 & 7.02 & 211 \\
\hline 1960 & April 11 & 13.28 & 4,160 & 1974 & April 16 & 12.43 & 3,190 \\
\hline 1961 & March 25 & 7.46 & 300 & 1975 & April 12 & 7.80 & 347 \\
\hline 1962 & April 18 & 12.79 & 3,100 & 1976 & April 2 & 12.39 & 2,450 \\
\hline 1963 & July 26 & 10.11 & 1,310 & 1977 & May 19 & 7.57 & 368 \\
\hline 1964 & April 9 & 7.90 & 337 & 1978 & April 6 & 10.06 & 1,600 \\
\hline 1965 & April 10 & 13.36 & 2,770 & 1979 & April 19 & 12.44 & 3,200 \\
\hline 1966 & April 1 & 11.62 & 2,380 & 1980 & March 31 & 6.21 & 140 \\
\hline 1967 & May 6 & 11.47 & 2,230 & 1981 & March 12 & 6.98 & 200 \\
\hline 1968 & March 25 & 7.82 & 270 & 1982 & March 30 & 8.29 & 464 \\
\hline 1969 & April 9 & 12.76 & 6,000 & & & & \\
\hline \multicolumn{8}{|c|}{ Annual peak discharge, from highest to lowest, and corresponding gage height } \\
\hline 1970 & April 25 & 13.95 & 6,600 & 1959 & April 3 & 9.38 & 996 \\
\hline 1969 & April 9 & 12.76 & 6,000 & 1972 & March 17 & 9.56 & 626 \\
\hline 1971 & April 9 & 13.26 & 4,480 & 1982 & March 30 & 8.29 & 464 \\
\hline 1960 & April 11 & 13.28 & 4,160 & 1957 & August 1 & 7.73 & 447 \\
\hline 1979 & April 19 & 12.44 & 3,200 & 1977 & May 19 & 7.57 & 368 \\
\hline 1974 & April 16 & 12.43 & 3,190 & 1975 & April 12 & 7.80 & 347 \\
\hline 1962 & April 18 & 12.79 & 3,100 & 1964 & April 9 & 7.90 & 337 \\
\hline 1956 & April 21 & 10.15 & 2,800 & 1961 & March 25 & 7.46 & 300 \\
\hline 1965 & April 10 & 13.36 & 2,770 & 1968 & March 25 & 7.82 & 270 \\
\hline 1976 & April 2 & 12.39 & 2,450 & 1973 & August 9 & 7.02 & 211 \\
\hline 1966 & April 1 & 11.62 & 2,380 & 1981 & March 12 & 6.98 & 200 \\
\hline 1967 & May 6 & 11.47 & 2,230 & 1980 & March 31 & 6.21 & 140 \\
\hline 1978 & April 6 & 10.06 & 1,600 & 1958 & July 5 & 6.75 & 20.0 \\
\hline 1963 & July 26 & 10.11 & 1,310 & & & & \\
\hline
\end{tabular}




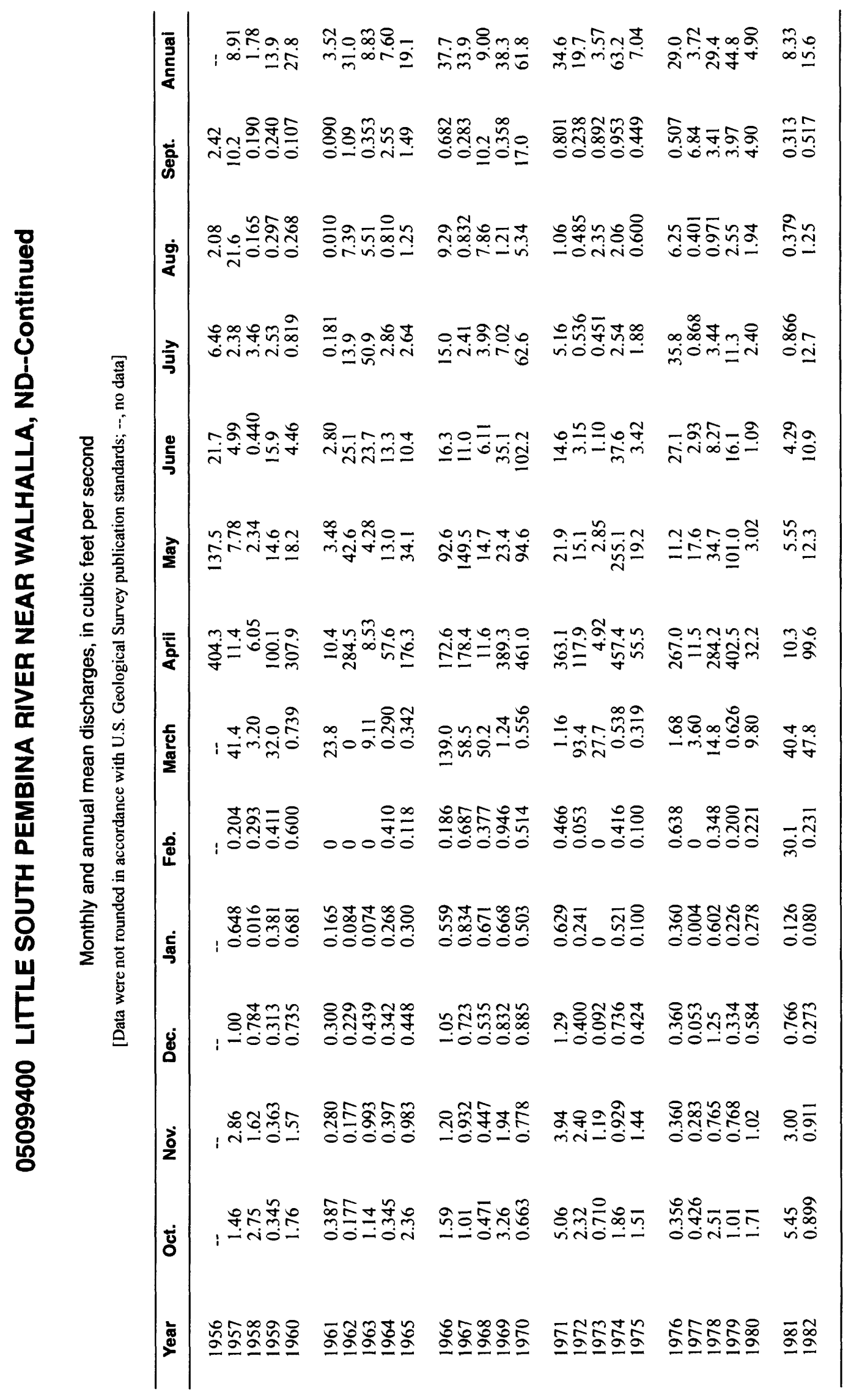


LOCATION.--Lat 48 54'50', long 97 $55^{\prime} 00^{\prime}$ ', in NE $1 /{ }_{4} \mathrm{NE}^{1} / 4 \mathrm{sec} .29$, T.163 N., R.56 W., Pembina County, Hydrologic Unit 09020313, on left bank at downstream side of bridge on State Highway 32, at south edge of Walhalla, and $7 \mathrm{mi}$ downstream from Little South Pembina River.

DRAINAGE AREA.--3,350 $\mathrm{mi}^{2}$, approximately.

PERIOD OF RECORD.--October 1939 to September 1990, October 1993 to September 1994. Prior to October 1963, published as "near Walhalla."

GAGE.-- Water-stage recorder. Datum of gage is $934 \mathrm{ft}$ above sea level, from topographic map. Prior to Nov. 10, 1943, nonrecording gage and Nov. 10, 1943, to Sept. 30, 1963, water-stage recorder at site $5.5 \mathrm{mi}$ upstream at different datum.

EXTREMES FOR PERIOD OF RECORD.--Maximum discharge, 20,400 $\mathrm{ft}^{3} / \mathrm{s}$, Apr. 18, 1950, gage height, $19.2 \mathrm{ft}$ former site and datum, $16.2 \mathrm{ft}$ present site and datum, from rating curve extended above $7,000 \mathrm{ft}^{3} / \mathrm{s}$ on basis of contracted-opening measurement of peak flow; no flow at times in some years.

Annual mean discharge

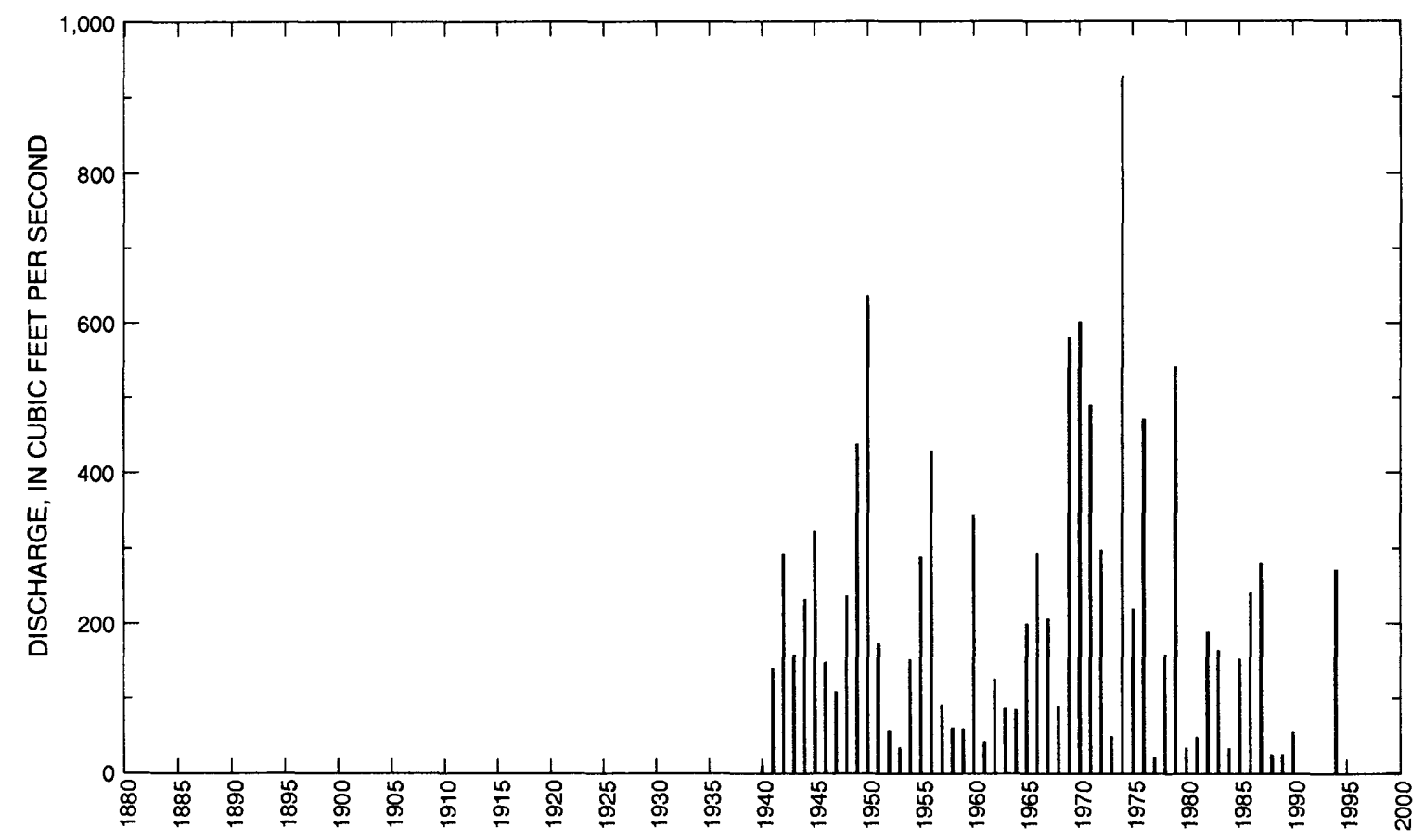




\section{PEMBINA RIVER AT WALHALLA, ND--Continued}

Statistics of monthly and annual mean discharges

[m, more than 1 year of occurrence]

\begin{tabular}{|c|c|c|c|c|c|c|c|c|}
\hline \multirow[b]{2}{*}{ Month } & \multicolumn{2}{|c|}{ Maximum } & \multicolumn{2}{|c|}{ Minimum } & \multicolumn{4}{|c|}{ Mean } \\
\hline & $\begin{array}{c}\text { Discharge } \\
\left(\mathrm{ft}^{3} / \mathrm{s}\right)\end{array}$ & $\begin{array}{l}\text { Water year } \\
\text { of } \\
\text { occurrence }\end{array}$ & $\begin{array}{c}\text { Discharge } \\
\left(\mathrm{ft}^{3} / \mathrm{s}\right)\end{array}$ & $\begin{array}{c}\text { Water year } \\
\text { of } \\
\text { occurrence }\end{array}$ & $\begin{array}{c}\text { Discharge } \\
\left(\mathrm{ft}^{3} / \mathrm{s}\right)\end{array}$ & $\begin{array}{c}\text { Standard } \\
\text { deviation } \\
\left(\mathrm{ft}^{3} / \mathrm{s}\right)\end{array}$ & $\begin{array}{l}\text { Coeffi- } \\
\text { cient of } \\
\text { variation }\end{array}$ & $\begin{array}{l}\text { Percentage } \\
\text { of annual } \\
\text { discharge }\end{array}$ \\
\hline October & 369 & 1969 & 0.042 & 1940 & 56.3 & 67.4 & 1.20 & 2.13 \\
\hline November & 213 & 1945 & 0.147 & 1941 & 36.6 & 43.8 & 1.20 & 1.38 \\
\hline December & 91.8 & 1994 & 0 & 1941 & 17.6 & 21.3 & 1.21 & 0.67 \\
\hline January & 51.2 & 1960 & 0 & $\mathrm{~m}$ & 9.87 & 11.6 & 1.17 & 0.37 \\
\hline February & 41.5 & 1945 & 0 & $\mathrm{~m}$ & 8.03 & 9.63 & 1.20 & 0.30 \\
\hline March & 804 & 1945 & 0 & 1940 & 106 & 179 & 1.69 & 4.01 \\
\hline April & 3,700 & 1974 & 49.6 & 1977 & 985 & 960 & 0.97 & 37.3 \\
\hline May & 4,670 & 1974 & 18.8 & 1940 & 748 & 935 & 1.25 & 28.4 \\
\hline June & 1930 & 1974 & 2.83 & 1940 & 332 & 362 & 1.09 & 12.6 \\
\hline July & 814 & 1970 & 0.735 & 1940 & 168 & 185 & 1.10 & 6.35 \\
\hline August & 785 & 1944 & 0.103 & 1961 & 100 & 130 & 1.29 & 3.80 \\
\hline September & 432 & 1944 & 0 & 1940 & 72.1 & 84.7 & 1.18 & 2.73 \\
\hline Annual & 928 & 1974 & 9.77 & 1940 & 220 & 194 & 0.88 & 100 \\
\hline
\end{tabular}

Annual flow duration

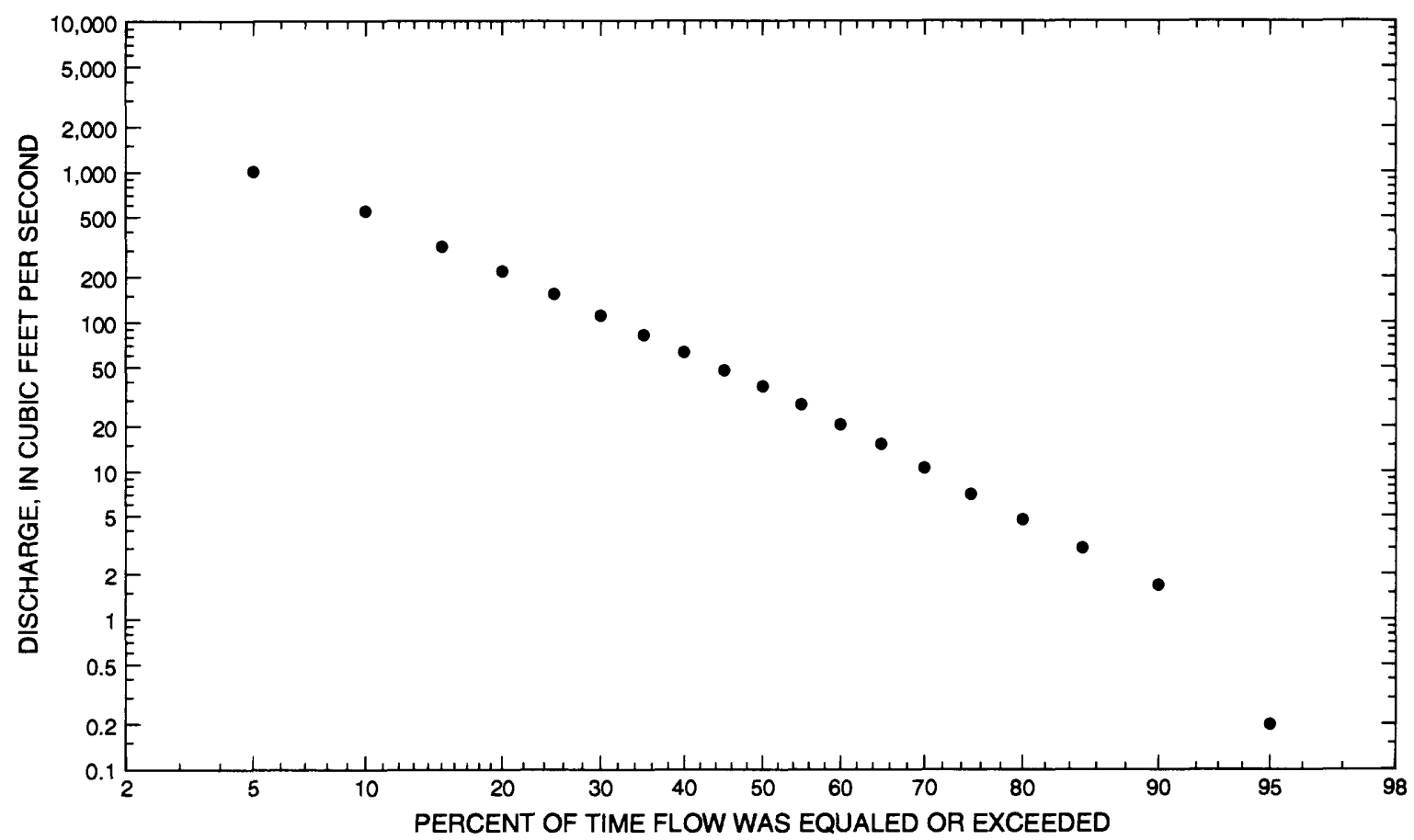




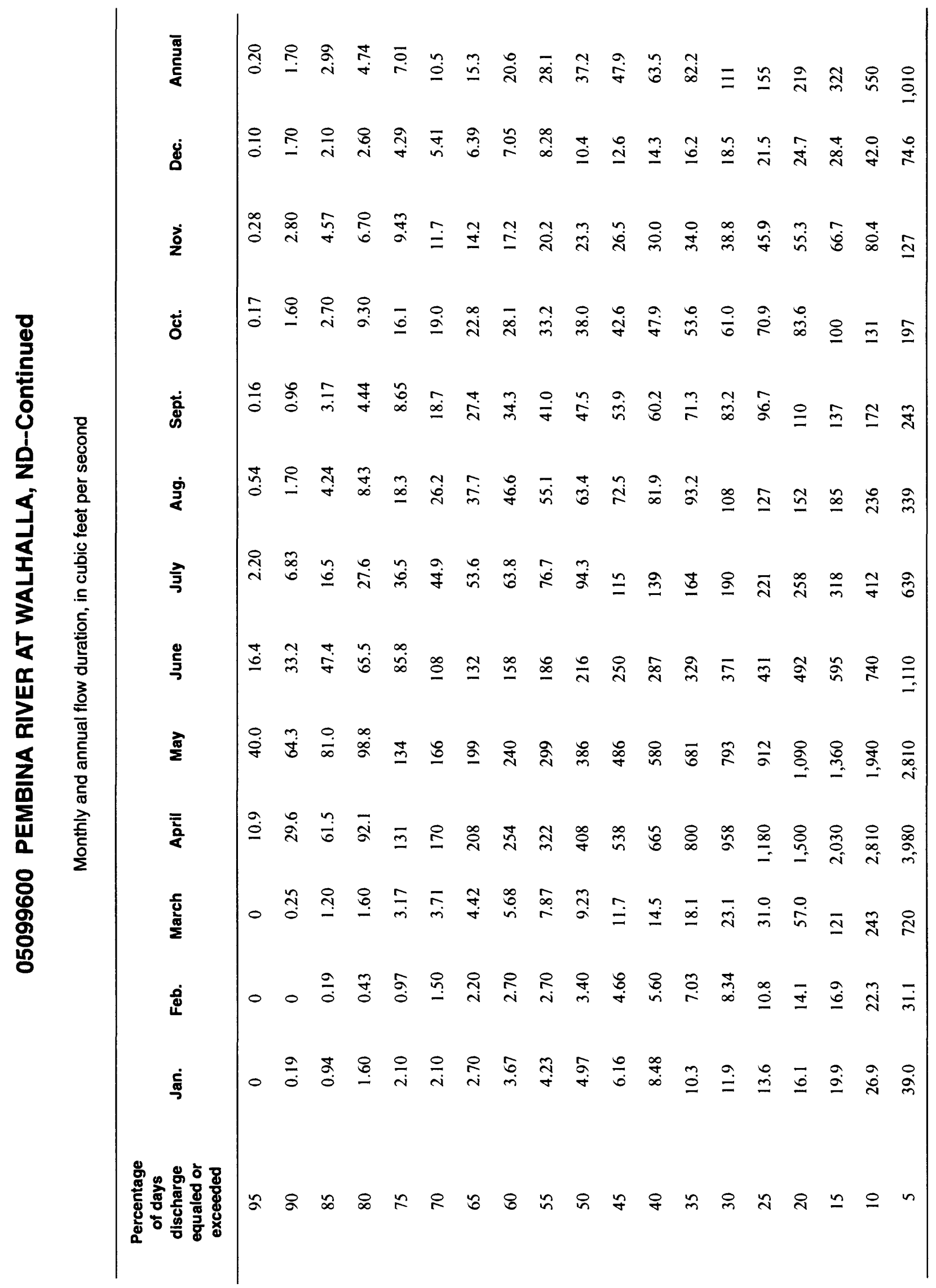




\section{PEMBINA RIVER AT WALHALLA, ND--Continued}

Probability of occurrence of annual high discharges

[ng, statistic not given]

\begin{tabular}{|c|c|c|c|c|c|c|c|}
\hline \multirow[b]{2}{*}{$\begin{array}{l}\text { Exceedance } \\
\text { probability }\end{array}$} & \multirow{2}{*}{$\begin{array}{l}\text { Recurrence } \\
\text { intervai } \\
\text { (years) }\end{array}$} & \multicolumn{2}{|c|}{$\begin{array}{l}\text { Maximum } \\
\text { instantaneous } \\
\left(\mathrm{ft}^{3} / \mathrm{s}\right)\end{array}$} & \multicolumn{4}{|c|}{$\begin{array}{l}\text { Maximum mean discharge } \\
\qquad\left(\mathrm{ft}^{3} / \mathrm{s}\right)\end{array}$} \\
\hline & & $1940-63$ & ${ }^{1}$ 1964-94 & 3-day period & 7-day period & 15-day period & 30-day period \\
\hline 0.99 & 1.01 & 201 & 111 & 87.0 & 72.0 & 60.1 & 44.6 \\
\hline 0.95 & 1.05 & 414 & 282 & 208 & 175 & 144 & 110 \\
\hline 0.90 & 1.11 & 605 & 454 & 326 & 276 & 225 & 174 \\
\hline 0.80 & 1.25 & 950 & 798 & 556 & 472 & 381 & 300 \\
\hline 0.50 & 2 & 2,220 & 2,240 & 1,490 & 1,260 & 1,000 & 809 \\
\hline 0.20 & 5 & 5,040 & 5,910 & 3,790 & 3,180 & 2,480 & 2,050 \\
\hline 0.10 & 10 & 7,680 & 9,590 & 6,060 & 5,040 & 3,900 & 3,240 \\
\hline 0.04 & 25 & 12,000 & 15,800 & 9,860 & 8,100 & 6,220 & 5,210 \\
\hline 0.02 & 50 & 15,800 & 21,600 & 13,400 & 10,900 & 8,330 & 7,000 \\
\hline 0.01 & 100 & 20,400 & 28,400 & 17,600 & 14,200 & 10,800 & 9,070 \\
\hline 0.005 & 200 & 25,500 & 36,400 & 22,500 & 17,900 & 13,500 & 11,400 \\
\hline 0.002 & 500 & 33,600 & 48,800 & ng & ng & ng & ng \\
\hline
\end{tabular}

${ }^{1}$ Historic adjustment made to Log Pearson Type IIl peak frequency.

Probability of occurrence of annual low discharges

\begin{tabular}{|c|c|c|c|c|c|c|c|c|c|c|}
\hline \multirow[b]{3}{*}{$\begin{array}{l}\text { Non- } \\
\text { exceed- } \\
\text { ance } \\
\text { prob- } \\
\text { ability }\end{array}$} & \multirow[b]{3}{*}{$\begin{array}{c}\text { Recur- } \\
\text { rence } \\
\text { inter- } \\
\text { val } \\
\text { (years) }\end{array}$} & \multicolumn{9}{|c|}{ Minimum mean discharge $\left(\mathrm{ft}^{3} / \mathrm{s}\right)$} \\
\hline & & \multicolumn{9}{|c|}{ Number of consecutive days } \\
\hline & & 1 & 3 & 7 & 14 & 30 & 60 & 90 & 120 & 183 \\
\hline 0.05 & 20 & 0 & 0 & 0 & 0 & 0 & 0 & 0.036 & 0.375 & 0.589 \\
\hline 0.10 & 10 & 0 & 0 & 0 & 0 & 0 & 0.332 & 0.405 & 1.02 & 1.62 \\
\hline 0.20 & 5 & 0.153 & 0.156 & 0.184 & 0.271 & 0.427 & 0.922 & 1.56 & 2.55 & 4.54 \\
\hline 0.50 & 2 & 1.99 & 2.05 & 2.25 & 2.47 & 3.06 & 3.64 & 6.88 & 8.66 & 18.8 \\
\hline
\end{tabular}




\section{PEMBINA RIVER AT WALHALLA, ND--Continued}

Probability of occurrence of seasonal low discharges

\begin{tabular}{|c|c|c|c|c|c|c|c|c|c|}
\hline \multirow[b]{3}{*}{$\begin{array}{c}\text { Non- } \\
\text { exceedance } \\
\text { probability }\end{array}$} & \multirow[b]{3}{*}{$\begin{array}{l}\text { Recurrence } \\
\text { interval } \\
\text { (years) }\end{array}$} & \multicolumn{8}{|c|}{ Minimum mean discharge $\left(\mathrm{ft}^{3} / \mathrm{s}\right)$} \\
\hline & & \multicolumn{8}{|c|}{ Number of consecutive days } \\
\hline & & 1 & 7 & 14 & 30 & 1 & 7 & 14 & 30 \\
\hline & & \multicolumn{4}{|c|}{ December-January-February } & \multicolumn{4}{|c|}{ March-April-May } \\
\hline 0.05 & 20 & 0 & 0 & 0 & 0 & 0 & 0 & 0 & 0.304 \\
\hline 0.10 & 10 & 0 & 0 & 0 & 0 & 0 & 0 & 0 & 1.14 \\
\hline 0.20 & 5 & 0.238 & 0.265 & 0.305 & 0.587 & 0.721 & 0.837 & 0.903 & 4.12 \\
\hline \multirow[t]{2}{*}{0.50} & 2 & 2.86 & 3.01 & 3.17 & 3.56 & 4.07 & 4.77 & 5.78 & 27.9 \\
\hline & & \multicolumn{4}{|c|}{ June-July-August } & \multicolumn{4}{|c|}{ September-October-November } \\
\hline 0.05 & 20 & 0.248 & 0.326 & 0.414 & 0.626 & 0.160 & 0.170 & 0.265 & 0.369 \\
\hline 0.10 & 10 & 1.08 & 1.36 & 1.68 & 2.22 & 0.612 & 0.672 & 0.931 & 1.30 \\
\hline 0.20 & 5 & 4.29 & 5.16 & 6.25 & 8.20 & 2.12 & 2.39 & 3.01 & 4.16 \\
\hline 0.50 & 2 & 29.5 & 33.2 & 38.5 & 52.5 & 11.4 & 13.4 & 15.4 & 20.4 \\
\hline
\end{tabular}




\section{PEMBINA RIVER AT WALHALLA, ND--Continued}

Annual peak discharge and corresponding gage height

\begin{tabular}{|c|c|c|c|c|c|c|c|}
\hline $\begin{array}{l}\text { Water } \\
\text { year }\end{array}$ & Date & $\begin{array}{c}\text { Gage } \\
\text { height } \\
\text { (feet) }\end{array}$ & $\begin{array}{c}\text { Peak } \\
\text { discharge } \\
\left(\mathrm{ft}^{3} / \mathrm{s}\right)\end{array}$ & $\begin{array}{l}\text { Water } \\
\text { year }\end{array}$ & Date & $\begin{array}{c}\text { Gage } \\
\text { height } \\
\text { (feet) }\end{array}$ & $\begin{array}{c}\text { Peak } \\
\text { discharge } \\
\left(\mathrm{ft}^{3} / \mathrm{s}\right)\end{array}$ \\
\hline \multicolumn{8}{|c|}{ Annual peak discharge, by year, and corresponding gage height } \\
\hline 1940 & April 19 & 5.52 & 1,000 & 1966 & March 31 & 14.82 & 4,700 \\
\hline 1941 & April 10 & 11.03 & 3,200 & 1967 & April 21 & 12.10 & 4,530 \\
\hline 1942 & April 5 & 12.45 & 5,000 & 1968 & March 18 & 9.40 & 1,400 \\
\hline 1943 & June 3 & 6.15 & 1,420 & 1969 & April 20 & 14.58 & 8,440 \\
\hline 1944 & August 4 & 6.28 & 1,470 & 1970 & April 26 & 15.08 & 10,200 \\
\hline 1945 & March 26 & 9.20 & 3,020 & 1971 & April 11 & 14.56 & 10,200 \\
\hline 1946 & March 19 & 9.24 & 2,900 & 1972 & April 9 & 9.81 & 3,160 \\
\hline 1947 & April 3 & 5.83 & 1,280 & 1973 & August 9 & 3.79 & 358 \\
\hline 1948 & April 19 & 14.94 & 7,280 & 1974 & April 27 & 15.19 & 13,800 \\
\hline 1949 & April 19 & 13.18 & 5,840 & 1975 & May 17 & 13.38 & 1,470 \\
\hline 1950 & April 18 & 19.20 & 20,400 & 1976 & April 17 & 9.81 & 4,530 \\
\hline 1951 & April 5 & 8.22 & 2,310 & 1977 & May 19 & 4.31 & 610 \\
\hline 1952 & March 31 & 7.28 & 700 & 1978 & April 6 & 9.97 & 3,800 \\
\hline 1953 & June 3 & 4.48 & 642 & 1979 & April 20 & 13.32 & 9,130 \\
\hline 1954 & July 6 & 5.45 & 1,160 & 1980 & April 7 & 3.83 & 400 \\
\hline 1955 & April 2 & 10.75 & 3,400 & 1981 & April 1 & 3.16 & 260 \\
\hline 1956 & April 25 & 12.89 & 5,990 & 1982 & June 7 & 6.96 & 2,100 \\
\hline 1957 & March 23 & 6.22 & 725 & 1983 & April 8 & 6.32 & 1,660 \\
\hline 1958 & April 6 & 6.52 & 500 & 1984 & June 16 & 3.23 & 374 \\
\hline 1959 & April 3 & 7.90 & 2,000 & 1985 & March 19 & 8.57 & 1,860 \\
\hline 1960 & April 12 & 12.50 & 5,690 & 1986 & March 22 & 9.31 & 2,800 \\
\hline 1961 & April 9 & 3.46 & 334 & 1987 & April 5 & 10.39 & 5,300 \\
\hline 1962 & April 19 & 11.74 & 5,110 & 1988 & April 4 & 2.98 & 321 \\
\hline 1963 & July 26 & 7.72 & 2,340 & 1989 & April 16 & 6.67 & 1,000 \\
\hline 1964 & June 19 & 5.43 & 796 & 1990 & April 1 & 7.64 & 1,200 \\
\hline 1965 & April 11 & 12.68 & 4,960 & 1994 & March 22 & 7.03 & 1,800 \\
\hline \multicolumn{8}{|c|}{ Annual peak discharge, from highest to lowest, and corresponding gage height } \\
\hline 1950 & April 18 & 19.20 & 20,400 & 1955 & April 2 & 10.75 & 3,400 \\
\hline 1974 & April 27 & 15.19 & 13,800 & 1941 & April 10 & 11.03 & 3,200 \\
\hline 1970 & April 26 & 15.08 & 10,200 & 1972 & April 9 & 9.81 & 3,160 \\
\hline 1971 & April 11 & 14.56 & 10,200 & 1945 & March 26 & 9.20 & 3,020 \\
\hline 1979 & April 20 & 13.32 & 9,130 & 1946 & March 19 & 9.24 & 2,900 \\
\hline 1969 & April 20 & 14.58 & 8,440 & 1986 & March 22 & 9.31 & 2,800 \\
\hline 1948 & April 19 & 14.94 & 7,280 & 1963 & July 26 & 7.72 & 2,340 \\
\hline 1956 & April 25 & 12.89 & 5,990 & 1951 & April 5 & 8.22 & 2,310 \\
\hline 1949 & April 19 & 13.18 & 5,840 & 1982 & June 7 & 6.96 & 2,100 \\
\hline 1960 & April 12 & 12.50 & 5,690 & 1959 & April 3 & 7.90 & 2,000 \\
\hline 1987 & April 5 & 10.39 & 5,300 & 1985 & March 19 & 8.57 & 1,860 \\
\hline 1962 & April 19 & 11.74 & 5,110 & 1994 & March 22 & 7.03 & 1,800 \\
\hline 1942 & April 5 & 12.45 & 5,000 & 1983 & April 8 & 6.32 & 1,660 \\
\hline 1965 & April 11 & 12.68 & 4,960 & 1944 & August 4 & 6.28 & 1,470 \\
\hline 1966 & March 31 & 14.82 & 4,700 & 1975 & May 17 & 13.38 & 1,470 \\
\hline 1967 & April 21 & 12.10 & 4,530 & 1943 & June 3 & 6.15 & 1,420 \\
\hline 1976 & April 17 & 9.81 & 4,530 & 1968 & March 18 & 9.40 & 1,400 \\
\hline 1978 & April 6 & 9.97 & 3,800 & 1947 & April 3 & 5.83 & 1,280 \\
\hline
\end{tabular}




\section{PEMBINA RIVER AT WALHALLA, ND--Continued}

Annual peak discharge and corresponding gage height-Continued

\begin{tabular}{llllllll}
\hline $\begin{array}{l}\text { Water } \\
\text { year }\end{array}$ & Date & $\begin{array}{c}\text { Gage } \\
\text { height } \\
\text { (feet) }\end{array}$ & $\begin{array}{c}\text { Peak } \\
\text { discharge } \\
\left(\mathbf{f t}^{\mathbf{3}} \mathbf{s}\right)\end{array}$ & $\begin{array}{c}\text { Water } \\
\text { year }\end{array}$ & Date & $\begin{array}{c}\text { Gage } \\
\text { height } \\
\text { (feet) }\end{array}$ & $\begin{array}{c}\text { Peak } \\
\text { discharge } \\
\left(\mathbf{f t}^{\mathbf{3}} / \mathbf{s}\right)\end{array}$ \\
\hline \multicolumn{7}{c}{ Annual peak discharge, from highest to lowest, and corresponding gage height-Continued } \\
1990 & April 1 & 7.64 & 1,200 & 1977 & May 19 & 4.31 & 610 \\
1954 & July 6 & 5.45 & 1,160 & 1958 & April 6 & 6.52 & 500 \\
1940 & April 19 & 5.52 & 1,000 & 1980 & April 7 & 3.83 & 400 \\
1989 & April 16 & 6.67 & 1,000 & 1984 & June 16 & 3.23 & 374 \\
1964 & June 19 & 5.43 & 796 & 1973 & August 9 & 3.79 & 358 \\
1957 & March 23 & 6.22 & 725 & 1961 & April 9 & 3.46 & 334 \\
1952 & March 31 & 7.28 & 700 & 1988 & April 4 & 2.98 & 321 \\
1953 & June 3 & 4.48 & 642 & 1981 & April 1 & 3.16 & 260 \\
\hline
\end{tabular}


Farnt nanam a-oor

ämi mon

ปลิก

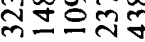

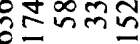

moorn

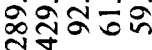

ज्ञ

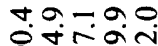

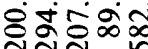

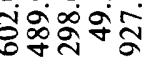

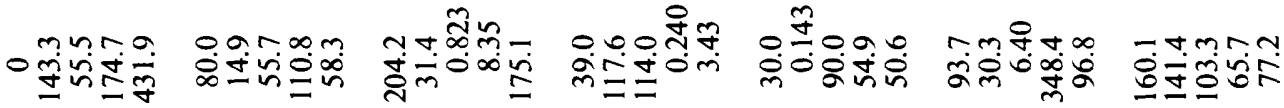

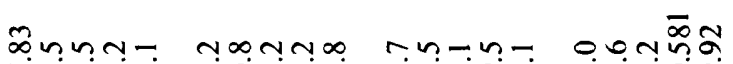

กับูณ

๓ก่ลู่อ

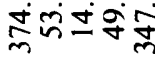

ninis०

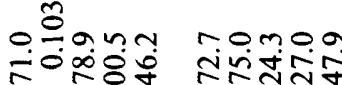

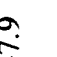

omoon

$\tilde{n}$

ก0not $0-0000-0.00$

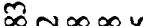
igidis

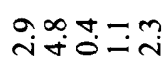

0,0

$-\infty .009$

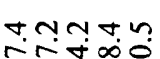

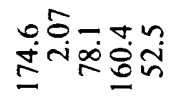

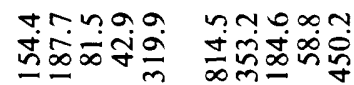

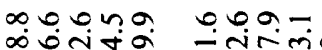

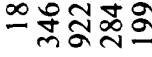

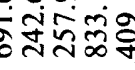

.

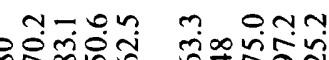

ํํำลำ

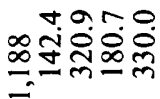

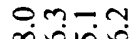

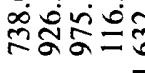

힐

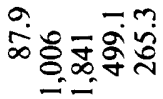

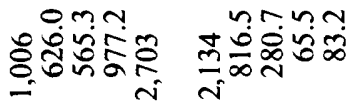

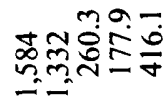

여ำำำ

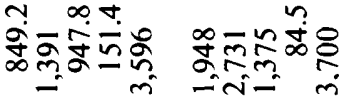

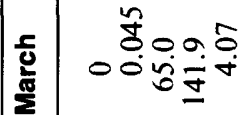

rros

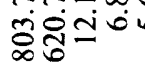

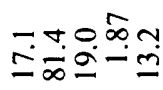

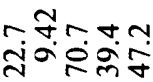

우궁

\$0000

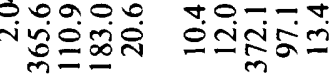

ミะ

$n 8 x=\overline{1}$

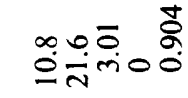

웅유

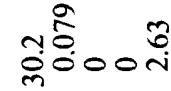

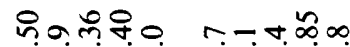
000 vim

o0तifi

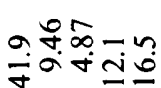

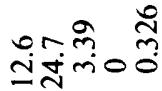

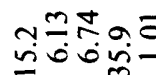

กำำกับ

mabar nNata

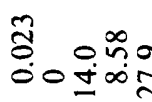

ตलกิनm

$\sim-\infty \sim ⿻ n$

$\stackrel{n}{\sim} \underset{\sim}{-\infty}=$

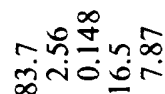

$4-80 \bar{m}$

r+a

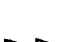

oे

的우으.

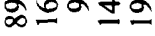

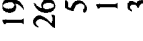

=o

moog

กี่สิดั

0 ำล

얻ㅇ

$\forall \infty, \pi \frac{1}{4}$

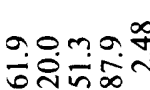

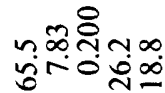

taomo orodत्रतज़ min=min 的少

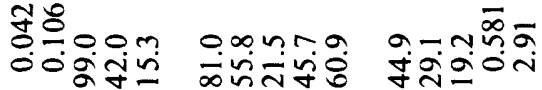

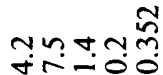

ㅇํำ? 的实 ल당ำ

$0.700 \pi$ monos

ठ்

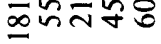

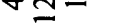

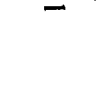

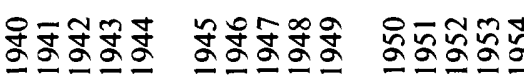

๙ูํํํํำ

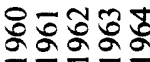

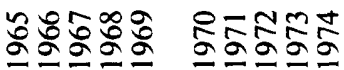




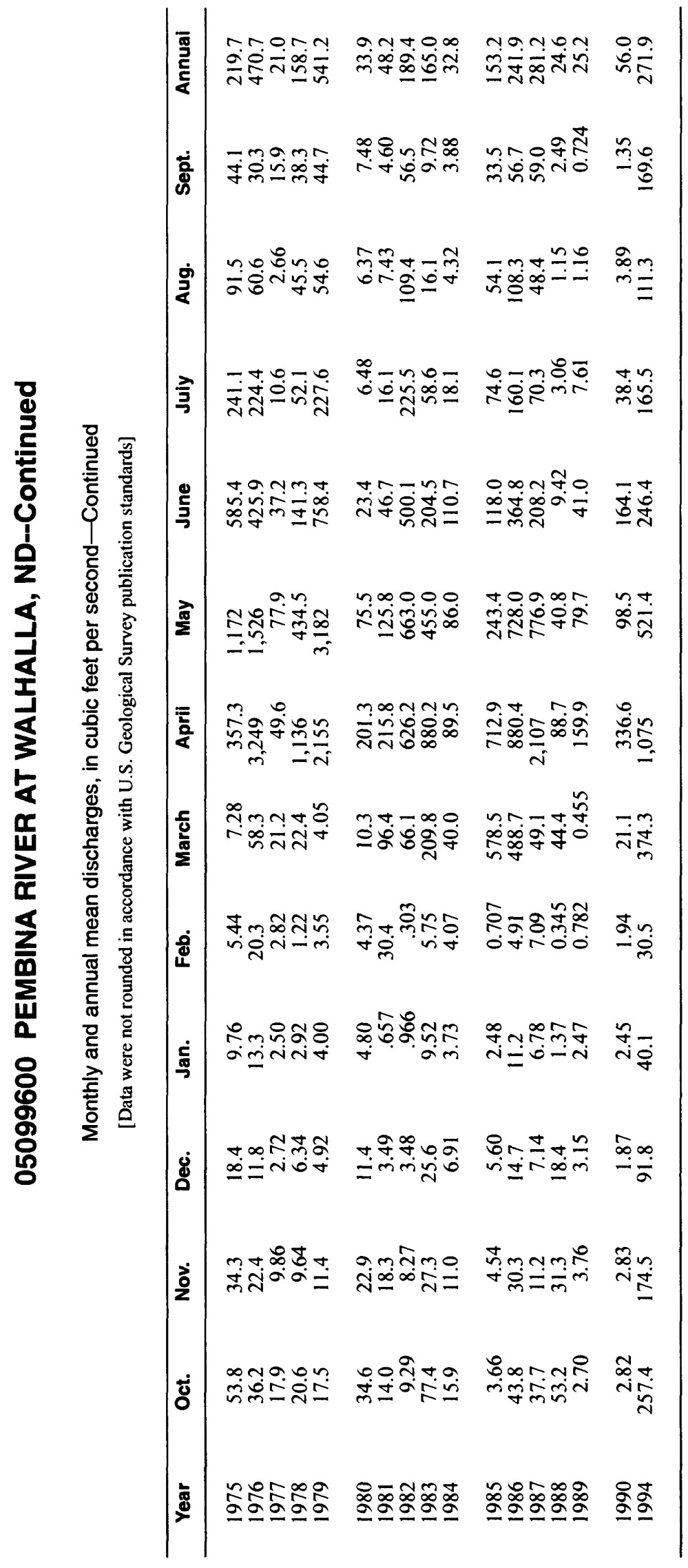




\section{PEMBINA RIVER AT NECHE, ND}

LOCATION.--Lat 48 59'20', long 97'33'05”, in SE $1 /{ }_{4} \mathrm{NW}^{1} / 4$ sec.31, T.164 N., R.53 W., Pembina County, Hydrologic Unit 09020313 , on right bank 0.3 mi east of State Highway 18, and at north edge of Neche.

DRAINAGE AREA.--3,410 $\mathrm{mi}^{2}$, approximately.

PERIOD OF RECORD.--May 1903 to September 1908, June 1909 to September 1915, April 1919 to current year. Monthly discharge only for some periods, published in WSP 1308.

GAGE.--Water-stage recorder and concrete control. Datum of gage is $809.69 \mathrm{ft}$ above sea level. Prior to May 24, 1932, nonrecording gage at Burlington Northern Railway bridge 1 mi upstream, at same datum. May 25, 1932, to Apr. 17, 1939, nonrecording gage on bridge on State Highway 18, $500 \mathrm{ft}$ downstream from railway bridge, at same datum.

EXTREMES FOR PERIOD OF RECORD.--Maximum discharge, 10,700 $\mathrm{ft}^{3} / \mathrm{s}$, Apr. 20, 1950, from rating curve extended above $5,300 \mathrm{ft}^{3} / \mathrm{s}$, gage height, $21.58 \mathrm{ft}$, backwater from ice; maximum gage height, $23.64 \mathrm{ft}$, Apr. 20, 1979, backwater from ice; no flow at times.

Annual mean discharge

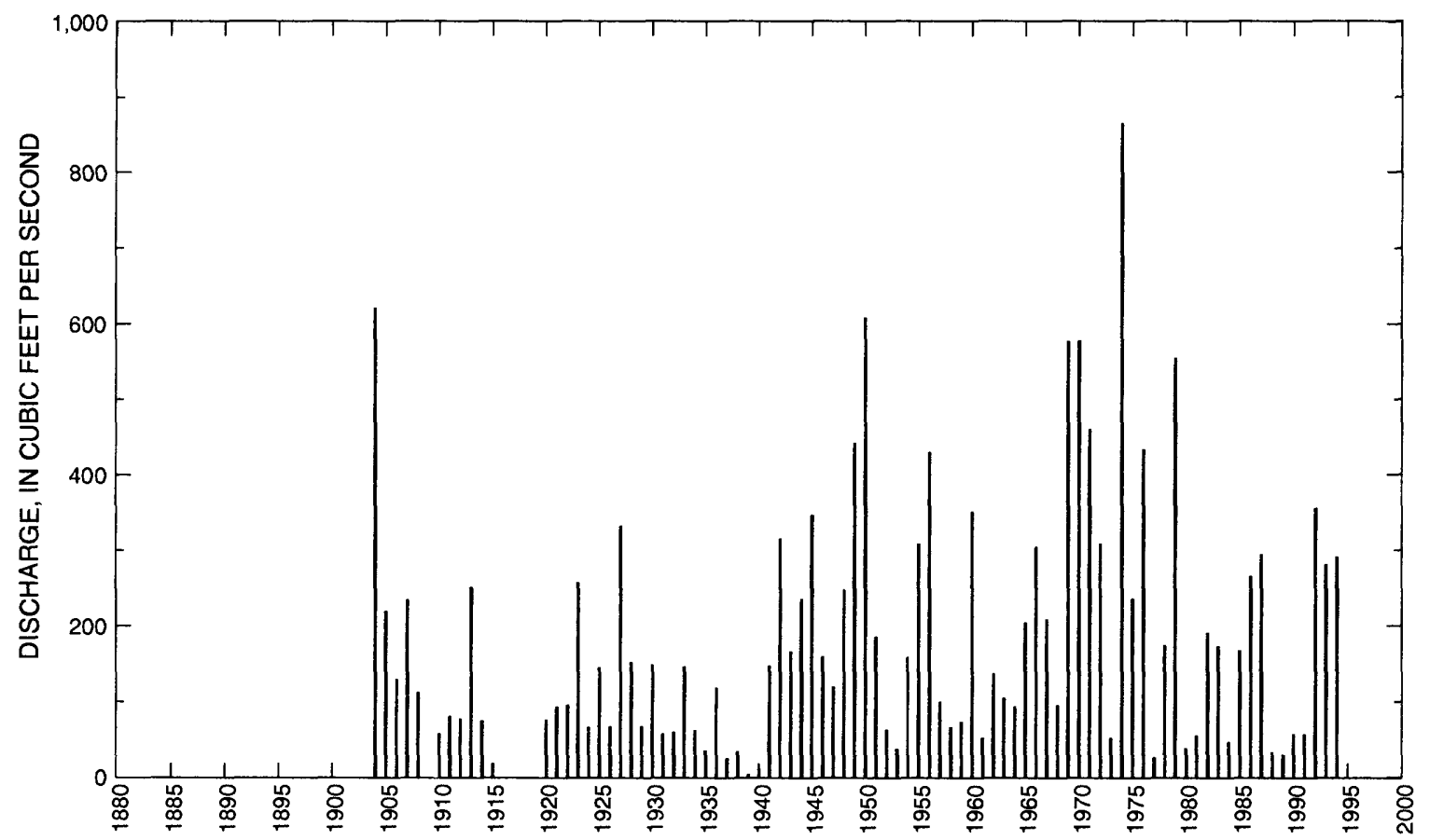




\section{PEMBINA RIVER AT NECHE, ND--Continued}

Statistics of monthly and annual mean discharges

[m, more than I year of occurrence]

\begin{tabular}{|c|c|c|c|c|c|c|c|c|}
\hline \multirow[b]{2}{*}{ Month } & \multicolumn{2}{|c|}{ Maximum } & \multicolumn{2}{|c|}{ Minimum } & \multicolumn{4}{|c|}{ Mean } \\
\hline & $\begin{array}{c}\text { Discharge } \\
\left(\mathrm{ft}^{3} / \mathrm{s}\right)\end{array}$ & $\begin{array}{c}\text { Water year } \\
\text { of } \\
\text { occurrence }\end{array}$ & $\begin{array}{c}\text { Discharge } \\
\left(\mathrm{ft}^{3} / \mathrm{s}\right)\end{array}$ & $\begin{array}{c}\text { Water year } \\
\text { of } \\
\text { occurrence }\end{array}$ & $\begin{array}{c}\text { Discharge } \\
\left(\mathrm{ft}^{3} / \mathrm{s}\right)\end{array}$ & $\begin{array}{l}\text { Standard } \\
\text { deviation } \\
\left(\mathrm{ft}^{3} / \mathrm{s}\right)\end{array}$ & $\begin{array}{l}\text { Coeffi- } \\
\text { cient of } \\
\text { variation }\end{array}$ & $\begin{array}{l}\text { Percentage } \\
\text { of annual } \\
\text { discharge }\end{array}$ \\
\hline October & 378 & 1969 & 0 & $\mathrm{~m}$ & 67.4 & 71.2 & 1.05 & 2.99 \\
\hline November & 234 & 1945 & 0 & $\mathrm{~m}$ & 43.3 & 45.5 & 1.05 & 1.92 \\
\hline December & 130 & 1905 & 0 & $\mathrm{~m}$ & 20.0 & 24.8 & 1.24 & 0.88 \\
\hline January & 66.0 & 1960 & 0 & $\mathrm{~m}$ & 10.3 & 13.0 & 1.26 & 0.46 \\
\hline February & 49.5 & 1945 & 0 & $\mathrm{~m}$ & 7.33 & 9.36 & 1.28 & 0.32 \\
\hline March & 741 & 1945 & 0 & $\mathrm{~m}$ & 88.6 & 152 & 1.72 & 3.92 \\
\hline April & 3,150 & 1969 & 24.7 & 1939 & 766 & 749 & 0.98 & 33.9 \\
\hline May & 4,620 & 1974 & 11.8 & 1939 & 619 & 800 & 1.29 & 27.4 \\
\hline June & 1,780 & 1974 & 6.56 & 1940 & 304 & 333 & 1.10 & 13.5 \\
\hline July & 839 & 1904 & 0 & 1940 & 161 & 179 & 1.11 & 7.12 \\
\hline August & 946 & 1993 & 0 & 1939 & 96.5 & 149 & 1.54 & 4.27 \\
\hline September & 648 & 1993 & 0 & $\mathrm{~m}$ & 74.7 & 103 & 1.38 & 3.31 \\
\hline Annual & 865 & 1974 & 3.96 & 1939 & 190 & 169 & 0.89 & 100 \\
\hline
\end{tabular}

Annual flow duration

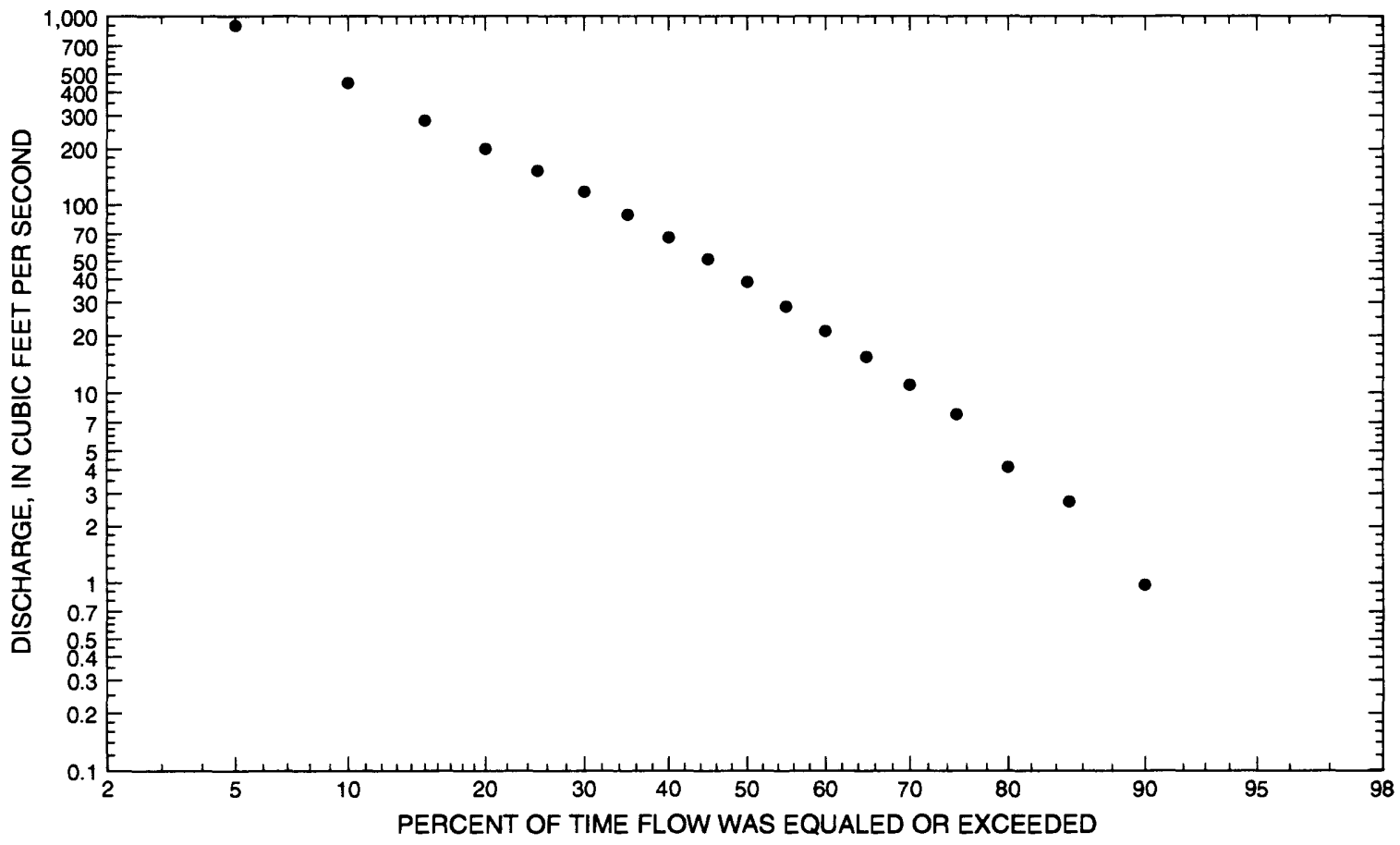




\section{PEMBINA RIVER AT NECHE, ND--Continued}

Probability of occurrence of annual high discharges

[ng, statistic not given]

\begin{tabular}{|c|c|c|c|c|c|c|}
\hline \multirow[b]{2}{*}{$\begin{array}{l}\text { Exceedance } \\
\text { probability }\end{array}$} & \multirow[b]{2}{*}{$\begin{array}{l}\text { Recurrence } \\
\text { intervai } \\
\text { (years) }\end{array}$} & \multirow[b]{2}{*}{$\begin{array}{c}\text { Maximum } \\
\text { instantaneous } \\
\left(\mathrm{ft}^{3} / \mathbf{s}\right)\end{array}$} & \multicolumn{4}{|c|}{$\begin{array}{l}\text { Maximum mean discharge } \\
\qquad\left(\mathrm{ft}^{3} / \mathrm{s}\right)\end{array}$} \\
\hline & & & 3-day period & 7-day period & 15-day period & 30-day period \\
\hline 0.99 & 1.01 & ng & 69.9 & 58.8 & 49.6 & 40.0 \\
\hline 0.95 & 1.05 & 264 & 172 & 146 & 120 & 95.6 \\
\hline 0.90 & 1.11 & 391 & 272 & 231 & 188 & 149 \\
\hline 0.80 & 1.25 & 623 & 463 & 396 & 317 & 252 \\
\hline 0.50 & 2 & 1,470 & 1,210 & 1,040 & 820 & 656 \\
\hline 0.20 & 5 & 3,360 & 2,950 & 2,530 & 1,980 & 1,600 \\
\hline 0.10 & 10 & 5,100 & 4,550 & 3,890 & 3,050 & 2,490 \\
\hline 0.04 & 25 & 7,860 & 7,070 & 6,030 & 4,730 & 3,910 \\
\hline 0.02 & 50 & 10,300 & 9,280 & 7,910 & 6,220 & 5,190 \\
\hline 0.01 & 100 & 13,200 & 11,800 & 10,000 & 7,900 & 6,650 \\
\hline 0.005 & 200 & 16,400 & 14,500 & 12,300 & 9,780 & 8,300 \\
\hline 0.002 & 500 & 21,300 & ng & $n g$ & $n g$ & ng \\
\hline
\end{tabular}

Probability of occurrence of annual low discharges

\begin{tabular}{|c|c|c|c|c|c|c|c|c|c|c|}
\hline \multirow[b]{3}{*}{$\begin{array}{l}\text { Non- } \\
\text { exceed- } \\
\text { ance } \\
\text { prob- } \\
\text { ability }\end{array}$} & \multirow[b]{3}{*}{$\begin{array}{c}\text { Recur- } \\
\text { rence } \\
\text { inter- } \\
\text { vai } \\
\text { (years) }\end{array}$} & \multicolumn{9}{|c|}{ Minimum mean discharge $\left(\mathrm{ft}^{3} / \mathrm{s}\right)$} \\
\hline & & \multicolumn{9}{|c|}{ Number of consecutive days } \\
\hline & & 1 & 3 & 7 & 14 & 30 & 60 & 90 & 120 & 183 \\
\hline 0.05 & 20 & 0 & 0 & 0 & 0 & 0 & 0 & 0 & 0.057 & 0.293 \\
\hline 0.10 & 10 & 0 & 0 & 0 & 0 & 0 & 0 & 0.096 & 0.383 & 1.43 \\
\hline 0.20 & 5 & 0 & 0 & 0 & 0 & 0.091 & 0.240 & 0.593 & 1.41 & 4.28 \\
\hline 0.50 & 2 & 2.78 & 2.97 & 3.02 & 3.19 & 3.32 & 3.97 & 4.87 & 7.76 & 18.8 \\
\hline
\end{tabular}




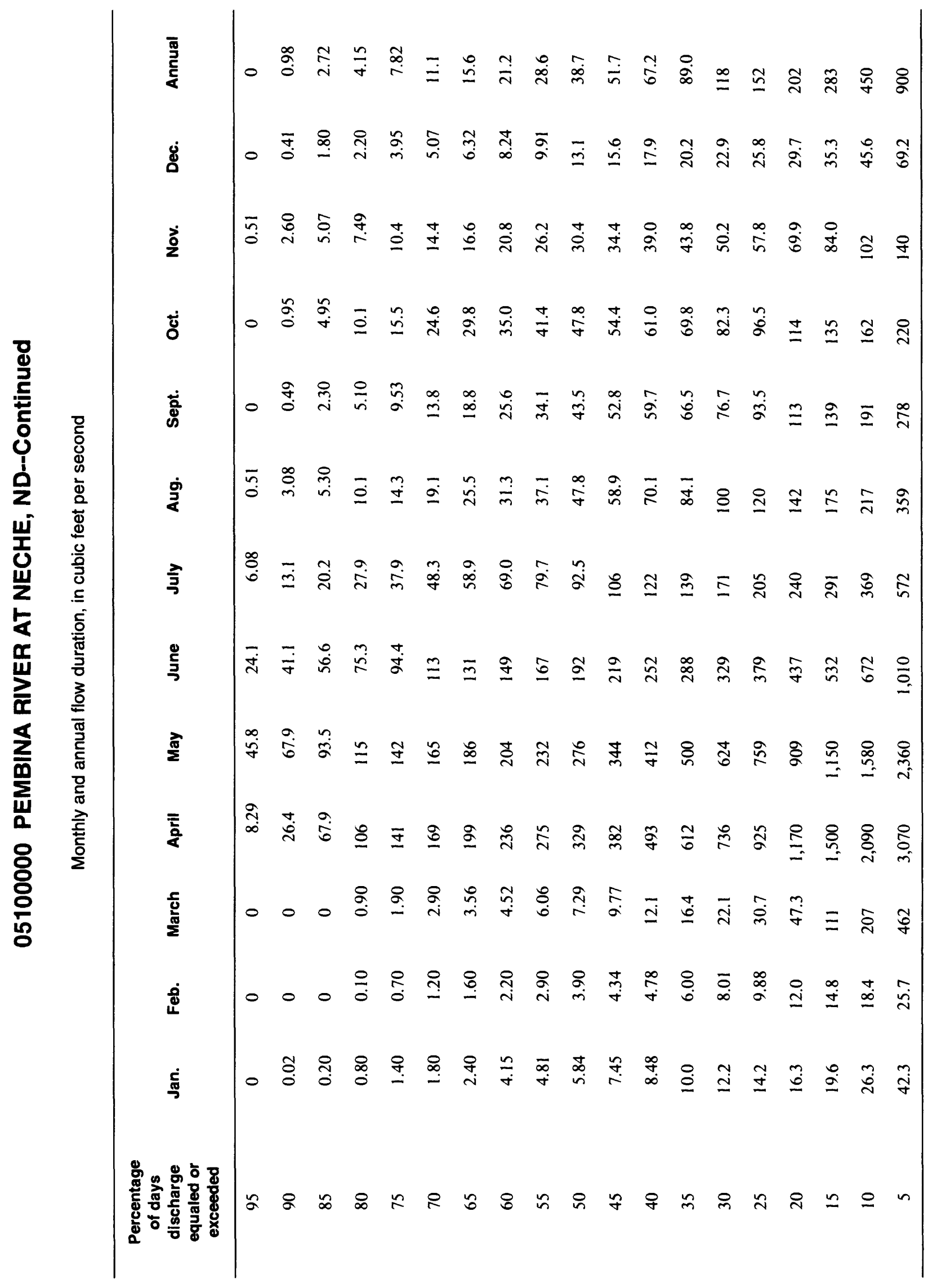




\section{PEMBINA RIVER AT NECHE, ND--Continued}

Probability of occurrence of seasonal low discharges

\begin{tabular}{|c|c|c|c|c|c|c|c|c|c|}
\hline \multirow[b]{3}{*}{$\begin{array}{c}\text { Non- } \\
\text { exceedance } \\
\text { probability }\end{array}$} & \multirow[b]{3}{*}{$\begin{array}{c}\text { Recurrence } \\
\text { intervai } \\
\text { (years) }\end{array}$} & \multicolumn{8}{|c|}{ Minimum mean discharge $\left(\mathrm{ft}^{3} / \mathrm{s}\right)$} \\
\hline & & \multicolumn{8}{|c|}{ Number of consecutive days } \\
\hline & & 1 & 7 & 14 & 30 & 1 & 7 & 14 & 30 \\
\hline & & \multicolumn{4}{|c|}{ December-January-February } & \multicolumn{4}{|c|}{ March-April-May } \\
\hline 0.05 & 20 & 0 & 0 & 0 & 0 & 0 & 0 & 0 & 0 \\
\hline 0.10 & 10 & 0 & 0 & 0 & 0 & 0 & 0 & 0 & 0.425 \\
\hline 0.20 & 5 & 0.108 & 0.112 & 0.163 & 0.229 & 0.078 & 0.110 & 0.145 & 2.28 \\
\hline \multirow[t]{2}{*}{0.50} & 2 & 3.10 & 3.21 & 3.34 & 3.64 & 4.45 & 5.10 & 5.66 & 18.1 \\
\hline & & \multicolumn{4}{|c|}{ June-July-August } & \multicolumn{4}{|c|}{ September-October-November } \\
\hline 0.05 & 20 & 0 & 0.128 & 0.330 & 0.636 & 0 & 0 & 0 & 0 \\
\hline 0.10 & 10 & 1.10 & ${ }^{1} 1.40$ & 1.80 & 2.60 & 0.032 & 0.154 & 0.332 & 0.521 \\
\hline 0.20 & 5 & 4.31 & 4.86 & 6.31 & 9.19 & 1.39 & 1.93 & 2.56 & 3.50 \\
\hline 0.50 & 2 & 25.1 & 32.8 & 34.6 & 48.9 & 12.3 & 15.6 & 18.7 & 26.1 \\
\hline
\end{tabular}

${ }^{1}$ Graphical interpretation. 


\section{PEMBINA RIVER AT NECHE, ND--Continued}

Annual peak discharge and corresponding gage height

$$
[--, \text { no data }]
$$

\begin{tabular}{|c|c|c|c|c|c|c|c|}
\hline $\begin{array}{l}\text { Water } \\
\text { year }\end{array}$ & Date & $\begin{array}{c}\text { Gage } \\
\text { height } \\
\text { (feet) }\end{array}$ & $\begin{array}{c}\text { Peak } \\
\text { discharge } \\
\left(\mathrm{ft}^{3} / \mathrm{s}\right)\end{array}$ & $\begin{array}{c}\text { Water } \\
\text { year }\end{array}$ & Date & $\begin{array}{c}\text { Gage } \\
\text { height } \\
\text { (feet) }\end{array}$ & $\begin{array}{c}\text { Peak } \\
\text { discharge } \\
\left(\mathrm{ft}^{3} / \mathrm{s}\right)\end{array}$ \\
\hline \multicolumn{8}{|c|}{ Annual peak discharge, by year, and corresponding gage height } \\
\hline 1904 & May 2 & 20.90 & 4,300 & 1952 & April 3 & 8.18 & 550 \\
\hline 1905 & April 5 & 9.80 & 1,370 & 1953 & June 10 & 6.74 & 250 \\
\hline 1906 & April 4 & 9.00 & 800 & 1954 & July 7 & 7.97 & 846 \\
\hline 1907 & May 14 & 13.60 & 2,190 & 1955 & April 5 & 20.11 & 2,700 \\
\hline 1908 & April 10 & 7.70 & 927 & 1956 & April 27 & 20.90 & 5,200 \\
\hline 1910 & March 15 & 6.50 & 685 & 1957 & March 27 & 7.63 & 661 \\
\hline 1911 & March 23 & 8.90 & 900 & 1958 & April 7 & 7.17 & 442 \\
\hline 1912 & July 29 & 8.50 & 1,000 & 1959 & April 5 & 14.40 & 1,800 \\
\hline 1913 & April 7 & 21.50 & 3,870 & 1960 & April 14 & 21.00 & 4,040 \\
\hline 1914 & April 18 & 4.80 & 388 & 1961 & April 3 & 9.49 & 372 \\
\hline 1915 & April 7 & 6.20 & 180 & 1962 & April 21 & 20.97 & 3,650 \\
\hline 1919 & April 15 & 15.10 & 2,430 & 1963 & July 28 & 10.55 & 1,150 \\
\hline 1920 & April 19 & 7.10 & 361 & 1964 & April 17 & 10.07 & 1,080 \\
\hline 1921 & April 13 & 7.40 & 733 & 1965 & April 13 & 19.21 & 3,600 \\
\hline 1922 & April 7 & -- & 1,300 & 1966 & April 3 & 21.20 & 4,300 \\
\hline 1923 & April 20 & 17.80 & 3,120 & 1967 & April 23 & 16.83 & 2,900 \\
\hline 1924 & April 20 & 6.70 & 674 & 1968 & March 28 & 9.34 & 734 \\
\hline 1925 & March 28 & 18.30 & 2,350 & 1969 & April 21 & 21.32 & 7,360 \\
\hline 1926 & July 6 & 5.00 & 318 & 1970 & April 27 & 21.47 & 7,070 \\
\hline 1927 & May 12 & 17.80 & 3,110 & 1971 & April 12 & 22.22 & 7,350 \\
\hline 1928 & March 25 & -- & 1,270 & 1972 & April 16 & 15.87 & 2,550 \\
\hline 1929 & March 21 & 9.00 & 750 & 1973 & March 27 & 8.27 & 224 \\
\hline 1930 & April 8 & 19.00 & 2,900 & 1974 & April 28 & 22.92 & 10,300 \\
\hline 1931 & April 9 & 13.00 & 1,580 & 1975 & May 18 & 11.03 & 1,500 \\
\hline 1932 & April 9 & 13.60 & 1,240 & 1976 & April 19 & 19.87 & 4,430 \\
\hline 1933 & May 26 & -- & 1,180 & 1977 & May 21 & 9.26 & 261 \\
\hline 1934 & April 9 & 9.76 & 780 & 1978 & April 11 & 19.31 & 3,800 \\
\hline 1935 & June 18 & 5.38 & 364 & 1979 & April 20 & 23.64 & 9,500 \\
\hline 1936 & April 15 & 17.34 & 2,530 & 1980 & April 7 & 8.85 & 435 \\
\hline 1937 & June 8 & 4.32 & 237 & 1981 & April 1 & 9.28 & 285 \\
\hline 1938 & March 20 & 8.11 & 730 & 1982 & June 8 & 11.68 & 1,520 \\
\hline 1939 & April 4 & 6.30 & 52.0 & 1983 & April 9 & 11.86 & 1,630 \\
\hline 1940 & April 20 & 7.97 & 816 & 1984 & April 7 & 9.35 & 312 \\
\hline 1941 & April 14 & 18.23 & 2,830 & 1985 & March 20 & 13.97 & 1,750 \\
\hline 1942 & April 7 & 19.96 & 3,550 & 1986 & March 24 & 14.83 & 2,390 \\
\hline 1943 & March 27 & 12.82 & 1,400 & 1987 & April 7 & 21.41 & 5,510 \\
\hline 1944 & August 6 & -- & 1,200 & 1988 & April 6 & 8.74 & 420 \\
\hline 1945 & March 29 & 16.54 & 2,440 & 1989 & April 17 & 10.08 & 1,000 \\
\hline 1946 & March 24 & 16.27 & 2,070 & 1990 & April 3 & 10.50 & 1,000 \\
\hline 1947 & April 11 & 10.19 & 1,320 & 1991 & June 16 & 10.22 & 888 \\
\hline 1948 & April 21 & 20.36 & 3,770 & 1992 & April 1 & 19.59 & 3,900 \\
\hline 1949 & April 22 & 20.83 & 5,010 & 1993 & July 30 & 18.10 & 3,580 \\
\hline 1950 & April 20 & 21.58 & 10,700 & 1994 & April 2 & 13.24 & 2,040 \\
\hline 1951 & April 7 & 14.95 & 2,000 & & & & \\
\hline
\end{tabular}




\section{PEMBINA RIVER AT NECHE, ND--Continued}

Annual peak discharge and corresponding gage height-Continued

$$
\text { [--, no data] }
$$

\begin{tabular}{|c|c|c|c|c|c|c|c|}
\hline $\begin{array}{l}\text { Water } \\
\text { year }\end{array}$ & Date & $\begin{array}{c}\text { Gage } \\
\text { height } \\
\text { (feet) }\end{array}$ & $\begin{array}{c}\text { Peak } \\
\text { discharge } \\
\left(\mathrm{ft}^{3} / \mathrm{s}\right) \\
\end{array}$ & $\begin{array}{l}\text { Water } \\
\text { year }\end{array}$ & Date & $\begin{array}{c}\text { Gage } \\
\text { height } \\
\text { (feet) }\end{array}$ & $\begin{array}{c}\text { Peak } \\
\text { discharge } \\
\left(\mathrm{ft}^{3} / \mathbf{s}\right)\end{array}$ \\
\hline \multicolumn{8}{|c|}{ Annual peak discharge, from highest to lowest, and corresponding gage height } \\
\hline 1950 & April 20 & 21.58 & 10,700 & 1905 & April 5 & 9.80 & 1,370 \\
\hline 1974 & April 28 & 22.92 & 10,300 & 1947 & April 11 & 10.19 & 1,320 \\
\hline 1979 & April 20 & 23.64 & 9,500 & 1922 & April 7 & -- & 1,300 \\
\hline 1969 & April 21 & 21.32 & 7,360 & 1928 & March 25 & -- & 1,270 \\
\hline 1971 & April 12 & 22.22 & 7,350 & 1932 & April 9 & 13.60 & 1,240 \\
\hline 1970 & April 27 & 21.47 & 7,070 & 1944 & August 6 & -- & 1,200 \\
\hline 1987 & April 7 & 21.41 & 5,510 & 1933 & May 26 & -- & 1,180 \\
\hline 1956 & April 27 & 20.90 & 5,200 & 1963 & July 28 & 10.55 & 1,150 \\
\hline 1949 & April 22 & 20.83 & 5,010 & 1964 & April 17 & 10.07 & 1,080 \\
\hline 1976 & April 19 & 19.87 & 4,430 & 1912 & July 29 & 8.50 & 1,000 \\
\hline 1904 & May 2 & 20.90 & 4,300 & 1989 & April 17 & 10.08 & 1,000 \\
\hline 1966 & April 3 & 21.20 & 4,300 & 1990 & April 3 & 10.50 & 1,000 \\
\hline 1960 & April 14 & 21.00 & 4,040 & 1908 & April 10 & 7.70 & 927 \\
\hline 1992 & April 1 & 19.59 & 3,900 & 1911 & March 23 & 8.90 & 900 \\
\hline 1913 & April 7 & 21.50 & 3,870 & 1991 & June 16 & 10.22 & 888 \\
\hline 1978 & April 11 & 19.31 & 3,800 & 1954 & July 7 & 7.97 & 846 \\
\hline 1948 & April 21 & 20.36 & 3,770 & 1940 & April 20 & 7.97 & 816 \\
\hline 1962 & April 21 & 20.97 & 3,650 & 1906 & April 4 & 9.00 & 800 \\
\hline 1965 & April 13 & 19.21 & 3,600 & 1934 & April 9 & 9.76 & 780 \\
\hline 1993 & July 30 & 18.10 & 3,580 & 1929 & March 21 & 9.00 & 750 \\
\hline 1942 & April 7 & 19.96 & 3,550 & 1968 & March 28 & 9.34 & 734 \\
\hline 1923 & April 20 & 17.80 & 3,120 & 1921 & April 13 & 7.40 & 733 \\
\hline 1927 & May 12 & 17.80 & 3,110 & 1938 & March 20 & 8.11 & 730 \\
\hline 1930 & April 8 & 19.00 & 2,900 & 1910 & March 15 & 6.50 & 685 \\
\hline 1967 & April 23 & 16.83 & 2,900 & 1924 & April 20 & 6.70 & 674 \\
\hline 1941 & April 14 & 18.23 & 2,830 & 1957 & March 27 & 7.63 & 661 \\
\hline 1955 & April 5 & 20.11 & 2,700 & 1952 & April 3 & 8.18 & 550 \\
\hline 1972 & April 16 & 15.87 & 2,550 & 1958 & April 7 & 7.17 & 442 \\
\hline 1936 & April 15 & 17.34 & 2,530 & 1980 & April 7 & 8.85 & 435 \\
\hline 1945 & March 29 & 16.54 & 2,440 & 1988 & April 6 & 8.74 & 420 \\
\hline 1919 & April 15 & 15.10 & 2,430 & 1914 & April 18 & 4.80 & 388 \\
\hline 1986 & March 24 & 14.83 & 2,390 & 1961 & April 3 & 9.49 & 372 \\
\hline 1925 & March 28 & 18.30 & 2,350 & 1935 & June 18 & 5.38 & 364 \\
\hline 1907 & May 14 & 13.60 & 2,190 & 1920 & April 19 & 7.10 & 361 \\
\hline 1946 & March 24 & 16.27 & 2,070 & 1926 & July 6 & 5.00 & 318 \\
\hline 1994 & April 2 & 13.24 & 2,040 & 1984 & April 7 & 9.35 & 312 \\
\hline 1951 & April 7 & 14.95 & 2,000 & 1981 & April 1 & 9.28 & 285 \\
\hline 1959 & April 5 & 14.40 & 1,800 & 1977 & May 21 & 9.26 & 261 \\
\hline 1985 & March 20 & 13.97 & 1,750 & 1953 & June 10 & 6.74 & 250 \\
\hline 1983 & April 9 & 11.86 & 1,630 & 1937 & June 8 & 4.32 & 237 \\
\hline 1931 & April 9 & 13.00 & 1,580 & 1973 & March 27 & 8.27 & 224 \\
\hline 1982 & June 8 & 11.68 & 1,520 & 1915 & April 7 & 6.20 & 180 \\
\hline 1975 & May 18 & 11.03 & 1,500 & 1939 & April 4 & 6.30 & 52.0 \\
\hline 1943 & March 27 & 12.82 & 1,400 & & & & \\
\hline
\end{tabular}




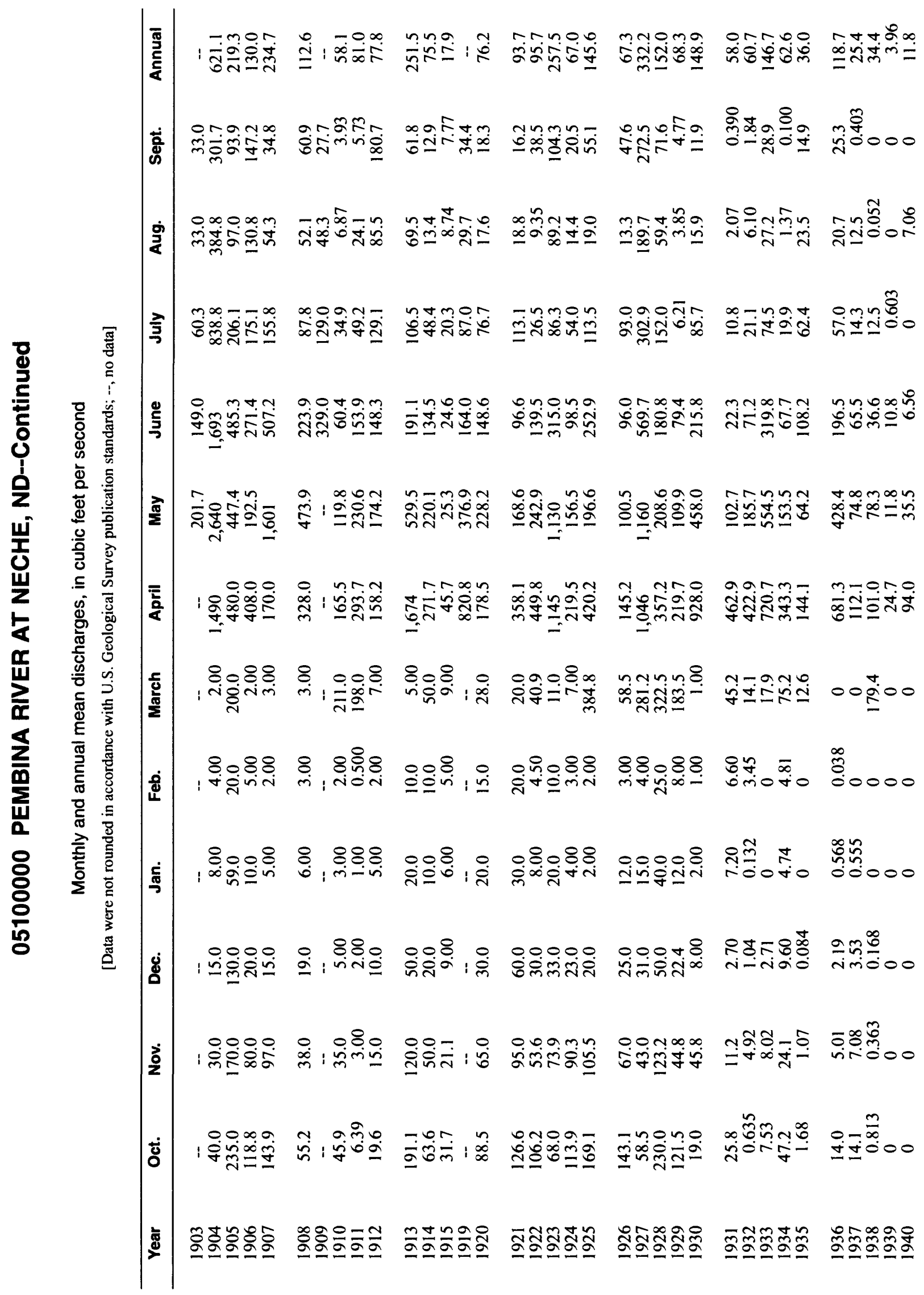




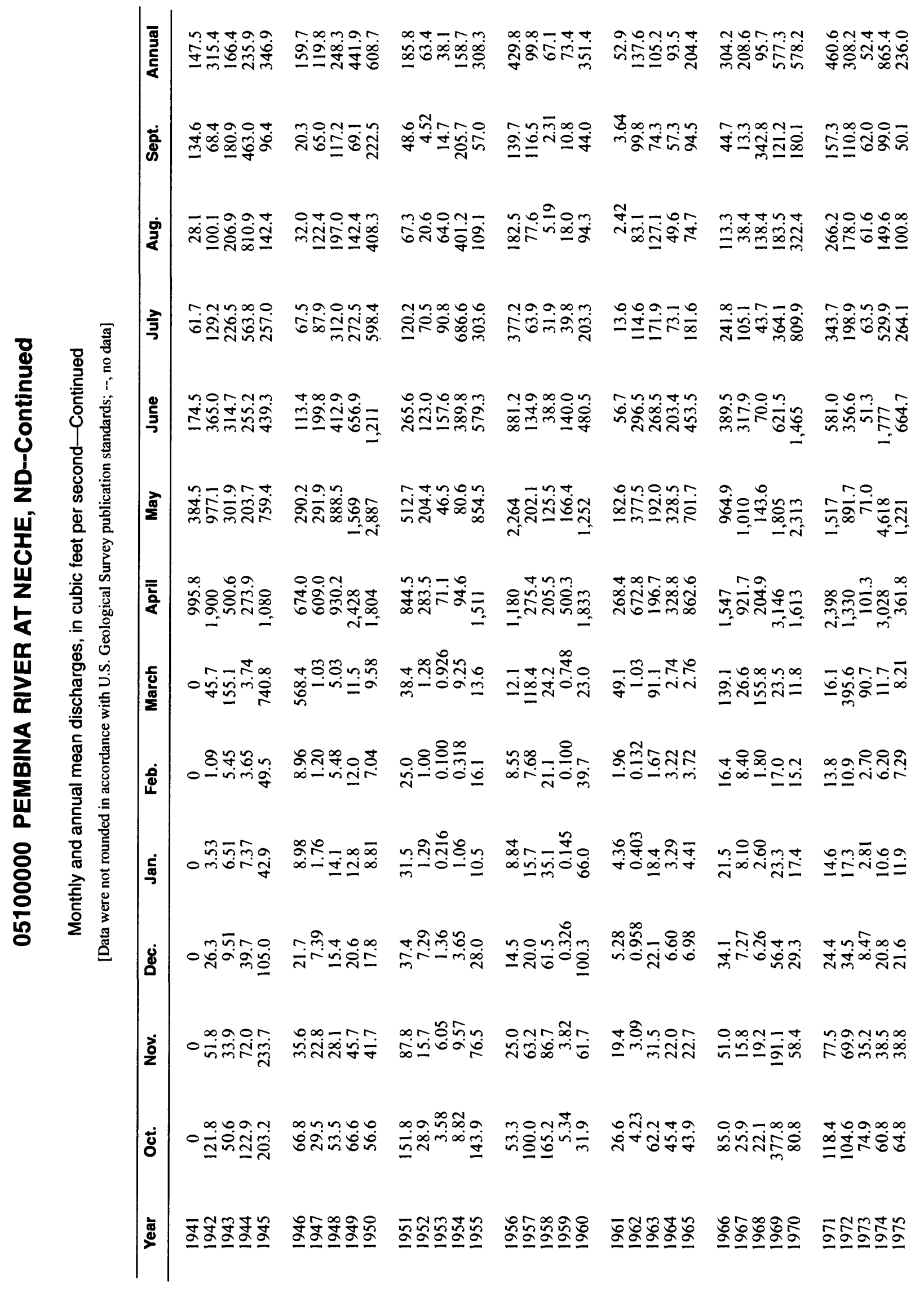




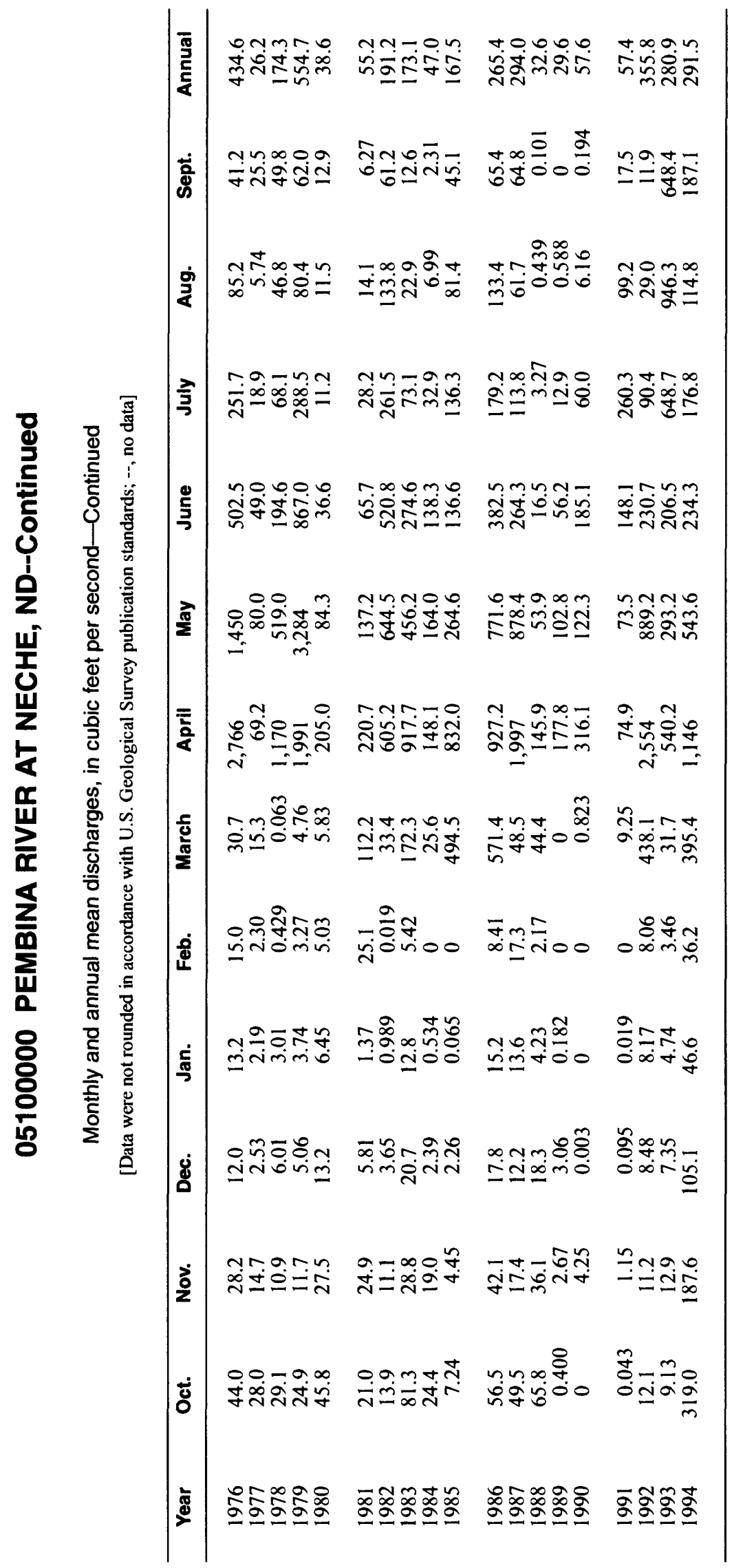




\section{HERZOG CREEK NEAR CONCRETE, ND}

LOCATION.--Lat $48^{\circ} 45^{\prime} 13^{\prime}$, long 97'54'22”, in SE $1 / 4$ sec.20, T.161 N., R.56 W., Pembina County, Hydrologic Unit 09020313 , on left bank $1.7 \mathrm{mi}$ northeast of Concrete and $1.7 \mathrm{mi}$ upstream from mouth.

DRAINAGE AREA.--18.9 $\mathrm{mi}^{2}$.

PERIOD OF RECORD.--June 1954 to September 1977.

GAGE.--Water-stage recorder and concrete control. Datum of gage is $1,108.95 \mathrm{ft}$ above mean sea level (levels by Soil Conservation Service). Prior to Sept. 15, 1971, recording gage at site $0.5 \mathrm{mi}$ downstream at same datum.

EXTREMES FOR PERIOD OF RECORD.--Maximum discharge observed, $260 \mathrm{ft}^{3} / \mathrm{s}$, Apr. 2, 1955, gage height, $9.74 \mathrm{ft}$, from floodmarks, backwater from ice; maximum gage height, $12.59 \mathrm{ft}$, Mar. 25, 1976, backwater from ice; no flow at times each year.

Annual mean discharge

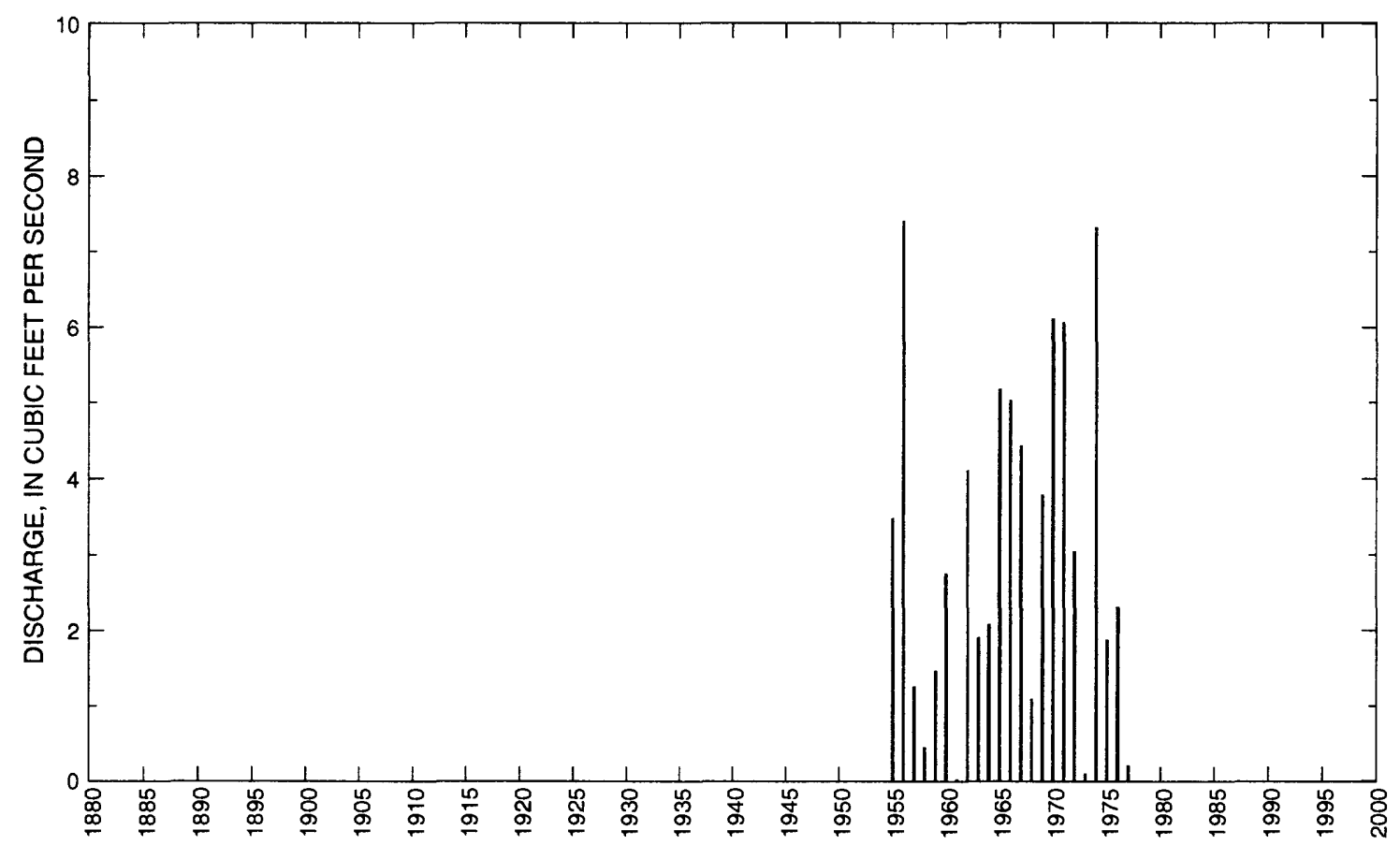




\section{HERZOG CREEK NEAR CONCRETE, ND--Continued}

Statistics of monthly and annual mean discharges

[m, more than 1 year of occurrence]

\begin{tabular}{|c|c|c|c|c|c|c|c|c|}
\hline \multirow[b]{2}{*}{ Month } & \multicolumn{2}{|c|}{ Maximum } & \multicolumn{2}{|c|}{ Minimum } & \multicolumn{4}{|c|}{ Mean } \\
\hline & $\begin{array}{c}\text { Discharge } \\
\left(\mathrm{ft}^{3} / \mathrm{s}\right)\end{array}$ & $\begin{array}{c}\text { Water year } \\
\text { of } \\
\text { occurrence }\end{array}$ & $\begin{array}{c}\text { Discharge } \\
\left(\mathrm{ft}^{3} / \mathrm{s}\right)\end{array}$ & $\begin{array}{c}\text { Water year } \\
\text { of } \\
\text { occurrence }\end{array}$ & $\begin{array}{c}\text { Discharge } \\
\left(\mathrm{ft}^{3} / \mathrm{s}\right)\end{array}$ & $\begin{array}{c}\text { Standard } \\
\text { deviation } \\
\left(\mathrm{ft}^{3} / \mathrm{s}\right)\end{array}$ & $\begin{array}{l}\text { Coeffi- } \\
\text { cient of } \\
\text { variation }\end{array}$ & $\begin{array}{l}\text { Percentage } \\
\text { of annual } \\
\text { discharge }\end{array}$ \\
\hline October & 8.80 & 1974 & 0 & $\mathrm{~m}$ & 1.76 & 2.64 & 1.50 & 4.70 \\
\hline November & 2.20 & 1972 & 0 & $\mathrm{~m}$ & 0.550 & 0.69 & 1.25 & 1.48 \\
\hline December & 1.80 & 1963 & 0 & $\mathrm{~m}$ & 0.160 & 0.37 & 2.37 & 0.42 \\
\hline January & 1.04 & 1963 & 0 & $\mathrm{~m}$ & 0.060 & 0.22 & 3.76 & 0.15 \\
\hline February & 0.343 & 1958 & 0 & $\mathrm{~m}$ & 0.030 & 0.08 & 3.11 & 0.07 \\
\hline March & 11.6 & 1966 & 0 & $\mathrm{~m}$ & 1.38 & 2.83 & 2.05 & 3.69 \\
\hline April & 48.6 & 1971 & 0.050 & 1973 & 19.4 & 16.6 & 0.86 & 51.7 \\
\hline May & 37.7 & 1974 & 0 & 1961 & 8.94 & 11.4 & 1.27 & 23.9 \\
\hline June & 10.2 & 1962 & 0 & 1961 & 2.83 & 3.09 & 1.09 & 7.55 \\
\hline July & 5.48 & 1966 & 0 & $\mathrm{~m}$ & 1.23 & 1.64 & 1.33 & 3.29 \\
\hline August & 2.27 & 1964 & 0 & $\mathrm{~m}$ & 0.240 & 0.52 & 2.17 & 0.64 \\
\hline September & 6.73 & 1972 & 0 & $\mathrm{~m}$ & 0.920 & 1.79 & 1.93 & 2.47 \\
\hline Annual & 7.41 & 1956 & 0.018 & 1961 & 3.11 & 2.28 & 0.73 & 100 \\
\hline
\end{tabular}

Annual flow duration

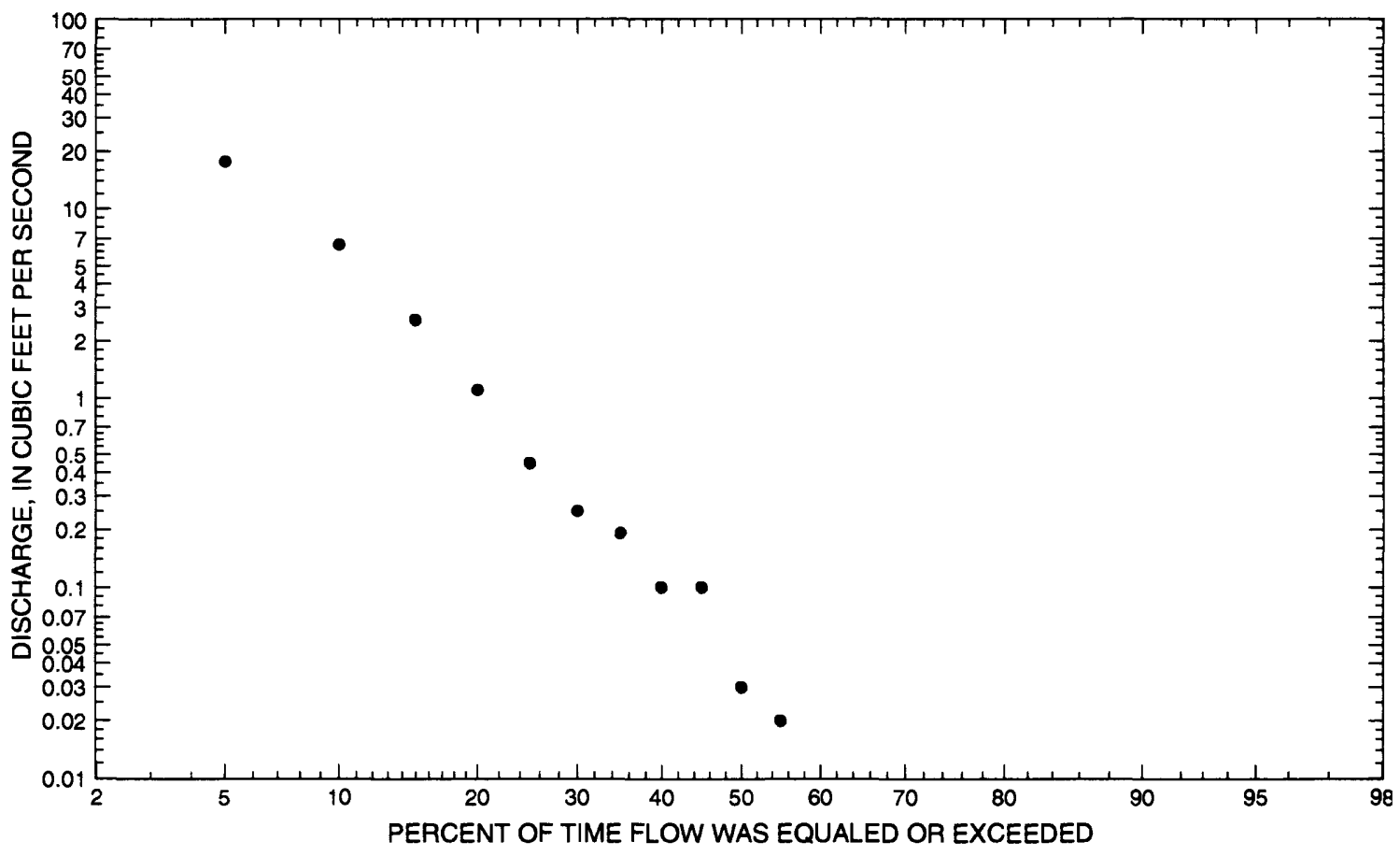




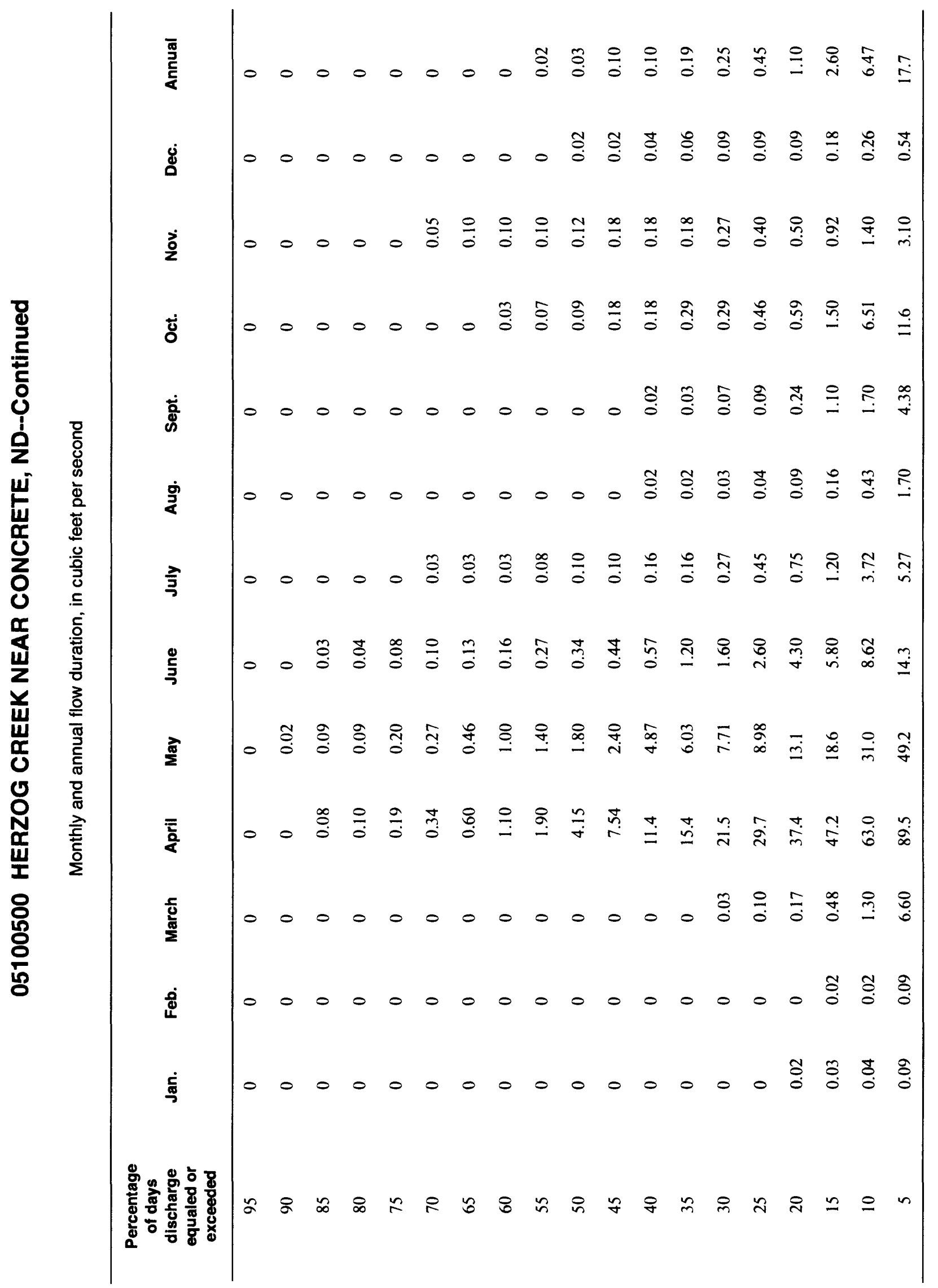




\section{HERZOG CREEK NEAR CONCRETE, ND--Continued}

Probability of occurrence of annual high discharges

[ng, statistic not given]

\begin{tabular}{|c|c|c|c|c|c|c|}
\hline \multirow[b]{2}{*}{$\begin{array}{l}\text { Exceedance } \\
\text { probability }\end{array}$} & \multirow[b]{2}{*}{$\begin{array}{l}\text { Recurrence } \\
\text { interval } \\
\text { (years) }\end{array}$} & \multirow[b]{2}{*}{$\begin{array}{c}\text { Maximum } \\
\text { instantaneous } \\
\left(\mathrm{ft}^{3} / \mathrm{s}\right)\end{array}$} & \multicolumn{4}{|c|}{$\begin{array}{l}\text { Maximum mean discharge } \\
\left(\mathrm{ft}^{3} / \mathrm{s}\right)\end{array}$} \\
\hline & & & 3-day period & 7-day period & 15-day period & 30-day period \\
\hline 0.99 & 1.01 & ng & 0.248 & 0.182 & 0.124 & 0.095 \\
\hline 0.95 & 1.05 & 13.3 & 2.21 & 1.57 & 1.03 & 0.688 \\
\hline 0.90 & 1.11 & 20.1 & 5.71 & 4.05 & 2.64 & 1.69 \\
\hline 0.80 & 1.25 & 32.0 & 14.9 & 10.7 & 7.05 & 4.38 \\
\hline 0.50 & 2 & 70.5 & 54.9 & 41.7 & 29.0 & 18.1 \\
\hline 0.20 & 5 & 137 & 112 & 92.2 & 69.8 & 46.9 \\
\hline 0.10 & 10 & 185 & 135 & 117 & 93.0 & 65.8 \\
\hline 0.04 & 25 & 247 & 152 & 137 & 114 & 85.5 \\
\hline 0.02 & 50 & 293 & 158 & 146 & 124 & 96.7 \\
\hline 0.01 & 100 & 337 & 162 & 151 & 132 & 105 \\
\hline 0.005 & 200 & 379 & 164 & 154 & 136 & 112 \\
\hline 0.002 & 500 & 434 & ng & $\mathrm{ng}$ & ng & ng \\
\hline
\end{tabular}

Probability of occurrence of annual low discharges

[ng, statistic not given]

\begin{tabular}{|c|c|c|c|c|c|c|c|c|c|c|}
\hline \multirow{3}{*}{$\begin{array}{l}\text { Non- } \\
\text { exceed- } \\
\text { ance } \\
\text { prob- } \\
\text { ability }\end{array}$} & \multirow{3}{*}{$\begin{array}{l}\text { Recur- } \\
\text { rence } \\
\text { inter- } \\
\text { val } \\
\text { (years) }\end{array}$} & \multicolumn{9}{|c|}{ Minimum mean discharge $\left(\mathrm{ft}^{3} / \mathrm{s}\right)$} \\
\hline & & \multicolumn{9}{|c|}{ Number of consecutive days } \\
\hline & & 1 & 3 & 7 & 14 & 30 & 60 & 90 & 120 & 183 \\
\hline 0.05 & 20 & $\mathrm{ng}$ & $\mathrm{ng}$ & $\mathrm{ng}$ & $\mathrm{ng}$ & $\mathrm{ng}$ & $\mathrm{ng}$ & 0 & 0 & 0 \\
\hline 0.10 & 10 & $\mathrm{ng}$ & $\mathrm{ng}$ & $\mathrm{ng}$ & $\mathrm{ng}$ & $\mathrm{ng}$ & ng & 0 & 0 & 0 \\
\hline 0.20 & 5 & $\mathrm{ng}$ & $\mathrm{ng}$ & $\mathrm{ng}$ & $\mathrm{ng}$ & $\mathrm{ng}$ & $\mathrm{ng}$ & 0 & 0 & 0.034 \\
\hline 0.50 & 2 & $\mathrm{ng}$ & $\mathrm{ng}$ & $\mathrm{ng}$ & $\mathrm{ng}$ & $\mathrm{ng}$ & $\mathrm{ng}$ & 0 & 0.016 & 0.203 \\
\hline
\end{tabular}




\section{HERZOG CREEK NEAR CONCRETE, ND--Continued}

Probability of occurrence of seasonal low discharges

[ng, statistic not given]

\begin{tabular}{|c|c|c|c|c|c|c|c|c|c|}
\hline \multirow[b]{3}{*}{$\begin{array}{c}\text { Non- } \\
\text { exceedance } \\
\text { probability }\end{array}$} & \multirow[b]{3}{*}{$\begin{array}{c}\text { Recurrence } \\
\text { intervai } \\
\text { (years) }\end{array}$} & \multicolumn{8}{|c|}{ Minimum mean discharge $\left(\mathrm{ft}^{3} / \mathrm{s}\right)$} \\
\hline & & \multicolumn{8}{|c|}{ Number of consecutive days } \\
\hline & & 1 & 7 & 14 & 30 & 1 & 7 & 14 & 30 \\
\hline & & \multicolumn{4}{|c|}{ December-January-February } & \multicolumn{4}{|c|}{ March-April-May } \\
\hline 0.05 & 20 & 0 & $\mathrm{ng}$ & 0 & 0 & $\mathrm{ng}$ & 0 & 0 & 0 \\
\hline 0.10 & 10 & 0 & $\mathrm{ng}$ & 0 & 0 & $\mathrm{ng}$ & 0 & 0 & 0 \\
\hline 0.20 & 5 & 0 & $\mathrm{ng}$ & 0 & 0 & $\mathrm{ng}$ & 0 & 0 & 0 \\
\hline \multirow[t]{2}{*}{0.50} & 2 & 0 & $\mathrm{ng}$ & 0 & 0 & $\mathrm{ng}$ & 0 & 0 & 0.036 \\
\hline & & \multicolumn{4}{|c|}{ June-July-August } & \multicolumn{4}{|c|}{ September-October-November } \\
\hline 0.05 & 20 & 0 & 0 & 0 & 0 & 0 & 0 & 0 & 0 \\
\hline 0.10 & 10 & 0 & 0 & 0 & 0 & 0 & 0 & 0 & 0 \\
\hline 0.20 & 5 & 0 & 0 & 0 & 0 & 0 & 0 & 0 & 0 \\
\hline 0.50 & 2 & 0 & 0 & 0 & 0.022 & 0 & 0 & 0 & 0 \\
\hline
\end{tabular}




\section{HERZOG CREEK NEAR CONCRETE, ND--Continued}

\section{Annual peak discharge and corresponding gage height}

\begin{tabular}{|c|c|c|c|c|c|c|c|}
\hline $\begin{array}{l}\text { Water } \\
\text { year }\end{array}$ & Date & $\begin{array}{c}\text { Gage } \\
\text { height } \\
\text { (feet) }\end{array}$ & $\begin{array}{c}\text { Peak } \\
\text { discharge } \\
\left(\mathrm{ft}^{3} / \mathrm{s}\right)\end{array}$ & $\begin{array}{l}\text { Water } \\
\text { year }\end{array}$ & Date & $\begin{array}{c}\text { Gage } \\
\text { height } \\
\text { (feet) }\end{array}$ & $\begin{array}{c}\text { Peak } \\
\text { discharge } \\
\left(f^{3} / s\right)\end{array}$ \\
\hline \multicolumn{8}{|c|}{ Annual peak discharge, by year, and corresponding gage height } \\
\hline 1955 & April 2 & 9.74 & 260 & 1967 & May 7 & 6.52 & 94.0 \\
\hline 1956 & April 25 & 8.38 & 243 & 1968 & June 25 & 3.61 & 8.60 \\
\hline 1957 & March 21 & 7.54 & 90.0 & 1969 & April 10 & 7.29 & 129 \\
\hline 1958 & March 26 & 5.22 & 20.0 & 1970 & April 25 & 6.65 & 106 \\
\hline 1959 & April 6 & 4.79 & 35.0 & 1971 & April 9 & 7.92 & 128 \\
\hline 1960 & April 13 & 6.66 & 100 & 1972 & April 14 & 10.29 & 94.0 \\
\hline 1961 & April 1 & 3.12 & 2.00 & 1973 & August 9 & 9.89 & 16.0 \\
\hline 1962 & April 20 & 6.67 & 102 & 1974 & April 20 & 10.85 & 122 \\
\hline 1963 & July 26 & 5.81 & 63.0 & 1975 & April 29 & 10.27 & 53.0 \\
\hline 1964 & June 19 & 5.25 & 46.0 & 1976 & April 6 & 10.64 & 44.0 \\
\hline 1965 & April 11 & 7.41 & 115 & 1977 & October 26 & 9.83 & 36.0 \\
\hline 1966 & May 5 & 6.24 & 79.0 & & & & \\
\hline \multicolumn{8}{|c|}{ Annual peak discharge, from highest to lowest, and corresponding gage height } \\
\hline 1955 & April 2 & 9.74 & 260 & 1966 & May 5 & 6.24 & 79.0 \\
\hline 1956 & April 25 & 8.38 & 243 & 1963 & July 26 & 5.81 & 63.0 \\
\hline 1969 & April 10 & 7.29 & 129 & 1975 & April 29 & 10.27 & 53.0 \\
\hline 1971 & April 9 & 7.92 & 128 & 1964 & June 19 & 5.25 & 46.0 \\
\hline 1974 & April 20 & 10.85 & 122 & 1976 & April 6 & 10.64 & 44.0 \\
\hline 1965 & April 11 & 7.41 & 115 & 1977 & October 26 & 9.83 & 36.0 \\
\hline 1970 & April 25 & 6.65 & 106 & 1959 & April 6 & 4.79 & 35.0 \\
\hline 1962 & April 20 & 6.67 & 102 & 1958 & March 26 & 5.22 & 20.0 \\
\hline 1960 & April 13 & 6.66 & 100 & 1973 & August 9 & 9.89 & 16.0 \\
\hline 1967 & May 7 & 6.52 & 94.0 & 1968 & June 25 & 3.61 & 8.60 \\
\hline 1972 & April 14 & 10.29 & 94.0 & 1961 & April 1 & 3.12 & 2.00 \\
\hline 1957 & March 21 & 7.54 & 90.0 & & & & \\
\hline
\end{tabular}




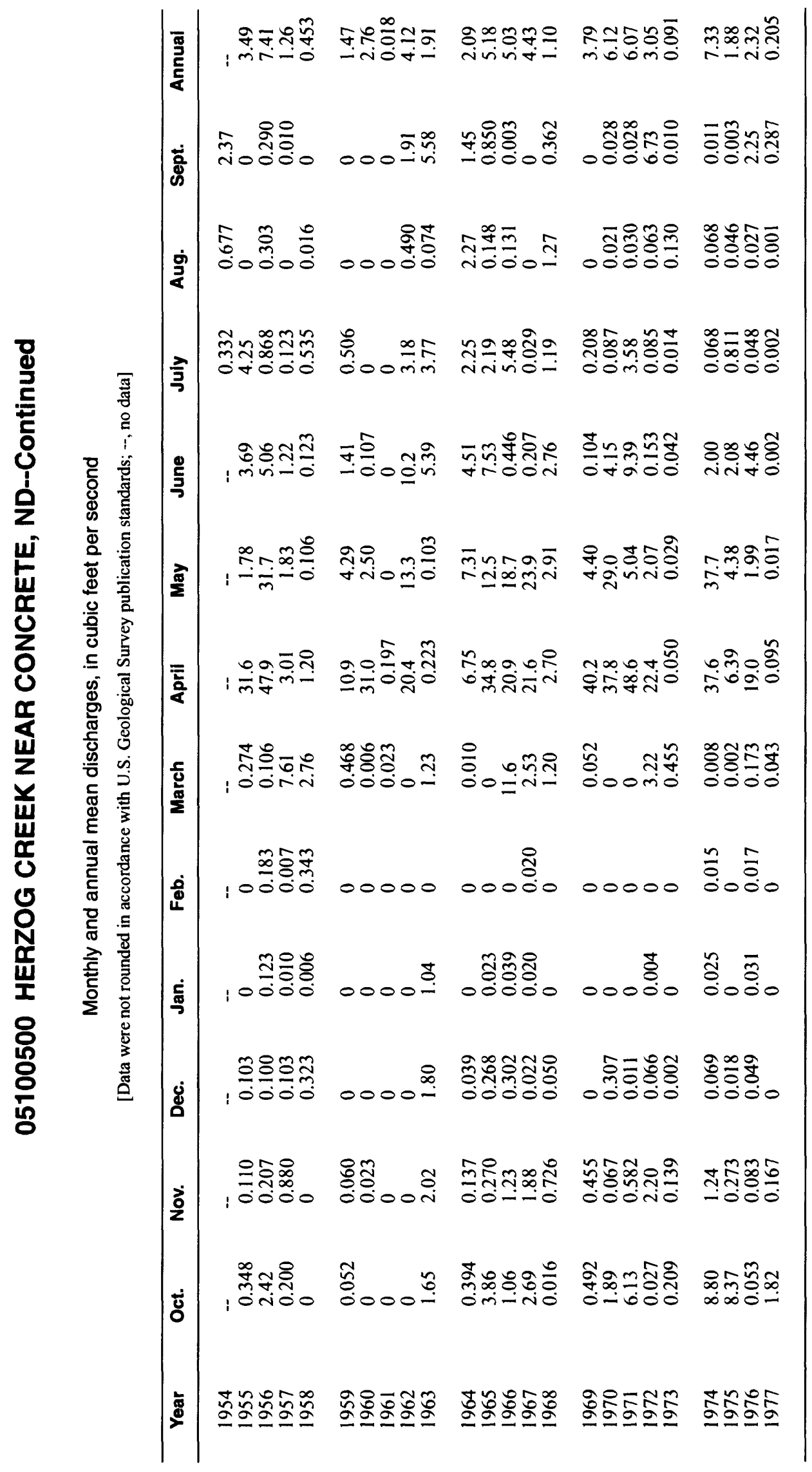




\section{TONGUE RIVER AT AKRA, ND}

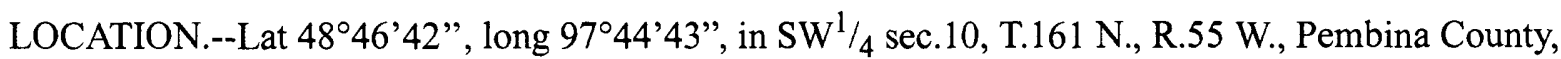
Hydrologic Unit 09020313, on left bank $300 \mathrm{ft}$ downstream from Renwick Dam, 0.9 mi northwest of Akra, and $6 \mathrm{mi}$ west of Cavalier.

DRAINAGE AREA.--160 $\mathrm{mi}^{2}$.

PERIOD OF RECORD.--April to June 1950 (WSP 1137-B), October 1951 to current year. Seasonal record since 1983 .

GAGE.--Water-stage recorder. Datum of gage is $930.00 \mathrm{ft}$ above sea level. Prior to July 10, 1954, nonrecording gage $1.2 \mathrm{mi}$ downstream at datum $30.00 \mathrm{ft}$ lower. July 23,1954 , to Dec. 19, 1973, water stage recorder $2.7 \mathrm{mi}$ downstream at datum $9.10 \mathrm{ft}$ lower.

EXTREMES FOR PERIOD OF RECORD.--Maximum discharge, 11,800 $\mathrm{ft}^{3} / \mathrm{s}$, Apr. 18, 1950; gage height, $48.70 \mathrm{ft}$; no flow at times.

Annual mean discharge

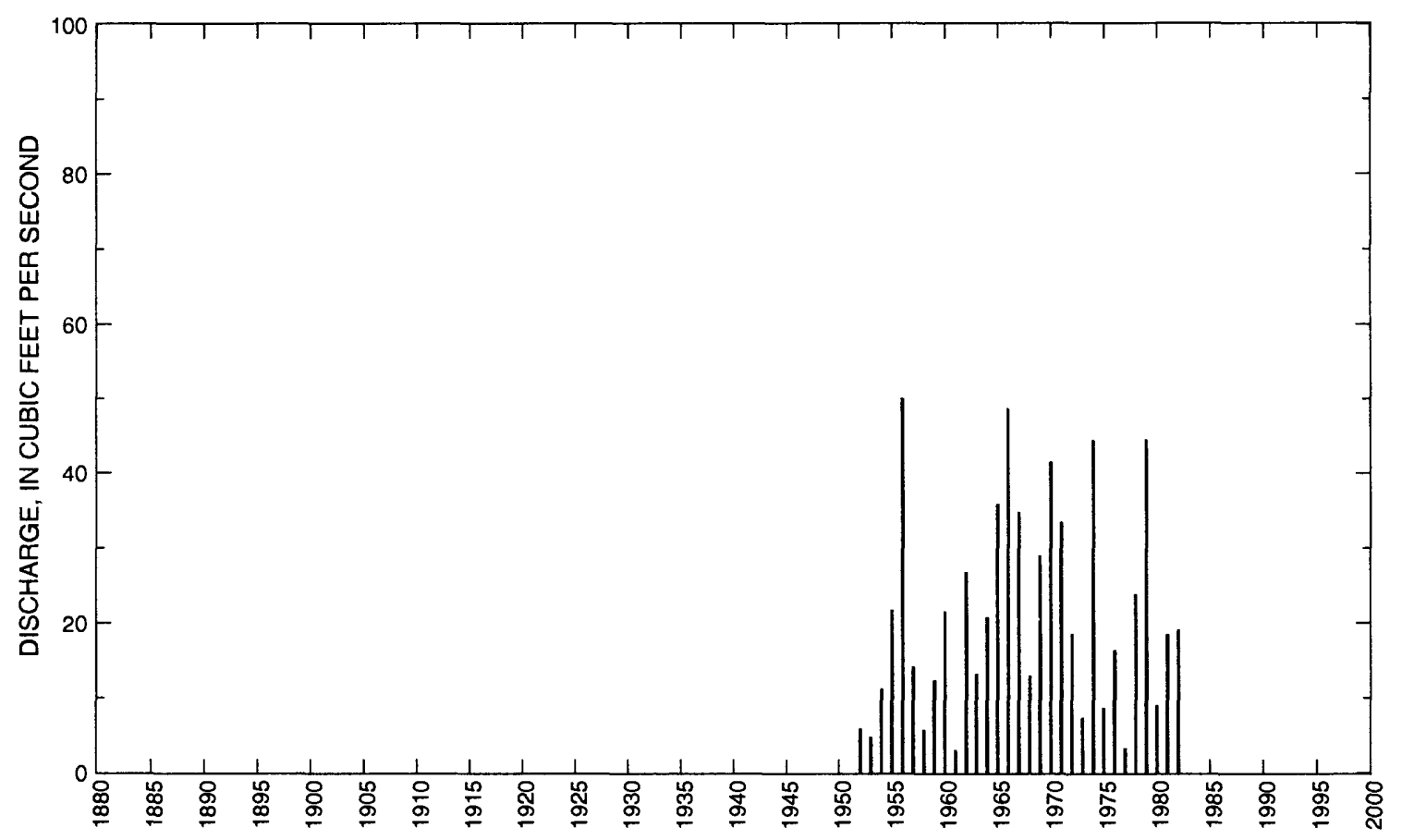




\section{TONGUE RIVER AT AKRA, ND--Continued}

Post-regulation period, 1955-94

Statistics of monthly and annual mean discharges, post-regulation period

\begin{tabular}{|c|c|c|c|c|c|c|c|c|}
\hline \multirow[b]{2}{*}{ Month } & \multicolumn{2}{|c|}{ Maximum } & \multicolumn{2}{|c|}{ Minimum } & \multicolumn{4}{|c|}{ Mean } \\
\hline & $\begin{array}{c}\text { Discharge } \\
\left(\mathrm{ft}^{3} / \mathrm{s}\right)\end{array}$ & $\begin{array}{l}\text { Water year } \\
\text { of } \\
\text { occurrence }\end{array}$ & $\begin{array}{c}\text { Discharge } \\
\left(\mathrm{ft}^{3} / \mathrm{s}\right)\end{array}$ & $\begin{array}{c}\text { Water year } \\
\text { of } \\
\text { occurrence }\end{array}$ & $\begin{array}{c}\text { Discharge } \\
\left(\mathrm{ft}^{3} / \mathrm{s}\right)\end{array}$ & $\begin{array}{l}\text { Standard } \\
\text { deviation } \\
\left(\mathrm{ft}^{3} / \mathrm{s}\right)\end{array}$ & $\begin{array}{l}\text { Coeffi- } \\
\text { cient of } \\
\text { variation }\end{array}$ & $\begin{array}{l}\text { Percentage } \\
\text { of annual } \\
\text { discharge }\end{array}$ \\
\hline October & 30.1 & 1981 & 0.513 & 1962 & 6.70 & 6.60 & 0.98 & 2.69 \\
\hline November & 22.7 & 1981 & 0.560 & 1976 & 7.26 & 5.17 & 0.71 & 2.92 \\
\hline December & 12.9 & 1971 & 0.700 & 1976 & 4.82 & 3.25 & 0.68 & 1.94 \\
\hline January & 7.27 & 1971 & 1.23 & 1955 & 3.42 & 1.70 & 0.50 & 1.37 \\
\hline February & 18.7 & 1981 & 0.571 & 1962 & 3.91 & 3.58 & 0.92 & 1.57 \\
\hline March & 135 & 1966 & 0.216 & 1964 & 21.5 & 27.6 & 1.28 & 8.65 \\
\hline April & 294 & 1956 & 0.428 & 1991 & 108 & 93.2 & 0.86 & 43.3 \\
\hline May & 225 & 1974 & 1.63 & 1980 & 49.2 & 63.9 & 1.30 & 19.8 \\
\hline June & 78.7 & 1964 & 0.473 & 1988 & 18.2 & 19.5 & 1.07 & 7.33 \\
\hline July & 107 & 1993 & 0.086 & 1978 & 11.5 & 17.6 & 1.53 & 4.63 \\
\hline August & 144 & 1993 & 0.208 & 1988 & 7.99 & 22.6 & 2.83 & 3.21 \\
\hline September & 28.3 & 1980 & 0.096 & 1989 & 6.51 & 7.56 & 1.16 & 2.62 \\
\hline Annual & 50.1 & 1956 & 3.11 & 1961 & 22.9 & 14.1 & 0.62 & 100 \\
\hline
\end{tabular}

Annual flow duration

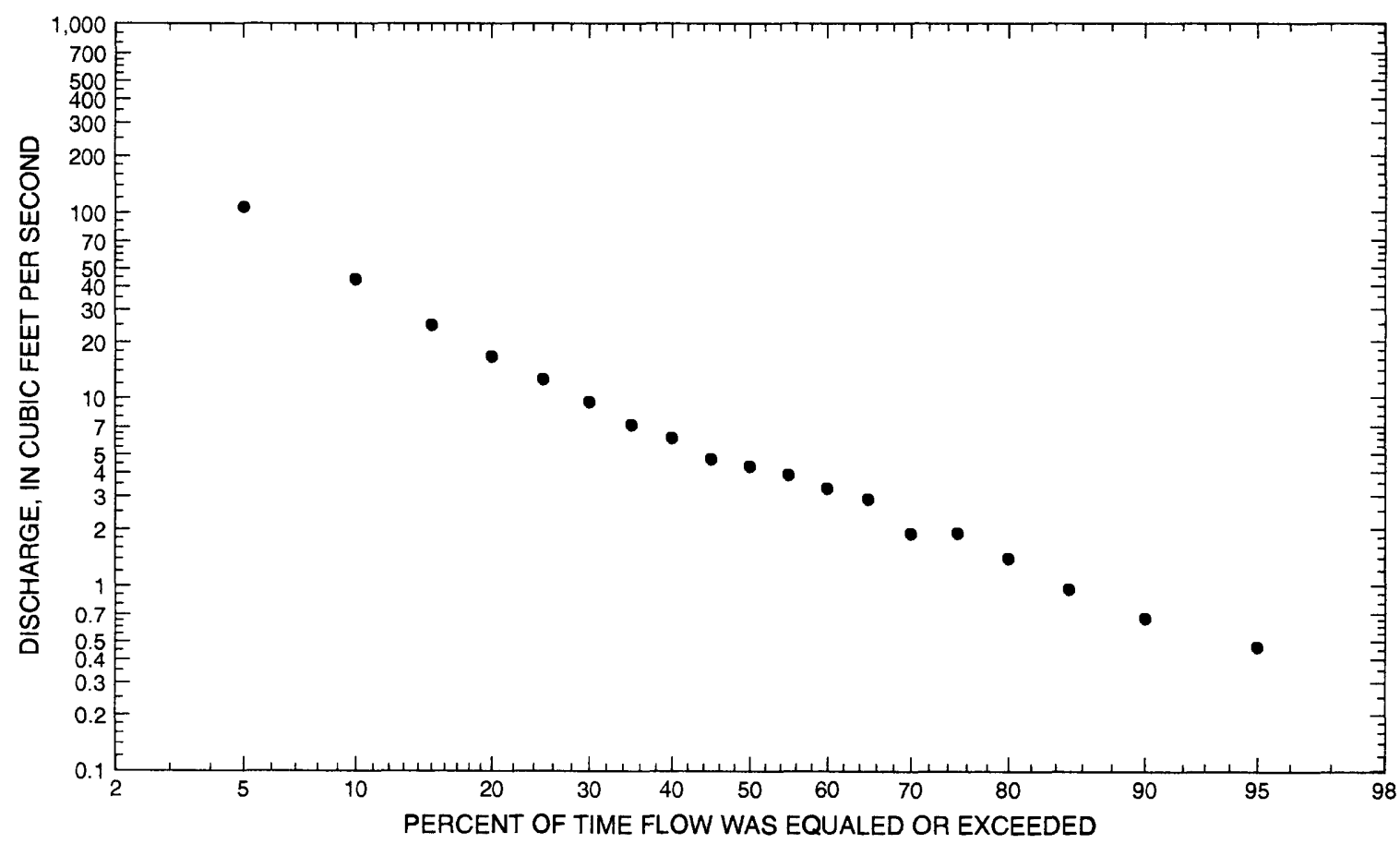




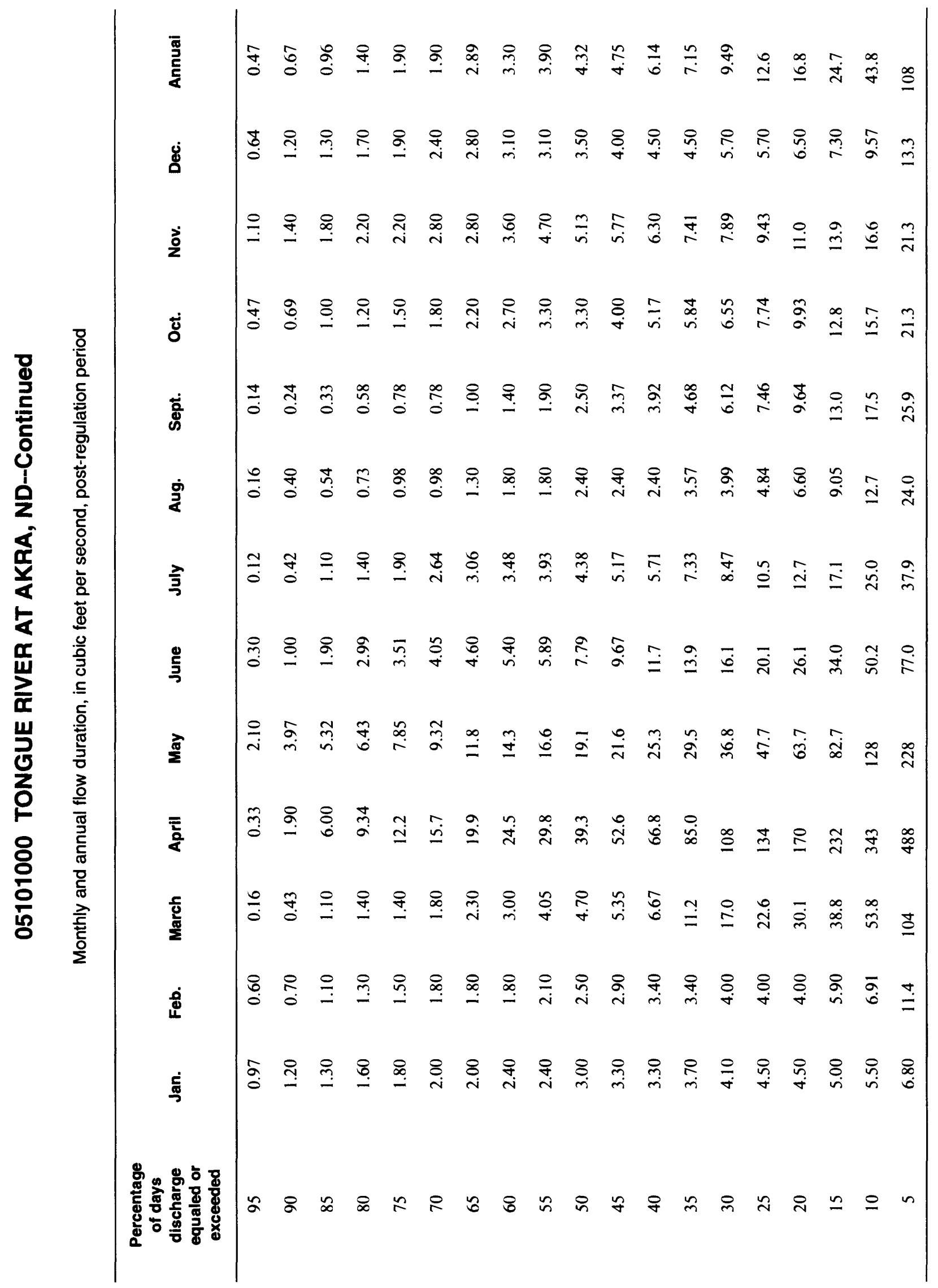




\section{TONGUE RIVER AT AKRA, ND--Continued}

Probability of occurrence of annual high discharges, post-regulation period

[ng, statistic not given]

\begin{tabular}{|c|c|c|c|c|c|c|}
\hline \multirow[b]{2}{*}{$\begin{array}{l}\text { Exceedance } \\
\text { probability }\end{array}$} & \multirow[b]{2}{*}{$\begin{array}{l}\text { Recurrence } \\
\text { interval } \\
\text { (years) }\end{array}$} & \multirow[b]{2}{*}{$\begin{array}{c}\text { Maximum } \\
\text { instantaneous } \\
\left(\mathrm{ft}^{3} / \mathrm{s}\right)\end{array}$} & \multicolumn{4}{|c|}{$\begin{array}{l}\text { Maximum mean discharge } \\
\qquad\left(\mathrm{ft}^{3} / \mathrm{s}\right)\end{array}$} \\
\hline & & & 3-day period & 7-day period & 15-day period & 30-day period \\
\hline 0.99 & 1.01 & 11.7 & 15.4 & 10.9 & 8.45 & 5.87 \\
\hline 0.95 & 1.05 & 32.2 & 43.4 & 32.3 & 23.9 & 16.4 \\
\hline 0.90 & 1.11 & 52.8 & 71.1 & 54.2 & 39.6 & 27.1 \\
\hline 0.80 & 1.25 & 92.8 & 122 & 95.8 & 69.4 & 47.3 \\
\hline 0.50 & 2 & 244 & 296 & 242 & 177 & 121 \\
\hline 0.20 & 5 & 556 & 590 & 502 & 380 & 261 \\
\hline 0.10 & 10 & 811 & 789 & 682 & 532 & 368 \\
\hline 0.04 & 25 & 1,170 & 1,030 & 900 & 727 & 507 \\
\hline 0.02 & 50 & 1,450 & 1,190 & 1,050 & 870 & 610 \\
\hline 0.01 & 100 & 1,740 & 1,330 & 1,190 & 1,010 & 711 \\
\hline 0.005 & 200 & 2,030 & 1,460 & 1,310 & 1,140 & 808 \\
\hline 0.002 & 500 & 2,410 & ng & ng & $\mathrm{ng}$ & ng \\
\hline
\end{tabular}

Probability of occurrence of annual low discharges, post-regulation period

\begin{tabular}{|c|c|c|c|c|c|c|c|c|c|c|}
\hline \multirow[b]{3}{*}{$\begin{array}{l}\text { Non- } \\
\text { exceed- } \\
\text { ance } \\
\text { prob- } \\
\text { ability }\end{array}$} & \multirow[b]{3}{*}{$\begin{array}{l}\text { Recur- } \\
\text { rence } \\
\text { inter- } \\
\text { vai } \\
\text { (years) }\end{array}$} & \multicolumn{9}{|c|}{ Minimum mean discharge $\left(\mathrm{ft}^{3} / \mathrm{s}\right)$} \\
\hline & & \multicolumn{9}{|c|}{ Number of consecutive days } \\
\hline & & 1 & 3 & 7 & 14 & 30 & 60 & 90 & 120 & 183 \\
\hline 0.05 & 20 & 0.037 & 0.056 & 0.065 & 0.076 & 0.151 & 0.336 & 0.484 & 0.633 & 1.18 \\
\hline 0.10 & 10 & 0.063 & 0.088 & 0.102 & 0.126 & 0.231 & 0.467 & 0.673 & 0.942 & 1.58 \\
\hline 0.20 & 5 & 0.114 & 0.149 & 0.172 & 0.226 & 0.373 & 0.683 & 0.980 & 1.44 & 2.20 \\
\hline 0.50 & 2 & 0.331 & 0.392 & 0.448 & 0.611 & 0.855 & 1.33 & 1.88 & 2.72 & 3.87 \\
\hline
\end{tabular}




\section{TONGUE RIVER AT AKRA, ND--Continued}

Probability of occurrence of seasonal low discharges, post-regulation period

\begin{tabular}{|c|c|c|c|c|c|c|c|c|c|}
\hline \multirow[b]{3}{*}{$\begin{array}{c}\text { Non- } \\
\text { exceedance } \\
\text { probability }\end{array}$} & \multirow[b]{3}{*}{$\begin{array}{c}\text { Recurrence } \\
\text { interval } \\
\text { (years) }\end{array}$} & \multicolumn{8}{|c|}{ Minimum mean discharge $\left(\mathrm{ft}^{3} / \mathrm{s}\right)$} \\
\hline & & \multicolumn{8}{|c|}{ Number of consecutive days } \\
\hline & & 1 & 7 & 14 & 30 & 1 & 7 & 14 & 30 \\
\hline & & \multicolumn{4}{|c|}{ December-January-February } & \multicolumn{4}{|c|}{ March-April-May } \\
\hline 0.05 & 20 & 0.396 & 0.414 & 0.509 & 0.704 & 0.037 & 0.064 & 0.103 & 0.423 \\
\hline 0.10 & 10 & 0.555 & 0.579 & 0.667 & 0.906 & 0.087 & 0.139 & 0.225 & 0.834 \\
\hline 0.20 & 5 & 0.819 & 0.852 & 0.950 & 1.22 & 0.229 & 0.335 & 0.543 & 1.79 \\
\hline \multirow[t]{2}{*}{0.50} & 2 & 1.63 & 1.69 & 1.77 & 2.10 & 1.11 & 1.48 & 2.44 & 6.52 \\
\hline & & \multicolumn{4}{|c|}{ June-July-August } & \multicolumn{4}{|c|}{ September-October-November } \\
\hline 0.05 & 20 & 0.047 & 0.067 & 0.094 & 0.207 & 0.067 & 0.142 & 0.174 & 0.270 \\
\hline 0.10 & 10 & 0.096 & 0.137 & 0.188 & 0.378 & 0.123 & 0.218 & 0.264 & 0.424 \\
\hline 0.20 & 5 & 0.209 & 0.296 & 0.400 & 0.738 & 0.243 & 0.368 & 0.440 & 0.728 \\
\hline 0.50 & 2 & 0.720 & 1.00 & 1.36 & 2.24 & 0.775 & 1.01 & 1.20 & 2.01 \\
\hline
\end{tabular}




\section{TONGUE RIVER AT AKRA, ND--Continued}

Annual peak discharge and corresponding gage height

$$
[--, \text { no data }]
$$

\begin{tabular}{|c|c|c|c|c|c|c|c|}
\hline $\begin{array}{l}\text { Water } \\
\text { year }\end{array}$ & Date & $\begin{array}{c}\text { Gage } \\
\text { height } \\
\text { (feet) }\end{array}$ & $\begin{array}{c}\text { Peak } \\
\text { discharge } \\
\left(\mathrm{ft}^{3} / \mathrm{s}\right)\end{array}$ & $\begin{array}{l}\text { Water } \\
\text { year }\end{array}$ & Date & $\begin{array}{c}\text { Gage } \\
\text { height } \\
\text { (feet) }\end{array}$ & $\begin{array}{c}\text { Peak } \\
\text { discharge } \\
\left(\mathrm{ft}^{3} / \mathrm{s}\right)\end{array}$ \\
\hline \multicolumn{8}{|c|}{ Annual peak discharge, by year, and corresponding gage height } \\
\hline 1950 & April 18 & 48.70 & 11,800 & 1973 & March 24 & 9.85 & 118 \\
\hline 1952 & April 1 & 38.48 & 260 & 1974 & April 22 & 15.61 & 595 \\
\hline 1953 & May 31 & 35.86 & 178 & 1975 & April 25 & 10.38 & 76.0 \\
\hline 1954 & June 12 & 35.53 & 187 & 1976 & April 9 & 13.31 & 313 \\
\hline 1955 & April 2 & 13.23 & 700 & 1977 & July 18 & 10.40 & 64.0 \\
\hline 1956 & April 19 & 14.23 & 1,350 & 1978 & April 10 & 14.28 & 429 \\
\hline 1957 & March 22 & 6.12 & 340 & 1979 & April 22 & 16.75 & 900 \\
\hline 1958 & July 5 & 2.38 & 78.0 & 1980 & April 6 & 11.86 & 180 \\
\hline 1959 & April 5 & 9.04 & 485 & 1981 & March 25 & 10.73 & 76.0 \\
\hline 1960 & April 14 & 8.67 & 654 & 1982 & April 14 & 12.95 & 308 \\
\hline 1961 & March 27 & 4.56 & 60.0 & 1983 & April 8 & 12.89 & 354 \\
\hline 1962 & April 23 & 7.03 & 473 & 1984 & April 14 & 12.89 & 33.0 \\
\hline 1963 & June 12 & 4.57 & 210 & 1985 & March 20 & 12.13 & 243 \\
\hline 1964 & June 20 & 5.38 & 286 & 1986 & March 25 & 12.35 & 275 \\
\hline 1965 & April 14 & 8.10 & 580 & 1987 & April 8 & 13.79 & 480 \\
\hline 1966 & April 2 & 7.35 & 492 & 1988 & April 6 & 8.71 & 38.0 \\
\hline 1967 & May 9 & 6.28 & 412 & 1989 & April 24 & 9.48 & 49.0 \\
\hline 1968 & March 27 & 5.55 & 160 & 1990 & April 24 & 8.82 & 15.0 \\
\hline 1969 & April 14 & 7.85 & 606 & 1991 & June 19 & 9.45 & 35.0 \\
\hline 1970 & April 29 & 7.99 & 567 & 1992 & March 9 & 10.77 & 80.0 \\
\hline 1971 & April 12 & 7.75 & 568 & 1993 & July 29 & 14.11 & 492 \\
\hline 1972 & April 16 & 5.79 & 325 & 1994 & April 12 & 14.11 & 138 \\
\hline \multicolumn{8}{|c|}{ Annual peak discharge, from highest to lowest, and corresponding gage height } \\
\hline 1950 & April 18 & 48.70 & 11,800 & 1976 & April 9 & 13.31 & 313 \\
\hline 1956 & April 19 & 14.23 & 1,350 & 1982 & April 14 & 12.95 & 308 \\
\hline 1979 & April 22 & 16.75 & 900 & 1964 & June 20 & 5.38 & 286 \\
\hline 1955 & April 2 & 13.23 & 700 & 1986 & March 25 & 12.35 & 275 \\
\hline 1960 & April 14 & 8.67 & 654 & 1952 & April 1 & 38.48 & 260 \\
\hline 1969 & April 14 & 7.85 & 606 & 1985 & March 20 & 12.13 & 243 \\
\hline 1974 & April 22 & 15.61 & 595 & 1963 & June 12 & 4.57 & 210 \\
\hline 1965 & April 14 & 8.10 & 580 & 1954 & June 12 & 35.53 & 187 \\
\hline 1971 & April 12 & 7.75 & 568 & 1980 & April 6 & 11.86 & 180 \\
\hline 1970 & April 29 & 7.99 & 567 & 1953 & May 31 & 35.86 & 178 \\
\hline 1966 & April 2 & 7.35 & 492 & 1968 & March 27 & 5.55 & 160 \\
\hline 1993 & July 29 & 14.11 & 492 & 1994 & April 12 & 14.11 & 138 \\
\hline 1959 & April 5 & 9.04 & 485 & 1973 & March 24 & 9.85 & 118 \\
\hline 1987 & April 8 & 13.79 & 480 & 1992 & March 9 & 10.77 & 80.0 \\
\hline 1962 & April 23 & 7.03 & 473 & 1958 & July 5 & 2.38 & 78.0 \\
\hline 1978 & April 10 & 14.28 & 429 & 1975 & April 25 & 10.38 & 76.0 \\
\hline 1967 & May 9 & 6.28 & 412 & 1981 & March 25 & 10.73 & 76.0 \\
\hline 1983 & April 8 & 12.89 & 354 & 1977 & July 18 & 10.40 & 64.0 \\
\hline 1957 & March 22 & 6.12 & 340 & 1961 & March 27 & 4.56 & 60.0 \\
\hline 1972 & April 16 & 5.79 & 325 & 1989 & April 24 & 9.48 & 49.0 \\
\hline
\end{tabular}




\section{TONGUE RIVER AT AKRA, ND-Continued}

Annual peak discharge and corresponding gage height--Continued

$[--$, no data $]$

\begin{tabular}{|c|c|c|c|c|c|c|c|}
\hline $\begin{array}{l}\text { Water } \\
\text { year }\end{array}$ & Date & $\begin{array}{c}\text { Gage } \\
\text { height } \\
\text { (feet) }\end{array}$ & $\begin{array}{c}\text { Peak } \\
\text { discharge } \\
\left(\mathrm{ft}^{3} / \mathrm{s}\right)\end{array}$ & $\begin{array}{c}\text { Water } \\
\text { year }\end{array}$ & Date & $\begin{array}{l}\text { Gage } \\
\text { height } \\
\text { (feet) }\end{array}$ & $\begin{array}{c}\text { Peak } \\
\text { discharge } \\
\left(\mathrm{ft}^{3} / \mathrm{s}\right)\end{array}$ \\
\hline \multicolumn{8}{|c|}{ Annual peak discharge, from highest to lowest, and corresponding gage height-Continued } \\
\hline 1988 & April 6 & 8.71 & 38.0 & 1984 & April 14 & 12.89 & 33.0 \\
\hline 1991 & June 19 & 9.45 & 35.0 & 1990 & April 24 & 8.82 & 15.0 \\
\hline
\end{tabular}


i

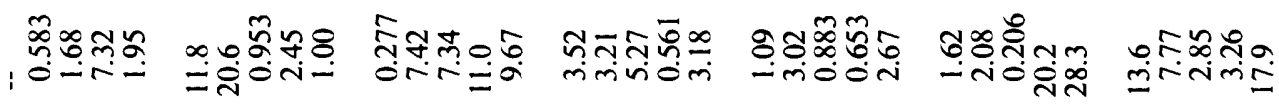

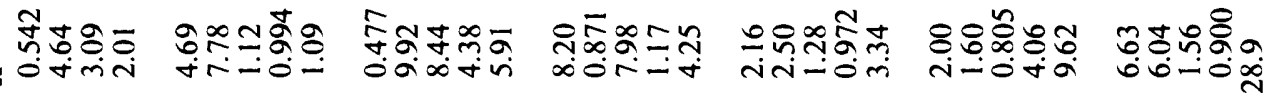

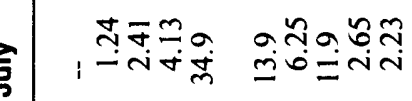

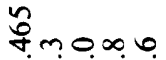

$+\operatorname{tg} 8 \because$

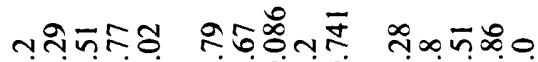
-

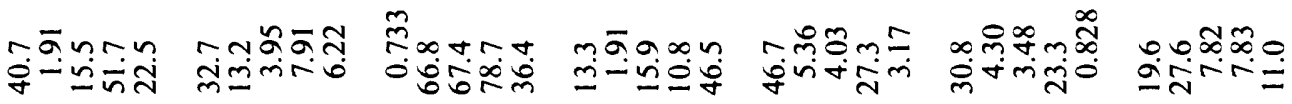

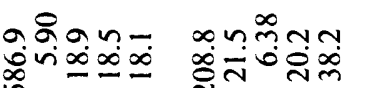

nักติ๐-

西

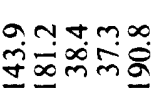

$\infty 0 \tilde{n} \infty 0$

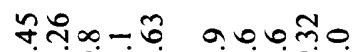
吕- 穴 Nm

$\infty$ mo

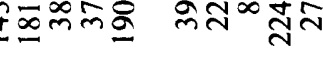

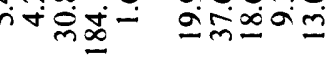

anton nuboo

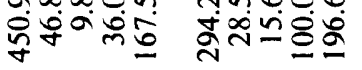

กิด

$-0 m+0$

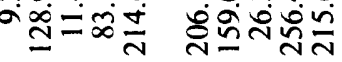

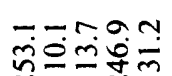

mô̆- man-0

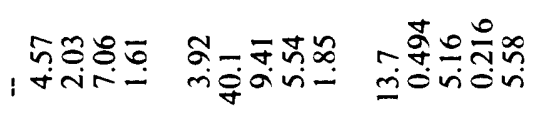

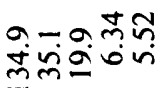

$\infty a 0+\hat{0}$

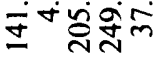

รล์ ลส่น

:

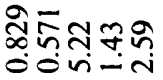

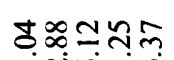

=าณกิ

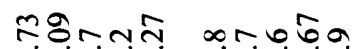

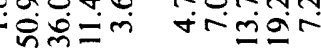

๙ับำำ

กั้กิก

$q \infty \bar{N}=\mathbb{T}$

กํํㅎㅇ

๓ะบษ์

กิำํิต

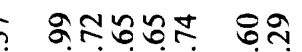

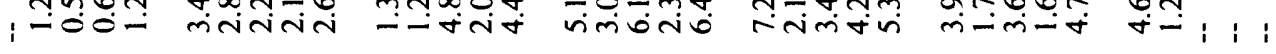

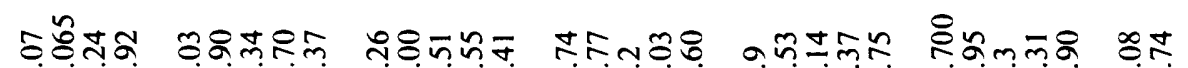
i $\cos -1$ जivini-im

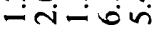

arogir inimito $\dot{0-0}-\dot{0}$ ज่ं i i

চ is

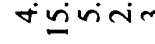
minging

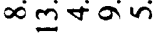

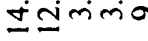

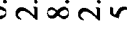

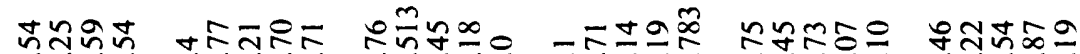

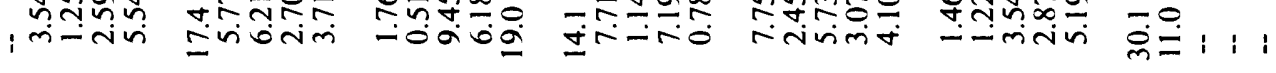




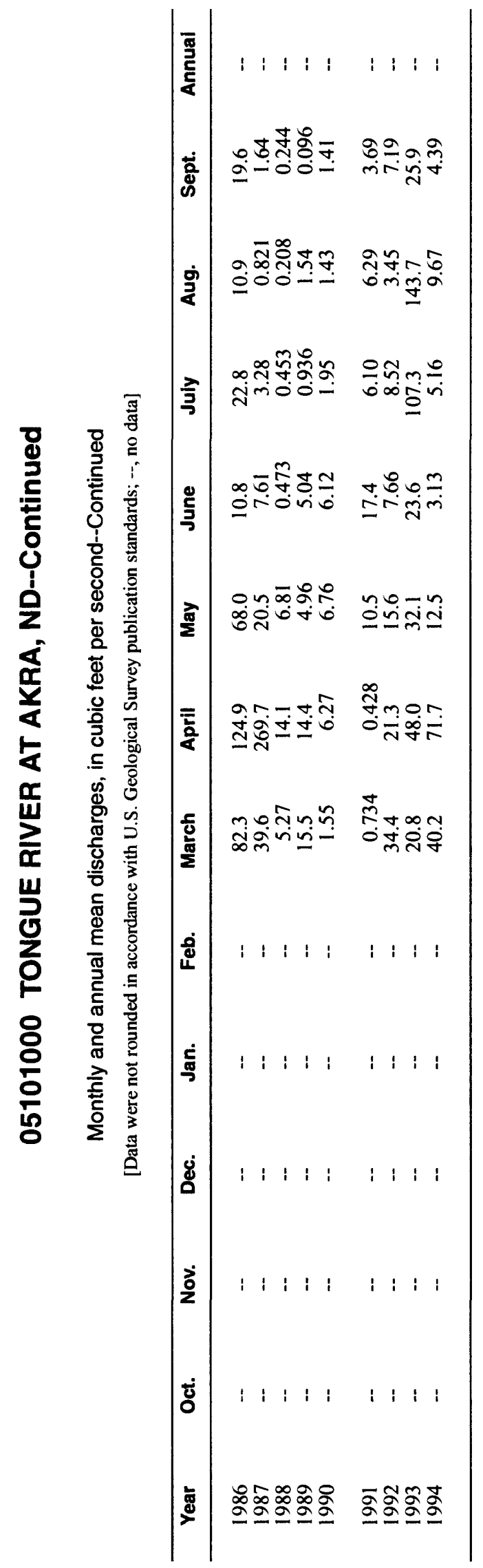




\section{TONGUE RIVER AT CAVALIER, ND}

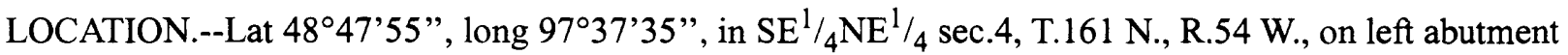
of dam $0.5 \mathrm{mi}$ upstream from State Highway 5 in Cavalier.

DRAINAGE AREA.-- $167 \mathrm{mi}^{2}$.

PERIOD OF RECORD.--October 1938 to October 1951.

GAGE.--Staff gage and concrete control. Datum of gage is $880.98 \mathrm{ft}$ above mean sea level, datum of 1929, Emerson-Crookston supplementary adjustment of 1941. Prior to July 21, 1946, wire-weight or chain gage at site $0.5 \mathrm{mi}$ downstream at datum $11.74 \mathrm{ft}$ lower.

EXTREMES FOR PERIOD OF RECORD.--Maximum discharge (not including bypass flow), $1,340 \mathrm{ft}^{3} / \mathrm{s}$, May 8, 1950, gage height, $4.58 \mathrm{ft}$; no flow for several months in some years. During the floods of April and May 1950 and probably during flood of April 1948, large amounts of water bypassed the gage and are not included in the records. Maximum discharge including bypass flow, $11,800 \mathrm{ft}^{3} / \mathrm{s}$, Apr. 18, 1950, on basis of contracted-opening measurement of peak flow at site about 5 mi upstream.

Annual mean discharge

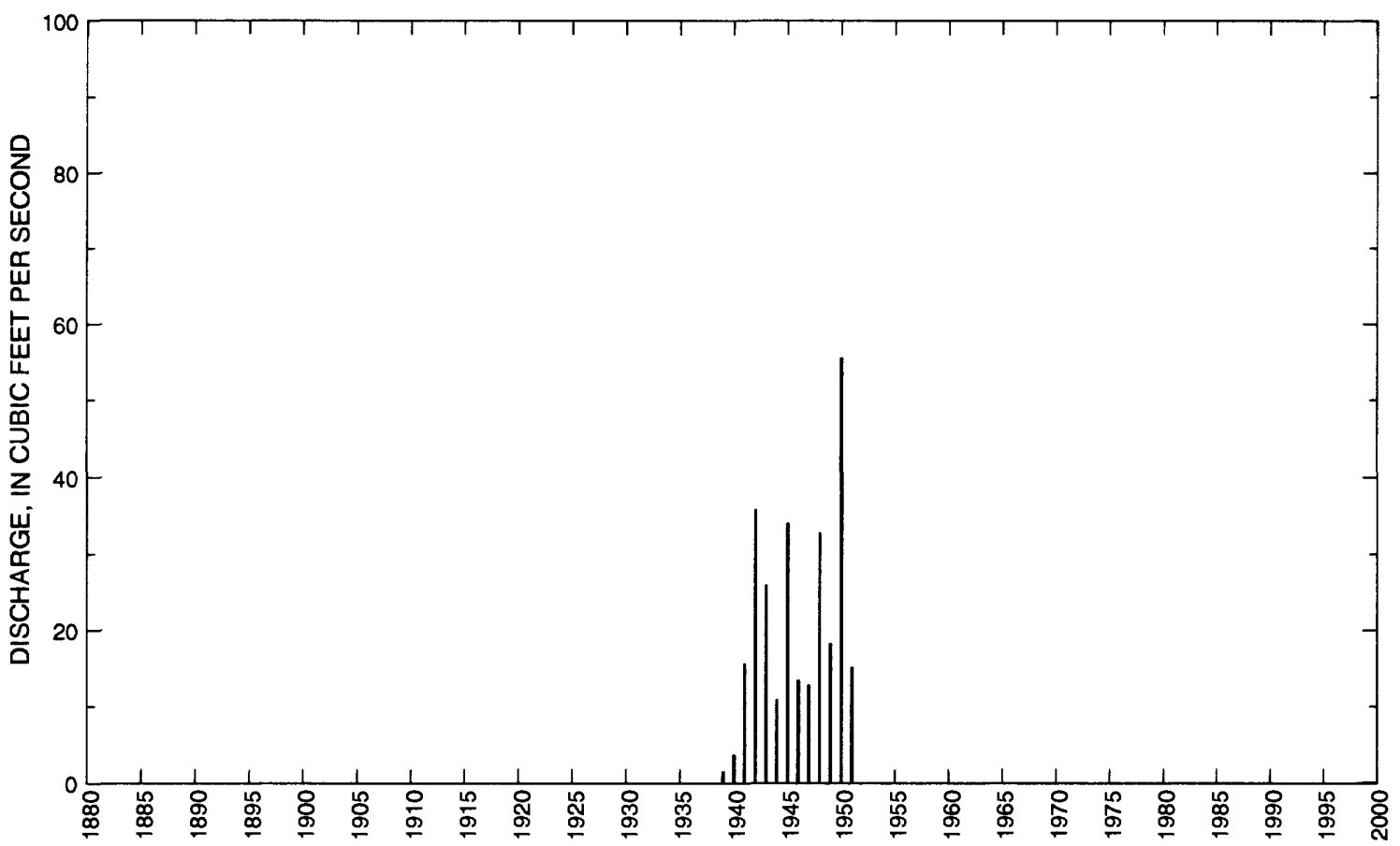




\section{TONGUE RIVER AT CAVALIER ND--Continued}

Statistics of monthly and annual mean discharges

[m, more than 1 year of occurrence]

\begin{tabular}{|c|c|c|c|c|c|c|c|c|}
\hline \multirow[b]{2}{*}{ Month } & \multicolumn{2}{|c|}{ Maximum } & \multicolumn{2}{|c|}{ Minimum } & \multicolumn{4}{|c|}{ Mean } \\
\hline & $\begin{array}{c}\text { Discharge } \\
\left(\mathrm{ft}^{3} / \mathrm{s}\right)\end{array}$ & $\begin{array}{c}\text { Water year } \\
\text { of } \\
\text { occurrence }\end{array}$ & $\begin{array}{c}\text { Discharge } \\
\left(\mathrm{ft}^{3} / \mathrm{s}\right)\end{array}$ & $\begin{array}{c}\text { Water year } \\
\text { of } \\
\text { occurrence }\end{array}$ & $\begin{array}{c}\text { Discharge } \\
\left(\mathrm{ft}^{3} / \mathrm{s}\right)\end{array}$ & $\begin{array}{c}\text { Standard } \\
\text { deviation } \\
\left(\mathrm{ft}^{3} / \mathbf{s}\right)\end{array}$ & $\begin{array}{l}\text { Coeffi- } \\
\text { cient of } \\
\text { variation }\end{array}$ & $\begin{array}{l}\text { Percentage } \\
\text { of annuai } \\
\text { discharge }\end{array}$ \\
\hline October & 14.3 & 1942 & 0 & $\mathrm{~m}$ & 3.62 & 3.76 & 1.04 & 1.42 \\
\hline November & 45.3 & 1945 & 0 & $\mathrm{~m}$ & 5.90 & 12.0 & 2.03 & 2.31 \\
\hline December & 10.0 & 1945 & 0 & $\mathrm{~m}$ & 1.98 & 2.64 & 1.33 & 0.78 \\
\hline January & 3.65 & 1945 & 0 & $\mathrm{~m}$ & 0.970 & 1.10 & 1.13 & 0.38 \\
\hline February & 2.83 & 1945 & 0 & $\mathrm{~m}$ & 0.510 & 0.81 & 1.57 & 0.20 \\
\hline March & 184 & 1945 & 0 & $\mathrm{~m}$ & 30.2 & 54.4 & 1.80 & 11.8 \\
\hline April & 273 & 1948 & 14.2 & 1939 & 117 & 85.2 & 0.73 & 45.9 \\
\hline May & 406 & 1950 & 2.87 & 1939 & 53.7 & 108 & 2.00 & 21.0 \\
\hline June & 98.2 & 1943 & 1.24 & 1940 & 22.8 & 26.3 & 1.15 & 8.92 \\
\hline July & 30.9 & 1943 & 0.087 & 1939 & 8.57 & 9.41 & 1.10 & 3.35 \\
\hline August & 20.9 & 1942 & 0 & 1939 & 4.39 & 5.82 & 1.33 & 1.72 \\
\hline September & 23.5 & 1944 & 0 & 1939 & 5.58 & 7.41 & 1.33 & 2.19 \\
\hline Annual & 55.6 & 1950 & 1.57 & 1939 & 21.3 & 15.1 & 0.71 & 100 \\
\hline
\end{tabular}

Annual flow duration

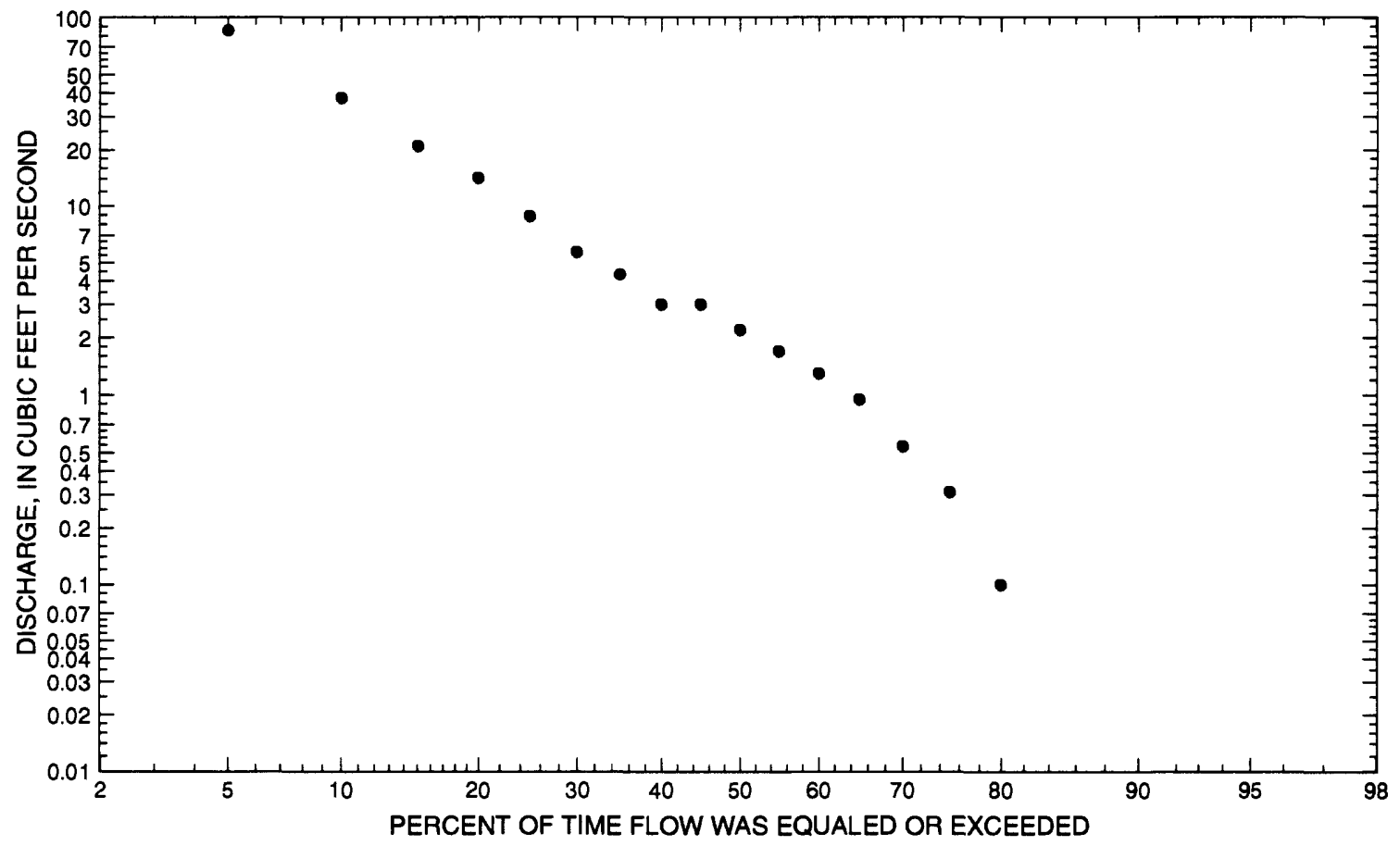




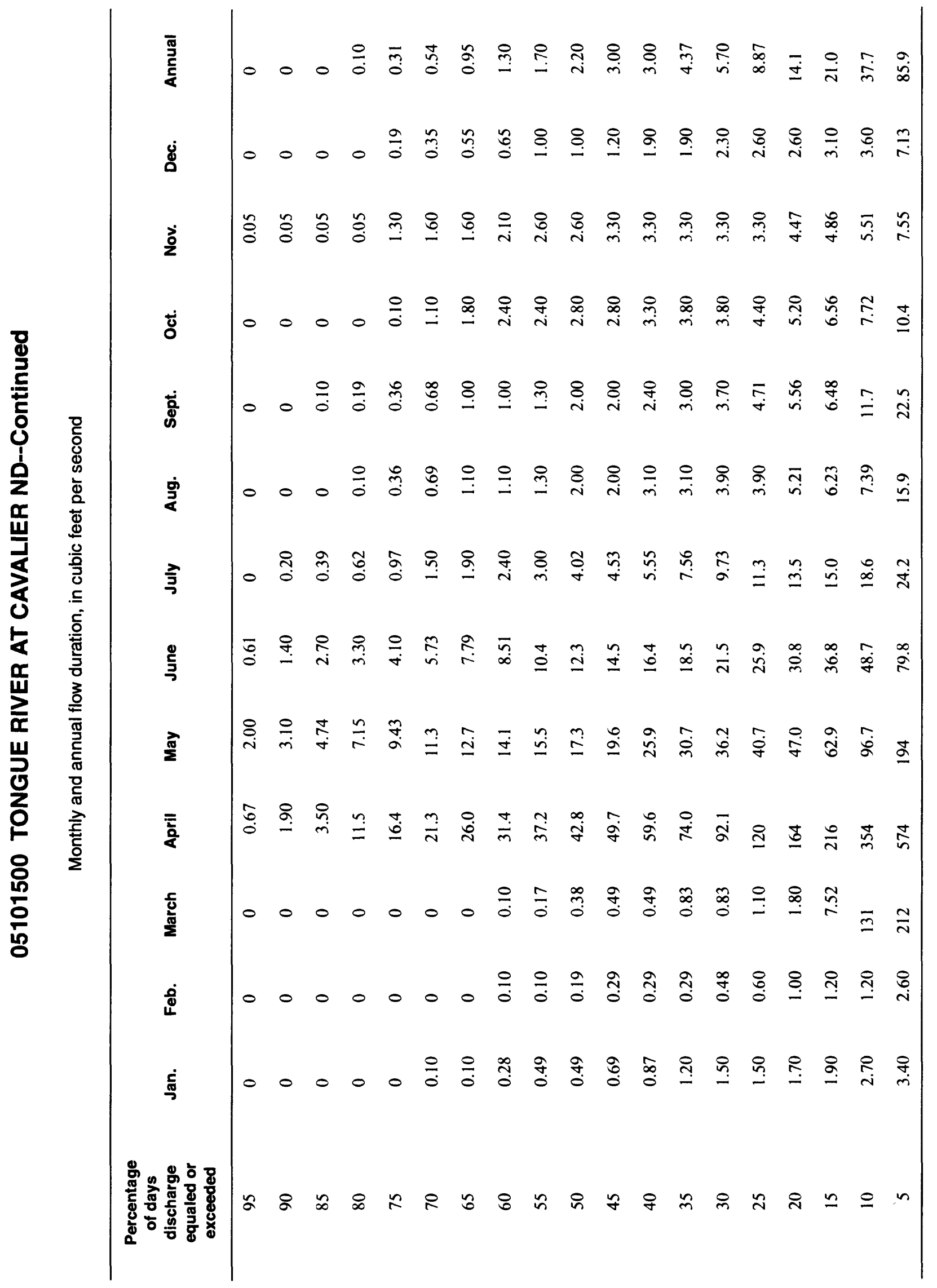




\section{TONGUE RIVER AT CAVALIER ND--Continued}

Probability of occurrence of annual high discharges

[ng, statistic not given]

\begin{tabular}{ccccccc}
\hline & & & \multicolumn{5}{c}{$\begin{array}{c}\text { Maximum mean discharge } \\
\left(\mathbf{f t}^{3} / \mathbf{s}\right)\end{array}$} \\
\cline { 5 - 7 } $\begin{array}{c}\text { Exceedance } \\
\text { probability }\end{array}$ & $\begin{array}{c}\text { Recurrence } \\
\text { interval } \\
\text { (years) }\end{array}$ & $\begin{array}{c}\text { Maximum } \\
\text { instantaneous } \\
\left(\mathbf{f t}^{3} / \mathbf{s}\right)\end{array}$ & 3-day period & 7-day period & 15-day period & 30-day period \\
\hline 0.99 & 1.01 & $\mathrm{ng}$ & 13.9 & 12.0 & 7.39 & 7.75 \\
0.95 & 1.05 & $\mathrm{ng}$ & 58.6 & 44.1 & 28.9 & 21.9 \\
0.90 & 1.11 & 327 & 110 & 79.2 & 53.2 & 35.8 \\
0.80 & 1.25 & 436 & 208 & 146 & 100 & 61.1 \\
0.50 & 2 & 720 & 500 & 353 & 251 & 144 \\
0.20 & 5 & 1,120 & 813 & 617 & 446 & 275 \\
0.10 & 10 & 1,370 & 933 & 742 & 538 & 359 \\
0.04 & 25 & 1,680 & 1,020 & 848 & 616 & 454 \\
0.02 & 50 & 1,900 & 1,050 & 900 & 653 & 514 \\
0.01 & 100 & 2,110 & 1,070 & 935 & 678 & 567 \\
0.005 & 200 & 2,300 & 1,080 & 958 & 695 & 613 \\
0.002 & 500 & 2,550 & $\mathrm{ng}$ & $\mathrm{ng}$ & $\mathrm{ng}$ & $\mathrm{ng}$ \\
\hline
\end{tabular}

Probability of occurrence of annual low discharges

Minimum mean discharge $\left(\mathrm{ft}^{3} / \mathrm{s}\right)$

Number of consecutive days

\begin{tabular}{ccccccccccc}
$\begin{array}{c}\text { Non- } \\
\text { exceed- } \\
\text { ance } \\
\text { prob- } \\
\text { ability }\end{array}$ & $\begin{array}{c}\text { Recur- } \\
\text { rence } \\
\text { inter- } \\
\text { val } \\
\text { (years) }\end{array}$ & $\mathbf{1}$ & $\mathbf{3}$ & $\mathbf{7}$ & $\mathbf{1 4}$ & $\mathbf{3 0}$ & $\mathbf{6 0}$ & $\mathbf{9 0}$ & $\mathbf{1 2 0}$ & $\mathbf{1 8 3}$ \\
\hline 0.05 & 20 & 0 & 0 & 0 & 0 & 0 & 0 & 0 & 0 & 0 \\
0.10 & 10 & 0 & 0 & 0 & 0 & 0 & 0 & 0 & 0 & 0 \\
0.20 & 5 & 0 & 0 & 0 & 0 & 0 & 0 & 0.014 & 0.229 & 0.750 \\
0.50 & 2 & 0 & 0 & 0 & 0.121 & 0.145 & 0.310 & 0.497 & 0.699 & 1.77 \\
\hline
\end{tabular}




\section{TONGUE RIVER AT CAVALIER ND--Continued}

Probability of occurrence of seasonal low discharges

\begin{tabular}{|c|c|c|c|c|c|c|c|c|c|}
\hline \multirow[b]{3}{*}{$\begin{array}{c}\text { Non- } \\
\text { exceedance } \\
\text { probabllity }\end{array}$} & \multirow[b]{3}{*}{$\begin{array}{l}\text { Recurrence } \\
\text { interval } \\
\text { (years) }\end{array}$} & \multicolumn{8}{|c|}{ Minimum mean discharge $\left(\mathrm{ft}^{3} / \mathrm{s}\right)$} \\
\hline & & \multicolumn{8}{|c|}{ Number of consecutive days } \\
\hline & & 1 & 7 & 14 & 30 & 1 & 7 & 14 & 30 \\
\hline & & \multicolumn{4}{|c|}{ December-January-February } & \multicolumn{4}{|c|}{ March-April-May } \\
\hline 0.05 & 20 & 0 & 0 & 0 & 0 & 0 & 0 & 0 & 0 \\
\hline 0.10 & 10 & 0 & 0 & 0 & 0 & 0 & 0 & 0 & 0 \\
\hline 0.20 & 5 & 0 & 0 & 0 & 0 & 0 & 0 & 0 & 0 \\
\hline \multirow[t]{2}{*}{0.50} & 2 & 0.170 & 0.179 & 0.183 & 0.226 & 0.179 & 0.183 & ${ }^{1} 0.800$ & 1.43 \\
\hline & & \multicolumn{4}{|c|}{ June-July-August } & \multicolumn{4}{|c|}{ September-October-November } \\
\hline 0.05 & 20 & 0 & 0 & 0 & 0 & 0 & 0 & 0 & 0 \\
\hline 0.10 & 10 & 0 & 0 & 0 & 0.028 & 0 & 0 & 0 & 0 \\
\hline 0.20 & 5 & 0 & 0 & 0 & 0.233 & 0.055 & 0.090 & 0.119 & 0.260 \\
\hline 0.50 & 2 & 0.659 & 1.04 & 1.31 & 1.65 & 0.269 & 0.702 & 1.28 & 2.24 \\
\hline
\end{tabular}

${ }^{\mathrm{I}}$ Graphical interpretation. 


\section{TONGUE RIVER AT CAVALIER ND--Continued}

Annual peak discharge and corresponding gage height

$$
[--, \text { no data }]
$$

\begin{tabular}{|c|c|c|c|c|c|c|c|}
\hline $\begin{array}{l}\text { Water } \\
\text { year }\end{array}$ & Date & $\begin{array}{c}\text { Gage } \\
\text { height } \\
\text { (feet) }\end{array}$ & $\begin{array}{c}\text { Peak } \\
\text { discharge } \\
\left(\mathrm{ft}^{3} / \mathrm{s}\right)\end{array}$ & $\begin{array}{l}\text { Water } \\
\text { year }\end{array}$ & Date & $\begin{array}{c}\text { Gage } \\
\text { height } \\
\text { (feet) }\end{array}$ & $\begin{array}{c}\text { Peak } \\
\text { discharge } \\
\left(\mathrm{ft}^{3} / \mathrm{s}\right)\end{array}$ \\
\hline \multicolumn{8}{|c|}{ Annual peak discharge, by year, and corresponding gage height } \\
\hline 1939 & April I & 4.10 & 35.0 & 1945 & March 27 & -- & 934 \\
\hline 1940 & April 20 & 6.77 & 288 & 1946 & March 22 & 10.02 & 700 \\
\hline 1941 & April 11 & 12.26 & 1,080 & 1948 & April 21 & 4.38 & 1,300 \\
\hline 1942 & April 14 & -- & 1,080 & 1949 & April 10 & -- & 990 \\
\hline 1943 & March 27 & -- & 500 & 1950 & May 8 & 4.58 & 1,340 \\
\hline 1944 & April 12 & -- & 450 & 1951 & April 5 & 2.60 & 420 \\
\hline \multicolumn{8}{|c|}{ Annual peak discharge, from highest to lowest, and corresponding gage height } \\
\hline 1950 & May 8 & 4.58 & 1,340 & 1946 & March 22 & 10.02 & 700 \\
\hline 1948 & April 21 & 4.38 & 1,300 & 1943 & March 27 & -- & 500 \\
\hline 1941 & April 11 & 12.26 & 1,080 & 1944 & April 12 & -- & 450 \\
\hline 1942 & April 14 & - & 1,080 & 1951 & April 5 & 2.60 & 420 \\
\hline 1949 & April 10 & -- & 990 & 1940 & April 20 & 6.77 & 288 \\
\hline 1945 & March 27 & .. & 934 & 1939 & April 1 & 4.10 & 35.0 \\
\hline
\end{tabular}




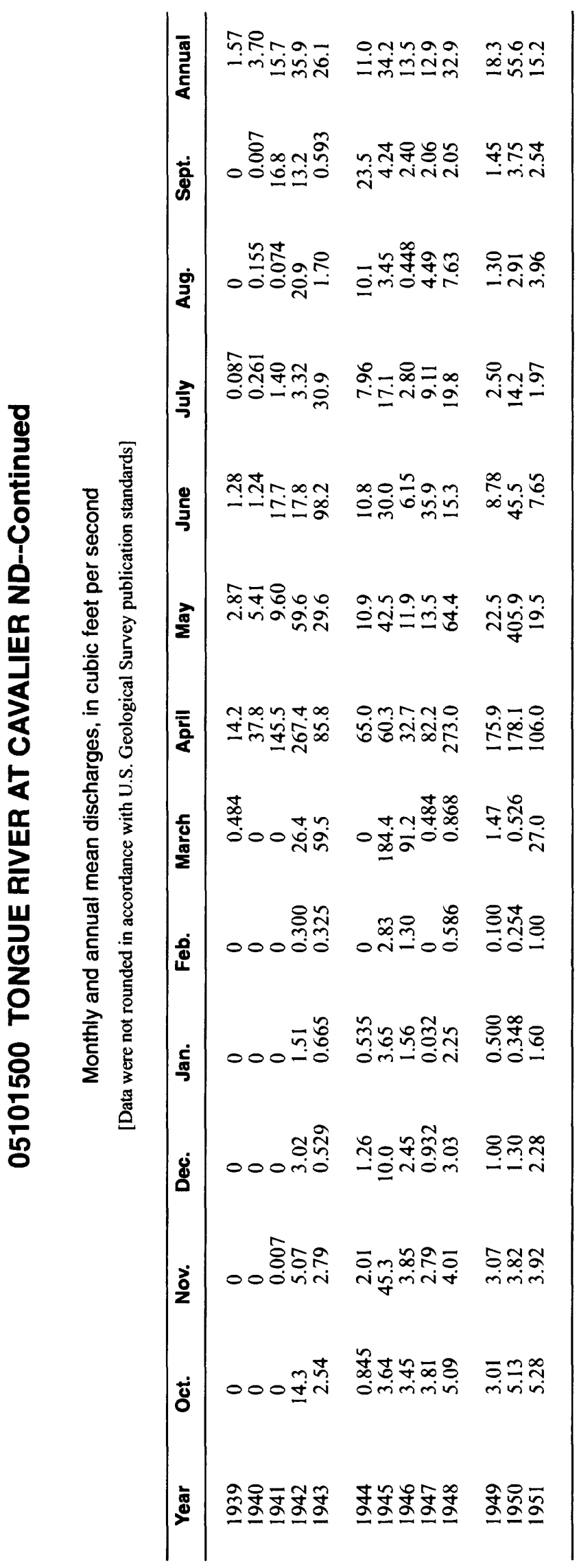




\section{RED RIVER OF THE NORTH AT EMERSON, MB}

LOCATION.--Lat 4900'30", long 97²'40”, in sec.2, T.1, R.2 E., Hydrologic Unit 09020311, on right bank 1,500 ft downstream from Canadian National Railway bridge in Emerson, 0.8 mi downstream from international boundary, $3.6 \mathrm{mi}$ downstream from Pembina River, and at mile 154.3.

DRAINAGE AREA.--40,200 $\mathrm{mi}^{2}$, approximately, includes $3,800 \mathrm{mi}^{2}$ in closed basins.

PERIOD OF RECORD.--March to November 1902 (gage heights only), May 1912 to September 1929 (monthly discharge only, published in WSP 1308), October 1929 to current year.

GAGE.--Water-stage recorder. Datum of gage is Geodetic Survey of Canada Datum of 1929. See WSP 1728 or 1913 for history of changes prior to Apr. 10, 1953.

EXTREMES FOR PERIOD OF RECORD.--Maximum discharge, 95,500 $\mathrm{ft}^{3} / \mathrm{s}$, May 13, 1950, gage height, $90.89 \mathrm{ft}$; maximum gage height, $91.19 \mathrm{ft}$, May 1, 1979; minimum daily discharge, $0.9 \mathrm{ft}^{3} / \mathrm{s}$, Feb. 6-8, 1937.

Annual mean discharge

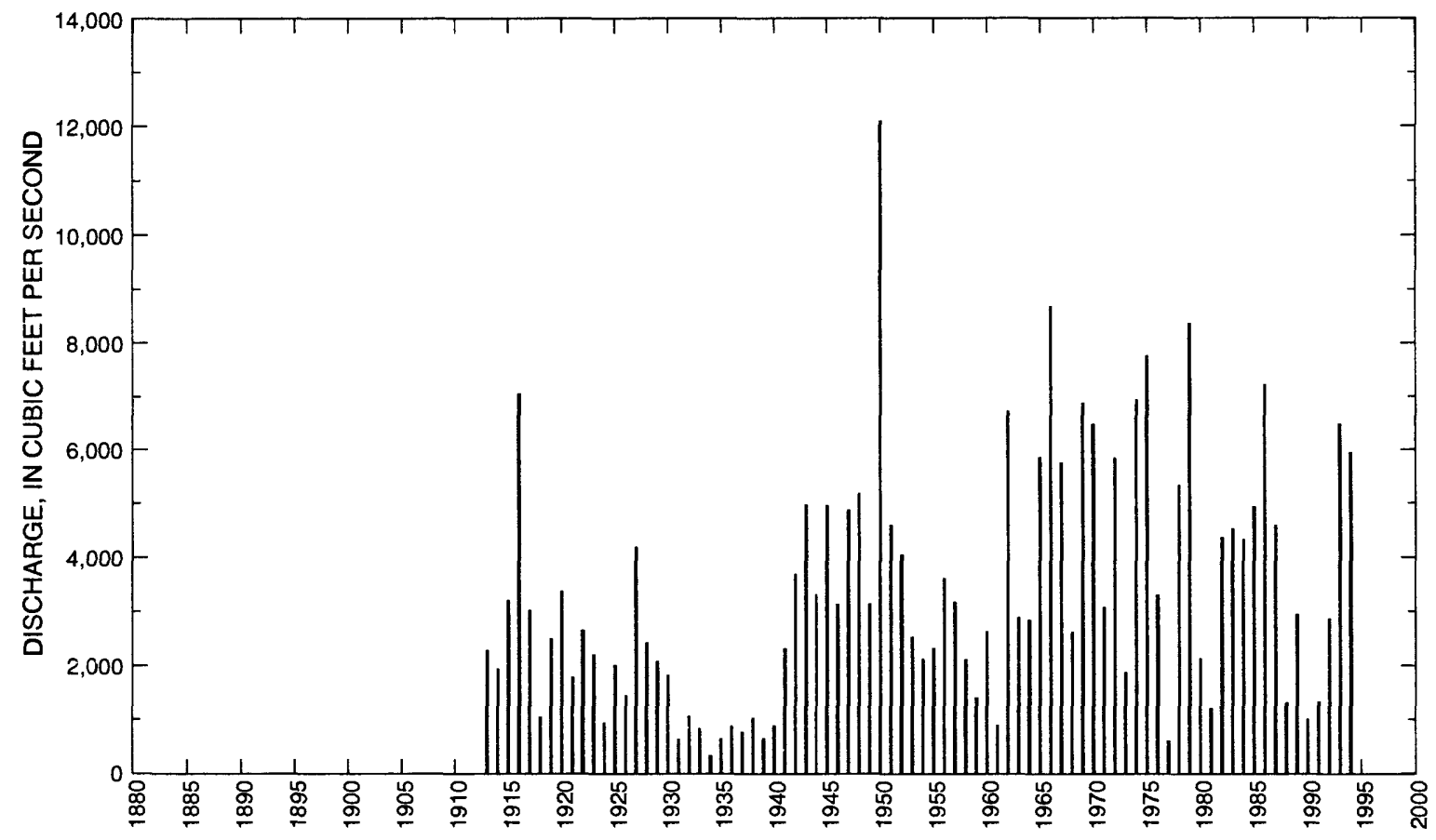




\section{RED RIVER OF THE NORTH AT EMERSON, MB--Continued}

Statistics of monthly and annual mean discharges

\begin{tabular}{|c|c|c|c|c|c|c|c|c|}
\hline \multirow[b]{2}{*}{ Month } & \multicolumn{2}{|c|}{ Maximum } & \multicolumn{2}{|c|}{ Minimum } & \multicolumn{4}{|c|}{ Mean } \\
\hline & $\begin{array}{c}\text { Discharge } \\
\left(\mathrm{ft}^{3} / \mathrm{s}\right)\end{array}$ & $\begin{array}{c}\text { Water year } \\
\text { of } \\
\text { occurrence }\end{array}$ & $\begin{array}{c}\text { Discharge } \\
\left(\mathrm{ft}^{3} / \mathrm{s}\right)\end{array}$ & $\begin{array}{c}\text { Water year } \\
\text { of } \\
\text { occurrence }\end{array}$ & $\begin{array}{c}\text { Discharge } \\
\left(\mathrm{ft}^{3} / \mathrm{s}\right)\end{array}$ & $\begin{array}{c}\text { Standard } \\
\text { deviation } \\
\left(\mathrm{ft}^{3} / \mathbf{s}\right)\end{array}$ & $\begin{array}{l}\text { Coeffi- } \\
\text { cient of } \\
\text { variation }\end{array}$ & $\begin{array}{l}\text { Percentage } \\
\text { of annuai } \\
\text { discharge }\end{array}$ \\
\hline October & 4,530 & 1986 & 28.6 & 1937 & 1,460 & 1,190 & 0.81 & 3.59 \\
\hline November & 5,160 & 1972 & 23.7 & 1937 & 1,290 & 967 & 0.75 & 3.17 \\
\hline December & 2,760 & 1966 & 33.3 & 1937 & 949 & 691 & 0.73 & 2.34 \\
\hline January & 2,050 & 1951 & 7.05 & 1937 & 786 & 564 & 0.72 & 1.94 \\
\hline February & 1,910 & 1952 & 1.21 & 1937 & 749 & 518 & 0.69 & 1.85 \\
\hline March & 9,360 & 1983 & 2.25 & 1937 & 2,060 & 2,130 & 1.03 & 5.06 \\
\hline April & 45,800 & 1966 & 1,280 & 1938 & 12,600 & 9,070 & 0.72 & 30.9 \\
\hline May & 72,800 & 1950 & 663 & 1934 & 8,300 & 11,000 & 1.32 & 20.4 \\
\hline June & 25,400 & 1962 & 196 & 1934 & 4,910 & 4,470 & 0.91 & 12.1 \\
\hline July & 28,000 & 1975 & 121 & 1936 & 3,920 & 4,190 & 1.07 & 9.66 \\
\hline August & 27,000 & 1993 & 46.6 & 1934 & 2,040 & 3,170 & 1.55 & 5.02 \\
\hline September & 10,000 & 1993 & 23.6 & 1934 & 1,610 & 1,620 & 1.00 & 3.97 \\
\hline Annual & 12,100 & 1950 & 333 & 1934 & 3,390 & 2,300 & 0.68 & 100 \\
\hline
\end{tabular}

Annual flow duration

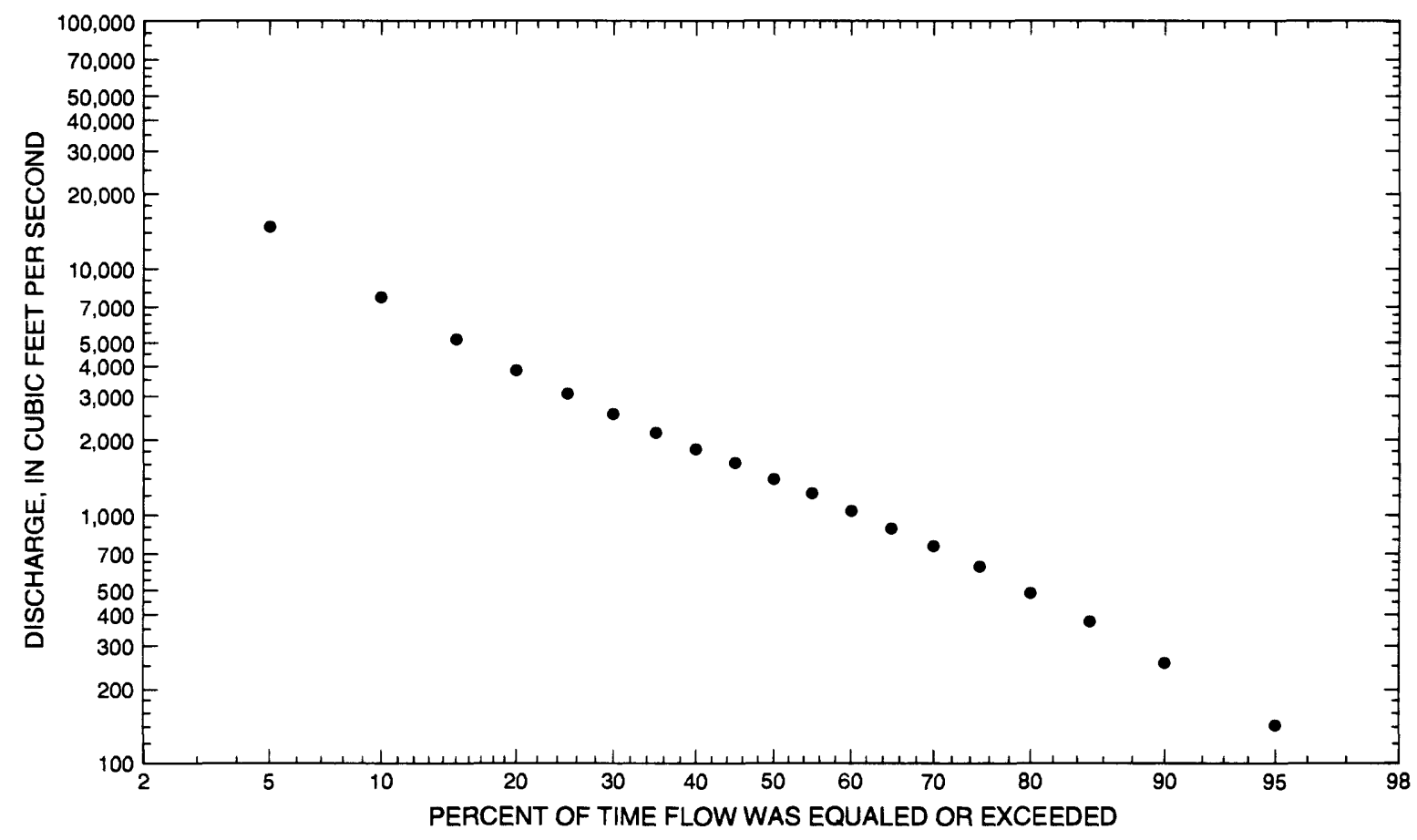




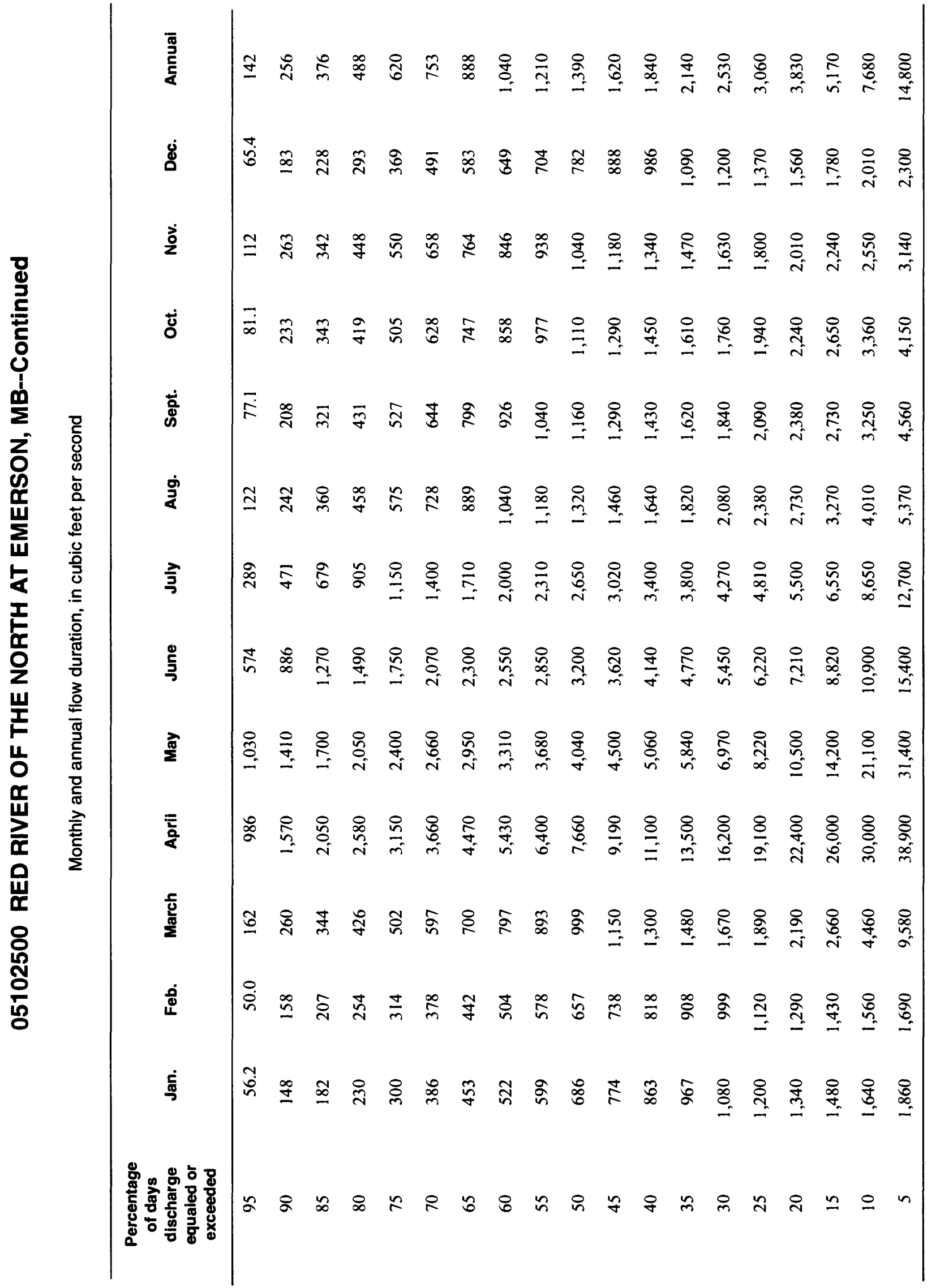




\section{RED RIVER OF THE NORTH AT EMERSON, MB--Continued}

Probability of occurrence of annual high discharges

[ng, statistic not given]

\begin{tabular}{|c|c|c|c|c|c|c|}
\hline \multirow[b]{2}{*}{$\begin{array}{l}\text { Exceedance } \\
\text { probability }\end{array}$} & \multirow[b]{2}{*}{$\begin{array}{l}\text { Recurrence } \\
\text { intervai } \\
\text { (years) }\end{array}$} & \multirow[b]{2}{*}{$\begin{array}{l}\text { Maximum } \\
\text { instantaneous } \\
\left(\mathrm{ft}^{3} / \mathrm{s}\right)\end{array}$} & \multicolumn{4}{|c|}{$\begin{array}{l}\text { Maximum mean discharge } \\
\qquad\left(\mathrm{ft}^{3} / \mathrm{s}\right)\end{array}$} \\
\hline & & & 3-day period & 7-day period & 15-day period & 30-day period \\
\hline 0.99 & 1.01 & 3,040 & 2,890 & 2,630 & 2,150 & 1,670 \\
\hline 0.95 & 1.05 & 5,530 & 5,330 & 4,900 & 4,030 & 3,050 \\
\hline 0.90 & 1.11 & 7,500 & 7,260 & 6,720 & 5,560 & 4,180 \\
\hline 0.80 & 1.25 & 10,700 & 10,400 & 9,710 & 8,110 & 6,090 \\
\hline 0.50 & 2 & 20,100 & 19,700 & 18,800 & 16,100 & 12,300 \\
\hline 0.20 & 5 & 35,900 & 35,400 & 34,300 & 30,700 & 24,200 \\
\hline 0.10 & 10 & 47,500 & 46,900 & 45,900 & 42,100 & 34,200 \\
\hline 0.04 & 25 & 63,200 & 62,400 & 61,700 & 58,400 & 49,200 \\
\hline 0.02 & 50 & 75,300 & 74,400 & 74,000 & 71,600 & 61,900 \\
\hline 0.01 & 100 & 87,700 & 86,600 & 86,700 & 85,700 & 76,000 \\
\hline 0.005 & 200 & 100,000 & 99,000 & 99,700 & 100,000 & 91,500 \\
\hline 0.002 & 500 & 118,000 & ng & ng & ng & ng \\
\hline
\end{tabular}

Probability of occurrence of annual low discharges

\begin{tabular}{|c|c|c|c|c|c|c|c|c|c|c|}
\hline \multirow[b]{3}{*}{$\begin{array}{l}\text { Non- } \\
\text { exceed- } \\
\text { ance } \\
\text { prob- } \\
\text { ability }\end{array}$} & \multirow[b]{3}{*}{$\begin{array}{c}\text { Recur- } \\
\text { rence } \\
\text { inter- } \\
\text { val } \\
\text { (years) }\end{array}$} & \multicolumn{9}{|c|}{ Minimum mean discharge $\left(\mathrm{ft}^{3} / \mathrm{s}\right)$} \\
\hline & & \multicolumn{9}{|c|}{ Number of consecutive days } \\
\hline & & 1 & 3 & 7 & 14 & 30 & 60 & 90 & 120 & 183 \\
\hline 0.05 & 20 & 27.6 & 29.4 & 31.6 & 33.8 & 37.5 & 43.8 & 57.5 & 80.1 & 97.9 \\
\hline 0.10 & 10 & 66.0 & 69.8 & 74.3 & 78.8 & 87.4 & 101 & 118 & 147 & 179 \\
\hline 0.20 & 5 & 159 & 167 & 175 & 185 & 204 & 231 & 248 & 280 & 340 \\
\hline 0.50 & 2 & 520 & 535 & 554 & 582 & 627 & 685 & 696 & 729 & 886 \\
\hline
\end{tabular}




\section{RED RIVER OF THE NORTH AT EMERSON, MB--Continued}

Probability of occurrence of seasonal low discharges

\begin{tabular}{|c|c|c|c|c|c|c|c|c|c|}
\hline \multirow[b]{3}{*}{$\begin{array}{c}\text { Non- } \\
\text { exceedance } \\
\text { probability }\end{array}$} & \multirow[b]{3}{*}{$\begin{array}{c}\text { Recurrence } \\
\text { interval } \\
\text { (years) }\end{array}$} & \multicolumn{8}{|c|}{ Minimum mean discharge $\left(\mathrm{ft}^{3} / \mathrm{s}\right)$} \\
\hline & & \multicolumn{8}{|c|}{ Number of consecutive days } \\
\hline & & 1 & 7 & 14 & 30 & 1 & 7 & 14 & 30 \\
\hline & & \multicolumn{4}{|c|}{ December-January-February } & \multicolumn{4}{|c|}{ March-April-May } \\
\hline 0.05 & 20 & 34.2 & 35.9 & 38.0 & 41.2 & 53.9 & 59.0 & 63.1 & 115 \\
\hline 0.10 & 10 & 82.0 & 85.6 & 89.6 & 96.6 & 126 & 138 & 146 & 261 \\
\hline 0.20 & 5 & 197 & 204 & 211 & 225 & 288 & 314 & 332 & 583 \\
\hline \multirow[t]{2}{*}{0.50} & 2 & 621 & 638 & 653 & 684 & 784 & 839 & 918 & 1,600 \\
\hline & & \multicolumn{4}{|c|}{ June-July-August } & \multicolumn{4}{|c|}{ September-October-November } \\
\hline 0.05 & 20 & 90.9 & 104 & 115 & 146 & 59.2 & 66.1 & 71.9 & 85.8 \\
\hline 0.10 & 10 & 167 & 187 & 204 & 253 & 113 & 126 & 137 & 163 \\
\hline 0.20 & 5 & 324 & 352 & 382 & 462 & 224 & 249 & 273 & 321 \\
\hline 0.50 & 2 & 918 & 968 & 1,050 & 1,240 & 640 & 704 & 773 & 890 \\
\hline
\end{tabular}


05102500 RED RIVER OF THE NORTH AT EMERSON, MB--Continued

Annual peak discharge and corresponding gage height

$[--$, no data]

\begin{tabular}{|c|c|c|c|c|c|c|c|}
\hline $\begin{array}{l}\text { Water } \\
\text { year }\end{array}$ & Date & $\begin{array}{c}\text { Gage } \\
\text { height } \\
\text { (feet) }{ }^{1}\end{array}$ & $\begin{array}{c}\text { Peak } \\
\text { discharge } \\
\left(\mathrm{ft}^{3} / \mathrm{s}\right)\end{array}$ & $\begin{array}{l}\text { Water } \\
\text { year }\end{array}$ & Date & $\begin{array}{l}\text { Gage } \\
\text { height } \\
\text { (feet) }{ }^{1}\end{array}$ & $\begin{array}{c}\text { Peak } \\
\text { discharge } \\
\left(\mathrm{ft}^{3} / \mathrm{s}\right)\end{array}$ \\
\hline \multicolumn{8}{|c|}{ Annual peak discharge, by year, and corresponding gage height } \\
\hline${ }^{2} 1861$ & -- & 95.00 & -- & 1953 & June 28 & 63.70 & 14,500 \\
\hline${ }^{2} 1882$ & -- & 90.00 & -- & 1954 & April 17 & -- & 11,500 \\
\hline${ }^{2} 1897$ & -- & 91.00 & -- & 1955 & April 10 & 72.25 & 24,000 \\
\hline 1913 & April 11 & 74.52 & 25,600 & 1956 & April 27 & 81.02 & 33,800 \\
\hline 1914 & June 19 & 58.36 & 7,260 & 1957 & July 4 & 65.37 & 15,300 \\
\hline 1915 & July 9 & 69.06 & 20,100 & 1958 & July 12 & 57.17 & 7,940 \\
\hline 1916 & April 24 & 85.74 & 46,200 & 1959 & April 10 & -- & 15,700 \\
\hline 1917 & April 12 & -- & 25,900 & 1960 & April 13 & 77.65 & 30,500 \\
\hline 1918 & April 3 & -- & 4,990 & 1961 & March 31 & 57.26 & 4,320 \\
\hline 1919 & July 12 & -- & 13,400 & 1962 & April 25 & 81.93 & 33,400 \\
\hline 1920 & April 16 & -- & 26,700 & 1963 & April 13 & 64.14 & 13,800 \\
\hline 1921 & April 15 & -- & 12,800 & 1964 & June 25 & 66.82 & 17,500 \\
\hline 1922 & April 14 & 69.40 & 18,900 & 1965 & April 26 & 85.19 & 46,200 \\
\hline 1923 & April 25 & 74.98 & 26,000 & 1966 & April 11 & 89.15 & 66,800 \\
\hline 1924 & April 28 & 57.25 & 6,320 & 1967 & April 9 & 80.79 & 33,600 \\
\hline 1925 & June 21 & -- & 17,500 & 1968 & July 24 & 64.12 & 13,900 \\
\hline 1926 & April 1 & 61.02 & 8,000 & 1969 & April 26 & 87.52 & 54,700 \\
\hline 1927 & May 16 & 71.58 & 20,500 & 1970 & April 29 & 84.67 & 39,600 \\
\hline 1928 & April 6 & 67.91 & 16,800 & 1971 & April 16 & -- & 26,600 \\
\hline 1929 & April 1 & -- & 19,200 & 1972 & April 24 & 78.16 & 30,700 \\
\hline 1930 & April 10 & 72.51 & 20,800 & 1973 & March 27 & -- & 14,700 \\
\hline 1931 & April 10 & 59.29 & 7,940 & 1974 & April 28 & 86.51 & 43,500 \\
\hline 1932 & April 15 & 71.64 & 18,900 & 1975 & May 8 & 84.32 & 42,800 \\
\hline 1933 & April 9 & -- & 11,000 & 1976 & April 6 & 79.06 & 32,900 \\
\hline 1934 & April 13 & -- & 4,800 & 1977 & April 10 & 53.75 & 4,590 \\
\hline 1935 & April 3 & 59.65 & 5,470 & 1978 & April 18 & 86.89 & 50,600 \\
\hline 1936 & April 21 & 68.16 & 18,000 & 1979 & May 1 & 91.19 & 92,700 \\
\hline 1937 & May 7 & 56.55 & 5,840 & 1980 & April 10 & 73.54 & 21,700 \\
\hline 1938 & May 20 & -- & 7,530 & 1981 & July 4 & 55.19 & 6,150 \\
\hline 1939 & April 10 & 60.77 & 6,700 & 1982 & April 18 & 81.15 & 34,000 \\
\hline 1940 & April 21 & 66.84 & 14,600 & 1983 & April 9 & 77.29 & 25,800 \\
\hline 1941 & April 16 & 76.94 & 27,800 & 1984 & April 8 & -- & 30,200 \\
\hline 1942 & April 10 & 78.77 & 27,900 & 1985 & March 29 & -- & 16,700 \\
\hline 1943 & April 20 & 77.54 & 29,500 & 1986 & April 7 & -- & 34,200 \\
\hline 1944 & April 19 & 66.82 & 12,300 & 1987 & April 9 & - & 37,400 \\
\hline 1945 & April 4 & - & 29,400 & 1988 & April 8 & 64.89 & 15,700 \\
\hline 1946 & April 5 & -- & 24,100 & 1989 & April 23 & 84.30 & 42,700 \\
\hline 1947 & April 28 & 76.07 & 28,400 & 1990 & April 10 & 60.90 & 5,510 \\
\hline 1948 & April 27 & 87.62 & 51,800 & 1991 & July 12 & 56.15 & 5,690 \\
\hline 1949 & April 15 & 77.13 & 29,200 & 1992 & April 4 & 74.19 & 15,800 \\
\hline 1950 & May 13 & 90.89 & 95,500 & 1993 & August 16 & 79.02 & 31,900 \\
\hline 1951 & April 15 & 74.55 & 26,600 & 1994 & April 9 & 75.90 & 26,900 \\
\hline 1952 & April 24 & -- & 24,200 & & & & \\
\hline
\end{tabular}




\section{RED RIVER OF THE NORTH AT EMERSON, MB--Continued}

Annual peak discharge and corresponding gage height-Continued

$[--$, no data $]$

\begin{tabular}{|c|c|c|c|c|c|c|c|}
\hline $\begin{array}{l}\text { Water } \\
\text { year }\end{array}$ & Date & $\begin{array}{c}\text { Gage } \\
\text { height } \\
\text { (feet) }\end{array}$ & $\begin{array}{c}\text { Peak } \\
\text { discharge } \\
\left(\mathrm{ft}^{3} / \mathrm{s}\right)\end{array}$ & $\begin{array}{l}\text { Water } \\
\text { year }\end{array}$ & Date & $\begin{array}{c}\text { Gage } \\
\text { height } \\
\text { (feet) }{ }^{1}\end{array}$ & $\begin{array}{c}\text { Peak } \\
\text { discharge } \\
\left(\mathrm{ft}^{3} / \mathrm{s}\right)\end{array}$ \\
\hline \multicolumn{8}{|c|}{ Annual peak discharge, from highest to lowest, and corresponding gage height } \\
\hline 1950 & May 13 & 90.89 & 95,500 & 1915 & July 9 & 69.06 & 20,100 \\
\hline 1979 & May 1 & 91.19 & 92,700 & 1929 & April 1 & -- & 19,200 \\
\hline 1966 & April 11 & 89.15 & 66,800 & 1922 & April 14 & 69.40 & 18,900 \\
\hline 1969 & April 26 & 87.52 & 54,700 & 1932 & April 15 & 71.64 & 18,900 \\
\hline 1948 & April 27 & 87.62 & 51,800 & 1936 & April 21 & 68.16 & 18,000 \\
\hline 1978 & April 18 & 86.89 & 50,600 & 1925 & June 21 & -- & 17,500 \\
\hline 1916 & April 24 & 85.74 & 46,200 & 1964 & June 25 & 66.82 & 17,500 \\
\hline 1965 & April 26 & 85.19 & 46,200 & 1928 & April 6 & 67.91 & 16,800 \\
\hline 1974 & April 28 & 86.51 & 43,500 & 1985 & March 29 & -- & 16,700 \\
\hline 1975 & May 8 & 84.32 & 42,800 & 1992 & April 4 & 74.19 & 15,800 \\
\hline 1989 & April 23 & 84.30 & 42,700 & 1959 & April 10 & -- & 15,700 \\
\hline 1970 & April 29 & 84.67 & 39,600 & 1988 & April 8 & 64.89 & 15,700 \\
\hline 1987 & April 9 & -- & 37,400 & 1957 & July 4 & 65.37 & 15,300 \\
\hline 1986 & April 7 & -- & 34,200 & 1973 & March 27 & -- & 14,700 \\
\hline 1982 & April 18 & 81.15 & 34,000 & 1940 & April 21 & 66.84 & 14,600 \\
\hline 1956 & April 27 & 81.02 & 33,800 & 1953 & June 28 & 63.70 & 14,500 \\
\hline 1967 & April 9 & 80.79 & 33,600 & 1968 & July 24 & 64.12 & 13,900 \\
\hline 1962 & April 25 & 81.93 & 33,400 & 1963 & April 13 & 64.14 & 13,800 \\
\hline 1976 & April 6 & 79.06 & 32,900 & 1919 & July 12 & - & 13,400 \\
\hline 1993 & August 16 & 79.02 & 31,900 & 1921 & April 15 & - & 12,800 \\
\hline 1972 & April 24 & 78.16 & 30,700 & 1944 & April 19 & 66.82 & 12,300 \\
\hline 1960 & April 13 & 77.65 & 30,500 & 1954 & April 17 & -- & 11,500 \\
\hline 1984 & April 8 & -- & 30,200 & 1933 & April 9 & -- & 11,000 \\
\hline 1943 & April 20 & 77.54 & 29,500 & 1926 & April 1 & 61.02 & 8,000 \\
\hline 1945 & April 4 & -- & 29,400 & 1931 & April 10 & 59.29 & 7,940 \\
\hline 1949 & April 15 & 77.13 & 29,200 & 1958 & July 12 & 57.17 & 7,940 \\
\hline 1947 & April 28 & 76.07 & 28,400 & 1938 & May 20 & - & 7,530 \\
\hline 1942 & April 10 & 78.77 & 27,900 & 1914 & June 19 & 58.36 & 7,260 \\
\hline 1941 & April 16 & 76.94 & 27,800 & 1939 & April 10 & 60.77 & 6,700 \\
\hline 1994 & April 9 & 75.90 & 26,900 & 1924 & April 28 & 57.25 & 6,320 \\
\hline 1920 & April 16 & -- & 26,700 & 1981 & July 4 & 55.19 & 6,150 \\
\hline 1951 & April 15 & 74.55 & 26,600 & 1937 & May 7 & 56.55 & 5,840 \\
\hline 1971 & April 16 & -- & 26,600 & 1991 & July 12 & 56.15 & 5,690 \\
\hline 1923 & April 25 & 74.98 & 26,000 & 1990 & April 10 & 60.90 & 5,510 \\
\hline 1917 & April 12 & -- & 25,900 & 1935 & April 3 & 59.65 & 5,470 \\
\hline 1983 & April 9 & 77.29 & 25,800 & 1918 & April 3 & -- & 4,990 \\
\hline 1913 & April 11 & 74.52 & 25,600 & 1934 & April 13 & -- & 4,800 \\
\hline 1952 & April 24 & -- & 24,200 & 1977 & April 10 & 53.75 & 4,590 \\
\hline 1946 & April 5 & -- & 24,100 & 1961 & March 31 & 57.26 & 4,320 \\
\hline 1955 & April 10 & 72.25 & 24,000 & ${ }^{2} 1861$ & -- & 95.00 & -- \\
\hline 1980 & April 10 & 73.54 & 21,700 & ${ }^{2} 1882$ & -- & 90.00 & -- \\
\hline 1930 & April 10 & 72.51 & 20,800 & ${ }^{2} 1897$ & -- & 91.00 & -- \\
\hline 1927 & May 16 & 71.58 & 20,500 & & & & \\
\hline
\end{tabular}

${ }^{1}$ Datum is 700 feet Geodetic Survey of Canada Datum of 1929.

${ }^{2}$ Data source unknown. Not used in statistics. 


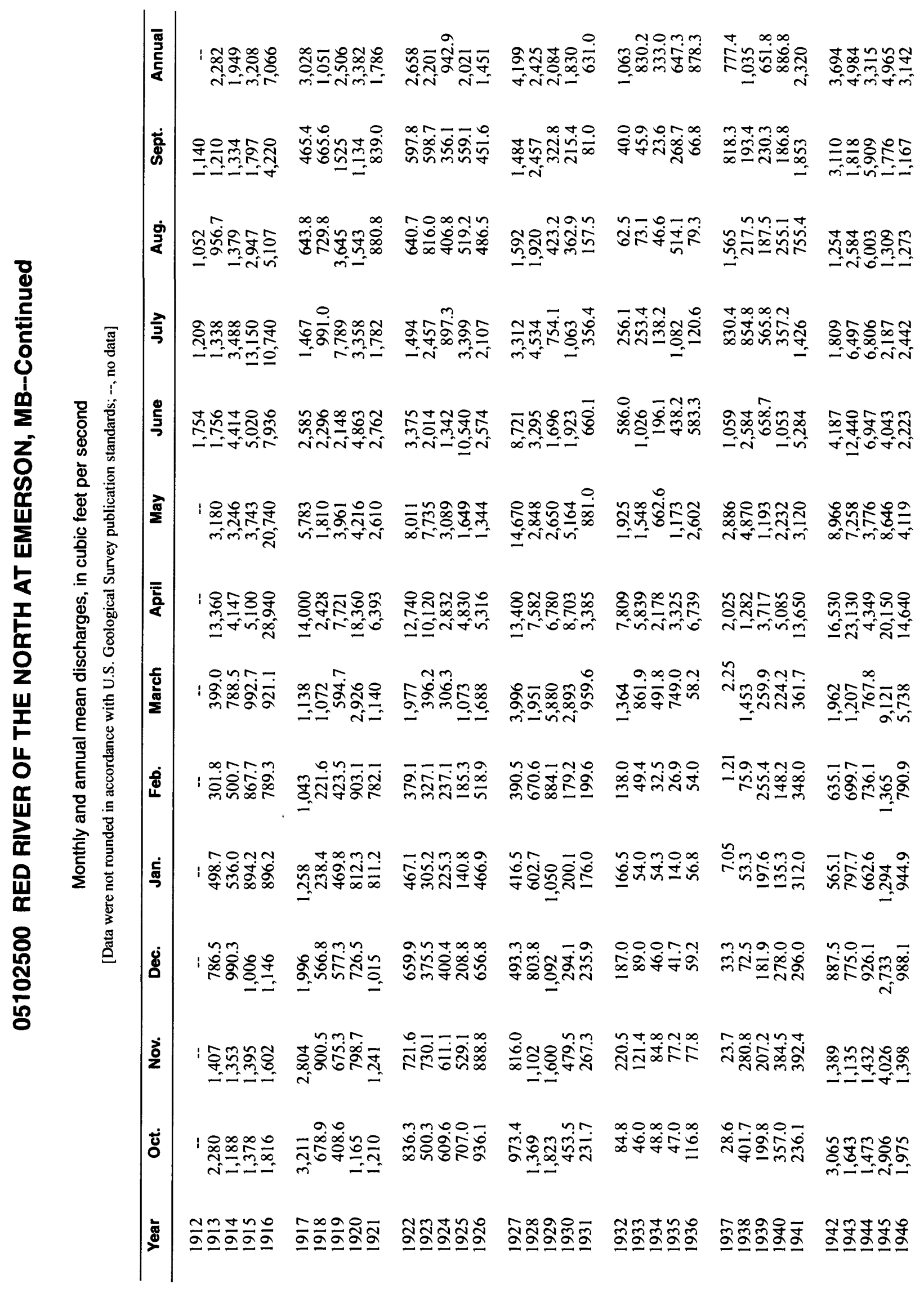




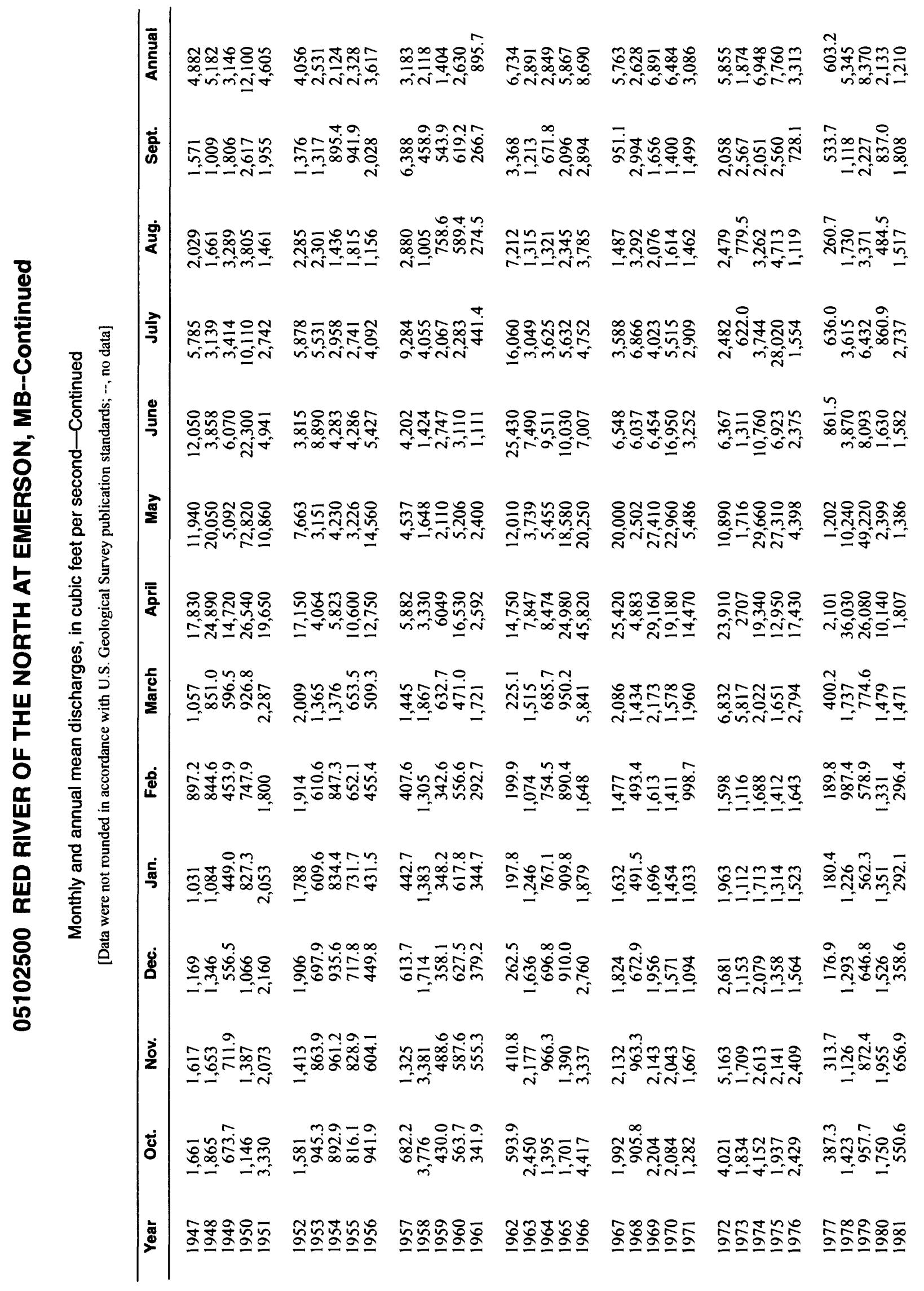




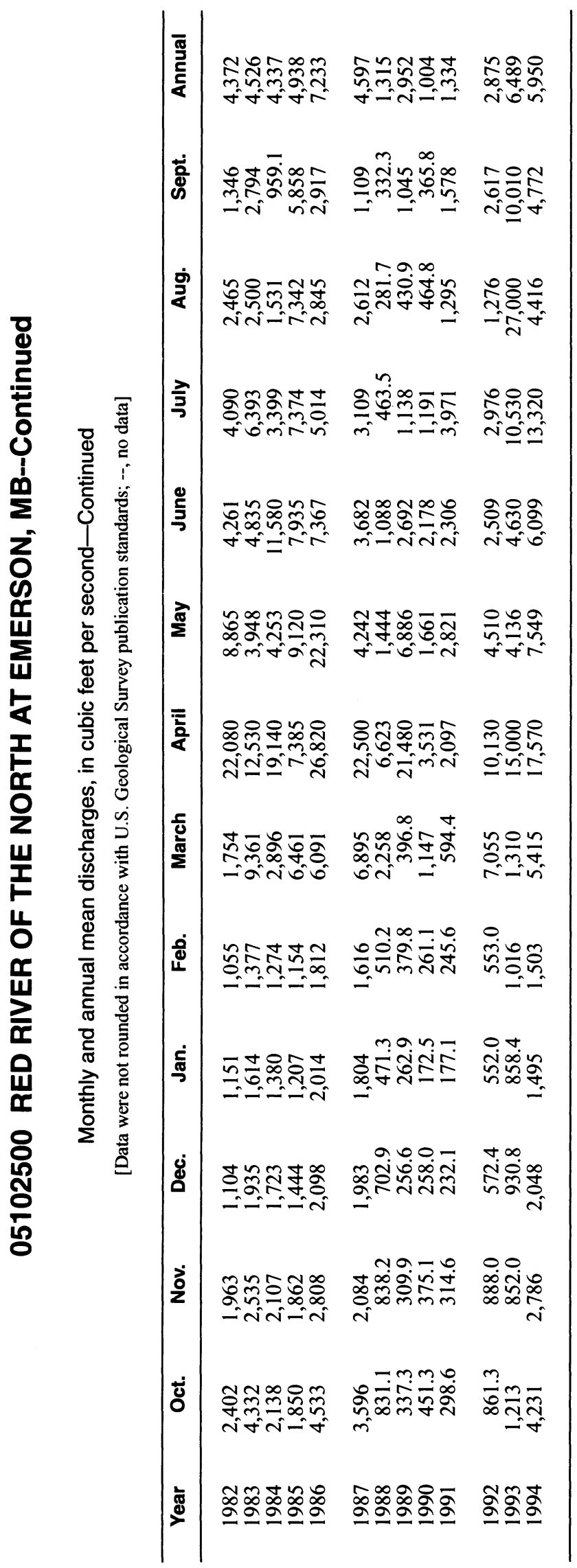

